Wright State University

CORE Scholar

2012

\title{
Measurement of Static and Dynamic Performance Characteristics of Electric Propulsion Systems
}

Aron Jon Brezina

Wright State University

Follow this and additional works at: https://corescholar.libraries.wright.edu/etd_all

Part of the Mechanical Engineering Commons

\section{Repository Citation}

Brezina, Aron Jon, "Measurement of Static and Dynamic Performance Characteristics of Electric Propulsion Systems" (2012). Browse all Theses and Dissertations. 565.

https://corescholar.libraries.wright.edu/etd_all/565

This Thesis is brought to you for free and open access by the Theses and Dissertations at CORE Scholar. It has been accepted for inclusion in Browse all Theses and Dissertations by an authorized administrator of CORE Scholar. For more information, please contact library-corescholar@wright.edu. 


\section{WRIGHT STATE UNIVERSITY}

GRADUATE SCHOOL

April 13,2012

I HEREBY RECOMMEND THAT THE THESIS PREPARED UNDER MY SUPERVISION BY Aron Jon Brezina ENTITLED Measurement of Static and Dynamic Performance Characteristics of Electric Propulsion Systems BE ACCEPTED IN PARTIAL FULFILLMENT OF THE REQUIREMENTS FOR THE DEGREE OF Master of Science in Engineering.

Committee on

Final Examination
Scott K. Thomas, Ph.D. Thesis Director

Haibo Dong, Ph.D.

Zifeng Yang, Ph.D.

Mitch Wolff, Ph.D.

Andrew Hsu, Ph.D.

Dean, Graduate School

George Huang, Ph.D. Chair Department of Mechanical and

Materials Engineering College of Engineering and Computer Science 


\begin{abstract}
Brezina, Aron Jon. M.S., Egr., Wright State University, 2012.

Measurement of Static and Dynamic Performance Characteristics of Electric Propulsion Systems.
\end{abstract}

Today's unmanned aerial vehicles are being utilized by numerous groups around the world for various missions. Most of the smaller vehicles that have been developed use commercially-off-the-shelf parts, and little information about the performance characteristics of the propulsion systems is available in the archival literature. In light of this, the aim of the present research was to determine the performance of various smallscale propellers in the 4.0 to 6.0 inch diameter range driven by an electric motor. An experimental test stand was designed and constructed in which the propeller/electric motor was mounted in a wind tunnel for both static and dynamic testing. Both static and dynamic results from the present experiment were compared to those from previous studies. For static testing, the coefficient of thrust, the coefficient of propeller power, and the overall efficiency, defined as the ratio of the propeller output power to the electrical input power, were plotted versus the propeller rotational speed. For dynamic testing, the rotational speed of the propeller was held constant at regular intervals while the freestream airspeed was increased from zero to the windmill state. The coefficient of thrust, the coefficient of power, the propeller efficiency and the overall efficiency were plotted versus the advance ratio for various rotational speeds. The thrust and torque were found to increase with rotational speed, propeller pitch and diameter, and decrease with airspeed. Using the present data and data from the archival and non-archival sources, it was found that the coefficient of thrust increases with propeller diameter for square 
propellers where $D=P$. The coefficient of thrust for a family of propellers (same manufacturer and application) was found to have a good correlation from static conditions to the windmill state. While the propeller efficiency was well correlated for this family of propellers, the goodness of fit parameter was improved by modifying the propeller efficiency with $D / P$. 


\section{TABLE OF CONTENTS}

Page

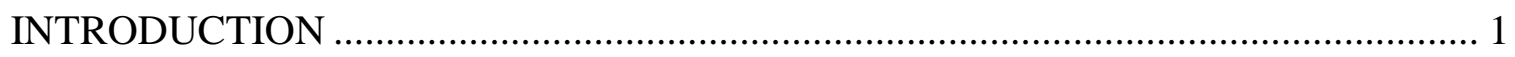

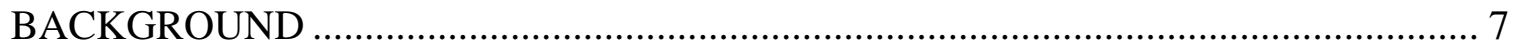

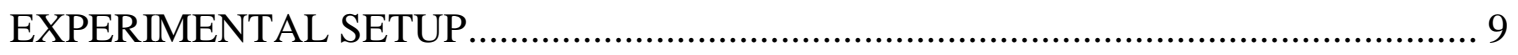

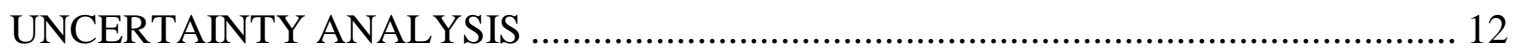

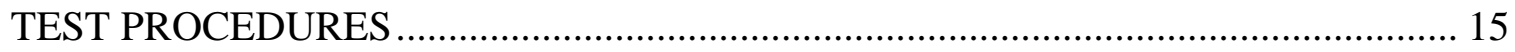

Static Test Procedure ................................................................................... 16

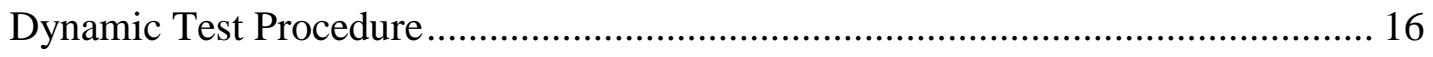

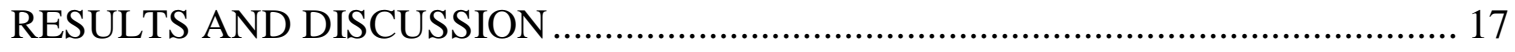

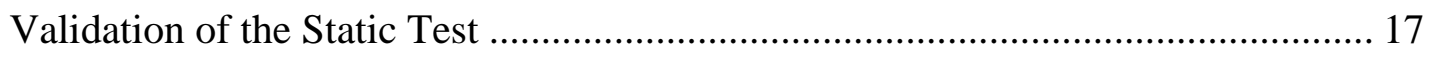

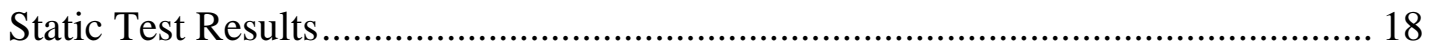

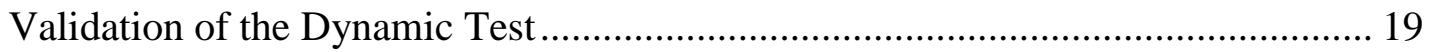

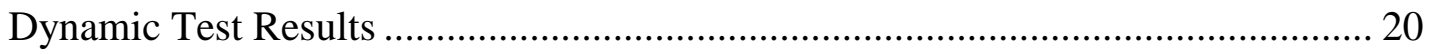

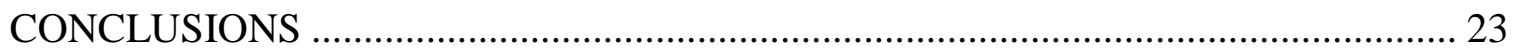

APPENDIX A: CALIBRATION PROCEDURES AND DATA SHEETS .................... 45

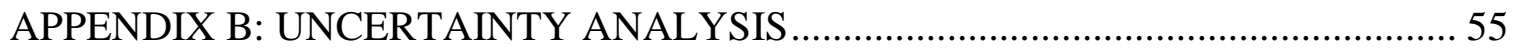

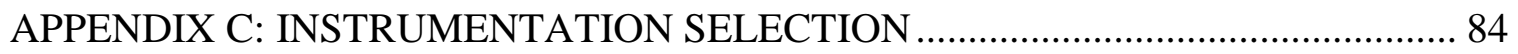

APPENDIX D: WIND TUNNEL BOUNDARY LAYER MEASUREMENT .............. 98

APPENDIX E: EXPERIMENTAL PROCEDURES ............................................ 101

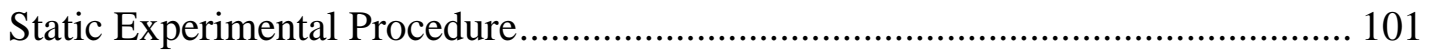

Dynamic Experimental Procedure ........................................................... 102 
APPENDIX F: SUMMARY OF STATIC TEST DATA .......................................... 104

APPENDIX G: SUMMARY OF DYNAMIC TEST DATA …................................. 175

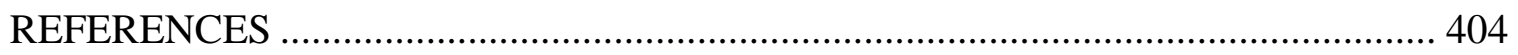




\section{LIST OF FIGURES}

Page

Figure 1: Assembly of Motor, Torque Cell and Load Cell: (a) Solid Model

Representation; (b) Photograph. .25

Figure 2: Schematic Diagram of the Experimental Setup. .26

Figure 3: Typical Static Test Results (Graupner $4.7 \times 4.7$ inch Propeller): (a) Thrust and Torque Versus Rotational Speed, (b) Coefficient of Thrust, Power and Total Efficiency Versus Rotational Speed.

Figure 4: Comparison of Three Identical Propellers (Graupner $4.7 \times 4.7$ ): (a) Static

Coefficient of Thrust, (b) Static Coefficient of Power, (c) Static Total Efficiency. .....28

Figure 5: Comparison of the Present Results to Deters and Selig (2008) (GWS $4.5 \times$

3.0 and GWS 5.0 × 4.3 Propellers): (a) Static Coefficient of Thrust, (b) Static

Coefficient of Power.

Figure 6: The Effect of Varying Propeller Pitch While Holding Diameter Constant: (a)

Static Coefficient of Thrust, (b) Static Coefficient of Power, (c) Static Total

Efficiency. .30

Figure 7: The Effect of Varying Propeller Diameter While Holding Pitch Constant: (a)

Static Coefficient of Thrust, (b) Static Coefficient of Power, (c) Static Total

Efficiency.

Figure 8: Typical Dynamic Test Results (Graupner $4.7 \times 4.7$ inch Propeller): (a)Thrust and Torque Versus Airspeed for Various Rotational Speeds, (b) Coefficient of Thrust, Power and Propeller Efficiency Versus Advance Ratio. 
Figure 9: Comparison of Present Results to Selig (2012) (APC $8.0 \times 3.8$ SF): (a)

Dynamic Coefficient of Thrust, (b) Dynamic Coefficient of Power, (c) Propeller

Efficiency.

Figure 10: Comparison of Present Results to Ol et al. (2008) (APC $6.0 \times 4.0)$ : (a)

Dynamic Coefficient of Thrust, (b) Dynamic Coefficient of Torque, (c) Propeller

Efficiency.

Figure 11: The Effect of Varying Propeller Pitch While Holding Diameter Constant:

(a) Dynamic Coefficient of Thrust, (b) Dynamic Coefficient of Power, (c) Propeller

Efficiency.

Figure 12: The Effect of Varying Propeller Diameter While Holding Pitch Constant:

(a) Dynamic Coefficient of Thrust, (b) Dynamic Coefficient of Power, (c) Propeller

Efficiency.

Figure 13: Coefficient of Thrust Versus Advance Ratio for Square Propellers $(D / P=$

1.0) with Diameter Ranging from $4.0 \leq D \leq 18$ inches.

Figure 14: Coefficient of Thrust Versus Advance Ratio for the APC Sport 400 Electric

Propellers $\left(\Delta C_{\mathrm{T}} \leq 20 \%\right)$ : (a) Original Representation of $C_{\mathrm{T}}$; (b) $C_{\mathrm{T}}$ Modified by the

Diameter to Pitch Ratio, (c) $C_{\mathrm{T}}$ and $J$ Modified by the Diameter to Pitch Ratio

Figure 14: Coefficient of Power Versus Advance Ratio for the APC Sport 400 Electric

Propellers $\left(\Delta C_{\mathrm{P}} \leq 20 \%\right)$ : (a) Original Representation of $C_{\mathrm{P}}$; (b) $C_{\mathrm{P}}$ Modified by the

Diameter to Pitch Ratio.

Figure 15: Propeller Efficiency Versus Advance Ratio for the APC Sport 400 Electric

Propellers $\left(\Delta \eta_{\mathrm{P}} \leq 20 \%\right)$ : (a) Original Representation of $\eta_{\mathrm{P}}$; (b) Advance Ratio

Modified by the Diameter to Pitch Ratio. 


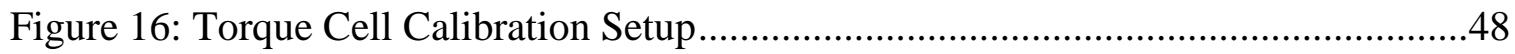

Figure 17: Torque Cell Calibration Equation ...............................................................49

Figure 18: Schematic of Load Cell Calibration ..........................................................49

Figure 19: Load Cell Calibration Setup …………………...............................................50

Figure 20: Calibration Equation of Load Cell ...............................................................50

Figure 21: Fixture Drag versus Free-Stream Velocity..................................................51

Figure 22: Vaisala PTB110 Barometer Calibration...........................................................52

Figure 23: Monarch Instruments ACT-3X Calibration ...................................................53

Figure 24: MKS 226A Differential Pressure Transducer Calibration ................................54

Figure 25: Solidworks Model of Static Test Stand .........................................................90

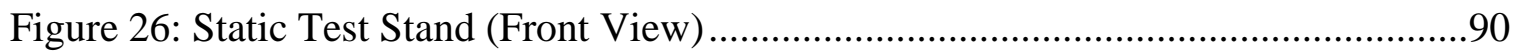

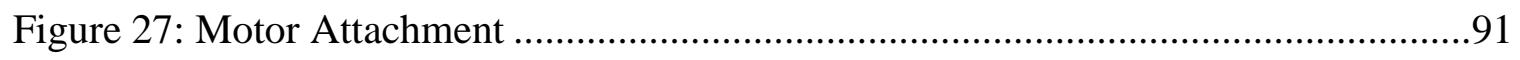

Figure 28: Top View of Test Stand............................................................................

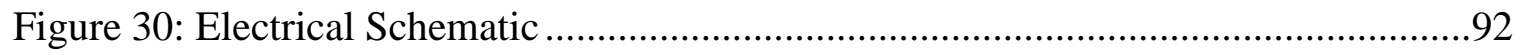

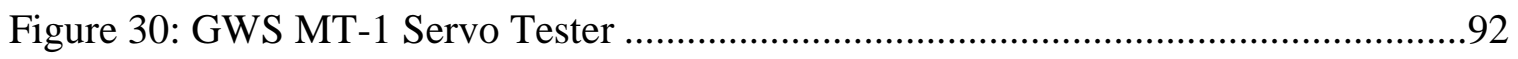

Figure 31: Full Throttle Thrust versus Time for Various Propellers .................................93

Figure 32: Full Throttle Torque versus Time for Various Propellers ................................93

Figure 33: Temperature versus Time for Various Propellers ...........................................94

Figure 34: RPM versus Time for Various Propellers ……………....................................94

Figure 35: Voltage versus Time for Various Propellers ………………..........................95

Figure 36: Current versus Time for Various Propellers..................................................95

Figure 37: Stepped Throttle Voltage versus Time for Various Propellers .........................96

Figure 38: Stepped Throttle Thrust versus RPM for Various Propellers............................96 
Figure 39: Pitot Tube Placement

Figure 40: Velocity Profile at Different Airspeeds

.100

Figure 41: APC 4.1 x 4.1 Static Test Results: (a) Coefficient of Thrust, (b) Coefficient of Power, (c) Total Efficiency. 105

Figure 42: APC 4.2 x 2.0 Static Test Results: (a) Coefficient of Thrust, (b) Coefficient of Power, (c) Total Efficiency. .107

Figure 43: APC 4.2 x 4.0 Static Test Results: (a) Coefficient of Thrust, (b) Coefficient of Power, (c) Total Efficiency. .109

Figure 44: APC 4.5 x 4.1 Static Test Results: (a) Coefficient of Thrust, (b) Coefficient of Power, (c) Total Efficiency. .111

Figure 45: APC 4.7 x 4.25 Static Test Results: (a) Coefficient of Thrust, (b) Coefficient of Power, (c) Total Efficiency.

Figure 46: APC 4.75 x 4.75 Carbon Fiber Static Test Results: (a) Coefficient of

Thrust, (b) Coefficient of Power, (c) Total Efficiency.

Figure 47: APC 4.75 x 4.75 Static Test Results: (a) Coefficient of Thrust, (b)

Coefficient of Power, (c) Total Efficiency.

Figure 48: APC 4.75 x 5.5 Static Test Results: (a) Coefficient of Thrust, (b)

Coefficient of Power, (c) Total Efficiency.

Figure 49: APC 5.1 x 4.5 Static Test Results: (a) Coefficient of Thrust, (b) Coefficient

of Power, (c) Total Efficiency.

Figure 50: APC 5.25 x 4.75 Static Test Results: (a) Coefficient of Thrust, (b)

Coefficient of Power, (c) Total Efficiency. 
Figure 51: APC 5.5 x 2.0 Static Test Results: (a) Coefficient of Thrust, (b) Coefficient

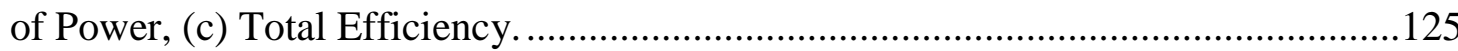

Figure 52: APC 5.5 x 4.5 Static Test Results: (a) Coefficient of Thrust, (b) Coefficient of Power, (c) Total Efficiency.

Figure 53: APC 6.0 x 2.0 Static Test Results: (a) Coefficient of Thrust, (b) Coefficient of Power, (c) Total Efficiency.

Figure 54: APC 6.0 x 4.0 Static Test Results: (a) Coefficient of Thrust, (b) Coefficient of Power, (c) Total Efficiency. 131

Figure 55: APC 8.0 x 3.8 Static Test Results: (a) Coefficient of Thrust, (b) Coefficient of Power, (c) Total Efficiency.

Figure 56: Graupner 4.0 x 3.0 Static Test Results: (a) Coefficient of Thrust, (b)

Coefficient of Power, (c) Total Efficiency.

Figure 57: Graupner 4.7 x 4.0 Static Test Results: (a) Coefficient of Thrust, (b)

Coefficient of Power, (c) Total Efficiency.

Figure 58: Graupner 4.7 x 4.7 Static Test Results (Prop 1 Test 1): (a) Coefficient of

Thrust, (b) Coefficient of Power, (c) Total Efficiency.

Figure 59: Graupner 4.7 x 4.7 Static Test Results (Prop 1 Test 2): (a) Coefficient of

Thrust, (b) Coefficient of Power, (c) Total Efficiency.

Figure 60: Graupner 4.7 x 4.7 Static Test Results (Prop 1 Test 3): (a) Coefficient of

Thrust, (b) Coefficient of Power, (c) Total Efficiency.

Figure 61: Graupner 4.7 x 4.7 Static Test Results (Prop 2 Test 1): (a) Coefficient of

Thrust, (b) Coefficient of Power, (c) Total Efficiency. 145 
Figure 62: Graupner 4.7 x 4.7 Static Test Results (Prop 2 Test 2): (a) Coefficient of

Thrust, (b) Coefficient of Power, (c) Total Efficiency.

Figure 63: Graupner 4.7 x 4.7 Static Test Results (Prop 2 Test 3): (a) Coefficient of

Thrust, (b) Coefficient of Power, (c) Total Efficiency.

Figure 64: Graupner 4.7 x 4.7 Static Test Results (Prop 3 Test 1): (a) Coefficient of

Thrust, (b) Coefficient of Power, (c) Total Efficiency.

Figure 65: Graupner 4.7 x 4.7 Static Test Results (Prop 3 Test 2): (a) Coefficient of

Thrust, (b) Coefficient of Power, (c) Total Efficiency.

Figure 66: Graupner 4.7 x 4.7 Static Test Results (Prop 3 Test 3): (a) Coefficient of

Thrust, (b) Coefficient of Power, (c) Total Efficiency.

Figure 67: Graupner 5.5 x 4.3 Static Test Results: (a) Coefficient of Thrust, (b)

Coefficient of Power, (c) Total Efficiency.

Figure 68: Graupner 5.5 x 5.5 Static Test Results: (a) Coefficient of Thrust, (b)

Coefficient of Power, (c) Total Efficiency.

Figure 69: GWS 4.0 x 2.5 Static Test Results: (a) Coefficient of Thrust, (b) Coefficient

of Power, (c) Total Efficiency. .161

Figure 70: GWS 4.0 x 4.0 Static Test Results: (a) Coefficient of Thrust, (b) Coefficient

of Power, (c) Total Efficiency.

Figure 71: GWS 4.5 x 3.0 Static Test Results: (a) Coefficient of Thrust, (b) Coefficient

of Power, (c) Total Efficiency. 165

Figure 72: GWS 5.0 x 3.0 Static Test Results: (a) Coefficient of Thrust, (b) Coefficient

of Power, (c) Total Efficiency. 
Figure 73: GWS 5.0 x 4.3 Static Test Results: (a) Coefficient of Thrust, (b) Coefficient

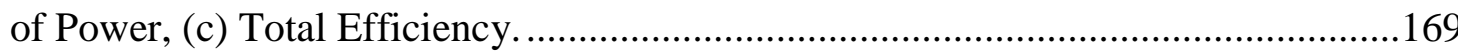

Figure 74: Summary of All Static Coefficients of Thrust.............................................172

Figure 75: Summary of All Static Coefficients of Power...............................................173

Figure 76: Summary of All Static Total Efficiencies ……….........................................174

Figure 77: APC 4.1 x 4.1 Dynamic Test Results: (a) Coefficient of Thrust, (b)

Coefficient of Power, (c) Propeller Efficiency, (d) Total Efficiency.

Figure 78: APC 4.2 x 2.0 Dynamic Test Results: (a) Coefficient of Thrust, (b)

Coefficient of Power, (c) Propeller Efficiency, (d) Total Efficiency. 186

Figure 79: APC 4.2 x 4.0 Dynamic Test Results: (a) Coefficient of Thrust, (b)

Coefficient of Power, (c) Propeller Efficiency, (d) Total Efficiency.

Figure 80: APC 4.5 x 4.1 Dynamic Test Results: (a) Coefficient of Thrust, (b)

Coefficient of Power, (c) Propeller Efficiency, (d) Total Efficiency.

Figure 81: APC 4.7 x 4.25 Dynamic Test Results: (a) Coefficient of Thrust, (b)

Coefficient of Power, (c) Propeller Efficiency, (d) Total Efficiency.

Figure 82: APC 4.75 x 4.75 Dynamic Test Results: (a) Coefficient of Thrust, (b)

Coefficient of Power, (c) Propeller Efficiency, (d) Total Efficiency.

Figure 83: APC 4.75 x 4.75 Carbon Fiber Dynamic Test Results: (a) Coefficient of

Thrust, (b) Coefficient of Power, (c) Propeller Efficiency, (d) Total Efficiency........233

Figure 84: APC 4.75 x 5.5 Dynamic Test Results: (a) Coefficient of Thrust, (b)

Coefficient of Power, (c) Propeller Efficiency, (d) Total Efficiency. .242

Figure 85: APC 5.1 x 4.5 Dynamic Test Results: (a) Coefficient of Thrust, (b)

Coefficient of Power, (c) Propeller Efficiency, (d) Total Efficiency. .251 
Figure 86: APC 5.25 x 4.75 Dynamic Test Results: (a) Coefficient of Thrust, (b)

Coefficient of Power, (c) Propeller Efficiency, (d) Total Efficiency. .260

Figure 87: APC 5.5 x 2.0 Dynamic Test Results: (a) Coefficient of Thrust, (b)

Coefficient of Power, (c) Propeller Efficiency, (d) Total Efficiency.

Figure 88: APC 5.5 x 4.5 Dynamic Test Results: (a) Coefficient of Thrust, (b)

Coefficient of Power, (c) Propeller Efficiency, (d) Total Efficiency. .278

Figure 89: APC 6.0 x 2.0 Dynamic Test Results: (a) Coefficient of Thrust, (b)

Coefficient of Power, (c) Propeller Efficiency, (d) Total Efficiency.

Figure 90: APC 6.0 x 4.0 Dynamic Test Results: (a) Coefficient of Thrust, (b)

Coefficient of Power, (c) Propeller Efficiency, (d) Total Efficiency.

Figure 91: APC 8.0 x 3.8 Dynamic Test Results: (a) Coefficient of Thrust, (b)

Coefficient of Power, (c) Propeller Efficiency, (d) Total Efficiency.

Figure 92: Graupner 4.0 x 3.0 Dynamic Test Results: (a) Coefficient of Thrust, (b)

Coefficient of Power, (c) Propeller Efficiency, (d) Total Efficiency.

Figure 93: Graupner 4.7 x 4.0 Dynamic Test Results: (a) Coefficient of Thrust, (b)

Coefficient of Power, (c) Propeller Efficiency, (d) Total Efficiency.

Figure 94: Graupner 4.7 x 4.7 Dynamic Test Results: (a) Coefficient of Thrust, (b)

Coefficient of Power, (c) Propeller Efficiency, (d) Total Efficiency.

Figure 95: Graupner 5.5 x 4.3 Dynamic Test Results: (a) Coefficient of Thrust, (b)

Coefficient of Power, (c) Propeller Efficiency, (d) Total Efficiency.

Figure 96: Graupner 5.5 x 5.5 Dynamic Test Results: (a) Coefficient of Thrust, (b)

Coefficient of Power, (c) Propeller Efficiency, (d) Total Efficiency. 
Figure 97: GWS 4.0 x 2.5 Dynamic Test Results: (a) Coefficient of Thrust, (b) Coefficient of Power, (c) Propeller Efficiency, (d) Total Efficiency. 360

Figure 98: GWS 4.0 x 4.0 Dynamic Test Results: (a) Coefficient of Thrust, (b)

Coefficient of Power, (c) Propeller Efficiency, (d) Total Efficiency.

Figure 99: GWS 4.5 x 3.0 Dynamic Test Results: (a) Coefficient of Thrust, (b)

Coefficient of Power, (c) Propeller Efficiency, (d) Total Efficiency.

Figure 100: GWS 5.0 x 3.0 Dynamic Test Results: (a) Coefficient of Thrust, (b)

Coefficient of Power, (c) Propeller Efficiency, (d) Total Efficiency.

Figure 101: GWS 5.0 x 4.3 Dynamic Test Results: (a) Coefficient of Thrust, (b)

Coefficient of Power, (c) Propeller Efficiency, (d) Total Efficiency. 


\section{LIST OF TABLES}

Page

Table 1: Uncertainties of Primary Measurement Devices and Calibration Sources........ 41

Table 2: Summary of Propeller Measurements. ................................................... 42

Table 3: Summary of Propeller Measurements, cont.................................................. 43

Table 4: Summary of Propeller Measurements, cont.................................................. 44

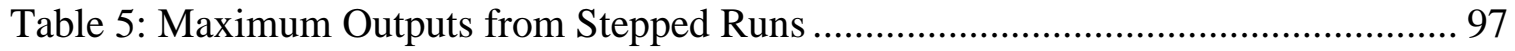

Table 6: APC 4.1 x 4.1 Static Measured Values........................................................ 106

Table 7: APC 4.1 x 4.1 Static Calculated Values ................................................... 106

Table 8: APC 4.2 x 2.0 Static Measured Values...................................................... 108

Table 9: APC 4.2 x 2.0 Static Calculated Values ................................................... 108

Table 10: APC 4.2 x 4.0 Static Measured Values....................................................... 110

Table 11: APC 4.2 x 4.0 Static Calculated Values ................................................... 110

Table 12: APC 4.5 x 4.1 Static Measured Values....................................................... 112

Table 13: APC 4.5 x 4.1 Static Calculated Values ................................................... 112

Table 14: APC 4.7 x 4.25 Static Measured Values..................................................... 114

Table 15: APC 4.7 x 4.25 Static Calculated Values ................................................... 114

Table 16: APC 4.75 x 4.75 Carbon Fiber Static Measured Values .............................. 116

Table 17: APC 4.75 x 4.75 Carbon Fiber Static Calculated Values .............................. 116

Table 18: APC 4.75 x 4.75 Static Measured Values.................................................. 118

Table 19: APC 4.75 x 4.75 Static Calculated Values ................................................... 118

Table 20: APC 4.75 x 5.5 Static Measured Values.................................................... 120

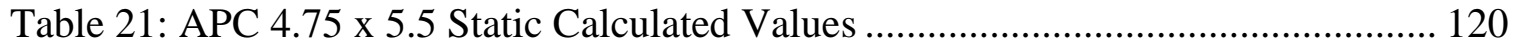

Table 22: APC 5.1 x 4.5 Static Measured Values........................................................ 122 
Table 23: APC 5.1 x 4.5 Static Calculated Values .................................................. 122

Table 24: APC 5.25 x 4.75 Static Measured Values.................................................. 124

Table 25: APC 5.25 x 4.75 Static Calculated Values ................................................ 124

Table 26: APC 5.5 x 2.0 Static Measured Values....................................................... 126

Table 27: APC 5.5 x 2.0 Static Calculated Values ................................................. 126

Table 28: APC 5.5 x 4.5 Static Measured Values..................................................... 128

Table 29: APC 5.5 x 4.5 Static Calculated Values ................................................... 128

Table 30: APC 6.0 x 2.0 Static Measured Values...................................................... 130

Table 31: APC 6.0 x 2.0 Static Calculated Values .................................................. 130

Table 32: APC 6.0 x 4.0 Static Measured Values...................................................... 132

Table 33: APC 6.0 x 4.0 Static Calculated Values ..................................................... 132

Table 34: APC 8.0 x 3.8 Static Measured Values..................................................... 134

Table 35: APC 8.0 x 3.8 Static Calculated Values .................................................. 134

Table 36: Graupner 4.0 x 3.0 Static Measured Values ............................................ 136

Table 37: Graupner 4.0 x 3.0 Static Calculated Values ............................................ 136

Table 38: Graupner 4.7 x 4.0 Static Measured Values ............................................ 138

Table 39: Graupner 4.7 x 4.0 Static Calculated Values ............................................. 138

Table 40: Graupner 4.7 x 4.7 Static Measured Values (Prop 1 Test 1) ......................... 140

Table 41: Graupner 4.7 x 4.7 Static Calculated Values (Prop 1 Test 1) ....................... 140

Table 42: Graupner 4.7 x 4.7 Static Measured Values (Prop 1 Test 2) ......................... 142

Table 43: Graupner 4.7 x 4.7 Static Calculated Values (Prop 1 Test 2) ....................... 142

Table 44: Graupner 4.7 x 4.7 Static Measured Values (Prop 1 Test 3) ........................ 144

Table 45: Graupner 4.7 x 4.7 Static Calculated Values (Prop 1 Test 3)....................... 144 
Table 46: Graupner 4.7 x 4.7 Static Measured Values (Prop 2 Test 1) ......................... 146

Table 47: Graupner 4.7 x 4.7 Static Calculated Values (Prop 2 Test 1)....................... 146

Table 48: Graupner 4.7 x 4.7 Static Measured Values (Prop 2 Test 2) ........................ 148

Table 49: Graupner 4.7 x 4.7 Static Calculated Values (Prop 2 Test 2)....................... 148

Table 50: Graupner 4.7 x 4.7 Static Measured Values (Prop 2 Test 3) ........................ 150

Table 51: Graupner 4.7 x 4.7 Static Calculated Values (Prop 2 Test 3)....................... 150

Table 52: Graupner 4.7 x 4.7 Static Measured Values (Prop 3 Test 1) ........................ 152

Table 53: Graupner 4.7 x 4.7 Static Measured Values (Prop 3 Test 1) ......................... 152

Table 54: Graupner 4.7 x 4.7 Static Measured Values (Prop 3 Test 2) ......................... 154

Table 55: Graupner 4.7 x 4.7 Static Calculated Values (Prop 3 Test 2) ........................ 154

Table 56: Graupner 4.7 x 4.7 Static Measured Values (Prop 3 Test 3) ........................ 156

Table 57: Graupner 4.7 x 4.7 Static Calculated Values (Prop 3 Test 3) ....................... 156

Table 58: Graupner 5.5 x 4.3 Static Measured Values ........................................... 158

Table 59: Graupner 5.5 x 4.3 Static Calculated Values ........................................... 158

Table 60: Graupner 5.5 x 5.5 Static Measured Values ............................................. 160

Table 61: Graupner 5.5 x 5.5 Static Calculated Values ........................................... 160

Table 62: GWS 4.0 x 2.5 Static Measured Values ................................................. 162

Table 63: GWS 4.0 x 2.5 Static Calculated Values .................................................. 162

Table 64: GWS 4.0 x 4.0 Static Measured Values .................................................. 164

Table 65: GWS 4.0 x 4.0 Static Calculated Values .................................................. 164

Table 66: GWS 4.5 x 3.0 Static Measured Values ................................................... 166

Table 67: GWS 4.5 x 3.0 Static Calculated Values ................................................ 166

Table 68: GWS 5.0 x 3.0 Static Measured Values .................................................. 168 
Table 69: GWS 5.0 x 3.0 Static Calculated Values ................................................. 168

Table 70: GWS 5.0 x 4.3 Static Measured Values .................................................. 170

Table 71: GWS 5.0 x 4.3 Static Calculated Values .................................................. 171

Table 72: APC 4.1 x 4.1 Dynamic Measured Values - 9989 RPM.............................. 177

Table 73: APC 4.1 x 4.1 Dynamic Calculated Values - 9989 RPM ........................... 178

Table 74: APC 4.1 x 4.1 Dynamic Measured Values - 14010 RPM............................ 179

Table 75: APC 4.1 x 4.1 Dynamic Calculated Values - 14010 RPM .......................... 180

Table 76: APC 4.1 x 4.1 Dynamic Measured Values - 18035 RPM............................. 181

Table 77: APC 4.1 x 4.1 Dynamic Calculated Values - 18035 RPM .......................... 182

Table 78: APC 4.1 x 4.1 Dynamic Measured Values - 22460 RPM............................ 183

Table 79: APC 4.1 x 4.1 Dynamic Calculated Values - 22460 RPM .......................... 184

Table 80: APC 4.2 x 2.0 Dynamic Measured Values - 7988 RPM............................. 186

Table 81: APC 4.2 x 2.0 Dynamic Calculated Values - 7988 RPM …....................... 187

Table 82: APC 4.2 x 2.0 Dynamic Measured Values - 11999 RPM............................. 188

Table 83: APC 4.2 x 2.0 Dynamic Calculated Values - 11999 RPM .......................... 189

Table 84: APC 4.2 x 2.0 Dynamic Measured Values - 16023 RPM............................. 190

Table 85: APC 4.2 x 2.0 Dynamic Calculated Values - 16023 RPM .......................... 191

Table 86: APC 4.2 x 2.0 Dynamic Measured Values - 20004 RPM............................. 192

Table 87: APC 4.2 x 2.0 Dynamic Calculated Values - 20004 RPM .......................... 193

Table 88: APC 4.2 x 4.0 Dynamic Measured Values - 10012 RPM............................ 195

Table 89: APC 4.2 x 4.0 Dynamic Calculated Values - 10012 RPM .......................... 196

Table 90: APC 4.2 x 4.0 Dynamic Measured Values - 14007 RPM............................. 197

Table 91: APC 4.2 x 4.0 Dynamic Calculated Values - 14007 RPM .......................... 198 
Table 92: APC 4.2 x 4.0 Dynamic Measured Values - 17997 RPM............................. 199

Table 93: APC 4.2 x 4.0 Dynamic Calculated Values - 17997 RPM .......................... 200

Table 94: APC 4.2 x 4.0 Dynamic Measured Values - 21495 RPM............................ 201

Table 95: APC 4.2 x 4.0 Dynamic Calculated Values - 21495 RPM ......................... 202

Table 96: APC 4.5 x 4.1 Dynamic Measured Values - 8033 RPM.............................. 204

Table 97: APC 4.5 x 4.1 Dynamic Calculated Values - 8033 RPM …........................ 205

Table 98: APC 4.5 x 4.1 Dynamic Measured Values - 12031 RPM............................ 206

Table 99: APC 4.5 x 4.1 Dynamic Calculated Values - 12031 RPM .......................... 207

Table 100: APC 4.5 x 4.1 Dynamic Measured Values - 16029 RPM.......................... 208

Table 101: APC 4.5 x 4.1 Dynamic Calculated Values - 16029 RPM ........................ 209

Table 102: APC 4.5 x 4.1 Dynamic Measured Values - 20027 RPM.......................... 210

Table 103: APC 4.5 x 4.1 Dynamic Calculated Values - 20027 RPM ........................ 211

Table 104: APC 4.7 x 4.25 Dynamic Measured Values - 8024 RPM.......................... 213

Table 105: APC 4.7 x 4.25 Dynamic Calculated Values - 8024 RPM ........................ 214

Table 106: APC 4.7 x 4.25 Dynamic Measured Values - 12018 RPM......................... 215

Table 107: APC 4.7 x 4.25 Dynamic Calculated Values - 12018 RPM ...................... 216

Table 108: APC 4.7 x 4.25 Dynamic Measured Values - 16009 RPM........................ 217

Table 109: APC 4.7 x 4.25 Dynamic Calculated Values - 16009 RPM ....................... 218

Table 110: APC 4.7 x 4.25 Dynamic Measured Values - 19973 RPM........................ 219

Table 111: APC 4.7 x 4.25 Dynamic Calculated Values - 19973 RPM ...................... 220

Table 112: APC 4.75 x 4.75 Dynamic Measured Values - 10010 RPM....................... 222

Table 113: APC 4.75 x 4.75 Dynamic Calculated Values - 10010 RPM ..................... 223

Table 114: APC 4.75 x 4.75 Dynamic Measured Values - 12009 RPM........................ 224 
Table 115: APC 4.75 x 4.75 Dynamic Calculated Values - 12009 RPM .................... 225

Table 116: APC 4.75 x 4.75 Dynamic Measured Values - 14009 RPM....................... 226

Table 117: APC 4.75 x 4.75 Dynamic Calculated Values - 14009 RPM ..................... 227

Table 118: APC 4.75 x 4.75 Dynamic Measured Values - 15997 RPM....................... 228

Table 119: APC 4.75 x 4.75 Dynamic Calculated Values - 15997 RPM .................... 229

Table 120: APC 4.75 x 4.75 Dynamic Measured Values - 18016 RPM....................... 230

Table 121: APC 4.75 x 4.75 Dynamic Calculated Values - 18016 RPM .................... 231

Table 122: APC 4.75 x 4.75 Carbon Fiber Dynamic Measured Values - 10038 RPM . 233

Table 123: APC 4.75 x 4.75 Carbon Fiber Dynamic Calculated Values - 10038 RPM 234

Table 124: APC 4.75 x 4.75 Carbon Fiber Dynamic Measured Values - 14015 RPM . 235

Table 125: APC 4.75 x 4.75 Carbon Fiber Dynamic Calculated Values - 14015 RPM 236

Table 126: APC 4.75 x 4.75 Carbon Fiber Dynamic Measured Values - 18006 RPM . 237

Table 127: APC 4.75 x 4.75 Carbon Fiber Dynamic Calculated Values - 18006 RPM 238

Table 128: APC 4.75 x 4.75 Carbon Fiber Dynamic Measured Values - 22071 RPM . 239

Table 129: APC 4.75 x 4.75 Carbon Fiber Dynamic Calculated Values - 22071 RPM 240

Table 130: APC 4.75 x 5.5 Dynamic Measured Values - 9993 RPM.......................... 242

Table 131: APC 4.75 x 5.5 Dynamic Calculated Values - 9993 RPM ........................ 243

Table 132: APC 4.75 x 5.5 Dynamic Measured Values - 12014 RPM......................... 244

Table 133: APC 4.75 x 5.5 Dynamic Calculated Values - 12014 RPM ...................... 245

Table 134: APC 4.75 x 5.5 Dynamic Measured Values - 14004 RPM......................... 246

Table 135: APC 4.75 x 5.5 Dynamic Calculated Values - 14004 RPM ....................... 247

Table 136: APC 4.75 x 5.5 Dynamic Measured Values - 15990 RPM........................ 248

Table 137: APC 4.75 x 5.5 Dynamic Calculated Values - 15990 RPM ...................... 249 
Table 138: APC 5.1 x 4.5 Dynamic Measured Values - 10000 RPM.......................... 251

Table 139: APC 5.1 x 4.5 Dynamic Calculated Values - 10000 RPM ........................ 252

Table 140: APC 5.1 x 4.5 Dynamic Measured Values - 11995 RPM........................... 253

Table 141: APC 5.1 x 4.5 Dynamic Calculated Values - 11995 RPM ......................... 254

Table 142: APC 5.1 x 4.5 Dynamic Measured Values - 14006 RPM.......................... 255

Table 143: APC 5.1 x 4.5 Dynamic Calculated Values - 14006 RPM ........................ 256

Table 144: APC 5.1 x 4.5 Dynamic Measured Values - 15974 RPM.......................... 257

Table 145: APC 5.1 x 4.5 Dynamic Calculated Values - 15974 RPM ......................... 258

Table 146: APC 5.25 x 4.75 Dynamic Measured Values - 9999 RPM......................... 260

Table 147: APC 5.25 x 4.75 Dynamic Calculated Values - 9999 RPM ....................... 261

Table 148: APC 5.25 x 4.75 Dynamic Measured Values - 12000 RPM....................... 262

Table 149: APC 5.25 x 4.75 Dynamic Calculated Values - 12000 RPM ..................... 263

Table 150: APC 5.25 x 4.75 Dynamic Measured Values - 14017 RPM........................ 264

Table 151: APC 5.25 x 4.75 Dynamic Calculated Values - 14017 RPM .................... 265

Table 152: APC 5.25 x 4.75 Dynamic Measured Values - 15988 RPM....................... 266

Table 153: APC 5.25 x 4.75 Dynamic Calculated Values - 15988 RPM .................... 267

Table 154: APC 5.5 x 2.0 Dynamic Measured Values - 12034 RPM.......................... 269

Table 155: APC 5.5 x 2.0 Dynamic Calculated Values - 12034 RPM ........................ 270

Table 156: APC 5.5 x 2.0 Dynamic Measured Values - 14007 RPM.......................... 271

Table 157: APC 5.5 x 2.0 Dynamic Calculated Values - 14007 RPM ........................ 272

Table 158: APC 5.5 x 2.0 Dynamic Measured Values - 16005 RPM......................... 273

Table 159: APC 5.5 x 2.0 Dynamic Calculated Values - 16005 RPM ........................ 274

Table 160: APC 5.5 x 2.0 Dynamic Measured Values - 18016 RPM.......................... 275 
Table 161: APC 5.5 x 2.0 Dynamic Calculated Values - 18016 RPM ........................ 276

Table 162: APC 5.5 x 4.5 Dynamic Measured Values - 10014 RPM.......................... 278

Table 163: APC 5.5 x 4.5 Dynamic Calculated Values - 10014 RPM ........................ 279

Table 164: APC 5.5 x 4.5 Dynamic Measured Values - 12007 RPM........................... 280

Table 165: APC 5.5 x 4.5 Dynamic Calculated Values - 12007 RPM ........................ 281

Table 166: APC 5.5 x 4.5 Dynamic Measured Values - 13992 RPM........................... 282

Table 167: APC 5.5 x 4.5 Dynamic Calculated Values - 13992 RPM ........................ 283

Table 168: APC 5.5 x 4.5 Dynamic Measured Values - 15993 RPM........................... 284

Table 169: APC 5.5 x 4.5 Dynamic Calculated Values - 15993 RPM ........................ 285

Table 170: APC 6.0 x 2.0 Dynamic Measured Values - 11976 RPM.......................... 287

Table 171: APC 6.0 x 2.0 Dynamic Calculated Values - 11976 RPM ......................... 288

Table 172: APC 6.0 x 2.0 Dynamic Measured Values - 13976 RPM.......................... 289

Table 173: APC 6.0 x 2.0 Dynamic Calculated Values - 13976 RPM ........................ 290

Table 174: APC 6.0 x 2.0 Dynamic Measured Values - 16009 RPM.......................... 291

Table 175: APC 6.0 x 2.0 Dynamic Calculated Values - 16009 RPM ........................ 292

Table 176: APC 6.0 x 2.0 Dynamic Measured Values - 18011 RPM.......................... 293

Table 177: APC 6.0 x 2.0 Dynamic Calculated Values - 18011 RPM ......................... 294

Table 178: APC 6.0 x 4.0 Dynamic Measured Values - 8020 RPM Sweep Up ........... 296

Table 179: APC 6.0 x 4.0 Dynamic Calculated Values - 8020 RPM Sweep Up.......... 297

Table 180: APC 6.0 x 4.0 Dynamic Measured Values - 7992 RPM Sweep Down ....... 298

Table 181: APC 6.0 x 4.0 Dynamic Calculated Values - 7992 RPM Sweep Down..... 299

Table 182: APC 6.0 x 4.0 Dynamic Measured Values - 9995 RPM............................ 300

Table 183: APC 6.0 x 4.0 Dynamic Calculated Values - 9995 RPM ......................... 301 
Table 184: APC 6.0 x 4.0 Dynamic Measured Values - 12022 RPM.......................... 302

Table 185: APC 6.0 x 4.0 Dynamic Calculated Values - 12022 RPM ........................ 303

Table 186: APC 6.0 x 4.0 Dynamic Measured Values - 13992 RPM........................... 304

Table 187: APC 6.0 x 4.0 Dynamic Calculated Values - 13992 RPM ........................ 305

Table 188: APC 6.0 x 4.0 Dynamic Measured Values - 16002 RPM.......................... 306

Table 189: APC 6.0 x 4.0 Dynamic Calculated Values - 16002 RPM ........................ 307

Table 190: APC 8.0 x 3.8 Dynamic Measured Values - 4033 RPM............................. 309

Table 191: APC 8.0 x 3.8 Dynamic Calculated Values - 4033 RPM ........................... 310

Table 192: APC 8.0 x 3.8 Dynamic Measured Values - 6897 RPM............................. 310

Table 193: APC 8.0 x 3.8 Dynamic Calculated Values - 6897 RPM ......................... 311

Table 194: Graupner 4.0 x 3.0 Dynamic Measured Values - 10016 RPM ................... 313

Table 195: Graupner 4.0 x 3.0 Dynamic Calculated Values - 10016 RPM .................. 314

Table 196: Graupner 4.0 x 3.0 Dynamic Measured Values - 14019 RPM ................... 315

Table 197: Graupner 4.0 x 3.0 Dynamic Calculated Values - 14019 RPM.................. 316

Table 198: Graupner 4.0 x 3.0 Dynamic Measured Values -18026 RPM .................... 317

Table 199: Graupner 4.0 x 3.0 Dynamic Calculated Values - 18026 RPM.................. 318

Table 200: Graupner 4.0 x 3.0 Dynamic Measured Values - 22017 RPM ................... 319

Table 201: Graupner 4.0 x 3.0 Dynamic Calculated Values - 22017 RPM.................. 320

Table 202: Graupner 4.7 x 4.0 Dynamic Measured Values - 7981 RPM .................... 322

Table 203: Graupner 4.7 x 4.0 Dynamic Calculated Values - 7981 RPM .................... 323

Table 204: Graupner 4.7 x 4.0 Dynamic Measured Values - 12006 RPM ................... 324

Table 205: Graupner 4.7 x 4.0 Dynamic Calculated Values - 12006 RPM .................. 325

Table 206: Graupner 4.7 x 4.0 Dynamic Measured Values - 15997 RPM ................... 326 
Table 207: Graupner 4.7 x 4.0 Dynamic Calculated Values - 15997 RPM.................. 327

Table 208: Graupner 4.7 x 4.0 Dynamic Measured Values - 20027 RPM ................... 328

Table 209: Graupner 4.7 x 4.0 Dynamic Calculated Values - 20027 RPM.................. 329

Table 210: Graupner 4.7 x 4.7 Dynamic Measured Values - 10023 RPM ................... 331

Table 211: Graupner 4.7 x 4.7 Dynamic Calculated Values - 10023 RPM.................. 332

Table 212: Graupner 4.7 x 4.7 Dynamic Measured Values - 14006 RPM ................... 333

Table 213: Graupner 4.7 x 4.7 Dynamic Calculated Values - 14006 RPM.................. 334

Table 214: Graupner 4.7 x 4.7 Dynamic Measured Values - 18002 RPM ................... 335

Table 215: Graupner 4.7 x 4.7 Dynamic Calculated Values - 18002 RPM.................. 336

Table 216: Graupner 4.7 x 4.7 Dynamic Measured Values - 20026 RPM ................... 337

Table 217: Graupner 4.7 x 4.7 Dynamic Calculated Values - 20026 RPM.................. 338

Table 218: Graupner 5.5 x 4.3 Dynamic Measured Values - 11993 RPM ................... 340

Table 219: Graupner 5.5 x 4.3 Dynamic Calculated Values - 11993 RPM.................. 341

Table 220: Graupner 5.5 x 4.3 Dynamic Measured Values - 13998 RPM ................... 342

Table 221: Graupner 5.5 x 4.3 Dynamic Calculated Values - 13998 RPM.................. 343

Table 222: Graupner 5.5 x 4.3 Dynamic Measured Values - 15988 RPM ................... 344

Table 223: Graupner 5.5 x 4.3 Dynamic Calculated Values - 15988 RPM.................. 345

Table 224: Graupner 5.5 x 4.3 Dynamic Measured Values - 17953 RPM ................... 346

Table 225: Graupner 5.5 x 4.3 Dynamic Calculated Values - 17953 RPM.................. 347

Table 226: Graupner 5.5 x 5.5 Dynamic Measured Values - 8001 RPM ..................... 349

Table 227: Graupner 5.5 x 5.5 Dynamic Calculated Values - 8001 RPM.................... 350

Table 228: Graupner 5.5 x 5.5 Dynamic Measured Values - 9999 RPM .................... 351

Table 229: Graupner 5.5 x 5.5 Dynamic Calculated Values - 9999 RPM.................... 352 
Table 230: Graupner 5.5 x 5.5 Dynamic Measured Values - 12010 RPM ................... 353

Table 231: Graupner 5.5 x 5.5 Dynamic Calculated Values - 12010 RPM.................. 354

Table 232: Graupner 5.5 x 5.5 Dynamic Measured Values - 13992 RPM ................... 355

Table 233: Graupner 5.5 x 5.5 Dynamic Calculated Values - 13992 RPM................... 356

Table 234: Graupner 5.5 x 5.5 Dynamic Measured Values - 16015 RPM ................... 357

Table 235: Graupner 5.5 x 5.5 Dynamic Calculated Values - 16015 RPM.................. 358

Table 236: GWS 4.0 x 2.5 Dynamic Measured Values - 8133 RPM ......................... 360

Table 237: GWS 4.0 x 2.5 Dynamic Calculated Values - 8133 RPM ......................... 361

Table 238: GWS 4.0 x 2.5 Dynamic Measured Values - 12040 RPM ........................ 362

Table 239: GWS 4.0 x 2.5 Dynamic Calculated Values - 12040 RPM ....................... 363

Table 240: GWS 4.0 x 2.5 Dynamic Measured Values - 16020 RPM ......................... 364

Table 241: GWS 4.0 x 2.5 Dynamic Calculated Values - 16020 RPM ....................... 365

Table 242: GWS 4.0 x 2.5 Dynamic Measured Values - 20020 RPM ........................ 366

Table 243: GWS 4.0 x 2.5 Dynamic Calculated Values - 20020 RPM ....................... 367

Table 244: GWS 4.0 x 4.0 Dynamic Measured Values - 5995 RPM .......................... 369

Table 245: GWS 4.0 x 4.0 Dynamic Calculated Values - 5995 RPM ........................ 370

Table 246: GWS 4.0 x 4.0 Dynamic Measured Values - 9988 RPM ......................... 371

Table 247: GWS 4.0 x 4.0 Dynamic Calculated Values - 9988 RPM ......................... 372

Table 248: GWS 4.0 x 4.0 Dynamic Measured Values - 14014 RPM ....................... 373

Table 249: GWS 4.0 x 4.0 Dynamic Calculated Values - 14014 RPM ........................ 374

Table 250: GWS 4.0 x 4.0 Dynamic Measured Values - 18018 RPM ........................ 375

Table 251: GWS 4.0 x 4.0 Dynamic Calculated Values - 18018 RPM ....................... 376

Table 252: GWS 4.5 x 3.0 Dynamic Measured Values - 9993 RPM …...................... 378 
Table 253: GWS 4.5 x 3.0 Dynamic Calculated Values - 9993 RPM ......................... 379

Table 254: GWS 4.5 x 3.0 Dynamic Measured Values - 12002 RPM ........................ 380

Table 255: GWS 4.5 x 3.0 Dynamic Calculated Values - 12002 RPM ........................ 381

Table 256: GWS 4.5 x 3.0 Dynamic Measured Values - 14011 RPM ........................ 382

Table 257: GWS 4.5 x 3.0 Dynamic Calculated Values - 14011 RPM ....................... 383

Table 258: GWS 4.5 x 3.0 Dynamic Measured Values - 15996 RPM ........................ 384

Table 259: GWS 4.5 x 3.0 Dynamic Calculated Values - 15996 RPM …................... 385

Table 260: GWS 5.0 x 3.0 Dynamic Measured Values - 8019 RPM .......................... 387

Table 261: GWS 5.0 x 3.0 Dynamic Calculated Values - 8019 RPM ......................... 388

Table 262: GWS 5.0 x 3.0 Dynamic Measured Values - 12024 RPM ........................ 389

Table 263: GWS 5.0 x 3.0 Dynamic Calculated Values - 12024 RPM ........................ 390

Table 264: GWS 5.0 x 3.0 Dynamic Measured Values - 15998 RPM ........................ 391

Table 265: GWS 5.0 x 3.0 Dynamic Calculated Values - 15998 RPM ....................... 392

Table 266: GWS 5.0 x 3.0 Dynamic Measured Values - 20000 RPM ........................ 393

Table 267: GWS 5.0 x 3.0 Dynamic Calculated Values - 20000 RPM ........................ 394

Table 268: GWS 5.0 x 4.3 Dynamic Measured Values - 8021 RPM ......................... 396

Table 269: GWS 5.0 x 4.3 Dynamic Calculated Values - 8021 RPM ......................... 397

Table 270: GWS 5.0 x 4.3 Dynamic Measured Values - 11986 RPM ........................ 398

Table 271: GWS 5.0 x 4.3 Dynamic Calculated Values - 11986 RPM ....................... 399

Table 272: GWS 5.0 x 4.3 Dynamic Measured Values - 16006 RPM ........................ 400

Table 273: GWS 5.0 x 4.3 Dynamic Calculated Values - 16006 RPM ....................... 401

Table 274: GWS 5.0 x 4.3 Dynamic Measured Values - 19971 RPM ........................ 402

Table 275: GWS 5.0 x 4.3 Dynamic Calculated Values - 19971 RPM ....................... 403 


\section{NOMENCLATURE}

\begin{tabular}{|c|c|}
\hline$A_{\mathrm{P}}$ & propeller disk area, $\mathrm{m}^{2}$ \\
\hline$A_{\mathrm{WT}}$ & wind tunnel test section area, $\mathrm{m}^{2}$ \\
\hline$C_{0.75}$ & propeller chord length at the $75 \%$ radius, $\mathrm{m}$ \\
\hline$C_{\mathrm{P}}$ & coefficient of propeller power \\
\hline$C_{\mathrm{Q}}$ & coefficient of torque \\
\hline$C_{\mathrm{T}}$ & coefficient of thrust \\
\hline$D$ & propeller diameter, $\mathrm{m}$ \\
\hline$F_{D}$ & fixture drag, $\mathrm{N}$ \\
\hline$H$ & height of Pitot tube from bottom of wind tunnel, $m$ \\
\hline$I$ & electrical motor current, Amperes \\
\hline$J$ & advance ratio \\
\hline$K_{\mathrm{v}}$ & motor velocity constant, RPM/Volt \\
\hline$n$ & propeller rotational speed, rev/s \\
\hline$P$ & propeller pitch, m \\
\hline$P_{\mathrm{atm}}$ & atmospheric pressure, $\mathrm{Pa}$ \\
\hline$P_{\text {diff }}$ & Pitot tube differential pressure, $\mathrm{Pa}$ \\
\hline$P_{\mathrm{e}}$ & electrical input power, $\mathrm{W}$ \\
\hline$P_{\mathrm{P}}$ & propeller output power, W \\
\hline$Q$ & torque, $\mathrm{N}-\mathrm{m}$ \\
\hline$R_{0.75}$ & propeller $75 \%$ radius, $m$ \\
\hline$R$ & particular gas constant, $\mathrm{J} /(\mathrm{kg}-\mathrm{K})$ \\
\hline $\operatorname{Re}_{0.75}$ & Reynolds number at the $75 \%$ radius of the propeller \\
\hline$T$ & measured thrust, $\mathrm{N}$ \\
\hline$T^{\prime}$ & corrected thrust, $\mathrm{N}$ \\
\hline$T_{\mathrm{atm}}$ & atmospheric temperature, $\mathrm{K}$ \\
\hline$T_{\text {ref }}$ & reference temperature, $\mathrm{K}$ \\
\hline$V$ & electric motor voltage, Volts \\
\hline$V_{\infty}$ & free-stream velocity, $\mathrm{m} / \mathrm{s}$ \\
\hline$V_{\infty}^{\prime}$ & corrected free-stream velocity, $\mathrm{m} / \mathrm{s}$ \\
\hline
\end{tabular}




$\begin{array}{ll}V_{\mathrm{P}} & \text { propeller velocity, } \mathrm{m} / \mathrm{s} \\ V_{\mathrm{T}} & \text { total velocity, } \mathrm{m} / \mathrm{s} \\ W & \text { wind tunnel test section width and height, } \mathrm{m} \\ \Delta & \text { uncertainty } \\ \eta_{\mathrm{P}} & \text { propeller efficiency } \\ \eta_{\mathrm{T}} & \text { overall propulsion system efficiency } \\ \mu & \text { absolute viscosity, } \mathrm{kg} /(\mathrm{m}-\mathrm{s}) \\ \mu_{\mathrm{ref}} & \text { reference absolute viscosity evaluated at } T_{\mathrm{ref}}, \mathrm{kg} /(\mathrm{m}-\mathrm{s}) \\ \rho & \text { density, } \mathrm{kg} / \mathrm{m}^{3} \\ \tau_{4} & \text { Glauert correction variable }\end{array}$




\section{ACKNOWLEDGEMENTS}

First off I want to thank my thesis advisor, Dr. Scott Thomas for all of his time and financial support over the last two years of this project. I am very grateful for the precedence that the project and I received in his life. Even with Aero Design, multiple senior design groups, and teaching duties, there was always time to sit down and talk about what was going on. Whenever I needed a course correction, literally and figuratively, he was always there for me. He has had a huge influence on me as an Engineer and as a person and that is something I can take with me for the rest of my life.

I would also like to thank Mike Rottmayer from Wright Patterson for his support of the project and the generous use of their lab equipment. Without their equipment the project would not have been successful. The support I received from Greg Wilt and the Department of Mechanical and Materials Engineering was also very helpful. From moving things in lab to finding the tools I needed, Greg helped me tremendously in setting up my experiment in the wind tunnel.

Last but not least I would like to thank my friends and family for their help and positive support. Without their constant encouragement none of this would have been possible. More specifically I would like to thank my brother Dan for coming up from school and helping me collect data and keep me company during the long tests. I would also like to thank my Dad for his help designing the fixtures for the experiment and my Mom for being there whenever I needed someone to talk to about anything and everything. Lastly I thank Lisa for her understanding and for putting up with my long hours and time spent away from her. We have both been through a lot in the last year and I don't think we would have made it without each other's support. 


\section{INTRODUCTION}

Interest in the performance of small propellers operating at low Reynolds numbers has grown recently. The aerospace industry has developed numerous unmanned aerial vehicles (UAVs) and has kept most of the data about the propulsion systems proprietary. Very little information is available in the archival literature about the performance characteristics of these motor and propeller combinations. The present research and others like it have aimed to gather and compare information about these small propulsion systems so that proper motor and propeller combinations can be selected for a given mission profile. Several papers were reviewed that relate directly to the present work and provide direction for the research.

Brandt and Selig (2011) experimentally determined efficiency as well as coefficients of thrust and power for low Reynolds number propellers. The parametric ranges were as follows: Propeller diameter $9 \leq D \leq 11$ inches, propeller rotational speed $1500 \leq n \leq 7500 \mathrm{RPM}$, and the incoming air velocity $V_{\infty}^{\prime}$ ranged from zero (static) to the windmill state of each propeller, i.e., that point at which the propeller generates zero thrust. A test stand was built inside the UIUC wind tunnel to measure thrust, torque, and propeller rotational speed. Freestream air velocity was measured using a Pitot tube and one of two differential pressure transducers depending on the airspeed range. Velocity corrections were applied to account for the change in upstream airspeed at the Pitot tube created by the propeller as well as the pressure change created by the fairing and the constriction of the propeller slipstream caused by the walls. In total, 79 propellers from four different manufacturers were tested to find the coefficient of thrust, the coefficient of 
power and the propeller efficiency, all of which were plotted against advance ratio. The designs of the propellers ranged from those for electric motors to those used for fuelpowered engines. For each test, the rotational speed of each propeller was fixed while the freestream airspeed was varied. Four different values of propeller rotational speed $(n=$ $3000,4000,5000$, and 6000 RPM) were tested for each of the propellers. The results show that the propeller efficiency increases with the propeller speed. This is primarily due to the increase in Reynolds number as the propeller spins faster. Overall, the propeller efficiency ranged from $28 \leq \eta_{\mathrm{P}} \leq 65 \%$. The propellers were also tested statically, but the data is only available in the UIUC propeller database (Selig, 2012).

Gamble (2009) designed an intricate LabVIEW program to automatically collect data and generate propeller performance plots. A dynamometer was constructed using beam-type load cells to measure thrust and torque. The development of the LabVIEW program was detailed as well as a procedure for carrying out the experiment. Propellers were tested for repeatability by performing identical experiments over several days with two identical propellers. The results primarily focus on the effect of the Reynolds number on thrust and power coefficients and efficiency versus advance ratio. Thrust versus velocity was compared for propellers with constant diameter and varying pitch. Lastly, advance ratio was modified by replacing diameter with pitch in the equation for advance ratio. The optimal advance ratio is shown using this technique. This allows for the optimal pitch of a model propeller to be selected to achieve maximum efficiency. The diameter can then be chosen from plots of thrust versus velocity to produce the required thrust for the airframe. 
Deters and Selig (2008) performed static tests on smaller propellers ranging from $2.5 \leq D \leq 5$ inches in diameter. Static coefficients of thrust and power as well as the figure of merit $\left(\mathrm{FOM}=C_{\mathrm{T}}^{3 / 2} / \sqrt{2} C_{\mathrm{P}}\right.$, typically used to measure the efficiency of helicopters) using modified coefficients of thrust and power that use disk area and tip speed were determined experimentally using a test stand specifically designed to test this size range of propellers. It was noted that the figure of merit should only be used for comparison when the disk loading is the same. The test stand utilized a $0.3 \mathrm{~kg}$ load cell and a $25 \mathrm{oz}$-in torque transducer to measure thrust and torque, respectively. Propeller rotational speeds ranging from $2500 \leq n \leq 27,000$ RPM were measured using an infrared detector. The test stand is not shown photographically, but a basic schematic is given indicating the locations of the components and a fairing surrounding the load cell and torque transducer. Calibrations of the components were performed and data was collected using a data acquisition board. The geometry of each propeller was found using PropellerScanner software (Hepperle, 2003) to find the chord and twist distribution. This was used to calculate the Reynolds number at the $75 \%$ chord location. Results show that over the rotational speed range tested, the figure of merit remained fairly constant throughout the test. The results also show that a larger diameter propeller is more efficient than a smaller one, and a propeller with a lower pitch is more efficient than one with a higher pitch.

Ol et al. (2008) took a more analytical approach to studying small propellers operating at low Reynolds numbers. Iterative methods were used to calculate the coefficient of thrust, the coefficient of torque, and the propeller efficiency using propeller momentum theory and blade-element methods. Propellers were discretized by cutting and 
tracing sections as well as digital scans. Leading and trailing edges were fitted to the UIUC propeller library so that the resulting analysis in XFOIL would successfully converge. The iterative process for thrust was dependent on the various Reynolds numbers across the propeller blade at a given rotational speed. Two separate experimental setups were constructed to compare the numerical results. Propellers in the $6 \leq D \leq 12$ inch range were tested in the Langley Research Center Basic Aerodynamics Research Tunnel (BART) and larger propellers in the $14 \leq D \leq 20$ inch range were tested in the AFRL Vertical Wind Tunnel (VWT). Two different efficiencies were studied: The first was the propulsive efficiency where propeller speed was held constant and the forces and moments were shown versus rotational speed and airspeed. Static tests were performed with the wind tunnel sides open to alleviate the induced airflow velocity inside the wind tunnel. Blockage corrections were applied to BART tests but not to VWT tests, since the tunnel diameter of the VWT was greater than five times the diameter of the propellers tested. Drag on the test stand was corrected by sweeping tunnel velocity and generating curve fits that were used to adjust the actual data. A large sensitivity to twist distribution was observed in the tests and the analysis. $\mathrm{Ol}$ et al. postulated that plots of torque coefficient versus advance ratio are sometimes misleading because they do not account for Reynolds number effects. It was also shown that when the ratio of diameter to pitch is scaled $(10 \times 10$ to $12 \times 12$, for example) the experimental data fits together well within the bounds of error. Modifications to the dimensionless terms to factor in propeller pitch were presented, however more research was deemed necessary to apply this theory.

Corrigan and Altman (2008) examined different methods for wind tunnel blockage corrections. These methods included the Glauert (1926) correction as well as a 
correction by Hackett et al. (1979). These methods were described in detail and their applications were shown. A wind tunnel experiment was designed and constructed to record the necessary variables to calculate total system efficiency, defined as propulsive efficiency divided by electrical efficiency. This is in contrast to other works that primarily explored propeller efficiency. The stand was constructed using a beam-type load cell and a reaction torque sensor. Three propellers $(D=10,12$, and 14 inches) were tested using different motors for each propeller. Static pressure taps were used on the wall of the wind tunnel test section to record the changes in pressure forward and behind the propeller disk plane for the velocity corrections. The Glauert method did not provide sufficient correction for large blockage conditions. The Hackett method yielded more correction at higher airspeeds and larger propeller diameters, but the method could not be validated and therefore further work was found to be necessary.

Merchant and Miller (2006) performed dynamic tests on propellers in the $6 \leq D \leq$ 22 inch range. A test stand was constructed to record propeller performance parameters, where the thrust and torque were collected by a combined thrust/torque cell. The load and torque cell was calibrated using dead weights in the axial (thrust) and transverse (torque) directions. Wind tunnel velocity was measured directly using a Pitot probe and a differential pressure transducer. Since the propellers were large compared to the test section, blockage corrections developed by Glauert (1926) were applied to the results. Readings were taken at wind-off-zero conditions before and after each test. These values were then averaged and subtracted from the test data to account for zero drift and temperature effects. Data was collected at constant propeller rotational speeds and the wind tunnel velocity was varied to sweep through values of advance ratio. The results 
were compared to other works and were shown to be acceptable. The setup was also tested for variations in flow angularity. Pitch and yaw variations between -3 and +3 arc degrees were examined and it was shown that only the coefficient of thrust was affected by a change in pitch. However, it was shown that pitch variations of -3 and +3 degrees yielded the same results, which indicated that the system was symmetric in the pitch direction. Lastly, two identical propellers made by the same manufacturer were tested and compared, which showed that for some propellers there may be significant differences in performance due to manufacturing. Very limited results were presented, however, and the results shown only give a small sample of the entire test range.

The objective of the present research was to determine the performance of various commercially-available small-scale propellers driven by an electric motor. An experimental test stand was designed and constructed in which the electric motor was mounted in a wind tunnel at Wright State University for both static and dynamic testing. The freestream airspeed was varied from zero to the windmill state for each propeller. The rotational speed was varied over the operational range recommended by the propeller manufacturers, while ensuring that the electric motor did not overheat. The primary measurement devices were calibrated, and an extensive uncertainty analysis was performed. The results from the present experiment were compared to those from previous studies for both static and dynamic data. For static testing, the coefficient of thrust, the coefficient of propeller power, and the overall efficiency, defined as the propeller output compared to the electrical power input, were plotted versus the propeller rotational speed. For dynamic testing, the rotational speed of the propeller was held constant at regular intervals while the freestream airspeed was increased from zero to the 
maximum. The coefficient of thrust, the coefficient of power, the propeller efficiency and the overall efficiency were plotted versus the advance ratio for various rotational speeds.

\section{BACKGROUND}

The performance characteristics to be determined by the experimental setup are as follows. The coefficients of thrust, torque and propeller power, and the propeller efficiency are (Merchant and Miller, 2006):

$$
C_{\mathrm{T}}=\frac{T^{\prime}}{\rho n^{2} D^{4}}, \quad C_{\mathrm{Q}}=\frac{Q}{\rho n^{2} D^{5}}, \quad C_{\mathrm{P}}=\frac{P_{\mathrm{P}}}{\rho n^{3} D^{5}}, \quad \eta_{\mathrm{P}}=\frac{J C_{\mathrm{T}}}{C_{\mathrm{P}}}
$$

The three performance coefficients and the propeller efficiency defined above are typically plotted against the advance ratio for dynamic testing:

$$
J=\frac{V_{\infty}^{\prime}}{n D}
$$

where the corrected freestream velocity is (Glauert, 1926):

$$
V_{\infty}^{\prime}=V_{\infty}\left[1-\frac{\tau_{4}\left(\frac{A_{\mathrm{P}}}{A_{\mathrm{WT}}}\right)}{2 \sqrt{1+2 \tau_{4}}}\right]
$$

The uncorrected freestream velocity is:

$$
V_{\infty}=\sqrt{\frac{2 P_{\text {diff }}}{\rho}}
$$

The Glauert correction variable is:

$$
\tau_{4}=\frac{T^{\prime}}{\rho A_{\mathrm{P}} V_{\infty}^{2}}
$$


The propeller disk area and wind tunnel area are, respectively:

$$
A_{\mathrm{P}}=\frac{\pi D^{2}}{4}, A_{\mathrm{WT}}=W^{2}
$$

The corrected thrust is defined as the measured thrust minus the drag force due to the flow of air over the motor, torque cell and load cell (Selig and Ananda, 2011):

$$
T^{\prime}=T-F_{D}
$$

The overall propulsion system efficiency is the ratio of the propeller output power to the electrical input power:

$$
\eta_{\mathrm{T}}=\frac{P_{\mathrm{P}}}{P_{\mathrm{e}}}=\frac{2 \pi n Q}{V I}
$$

The density of air is given by the perfect gas law:

$$
\rho=\frac{P_{\mathrm{atm}}}{R T_{\mathrm{atm}}}
$$

The Reynolds number at the $75 \%$ radius of the propeller is defined as follows for the static and dynamic tests:

$$
\operatorname{Re}_{0.75, \mathrm{~S}}=\frac{\rho V_{\mathrm{P}} C_{0.75}}{\mu}, \quad \operatorname{Re}_{0.75, \mathrm{D}}=\frac{\rho V_{\mathrm{T}} C_{0.75}}{\mu}
$$

where the propeller velocity and total velocity (Merchant and Miller, 2006) are given by:

$$
V_{\mathrm{P}}=2 \pi n R_{0.75}, \quad V_{\mathrm{T}}=\sqrt{\left(V_{\infty}^{\prime}\right)^{2}+V_{\mathrm{P}}^{2}}
$$

The absolute viscosity of air is a function of absolute temperature (NACA, 1953):

$$
\mu=\mu_{\mathrm{ref}}\left(\frac{T_{\mathrm{atm}}}{T_{\mathrm{ref}}}\right)^{0.76}
$$




\section{EXPERIMENTAL SETUP}

The objective of the present experiment was to determine the performance characteristics of small electric motor/propeller combinations from static conditions to the windmill state. Initially, a simple bench-top static test rig was designed and constructed to properly size the load cell and torque cell used in the experiment. The design, construction and test results from the bench-top static test rig are discussed in detail in Appendix C.

The overall design of the dynamic test rig is shown in Figure 2. The electric motor was directly attached to a 25 oz-in torque cell (Transducer Techniques, Model RTS-25), which is able to withstand $10 \mathrm{~kg}$ in thrust and $1.7 \mathrm{~kg}$ in shear. The torque cell was in turn mounted onto a 1-kg single point beam-type load cell (Transducer Techniques, Model LSP-1). Each cell was driven by a signal conditioner (Transducer Techniques, Model TMO-1) that produced a 0 to 5 Volt linear output. The assembly of the motor, torque cell and load cell is shown in Figure 1. The motor is held in place with a custom-designed clam-shell clamp, in which fins were incorporated to increase the convective heat transfer from the electric motor to the air. This complex design was cut from a plate of 6061 aluminum using the wire electrical discharge machine (EDM) in the Micro Air Vehicle Lab at Wright State University.

The load cell was attached to a section of 1.25-inch square aluminum tubing, which acted as a riser to place the propeller in the middle of the test section. The bottom of the riser was connected to an optical breadboard table (Melles-Griot, Model BBSS-25610-1219) using flanges of angle aluminum. 
A hole was milled in the acrylic floor of the wind tunnel for the aluminum riser to pass through. The low-speed wind tunnel at Wright State University is an open circuit design capable of producing speeds from 0.6 to $36 \mathrm{~m} / \mathrm{s}$ with a contraction ratio of 6.25:1. The square entrance of the wind tunnel has a $3.8 \mathrm{~m}^{2}$ opening with an aluminum hexagonal honeycomb section that serves as a flow straightener. The height and width of the square test section is $W=0.6096 \mathrm{~m}$, and its length is $2.438 \mathrm{~m}$. Doors on one side of the test section allow for an entire wall to be opened for easy access. The diffuser is connected to an axial flow fan driven by a 20-hp electrical motor.

The data acquisition system used to collect data from the instrumentation consisted of a DAQ board (National Instruments, Model SCC-68) and a DAQ card (National Instruments, Model PCI-6221) installed in a PC. Shielded wires were used to connect the outputs of the transducers to the DAQ board. The electric motor driving the propeller was energized using a precision DC power supply (Hewlett-Packard, Model 6012B). A servo tester (GWS, Model MT-1) was used to control the rotational speed of the propeller (Corrigan and Altman, 2008). The voltage supplied to the electric motor was measured using a digital multi-meter (National Instruments, Model USB-4065). To measure the current, a DC Hall effect current transducer (CR Magnetics, Model CR521030 ) with a range of 0 to $30 \mathrm{~A}$ was placed in-line between the power supply and the motor speed controller.

A remote optical sensor (Monarch Instrument, Model ROS-W) connected to a panel meter (Monarch Instrument, Model ACT-3X) was used to measure propeller rotational speed. Reflective tape supplied with the sensor was placed near the hub on the leeward side of the propeller so that the optical sensor did not have to be adjusted 
between runs. A roughly $5 \times 5 \mathrm{~mm}$ piece of tape on each blade proved sufficient to get a good signal. The optical sensor was attached to the aluminum riser and aimed at the reflective tape.

Atmospheric pressure was measured to determine the density and absolute viscosity of the air. To record atmospheric pressure, a barometer (Vaisala, Model PTB110) capable of measuring 500 to 1100 mbar with accuracy of \pm 0.3 mbar was used. The differential pressure produced by the Pitot tube was measured using a differential pressure manometer (MKS, Model 226A) capable of reading a pressure differential of five Torr with an accuracy of $0.30 \%$ of the reading. The height of the Pitot tube from the floor of the wind tunnel was selected by traversing the boundary layer thickness using the Pitot tube as outlined in Appendix D. The height was set to $H=2.5$ inches, and the Pitot tube was made parallel to the wind tunnel walls by using a bubble level and a custommade jig.

The temperature of the motor was measured using a Type $\mathrm{T}$ thermocouple while the temperature of the air inside the wind tunnel was measured using a Type E thermocouple probe (Omega, Model EMQSS-125G-12). The Type T thermocouple junction was placed on the center of the motor and held in place by the aluminum clamshell clamp. Thermally conductive paste was placed on the thermocouple to aid in the transfer of heat. The Type E probe was mounted in the floor of the wind tunnel ahead of the motor/propeller so that the sensing junction extended into the airflow. The thermocouples were connected to thermocouple modules (National Instruments, Model SCC-TC01) on the data acquisition board. The signals from the eight sensors were read 
using custom-designed LabVIEW virtual instruments. The experimental data was reduced and tabulated using Excel.

The twenty-three propellers selected for analysis ranged from $4.0 \leq D \leq 6.0$ inches in diameter and $2.0 \leq P \leq 5.5$ inches in pitch. Some of the propellers were selected to overlap with previous research so that the procedures and test setup used for the measurements could be compared and validated. The GWS $4.5 \times 3.0$ and $5.0 \times 4.3$ inch propellers were tested statically and compared to Deters and Selig (2008). An APC $8.0 \times$ 3.8 inch Slow Flyer was tested dynamically and compared to the results posted on the UIUC Propeller Database (Selig, 2012), while an APC $6.0 \times 4.0$ inch propeller was also tested dynamically and compared to the results presented by Ol et al. (2008). In order to accurately determine the diameter, $75 \%$ radius, and chord length at the $75 \%$ radius, three propellers of each type were measured using calipers. The three measurements for each of the propellers were then averaged and used in the calculations, as shown in Table 2, Table 3, and Table 4.

\section{UNCERTAINTY ANALYSIS}

The uncertainties of all of the calculated results described in the above equations were determined using the root-sum-square uncertainty method (Kline and McClintock, 1953). Prior to conducting the experiments, three length measurements were made: Propeller diameter, $D$; propeller chord length at the $75 \%$ radius, $C_{0.75}$; and wind tunnel width, $W$. During experimentation, eight primary measurements were made using a data acquisition system: Uncorrected thrust, $T$; torque, $Q$; propeller rotational speed, $n$; 
atmospheric pressure, $P_{\mathrm{atm}}$; atmospheric temperature, $T_{\mathrm{atm}}$; Pitot tube pressure difference, $P_{\text {diff }}$; motor voltage, $V$; and motor amperage, $I$. The load cell, the torque cell, and the thermocouple used to measure the atmospheric temperature were calibrated in-house. In general, the calibration uncertainty is comprised of the uncertainty of the calibration standard and the difference between the prediction by the best-fit calibration line and the collected data point:

$$
\Delta U_{\mathrm{CAL}}=\Delta U_{\mathrm{CS}}+\left|(m x+b)-U_{\mathrm{DATA}}\right|
$$

The measurement uncertainty can then be estimated to be the sum of the calibration uncertainty and the confidence interval of the collected data set at a confidence level of 99\%:

$$
\Delta U=\Delta U_{\mathrm{CAL}}+\Delta U_{99}
$$

The type $\mathrm{E}$ thermocouple probe used to measure the atmospheric temperature was calibrated over the anticipated ambient air temperature range in the wind tunnel room of 15 to $30^{\circ} \mathrm{C}$ in intervals of $5^{\circ} \mathrm{C}$. In order to calibrate the torque cell, two identical arms were attached to the sides of the motor clamp so that the torque cell could be calibrated in both directions of rotation simultaneously. Varying weights were hung from one of the arms to calibrate in the clockwise direction, and then the process was repeated for the counterclockwise direction. The load cell used to measure thrust was calibrated in situ as follows. A strand of fishing line was attached to the front of the propeller using aircraft wire. This strand was then passed over a smooth cylinder with bearings mounted in the wind tunnel. Varying weights were suspended from the fishing line over the expected range of thrust. 
The drag of the fixture was measured versus airspeed by removing the propeller and replacing it with just a propeller hub with the blades removed. The airspeed was increased systematically while data was collected from the load cell and the Pitot tube. The free-stream velocity was then calculated and the measured drag was plotted against the velocity. A second-order regression was applied to the points and this equation was used in the calculation of the corrected thrust.

Table 1 gives the uncertainties for each device or transducer used to collect the data. A complete discussion of the calibration process and a list of calibration certificates are given in Appendix A. The principal equations used for determining the uncertainties of the computed quantities shown in the graphs in the Results and Discussion section are shown below. A complete listing of equations used is provided in Appendix B.

\section{Coefficient of Thrust:}

$$
\Delta C_{T}=\left[\left(\frac{\Delta T}{\rho n^{2} D^{4}}\right)^{2}+\left(\frac{-T \Delta \rho}{\rho^{2} n^{2} D^{4}}\right)^{2}+\left(\frac{-2 T \Delta n}{\rho n^{3} D^{4}}\right)^{2}+\left(\frac{-4 T \Delta D}{\rho n^{2} D^{5}}\right)^{2}\right]^{\frac{1}{2}}
$$

Coefficient of Torque:

$$
\Delta C_{Q}=\left[\left(\frac{\Delta Q}{\rho n^{2} D^{5}}\right)^{2}+\left(\frac{-Q \Delta \rho}{\rho^{2} n^{2} D^{5}}\right)^{2}+\left(\frac{-2 Q \Delta n}{\rho n^{3} D^{5}}\right)^{2}+\left(\frac{-5 Q \Delta D}{\rho n^{2} D^{6}}\right)^{2}\right]^{\frac{1}{2}}
$$

Coefficient of Power:

$$
\Delta C_{P}=\left[\left(\frac{\Delta P_{P}}{\rho n^{3} D^{5}}\right)^{2}+\left(\frac{-P_{P} \Delta \rho}{\rho^{2} n^{3} D^{5}}\right)^{2}+\left(\frac{-3 P_{P} \Delta n}{\rho n^{4} D^{5}}\right)^{2}+\left(\frac{-5 P_{P} \Delta D}{\rho n^{3} D^{6}}\right)^{2}\right]^{\frac{1}{2}}
$$

Propeller Efficiency:

$$
\Delta \eta_{\mathrm{P}}=\left[\left(\frac{C_{\mathrm{T}} \Delta J}{C_{\mathrm{P}}}\right)^{2}+\left(\frac{\Delta C_{\mathrm{T}} J}{C_{\mathrm{P}}}\right)^{2}+\left(\frac{-J C_{\mathrm{T}} \Delta C_{\mathrm{P}}}{C_{\mathrm{P}}^{2}}\right)^{2}\right]^{\frac{1}{2}}
$$


Advance Ratio:

$$
\Delta J=\left[\left(\frac{\Delta V_{\infty}^{\prime}}{n D}\right)^{2}+\left(\frac{-V_{\infty}^{\prime} \Delta n}{n^{2} D}\right)^{2}+\left(\frac{-V_{\infty}^{\prime} \Delta D}{n D^{2}}\right)^{2}\right]^{\frac{1}{2}}
$$

Overall Propulsion Efficiency:

$$
\Delta \eta_{\mathrm{T}}=\left[\left(\frac{2 \pi n \Delta Q}{V I}\right)^{2}+\left(\frac{2 \pi Q \Delta n}{V I}\right)^{2}+\left(\frac{-2 \pi n Q \Delta V_{\mathrm{e}}}{V^{2} I}\right)^{2}+\left(\frac{-2 \pi n Q \Delta I}{V I^{2}}\right)^{2}\right]^{\frac{1}{2}}
$$

Reynolds Number at 75\% Propeller Radius (Static Testing):

$$
\Delta \operatorname{Re}_{0.75, \mathrm{~S}}=\left[\left(\frac{C_{0.75} V_{\mathrm{P}} \Delta \rho}{\mu}\right)^{2}+\left(\frac{C_{0.75} \rho \Delta V_{\mathrm{P}}}{\mu}\right)^{2}+\left(\frac{\rho V_{\mathrm{P}} \Delta C_{0.75}}{\mu}\right)^{2}+\left(\frac{-C_{0.75} \rho V_{\mathrm{P}} \Delta \mu}{\mu^{2}}\right)^{2}\right]^{\frac{1}{2}}
$$

Reynolds Number at 75\% Propeller Radius (Dynamic Testing):

$$
\Delta \operatorname{Re}_{0.75, \mathrm{D}}=\left[\left(\frac{C_{0.75} V_{\mathrm{t}} \Delta \rho}{\mu}\right)^{2}+\left(\frac{C_{0.75} \rho \Delta V_{\mathrm{t}}}{\mu}\right)^{2}+\left(\frac{\rho V_{\mathrm{t}} \Delta C_{0.75}}{\mu}\right)^{2}+\left(\frac{-C_{0.75} \rho V_{\mathrm{t}} \Delta \mu}{\mu^{2}}\right)^{2}\right]^{\frac{1}{2}}
$$

\section{TEST PROCEDURES}

In order to perform the experiments consistently, it was necessary to follow detailed procedures to collect data. Two separate procedures were developed for the static and dynamic tests. For all of the tests, the power supply driving the motor controller for the propeller motor was turned on and set to a nominal output of 11.1 Volts, which matches the voltage output of a standard 3-cell battery. Then, the data acquisition system and the signal conditioners driving the sensors were powered up for the warm-up periods recommended by the manufacturers. A file name and location were chosen in the data acquisition software that identified such parameters as propeller diameter, pitch, and 
propeller rotational speed. A complete description of the experimental procedures used is provided in Appendix E.

\section{Static Test Procedure}

After the warm-up period, the load cell and torque cell were zeroed by adjusting the balance potentiometers on the signal conditioners so that the voltage outputs were as close as possible to zero. At this point, five hundred data points were collected with the propeller off in order to obtain a baseline for the actual value of zero for the load cell and torque cell. The propeller was then set to the first desired speed setting and one thousand data points were collected. The propeller was then turned off and another set of 500 data points was acquired. The average values for thrust and torque from the two propeller-off states were averaged and this value was used to correct the thrust and torque measurements to account for zero drift and temperature effects (Merchant and Miller, 2008). The process was then repeated for increased values of rotational speed until the maximum speed was achieved.

\section{Dynamic Test Procedure}

After the warm-up period, the differential pressure transducer reading the Pitot tube, the load cell, and the torque cell were zeroed. Five hundred data points were taken with the propeller motor off and the wind tunnel motor off. At the end of the first five hundred points, the propeller motor was set to the desired rotational speed setting and the wind tunnel airspeed was set to the first desired setting. After the system reached steady state, one thousand data points were acquired. Next, the wind tunnel airspeed setting was changed and the propeller rotational speed was adjusted to match the original setting. 
This process was repeated until the windmill state of the propeller was reached. The propeller motor and the wind tunnel motor were both stopped at this point, and then five hundred data points were collected in order to again account for drift in the sensors. Data sets were collected for approximately ten wind tunnel airspeed settings for each of the four rotational speed settings for each propeller tested.

\section{RESULTS AND DISCUSSION}

To ensure that the collected data was repeatable and correct, tests were necessary to validate the static and dynamic results. The first type of test checked for repeatability of the same propeller as well as the repeatability across three identical propellers. The second type of test was to compare the results of the present experiment to published results from researchers using the same propeller. A complete summary of all of the data for the static and dynamic tests are provided in Appendix $F$ and Appendix $G$, respectively.

\section{Validation of the Static Test}

In order to check the repeatability of the experiment, three identical Graupner 4.7 $\times 4.7$ inch propellers were tested under static conditions three times each, thus creating a total of nine sets of data. This was done to determine the repeatability of the experiment for multiple runs of the same propeller as well as establishing whether manufacturing variability affected the performance of identical propellers. Figure 3 shows typical results for a static propeller, where both the thrust and torque increase monotonically with 
rotational speed. Figure 4 shows that the repeatability of the reduced data (coefficient of thrust, coefficient of power and total efficiency) was excellent. The data from all nine tests fall within the uncertainty bounds for the first run. The duplicate propellers also fall directly in line, meaning that, at least for this type of propeller, manufacturing differences can be neglected. The uncertainties of the coefficients of thrust and power increased significantly at the lowest propeller rotational speed. This was driven by the uncertainty of the load cell and the torque cell at relatively small values of thrust and torque. Also, it was noted that at a rotational speed of $n=6000 \mathrm{rev} / \mathrm{min}$, excessive vibrations were encountered, so that test was halted.

Static tests were performed on two propellers (GWS $4.5 \times 3.0$ and GWS $5.0 \times$ 4.3) which matched tests performed by Deters and Selig (2008). The coefficient of thrust and the coefficient of power were compared to data provided by Deters and Selig as shown in Figure 5, where the results for both propellers show good agreement.

\section{Static Test Results}

Having established the validity of the experimental results, data was collected for all of the propellers shown in Table 2 - Table 4. Figure 6 shows a comparison between propellers with constant diameter and varying pitch, while Figure 7 gives a comparison between propellers with varying diameter and constant pitch. Each figure shows the coefficient of thrust, coefficient of power, and propeller efficiency. In Figure 6, the coefficients of thrust and power are relatively constant while the propeller efficiency increased with propeller rotational speed. The effect of reducing the pitch significantly decreased all three measures of performance. This same trend can be found in the data provided by Deters and Selig (2008) for the coefficient of thrust and coefficient of power 
for the GWS $4.0 \times 4.0$ propeller versus that for the GWS $4.0 \times 2.5$ propeller. In Figure 7 , the variation of the three performance parameters with varying propeller diameter is also shown to be significant, where increasing the diameter decreased the thrust coefficient and power coefficient but increased the propeller efficiency. This trend is also apparent in the data reported by Deters and Selig for the following propellers: GWS $3.0 \times 3.0$, GWS $4.5 \times 3.0$, and GWS $5.0 \times 3.0$.

\section{Validation of the Dynamic Test}

The dynamic test procedure and experiment were validated similarly to the static experiment. Figure 8 shows typical dynamic results for the thrust and torque generated by one propeller over the full range of airspeed and various levels of rotational speed. Both the thrust and torque increase with rotational speed and decrease with airspeed, as expected. The APC $8.0 \times 3.8$ inch Slow Flyer propeller was tested at nominal propeller rotational speeds of $n=4000$ and $7000 \mathrm{rpm}$, and the results for the coefficient of thrust, the coefficient of power, and the propeller efficiency versus advance ratio were compared to those reported on the UIUC propeller database (Selig, 2012), as shown in Figure 9. In general, the coefficient of thrust and coefficient of power decrease with advance ratio, whereas the propeller efficiency reaches a peak value, as shown in Figure 9(c). The agreement with the data from the UIUC database is excellent for both rotational speeds, even where the propeller efficiency drops off steeply with advance ratio.

To further validate the dynamic results, an APC $6.0 \times 4.0$ inch propeller was tested at nominal propeller rotational speeds of $n=8000$ to $16000 \mathrm{rpm}$ by intervals of $2000 \mathrm{rpm}$ and compared to the results for the coefficient of thrust, the coefficient of torque, and propeller efficiency versus advance ratio reported by $\mathrm{Ol}$ et al. (2008), as 
shown in Figure 10. Again, the coefficients of thrust and torque decrease with advance ratio and the propeller efficiency increases to a peak and then decreases. Since the exact propeller rotational speed tested by $\mathrm{Ol}$ et al. is unclear, it can only be compared to the trends in the data. The present data agrees with that shown by $\mathrm{Ol}$ et al. and the trends are similar. At a rotational speed of $n=8000 \mathrm{rpm}$, the propeller was tested by sweeping the advance ratio from low to high values, and then sweeping from high to low values to examine the potential for hysteresis in the experiment. As can be seen, there is not a noticeable difference between these two sets of data.

\section{Dynamic Test Results}

With the dynamic results validated, data was collected for all of the propellers. Similarly to the static tests, comparisons were drawn between propellers with constant diameter and varying pitch in Figure 11 and between propellers with constant pitch and varying diameter in Figure 12, both at a nominal rotational speed of $n=16000 \mathrm{rpm}$. In Figure 11, propellers with larger pitch generally had larger coefficients of thrust and power, and the windmill state occurred at higher values of the advance ratio, which indicates that larger pitch values tend to allow for higher airspeed. The results for the propeller efficiency given in Figure 11(c) shows that the efficiency decreases with increasing pitch for lower values of advance ratio, and the peak efficiency occurs at higher values of advance ratio. An increase in pitch essentially means that the angle of attack of the airfoil is higher, which should increase both thrust and torque prior to reaching stall. In Figure 12, increasing the propeller diameter for a given pitch tends to decrease the coefficient of thrust and the coefficient of power, and the propeller efficiency increases with diameter for lower values of advance ratio. Increasing the 
diameter for a given rotational speed and airspeed actually increases the thrust and torque due to the increased wingspan of the propeller, but this effect is negated due to the factor of $D^{4}$ in the denominator of $C_{\mathrm{T}}$ and the factor of $D^{5}$ in the denominator of the $C_{\mathrm{P}}$.

Figure 13 presents results for square propellers, where $D=P$, from $\mathrm{Ol}$ et al. (2008), Selig (2012), and the present experiment for a fairly wide range in propeller diameter (4.0 $\leq D \leq 18$ inches). Ol et al. had conjectured that the coefficient of thrust should collapse for square propellers. The results are grouped from small to large propeller diameter, where the three researchers essentially covered different diameter ranges. In general, the coefficient of thrust appears to increase with diameter, but more data would be required to make a definitive statement.

Figure 14 presents the coefficient of thrust for the same family of APC propellers (Speed 400 Electric). In this case, the diameter to pitch ratio for this group of propellers has a relatively small range $(0.86 \leq D / P \leq 1.5)$. However, it was found that the other types of propellers (Free Flight, Sport, Thin Electric, Slow Flyer) have noticeably different blade shapes, which could induce variations in the results simply due to the geometry of the propeller which could not be accounted for by using $D / P$ alone. Figure 14(a) shows the coefficient of thrust versus advance ratio for all of the collected data for the APC Speed 400 Electric propellers with an uncertainty level of $\Delta C_{\mathrm{T}} \leq 20 \%$. As can be seen, the results are not correlated well, as witnessed by the low goodness of fit parameter, $R^{2}=0.539$. As the uncertainty requirement becomes more restrictive, less data points are permitted to be graphed, which should increase the accuracy of the predictive best-fit line. However, this in itself becomes problematic, since more data points (for the $\Delta C_{\mathrm{T}}=20 \%$ case, for example) would provide more confidence in the resulting best-fit 
line. It is entirely possible that, if the uncertainty requirement should become too restrictive, the best-fit line could become erratic due to an insufficient number of data points. Conversely, if the uncertainty requirement becomes too lax, the accuracy of the predictive curve would suffer due to inclusion of obviously erroneous data points. It should be noted that many of the data points near the windmill state, where the thrust approaches zero, will not appear in these graphs due to the fact that the values of thrust are much lower than the uncertainty. Figure 14(b) shows the coefficient of thrust modified by $D / P$ plotted against advance ratio. This change to coefficient of thrust improves the goodness of fit parameter to $\mathrm{R}^{2}=0.680$. This gives a better correlation than (a) but this can be improved by modifying the advance ratio by $D / P$ as seen in Figure 14 (c). This improves the goodness of fit parameter to a value of $R^{2}=0.720$. This is a large improvement over the original data and shows that the data fits together better when modified by the diameter to pitch ratio.

Figure 15(a) similarly shows the coefficient of power versus advance ratio for all of the collected data with an uncertainty level of $\Delta C_{\mathrm{P}} \leq 20 \%$. As can be seen, the results are not correlated well, as witnessed by the very low goodness of fit parameter, $\mathrm{R}^{2}=$ 0.059. Figure 15(b) shows a coefficient of power that is modified by the diameter to pitch ratio squared, which improves the goodness of fit parameter to $R^{2}=0.538$. This is still relatively low, and probably should not be used in most engineering analyses. Figure 16 shows a similar comparison for the propeller efficiency versus the advance ratio. Here, the diameter to pitch ratio was used to modify the original advance ratio to increase the goodness of fit parameter from $\mathrm{R}^{2}=0.938$ to 0.983 , which is deemed to be very accurate 
for most applications, especially for advance ratios less than $J \leq 0.4$, which is near the peak efficiency predicted by the best-fit curve.

\section{CONCLUSIONS}

Twenty-three propellers in the range of $4.0 \leq D \leq 6.0$ inches in diameter and $2.0 \leq$ $P \leq 5.5$ inches in pitch were tested statically and dynamically in the Wright State University wind tunnel over a wide range of propeller rotational speeds and air speeds. A detailed experimental procedure for both cases was employed and an extensive uncertainty analysis was performed on the resulting data. The experiments were validated by comparing the results to previous works. The repeatability of the experimental results and the repeatability of the manufacture of the propellers were proven by testing three duplicate propellers three times each. Static tests were performed by varying propeller speed from $n=4000 \mathrm{rpm}$ to the maximum speed limited by the manufacturer's specifications or the maximum motor temperature. Dynamic tests were performed by holding the propeller speed constant and varying the wind tunnel airspeed and thus varying the advance ratio.

For a given airspeed and rotational speed, the thrust and torque both increased with propeller pitch and diameter, as expected. Propeller efficiencies ranged from $24 \%$ to $52 \%$ for some of the more efficient designs. It was found that the coefficient of thrust for square propellers, where $D=P$, increased with the propeller diameter. The coefficient of thrust for a family of propellers (same manufacturer and application) was found to have a good correlation that was well predicted over a range of diameter to pitch ratio of $0.86 \leq$ 
$D / P \leq 1.5$. Results for different propeller manufacturers and even the same manufacturer with higher values of $D / P$ did not correlate well, possibly due to variations of the propeller blade geometry that was not accounted for by $D / P$ alone. The correlation of the coefficient of thrust versus advance ratio plot was improved by modifying both the coefficient of thrust and advance ratio by the diameter to pitch ratio. The coefficient of power for this family of propellers was not found to be well correlated by either the original definition of the coefficient, or with a power coefficient that was modified by $D / P$ squared. The propeller efficiency of the above-mentioned set of propellers was plotted using the original expression for propeller efficiency versus advance ratio as well as a modified advance ratio, and it was determined that the correlation was improved substantially by using the modified advance ratio expression. The data reported here will serve to add to the database of work produced by others by filling in a critical range in propeller diameters previously untested dynamically. The results will provide future aircraft designers and researchers much needed information about the propellers and propulsion systems needed to create new aircraft designs or modifying existing designs. 


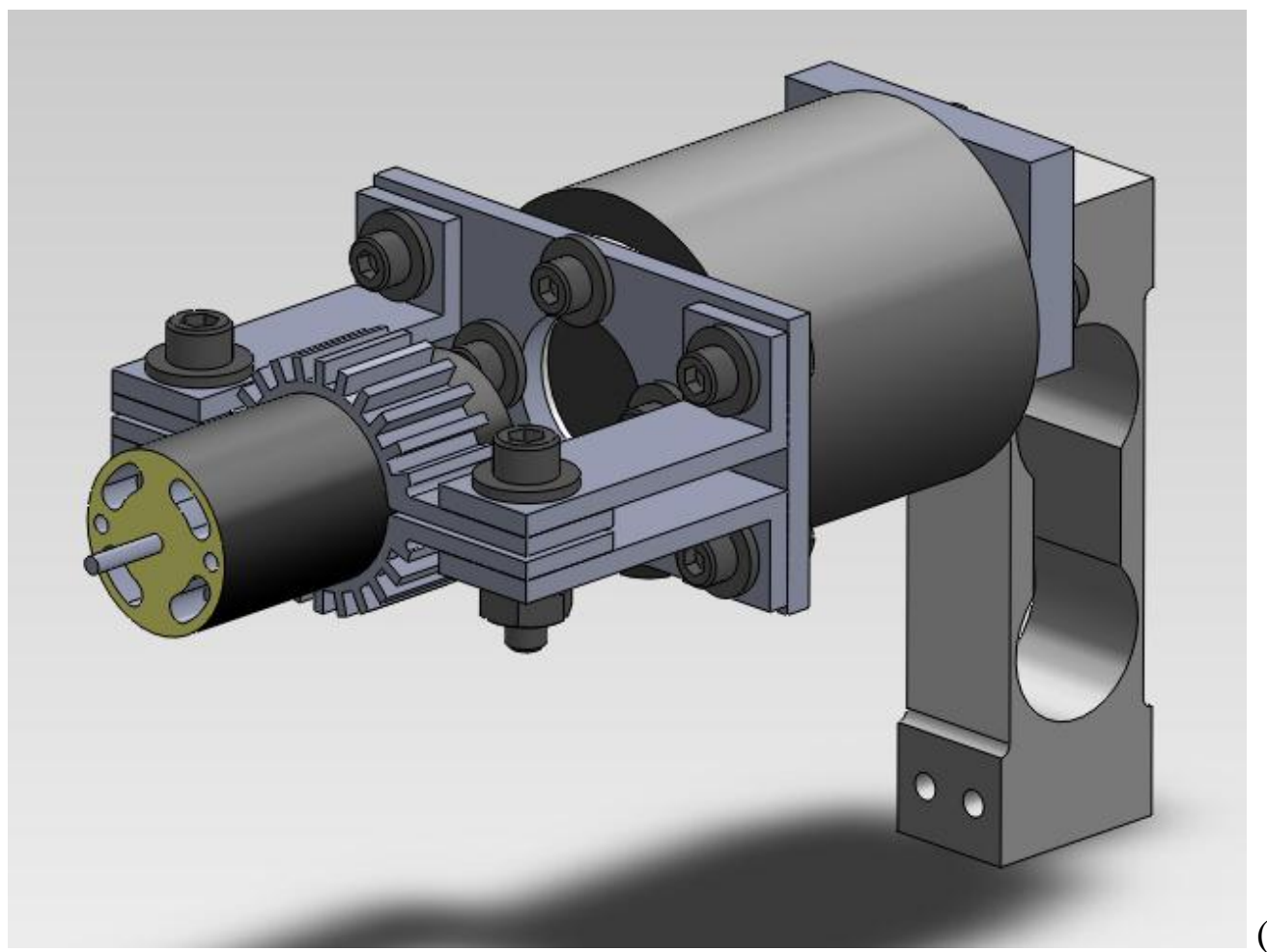

(a)

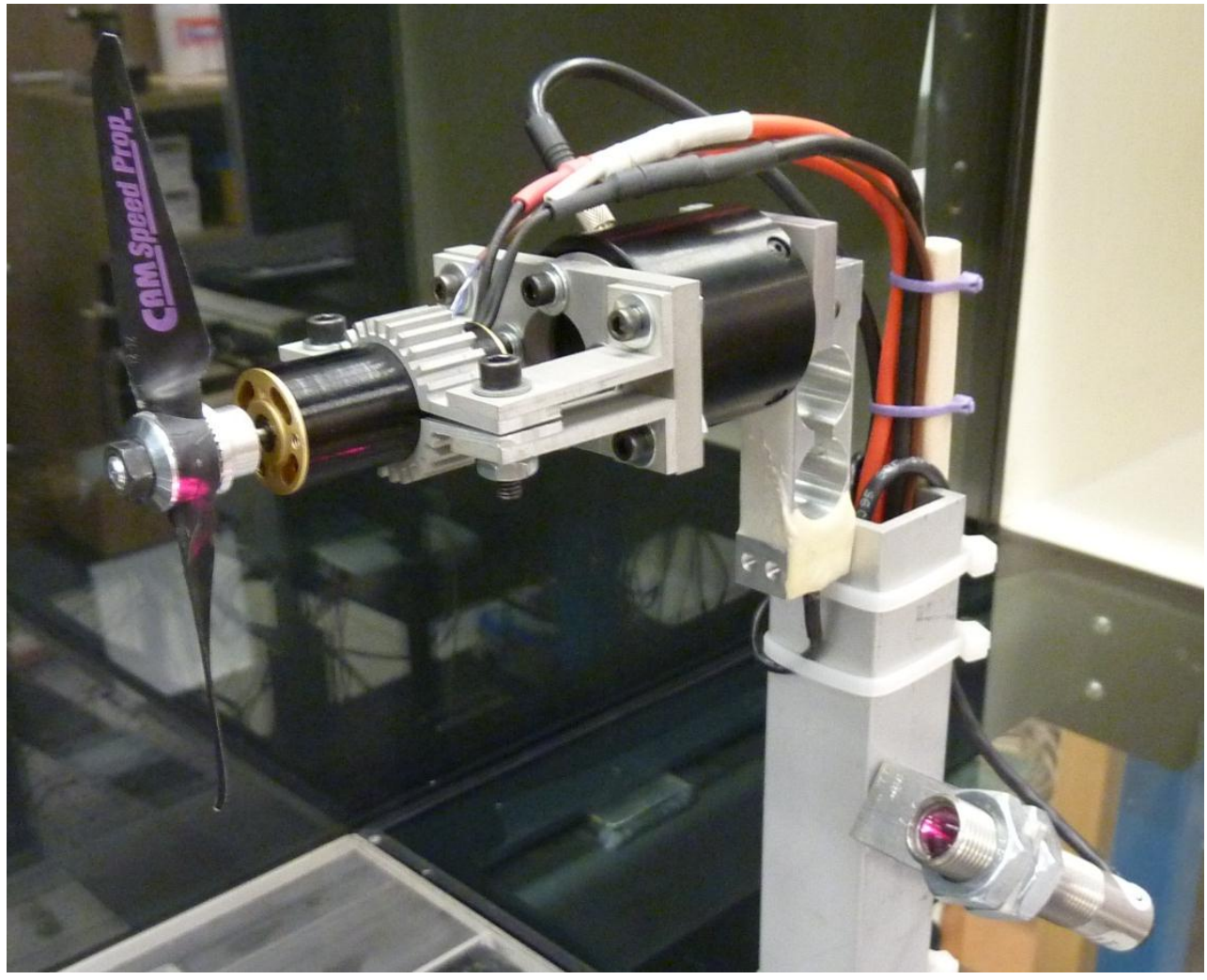

(b)

Figure 1: Assembly of Motor, Torque Cell and Load Cell: (a) Solid Model Representation; (b) Photograph. 


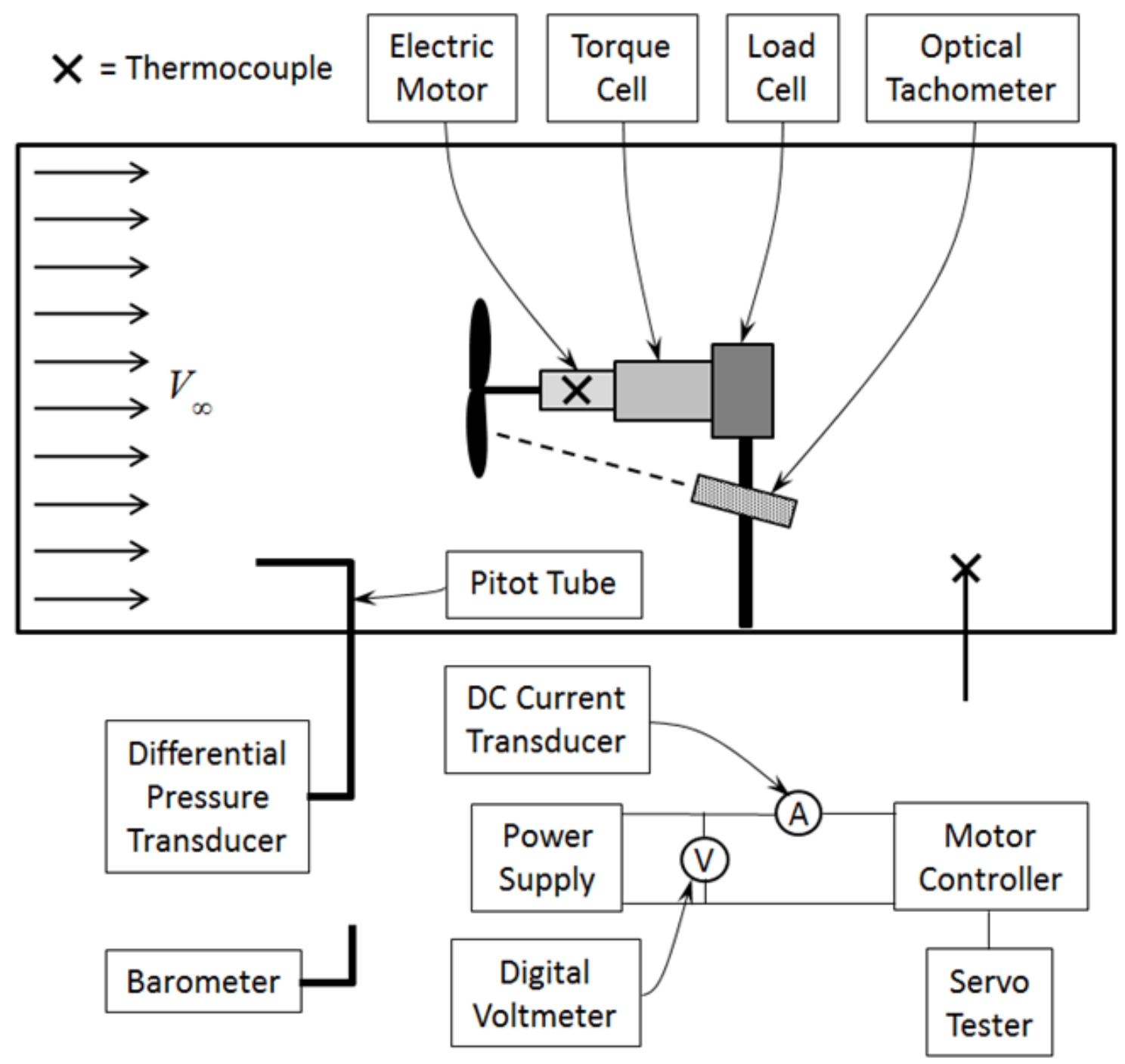

Figure 2: Schematic Diagram of the Experimental Setup. 


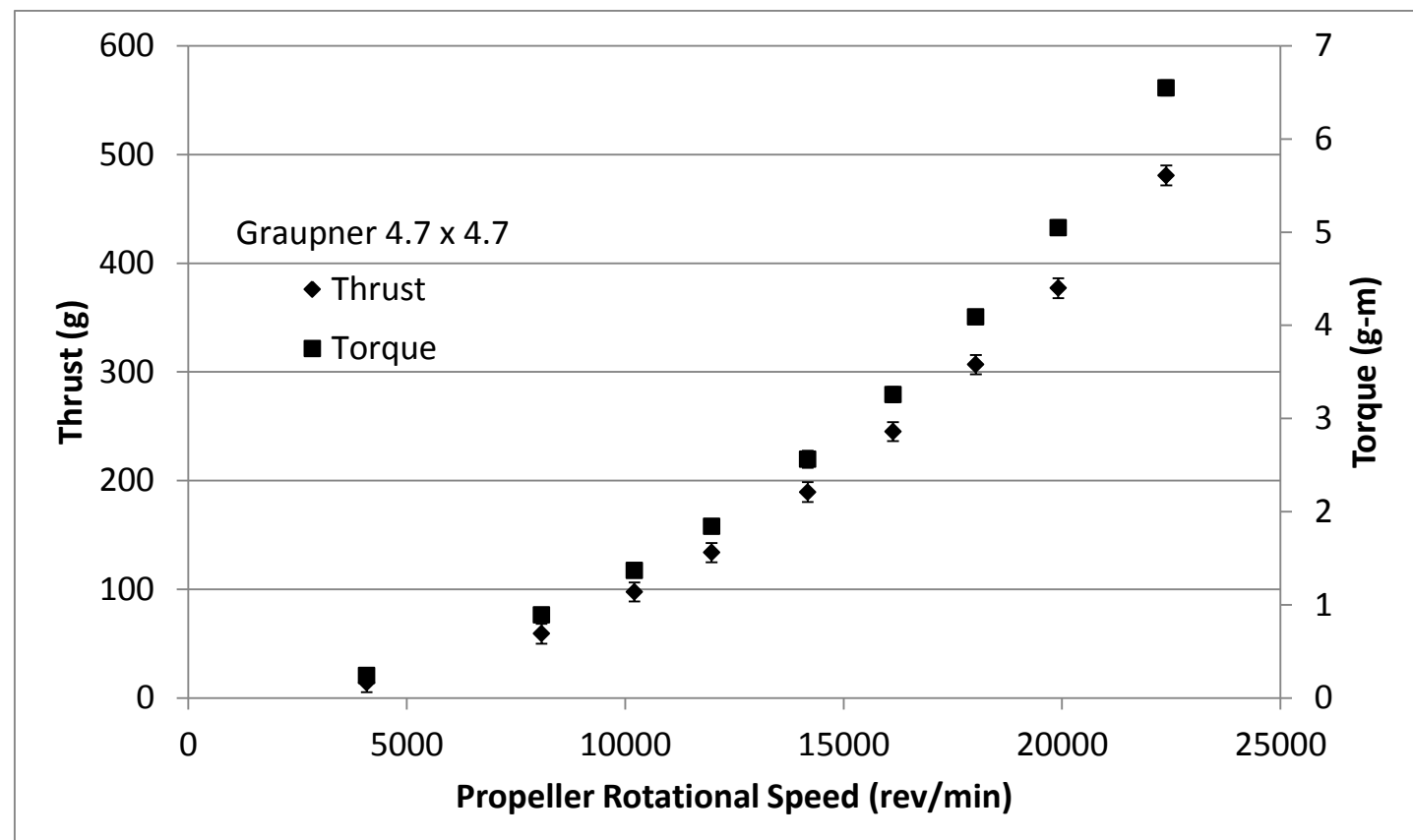

(a)

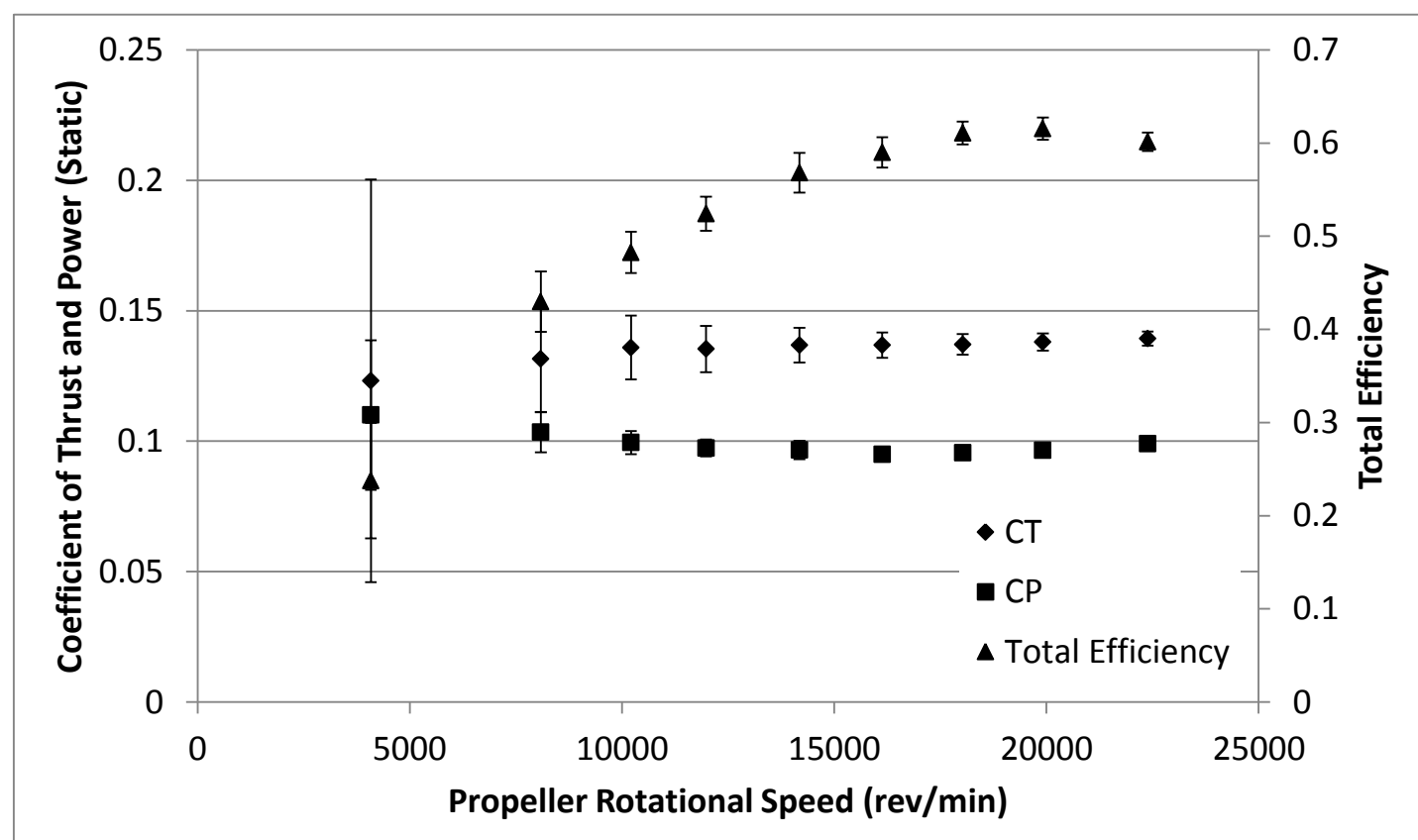

(b)

Figure 3: Typical Static Test Results (Graupner $4.7 \times 4.7$ inch Propeller): (a) Thrust and Torque Versus Rotational Speed, (b) Coefficient of Thrust, Power and Total Efficiency Versus Rotational Speed. 


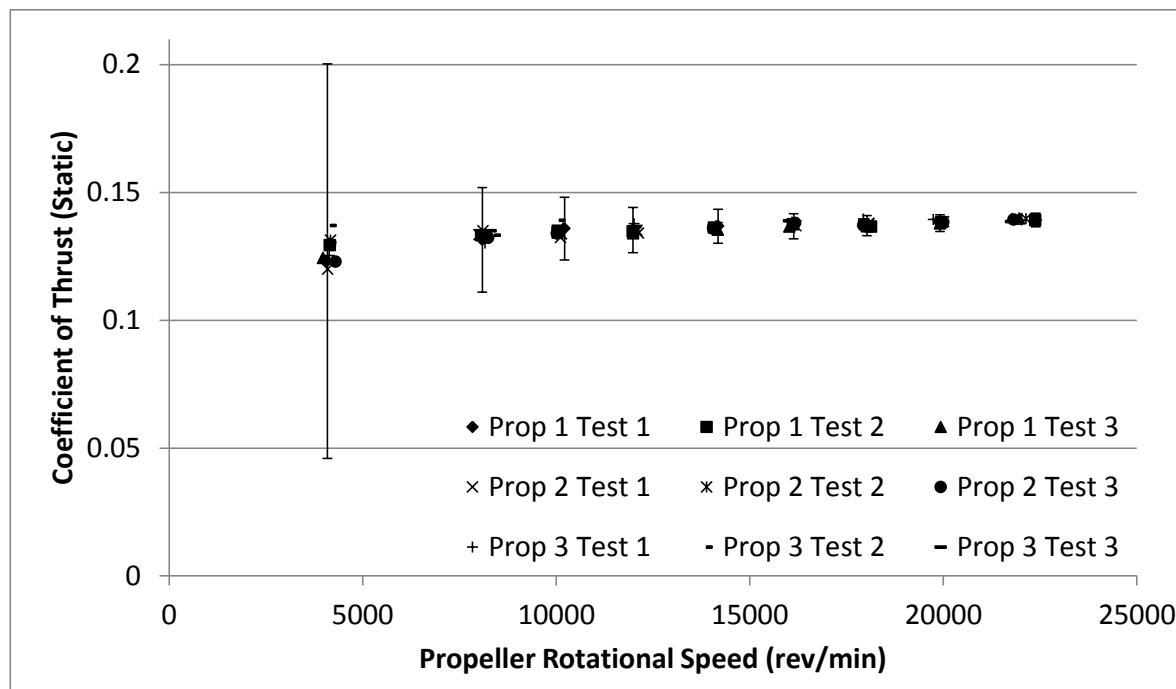

(a)

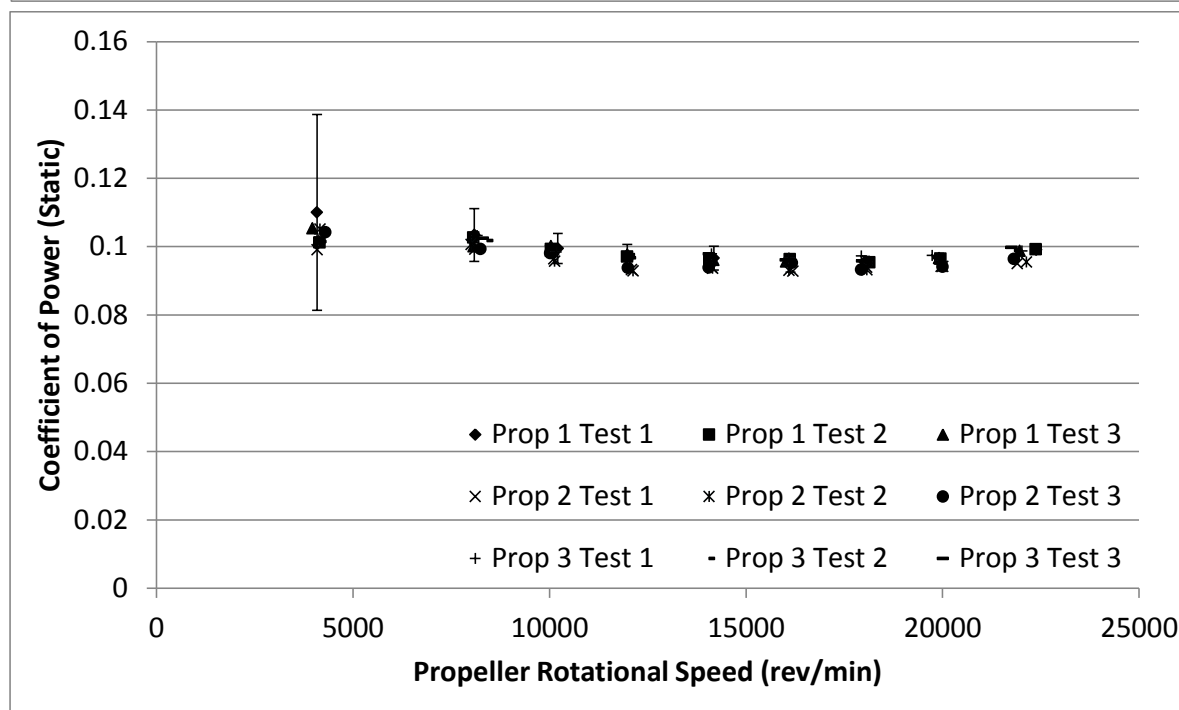

(b)

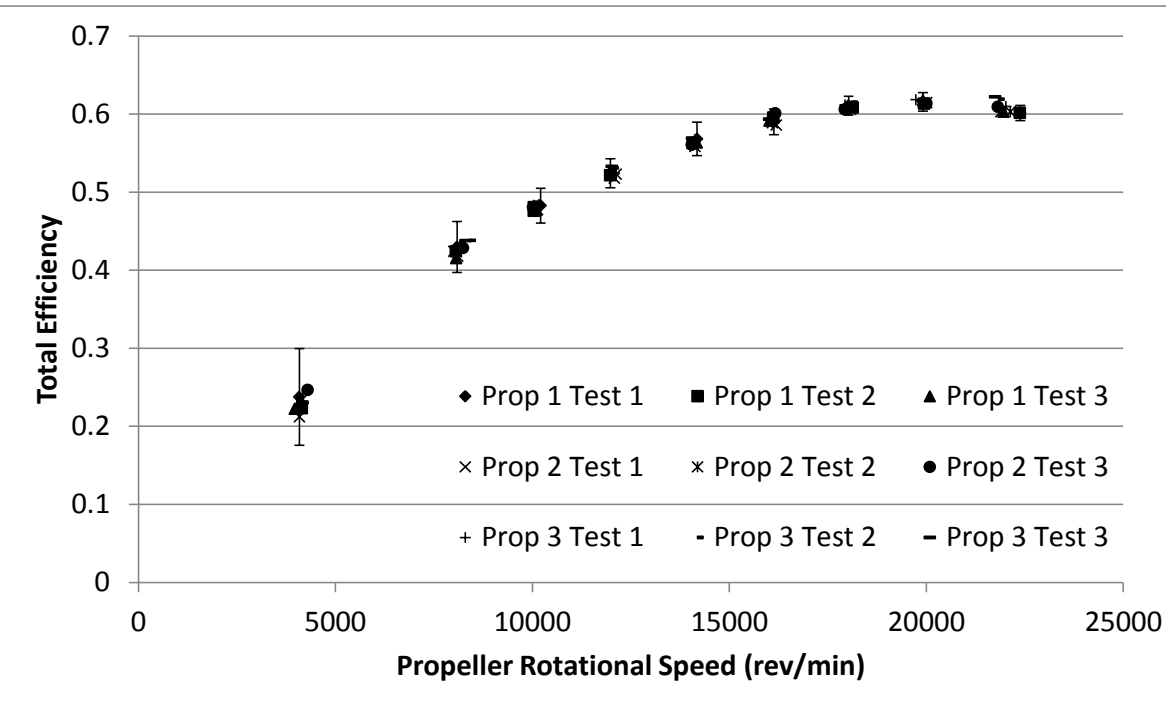

(c)

Figure 4: Comparison of Three Identical Propellers (Graupner $4.7 \times 4.7$ ): (a) Static Coefficient of Thrust, (b) Static Coefficient of Power, (c) Static Total Efficiency. 


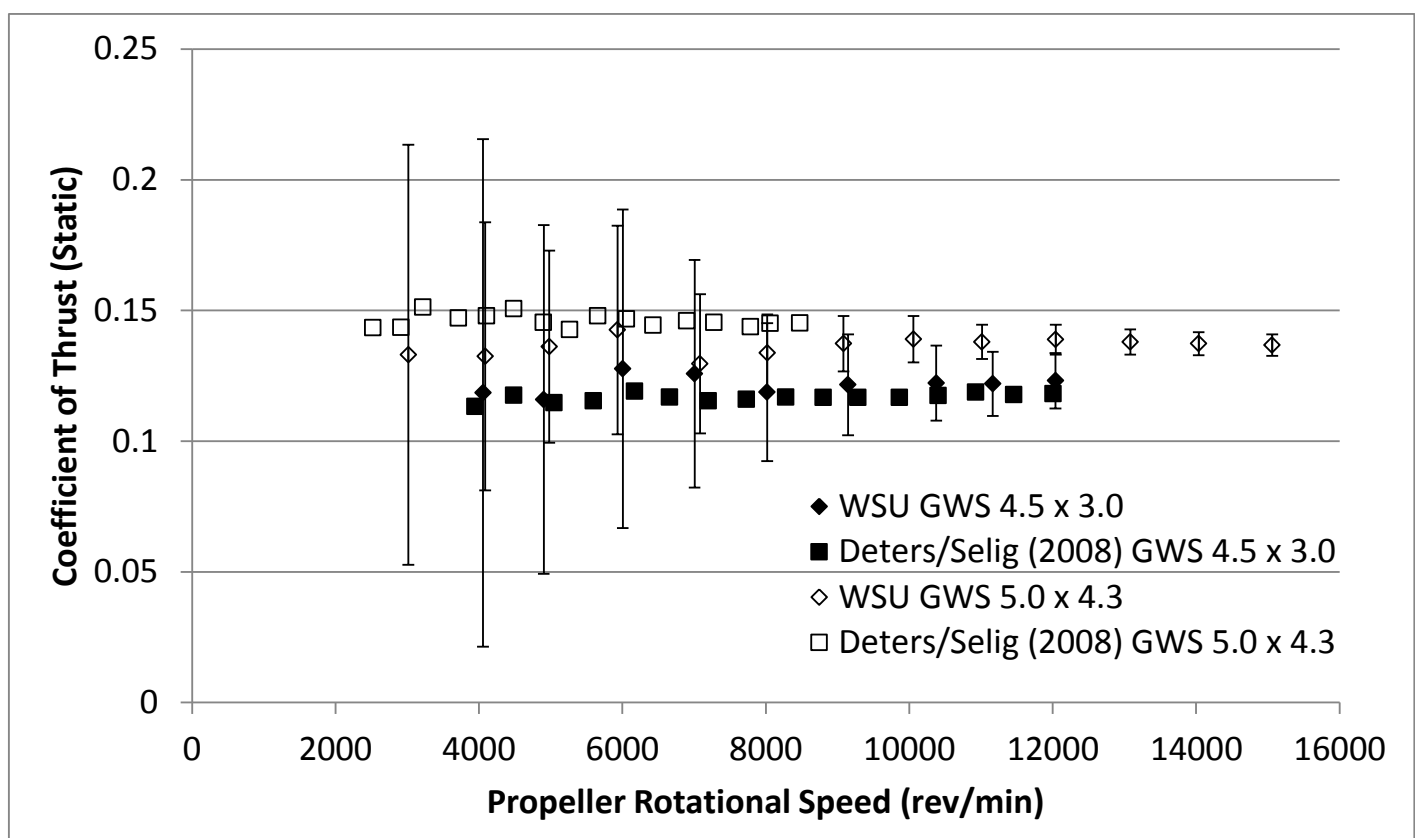

(a)

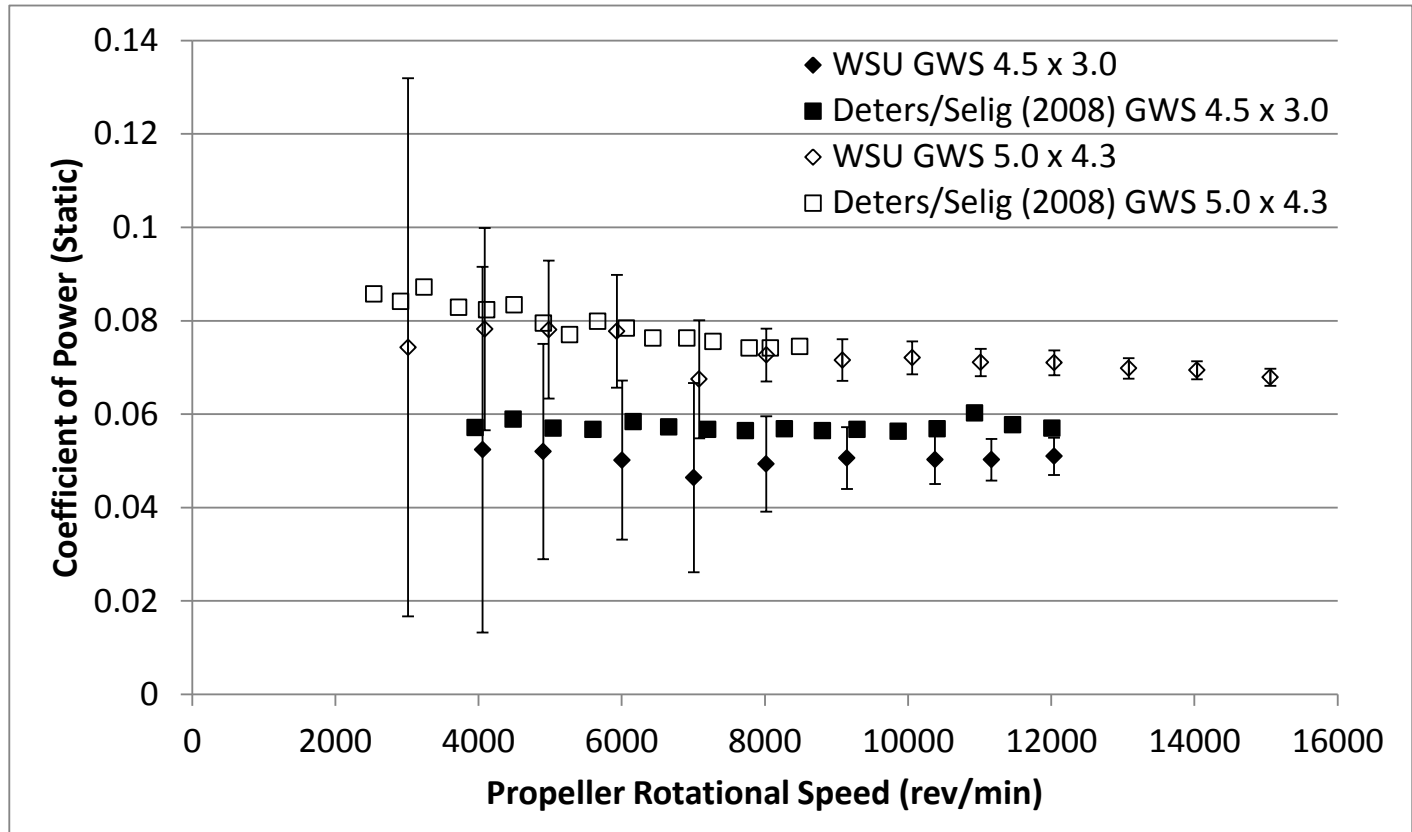

(b)

Figure 5: Comparison of the Present Results to Deters and Selig (2008) (GWS $4.5 \times 3.0$ and GWS $5.0 \times 4.3$ Propellers): (a) Static Coefficient of Thrust, (b) Static Coefficient of Power. 


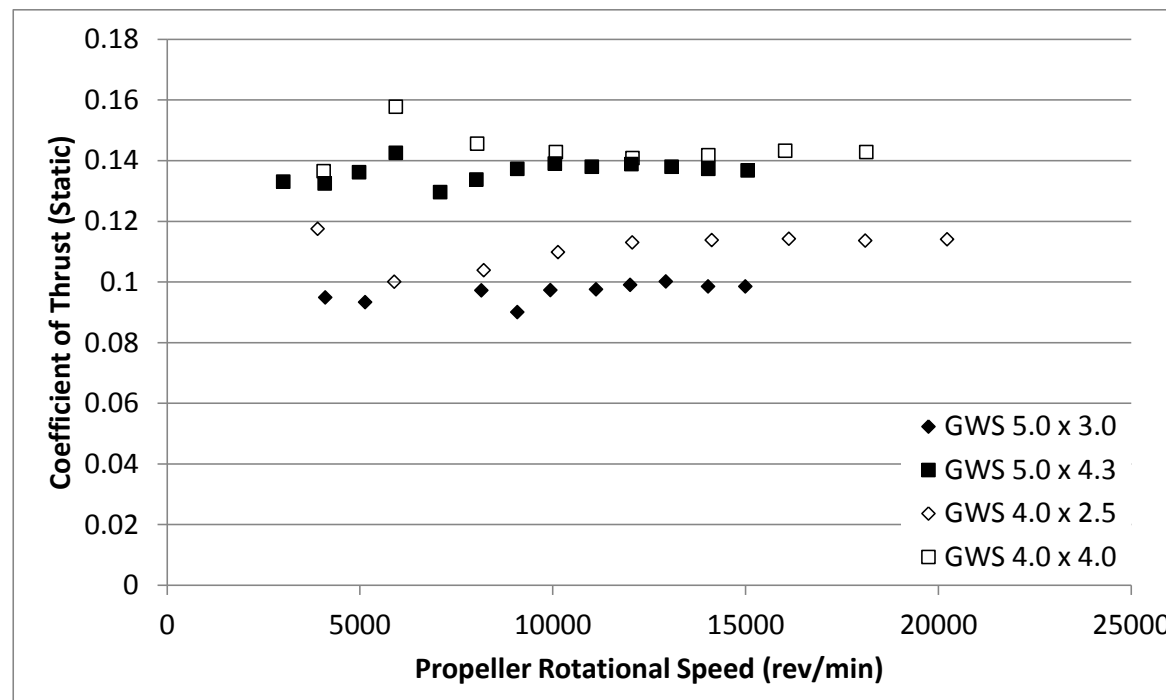

(a)

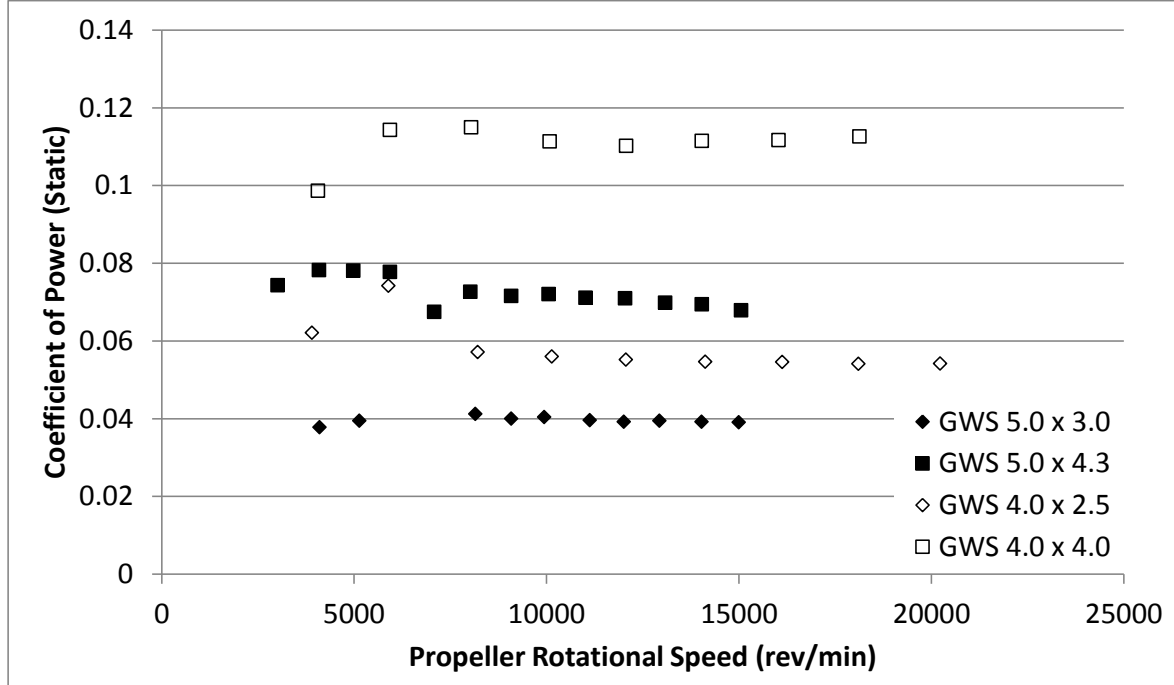

(b)

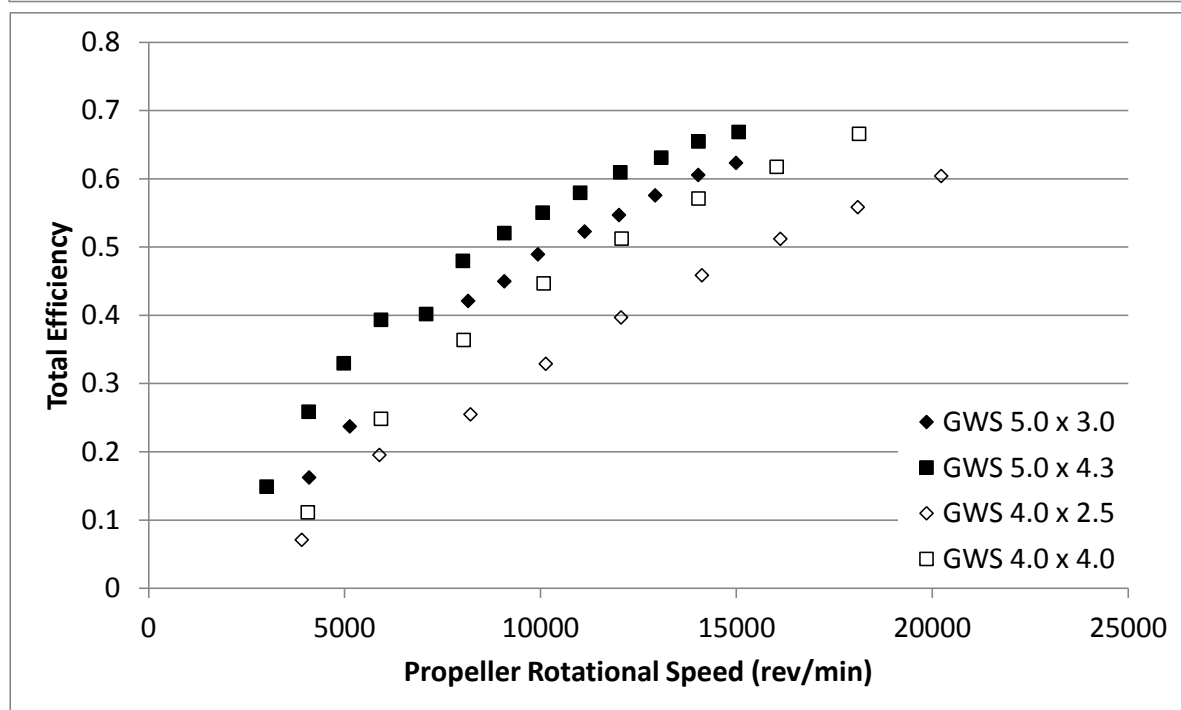

(c)

Figure 6: The Effect of Varying Propeller Pitch While Holding Diameter Constant: (a) Static Coefficient of Thrust, (b) Static Coefficient of Power, (c) Static Total Efficiency. 


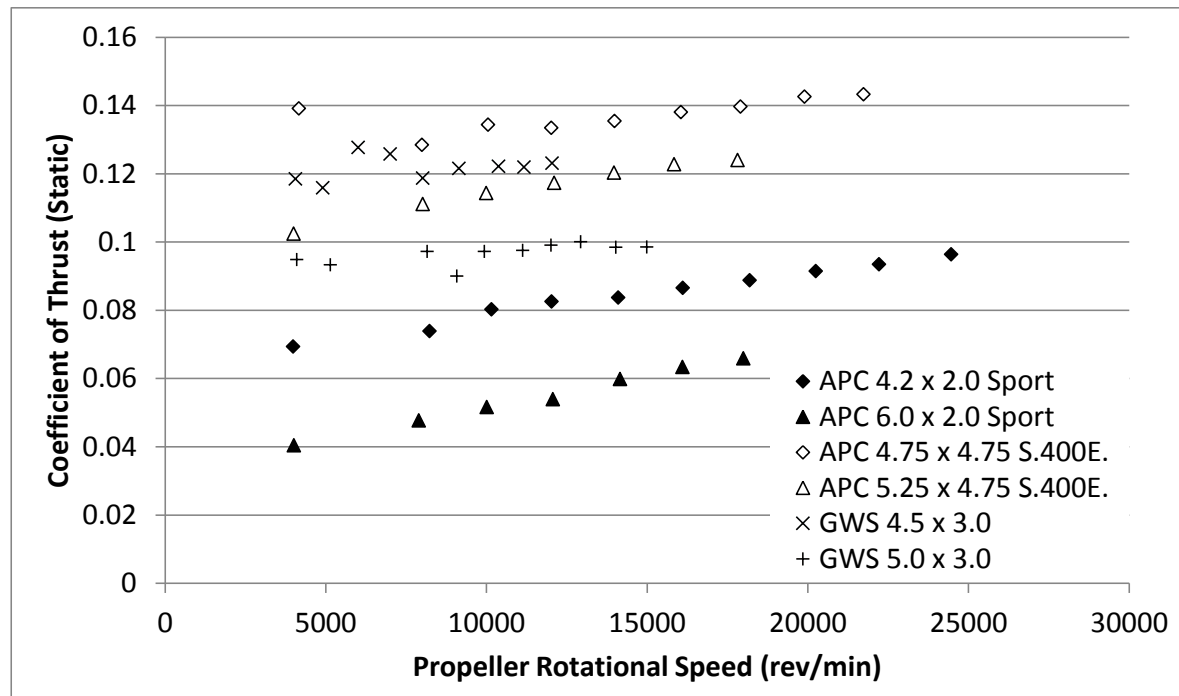

(a)

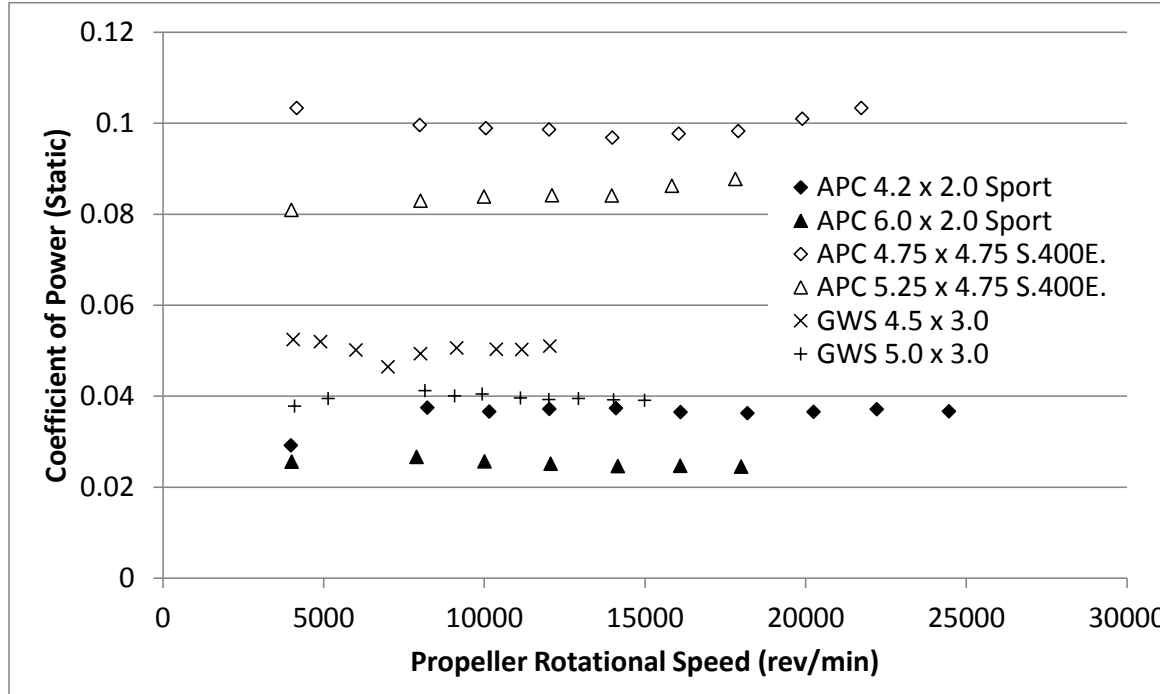

(b)

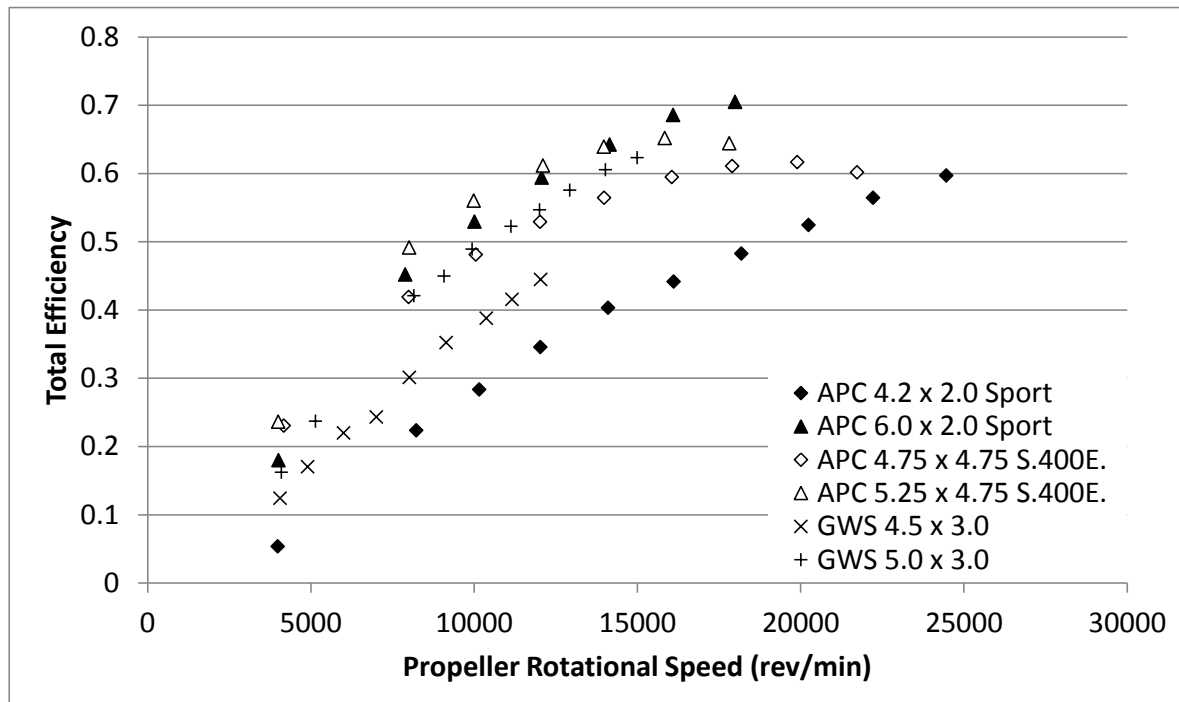

(c)

Figure 7: The Effect of Varying Propeller Diameter While Holding Pitch Constant: (a) Static Coefficient of Thrust, (b) Static Coefficient of Power, (c) Static Total Efficiency. 


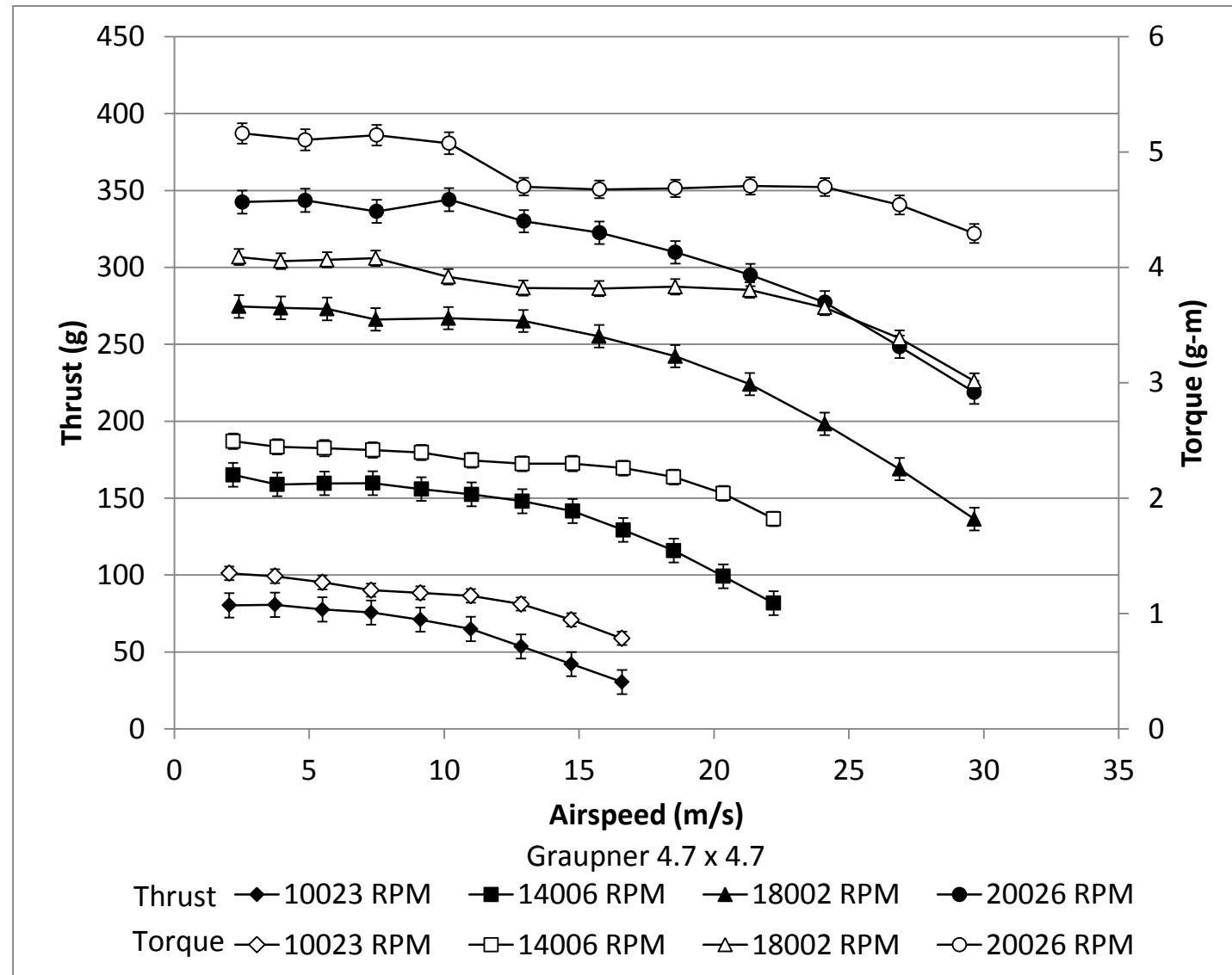

(a)

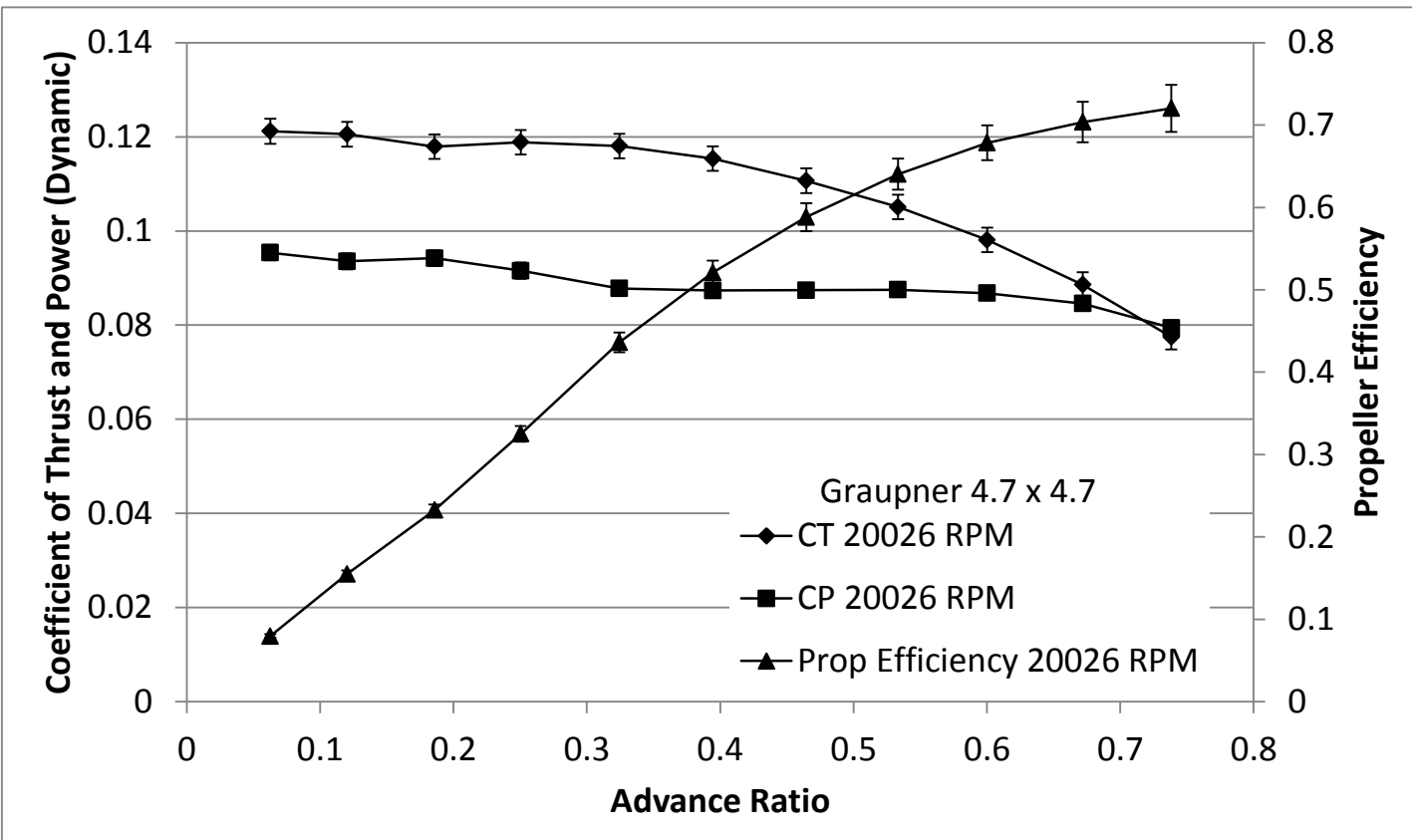

(b)

Figure 8: Typical Dynamic Test Results (Graupner $4.7 \times 4.7$ inch Propeller): (a)Thrust and Torque Versus Airspeed for Various Rotational Speeds, (b) Coefficient of Thrust, Power and Propeller Efficiency Versus Advance Ratio. 


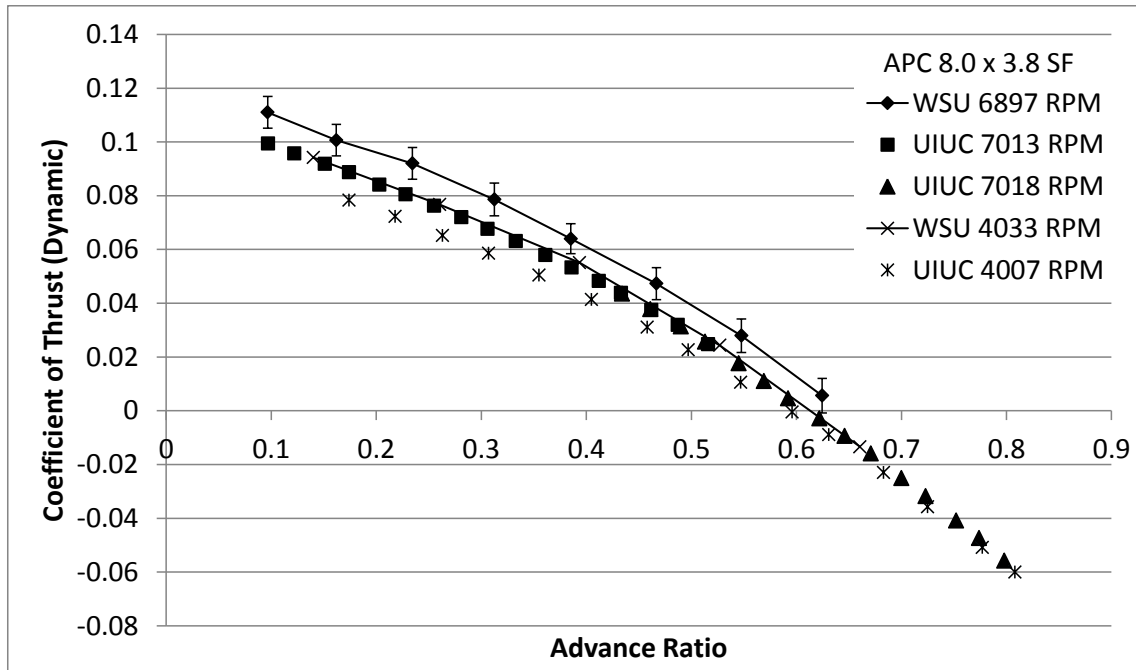

(a)

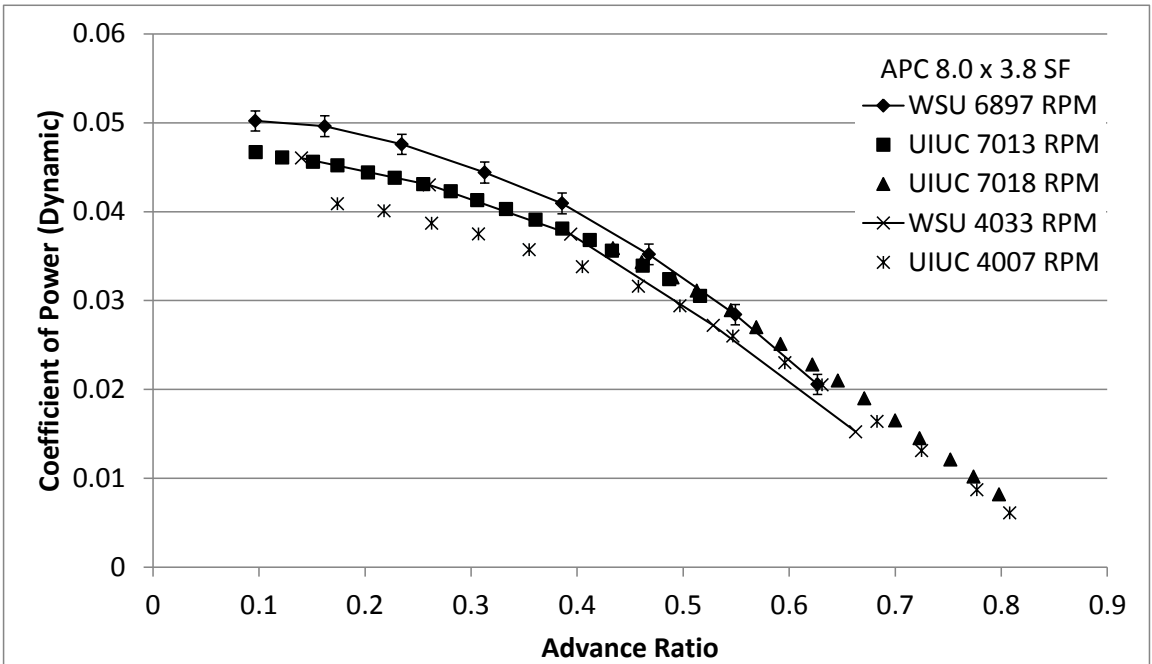

(b)

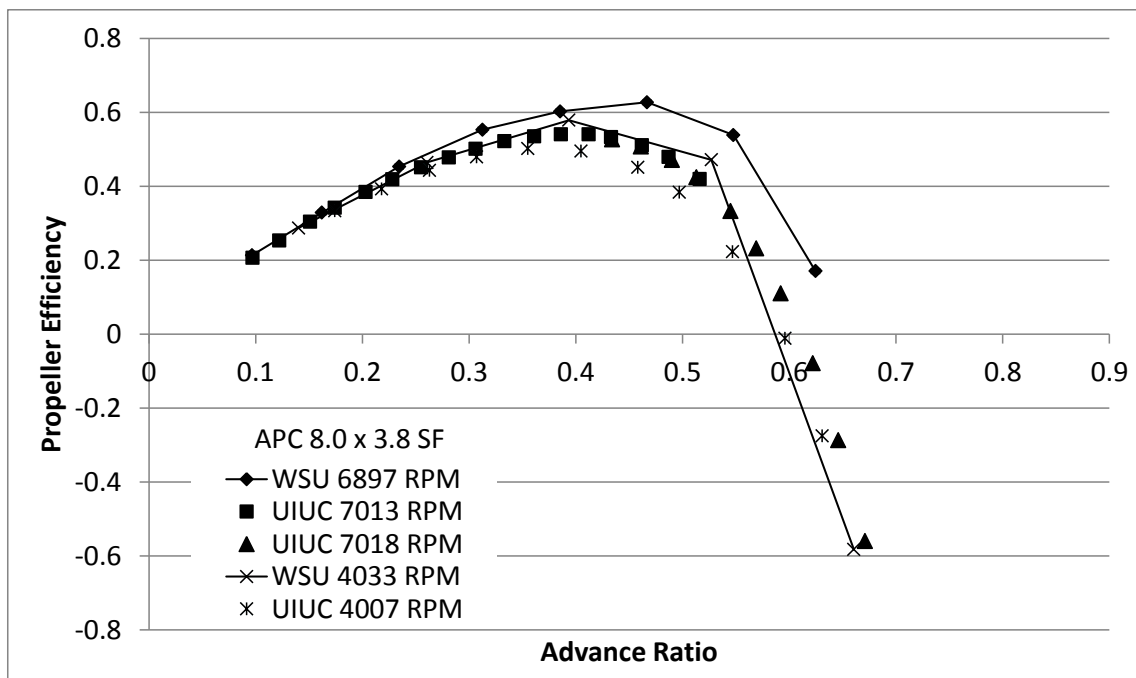

(c)

Figure 9: Comparison of Present Results to Selig (2012) (APC 8.0 × 3.8 SF): (a) Dynamic Coefficient of Thrust, (b) Dynamic Coefficient of Power, (c) Propeller Efficiency. 


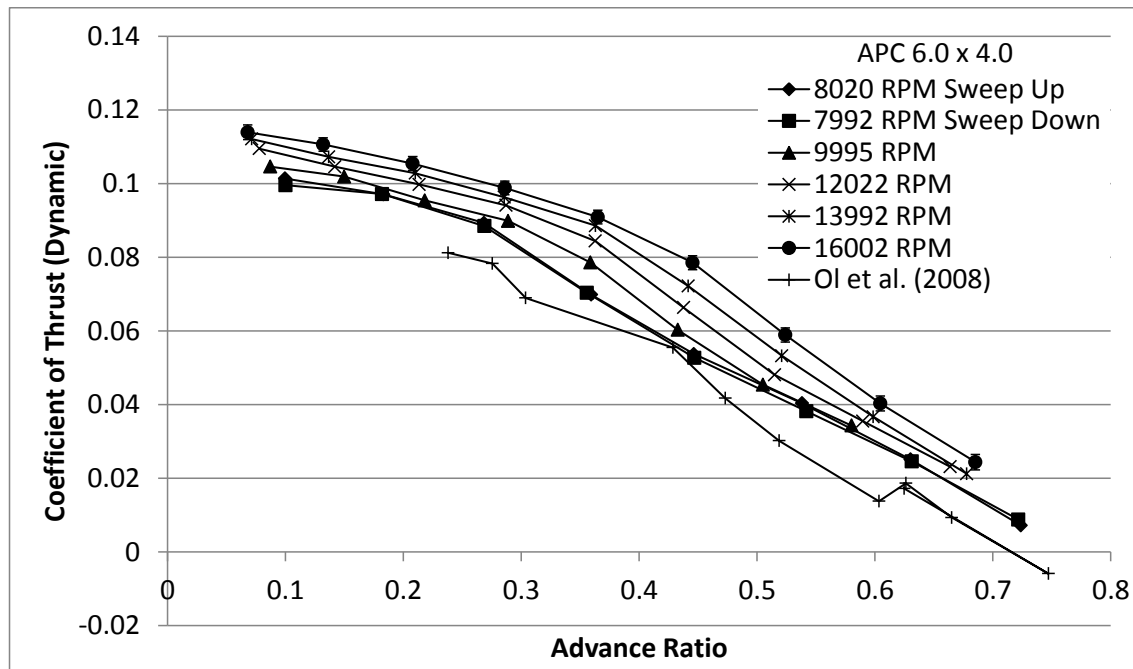

(a)

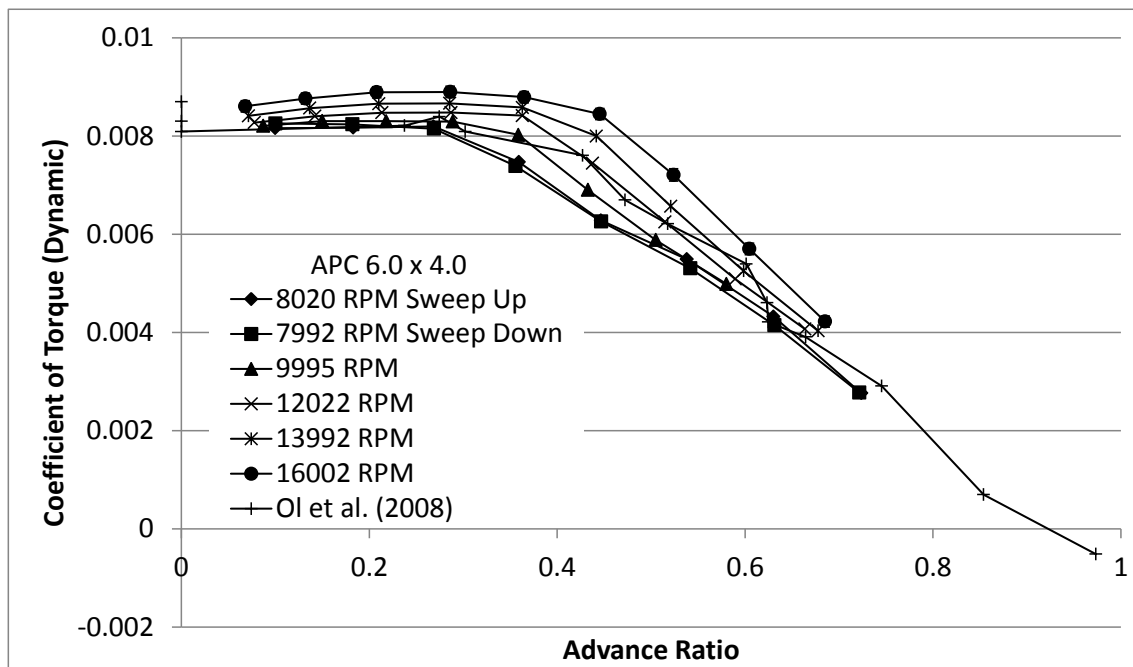

(b)

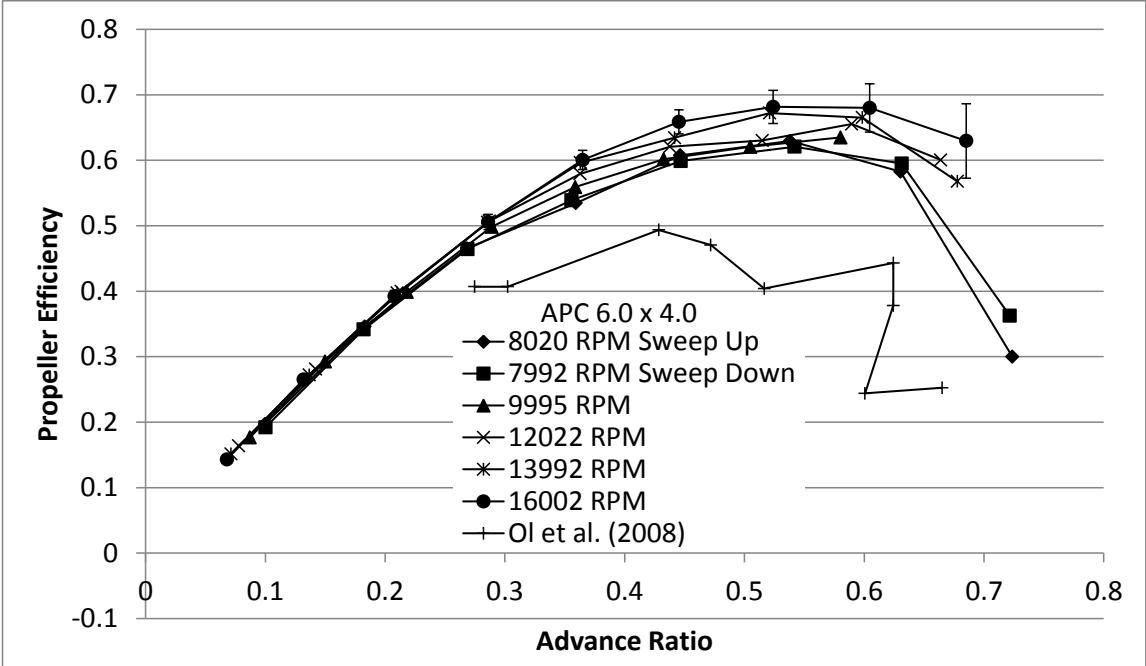

(c)

Figure 10: Comparison of Present Results to Ol et al. (2008) (APC $6.0 \times 4.0)$ : (a) Dynamic Coefficient of Thrust, (b) Dynamic Coefficient of Torque, (c) Propeller Efficiency. 


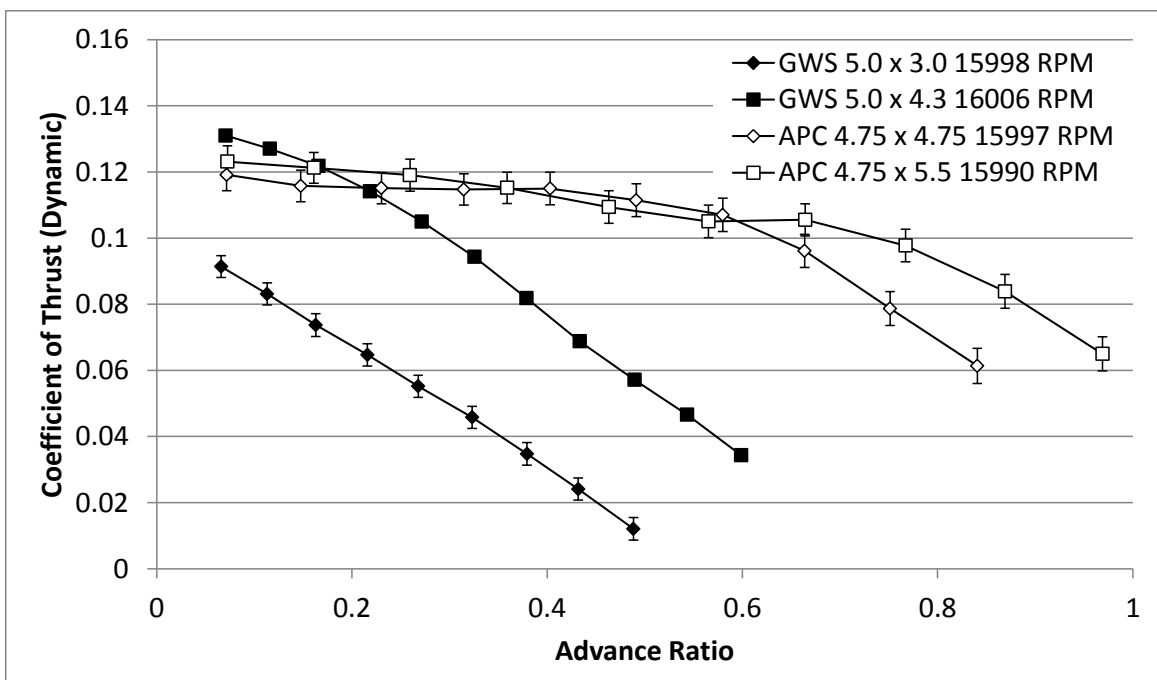

(a)

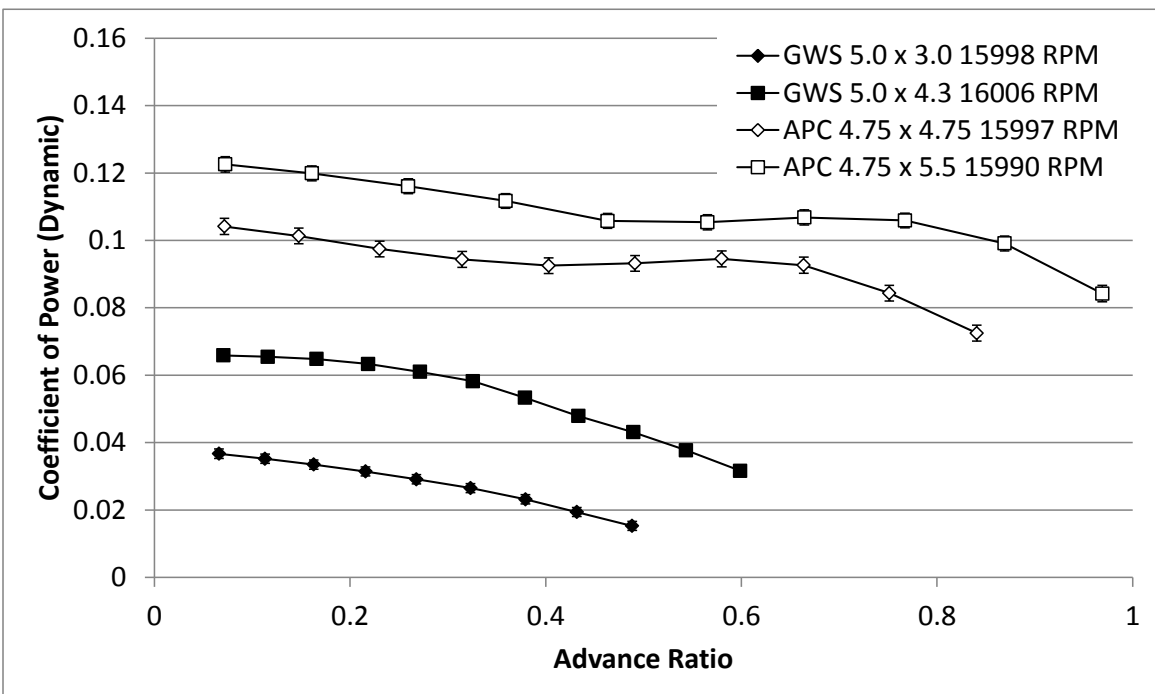

(b)

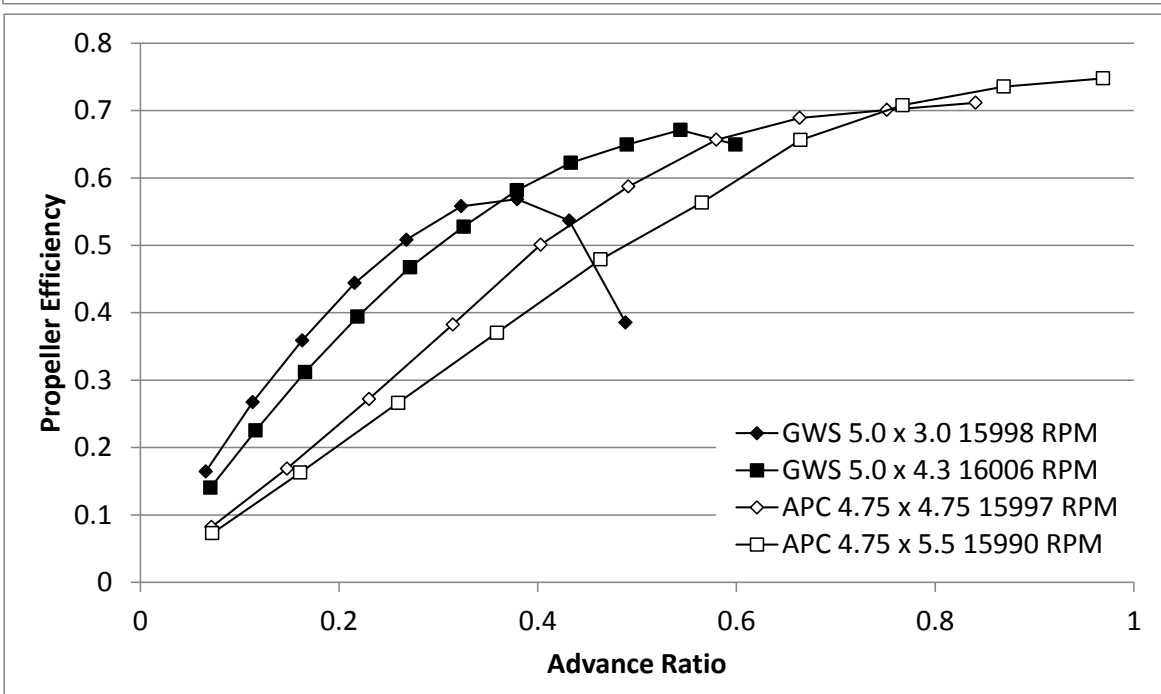

(c)

Figure 11: The Effect of Varying Propeller Pitch While Holding Diameter Constant: (a)

Dynamic Coefficient of Thrust, (b) Dynamic Coefficient of Power, (c) Propeller Efficiency. 


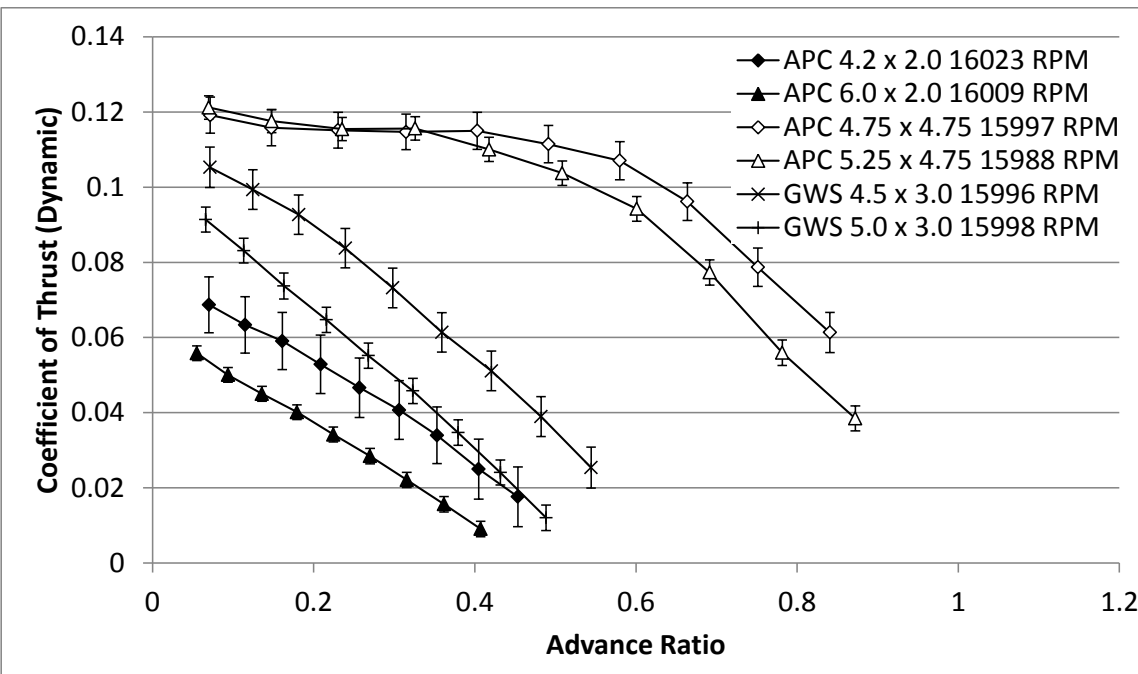

(a)
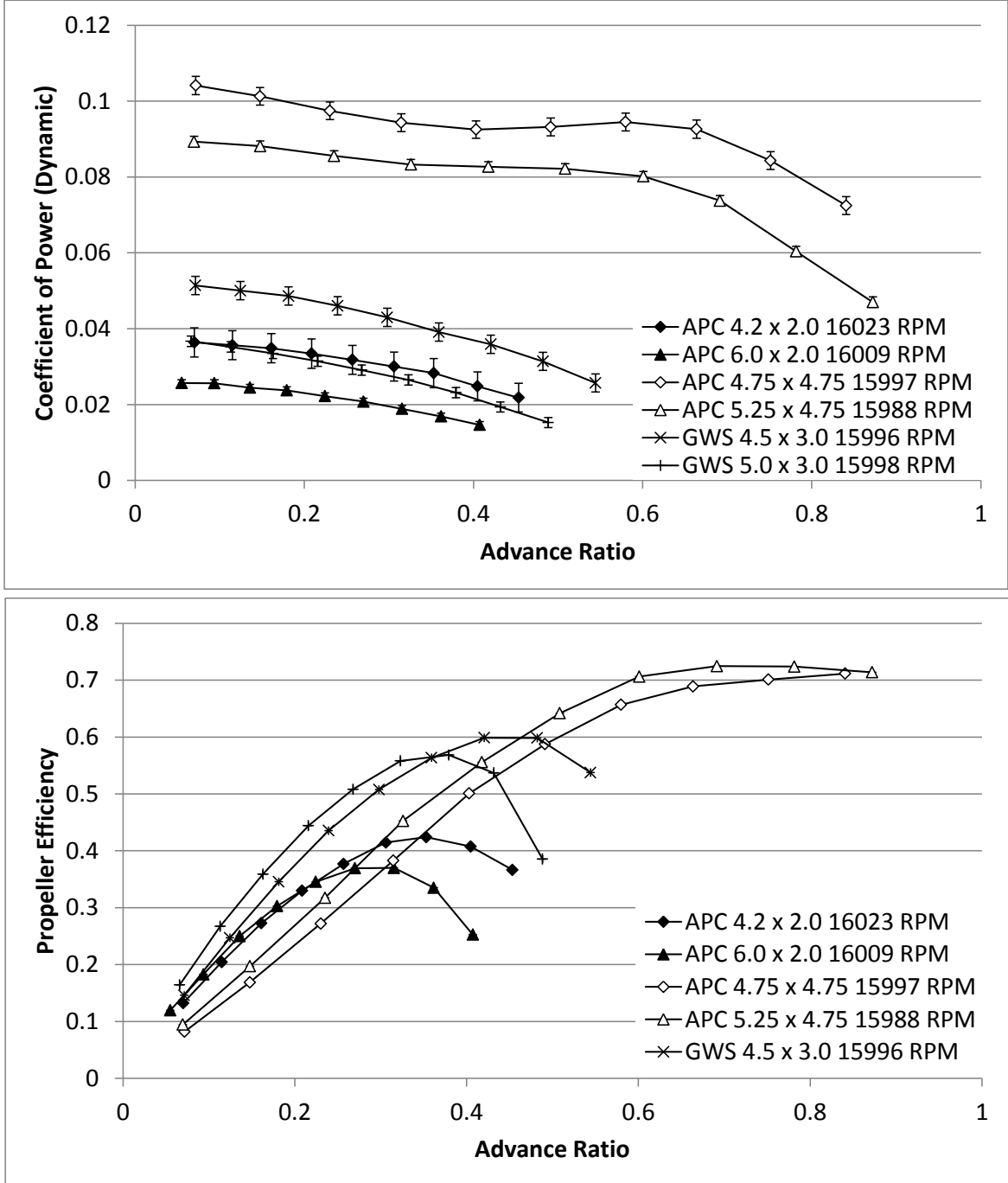

(b)

(c)

Figure 12: The Effect of Varying Propeller Diameter While Holding Pitch Constant: (a)

Dynamic Coefficient of Thrust, (b) Dynamic Coefficient of Power, (c) Propeller Efficiency. 


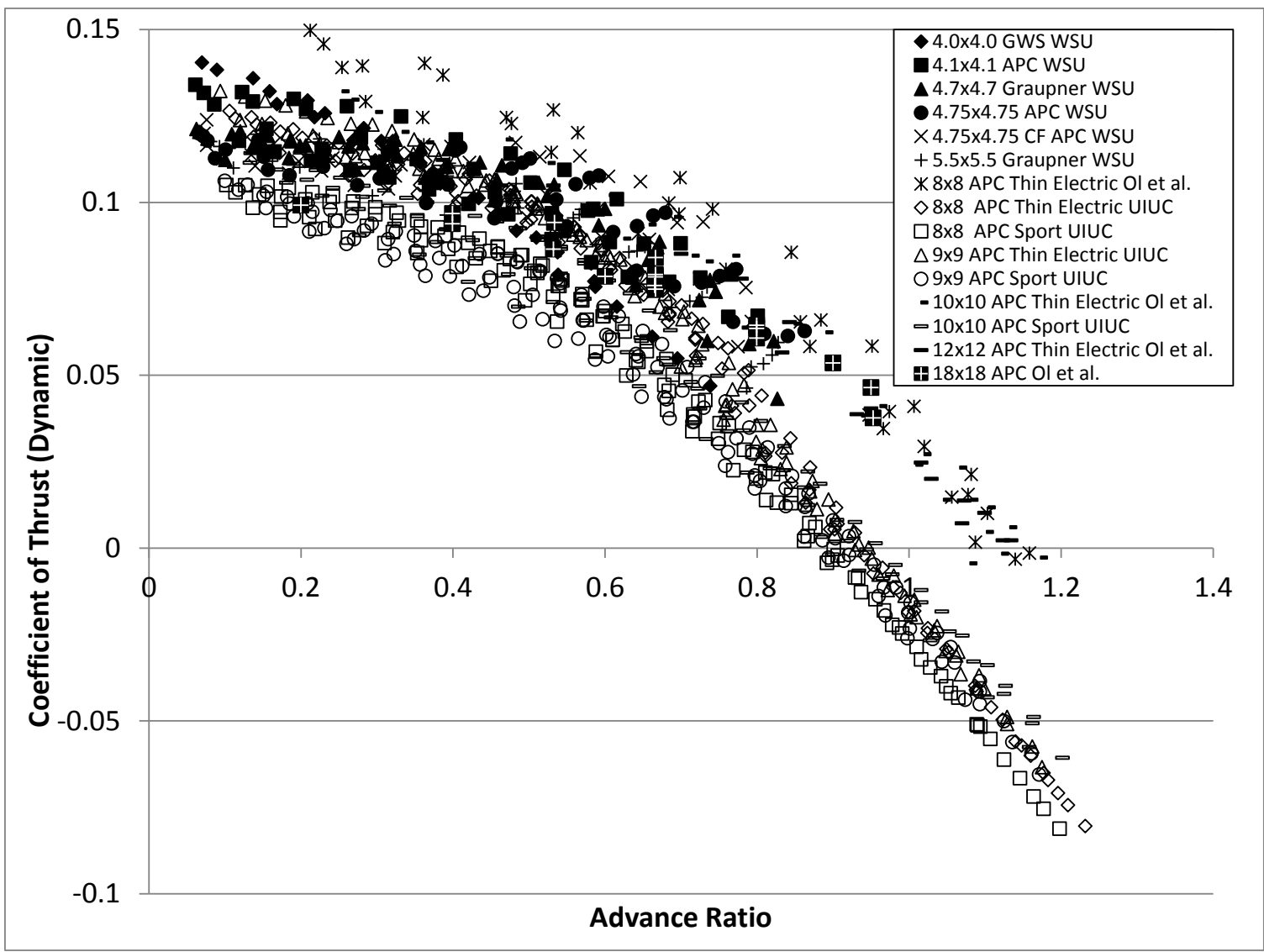

Figure 13: Coefficient of Thrust Versus Advance Ratio for Square Propellers $(D / P=1.0)$ with Diameter Ranging from $4.0 \leq D \leq 18$ inches. 

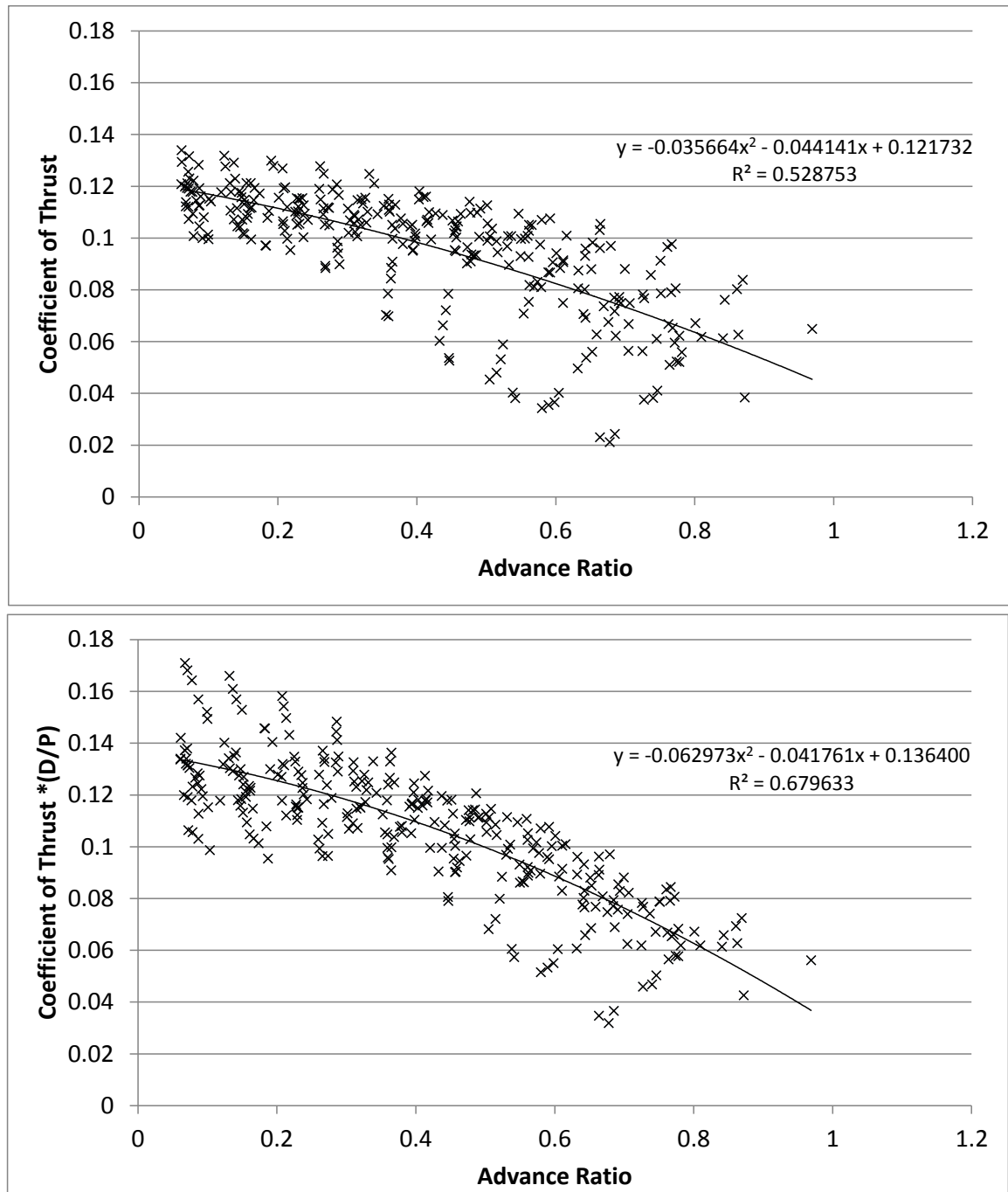

(a)

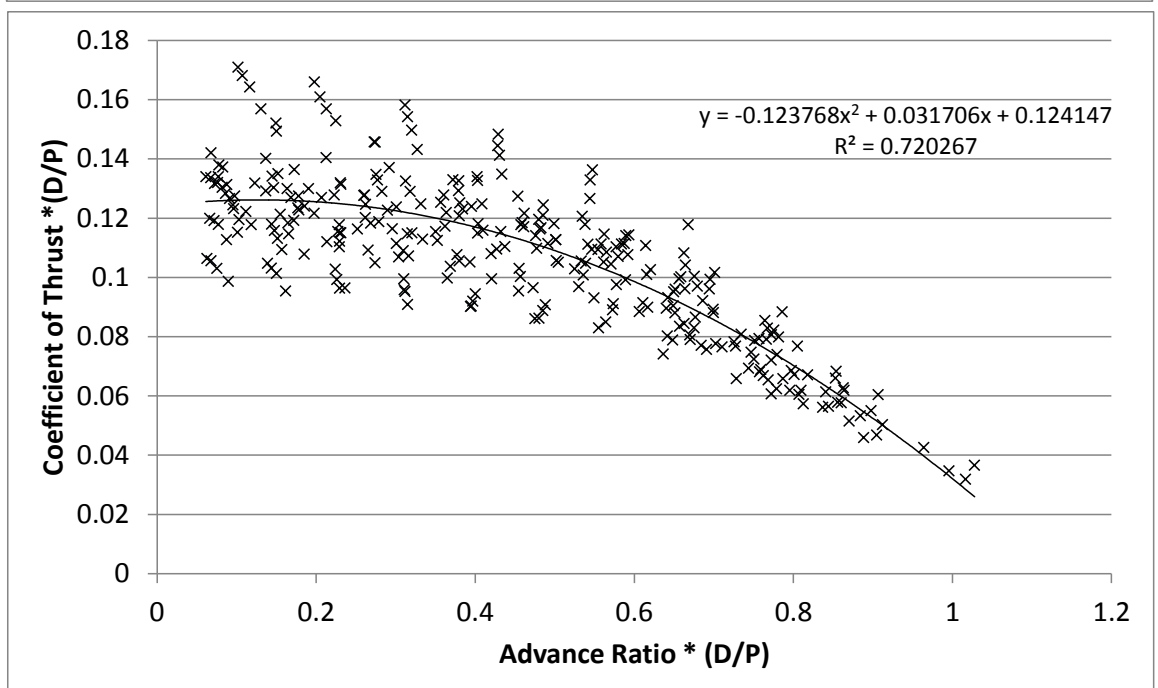

(b)

(c)

Figure 14: Coefficient of Thrust Versus Advance Ratio for the APC Sport 400 Electric Propellers $\left(\Delta C_{\mathrm{T}} \leq 20 \%\right)$ : (a) Original Representation of $C_{\mathrm{T}}$; (b) $C_{\mathrm{T}}$ Modified by the Diameter to Pitch Ratio, (c) $C_{\mathrm{T}}$ and $J$ Modified by the Diameter to Pitch Ratio 


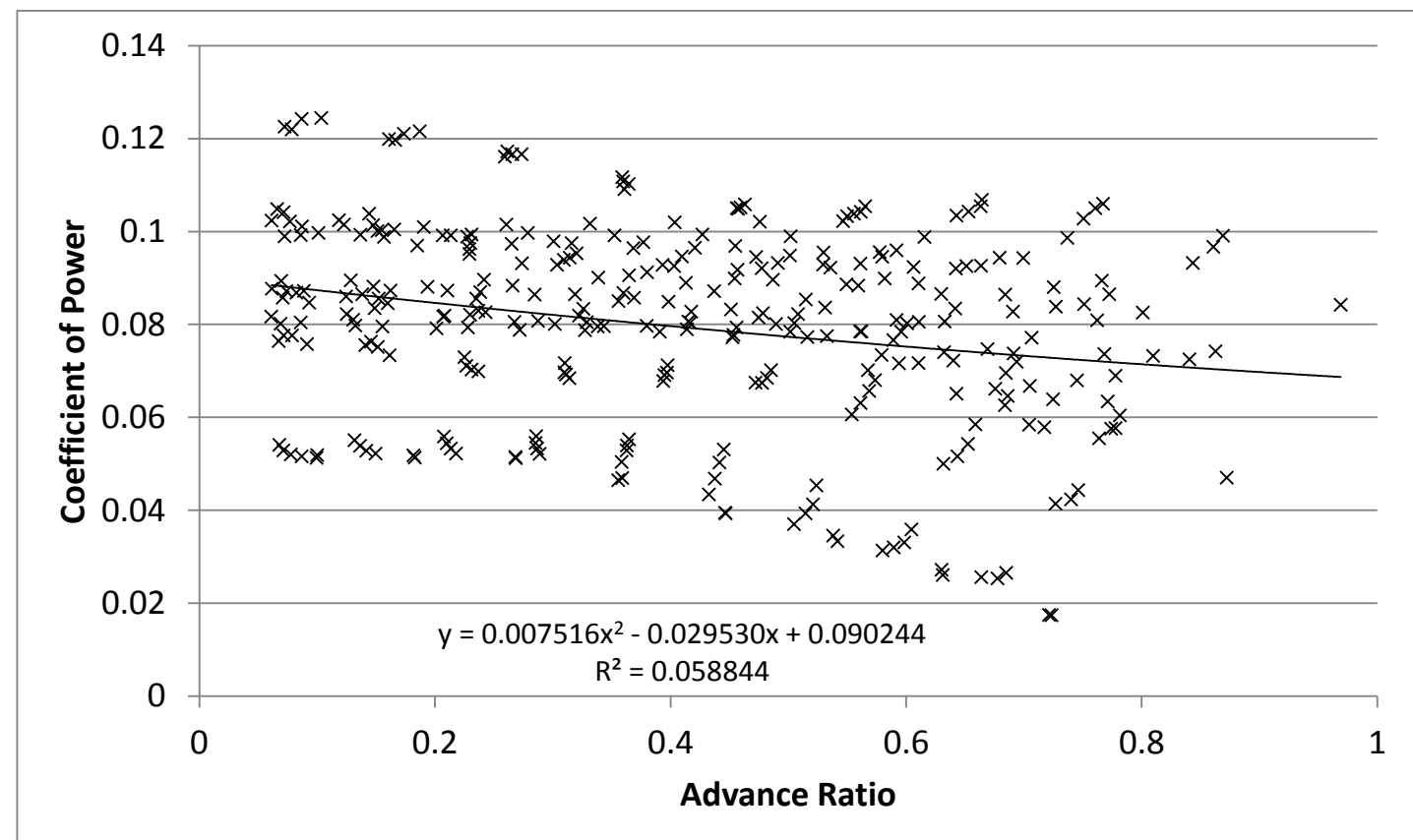

(a)

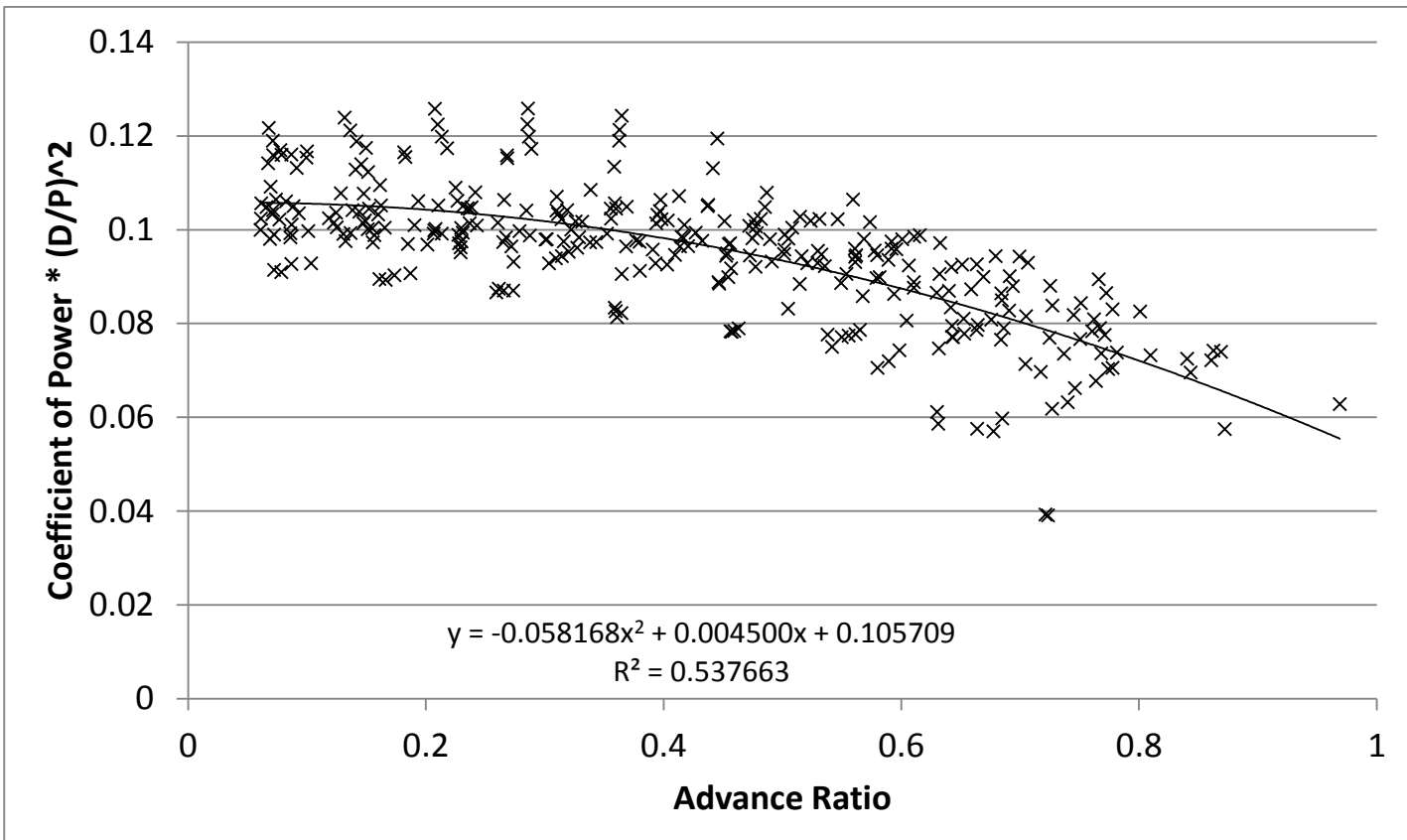

(b)

Figure 15: Coefficient of Power Versus Advance Ratio for the APC Sport 400 Electric Propellers $\left(\Delta C_{\mathrm{P}} \leq 20 \%\right)$ : (a) Original Representation of $C_{\mathrm{P}}$; (b) $C_{\mathrm{P}}$ Modified by the Diameter to Pitch Ratio. 


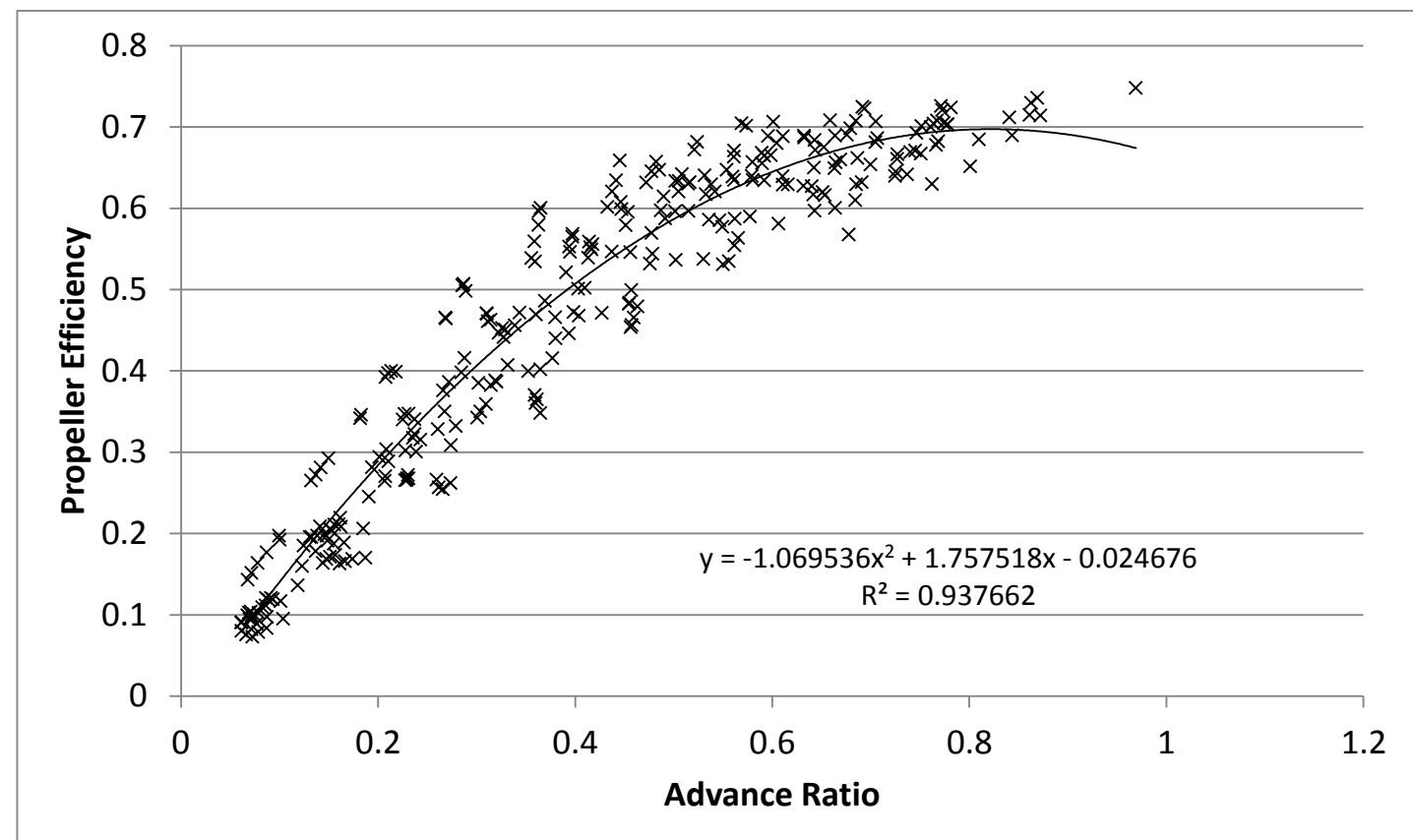

(a)

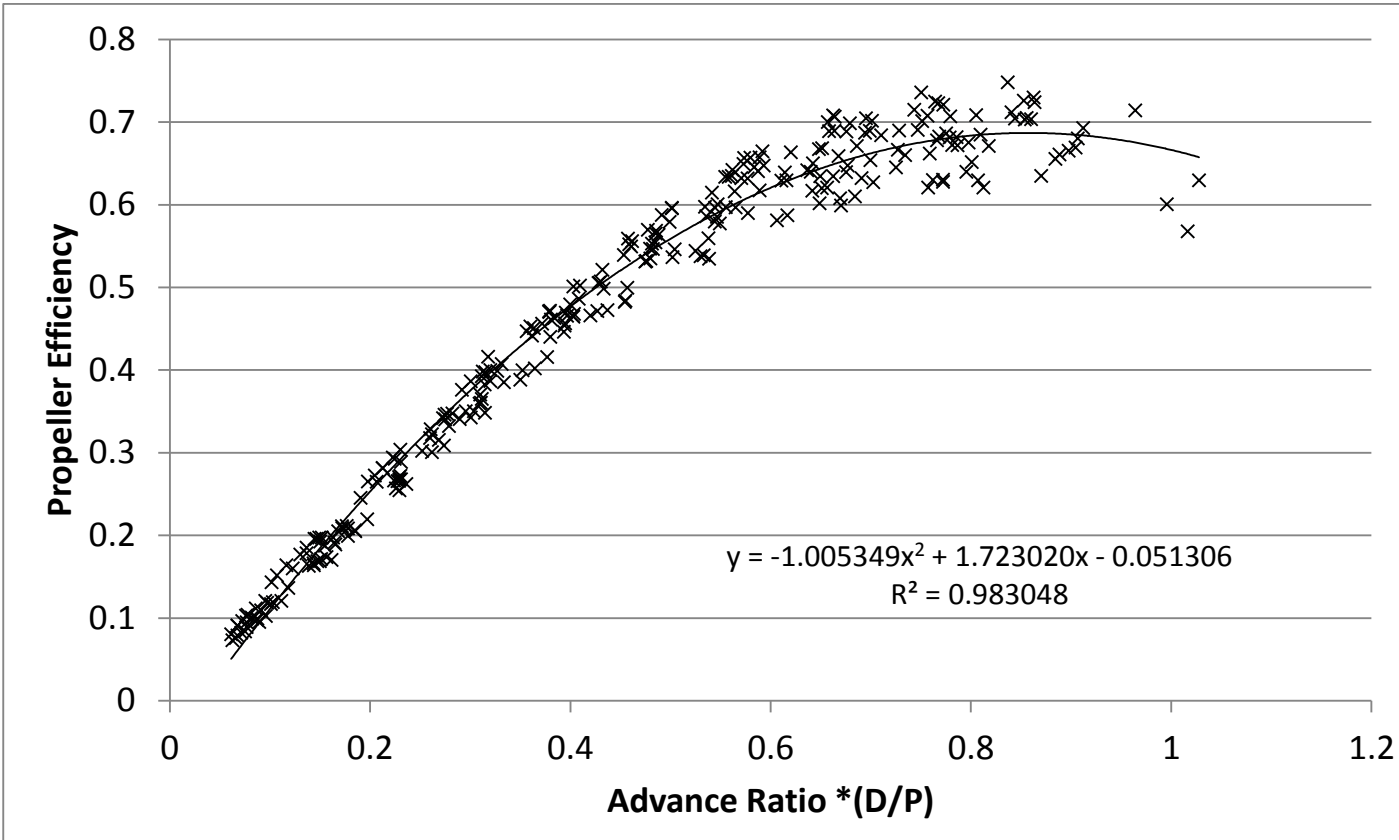

(b)

Figure 16: Propeller Efficiency Versus Advance Ratio for the APC Sport 400 Electric Propellers $\left(\Delta \eta_{\mathrm{P}} \leq 20 \%\right)$ : (a) Original Representation of $\eta_{\mathrm{P}}$; (b) Advance Ratio Modified by the Diameter to Pitch Ratio. 
Table 1: Uncertainties of Primary Measurement Devices and Calibration Sources.

\begin{tabular}{|c|c|c|}
\hline Variable & Measurement Device & Uncertainty \\
\hline Thrust, $T$ & $\begin{array}{l}\text { Transducer Techniques LSP 1kg } \\
\text { Load Cell }\end{array}$ & $\Delta T_{\text {cal }}= \pm 7.70 \mathrm{~g}$ \\
\hline Torque, $Q$ & $\begin{array}{c}\text { Transducer Techniques RTS } 25 \\
\text { oz-in Reaction Torque Sensor }\end{array}$ & $\Delta Q_{\mathrm{cal}}= \pm 0.0498 \mathrm{~g}-\mathrm{m}$ \\
\hline $\begin{array}{c}\text { Atmospheric } \\
\text { Temperature, } T_{\text {atm }}\end{array}$ & Omega Type E Thermocouple & $\Delta T_{\mathrm{atm}, \mathrm{cal}}= \pm 0.0334^{\circ} \mathrm{C}$ \\
\hline Calibration Mass & Ohaus Digital Scale & $\Delta m= \pm 1.00 \times 10^{-3} \mathrm{~g}$ \\
\hline Propeller Diameter, $D$ & Digital Vernier Calipers & $\Delta D= \pm 1.00 \times 10^{-5} \mathrm{~m}$ \\
\hline $\begin{array}{c}\text { Propeller Chord Length } \\
\text { at } 75 \% \text { Radius, } C_{0.75}\end{array}$ & Digital Vernier Calipers & $\Delta C_{0.75}= \pm 1.00 \times 10^{-5} \mathrm{~m}$ \\
\hline $\begin{array}{l}\text { Propeller Rotational } \\
\text { Speed, } n\end{array}$ & $\begin{array}{l}\text { Monarch Instruments Remote } \\
\text { Optical Sensor (ROS) and ACT } \\
\text { 3x Panel Tachometer }\end{array}$ & $\Delta n= \pm 1 \mathrm{RPM}$ \\
\hline Motor Voltage, $V$ & $\begin{array}{c}\text { National Instruments USB-4065 } \\
\text { Digital Multi-Meter }\end{array}$ & $\Delta V= \pm 1.00 \times 10^{-3} \mathrm{~V}$ \\
\hline Motor Current, $I$ & $\begin{array}{l}\text { CR Magnetics CR5210-30 } \\
\text { Current Transducer }\end{array}$ & $\Delta I= \pm(1 \% \times$ Reading $)$ \\
\hline $\begin{array}{c}\text { Atmospheric Pressure, } \\
P_{\text {abs }}\end{array}$ & Vaisala PTB110 Barometer & $\Delta P_{\mathrm{atm}}= \pm 30.0 \mathrm{~Pa}$ \\
\hline $\begin{array}{l}\text { Pitot Tube Differential } \\
\text { Pressure, } P_{\text {diff }}\end{array}$ & $\begin{array}{l}\text { MKS 226A Differential Pressure } \\
\text { Manometer }\end{array}$ & $\Delta P_{\text {diff }}= \pm(0.3 \% \times$ Reading $)$ \\
\hline
\end{tabular}


Table 2: Summary of Propeller Measurements.

\begin{tabular}{|c|c|c|c|c|c|}
\hline Manufacturer & $\begin{array}{l}\text { Nominal } D / P \\
\quad(\text { in } \times \text { in }) \\
(\mathrm{mm} \times \mathbf{m m})\end{array}$ & $\begin{array}{c}\text { Propeller } \\
\text { Number }\end{array}$ & $\begin{array}{c}D \\
(\mathbf{m m})\end{array}$ & $\begin{array}{l}R_{\mathbf{7 5} \%} \\
(\mathbf{m m})\end{array}$ & $\begin{array}{l}C_{75 \%} \\
(\mathbf{m m})\end{array}$ \\
\hline \multirow{4}{*}{ APC } & \multirow{4}{*}{$\begin{array}{c}4.10 \times 4.10 \\
\text { Speed } 400 \text { Electric } \\
(104.1 \times 104.1)\end{array}$} & 1 & 103.62 & 38.86 & 8.09 \\
\hline & & 2 & 103.65 & 38.87 & 8.09 \\
\hline & & 3 & 103.67 & 38.88 & 8.11 \\
\hline & & Average & 103.65 & 38.87 & 8.10 \\
\hline \multirow{4}{*}{ APC } & \multirow{4}{*}{$\begin{array}{c}4.20 \times 2.00 \\
\text { Sport } \\
(106.7 \times 50.8)\end{array}$} & 1 & 105.78 & 39.67 & 8.76 \\
\hline & & 2 & 105.81 & 39.68 & 8.65 \\
\hline & & 3 & 105.79 & 39.67 & 8.67 \\
\hline & & Average & 105.79 & 39.67 & 8.69 \\
\hline \multirow{4}{*}{ APC } & \multirow{4}{*}{$\begin{array}{c}4.20 \times 4.00 \\
\text { Free Flight } \\
(106.7 \times 101.6)\end{array}$} & 1 & 106.21 & 39.83 & 8.82 \\
\hline & & 2 & 106.60 & 39.98 & 8.76 \\
\hline & & 3 & 106.18 & 39.82 & 8.76 \\
\hline & & Average & 106.33 & 39.88 & 8.78 \\
\hline \multirow{4}{*}{ APC } & \multirow{4}{*}{$\begin{array}{c}4.50 \times 4.10 \\
\text { Speed } 400 \text { Electric } \\
(114.3 \times 104.1)\end{array}$} & 1 & 113.95 & 42.73 & 8.65 \\
\hline & & 2 & 113.89 & 42.71 & 8.55 \\
\hline & & 3 & 113.80 & 42.68 & 8.62 \\
\hline & & Average & 113.88 & 42.71 & 8.61 \\
\hline \multirow{4}{*}{ APC } & \multirow{4}{*}{$\begin{array}{c}4.70 \times 4.25 \\
\text { Speed } 400 \text { Electric } \\
(119.4 \times 108.0)\end{array}$} & 1 & 120.31 & 45.12 & 8.45 \\
\hline & & 2 & 120.27 & 45.10 & 8.42 \\
\hline & & 3 & 120.02 & 45.01 & 8.39 \\
\hline & & Average & 120.20 & 45.08 & 8.42 \\
\hline \multirow{4}{*}{ APC } & \multirow{4}{*}{$\begin{array}{c}4.75 \times 4.75 \\
\text { Speed } 400 \text { Electric } \\
(120.7 \times 120.7)\end{array}$} & 1 & 120.12 & 45.05 & 8.16 \\
\hline & & 2 & 119.91 & 44.97 & 8.28 \\
\hline & & 3 & 119.58 & 44.84 & 8.14 \\
\hline & & Average & 119.87 & 44.95 & 8.19 \\
\hline \multirow{4}{*}{ APC } & \multirow{4}{*}{$\begin{array}{c}4.75 \times 5.50 \\
\text { Speed } 400 \text { Electric } \\
(120.7 \times 139.7)\end{array}$} & 1 & 119.87 & 44.95 & 8.00 \\
\hline & & 2 & 119.90 & 44.96 & 8.03 \\
\hline & & 3 & 119.99 & 45.00 & 8.29 \\
\hline & & Average & 119.92 & 44.97 & 8.11 \\
\hline \multirow{4}{*}{ APC } & \multirow{4}{*}{$\begin{array}{c}5.10 \times 4.50 \mathrm{E} \\
\text { Thin Electric } \\
(129.5 \times 114.3)\end{array}$} & 1 & 129.37 & 48.51 & 15.17 \\
\hline & & 2 & 129.39 & 48.52 & 15.27 \\
\hline & & 3 & 129.44 & 48.54 & 15.29 \\
\hline & & Average & 129.40 & 48.52 & 15.24 \\
\hline \multirow{4}{*}{ APC } & \multirow{4}{*}{$\begin{array}{c}5.25 \times 4.75 \\
\text { Speed } 400 \text { Electric } \\
(133.4 \times 120.7)\end{array}$} & 1 & 132.75 & 49.78 & 9.33 \\
\hline & & 2 & 132.65 & 49.74 & 9.30 \\
\hline & & 3 & 132.79 & 49.80 & 9.32 \\
\hline & & Average & 132.73 & 49.77 & 9.32 \\
\hline \multirow{4}{*}{ APC } & \multirow{4}{*}{$\begin{array}{c}5.50 \times 2.00 \\
\text { Free Flight } \\
(139.7 \times 50.8)\end{array}$} & 1 & 139.30 & 52.24 & 9.78 \\
\hline & & 2 & 139.21 & 52.20 & 9.81 \\
\hline & & 3 & 139.23 & 52.21 & 9.84 \\
\hline & & Average & 139.25 & 52.22 & 9.81 \\
\hline
\end{tabular}


Table 3: Summary of Propeller Measurements, cont.

\begin{tabular}{|c|c|c|c|c|c|}
\hline Manufacturer & $\begin{array}{l}\text { Nominal } D / P \\
\quad(\mathrm{in} \times \mathrm{in}) \\
(\mathrm{mm} \times \mathrm{mm})\end{array}$ & $\begin{array}{l}\text { Propeller } \\
\text { Number }\end{array}$ & $\begin{array}{c}D \\
(\mathbf{m m})\end{array}$ & $\begin{array}{l}R_{75 \%} \\
(\mathbf{m m})\end{array}$ & $\begin{array}{l}C_{75 \%} \\
(\mathbf{m m})\end{array}$ \\
\hline \multirow{4}{*}{ APC } & \multirow{4}{*}{$\begin{array}{c}5.50 \times 4.50 \\
\text { Speed } 400 \text { Electric } \\
(139.7 \times 114.3)\end{array}$} & 1 & 139.30 & 52.24 & 9.60 \\
\hline & & 2 & 139.46 & 52.30 & 9.53 \\
\hline & & 3 & 139.47 & 52.30 & 9.55 \\
\hline & & Average & 139.41 & 52.28 & 9.56 \\
\hline \multirow{4}{*}{ APC } & \multirow{4}{*}{$\begin{array}{c}6.00 \times 2.00 \\
\text { Sport } \\
(152.4 \times 50.8)\end{array}$} & 1 & 152.25 & 57.09 & 8.69 \\
\hline & & 2 & 152.19 & 57.07 & 9.06 \\
\hline & & 3 & 152.14 & 57.05 & 8.87 \\
\hline & & Average & 152.19 & 57.07 & 8.87 \\
\hline \multirow{4}{*}{ APC } & \multirow{4}{*}{$\begin{array}{c}6.00 \times 4.00 \mathrm{E} \\
\text { Speed } 400 \text { Electric } \\
(152.4 \times 101.6)\end{array}$} & 1 & 152.09 & 57.03 & 10.35 \\
\hline & & 2 & 151.74 & 56.90 & 10.26 \\
\hline & & 3 & 151.83 & 56.94 & 10.51 \\
\hline & & Average & 151.89 & 56.96 & 10.37 \\
\hline APC & $\begin{array}{c}8.00 \times 3.8 \\
\text { Slow Flyer } \\
(203.2 \times 96.5) \\
\end{array}$ & 1 & 203.65 & 76.37 & 20.79 \\
\hline \multirow{4}{*}{ Graupner } & \multirow{4}{*}{$\begin{array}{c}4.00 \times 3.00 \\
\text { Cam Speed } \\
(101.6 \times 76.2)\end{array}$} & 1 & 99.77 & 37.41 & 9.60 \\
\hline & & 2 & 100.06 & 37.52 & 9.40 \\
\hline & & 3 & 99.94 & 37.48 & 9.38 \\
\hline & & Average & 99.92 & 37.47 & 9.46 \\
\hline \multirow{4}{*}{ Graupner } & \multirow{4}{*}{$\begin{array}{c}4.70 \times 4.00 \\
\text { Cam Speed } \\
(119.4 \times 101.6)\end{array}$} & 1 & 119.16 & 44.69 & 9.47 \\
\hline & & 2 & 119.16 & 44.69 & 9.49 \\
\hline & & 3 & 118.96 & 44.61 & 9.39 \\
\hline & & Average & 119.09 & 44.66 & 9.45 \\
\hline \multirow{4}{*}{ Graupner } & \multirow{4}{*}{$\begin{array}{c}4.70 \times 4.70 \\
\text { Cam Speed } \\
(119.4 \times 119.4)\end{array}$} & 1 & 120.22 & 45.08 & 8.96 \\
\hline & & 2 & 120.22 & 45.08 & 8.95 \\
\hline & & 3 & 120.49 & 45.18 & 9.01 \\
\hline & & Average & 120.31 & 45.11 & 8.97 \\
\hline \multirow{4}{*}{ Graupner } & \multirow{4}{*}{$\begin{array}{c}5.50 \times 4.30 \\
\text { Cam Speed } \\
(139.7 \times 109.2)\end{array}$} & 1 & 141.11 & 52.92 & 10.58 \\
\hline & & 2 & 141.08 & 52.91 & 10.42 \\
\hline & & 3 & 141.13 & 52.92 & 10.52 \\
\hline & & Average & 141.11 & 52.92 & 10.51 \\
\hline \multirow{4}{*}{ Graupner } & \multirow{4}{*}{$\begin{array}{c}5.50 \times 5.50 \\
\text { Cam Speed } \\
(139.7 \times 139.7)\end{array}$} & 1 & 139.60 & 52.35 & 9.82 \\
\hline & & 2 & 140.37 & 52.64 & 9.97 \\
\hline & & 3 & 140.17 & 52.56 & 9.91 \\
\hline & & Average & 140.05 & 52.52 & 9.90 \\
\hline \multirow{4}{*}{ GWS } & \multirow{4}{*}{$\begin{array}{c}4.00 \times 2.50 \\
(101.6 \times 63.5)\end{array}$} & 1 & 101.57 & 38.09 & 12.39 \\
\hline & & 2 & 101.57 & 38.09 & 12.34 \\
\hline & & 3 & 101.53 & 38.07 & 12.40 \\
\hline & & Average & 101.56 & 38.08 & 12.38 \\
\hline
\end{tabular}


Table 4: Summary of Propeller Measurements, cont.

\begin{tabular}{|c|c|c|c|c|c|}
\hline Manufacturer & $\begin{array}{c}\text { Nominal } D / P \\
(\text { in } \times \text { in }) \\
(\mathbf{m m} \times \mathbf{m m}) \\
\end{array}$ & $\begin{array}{l}\text { Propeller } \\
\text { Number }\end{array}$ & $\begin{array}{c}D \\
(\mathbf{m m})\end{array}$ & $\begin{array}{l}R_{\mathbf{7 5} \%} \\
(\mathbf{m m})\end{array}$ & $\begin{array}{l}C_{75 \%} \\
(\mathbf{m m})\end{array}$ \\
\hline \multirow{4}{*}{ GWS } & \multirow{4}{*}{$\begin{array}{c}4.00 \times 4.00 \\
(101.6 \times 101.6)\end{array}$} & 1 & 101.98 & 38.24 & 10.38 \\
\hline & & 2 & 102.00 & 38.25 & 10.44 \\
\hline & & 3 & 101.99 & 38.25 & 10.48 \\
\hline & & Average & 101.99 & 38.25 & 10.43 \\
\hline \multirow{4}{*}{ GWS } & \multirow{4}{*}{$\begin{array}{c}4.50 \times 3.00 \\
(114.3 \times 76.2)\end{array}$} & 1 & 114.32 & 42.87 & 11.09 \\
\hline & & 2 & 114.26 & 42.85 & 10.71 \\
\hline & & 3 & 114.28 & 42.86 & 10.77 \\
\hline & & Average & 114.29 & 42.86 & 10.86 \\
\hline \multirow{4}{*}{ GWS } & \multirow{4}{*}{$\begin{array}{c}5.00 \times 3.00 \\
(127.0 \times 76.2)\end{array}$} & 1 & 127.23 & 47.71 & 12.43 \\
\hline & & 2 & 127.16 & 47.69 & 12.36 \\
\hline & & 3 & 127.16 & 47.69 & 12.39 \\
\hline & & Average & 127.18 & 47.70 & 12.40 \\
\hline \multirow{4}{*}{ GWS } & \multirow{4}{*}{$\begin{array}{c}5.00 \times 4.30 \\
(127.0 \times 109.2)\end{array}$} & 1 & 126.89 & 47.58 & 12.69 \\
\hline & & 2 & 126.83 & 47.56 & 12.53 \\
\hline & & 3 & 127.03 & 47.64 & 12.51 \\
\hline & & Average & 126.92 & 47.59 & 12.58 \\
\hline
\end{tabular}




\section{APPENDIX A: CALIBRATION PROCEDURES AND DATA SHEETS}

The thermocouples were calibrated over their expected operating ranges. The Type $\mathrm{T}$ thermocouple was calibrated over the anticipated range for the motor temperature of 15 to $70^{\circ} \mathrm{C}$ in intervals of $5^{\circ} \mathrm{C}$. The Type $\mathrm{E}$ thermocouple probe was calibrated over the anticipated air temperature range of 15 to $30^{\circ} \mathrm{C}$ in intervals of $5^{\circ} \mathrm{C}$. The two thermocouples were placed into a recirculating constant temperature bath along with a NIST-traceable precision resistance temperature detector (RTD) with a resolution of $\pm 0.001^{\circ} \mathrm{C}$. With the bath temperature set, the RTD and LabVIEW VI were used to determine that steady state had occurred. When steady state was verified, 500 data points for the thermocouples and 150 data points for the RTD were collected. These values for data points required roughly the same amount of time since the RTD uses a slower data transfer rate. Four sources of error were used in accounting for the uncertainty of each thermocouple. These included the uncertainty of the RTD, the $95 \%$ confidence interval of the RTD average temperature, the $95 \%$ confidence interval of the thermocouple average temperature, and the deviation of the actual thermocouple data average with the highest deviation from the linear fit prediction equation.

In order to calibrate the torque cell, two identical arms were cut from the $1 / 8^{\text {th }}$ inch birch plywood using the laser cutter that would attach to the sides of the motor clamp. The two arms were made identical by adding weight to one arm at the center of gravity. Two arms were created so that the torque cell could be calibrated in both directions of rotation simultaneously. This gives a much better idea of the calibration line around the zero load point. Strings were strung through the arms at a distance of nine inches from the center of rotation. Nine inches was selected because the largest weight in 
the calibration set was 50 grams and this weight at nine inches is produces a torque close to the maximum input of the cell. The setup of the torque cell calibration is shown in Figure 17.

The torque sensor was powered and left to warm up for fifteen minutes. Then it was zeroed manually using the signal conditioner and the maximum output was set to five volts. The torque arms were then attached to the motor clamp and the torque produced served as the first data set of five hundred data points. Weights were added until the maximum value of torque was applied, taking five hundred data points at each weight. Lastly the weight was removed and another five hundred points were collected. The sets of data before and after the weight are averaged and this value is subtracted from the average from the calibration data. This is done since the transducer cannot truly be zeroed out and may be slightly more or less than zero. It also helps to account for any drift during the calibration. The cell was then re-zeroed if necessary and the process was repeated for weights from five to fifty grams. Once the cell was calibrated in the clockwise direction, the process was repeated for the opposing direction. These calibrations were replicated twice in each direction to show repeatability and aid in the estimation of uncertainty. The adjusted calibration values were then plotted against the calculated values of torque produced by the overhung weight. A linear best fit line with an intercept at $\mathrm{y}=0$ was applied to the data to convert volts to torque. Having a zero intercept allows the torque sensor to be zeroed regularly without calibration. The calibration line of the torque cell is shown in Figure 18.

The load cell used to measure thrust was calibrated in situ as follows. The load cell was powered and left to warm up for fifteen minutes per the manufacturer's 
specifications. A strand of fishing line was attached to the front of the propeller using aircraft wire. This strand was then passed over a frictionless cylinder as shown in schematic in Figure 19. Figure 20 shows the actual calibration setup.

The signal conditioner of the load cell was zeroed manually. Five hundred data points were collected after the load cell was zeroed. The weight was attached to the fishing line, and one thousand data points were collected. The weight was then removed from the fishing line, and five hundred more data points were collected. The five hundred points before and after the weights were applied were averaged and just to adjust the average of the calibration data. This entire calibration was repeated three times to show repeatability and to incorporate into the uncertainty of the cell. The average of the five hundred data points collected were averaged and plotted as shown in Figure 21.

With the load and torque cells calibrated, the last step was to record the drag of the fixture versus airspeed. To measure the drag the propeller was removed and replaced with just a propeller hub with the blades removed. The load cell was then left to warm up for at least fifteen minutes and the differential pressure transducer was left to warm up for at least thirty minutes. After warming up both devices were zeroed. Next, five hundred data points were collected with the wind off. The wind tunnel was then activated and set to $3.5 \mathrm{~Hz}$. Once steady state airspeed was achieved, one thousand data points were collected. The wind tunnel was then increased by $3.5 \mathrm{~Hz}$ and another thousand points were collected. This process was repeated until the wind tunnel was set to $35 \mathrm{~Hz}$. The wind tunnel was then turned off and five hundred data points were collected. The two sets of five hundred points were averaged and then averaged together. This average was subtracted from the recorded values. This was done for both the load cell and the 
differential pressure transducer. The free-stream velocity was calculated and plotted against the measured drag. A second order regression was applied to the points. The drag versus airspeed plot is shown in Figure 22.

The calibration data sheets supplied by manufacturers are shown in Figure 23, Figure 24 and Figure 25.

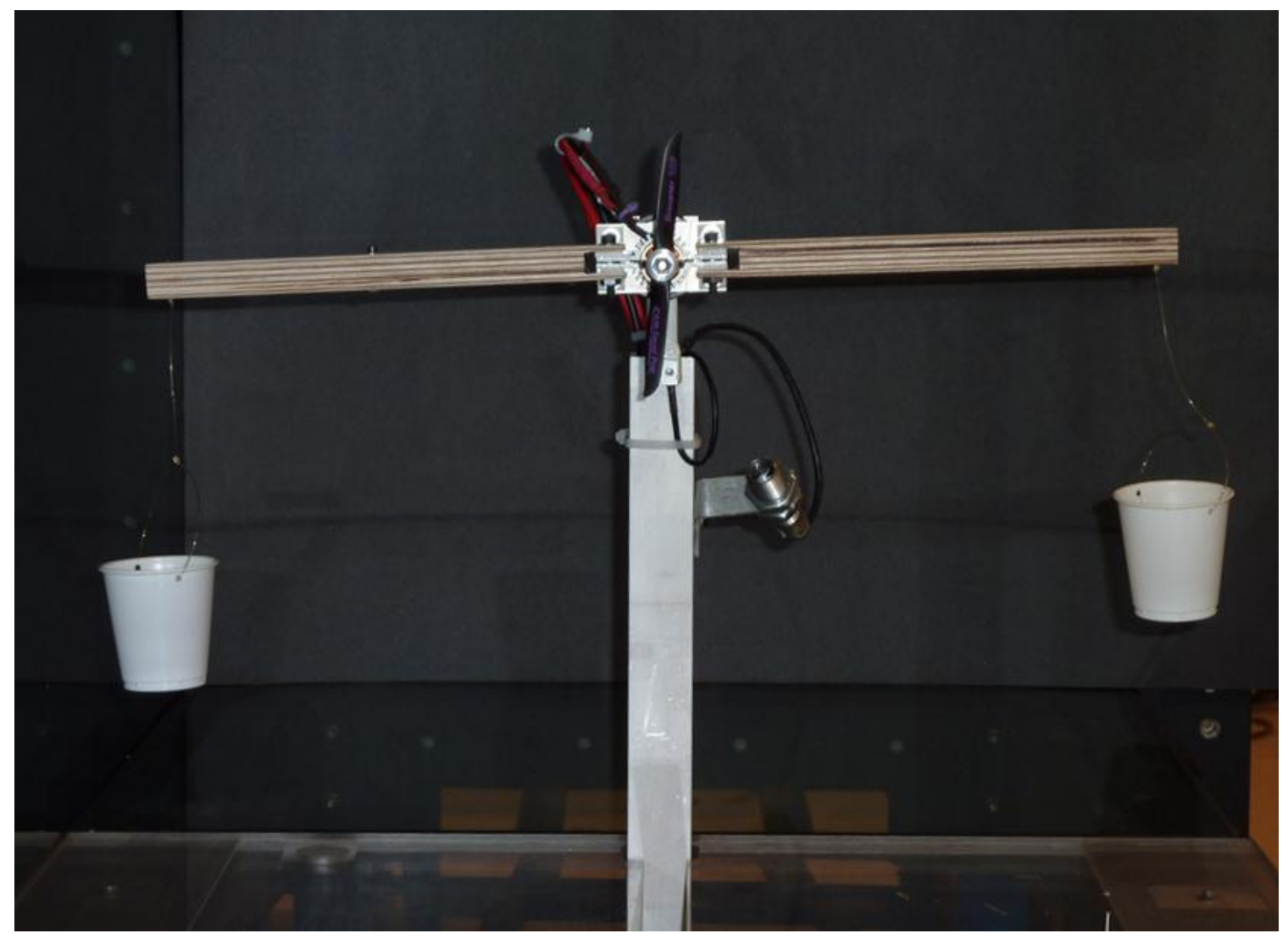

Figure 17: Torque Cell Calibration Setup 


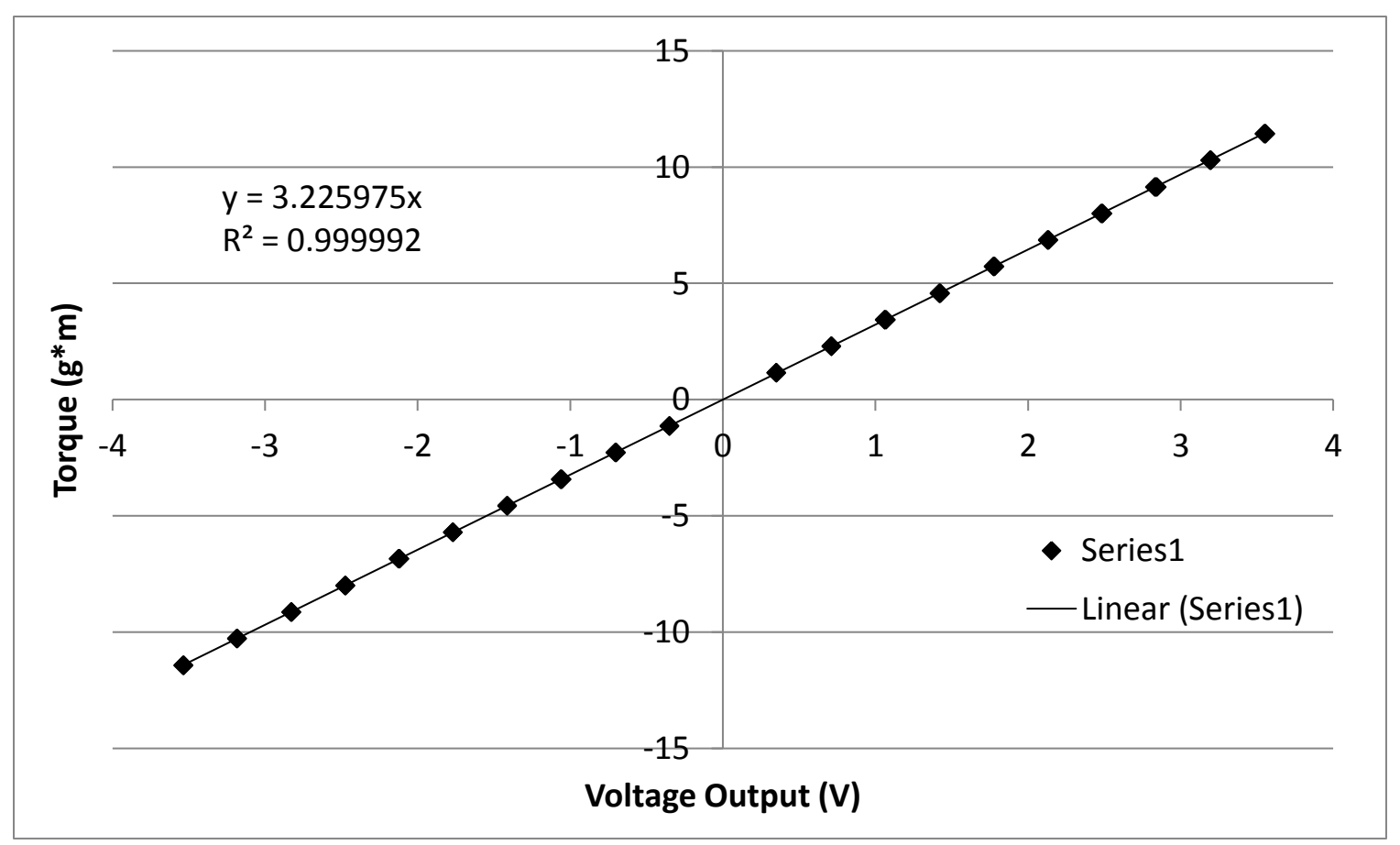

Figure 18: Torque Cell Calibration Equation

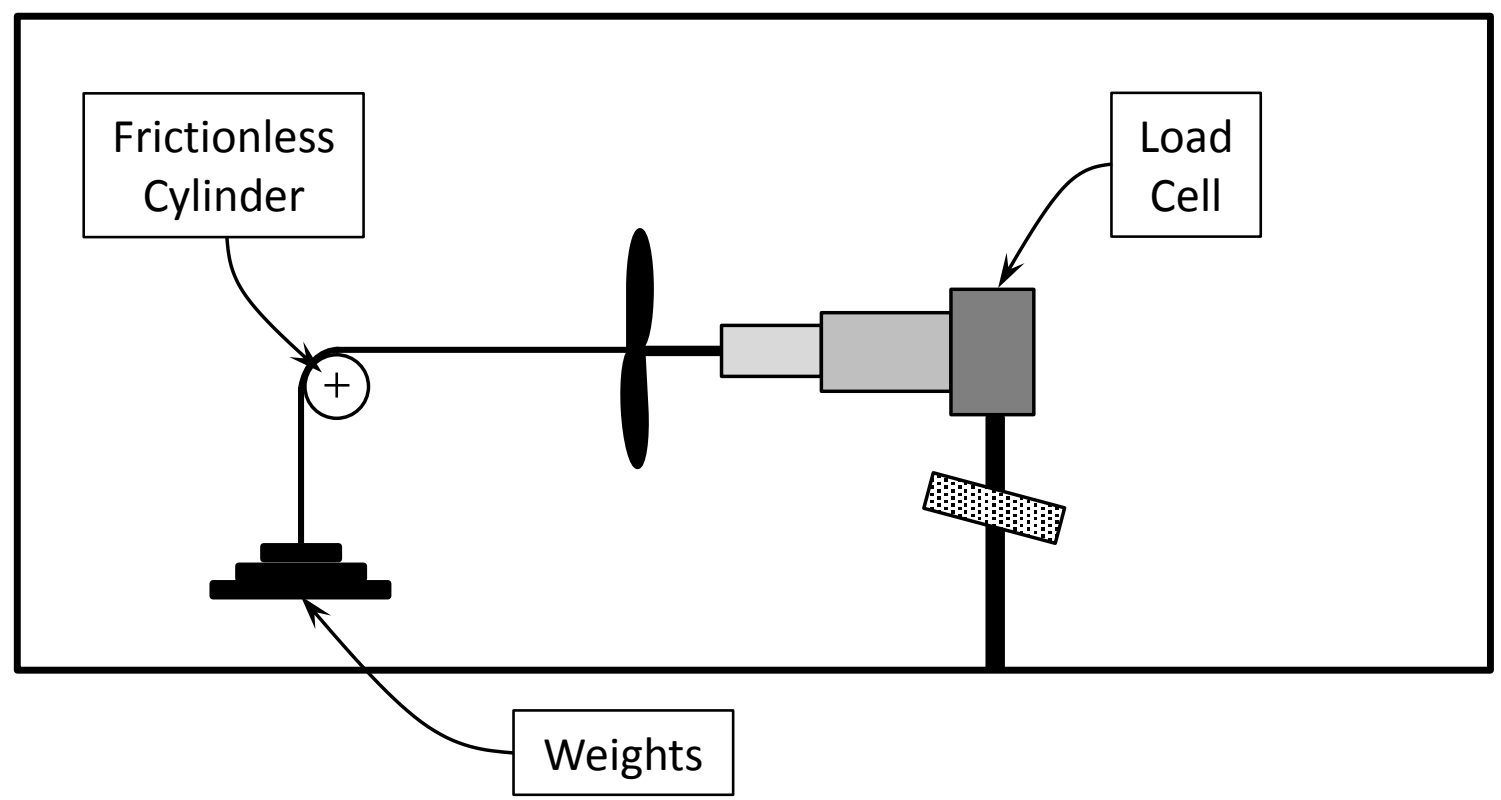

Figure 19: Schematic of Load Cell Calibration 


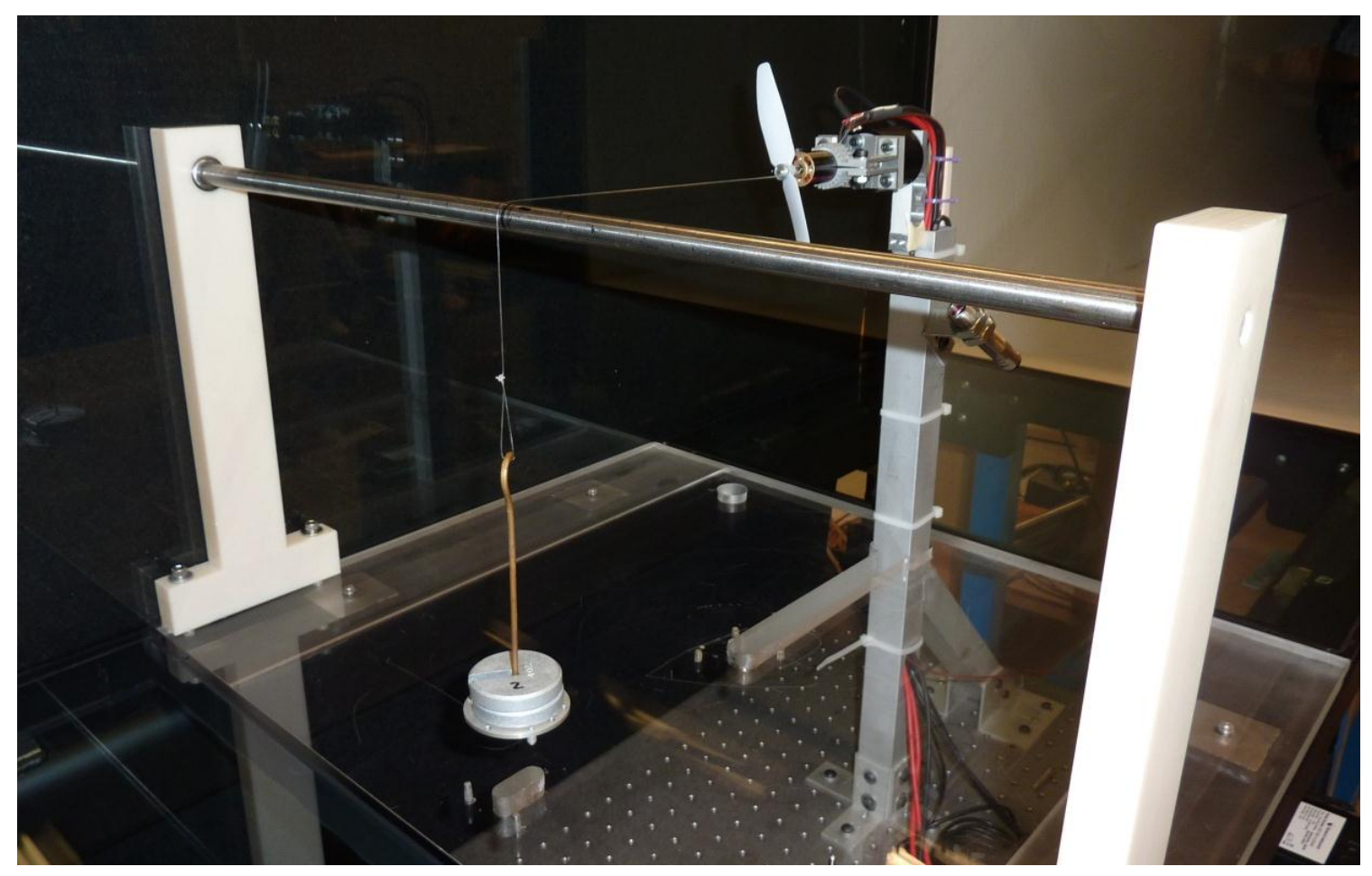

Figure 20: Load Cell Calibration Setup

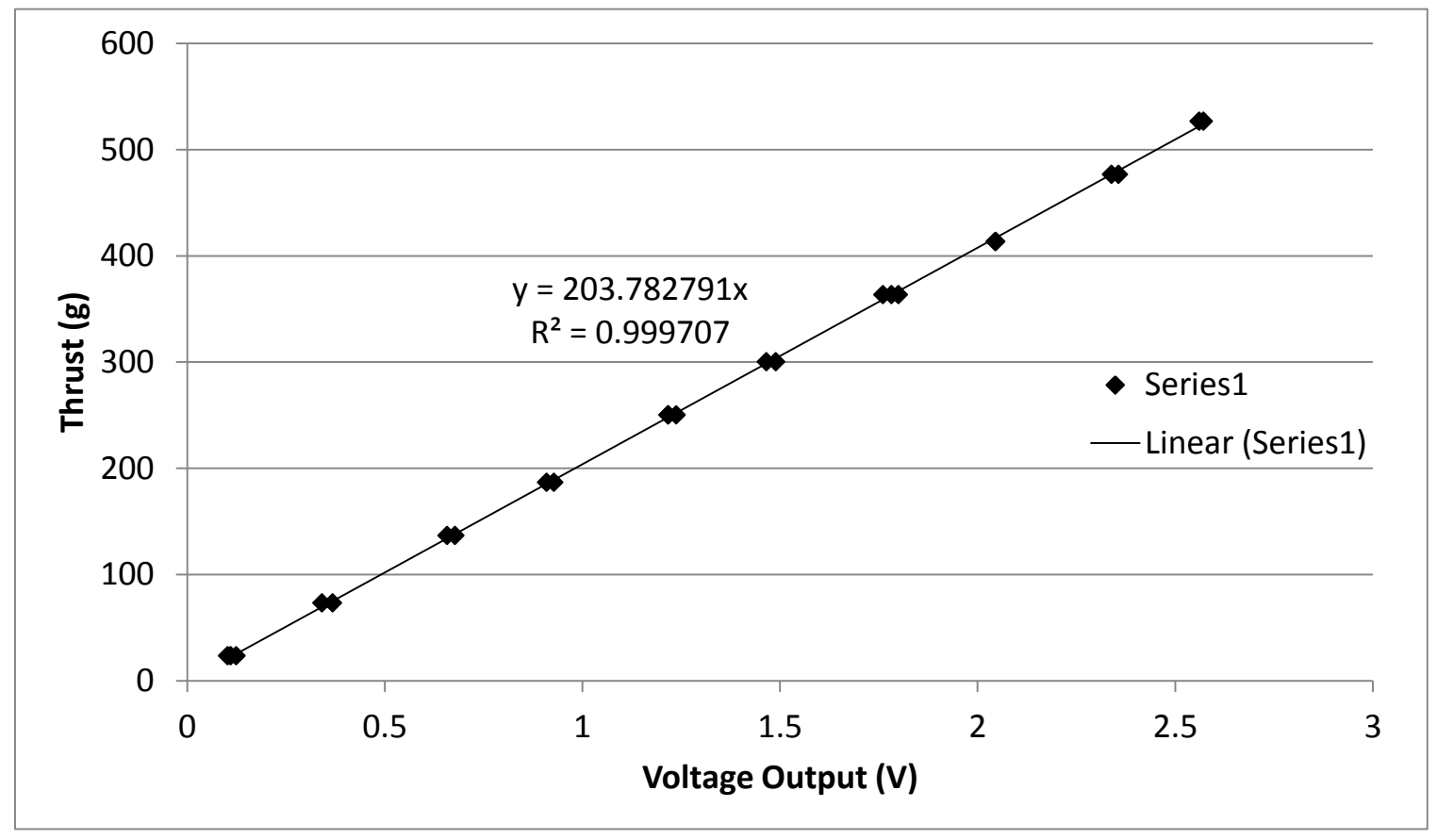

Figure 21: Calibration Equation of Load Cell 


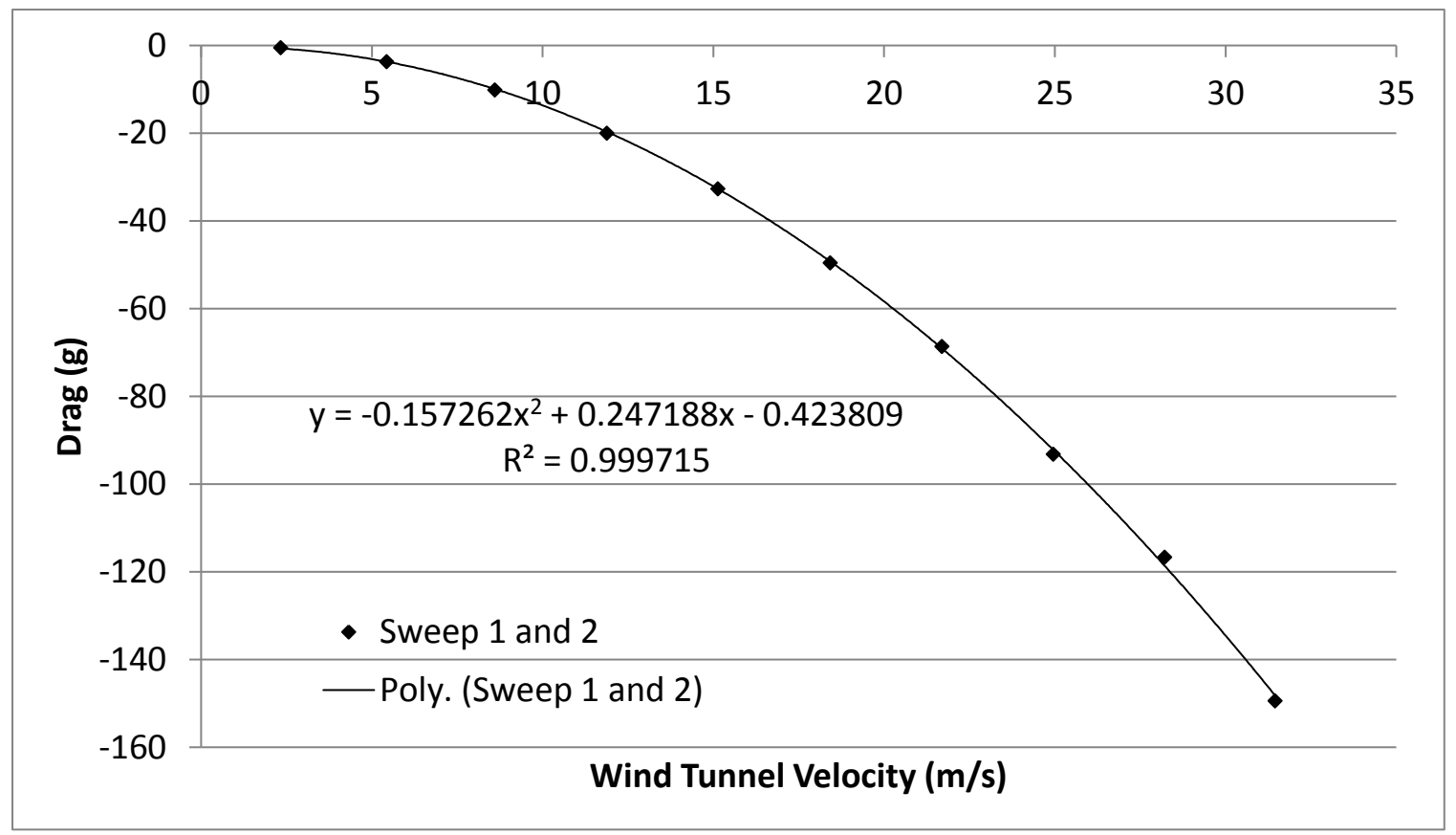

Figure 22: Fixture Drag versus Free-Stream Velocity 


\title{
VAISALA
}

\section{CALIBRATION CERTIFICATE}

\author{
Instrument \\ Serial number \\ PTB110 Barometer \\ G3720001 \\ Manufacturer Vaisala Oyj, Finland \\ Calibration date 13th September 2011
}

This instrument has been calibrated against a Vaisala PTB220 factory working standard. The Vaisala PTB220 is traceable to the National Institute of Standards and Technology (NIST, USA) via Vaisala Measurement Standards Laboratory (MSL). Vaisala MSL has been accredited by FINAS according to ISO/IEC 17025 standard.

At the time of shipment, the instrument described above was within its operating specifications.

Calibration results

\begin{tabular}{|c|c|c|c|c|}
\hline $\begin{array}{c}\text { Reference pressure } \\
\mathrm{hPa}\end{array}$ & $\begin{array}{c}\text { Calculated pressure } \\
\mathrm{hPa}\end{array}$ & $\begin{array}{c}\text { Observed voltage } \\
\text { Vdc }\end{array}$ & $\begin{array}{c}\text { Correction } \\
\mathrm{hPa}\end{array}$ & $\begin{array}{c}\text { Uncertainty } \\
\mathrm{hPa}\end{array}$ \\
\hline 510.3 & 510.3 & 0.086 & 0.0 & \pm 0.15 \\
\hline 610.1 & 610.1 & 0.918 & 0.0 & \pm 0.15 \\
\hline 699.8 & 699.8 & 1.665 & 0.0 & \pm 0.15 \\
\hline 810.0 & 810.0 & 2.584 & 0.0 & \pm 0.15 \\
\hline 900.0 & 900.0 & 3.333 & 0.0 & \pm 0.15 \\
\hline 1000.0 & 1000.0 & 4.166 & 0.0 & \pm 0.15 \\
\hline 1059.9 & 1059.9 & 4.666 & 0.0 & \pm 0.15 \\
\hline 1100.0 & 1100.0 & 5.000 & 0.0 & \pm 0.15 \\
\hline
\end{tabular}

"To obtain the true pressure, add the correction to the barometer reading. Interpolated corrections may be used at intermediate readings of the scale of the barometer.

**The calibration uncertainty given at $95 \%$ confidence level, $k=2$

Equipment used in calibration

Equipm

HP34970A

Serial number

PTB220

EM 13409

PA 9575

Calibration date

2011-04-06

2011-05-09

Certificate number

Ambient conditions

Humidity: $45 \pm 5 \%$ RH

Temperature: $23 \pm 2{ }^{\circ} \mathrm{C}$

Howith knmer

Technician

Vaisala Oyj | PO Box 26, Fl.00421 Helsinki, Finlan

Phone +3589 89491 | Fax +358989492227

Email firstname.lastname a vaisala.com | wwwivaisala.com

Domicile Vantaa, Finland | VAT F101244162 | Business 10 0124416.2

Figure 23: Vaisala PTB110 Barometer Calibration 
Certificate of Calibration

$\begin{array}{llll}\text { Model: } & \text { ACT3X } & \text { Serial Number: } & 1480790 \\ \text { Date Calibrated: } & 07 / 08 / 2011 & \text { Calibration Expires: } & 01 / 08 / 2013 \\ \text { Temperature: } & 22 \quad \text { (Celsius) } & \text { Pulses Per Revolution: } & 1 \\ \text { Scale: } & 1 & & \end{array}$

This instrument has been calibrated using standards and instruments which are traceable

to the National Institute of Standards and Technology and meet the requirements of

MIL-STD-45662A. This certificate may only be duplicated in full unless approved by us in

writing. This certificate is only valid for the instrument mentioned above and the modes

listed below. The instrument was found to be within specification.

Equipment used in calibration:

$\begin{array}{lcccc}\text { MANUFACTURER } & \text { MODEL \# } & \text { SERIAL \# } & \text { CALIBRATION DATE } & \text { EXP DATE } \\ \text { TEKTRONIX } & \text { AFG3021 } & \text { CO30708 } & 12 / 15 / 2010 & 12 / 152011 \\ \text { Tachometer Calibration (The generated signal is measured by the Instrument) }\end{array}$

Tachometer Calibration (The generated signal is measured by the Instrument)

TEST RESULT

\begin{tabular}{|c|c|c|c|c|c|}
\hline RPM Set & Displayed RPM & Accep & able & RPM Range & Result \\
\hline 5 & 5.000 & 4.995 & to & 5.005 & PASS \\
\hline 50 & 50.000 & 49.95 & to & 50.05 & PASS \\
\hline 500 & 500.000 & 499.5 & to & 500.5 & PASS \\
\hline 5000 & 5000.003 & 4995 & to & 5005 & PASS \\
\hline 50000 & 50000.030 & 49950 & to & 50050 & PASS \\
\hline 100000 & 100000.200 & 99900 & to & 100100 & PASS \\
\hline 500000 & 499999.900 & 499500 & to & 500500 & pASS \\
\hline
\end{tabular}

Calibrated by: Monarch Calibration Lab. 15 Columbia Drive. Amherst, NH 03031

Note:

Technician : UF

W F

Date: $7 \cdot 8 \cdot 11$

Document \#: CAL-500-002 Rev 3.2 Page 1 of 1 A 10076

Certificate Serial \#:

Figure 24: Monarch Instruments ACT-3X Calibration 


\begin{tabular}{|c|c|c|c|c|}
\hline \multicolumn{2}{|c|}{ Unit Type: $226 \mathrm{A05TBBBBSU7T1}$} & \multirow{2}{*}{$\begin{array}{l}\text { Unit Range: } \\
\text { Voltage UUT }\end{array}$} & \multirow{2}{*}{$\begin{array}{l}5 \text { Pre } \\
\text { Error }(m V)\end{array}$} & \multirow{2}{*}{$\begin{array}{l}\text { Torr } \\
\text { Error (\%) }\end{array}$} \\
\hline Pressure Std & Voltage Std & & & \\
\hline 0.00 & 0.00 & 0.00 & 0.00 & 0.00 \\
\hline 0.50 & 0.50 & 0.50 & 0.00 & 0.00 \\
\hline 0.99 & 0.99 & 0.99 & -1.00 & -0.10 \\
\hline 2.01 & 2.01 & 2.01 & -2.00 & -0.10 \\
\hline 3.02 & 3.02 & 3.02 & -3.00 & -0.10 \\
\hline 4.03 & 4.03 & 4.02 & -4.00 & -0.10 \\
\hline 5.03 & 5.03 & 5.03 & 6.00 & 0.12 \\
\hline
\end{tabular}

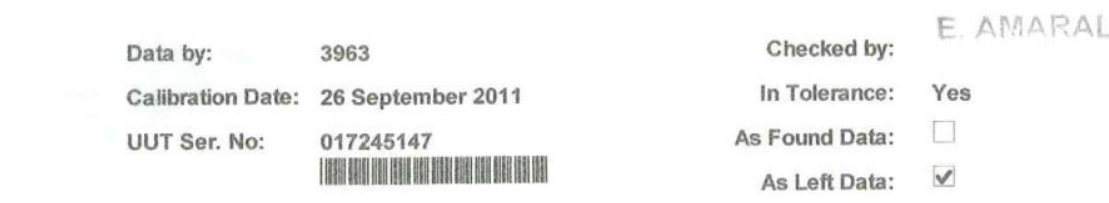

Notes: 1) Temperature regulated units must be on for a minimum of four hours prior to making any adjustments.

2) This calibration was performed in compliance with ISO/IEC 17025:2000 requirements.

3) The allowable specification for the unit is 0.3 Percent of Reading

4) This calibration was performed per the latest revision of MKS Calibration Procedure 1026576A.

5) The environment conditions were controlled to the extent necessary during this calibration.

6) These calibration results relate only to the item calibrated.

7) This report shall only be reproduced in full

End of Report

\section{Figure 25: MKS 226A Differential Pressure Transducer Calibration}




\section{APPENDIX B: UNCERTAINTY ANALYSIS}

In order to determine the uncertainty of the propeller performance coefficients it was first necessary to find the uncertainty of the measured values since they factor into the final uncertainty. The uncertainty of the thrust calibration $\left(\Delta T_{\text {cal }}\right)$ included the minimum resolution/uncertainty of the scale used to weigh the suspended mass $( \pm 0.001$ g) plus the largest $99 \%$ confidence interval of all the thrust measurements taken during the calibration plus the largest deviation of any calibration point from the calibration line. The final thrust uncertainty $(\Delta T)$ was defined as the thrust calibration uncertainty from above plus the $99 \%$ confidence interval of thrust for the test. For dynamic cases the uncertainty of the drag was also added to the thrust uncertainty. The drag uncertainty was found similarly by adding the largest $99 \%$ confidence interval of the drag calibration and the largest deviation from the drag calibration line.

The uncertainty of the torque $(\Delta Q)$ was defined similarly. Atmospheric temperature uncertainty $\left(\Delta T_{\text {atm,cal }}\right)$ included the resolution/uncertainty of the RTD used in the calibration $\left( \pm 0.01{ }^{\circ} \mathrm{C}\right)$ plus the $99 \%$ confidence interval of the RTD reading plus the largest deviation from the calibration line for any thermocouple reading. The final atmospheric temperature uncertainty $\left(\Delta T_{\mathrm{atm}}\right)$ was defined as the calibration uncertainty plus the $99 \%$ confidence interval from the temperature reading during the test.

To determine the uncertainties of calculated quantities such as air density, propeller power, electrical power, coefficient of power, thrust, and torque, the root sum square method was used. These equations were derived by hand and are presented below. The units were also checked by hand to make sure the equation was derived correctly. However, only some equations relate to static tests and others relate only to dynamic 
tests. The uncertainties associated with the static tests are as follows with the equations only used for static tests noted.

Air Density:

$$
\Delta \rho=\left[\left(\frac{\Delta P_{\mathrm{atm}}}{R T_{\mathrm{atm}}}\right)^{2}+\left(\frac{-P_{\mathrm{atm}} \Delta T_{\mathrm{atm}}}{R T_{\mathrm{atm}}^{2}}\right)^{2}\right]^{\frac{1}{2}}
$$

Propeller Power:

$$
\Delta P_{\mathrm{P}}=\left[(2 \pi n \Delta Q)^{2}+(2 \pi Q \Delta n)^{2}\right]^{\frac{1}{2}}
$$

Electrical Power:

$$
\Delta P_{\mathrm{e}}=\left[\left(I \Delta V_{\mathrm{e}}\right)^{2}+\left(V_{\mathrm{e}} \Delta I\right)^{2}\right]^{\frac{1}{2}}
$$

Total Efficiency:

$$
\Delta \eta_{\mathrm{T}}=\left[\left(\frac{2 \pi n \Delta Q}{V I}\right)^{2}+\left(\frac{Q 2 \pi \Delta n}{V I}\right)^{2}+\left(\frac{-2 \pi n Q \Delta V_{\mathrm{e}}}{V^{2} I}\right)^{2}+\left(\frac{-2 \pi n Q \Delta I}{V I^{2}}\right)^{2}\right]^{\frac{1}{2}}
$$

Coefficient of Thrust:

$$
\Delta C_{T}=\left[\left(\frac{\Delta T}{\rho n^{2} D^{4}}\right)^{2}+\left(\frac{-T \Delta \rho}{\rho^{2} n^{2} D^{4}}\right)^{2}+\left(\frac{-2 T \Delta n}{\rho n^{3} D^{4}}\right)^{2}+\left(\frac{-4 T \Delta D}{\rho n^{2} D^{5}}\right)^{2}\right]^{\frac{1}{2}}
$$

Coefficient of Power:

$$
\Delta C_{P}=\left[\left(\frac{\Delta P_{P}}{\rho n^{3} D^{5}}\right)^{2}+\left(\frac{-P_{P} \Delta \rho}{\rho^{2} n^{3} D^{5}}\right)^{2}+\left(\frac{-3 P_{P} \Delta n}{\rho n^{4} D^{5}}\right)^{2}+\left(\frac{-5 P_{P} \Delta D}{\rho n^{3} D^{6}}\right)^{2}\right]^{\frac{1}{2}}
$$

\section{Coefficient of Torque:}

$$
\Delta C_{Q}=\left[\left(\frac{\Delta Q}{\rho n^{2} D^{5}}\right)^{2}+\left(\frac{-Q \Delta \rho}{\rho^{2} n^{2} D^{5}}\right)^{2}+\left(\frac{-2 Q \Delta n}{\rho n^{3} D^{5}}\right)^{2}+\left(\frac{-5 Q \Delta D}{\rho n^{2} D^{6}}\right)^{2}\right]^{\frac{1}{2}}
$$


Viscosity of Air:

$$
\Delta \mu=\frac{0.76 \mu_{\mathrm{ref}}}{\left(\frac{T_{\mathrm{atm}}}{T_{\mathrm{ref}}}\right)^{.24} T_{\mathrm{ref}}} * \Delta T_{\mathrm{atm}}
$$

Propeller Velocity: (Static Only)

$$
V_{P}=\left[\left(2 \pi n \Delta R_{0.75}\right)^{2}+\left(2 \pi R_{0.75} \Delta n\right)^{2}\right]^{\frac{1}{2}}
$$

Reynolds Number at 75\% Propeller Radius: (Static Only)

$$
\Delta \operatorname{Re}_{0.75}=\left[\left(\frac{C_{0.75} V_{\mathrm{P}} \Delta \rho}{\mu}\right)^{2}+\left(\frac{C_{0.75} \rho \Delta V_{\mathrm{P}}}{\mu}\right)^{2}+\left(\frac{\rho V_{\mathrm{P}} \Delta C_{0.75}}{\mu}\right)^{2}+\left(\frac{-C_{0.75} \rho V_{\mathrm{P}} \Delta \mu}{\mu^{2}}\right)^{2}\right]^{\frac{1}{2}}
$$

The following equations are used only for the dynamic tests since they require a free-stream velocity.

Uncorrected Free-Stream Velocity:

$$
\Delta V_{\infty}=\left[\frac{1}{2 \rho P_{\text {diff }}}\left(\Delta P_{\text {diff }}\right)^{2}+\frac{2 P_{\text {diff }}}{\rho}\left(\frac{-\Delta \rho}{2 \rho}\right)^{2}\right]^{\frac{1}{2}}
$$

Glauert Correction Variable:

$$
\Delta \tau_{4}=\left[\left(\frac{\Delta T}{\rho A_{\mathrm{P}} V_{\infty}^{2}}\right)^{2}+\left(\frac{-T \Delta \rho}{\rho^{2} A_{\mathrm{P}} V_{\infty}^{2}}\right)^{2}+\left(\frac{-T \Delta A_{\mathrm{P}}}{\rho A_{\mathrm{P}}^{2} V_{\infty}^{2}}\right)^{2}+\left(\frac{-2 T \Delta V_{\infty}}{\rho A_{\mathrm{P}} V_{\infty}^{3}}\right)^{2}\right]^{\frac{1}{2}}
$$


Corrected Free-Stream Velocity:

$$
\begin{gathered}
\Delta V_{\infty}^{\prime}=\left[\left(\left(1-\frac{A_{\mathrm{P}} \tau_{4}}{2 A_{\mathrm{WT}} \sqrt{2 \tau_{4}+1}}\right) \Delta V_{\infty}\right)^{2}+\left(\frac{-A_{\mathrm{P}}\left(\tau_{4}+1\right) V_{\infty} \Delta \tau_{4}}{2 A_{\mathrm{WT}}\left(2 \tau_{4}+1\right)^{3 / 2}}\right)^{2}\right. \\
\left.+\left(\frac{-\tau_{4} V_{\infty} \Delta A_{\mathrm{P}}}{2 A_{\mathrm{WT}} \sqrt{2 \tau_{4}+1}}\right)^{2}\right]^{\frac{1}{2}} \\
\Delta A_{\mathrm{P}}=\frac{\pi D \Delta D}{2}
\end{gathered}
$$

Total Velocity:

$$
\Delta V_{\mathrm{t}}=\left[\left(\frac{V_{\infty}^{\prime 2} \Delta V_{\infty}^{\prime 2}}{V_{\infty}^{\prime 2}+4 n^{2} \pi^{2} R_{0.75}^{2}}\right)^{2}+\left(\frac{16 n^{2} \pi^{4} R_{0.75}^{4} \Delta n^{2}}{4 n^{2} \pi^{2} R_{0.75}^{2}+V_{\infty}^{\prime 2}}\right)^{2}+\left(\frac{16 n^{4} \pi^{4} R_{0.75}^{2} \Delta R_{0.75}^{2}}{4 n^{2} \pi^{2} R_{0.75}^{2}+V_{\infty}^{\prime 2}}\right)^{2}\right]^{\frac{1}{2}}
$$

Advance Ratio:

$$
\Delta J=\left[\left(\frac{\Delta V_{\infty}^{\prime}}{n D}\right)^{2}+\left(\frac{-V_{\infty}^{\prime} \Delta n}{n^{2} D}\right)^{2}+\left(\frac{-V_{\infty}^{\prime} \Delta D}{n D^{2}}\right)^{2}\right]^{\frac{1}{2}}
$$

Propeller Efficiency:

$$
\Delta \eta_{\mathrm{P}}=\left[\left(\frac{C_{\mathrm{T}} \Delta J}{C_{\mathrm{P}}}\right)^{2}+\left(\frac{\Delta C_{\mathrm{T}} J}{C_{\mathrm{P}}}\right)^{2}+\left(\frac{-J C_{\mathrm{T}} \Delta C_{\mathrm{P}}}{C_{\mathrm{P}}^{2}}\right)^{2}\right]^{\frac{1}{2}}
$$

Reynolds Number at $75 \%$ Propeller Radius:

$$
\Delta \operatorname{Re}_{0.75}=\left[\left(\frac{C_{0.75} V_{\mathrm{t}} \Delta \rho}{\mu}\right)^{2}+\left(\frac{C_{0.75} \rho \Delta V_{\mathrm{t}}}{\mu}\right)^{2}+\left(\frac{\rho V_{\mathrm{t}} \Delta C_{0.75}}{\mu}\right)^{2}+\left(\frac{-C_{0.75} \rho V_{\mathrm{t}} \Delta \mu}{\mu^{2}}\right)^{2}\right]^{\frac{1}{2}}
$$

Corrected Thrust:

$$
\Delta T^{\prime}=\left[(\Delta T)^{2}+\left(\Delta F_{\mathrm{D}}\right)^{2}\right]^{\frac{1}{2}}
$$




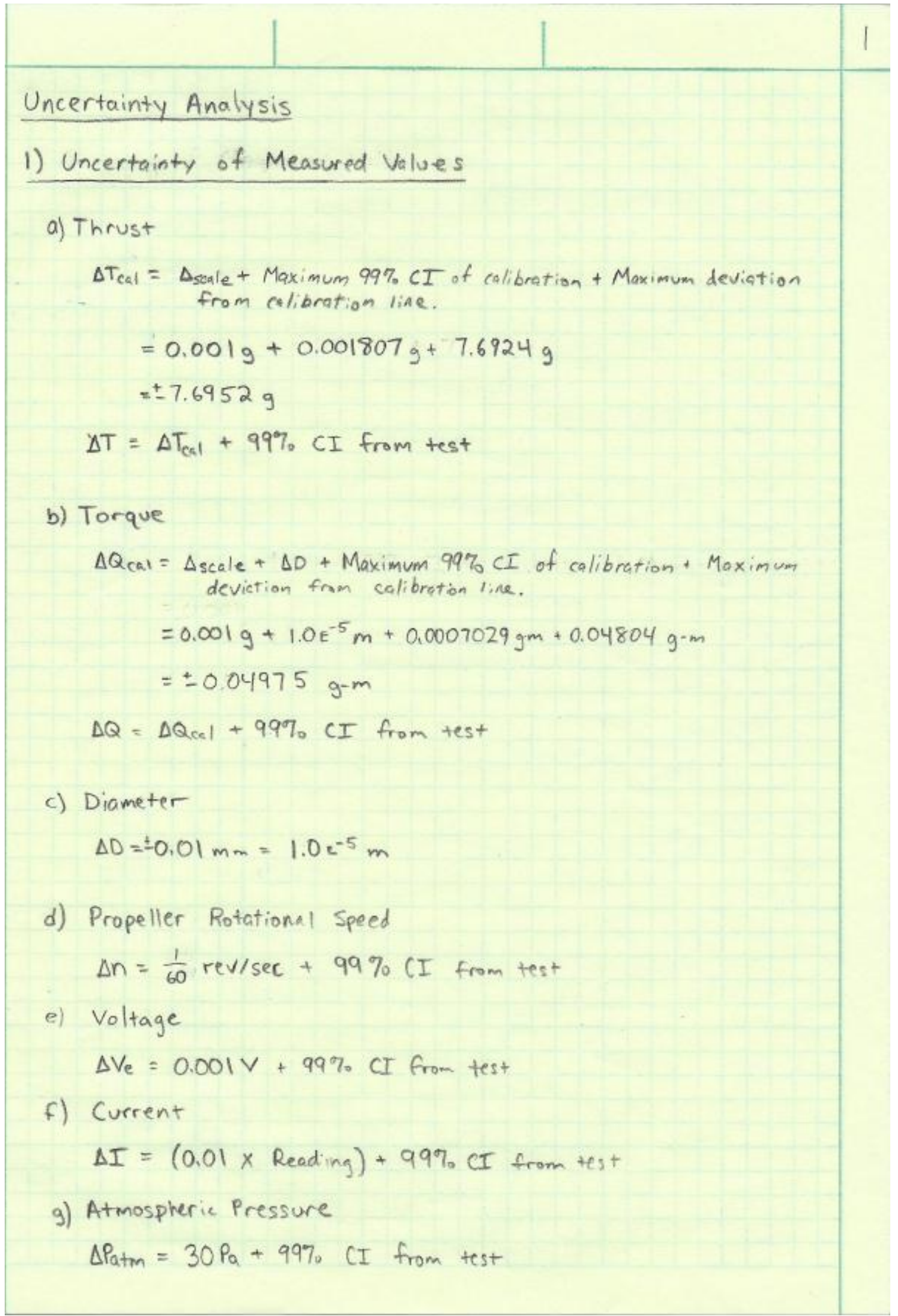




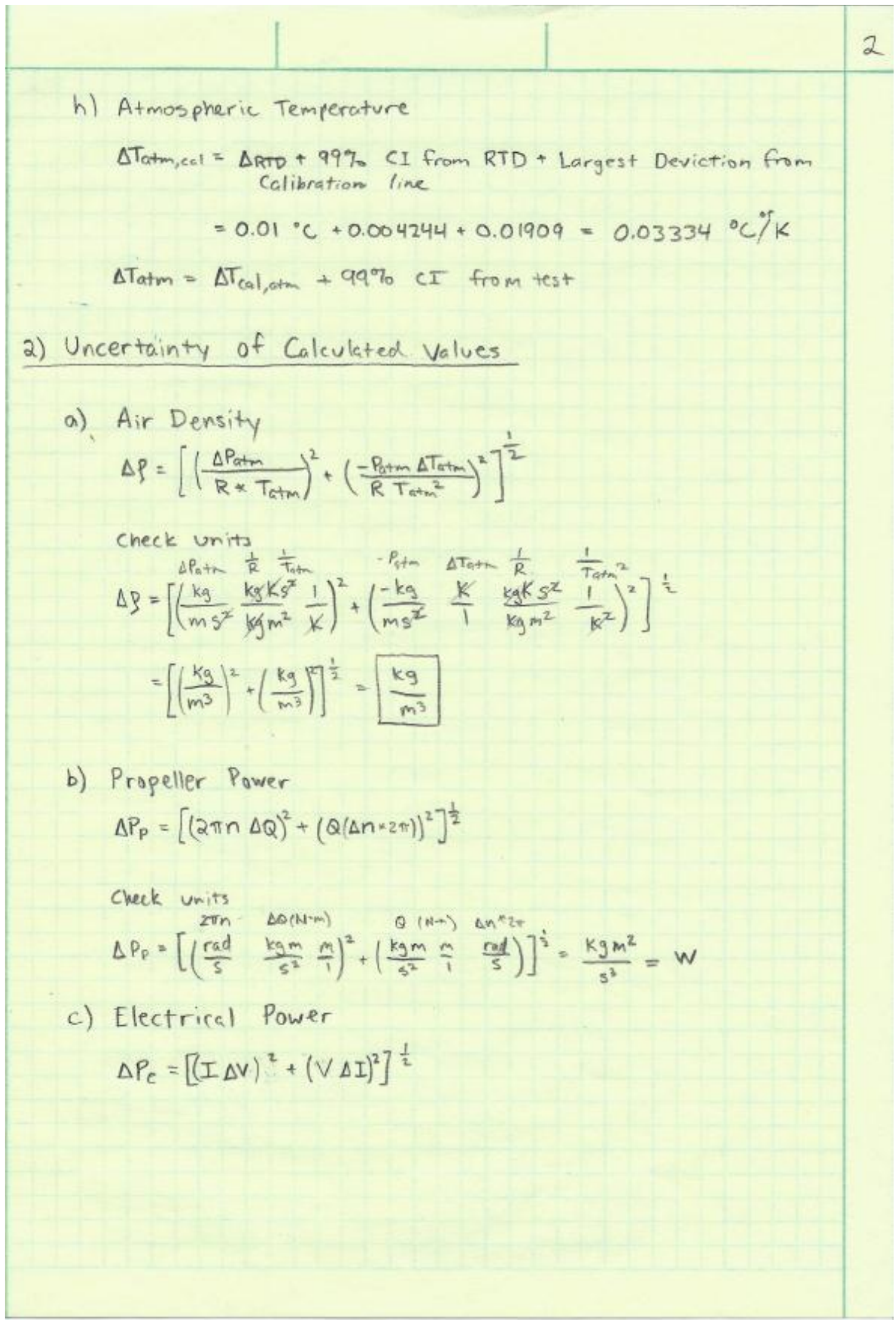




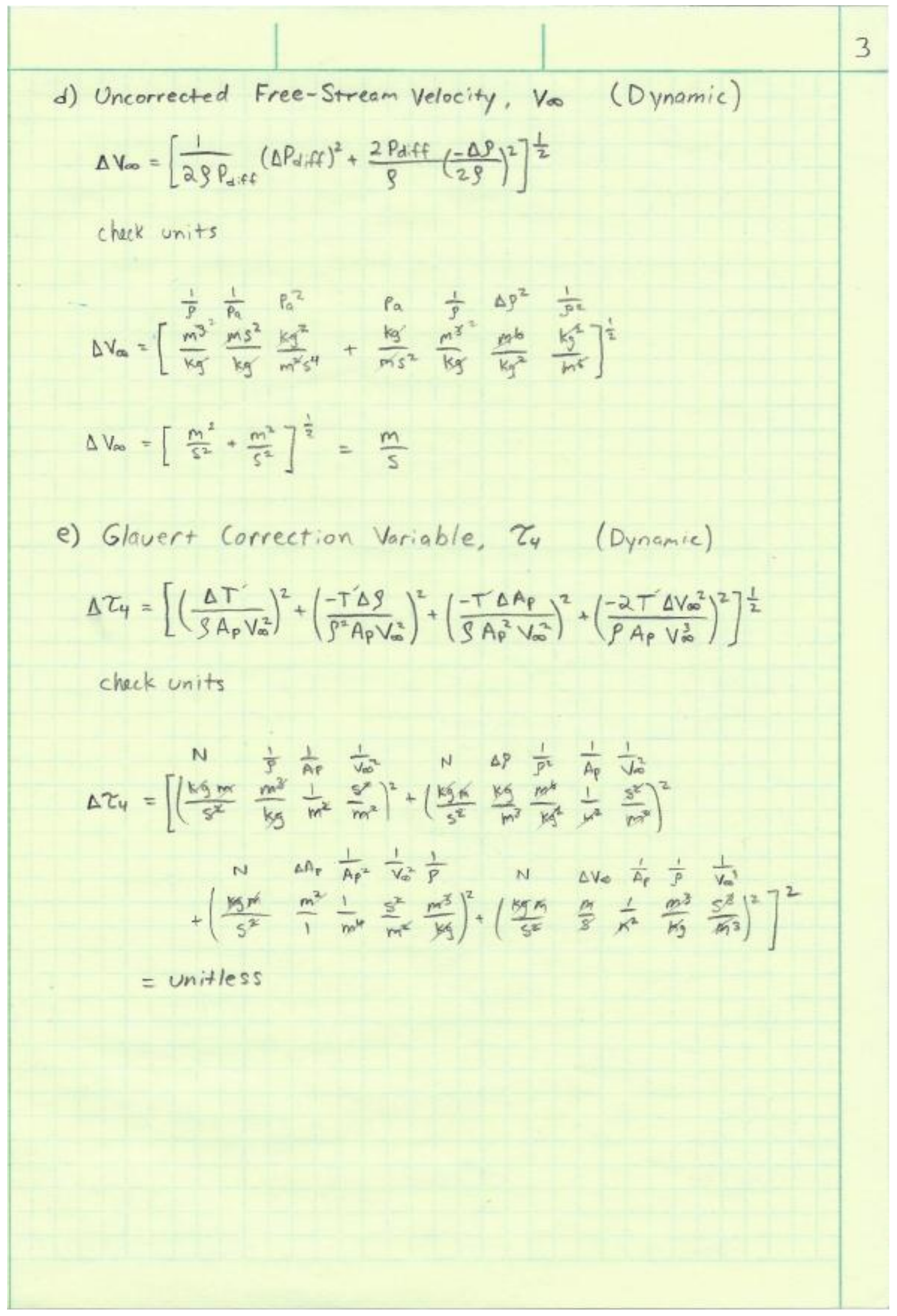




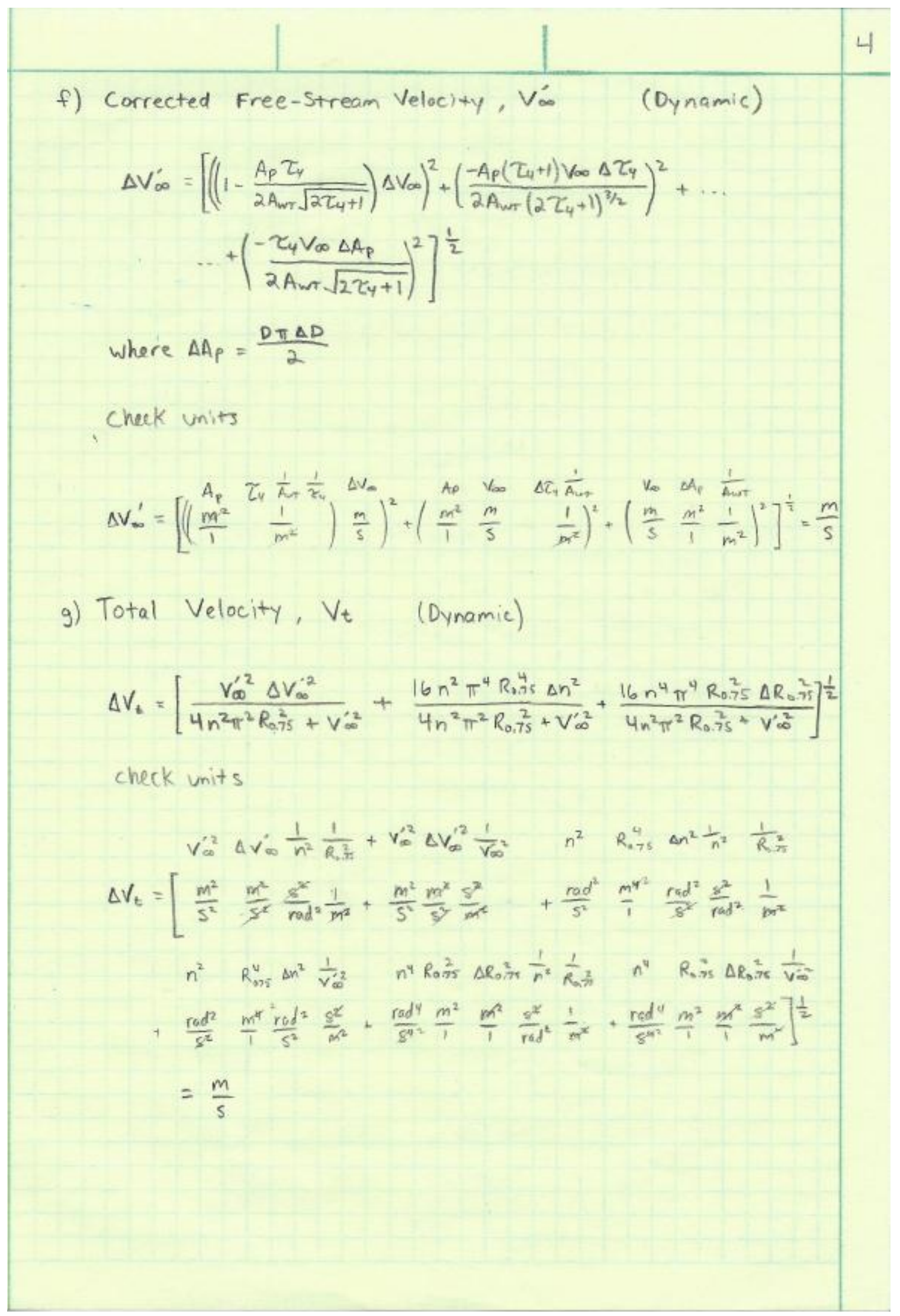




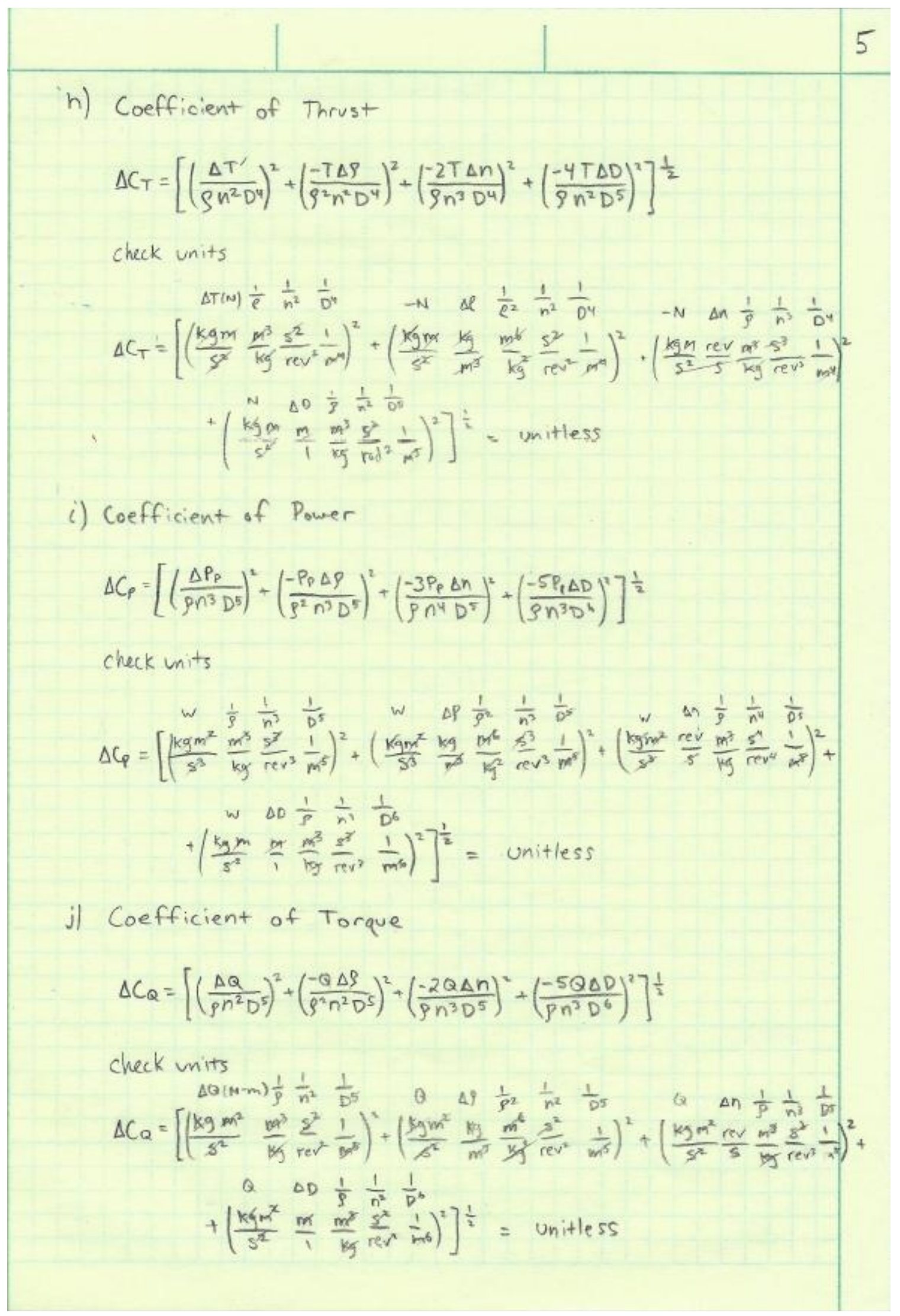




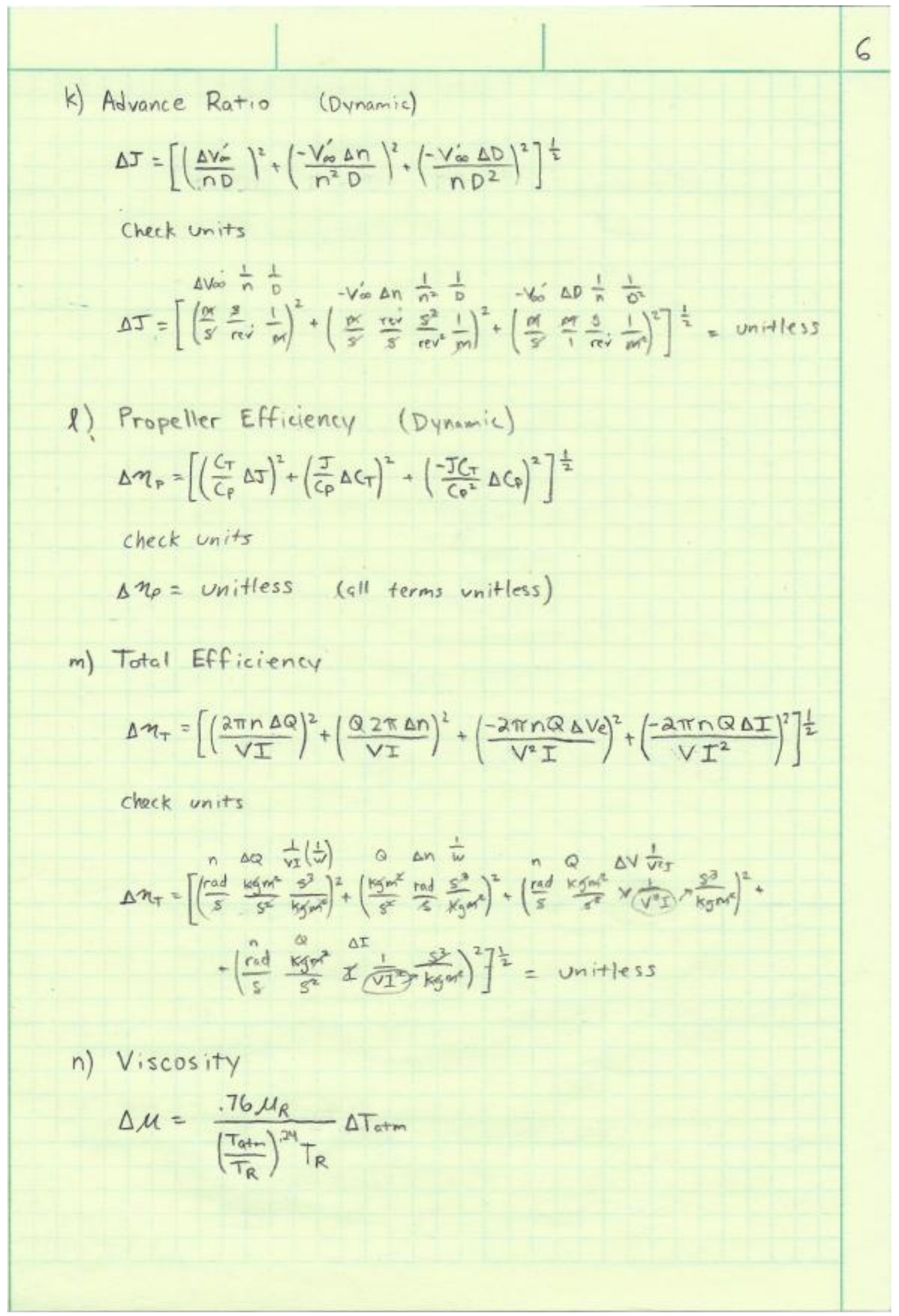




0) Propeller Velocity, $V_{p}=2 \pi n$
$\Delta V_{p}=\left[\left(2 \pi n \Delta R_{0.75}\right)^{2}+\left(R_{0.75} 2 \pi \Delta n\right)^{i}\right]$
check units
$\Delta V_{p}=\left[\left(\frac{\text { rad }}{\mathrm{s}} m\right)^{2}+\left(m \frac{\mathrm{rad}}{\mathrm{s}}\right)^{2}\right]^{\frac{1}{2}}=\frac{m}{\mathrm{~s}}$

P). Reynolds Number (Static) $R e=\frac{\rho V_{p} C_{0.75}}{\mu}$

$$
\Delta \operatorname{Re}_{0.75}=\left[\left(\frac{\left(0.75 V_{p} \Delta \rho\right.}{\mu}\right)^{2}+\left(\frac{C_{0.75} \rho \Delta V_{p}}{\mu}\right)^{2}+\left(\frac{\rho V_{p} \Delta C_{0.75}}{\mu}\right)^{2}+\left(\frac{-C_{0.75} \rho V_{p} \Delta \mu}{\mu^{2}}\right)^{2}\right]^{\frac{1}{2}}
$$

check units

$$
\begin{aligned}
& C_{0.75} V_{p} \Delta \rho \frac{1}{\mu} \quad \operatorname{coss} \rho \quad \Delta V_{p} \frac{1}{x} \quad p \quad V_{p} \Delta C_{a, 3 s} \frac{1}{\mu}
\end{aligned}
$$

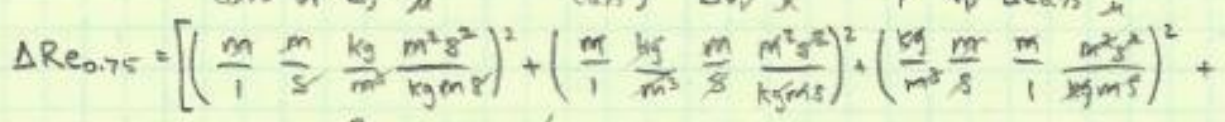

$$
\begin{aligned}
& \left.+\left(\frac{m}{1} \frac{k g}{m^{2}} \frac{m}{s} \frac{k g m s}{m^{2} s^{2}} \frac{m^{4} s^{4}}{k_{g}^{2} m^{2} s^{2}}\right)^{2}\right]^{\frac{1}{2}}=\text { unites }
\end{aligned}
$$

q) Reynolds Number (Dynamic) $R_{e}=\frac{\rho V_{t} C_{0.75}}{\mu}$
$\Delta \operatorname{Re}_{0.75}=\left[\left(\frac{C_{0.75} V_{t} \Delta \rho}{\mu}\right)^{2}+\left(\frac{C_{0.75} \rho \Delta V_{t}}{\mu}\right)^{2}+\left(\frac{\rho V_{t} \Delta C_{0.75}}{\mu}\right)^{2}+\left(\frac{-C_{0.75} \rho V_{t} \Delta \mu}{\mu}\right)^{2}\right]^{\frac{1}{2}}$

check units

some as static case

65 


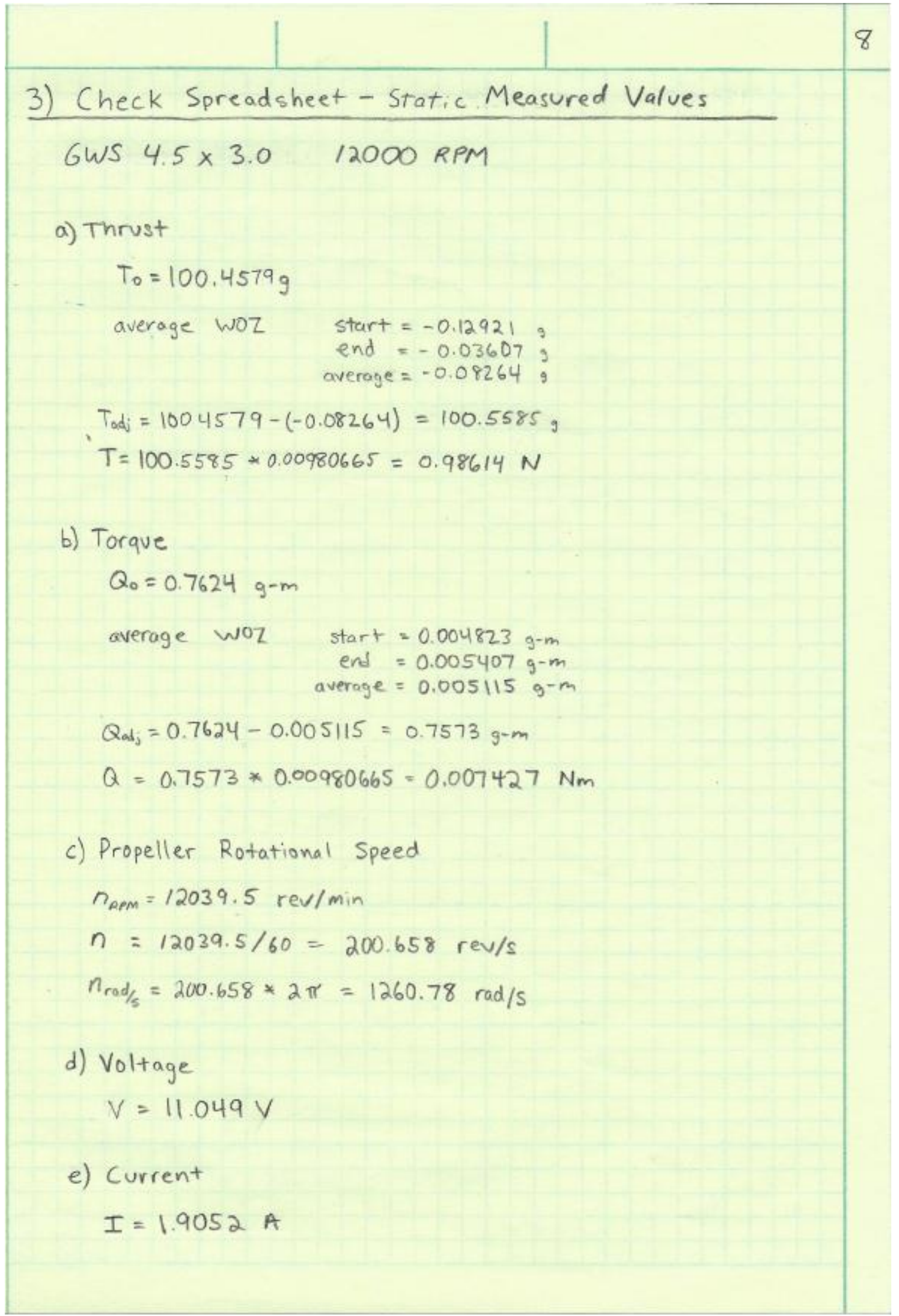




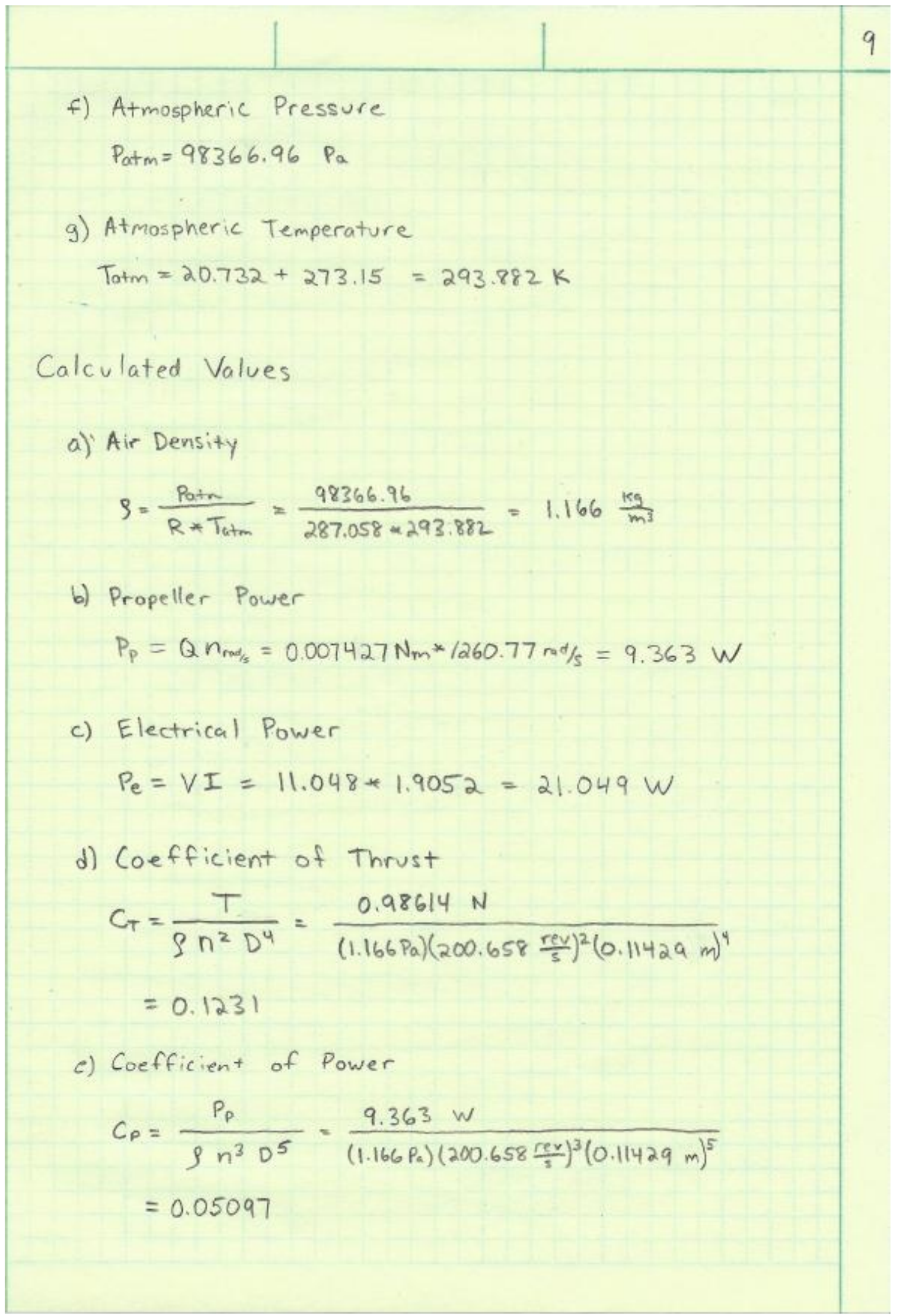




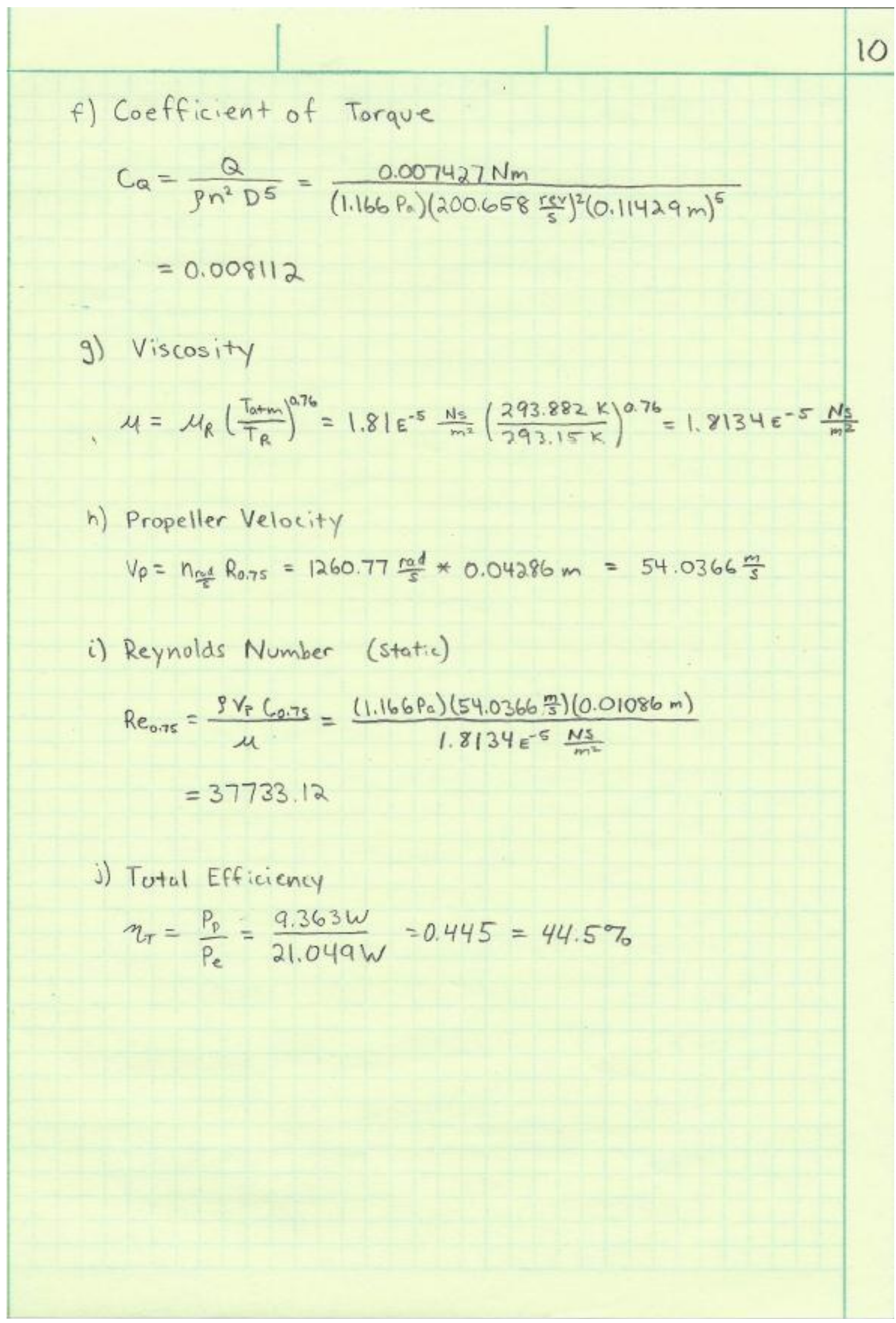




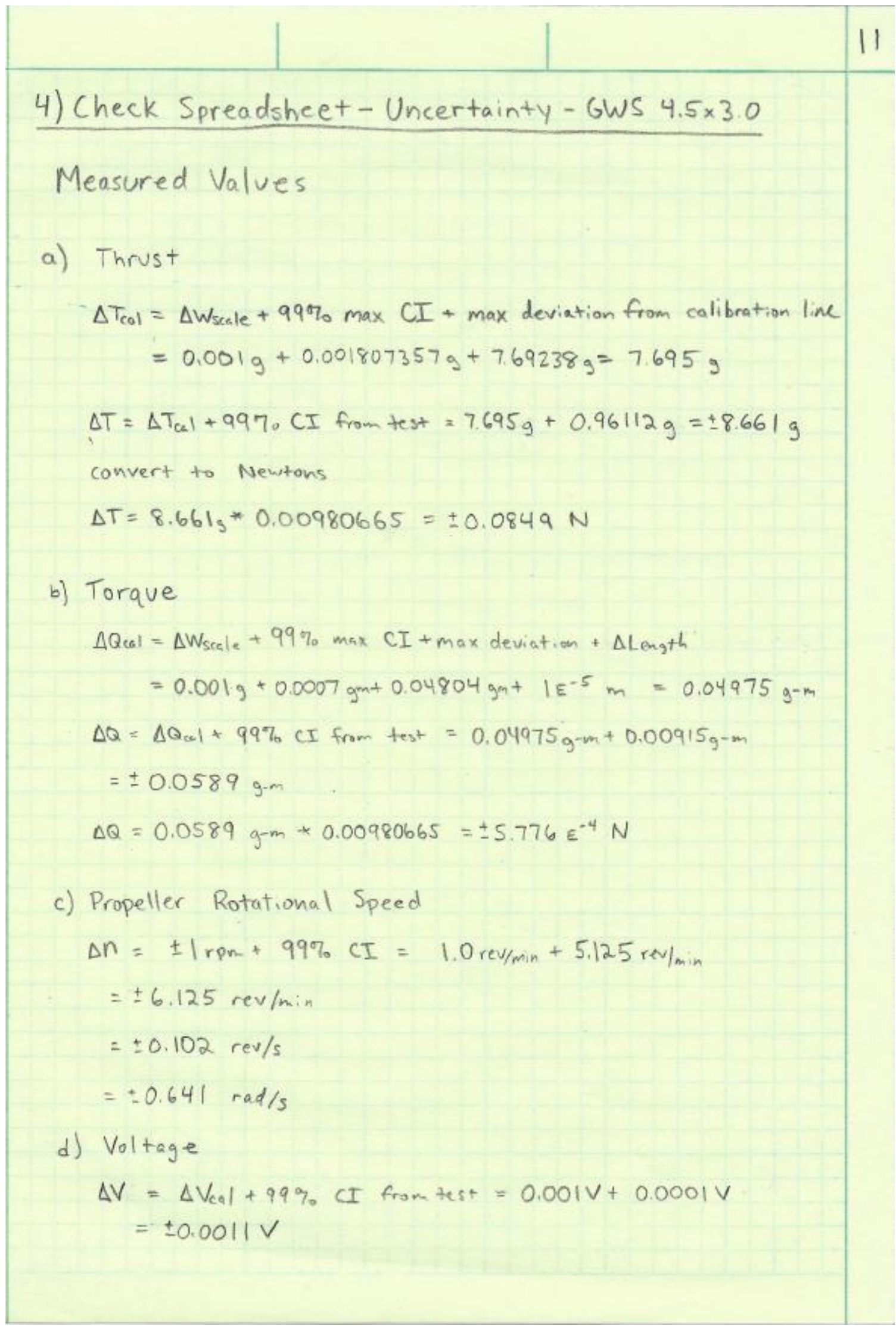




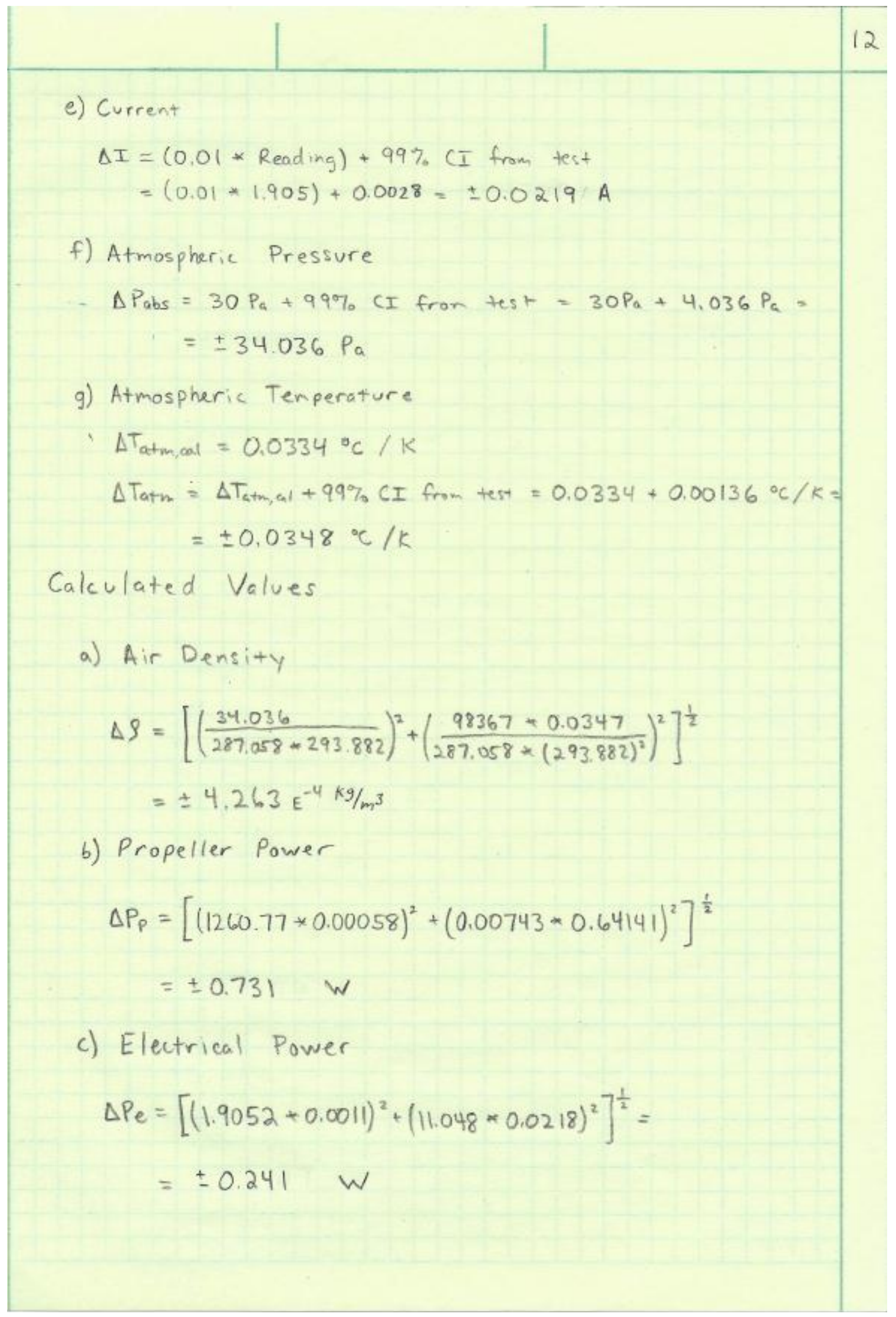




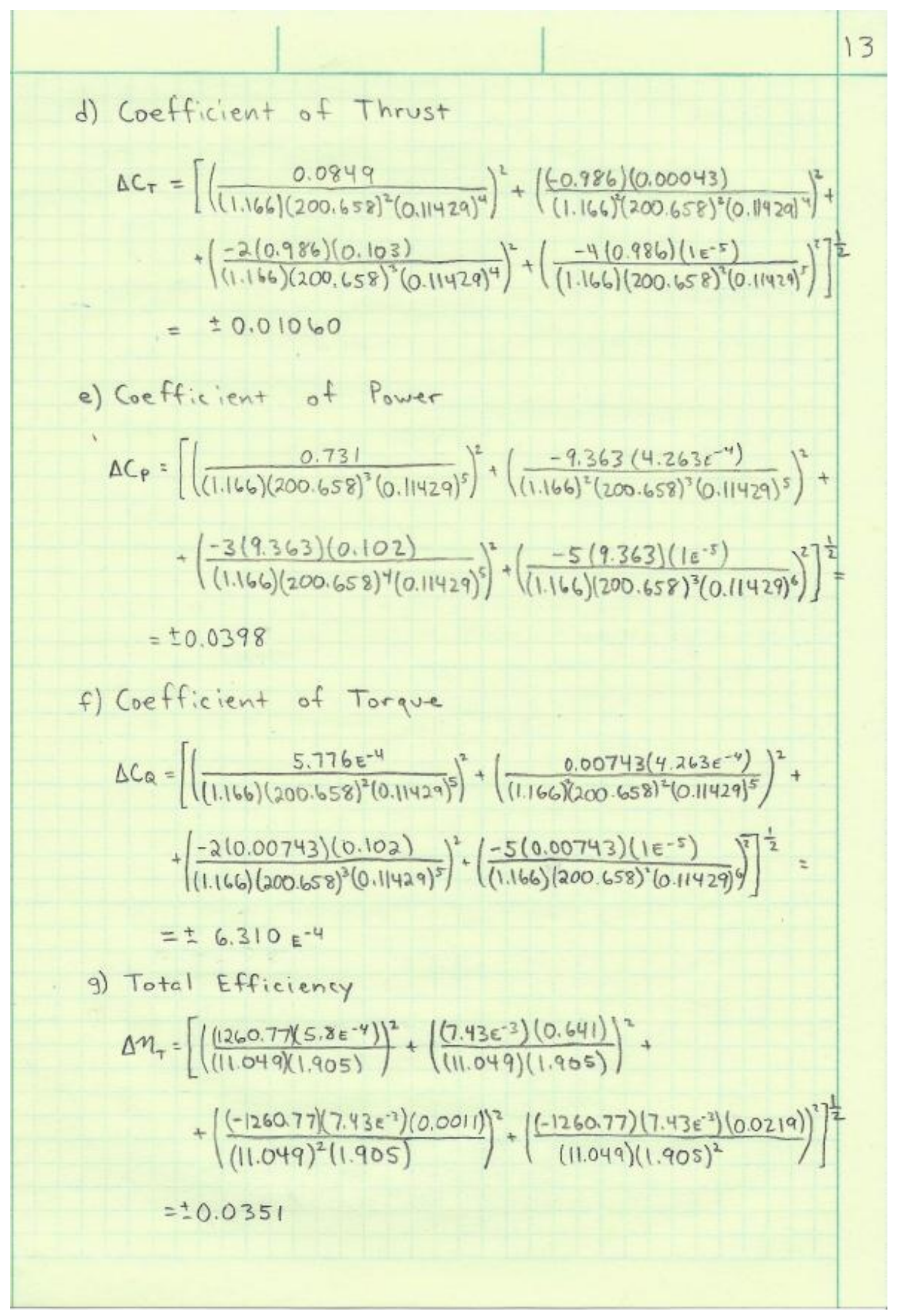




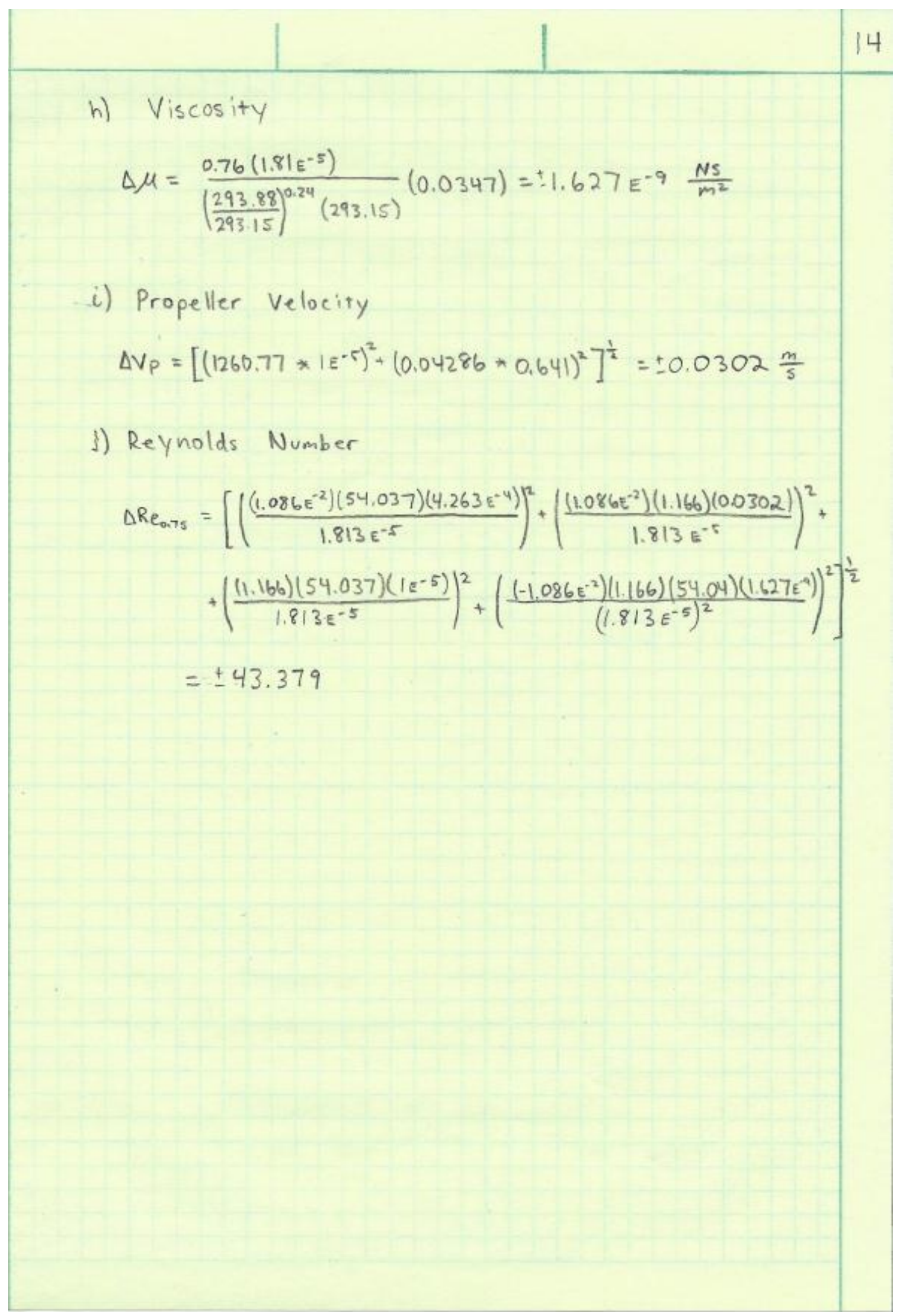




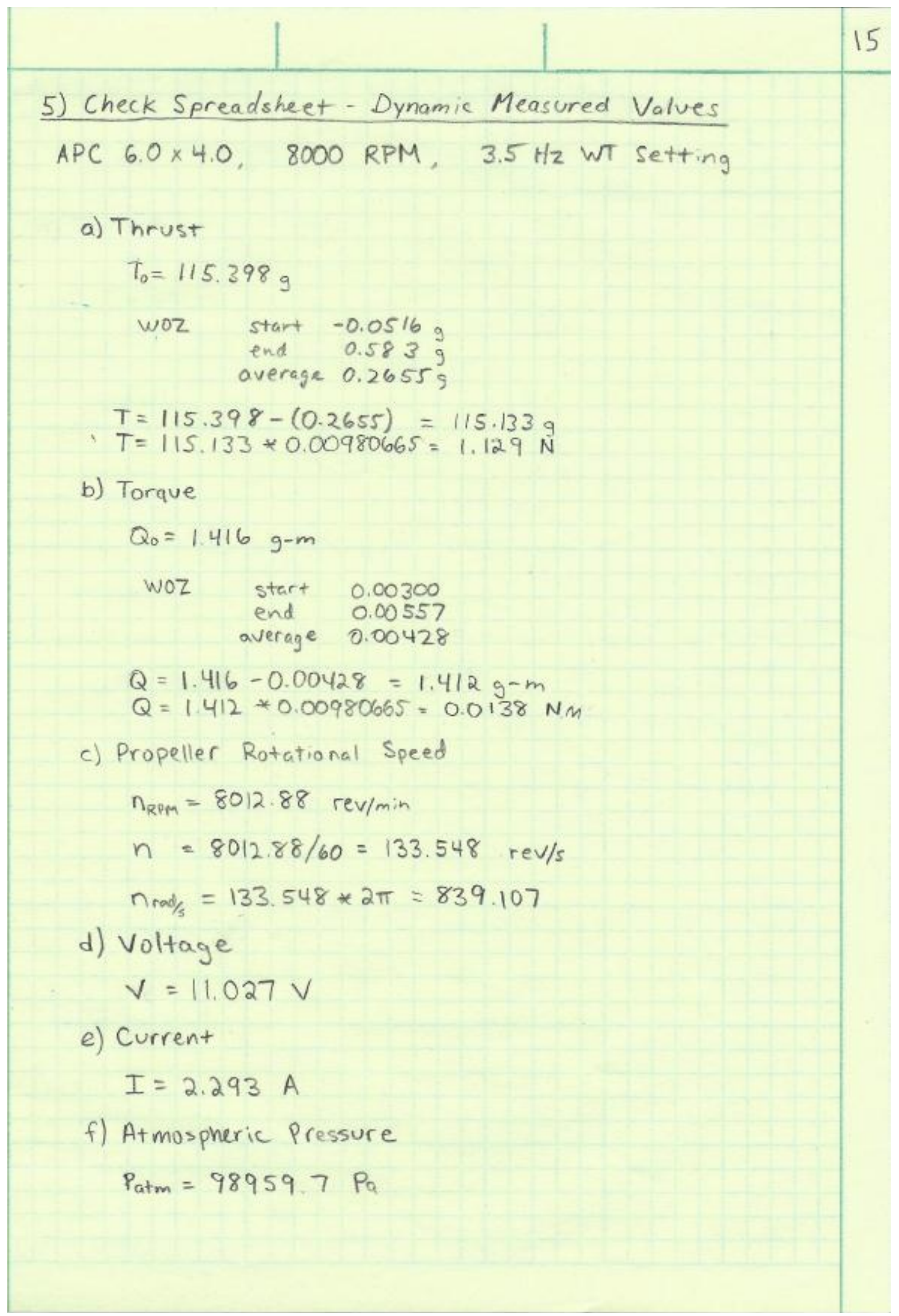




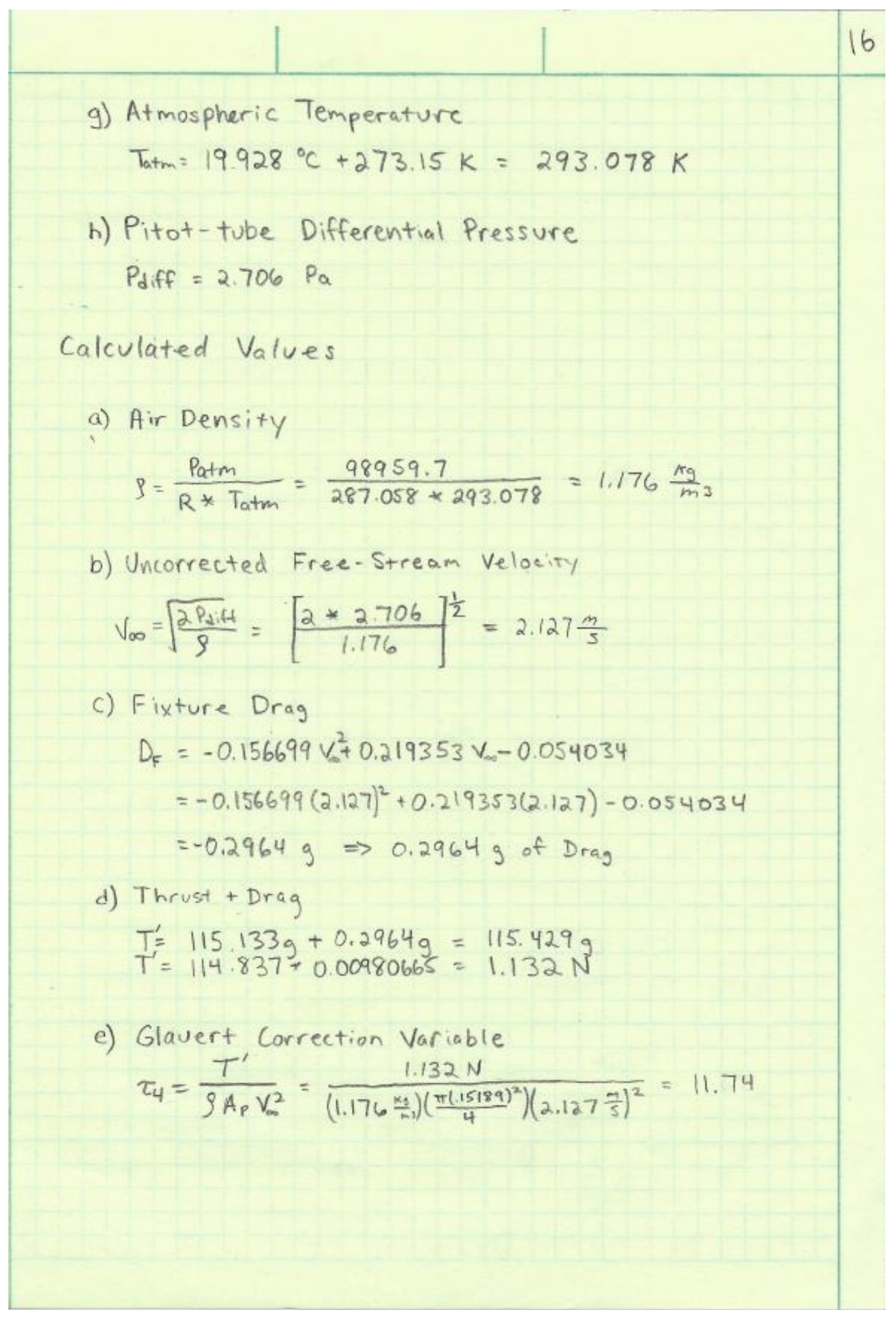









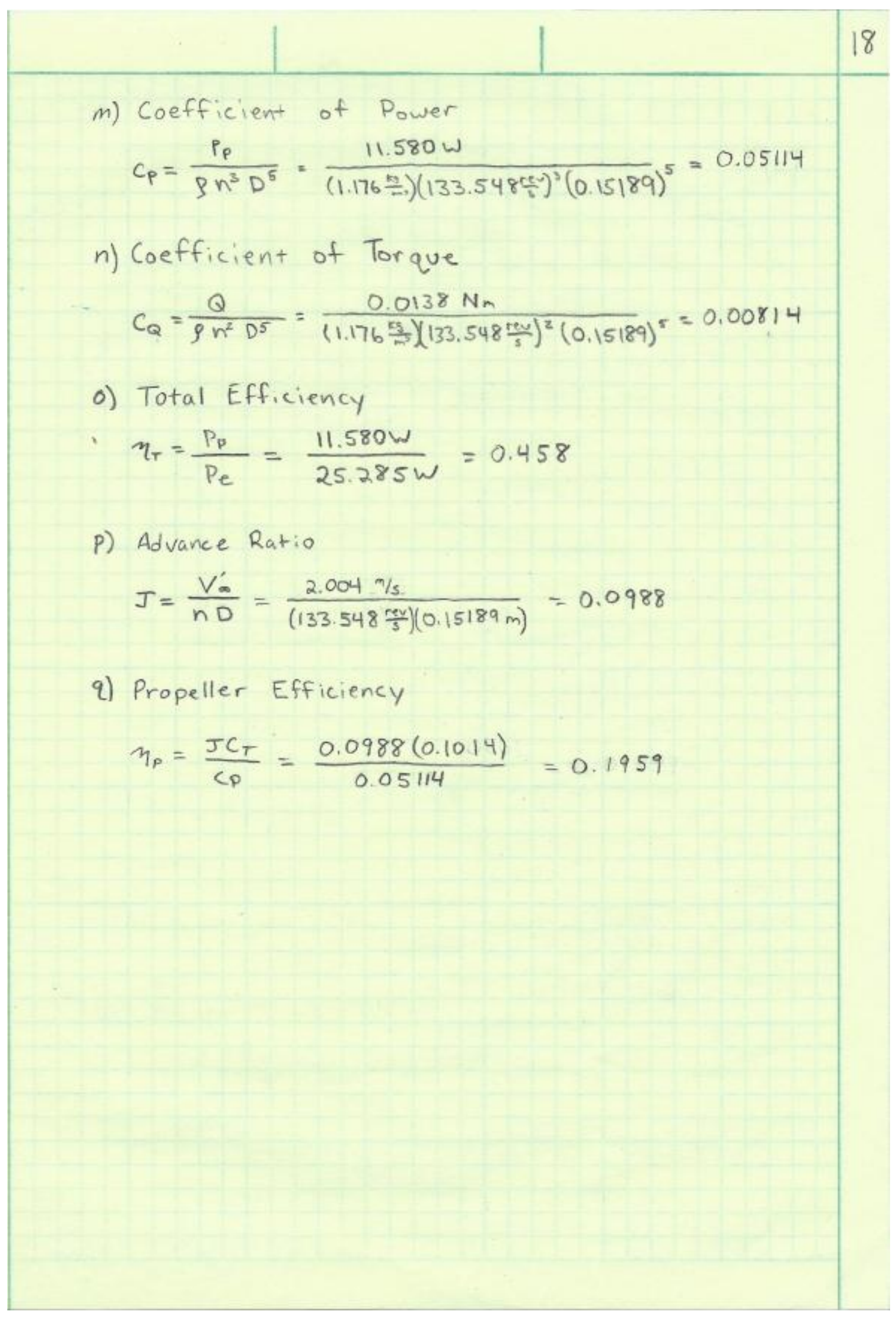




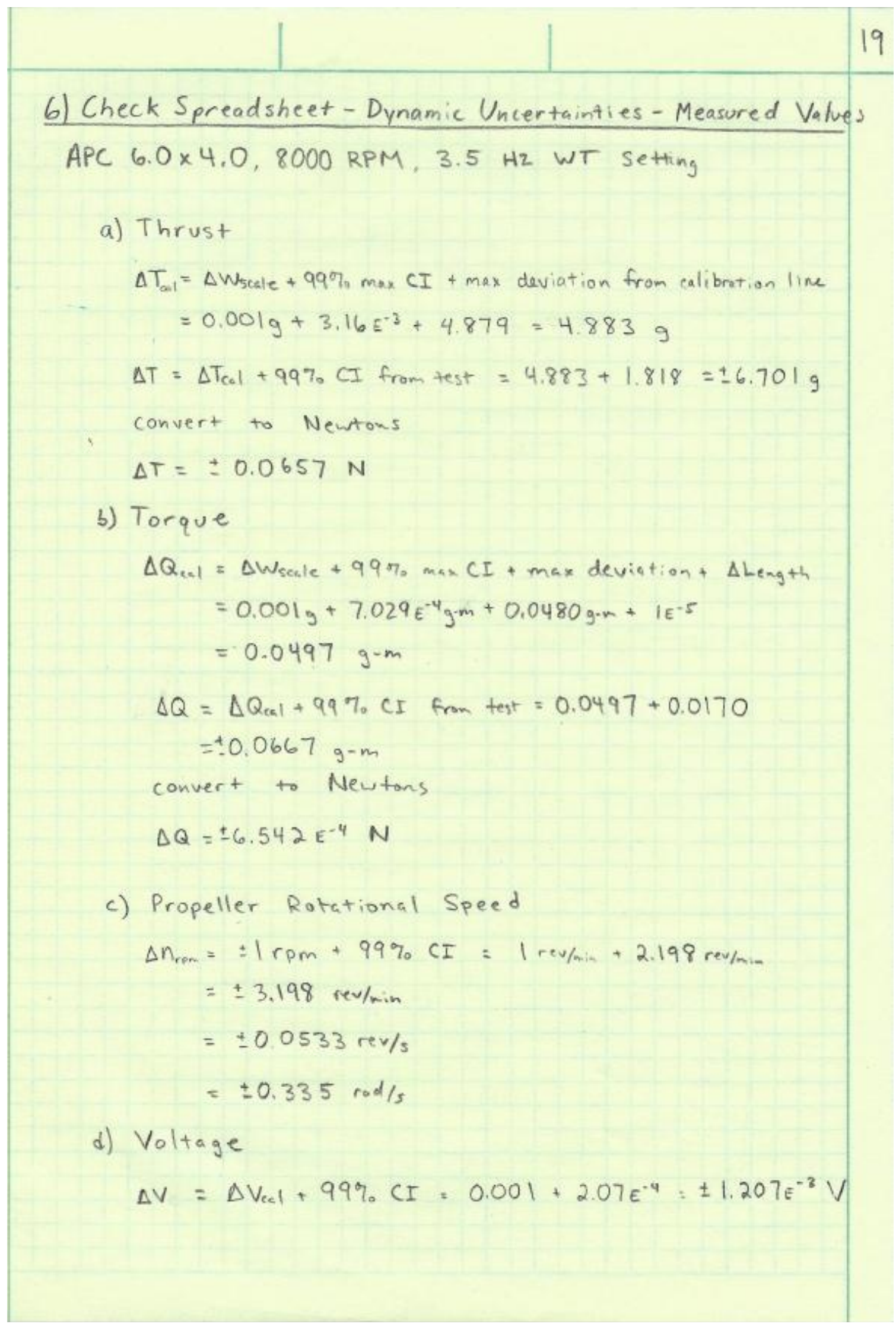




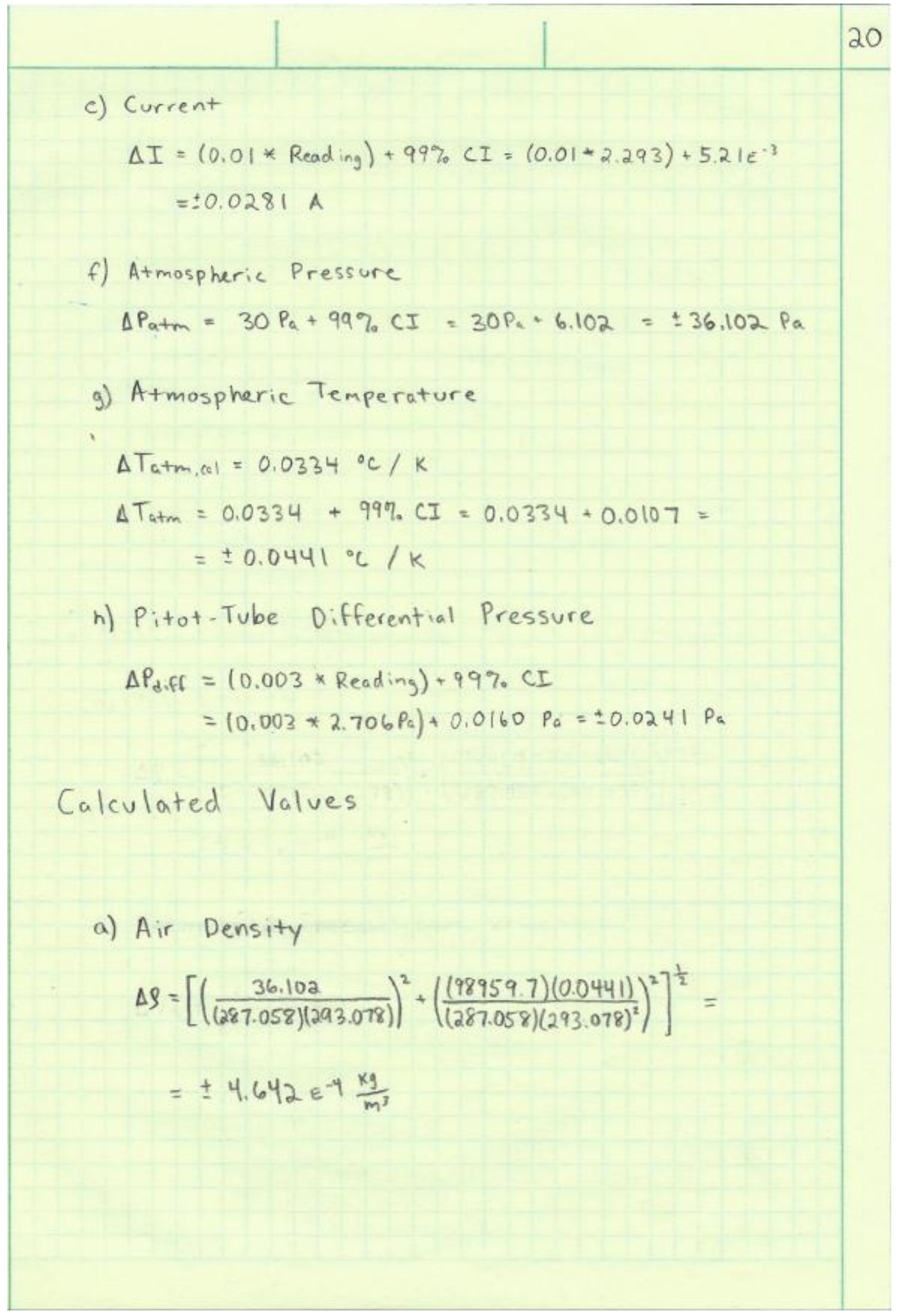




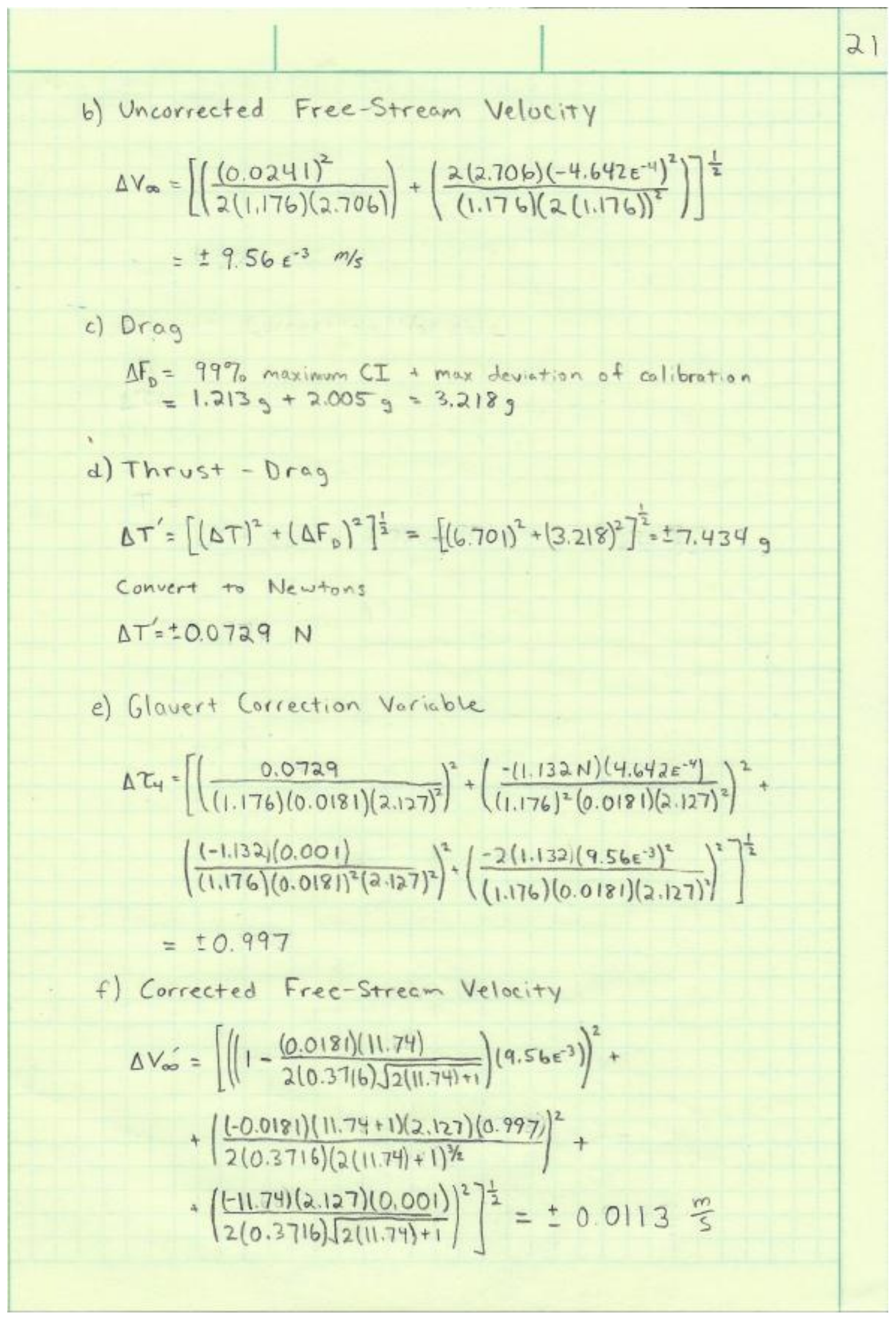


g) Total Velocity

$$
\begin{aligned}
\Delta v_{t} & =\left[\left(\frac{(2.004)^{2}(0.0113)^{2}}{4(133.548)^{2} \pi^{2}(0.05696)^{2}+(2.004)^{2}}\right)+\right. \\
& +\left(\frac{16(133.548)^{2} \pi^{4}(0.05696)^{4}(0.0533)^{2}}{4(133.548)^{2} \pi^{2}(0.05696)^{2}+(2004)^{2}}\right) \\
& \left.+\left(\frac{16(133.548)^{4} \pi^{4}(0.05696)^{2}\left(16^{-5}\right)^{2}}{4(133.548)^{2} \pi^{2}(0.05696)^{2}+(2.004)^{2}}\right)\right]^{\frac{1}{2}}= \pm 0.0208 \frac{\mathrm{m}}{\mathrm{s}}
\end{aligned}
$$

h) Coefficient of Thrust

$$
\begin{aligned}
\Delta C_{T}= & {\left[\left(\frac{0.0729}{(1.176)(133.548)^{2}(0.15189)^{4}}\right)^{2}+\left(\frac{-(1.132)\left(4.642 \epsilon^{-9}\right)}{(1.176)^{2}(133.548)^{2}(0.15189)^{4}}\right)^{2}+\right.} \\
& \left.+\left(\frac{-2(1.132)(0.0533)}{(1.176)(133.548)^{3}(0.15189)^{4}}\right)^{2}+\left(\frac{-4(1.132)\left(11 \epsilon^{-5}\right)}{(1.176)(133.548)^{2}(0.15189)^{5}}\right)^{2}\right]^{\frac{1}{2}}= \\
= & \pm 0.00653
\end{aligned}
$$

i) Propeller Power

$$
\Delta P_{P}=\left[\left(839.107 * 6.542 \mathrm{E}^{-4}\right)^{2}+(0.0138 \times 0.335)^{2}\right]^{\frac{1}{2}}= \pm 0.5490 \mathrm{~W}
$$

j) Electrical Power

$$
\Delta P_{e}=\left[\left(2.293 * 1.207 e^{-3}\right)^{2}+(11.027 * 0.0281)^{2}\right]^{\frac{1}{2}}= \pm 0.3100 \mathrm{~W}
$$

k) Viscosity

$$
\Delta \mu=\frac{0.76\left(1.81 E^{-5}\right)}{\left(\frac{293.078}{293.150}\right)^{0.24}(293.150)}(0.0441)= \pm 2.069 E^{-9} \frac{\mathrm{Ns}_{5}}{\mathrm{~m}^{2}}
$$


1) Coefficient of Power

$$
\begin{aligned}
\Delta C_{p} & =\left[\left(\frac{0.549}{(1.176)(133.548)^{3}(0.15189)^{5}}\right)^{2}+\left(\frac{-11.580\left(4.642 E^{-4}\right)}{(1.176)^{2}(133.548)^{3}(0.15189)^{5}}\right)^{2}+\right. \\
& \left.+\left(\frac{-3(11.580)(0.0533)}{(1.176)(133.548)^{4}(0.15189)^{5}}\right)^{2}+\left(\frac{-5(11.580)\left(1 E^{-5}\right)}{(1.176)(133.548)^{3}(0.15189)^{5}}\right)^{2}\right]^{\frac{1}{2}}= \\
& = \pm 2.425 E^{-3}
\end{aligned}
$$

m) Coefficient of Torque

$$
\begin{aligned}
\Delta C_{Q} & =\left[\left(\frac{6.542 E^{-4}}{(1.176)(133.548)^{2}(0.15189)^{5}}\right)^{2}+\left(\frac{(0.0138)\left(4.642 \epsilon^{-4}\right)}{(1.176)^{2}(133.548)^{2}(0.15189)^{5}}\right)^{2}+\right. \\
& \left.+\left(\frac{-2(0.0138)(0.0533)}{(1.176)(133.548)^{2}(0.15189)^{5}}\right)^{2}+\left(\frac{-5(0.0138)\left(1 \epsilon^{-5}\right)}{(1.176)(133.548)^{2}(0.15189)^{5}}\right)^{2}\right]^{\frac{1}{2}}= \\
& = \pm 3.860 \epsilon^{-4}
\end{aligned}
$$

n) Total Efficiency

$$
\begin{aligned}
\Delta \eta_{T} & =\left[\left(\frac{(839.107)\left(6.542 \varepsilon^{-4}\right)}{(11.027)(2.293)}\right)^{2}+\left(\frac{(0.0138)(0.335)}{(11.027)(2.293)}\right)^{2}+\right. \\
& \left.+\left(\frac{(-839.107)(0.0138)\left(1.207 \varepsilon^{-3}\right)}{(11.027)^{2}(2.293)}\right)^{2}+\left(\frac{(8.839 .107)(0.0138)(0.0281)}{(11.027)(2.293)^{2}}\right)^{2}\right]^{\frac{1}{2}}= \\
& = \pm 0.0224
\end{aligned}
$$

0) Advance Ratio

$$
\begin{aligned}
\Delta J & =\left[\left(\frac{0.0113}{(133.548)(0.15189)}\right)^{2}+\left(\frac{(2.004)(0.0533)}{(133.548)^{2}(0.15189)}\right)^{2}+\left(\frac{(2.004)\left(16^{-5}\right)}{(133.548)(0.15189)^{2}}\right)^{2}\right]^{\frac{1}{2}}= \\
& = \pm 0.000559
\end{aligned}
$$




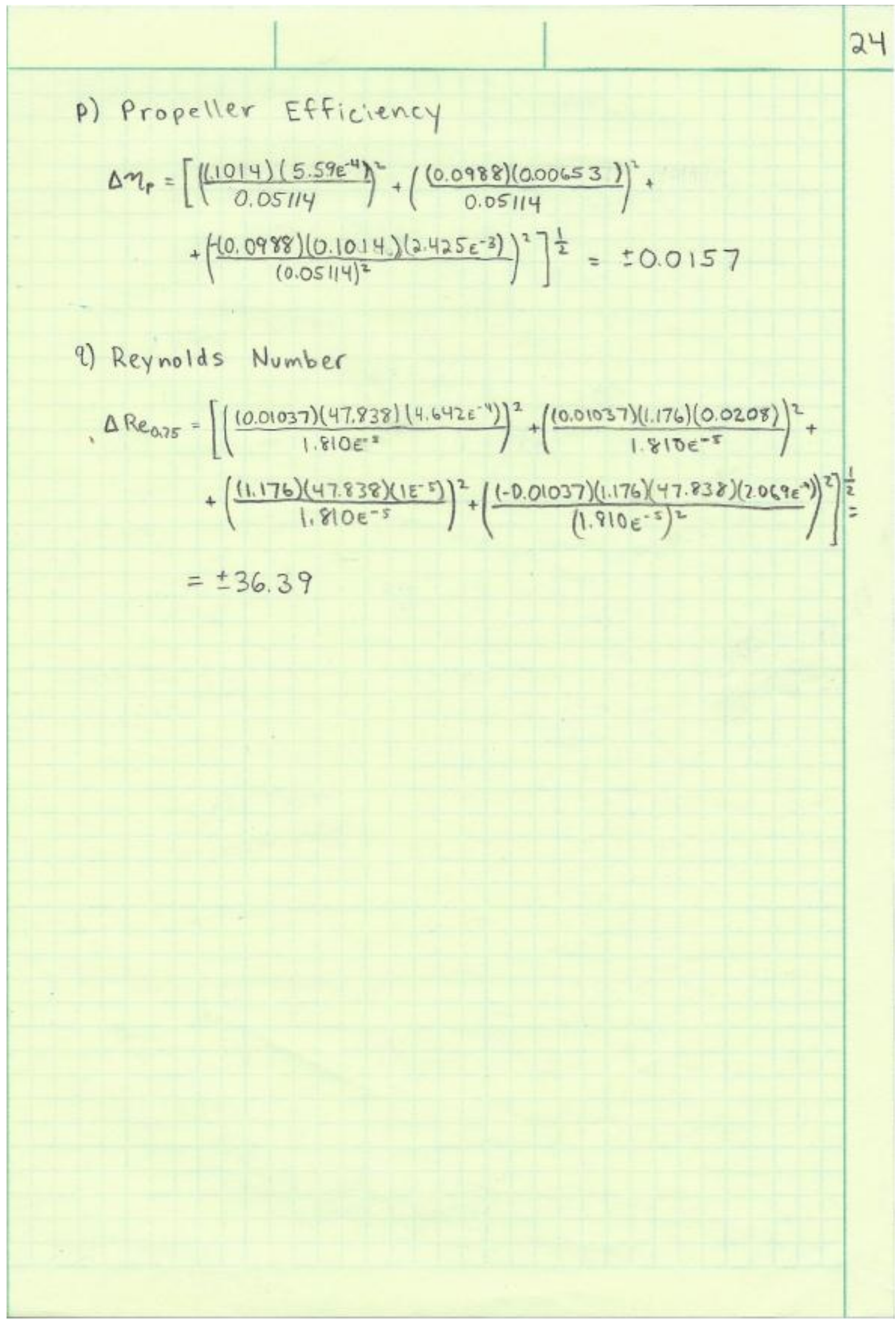




\section{APPENDIX C: INSTRUMENTATION SELECTION}

In order to determine required size and accuracy of the load and torque cells for the wind tunnel test stand, a simple static test stand was constructed. Testing the performance of the motor and propellers statically proved to be the simplest and least expensive method for finding the maximum value of thrust and torque simultaneously so that ranges for the sensors could be selected. Knowing the maximum values also is useful in determining the minimum resolution of the load and torque cell. In order to meet our requirements for accuracy, the minimum value to measure was $1 / 100$ of the maximum value. This requirement can easily be seen in the thrust measurement in the wind tunnel. When there is no incoming velocity, the thrust is at its maximum. As the free stream velocity increases, eventually the thrust will go to zero at the windmill state. In order to achieve accuracy over this range, the resolution of $1 / 100$ was required to measure the small velocities.

The stand was designed in Solidworks as individual pieces that could be cut using the laser cutter at Wright State and assembled to ensure proper fit. The pieces were individually designed to include flanges to join the pieces together and to make the structure stronger. Once cut, the pieces were glued together to create box beams. The design of the stand is shown in Figure 26.

The thrust measurement was found by simply mounting the motor and propeller under the crossbeam to drive the whole stand into a digital scale. The motor that was used was a GWS $2300 \mathrm{Kv} 20 \mathrm{~mm}$ brushless in-runner. It was determined using the propeller size, weight, wingspan, and mission profile of the WASP airframe. Since the only mounting points on the motor were on the front, a one inch wide strip of sheet metal was 
bent into a $U$ shape so that the motor could be joined to the stand. A piece of aluminum plate was used to join the motor to a model engine crankshaft and its housing. This housing contained thrust bearings to allow thrust, as well as torque, to be transferred through the shaft. The crankshaft housing was attached to the top of the crossbeam using socket head cap screws so that part of the crankshaft extended above the beam. The torque arm was laser cut to give a nine inch distance from the center of rotation to a 0 100 gram spring scale. This torque force, multiplied by the length of the arm, gives the torque of the motor. The front view of the setup is shown below in Figure 27 and a close up of the motor attachment is given in Figure 28. The top view of the stand is shown in Figure 29.

Three propellers were tested using the stand. These included a $4.75 \times 4.75$ inch carbon fiber propeller made by APC, as well as a $4.7 \times 4.25$ and a $4.5 \times 4.1$ inch propeller, both made from plastic composite by APC. The propeller was connected directly to the motor using a two millimeter prop adapter. The battery selected was a three cell (in series) lithium polymer battery rated for 11.1 volts and $2000 \mathrm{mAh}$ with a 20C discharge rate. The speed controller was a Castle Thunderbird model rated for 36 Amps. These were connected to a receiver and a transmitter was used to control the throttle.

Voltage and current measurements from the battery were necessary to determine power consumption and to calculate motor efficiency. Current was found by using a clamp-on ammeter and the voltage was found by breaking the circuit and measuring across the battery using a voltmeter. The rotational speed of the propeller was found by using a propeller tachometer that measures the number of shadows that pass over a 
sensor. Lastly, motor temperature was monitored at the center of the length of the motor using a Type $\mathrm{T}$ thermocouple. The electrical schematic is shown in Figure 30. To ensure safety, a shield was made from $3 / 8^{\text {th }}$ inch Plexiglas to separate the operator from any moving components. It was constructed in a tri-fold design to store easily and to protect the operator on three sides around the propeller.

After the test was completed using the battery to produce power, a power supply was used to maintain nearly a constant voltage throughout the test. The power supply was a 0-60 volt, 0-50 amp Hewlett Packard model 6032A. Having the constant voltage is very important in the wind tunnel to get a constant output from the motor to ensure steady state performance as well as being able to perform extended tests. This power supply was tested on the static test stand before moving it to the wind tunnel to ensure it worked properly. In order to make sure the throttle position was set at an exact and repeatable value, a GWS MC-1 servo tester was used. It allowed for the exact value of throttle position to be displayed by the LCD screen, thus giving better repeatability than a dial. It is shown in Figure 31 set at $60 \%$ throttle.

Since all of the components of the setup were not able to be connected to a computer or the voltage was too high for the available data acquisition system, the data was recorded using two high definition camcorders. One camera recorded thrust, current, volts, RPM, and temperature while the other recorded the torque. The video was then played back and the data was manually entered into Excel where it was manipulated and plotted.

The experimental procedure is as follows: First, the electronic speed controller must be connected to the motor. Next, the receiver or servo tester was connected to the 
speed controller making sure the throttle position was set to zero on the transmitter or servo tester. Care was taken to ensure that the battery was fully charged prior to each run so that the results could be as identical as possible. Temperature was monitored using a type $\mathrm{T}$ thermocouple placed on the outside of the motor at its center of length. An upper limit on temperature was set at 60 degrees $\mathrm{C}$ to prevent damage to the motor. The clampon ammeter was then zeroed and all readouts were placed in the view of a camera. The other camera was placed above the spring scale to measure the torque. With all readouts on and all components safely away from the propeller, the power source was connected safely and the test started. First, the transmitter or servo tester was quickly turned to full throttle and then back to zero. This ensures full throttle is set at 100. Otherwise, full power will occur at about $80 \%$. Both cameras were set to record and the size of the propeller and date of the test were stated in the video.

Two tests were performed on each propeller. The first test was a full throttle run to see how long it would run and the performance over the lifecycle of the battery. The second test was a stepped run where the throttle would be increased one click on the transmitter every ten seconds until full throttle and then decreased one click at a time. For the full throttle runs the power was increased steadily until the throttle was set to $100 \%$. The full throttle test was ended when the speed controller began to cut power to the motor due to low battery voltage. After the cameras were turned off, a new battery was connected and the power was set at about $10 \%$ to keep cool air moving over the motor. For the stepped runs, the throttle was increased by one click on the transmitter every ten seconds until full throttle and then decreased one click every ten seconds until zero 
throttle. The data was played back to be entered into Excel every five seconds for the full throttle runs and every two seconds for the stepped throttle runs.

For each of the two tests there was measured raw data, calculated data, and reduced data. The measured raw data included the thrust in grams, torque force in grams, voltage, current, temperature, and propeller speed in revolutions per minute. The calculated data included the motor torque in $\mathrm{Nm}$ and motor power in Watts. The reduced data included coefficient of torque, thrust, and power, as well as efficiency.

The first static test was a full throttle run until the battery voltage dropped and the speed controller began to cut the power. The second test was a stepped run where the throttle was increased by one click on the transmitter every ten seconds until full throttle was reached. It was then decreased in the same manner until the throttle was zero. Each test was completed for all three propeller sizes discussed previously.

The full throttle runs can tell a lot about the performance of the propeller and how each of the measured quantities interacts with each other. However, the main interest is the maximum values so that transducers can be selected for use in the wind tunnel so that data can be collected electronically. Figure 32 shows the thrust versus time for all three propellers while Figure 33 shows the torque.

Looking at the thrust graph shows that the $4.75 \times 4.75$ carbon fiber propeller ran out of power first yet produced the least amount of thrust. It did however produce the most torque due to it being the largest. The two other propellers performed similarly in thrust, but the $4.5 \times 4.1$ propeller produced noticeably less torque.

The other measured quantities are plotted versus time. The temperature versus time plot is given in Figure 34 while the RPM versus time, Volts versus time, and current 
versus time are given by Figure 35 to Figure 37, respectively. One big piece of information to come from these graphs is the relationship between temperature and thrust output. It is seen that as the temperature increases, the thrust, torque, RPM, and current draw all increase. This tells us that to achieve accurate results in the wind tunnel the motor must be allowed to reach steady state temperature before data is collected. The jump at the end of the temperature plot occurs after the motor is turned off and convective cooling from the propeller has stopped.

Looking at the results from the stepped runs can give further insight into the system performance. The voltage versus time plot is shown by Figure 38 and shows that as the throttle is decreased, the voltage does not recover to its previous value. This is due to the battery discharging and not being able to supply the same power as it had when the throttle is increasing. This tells us that a power supply is very important for even short tests.

Another point of information is the thrust versus RPM graph given by Figure 39. It shows how much thrust can be seen at various RPM levels. The $4.7 \times 4.25$ propeller follows the same line as the $4.75 \times 4.75$ carbon fiber propeller, but is able to spin faster and produce thrust beyond the carbon fiber propeller.

Since the main objective was to find the maximum thrust and torque so that load and torque cells could be selected for the dynamic tests in the wind tunnel. Table 5 shows the maximum values for each of the stepped runs. The highest trust of $2.61 \mathrm{~N}$ corresponds to a thrust of 266.2 grams. Therefore a $1 \mathrm{~kg}$ load cell proved to be suitable for our needs while leaving room to test larger propellers that may have more thrust. 


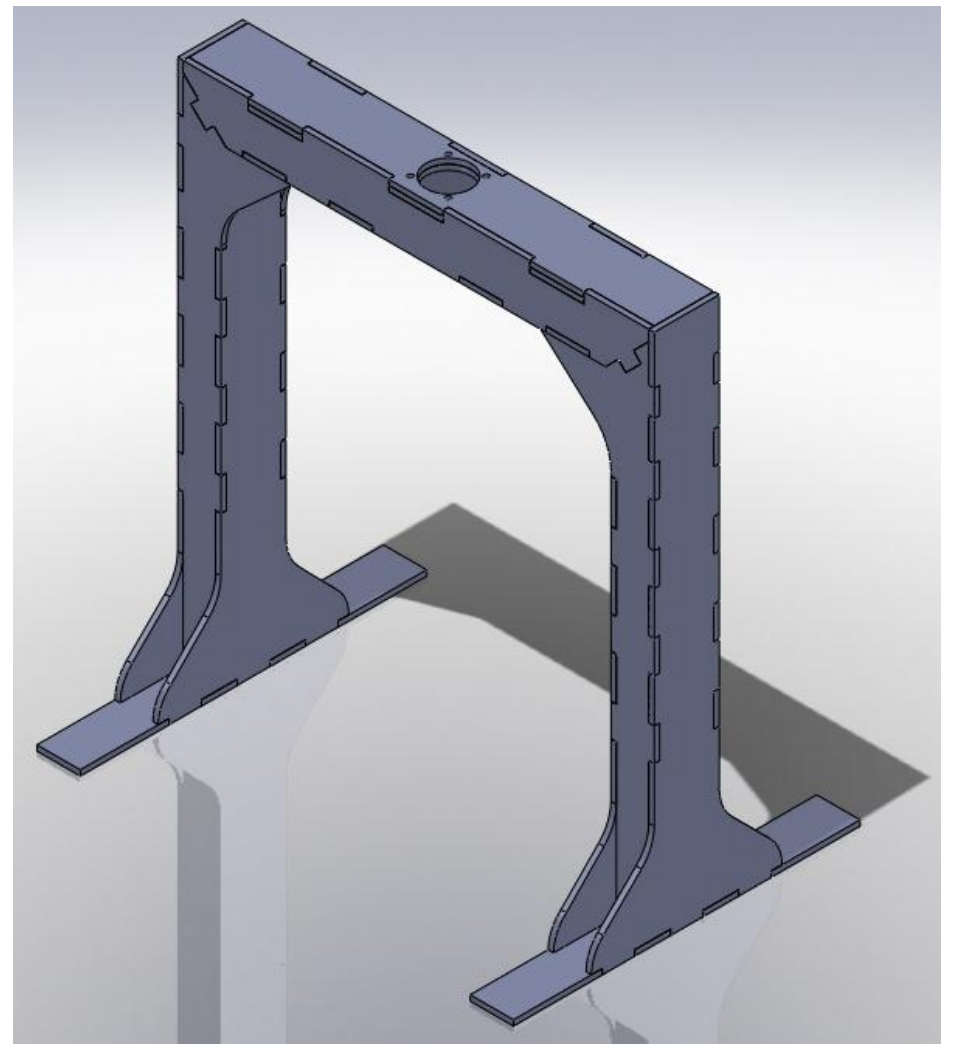

Figure 26: Solidworks Model of Static Test Stand

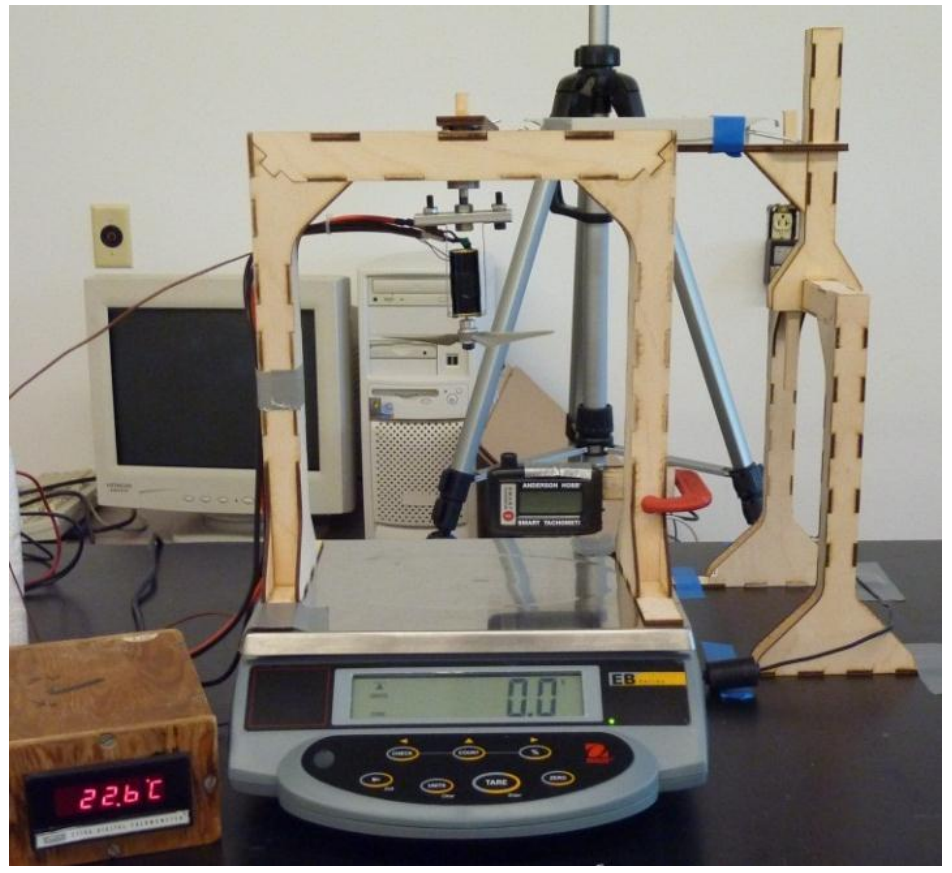

Figure 27: Static Test Stand (Front View) 


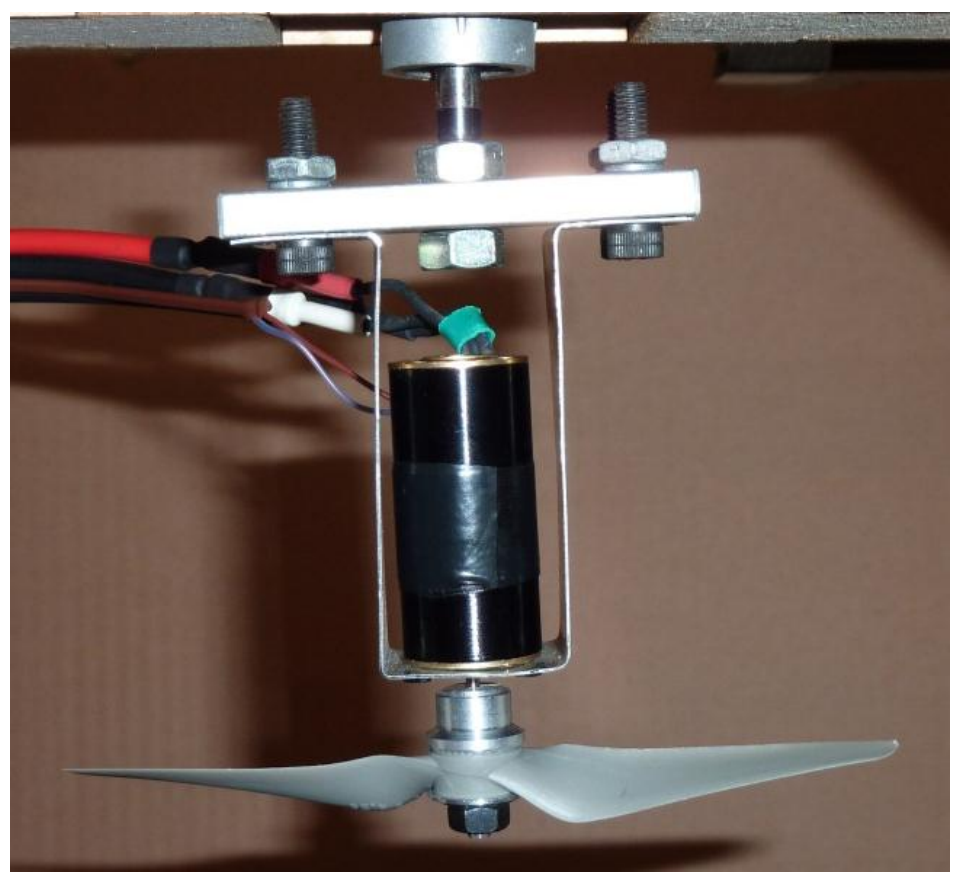

Figure 28: Motor Attachment

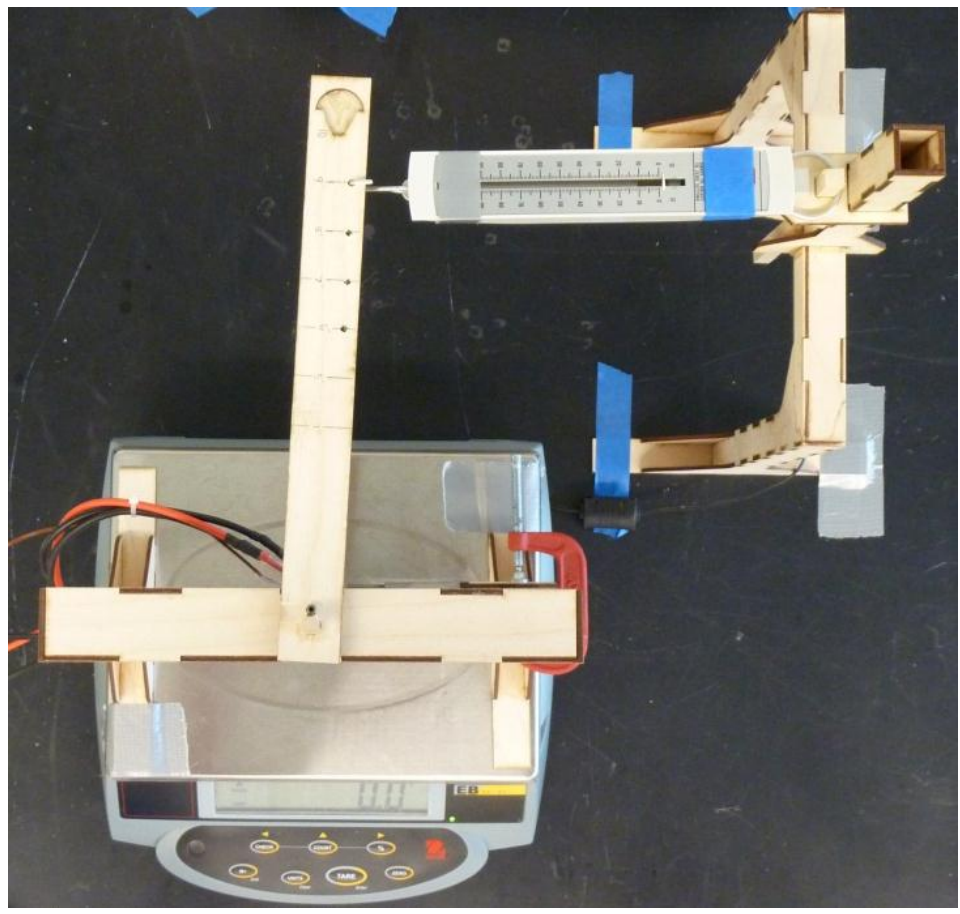

Figure 29: Top View of Test Stand 


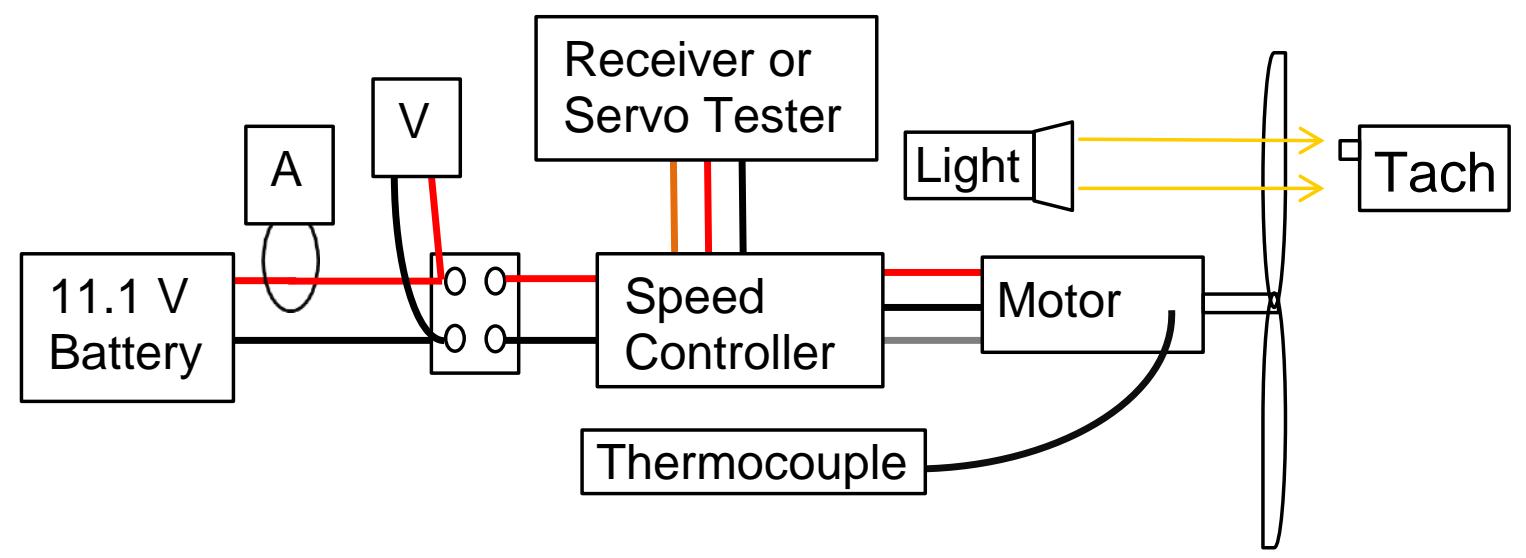

Figure 30: Electrical Schematic

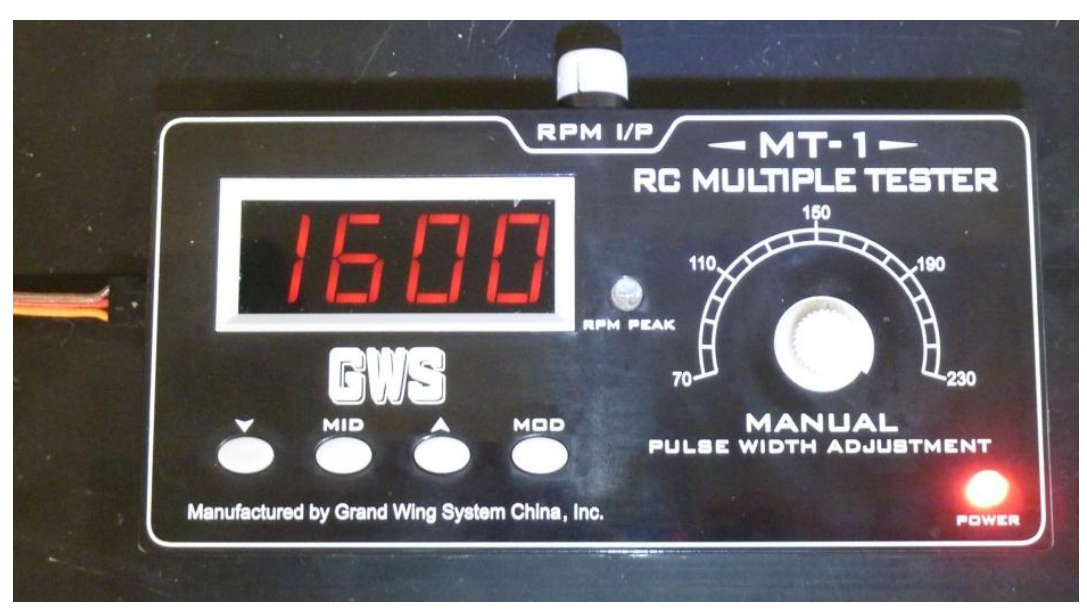

Figure 31: GWS MT-1 Servo Tester 


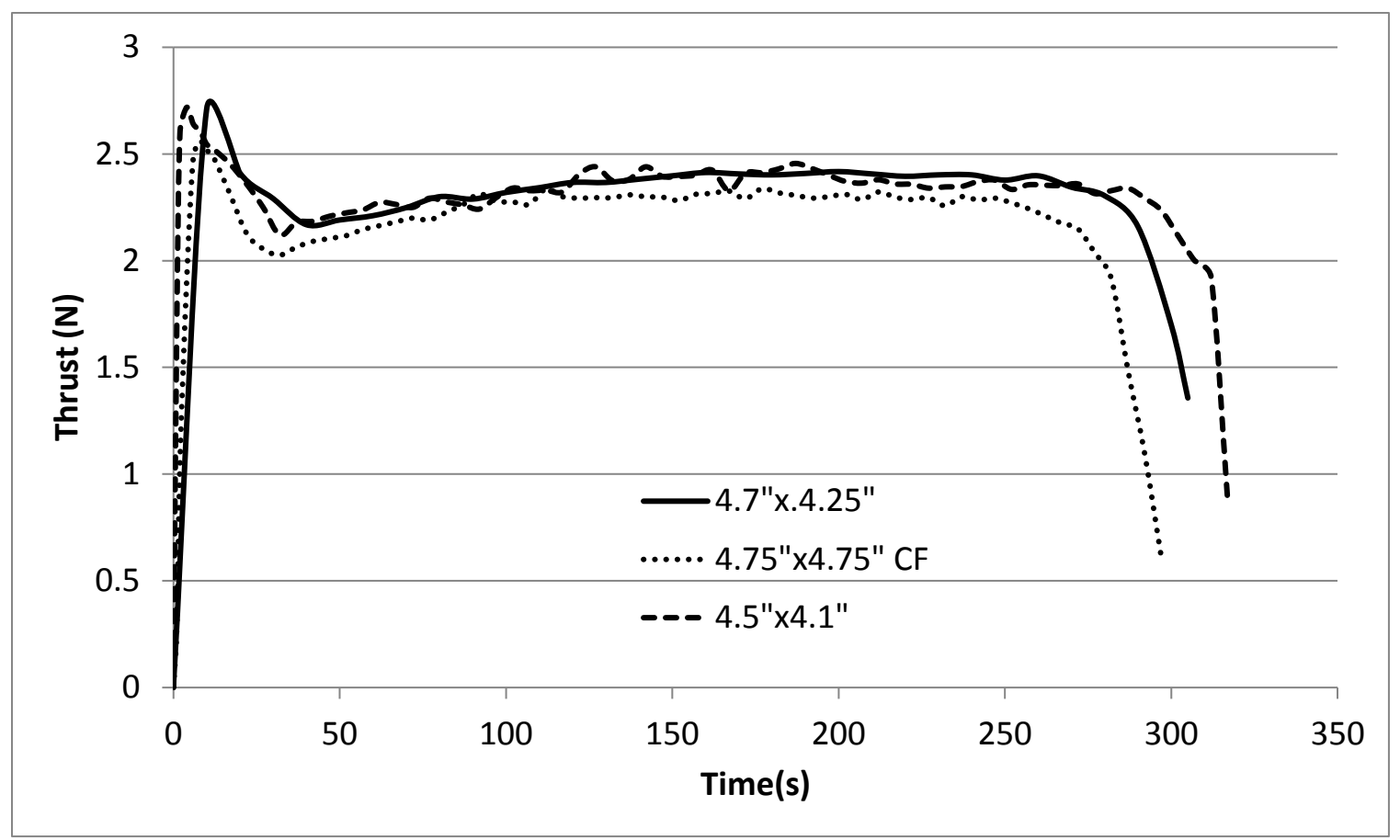

Figure 32: Full Throttle Thrust versus Time for Various Propellers

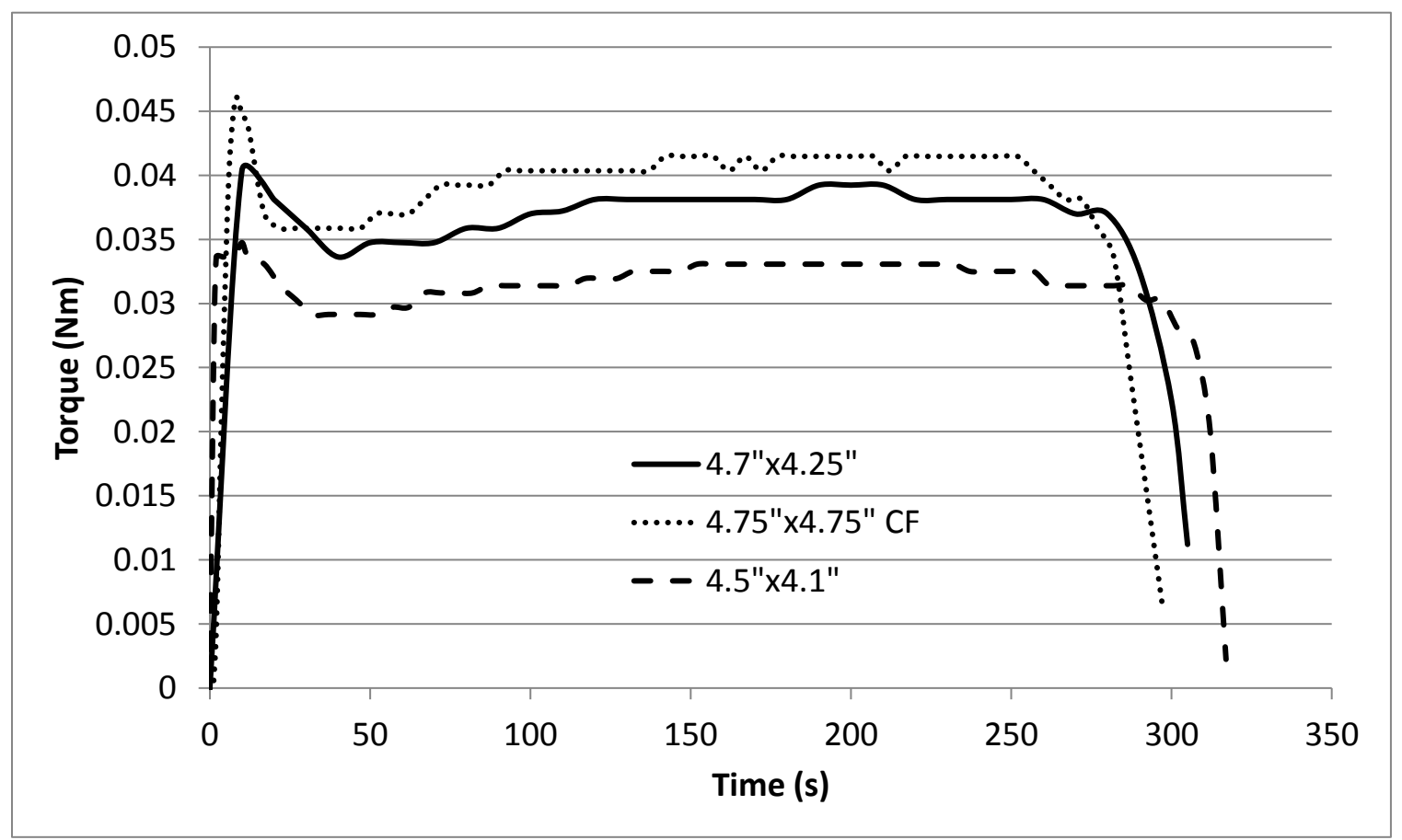

Figure 33: Full Throttle Torque versus Time for Various Propellers 


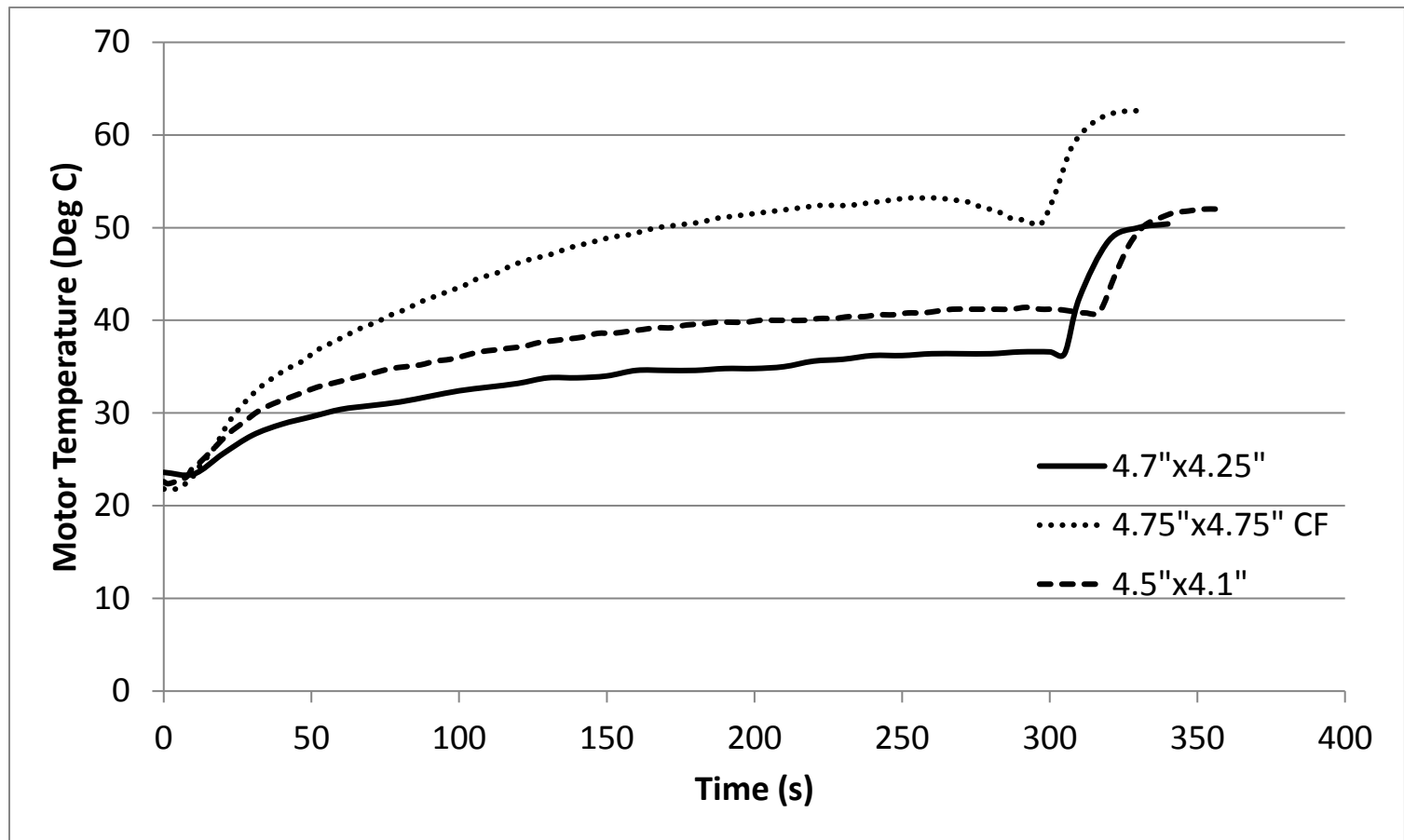

Figure 34: Temperature versus Time for Various Propellers

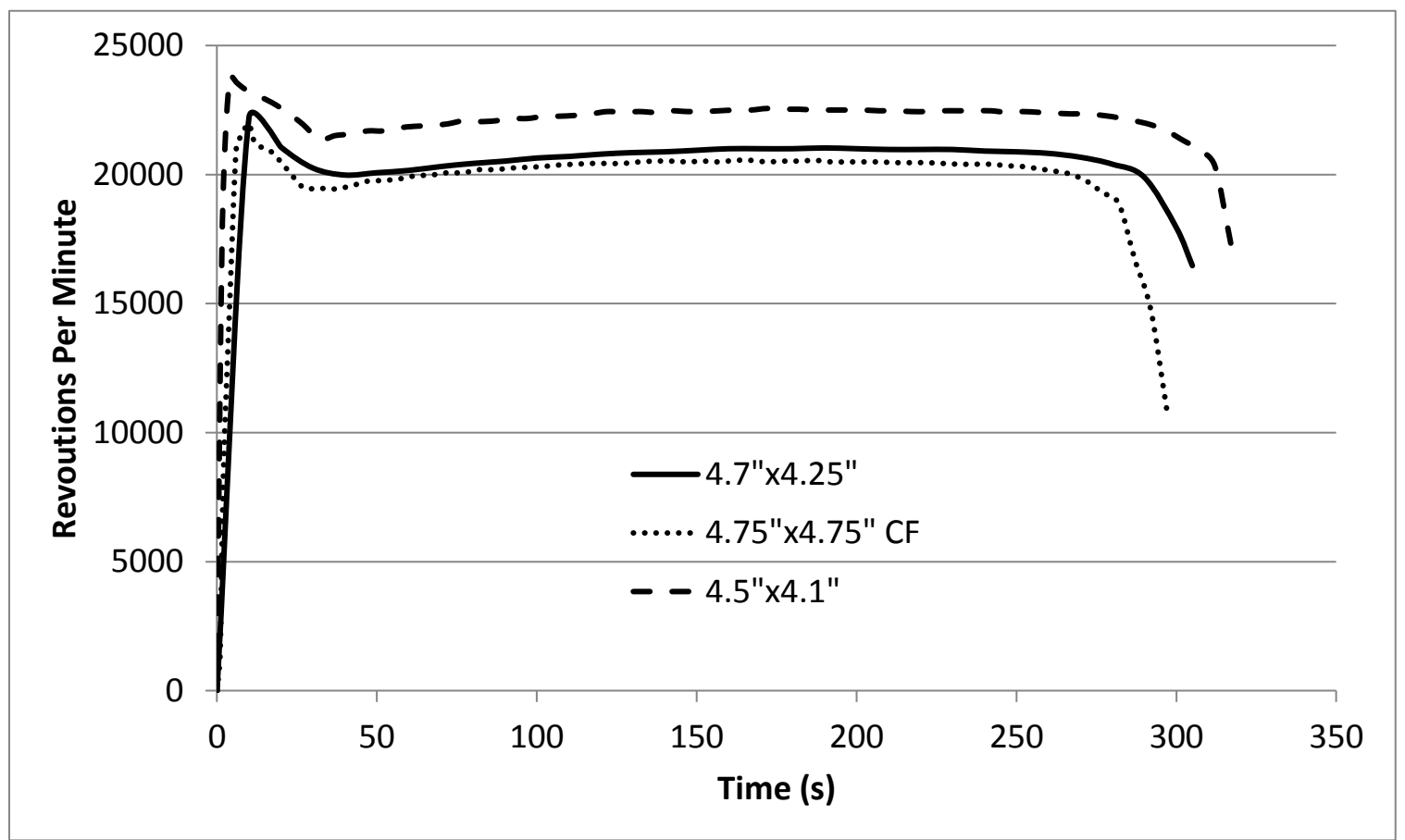

Figure 35: RPM versus Time for Various Propellers 


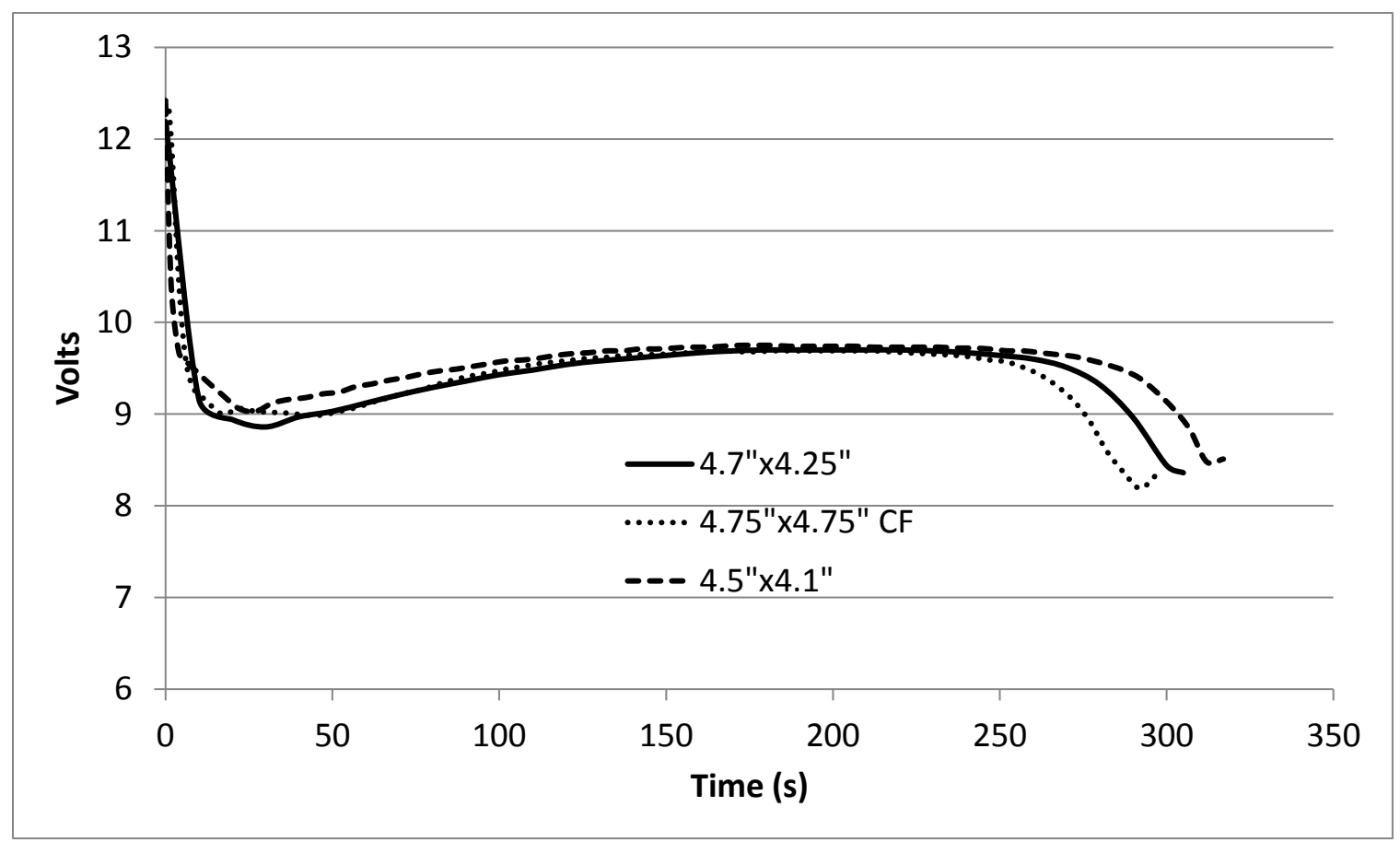

Figure 36: Voltage versus Time for Various Propellers

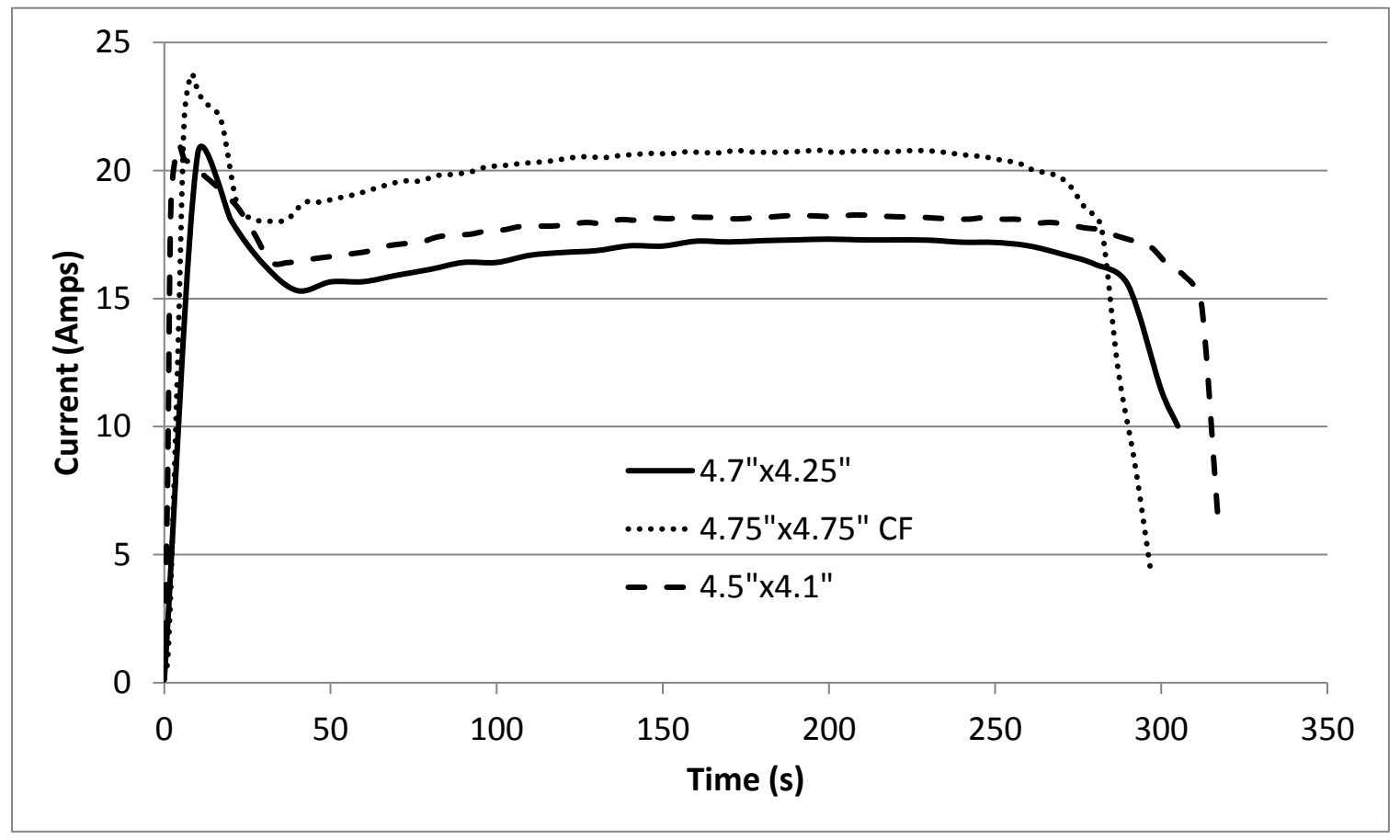

Figure 37: Current versus Time for Various Propellers 


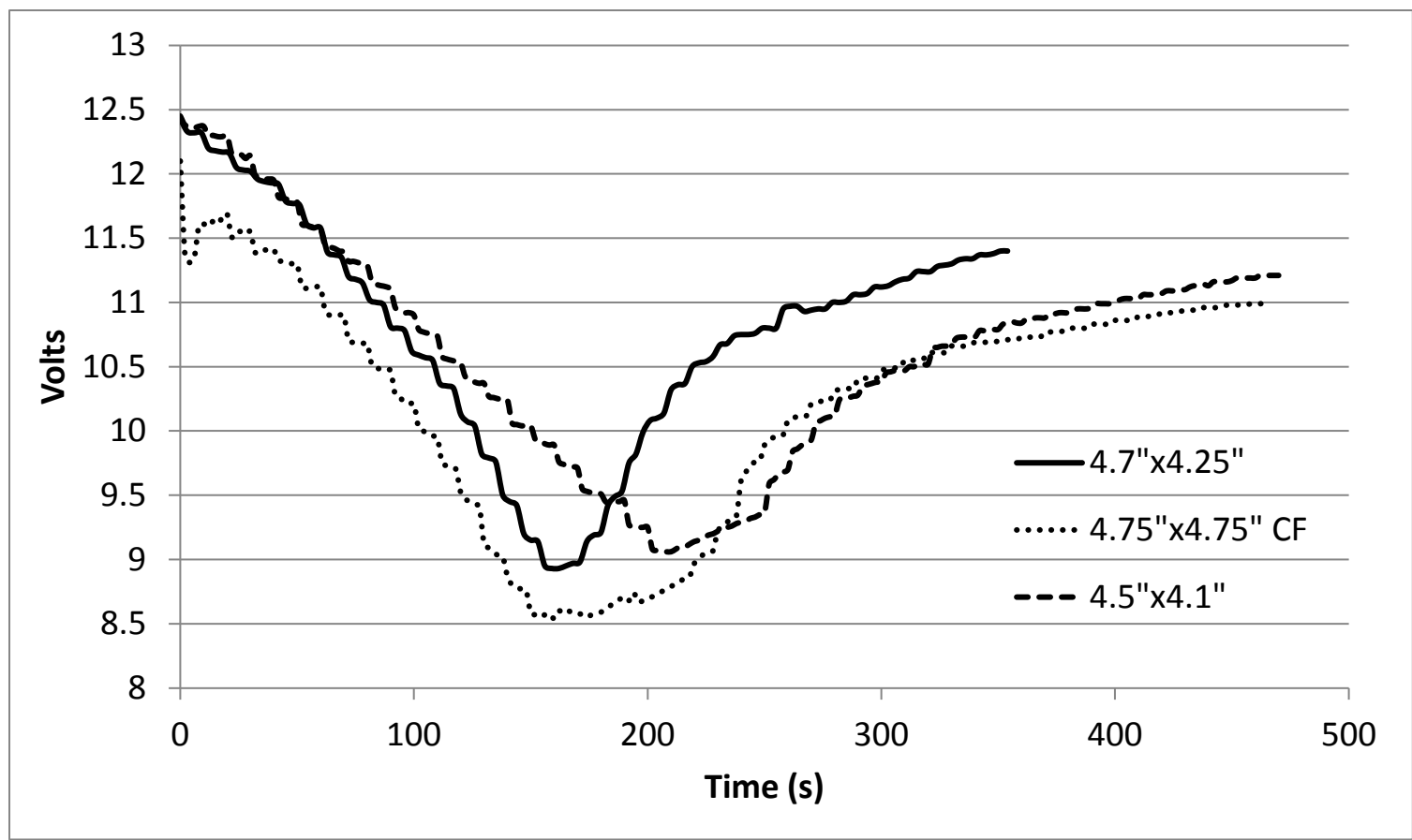

Figure 38: Stepped Throttle Voltage versus Time for Various Propellers

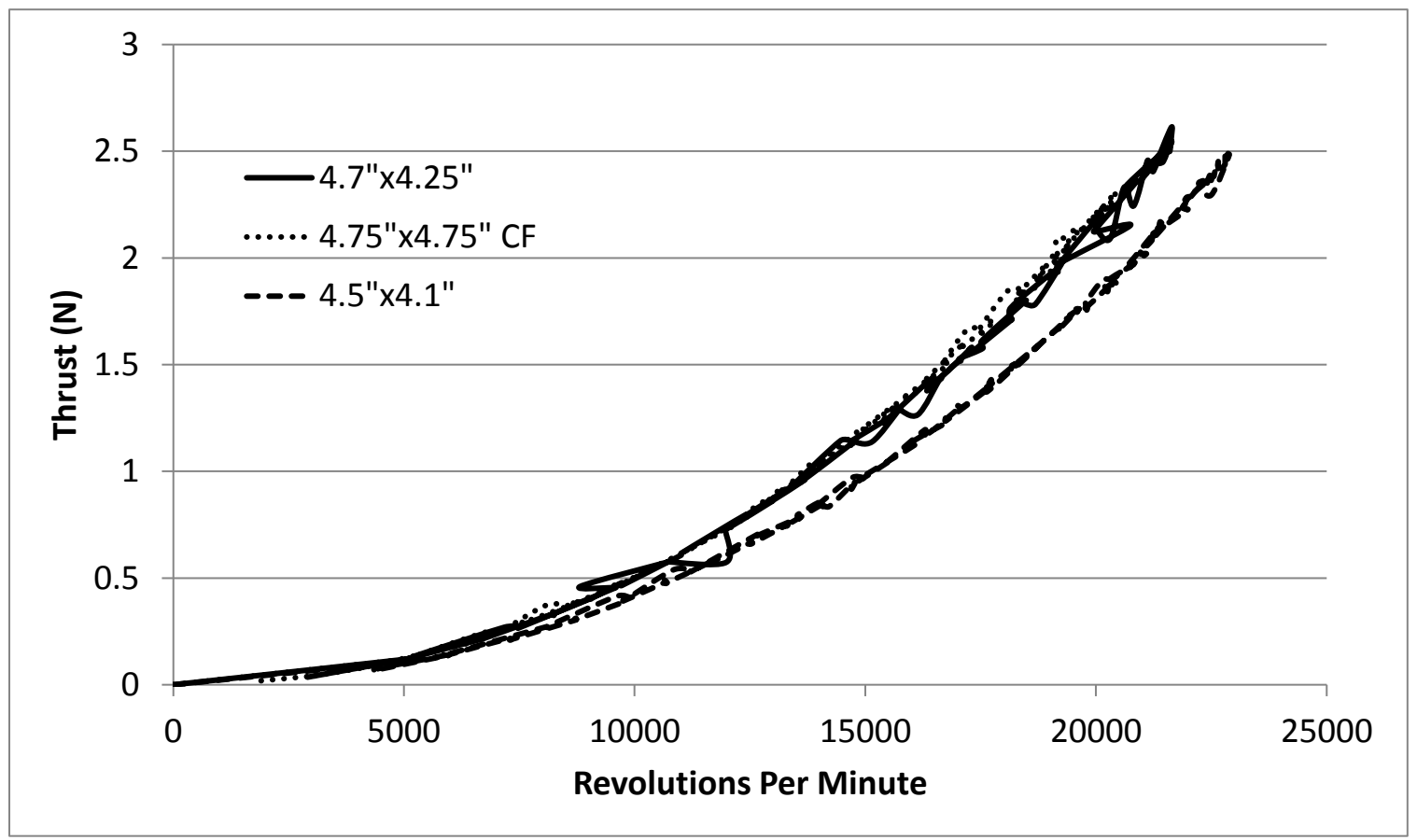

Figure 39: Stepped Throttle Thrust versus RPM for Various Propellers 
Table 5: Maximum Outputs from Stepped Runs

\begin{tabular}{|c|c|c|c|c|c|c|c|}
\hline Propeller & $\begin{array}{c}\text { Max } \\
\text { Thrust } \\
(\mathbf{N})\end{array}$ & $\begin{array}{c}\text { Max } \\
\text { Torque } \\
(\mathbf{N m})\end{array}$ & $\begin{array}{c}\text { Max } \\
\text { RPM }\end{array}$ & $\begin{array}{c}\text { Max } \\
\text { Temp } \\
\left({ }^{\circ} \mathbf{C}\right)\end{array}$ & $\begin{array}{c}\text { Max } \\
\text { Current } \\
(\mathbf{A})\end{array}$ & $\begin{array}{c}\text { Max } \\
\text { Power } \\
(\mathbf{W})\end{array}$ & Efficiency \\
\hline APC 4.7 x 4.25 & 2.61 & .0347 & 21630 & 42.6 & 20.18 & 181.22 & $43.68 \%$ \\
\hline APC 4.75 x 4.75 CF & 2.30 & .0414 & 20430 & 42.6 & 21.84 & 193.72 & $47.57 \%$ \\
\hline APC 4.5 x 4.1 & 2.49 & .0342 & 22890 & 41.2 & 20.08 & 187.75 & $45.04 \%$ \\
\hline
\end{tabular}




\section{APPENDIX D: WIND TUNNEL BOUNDARY LAYER MEASUREMENT}

In order to determine the location to place the Pitot-static tube inside of the wind tunnel to measure flow velocity, it was necessary to know the height of the boundary layer. If the Pitot tube is placed within the boundary layer of the walls of the wind tunnel, the measured velocity at the point will be smaller than the actual velocity at the centerline of the wind tunnel. If the placement is too high it may interfere with the continuity of the freestream airflow into the propeller. Another reason that the Pitot tube was not placed at the centerline was seen when the propeller was running. The propeller generates an inflow of air which was being recorded by the Pitot tube. In an effort to minimize this effect, the Pitot tube is placed lower in the test section. To determine the optimal location, the Pitot tube was placed at heights ranging from $0.5 \leq H \leq 4.5$ inches by 0.5 inches from the bottom of the wind tunnel. The centerline of the Pitot tube was set at 0.5 inches using a square from the floor of the wind tunnel. The tube was then made parallel to the walls using a custom gauge and a bubble level. Figure 40 shows the Pitot tube placement relative to the test stand in the wind tunnel.

The differential pressure manometer was then turned on and left to warm up for 30 minutes. After warm-up, the manometer was zeroed and then tests could be run. At each location the wind tunnel fan motor was varied from 5 to $35 \mathrm{~Hz}$ by $5 \mathrm{~Hz}$ increments to see the velocity profile at several airspeeds. Five hundred data points were collected for air temperature, atmospheric pressure, and Pitot-tube differential pressure to calculate the air velocity at each wind tunnel setting. 
After the velocity was swept through, the Pitot tube was raised another half inch and the test repeated until all heights were sampled. Figure 41 shows the velocity profile of the wind tunnel at several different airspeeds. It also shows the point at which the measured velocity reaches $99 \%$ of the freestream velocity measurement. The results show that the measured velocity reaches $99 \%$ freestream velocity at about 1.5 inches from the floor. The slope of the velocity profile levels off around this height. To ensure freestream conditions, the Pitot tube is placed at 2.5 inches from the floor for all subsequent propeller tests.

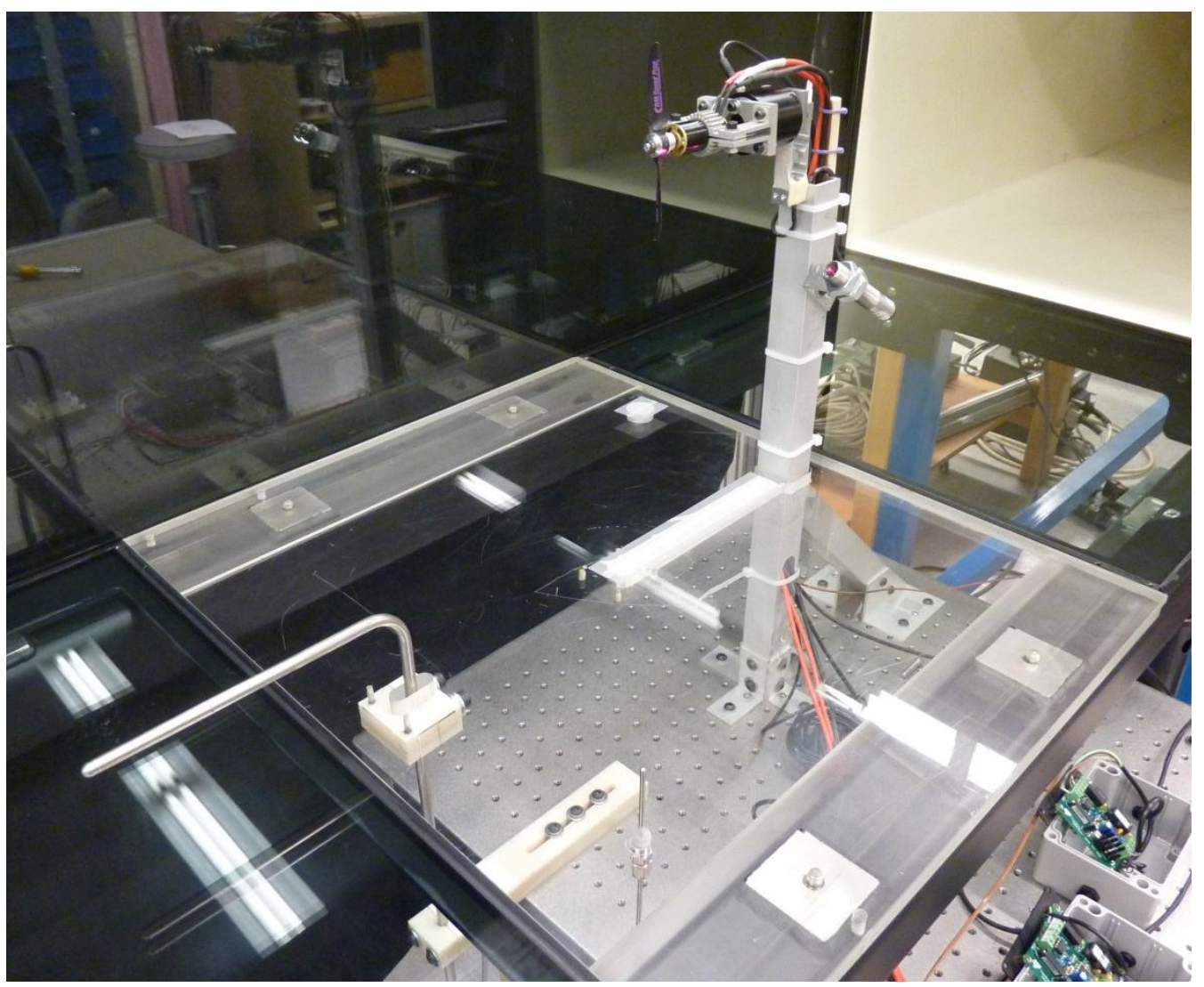

Figure 40: Pitot Tube Placement 


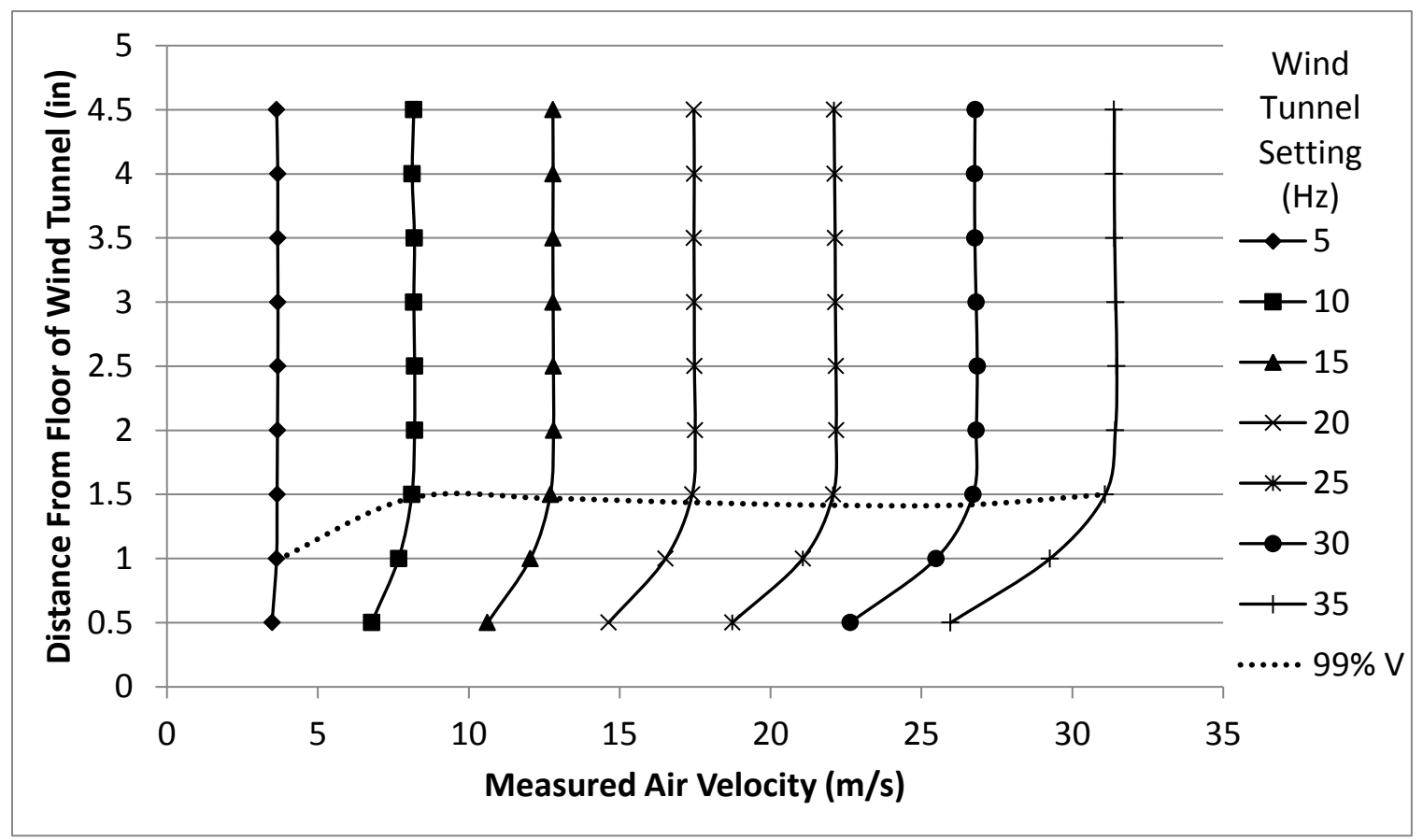

Figure 41: Velocity Profile at Different Airspeeds 


\section{APPENDIX E: EXPERIMENTAL PROCEDURES}

In order to perform the experiments, it was necessary to follow a detailed procedure to collect data. These procedures were designed to ensure that the tests were as repeatable and accurate as possible. Two separate procedures have been developed for static tests as well dynamic tests. These procedures begin by connecting the outputs from the transducers to the DAQ board. LabVIEW was then configured so that the DAQ board and the thermocouple modules were recognized by the DAQ card. The locations of each component were input into the LabVIEW VI. Reflective tape was then placed on the propeller near the hub so that the optical sensor could read the rotational speed. The propeller was then attached to the motor using an adapter for the given propeller. The power supply was turned on and set to an output of 11.1 Volts. From this point on, two separate procedures are followed for the static and dynamic cases.

\section{Static Experimental Procedure}

The first step in the static procedure was to power the load cell, torque cell, tachometer, barometer, and current transducer where they were left to warm up for a minimum of fifteen minutes. Once warmed up, the propeller was quickly turned up to $100 \%$ throttle and then back to zero. This sets the speed controller so that $100 \%$ actually corresponds to $100 \%$ throttle. Then the load cell and torque cell were zeroed by adjusting

the balance potentiometer on the signal conditioner so that the output as shown on a multimeter read as close as possible to zero. A file name and location were chosen such that it could be found easily later and identified such parameters as propeller diameter and pitch as well as rpm setting. At this point, five hundred data points were collected 
with the propeller off. This is to get a baseline for the actual value of zero for the load cell and torque cell. Staring at 500 data points, the propeller was then set to the first desired speed and one thousand data points were collected. The one thousand data points started at 600 and ended at 1600 total data points. The section between 500 and 600 was later removed during post-processing. The propeller was then turned off at 1600 points and the next set of 500 data points started at 1650 . The average value for thrust and torque from the two zero thrust states were averaged and this value was used correct the thrust and torque measurements from the actual tests by subtraction. The process was then repeated for increased values of rotational speed until the maximum speed was achieved. The change in speed from step to step depended on the maximum achievable speed.

\section{Dynamic Experimental Procedure}

The procedure for the dynamic experiment was similar to that of the static experiment. In addition to warming up the load and torque cells for fifteen minutes, the differential pressure transducer was left to warm up for thirty minutes. It was also zeroed similarly to the load and torque cells. When zeroing the differential pressure transducer the doors of the test section were closed to ensure air drafts from the room were not acting on the Pitot tube. The tube was checked to make sure it was still level and parallel to the walls of the wind tunnel. The motor power supply was activated and again set to 11.1 Volts. An easily recognizable file name was selected and the test was started. Five hundred data points were taken with the propeller off and wind tunnel off. At the end of the first five hundred points, the propeller was set to the desired rpm setting and the wind tunnel was set to the first desired setting and activated. After one hundred data points had passed, one thousand data points were collected. Next the wind tunnel was set to the next 
setting and the propeller rpm was adjusted if necessary to match the previous setting. This adjustment to the next setting was given one hundred points to ensure that all changes could be easily removed from the data file. This process was repeated until the windmill state of the propeller was achieved. When the last of the wind tunnel settings was achieved, the propeller and the wind tunnel were stopped over the range of one hundred data points and then five hundred data points were collected. A total of around ten wind tunnel settings were desired for each of the four propeller rpm settings for each propeller tested. 


\section{APPENDIX F: SUMMARY OF STATIC TEST DATA}

The following pages present the data collected during the static tests. This data includes measured data and calculated values and their associated uncertainties. 


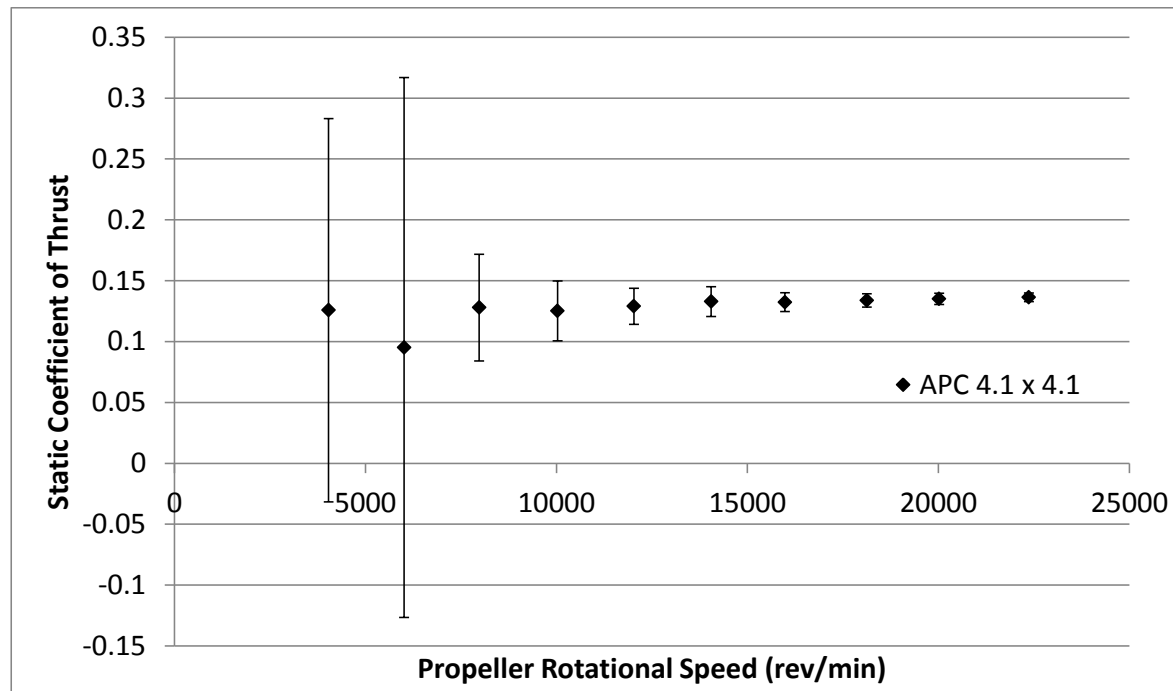

(a)

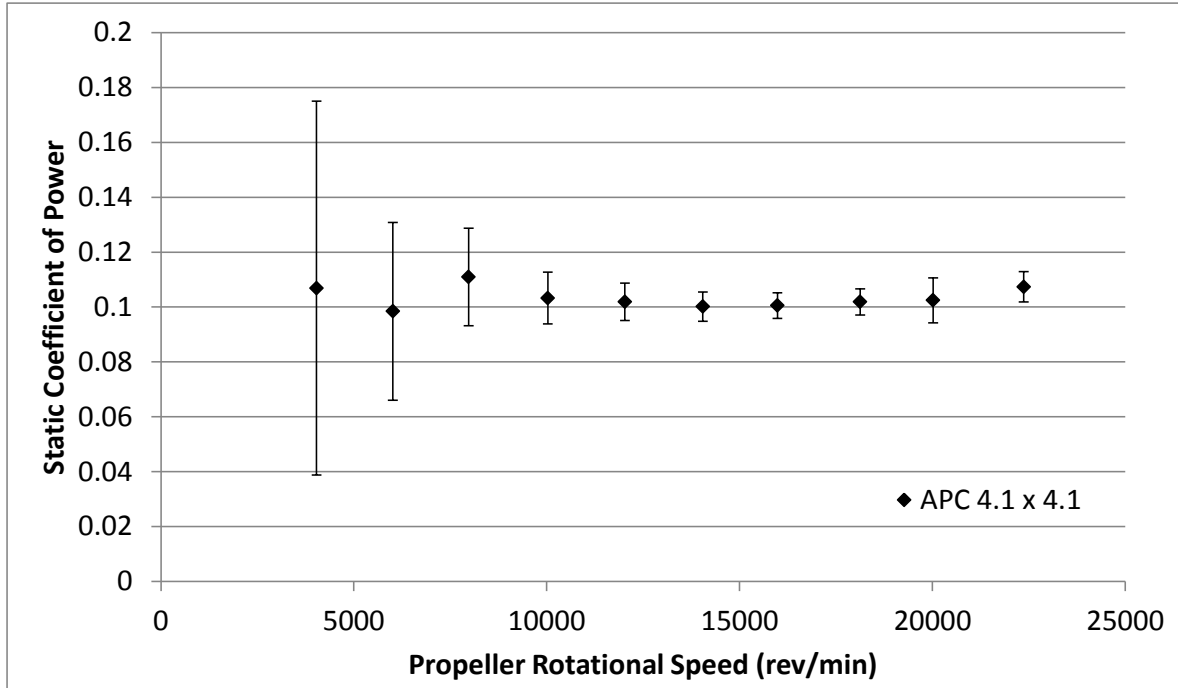

(b)

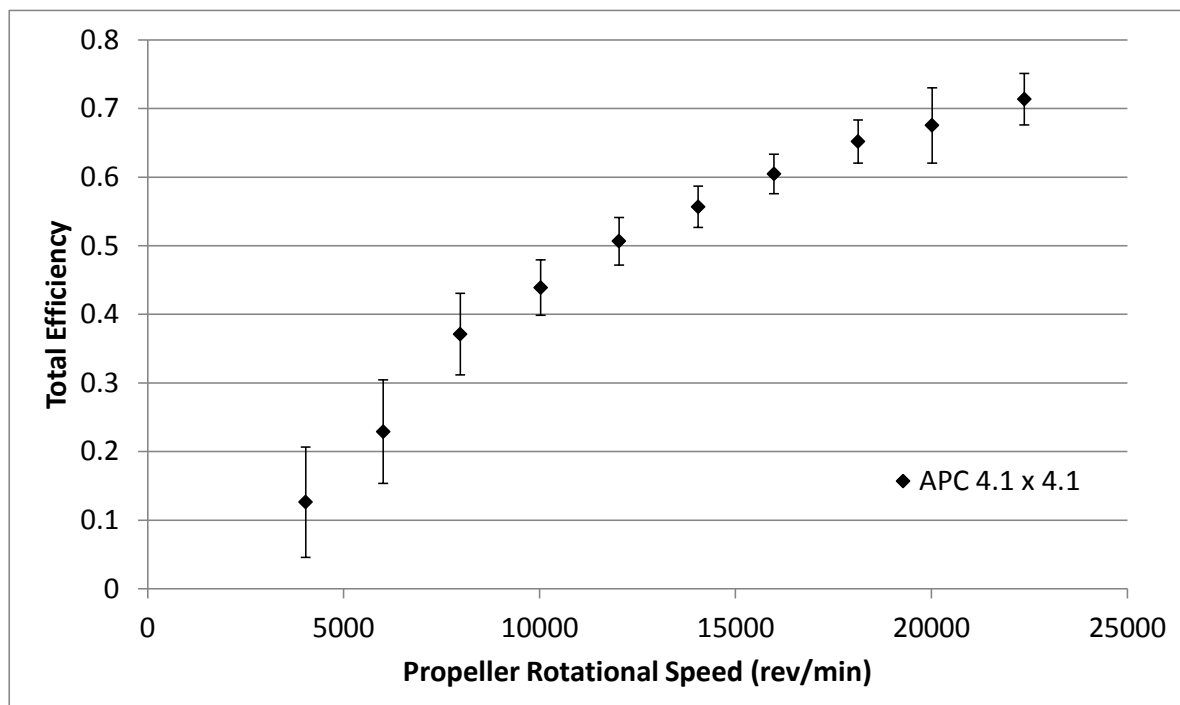

(c)

Figure 42: APC 4.1 x 4.1 Static Test Results: (a) Coefficient of Thrust, (b) Coefficient of Power, (c) Total Efficiency. 
Table 6: APC 4.1 x 4.1 Static Measured Values

\begin{tabular}{|c|c|c|c|c|c|c|c|c|}
\hline$n(\mathrm{RPM})$ & $T(\mathrm{~g})$ & $\Delta T(\mathrm{~g})$ & $Q(\mathrm{~g}-\mathrm{m})$ & $\Delta Q(\mathrm{~g}-\mathrm{m})$ & $V(\mathrm{~V})$ & $I(\mathrm{~A})$ & $T_{\text {atm }}\left({ }^{\circ} \mathrm{C}\right)$ & $P_{\text {atm }}(\mathrm{Pa})$ \\
\hline $4.033 \mathrm{E}+03$ & $7.766 \mathrm{E}+00$ & $9.72 \mathrm{E}+00$ & $1.089 \mathrm{E}-01$ & $6.93 \mathrm{E}-02$ & $1.111 \mathrm{E}+01$ & $3.215 \mathrm{E}-01$ & $2.263 \mathrm{E}+01$ & $9.858 \mathrm{E}+04$ \\
\hline $6.011 \mathrm{E}+03$ & $1.304 \mathrm{E}+01$ & $3.04 \mathrm{E}+01$ & $2.227 \mathrm{E}-01$ & $7.33 \mathrm{E}-02$ & $1.110 \mathrm{E}+01$ & $5.410 \mathrm{E}-01$ & $2.264 \mathrm{E}+01$ & $9.858 \mathrm{E}+04$ \\
\hline $7.976 \mathrm{E}+03$ & $3.090 \mathrm{E}+01$ & $1.06 \mathrm{E}+01$ & $4.420 \mathrm{E}-01$ & $7.07 \mathrm{E}-02$ & $1.109 \mathrm{E}+01$ & $8.796 \mathrm{E}-01$ & $2.262 \mathrm{E}+01$ & $9.858 \mathrm{E}+04$ \\
\hline $1.003 \mathrm{E}+04$ & $4.778 \mathrm{E}+01$ & $9.40 \mathrm{E}+00$ & $6.504 \mathrm{E}-01$ & $5.93 \mathrm{E}-02$ & $1.108 \mathrm{E}+01$ & $1.378 \mathrm{E}+00$ & $2.259 \mathrm{E}+01$ & $9.858 \mathrm{E}+04$ \\
\hline $1.203 \mathrm{E}+04$ & $7.087 \mathrm{E}+01$ & $8.17 \mathrm{E}+00$ & $9.234 \mathrm{E}-01$ & $6.21 \mathrm{E}-02$ & $1.106 \mathrm{E}+01$ & $2.036 \mathrm{E}+00$ & $2.263 \mathrm{E}+01$ & $9.858 \mathrm{E}+04$ \\
\hline $1.405 \mathrm{E}+04$ & $9.956 \mathrm{E}+01$ & $9.16 \mathrm{E}+00$ & $1.238 \mathrm{E}+00$ & $6.58 \mathrm{E}-02$ & $1.103 \mathrm{E}+01$ & $2.908 \mathrm{E}+00$ & $2.262 \mathrm{E}+01$ & $9.859 \mathrm{E}+04$ \\
\hline $1.598 \mathrm{E}+04$ & $1.283 \mathrm{E}+02$ & $7.46 \mathrm{E}+00$ & $1.608 \mathrm{E}+00$ & $7.45 \mathrm{E}-02$ & $1.101 \mathrm{E}+01$ & $3.966 \mathrm{E}+00$ & $2.270 \mathrm{E}+01$ & $9.859 \mathrm{E}+04$ \\
\hline $1.813 \mathrm{E}+04$ & $1.669 \mathrm{E}+02$ & $6.80 \mathrm{E}+00$ & $2.098 \mathrm{E}+00$ & $9.81 \mathrm{E}-02$ & $1.096 \mathrm{E}+01$ & $5.465 \mathrm{E}+00$ & $2.259 \mathrm{E}+01$ & $9.860 \mathrm{E}+04$ \\
\hline $2.002 \mathrm{E}+04$ & $2.056 \mathrm{E}+02$ & $7.11 \mathrm{E}+00$ & $2.572 \mathrm{E}+00$ & $2.07 \mathrm{E}-01$ & $1.092 \mathrm{E}+01$ & $7.172 \mathrm{E}+00$ & $2.260 \mathrm{E}+01$ & $9.860 \mathrm{E}+04$ \\
\hline $2.237 \mathrm{E}+04$ & $2.591 \mathrm{E}+02$ & $6.67 \mathrm{E}+00$ & $3.366 \mathrm{E}+00$ & $1.73 \mathrm{E}-01$ & $1.084 \mathrm{E}+01$ & $1.000 \mathrm{E}+01$ & $2.261 \mathrm{E}+01$ & $9.860 \mathrm{E}+04$ \\
\hline
\end{tabular}

Table 7: APC 4.1 x 4.1 Static Calculated Values

\begin{tabular}{|c|c|c|c|c|c|c|c|}
\hline$n(\mathrm{RPM})$ & $\rho\left(\mathrm{kg} / \mathrm{m}^{\wedge} 3\right)$ & $P_{\mathrm{P}}(\mathrm{W})$ & $\Delta P_{\mathrm{P}}(\mathrm{W})$ & $P_{\mathrm{e}}(\mathrm{W})$ & $\Delta P_{\mathrm{e}}(\mathrm{W})$ & $C_{\mathrm{T}}$ & $\Delta C_{\mathrm{T}}$ \\
\hline $4.033 \mathrm{E}+03$ & $1.161 \mathrm{E}+00$ & $4.508 \mathrm{E}-01$ & $2.87 \mathrm{E}-01$ & $3.571 \mathrm{E}+00$ & $5.72 \mathrm{E}-02$ & $1.258 \mathrm{E}-01$ & $1.57 \mathrm{E}-01$ \\
\hline $6.011 \mathrm{E}+03$ & $1.161 \mathrm{E}+00$ & $1.375 \mathrm{E}+00$ & $4.53 \mathrm{E}-01$ & $6.005 \mathrm{E}+00$ & $8.19 \mathrm{E}-02$ & $9.509 \mathrm{E}-02$ & $2.22 \mathrm{E}-01$ \\
\hline $7.976 \mathrm{E}+03$ & $1.161 \mathrm{E}+00$ & $3.620 \mathrm{E}+00$ & $5.79 \mathrm{E}-01$ & $9.756 \mathrm{E}+00$ & $1.22 \mathrm{E}-01$ & $1.280 \mathrm{E}-01$ & $4.38 \mathrm{E}-02$ \\
\hline $1.003 \mathrm{E}+04$ & $1.161 \mathrm{E}+00$ & $6.699 \mathrm{E}+00$ & $6.11 \mathrm{E}-01$ & $1.526 \mathrm{E}+01$ & $1.79 \mathrm{E}-01$ & $1.251 \mathrm{E}-01$ & $2.46 \mathrm{E}-02$ \\
\hline $1.203 \mathrm{E}+04$ & $1.161 \mathrm{E}+00$ & $1.141 \mathrm{E}+01$ & $7.67 \mathrm{E}-01$ & $2.252 \mathrm{E}+01$ & $2.56 \mathrm{E}-01$ & $1.290 \mathrm{E}-01$ & $1.49 \mathrm{E}-02$ \\
\hline $1.405 \mathrm{E}+04$ & $1.161 \mathrm{E}+00$ & $1.786 \mathrm{E}+01$ & $9.49 \mathrm{E}-01$ & $3.209 \mathrm{E}+01$ & $3.57 \mathrm{E}-01$ & $1.328 \mathrm{E}-01$ & $1.22 \mathrm{E}-02$ \\
\hline $1.598 \mathrm{E}+04$ & $1.161 \mathrm{E}+00$ & $2.639 \mathrm{E}+01$ & $1.22 \mathrm{E}+00$ & $4.365 \mathrm{E}+01$ & $\mathrm{E}-01$ & $1.323 \mathrm{E}-01$ & $7.70 \mathrm{E}-03$ \\
\hline $1.813 \mathrm{E}+04$ & $1.161 \mathrm{E}+00$ & $3.905 \mathrm{E}+01$ & $1.83 \mathrm{E}+00$ & +01 & -01 & -01 & $5.45 \mathrm{E}-03$ \\
\hline $2.002 \mathrm{E}+04$ & $1.161 \mathrm{E}+00$ & $5.287 \mathrm{E}+01$ & $4.25 \mathrm{E}+00$ & $7.829 \mathrm{E}+01$ & $8.55 \mathrm{E}-01$ & $1.351 \mathrm{E}-01$ & $4.67 \mathrm{E}-03$ \\
\hline $2.237 \mathrm{E}+04$ & $1.161 \mathrm{E}+00$ & $7.732 \mathrm{E}+01$ & $3.98 \mathrm{E}+00$ & $1.084 \mathrm{E}+02$ & $1.16 \mathrm{E}+00$ & $1.363 \mathrm{E}-01$ & $3.51 \mathrm{E}-03$ \\
\hline$C_{\mathrm{P}}$ & $\Delta C_{\mathrm{P}}$ & $C_{\mathrm{Q}}$ & $\Delta C_{\mathrm{Q}}$ & $\eta_{\mathrm{T}}$ & $\Delta \eta_{\mathrm{T}}$ & $R e_{0.75}$ & $\Delta R e_{0.75}$ \\
\hline $1.069 \mathrm{E}-01$ & $6.81 \mathrm{E}-02$ & $1.701 \mathrm{E}-02$ & $1.08 \mathrm{E}-02$ & $1.262 \mathrm{E}-01$ & 8.04E-02 & $8.471 \mathrm{E}+03$ & $1.26 \mathrm{E}+01$ \\
\hline 9.842E-02 & $3.24 \mathrm{E}-02$ & $1.566 \mathrm{E}-02$ & $5.16 \mathrm{E}-03$ & $2.289 \mathrm{E}-01$ & $7.54 \mathrm{E}-02$ & $1.263 \mathrm{E}+04$ & $2.01 \mathrm{E}+01$ \\
\hline $1.110 \mathrm{E}-01$ & $1.77 \mathrm{E}-02$ & $1.766 \mathrm{E}-02$ & $2.82 \mathrm{E}-03$ & $3.711 \mathrm{E}-01$ & $5.95 \mathrm{E}-02$ & $1.676 \mathrm{E}+04$ & $2.44 \mathrm{E}+01$ \\
\hline $1.033 \mathrm{E}-01$ & $9.42 \mathrm{E}-03$ & $1.643 \mathrm{E}-02$ & $1.50 \mathrm{E}-03$ & $4.390 \mathrm{E}-01$ & 4.04E-02 & $2.107 \mathrm{E}+04$ & $3.04 \mathrm{E}+01$ \\
\hline $1.019 \mathrm{E}-01$ & $6.85 \mathrm{E}-03$ & $1.622 \mathrm{E}-02$ & $1.09 \mathrm{E}-03$ & $5.065 \mathrm{E}-01$ & $3.45 \mathrm{E}-02$ & $2.527 \mathrm{E}+04$ & $3.54 \mathrm{E}+01$ \\
\hline $1.002 \mathrm{E}-01$ & $5.32 \mathrm{E}-03$ & $1.594 \mathrm{E}-02$ & $8.47 \mathrm{E}-04$ & $5.568 \mathrm{E}-01$ & $3.02 \mathrm{E}-02$ & $2.952 \mathrm{E}+04$ & $4.03 \mathrm{E}+01$ \\
\hline $1.005 \mathrm{E}-01$ & $4.66 \mathrm{E}-03$ & $1.600 \mathrm{E}-02$ & $7.41 \mathrm{E}-04$ & $6.047 \mathrm{E}-01$ & $2.88 \mathrm{E}-02$ & $3.357 \mathrm{E}+04$ & $4.53 \mathrm{E}+01$ \\
\hline $1.019 \mathrm{E}-01$ & $4.77 \mathrm{E}-03$ & $1.622 \mathrm{E}-02$ & $7.58 \mathrm{E}-04$ & $6.519 \mathrm{E}-01$ & $3.14 \mathrm{E}-02$ & $3.810 \mathrm{E}+04$ & $5.13 \mathrm{E}+01$ \\
\hline $1.024 \mathrm{E}-01$ & $8.23 \mathrm{E}-03$ & $1.701 \mathrm{E}-02$ & $1.31 \mathrm{E}-03$ & $6.754 \mathrm{E}-01$ & $5.47 \mathrm{E}-02$ & $4.207 \mathrm{E}+04$ & $5.63 \mathrm{E}+01$ \\
\hline $1.074 \mathrm{E}-01$ & $5.53 \mathrm{E}-03$ & $1.709 \mathrm{E}-02$ & $8.80 \mathrm{E}-04$ & $7.136 \mathrm{E}-01$ & $3.75 \mathrm{E}-02$ & $4.701 \mathrm{E}+04$ & $6.28 \mathrm{E}+01$ \\
\hline
\end{tabular}




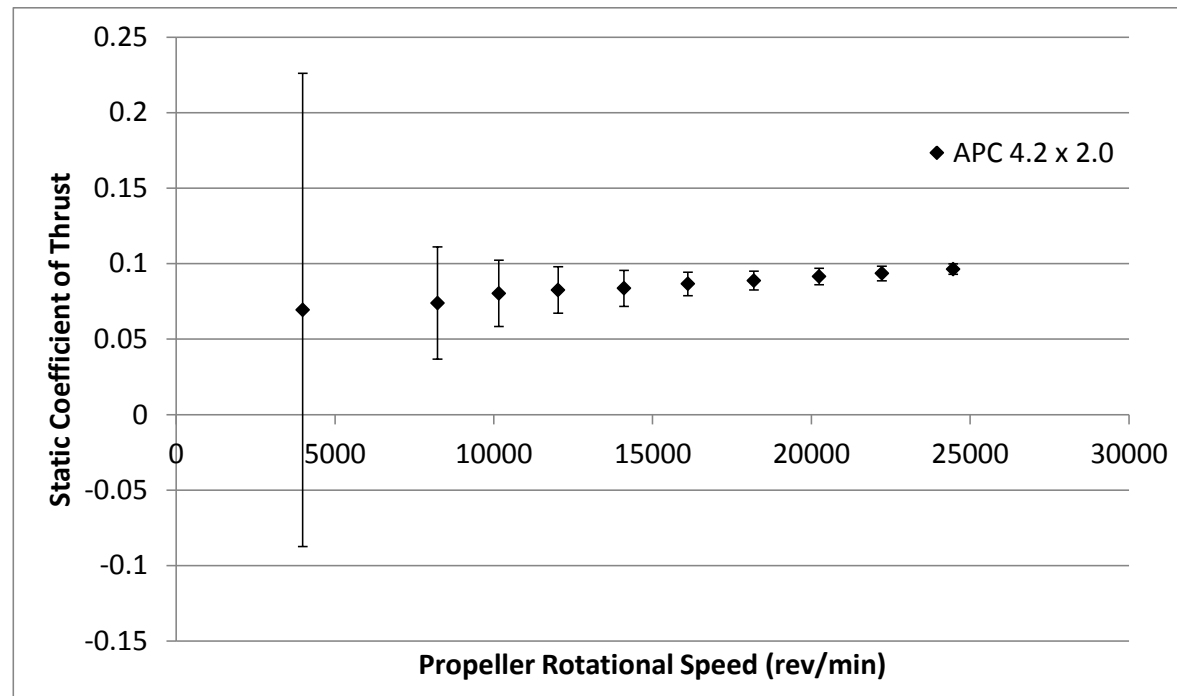

(a)

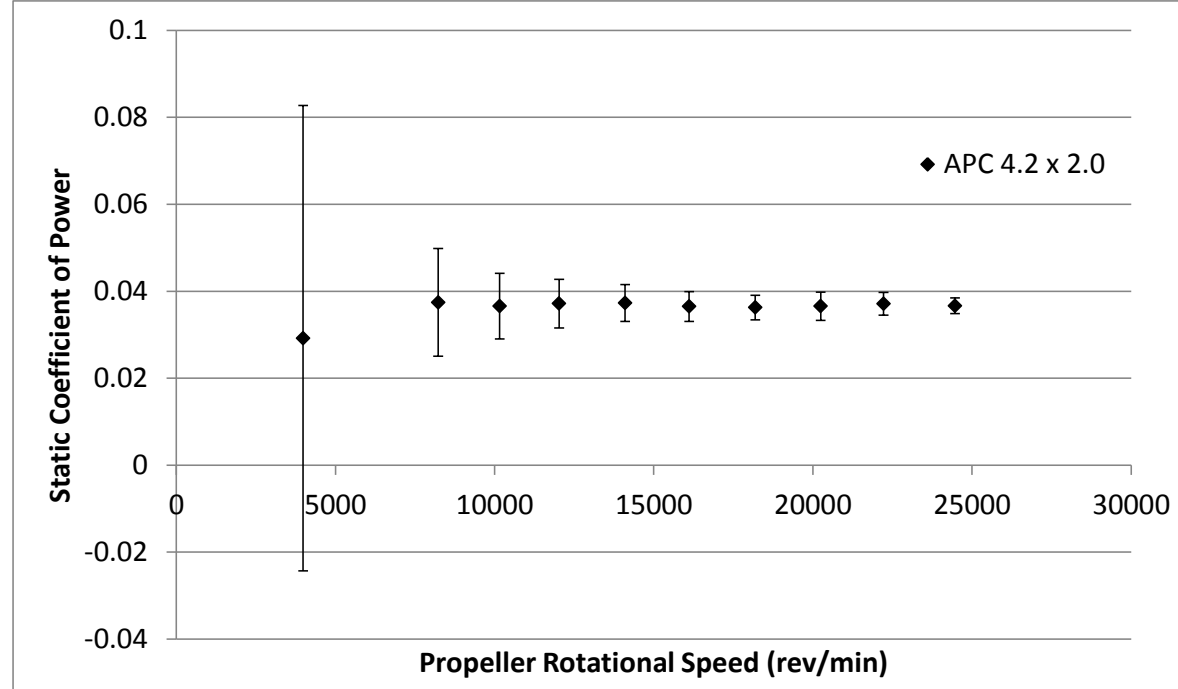

(b)

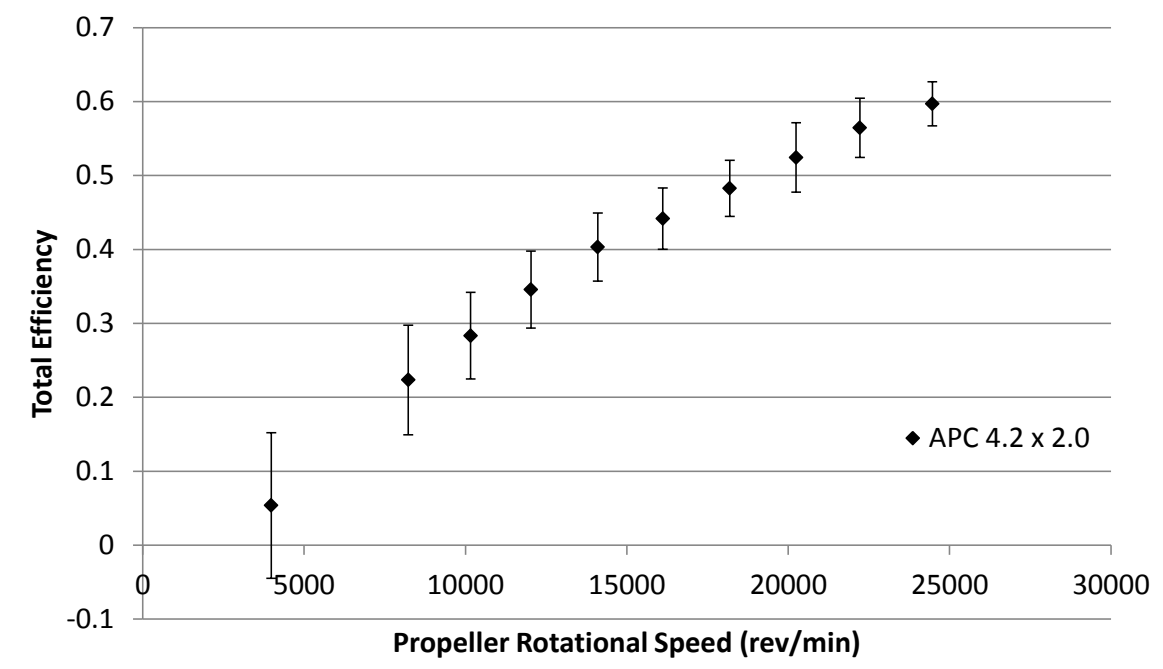

(c)

Figure 43: APC 4.2 x 2.0 Static Test Results: (a) Coefficient of Thrust, (b) Coefficient of Power, (c) Total Efficiency. 
Table 8: APC 4.2 × 2.0 Static Measured Values

\begin{tabular}{|c|c|c|c|c|c|c|c|c|}
\hline$n(\mathrm{RPM})$ & $T(\mathrm{~g})$ & $\Delta T(\mathrm{~g})$ & $Q(\mathrm{~g}-\mathrm{m})$ & $\Delta Q(\mathrm{~g}-\mathrm{m})$ & $V(\mathrm{~V})$ & $I(\mathrm{~A})$ & $T_{\text {atm }}\left({ }^{\circ} \mathrm{C}\right)$ & $P_{\text {atm }}(\mathrm{Pa})$ \\
\hline $3.982 \mathrm{E}+03$ & $4.579 \mathrm{E}+00$ & $1.03 \mathrm{E}+01$ & $3.243 \mathrm{E}-02$ & $5.95 \mathrm{E}-02$ & $1.108 \mathrm{E}+01$ & $2.227 \mathrm{E}-01$ & $2.114 \mathrm{E}+01$ & $9.908 \mathrm{E}+04$ \\
\hline $8.228 \mathrm{E}+03$ & $2.083 \mathrm{E}+01$ & $1.05 \mathrm{E}+01$ & $1.776 \mathrm{E}-01$ & $5.88 \mathrm{E}-02$ & $1.107 \mathrm{E}+01$ & $6.063 \mathrm{E}-01$ & $2.100 \mathrm{E}+01$ & $9.908 \mathrm{E}+04$ \\
\hline $1.016 \mathrm{E}+04$ & $3.450 \mathrm{E}+01$ & $9.45 \mathrm{E}+00$ & $2.648 \mathrm{E}-01$ & $5.45 \mathrm{E}-02$ & $1.107 \mathrm{E}+01$ & $8.807 \mathrm{E}-01$ & $2.097 \mathrm{E}+01$ & $9.908 \mathrm{E}+04$ \\
\hline $1.203 \mathrm{E}+04$ & $4.972 \mathrm{E}+01$ & $9.28 \mathrm{E}+00$ & $3.769 \mathrm{E}-01$ & $5.66 \mathrm{E}-02$ & $1.106 \mathrm{E}+01$ & $1.217 \mathrm{E}+00$ & $2.090 \mathrm{E}+01$ & $9.908 \mathrm{E}+04$ \\
\hline $1.410 \mathrm{E}+04$ & $6.929 \mathrm{E}+01$ & $9.89 \mathrm{E}+00$ & $5.202 \mathrm{E}-01$ & $5.91 \mathrm{E}-02$ & $1.105 \mathrm{E}+01$ & $1.690 \mathrm{E}+00$ & $2.086 \mathrm{E}+01$ & $9.908 \mathrm{E}+04$ \\
\hline $1.611 \mathrm{E}+04$ & $9.363 \mathrm{E}+01$ & $8.42 \mathrm{E}+00$ & $6.644 \mathrm{E}-01$ & $6.20 \mathrm{E}-02$ & $1.104 \mathrm{E}+01$ & $2.255 \mathrm{E}+00$ & $2.085 \mathrm{E}+01$ & $9.907 \mathrm{E}+04$ \\
\hline $1.819 \mathrm{E}+04$ & $1.223 \mathrm{E}+02$ & $8.63 \mathrm{E}+00$ & $8.413 \mathrm{E}-01$ & $6.55 \mathrm{E}-02$ & $1.102 \mathrm{E}+01$ & $2.953 \mathrm{E}+00$ & $2.081 \mathrm{E}+01$ & $9.907 \mathrm{E}+04$ \\
\hline $2.025 \mathrm{E}+04$ & $1.561 \mathrm{E}+02$ & $9.28 \mathrm{E}+00$ & $1.051 \mathrm{E}+00$ & $9.35 \mathrm{E}-02$ & $1.100 \mathrm{E}+01$ & $3.785 \mathrm{E}+00$ & $2.082 \mathrm{E}+01$ & $9.908 \mathrm{E}+04$ \\
\hline $2.222 \mathrm{E}+04$ & $1.923 \mathrm{E}+02$ & $9.92 \mathrm{E}+00$ & $1.286 \mathrm{E}+00$ & $9.02 \mathrm{E}-02$ & $1.098 \mathrm{E}+01$ & $4.732 \mathrm{E}+00$ & $2.082 \mathrm{E}+01$ & $9.908 \mathrm{E}+04$ \\
\hline $2.447 \mathrm{E}+04$ & $2.403 \mathrm{E}+02$ & $8.54 \mathrm{E}+00$ & $1.539 \mathrm{E}+00$ & $7.46 \mathrm{E}-02$ & $1.096 \mathrm{E}+01$ & $5.911 \mathrm{E}+00$ & $2.087 \mathrm{E}+01$ & $9.908 \mathrm{E}+04$ \\
\hline
\end{tabular}

Table 9: APC 4.2 x 2.0 Static Calculated Values

\begin{tabular}{|c|c|c|c|c|c|c|c|}
\hline$n(\mathrm{RPM})$ & $\rho\left(\mathrm{kg} / \mathrm{m}^{\wedge} 3\right)$ & $P_{\mathrm{P}}(\mathrm{W})$ & $\Delta P_{\mathrm{P}}(\mathrm{W})$ & $P_{\mathrm{e}}(\mathrm{W})$ & $\Delta P_{\mathrm{e}}(\mathrm{W})$ & $C_{\mathrm{T}}$ & $\Delta C_{\mathrm{T}}$ \\
\hline $3.982 \mathrm{E}+03$ & $1.173 \mathrm{E}+00$ & $1.326 \mathrm{E}-01$ & $2.43 \mathrm{E}-01$ & $2.468 \mathrm{E}+00$ & $4.75 \mathrm{E}-02$ & $6.940 \mathrm{E}-02$ & $1.57 \mathrm{E}-01$ \\
\hline $8.228 \mathrm{E}+03$ & $1.173 \mathrm{E}+00$ & $1.501 \mathrm{E}+00$ & $4.97 \mathrm{E}-01$ & $6.714 \mathrm{E}+00$ & $8.91 \mathrm{E}-02$ & $7.390 \mathrm{E}-02$ & $3.72 \mathrm{E}-02$ \\
\hline $1.016 \mathrm{E}+04$ & $1.174 \mathrm{E}+00$ & $2.763 \mathrm{E}+00$ & $5.69 \mathrm{E}-01$ & $9.748 \mathrm{E}+00$ & $1.21 \mathrm{E}-01$ & $8.028 \mathrm{E}-02$ & $2.20 \mathrm{E}-02$ \\
\hline $1.203 \mathrm{E}+04$ & $1.174 \mathrm{E}+00$ & $4.655 \mathrm{E}+00$ & $6.99 \mathrm{E}-01$ & $1.346 \mathrm{E}+01$ & $1.61 \mathrm{E}-01$ & $8.254 \mathrm{E}-02$ & $1.54 \mathrm{E}-02$ \\
\hline $1.410 \mathrm{E}+04$ & $1.174 \mathrm{E}+00$ & $7.533 \mathrm{E}+00$ & $8.56 \mathrm{E}-01$ & $1.868 \mathrm{E}+01$ & $2.15 \mathrm{E}-01$ & $8.368 \mathrm{E}-02$ & $1.19 \mathrm{E}-02$ \\
\hline $1.611 \mathrm{E}+04$ & $1.174 \mathrm{E}+00$ & $1.099 \mathrm{E}+01$ & $1.03 \mathrm{E}+00$ & $2.490 \mathrm{E}+01$ & $2.83 \mathrm{E}-01$ & $8.658 \mathrm{E}-02$ & $7.79 \mathrm{E}-03$ \\
\hline $1.819 \mathrm{E}+04$ & $1.174 \mathrm{E}+00$ & $1.572 \mathrm{E}+01$ & $1.22 \mathrm{E}+00$ & $3.255 \mathrm{E}+01$ & $3.62 \mathrm{E}-01$ & $8.877 \mathrm{E}-02$ & $\mathrm{E}-03$ \\
\hline $2.025 \mathrm{E}+04$ & $1.174 \mathrm{E}+00$ & $2.185 \mathrm{E}+01$ & $1.94 \mathrm{E}+00$ & $4.165 \mathrm{E}+01$ & -01 & -02 & E-03 \\
\hline $2.222 \mathrm{E}+04$ & $1.174 \mathrm{E}+00$ & $2.934 \mathrm{E}+01$ & $2.06 \mathrm{E}+00$ & $5.197 \mathrm{E}+01$ & $5.81 \mathrm{E}-01$ & $9.350 \mathrm{E}-02$ & $4.82 \mathrm{E}-03$ \\
\hline $2.447 \mathrm{E}+04$ & $1.174 \mathrm{E}+00$ & $3.867 \mathrm{E}+01$ & $1.87 \mathrm{E}+00$ & $6.477 \mathrm{E}+01$ & 7.35E-01 & $9.637 \mathrm{E}-02$ & $3.43 \mathrm{E}-03$ \\
\hline$C_{\mathrm{P}}$ & $\Delta C_{\mathrm{P}}$ & $C_{\mathrm{Q}}$ & $\Delta C_{\mathrm{Q}}$ & $\eta_{\mathrm{T}}$ & $\Delta \eta_{\mathrm{T}}$ & $R e_{0.75}$ & $\Delta R e_{0.75}$ \\
\hline $2.919 \mathrm{E}-02$ & $5.35 \mathrm{E}-02$ & $4.646 \mathrm{E}-03$ & $8.52 \mathrm{E}-03$ & $5.372 \mathrm{E}-02$ & $9.85 \mathrm{E}-02$ & $9.288 \mathrm{E}+03$ & $1.31 \mathrm{E}+01$ \\
\hline $3.744 \mathrm{E}-02$ & $1.24 \mathrm{E}-02$ & $5.958 \mathrm{E}-03$ & $1.97 \mathrm{E}-03$ & $2.235 \mathrm{E}-01$ & 7.41E-02 & $1.921 \mathrm{E}+04$ & $2.53 \mathrm{E}+01$ \\
\hline $3.659 \mathrm{E}-02$ & $7.54 \mathrm{E}-03$ & $5.824 \mathrm{E}-03$ & $1.20 \mathrm{E}-03$ & $2.834 \mathrm{E}-01$ & $5.85 \mathrm{E}-02$ & $2.372 \mathrm{E}+04$ & $3.07 \mathrm{E}+01$ \\
\hline $3.716 \mathrm{E}-02$ & $5.58 \mathrm{E}-03$ & $5.914 \mathrm{E}-03$ & $8.88 \mathrm{E}-04$ & $3.458 \mathrm{E}-01$ & $5.21 \mathrm{E}-02$ & $2.809 \mathrm{E}+04$ & $3.60 \mathrm{E}+01$ \\
\hline $3.731 \mathrm{E}-02$ & 4.24E-03 & $5.939 \mathrm{E}-03$ & $6.75 \mathrm{E}-04$ & $4.033 \mathrm{E}-01$ & 4.61E-02 & $3.294 \mathrm{E}+04$ & $4.16 \mathrm{E}+01$ \\
\hline $3.649 \mathrm{E}-02$ & $3.40 \mathrm{E}-03$ & $5.808 \mathrm{E}-03$ & 5.42E-04 & $4.416 \mathrm{E}-01$ & $4.15 \mathrm{E}-02$ & $3.764 \mathrm{E}+04$ & $4.78 \mathrm{E}+01$ \\
\hline $3.626 \mathrm{E}-02$ & $2.82 \mathrm{E}-03$ & $5.771 \mathrm{E}-03$ & 4.49E-04 & $4.828 \mathrm{E}-01$ & $3.80 \mathrm{E}-02$ & $4.250 \mathrm{E}+04$ & $5.41 \mathrm{E}+01$ \\
\hline $3.655 \mathrm{E}-02$ & $3.25 \mathrm{E}-03$ & $5.817 \mathrm{E}-03$ & $5.18 \mathrm{E}-04$ & $5.245 \mathrm{E}-01$ & 4.71E-02 & $4.731 \mathrm{E}+04$ & $6.00 \mathrm{E}+01$ \\
\hline $3.713 \mathrm{E}-02$ & $2.60 \mathrm{E}-03$ & $4.646 \mathrm{E}-03$ & 4.14E-04 & $5.646 \mathrm{E}-01$ & 4.01E-02 & $5.193 \mathrm{E}+04$ & $6.51 \mathrm{E}+01$ \\
\hline $3.667 \mathrm{E}-02$ & $1.78 \mathrm{E}-03$ & $5.836 \mathrm{E}-03$ & $2.83 \mathrm{E}-04$ & $5.971 \mathrm{E}-01$ & $2.97 \mathrm{E}-02$ & $5.716 \mathrm{E}+04$ & $7.16 \mathrm{E}+01$ \\
\hline
\end{tabular}




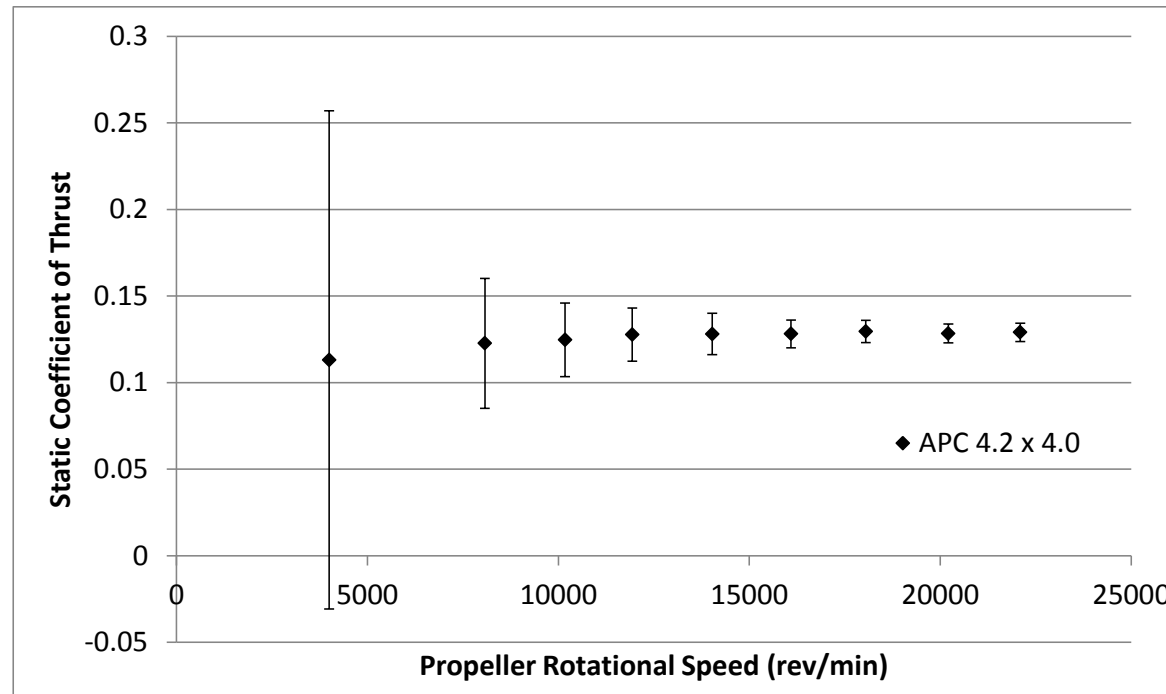

(a)

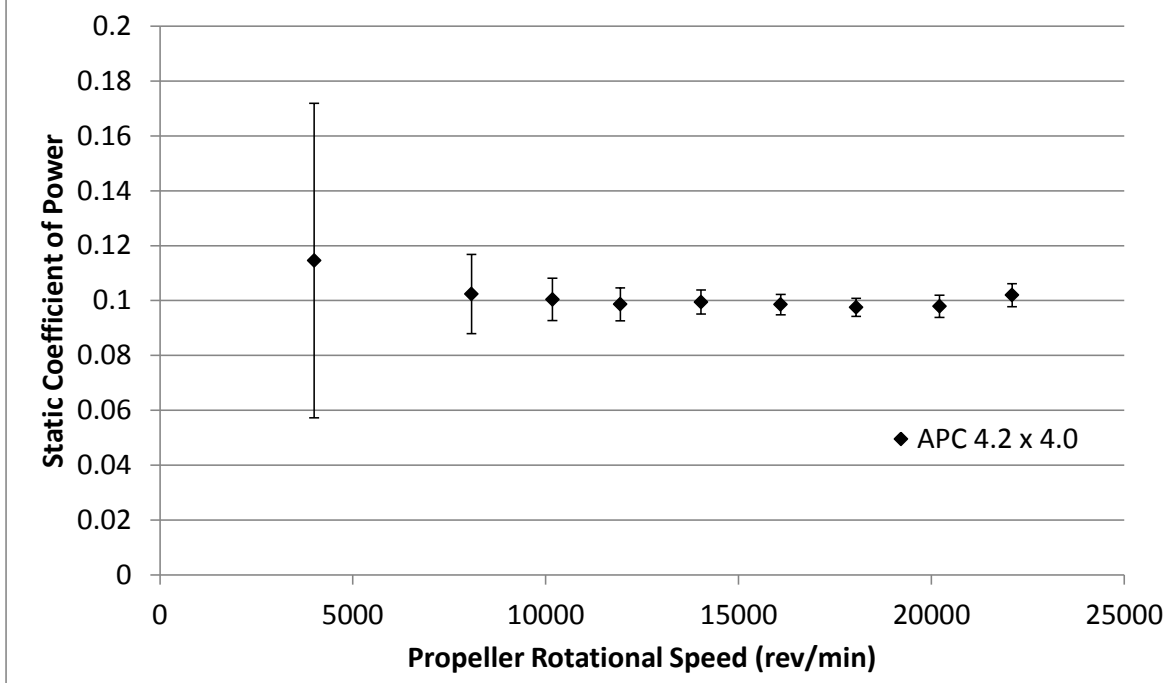

(b)

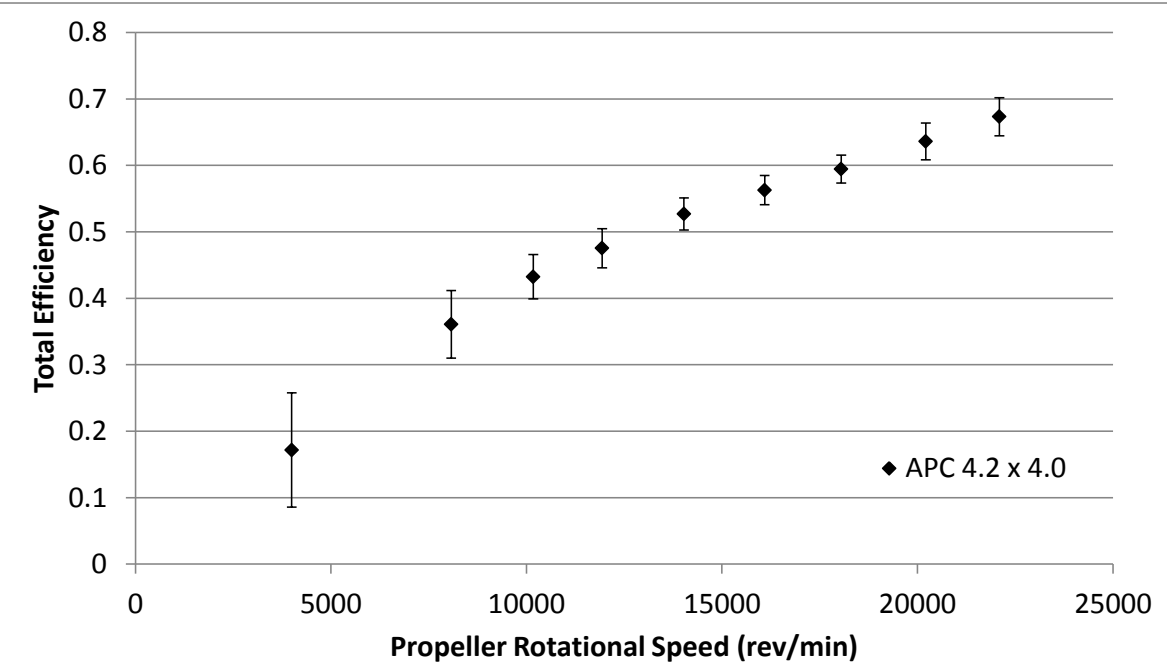

(c)

Figure 44: APC 4.2 x 4.0 Static Test Results: (a) Coefficient of Thrust, (b) Coefficient of Power, (c) Total Efficiency. 
Table 10: APC 4.2 x 4.0 Static Measured Values

\begin{tabular}{|c|c|c|c|c|c|c|c|c|}
\hline$n(\mathrm{RPM})$ & $T(\mathrm{~g})$ & $\Delta T(\mathrm{~g})$ & $Q(\mathrm{~g}-\mathrm{m})$ & $\Delta Q(\mathrm{~g}-\mathrm{m})$ & $V(\mathrm{~V})$ & $I(\mathrm{~A})$ & $T_{\mathrm{atm}}\left({ }^{\circ} \mathrm{C}\right)$ & $P_{\mathrm{atm}}(\mathrm{Pa})$ \\
\hline $3.998 \mathrm{E}+03$ & $7.670 \mathrm{E}+00$ & $9.76 \mathrm{E}+00$ & $1.315 \mathrm{E}-01$ & $6.58 \mathrm{E}-02$ & $1.108 \mathrm{E}+01$ & $2.838 \mathrm{E}-01$ & $2.135 \mathrm{E}+01$ & $9.907 \mathrm{E}+04$ \\
\hline $8.076 \mathrm{E}+03$ & $3.397 \mathrm{E}+01$ & $1.04 \mathrm{E}+01$ & $4.798 \mathrm{E}-01$ & $6.75 \mathrm{E}-02$ & $1.107 \mathrm{E}+01$ & $9.969 \mathrm{E}-01$ & $2.119 \mathrm{E}+01$ & $9.907 \mathrm{E}+04$ \\
\hline $1.018 \mathrm{E}+04$ & $5.484 \mathrm{E}+01$ & $9.34 \mathrm{E}+00$ & $7.473 \mathrm{E}-01$ & $5.71 \mathrm{E}-02$ & $1.105 \mathrm{E}+01$ & $1.634 \mathrm{E}+00$ & $2.110 \mathrm{E}+01$ & $9.907 \mathrm{E}+04$ \\
\hline $1.194 \mathrm{E}+04$ & $7.728 \mathrm{E}+01$ & $9.34 \mathrm{E}+00$ & $1.010 \mathrm{E}+00$ & $6.15 \mathrm{E}-02$ & $1.104 \mathrm{E}+01$ & $2.360 \mathrm{E}+00$ & $2.108 \mathrm{E}+01$ & $9.908 \mathrm{E}+04$ \\
\hline $1.403 \mathrm{E}+04$ & $1.071 \mathrm{E}+02$ & $9.96 \mathrm{E}+00$ & $1.407 \mathrm{E}+00$ & $6.24 \mathrm{E}-02$ & $1.101 \mathrm{E}+01$ & $3.494 \mathrm{E}+00$ & $2.109 \mathrm{E}+01$ & $9.907 \mathrm{E}+04$ \\
\hline $1.609 \mathrm{E}+04$ & $1.410 \mathrm{E}+02$ & $8.78 \mathrm{E}+00$ & $1.835 \mathrm{E}+00$ & $6.89 \mathrm{E}-02$ & $1.098 \mathrm{E}+01$ & $4.909 \mathrm{E}+00$ & $2.104 \mathrm{E}+01$ & $9.907 \mathrm{E}+04$ \\
\hline $1.805 \mathrm{E}+04$ & $1.794 \mathrm{E}+02$ & $8.97 \mathrm{E}+00$ & $2.285 \mathrm{E}+00$ & $7.65 \mathrm{E}-02$ & $1.094 \mathrm{E}+01$ & $6.512 \mathrm{E}+00$ & $2.094 \mathrm{E}+01$ & $9.908 \mathrm{E}+04$ \\
\hline $2.021 \mathrm{E}+04$ & $2.228 \mathrm{E}+02$ & $9.51 \mathrm{E}+00$ & $2.876 \mathrm{E}+00$ & $1.20 \mathrm{E}-01$ & $1.090 \mathrm{E}+01$ & $8.615 \mathrm{E}+00$ & $2.097 \mathrm{E}+01$ & $9.908 \mathrm{E}+04$ \\
\hline $2.210 \mathrm{E}+04$ & $2.677 \mathrm{E}+02$ & $1.11 \mathrm{E}+01$ & $3.579 \mathrm{E}+00$ & $1.46 \mathrm{E}-01$ & $1.084 \mathrm{E}+01$ & $1.113 \mathrm{E}+01$ & $2.096 \mathrm{E}+01$ & $9.908 \mathrm{E}+04$ \\
\hline
\end{tabular}

Table 11: APC 4.2 x 4.0 Static Calculated Values

\begin{tabular}{|c|c|c|c|c|c|c|c|}
\hline$n(\mathrm{RPM})$ & $\rho\left(\mathrm{kg} / \mathrm{m}^{\wedge} 3\right)$ & $P_{\mathrm{P}}(\mathrm{W})$ & $\Delta P_{\mathrm{P}}(\mathrm{W})$ & $P_{\mathrm{e}}(\mathrm{W})$ & $\Delta P_{\mathrm{e}}(\mathrm{W})$ & $C_{\mathrm{T}}$ & $\Delta C_{\mathrm{T}}$ \\
\hline $3.998 \mathrm{E}+03$ & $1.172 \mathrm{E}+00$ & $5.397 \mathrm{E}-01$ & $2.70 \mathrm{E}-01$ & $3.145 \mathrm{E}+00$ & $5.40 \mathrm{E}-02$ & $1.131 \mathrm{E}-01$ & $1.44 \mathrm{E}-01$ \\
\hline $8.076 \mathrm{E}+03$ & $1.173 \mathrm{E}+00$ & $3.979 \mathrm{E}+00$ & $5.60 \mathrm{E}-01$ & $1.103 \mathrm{E}+01$ & $1.35 \mathrm{E}-01$ & $1.227 \mathrm{E}-01$ & $3.75 \mathrm{E}-02$ \\
\hline $1.018 \mathrm{E}+04$ & $1.173 \mathrm{E}+00$ & $7.809 \mathrm{E}+00$ & $5.97 \mathrm{E}-01$ & $1.806 \mathrm{E}+01$ & $2.11 \mathrm{E}-01$ & $1.247 \mathrm{E}-01$ & $2.12 \mathrm{E}-02$ \\
\hline $1.194 \mathrm{E}+04$ & $1.173 \mathrm{E}+00$ & $1.238 \mathrm{E}+01$ & $7.54 \mathrm{E}-01$ & $2.605 \mathrm{E}+01$ & $2.96 \mathrm{E}-01$ & $1.277 \mathrm{E}-01$ & $1.54 \mathrm{E}-02$ \\
\hline $1.403 \mathrm{E}+04$ & $1.173 \mathrm{E}+00$ & $2.027 \mathrm{E}+01$ & $8.99 \mathrm{E}-01$ & $3.847 \mathrm{E}+01$ & $4.27 \mathrm{E}-01$ & $1.281 \mathrm{E}-01$ & $1.19 \mathrm{E}-02$ \\
\hline $1.609 \mathrm{E}+04$ & $1.173 \mathrm{E}+00$ & $3.033 \mathrm{E}+01$ & $1.14 \mathrm{E}+00$ & $5.389 \mathrm{E}+01$ & $5.98 \mathrm{E}-01$ & $1.281 \mathrm{E}-01$ & $7.98 \mathrm{E}-03$ \\
\hline $1.805 \mathrm{E}+04$ & $1.174 \mathrm{E}+00$ & $4.237 \mathrm{E}+01$ & $1.42 \mathrm{E}+00$ & $7.126 \mathrm{E}+01$ & $7.87 \mathrm{E}-01$ & $1.295 \mathrm{E}-01$ & $6.48 \mathrm{E}-03$ \\
\hline $2.021 \mathrm{E}+04$ & $1.174 \mathrm{E}+00$ & $5.971 \mathrm{E}+01$ & $2.50 \mathrm{E}+00$ & $9.386 \mathrm{E}+01$ & $1.15 \mathrm{E}+00$ & $1.284 \mathrm{E}-01$ & $5.48 \mathrm{E}-03$ \\
\hline $2.210 \mathrm{E}+04$ & $1.174 \mathrm{E}+00$ & $8.121 \mathrm{E}+01$ & $3.31 \mathrm{E}+00$ & $1.206 \mathrm{E}+02$ & $1.42 \mathrm{E}+00$ & $1.290 \mathrm{E}-01$ & $5.35 \mathrm{E}-03$ \\
\hline$C_{\mathrm{P}}$ & $\Delta C_{\mathrm{P}}$ & $C_{\mathrm{Q}}$ & $\Delta C_{\mathrm{Q}}$ & $\eta_{\mathrm{T}}$ & $\Delta \eta_{\mathrm{T}}$ & $R e_{0.75}$ & $\Delta R e_{0.75}$ \\
\hline $1.145 \mathrm{E}-01$ & $5.73 \mathrm{E}-02$ & $1.823 \mathrm{E}-02$ & $9.12 \mathrm{E}-03$ & $1.716 \mathrm{E}-01$ & $8.59 \mathrm{E}-02$ & $9.458 \mathrm{E}+03$ & $1.35 \mathrm{E}+01$ \\
\hline $1.024 \mathrm{E}-01$ & $1.44 \mathrm{E}-02$ & $1.629 \mathrm{E}-02$ & $2.29 \mathrm{E}-03$ & $3.607 \mathrm{E}-01$ & $5.09 \mathrm{E}-02$ & $1.913 \mathrm{E}+04$ & $2.56 \mathrm{E}+01$ \\
\hline $1.004 \mathrm{E}-01$ & $7.68 \mathrm{E}-03$ & $1.598 \mathrm{E}-02$ & $1.22 \mathrm{E}-03$ & $4.323 \mathrm{E}-01$ & $3.34 \mathrm{E}-02$ & $2.411 \mathrm{E}+04$ & $3.11 \mathrm{E}+01$ \\
\hline $9.863 \mathrm{E}-02$ & $6.01 \mathrm{E}-03$ & $1.570 \mathrm{E}-02$ & $9.56 \mathrm{E}-04$ & $4.753 \mathrm{E}-01$ & $2.94 \mathrm{E}-02$ & $2.829 \mathrm{E}+04$ & $3.58 \mathrm{E}+01$ \\
\hline $9.945 \mathrm{E}-02$ & $4.41 \mathrm{E}-03$ & $1.583 \mathrm{E}-02$ & $7.02 \mathrm{E}-04$ & $5.269 \mathrm{E}-01$ & $2.41 \mathrm{E}-02$ & $3.324 \mathrm{E}+04$ & $4.21 \mathrm{E}+01$ \\
\hline $9.854 \mathrm{E}-02$ & $3.70 \mathrm{E}-03$ & $1.568 \mathrm{E}-02$ & $5.89 \mathrm{E}-04$ & $5.627 \mathrm{E}-01$ & $2.20 \mathrm{E}-02$ & $3.815 \mathrm{E}+04$ & $4.82 \mathrm{E}+01$ \\
\hline $9.753 \mathrm{E}-02$ & $3.27 \mathrm{E}-03$ & $1.552 \mathrm{E}-02$ & $5.20 \mathrm{E}-04$ & $5.945 \mathrm{E}-01$ & $2.10 \mathrm{E}-02$ & $4.281 \mathrm{E}+04$ & $5.39 \mathrm{E}+01$ \\
\hline $9.790 \mathrm{E}-02$ & $4.10 \mathrm{E}-03$ & $1.558 \mathrm{E}-02$ & $6.52 \mathrm{E}-04$ & $6.361 \mathrm{E}-01$ & $2.77 \mathrm{E}-02$ & $4.793 \mathrm{E}+04$ & $6.24 \mathrm{E}+01$ \\
\hline $1.020 \mathrm{E}-01$ & $4.16 \mathrm{E}-03$ & $1.823 \mathrm{E}-02$ & $6.62 \mathrm{E}-04$ & $6.732 \mathrm{E}-01$ & $2.86 \mathrm{E}-02$ & $5.240 \mathrm{E}+04$ & $6.63 \mathrm{E}+01$ \\
\hline
\end{tabular}




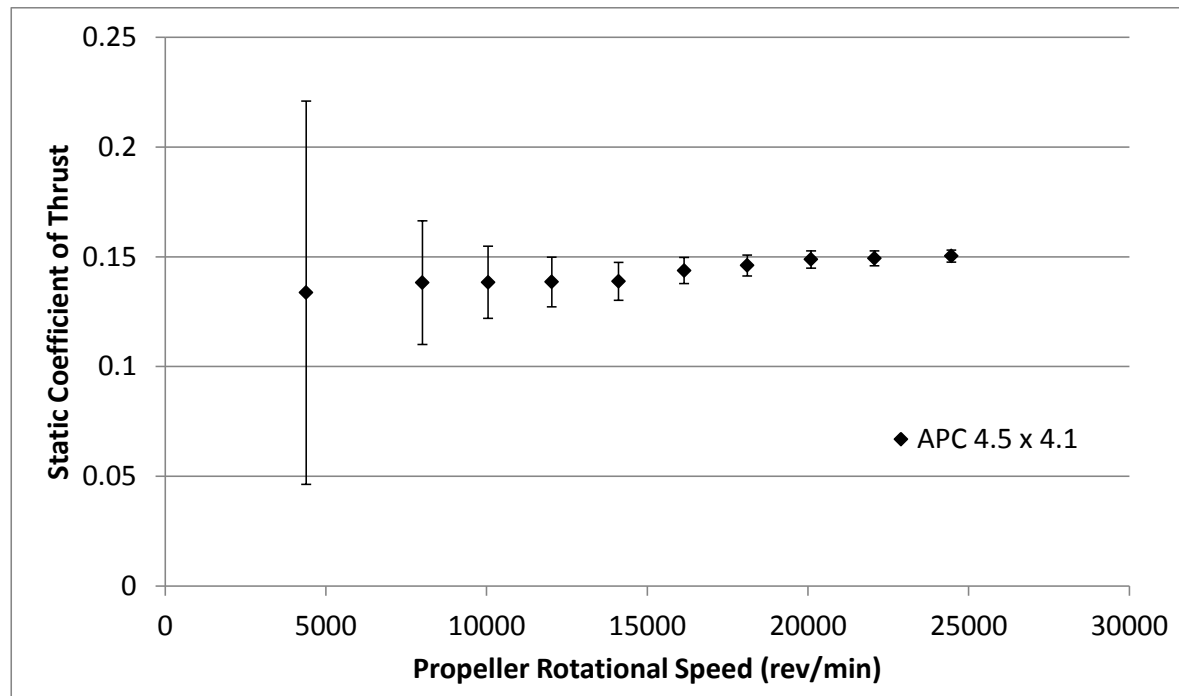

(a)

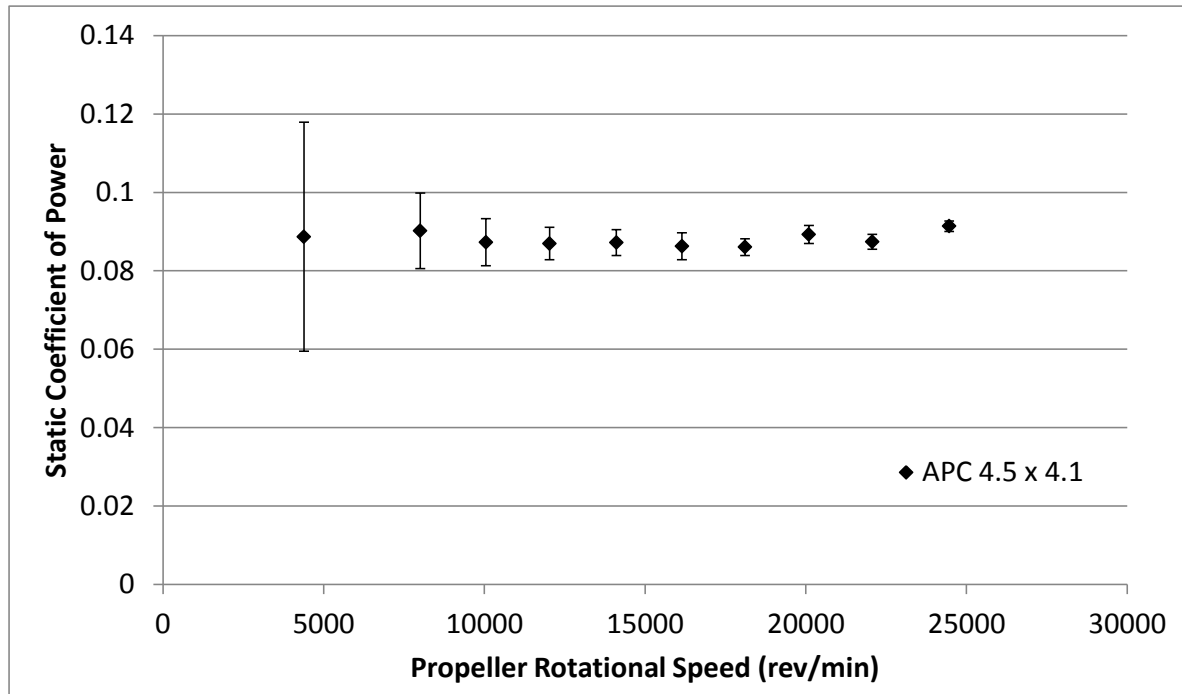

(b)

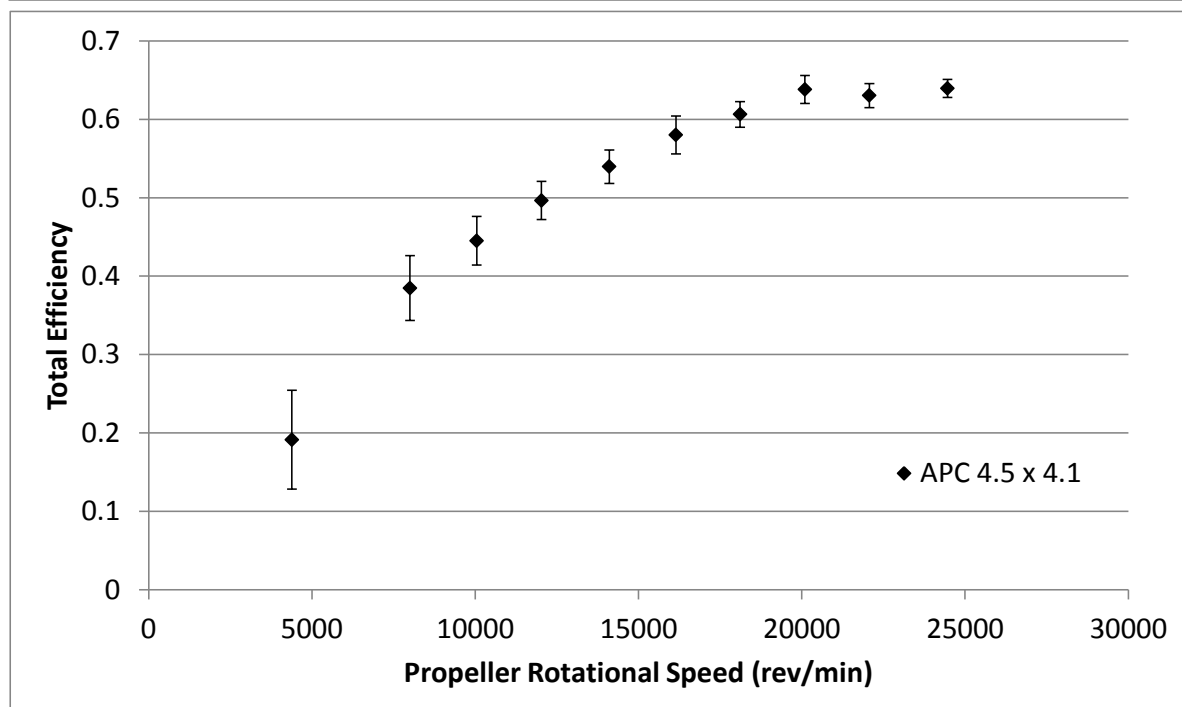

(c)

Figure 45: APC 4.5 x 4.1 Static Test Results: (a) Coefficient of Thrust, (b) Coefficient of Power, (c) Total Efficiency. 
Table 12: APC 4.5 x 4.1 Static Measured Values

\begin{tabular}{|c|c|c|c|c|c|c|c|c|}
\hline$n(\mathrm{RPM})$ & $T(\mathrm{~g})$ & $\Delta T(\mathrm{~g})$ & $Q(\mathrm{~g}-\mathrm{m})$ & $\Delta Q(\mathrm{~g}-\mathrm{m})$ & $V(\mathrm{~V})$ & $I(\mathrm{~A})$ & $T_{\mathrm{atm}}\left({ }^{\circ} \mathrm{C}\right)$ & $P_{\text {atm }}(\mathrm{Pa})$ \\
\hline $4.386 \mathrm{E}+03$ & $1.431 \mathrm{E}+01$ & $9.34 \mathrm{E}+00$ & $1.720 \mathrm{E}-01$ & $5.66 \mathrm{E}-02$ & $1.108 \mathrm{E}+01$ & $3.655 \mathrm{E}-01$ & $2.217 \mathrm{E}+01$ & $9.900 \mathrm{E}+04$ \\
\hline $8.004 \mathrm{E}+03$ & $4.925 \mathrm{E}+01$ & $1.00 \mathrm{E}+01$ & $5.825 \mathrm{E}-01$ & $6.21 \mathrm{E}-02$ & $1.106 \mathrm{E}+01$ & $1.125 \mathrm{E}+00$ & $2.217 \mathrm{E}+01$ & $9.899 \mathrm{E}+04$ \\
\hline $1.005 \mathrm{E}+04$ & $7.773 \mathrm{E}+01$ & $9.24 \mathrm{E}+00$ & $8.886 \mathrm{E}-01$ & $6.10 \mathrm{E}-02$ & $1.105 \mathrm{E}+01$ & $1.865 \mathrm{E}+00$ & $2.210 \mathrm{E}+01$ & $9.899 \mathrm{E}+04$ \\
\hline $1.203 \mathrm{E}+04$ & $1.115 \mathrm{E}+02$ & $9.10 \mathrm{E}+00$ & $1.269 \mathrm{E}+00$ & $6.03 \mathrm{E}-02$ & $1.102 \mathrm{E}+01$ & $2.863 \mathrm{E}+00$ & $2.210 \mathrm{E}+01$ & $9.898 \mathrm{E}+04$ \\
\hline $1.411 \mathrm{E}+04$ & $1.537 \mathrm{E}+02$ & $9.59 \mathrm{E}+00$ & $1.750 \mathrm{E}+00$ & $6.66 \mathrm{E}-02$ & $1.099 \mathrm{E}+01$ & $4.274 \mathrm{E}+00$ & $2.209 \mathrm{E}+01$ & $9.899 \mathrm{E}+04$ \\
\hline $1.615 \mathrm{E}+04$ & $2.085 \mathrm{E}+02$ & $8.63 \mathrm{E}+00$ & $2.269 \mathrm{E}+00$ & $9.05 \mathrm{E}-02$ & $1.095 \mathrm{E}+01$ & $5.922 \mathrm{E}+00$ & $2.209 \mathrm{E}+01$ & $9.898 \mathrm{E}+04$ \\
\hline $1.811 \mathrm{E}+04$ & $2.666 \mathrm{E}+02$ & $8.66 \mathrm{E}+00$ & $2.846 \mathrm{E}+00$ & $7.00 \mathrm{E}-02$ & $1.091 \mathrm{E}+01$ & $8.005 \mathrm{E}+00$ & $2.206 \mathrm{E}+01$ & $9.898 \mathrm{E}+04$ \\
\hline $2.010 \mathrm{E}+04$ & $3.343 \mathrm{E}+02$ & $8.88 \mathrm{E}+00$ & $3.636 \mathrm{E}+00$ & $9.25 \mathrm{E}-02$ & $1.084 \mathrm{E}+01$ & $1.084 \mathrm{E}+01$ & $2.203 \mathrm{E}+01$ & $9.896 \mathrm{E}+04$ \\
\hline $2.207 \mathrm{E}+04$ & $4.046 \mathrm{E}+02$ & $9.22 \mathrm{E}+00$ & $4.294 \mathrm{E}+00$ & $9.23 \mathrm{E}-02$ & $1.076 \mathrm{E}+01$ & $1.435 \mathrm{E}+01$ & $2.198 \mathrm{E}+01$ & $9.896 \mathrm{E}+04$ \\
\hline $2.446 \mathrm{E}+04$ & $5.006 \mathrm{E}+02$ & $9.16 \mathrm{E}+00$ & $5.517 \mathrm{E}+00$ & $8.02 \mathrm{E}-02$ & $1.062 \mathrm{E}+01$ & $2.042 \mathrm{E}+01$ & $2.197 \mathrm{E}+01$ & $9.896 \mathrm{E}+04$ \\
\hline
\end{tabular}

Table 13: APC $4.5 \times 4.1$ Static Calculated Values

\begin{tabular}{|c|c|c|c|c|c|c|c|}
\hline$n(\mathrm{RPM})$ & $\rho\left(\mathrm{kg} / \mathrm{m}^{\wedge} 3\right)$ & $P_{\mathrm{P}}(\mathrm{W})$ & $\Delta P_{\mathrm{P}}(\mathrm{W})$ & $P_{\mathrm{e}}(\mathrm{W})$ & $\Delta P_{\mathrm{e}}(\mathrm{W})$ & $C_{\mathrm{T}}$ & $\Delta C_{\mathrm{T}}$ \\
\hline $4.386 \mathrm{E}+03$ & $1.168 \mathrm{E}+00$ & $7.749 \mathrm{E}-01$ & $2.55 \mathrm{E}-01$ & $4.049 \mathrm{E}+00$ & $6.32 \mathrm{E}-02$ & $1.337 \mathrm{E}-01$ & $8.73 \mathrm{E}-02$ \\
\hline $8.004 \mathrm{E}+03$ & $1.168 \mathrm{E}+00$ & $4.788 \mathrm{E}+00$ & $5.11 \mathrm{E}-01$ & $1.244 \mathrm{E}+01$ & $1.51 \mathrm{E}-01$ & $1.382 \mathrm{E}-01$ & $2.81 \mathrm{E}-02$ \\
\hline $1.005 \mathrm{E}+04$ & $1.168 \mathrm{E}+00$ & $9.169 \mathrm{E}+00$ & $6.30 \mathrm{E}-01$ & $2.060 \mathrm{E}+01$ & $E-01$ & E-01 & E-02 \\
\hline $1.203 \mathrm{E}+04$ & $1.168 \mathrm{E}+00$ & $1.567 \mathrm{E}+01$ & $7.45 \mathrm{E}-01$ & $3.156 \mathrm{E}+01$ & $3.53 \mathrm{E}-01$ & $1.385 \mathrm{E}-01$ & $1.13 \mathrm{E}-02$ \\
\hline $1.411 \mathrm{E}+04$ & $1.168 \mathrm{E}+00$ & $2.535 \mathrm{E}+01$ & $9.65 \mathrm{E}-01$ & $4.698 \mathrm{E}+01$ & $5.18 \mathrm{E}-01$ & $1.388 \mathrm{E}-01$ & $8.67 \mathrm{E}-03$ \\
\hline $1.615 \mathrm{E}+04$ & $1.168 \mathrm{E}+00$ & $3.763 \mathrm{E}+01$ & $1.50 \mathrm{E}+00$ & $6.487 \mathrm{E}+01$ & 7.39E-01 & $1.437 \mathrm{E}-01$ & $5.95 \mathrm{E}-03$ \\
\hline $1.811 \mathrm{E}+04$ & $1.168 \mathrm{E}+00$ & $5.294 \mathrm{E}+01$ & $1.30 \mathrm{E}+00$ & $8.731 \mathrm{E}+01$ & $9.65 \mathrm{E}-01$ & $1.460 \mathrm{E}-01$ & $4.75 \mathrm{E}-03$ \\
\hline $2.010 \mathrm{E}+04$ & $1.168 \mathrm{E}+00$ & $7.504 \mathrm{E}+01$ & $1.91 \mathrm{E}+00$ & $1.176 \mathrm{E}+02$ & $1.39 \mathrm{E}+00$ & $1.488 \mathrm{E}-01$ & $3.95 \mathrm{E}-03$ \\
\hline $2.207 \mathrm{E}+04$ & $1.168 \mathrm{E}+00$ & $9.733 \mathrm{E}+01$ & $2.09 \mathrm{E}+00$ & $1.544 \mathrm{E}+02$ & $1.78 \mathrm{E}+00$ & $1.493 \mathrm{E}-01$ & $3.40 \mathrm{E}-03$ \\
\hline $2.446 \mathrm{E}+04$ & $1.168 \mathrm{E}+00$ & $1.386 \mathrm{E}+02$ & $2.02 \mathrm{E}+00$ & $2.168 \mathrm{E}+02$ & $2.26 \mathrm{E}+00$ & $1.503 \mathrm{E}-01$ & $2.75 \mathrm{E}-03$ \\
\hline$C_{\mathrm{P}}$ & $\Delta C_{\mathrm{P}}$ & $C_{\mathrm{Q}}$ & $\Delta C_{\mathrm{Q}}$ & $\eta_{\mathrm{T}}$ & $\Delta \eta_{\mathrm{T}}$ & $R e_{0.75}$ & $\Delta R e_{0.75}$ \\
\hline $8.869 \mathrm{E}-02$ & $2.92 \mathrm{E}-02$ & $1.412 \mathrm{E}-02$ & $4.65 \mathrm{E}-03$ & $1.914 \mathrm{E}-01$ & $6.31 \mathrm{E}-02$ & $1.084 \mathrm{E}+04$ & $1.53 \mathrm{E}+01$ \\
\hline $9.019 \mathrm{E}-02$ & $9.62 \mathrm{E}-03$ & $1.435 \mathrm{E}-02$ & $1.53 \mathrm{E}-03$ & $3.847 \mathrm{E}-01$ & $4.13 \mathrm{E}-02$ & $1.977 \mathrm{E}+04$ & $2.87 \mathrm{E}+01$ \\
\hline $8.729 \mathrm{E}-02$ & $6.00 \mathrm{E}-03$ & $1.389 \mathrm{E}-02$ & $9.54 \mathrm{E}-04$ & $4.451 \mathrm{E}-01$ & $3.10 \mathrm{E}-02$ & $2.483 \mathrm{E}+04$ & $3.20 \mathrm{E}+01$ \\
\hline $8.695 \mathrm{E}-02$ & $4.13 \mathrm{E}-03$ & $1.384 \mathrm{E}-02$ & $6.58 \mathrm{E}-04$ & $4.965 \mathrm{E}-01$ & $2.42 \mathrm{E}-02$ & $2.973 \mathrm{E}+04$ & $4.00 \mathrm{E}+01$ \\
\hline 8.719E-02 & $3.32 \mathrm{E}-03$ & $1.388 \mathrm{E}-02$ & $5.29 \mathrm{E}-04$ & $5.397 \mathrm{E}-01$ & $2.14 \mathrm{E}-02$ & $3.487 \mathrm{E}+04$ & $4.68 \mathrm{E}+01$ \\
\hline $8.629 \mathrm{E}-02$ & $3.44 \mathrm{E}-03$ & $1.373 \mathrm{E}-02$ & $5.48 \mathrm{E}-04$ & $5.800 \mathrm{E}-01$ & $2.41 \mathrm{E}-02$ & $3.991 \mathrm{E}+04$ & $5.14 \mathrm{E}+01$ \\
\hline $8.605 \mathrm{E}-02$ & $2.12 \mathrm{E}-03$ & $1.370 \mathrm{E}-02$ & $3.37 \mathrm{E}-04$ & $6.064 \mathrm{E}-01$ & $1.63 \mathrm{E}-02$ & $4.477 \mathrm{E}+04$ & $5.93 \mathrm{E}+01$ \\
\hline 8.926E-02 & $2.28 \mathrm{E}-03$ & $1.421 \mathrm{E}-02$ & $3.62 \mathrm{E}-04$ & $6.382 \mathrm{E}-01$ & 1.79E-02 & $4.968 \mathrm{E}+04$ & $6.61 \mathrm{E}+01$ \\
\hline $8.740 \mathrm{E}-02$ & $1.88 \mathrm{E}-03$ & $1.412 \mathrm{E}-02$ & $2.99 \mathrm{E}-04$ & $6.303 \mathrm{E}-01$ & $1.54 \mathrm{E}-02$ & $5.457 \mathrm{E}+04$ & $6.85 \mathrm{E}+01$ \\
\hline $9.139 \mathrm{E}-02$ & $1.33 \mathrm{E}-03$ & $1.455 \mathrm{E}-02$ & $2.12 \mathrm{E}-04$ & $6.394 \mathrm{E}-01$ & $1.14 \mathrm{E}-02$ & $6.049 \mathrm{E}+04$ & $7.84 \mathrm{E}+01$ \\
\hline
\end{tabular}




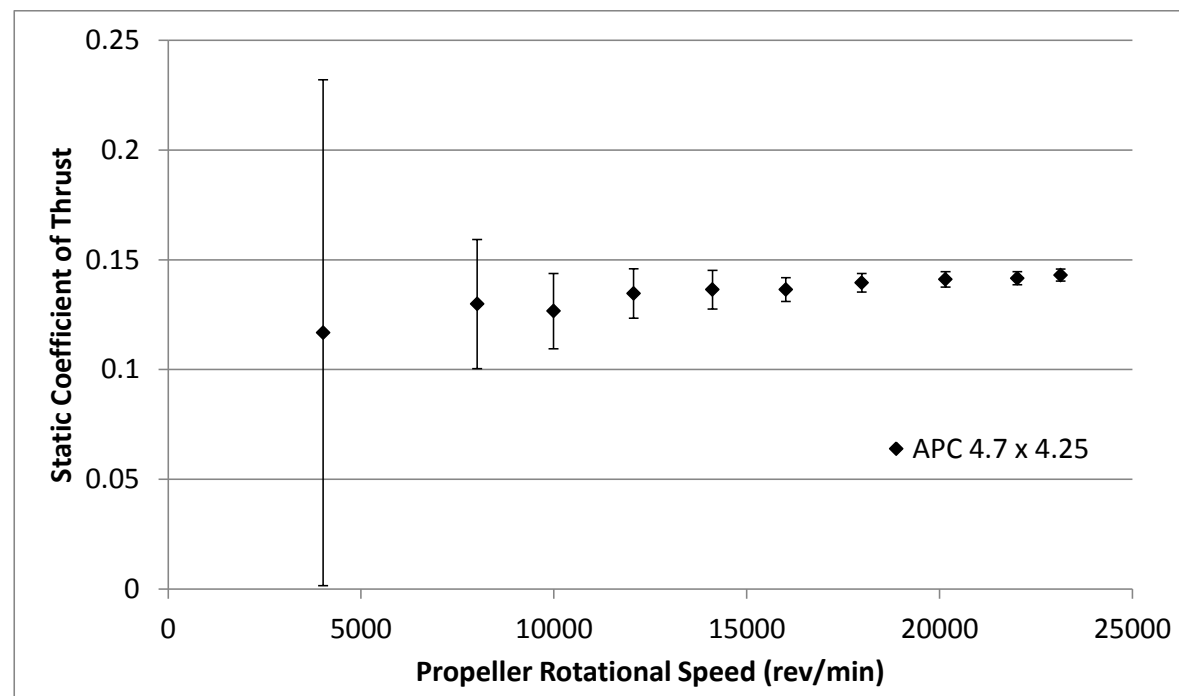

(a)

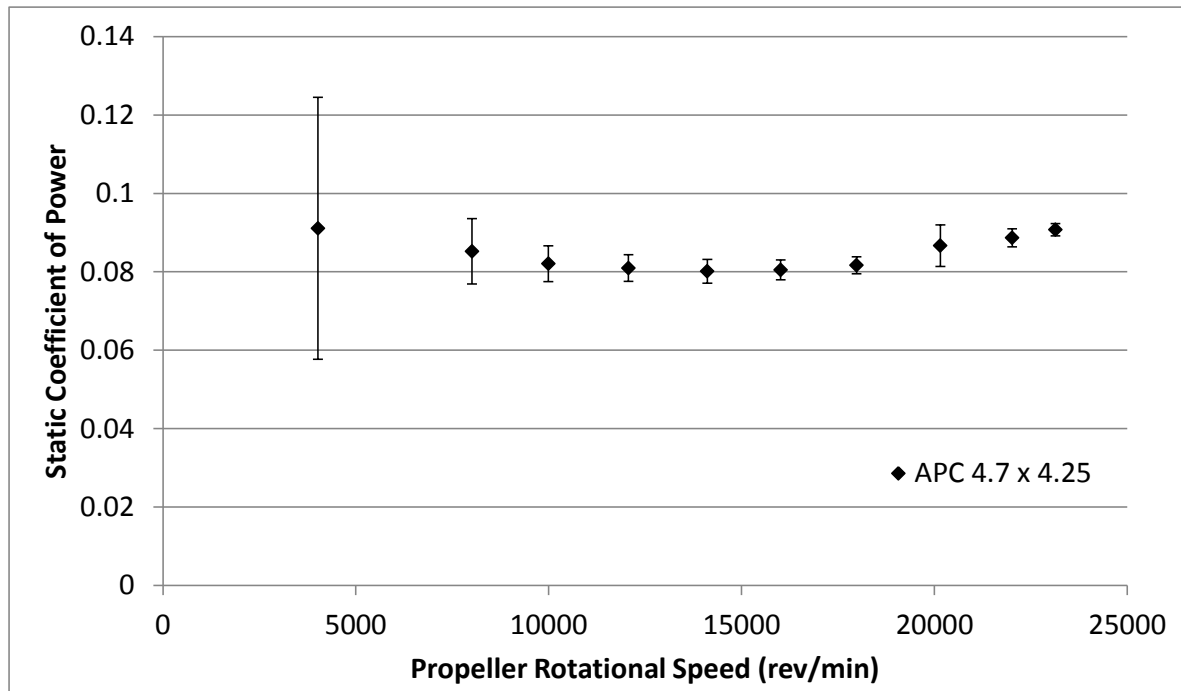

(b)

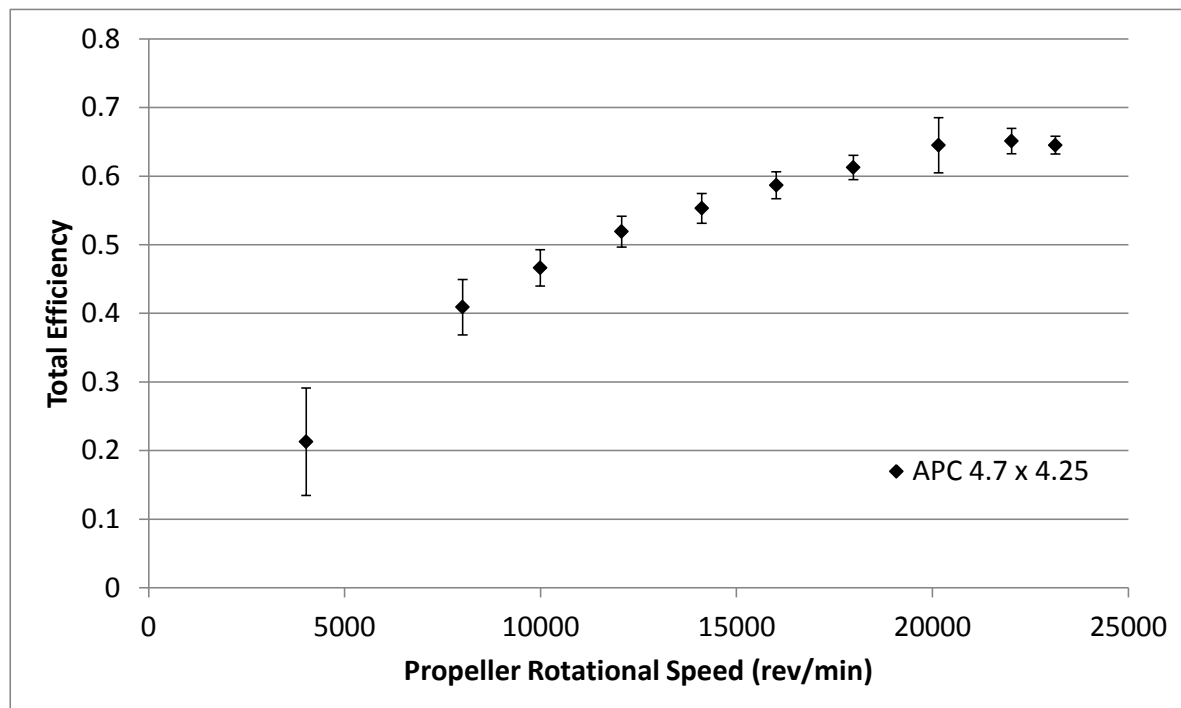

(c)

Figure 46: APC 4.7 x 4.25 Static Test Results: (a) Coefficient of Thrust, (b) Coefficient of Power, (c) Total Efficiency. 
Table 14: APC 4.7 x 4.25 Static Measured Values

\begin{tabular}{|c|c|c|c|c|c|c|c|c|}
\hline$n(\mathrm{RPM})$ & $T(\mathrm{~g})$ & $\Delta T(\mathrm{~g})$ & $Q(\mathrm{~g}-\mathrm{m})$ & $\Delta Q(\mathrm{~g}-\mathrm{m})$ & $V(\mathrm{~V})$ & $I(\mathrm{~A})$ & $T_{\text {atm }}\left({ }^{\circ} \mathrm{C}\right)$ & $P_{\text {atm }}(\mathrm{Pa})$ \\
\hline $4.019 \mathrm{E}+03$ & $1.305 \mathrm{E}+01$ & $1.29 \mathrm{E}+01$ & $1.948 \mathrm{E}-01$ & $7.15 \mathrm{E}-02$ & $1.108 \mathrm{E}+01$ & $3.407 \mathrm{E}-01$ & $2.162 \mathrm{E}+01$ & $9.906 \mathrm{E}+04$ \\
\hline $8.013 \mathrm{E}+03$ & $5.766 \mathrm{E}+01$ & $1.31 \mathrm{E}+01$ & $7.241 \mathrm{E}-01$ & $7.08 \mathrm{E}-02$ & $1.106 \mathrm{E}+01$ & $1.317 \mathrm{E}+00$ & $2.181 \mathrm{E}+01$ & $9.906 \mathrm{E}+04$ \\
\hline $9.993 \mathrm{E}+03$ & $8.742 \mathrm{E}+01$ & $1.18 \mathrm{E}+01$ & $1.084 \mathrm{E}+00$ & $6.05 \mathrm{E}-02$ & $1.104 \mathrm{E}+01$ & $2.161 \mathrm{E}+00$ & $2.195 \mathrm{E}+01$ & $9.905 \mathrm{E}+04$ \\
\hline $1.207 \mathrm{E}+04$ & $1.356 \mathrm{E}+02$ & $1.13 \mathrm{E}+01$ & $1.560 \mathrm{E}+00$ & $6.54 \mathrm{E}-02$ & $1.101 \mathrm{E}+01$ & $3.382 \mathrm{E}+00$ & $2.196 \mathrm{E}+01$ & $9.905 \mathrm{E}+04$ \\
\hline $1.411 \mathrm{E}+04$ & $1.879 \mathrm{E}+02$ & $1.21 \mathrm{E}+01$ & $2.112 \mathrm{E}+00$ & $7.99 \mathrm{E}-02$ & $1.097 \mathrm{E}+01$ & $5.042 \mathrm{E}+00$ & $2.193 \mathrm{E}+01$ & $9.905 \mathrm{E}+04$ \\
\hline $1.602 \mathrm{E}+04$ & $2.420 \mathrm{E}+02$ & $9.61 \mathrm{E}+00$ & $2.731 \mathrm{E}+00$ & $8.59 \mathrm{E}-02$ & $1.093 \mathrm{E}+01$ & $7.005 \mathrm{E}+00$ & $2.199 \mathrm{E}+01$ & $9.905 \mathrm{E}+04$ \\
\hline $1.798 \mathrm{E}+04$ & $3.119 \mathrm{E}+02$ & $9.52 \mathrm{E}+00$ & $3.493 \mathrm{E}+00$ & $9.30 \mathrm{E}-02$ & $1.087 \mathrm{E}+01$ & $9.685 \mathrm{E}+00$ & $2.191 \mathrm{E}+01$ & $9.904 \mathrm{E}+04$ \\
\hline $2.016 \mathrm{E}+04$ & $3.964 \mathrm{E}+02$ & $9.87 \mathrm{E}+00$ & $4.658 \mathrm{E}+00$ & $2.85 \mathrm{E}-01$ & $1.078 \mathrm{E}+01$ & $1.387 \mathrm{E}+01$ & $2.190 \mathrm{E}+01$ & $9.903 \mathrm{E}+04$ \\
\hline $2.202 \mathrm{E}+04$ & $4.745 \mathrm{E}+02$ & $1.00 \mathrm{E}+01$ & $5.686 \mathrm{E}+00$ & $1.49 \mathrm{E}-01$ & $1.067 \mathrm{E}+01$ & $1.852 \mathrm{E}+01$ & $2.191 \mathrm{E}+01$ & $9.902 \mathrm{E}+04$ \\
\hline $2.314 \mathrm{E}+04$ & $5.294 \mathrm{E}+02$ & $1.00 \mathrm{E}+01$ & $6.426 \mathrm{E}+00$ & $1.11 \mathrm{E}-01$ & $1.057 \mathrm{E}+01$ & $2.239 \mathrm{E}+01$ & $2.189 \mathrm{E}+01$ & $9.902 \mathrm{E}+04$ \\
\hline
\end{tabular}

Table 15: APC 4.7 x 4.25 Static Calculated Values

\begin{tabular}{|c|c|c|c|c|c|c|c|}
\hline$n(\mathrm{RPM})$ & $\rho\left(\mathrm{kg} / \mathrm{m}^{\wedge} 3\right)$ & $P_{\mathrm{P}}(\mathrm{W})$ & $\Delta P_{\mathrm{P}}(\mathrm{W})$ & $P_{\mathrm{e}}(\mathrm{W})$ & $\Delta P_{\mathrm{e}}(\mathrm{W})$ & $C_{\mathrm{T}}$ & $\Delta C_{\mathrm{T}}$ \\
\hline $4.019 \mathrm{E}+03$ & $1.171 \mathrm{E}+00$ & $8.039 \mathrm{E}-01$ & $2.95 \mathrm{E}-01$ & $3.776 \mathrm{E}+00$ & $6.10 \mathrm{E}-02$ & $1.168 \mathrm{E}-01$ & $1.15 \mathrm{E}-01$ \\
\hline $8.013 \mathrm{E}+03$ & $1.170 \mathrm{E}+00$ & $5.958 \mathrm{E}+00$ & $5.83 \mathrm{E}-01$ & $1.457 \mathrm{E}+01$ & $1.74 \mathrm{E}-01$ & $1.298 \mathrm{E}-01$ & $2.94 \mathrm{E}-02$ \\
\hline $9.993 \mathrm{E}+03$ & $1.169 \mathrm{E}+00$ & $1.112 \mathrm{E}+01$ & $6.21 \mathrm{E}-01$ & $2.386 \mathrm{E}+01$ & $2.72 \mathrm{E}-01$ & $1.266 \mathrm{E}-01$ & $1.71 \mathrm{E}-02$ \\
\hline $1.207 \mathrm{E}+04$ & $1.169 \mathrm{E}+00$ & $1.933 \mathrm{E}+01$ & $8.11 \mathrm{E}-01$ & $3.724 \mathrm{E}+01$ & 4.14E-01 & $1.347 \mathrm{E}-01$ & $1.12 \mathrm{E}-02$ \\
\hline $1.411 \mathrm{E}+04$ & $1.169 \mathrm{E}+00$ & $3.061 \mathrm{E}+01$ & $1.16 \mathrm{E}+00$ & $5.534 \mathrm{E}+01$ & $6.09 \mathrm{E}-01$ & $1.364 \mathrm{E}-01$ & $8.82 \mathrm{E}-03$ \\
\hline $1.602 \mathrm{E}+04$ & $1.169 \mathrm{E}+00$ & $4.491 \mathrm{E}+01$ & $41 \mathrm{E}+00$ & $7.655 \mathrm{E}+01$ & -01 & -01 & $5.42 \mathrm{E}-03$ \\
\hline $1.798 \mathrm{E}+04$ & $1.169 \mathrm{E}+00$ & $6.450 \mathrm{E}+01$ & $1.72 \mathrm{E}+00$ & $1.053 \mathrm{E}+02$ & $1.15 \mathrm{E}+00$ & $1.395 \mathrm{E}-01$ & $4.26 \mathrm{E}-03$ \\
\hline $2.016 \mathrm{E}+04$ & $1.169 \mathrm{E}+00$ & $9.642 \mathrm{E}+01$ & $5.90 \mathrm{E}+00$ & 1.495 & +00 & -01 & $3.51 \mathrm{E}-03$ \\
\hline $2.202 \mathrm{E}+04$ & $1.169 \mathrm{E}+00$ & $1.286 \mathrm{E}+02$ & $3.37 \mathrm{E}+00$ & $1.975 \mathrm{E}+02$ & $2.07 \mathrm{E}+00$ & $1.416 \mathrm{E}-01$ & $2.99 \mathrm{E}-03$ \\
\hline $2.314 \mathrm{E}+04$ & $1.169 \mathrm{E}+00$ & $1.527 \mathrm{E}+02$ & $2.63 \mathrm{E}+00$ & $2.367 \mathrm{E}+02$ & $2.48 \mathrm{E}+00$ & $1.430 \mathrm{E}-01$ & $2.70 \mathrm{E}-03$ \\
\hline$C_{\mathrm{P}}$ & $\Delta C_{\mathrm{P}}$ & $C_{\mathrm{Q}}$ & $\Delta C_{\mathrm{Q}}$ & $\eta_{\mathrm{T}}$ & $\Delta \eta_{\mathrm{T}}$ & $R e_{0.75}$ & $\Delta R e_{0.75}$ \\
\hline $9.109 \mathrm{E}-02$ & $3.34 \mathrm{E}-02$ & $1.450 \mathrm{E}-02$ & 5.32E-03 & $2.129 \mathrm{E}-01$ & 7.82E-02 & $1.029 \mathrm{E}+04$ & $1.60 \mathrm{E}+01$ \\
\hline $8.522 \mathrm{E}-02$ & $8.34 \mathrm{E}-03$ & $1.356 \mathrm{E}-02$ & $1.33 \mathrm{E}-03$ & $4.090 \mathrm{E}-01$ & 4.03E-02 & $2.049 \mathrm{E}+04$ & $2.80 \mathrm{E}+01$ \\
\hline 8.207E-02 & $4.58 \mathrm{E}-03$ & $1.306 \mathrm{E}-02$ & 7.30E-04 & $4.662 \mathrm{E}-01$ & $2.66 \mathrm{E}-02$ & $2.553 \mathrm{E}+04$ & $3.34 \mathrm{E}+01$ \\
\hline $8.096 \mathrm{E}-02$ & $3.40 \mathrm{E}-03$ & $1.288 \mathrm{E}-02$ & 5.40E-04 & $5.191 \mathrm{E}-01$ & $2.25 \mathrm{E}-02$ & $3.083 \mathrm{E}+04$ & $4.05 \mathrm{E}+01$ \\
\hline $8.013 \mathrm{E}-02$ & $3.03 \mathrm{E}-03$ & $1.275 \mathrm{E}-02$ & $4.83 \mathrm{E}-04$ & $5.531 \mathrm{E}-01$ & $2.18 \mathrm{E}-02$ & $3.606 \mathrm{E}+04$ & $4.70 \mathrm{E}+01$ \\
\hline $8.050 \mathrm{E}-02$ & $2.53 \mathrm{E}-03$ & $1.281 \mathrm{E}-02$ & $4.03 \mathrm{E}-04$ & $5.867 \mathrm{E}-01$ & $1.96 \mathrm{E}-02$ & $4.091 \mathrm{E}+04$ & $5.30 \mathrm{E}+01$ \\
\hline $8.164 \mathrm{E}-02$ & $2.18 \mathrm{E}-03$ & $1.299 \mathrm{E}-02$ & $3.46 \mathrm{E}-04$ & $6.127 \mathrm{E}-01$ & $1.76 \mathrm{E}-02$ & $4.595 \mathrm{E}+04$ & $5.93 \mathrm{E}+01$ \\
\hline 8.667E-02 & $5.31 \mathrm{E}-03$ & $1.379 \mathrm{E}-02$ & $8.45 \mathrm{E}-04$ & $6.451 \mathrm{E}-01$ & 4.02E-02 & $5.151 \mathrm{E}+04$ & $6.62 \mathrm{E}+01$ \\
\hline 8.867E-02 & $2.33 \mathrm{E}-03$ & $1.450 \mathrm{E}-02$ & $3.70 \mathrm{E}-04$ & $6.510 \mathrm{E}-01$ & $1.84 \mathrm{E}-02$ & $5.626 \mathrm{E}+04$ & $7.20 \mathrm{E}+01$ \\
\hline $9.074 \mathrm{E}-02$ & $1.56 \mathrm{E}-03$ & $1.444 \mathrm{E}-02$ & $2.49 \mathrm{E}-04$ & $6.451 \mathrm{E}-01$ & $1.30 \mathrm{E}-02$ & $5.913 \mathrm{E}+04$ & $7.61 \mathrm{E}+01$ \\
\hline
\end{tabular}




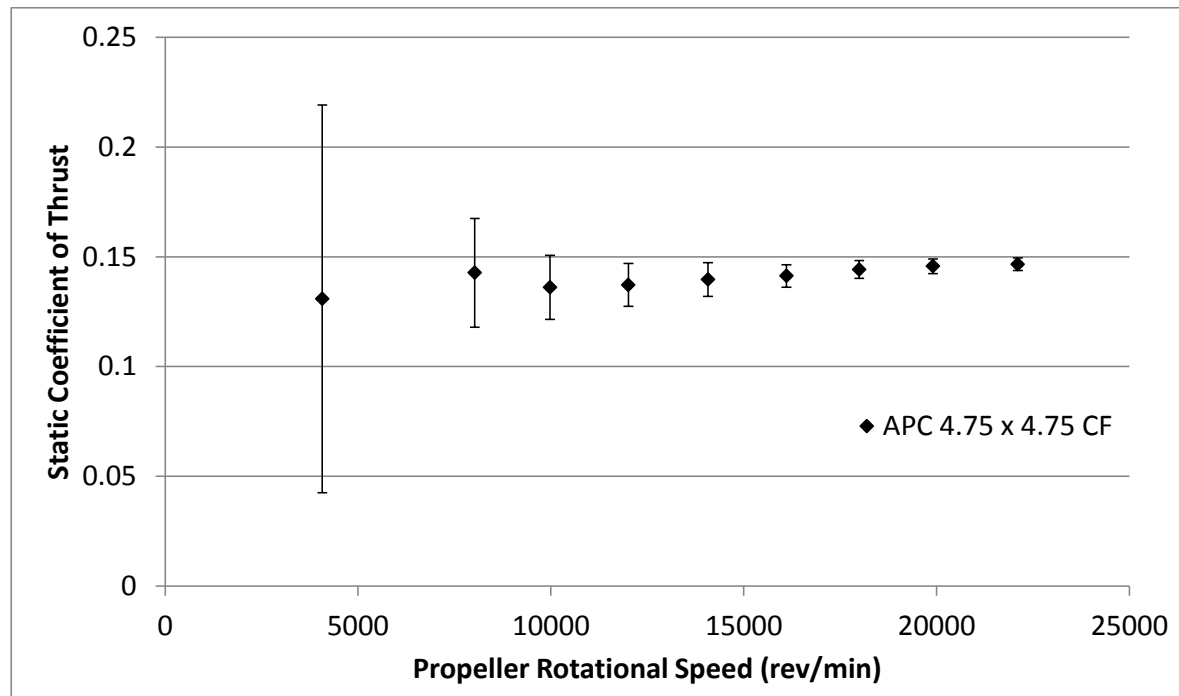

(a)

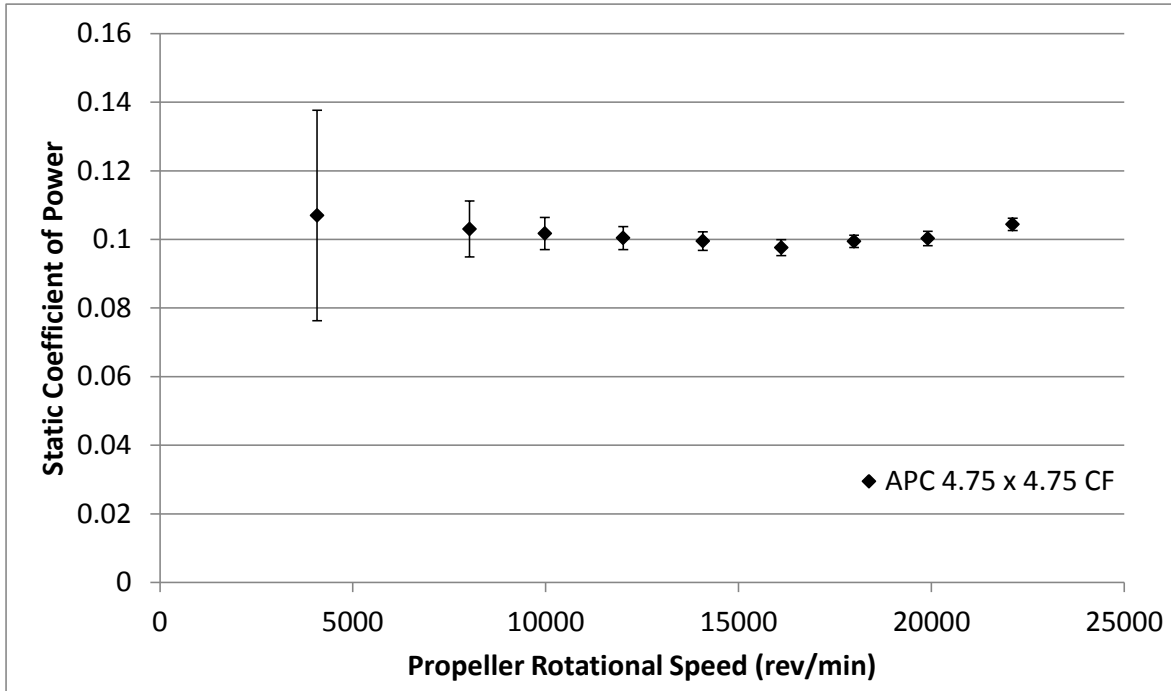

(b)

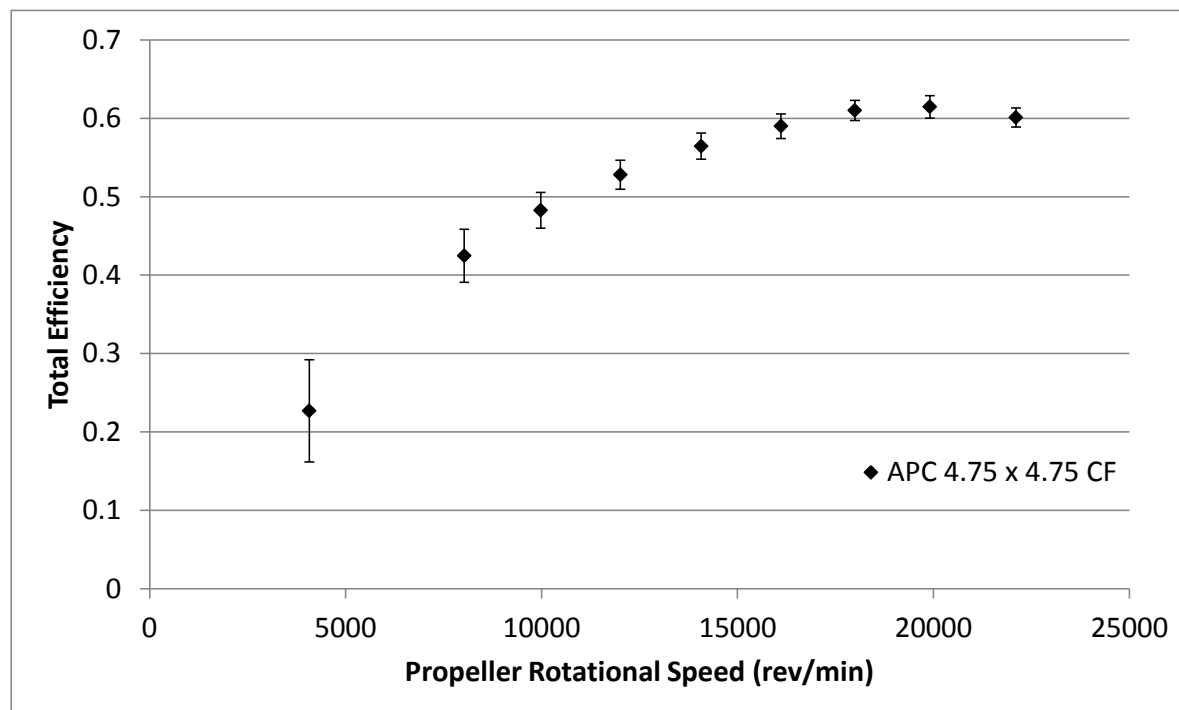

(c)

Figure 47: APC 4.75 x 4.75 Carbon Fiber Static Test Results: (a) Coefficient of Thrust, (b) Coefficient of Power, (c) Total Efficiency. 
Table 16: APC 4.75 x 4.75 Carbon Fiber Static Measured Values

\begin{tabular}{|c|c|c|c|c|c|c|c|c|}
\hline$n(\mathrm{RPM})$ & $T(\mathrm{~g})$ & $\Delta T(\mathrm{~g})$ & $Q(\mathrm{~g}-\mathrm{m})$ & $\Delta Q(\mathrm{~g}-\mathrm{m})$ & $V(\mathrm{~V})$ & $I(\mathrm{~A})$ & $T_{\mathrm{atm}}\left({ }^{\circ} \mathrm{C}\right)$ & $P_{\mathrm{atm}}(\mathrm{Pa})$ \\
\hline $4.074 \mathrm{E}+03$ & $1.485 \mathrm{E}+01$ & $1.00 \mathrm{E}+01$ & $2.317 \mathrm{E}-01$ & $6.64 \mathrm{E}-02$ & $1.108 \mathrm{E}+01$ & $3.856 \mathrm{E}-01$ & $2.202 \mathrm{E}+01$ & $9.907 \mathrm{E}+04$ \\
\hline $8.029 \mathrm{E}+03$ & $6.291 \mathrm{E}+01$ & $1.09 \mathrm{E}+01$ & $8.667 \mathrm{E}-01$ & $6.85 \mathrm{E}-02$ & $1.105 \mathrm{E}+01$ & $1.522 \mathrm{E}+00$ & $2.192 \mathrm{E}+01$ & $9.908 \mathrm{E}+04$ \\
\hline $9.980 \mathrm{E}+03$ & $9.265 \mathrm{E}+01$ & $9.94 \mathrm{E}+00$ & $1.322 \mathrm{E}+00$ & $6.08 \mathrm{E}-02$ & $1.103 \mathrm{E}+01$ & $2.543 \mathrm{E}+00$ & $2.198 \mathrm{E}+01$ & $9.908 \mathrm{E}+04$ \\
\hline $1.201 \mathrm{E}+04$ & $1.354 \mathrm{E}+02$ & $9.63 \mathrm{E}+00$ & $1.890 \mathrm{E}+00$ & $6.27 \mathrm{E}-02$ & $1.100 \mathrm{E}+01$ & $4.016 \mathrm{E}+00$ & $2.195 \mathrm{E}+01$ & $9.908 \mathrm{E}+04$ \\
\hline $1.408 \mathrm{E}+04$ & $1.892 \mathrm{E}+02$ & $1.04 \mathrm{E}+01$ & $2.572 \mathrm{E}+00$ & $7.03 \mathrm{E}-02$ & $1.095 \mathrm{E}+01$ & $6.013 \mathrm{E}+00$ & $2.196 \mathrm{E}+01$ & $9.908 \mathrm{E}+04$ \\
\hline $1.611 \mathrm{E}+04$ & $2.509 \mathrm{E}+02$ & $9.08 \mathrm{E}+00$ & $3.307 \mathrm{E}+00$ & $7.90 \mathrm{E}-02$ & $1.089 \mathrm{E}+01$ & $8.512 \mathrm{E}+00$ & $2.190 \mathrm{E}+01$ & $9.907 \mathrm{E}+04$ \\
\hline $1.800 \mathrm{E}+04$ & $3.195 \mathrm{E}+02$ & $8.98 \mathrm{E}+00$ & $4.205 \mathrm{E}+00$ & $7.56 \mathrm{E}-02$ & $1.082 \mathrm{E}+01$ & $1.177 \mathrm{E}+01$ & $2.188 \mathrm{E}+01$ & $9.907 \mathrm{E}+04$ \\
\hline $1.991 \mathrm{E}+04$ & $3.951 \mathrm{E}+02$ & $9.11 \mathrm{E}+00$ & $5.187 \mathrm{E}+00$ & $1.07 \mathrm{E}-01$ & $1.072 \mathrm{E}+01$ & $1.610 \mathrm{E}+01$ & $2.184 \mathrm{E}+01$ & $9.906 \mathrm{E}+04$ \\
\hline $2.211 \mathrm{E}+04$ & $4.903 \mathrm{E}+02$ & $9.45 \mathrm{E}+00$ & $6.658 \mathrm{E}+00$ & $1.14 \mathrm{E}-01$ & $1.053 \mathrm{E}+01$ & $2.389 \mathrm{E}+01$ & $2.184 \mathrm{E}+01$ & $9.906 \mathrm{E}+04$ \\
\hline
\end{tabular}

Table 17: APC 4.75 x 4.75 Carbon Fiber Static Calculated Values

\begin{tabular}{|c|c|c|c|c|c|c|c|}
\hline$n(\mathrm{RPM})$ & $\rho\left(\mathrm{kg} / \mathrm{m}^{\wedge} 3\right)$ & $P_{\mathrm{P}}(\mathrm{W})$ & $\Delta P_{\mathrm{P}}(\mathrm{W})$ & $P_{\mathrm{e}}(\mathrm{W})$ & $\Delta P_{\mathrm{e}}(\mathrm{W})$ & $C_{\mathrm{T}}$ & $\Delta C_{\mathrm{T}}$ \\
\hline $4.074 \mathrm{E}+03$ & $1.169 \mathrm{E}+00$ & $9.695 \mathrm{E}-01$ & $2.78 \mathrm{E}-01$ & $4.273 \mathrm{E}+00$ & $6.77 \mathrm{E}-02$ & $1.308 \mathrm{E}-01$ & $8.83 \mathrm{E}-02$ \\
\hline $8.029 \mathrm{E}+03$ & $1.170 \mathrm{E}+00$ & $7.146 \mathrm{E}+00$ & $5.65 \mathrm{E}-01$ & $1.683 \mathrm{E}+01$ & $1.98 \mathrm{E}-01$ & $1.427 \mathrm{E}-01$ & $2.47 \mathrm{E}-02$ \\
\hline $9.980 \mathrm{E}+03$ & $1.170 \mathrm{E}+00$ & $1.354 \mathrm{E}+01$ & $6.23 \mathrm{E}-01$ & $2.805 \mathrm{E}+01$ & $3.19 \mathrm{E}-01$ & $1.360 \mathrm{E}-01$ & $1.46 \mathrm{E}-02$ \\
\hline $1.201 \mathrm{E}+04$ & $1.170 \mathrm{E}+00$ & $2.332 \mathrm{E}+01$ & $7.74 \mathrm{E}-01$ & $4.417 \mathrm{E}+01$ & $4.88 \mathrm{E}-01$ & $1.372 \mathrm{E}-01$ & $9.76 \mathrm{E}-03$ \\
\hline $1.408 \mathrm{E}+04$ & $1.170 \mathrm{E}+00$ & $3.718 \mathrm{E}+01$ & $1.02 \mathrm{E}+00$ & $6.586 \mathrm{E}+01$ & $7.44 \mathrm{E}-01$ & $1.396 \mathrm{E}-01$ & $7.68 \mathrm{E}-03$ \\
\hline $1.611 \mathrm{E}+04$ & $1.170 \mathrm{E}+00$ & $5.471 \mathrm{E}+01$ & $1.31 \mathrm{E}+00$ & $9.273 \mathrm{E}+01$ & $1.05 \mathrm{E}+00$ & $1.413 \mathrm{E}-01$ & $5.11 \mathrm{E}-03$ \\
\hline $1.800 \mathrm{E}+04$ & $1.170 \mathrm{E}+00$ & $7.771 \mathrm{E}+01$ & $1.40 \mathrm{E}+00$ & $1.274 \mathrm{E}+02$ & $1.36 \mathrm{E}+00$ & $1.442 \mathrm{E}-01$ & $4.06 \mathrm{E}-03$ \\
\hline $1.991 \mathrm{E}+04$ & $1.170 \mathrm{E}+00$ & $1.061 \mathrm{E}+02$ & $2.19 \mathrm{E}+00$ & $1.726 \mathrm{E}+02$ & $1.84 \mathrm{E}+00$ & $1.457 \mathrm{E}-01$ & $3.36 \mathrm{E}-03$ \\
\hline $2.211 \mathrm{E}+04$ & $1.170 \mathrm{E}+00$ & $1.512 \mathrm{E}+02$ & $2.60 \mathrm{E}+00$ & $2.516 \mathrm{E}+02$ & $2.62 \mathrm{E}+00$ & $1.466 \mathrm{E}-01$ & $2.83 \mathrm{E}-03$ \\
\hline$C_{\mathrm{P}}$ & $\Delta C_{\mathrm{P}}$ & $C_{\mathrm{Q}}$ & $\Delta C_{\mathrm{Q}}$ & $\eta_{\mathrm{T}}$ & $\Delta \eta_{\mathrm{T}}$ & $R e_{0.75}$ & $\Delta R e_{0.75}$ \\
\hline $1.070 \mathrm{E}-01$ & $3.07 \mathrm{E}-02$ & $1.703 \mathrm{E}-02$ & $4.88 \mathrm{E}-03$ & $2.269 \mathrm{E}-01$ & $6.52 \mathrm{E}-02$ & $1.009 \mathrm{E}+04$ & $1.55 \mathrm{E}+01$ \\
\hline $1.030 \mathrm{E}-01$ & $8.14 \mathrm{E}-03$ & $1.640 \mathrm{E}-02$ & $1.30 \mathrm{E}-03$ & $4.246 \mathrm{E}-01$ & $3.39 \mathrm{E}-02$ & $1.990 \mathrm{E}+04$ & $2.86 \mathrm{E}+01$ \\
\hline $1.017 \mathrm{E}-01$ & $4.68 \mathrm{E}-03$ & $1.619 \mathrm{E}-02$ & $7.45 \mathrm{E}-04$ & $4.828 \mathrm{E}-01$ & $2.29 \mathrm{E}-02$ & $2.473 \mathrm{E}+04$ & $3.33 \mathrm{E}+01$ \\
\hline $1.004 \mathrm{E}-01$ & $3.33 \mathrm{E}-03$ & $1.598 \mathrm{E}-02$ & $5.30 \mathrm{E}-04$ & $5.280 \mathrm{E}-01$ & $1.85 \mathrm{E}-02$ & $2.978 \mathrm{E}+04$ & $4.02 \mathrm{E}+01$ \\
\hline $9.949 \mathrm{E}-02$ & $2.72 \mathrm{E}-03$ & $1.583 \mathrm{E}-02$ & $4.33 \mathrm{E}-04$ & $5.645 \mathrm{E}-01$ & $1.67 \mathrm{E}-02$ & $3.489 \mathrm{E}+04$ & $4.70 \mathrm{E}+01$ \\
\hline $9.761 \mathrm{E}-02$ & $2.34 \mathrm{E}-03$ & $1.554 \mathrm{E}-02$ & $3.72 \mathrm{E}-04$ & $5.900 \mathrm{E}-01$ & $1.56 \mathrm{E}-02$ & $3.994 \mathrm{E}+04$ & $5.29 \mathrm{E}+01$ \\
\hline $9.947 \mathrm{E}-02$ & $1.79 \mathrm{E}-03$ & $1.583 \mathrm{E}-02$ & $2.85 \mathrm{E}-04$ & $6.101 \mathrm{E}-01$ & $1.28 \mathrm{E}-02$ & $4.462 \mathrm{E}+04$ & $5.89 \mathrm{E}+01$ \\
\hline $1.003 \mathrm{E}-01$ & $2.07 \mathrm{E}-03$ & $1.596 \mathrm{E}-02$ & $3.30 \mathrm{E}-04$ & $6.146 \mathrm{E}-01$ & $1.43 \mathrm{E}-02$ & $4.937 \mathrm{E}+04$ & $6.51 \mathrm{E}+01$ \\
\hline $1.044 \mathrm{E}-01$ & $1.80 \mathrm{E}-03$ & $1.703 \mathrm{E}-02$ & $2.86 \mathrm{E}-04$ & $6.010 \mathrm{E}-01$ & $1.21 \mathrm{E}-02$ & $5.483 \mathrm{E}+04$ & $7.30 \mathrm{E}+01$ \\
\hline
\end{tabular}




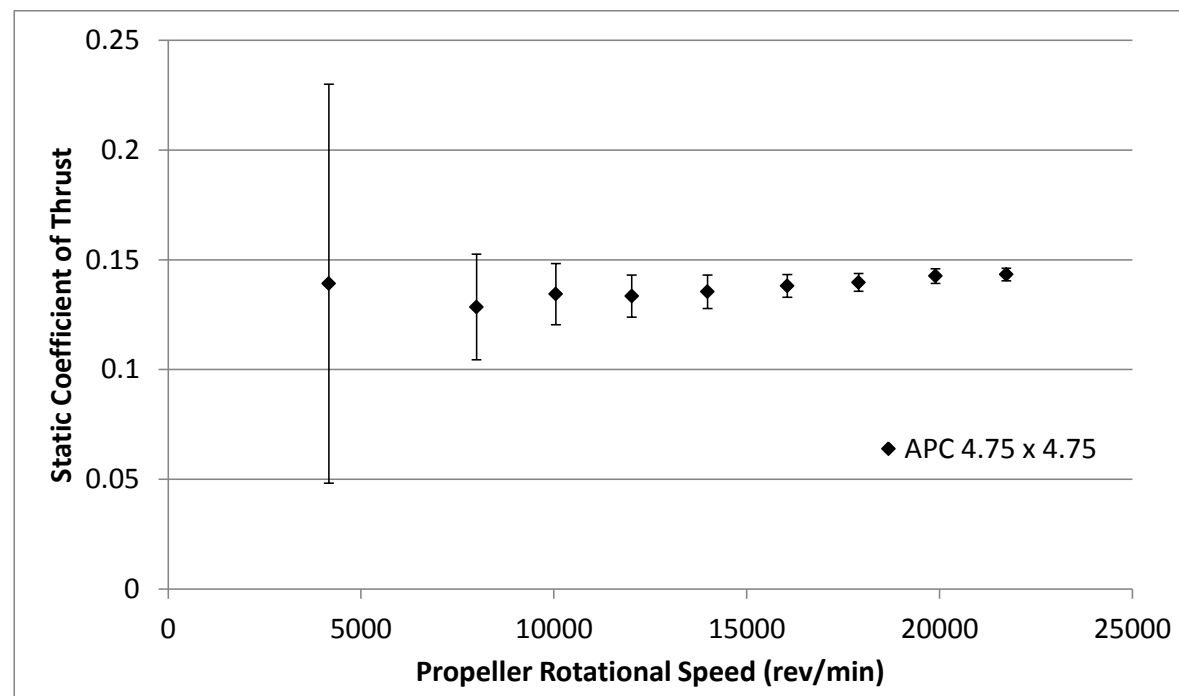

(a)

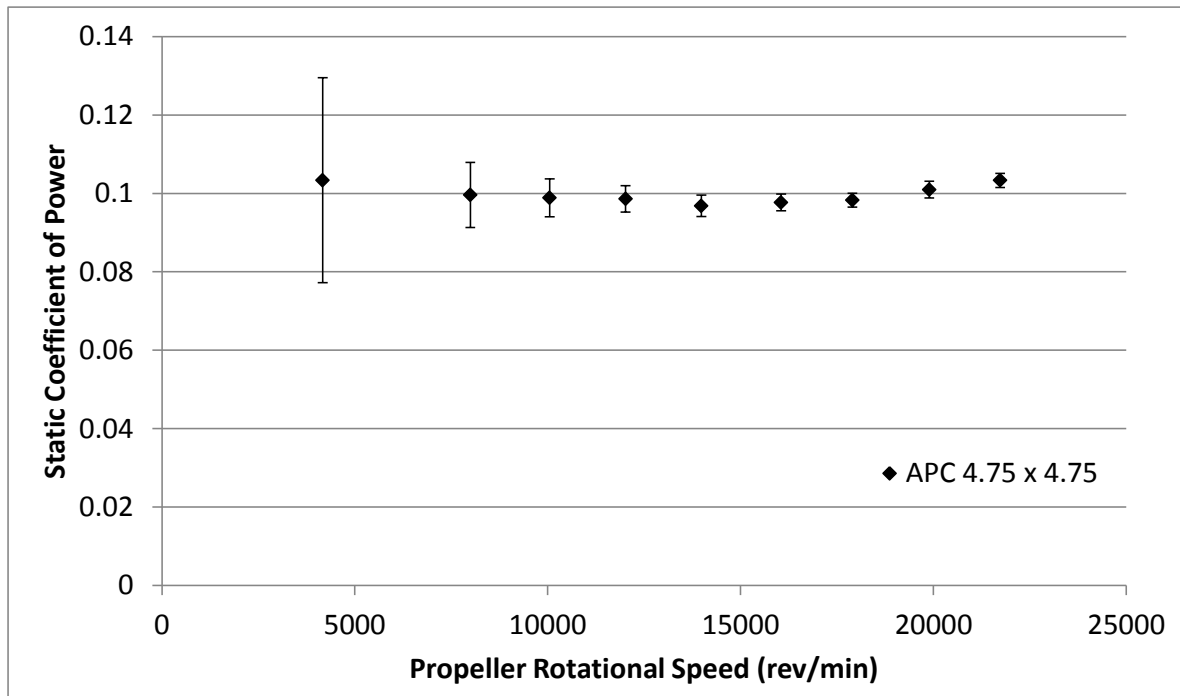

(b)

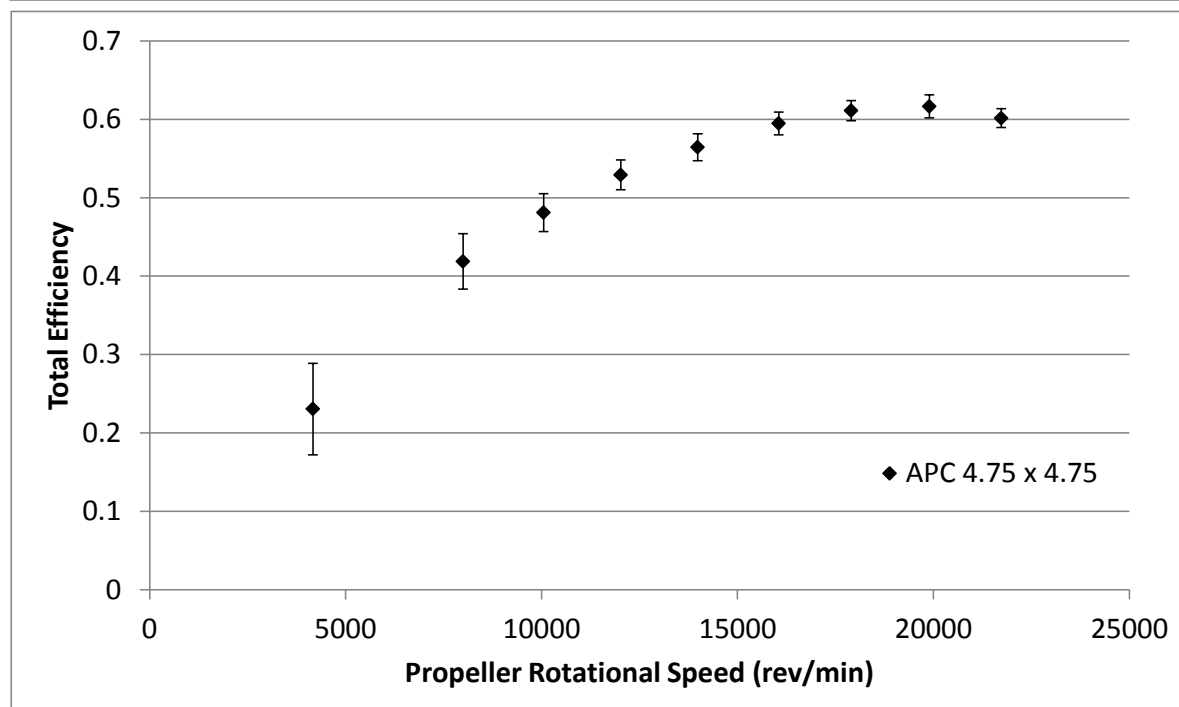

(c)

Figure 48: APC 4.75 x 4.75 Static Test Results: (a) Coefficient of Thrust, (b) Coefficient of Power, (c) Total Efficiency. 
Table 18: APC 4.75 x 4.75 Static Measured Values

\begin{tabular}{|c|c|c|c|c|c|c|c|c|}
\hline$n(\mathrm{RPM})$ & $T(\mathrm{~g})$ & $\Delta T(\mathrm{~g})$ & $Q(\mathrm{~g}-\mathrm{m})$ & $\Delta Q(\mathrm{~g}-\mathrm{m})$ & $V(\mathrm{~V})$ & $I(\mathrm{~A})$ & $T_{\mathrm{atm}}\left({ }^{\circ} \mathrm{C}\right)$ & $P_{\mathrm{atm}}(\mathrm{Pa})$ \\
\hline $4.167 \mathrm{E}+03$ & $1.642 \mathrm{E}+01$ & $1.07 \mathrm{E}+01$ & $2.327 \mathrm{E}-01$ & $5.89 \mathrm{E}-02$ & $1.109 \mathrm{E}+01$ & $3.894 \mathrm{E}-01$ & $2.224 \mathrm{E}+01$ & $9.855 \mathrm{E}+04$ \\
\hline $7.996 \mathrm{E}+03$ & $5.622 \mathrm{E}+01$ & $1.05 \mathrm{E}+01$ & $8.313 \mathrm{E}-01$ & $6.93 \mathrm{E}-02$ & $1.105 \mathrm{E}+01$ & $1.475 \mathrm{E}+00$ & $2.176 \mathrm{E}+01$ & $9.905 \mathrm{E}+04$ \\
\hline $1.005 \mathrm{E}+04$ & $9.291 \mathrm{E}+01$ & $9.64 \mathrm{E}+00$ & $1.304 \mathrm{E}+00$ & $6.40 \mathrm{E}-02$ & $1.103 \mathrm{E}+01$ & $2.537 \mathrm{E}+00$ & $2.179 \mathrm{E}+01$ & $9.906 \mathrm{E}+04$ \\
\hline $1.202 \mathrm{E}+04$ & $1.320 \mathrm{E}+02$ & $9.49 \mathrm{E}+00$ & $1.860 \mathrm{E}+00$ & $6.35 \mathrm{E}-02$ & $1.100 \mathrm{E}+01$ & $3.945 \mathrm{E}+00$ & $2.175 \mathrm{E}+01$ & $9.906 \mathrm{E}+04$ \\
\hline $1.399 \mathrm{E}+04$ & $1.813 \mathrm{E}+02$ & $1.02 \mathrm{E}+01$ & $2.472 \mathrm{E}+00$ & $6.94 \mathrm{E}-02$ & $1.096 \mathrm{E}+01$ & $5.742 \mathrm{E}+00$ & $2.176 \mathrm{E}+01$ & $9.906 \mathrm{E}+04$ \\
\hline $1.605 \mathrm{E}+04$ & $2.435 \mathrm{E}+02$ & $9.10 \mathrm{E}+00$ & $3.287 \mathrm{E}+00$ & $7.12 \mathrm{E}-02$ & $1.090 \mathrm{E}+01$ & $8.358 \mathrm{E}+00$ & $2.179 \mathrm{E}+01$ & $9.906 \mathrm{E}+04$ \\
\hline $1.790 \mathrm{E}+04$ & $3.064 \mathrm{E}+02$ & $8.88 \mathrm{E}+00$ & $4.113 \mathrm{E}+00$ & $7.51 \mathrm{E}-02$ & $1.083 \mathrm{E}+01$ & $1.143 \mathrm{E}+01$ & $2.171 \mathrm{E}+01$ & $9.906 \mathrm{E}+04$ \\
\hline $1.990 \mathrm{E}+04$ & $3.863 \mathrm{E}+02$ & $9.01 \mathrm{E}+00$ & $5.219 \mathrm{E}+00$ & $1.11 \mathrm{E}-01$ & $1.072 \mathrm{E}+01$ & $1.613 \mathrm{E}+01$ & $2.176 \mathrm{E}+01$ & $9.906 \mathrm{E}+04$ \\
\hline $2.173 \mathrm{E}+04$ & $4.633 \mathrm{E}+02$ & $9.27 \mathrm{E}+00$ & $6.372 \mathrm{E}+00$ & $1.10 \mathrm{E}-01$ & $1.057 \mathrm{E}+01$ & $2.237 \mathrm{E}+01$ & $2.174 \mathrm{E}+01$ & $9.907 \mathrm{E}+04$ \\
\hline
\end{tabular}

Table 19: APC 4.75 x 4.75 Static Calculated Values

\begin{tabular}{|c|c|c|c|c|c|c|c|}
\hline$n(\mathrm{RPM})$ & $\rho\left(\mathrm{kg} / \mathrm{m}^{\wedge} 3\right)$ & $P_{\mathrm{P}}(\mathrm{W})$ & $\Delta P_{\mathrm{P}}(\mathrm{W})$ & $P_{\mathrm{e}}(\mathrm{W})$ & $\Delta P_{\mathrm{e}}(\mathrm{W})$ & $C_{\mathrm{T}}$ & $\Delta C_{\mathrm{T}}$ \\
\hline $4.167 \mathrm{E}+03$ & $1.162 \mathrm{E}+00$ & $9.956 \mathrm{E}-01$ & $2.52 \mathrm{E}-01$ & $4.319 \mathrm{E}+00$ & $6.71 \mathrm{E}-02$ & $1.391 \mathrm{E}-01$ & $9.08 \mathrm{E}-02$ \\
\hline $7.996 \mathrm{E}+03$ & $1.170 \mathrm{E}+00$ & $6.827 \mathrm{E}+00$ & $5.69 \mathrm{E}-01$ & $1.630 \mathrm{E}+01$ & $1.92 \mathrm{E}-01$ & $1.285 \mathrm{E}-01$ & $2.41 \mathrm{E}-02$ \\
\hline $1.005 \mathrm{E}+04$ & $1.170 \mathrm{E}+00$ & $1.347 \mathrm{E}+01$ & $6.61 \mathrm{E}-01$ & $2.799 \mathrm{E}+01$ & $3.17 \mathrm{E}-01$ & $1.344 \mathrm{E}-01$ & $1.39 \mathrm{E}-02$ \\
\hline $1.202 \mathrm{E}+04$ & $1.170 \mathrm{E}+00$ & $2.297 \mathrm{E}+01$ & $7.84 \mathrm{E}-01$ & $4.340 \mathrm{E}+01$ & $4.79 \mathrm{E}-01$ & $1.335 \mathrm{E}-01$ & $9.60 \mathrm{E}-03$ \\
\hline $1.399 \mathrm{E}+04$ & $1.170 \mathrm{E}+00$ & $3.551 \mathrm{E}+01$ & $9.97 \mathrm{E}-01$ & $6.292 \mathrm{E}+01$ & $7.08 \mathrm{E}-01$ & $1.354 \mathrm{E}-01$ & $7.61 \mathrm{E}-03$ \\
\hline $1.605 \mathrm{E}+04$ & $1.170 \mathrm{E}+00$ & $5.419 \mathrm{E}+01$ & $1.17 \mathrm{E}+00$ & $9.110 \mathrm{E}+01$ & $1.02 \mathrm{E}+00$ & $1.381 \mathrm{E}-01$ & $5.16 \mathrm{E}-03$ \\
\hline $1.790 \mathrm{E}+04$ & $1.170 \mathrm{E}+00$ & $7.561 \mathrm{E}+01$ & $1.38 \mathrm{E}+00$ & $1.237 \mathrm{E}+02$ & $1.33 \mathrm{E}+00$ & $1.397 \mathrm{E}-01$ & $4.05 \mathrm{E}-03$ \\
\hline $1.990 \mathrm{E}+04$ & $1.170 \mathrm{E}+00$ & $1.066 \mathrm{E}+02$ & $2.26 \mathrm{E}+00$ & $1.730 \mathrm{E}+02$ & $1.84 \mathrm{E}+00$ & $1.426 \mathrm{E}-01$ & $3.33 \mathrm{E}-03$ \\
\hline $2.173 \mathrm{E}+04$ & $1.170 \mathrm{E}+00$ & $1.422 \mathrm{E}+02$ & $2.45 \mathrm{E}+00$ & $2.364 \mathrm{E}+02$ & $2.47 \mathrm{E}+00$ & $1.433 \mathrm{E}-01$ & $2.87 \mathrm{E}-03$ \\
\hline$C_{\mathrm{P}}$ & $\Delta C_{\mathrm{P}}$ & $C_{\mathrm{Q}}$ & $\Delta C_{\mathrm{Q}}$ & $\eta_{\mathrm{T}}$ & $\Delta \eta_{\mathrm{T}}$ & $R e_{0.75}$ & $\Delta R e_{0.75}$ \\
\hline $1.033 \mathrm{E}-01$ & $2.61 \mathrm{E}-02$ & $1.645 \mathrm{E}-02$ & $4.16 \mathrm{E}-03$ & $2.305 \mathrm{E}-01$ & $5.84 \mathrm{E}-02$ & $1.026 \mathrm{E}+04$ & $1.53 \mathrm{E}+01$ \\
\hline $9.960 \mathrm{E}-02$ & $8.31 \mathrm{E}-03$ & $1.585 \mathrm{E}-02$ & $1.32 \mathrm{E}-03$ & $4.188 \mathrm{E}-01$ & $3.53 \mathrm{E}-02$ & $1.984 \mathrm{E}+04$ & $3.12 \mathrm{E}+01$ \\
\hline $9.887 \mathrm{E}-02$ & $4.85 \mathrm{E}-03$ & $1.574 \mathrm{E}-02$ & $7.72 \mathrm{E}-04$ & $4.810 \mathrm{E}-01$ & $2.42 \mathrm{E}-02$ & $2.494 \mathrm{E}+04$ & $3.35 \mathrm{E}+01$ \\
\hline $9.860 \mathrm{E}-02$ & $3.37 \mathrm{E}-03$ & $1.569 \mathrm{E}-02$ & $5.36 \mathrm{E}-04$ & $5.292 \mathrm{E}-01$ & $1.90 \mathrm{E}-02$ & $2.983 \mathrm{E}+04$ & $4.19 \mathrm{E}+01$ \\
\hline $9.682 \mathrm{E}-02$ & $2.72 \mathrm{E}-03$ & $1.541 \mathrm{E}-02$ & $4.33 \mathrm{E}-04$ & $5.644 \mathrm{E}-01$ & $1.71 \mathrm{E}-02$ & $3.470 \mathrm{E}+04$ & $4.89 \mathrm{E}+01$ \\
\hline $9.770 \mathrm{E}-02$ & $2.12 \mathrm{E}-03$ & $1.555 \mathrm{E}-02$ & $3.37 \mathrm{E}-04$ & $5.949 \mathrm{E}-01$ & $1.45 \mathrm{E}-02$ & $3.982 \mathrm{E}+04$ & $5.26 \mathrm{E}+01$ \\
\hline $9.827 \mathrm{E}-02$ & $1.80 \mathrm{E}-03$ & $1.564 \mathrm{E}-02$ & $2.86 \mathrm{E}-04$ & $6.111 \mathrm{E}-01$ & $1.29 \mathrm{E}-02$ & $4.443 \mathrm{E}+04$ & $5.86 \mathrm{E}+01$ \\
\hline $1.010 \mathrm{E}-01$ & $2.15 \mathrm{E}-03$ & $1.607 \mathrm{E}-02$ & $3.41 \mathrm{E}-04$ & $6.165 \mathrm{E}-01$ & $1.46 \mathrm{E}-02$ & $4.936 \mathrm{E}+04$ & $6.74 \mathrm{E}+01$ \\
\hline $1.033 \mathrm{E}-01$ & $1.78 \mathrm{E}-03$ & $1.645 \mathrm{E}-02$ & $2.83 \mathrm{E}-04$ & $6.016 \mathrm{E}-01$ & $1.21 \mathrm{E}-02$ & $5.393 \mathrm{E}+04$ & $7.13 \mathrm{E}+01$ \\
\hline
\end{tabular}




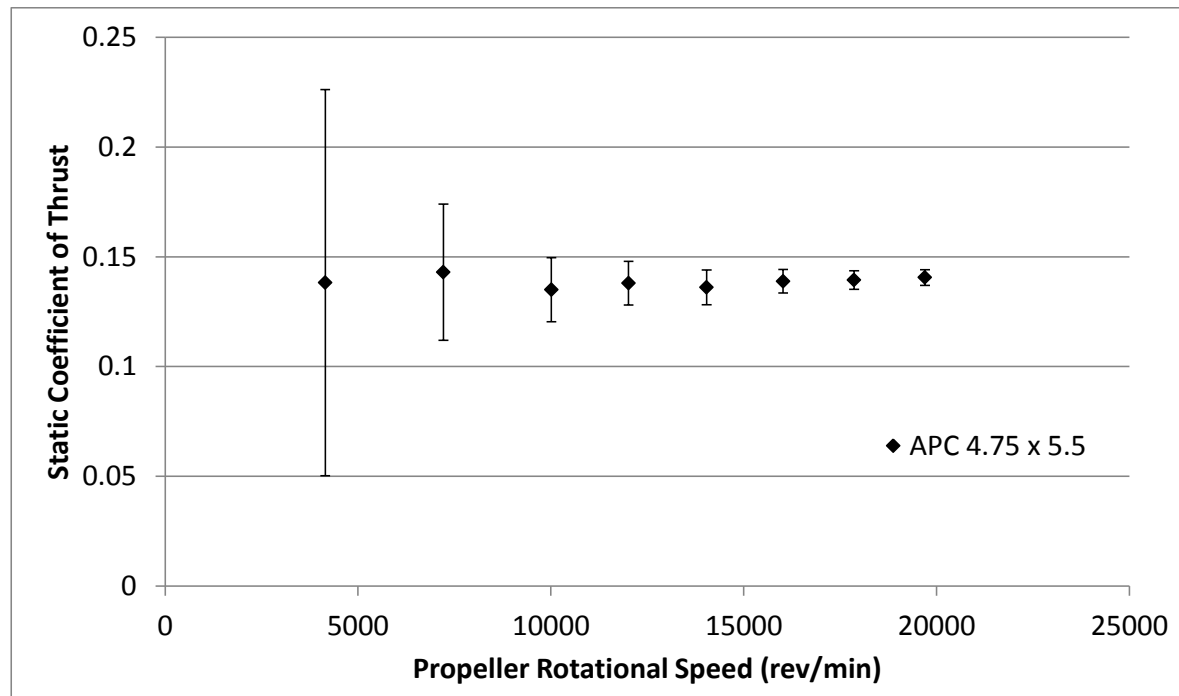

(a)

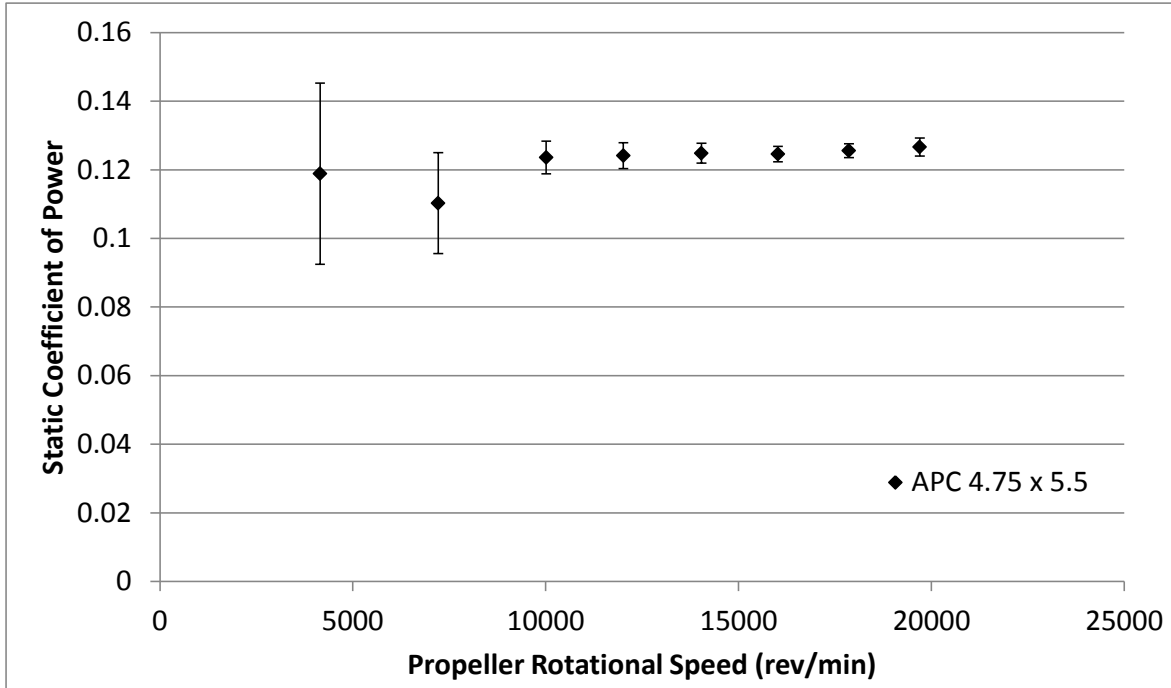

(b)

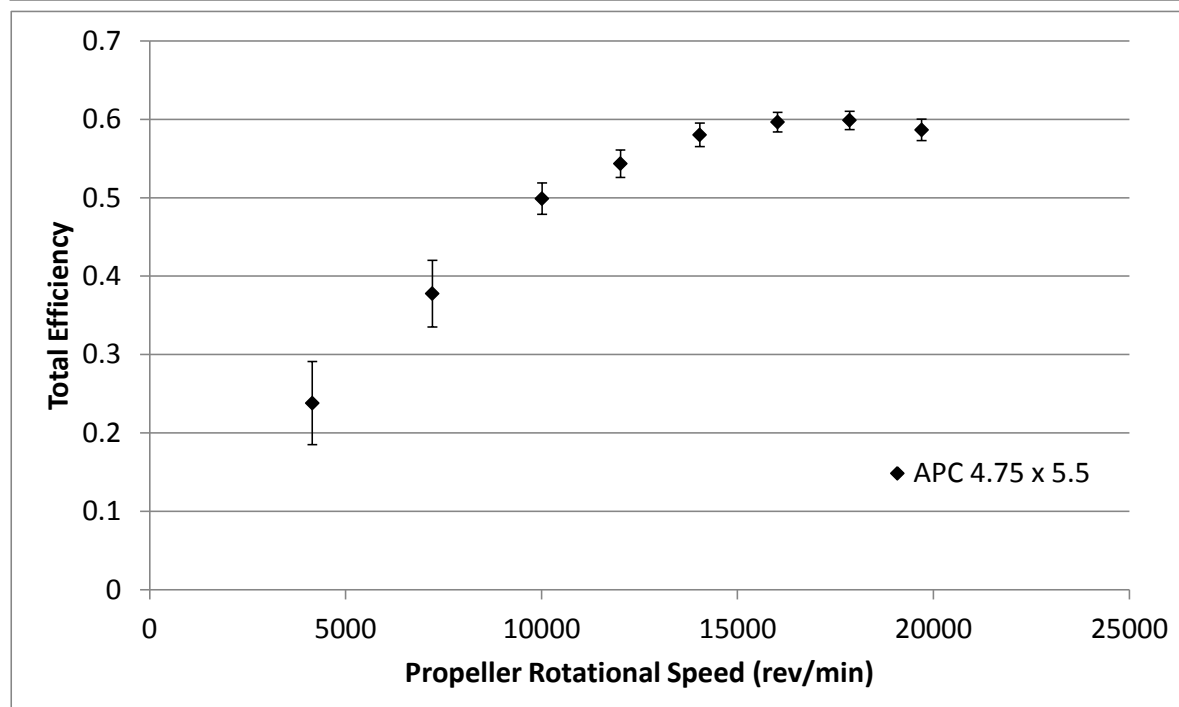

(c)

Figure 49: APC 4.75 x 5.5 Static Test Results: (a) Coefficient of Thrust, (b) Coefficient of Power, (c) Total Efficiency. 
Table 20: APC 4.75 x 5.5 Static Measured Values

\begin{tabular}{|c|c|c|c|c|c|c|c|c|}
\hline$n(\mathrm{RPM})$ & $T(\mathrm{~g})$ & $\Delta T(\mathrm{~g})$ & $Q(\mathrm{~g}-\mathrm{m})$ & $\Delta Q(\mathrm{~g}-\mathrm{m})$ & $V(\mathrm{~V})$ & $I(\mathrm{~A})$ & $T_{\text {atm }}\left({ }^{\circ} \mathrm{C}\right)$ & $P_{\text {atm }}(\mathrm{Pa})$ \\
\hline $4.151 \mathrm{E}+03$ & $1.628 \mathrm{E}+01$ & $1.04 \mathrm{E}+01$ & $2.672 \mathrm{E}-01$ & $5.94 \mathrm{E}-02$ & $1.108 \mathrm{E}+01$ & $4.321 \mathrm{E}-01$ & $2.236 \mathrm{E}+01$ & $9.896 \mathrm{E}+04$ \\
\hline $7.215 \mathrm{E}+03$ & $5.101 \mathrm{E}+01$ & $1.07 \mathrm{E}+01$ & $7.509 \mathrm{E}-01$ & $7.71 \mathrm{E}-02$ & $1.106 \mathrm{E}+01$ & $1.332 \mathrm{E}+00$ & $2.175 \mathrm{E}+01$ & $9.905 \mathrm{E}+04$ \\
\hline $1.001 \mathrm{E}+04$ & $9.258 \mathrm{E}+01$ & $1.00 \mathrm{E}+01$ & $1.617 \mathrm{E}+00$ & $6.23 \mathrm{E}-02$ & $1.102 \mathrm{E}+01$ & $3.026 \mathrm{E}+00$ & $2.229 \mathrm{E}+01$ & $9.899 \mathrm{E}+04$ \\
\hline $1.202 \mathrm{E}+04$ & $1.362 \mathrm{E}+02$ & $9.84 \mathrm{E}+00$ & $2.340 \mathrm{E}+00$ & $7.08 \mathrm{E}-02$ & $1.098 \mathrm{E}+01$ & $4.839 \mathrm{E}+00$ & $2.215 \mathrm{E}+01$ & $9.899 \mathrm{E}+04$ \\
\hline $1.404 \mathrm{E}+04$ & $1.835 \mathrm{E}+02$ & $1.07 \mathrm{E}+01$ & $3.212 \mathrm{E}+00$ & $7.43 \mathrm{E}-02$ & $1.092 \mathrm{E}+01$ & $7.306 \mathrm{E}+00$ & $2.220 \mathrm{E}+01$ & $9.899 \mathrm{E}+04$ \\
\hline $1.602 \mathrm{E}+04$ & $2.438 \mathrm{E}+02$ & $9.41 \mathrm{E}+00$ & $4.176 \mathrm{E}+00$ & $7.48 \mathrm{E}-02$ & $1.085 \mathrm{E}+01$ & $1.062 \mathrm{E}+01$ & $2.220 \mathrm{E}+01$ & $9.898 \mathrm{E}+04$ \\
\hline $1.786 \mathrm{E}+04$ & $3.043 \mathrm{E}+02$ & $9.22 \mathrm{E}+00$ & $5.231 \mathrm{E}+00$ & $8.46 \mathrm{E}-02$ & $1.075 \mathrm{E}+01$ & $1.492 \mathrm{E}+01$ & $2.220 \mathrm{E}+01$ & $9.898 \mathrm{E}+04$ \\
\hline $1.970 \mathrm{E}+04$ & $3.732 \mathrm{E}+02$ & $9.35 \mathrm{E}+00$ & $6.418 \mathrm{E}+00$ & $1.32 \mathrm{E}-01$ & $1.061 \mathrm{E}+01$ & $2.087 \mathrm{E}+01$ & $2.214 \mathrm{E}+01$ & $9.900 \mathrm{E}+04$ \\
\hline
\end{tabular}

Table 21: APC 4.75 x 5.5 Static Calculated Values

\begin{tabular}{|c|c|c|c|c|c|c|c|}
\hline$n(\mathrm{RPM})$ & $\rho\left(\mathrm{kg} / \mathrm{m}^{\wedge} 3\right)$ & $P_{\mathrm{P}}(\mathrm{W})$ & $\Delta P_{\mathrm{P}}(\mathrm{W})$ & $P_{\mathrm{e}}(\mathrm{W})$ & $\Delta P_{\mathrm{e}}(\mathrm{W})$ & $C_{\mathrm{T}}$ & $\Delta C_{\mathrm{T}}$ \\
\hline $4.151 \mathrm{E}+03$ & $1.167 \mathrm{E}+00$ & $1.139 \mathrm{E}+00$ & $2.53 \mathrm{E}-01$ & $4.787 \mathrm{E}+00$ & $7.12 \mathrm{E}-02$ & $1.382 \mathrm{E}-01$ & $8.79 \mathrm{E}-02$ \\
\hline $7.215 \mathrm{E}+03$ & $1.170 \mathrm{E}+00$ & $5.564 \mathrm{E}+00$ & $5.91 \mathrm{E}-01$ & $1.473 \mathrm{E}+01$ & $5.35 \mathrm{E}-01$ & $1.430 \mathrm{E}-01$ & $3.10 \mathrm{E}-02$ \\
\hline $1.001 \mathrm{E}+04$ & $1.167 \mathrm{E}+00$ & $1.664 \mathrm{E}+01$ & $6.41 \mathrm{E}-01$ & $3.335 \mathrm{E}+01$ & $3.76 \mathrm{E}-01$ & $1.350 \mathrm{E}-01$ & $1.46 \mathrm{E}-02$ \\
\hline $1.202 \mathrm{E}+04$ & $1.168 \mathrm{E}+00$ & $2.887 \mathrm{E}+01$ & $8.74 \mathrm{E}-01$ & $5.312 \mathrm{E}+01$ & $5.94 \mathrm{E}-01$ & $1.379 \mathrm{E}-01$ & $9.97 \mathrm{E}-03$ \\
\hline $1.404 \mathrm{E}+04$ & $1.168 \mathrm{E}+00$ & $4.630 \mathrm{E}+01$ & $1.07 \mathrm{E}+00$ & $7.980 \mathrm{E}+01$ & $9.03 \mathrm{E}-01$ & $1.361 \mathrm{E}-01$ & $7.93 \mathrm{E}-03$ \\
\hline $1.602 \mathrm{E}+04$ & $1.168 \mathrm{E}+00$ & $6.873 \mathrm{E}+01$ & $1.23 \mathrm{E}+00$ & $1.152 \mathrm{E}+02$ & $1.27 \mathrm{E}+00$ & $1.388 \mathrm{E}-01$ & $5.36 \mathrm{E}-03$ \\
\hline $1.786 \mathrm{E}+04$ & $1.167 \mathrm{E}+00$ & $9.597 \mathrm{E}+01$ & $1.55 \mathrm{E}+00$ & $1.603 \mathrm{E}+02$ & $1.71 \mathrm{E}+00$ & $1.394 \mathrm{E}-01$ & $4.22 \mathrm{E}-03$ \\
\hline $1.970 \mathrm{E}+04$ & $1.168 \mathrm{E}+00$ & $1.298 \mathrm{E}+02$ & $2.68 \mathrm{E}+00$ & $2.214 \mathrm{E}+02$ & $2.34 \mathrm{E}+00$ & $1.405 \mathrm{E}-01$ & $3.53 \mathrm{E}-03$ \\
\hline$C_{\mathrm{P}}$ & $\Delta C_{\mathrm{P}}$ & $C_{\mathrm{Q}}$ & $\Delta C_{\mathrm{Q}}$ & $\eta_{\mathrm{T}}$ & $\Delta \eta_{\mathrm{T}}$ & $R e_{0.75}$ & $\Delta R e_{0.75}$ \\
\hline $1.189 \mathrm{E}-01$ & $2.64 \mathrm{E}-02$ & $1.892 \mathrm{E}-02$ & $4.21 \mathrm{E}-03$ & $2.380 \mathrm{E}-01$ & $5.30 \mathrm{E}-02$ & $1.016 \mathrm{E}+04$ & $1.49 \mathrm{E}+01$ \\
\hline $1.103 \mathrm{E}-01$ & $1.47 \mathrm{E}-02$ & $1.755 \mathrm{E}-02$ & $2.04 \mathrm{E}-03$ & $3.776 \mathrm{E}-01$ & $4.24 \mathrm{E}-02$ & $1.773 \mathrm{E}+04$ & $4.79 \mathrm{E}+02$ \\
\hline $1.236 \mathrm{E}-01$ & $4.77 \mathrm{E}-03$ & $1.967 \mathrm{E}-02$ & $7.58 \mathrm{E}-04$ & $4.988 \mathrm{E}-01$ & $2.00 \mathrm{E}-02$ & $2.452 \mathrm{E}+04$ & $3.32 \mathrm{E}+01$ \\
\hline $1.241 \mathrm{E}-01$ & $3.76 \mathrm{E}-03$ & $1.976 \mathrm{E}-02$ & $5.98 \mathrm{E}-04$ & $5.435 \mathrm{E}-01$ & $1.75 \mathrm{E}-02$ & $2.944 \mathrm{E}+04$ & $4.08 \mathrm{E}+01$ \\
\hline $1.248 \mathrm{E}-01$ & $2.90 \mathrm{E}-03$ & $1.987 \mathrm{E}-02$ & $4.60 \mathrm{E}-04$ & $5.802 \mathrm{E}-01$ & $1.49 \mathrm{E}-02$ & $3.439 \mathrm{E}+04$ & $4.84 \mathrm{E}+01$ \\
\hline $1.246 \mathrm{E}-01$ & $2.24 \mathrm{E}-03$ & $1.983 \mathrm{E}-02$ & $3.56 \mathrm{E}-04$ & $5.964 \mathrm{E}-01$ & $1.25 \mathrm{E}-02$ & $3.925 \mathrm{E}+04$ & $5.23 \mathrm{E}+01$ \\
\hline $1.256 \mathrm{E}-01$ & $2.03 \mathrm{E}-03$ & $1.999 \mathrm{E}-02$ & $3.24 \mathrm{E}-04$ & $5.986 \mathrm{E}-01$ & $1.16 \mathrm{E}-02$ & $4.376 \mathrm{E}+04$ & $5.82 \mathrm{E}+01$ \\
\hline $1.266 \mathrm{E}-01$ & $2.64 \mathrm{E}-03$ & $2.016 \mathrm{E}-02$ & $4.17 \mathrm{E}-04$ & $5.865 \mathrm{E}-01$ & $1.36 \mathrm{E}-02$ & $4.828 \mathrm{E}+04$ & $7.47 \mathrm{E}+01$ \\
\hline
\end{tabular}




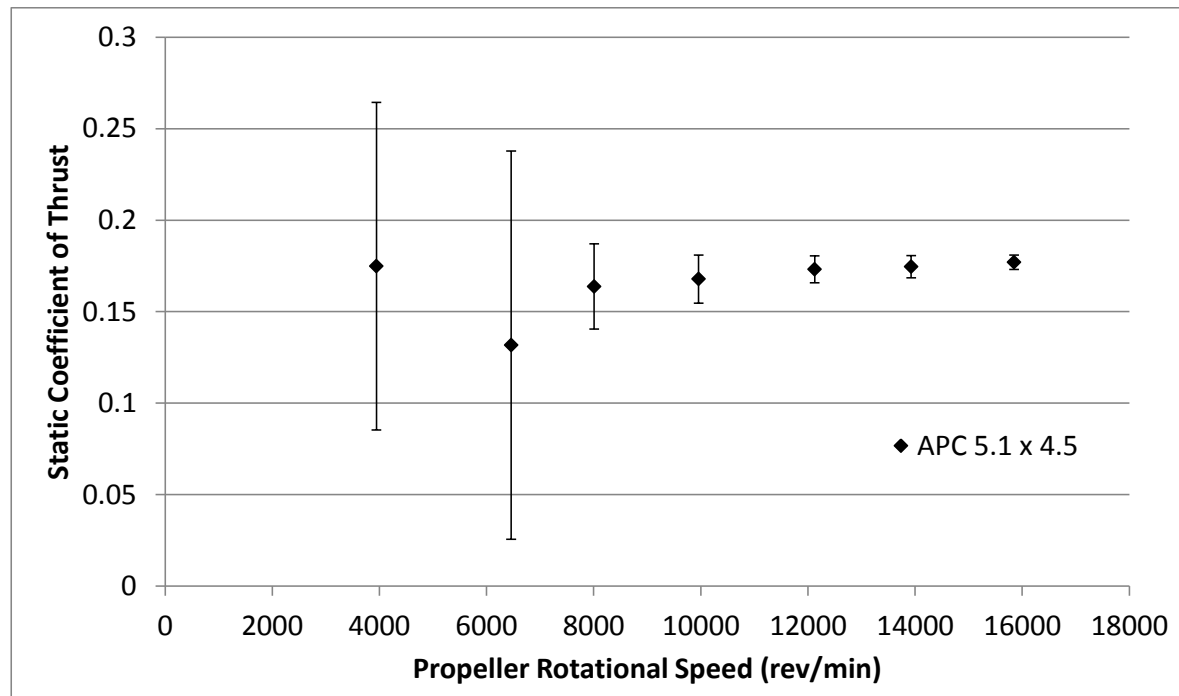

(a)

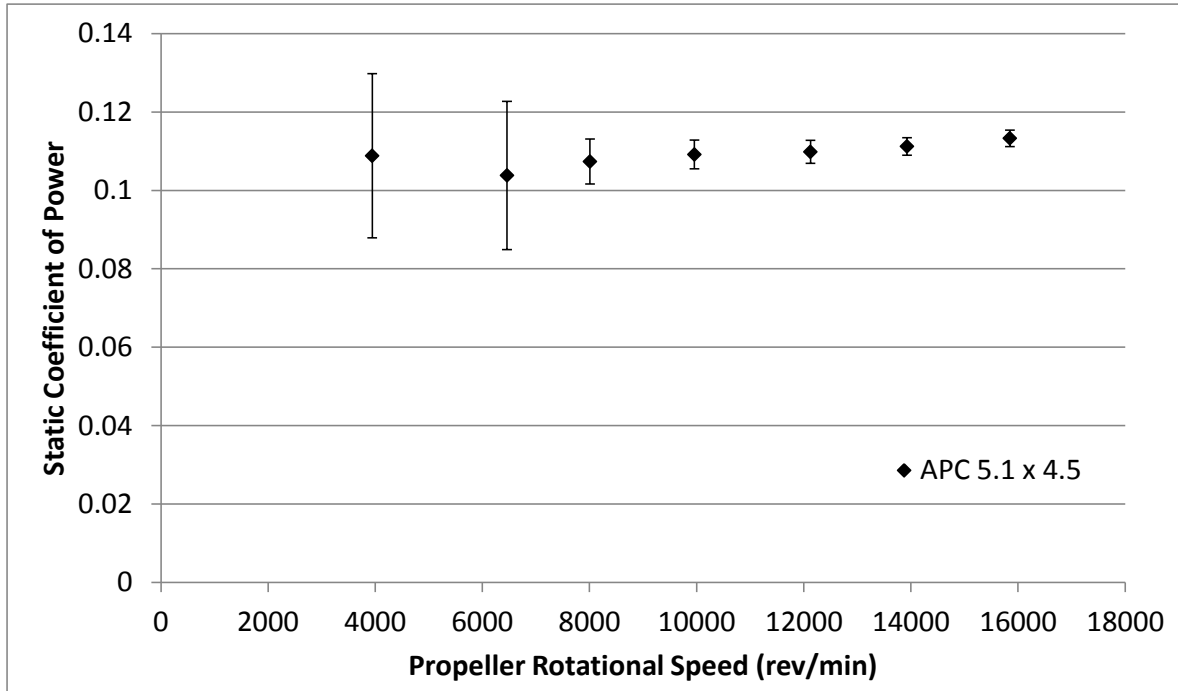

(b)

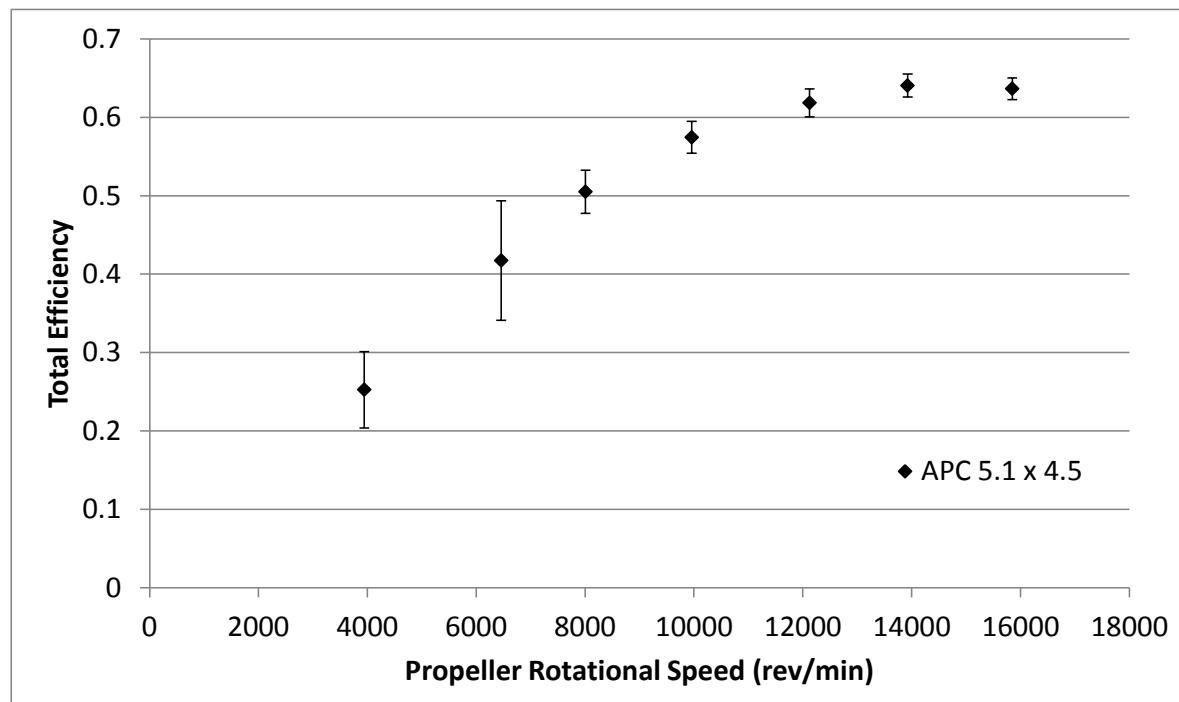

(c)

Figure 50: APC 5.1 x 4.5 Static Test Results: (a) Coefficient of Thrust, (b) Coefficient of Power, (c) Total Efficiency. 
Table 22: APC 5.1 x 4.5 Static Measured Values

\begin{tabular}{|c|c|c|c|c|c|c|c|c|}
\hline$n(\mathrm{RPM})$ & $T(\mathrm{~g})$ & $\Delta T(\mathrm{~g})$ & $Q(\mathrm{~g}-\mathrm{m})$ & $\Delta Q(\mathrm{~g}-\mathrm{m})$ & $V(\mathrm{~V})$ & $I(\mathrm{~A})$ & $T_{\text {atm }}\left({ }^{\circ} \mathrm{C}\right)$ & $P_{\text {atm }}(\mathrm{Pa})$ \\
\hline $3.944 \mathrm{E}+03$ & $2.508 \mathrm{E}+01$ & $1.28 \mathrm{E}+01$ & $3.215 \mathrm{E}-01$ & $6.18 \mathrm{E}-02$ & $1.110 \mathrm{E}+01$ & $4.643 \mathrm{E}-01$ & $2.265 \mathrm{E}+01$ & $9.862 \mathrm{E}+04$ \\
\hline $6.459 \mathrm{E}+03$ & $5.070 \mathrm{E}+01$ & $4.09 \mathrm{E}+01$ & $8.229 \mathrm{E}-01$ & $1.50 \mathrm{E}-01$ & $1.108 \mathrm{E}+01$ & $1.180 \mathrm{E}+00$ & $2.261 \mathrm{E}+01$ & $9.862 \mathrm{E}+04$ \\
\hline $8.009 \mathrm{E}+03$ & $9.692 \mathrm{E}+01$ & $1.38 \mathrm{E}+01$ & $1.309 \mathrm{E}+00$ & $6.96 \mathrm{E}-02$ & $1.106 \mathrm{E}+01$ & $1.927 \mathrm{E}+00$ & $2.260 \mathrm{E}+01$ & $9.862 \mathrm{E}+04$ \\
\hline $9.960 \mathrm{E}+03$ & $1.536 \mathrm{E}+02$ & $1.21 \mathrm{E}+01$ & $2.058 \mathrm{E}+00$ & $6.91 \mathrm{E}-02$ & $1.102 \mathrm{E}+01$ & $3.324 \mathrm{E}+00$ & $2.257 \mathrm{E}+01$ & $9.863 \mathrm{E}+04$ \\
\hline $1.213 \mathrm{E}+04$ & $2.349 \mathrm{E}+02$ & $1.00 \mathrm{E}+01$ & $3.070 \mathrm{E}+00$ & $8.13 \mathrm{E}-02$ & $1.096 \mathrm{E}+01$ & $5.640 \mathrm{E}+00$ & $2.251 \mathrm{E}+01$ & $9.863 \mathrm{E}+04$ \\
\hline $1.393 \mathrm{E}+04$ & $3.126 \mathrm{E}+02$ & $1.08 \mathrm{E}+01$ & $4.102 \mathrm{E}+00$ & $8.20 \mathrm{E}-02$ & $1.088 \mathrm{E}+01$ & $8.419 \mathrm{E}+00$ & $2.249 \mathrm{E}+01$ & $9.864 \mathrm{E}+04$ \\
\hline $1.585 \mathrm{E}+04$ & $4.104 \mathrm{E}+02$ & $9.01 \mathrm{E}+00$ & $5.409 \mathrm{E}+00$ & $1.02 \mathrm{E}-01$ & $1.075 \mathrm{E}+01$ & $1.286 \mathrm{E}+01$ & $2.254 \mathrm{E}+01$ & $9.864 \mathrm{E}+04$ \\
\hline
\end{tabular}

Table 23: APC 5.1 x 4.5 Static Calculated Values

\begin{tabular}{|c|c|c|c|c|c|c|c|}
\hline$n(\mathrm{RPM})$ & $\rho\left(\mathrm{kg} / \mathrm{m}^{\wedge} 3\right)$ & $P_{\mathrm{P}}(\mathrm{W})$ & $\Delta P_{\mathrm{P}}(\mathrm{W})$ & $P_{\mathrm{e}}(\mathrm{W})$ & $\Delta P_{\mathrm{e}}(\mathrm{W})$ & $C_{\mathrm{T}}$ & $\Delta C_{\mathrm{T}}$ \\
\hline $3.944 \mathrm{E}+03$ & $1.161 \mathrm{E}+00$ & $1.302 \mathrm{E}+00$ & $2.50 \mathrm{E}-01$ & $5.155 \mathrm{E}+00$ & $7.41 \mathrm{E}-02$ & $1.749 \mathrm{E}-01$ & $8.96 \mathrm{E}-02$ \\
\hline $6.459 \mathrm{E}+03$ & $1.162 \mathrm{E}+00$ & $5.459 \mathrm{E}+00$ & $9.95 \mathrm{E}-01$ & $1.308 \mathrm{E}+01$ & $1.60 \mathrm{E}-01$ & $1.317 \mathrm{E}-01$ & $1.06 \mathrm{E}-01$ \\
\hline $8.009 \mathrm{E}+03$ & $1.162 \mathrm{E}+00$ & $1.076 \mathrm{E}+01$ & $5.72 \mathrm{E}-01$ & $2.131 \mathrm{E}+01$ & $2.47 \mathrm{E}-01$ & $1.638 \mathrm{E}-01$ & $2.34 \mathrm{E}-02$ \\
\hline $9.960 \mathrm{E}+03$ & $1.162 \mathrm{E}+00$ & $2.105 \mathrm{E}+01$ & $7.06 \mathrm{E}-01$ & $3.664 \mathrm{E}+01$ & $4.16 \mathrm{E}-01$ & $1.678 \mathrm{E}-01$ & $1.32 \mathrm{E}-02$ \\
\hline $1.213 \mathrm{E}+04$ & $1.162 \mathrm{E}+00$ & $3.823 \mathrm{E}+01$ & $1.01 \mathrm{E}+00$ & $6.180 \mathrm{E}+01$ & $7.10 \mathrm{E}-01$ & $1.731 \mathrm{E}-01$ & $7.37 \mathrm{E}-03$ \\
\hline $1.393 \mathrm{E}+04$ & $1.162 \mathrm{E}+00$ & $5.867 \mathrm{E}+01$ & $1.17 \mathrm{E}+00$ & $9.160 \mathrm{E}+01$ & $1.00 \mathrm{E}+00$ & $1.746 \mathrm{E}-01$ & $6.06 \mathrm{E}-03$ \\
\hline $1.585 \mathrm{E}+04$ & $1.162 \mathrm{E}+00$ & $8.804 \mathrm{E}+01$ & $1.65 \mathrm{E}+00$ & $1.383 \mathrm{E}+02$ & $1.48 \mathrm{E}+00$ & $1.770 \mathrm{E}-01$ & $3.89 \mathrm{E}-03$ \\
\hline$C_{\mathrm{P}}$ & $\Delta C_{\mathrm{P}}$ & $C_{\mathrm{Q}}$ & $\Delta C_{\mathrm{Q}}$ & $\eta_{\mathrm{T}}$ & $\Delta \eta_{\mathrm{T}}$ & $R e_{0.75}$ & $\Delta R e_{0.75}$ \\
\hline $1.088 \mathrm{E}-01$ & $2.09 \mathrm{E}-02$ & $1.732 \mathrm{E}-02$ & $3.33 \mathrm{E}-03$ & $2.526 \mathrm{E}-01$ & $4.87 \mathrm{E}-02$ & $1.946 \mathrm{E}+04$ & $2.38 \mathrm{E}+01$ \\
\hline $1.038 \mathrm{E}-01$ & $1.89 \mathrm{E}-02$ & $1.652 \mathrm{E}-02$ & $3.01 \mathrm{E}-03$ & $4.173 \mathrm{E}-01$ & $7.63 \mathrm{E}-02$ & $3.188 \mathrm{E}+04$ & $3.65 \mathrm{E}+01$ \\
\hline $1.074 \mathrm{E}-01$ & $5.72 \mathrm{E}-03$ & $1.709 \mathrm{E}-02$ & $9.09 \mathrm{E}-04$ & $5.050 \mathrm{E}-01$ & $2.75 \mathrm{E}-02$ & $3.954 \mathrm{E}+04$ & $4.69 \mathrm{E}+01$ \\
\hline $1.092 \mathrm{E}-01$ & $3.67 \mathrm{E}-03$ & $1.737 \mathrm{E}-02$ & $5.83 \mathrm{E}-04$ & $5.744 \mathrm{E}-01$ & $2.04 \mathrm{E}-02$ & $4.918 \mathrm{E}+04$ & $4.45 \mathrm{E}+01$ \\
\hline $1.098 \mathrm{E}-01$ & $2.92 \mathrm{E}-03$ & $1.748 \mathrm{E}-02$ & $4.63 \mathrm{E}-04$ & $6.186 \mathrm{E}-01$ & $1.79 \mathrm{E}-02$ & $5.990 \mathrm{E}+04$ & $6.05 \mathrm{E}+01$ \\
\hline $1.112 \mathrm{E}-01$ & $2.24 \mathrm{E}-03$ & $1.770 \mathrm{E}-02$ & $3.55 \mathrm{E}-04$ & $6.405 \mathrm{E}-01$ & $1.46 \mathrm{E}-02$ & $6.881 \mathrm{E}+04$ & $6.82 \mathrm{E}+01$ \\
\hline $1.133 \mathrm{E}-01$ & $2.13 \mathrm{E}-03$ & $1.803 \mathrm{E}-02$ & $3.39 \mathrm{E}-04$ & $6.365 \mathrm{E}-01$ & $1.38 \mathrm{E}-02$ & $7.828 \mathrm{E}+04$ & $6.52 \mathrm{E}+01$ \\
\hline
\end{tabular}




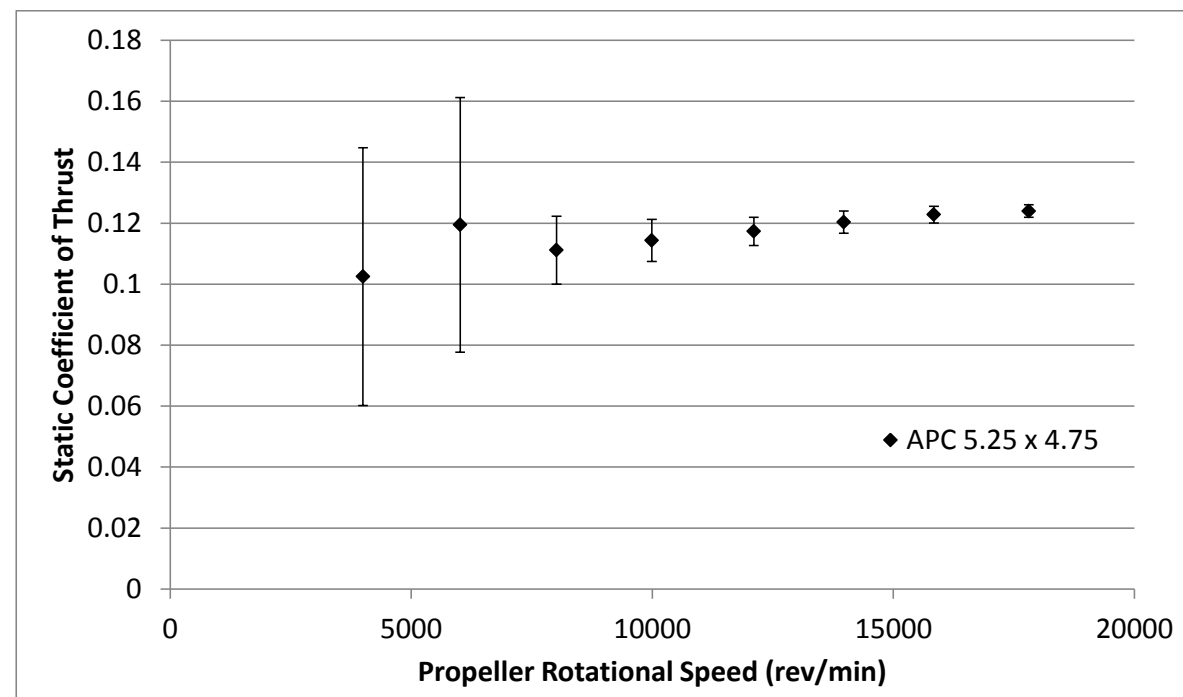

(a)

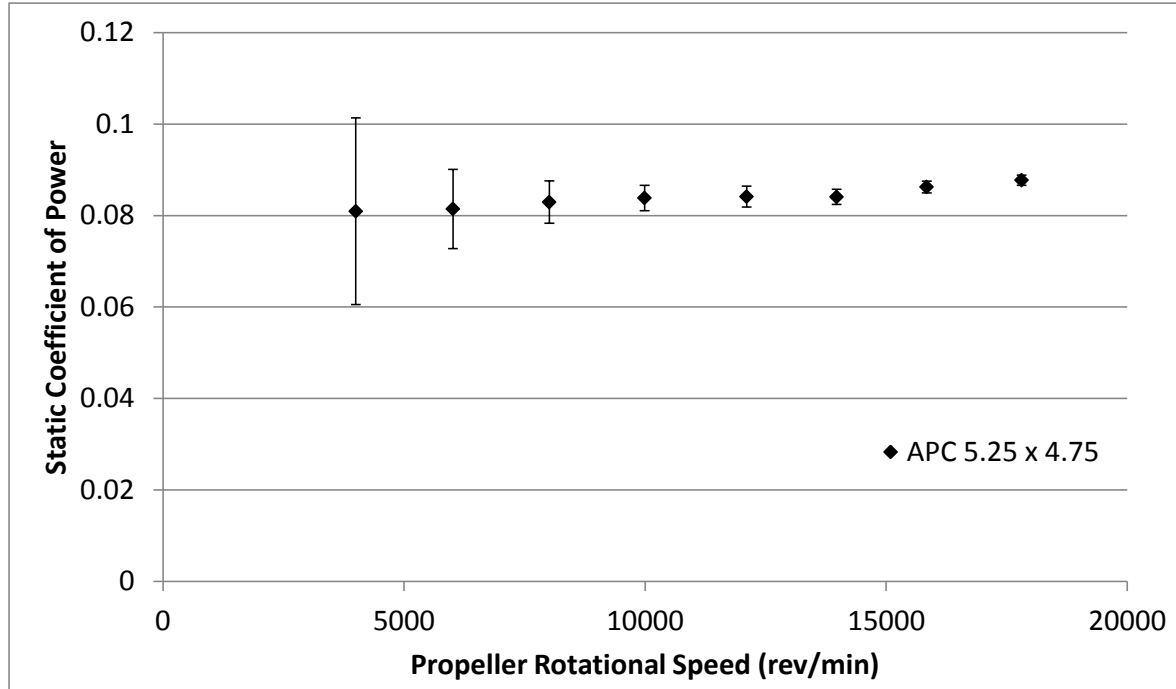

(b)

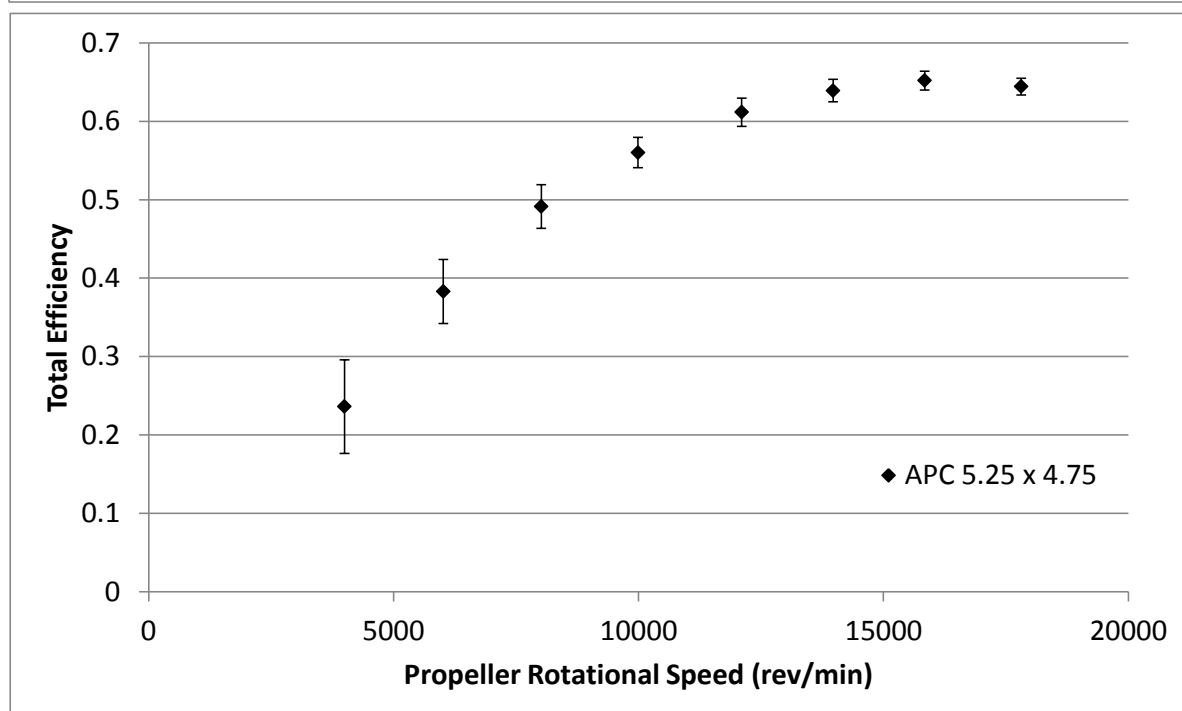

(c)

Figure 51: APC 5.25 x 4.75 Static Test Results: (a) Coefficient of Thrust, (b) Coefficient of Power, (c) Total Efficiency. 
Table 24: APC 5.25 x 4.75 Static Measured Values

\begin{tabular}{|c|c|c|c|c|c|c|c|c|}
\hline$n(\mathrm{RPM})$ & $T(\mathrm{~g})$ & $\Delta T(\mathrm{~g})$ & $Q(\mathrm{~g}-\mathrm{m})$ & $\Delta Q(\mathrm{~g}-\mathrm{m})$ & $V(\mathrm{~V})$ & $I(\mathrm{~A})$ & $T_{\text {atm }}\left({ }^{\circ} \mathrm{C}\right)$ & $P_{\text {atm }}(\mathrm{Pa})$ \\
\hline $3.999 \mathrm{E}+03$ & $1.681 \mathrm{E}+01$ & $6.93 \mathrm{E}+00$ & $2.803 \mathrm{E}-01$ & $7.07 \mathrm{E}-02$ & $1.110 \mathrm{E}+01$ & $4.390 \mathrm{E}-01$ & $2.204 \mathrm{E}+01$ & $9.882 \mathrm{E}+04$ \\
\hline $6.015 \mathrm{E}+03$ & $4.433 \mathrm{E}+01$ & $1.55 \mathrm{E}+01$ & $6.384 \mathrm{E}-01$ & $6.78 \mathrm{E}-02$ & $1.109 \mathrm{E}+01$ & $9.284 \mathrm{E}-01$ & $2.197 \mathrm{E}+01$ & $9.882 \mathrm{E}+04$ \\
\hline $8.015 \mathrm{E}+03$ & $7.316 \mathrm{E}+01$ & $7.33 \mathrm{E}+00$ & $1.153 \mathrm{E}+00$ & $6.39 \mathrm{E}-02$ & $1.107 \mathrm{E}+01$ & $1.745 \mathrm{E}+00$ & $2.208 \mathrm{E}+01$ & $9.878 \mathrm{E}+04$ \\
\hline $9.990 \mathrm{E}+03$ & $1.170 \mathrm{E}+02$ & $7.05 \mathrm{E}+00$ & $1.812 \mathrm{E}+00$ & $5.96 \mathrm{E}-02$ & $1.103 \mathrm{E}+01$ & $3.009 \mathrm{E}+00$ & $2.197 \mathrm{E}+01$ & $9.881 \mathrm{E}+04$ \\
\hline $1.211 \mathrm{E}+04$ & $1.763 \mathrm{E}+02$ & $6.96 \mathrm{E}+00$ & $2.671 \mathrm{E}+00$ & $7.20 \mathrm{E}-02$ & $1.098 \mathrm{E}+01$ & $4.944 \mathrm{E}+00$ & $2.196 \mathrm{E}+01$ & $9.880 \mathrm{E}+04$ \\
\hline $1.397 \mathrm{E}+04$ & $2.410 \mathrm{E}+02$ & $7.29 \mathrm{E}+00$ & $3.556 \mathrm{E}+00$ & $6.99 \mathrm{E}-02$ & $1.092 \mathrm{E}+01$ & $7.310 \mathrm{E}+00$ & $2.196 \mathrm{E}+01$ & $9.880 \mathrm{E}+04$ \\
\hline $1.584 \mathrm{E}+04$ & $3.160 \mathrm{E}+02$ & $6.98 \mathrm{E}+00$ & $4.687 \mathrm{E}+00$ & $6.93 \mathrm{E}-02$ & $1.082 \mathrm{E}+01$ & $1.082 \mathrm{E}+01$ & $2.201 \mathrm{E}+01$ & $9.879 \mathrm{E}+04$ \\
\hline $1.781 \mathrm{E}+04$ & $4.032 \mathrm{E}+02$ & $6.69 \mathrm{E}+00$ & $6.024 \mathrm{E}+00$ & $7.58 \mathrm{E}-02$ & $1.066 \mathrm{E}+01$ & $1.604 \mathrm{E}+01$ & $2.207 \mathrm{E}+01$ & $9.879 \mathrm{E}+04$ \\
\hline
\end{tabular}

Table 25: APC 5.25 x 4.75 Static Calculated Values

\begin{tabular}{|c|c|c|c|c|c|c|c|}
\hline$n(\mathrm{RPM})$ & $\rho\left(\mathrm{kg} / \mathrm{m}^{\wedge} 3\right)$ & $P_{\mathrm{P}}(\mathrm{W})$ & $\Delta P_{\mathrm{P}}(\mathrm{W})$ & $P_{\mathrm{e}}(\mathrm{W})$ & $\Delta P_{\mathrm{e}}(\mathrm{W})$ & $C_{\mathrm{T}}$ & $\Delta C_{\mathrm{T}}$ \\
\hline $3.999 \mathrm{E}+03$ & $1.166 \mathrm{E}+00$ & $1.151 \mathrm{E}+00$ & $2.90 \mathrm{E}-01$ & $4.875 \mathrm{E}+00$ & $7.36 \mathrm{E}-02$ & $1.025 \mathrm{E}-01$ & $4.23 \mathrm{E}-02$ \\
\hline $6.015 \mathrm{E}+03$ & $1.166 \mathrm{E}+00$ & $3.944 \mathrm{E}+00$ & $4.19 \mathrm{E}-01$ & $1.030 \mathrm{E}+01$ & $1.30 \mathrm{E}-01$ & $1.195 \mathrm{E}-01$ & $4.18 \mathrm{E}-02$ \\
\hline $8.015 \mathrm{E}+03$ & $1.166 \mathrm{E}+00$ & $9.493 \mathrm{E}+00$ & $5.26 \mathrm{E}-01$ & $1.932 \mathrm{E}+01$ & $2.24 \mathrm{E}-01$ & $1.111 \mathrm{E}-01$ & $1.11 \mathrm{E}-02$ \\
\hline $9.990 \mathrm{E}+03$ & $1.166 \mathrm{E}+00$ & $1.860 \mathrm{E}+01$ & $6.11 \mathrm{E}-01$ & $3.319 \mathrm{E}+01$ & $3.73 \mathrm{E}-01$ & $1.143 \mathrm{E}-01$ & $6.89 \mathrm{E}-03$ \\
\hline $1.211 \mathrm{E}+04$ & $1.166 \mathrm{E}+00$ & $3.321 \mathrm{E}+01$ & $8.96 \mathrm{E}-01$ & $5.430 \mathrm{E}+01$ & $6.34 \mathrm{E}-01$ & $1.173 \mathrm{E}-01$ & $4.63 \mathrm{E}-03$ \\
\hline $1.397 \mathrm{E}+04$ & $1.166 \mathrm{E}+00$ & $5.102 \mathrm{E}+01$ & $1.00 \mathrm{E}+00$ & $7.982 \mathrm{E}+01$ & $8.87 \mathrm{E}-01$ & $1.204 \mathrm{E}-01$ & $3.65 \mathrm{E}-03$ \\
\hline $1.584 \mathrm{E}+04$ & $1.166 \mathrm{E}+00$ & $7.627 \mathrm{E}+01$ & $1.13 \mathrm{E}+00$ & $1.170 \mathrm{E}+02$ & $1.26 \mathrm{E}+00$ & $1.228 \mathrm{E}-01$ & $2.72 \mathrm{E}-03$ \\
\hline $1.781 \mathrm{E}+04$ & $1.166 \mathrm{E}+00$ & $1.102 \mathrm{E}+02$ & $1.39 \mathrm{E}+00$ & $1.710 \mathrm{E}+02$ & $1.86 \mathrm{E}+00$ & $1.240 \mathrm{E}-01$ & $2.06 \mathrm{E}-03$ \\
\hline$C_{\mathrm{P}}$ & $\Delta C_{\mathrm{P}}$ & $C_{\mathrm{Q}}$ & $\Delta C_{\mathrm{Q}}$ & $\eta_{\mathrm{T}}$ & $\Delta \eta_{\mathrm{T}}$ & $R e_{0.75}$ & $\Delta R e_{0.75}$ \\
\hline $8.093 \mathrm{E}-02$ & $2.04 \mathrm{E}-02$ & $1.288 \mathrm{E}-02$ & $3.25 \mathrm{E}-03$ & $2.362 \mathrm{E}-01$ & $5.97 \mathrm{E}-02$ & $1.245 \mathrm{E}+04$ & $1.76 \mathrm{E}+01$ \\
\hline $8.144 \mathrm{E}-02$ & $8.65 \mathrm{E}-03$ & $1.296 \mathrm{E}-02$ & $1.38 \mathrm{E}-03$ & $3.830 \mathrm{E}-01$ & $4.09 \mathrm{E}-02$ & $1.874 \mathrm{E}+04$ & $3.27 \mathrm{E}+01$ \\
\hline $8.295 \mathrm{E}-02$ & $4.61 \mathrm{E}-03$ & $1.320 \mathrm{E}-02$ & $7.32 \mathrm{E}-04$ & $4.915 \mathrm{E}-01$ & $2.78 \mathrm{E}-02$ & $2.494 \mathrm{E}+04$ & $3.92 \mathrm{E}+01$ \\
\hline $8.384 \mathrm{E}-02$ & $2.76 \mathrm{E}-03$ & $1.334 \mathrm{E}-02$ & $4.39 \mathrm{E}-04$ & $5.602 \mathrm{E}-01$ & $1.95 \mathrm{E}-02$ & $3.111 \mathrm{E}+04$ & $3.87 \mathrm{E}+01$ \\
\hline $8.413 \mathrm{E}-02$ & $2.28 \mathrm{E}-03$ & $1.339 \mathrm{E}-02$ & $3.61 \mathrm{E}-04$ & $6.117 \mathrm{E}-01$ & $1.80 \mathrm{E}-02$ & $3.770 \mathrm{E}+04$ & $5.06 \mathrm{E}+01$ \\
\hline $8.407 \mathrm{E}-02$ & $1.66 \mathrm{E}-03$ & $1.338 \mathrm{E}-02$ & $2.64 \mathrm{E}-04$ & $6.392 \mathrm{E}-01$ & $1.44 \mathrm{E}-02$ & $4.352 \mathrm{E}+04$ & $5.72 \mathrm{E}+01$ \\
\hline $8.624 \mathrm{E}-02$ & $1.28 \mathrm{E}-03$ & $1.373 \mathrm{E}-02$ & $2.03 \mathrm{E}-04$ & $6.520 \mathrm{E}-01$ & $1.19 \mathrm{E}-02$ & $4.932 \mathrm{E}+04$ & $5.89 \mathrm{E}+01$ \\
\hline $8.771 \mathrm{E}-02$ & $1.11 \mathrm{E}-03$ & $1.396 \mathrm{E}-02$ & $1.76 \mathrm{E}-04$ & $6.443 \mathrm{E}-01$ & $1.07 \mathrm{E}-02$ & $5.543 \mathrm{E}+04$ & $6.63 \mathrm{E}+01$ \\
\hline
\end{tabular}




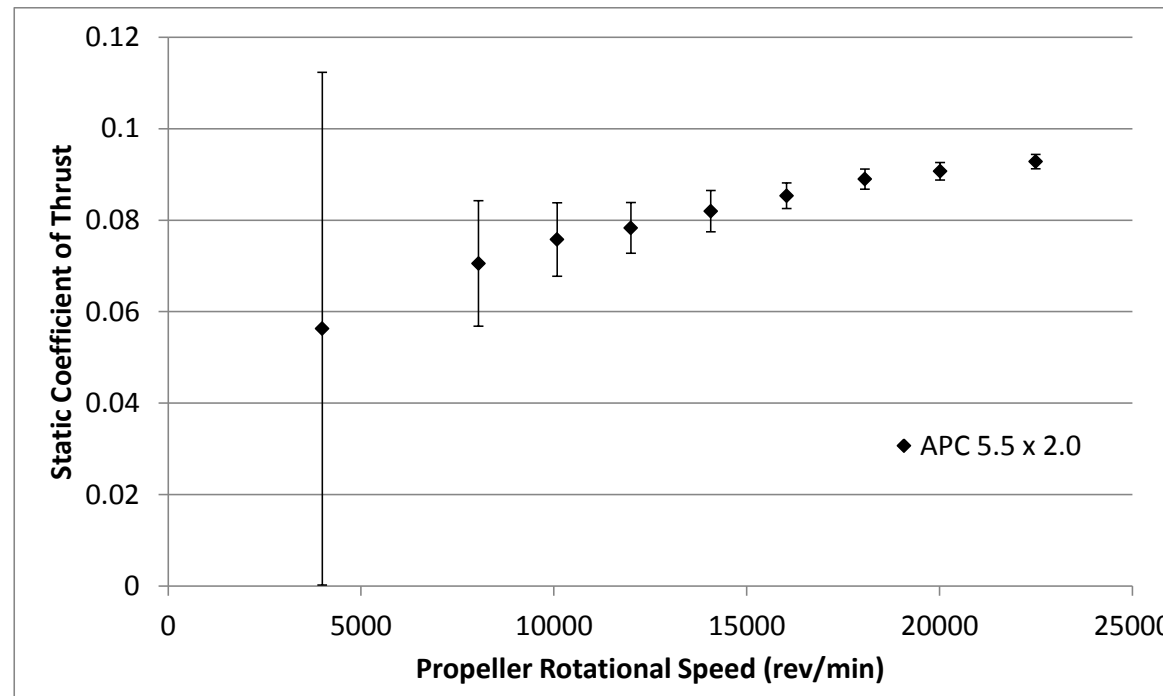

(a)

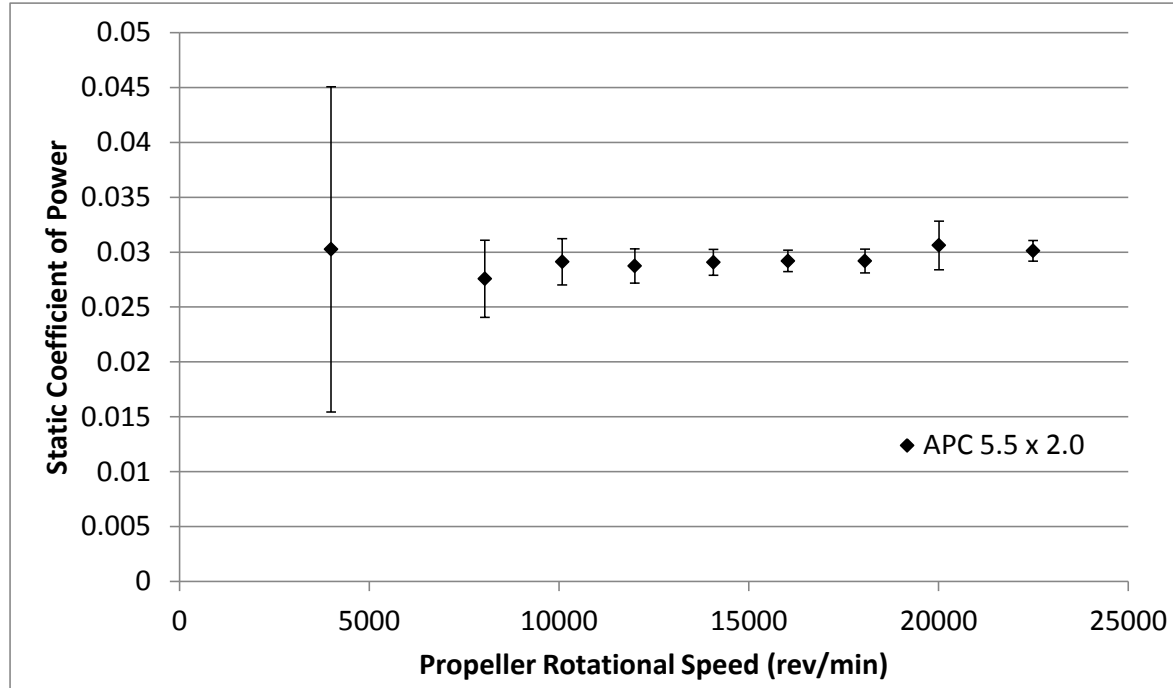

(b)

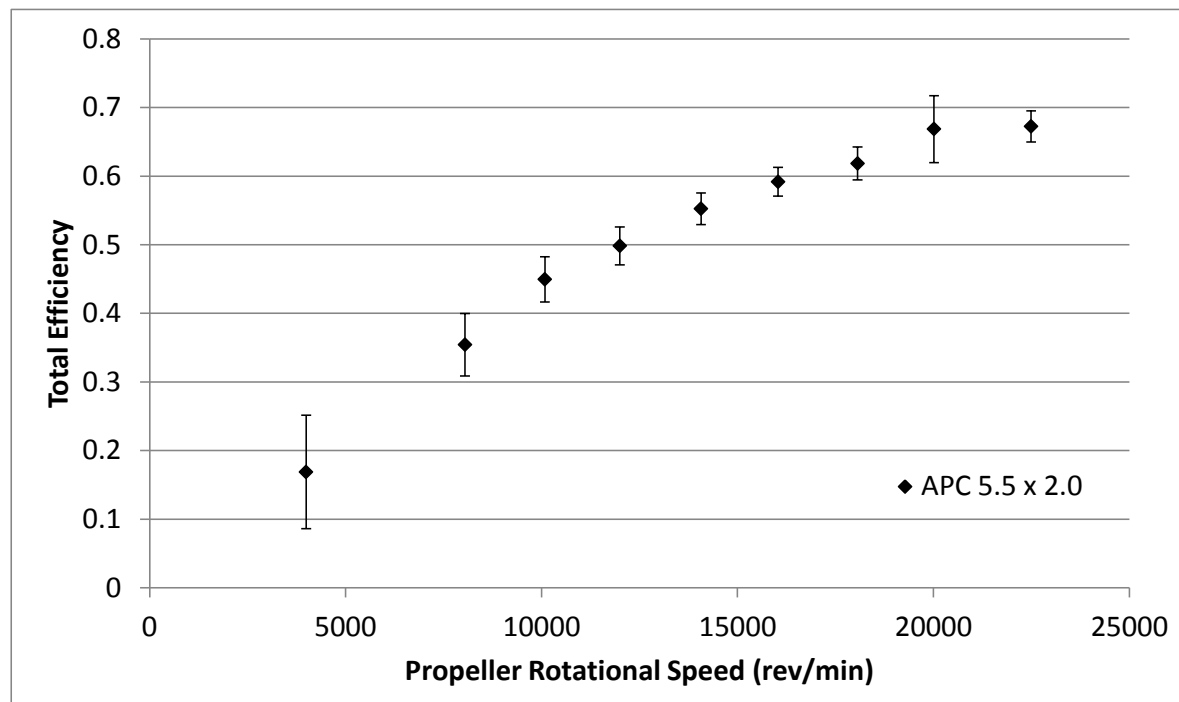

(c)

Figure 52: APC 5.5 x 2.0 Static Test Results: (a) Coefficient of Thrust, (b) Coefficient of Power, (c) Total Efficiency. 
Table 26: APC 5.5 x 2.0 Static Measured Values

\begin{tabular}{|c|c|c|c|c|c|c|c|c|}
\hline$n(\mathrm{RPM})$ & $T(\mathrm{~g})$ & $\Delta T(\mathrm{~g})$ & $Q(\mathrm{~g}-\mathrm{m})$ & $\Delta Q(\mathrm{~g}-\mathrm{m})$ & $V(\mathrm{~V})$ & $I(\mathrm{~A})$ & $T_{\mathrm{atm}}\left({ }^{\circ} \mathrm{C}\right)$ & $P_{\mathrm{atm}}(\mathrm{Pa})$ \\
\hline $3.995 \mathrm{E}+03$ & $1.116 \mathrm{E}+01$ & $1.11 \mathrm{E}+01$ & $1.330 \mathrm{E}-01$ & $6.51 \mathrm{E}-02$ & $1.108 \mathrm{E}+01$ & $2.917 \mathrm{E}-01$ & $2.230 \mathrm{E}+01$ & $9.897 \mathrm{E}+04$ \\
\hline $8.047 \mathrm{E}+03$ & $5.678 \mathrm{E}+01$ & $1.10 \mathrm{E}+01$ & $4.917 \mathrm{E}-01$ & $6.27 \mathrm{E}-02$ & $1.106 \mathrm{E}+01$ & $1.037 \mathrm{E}+00$ & $2.222 \mathrm{E}+01$ & $9.897 \mathrm{E}+04$ \\
\hline $1.009 \mathrm{E}+04$ & $9.582 \mathrm{E}+01$ & $1.02 \mathrm{E}+01$ & $8.162 \mathrm{E}-01$ & $5.92 \mathrm{E}-02$ & $1.105 \mathrm{E}+01$ & $1.702 \mathrm{E}+00$ & $2.220 \mathrm{E}+01$ & $9.897 \mathrm{E}+04$ \\
\hline $1.200 \mathrm{E}+04$ & $1.402 \mathrm{E}+02$ & $9.91 \mathrm{E}+00$ & $1.140 \mathrm{E}+00$ & $6.18 \mathrm{E}-02$ & $1.103 \mathrm{E}+01$ & $2.555 \mathrm{E}+00$ & $2.212 \mathrm{E}+01$ & $9.896 \mathrm{E}+04$ \\
\hline $1.407 \mathrm{E}+04$ & $2.018 \mathrm{E}+02$ & $1.11 \mathrm{E}+01$ & $1.586 \mathrm{E}+00$ & $6.40 \mathrm{E}-02$ & $1.100 \mathrm{E}+01$ & $3.770 \mathrm{E}+00$ & $2.213 \mathrm{E}+01$ & $9.896 \mathrm{E}+04$ \\
\hline $1.604 \mathrm{E}+04$ & $2.729 \mathrm{E}+02$ & $9.01 \mathrm{E}+00$ & $2.069 \mathrm{E}+00$ & $6.95 \mathrm{E}-02$ & $1.097 \mathrm{E}+01$ & $5.250 \mathrm{E}+00$ & $2.212 \mathrm{E}+01$ & $9.897 \mathrm{E}+04$ \\
\hline $1.807 \mathrm{E}+04$ & $3.613 \mathrm{E}+02$ & $8.98 \mathrm{E}+00$ & $2.626 \mathrm{E}+00$ & $9.73 \mathrm{E}-02$ & $1.093 \mathrm{E}+01$ & $7.211 \mathrm{E}+00$ & $2.207 \mathrm{E}+01$ & $9.897 \mathrm{E}+04$ \\
\hline $2.002 \mathrm{E}+04$ & $4.521 \mathrm{E}+02$ & $9.44 \mathrm{E}+00$ & $3.382 \mathrm{E}+00$ & $2.44 \mathrm{E}-01$ & $1.087 \mathrm{E}+01$ & $9.562 \mathrm{E}+00$ & $2.199 \mathrm{E}+01$ & $9.897 \mathrm{E}+04$ \\
\hline $2.249 \mathrm{E}+04$ & $5.843 \mathrm{E}+02$ & $9.79 \mathrm{E}+00$ & $4.202 \mathrm{E}+00$ & $1.32 \mathrm{E}-01$ & $1.079 \mathrm{E}+01$ & $1.338 \mathrm{E}+01$ & $2.198 \mathrm{E}+01$ & $9.897 \mathrm{E}+04$ \\
\hline
\end{tabular}

Table 27: APC 5.5 x 2.0 Static Calculated Values

\begin{tabular}{|c|c|c|c|c|c|c|c|}
\hline$n(\mathrm{RPM})$ & $\rho\left(\mathrm{kg} / \mathrm{m}^{\wedge} 3\right)$ & $P_{\mathrm{P}}(\mathrm{W})$ & $\Delta P_{\mathrm{P}}(\mathrm{W})$ & $P_{\mathrm{e}}(\mathrm{W})$ & $\Delta P_{\mathrm{e}}(\mathrm{W})$ & $C_{\mathrm{T}}$ & $\Delta C_{\mathrm{T}}$ \\
\hline $3.995 \mathrm{E}+03$ & $1.167 \mathrm{E}+00$ & $5.454 \mathrm{E}-01$ & $2.67 \mathrm{E}-01$ & $3.233 \mathrm{E}+00$ & $5.52 \mathrm{E}-02$ & $5.627 \mathrm{E}-02$ & $5.61 \mathrm{E}-02$ \\
\hline $8.047 \mathrm{E}+03$ & $1.167 \mathrm{E}+00$ & $4.064 \mathrm{E}+00$ & $5.18 \mathrm{E}-01$ & $1.147 \mathrm{E}+01$ & $1.41 \mathrm{E}-01$ & $7.054 \mathrm{E}-02$ & $1.37 \mathrm{E}-02$ \\
\hline $1.009 \mathrm{E}+04$ & $1.167 \mathrm{E}+00$ & $8.454 \mathrm{E}+00$ & $6.14 \mathrm{E}-01$ & $1.881 \mathrm{E}+01$ & $2.18 \mathrm{E}-01$ & $7.576 \mathrm{E}-02$ & $8.04 \mathrm{E}-03$ \\
\hline $1.200 \mathrm{E}+04$ & $1.168 \mathrm{E}+00$ & $1.405 \mathrm{E}+01$ & $7.62 \mathrm{E}-01$ & $2.819 \mathrm{E}+01$ & $3.17 \mathrm{E}-01$ & $7.831 \mathrm{E}-02$ & $5.54 \mathrm{E}-03$ \\
\hline $1.407 \mathrm{E}+04$ & $1.168 \mathrm{E}+00$ & $2.292 \mathrm{E}+01$ & $9.25 \mathrm{E}-01$ & $4.148 \mathrm{E}+01$ & $4.58 \mathrm{E}-01$ & $8.197 \mathrm{E}-02$ & $4.51 \mathrm{E}-03$ \\
\hline $1.604 \mathrm{E}+04$ & $1.168 \mathrm{E}+00$ & $3.408 \mathrm{E}+01$ & $1.14 \mathrm{E}+00$ & $5.759 \mathrm{E}+01$ & $6.29 \mathrm{E}-01$ & $8.534 \mathrm{E}-02$ & $2.82 \mathrm{E}-03$ \\
\hline $1.807 \mathrm{E}+04$ & $1.168 \mathrm{E}+00$ & $4.872 \mathrm{E}+01$ & $1.81 \mathrm{E}+00$ & $7.878 \mathrm{E}+01$ & $8.77 \mathrm{E}-01$ & $8.899 \mathrm{E}-02$ & $2.21 \mathrm{E}-03$ \\
\hline $2.002 \mathrm{E}+04$ & $1.168 \mathrm{E}+00$ & $6.951 \mathrm{E}+01$ & $5.02 \mathrm{E}+00$ & $1.040 \mathrm{E}+02$ & $1.16 \mathrm{E}+00$ & $9.069 \mathrm{E}-02$ & $1.89 \mathrm{E}-03$ \\
\hline $2.249 \mathrm{E}+04$ & $1.168 \mathrm{E}+00$ & $9.708 \mathrm{E}+01$ & $3.06 \mathrm{E}+00$ & $1.444 \mathrm{E}+02$ & $1.68 \mathrm{E}+00$ & $9.281 \mathrm{E}-02$ & $1.56 \mathrm{E}-03$ \\
\hline$C_{\mathrm{P}}$ & $\Delta C_{\mathrm{P}}$ & $C_{\mathrm{Q}}$ & $\Delta C_{\mathrm{Q}}$ & $\eta_{\mathrm{T}}$ & $\Delta \eta_{\mathrm{T}}$ & $R e_{0.75}$ & $\Delta R e_{0.75}$ \\
\hline $3.025 \mathrm{E}-02$ & $1.48 \mathrm{E}-02$ & $4.815 \mathrm{E}-03$ & $2.36 \mathrm{E}-03$ & $1.687 \mathrm{E}-01$ & $8.26 \mathrm{E}-02$ & $1.373 \mathrm{E}+04$ & $1.74 \mathrm{E}+01$ \\
\hline $2.756 \mathrm{E}-02$ & $3.52 \mathrm{E}-03$ & $4.387 \mathrm{E}-03$ & $5.60 \mathrm{E}-04$ & $3.542 \mathrm{E}-01$ & $4.54 \mathrm{E}-02$ & $2.768 \mathrm{E}+04$ & $3.56 \mathrm{E}+01$ \\
\hline $2.912 \mathrm{E}-02$ & $2.11 \mathrm{E}-03$ & $4.634 \mathrm{E}-03$ & $3.36 \mathrm{E}-04$ & $4.495 \mathrm{E}-01$ & $3.30 \mathrm{E}-02$ & $3.470 \mathrm{E}+04$ & $4.03 \mathrm{E}+01$ \\
\hline $2.874 \mathrm{E}-02$ & $1.56 \mathrm{E}-03$ & $4.574 \mathrm{E}-03$ & $2.48 \mathrm{E}-04$ & $4.983 \mathrm{E}-01$ & $2.76 \mathrm{E}-02$ & $4.129 \mathrm{E}+04$ & $4.78 \mathrm{E}+01$ \\
\hline $2.907 \mathrm{E}-02$ & $1.17 \mathrm{E}-03$ & $4.626 \mathrm{E}-03$ & $1.87 \mathrm{E}-04$ & $5.525 \mathrm{E}-01$ & $2.31 \mathrm{E}-02$ & $4.842 \mathrm{E}+04$ & $5.71 \mathrm{E}+01$ \\
\hline $2.920 \mathrm{E}-02$ & $9.80 \mathrm{E}-04$ & $4.647 \mathrm{E}-03$ & $1.56 \mathrm{E}-04$ & $5.918 \mathrm{E}-01$ & $2.09 \mathrm{E}-02$ & $5.520 \mathrm{E}+04$ & $6.24 \mathrm{E}+01$ \\
\hline $2.919 \mathrm{E}-02$ & $1.08 \mathrm{E}-03$ & $4.645 \mathrm{E}-03$ & $1.72 \mathrm{E}-04$ & $6.184 \mathrm{E}-01$ & $2.39 \mathrm{E}-02$ & $6.220 \mathrm{E}+04$ & $7.22 \mathrm{E}+01$ \\
\hline $3.061 \mathrm{E}-02$ & $2.21 \mathrm{E}-03$ & $4.872 \mathrm{E}-03$ & $3.52 \mathrm{E}-04$ & $6.685 \mathrm{E}-01$ & $4.88 \mathrm{E}-02$ & $6.895 \mathrm{E}+04$ & $7.81 \mathrm{E}+01$ \\
\hline $3.012 \mathrm{E}-02$ & $9.50 \mathrm{E}-04$ & $4.815 \mathrm{E}-03$ & $1.51 \mathrm{E}-04$ & $6.725 \mathrm{E}-01$ & $2.26 \mathrm{E}-02$ & $7.749 \mathrm{E}+04$ & $8.66 \mathrm{E}+01$ \\
\hline
\end{tabular}




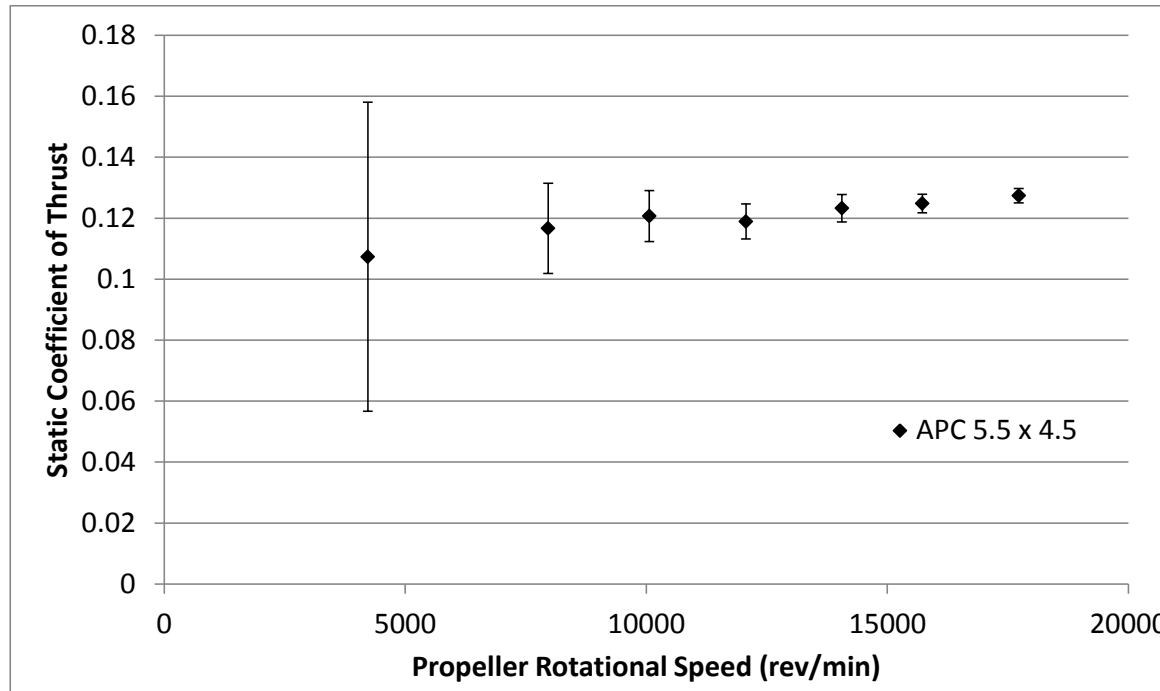

(a)

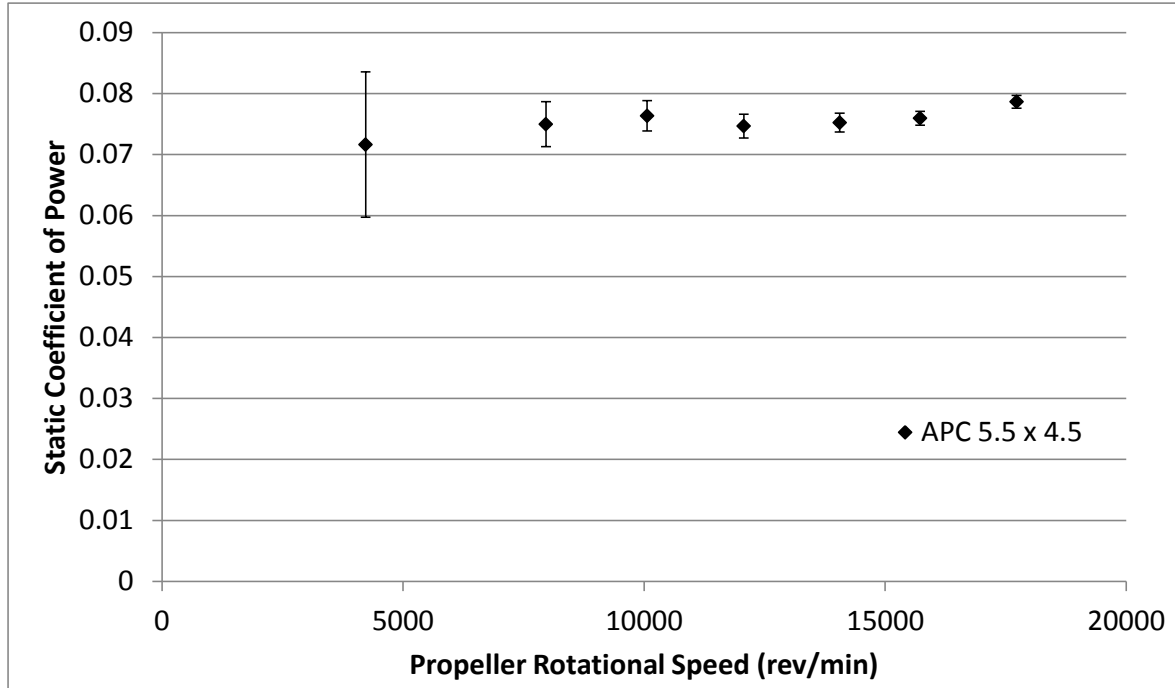

(b)

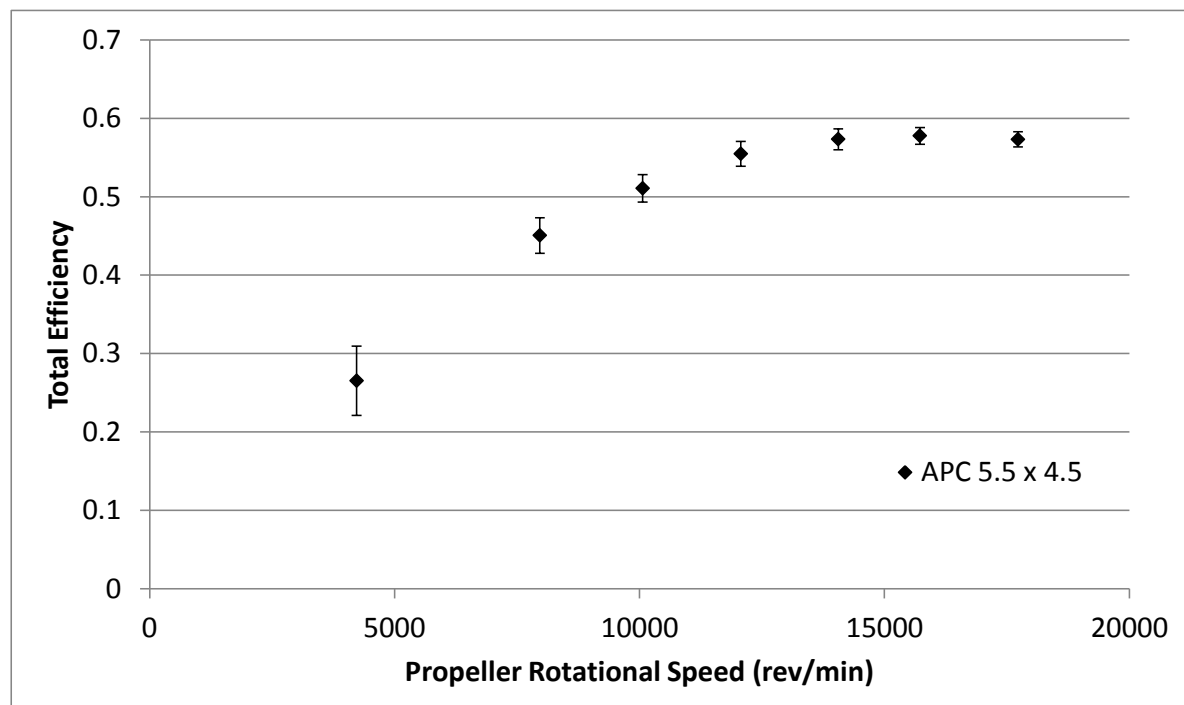

(c)

Figure 53: APC 5.5 x 4.5 Static Test Results: (a) Coefficient of Thrust, (b) Coefficient of Power, (c) Total Efficiency. 
Table 28: APC 5.5 x 4.5 Static Measured Values

\begin{tabular}{|c|c|c|c|c|c|c|c|c|}
\hline$n(\mathrm{RPM})$ & $T(\mathrm{~g})$ & $\Delta T(\mathrm{~g})$ & $Q(\mathrm{~g}-\mathrm{m})$ & $\Delta Q(\mathrm{~g}-\mathrm{m})$ & $V(\mathrm{~V})$ & $I(\mathrm{~A})$ & $T_{\text {atm }}\left({ }^{\circ} \mathrm{C}\right)$ & $P_{\text {atm }}(\mathrm{Pa})$ \\
\hline $4.225 \mathrm{E}+03$ & $2.397 \mathrm{E}+01$ & $1.13 \mathrm{E}+01$ & $3.549 \mathrm{E}-01$ & $5.91 \mathrm{E}-02$ & $1.108 \mathrm{E}+01$ & $5.241 \mathrm{E}-01$ & $2.170 \mathrm{E}+01$ & $9.898 \mathrm{E}+04$ \\
\hline $7.966 \mathrm{E}+03$ & $9.265 \mathrm{E}+01$ & $1.18 \mathrm{E}+01$ & $1.321 \mathrm{E}+00$ & $6.48 \mathrm{E}-02$ & $1.104 \mathrm{E}+01$ & $2.173 \mathrm{E}+00$ & $2.163 \mathrm{E}+01$ & $9.899 \mathrm{E}+04$ \\
\hline $1.006 \mathrm{E}+04$ & $1.530 \mathrm{E}+02$ & $1.06 \mathrm{E}+01$ & $2.148 \mathrm{E}+00$ & $6.96 \mathrm{E}-02$ & $1.100 \mathrm{E}+01$ & $3.953 \mathrm{E}+00$ & $2.157 \mathrm{E}+01$ & $9.898 \mathrm{E}+04$ \\
\hline $1.207 \mathrm{E}+04$ & $2.168 \mathrm{E}+02$ & $1.05 \mathrm{E}+01$ & $3.020 \mathrm{E}+00$ & $7.89 \mathrm{E}-02$ & $1.095 \mathrm{E}+01$ & $6.163 \mathrm{E}+00$ & $2.183 \mathrm{E}+01$ & $9.907 \mathrm{E}+04$ \\
\hline $1.406 \mathrm{E}+04$ & $3.054 \mathrm{E}+02$ & $1.11 \mathrm{E}+01$ & $4.136 \mathrm{E}+00$ & $8.48 \mathrm{E}-02$ & $1.087 \mathrm{E}+01$ & $9.583 \mathrm{E}+00$ & $2.138 \mathrm{E}+01$ & $9.907 \mathrm{E}+04$ \\
\hline $1.573 \mathrm{E}+04$ & $3.872 \mathrm{E}+02$ & $9.48 \mathrm{E}+00$ & $5.227 \mathrm{E}+00$ & $7.89 \mathrm{E}-02$ & $1.078 \mathrm{E}+01$ & $1.356 \mathrm{E}+01$ & $2.133 \mathrm{E}+01$ & $9.908 \mathrm{E}+04$ \\
\hline $1.773 \mathrm{E}+04$ & $5.020 \mathrm{E}+02$ & $9.39 \mathrm{E}+00$ & $6.878 \mathrm{E}+00$ & $9.13 \mathrm{E}-02$ & $1.061 \mathrm{E}+01$ & $2.058 \mathrm{E}+01$ & $2.129 \mathrm{E}+01$ & $9.908 \mathrm{E}+04$ \\
\hline
\end{tabular}

Table 29: APC 5.5 x 4.5 Static Calculated Values

\begin{tabular}{|c|c|c|c|c|c|c|c|}
\hline$n(\mathrm{RPM})$ & $\rho\left(\mathrm{kg} / \mathrm{m}^{\wedge} 3\right)$ & $P_{\mathrm{P}}(\mathrm{W})$ & $\Delta P_{\mathrm{P}}(\mathrm{W})$ & $P_{\mathrm{e}}(\mathrm{W})$ & $\Delta P_{\mathrm{e}}(\mathrm{W})$ & $C_{\mathrm{T}}$ & $\Delta C_{\mathrm{T}}$ \\
\hline $4.225 \mathrm{E}+03$ & $1.169 \mathrm{E}+00$ & $1.540 \mathrm{E}+00$ & $2.56 \mathrm{E}-01$ & $5.805 \mathrm{E}+00$ & $8.44 \mathrm{E}-02$ & $1.073 \mathrm{E}-01$ & $5.07 \mathrm{E}-02$ \\
\hline $7.966 \mathrm{E}+03$ & $1.170 \mathrm{E}+00$ & $1.081 \mathrm{E}+01$ & $5.30 \mathrm{E}-01$ & $2.399 \mathrm{E}+01$ & $2.77 \mathrm{E}-01$ & $1.167 \mathrm{E}-01$ & $1.48 \mathrm{E}-02$ \\
\hline $1.006 \mathrm{E}+04$ & $1.170 \mathrm{E}+00$ & $2.220 \mathrm{E}+01$ & $7.20 \mathrm{E}-01$ & $4.348 \mathrm{E}+01$ & $4.89 \mathrm{E}-01$ & $1.207 \mathrm{E}-01$ & $8.36 \mathrm{E}-03$ \\
\hline $1.207 \mathrm{E}+04$ & $1.170 \mathrm{E}+00$ & $3.743 \mathrm{E}+01$ & $9.78 \mathrm{E}-01$ & $6.748 \mathrm{E}+01$ & $7.73 \mathrm{E}-01$ & $1.189 \mathrm{E}-01$ & $5.75 \mathrm{E}-03$ \\
\hline $1.406 \mathrm{E}+04$ & $1.172 \mathrm{E}+00$ & $5.972 \mathrm{E}+01$ & $1.22 \mathrm{E}+00$ & $1.042 \mathrm{E}+02$ & $1.16 \mathrm{E}+00$ & $1.233 \mathrm{E}-01$ & $4.50 \mathrm{E}-03$ \\
\hline $1.573 \mathrm{E}+04$ & $1.172 \mathrm{E}+00$ & $8.443 \mathrm{E}+01$ & $1.27 \mathrm{E}+00$ & $1.462 \mathrm{E}+02$ & $1.57 \mathrm{E}+00$ & $1.248 \mathrm{E}-01$ & $3.06 \mathrm{E}-03$ \\
\hline $1.773 \mathrm{E}+04$ & $1.172 \mathrm{E}+00$ & $1.252 \mathrm{E}+02$ & $1.66 \mathrm{E}+00$ & $2.185 \mathrm{E}+02$ & $2.30 \mathrm{E}+00$ & $1.274 \mathrm{E}-01$ & $2.39 \mathrm{E}-03$ \\
\hline$C_{\mathrm{P}}$ & $\Delta C_{\mathrm{P}}$ & $C_{\mathrm{Q}}$ & $\Delta C_{\mathrm{Q}}$ & $\eta_{\mathrm{T}}$ & $\Delta \eta_{\mathrm{T}}$ & $R e_{0.75}$ & $\Delta R e_{0.75}$ \\
\hline $7.164 \mathrm{E}-02$ & $1.19 \mathrm{E}-02$ & $1.140 \mathrm{E}-02$ & $1.90 \mathrm{E}-03$ & $2.652 \mathrm{E}-01$ & $4.43 \mathrm{E}-02$ & $1.422 \mathrm{E}+04$ & $1.96 \mathrm{E}+01$ \\
\hline $7.498 \mathrm{E}-02$ & $3.68 \mathrm{E}-03$ & $1.193 \mathrm{E}-02$ & $5.86 \mathrm{E}-04$ & $4.506 \mathrm{E}-01$ & $2.27 \mathrm{E}-02$ & $2.683 \mathrm{E}+04$ & $3.26 \mathrm{E}+01$ \\
\hline $7.636 \mathrm{E}-02$ & $2.48 \mathrm{E}-03$ & $1.215 \mathrm{E}-02$ & $3.94 \mathrm{E}-04$ & $5.106 \mathrm{E}-01$ & $1.75 \mathrm{E}-02$ & $3.391 \mathrm{E}+04$ & $4.01 \mathrm{E}+01$ \\
\hline $7.465 \mathrm{E}-02$ & $1.95 \mathrm{E}-03$ & $1.188 \mathrm{E}-02$ & $3.11 \mathrm{E}-04$ & $5.547 \mathrm{E}-01$ & $1.58 \mathrm{E}-02$ & $4.064 \mathrm{E}+04$ & $4.77 \mathrm{E}+01$ \\
\hline $7.523 \mathrm{E}-02$ & $1.54 \mathrm{E}-03$ & $1.197 \mathrm{E}-02$ & $2.46 \mathrm{E}-04$ & $5.733 \mathrm{E}-01$ & $1.34 \mathrm{E}-02$ & $4.747 \mathrm{E}+04$ & $5.52 \mathrm{E}+01$ \\
\hline $7.596 \mathrm{E}-02$ & $1.15 \mathrm{E}-03$ & $1.209 \mathrm{E}-02$ & $1.83 \mathrm{E}-04$ & $5.777 \mathrm{E}-01$ & $1.07 \mathrm{E}-02$ & $5.312 \mathrm{E}+04$ & $6.31 \mathrm{E}+01$ \\
\hline $7.865 \mathrm{E}-02$ & $1.05 \mathrm{E}-03$ & $1.252 \mathrm{E}-02$ & $1.67 \mathrm{E}-04$ & $5.731 \mathrm{E}-01$ & $9.71 \mathrm{E}-03$ & $5.989 \mathrm{E}+04$ & $7.36 \mathrm{E}+01$ \\
\hline
\end{tabular}




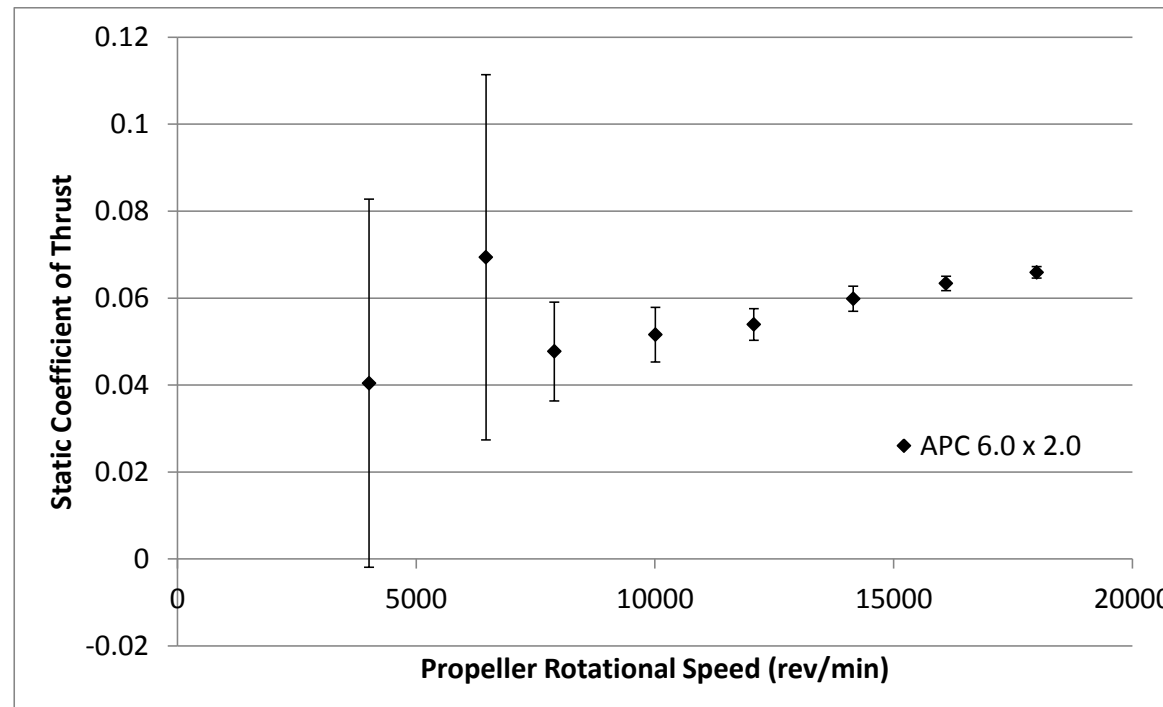

(a)

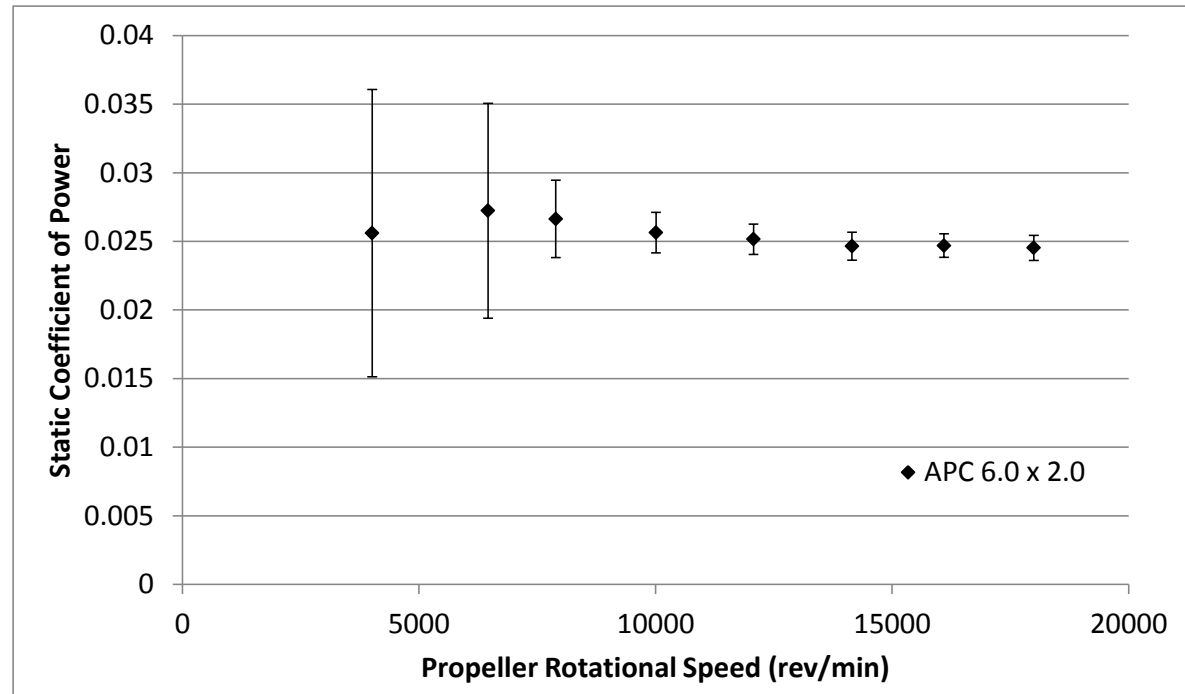

(b)

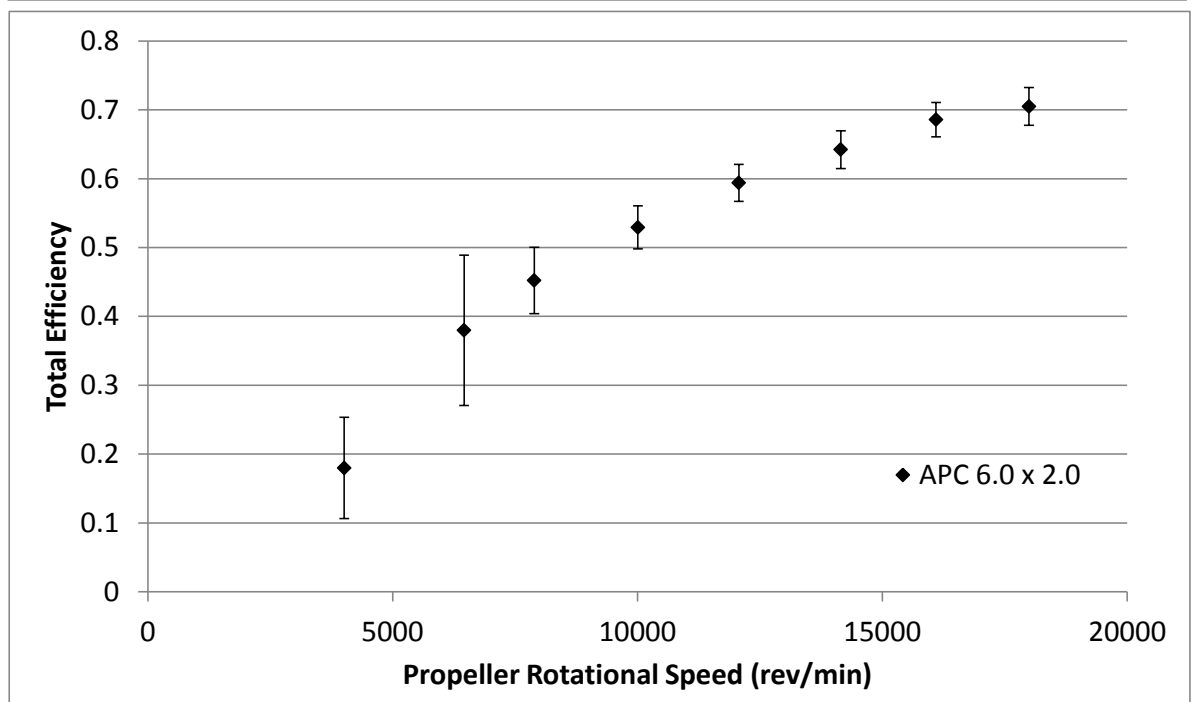

(c)

Figure 54: APC 6.0 x 2.0 Static Test Results: (a) Coefficient of Thrust, (b) Coefficient of Power, (c) Total Efficiency. 
Table 30: APC 6.0 x 2.0 Static Measured Values

\begin{tabular}{|c|c|c|c|c|c|c|c|c|}
\hline$n(\mathrm{RPM})$ & $T(\mathrm{~g})$ & $\Delta T(\mathrm{~g})$ & $Q(\mathrm{~g}-\mathrm{m})$ & $\Delta Q(\mathrm{~g}-\mathrm{m})$ & $V(\mathrm{~V})$ & $I(\mathrm{~A})$ & $T_{\text {atm }}\left({ }^{\circ} \mathrm{C}\right)$ & $P_{\text {atm }}(\mathrm{Pa})$ \\
\hline $4.011 \mathrm{E}+03$ & $1.143 \mathrm{E}+01$ & $1.20 \mathrm{E}+01$ & $1.753 \mathrm{E}-01$ & $7.17 \mathrm{E}-02$ & $1.111 \mathrm{E}+01$ & $3.618 \mathrm{E}-01$ & $2.398 \mathrm{E}+01$ & $9.862 \mathrm{E}+04$ \\
\hline $6.460 \mathrm{E}+03$ & $5.088 \mathrm{E}+01$ & $3.08 \mathrm{E}+01$ & $4.837 \mathrm{E}-01$ & $1.39 \mathrm{E}-01$ & $1.109 \mathrm{E}+01$ & $7.616 \mathrm{E}-01$ & $2.396 \mathrm{E}+01$ & $9.862 \mathrm{E}+04$ \\
\hline $7.893 \mathrm{E}+03$ & $5.225 \mathrm{E}+01$ & $1.24 \mathrm{E}+01$ & $7.063 \mathrm{E}-01$ & $7.48 \mathrm{E}-02$ & $1.108 \mathrm{E}+01$ & $1.142 \mathrm{E}+00$ & $2.393 \mathrm{E}+01$ & $9.860 \mathrm{E}+04$ \\
\hline $1.001 \mathrm{E}+04$ & $9.085 \mathrm{E}+01$ & $1.11 \mathrm{E}+01$ & $1.093 \mathrm{E}+00$ & $6.31 \mathrm{E}-02$ & $1.106 \mathrm{E}+01$ & $1.919 \mathrm{E}+00$ & $2.387 \mathrm{E}+01$ & $9.860 \mathrm{E}+04$ \\
\hline $1.207 \mathrm{E}+04$ & $1.382 \mathrm{E}+02$ & $9.30 \mathrm{E}+00$ & $1.560 \mathrm{E}+00$ & $6.84 \mathrm{E}-02$ & $1.104 \mathrm{E}+01$ & $2.951 \mathrm{E}+00$ & $2.389 \mathrm{E}+01$ & $9.860 \mathrm{E}+04$ \\
\hline $1.415 \mathrm{E}+04$ & $2.108 \mathrm{E}+02$ & $1.01 \mathrm{E}+01$ & $2.102 \mathrm{E}+00$ & $8.69 \mathrm{E}-02$ & $1.100 \mathrm{E}+01$ & $4.325 \mathrm{E}+00$ & $2.381 \mathrm{E}+01$ & $9.861 \mathrm{E}+04$ \\
\hline $1.610 \mathrm{E}+04$ & $2.888 \mathrm{E}+02$ & $7.43 \mathrm{E}+00$ & $2.725 \mathrm{E}+00$ & $9.45 \mathrm{E}-02$ & $1.095 \mathrm{E}+01$ & $5.997 \mathrm{E}+00$ & $2.373 \mathrm{E}+01$ & $9.860 \mathrm{E}+04$ \\
\hline $1.800 \mathrm{E}+04$ & $3.754 \mathrm{E}+02$ & $7.53 \mathrm{E}+00$ & $3.383 \mathrm{E}+00$ & $1.26 \mathrm{E}-01$ & $1.089 \mathrm{E}+01$ & $8.141 \mathrm{E}+00$ & $2.367 \mathrm{E}+01$ & $9.859 \mathrm{E}+04$ \\
\hline
\end{tabular}

Table 31: APC 6.0 x 2.0 Static Calculated Values

\begin{tabular}{|c|c|c|c|c|c|c|c|}
\hline$n(\mathrm{RPM})$ & $\rho\left(\mathrm{kg} / \mathrm{m}^{\wedge} 3\right)$ & $P_{\mathrm{P}}(\mathrm{W})$ & $\Delta P_{\mathrm{P}}(\mathrm{W})$ & $P_{\mathrm{e}}(\mathrm{W})$ & $\Delta P_{\mathrm{e}}(\mathrm{W})$ & $C_{\mathrm{T}}$ & $\Delta C_{\mathrm{T}}$ \\
\hline $4.011 \mathrm{E}+03$ & $1.156 \mathrm{E}+00$ & $7.223 \mathrm{E}-01$ & $2.95 \mathrm{E}-01$ & $4.018 \mathrm{E}+00$ & $6.30 \mathrm{E}-02$ & $4.043 \mathrm{E}-02$ & $4.23 \mathrm{E}-02$ \\
\hline $6.460 \mathrm{E}+03$ & $1.156 \mathrm{E}+00$ & $3.209 \mathrm{E}+00$ & $9.23 \mathrm{E}-01$ & $8.450 \mathrm{E}+00$ & $1.09 \mathrm{E}-01$ & $6.938 \mathrm{E}-02$ & $4.20 \mathrm{E}-02$ \\
\hline $7.893 \mathrm{E}+03$ & $1.156 \mathrm{E}+00$ & $5.725 \mathrm{E}+00$ & $6.06 \mathrm{E}-01$ & $1.266 \mathrm{E}+01$ & $1.54 \mathrm{E}-01$ & $4.773 \mathrm{E}-02$ & $1.14 \mathrm{E}-02$ \\
\hline $1.001 \mathrm{E}+04$ & $1.156 \mathrm{E}+00$ & $1.124 \mathrm{E}+01$ & $6.49 \mathrm{E}-01$ & $2.123 \mathrm{E}+01$ & $2.43 \mathrm{E}-01$ & $5.159 \mathrm{E}-02$ & $6.29 \mathrm{E}-03$ \\
\hline $1.207 \mathrm{E}+04$ & $1.156 \mathrm{E}+00$ & $1.934 \mathrm{E}+01$ & $8.48 \mathrm{E}-01$ & $3.257 \mathrm{E}+01$ & $3.66 \mathrm{E}-01$ & $5.395 \mathrm{E}-02$ & $3.63 \mathrm{E}-03$ \\
\hline $1.415 \mathrm{E}+04$ & $1.157 \mathrm{E}+00$ & $3.055 \mathrm{E}+01$ & $1.26 \mathrm{E}+00$ & $4.757 \mathrm{E}+01$ & $5.39 \mathrm{E}-01$ & $5.985 \mathrm{E}-02$ & $2.88 \mathrm{E}-03$ \\
\hline $1.610 \mathrm{E}+04$ & $1.157 \mathrm{E}+00$ & $4.505 \mathrm{E}+01$ & $1.56 \mathrm{E}+00$ & $6.569 \mathrm{E}+01$ & $7.48 \mathrm{E}-01$ & $6.338 \mathrm{E}-02$ & $1.63 \mathrm{E}-03$ \\
\hline $1.800 \mathrm{E}+04$ & $1.157 \mathrm{E}+00$ & $6.253 \mathrm{E}+01$ & $2.33 \mathrm{E}+00$ & $8.869 \mathrm{E}+01$ & $9.65 \mathrm{E}-01$ & $6.592 \mathrm{E}-02$ & $1.33 \mathrm{E}-03$ \\
\hline$C_{\mathrm{P}}$ & $\Delta C_{\mathrm{P}}$ & $C_{\mathrm{Q}}$ & $\Delta C_{\mathrm{Q}}$ & $\eta_{\mathrm{T}}$ & $\Delta \eta_{\mathrm{T}}$ & $R e_{0.75}$ & $\Delta R e_{0.75}$ \\
\hline $2.560 \mathrm{E}-02$ & $1.05 \mathrm{E}-02$ & $4.074 \mathrm{E}-03$ & $1.67 \mathrm{E}-03$ & $1.797 \mathrm{E}-01$ & $7.36 \mathrm{E}-02$ & $1.345 \mathrm{E}+04$ & $1.85 \mathrm{E}+01$ \\
\hline $2.723 \mathrm{E}-02$ & $7.83 \mathrm{E}-03$ & $4.334 \mathrm{E}-03$ & $1.25 \mathrm{E}-03$ & $3.798 \mathrm{E}-01$ & $1.09 \mathrm{E}-01$ & $2.166 \mathrm{E}+04$ & $3.24 \mathrm{E}+01$ \\
\hline $2.664 \mathrm{E}-02$ & $2.82 \mathrm{E}-03$ & $4.240 \mathrm{E}-03$ & $4.49 \mathrm{E}-04$ & $4.521 \mathrm{E}-01$ & $4.82 \mathrm{E}-02$ & $2.646 \mathrm{E}+04$ & $4.36 \mathrm{E}+01$ \\
\hline $2.563 \mathrm{E}-02$ & $1.48 \mathrm{E}-03$ & $4.080 \mathrm{E}-03$ & $2.35 \mathrm{E}-04$ & $5.293 \mathrm{E}-01$ & $3.11 \mathrm{E}-02$ & $3.357 \mathrm{E}+04$ & $4.22 \mathrm{E}+01$ \\
\hline $2.516 \mathrm{E}-02$ & $1.10 \mathrm{E}-03$ & $4.004 \mathrm{E}-03$ & $1.76 \mathrm{E}-04$ & $5.940 \mathrm{E}-01$ & $2.69 \mathrm{E}-02$ & $4.048 \mathrm{E}+04$ & $5.84 \mathrm{E}+01$ \\
\hline $2.464 \mathrm{E}-02$ & $1.02 \mathrm{E}-03$ & $3.922 \mathrm{E}-03$ & $1.62 \mathrm{E}-04$ & $6.422 \mathrm{E}-01$ & $2.75 \mathrm{E}-02$ & $4.748 \mathrm{E}+04$ & $6.81 \mathrm{E}+01$ \\
\hline $2.469 \mathrm{E}-02$ & $8.56 \mathrm{E}-04$ & $3.930 \mathrm{E}-03$ & $1.36 \mathrm{E}-04$ & $6.859 \mathrm{E}-01$ & $2.50 \mathrm{E}-02$ & $5.403 \mathrm{E}+04$ & $6.65 \mathrm{E}+01$ \\
\hline $2.453 \mathrm{E}-02$ & $9.16 \mathrm{E}-04$ & $3.904 \mathrm{E}-03$ & $1.46 \mathrm{E}-04$ & $7.050 \mathrm{E}-01$ & $2.74 \mathrm{E}-02$ & $6.041 \mathrm{E}+04$ & $7.90 \mathrm{E}+01$ \\
\hline
\end{tabular}




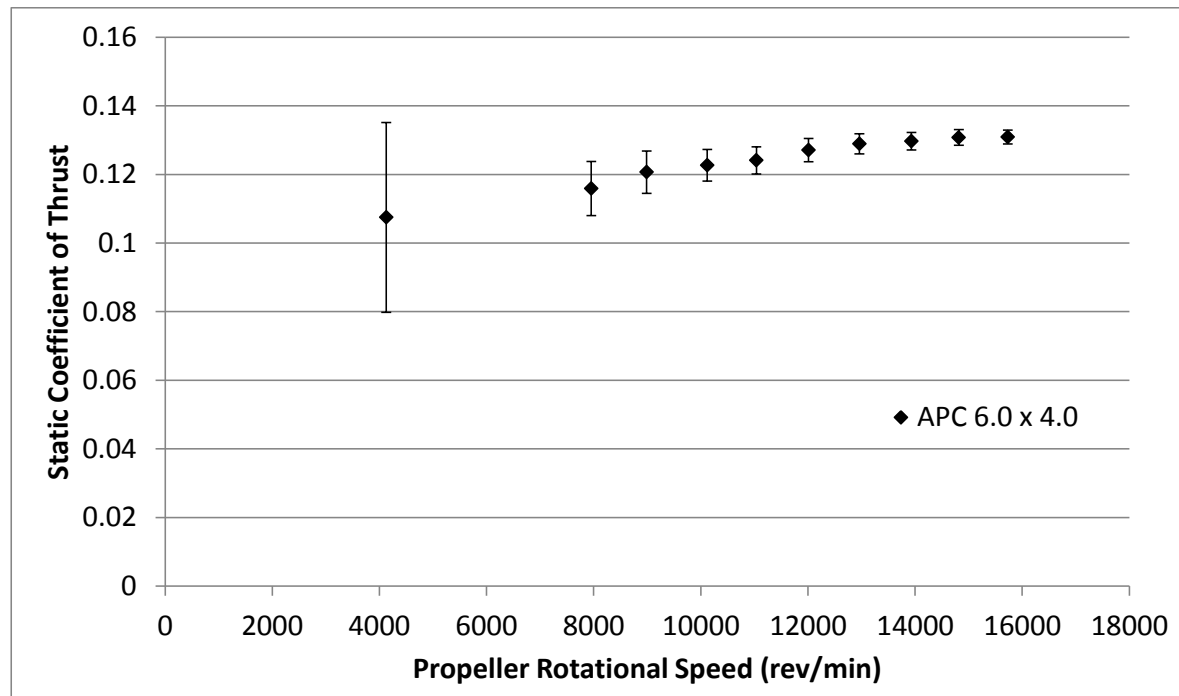

(a)

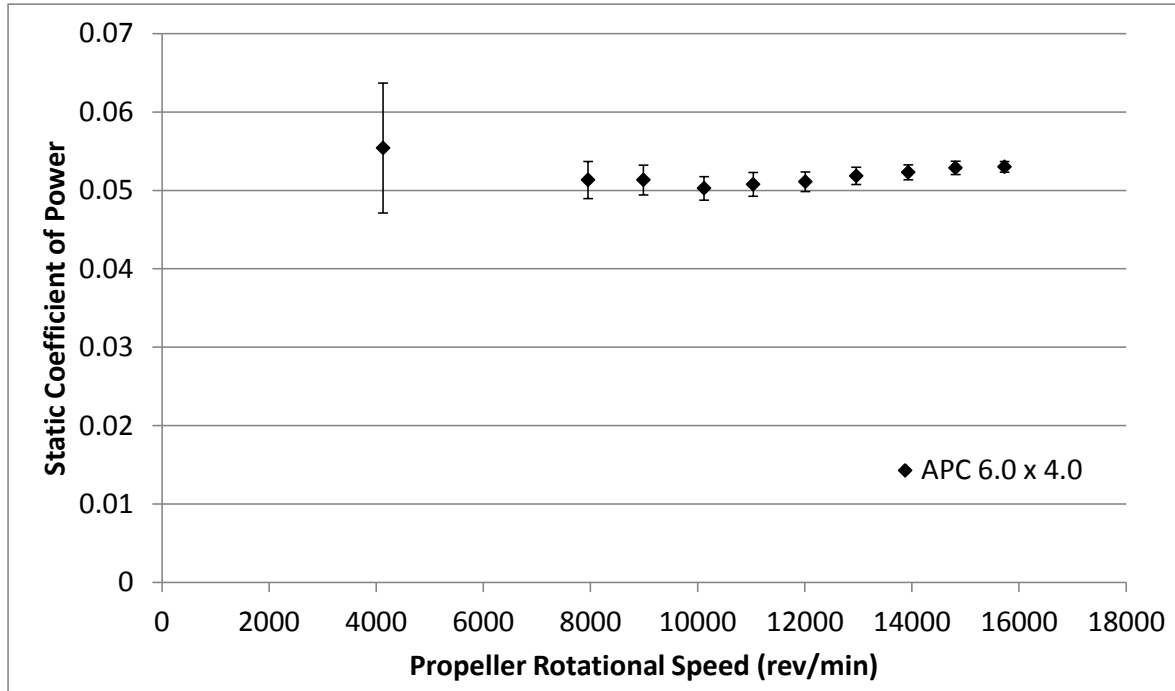

(b)

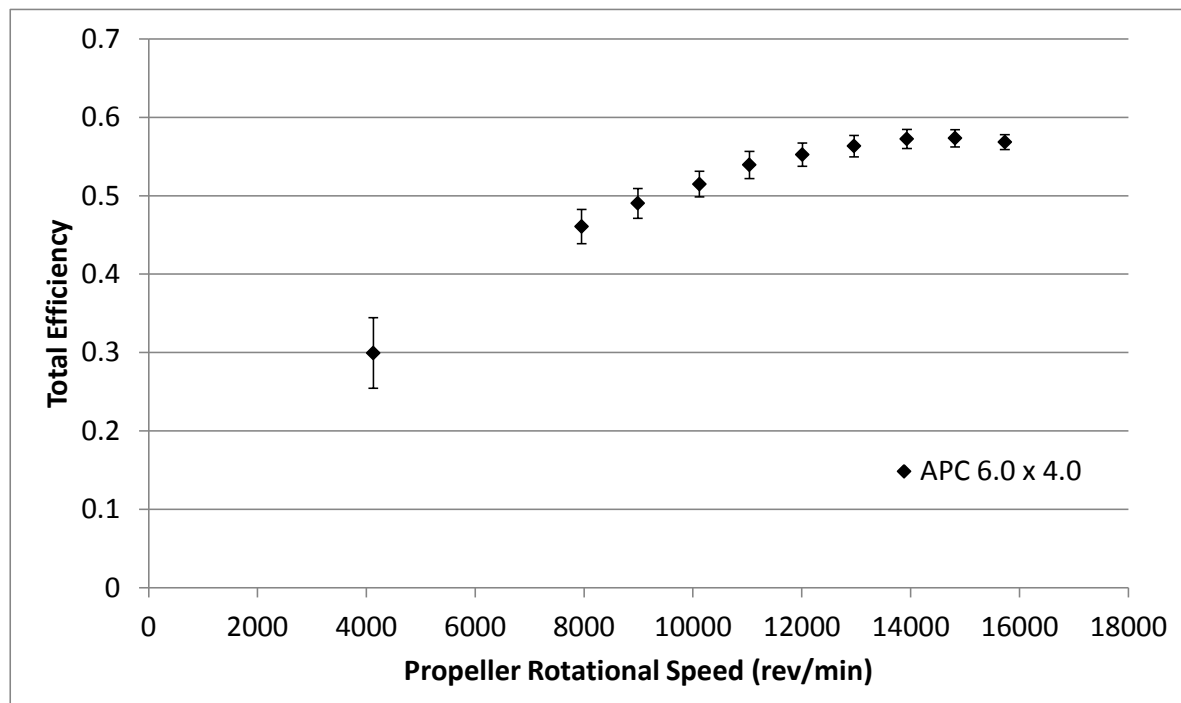

(c)

Figure 55: APC 6.0 x 4.0 Static Test Results: (a) Coefficient of Thrust, (b) Coefficient of Power, (c) Total Efficiency. 
Table 32: APC 6.0 x 4.0 Static Measured Values

\begin{tabular}{|c|c|c|c|c|c|c|c|c|}
\hline$n(\mathrm{RPM})$ & $T(\mathrm{~g})$ & $\Delta T(\mathrm{~g})$ & $Q(\mathrm{~g}-\mathrm{m})$ & $\Delta Q(\mathrm{~g}-\mathrm{m})$ & $V(\mathrm{~V})$ & $I(\mathrm{~A})$ & $T_{\text {atm }}\left({ }^{\circ} \mathrm{C}\right)$ & $P_{\text {atm }}(\mathrm{Pa})$ \\
\hline $4.128 \mathrm{E}+03$ & $3.234 \mathrm{E}+01$ & $8.32 \mathrm{E}+00$ & $4.031 \mathrm{E}-01$ & $6.03 \mathrm{E}-02$ & $1.108 \mathrm{E}+01$ & $5.153 \mathrm{E}-01$ & $2.160 \mathrm{E}+01$ & $9.908 \mathrm{E}+04$ \\
\hline $7.955 \mathrm{E}+03$ & $1.296 \mathrm{E}+02$ & $8.81 \mathrm{E}+00$ & $1.387 \mathrm{E}+00$ & $6.40 \mathrm{E}-02$ & $1.104 \mathrm{E}+01$ & $2.230 \mathrm{E}+00$ & $2.138 \mathrm{E}+01$ & $9.909 \mathrm{E}+04$ \\
\hline $8.989 \mathrm{E}+03$ & $1.722 \mathrm{E}+02$ & $8.79 \mathrm{E}+00$ & $1.770 \mathrm{E}+00$ & $6.57 \mathrm{E}-02$ & $1.102 \mathrm{E}+01$ & $3.025 \mathrm{E}+00$ & $2.144 \mathrm{E}+01$ & $9.907 \mathrm{E}+04$ \\
\hline $1.012 \mathrm{E}+04$ & $2.221 \mathrm{E}+02$ & $8.41 \mathrm{E}+00$ & $2.199 \mathrm{E}+00$ & $6.51 \mathrm{E}-02$ & $1.100 \mathrm{E}+01$ & $4.037 \mathrm{E}+00$ & $2.128 \mathrm{E}+01$ & $9.909 \mathrm{E}+04$ \\
\hline $1.104 \mathrm{E}+04$ & $2.670 \mathrm{E}+02$ & $8.51 \mathrm{E}+00$ & $2.642 \mathrm{E}+00$ & $7.87 \mathrm{E}-02$ & $1.097 \mathrm{E}+01$ & $5.060 \mathrm{E}+00$ & $2.144 \mathrm{E}+01$ & $9.906 \mathrm{E}+04$ \\
\hline $1.201 \mathrm{E}+04$ & $3.243 \mathrm{E}+02$ & $8.64 \mathrm{E}+00$ & $3.152 \mathrm{E}+00$ & $7.67 \mathrm{E}-02$ & $1.094 \mathrm{E}+01$ & $6.432 \mathrm{E}+00$ & $2.119 \mathrm{E}+01$ & $9.909 \mathrm{E}+04$ \\
\hline $1.296 \mathrm{E}+04$ & $3.830 \mathrm{E}+02$ & $8.70 \mathrm{E}+00$ & $3.724 \mathrm{E}+00$ & $7.90 \mathrm{E}-02$ & $1.091 \mathrm{E}+01$ & $8.068 \mathrm{E}+00$ & $2.113 \mathrm{E}+01$ & $9.910 \mathrm{E}+04$ \\
\hline $1.393 \mathrm{E}+04$ & $4.452 \mathrm{E}+02$ & $8.74 \mathrm{E}+00$ & $4.340 \mathrm{E}+00$ & $7.88 \mathrm{E}-02$ & $1.086 \mathrm{E}+01$ & $9.987 \mathrm{E}+00$ & $2.122 \mathrm{E}+01$ & $9.909 \mathrm{E}+04$ \\
\hline $1.482 \mathrm{E}+04$ & $5.078 \mathrm{E}+02$ & $8.95 \mathrm{E}+00$ & $4.961 \mathrm{E}+00$ & $7.96 \mathrm{E}-02$ & $1.081 \mathrm{E}+01$ & $1.218 \mathrm{E}+01$ & $2.118 \mathrm{E}+01$ & $9.910 \mathrm{E}+04$ \\
\hline $1.573 \mathrm{E}+04$ & $5.729 \mathrm{E}+02$ & $8.77 \mathrm{E}+00$ & $5.607 \mathrm{E}+00$ & $7.25 \mathrm{E}-02$ & $1.075 \mathrm{E}+01$ & $1.483 \mathrm{E}+01$ & $2.117 \mathrm{E}+01$ & $9.909 \mathrm{E}+04$ \\
\hline
\end{tabular}

Table 33: APC $6.0 \times 4.0$ Static Calculated Values

\begin{tabular}{|c|c|c|c|c|c|c|c|}
\hline$n(\mathrm{RPM})$ & $\rho\left(\mathrm{kg} / \mathrm{m}^{\wedge} 3\right)$ & $P_{\mathrm{P}}(\mathrm{W})$ & $\Delta P_{\mathrm{P}}(\mathrm{W})$ & $P_{\mathrm{e}}(\mathrm{W})$ & $\Delta P_{\mathrm{e}}(\mathrm{W})$ & $C_{\mathrm{T}}$ & $\Delta C_{\mathrm{T}}$ \\
\hline $4.128 \mathrm{E}+03$ & $1.171 \mathrm{E}+00$ & $1.709 \mathrm{E}+00$ & $2.56 \mathrm{E}-01$ & $5.708 \mathrm{E}+00$ & $8.27 \mathrm{E}-02$ & $1.075 \mathrm{E}-01$ & $2.77 \mathrm{E}-02$ \\
\hline $7.955 \mathrm{E}+03$ & $1.172 \mathrm{E}+00$ & $1.133 \mathrm{E}+01$ & $5.23 \mathrm{E}-01$ & $2.461 \mathrm{E}+01$ & $2.84 \mathrm{E}-01$ & $1.159 \mathrm{E}-01$ & $7.88 \mathrm{E}-03$ \\
\hline $8.989 \mathrm{E}+03$ & $1.171 \mathrm{E}+00$ & $1.634 \mathrm{E}+01$ & $6.06 \mathrm{E}-01$ & $3.333 \mathrm{E}+01$ & $3.82 \mathrm{E}-01$ & $1.207 \mathrm{E}-01$ & 6E-03 \\
\hline $1.012 \mathrm{E}+04$ & $1.172 \mathrm{E}+00$ & $2.286 \mathrm{E}+01$ & $6.76 \mathrm{E}-01$ & $4.440 \mathrm{E}+01$ & $5.01 \mathrm{E}-01$ & $1.227 \mathrm{E}-01$ & $4.65 \mathrm{E}-03$ \\
\hline $1.104 \mathrm{E}+04$ & $1.171 \mathrm{E}+00$ & $2.995 \mathrm{E}+01$ & $8.92 \mathrm{E}-01$ & $5.553 \mathrm{E}+01$ & $6.46 \mathrm{E}-01$ & $1.241 \mathrm{E}-01$ & $3.96 \mathrm{E}-03$ \\
\hline $1.201 \mathrm{E}+04$ & $1.173 \mathrm{E}+00$ & $3.888 \mathrm{E}+01$ & $9.47 \mathrm{E}-01$ & $7.038 \mathrm{E}+01$ & 7.98E-01 & $1.271 \mathrm{E}-01$ & $3.39 \mathrm{E}-03$ \\
\hline $1.296 \mathrm{E}+04$ & $1.173 \mathrm{E}+00$ & $4.957 \mathrm{E}+01$ & $1.05 \mathrm{E}+00$ & $8.799 \mathrm{E}+01$ & $1.01 \mathrm{E}+00$ & $1.289 \mathrm{E}-01$ & $2.93 \mathrm{E}-03$ \\
\hline $1.393 \mathrm{E}+04$ & $1.173 \mathrm{E}+00$ & $6.210 \mathrm{E}+01$ & $1.13 \mathrm{E}+00$ & $1.085 \mathrm{E}+02$ & $1.19 \mathrm{E}+00$ & $1.297 \mathrm{E}-01$ & $2.55 \mathrm{E}-03$ \\
\hline $1.482 \mathrm{E}+04$ & $1.173 \mathrm{E}+00$ & $7.548 \mathrm{E}+01$ & $1.21 \mathrm{E}+00$ & $1.317 \mathrm{E}+02$ & $1.43 \mathrm{E}+00$ & $1.308 \mathrm{E}-01$ & $2.31 \mathrm{E}-03$ \\
\hline $1.573 \mathrm{E}+04$ & $1.173 \mathrm{E}+00$ & $9.059 \mathrm{E}+01$ & $1.17 \mathrm{E}+00$ & $1.594 \mathrm{E}+02$ & $1.70 \mathrm{E}+00$ & $1.309 \mathrm{E}-01$ & $2.01 \mathrm{E}-03$ \\
\hline$C_{\mathrm{P}}$ & $\Delta C_{\mathrm{P}}$ & $C_{\mathrm{Q}}$ & $\Delta C_{\mathrm{Q}}$ & $\eta_{\mathrm{T}}$ & $\Delta \eta_{\mathrm{T}}$ & $R e_{0.75}$ & $\Delta R e_{0.75}$ \\
\hline $5.542 \mathrm{E}-02$ & $8.29 \mathrm{E}-03$ & $8.820 \mathrm{E}-03$ & $1.32 \mathrm{E}-03$ & $2.994 \mathrm{E}-01$ & $4.50 \mathrm{E}-02$ & $1.645 \mathrm{E}+04$ & $2.04 \mathrm{E}+01$ \\
\hline $5.133 \mathrm{E}-02$ & $2.37 \mathrm{E}-03$ & $8.169 \mathrm{E}-03$ & 3.77E-04 & $4.606 \mathrm{E}-01$ & $2.19 \mathrm{E}-02$ & $3.175 \mathrm{E}+04$ & $3.65 \mathrm{E}+01$ \\
\hline $5.132 \mathrm{E}-02$ & $1.91 \mathrm{E}-03$ & $8.168 \mathrm{E}-03$ & $3.03 \mathrm{E}-04$ & $4.903 \mathrm{E}-01$ & $1.90 \mathrm{E}-02$ & $3.585 \mathrm{E}+04$ & $4.04 \mathrm{E}+01$ \\
\hline $5.025 \mathrm{E}-02$ & $1.49 \mathrm{E}-03$ & $7.998 \mathrm{E}-03$ & $2.37 \mathrm{E}-04$ & $5.148 \mathrm{E}-01$ & $1.63 \mathrm{E}-02$ & $4.041 \mathrm{E}+04$ & $4.46 \mathrm{E}+01$ \\
\hline $5.078 \mathrm{E}-02$ & $1.51 \mathrm{E}-03$ & $8.082 \mathrm{E}-03$ & $2.41 \mathrm{E}-04$ & $5.393 \mathrm{E}-01$ & $1.72 \mathrm{E}-02$ & $4.403 \mathrm{E}+04$ & $4.89 \mathrm{E}+01$ \\
\hline $5.111 \mathrm{E}-02$ & $1.25 \mathrm{E}-03$ & $8.135 \mathrm{E}-03$ & $1.98 \mathrm{E}-04$ & $5.524 \mathrm{E}-01$ & $1.48 \mathrm{E}-02$ & $4.799 \mathrm{E}+04$ & $5.24 \mathrm{E}+01$ \\
\hline $5.184 \mathrm{E}-02$ & $1.10 \mathrm{E}-03$ & $8.250 \mathrm{E}-03$ & $1.75 \mathrm{E}-04$ & $5.633 \mathrm{E}-01$ & $1.36 \mathrm{E}-02$ & $5.181 \mathrm{E}+04$ & $5.68 \mathrm{E}+01$ \\
\hline $5.232 \mathrm{E}-02$ & $9.52 \mathrm{E}-04$ & $8.327 \mathrm{E}-03$ & $1.51 \mathrm{E}-04$ & $5.725 \mathrm{E}-01$ & $1.22 \mathrm{E}-02$ & $5.566 \mathrm{E}+04$ & $6.02 \mathrm{E}+01$ \\
\hline $5.286 \mathrm{E}-02$ & $8.50 \mathrm{E}-04$ & $8.820 \mathrm{E}-03$ & $1.35 \mathrm{E}-04$ & $5.732 \mathrm{E}-01$ & $1.11 \mathrm{E}-02$ & $5.921 \mathrm{E}+04$ & $6.50 \mathrm{E}+01$ \\
\hline $5.300 \mathrm{E}-02$ & 6.89E-04 & $8.436 \mathrm{E}-03$ & $1.09 \mathrm{E}-04$ & $5.685 \mathrm{E}-01$ & $9.54 \mathrm{E}-03$ & $6.286 \mathrm{E}+04$ & $6.93 \mathrm{E}+01$ \\
\hline
\end{tabular}




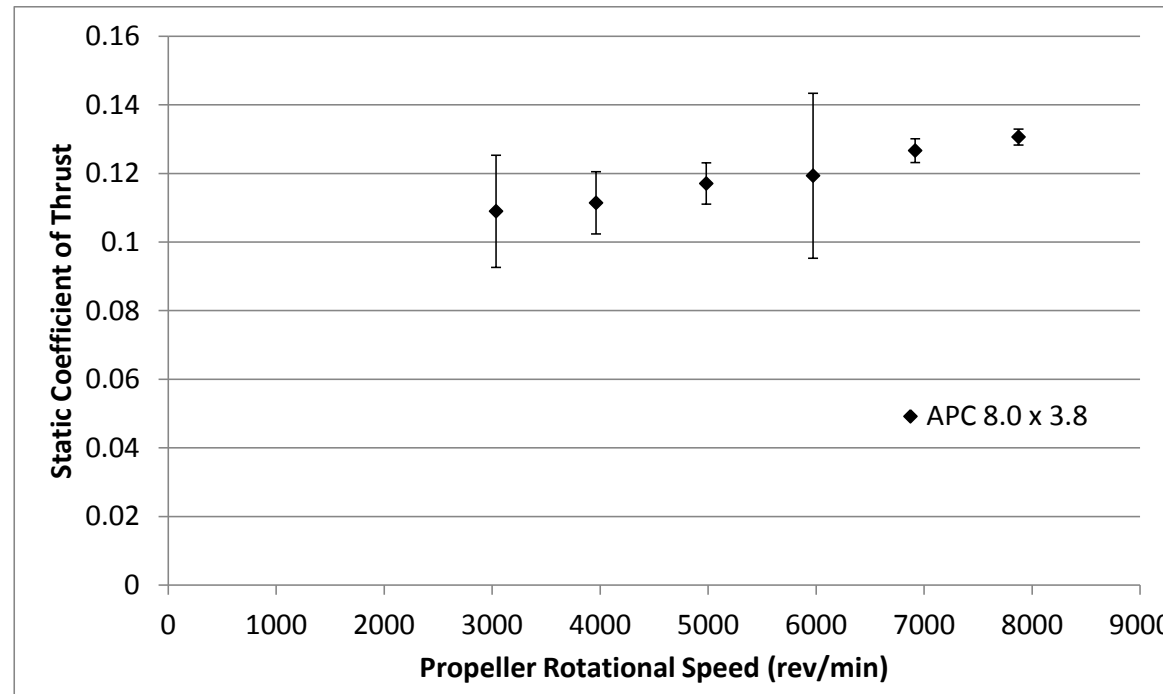

(a)

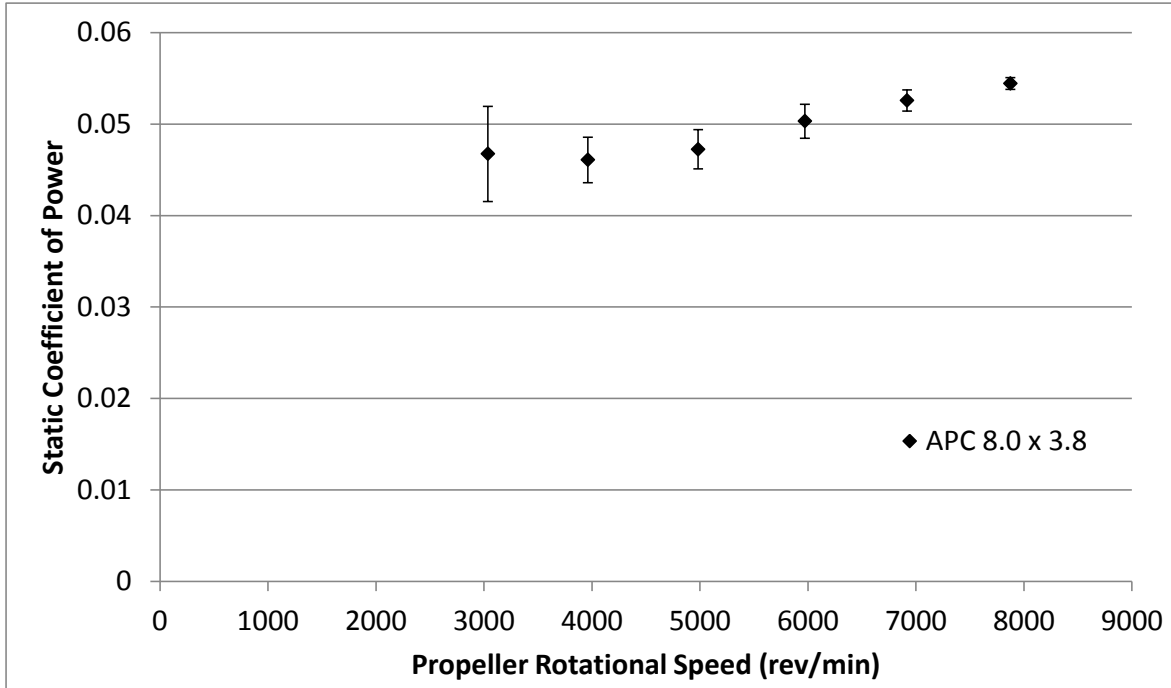

(b)

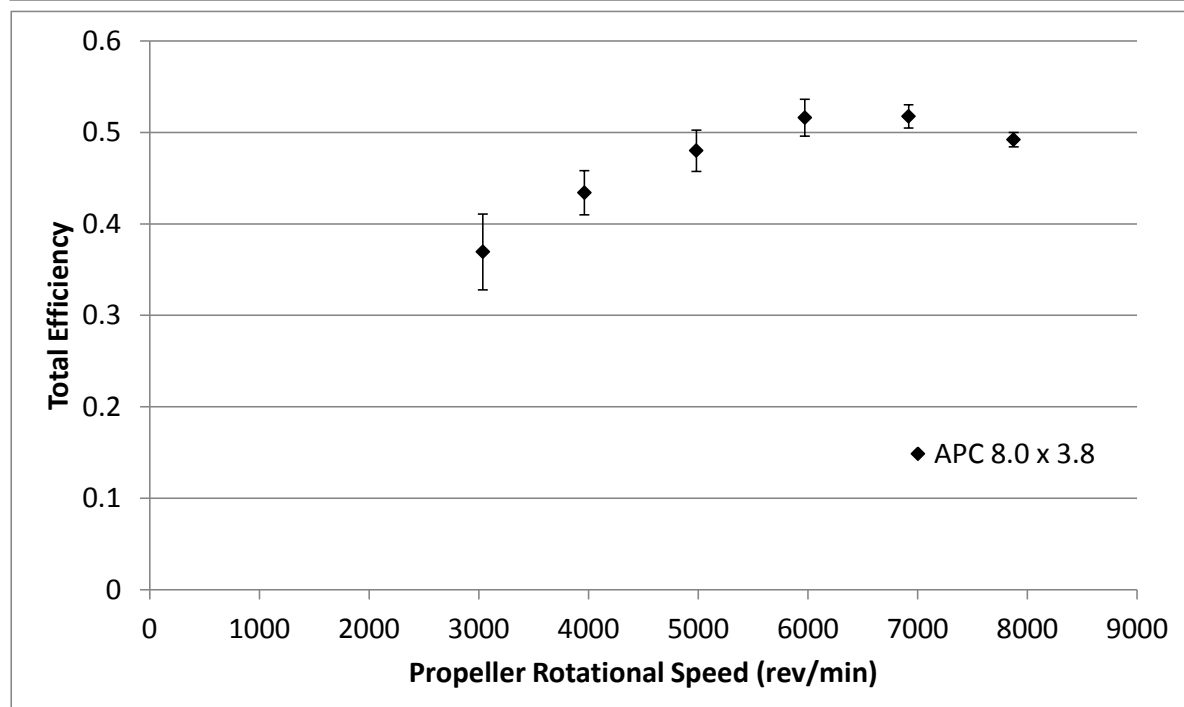

(c)

Figure 56: APC 8.0 x 3.8 Static Test Results: (a) Coefficient of Thrust, (b) Coefficient of Power, (c) Total Efficiency. 
Table 34: APC 8.0 x 3.8 Static Measured Values

\begin{tabular}{|c|c|c|c|c|c|c|c|c|}
\hline$n(\mathrm{RPM})$ & $T(\mathrm{~g})$ & $\Delta T(\mathrm{~g})$ & $Q(\mathrm{~g}-\mathrm{m})$ & $\Delta Q(\mathrm{~g}-\mathrm{m})$ & $V(\mathrm{~V})$ & $I(\mathrm{~A})$ & $T_{\text {atm }}\left({ }^{\circ} \mathrm{C}\right)$ & $P_{\mathrm{atm}}(\mathrm{Pa})$ \\
\hline $3.037 \mathrm{E}+03$ & $5.721 \mathrm{E}+01$ & $8.57 \mathrm{E}+00$ & $7.954 \mathrm{E}-01$ & $8.86 \mathrm{E}-02$ & $1.110 \mathrm{E}+01$ & $6.051 \mathrm{E}-01$ & $2.020 \mathrm{E}+01$ & $9.842 \mathrm{E}+04$ \\
\hline $3.963 \mathrm{E}+03$ & $9.955 \mathrm{E}+01$ & $8.09 \mathrm{E}+00$ & $1.334 \mathrm{E}+00$ & $7.18 \mathrm{E}-02$ & $1.109 \mathrm{E}+01$ & $1.128 \mathrm{E}+00$ & $2.022 \mathrm{E}+01$ & $9.834 \mathrm{E}+04$ \\
\hline $4.984 \mathrm{E}+03$ & $1.655 \mathrm{E}+02$ & $8.53 \mathrm{E}+00$ & $2.164 \mathrm{E}+00$ & $9.79 \mathrm{E}-02$ & $1.106 \mathrm{E}+01$ & $2.086 \mathrm{E}+00$ & $2.030 \mathrm{E}+01$ & $9.838 \mathrm{E}+04$ \\
\hline $5.972 \mathrm{E}+03$ & $2.422 \mathrm{E}+02$ & $4.88 \mathrm{E}+01$ & $3.310 \mathrm{E}+00$ & $1.22 \mathrm{E}-01$ & $1.102 \mathrm{E}+01$ & $3.567 \mathrm{E}+00$ & $2.024 \mathrm{E}+01$ & $9.838 \mathrm{E}+04$ \\
\hline $6.918 \mathrm{E}+03$ & $3.450 \mathrm{E}+02$ & $9.37 \mathrm{E}+00$ & $4.643 \mathrm{E}+00$ & $1.01 \mathrm{E}-01$ & $1.097 \mathrm{E}+01$ & $5.813 \mathrm{E}+00$ & $2.019 \mathrm{E}+01$ & $9.837 \mathrm{E}+04$ \\
\hline $7.875 \mathrm{E}+03$ & $4.613 \mathrm{E}+02$ & $8.14 \mathrm{E}+00$ & $6.233 \mathrm{E}+00$ & $7.21 \mathrm{E}-02$ & $1.088 \mathrm{E}+01$ & $9.415 \mathrm{E}+00$ & $2.011 \mathrm{E}+01$ & $9.841 \mathrm{E}+04$ \\
\hline
\end{tabular}

Table 35: APC 8.0 × 3.8 Static Calculated Values

\begin{tabular}{|r|c|c|c|c|c|c|c|}
\hline$n(\mathrm{RPM})$ & $\rho\left(\mathrm{kg} / \mathrm{m}^{\wedge} 3\right)$ & $P_{\mathrm{P}}(\mathrm{W})$ & $\Delta P_{\mathrm{P}}(\mathrm{W})$ & $P_{\mathrm{e}}(\mathrm{W})$ & $\Delta P_{\mathrm{e}}(\mathrm{W})$ & $C_{\mathrm{T}}$ & $\Delta C_{\mathrm{T}}$ \\
\hline $3.037 \mathrm{E}+03$ & $1.169 \mathrm{E}+00$ & $2.480 \mathrm{E}+00$ & $2.76 \mathrm{E}-01$ & $6.716 \mathrm{E}+00$ & $9.75 \mathrm{E}-02$ & $1.090 \mathrm{E}-01$ & $1.63 \mathrm{E}-02$ \\
\hline $3.963 \mathrm{E}+03$ & $1.168 \mathrm{E}+00$ & $5.430 \mathrm{E}+00$ & $2.92 \mathrm{E}-01$ & $1.251 \mathrm{E}+01$ & $1.67 \mathrm{E}-01$ & $1.114 \mathrm{E}-01$ & $9.06 \mathrm{E}-03$ \\
\hline $4.984 \mathrm{E}+03$ & $1.168 \mathrm{E}+00$ & $1.107 \mathrm{E}+01$ & $5.01 \mathrm{E}-01$ & $2.308 \mathrm{E}+01$ & $3.10 \mathrm{E}-01$ & $1.171 \mathrm{E}-01$ & $6.04 \mathrm{E}-03$ \\
\hline $5.972 \mathrm{E}+03$ & $1.168 \mathrm{E}+00$ & $2.030 \mathrm{E}+01$ & $7.51 \mathrm{E}-01$ & $3.933 \mathrm{E}+01$ & $4.88 \mathrm{E}-01$ & $1.193 \mathrm{E}-01$ & $2.40 \mathrm{E}-02$ \\
\hline $6.918 \mathrm{E}+03$ & $1.168 \mathrm{E}+00$ & $3.299 \mathrm{E}+01$ & $7.21 \mathrm{E}-01$ & $6.374 \mathrm{E}+01$ & $7.39 \mathrm{E}-01$ & $1.266 \mathrm{E}-01$ & $3.44 \mathrm{E}-03$ \\
\hline $7.875 \mathrm{E}+03$ & $1.169 \mathrm{E}+00$ & $5.041 \mathrm{E}+01$ & $5.85 \mathrm{E}-01$ & $1.024 \mathrm{E}+02$ & $1.14 \mathrm{E}+00$ & $1.306 \mathrm{E}-01$ & $2.32 \mathrm{E}-03$ \\
\hline$C_{\mathrm{P}}$ & $\Delta C_{\mathrm{P}}$ & $C_{\mathrm{Q}}$ & $\Delta C_{\mathrm{Q}}$ & $\eta_{\mathrm{T}}$ & $\Delta \eta_{\mathrm{T}}$ & $R e_{0.75}$ & $\Delta R e_{0.75}$ \\
\hline $4.673 \mathrm{E}-02$ & $5.21 \mathrm{E}-03$ & $7.438 \mathrm{E}-03$ & $8.29 \mathrm{E}-04$ & $3.693 \mathrm{E}-01$ & $4.15 \mathrm{E}-02$ & $3.259 \mathrm{E}+04$ & $3.92 \mathrm{E}+01$ \\
\hline $4.608 \mathrm{E}-02$ & $2.48 \mathrm{E}-03$ & $7.334 \mathrm{E}-03$ & $3.95 \mathrm{E}-04$ & $4.341 \mathrm{E}-01$ & $2.41 \mathrm{E}-02$ & $4.249 \mathrm{E}+04$ & $3.91 \mathrm{E}+01$ \\
\hline $4.724 \mathrm{E}-02$ & $2.14 \mathrm{E}-03$ & $7.518 \mathrm{E}-03$ & $3.40 \mathrm{E}-04$ & $4.799 \mathrm{E}-01$ & $2.27 \mathrm{E}-02$ & $5.343 \mathrm{E}+04$ & $4.77 \mathrm{E}+01$ \\
\hline $5.031 \mathrm{E}-02$ & $1.86 \mathrm{E}-03$ & $8.007 \mathrm{E}-03$ & $2.96 \mathrm{E}-04$ & $5.161 \mathrm{E}-01$ & $2.01 \mathrm{E}-02$ & $6.404 \mathrm{E}+04$ & $5.65 \mathrm{E}+01$ \\
\hline $5.259 \mathrm{E}-02$ & $1.16 \mathrm{E}-03$ & $8.370 \mathrm{E}-03$ & $1.83 \mathrm{E}-04$ & $5.175 \mathrm{E}-01$ & $1.28 \mathrm{E}-02$ & $7.420 \mathrm{E}+04$ & $7.30 \mathrm{E}+01$ \\
\hline $5.444 \mathrm{E}-02$ & $6.52 \mathrm{E}-04$ & $8.665 \mathrm{E}-03$ & $1.02 \mathrm{E}-04$ & $4.922 \mathrm{E}-01$ & $7.91 \mathrm{E}-03$ & $8.454 \mathrm{E}+04$ & $9.71 \mathrm{E}+01$ \\
\hline
\end{tabular}




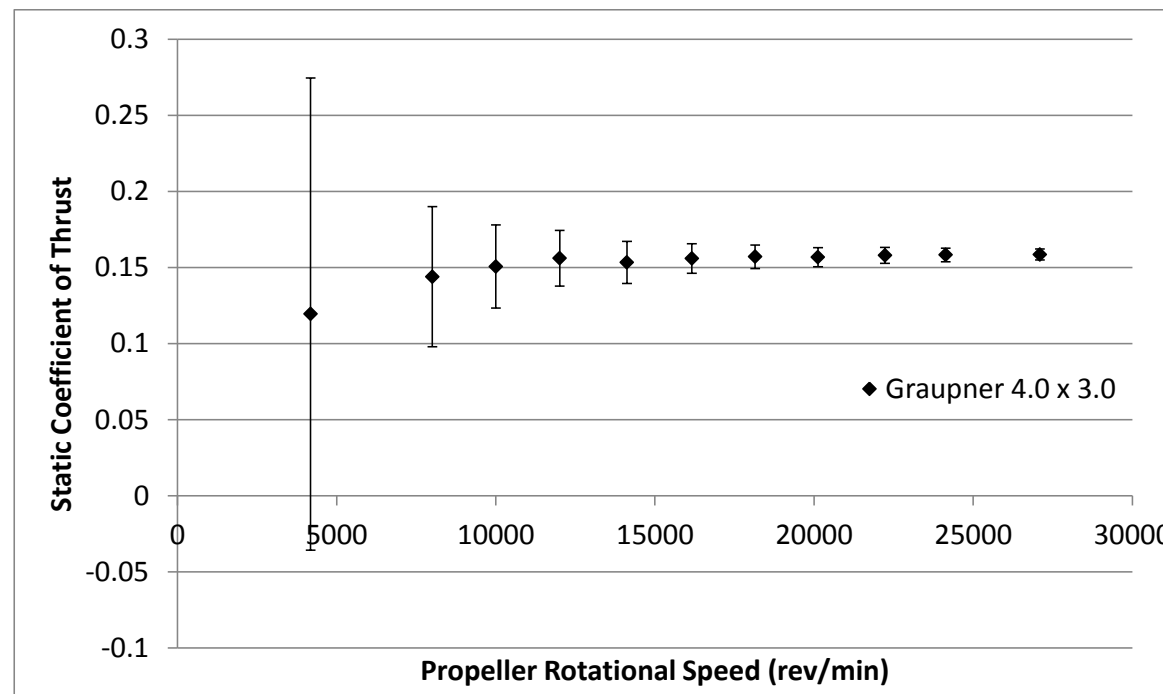

(a)

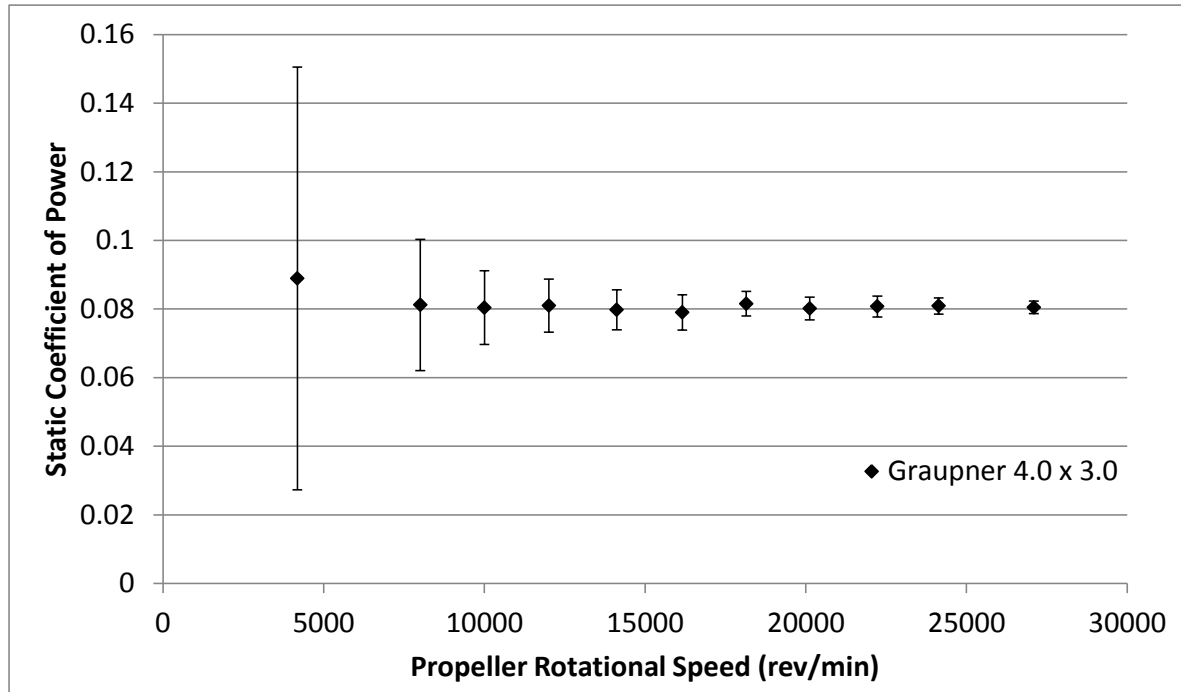

(b)

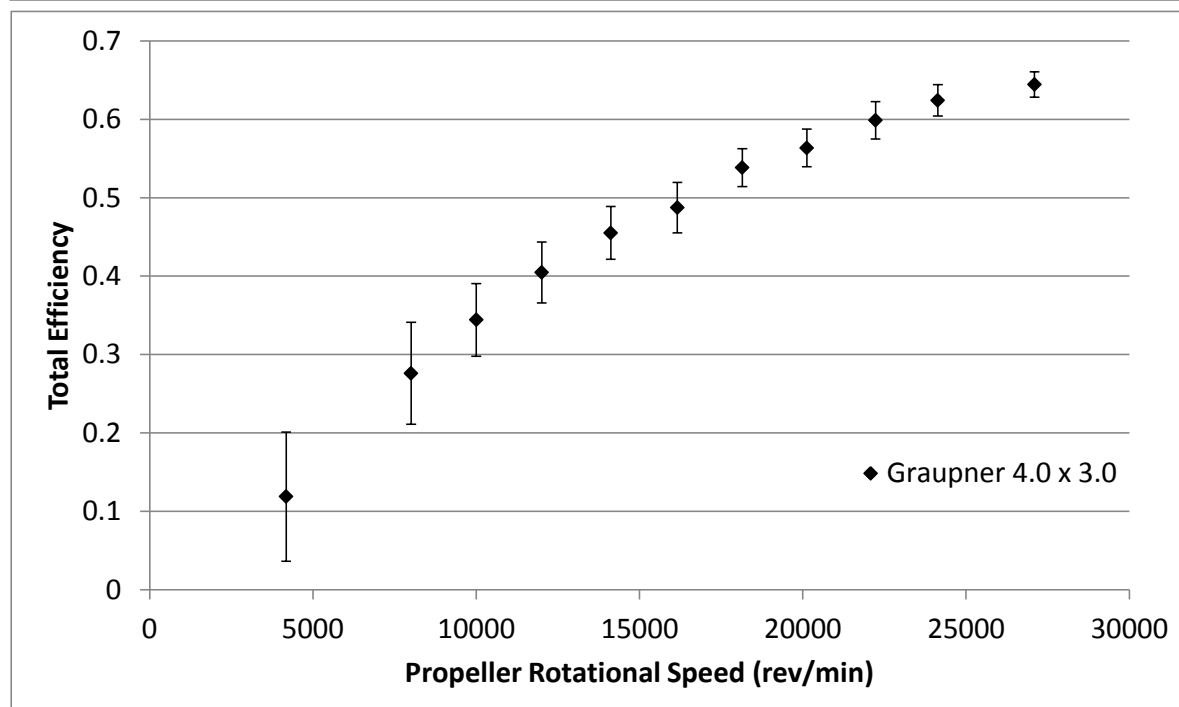

(c)

Figure 57: Graupner 4.0 x 3.0 Static Test Results: (a) Coefficient of Thrust, (b) Coefficient of Power, (c) Total Efficiency. 
Table 36: Graupner 4.0 x 3.0 Static Measured Values

\begin{tabular}{|c|c|c|c|c|c|c|c|c|}
\hline$n(\mathrm{RPM})$ & $T(\mathrm{~g})$ & $\Delta T(\mathrm{~g})$ & $Q(\mathrm{~g}-\mathrm{m})$ & $\Delta Q(\mathrm{~g}-\mathrm{m})$ & $V(\mathrm{~V})$ & $I(\mathrm{~A})$ & $T_{\text {atm }}\left({ }^{\circ} \mathrm{C}\right)$ & $P_{\text {atm }}(\mathrm{Pa})$ \\
\hline $4.181 \mathrm{E}+03$ & $6.878 \mathrm{E}+00$ & $8.93 \mathrm{E}+00$ & $8.140 \mathrm{E}-02$ & $5.64 \mathrm{E}-02$ & $1.108 \mathrm{E}+01$ & $2.655 \mathrm{E}-01$ & $2.218 \mathrm{E}+01$ & $9.891 \mathrm{E}+04$ \\
\hline $8.007 \mathrm{E}+03$ & $3.040 \mathrm{E}+01$ & $9.72 \mathrm{E}+00$ & $2.726 \mathrm{E}-01$ & $6.41 \mathrm{E}-02$ & $1.107 \mathrm{E}+01$ & $7.335 \mathrm{E}-01$ & $2.225 \mathrm{E}+01$ & $9.892 \mathrm{E}+04$ \\
\hline $1.000 \mathrm{E}+04$ & $4.965 \mathrm{E}+01$ & $9.01 \mathrm{E}+00$ & $4.214 \mathrm{E}-01$ & $5.64 \mathrm{E}-02$ & $1.106 \mathrm{E}+01$ & $1.137 \mathrm{E}+00$ & $2.225 \mathrm{E}+01$ & $9.893 \mathrm{E}+04$ \\
\hline $1.201 \mathrm{E}+04$ & $7.421 \mathrm{E}+01$ & $8.69 \mathrm{E}+00$ & $6.126 \mathrm{E}-01$ & $5.85 \mathrm{E}-02$ & $1.105 \mathrm{E}+01$ & $1.689 \mathrm{E}+00$ & $2.191 \mathrm{E}+01$ & $9.892 \mathrm{E}+04$ \\
\hline $1.412 \mathrm{E}+04$ & $1.008 \mathrm{E}+02$ & $9.09 \mathrm{E}+00$ & $8.342 \mathrm{E}-01$ & $6.10 \mathrm{E}-02$ & $1.103 \mathrm{E}+01$ & $2.409 \mathrm{E}+00$ & $2.189 \mathrm{E}+01$ & $9.892 \mathrm{E}+04$ \\
\hline $1.616 \mathrm{E}+04$ & $1.343 \mathrm{E}+02$ & $8.33 \mathrm{E}+00$ & $1.082 \mathrm{E}+00$ & $7.02 \mathrm{E}-02$ & $1.101 \mathrm{E}+01$ & $3.346 \mathrm{E}+00$ & $2.188 \mathrm{E}+01$ & $9.893 \mathrm{E}+04$ \\
\hline $1.815 \mathrm{E}+04$ & $1.706 \mathrm{E}+02$ & $8.36 \mathrm{E}+00$ & $1.409 \mathrm{E}+00$ & $6.17 \mathrm{E}-02$ & $1.099 \mathrm{E}+01$ & $4.439 \mathrm{E}+00$ & $2.182 \mathrm{E}+01$ & $9.891 \mathrm{E}+04$ \\
\hline $2.012 \mathrm{E}+04$ & $2.094 \mathrm{E}+02$ & $8.44 \mathrm{E}+00$ & $1.703 \mathrm{E}+00$ & $7.01 \mathrm{E}-02$ & $1.096 \mathrm{E}+01$ & $5.697 \mathrm{E}+00$ & $2.185 \mathrm{E}+01$ & $9.891 \mathrm{E}+04$ \\
\hline $2.223 \mathrm{E}+04$ & $2.574 \mathrm{E}+02$ & $8.47 \mathrm{E}+00$ & $2.092 \mathrm{E}+00$ & $7.92 \mathrm{E}-02$ & $1.092 \mathrm{E}+01$ & $7.303 \mathrm{E}+00$ & $2.183 \mathrm{E}+01$ & $9.891 \mathrm{E}+04$ \\
\hline $2.414 \mathrm{E}+04$ & $3.041 \mathrm{E}+02$ & $8.61 \mathrm{E}+00$ & $2.472 \mathrm{E}+00$ & $7.31 \mathrm{E}-02$ & $1.088 \mathrm{E}+01$ & $9.018 \mathrm{E}+00$ & $2.178 \mathrm{E}+01$ & $9.892 \mathrm{E}+04$ \\
\hline $2.710 \mathrm{E}+04$ & $3.841 \mathrm{E}+02$ & $8.62 \mathrm{E}+00$ & $3.101 \mathrm{E}+00$ & $7.03 \mathrm{E}-02$ & $1.081 \mathrm{E}+01$ & $1.239 \mathrm{E}+01$ & $2.175 \mathrm{E}+01$ & $9.892 \mathrm{E}+04$ \\
\hline
\end{tabular}

Table 37: Graupner 4.0 x 3.0 Static Calculated Values

\begin{tabular}{|c|c|c|c|c|c|c|c|}
\hline$n(\mathrm{RPM})$ & $\rho\left(\mathrm{kg} / \mathrm{m}^{\wedge} 3\right)$ & $P_{\mathrm{P}}(\mathrm{W})$ & $\Delta P_{\mathrm{P}}(\mathrm{W})$ & $P_{\mathrm{e}}(\mathrm{W})$ & $\Delta P_{\mathrm{e}}(\mathrm{W})$ & $C_{\mathrm{T}}$ & $\Delta C_{\mathrm{T}}$ \\
\hline $4.181 \mathrm{E}+03$ & $1.167 \mathrm{E}+00$ & $3.495 \mathrm{E}-01$ & $2.42 \mathrm{E}-01$ & $2.942 \mathrm{E}+00$ & $5.13 \mathrm{E}-02$ & $1.195 \mathrm{E}-01$ & $1.55 \mathrm{E}-01$ \\
\hline $8.007 \mathrm{E}+03$ & $1.167 \mathrm{E}+00$ & $2.241 \mathrm{E}+00$ & $5.27 \mathrm{E}-01$ & $8.122 \mathrm{E}+00$ & $1.05 \mathrm{E}-01$ & $1.440 \mathrm{E}-01$ & $4.60 \mathrm{E}-02$ \\
\hline $1.000 \mathrm{E}+04$ & $1.167 \mathrm{E}+00$ & $4.329 \mathrm{E}+00$ & 5.79E-01 & $1.258 \mathrm{E}+01$ & $1.52 \mathrm{E}-01$ & $1.507 \mathrm{E}-01$ & $2.73 \mathrm{E}-02$ \\
\hline $1.201 \mathrm{E}+04$ & $1.168 \mathrm{E}+00$ & $7.554 \mathrm{E}+00$ & $7.21 \mathrm{E}-01$ & $1.866 \mathrm{E}+01$ & $2.15 \mathrm{E}-01$ & $1.561 \mathrm{E}-01$ & $1.83 \mathrm{E}-02$ \\
\hline $1.412 \mathrm{E}+04$ & $1.168 \mathrm{E}+00$ & $1.210 \mathrm{E}+01$ & $8.84 \mathrm{E}-01$ & $2.658 \mathrm{E}+01$ & $3.00 \mathrm{E}-01$ & $1.533 \mathrm{E}-01$ & $1.38 \mathrm{E}-02$ \\
\hline $1.616 \mathrm{E}+04$ & $1.168 \mathrm{E}+00$ & $1.796 \mathrm{E}+01$ & $1.17 \mathrm{E}+00$ & $3.685 \mathrm{E}+01$ & $4.18 \mathrm{E}-01$ & $1.559 \mathrm{E}-01$ & $9.67 \mathrm{E}-03$ \\
\hline $1.815 \mathrm{E}+04$ & $1.168 \mathrm{E}+00$ & $2.626 \mathrm{E}+01$ & $1.15 \mathrm{E}+00$ & $4.878 \mathrm{E}+01$ & $5.37 \mathrm{E}-01$ & $1.570 \mathrm{E}-01$ & $7.69 \mathrm{E}-03$ \\
\hline $2.012 \mathrm{E}+04$ & $1.168 \mathrm{E}+00$ & $3.518 \mathrm{E}+01$ & $1.45 \mathrm{E}+00$ & $6.244 \mathrm{E}+01$ & $7.06 \mathrm{E}-01$ & $1.568 \mathrm{E}-01$ & $6.32 \mathrm{E}-03$ \\
\hline $2.223 \mathrm{E}+04$ & $1.168 \mathrm{E}+00$ & $4.776 \mathrm{E}+01$ & $1.81 \mathrm{E}+00$ & $7.978 \mathrm{E}+01$ & $9.59 \mathrm{E}-01$ & $1.579 \mathrm{E}-01$ & $5.20 \mathrm{E}-03$ \\
\hline $2.414 \mathrm{E}+04$ & $1.168 \mathrm{E}+00$ & $6.126 \mathrm{E}+01$ & $1.81 \mathrm{E}+00$ & $9.815 \mathrm{E}+01$ & $1.19 \mathrm{E}+00$ & $1.582 \mathrm{E}-01$ & $4.48 \mathrm{E}-03$ \\
\hline $2.710 \mathrm{E}+04$ & $1.169 \mathrm{E}+00$ & $8.628 \mathrm{E}+01$ & $1.96 \mathrm{E}+00$ & $9 \mathrm{E}+02$ & $1.44 \mathrm{E}+00$ & 1 & 03 \\
\hline$C_{\mathrm{P}}$ & $\Delta C_{\mathrm{P}}$ & $C_{\mathrm{Q}}$ & $\Delta C_{\mathrm{Q}}$ & $\eta_{\mathrm{T}}$ & $\Delta \eta_{\mathrm{T}}$ & $\operatorname{Re}_{0.75}$ & $\Delta R e_{0.75}$ \\
\hline $8.890 \mathrm{E}-02$ & $6.16 \mathrm{E}-02$ & $1.415 \mathrm{E}-02$ & $9.81 \mathrm{E}-03$ & $1.188 \mathrm{E}-01$ & $8.23 \mathrm{E}-02$ & $9.948 \mathrm{E}+03$ & $1.39 \mathrm{E}+01$ \\
\hline $8.118 \mathrm{E}-02$ & $1.91 \mathrm{E}-02$ & $1.292 \mathrm{E}-02$ & $3.04 \mathrm{E}-03$ & $2.760 \mathrm{E}-01$ & $6.50 \mathrm{E}-02$ & $1.904 \mathrm{E}+04$ & $2.42 \mathrm{E}+01$ \\
\hline $8.041 \mathrm{E}-02$ & $1.08 \mathrm{E}-02$ & $1.280 \mathrm{E}-02$ & $1.71 \mathrm{E}-03$ & $3.441 \mathrm{E}-01$ & $4.62 \mathrm{E}-02$ & $2.379 \mathrm{E}+04$ & $2.91 \mathrm{E}+01$ \\
\hline $8.101 \mathrm{E}-02$ & 7.73E-03 & $1.289 \mathrm{E}-02$ & $1.23 \mathrm{E}-03$ & 4.047E-01 & $3.89 \mathrm{E}-02$ & $2.862 \mathrm{E}+04$ & $3.46 \mathrm{E}+01$ \\
\hline 7.977E-02 & $5.83 \mathrm{E}-03$ & $1.270 \mathrm{E}-02$ & $9.28 \mathrm{E}-04$ & $4.552 \mathrm{E}-01$ & $3.37 \mathrm{E}-02$ & $3.366 \mathrm{E}+04$ & $4.04 \mathrm{E}+01$ \\
\hline $7.902 \mathrm{E}-02$ & $5.13 \mathrm{E}-03$ & $1.258 \mathrm{E}-02$ & $8.16 \mathrm{E}-04$ & $4.874 \mathrm{E}-01$ & $3.21 \mathrm{E}-02$ & $3.852 \mathrm{E}+04$ & $4.57 \mathrm{E}+01$ \\
\hline $8.155 \mathrm{E}-02$ & $3.57 \mathrm{E}-03$ & $1.298 \mathrm{E}-02$ & $5.69 \mathrm{E}-04$ & $5.384 \mathrm{E}-01$ & $2.43 \mathrm{E}-02$ & $4.328 \mathrm{E}+04$ & $5.12 \mathrm{E}+01$ \\
\hline $8.016 \mathrm{E}-02$ & $3.30 \mathrm{E}-03$ & $1.276 \mathrm{E}-02$ & $5.25 \mathrm{E}-04$ & $5.635 \mathrm{E}-01$ & $2.41 \mathrm{E}-02$ & $4.798 \mathrm{E}+04$ & $5.73 \mathrm{E}+01$ \\
\hline $8.073 \mathrm{E}-02$ & $3.06 \mathrm{E}-03$ & $1.415 \mathrm{E}-02$ & $4.86 \mathrm{E}-04$ & $5.987 \mathrm{E}-01$ & $2.38 \mathrm{E}-02$ & $5.300 \mathrm{E}+04$ & $6.25 \mathrm{E}+01$ \\
\hline $8.087 \mathrm{E}-02$ & $2.39 \mathrm{E}-03$ & $1.287 \mathrm{E}-02$ & $3.81 \mathrm{E}-04$ & $6.242 \mathrm{E}-01$ & $1.99 \mathrm{E}-02$ & $5.757 \mathrm{E}+04$ & $6.75 \mathrm{E}+01$ \\
\hline $8.048 \mathrm{E}-02$ & $1.83 \mathrm{E}-03$ & $1.281 \mathrm{E}-02$ & $2.91 \mathrm{E}-04$ & $6.444 \mathrm{E}-01$ & $1.62 \mathrm{E}-02$ & $6.464 \mathrm{E}+04$ & $7.56 \mathrm{E}+01$ \\
\hline
\end{tabular}




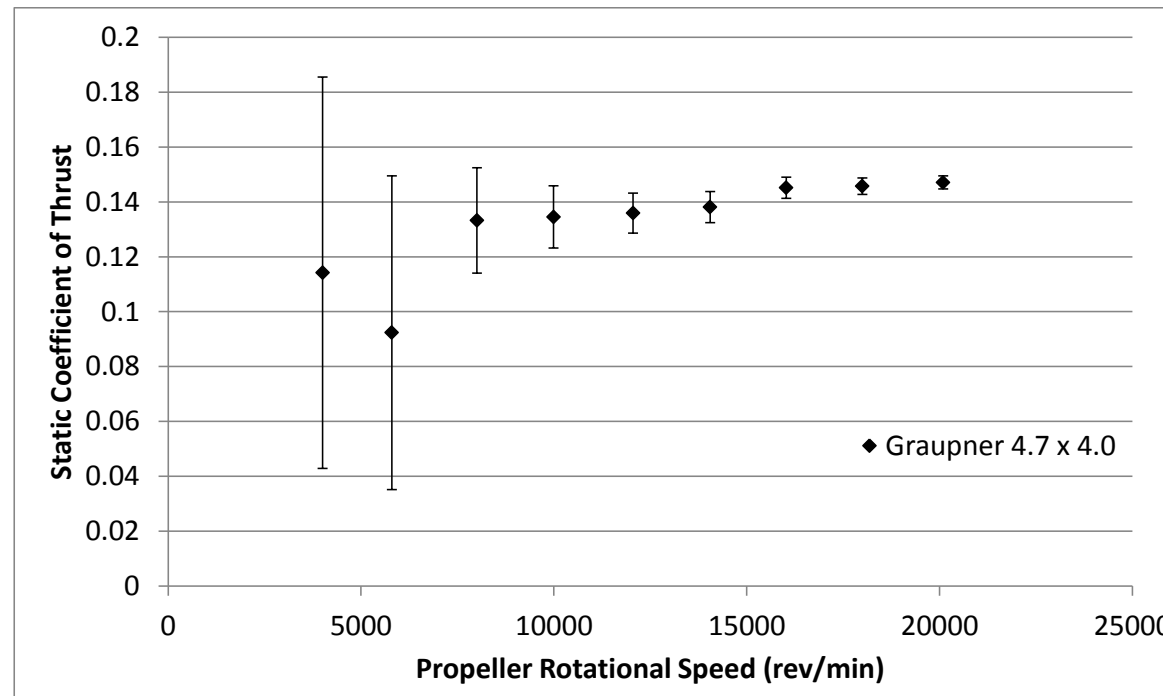

(a)

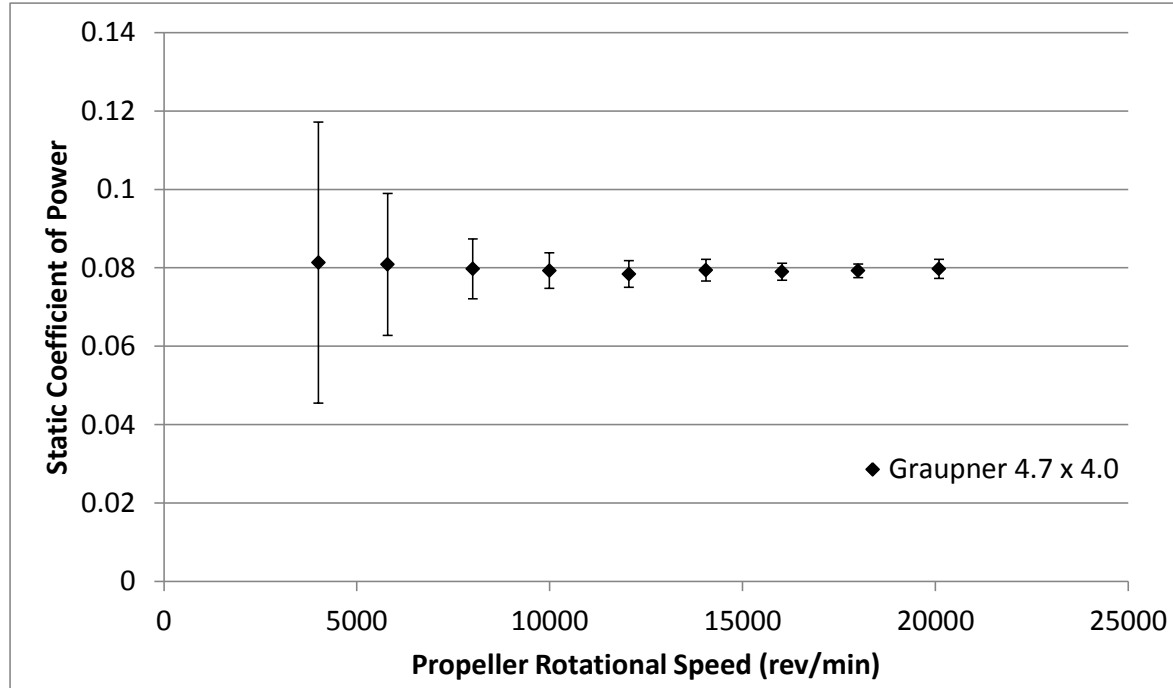

(b)

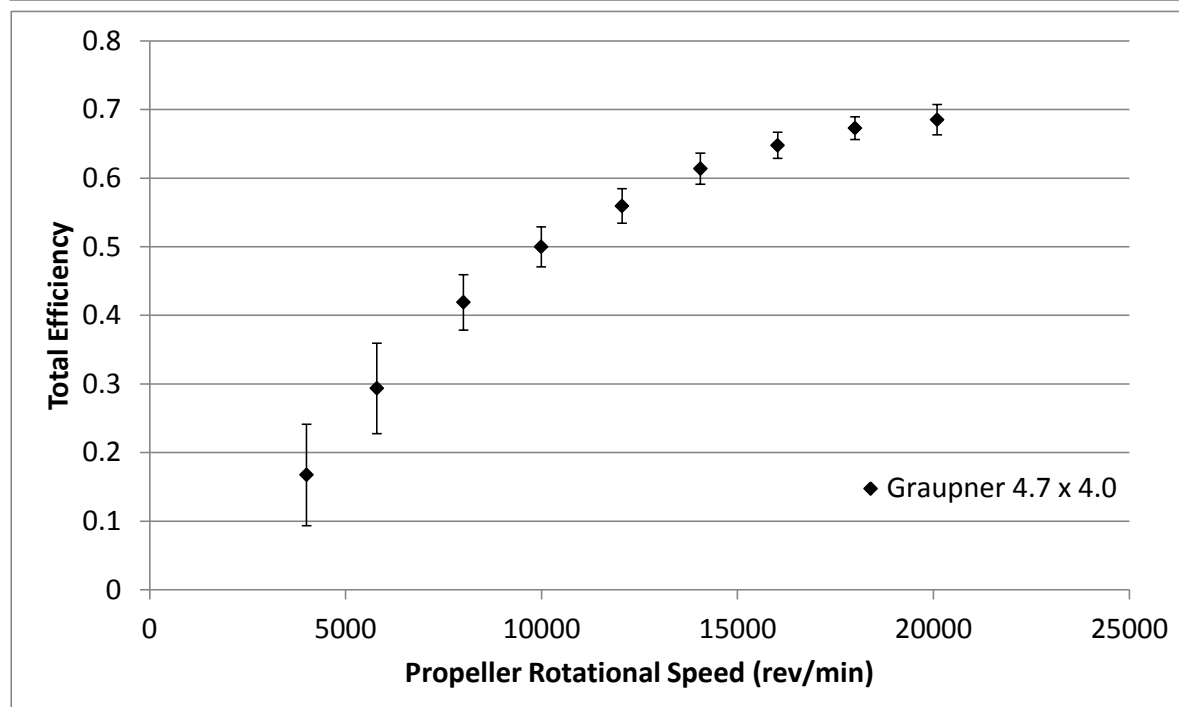

(c)

Figure 58: Graupner 4.7 x 4.0 Static Test Results: (a) Coefficient of Thrust, (b) Coefficient of Power, (c) Total Efficiency. 
Table 38: Graupner 4.7 x 4.0 Static Measured Values

\begin{tabular}{|c|c|c|c|c|c|c|c|c|}
\hline$n(\mathrm{RPM})$ & $T(\mathrm{~g})$ & $\Delta T(\mathrm{~g})$ & $Q(\mathrm{~g}-\mathrm{m})$ & $\Delta Q(\mathrm{~g}-\mathrm{m})$ & $V(\mathrm{~V})$ & $I(\mathrm{~A})$ & $T_{\text {atm }}\left({ }^{\circ} \mathrm{C}\right)$ & $P_{\text {atm }}(\mathrm{Pa})$ \\
\hline $4.004 \mathrm{E}+03$ & $1.208 \mathrm{E}+01$ & $7.55 \mathrm{E}+00$ & $1.631 \mathrm{E}-01$ & $7.19 \mathrm{E}-02$ & $1.111 \mathrm{E}+01$ & $3.608 \mathrm{E}-01$ & $2.331 \mathrm{E}+01$ & $9.856 \mathrm{E}+04$ \\
\hline $5.799 \mathrm{E}+03$ & $2.049 \mathrm{E}+01$ & $1.27 \mathrm{E}+01$ & $3.401 \mathrm{E}-01$ & $7.61 \mathrm{E}-02$ & $1.110 \mathrm{E}+01$ & $6.220 \mathrm{E}-01$ & $2.333 \mathrm{E}+01$ & $9.856 \mathrm{E}+04$ \\
\hline $8.008 \mathrm{E}+03$ & $5.639 \mathrm{E}+01$ & $8.11 \mathrm{E}+00$ & $6.394 \mathrm{E}-01$ & $6.12 \mathrm{E}-02$ & $1.108 \mathrm{E}+01$ & $1.132 \mathrm{E}+00$ & $2.332 \mathrm{E}+01$ & $9.855 \mathrm{E}+04$ \\
\hline $9.992 \mathrm{E}+03$ & $8.862 \mathrm{E}+01$ & $7.46 \mathrm{E}+00$ & $9.901 \mathrm{E}-01$ & $5.70 \mathrm{E}-02$ & $1.106 \mathrm{E}+01$ & $1.837 \mathrm{E}+00$ & $2.329 \mathrm{E}+01$ & $9.855 \mathrm{E}+04$ \\
\hline $1.205 \mathrm{E}+04$ & $1.303 \mathrm{E}+02$ & $6.96 \mathrm{E}+00$ & $1.425 \mathrm{E}+00$ & $6.19 \mathrm{E}-02$ & $1.104 \mathrm{E}+01$ & $2.858 \mathrm{E}+00$ & $2.334 \mathrm{E}+01$ & $9.854 \mathrm{E}+04$ \\
\hline $1.405 \mathrm{E}+04$ & $1.799 \mathrm{E}+02$ & $7.42 \mathrm{E}+00$ & $1.961 \mathrm{E}+00$ & $6.86 \mathrm{E}-02$ & $1.100 \mathrm{E}+01$ & $4.191 \mathrm{E}+00$ & $2.332 \mathrm{E}+01$ & $9.854 \mathrm{E}+04$ \\
\hline $1.603 \mathrm{E}+04$ & $2.459 \mathrm{E}+02$ & $6.48 \mathrm{E}+00$ & $2.536 \mathrm{E}+00$ & $6.91 \mathrm{E}-02$ & $1.095 \mathrm{E}+01$ & $5.885 \mathrm{E}+00$ & $2.334 \mathrm{E}+01$ & $9.854 \mathrm{E}+04$ \\
\hline $1.800 \mathrm{E}+04$ & $3.115 \mathrm{E}+02$ & $6.42 \mathrm{E}+00$ & $3.211 \mathrm{E}+00$ & $7.11 \mathrm{E}-02$ & $1.089 \mathrm{E}+01$ & $8.103 \mathrm{E}+00$ & $2.323 \mathrm{E}+01$ & $9.854 \mathrm{E}+04$ \\
\hline $2.010 \mathrm{E}+04$ & $3.920 \mathrm{E}+02$ & $6.39 \mathrm{E}+00$ & $4.028 \mathrm{E}+00$ & $1.23 \mathrm{E}-01$ & $1.080 \mathrm{E}+01$ & $1.123 \mathrm{E}+01$ & $2.330 \mathrm{E}+01$ & $9.856 \mathrm{E}+04$ \\
\hline
\end{tabular}

Table 39: Graupner 4.7 x 4.0 Static Calculated Values

\begin{tabular}{|c|c|c|c|c|c|c|c|}
\hline$n(\mathrm{RPM})$ & $\rho\left(\mathrm{kg} / \mathrm{m}^{\wedge} 3\right)$ & $P_{\mathrm{P}}(\mathrm{W})$ & $\Delta P_{\mathrm{P}}(\mathrm{W})$ & $P_{\mathrm{e}}(\mathrm{W})$ & $\Delta P_{\mathrm{e}}(\mathrm{W})$ & $C_{\mathrm{T}}$ & $\Delta C_{\mathrm{T}}$ \\
\hline $4.004 \mathrm{E}+03$ & $1.158 \mathrm{E}+00$ & $705 \mathrm{E}-01$ & $2.96 \mathrm{E}-01$ & $4.007 \mathrm{E}+00$ & $6.36 \mathrm{E}-02$ & $1.142 \mathrm{E}-01$ & $7.14 \mathrm{E}-02$ \\
\hline $5.799 \mathrm{E}+03$ & $1.158 \mathrm{E}+00$ & $2.026 \mathrm{E}+00$ & $4.53 \mathrm{E}-01$ & $6.903 \mathrm{E}+00$ & $9.52 \mathrm{E}-02$ & $9.235 \mathrm{E}-02$ & $5.72 \mathrm{E}-02$ \\
\hline $8.008 \mathrm{E}+03$ & $1.158 \mathrm{E}+00$ & $5.258 \mathrm{E}+00$ & $5.04 \mathrm{E}-01$ & $1.255 \mathrm{E}+01$ & $1.52 \mathrm{E}-01$ & $1.333 \mathrm{E}-01$ & $1.92 \mathrm{E}-02$ \\
\hline $9.992 \mathrm{E}+03$ & $1.158 \mathrm{E}+00$ & $1.016 \mathrm{E}+01$ & $5.84 \mathrm{E}-01$ & $2.033 \mathrm{E}+01$ & $2.35 \mathrm{E}-01$ & $1.345 \mathrm{E}-01$ & $1.13 \mathrm{E}-02$ \\
\hline $1.205 \mathrm{E}+04$ & $1.158 \mathrm{E}+00$ & $1.764 \mathrm{E}+01$ & $7.66 \mathrm{E}-01$ & $3.154 \mathrm{E}+01$ & $3.54 \mathrm{E}-01$ & $1.359 \mathrm{E}-01$ & $7.26 \mathrm{E}-03$ \\
\hline $1.405 \mathrm{E}+04$ & $1.158 \mathrm{E}+00$ & $2.830 \mathrm{E}+01$ & $9.91 \mathrm{E}-01$ & $4.610 \mathrm{E}+01$ & $5.15 \mathrm{E}-01$ & $1.381 \mathrm{E}-01$ & $5.69 \mathrm{E}-03$ \\
\hline $1.603 \mathrm{E}+04$ & $1.158 \mathrm{E}+00$ & $4.174 \mathrm{E}+01$ & $1.14 \mathrm{E}+00$ & $6.444 \mathrm{E}+01$ & -01 & $1.452 \mathrm{E}-01$ & -03 \\
\hline $1.800 \mathrm{E}+04$ & $1.158 \mathrm{E}+00$ & $5.935 \mathrm{E}+01$ & 1.3 & $2 \mathrm{E}+01$ & 01 & 01 & -03 \\
\hline $2.010 \mathrm{E}+04$ & $1.158 \mathrm{E}+00$ & $8.313 \mathrm{E}+01$ & $2.54 \mathrm{E}+00$ & $1.213 \mathrm{E}+02$ & $1.29 \mathrm{E}+00$ & $1.471 \mathrm{E}-01$ & $2.40 \mathrm{E}-03$ \\
\hline$C_{\mathrm{P}}$ & $\Delta C_{\mathrm{P}}$ & $C_{\mathrm{Q}}$ & $\Delta C_{\mathrm{Q}}$ & $\eta_{\mathrm{T}}$ & $\Delta \eta_{\mathrm{T}}$ & $R e_{0.75}$ & $\Delta R e_{0.75}$ \\
\hline $8.133 \mathrm{E}-02$ & $3.59 \mathrm{E}-02$ & $1.294 \mathrm{E}-02$ & $5.71 \mathrm{E}-03$ & $1.673 \mathrm{E}-01$ & $7.38 \mathrm{E}-02$ & $1.123 \mathrm{E}+04$ & $1.48 \mathrm{E}+01$ \\
\hline $8.087 \mathrm{E}-02$ & $1.81 \mathrm{E}-02$ & $1.287 \mathrm{E}-02$ & $2.88 \mathrm{E}-03$ & $2.935 \mathrm{E}-01$ & $6.58 \mathrm{E}-02$ & $1.626 \mathrm{E}+04$ & $2.44 \mathrm{E}+01$ \\
\hline 7.974E-02 & $7.64 \mathrm{E}-03$ & $1.269 \mathrm{E}-02$ & $1.22 \mathrm{E}-03$ & $4.189 \mathrm{E}-01$ & 4.04E-02 & $2.245 \mathrm{E}+04$ & $2.92 \mathrm{E}+01$ \\
\hline $7.930 \mathrm{E}-02$ & $4.56 \mathrm{E}-03$ & $1.262 \mathrm{E}-02$ & $7.26 \mathrm{E}-04$ & $4.998 \mathrm{E}-01$ & $2.93 \mathrm{E}-02$ & $2.802 \mathrm{E}+04$ & $3.37 \mathrm{E}+01$ \\
\hline $7.843 \mathrm{E}-02$ & $3.41 \mathrm{E}-03$ & $1.248 \mathrm{E}-02$ & $5.42 \mathrm{E}-04$ & $5.593 \mathrm{E}-01$ & $2.51 \mathrm{E}-02$ & $3.379 \mathrm{E}+04$ & $4.06 \mathrm{E}+01$ \\
\hline $7.939 \mathrm{E}-02$ & $2.78 \mathrm{E}-03$ & $1.264 \mathrm{E}-02$ & $4.42 \mathrm{E}-04$ & $6.138 \mathrm{E}-01$ & $2.26 \mathrm{E}-02$ & $3.939 \mathrm{E}+04$ & $4.72 \mathrm{E}+01$ \\
\hline $7.899 \mathrm{E}-02$ & $2.15 \mathrm{E}-03$ & $1.257 \mathrm{E}-02$ & $3.43 \mathrm{E}-04$ & $6.478 \mathrm{E}-01$ & $1.91 \mathrm{E}-02$ & $4.492 \mathrm{E}+04$ & $5.29 \mathrm{E}+01$ \\
\hline 7.923E-02 & $1.76 \mathrm{E}-03$ & $1.261 \mathrm{E}-02$ & $2.79 \mathrm{E}-04$ & $6.728 \mathrm{E}-01$ & $1.66 \mathrm{E}-02$ & $5.048 \mathrm{E}+04$ & $5.92 \mathrm{E}+01$ \\
\hline 7.974E-02 & $2.44 \mathrm{E}-03$ & $1.294 \mathrm{E}-02$ & $3.88 \mathrm{E}-04$ & $6.852 \mathrm{E}-01$ & $2.22 \mathrm{E}-02$ & $5.635 \mathrm{E}+04$ & $6.63 \mathrm{E}+01$ \\
\hline
\end{tabular}




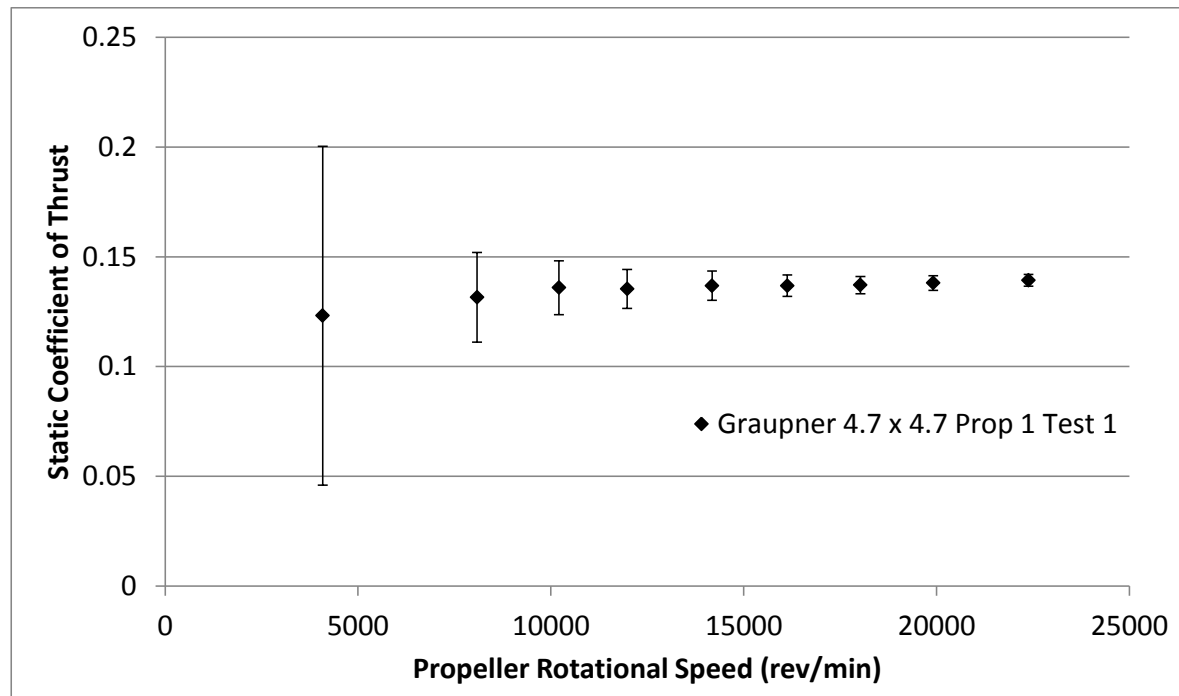

(a)

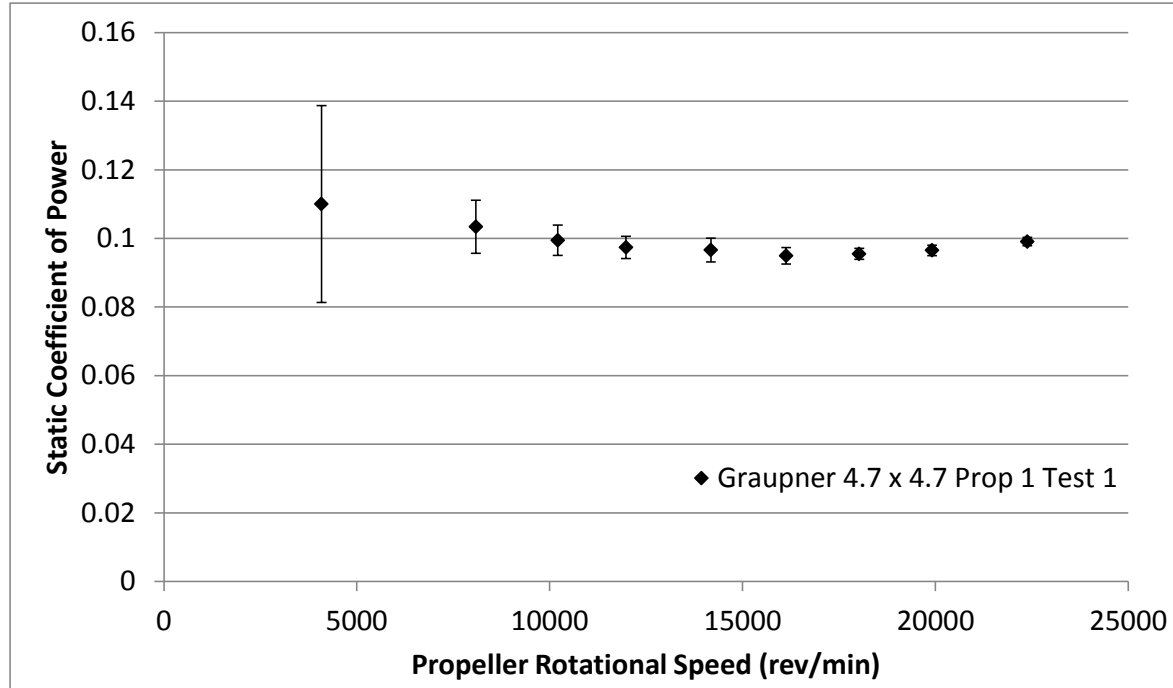

(b)

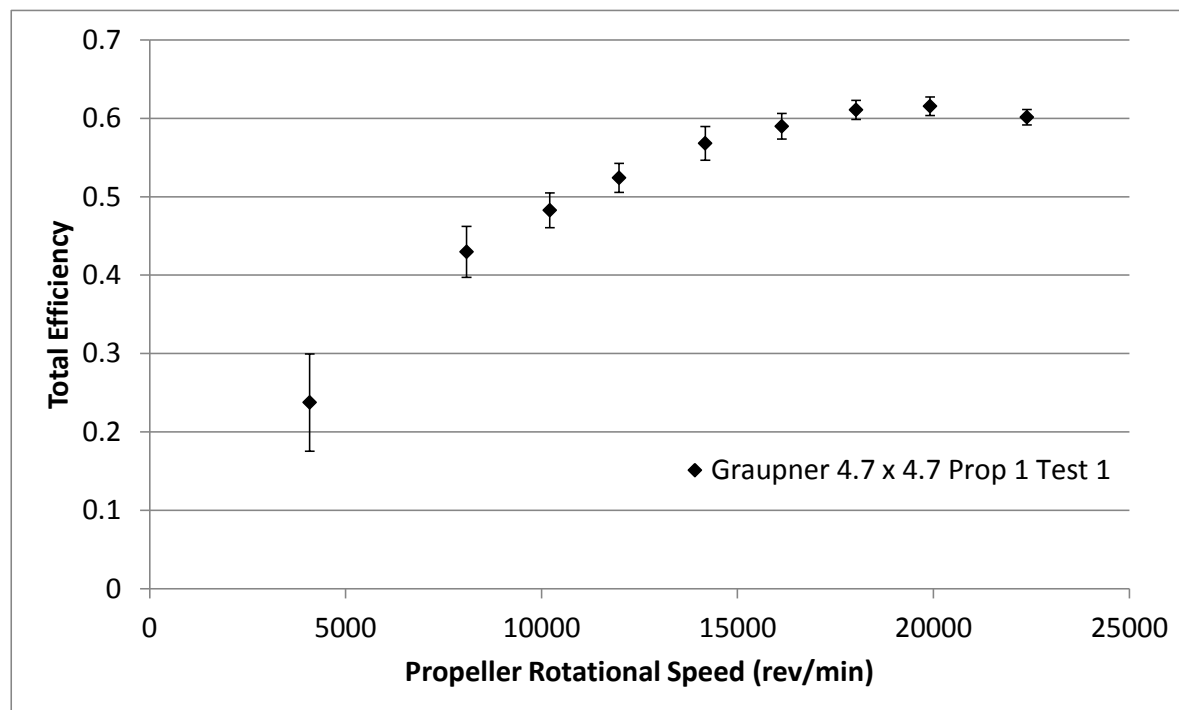

Figure 59: Graupner 4.7 x 4.7 Static Test Results (Prop 1 Test 1): (a) Coefficient of Thrust, (b) Coefficient of Power, (c) Total Efficiency. 
Table 40: Graupner 4.7 x 4.7 Static Measured Values (Prop 1 Test 1)

\begin{tabular}{|c|c|c|c|c|c|c|c|c|}
\hline$n(\mathrm{RPM})$ & $T(\mathrm{~g})$ & $\Delta T(\mathrm{~g})$ & $Q(\mathrm{~g}-\mathrm{m})$ & $\Delta Q(\mathrm{~g}-\mathrm{m})$ & $V(\mathrm{~V})$ & $I(\mathrm{~A})$ & $T_{\mathrm{atm}}\left({ }^{\circ} \mathrm{C}\right)$ & $P_{\mathrm{atm}}(\mathrm{Pa})$ \\
\hline $4.084 \mathrm{E}+03$ & $1.414 \mathrm{E}+01$ & $8.86 \mathrm{E}+00$ & $2.419 \mathrm{E}-01$ & $6.30 \mathrm{E}-02$ & $1.109 \mathrm{E}+01$ & $3.850 \mathrm{E}-01$ & $2.267 \mathrm{E}+01$ & $9.852 \mathrm{E}+04$ \\
\hline $8.087 \mathrm{E}+03$ & $5.922 \mathrm{E}+01$ & $9.21 \mathrm{E}+00$ & $8.916 \mathrm{E}-01$ & $6.67 \mathrm{E}-02$ & $1.107 \mathrm{E}+01$ & $1.557 \mathrm{E}+00$ & $2.265 \mathrm{E}+01$ & $9.853 \mathrm{E}+04$ \\
\hline $1.021 \mathrm{E}+04$ & $9.760 \mathrm{E}+01$ & $8.80 \mathrm{E}+00$ & $1.368 \mathrm{E}+00$ & $6.09 \mathrm{E}-02$ & $1.104 \mathrm{E}+01$ & $2.691 \mathrm{E}+00$ & $2.258 \mathrm{E}+01$ & $9.852 \mathrm{E}+04$ \\
\hline $1.198 \mathrm{E}+04$ & $1.338 \mathrm{E}+02$ & $8.77 \mathrm{E}+00$ & $1.843 \mathrm{E}+00$ & $6.16 \mathrm{E}-02$ & $1.101 \mathrm{E}+01$ & $3.927 \mathrm{E}+00$ & $2.257 \mathrm{E}+01$ & $9.852 \mathrm{E}+04$ \\
\hline $1.418 \mathrm{E}+04$ & $1.895 \mathrm{E}+02$ & $9.22 \mathrm{E}+00$ & $2.562 \mathrm{E}+00$ & $9.22 \mathrm{E}-02$ & $1.097 \mathrm{E}+01$ & $5.987 \mathrm{E}+00$ & $2.258 \mathrm{E}+01$ & $9.850 \mathrm{E}+04$ \\
\hline $1.613 \mathrm{E}+04$ & $2.451 \mathrm{E}+02$ & $8.76 \mathrm{E}+00$ & $3.257 \mathrm{E}+00$ & $8.25 \mathrm{E}-02$ & $1.092 \mathrm{E}+01$ & $8.378 \mathrm{E}+00$ & $2.259 \mathrm{E}+01$ & $9.849 \mathrm{E}+04$ \\
\hline $1.803 \mathrm{E}+04$ & $3.067 \mathrm{E}+02$ & $8.85 \mathrm{E}+00$ & $4.092 \mathrm{E}+00$ & $6.87 \mathrm{E}-02$ & $1.085 \mathrm{E}+01$ & $1.143 \mathrm{E}+01$ & $2.256 \mathrm{E}+01$ & $9.848 \mathrm{E}+04$ \\
\hline $1.992 \mathrm{E}+04$ & $3.770 \mathrm{E}+02$ & $9.01 \mathrm{E}+00$ & $5.047 \mathrm{E}+00$ & $8.01 \mathrm{E}-02$ & $1.076 \mathrm{E}+01$ & $1.559 \mathrm{E}+01$ & $2.252 \mathrm{E}+01$ & $9.848 \mathrm{E}+04$ \\
\hline $2.239 \mathrm{E}+04$ & $4.808 \mathrm{E}+02$ & $9.27 \mathrm{E}+00$ & $6.548 \mathrm{E}+00$ & $8.18 \mathrm{E}-02$ & $1.056 \mathrm{E}+01$ & $2.370 \mathrm{E}+01$ & $2.240 \mathrm{E}+01$ & $9.846 \mathrm{E}+04$ \\
\hline
\end{tabular}

Table 41: Graupner 4.7 x 4.7 Static Calculated Values (Prop 1 Test 1)

\begin{tabular}{|c|c|c|c|c|c|c|c|}
\hline$n(\mathrm{RPM})$ & $\rho\left(\mathrm{kg} / \mathrm{m}^{\wedge} 3\right)$ & $P_{\mathrm{P}}(\mathrm{W})$ & $\Delta P_{\mathrm{P}}(\mathrm{W})$ & $P_{\mathrm{e}}(\mathrm{W})$ & $\Delta P_{\mathrm{e}}(\mathrm{W})$ & $C_{\mathrm{T}}$ & $\Delta C_{\mathrm{T}}$ \\
\hline $4.084 \mathrm{E}+03$ & $1.160 \mathrm{E}+00$ & $1.015 \mathrm{E}+00$ & $2.64 \mathrm{E}-01$ & $4.270 \mathrm{E}+00$ & $6.75 \mathrm{E}-02$ & $1.231 \mathrm{E}-01$ & $7.72 \mathrm{E}-02$ \\
\hline $8.087 \mathrm{E}+03$ & $1.160 \mathrm{E}+00$ & $7.405 \mathrm{E}+00$ & $5.54 \mathrm{E}-01$ & $1.723 \mathrm{E}+01$ & $2.08 \mathrm{E}-01$ & $1.315 \mathrm{E}-01$ & $2.04 \mathrm{E}-02$ \\
\hline $1.021 \mathrm{E}+04$ & $1.161 \mathrm{E}+00$ & $1.434 \mathrm{E}+01$ & $6.39 \mathrm{E}-01$ & $2.972 \mathrm{E}+01$ & $3.38 \mathrm{E}-01$ & $1.359 \mathrm{E}-01$ & $1.23 \mathrm{E}-02$ \\
\hline $1.198 \mathrm{E}+04$ & $1.161 \mathrm{E}+00$ & $2.267 \mathrm{E}+01$ & $7.58 \mathrm{E}-01$ & $4.326 \mathrm{E}+01$ & $4.77 \mathrm{E}-01$ & $1.353 \mathrm{E}-01$ & $8.87 \mathrm{E}-03$ \\
\hline $1.418 \mathrm{E}+04$ & $1.160 \mathrm{E}+00$ & $3.731 \mathrm{E}+01$ & $1.34 \mathrm{E}+00$ & $6.567 \mathrm{E}+01$ & $7.53 \mathrm{E}-01$ & $1.368 \mathrm{E}-01$ & $6.66 \mathrm{E}-03$ \\
\hline $1.613 \mathrm{E}+04$ & $1.160 \mathrm{E}+00$ & $5.395 \mathrm{E}+01$ & $1.37 \mathrm{E}+00$ & $9.146 \mathrm{E}+01$ & $1.04 \mathrm{E}+00$ & $1.368 \mathrm{E}-01$ & $4.89 \mathrm{E}-03$ \\
\hline $1.803 \mathrm{E}+04$ & $1.160 \mathrm{E}+00$ & $7.575 \mathrm{E}+01$ & $1.27 \mathrm{E}+00$ & $1.240 \mathrm{E}+02$ & $1.33 \mathrm{E}+00$ & $1.371 \mathrm{E}-01$ & $3.96 \mathrm{E}-03$ \\
\hline $1.992 \mathrm{E}+04$ & $1.160 \mathrm{E}+00$ & $1.032 \mathrm{E}+02$ & $1.64 \mathrm{E}+00$ & $1.677 \mathrm{E}+02$ & $1.81 \mathrm{E}+00$ & $1.381 \mathrm{E}-01$ & $3.30 \mathrm{E}-03$ \\
\hline $2.239 \mathrm{E}+04$ & $1.161 \mathrm{E}+00$ & $1.505 \mathrm{E}+02$ & $1.88 \mathrm{E}+00$ & $2.503 \mathrm{E}+02$ & $2.61 \mathrm{E}+00$ & $1.393 \mathrm{E}-01$ & $2.69 \mathrm{E}-03$ \\
\hline$C_{\mathrm{P}}$ & $\Delta C_{\mathrm{P}}$ & $C_{\mathrm{Q}}$ & $\Delta C_{\mathrm{Q}}$ & $\eta_{\mathrm{T}}$ & $\Delta \eta_{\mathrm{T}}$ & $R e_{0.75}$ & $\Delta R e_{0.75}$ \\
\hline $1.100 \mathrm{E}-01$ & $2.87 \mathrm{E}-02$ & $1.751 \mathrm{E}-02$ & $4.56 \mathrm{E}-03$ & $2.376 \mathrm{E}-01$ & $6.20 \mathrm{E}-02$ & $1.102 \mathrm{E}+04$ & $1.65 \mathrm{E}+01$ \\
\hline $1.034 \mathrm{E}-01$ & $7.74 \mathrm{E}-03$ & $1.646 \mathrm{E}-02$ & $1.23 \mathrm{E}-03$ & $4.297 \mathrm{E}-01$ & $3.26 \mathrm{E}-02$ & $2.182 \mathrm{E}+04$ & $2.90 \mathrm{E}+01$ \\
\hline $9.945 \mathrm{E}-02$ & $4.43 \mathrm{E}-03$ & $1.583 \mathrm{E}-02$ & $7.05 \mathrm{E}-04$ & $4.826 \mathrm{E}-01$ & $2.22 \mathrm{E}-02$ & $2.756 \mathrm{E}+04$ & $3.46 \mathrm{E}+01$ \\
\hline $9.738 \mathrm{E}-02$ & $3.26 \mathrm{E}-03$ & $1.550 \mathrm{E}-02$ & $5.18 \mathrm{E}-04$ & $5.241 \mathrm{E}-01$ & $1.85 \mathrm{E}-02$ & $3.233 \mathrm{E}+04$ & $4.09 \mathrm{E}+01$ \\
\hline $9.663 \mathrm{E}-02$ & $3.48 \mathrm{E}-03$ & $1.538 \mathrm{E}-02$ & $5.54 \mathrm{E}-04$ & $5.681 \mathrm{E}-01$ & $2.15 \mathrm{E}-02$ & $3.826 \mathrm{E}+04$ & $4.77 \mathrm{E}+01$ \\
\hline $9.492 \mathrm{E}-02$ & $2.41 \mathrm{E}-03$ & $1.511 \mathrm{E}-02$ & $3.83 \mathrm{E}-04$ & $5.899 \mathrm{E}-01$ & $1.64 \mathrm{E}-02$ & $4.352 \mathrm{E}+04$ & $5.35 \mathrm{E}+01$ \\
\hline $9.550 \mathrm{E}-02$ & $1.61 \mathrm{E}-03$ & $1.520 \mathrm{E}-02$ & $2.55 \mathrm{E}-04$ & $6.108 \mathrm{E}-01$ & $1.22 \mathrm{E}-02$ & $4.864 \mathrm{E}+04$ & $5.97 \mathrm{E}+01$ \\
\hline $9.652 \mathrm{E}-02$ & $1.54 \mathrm{E}-03$ & $1.536 \mathrm{E}-02$ & $2.44 \mathrm{E}-04$ & $6.155 \mathrm{E}-01$ & $1.18 \mathrm{E}-02$ & $5.375 \mathrm{E}+04$ & $6.55 \mathrm{E}+01$ \\
\hline $9.907 \mathrm{E}-02$ & $1.24 \mathrm{E}-03$ & $1.751 \mathrm{E}-02$ & $1.97 \mathrm{E}-04$ & $6.014 \mathrm{E}-01$ & $9.78 \mathrm{E}-03$ & $6.045 \mathrm{E}+04$ & $7.42 \mathrm{E}+01$ \\
\hline
\end{tabular}




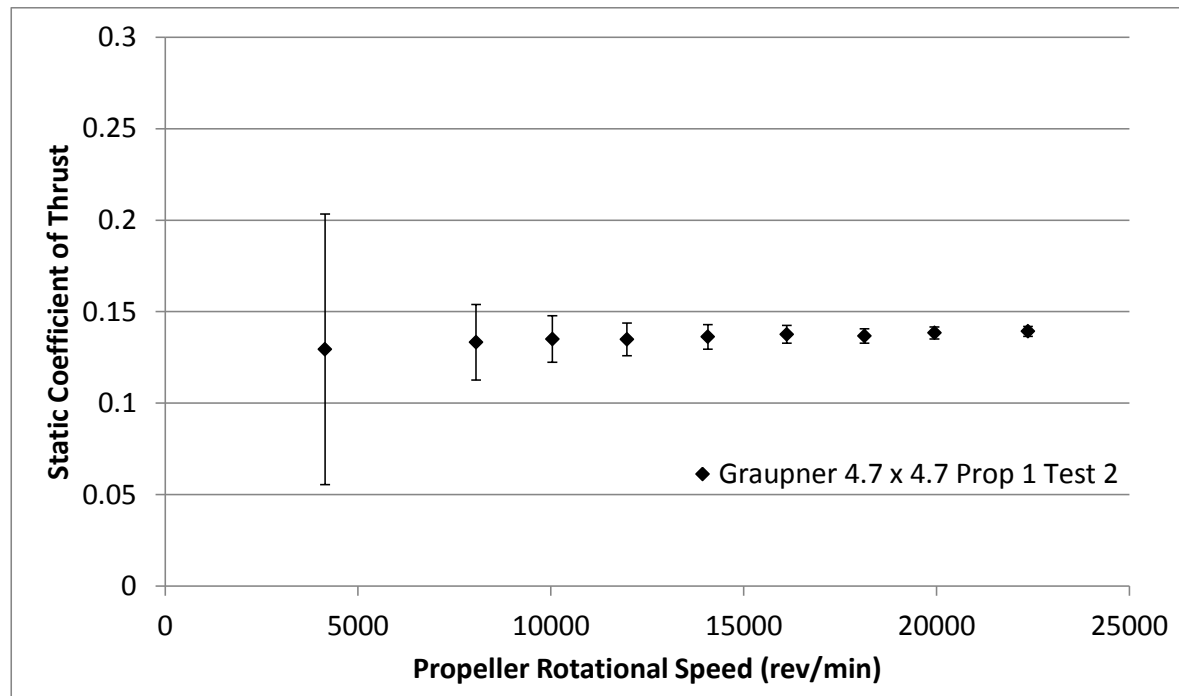

(a)

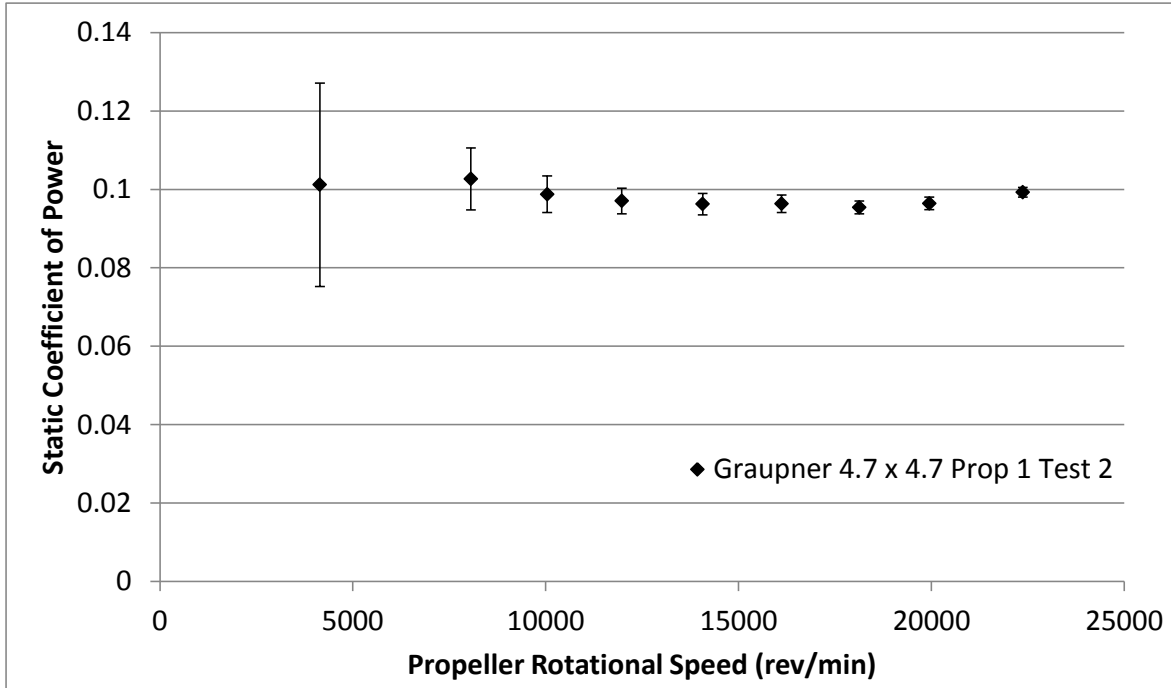

(b)

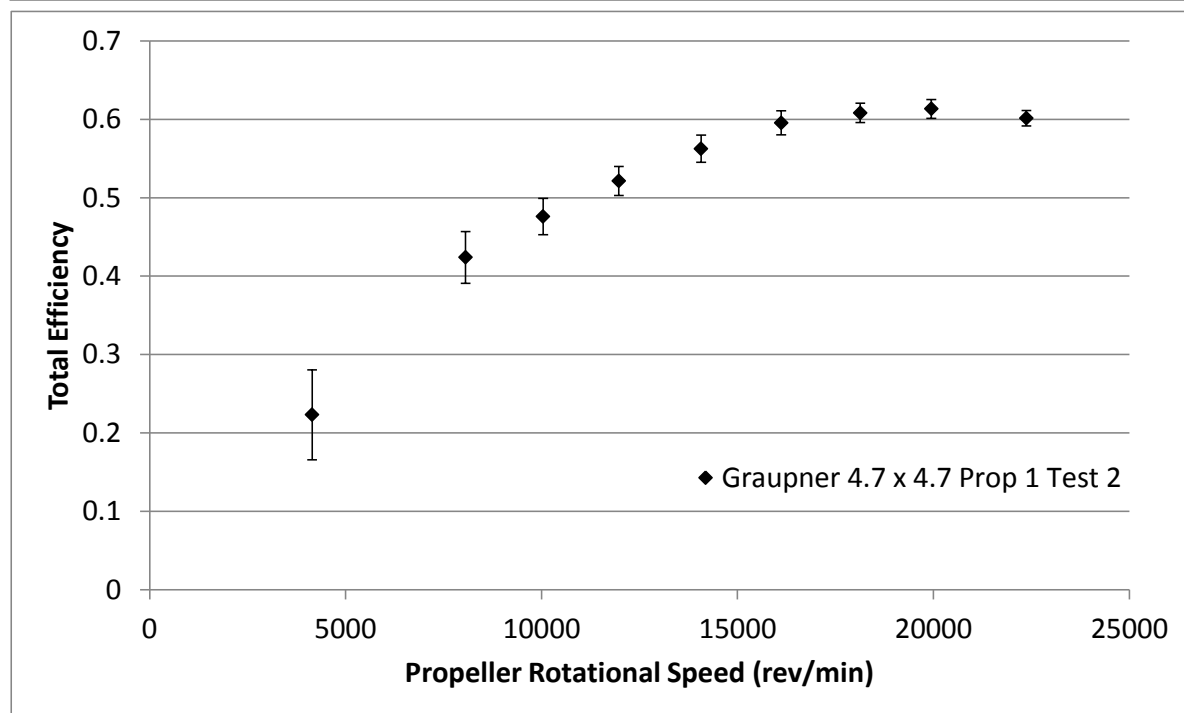

Figure 60: Graupner 4.7 x 4.7 Static Test Results (Prop 1 Test 2): (a) Coefficient of Thrust, (b) Coefficient of Power, (c) Total Efficiency. 
Table 42: Graupner 4.7 x 4.7 Static Measured Values (Prop 1 Test 2)

\begin{tabular}{|c|c|c|c|c|c|c|c|c|}
\hline$n(\mathrm{RPM})$ & $T(\mathrm{~g})$ & $\Delta T(\mathrm{~g})$ & $Q(\mathrm{~g}-\mathrm{m})$ & $\Delta Q(\mathrm{~g}-\mathrm{m})$ & $V(\mathrm{~V})$ & $I(\mathrm{~A})$ & $T_{\mathrm{atm}}\left({ }^{\circ} \mathrm{C}\right)$ & $P_{\mathrm{atm}}(\mathrm{Pa})$ \\
\hline $4.146 \mathrm{E}+03$ & $1.530 \mathrm{E}+01$ & $8.75 \mathrm{E}+00$ & $2.292 \mathrm{E}-01$ & $5.88 \mathrm{E}-02$ & $1.109 \mathrm{E}+01$ & $3.942 \mathrm{E}-01$ & $2.264 \mathrm{E}+01$ & $9.846 \mathrm{E}+04$ \\
\hline $8.062 \mathrm{E}+03$ & $5.964 \mathrm{E}+01$ & $9.25 \mathrm{E}+00$ & $8.796 \mathrm{E}-01$ & $6.76 \mathrm{E}-02$ & $1.107 \mathrm{E}+01$ & $1.553 \mathrm{E}+00$ & $2.256 \mathrm{E}+01$ & $9.846 \mathrm{E}+04$ \\
\hline $1.004 \mathrm{E}+04$ & $9.371 \mathrm{E}+01$ & $8.84 \mathrm{E}+00$ & $1.312 \mathrm{E}+00$ & $6.23 \mathrm{E}-02$ & $1.104 \mathrm{E}+01$ & $2.574 \mathrm{E}+00$ & $2.251 \mathrm{E}+01$ & $9.845 \mathrm{E}+04$ \\
\hline $1.197 \mathrm{E}+04$ & $1.331 \mathrm{E}+02$ & $8.80 \mathrm{E}+00$ & $1.834 \mathrm{E}+00$ & $6.15 \mathrm{E}-02$ & $1.101 \mathrm{E}+01$ & $3.928 \mathrm{E}+00$ & $2.247 \mathrm{E}+01$ & $9.845 \mathrm{E}+04$ \\
\hline $1.407 \mathrm{E}+04$ & $1.858 \mathrm{E}+02$ & $9.18 \mathrm{E}+00$ & $2.513 \mathrm{E}+00$ & $7.16 \mathrm{E}-02$ & $1.097 \mathrm{E}+01$ & $5.885 \mathrm{E}+00$ & $2.244 \mathrm{E}+01$ & $9.847 \mathrm{E}+04$ \\
\hline $1.612 \mathrm{E}+04$ & $2.462 \mathrm{E}+02$ & $8.77 \mathrm{E}+00$ & $3.300 \mathrm{E}+00$ & $7.63 \mathrm{E}-02$ & $1.092 \mathrm{E}+01$ & $8.403 \mathrm{E}+00$ & $2.246 \mathrm{E}+01$ & $9.847 \mathrm{E}+04$ \\
\hline $1.814 \mathrm{E}+04$ & $3.094 \mathrm{E}+02$ & $8.85 \mathrm{E}+00$ & $4.137 \mathrm{E}+00$ & $7.01 \mathrm{E}-02$ & $1.084 \mathrm{E}+01$ & $1.168 \mathrm{E}+01$ & $2.245 \mathrm{E}+01$ & $9.845 \mathrm{E}+04$ \\
\hline $1.995 \mathrm{E}+04$ & $3.792 \mathrm{E}+02$ & $9.03 \mathrm{E}+00$ & $5.061 \mathrm{E}+00$ & $8.37 \mathrm{E}-02$ & $1.075 \mathrm{E}+01$ & $1.573 \mathrm{E}+01$ & $2.238 \mathrm{E}+01$ & $9.846 \mathrm{E}+04$ \\
\hline $2.237 \mathrm{E}+04$ & $4.800 \mathrm{E}+02$ & $9.25 \mathrm{E}+00$ & $6.553 \mathrm{E}+00$ & $8.07 \mathrm{E}-02$ & $1.056 \mathrm{E}+01$ & $2.370 \mathrm{E}+01$ & $2.236 \mathrm{E}+01$ & $9.846 \mathrm{E}+04$ \\
\hline
\end{tabular}

Table 43: Graupner 4.7 x 4.7 Static Calculated Values (Prop 1 Test 2)

\begin{tabular}{|c|c|c|c|c|c|c|c|}
\hline$n(\mathrm{RPM})$ & $\rho\left(\mathrm{kg} / \mathrm{m}^{\wedge} 3\right)$ & $P_{\mathrm{P}}(\mathrm{W})$ & $\Delta P_{\mathrm{P}}(\mathrm{W})$ & $P_{\mathrm{e}}(\mathrm{W})$ & $\Delta P_{\mathrm{e}}(\mathrm{W})$ & $C_{\mathrm{T}}$ & $\Delta C_{\mathrm{T}}$ \\
\hline $4.146 \mathrm{E}+03$ & $1.160 \mathrm{E}+00$ & $9.757 \mathrm{E}-01$ & $2.50 \mathrm{E}-01$ & $4.372 \mathrm{E}+00$ & $6.77 \mathrm{E}-02$ & $1.294 \mathrm{E}-01$ & $7.40 \mathrm{E}-02$ \\
\hline $8.062 \mathrm{E}+03$ & $1.160 \mathrm{E}+00$ & $7.283 \mathrm{E}+00$ & $5.60 \mathrm{E}-01$ & $1.718 \mathrm{E}+01$ & $2.01 \mathrm{E}-01$ & $1.333 \mathrm{E}-01$ & $2.07 \mathrm{E}-02$ \\
\hline $1.004 \mathrm{E}+04$ & $1.160 \mathrm{E}+00$ & $1.353 \mathrm{E}+01$ & $6.43 \mathrm{E}-01$ & $2.843 \mathrm{E}+01$ & $3.23 \mathrm{E}-01$ & $1.350 \mathrm{E}-01$ & $1.27 \mathrm{E}-02$ \\
\hline $1.197 \mathrm{E}+04$ & $1.160 \mathrm{E}+00$ & $2.256 \mathrm{E}+01$ & $7.56 \mathrm{E}-01$ & $4.326 \mathrm{E}+01$ & $4.76 \mathrm{E}-01$ & $1.348 \mathrm{E}-01$ & $8.92 \mathrm{E}-03$ \\
\hline $1.407 \mathrm{E}+04$ & $1.160 \mathrm{E}+00$ & $3.632 \mathrm{E}+01$ & $1.03 \mathrm{E}+00$ & $6.457 \mathrm{E}+01$ & $7.35 \mathrm{E}-01$ & $1.362 \mathrm{E}-01$ & $6.73 \mathrm{E}-03$ \\
\hline $1.612 \mathrm{E}+04$ & $1.160 \mathrm{E}+00$ & $5.463 \mathrm{E}+01$ & $1.26 \mathrm{E}+00$ & $9.172 \mathrm{E}+01$ & $1.04 \mathrm{E}+00$ & $1.376 \mathrm{E}-01$ & $4.90 \mathrm{E}-03$ \\
\hline $1.814 \mathrm{E}+04$ & $1.160 \mathrm{E}+00$ & $7.706 \mathrm{E}+01$ & $1.31 \mathrm{E}+00$ & $1.267 \mathrm{E}+02$ & $1.38 \mathrm{E}+00$ & $1.366 \mathrm{E}-01$ & $3.91 \mathrm{E}-03$ \\
\hline $1.995 \mathrm{E}+04$ & $1.161 \mathrm{E}+00$ & $1.037 \mathrm{E}+02$ & $1.71 \mathrm{E}+00$ & $1.691 \mathrm{E}+02$ & $1.82 \mathrm{E}+00$ & $1.384 \mathrm{E}-01$ & $3.30 \mathrm{E}-03$ \\
\hline $2.237 \mathrm{E}+04$ & $1.161 \mathrm{E}+00$ & $1.506 \mathrm{E}+02$ & $1.85 \mathrm{E}+00$ & $2.504 \mathrm{E}+02$ & $2.60 \mathrm{E}+00$ & $1.392 \mathrm{E}-01$ & $2.69 \mathrm{E}-03$ \\
\hline$C_{\mathrm{P}}$ & $\Delta C_{\mathrm{P}}$ & $C_{\mathrm{Q}}$ & $\Delta C_{\mathrm{Q}}$ & $\eta_{\mathrm{T}}$ & $\Delta \eta_{\mathrm{T}}$ & $R e_{0.75}$ & $\Delta R e_{0.75}$ \\
\hline $1.012 \mathrm{E}-01$ & $2.59 \mathrm{E}-02$ & $1.611 \mathrm{E}-02$ & $4.13 \mathrm{E}-03$ & $2.232 \mathrm{E}-01$ & $5.73 \mathrm{E}-02$ & $1.118 \mathrm{E}+04$ & $1.62 \mathrm{E}+01$ \\
\hline $1.027 \mathrm{E}-01$ & $7.89 \mathrm{E}-03$ & $1.634 \mathrm{E}-02$ & $1.26 \mathrm{E}-03$ & $4.239 \mathrm{E}-01$ & $3.29 \mathrm{E}-02$ & $2.175 \mathrm{E}+04$ & $2.85 \mathrm{E}+01$ \\
\hline $9.877 \mathrm{E}-02$ & $4.69 \mathrm{E}-03$ & $1.572 \mathrm{E}-02$ & $7.47 \mathrm{E}-04$ & $4.760 \mathrm{E}-01$ & $2.33 \mathrm{E}-02$ & $2.709 \mathrm{E}+04$ & $3.42 \mathrm{E}+01$ \\
\hline $9.706 \mathrm{E}-02$ & $3.26 \mathrm{E}-03$ & $1.545 \mathrm{E}-02$ & $5.18 \mathrm{E}-04$ & $5.214 \mathrm{E}-01$ & $1.84 \mathrm{E}-02$ & $3.231 \mathrm{E}+04$ & $4.04 \mathrm{E}+01$ \\
\hline $9.625 \mathrm{E}-02$ & $2.74 \mathrm{E}-03$ & $1.532 \mathrm{E}-02$ & $4.36 \mathrm{E}-04$ & $5.626 \mathrm{E}-01$ & $1.73 \mathrm{E}-02$ & $3.799 \mathrm{E}+04$ & $4.72 \mathrm{E}+01$ \\
\hline $9.634 \mathrm{E}-02$ & $2.23 \mathrm{E}-03$ & $1.533 \mathrm{E}-02$ & $3.55 \mathrm{E}-04$ & $5.955 \mathrm{E}-01$ & $1.53 \mathrm{E}-02$ & $4.351 \mathrm{E}+04$ & $5.33 \mathrm{E}+01$ \\
\hline $9.542 \mathrm{E}-02$ & $1.62 \mathrm{E}-03$ & $1.519 \mathrm{E}-02$ & $2.57 \mathrm{E}-04$ & $6.082 \mathrm{E}-01$ & $1.22 \mathrm{E}-02$ & $4.895 \mathrm{E}+04$ & $5.99 \mathrm{E}+01$ \\
\hline $9.644 \mathrm{E}-02$ & $1.60 \mathrm{E}-03$ & $1.535 \mathrm{E}-02$ & $2.54 \mathrm{E}-04$ & $6.133 \mathrm{E}-01$ & $1.21 \mathrm{E}-02$ & $5.387 \mathrm{E}+04$ & $6.57 \mathrm{E}+01$ \\
\hline $9.926 \mathrm{E}-02$ & $1.23 \mathrm{E}-03$ & $1.611 \mathrm{E}-02$ & $1.95 \mathrm{E}-04$ & $6.014 \mathrm{E}-01$ & $9.69 \mathrm{E}-03$ & $6.042 \mathrm{E}+04$ & $7.44 \mathrm{E}+01$ \\
\hline
\end{tabular}




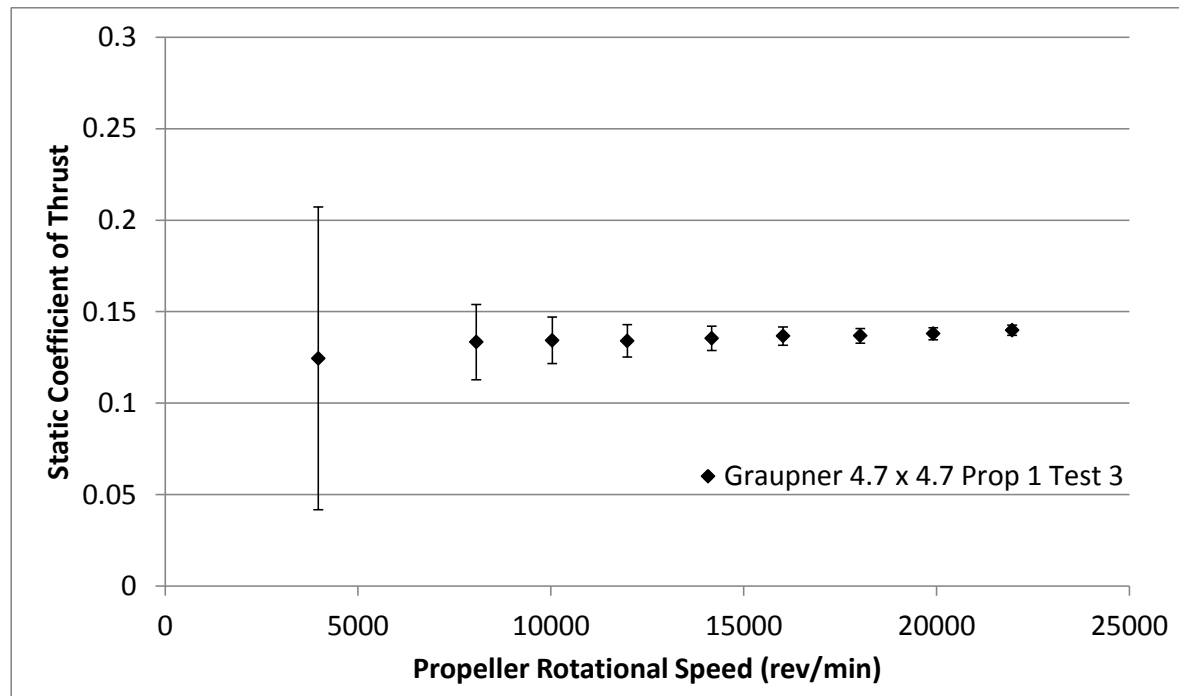

(a)

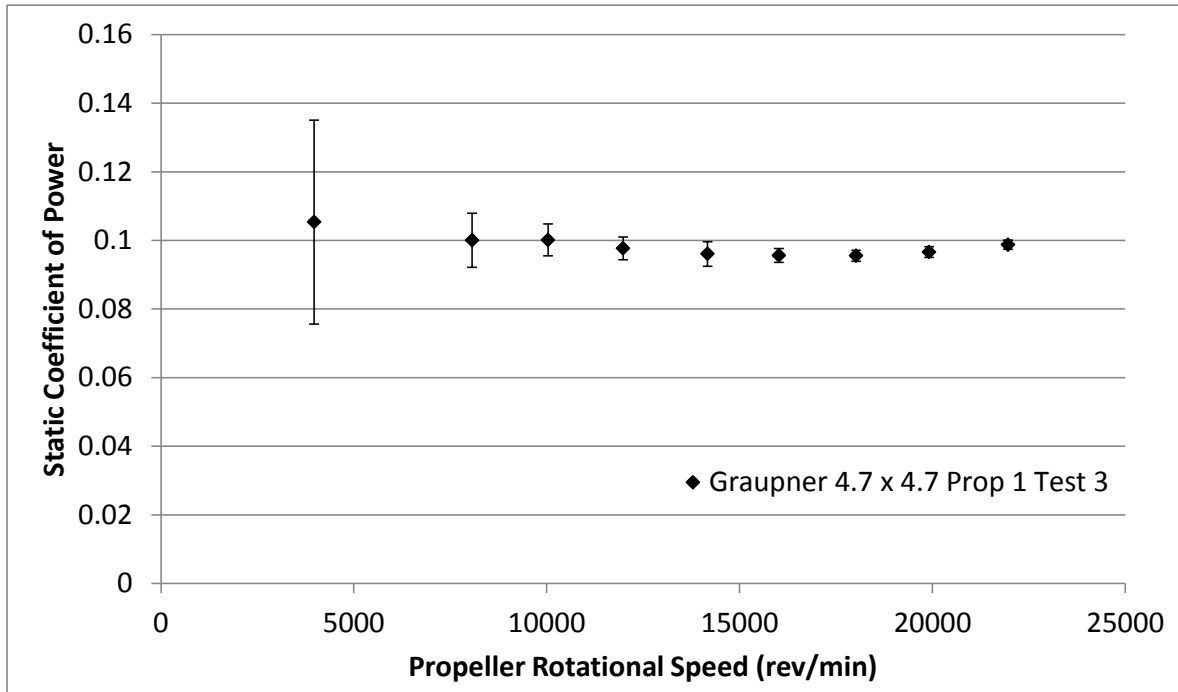

(b)

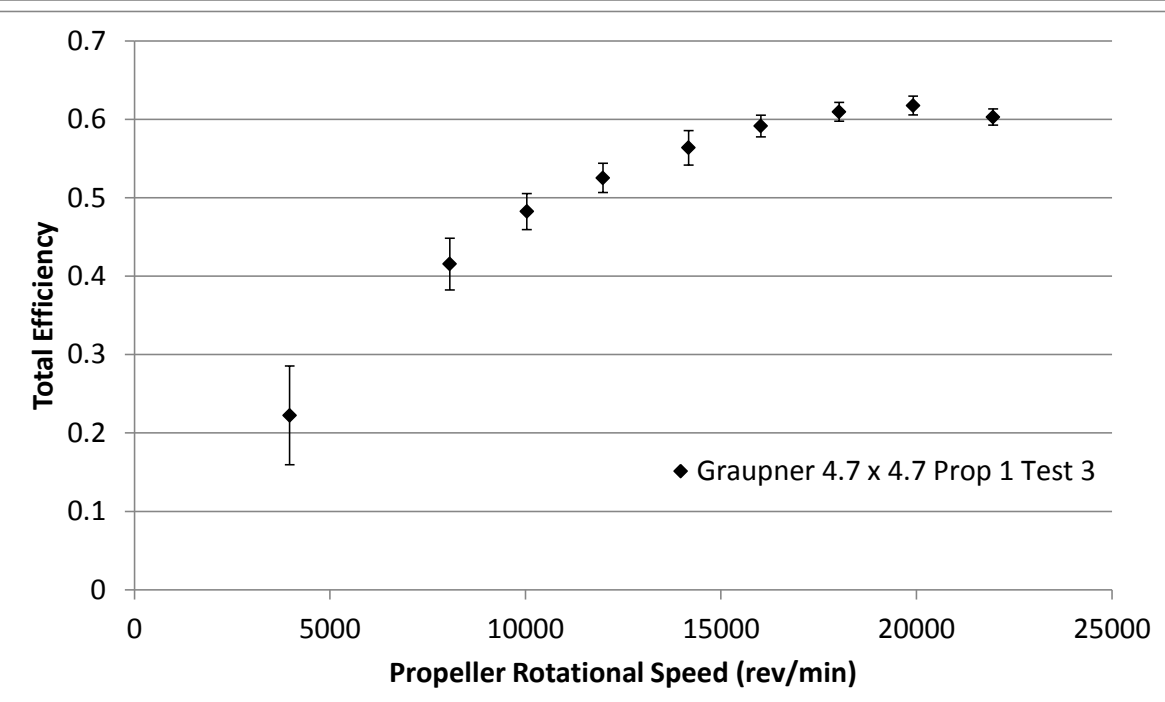

(c)

Figure 61: Graupner 4.7 x 4.7 Static Test Results (Prop 1 Test 3): (a) Coefficient of Thrust, (b) Coefficient of Power, (c) Total Efficiency. 
Table 44: Graupner 4.7 x 4.7 Static Measured Values (Prop 1 Test 3)

\begin{tabular}{|c|c|c|c|c|c|c|c|c|}
\hline$n(\mathrm{RPM})$ & $T(\mathrm{~g})$ & $\Delta T(\mathrm{~g})$ & $Q(\mathrm{~g}-\mathrm{m})$ & $\Delta Q(\mathrm{~g}-\mathrm{m})$ & $V(\mathrm{~V})$ & $I(\mathrm{~A})$ & $T_{\text {atm }}\left({ }^{\circ} \mathrm{C}\right)$ & $P_{\text {atm }}(\mathrm{Pa})$ \\
\hline $3.971 \mathrm{E}+03$ & $1.350 \mathrm{E}+01$ & $8.98 \mathrm{E}+00$ & $2.188 \mathrm{E}-01$ & $6.18 \mathrm{E}-02$ & $1.109 \mathrm{E}+01$ & $3.617 \mathrm{E}-01$ & $2.264 \mathrm{E}+01$ & $9.844 \mathrm{E}+04$ \\
\hline $8.066 \mathrm{E}+03$ & $5.972 \mathrm{E}+01$ & $9.21 \mathrm{E}+00$ & $8.579 \mathrm{E}-01$ & $6.74 \mathrm{E}-02$ & $1.107 \mathrm{E}+01$ & $1.546 \mathrm{E}+00$ & $2.251 \mathrm{E}+01$ & $9.843 \mathrm{E}+04$ \\
\hline $1.004 \mathrm{E}+04$ & $9.313 \mathrm{E}+01$ & $8.84 \mathrm{E}+00$ & $1.330 \mathrm{E}+00$ & $6.18 \mathrm{E}-02$ & $1.104 \mathrm{E}+01$ & $2.572 \mathrm{E}+00$ & $2.247 \mathrm{E}+01$ & $9.843 \mathrm{E}+04$ \\
\hline $1.198 \mathrm{E}+04$ & $1.325 \mathrm{E}+02$ & $8.76 \mathrm{E}+00$ & $1.849 \mathrm{E}+00$ & $6.24 \mathrm{E}-02$ & $1.101 \mathrm{E}+01$ & $3.932 \mathrm{E}+00$ & $2.244 \mathrm{E}+01$ & $9.844 \mathrm{E}+04$ \\
\hline $1.417 \mathrm{E}+04$ & $1.873 \mathrm{E}+02$ & $9.25 \mathrm{E}+00$ & $2.544 \mathrm{E}+00$ & $9.52 \mathrm{E}-02$ & $1.097 \mathrm{E}+01$ & $5.989 \mathrm{E}+00$ & $2.239 \mathrm{E}+01$ & $9.844 \mathrm{E}+04$ \\
\hline $1.602 \mathrm{E}+04$ & $2.415 \mathrm{E}+02$ & $8.78 \mathrm{E}+00$ & $3.236 \mathrm{E}+00$ & $6.71 \mathrm{E}-02$ & $1.092 \mathrm{E}+01$ & $8.245 \mathrm{E}+00$ & $2.239 \mathrm{E}+01$ & $9.844 \mathrm{E}+04$ \\
\hline $1.802 \mathrm{E}+04$ & $3.060 \mathrm{E}+02$ & $8.89 \mathrm{E}+00$ & $4.091 \mathrm{E}+00$ & $6.77 \mathrm{E}-02$ & $1.085 \mathrm{E}+01$ & $1.145 \mathrm{E}+01$ & $2.238 \mathrm{E}+01$ & $9.844 \mathrm{E}+04$ \\
\hline $1.992 \mathrm{E}+04$ & $3.769 \mathrm{E}+02$ & $9.01 \mathrm{E}+00$ & $5.055 \mathrm{E}+00$ & $8.08 \mathrm{E}-02$ & $1.075 \mathrm{E}+01$ & $1.557 \mathrm{E}+01$ & $2.234 \mathrm{E}+01$ & $9.846 \mathrm{E}+04$ \\
\hline $2.196 \mathrm{E}+04$ & $4.647 \mathrm{E}+02$ & $9.16 \mathrm{E}+00$ & $6.284 \mathrm{E}+00$ & $8.50 \mathrm{E}-02$ & $1.060 \mathrm{E}+01$ & $2.218 \mathrm{E}+01$ & $2.228 \mathrm{E}+01$ & $9.846 \mathrm{E}+04$ \\
\hline
\end{tabular}

Table 45: Graupner 4.7 x 4.7 Static Calculated Values (Prop 1 Test 3)

\begin{tabular}{|c|c|c|c|c|c|c|c|}
\hline$n(\mathrm{RPM})$ & $\rho\left(\mathrm{kg} / \mathrm{m}^{\wedge} 3\right)$ & $P_{\mathrm{P}}(\mathrm{W})$ & $\Delta P_{\mathrm{P}}(\mathrm{W})$ & $P_{\mathrm{e}}(\mathrm{W})$ & $\Delta P_{\mathrm{e}}(\mathrm{W})$ & $C_{\mathrm{T}}$ & $\Delta C_{\mathrm{T}}$ \\
\hline $3.971 \mathrm{E}+03$ & $1.159 \mathrm{E}+00$ & $8.923 \mathrm{E}-01$ & $2.52 \mathrm{E}-01$ & $4.012 \mathrm{E}+00$ & $6.44 \mathrm{E}-02$ & $1.244 \mathrm{E}-01$ & $8.28 \mathrm{E}-02$ \\
\hline $8.066 \mathrm{E}+03$ & $1.160 \mathrm{E}+00$ & $7.107 \mathrm{E}+00$ & $5.59 \mathrm{E}-01$ & $1.711 \mathrm{E}+01$ & $2.01 \mathrm{E}-01$ & $1.334 \mathrm{E}-01$ & $2.06 \mathrm{E}-02$ \\
\hline $1.004 \mathrm{E}+04$ & $1.160 \mathrm{E}+00$ & $1.370 \mathrm{E}+01$ & $6.37 \mathrm{E}-01$ & $2.841 \mathrm{E}+01$ & $3.22 \mathrm{E}-01$ & $1.343 \mathrm{E}-01$ & $1.27 \mathrm{E}-02$ \\
\hline $1.198 \mathrm{E}+04$ & $1.160 \mathrm{E}+00$ & $2.275 \mathrm{E}+01$ & $7.68 \mathrm{E}-01$ & $4.331 \mathrm{E}+01$ & $4.79 \mathrm{E}-01$ & $1.340 \mathrm{E}-01$ & $8.86 \mathrm{E}-03$ \\
\hline $1.417 \mathrm{E}+04$ & $1.160 \mathrm{E}+00$ & $3.703 \mathrm{E}+01$ & $1.39 \mathrm{E}+00$ & $6.569 \mathrm{E}+01$ & $7.52 \mathrm{E}-01$ & $1.354 \mathrm{E}-01$ & $6.69 \mathrm{E}-03$ \\
\hline $1.602 \mathrm{E}+04$ & $1.160 \mathrm{E}+00$ & $5.325 \mathrm{E}+01$ & $1.10 \mathrm{E}+00$ & $9.002 \mathrm{E}+01$ & $1.01 \mathrm{E}+00$ & $1.366 \mathrm{E}-01$ & $4.97 \mathrm{E}-03$ \\
\hline $1.802 \mathrm{E}+04$ & $1.160 \mathrm{E}+00$ & $7.573 \mathrm{E}+01$ & $1.25 \mathrm{E}+00$ & $1.242 \mathrm{E}+02$ & $1.34 \mathrm{E}+00$ & $1.368 \mathrm{E}-01$ & $3.98 \mathrm{E}-03$ \\
\hline $1.992 \mathrm{E}+04$ & $1.161 \mathrm{E}+00$ & $1.034 \mathrm{E}+02$ & $1.65 \mathrm{E}+00$ & $1.674 \mathrm{E}+02$ & $1.79 \mathrm{E}+00$ & $1.379 \mathrm{E}-01$ & $3.30 \mathrm{E}-03$ \\
\hline $2.196 \mathrm{E}+04$ & $1.161 \mathrm{E}+00$ & $1.417 \mathrm{E}+02$ & $1.92 \mathrm{E}+00$ & $2.351 \mathrm{E}+02$ & $2.47 \mathrm{E}+00$ & $1.398 \mathrm{E}-01$ & $2.76 \mathrm{E}-03$ \\
\hline$C_{\mathrm{P}}$ & $\Delta C_{\mathrm{P}}$ & $C_{\mathrm{Q}}$ & $\Delta C_{\mathrm{Q}}$ & $\eta_{\mathrm{T}}$ & $\Delta \eta_{\mathrm{T}}$ & $R e_{0.75}$ & $\Delta R e_{0.75}$ \\
\hline $1.053 \mathrm{E}-01$ & $2.97 \mathrm{E}-02$ & $1.677 \mathrm{E}-02$ & $4.73 \mathrm{E}-03$ & $2.224 \mathrm{E}-01$ & $6.29 \mathrm{E}-02$ & $1.070 \mathrm{E}+04$ & $1.55 \mathrm{E}+01$ \\
\hline $1.001 \mathrm{E}-01$ & $7.87 \mathrm{E}-03$ & $1.592 \mathrm{E}-02$ & $1.25 \mathrm{E}-03$ & $4.154 \mathrm{E}-01$ & $3.30 \mathrm{E}-02$ & $2.176 \mathrm{E}+04$ & $2.84 \mathrm{E}+01$ \\
\hline $1.001 \mathrm{E}-01$ & $4.66 \mathrm{E}-03$ & $1.594 \mathrm{E}-02$ & $7.41 \mathrm{E}-04$ & $4.824 \mathrm{E}-01$ & $2.31 \mathrm{E}-02$ & $2.708 \mathrm{E}+04$ & $3.39 \mathrm{E}+01$ \\
\hline $9.766 \mathrm{E}-02$ & $3.30 \mathrm{E}-03$ & $1.554 \mathrm{E}-02$ & $5.25 \mathrm{E}-04$ & $5.253 \mathrm{E}-01$ & $1.87 \mathrm{E}-02$ & $3.234 \mathrm{E}+04$ & $4.08 \mathrm{E}+01$ \\
\hline $9.606 \mathrm{E}-02$ & $3.60 \mathrm{E}-03$ & $1.529 \mathrm{E}-02$ & $5.72 \mathrm{E}-04$ & $5.637 \mathrm{E}-01$ & $2.21 \mathrm{E}-02$ & $3.826 \mathrm{E}+04$ & $4.75 \mathrm{E}+01$ \\
\hline $9.562 \mathrm{E}-02$ & $1.99 \mathrm{E}-03$ & $1.522 \mathrm{E}-02$ & $3.16 \mathrm{E}-04$ & $5.915 \mathrm{E}-01$ & $1.39 \mathrm{E}-02$ & $4.325 \mathrm{E}+04$ & $5.30 \mathrm{E}+01$ \\
\hline $9.552 \mathrm{E}-02$ & $1.58 \mathrm{E}-03$ & $1.520 \mathrm{E}-02$ & $2.52 \mathrm{E}-04$ & $6.096 \mathrm{E}-01$ & $1.20 \mathrm{E}-02$ & $4.866 \mathrm{E}+04$ & $5.98 \mathrm{E}+01$ \\
\hline $9.662 \mathrm{E}-02$ & $1.55 \mathrm{E}-03$ & $1.538 \mathrm{E}-02$ & $2.46 \mathrm{E}-04$ & $6.176 \mathrm{E}-01$ & $1.19 \mathrm{E}-02$ & $5.379 \mathrm{E}+04$ & $6.55 \mathrm{E}+01$ \\
\hline $9.876 \mathrm{E}-02$ & $1.34 \mathrm{E}-03$ & $1.677 \mathrm{E}-02$ & $2.13 \mathrm{E}-04$ & $6.030 \mathrm{E}-01$ & $1.03 \mathrm{E}-02$ & $5.934 \mathrm{E}+04$ & $7.33 \mathrm{E}+01$ \\
\hline
\end{tabular}




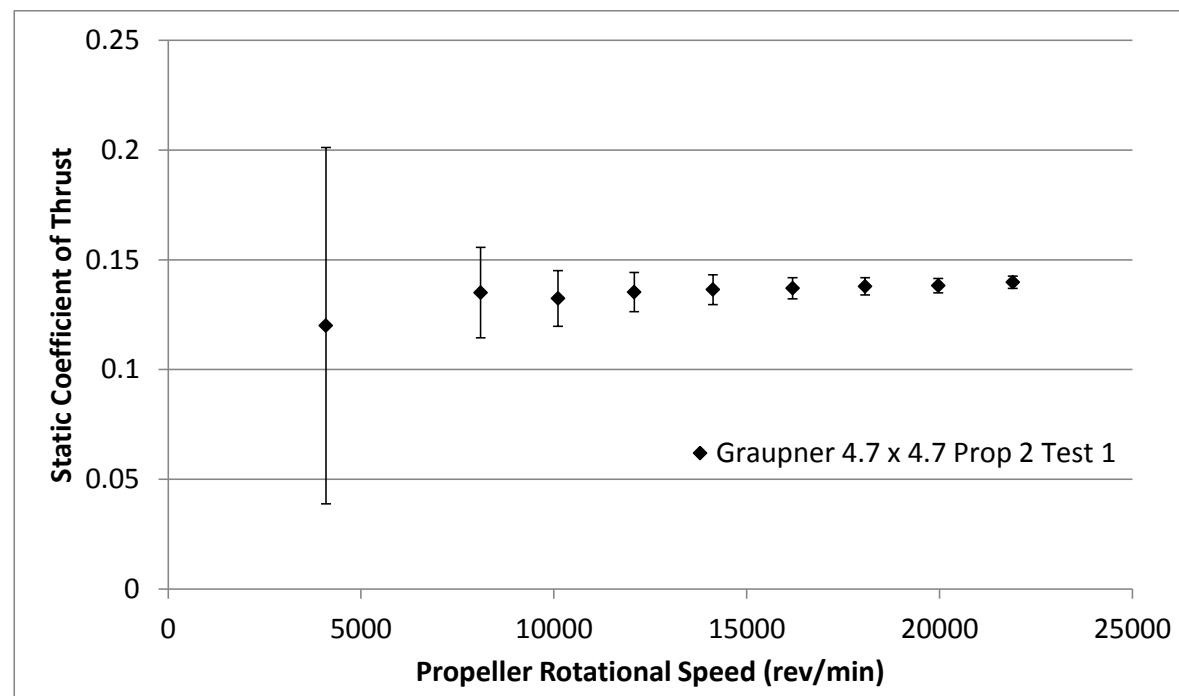

(a)

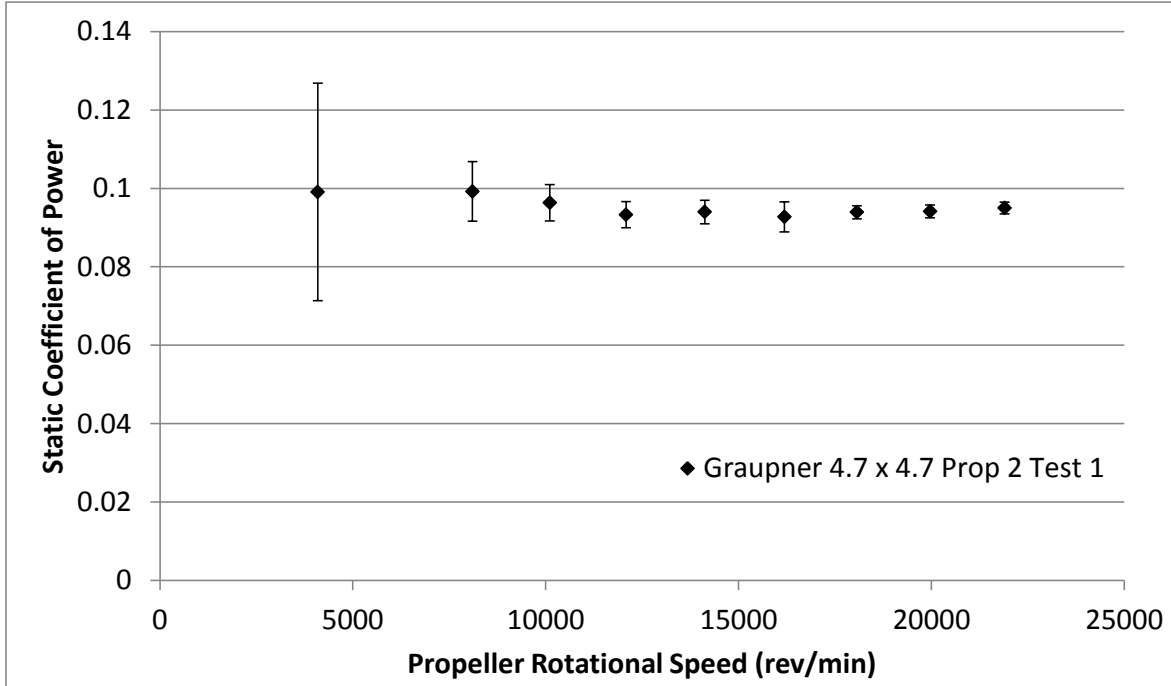

(b)

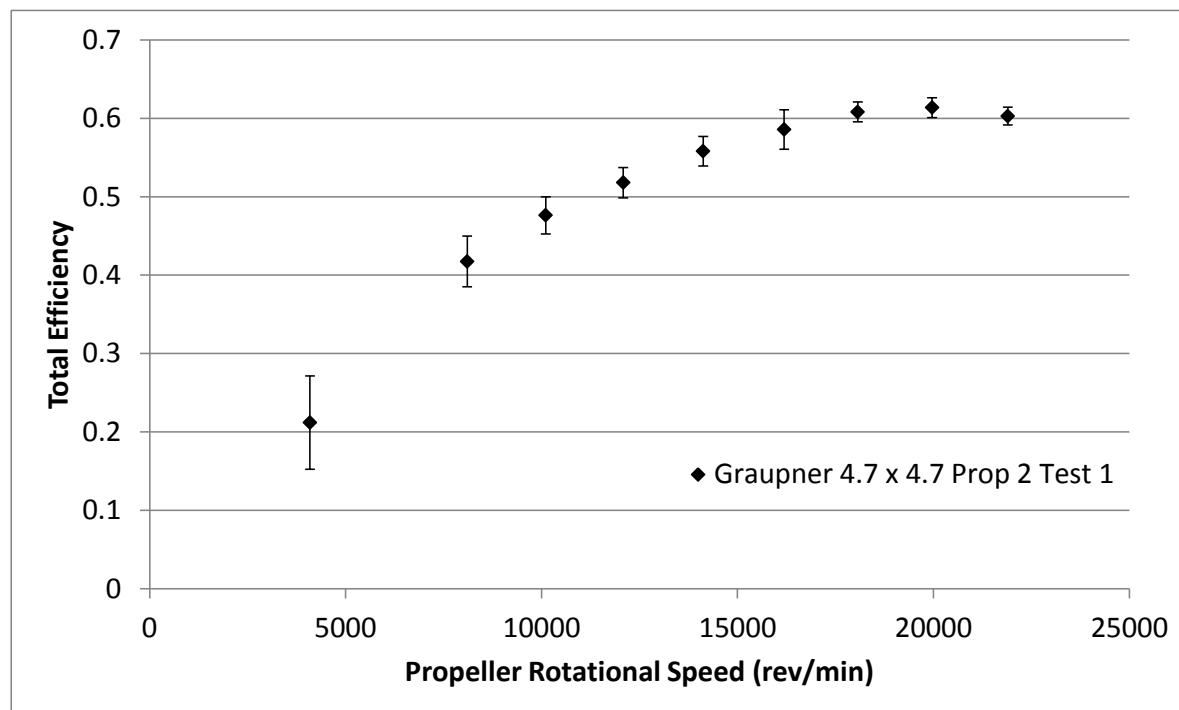

(c)

Figure 62: Graupner 4.7 x 4.7 Static Test Results (Prop 2 Test 1): (a) Coefficient of Thrust, (b) Coefficient of Power, (c) Total Efficiency. 
Table 46: Graupner 4.7 x 4.7 Static Measured Values (Prop 2 Test 1)

\begin{tabular}{|c|c|c|c|c|c|c|c|c|}
\hline$n(\mathrm{RPM})$ & $T(\mathrm{~g})$ & $\Delta T(\mathrm{~g})$ & $Q(\mathrm{~g}-\mathrm{m})$ & $\Delta Q(\mathrm{~g}-\mathrm{m})$ & $V(\mathrm{~V})$ & $I(\mathrm{~A})$ & $T_{\mathrm{atm}}\left({ }^{\circ} \mathrm{C}\right)$ & $P_{\mathrm{atm}}(\mathrm{Pa})$ \\
\hline $4.091 \mathrm{E}+03$ & $1.383 \mathrm{E}+01$ & $9.36 \mathrm{E}+00$ & $2.187 \mathrm{E}-01$ & $6.13 \mathrm{E}-02$ & $1.109 \mathrm{E}+01$ & $3.910 \mathrm{E}-01$ & $2.255 \mathrm{E}+01$ & $9.850 \mathrm{E}+04$ \\
\hline $8.103 \mathrm{E}+03$ & $6.109 \mathrm{E}+01$ & $9.33 \mathrm{E}+00$ & $8.595 \mathrm{E}-01$ & $6.57 \mathrm{E}-02$ & $1.107 \mathrm{E}+01$ & $1.548 \mathrm{E}+00$ & $2.239 \mathrm{E}+01$ & $9.848 \mathrm{E}+04$ \\
\hline $1.011 \mathrm{E}+04$ & $9.311 \mathrm{E}+01$ & $8.93 \mathrm{E}+00$ & $1.298 \mathrm{E}+00$ & $6.25 \mathrm{E}-02$ & $1.104 \mathrm{E}+01$ & $2.562 \mathrm{E}+00$ & $2.256 \mathrm{E}+01$ & $9.848 \mathrm{E}+04$ \\
\hline $1.209 \mathrm{E}+04$ & $1.360 \mathrm{E}+02$ & $9.01 \mathrm{E}+00$ & $1.797 \mathrm{E}+00$ & $6.41 \mathrm{E}-02$ & $1.102 \mathrm{E}+01$ & $3.908 \mathrm{E}+00$ & $2.254 \mathrm{E}+01$ & $9.849 \mathrm{E}+04$ \\
\hline $1.413 \mathrm{E}+04$ & $1.874 \mathrm{E}+02$ & $9.34 \mathrm{E}+00$ & $2.473 \mathrm{E}+00$ & $7.86 \mathrm{E}-02$ & $1.097 \mathrm{E}+01$ & $5.857 \mathrm{E}+00$ & $2.253 \mathrm{E}+01$ & $9.849 \mathrm{E}+04$ \\
\hline $1.619 \mathrm{E}+04$ & $2.473 \mathrm{E}+02$ & $8.78 \mathrm{E}+00$ & $3.206 \mathrm{E}+00$ & $1.32 \mathrm{E}-01$ & $1.092 \mathrm{E}+01$ & $8.334 \mathrm{E}+00$ & $2.254 \mathrm{E}+01$ & $9.847 \mathrm{E}+04$ \\
\hline $1.807 \mathrm{E}+04$ & $3.101 \mathrm{E}+02$ & $8.82 \mathrm{E}+00$ & $4.044 \mathrm{E}+00$ & $7.17 \mathrm{E}-02$ & $1.085 \mathrm{E}+01$ & $1.137 \mathrm{E}+01$ & $2.250 \mathrm{E}+01$ & $9.848 \mathrm{E}+04$ \\
\hline $1.997 \mathrm{E}+04$ & $3.796 \mathrm{E}+02$ & $9.03 \mathrm{E}+00$ & $4.949 \mathrm{E}+00$ & $8.74 \mathrm{E}-02$ & $1.076 \mathrm{E}+01$ & $1.537 \mathrm{E}+01$ & $2.252 \mathrm{E}+01$ & $9.847 \mathrm{E}+04$ \\
\hline $2.190 \mathrm{E}+04$ & $4.615 \mathrm{E}+02$ & $9.18 \mathrm{E}+00$ & $6.008 \mathrm{E}+00$ & $9.42 \mathrm{E}-02$ & $1.063 \mathrm{E}+01$ & $2.109 \mathrm{E}+01$ & $2.250 \mathrm{E}+01$ & $9.849 \mathrm{E}+04$ \\
\hline
\end{tabular}

Table 47: Graupner 4.7 x 4.7 Static Calculated Values (Prop 2 Test 1)

\begin{tabular}{|c|c|c|c|c|c|c|c|}
\hline$n(\mathrm{RPM})$ & $\rho\left(\mathrm{kg} / \mathrm{m}^{\wedge} 3\right)$ & $P_{\mathrm{P}}(\mathrm{W})$ & $\Delta P_{\mathrm{P}}(\mathrm{W})$ & $P_{\mathrm{e}}(\mathrm{W})$ & $\Delta P_{\mathrm{e}}(\mathrm{W})$ & $C_{\mathrm{T}}$ & $\Delta C_{\mathrm{T}}$ \\
\hline $4.091 \mathrm{E}+03$ & $1.160 \mathrm{E}+00$ & $9.189 \mathrm{E}-01$ & $2.57 \mathrm{E}-01$ & $4.337 \mathrm{E}+00$ & $6.76 \mathrm{E}-02$ & $1.200 \mathrm{E}-01$ & $8.12 \mathrm{E}-02$ \\
\hline $8.103 \mathrm{E}+03$ & $1.161 \mathrm{E}+00$ & $7.152 \mathrm{E}+00$ & $5.47 \mathrm{E}-01$ & $1.713 \mathrm{E}+01$ & $2.02 \mathrm{E}-01$ & $1.350 \mathrm{E}-01$ & $2.06 \mathrm{E}-02$ \\
\hline $1.011 \mathrm{E}+04$ & $1.160 \mathrm{E}+00$ & $1.348 \mathrm{E}+01$ & $6.49 \mathrm{E}-01$ & $2.830 \mathrm{E}+01$ & $3.21 \mathrm{E}-01$ & $1.324 \mathrm{E}-01$ & $1.27 \mathrm{E}-02$ \\
\hline $1.209 \mathrm{E}+04$ & $1.160 \mathrm{E}+00$ & $2.230 \mathrm{E}+01$ & $7.95 \mathrm{E}-01$ & $4.305 \mathrm{E}+01$ & $4.77 \mathrm{E}-01$ & $1.353 \mathrm{E}-01$ & $8.95 \mathrm{E}-03$ \\
\hline $1.413 \mathrm{E}+04$ & $1.160 \mathrm{E}+00$ & $3.587 \mathrm{E}+01$ & $1.14 \mathrm{E}+00$ & $6.427 \mathrm{E}+01$ & $7.32 \mathrm{E}-01$ & $1.364 \mathrm{E}-01$ & $6.80 \mathrm{E}-03$ \\
\hline $1.619 \mathrm{E}+04$ & $1.160 \mathrm{E}+00$ & $5.331 \mathrm{E}+01$ & $2.20 \mathrm{E}+00$ & $9.100 \mathrm{E}+01$ & $1.05 \mathrm{E}+00$ & $1.370 \mathrm{E}-01$ & $4.86 \mathrm{E}-03$ \\
\hline $1.807 \mathrm{E}+04$ & $1.160 \mathrm{E}+00$ & $7.505 \mathrm{E}+01$ & $1.33 \mathrm{E}+00$ & $1.234 \mathrm{E}+02$ & $1.33 \mathrm{E}+00$ & $1.379 \mathrm{E}-01$ & $3.92 \mathrm{E}-03$ \\
\hline $1.997 \mathrm{E}+04$ & $1.160 \mathrm{E}+00$ & $1.015 \mathrm{E}+02$ & $1.79 \mathrm{E}+00$ & $1.654 \mathrm{E}+02$ & $1.80 \mathrm{E}+00$ & $1.383 \mathrm{E}-01$ & $3.29 \mathrm{E}-03$ \\
\hline $2.190 \mathrm{E}+04$ & $1.160 \mathrm{E}+00$ & $1.351 \mathrm{E}+02$ & $2.12 \mathrm{E}+00$ & $2.242 \mathrm{E}+02$ & $2.36 \mathrm{E}+00$ & $1.397 \mathrm{E}-01$ & $2.78 \mathrm{E}-03$ \\
\hline$C_{\mathrm{P}}$ & $\Delta C_{\mathrm{P}}$ & $C_{\mathrm{Q}}$ & $\Delta C_{\mathrm{Q}}$ & $\eta_{\mathrm{T}}$ & $\Delta \eta_{\mathrm{T}}$ & $R e_{0.75}$ & $\Delta R e_{0.75}$ \\
\hline $9.909 \mathrm{E}-02$ & $2.78 \mathrm{E}-02$ & $1.577 \mathrm{E}-02$ & $4.42 \mathrm{E}-03$ & $2.119 \mathrm{E}-01$ & $5.94 \mathrm{E}-02$ & $1.104 \mathrm{E}+04$ & $1.52 \mathrm{E}+01$ \\
\hline $9.923 \mathrm{E}-02$ & $7.59 \mathrm{E}-03$ & $1.579 \mathrm{E}-02$ & $1.21 \mathrm{E}-03$ & $4.175 \mathrm{E}-01$ & $3.23 \mathrm{E}-02$ & $2.188 \mathrm{E}+04$ & $2.86 \mathrm{E}+01$ \\
\hline $9.636 \mathrm{E}-02$ & $4.64 \mathrm{E}-03$ & $1.534 \mathrm{E}-02$ & $7.38 \mathrm{E}-04$ & $4.762 \mathrm{E}-01$ & $2.35 \mathrm{E}-02$ & $2.727 \mathrm{E}+04$ & $3.46 \mathrm{E}+01$ \\
\hline $9.329 \mathrm{E}-02$ & $3.33 \mathrm{E}-03$ & $1.485 \mathrm{E}-02$ & $5.30 \mathrm{E}-04$ & $5.180 \mathrm{E}-01$ & $1.93 \mathrm{E}-02$ & $3.262 \mathrm{E}+04$ & $4.09 \mathrm{E}+01$ \\
\hline $9.397 \mathrm{E}-02$ & $2.99 \mathrm{E}-03$ & $1.496 \mathrm{E}-02$ & $4.75 \mathrm{E}-04$ & $5.582 \mathrm{E}-01$ & $1.88 \mathrm{E}-02$ & $3.813 \mathrm{E}+04$ & $4.74 \mathrm{E}+01$ \\
\hline $9.276 \mathrm{E}-02$ & $3.83 \mathrm{E}-03$ & $1.476 \mathrm{E}-02$ & $6.09 \mathrm{E}-04$ & $5.858 \mathrm{E}-01$ & $2.51 \mathrm{E}-02$ & $4.369 \mathrm{E}+04$ & $5.41 \mathrm{E}+01$ \\
\hline $9.391 \mathrm{E}-02$ & $1.67 \mathrm{E}-03$ & $1.495 \mathrm{E}-02$ & $2.65 \mathrm{E}-04$ & $6.082 \mathrm{E}-01$ & $1.26 \mathrm{E}-02$ & $4.878 \mathrm{E}+04$ & $5.94 \mathrm{E}+01$ \\
\hline $9.414 \mathrm{E}-02$ & $1.66 \mathrm{E}-03$ & $1.498 \mathrm{E}-02$ & $2.65 \mathrm{E}-04$ & $6.136 \mathrm{E}-01$ & $1.27 \mathrm{E}-02$ & $5.389 \mathrm{E}+04$ & $6.59 \mathrm{E}+01$ \\
\hline $9.499 \mathrm{E}-02$ & $1.49 \mathrm{E}-03$ & $1.577 \mathrm{E}-02$ & $2.37 \mathrm{E}-04$ & $6.028 \mathrm{E}-01$ & $1.14 \mathrm{E}-02$ & $5.912 \mathrm{E}+04$ & $7.26 \mathrm{E}+01$ \\
\hline
\end{tabular}




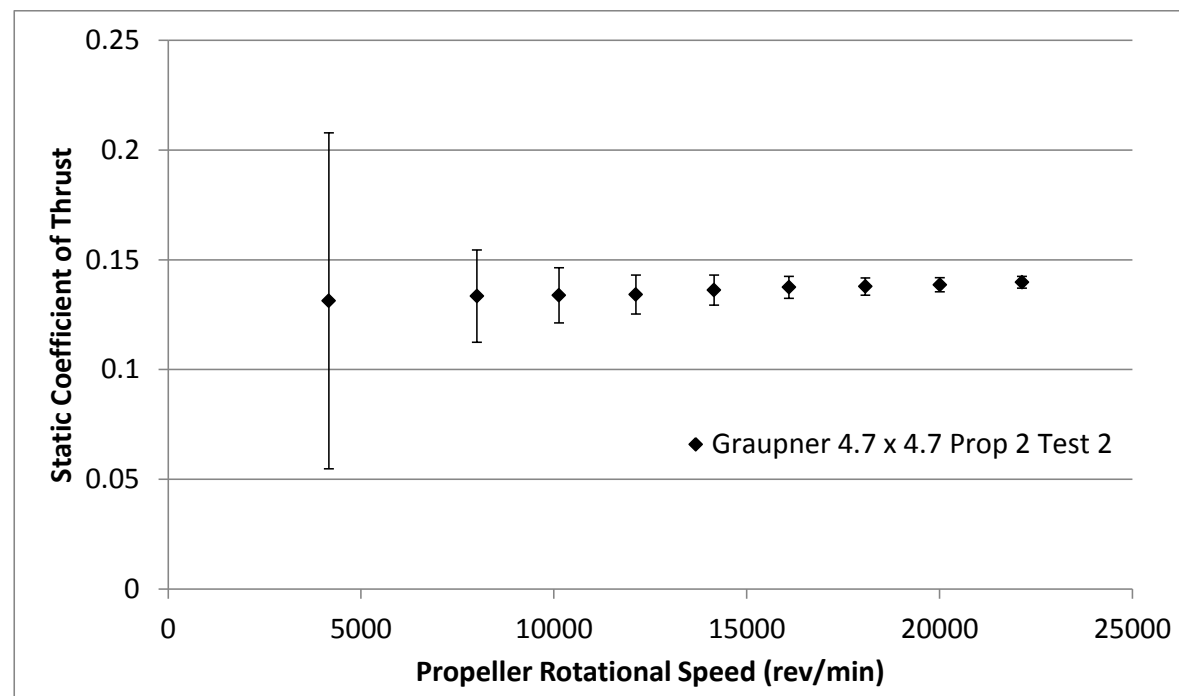

(a)

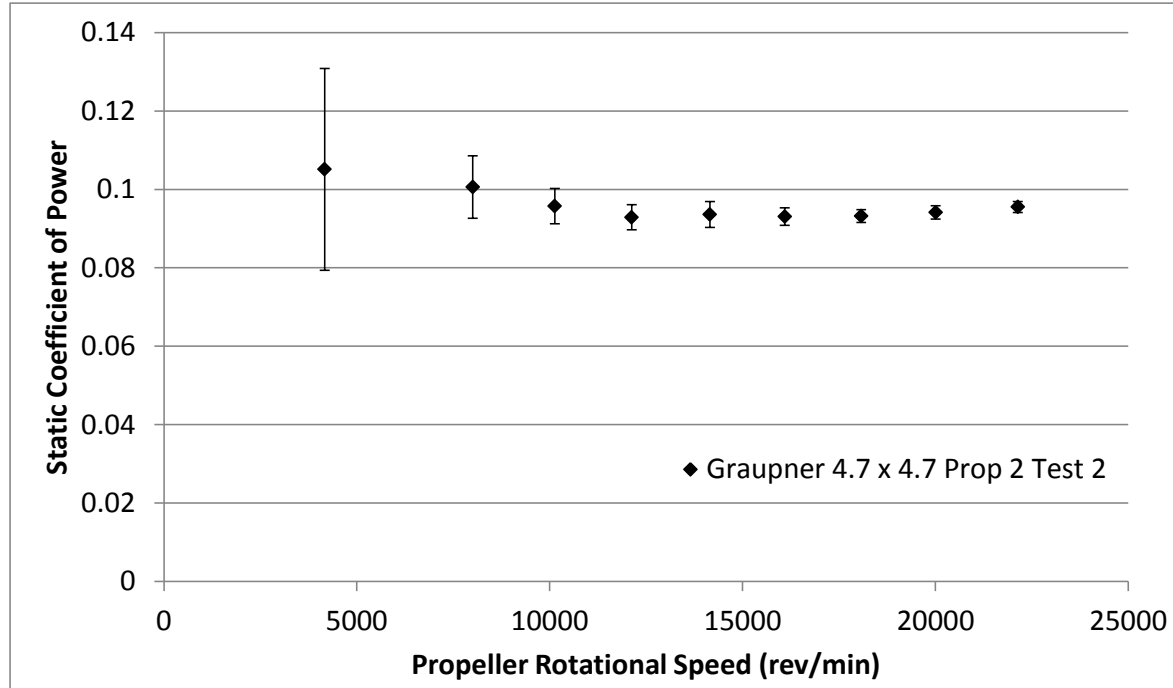

(b)

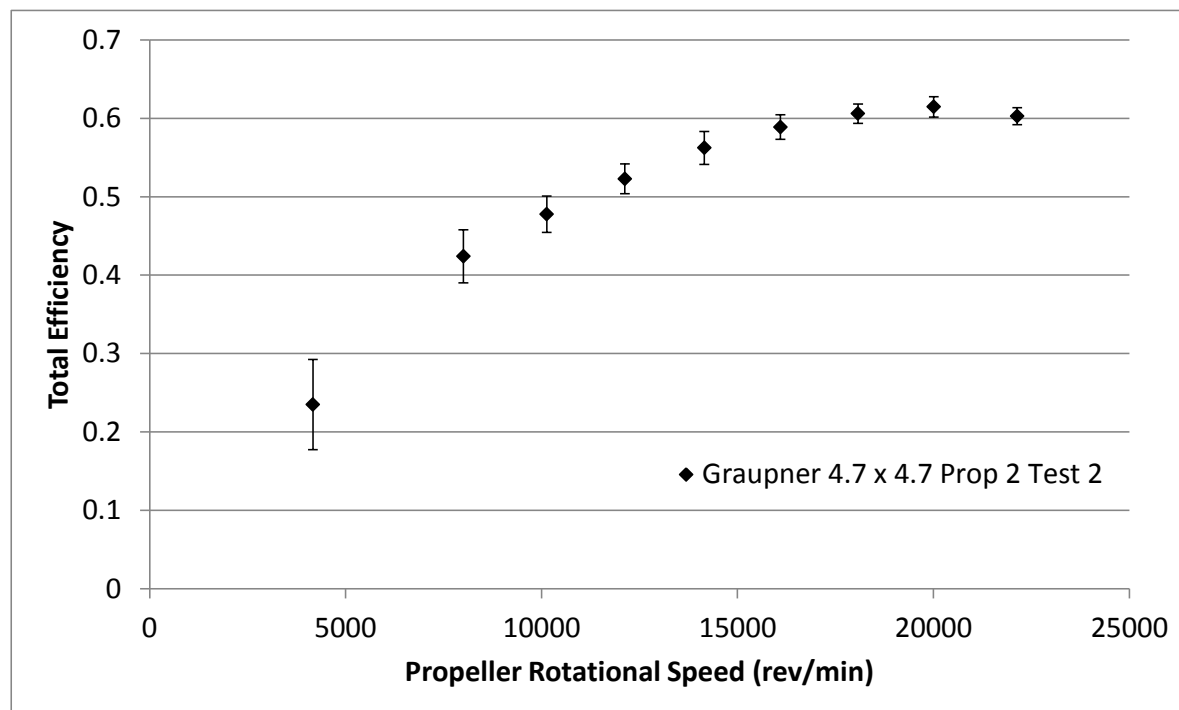

(c)

Figure 63: Graupner 4.7 x 4.7 Static Test Results (Prop 2 Test 2): (a) Coefficient of Thrust, (b) Coefficient of Power, (c) Total Efficiency. 
Table 48: Graupner 4.7 x 4.7 Static Measured Values (Prop 2 Test 2)

\begin{tabular}{|c|c|c|c|c|c|c|c|c|}
\hline$n(\mathrm{RPM})$ & $T(\mathrm{~g})$ & $\Delta T(\mathrm{~g})$ & $Q(\mathrm{~g}-\mathrm{m})$ & $\Delta Q(\mathrm{~g}-\mathrm{m})$ & $V(\mathrm{~V})$ & $I(\mathrm{~A})$ & $T_{\text {atm }}\left({ }^{\circ} \mathrm{C}\right)$ & $P_{\text {atm }}(\mathrm{Pa})$ \\
\hline $4.166 \mathrm{E}+03$ & $1.568 \mathrm{E}+01$ & $9.14 \mathrm{E}+00$ & $2.404 \mathrm{E}-01$ & $5.89 \mathrm{E}-02$ & $1.109 \mathrm{E}+01$ & $3.947 \mathrm{E}-01$ & $2.271 \mathrm{E}+01$ & $9.852 \mathrm{E}+04$ \\
\hline $8.007 \mathrm{E}+03$ & $5.891 \mathrm{E}+01$ & $9.29 \mathrm{E}+00$ & $8.501 \mathrm{E}-01$ & $6.74 \mathrm{E}-02$ & $1.107 \mathrm{E}+01$ & $1.490 \mathrm{E}+00$ & $2.260 \mathrm{E}+01$ & $9.851 \mathrm{E}+04$ \\
\hline $1.013 \mathrm{E}+04$ & $9.459 \mathrm{E}+01$ & $8.89 \mathrm{E}+00$ & $1.296 \mathrm{E}+00$ & $6.10 \mathrm{E}-02$ & $1.104 \mathrm{E}+01$ & $2.556 \mathrm{E}+00$ & $2.258 \mathrm{E}+01$ & $9.850 \mathrm{E}+04$ \\
\hline $1.213 \mathrm{E}+04$ & $1.358 \mathrm{E}+02$ & $8.96 \mathrm{E}+00$ & $1.802 \mathrm{E}+00$ & $6.25 \mathrm{E}-02$ & $1.102 \mathrm{E}+01$ & $3.896 \mathrm{E}+00$ & $2.258 \mathrm{E}+01$ & $9.850 \mathrm{E}+04$ \\
\hline $1.415 \mathrm{E}+04$ & $1.880 \mathrm{E}+02$ & $9.45 \mathrm{E}+00$ & $2.473 \mathrm{E}+00$ & $8.80 \mathrm{E}-02$ & $1.097 \mathrm{E}+01$ & $5.826 \mathrm{E}+00$ & $2.257 \mathrm{E}+01$ & $9.851 \mathrm{E}+04$ \\
\hline $1.610 \mathrm{E}+04$ & $2.453 \mathrm{E}+02$ & $8.88 \mathrm{E}+00$ & $3.179 \mathrm{E}+00$ & $7.63 \mathrm{E}-02$ & $1.092 \mathrm{E}+01$ & $8.170 \mathrm{E}+00$ & $2.254 \mathrm{E}+01$ & $9.851 \mathrm{E}+04$ \\
\hline $1.808 \mathrm{E}+04$ & $3.103 \mathrm{E}+02$ & $8.85 \mathrm{E}+00$ & $4.017 \mathrm{E}+00$ & $7.06 \mathrm{E}-02$ & $1.085 \mathrm{E}+01$ & $1.134 \mathrm{E}+01$ & $2.250 \mathrm{E}+01$ & $9.851 \mathrm{E}+04$ \\
\hline $2.001 \mathrm{E}+04$ & $3.823 \mathrm{E}+02$ & $8.91 \mathrm{E}+00$ & $4.972 \mathrm{E}+00$ & $9.06 \mathrm{E}-02$ & $1.076 \mathrm{E}+01$ & $1.545 \mathrm{E}+01$ & $2.249 \mathrm{E}+01$ & $9.851 \mathrm{E}+04$ \\
\hline $2.214 \mathrm{E}+04$ & $4.718 \mathrm{E}+02$ & $9.15 \mathrm{E}+00$ & $6.174 \mathrm{E}+00$ & $9.03 \mathrm{E}-02$ & $1.061 \mathrm{E}+01$ & $2.195 \mathrm{E}+01$ & $2.248 \mathrm{E}+01$ & $9.849 \mathrm{E}+04$ \\
\hline
\end{tabular}

Table 49: Graupner 4.7 x 4.7 Static Calculated Values (Prop 2 Test 2)

\begin{tabular}{|c|c|c|c|c|c|c|c|}
\hline$n(\mathrm{RPM})$ & $\rho\left(\mathrm{kg} / \mathrm{m}^{\wedge} 3\right)$ & $P_{\mathrm{P}}(\mathrm{W})$ & $\Delta P_{\mathrm{P}}(\mathrm{W})$ & $P_{\mathrm{e}}(\mathrm{W})$ & $\Delta P_{\mathrm{e}}(\mathrm{W})$ & $C_{\mathrm{T}}$ & $\Delta C_{\mathrm{T}}$ \\
\hline $4.166 \mathrm{E}+03$ & $1.160 \mathrm{E}+00$ & $1.028 \mathrm{E}+00$ & $2.52 \mathrm{E}-01$ & $4.378 \mathrm{E}+00$ & $6.73 \mathrm{E}-02$ & $1.313 \mathrm{E}-01$ & $7.66 \mathrm{E}-02$ \\
\hline $8.007 \mathrm{E}+03$ & $1.160 \mathrm{E}+00$ & $6.990 \mathrm{E}+00$ & $5.54 \mathrm{E}-01$ & $1.649 \mathrm{E}+01$ & $1.95 \mathrm{E}-01$ & $1.335 \mathrm{E}-01$ & $2.10 \mathrm{E}-02$ \\
\hline $1.013 \mathrm{E}+04$ & $1.160 \mathrm{E}+00$ & $1.349 \mathrm{E}+01$ & $6.35 \mathrm{E}-01$ & $2.823 \mathrm{E}+01$ & $3.21 \mathrm{E}-01$ & $1.338 \mathrm{E}-01$ & $1.26 \mathrm{E}-02$ \\
\hline $1.213 \mathrm{E}+04$ & $1.160 \mathrm{E}+00$ & $2.244 \mathrm{E}+01$ & $7.79 \mathrm{E}-01$ & $4.292 \mathrm{E}+01$ & $4.76 \mathrm{E}-01$ & $1.341 \mathrm{E}-01$ & $8.84 \mathrm{E}-03$ \\
\hline $1.415 \mathrm{E}+04$ & $1.160 \mathrm{E}+00$ & $3.595 \mathrm{E}+01$ & $1.28 \mathrm{E}+00$ & $6.393 \mathrm{E}+01$ & $7.33 \mathrm{E}-01$ & $1.362 \mathrm{E}-01$ & $6.85 \mathrm{E}-03$ \\
\hline $1.610 \mathrm{E}+04$ & $1.161 \mathrm{E}+00$ & $5.255 \mathrm{E}+01$ & $1.26 \mathrm{E}+00$ & $8.924 \mathrm{E}+01$ & $1.01 \mathrm{E}+00$ & $1.375 \mathrm{E}-01$ & $4.98 \mathrm{E}-03$ \\
\hline $1.808 \mathrm{E}+04$ & $1.161 \mathrm{E}+00$ & $7.458 \mathrm{E}+01$ & $1.31 \mathrm{E}+00$ & $1.231 \mathrm{E}+02$ & $1.33 \mathrm{E}+00$ & $1.378 \mathrm{E}-01$ & $3.93 \mathrm{E}-03$ \\
\hline $2.001 \mathrm{E}+04$ & $1.161 \mathrm{E}+00$ & $1.022 \mathrm{E}+02$ & $1.86 \mathrm{E}+00$ & $1.662 \mathrm{E}+02$ & $1.81 \mathrm{E}+00$ & $1.386 \mathrm{E}-01$ & $3.23 \mathrm{E}-03$ \\
\hline $2.214 \mathrm{E}+04$ & $1.161 \mathrm{E}+00$ & $1.404 \mathrm{E}+02$ & $2.05 \mathrm{E}+00$ & $2.329 \mathrm{E}+02$ & $2.45 \mathrm{E}+00$ & $1.397 \mathrm{E}-01$ & $2.71 \mathrm{E}-03$ \\
\hline$C_{\mathrm{P}}$ & $\Delta C_{\mathrm{P}}$ & $C_{\mathrm{Q}}$ & $\Delta C_{\mathrm{Q}}$ & $\eta_{\mathrm{T}}$ & $\Delta \eta_{\mathrm{T}}$ & $R e_{0.75}$ & $\Delta R e_{0.75}$ \\
\hline $1.051 \mathrm{E}-01$ & $2.57 \mathrm{E}-02$ & $1.673 \mathrm{E}-02$ & $4.10 \mathrm{E}-03$ & $2.349 \mathrm{E}-01$ & $5.76 \mathrm{E}-02$ & $1.123 \mathrm{E}+04$ & $1.56 \mathrm{E}+01$ \\
\hline $1.006 \mathrm{E}-01$ & $7.97 \mathrm{E}-03$ & $1.601 \mathrm{E}-02$ & $1.27 \mathrm{E}-03$ & $4.240 \mathrm{E}-01$ & $3.40 \mathrm{E}-02$ & $2.160 \mathrm{E}+04$ & $2.81 \mathrm{E}+01$ \\
\hline $9.572 \mathrm{E}-02$ & $4.51 \mathrm{E}-03$ & $1.523 \mathrm{E}-02$ & $7.17 \mathrm{E}-04$ & $4.777 \mathrm{E}-01$ & $2.31 \mathrm{E}-02$ & $2.734 \mathrm{E}+04$ & $3.46 \mathrm{E}+01$ \\
\hline $9.290 \mathrm{E}-02$ & $3.23 \mathrm{E}-03$ & $1.479 \mathrm{E}-02$ & $5.14 \mathrm{E}-04$ & $5.228 \mathrm{E}-01$ & $1.91 \mathrm{E}-02$ & $3.272 \mathrm{E}+04$ & $4.13 \mathrm{E}+01$ \\
\hline $9.361 \mathrm{E}-02$ & $3.33 \mathrm{E}-03$ & $1.490 \mathrm{E}-02$ & $5.30 \mathrm{E}-04$ & $5.623 \mathrm{E}-01$ & $2.10 \mathrm{E}-02$ & $3.820 \mathrm{E}+04$ & $4.75 \mathrm{E}+01$ \\
\hline $9.304 \mathrm{E}-02$ & $2.24 \mathrm{E}-03$ & $1.481 \mathrm{E}-02$ & $3.56 \mathrm{E}-04$ & $5.889 \mathrm{E}-01$ & $1.56 \mathrm{E}-02$ & $4.345 \mathrm{E}+04$ & $5.33 \mathrm{E}+01$ \\
\hline $9.320 \mathrm{E}-02$ & $1.64 \mathrm{E}-03$ & $1.483 \mathrm{E}-02$ & $2.61 \mathrm{E}-04$ & $6.059 \mathrm{E}-01$ & $1.25 \mathrm{E}-02$ & $4.881 \mathrm{E}+04$ & $5.96 \mathrm{E}+01$ \\
\hline $9.415 \mathrm{E}-02$ & $1.72 \mathrm{E}-03$ & $1.498 \mathrm{E}-02$ & $2.73 \mathrm{E}-04$ & $6.147 \mathrm{E}-01$ & $1.30 \mathrm{E}-02$ & $5.403 \mathrm{E}+04$ & $6.64 \mathrm{E}+01$ \\
\hline $9.551 \mathrm{E}-02$ & $1.40 \mathrm{E}-03$ & $1.673 \mathrm{E}-02$ & $2.22 \mathrm{E}-04$ & $6.028 \mathrm{E}-01$ & $1.09 \mathrm{E}-02$ & $5.977 \mathrm{E}+04$ & $7.30 \mathrm{E}+01$ \\
\hline
\end{tabular}




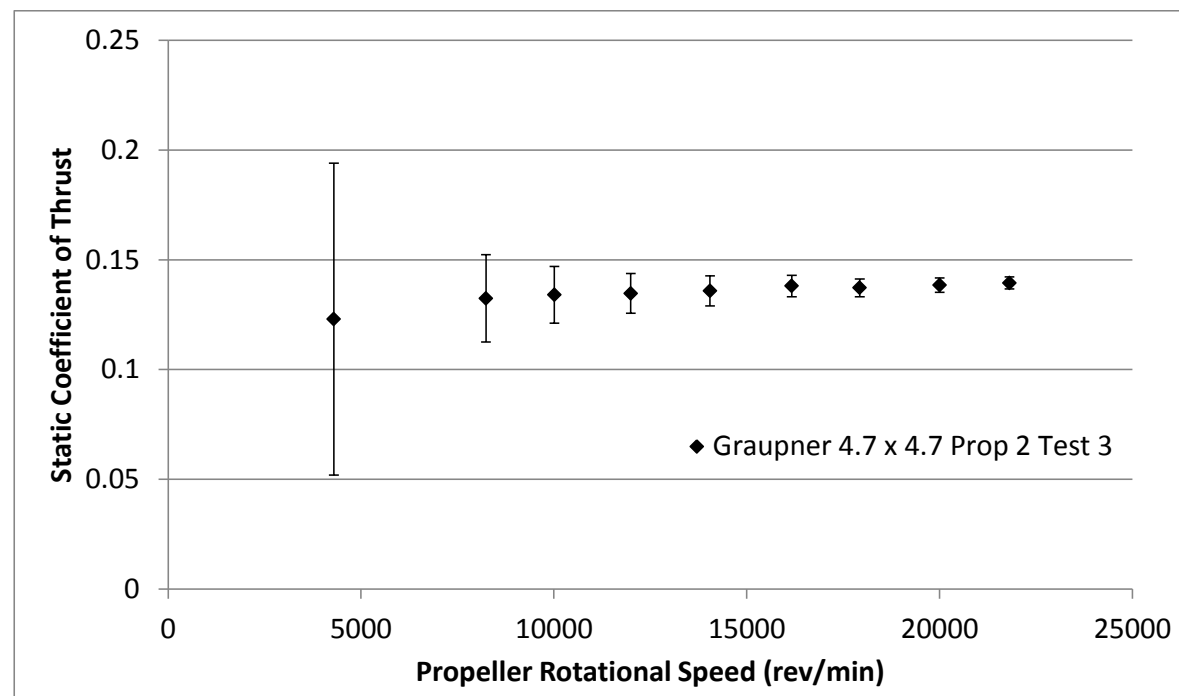

(a)

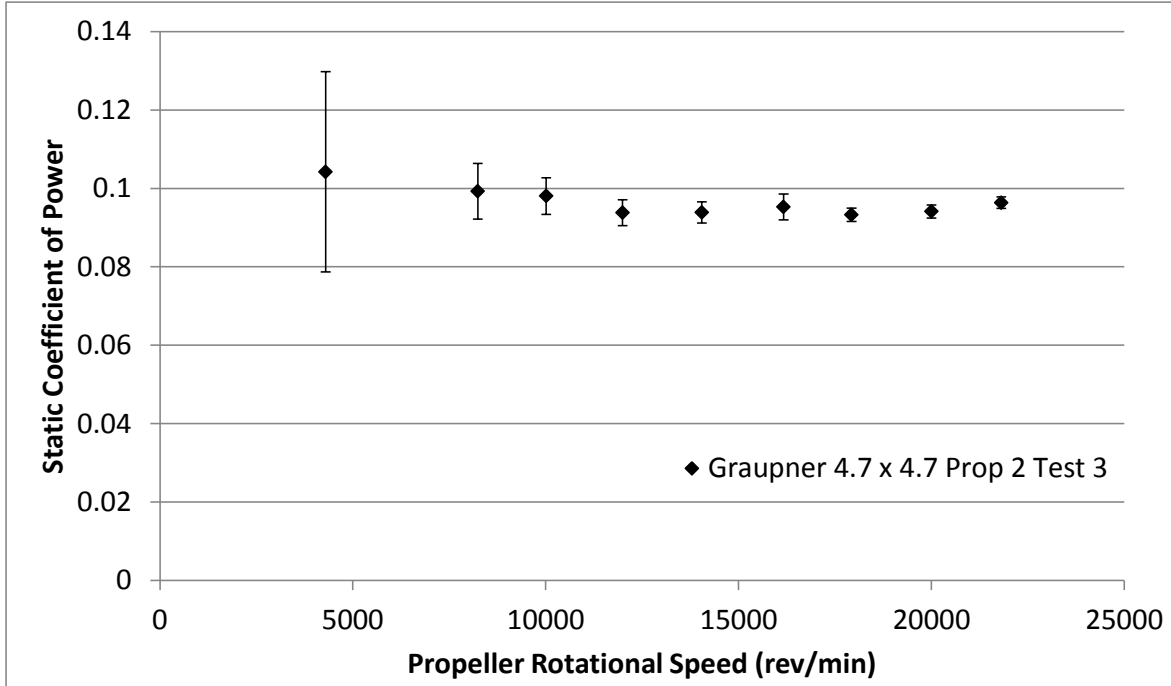

(b)

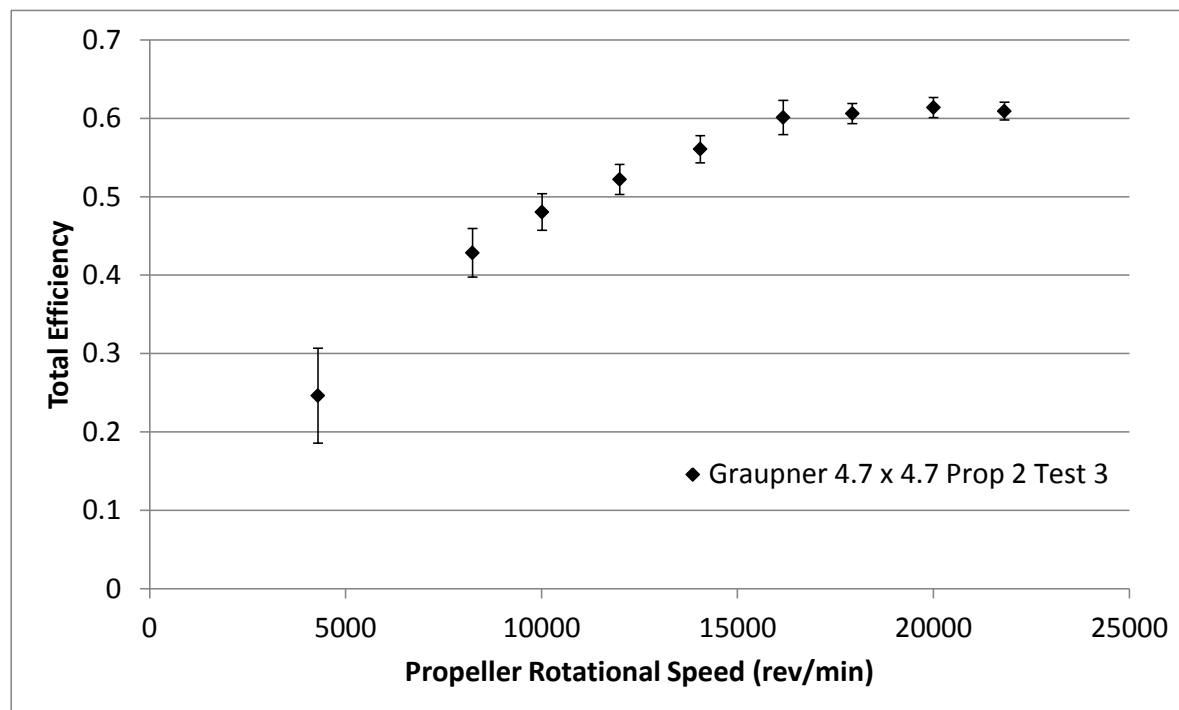

(c)

Figure 64: Graupner 4.7 x 4.7 Static Test Results (Prop 2 Test 3): (a) Coefficient of Thrust, (b) Coefficient of Power, (c) Total Efficiency. 
Table 50: Graupner 4.7 x 4.7 Static Measured Values (Prop 2 Test 3)

\begin{tabular}{|c|c|c|c|c|c|c|c|c|}
\hline$n(\mathrm{RPM})$ & $T(\mathrm{~g})$ & $\Delta T(\mathrm{~g})$ & $Q(\mathrm{~g}-\mathrm{m})$ & $\Delta Q(\mathrm{~g}-\mathrm{m})$ & $V(\mathrm{~V})$ & $I(\mathrm{~A})$ & $T_{\text {atm }}\left({ }^{\circ} \mathrm{C}\right)$ & $P_{\text {atm }}(\mathrm{Pa})$ \\
\hline $4.295 \mathrm{E}+03$ & $1.561 \mathrm{E}+01$ & $9.03 \mathrm{E}+00$ & $2.535 \mathrm{E}-01$ & $6.22 \mathrm{E}-02$ & $1.109 \mathrm{E}+01$ & $4.091 \mathrm{E}-01$ & $2.264 \mathrm{E}+01$ & $9.851 \mathrm{E}+04$ \\
\hline $8.240 \mathrm{E}+03$ & $6.192 \mathrm{E}+01$ & $9.31 \mathrm{E}+00$ & $8.889 \mathrm{E}-01$ & $6.34 \mathrm{E}-02$ & $1.107 \mathrm{E}+01$ & $1.587 \mathrm{E}+00$ & $2.260 \mathrm{E}+01$ & $9.852 \mathrm{E}+04$ \\
\hline $1.001 \mathrm{E}+04$ & $9.259 \mathrm{E}+01$ & $8.94 \mathrm{E}+00$ & $1.297 \mathrm{E}+00$ & $6.14 \mathrm{E}-02$ & $1.105 \mathrm{E}+01$ & $2.513 \mathrm{E}+00$ & $2.255 \mathrm{E}+01$ & $9.852 \mathrm{E}+04$ \\
\hline $1.200 \mathrm{E}+04$ & $1.335 \mathrm{E}+02$ & $9.00 \mathrm{E}+00$ & $1.780 \mathrm{E}+00$ & $6.26 \mathrm{E}-02$ & $1.102 \mathrm{E}+01$ & $3.814 \mathrm{E}+00$ & $2.254 \mathrm{E}+01$ & $9.851 \mathrm{E}+04$ \\
\hline $1.405 \mathrm{E}+04$ & $1.847 \mathrm{E}+02$ & $9.29 \mathrm{E}+00$ & $2.444 \mathrm{E}+00$ & $7.01 \mathrm{E}-02$ & $1.098 \mathrm{E}+01$ & $5.731 \mathrm{E}+00$ & $2.257 \mathrm{E}+01$ & $9.853 \mathrm{E}+04$ \\
\hline $1.617 \mathrm{E}+04$ & $2.486 \mathrm{E}+02$ & $8.82 \mathrm{E}+00$ & $3.285 \mathrm{E}+00$ & $1.13 \mathrm{E}-01$ & $1.092 \mathrm{E}+01$ & $8.312 \mathrm{E}+00$ & $2.251 \mathrm{E}+01$ & $9.850 \mathrm{E}+04$ \\
\hline $1.793 \mathrm{E}+04$ & $3.038 \mathrm{E}+02$ & $8.87 \mathrm{E}+00$ & $3.956 \mathrm{E}+00$ & $7.21 \mathrm{E}-02$ & $1.086 \mathrm{E}+01$ & $1.107 \mathrm{E}+01$ & $2.253 \mathrm{E}+01$ & $9.850 \mathrm{E}+04$ \\
\hline $2.000 \mathrm{E}+04$ & $3.815 \mathrm{E}+02$ & $8.97 \mathrm{E}+00$ & $4.967 \mathrm{E}+00$ & $8.85 \mathrm{E}-02$ & $1.076 \mathrm{E}+01$ & $1.546 \mathrm{E}+01$ & $2.252 \mathrm{E}+01$ & $9.850 \mathrm{E}+04$ \\
\hline $2.181 \mathrm{E}+04$ & $4.572 \mathrm{E}+02$ & $9.14 \mathrm{E}+00$ & $6.049 \mathrm{E}+00$ & $9.15 \mathrm{E}-02$ & $1.063 \mathrm{E}+01$ & $2.092 \mathrm{E}+01$ & $2.246 \mathrm{E}+01$ & $9.851 \mathrm{E}+04$ \\
\hline
\end{tabular}

Table 51: Graupner 4.7 x 4.7 Static Calculated Values (Prop 2 Test 3)

\begin{tabular}{|c|c|c|c|c|c|c|c|}
\hline$n(\mathrm{RPM})$ & $\rho\left(\mathrm{kg} / \mathrm{m}^{\wedge} 3\right)$ & $P_{\mathrm{P}}(\mathrm{W})$ & $\Delta P_{\mathrm{P}}(\mathrm{W})$ & $P_{\mathrm{e}}(\mathrm{W})$ & $\Delta P_{\mathrm{e}}(\mathrm{W})$ & $C_{\mathrm{T}}$ & $\Delta C_{\mathrm{T}}$ \\
\hline $4.295 \mathrm{E}+03$ & $1.160 \mathrm{E}+00$ & $1.118 \mathrm{E}+00$ & $2.74 \mathrm{E}-01$ & $4.537 \mathrm{E}+00$ & $6.85 \mathrm{E}-02$ & $1.230 \mathrm{E}-01$ & $7.11 \mathrm{E}-02$ \\
\hline $8.240 \mathrm{E}+03$ & $1.160 \mathrm{E}+00$ & $7.522 \mathrm{E}+00$ & $5.36 \mathrm{E}-01$ & $1.756 \mathrm{E}+01$ & $2.06 \mathrm{E}-01$ & $1.324 \mathrm{E}-01$ & $1.99 \mathrm{E}-02$ \\
\hline $1.001 \mathrm{E}+04$ & $1.161 \mathrm{E}+00$ & $1.334 \mathrm{E}+01$ & $6.32 \mathrm{E}-01$ & $2.776 \mathrm{E}+01$ & $3.16 \mathrm{E}-01$ & $1.340 \mathrm{E}-01$ & $1.29 \mathrm{E}-02$ \\
\hline $1.200 \mathrm{E}+04$ & $1.161 \mathrm{E}+00$ & $2.193 \mathrm{E}+01$ & $7.71 \mathrm{E}-01$ & $4.202 \mathrm{E}+01$ & $4.66 \mathrm{E}-01$ & $1.347 \mathrm{E}-01$ & $9.08 \mathrm{E}-03$ \\
\hline $1.405 \mathrm{E}+04$ & $1.161 \mathrm{E}+00$ & $3.526 \mathrm{E}+01$ & $1.01 \mathrm{E}+00$ & $6.290 \mathrm{E}+01$ & $7.10 \mathrm{E}-01$ & $1.358 \mathrm{E}-01$ & $6.84 \mathrm{E}-03$ \\
\hline $1.617 \mathrm{E}+04$ & $1.161 \mathrm{E}+00$ & $5.454 \mathrm{E}+01$ & $1.88 \mathrm{E}+00$ & $9.076 \mathrm{E}+01$ & $1.05 \mathrm{E}+00$ & $1.381 \mathrm{E}-01$ & $4.90 \mathrm{E}-03$ \\
\hline $1.793 \mathrm{E}+04$ & $1.161 \mathrm{E}+00$ & $7.285 \mathrm{E}+01$ & $1.33 \mathrm{E}+00$ & $1.202 \mathrm{E}+02$ & $1.30 \mathrm{E}+00$ & $1.372 \mathrm{E}-01$ & $4.01 \mathrm{E}-03$ \\
\hline $2.000 \mathrm{E}+04$ & $1.161 \mathrm{E}+00$ & $1.020 \mathrm{E}+02$ & $1.82 \mathrm{E}+00$ & $1.663 \mathrm{E}+02$ & $1.79 \mathrm{E}+00$ & $1.384 \mathrm{E}-01$ & $3.26 \mathrm{E}-03$ \\
\hline $2.181 \mathrm{E}+04$ & $1.161 \mathrm{E}+00$ & $1.355 \mathrm{E}+02$ & $2.05 \mathrm{E}+00$ & $2.224 \mathrm{E}+02$ & $2.35 \mathrm{E}+00$ & $1.395 \mathrm{E}-01$ & $2.79 \mathrm{E}-03$ \\
\hline$C_{\mathrm{P}}$ & $\Delta C_{\mathrm{P}}$ & $C_{\mathrm{Q}}$ & $\Delta C_{\mathrm{Q}}$ & $\eta_{\mathrm{T}}$ & $\Delta \eta_{\mathrm{T}}$ & $R e_{0.75}$ & $\Delta R e_{0.75}$ \\
\hline $1.042 \mathrm{E}-01$ & $2.56 \mathrm{E}-02$ & $1.659 \mathrm{E}-02$ & $4.07 \mathrm{E}-03$ & $2.464 \mathrm{E}-01$ & $6.05 \mathrm{E}-02$ & $1.159 \mathrm{E}+04$ & $1.63 \mathrm{E}+01$ \\
\hline $9.927 \mathrm{E}-02$ & $7.08 \mathrm{E}-03$ & $1.580 \mathrm{E}-02$ & $1.13 \mathrm{E}-03$ & $4.284 \mathrm{E}-01$ & $3.10 \mathrm{E}-02$ & $2.224 \mathrm{E}+04$ & $2.92 \mathrm{E}+01$ \\
\hline $9.805 \mathrm{E}-02$ & $4.65 \mathrm{E}-03$ & $1.560 \mathrm{E}-02$ & $7.39 \mathrm{E}-04$ & $4.805 \mathrm{E}-01$ & $2.34 \mathrm{E}-02$ & $2.703 \mathrm{E}+04$ & $3.44 \mathrm{E}+01$ \\
\hline $9.382 \mathrm{E}-02$ & $3.30 \mathrm{E}-03$ & $1.493 \mathrm{E}-02$ & $5.25 \mathrm{E}-04$ & $5.220 \mathrm{E}-01$ & $1.92 \mathrm{E}-02$ & $3.238 \mathrm{E}+04$ & $4.09 \mathrm{E}+01$ \\
\hline $9.386 \mathrm{E}-02$ & $2.69 \mathrm{E}-03$ & $1.494 \mathrm{E}-02$ & $4.29 \mathrm{E}-04$ & $5.607 \mathrm{E}-01$ & $1.73 \mathrm{E}-02$ & $3.793 \mathrm{E}+04$ & $4.79 \mathrm{E}+01$ \\
\hline $9.526 \mathrm{E}-02$ & $3.29 \mathrm{E}-03$ & $1.516 \mathrm{E}-02$ & $5.23 \mathrm{E}-04$ & $6.010 \mathrm{E}-01$ & $2.18 \mathrm{E}-02$ & $4.365 \mathrm{E}+04$ & $5.38 \mathrm{E}+01$ \\
\hline $9.329 \mathrm{E}-02$ & $1.70 \mathrm{E}-03$ & $1.485 \mathrm{E}-02$ & $2.71 \mathrm{E}-04$ & $6.060 \mathrm{E}-01$ & $1.28 \mathrm{E}-02$ & $4.840 \mathrm{E}+04$ & $5.92 \mathrm{E}+01$ \\
\hline $9.412 \mathrm{E}-02$ & $1.68 \mathrm{E}-03$ & $1.498 \mathrm{E}-02$ & $2.67 \mathrm{E}-04$ & $6.137 \mathrm{E}-01$ & $1.28 \mathrm{E}-02$ & $5.400 \mathrm{E}+04$ & $6.63 \mathrm{E}+01$ \\
\hline $9.637 \mathrm{E}-02$ & $1.46 \mathrm{E}-03$ & $1.659 \mathrm{E}-02$ & $2.32 \mathrm{E}-04$ & $6.092 \mathrm{E}-01$ & $1.12 \mathrm{E}-02$ & $5.891 \mathrm{E}+04$ & $7.26 \mathrm{E}+01$ \\
\hline
\end{tabular}




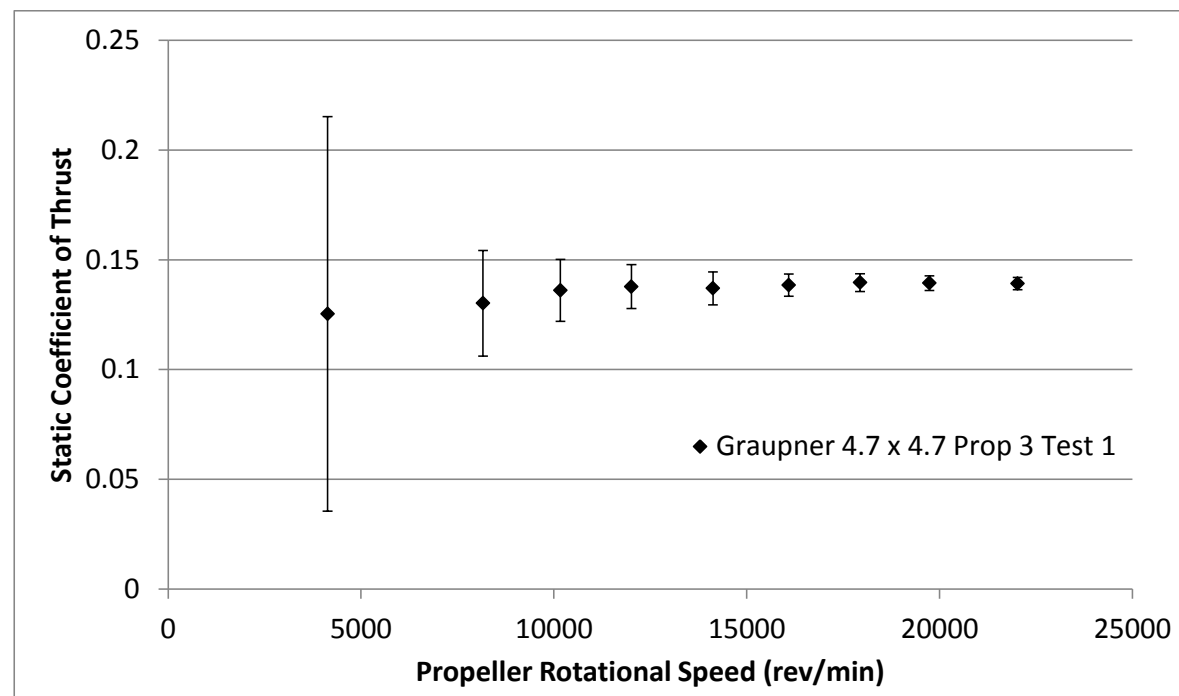

(a)

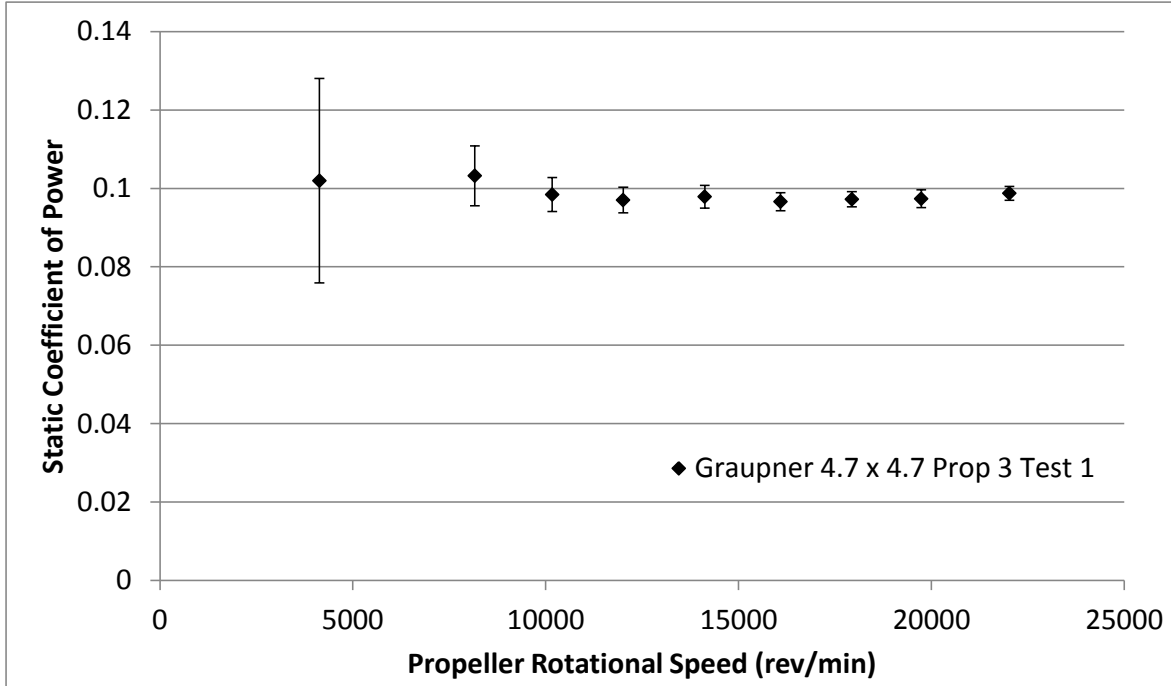

(b)

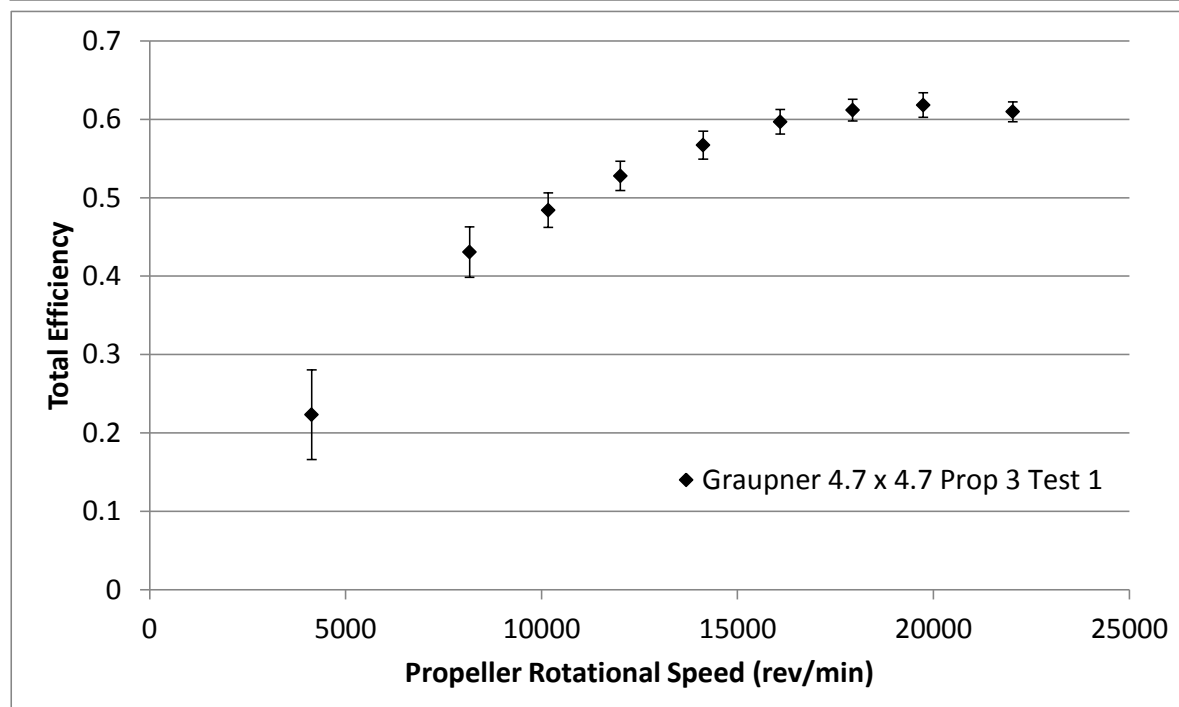

Figure 65: Graupner 4.7 x 4.7 Static Test Results (Prop 3 Test 1): (a) Coefficient of Thrust, (b) Coefficient of Power, (c) Total Efficiency. 
Table 52: Graupner 4.7 x 4.7 Static Measured Values (Prop 3 Test 1)

\begin{tabular}{|c|c|c|c|c|c|c|c|c|}
\hline$n(\mathrm{RPM})$ & $T(\mathrm{~g})$ & $\Delta T(\mathrm{~g})$ & $Q(\mathrm{~g}-\mathrm{m})$ & $\Delta Q(\mathrm{~g}-\mathrm{m})$ & $V(\mathrm{~V})$ & $I(\mathrm{~A})$ & $T_{\text {atm }}\left({ }^{\circ} \mathrm{C}\right)$ & $P_{\text {atm }}(\mathrm{Pa})$ \\
\hline $4.135 \mathrm{E}+03$ & $1.481 \mathrm{E}+01$ & $1.06 \mathrm{E}+01$ & $2.307 \mathrm{E}-01$ & $5.90 \mathrm{E}-02$ & $1.109 \mathrm{E}+01$ & $3.954 \mathrm{E}-01$ & $2.202 \mathrm{E}+01$ & $9.869 \mathrm{E}+04$ \\
\hline $8.164 \mathrm{E}+03$ & $5.994 \mathrm{E}+01$ & $1.11 \mathrm{E}+01$ & $9.099 \mathrm{E}-01$ & $6.71 \mathrm{E}-02$ & $1.107 \mathrm{E}+01$ & $1.601 \mathrm{E}+00$ & $2.192 \mathrm{E}+01$ & $9.861 \mathrm{E}+04$ \\
\hline $1.017 \mathrm{E}+04$ & $9.725 \mathrm{E}+01$ & $1.01 \mathrm{E}+01$ & $1.347 \mathrm{E}+00$ & $5.93 \mathrm{E}-02$ & $1.104 \mathrm{E}+01$ & $2.632 \mathrm{E}+00$ & $2.194 \mathrm{E}+01$ & $9.861 \mathrm{E}+04$ \\
\hline $1.201 \mathrm{E}+04$ & $1.373 \mathrm{E}+02$ & $9.98 \mathrm{E}+00$ & $1.852 \mathrm{E}+00$ & $6.24 \mathrm{E}-02$ & $1.102 \mathrm{E}+01$ & $3.929 \mathrm{E}+00$ & $2.192 \mathrm{E}+01$ & $9.859 \mathrm{E}+04$ \\
\hline $1.413 \mathrm{E}+04$ & $1.888 \mathrm{E}+02$ & $1.03 \mathrm{E}+01$ & $2.583 \mathrm{E}+00$ & $7.57 \mathrm{E}-02$ & $1.097 \mathrm{E}+01$ & $6.023 \mathrm{E}+00$ & $2.194 \mathrm{E}+01$ & $9.858 \mathrm{E}+04$ \\
\hline $1.609 \mathrm{E}+04$ & $2.475 \mathrm{E}+02$ & $9.06 \mathrm{E}+00$ & $3.309 \mathrm{E}+00$ & $7.84 \mathrm{E}-02$ & $1.092 \mathrm{E}+01$ & $8.390 \mathrm{E}+00$ & $2.193 \mathrm{E}+01$ & $9.857 \mathrm{E}+04$ \\
\hline $1.794 \mathrm{E}+04$ & $3.104 \mathrm{E}+02$ & $8.92 \mathrm{E}+00$ & $4.139 \mathrm{E}+00$ & $8.22 \mathrm{E}-02$ & $1.085 \mathrm{E}+01$ & $1.149 \mathrm{E}+01$ & $2.185 \mathrm{E}+01$ & $9.858 \mathrm{E}+04$ \\
\hline $1.974 \mathrm{E}+04$ & $3.752 \mathrm{E}+02$ & $9.00 \mathrm{E}+00$ & $5.019 \mathrm{E}+00$ & $1.16 \mathrm{E}-01$ & $1.077 \mathrm{E}+01$ & $1.528 \mathrm{E}+01$ & $2.190 \mathrm{E}+01$ & $9.859 \mathrm{E}+04$ \\
\hline $2.203 \mathrm{E}+04$ & $4.663 \mathrm{E}+02$ & $9.28 \mathrm{E}+00$ & $6.333 \mathrm{E}+00$ & $1.13 \mathrm{E}-01$ & $1.061 \mathrm{E}+01$ & $2.214 \mathrm{E}+01$ & $2.190 \mathrm{E}+01$ & $9.856 \mathrm{E}+04$ \\
\hline
\end{tabular}

Table 53: Graupner 4.7 x 4.7 Static Measured Values (Prop 3 Test 1)

\begin{tabular}{|c|c|c|c|c|c|c|c|}
\hline$n(\mathrm{RPM})$ & $\rho\left(\mathrm{kg} / \mathrm{m}^{\wedge} 3\right)$ & $P_{\mathrm{P}}(\mathrm{W})$ & $\Delta P_{\mathrm{P}}(\mathrm{W})$ & $P_{\mathrm{e}}(\mathrm{W})$ & $\Delta P_{\mathrm{e}}(\mathrm{W})$ & $C_{\mathrm{T}}$ & $\Delta C_{\mathrm{T}}$ \\
\hline $4.135 \mathrm{E}+03$ & $1.165 \mathrm{E}+00$ & $9.796 \mathrm{E}-01$ & $2.50 \mathrm{E}-01$ & $4.386 \mathrm{E}+00$ & $6.78 \mathrm{E}-02$ & $1.253 \mathrm{E}-01$ & 8.99E-02 \\
\hline $8.164 \mathrm{E}+03$ & $1.164 \mathrm{E}+00$ & $7.629 \mathrm{E}+00$ & $5.63 \mathrm{E}-01$ & $1.771 \mathrm{E}+01$ & $2.09 \mathrm{E}-01$ & 1.302E-01 & $2.41 \mathrm{E}-02$ \\
\hline $1.017 \mathrm{E}+04$ & $1.164 \mathrm{E}+00$ & $1.407 \mathrm{E}+01$ & $6.19 \mathrm{E}-01$ & $2.906 \mathrm{E}+01$ & $3.31 \mathrm{E}-01$ & $1.361 \mathrm{E}-01$ & $1.41 \mathrm{E}-02$ \\
\hline $1.201 \mathrm{E}+04$ & $1.164 \mathrm{E}+00$ & $2.285 \mathrm{E}+01$ & $7.70 \mathrm{E}-01$ & $4.329 \mathrm{E}+01$ & $4.78 \mathrm{E}-01$ & $1.378 \mathrm{E}-01$ & $1.00 \mathrm{E}-02$ \\
\hline $1.413 \mathrm{E}+04$ & $1.164 \mathrm{E}+00$ & $3.747 \mathrm{E}+01$ & $1.10 \mathrm{E}+00$ & $6.608 \mathrm{E}+01$ & 7.54E-01 & $1.370 \mathrm{E}-01$ & 7.48E-03 \\
\hline $1.609 \mathrm{E}+04$ & $1.164 \mathrm{E}+00$ & $5.468 \mathrm{E}+01$ & $1.30 \mathrm{E}+00$ & $9.162 \mathrm{E}+01$ & $1.03 \mathrm{E}+00$ & $1.384 \mathrm{E}-01$ & $5.07 \mathrm{E}-03$ \\
\hline $1.794 \mathrm{E}+04$ & $1.164 \mathrm{E}+00$ & $7.625 \mathrm{E}+01$ & $1.51 \mathrm{E}+00$ & $1.247 \mathrm{E}+02$ & $1.35 \mathrm{E}+00$ & $1.396 \mathrm{E}-01$ & $4.01 \mathrm{E}-03$ \\
\hline $1.974 \mathrm{E}+04$ & $1.164 \mathrm{E}+00$ & $1.018 \mathrm{E}+02$ & $2.36 \mathrm{E}+00$ & $1.646 \mathrm{E}+02$ & $1.79 \mathrm{E}+00$ & $1.394 \mathrm{E}-01$ & $3.34 \mathrm{E}-03$ \\
\hline $2.203 \mathrm{E}+04$ & $1.164 \mathrm{E}+00$ & $1.433 \mathrm{E}+02$ & $2.55 \mathrm{E}+00$ & $2.350 \mathrm{E}+02$ & $2.47 \mathrm{E}+00$ & $1.392 \mathrm{E}-01$ & $2.77 \mathrm{E}-03$ \\
\hline$C_{\mathrm{P}}$ & $\Delta C_{\mathrm{P}}$ & $C_{\mathrm{Q}}$ & $\Delta C_{\mathrm{Q}}$ & $\eta_{\mathrm{T}}$ & $\Delta \eta_{\mathrm{T}}$ & $R e_{0.75}$ & $\Delta R e_{0.75}$ \\
\hline $1.020 \mathrm{E}-01$ & $2.61 \mathrm{E}-02$ & $1.623 \mathrm{E}-02$ & $4.15 \mathrm{E}-03$ & $2.233 \mathrm{E}-01$ & $5.72 \mathrm{E}-02$ & $1.122 \mathrm{E}+04$ & $1.62 \mathrm{E}+01$ \\
\hline $1.032 \mathrm{E}-01$ & 7.61E-03 & 1.643E-02 & $1.21 \mathrm{E}-03$ & 4.307E-01 & $3.22 \mathrm{E}-02$ & $2.214 \mathrm{E}+04$ & $2.87 \mathrm{E}+01$ \\
\hline $9.841 \mathrm{E}-02$ & $4.33 \mathrm{E}-03$ & $1.566 \mathrm{E}-02$ & $6.90 \mathrm{E}-04$ & $4.841 \mathrm{E}-01$ & $2.20 \mathrm{E}-02$ & $2.758 \mathrm{E}+04$ & $3.53 \mathrm{E}+01$ \\
\hline $9.704 \mathrm{E}-02$ & $3.27 \mathrm{E}-03$ & $1.544 \mathrm{E}-02$ & $5.21 \mathrm{E}-04$ & $5.278 \mathrm{E}-01$ & $1.87 \mathrm{E}-02$ & $3.257 \mathrm{E}+04$ & $4.04 \mathrm{E}+01$ \\
\hline $9.787 \mathrm{E}-02$ & $2.87 \mathrm{E}-03$ & $1.558 \mathrm{E}-02$ & $4.57 \mathrm{E}-04$ & $5.671 \mathrm{E}-01$ & $1.78 \mathrm{E}-02$ & $3.829 \mathrm{E}+04$ & $4.71 \mathrm{E}+01$ \\
\hline $9.663 \mathrm{E}-02$ & $2.29 \mathrm{E}-03$ & $1.538 \mathrm{E}-02$ & $3.65 \mathrm{E}-04$ & $5.968 \mathrm{E}-01$ & $1.57 \mathrm{E}-02$ & $4.362 \mathrm{E}+04$ & $5.33 \mathrm{E}+01$ \\
\hline $9.721 \mathrm{E}-02$ & $1.93 \mathrm{E}-03$ & $1.547 \mathrm{E}-02$ & $3.08 \mathrm{E}-04$ & $6.117 \mathrm{E}-01$ & $1.38 \mathrm{E}-02$ & $4.866 \mathrm{E}+04$ & $5.93 \mathrm{E}+01$ \\
\hline $9.736 \mathrm{E}-02$ & $2.26 \mathrm{E}-03$ & $1.550 \mathrm{E}-02$ & $3.59 \mathrm{E}-04$ & $6.182 \mathrm{E}-01$ & $1.58 \mathrm{E}-02$ & $5.353 \mathrm{E}+04$ & $6.55 \mathrm{E}+01$ \\
\hline 9.872E-02 & $1.76 \mathrm{E}-03$ & $1.623 \mathrm{E}-02$ & $2.80 \mathrm{E}-04$ & $6.096 \mathrm{E}-01$ & $1.26 \mathrm{E}-02$ & $5.971 \mathrm{E}+04$ & $7.44 \mathrm{E}+01$ \\
\hline
\end{tabular}




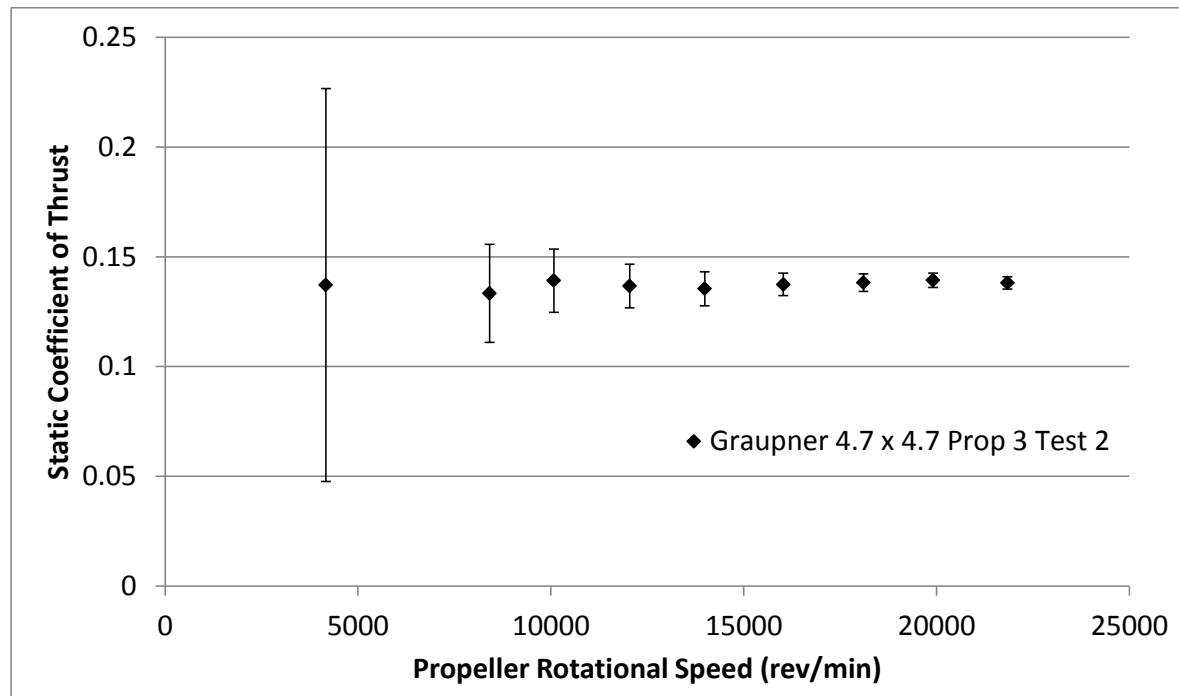

(a)

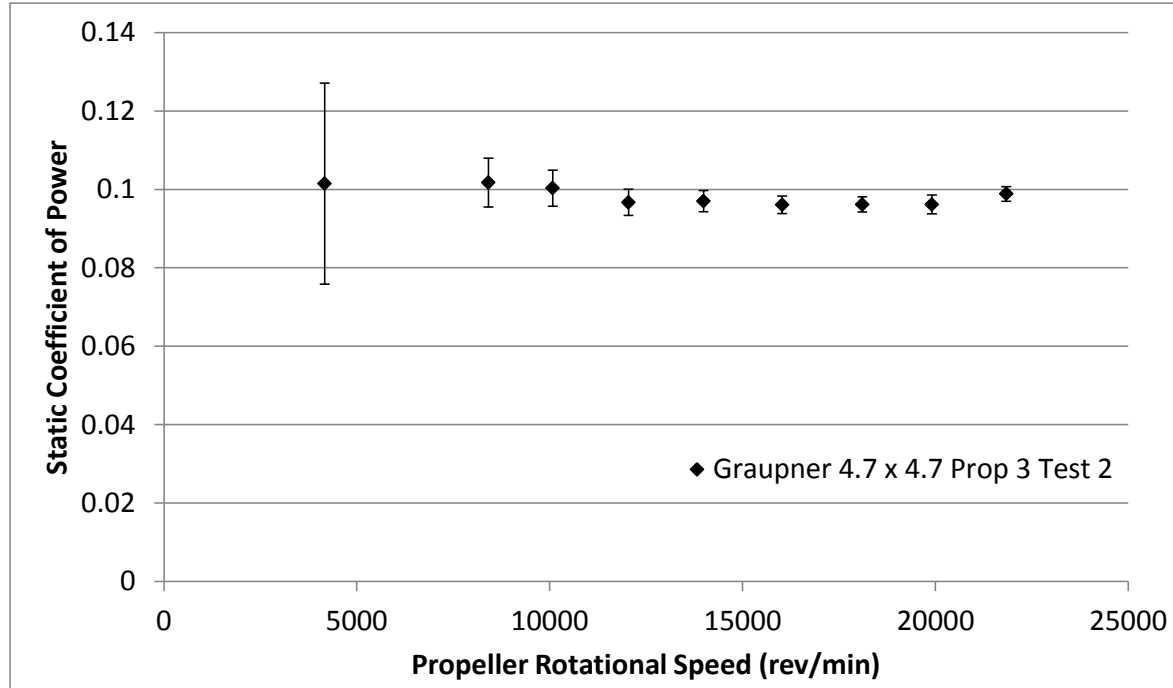

(b)

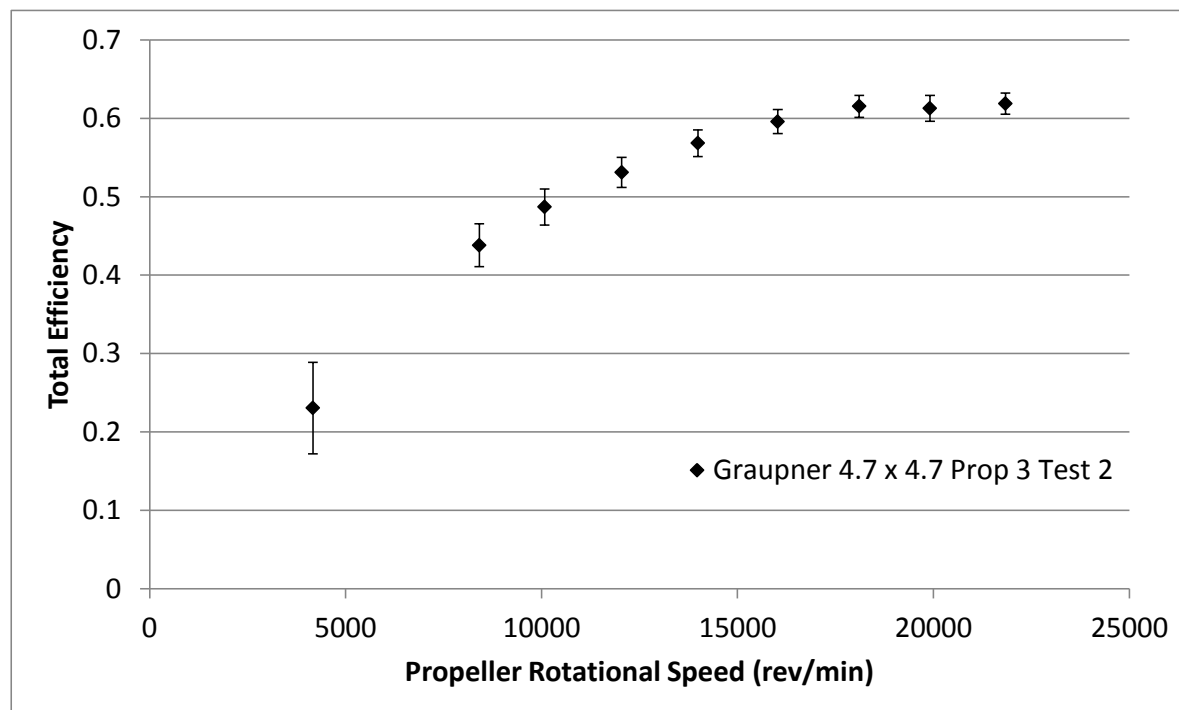

(c)

Figure 66: Graupner 4.7 x 4.7 Static Test Results (Prop 3 Test 2): (a) Coefficient of Thrust, (b) Coefficient of Power, (c) Total Efficiency. 
Table 54: Graupner 4.7 x 4.7 Static Measured Values (Prop 3 Test 2)

\begin{tabular}{|c|c|c|c|c|c|c|c|c|}
\hline$n(\mathrm{RPM})$ & $T(\mathrm{~g})$ & $\Delta T(\mathrm{~g})$ & $Q(\mathrm{~g}-\mathrm{m})$ & $\Delta Q(\mathrm{~g}-\mathrm{m})$ & $V(\mathrm{~V})$ & $I(\mathrm{~A})$ & $T_{\text {atm }}\left({ }^{\circ} \mathrm{C}\right)$ & $P_{\text {atm }}(\mathrm{Pa})$ \\
\hline $4.167 \mathrm{E}+03$ & $1.642 \mathrm{E}+01$ & $1.07 \mathrm{E}+01$ & $2.327 \mathrm{E}-01$ & $5.89 \mathrm{E}-02$ & $1.109 \mathrm{E}+01$ & $3.894 \mathrm{E}-01$ & $2.224 \mathrm{E}+01$ & $9.855 \mathrm{E}+04$ \\
\hline $8.412 \mathrm{E}+03$ & $6.511 \mathrm{E}+01$ & $1.09 \mathrm{E}+01$ & $9.515 \mathrm{E}-01$ & $5.85 \mathrm{E}-02$ & $1.106 \mathrm{E}+01$ & $1.696 \mathrm{E}+00$ & $2.207 \mathrm{E}+01$ & $9.854 \mathrm{E}+04$ \\
\hline $1.008 \mathrm{E}+04$ & $9.752 \mathrm{E}+01$ & $1.01 \mathrm{E}+01$ & $1.347 \mathrm{E}+00$ & $6.18 \mathrm{E}-02$ & $1.105 \mathrm{E}+01$ & $2.592 \mathrm{E}+00$ & $2.204 \mathrm{E}+01$ & $9.854 \mathrm{E}+04$ \\
\hline $1.204 \mathrm{E}+04$ & $1.368 \mathrm{E}+02$ & $9.94 \mathrm{E}+00$ & $1.854 \mathrm{E}+00$ & $6.34 \mathrm{E}-02$ & $1.102 \mathrm{E}+01$ & $3.920 \mathrm{E}+00$ & $2.201 \mathrm{E}+01$ & $9.852 \mathrm{E}+04$ \\
\hline $1.399 \mathrm{E}+04$ & $1.829 \mathrm{E}+02$ & $1.04 \mathrm{E}+01$ & $2.510 \mathrm{E}+00$ & $6.99 \mathrm{E}-02$ & $1.098 \mathrm{E}+01$ & $5.781 \mathrm{E}+00$ & $2.202 \mathrm{E}+01$ & $9.853 \mathrm{E}+04$ \\
\hline $1.603 \mathrm{E}+04$ & $2.436 \mathrm{E}+02$ & $9.08 \mathrm{E}+00$ & $3.262 \mathrm{E}+00$ & $7.61 \mathrm{E}-02$ & $1.092 \mathrm{E}+01$ & $8.248 \mathrm{E}+00$ & $2.196 \mathrm{E}+01$ & $9.852 \mathrm{E}+04$ \\
\hline $1.811 \mathrm{E}+04$ & $3.130 \mathrm{E}+02$ & $8.95 \mathrm{E}+00$ & $4.169 \mathrm{E}+00$ & $8.33 \mathrm{E}-02$ & $1.085 \mathrm{E}+01$ & $1.161 \mathrm{E}+01$ & $2.187 \mathrm{E}+01$ & $9.855 \mathrm{E}+04$ \\
\hline $1.991 \mathrm{E}+04$ & $3.814 \mathrm{E}+02$ & $8.92 \mathrm{E}+00$ & $5.042 \mathrm{E}+00$ & $1.25 \mathrm{E}-01$ & $1.076 \mathrm{E}+01$ & $1.563 \mathrm{E}+01$ & $2.184 \mathrm{E}+01$ & $9.854 \mathrm{E}+04$ \\
\hline $2.184 \mathrm{E}+04$ & $4.547 \mathrm{E}+02$ & $9.19 \mathrm{E}+00$ & $6.232 \mathrm{E}+00$ & $1.19 \mathrm{E}-01$ & $1.063 \mathrm{E}+01$ & $2.124 \mathrm{E}+01$ & $2.188 \mathrm{E}+01$ & $9.851 \mathrm{E}+04$ \\
\hline
\end{tabular}

Table 55: Graupner 4.7 x 4.7 Static Calculated Values (Prop 3 Test 2)

\begin{tabular}{|c|c|c|c|c|c|c|c|}
\hline$n(\mathrm{RPM})$ & $\rho\left(\mathrm{kg} / \mathrm{m}^{\wedge} 3\right)$ & $P_{\mathrm{P}}(\mathrm{W})$ & $\Delta P_{\mathrm{P}}(\mathrm{W})$ & $P_{\mathrm{e}}(\mathrm{W})$ & $\Delta P_{\mathrm{e}}(\mathrm{W})$ & $C_{\mathrm{T}}$ & $\Delta C_{\mathrm{T}}$ \\
\hline $4.167 \mathrm{E}+03$ & $1.162 \mathrm{E}+00$ & $9.956 \mathrm{E}-01$ & $2.52 \mathrm{E}-01$ & $4.319 \mathrm{E}+00$ & $6.71 \mathrm{E}-02$ & $1.371 \mathrm{E}-01$ & $8.95 \mathrm{E}-02$ \\
\hline $8.412 \mathrm{E}+03$ & $1.163 \mathrm{E}+00$ & $8.220 \mathrm{E}+00$ & $5.05 \mathrm{E}-01$ & $1.876 \mathrm{E}+01$ & $2.19 \mathrm{E}-01$ & $1.333 \mathrm{E}-01$ & $2.23 \mathrm{E}-02$ \\
\hline $1.008 \mathrm{E}+04$ & $1.163 \mathrm{E}+00$ & $1.394 \mathrm{E}+01$ & $6.40 \mathrm{E}-01$ & $2.863 \mathrm{E}+01$ & $3.29 \mathrm{E}-01$ & $1.391 \mathrm{E}-01$ & $1.45 \mathrm{E}-02$ \\
\hline $1.204 \mathrm{E}+04$ & $1.163 \mathrm{E}+00$ & $2.293 \mathrm{E}+01$ & $7.85 \mathrm{E}-01$ & $4.319 \mathrm{E}+01$ & $4.78 \mathrm{E}-01$ & $1.367 \mathrm{E}-01$ & $9.93 \mathrm{E}-03$ \\
\hline $1.399 \mathrm{E}+04$ & $1.163 \mathrm{E}+00$ & $3.606 \mathrm{E}+01$ & $1.00 \mathrm{E}+00$ & $6.345 \mathrm{E}+01$ & $7.13 \mathrm{E}-01$ & $1.354 \mathrm{E}-01$ & $7.74 \mathrm{E}-03$ \\
\hline $1.603 \mathrm{E}+04$ & $1.163 \mathrm{E}+00$ & $5.369 \mathrm{E}+01$ & $1.25 \mathrm{E}+00$ & $9.010 \mathrm{E}+01$ & $1.02 \mathrm{E}+00$ & $1.374 \mathrm{E}-01$ & $5.12 \mathrm{E}-03$ \\
\hline $1.811 \mathrm{E}+04$ & $1.164 \mathrm{E}+00$ & $7.752 \mathrm{E}+01$ & $1.55 \mathrm{E}+00$ & $1.260 \mathrm{E}+02$ & $1.38 \mathrm{E}+00$ & $1.382 \mathrm{E}-01$ & $3.96 \mathrm{E}-03$ \\
\hline $1.991 \mathrm{E}+04$ & $1.164 \mathrm{E}+00$ & $1.031 \mathrm{E}+02$ & $2.55 \mathrm{E}+00$ & $1.682 \mathrm{E}+02$ & $1.81 \mathrm{E}+00$ & $1.393 \mathrm{E}-01$ & $3.26 \mathrm{E}-03$ \\
\hline $2.184 \mathrm{E}+04$ & $1.163 \mathrm{E}+00$ & $1.398 \mathrm{E}+02$ & $2.67 \mathrm{E}+00$ & $2.258 \mathrm{E}+02$ & $2.38 \mathrm{E}+00$ & $1.381 \mathrm{E}-01$ & $2.79 \mathrm{E}-03$ \\
\hline$C_{\mathrm{P}}$ & $\Delta C_{\mathrm{P}}$ & $C_{\mathrm{Q}}$ & $\Delta C_{\mathrm{Q}}$ & $\eta_{\mathrm{T}}$ & $\Delta \eta_{\mathrm{T}}$ & $R e_{0.75}$ & $\Delta R e_{0.75}$ \\
\hline $1.015 \mathrm{E}-01$ & $2.57 \mathrm{E}-02$ & $1.615 \mathrm{E}-02$ & $4.09 \mathrm{E}-03$ & $2.305 \mathrm{E}-01$ & $5.84 \mathrm{E}-02$ & $1.127 \mathrm{E}+04$ & $1.59 \mathrm{E}+01$ \\
\hline $1.018 \mathrm{E}-01$ & $6.26 \mathrm{E}-03$ & $1.619 \mathrm{E}-02$ & $9.96 \mathrm{E}-04$ & $4.381 \mathrm{E}-01$ & $2.74 \mathrm{E}-02$ & $2.278 \mathrm{E}+04$ & $2.97 \mathrm{E}+01$ \\
\hline $1.003 \mathrm{E}-01$ & $4.61 \mathrm{E}-03$ & $1.597 \mathrm{E}-02$ & $7.33 \mathrm{E}-04$ & $4.869 \mathrm{E}-01$ & $2.30 \mathrm{E}-02$ & $2.729 \mathrm{E}+04$ & $3.47 \mathrm{E}+01$ \\
\hline $9.670 \mathrm{E}-02$ & $3.31 \mathrm{E}-03$ & $1.539 \mathrm{E}-02$ & $5.27 \mathrm{E}-04$ & $5.310 \mathrm{E}-01$ & $1.91 \mathrm{E}-02$ & $3.262 \mathrm{E}+04$ & $4.06 \mathrm{E}+01$ \\
\hline $9.703 \mathrm{E}-02$ & $2.71 \mathrm{E}-03$ & $1.544 \mathrm{E}-02$ & $4.30 \mathrm{E}-04$ & $5.682 \mathrm{E}-01$ & $1.71 \mathrm{E}-02$ & $3.789 \mathrm{E}+04$ & $4.69 \mathrm{E}+01$ \\
\hline $9.608 \mathrm{E}-02$ & $2.24 \mathrm{E}-03$ & $1.529 \mathrm{E}-02$ & $3.57 \mathrm{E}-04$ & $5.959 \mathrm{E}-01$ & $1.54 \mathrm{E}-02$ & $4.342 \mathrm{E}+04$ & $5.32 \mathrm{E}+01$ \\
\hline $9.616 \mathrm{E}-02$ & $1.92 \mathrm{E}-03$ & $1.530 \mathrm{E}-02$ & $3.06 \mathrm{E}-04$ & $6.153 \mathrm{E}-01$ & $1.40 \mathrm{E}-02$ & $4.909 \mathrm{E}+04$ & $5.96 \mathrm{E}+01$ \\
\hline $9.616 \mathrm{E}-02$ & $2.38 \mathrm{E}-03$ & $1.531 \mathrm{E}-02$ & $3.79 \mathrm{E}-04$ & $6.128 \mathrm{E}-01$ & $1.65 \mathrm{E}-02$ & $5.399 \mathrm{E}+04$ & $6.57 \mathrm{E}+01$ \\
\hline $9.884 \mathrm{E}-02$ & $1.89 \mathrm{E}-03$ & $1.615 \mathrm{E}-02$ & $3.00 \mathrm{E}-04$ & $6.188 \mathrm{E}-01$ & $1.35 \mathrm{E}-02$ & $5.918 \mathrm{E}+04$ & $7.28 \mathrm{E}+01$ \\
\hline
\end{tabular}




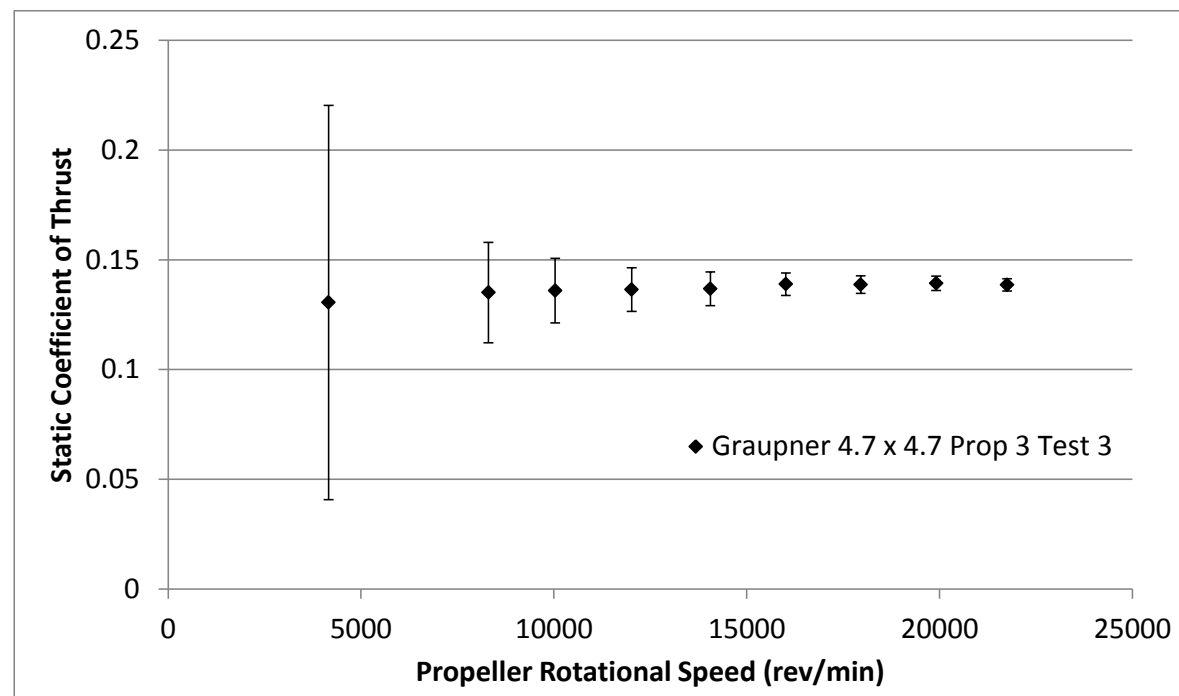

(a)

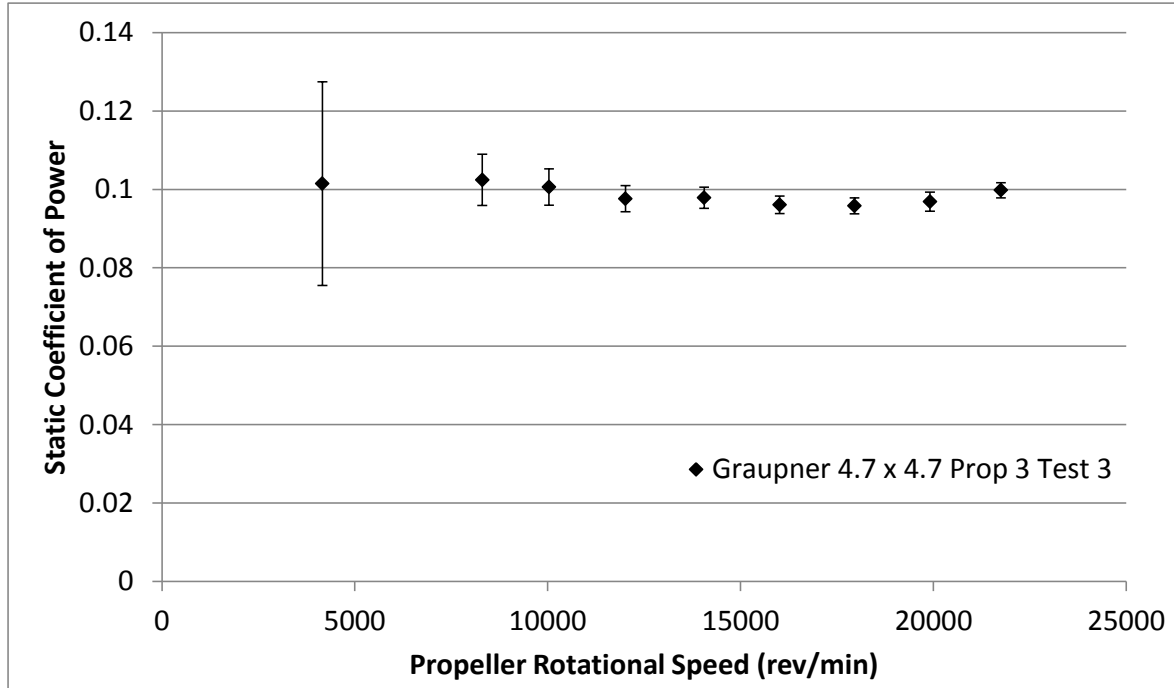

(b)

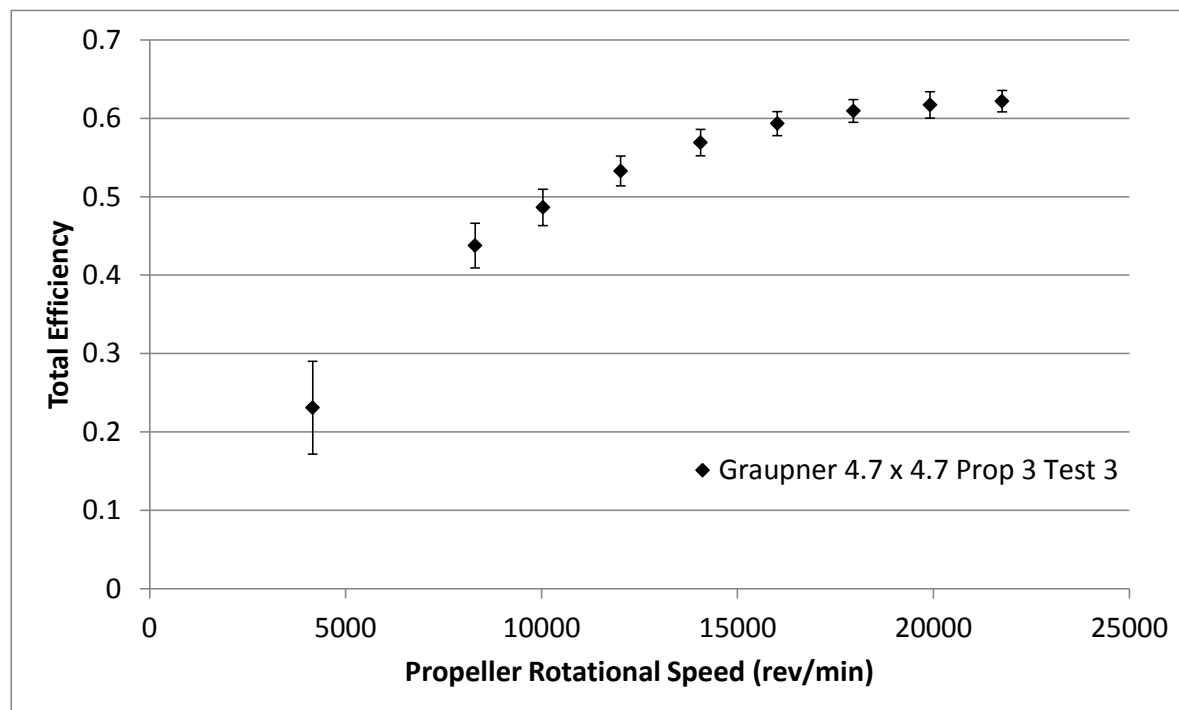

Figure 67: Graupner 4.7 x 4.7 Static Test Results (Prop 3 Test 3): (a) Coefficient of Thrust, (b) Coefficient of Power, (c) Total Efficiency. 
Table 56: Graupner 4.7 x 4.7 Static Measured Values (Prop 3 Test 3)

\begin{tabular}{|c|c|c|c|c|c|c|c|c|}
\hline$n(\mathrm{RPM})$ & $T(\mathrm{~g})$ & $\Delta T(\mathrm{~g})$ & $Q(\mathrm{~g}-\mathrm{m})$ & $\Delta Q(\mathrm{~g}-\mathrm{m})$ & $V(\mathrm{~V})$ & $I(\mathrm{~A})$ & $T_{\text {atm }}\left({ }^{\circ} \mathrm{C}\right)$ & $P_{\text {atm }}(\mathrm{Pa})$ \\
\hline $4.159 \mathrm{E}+03$ & $1.558 \mathrm{E}+01$ & $1.07 \mathrm{E}+01$ & $2.319 \mathrm{E}-01$ & $5.94 \mathrm{E}-02$ & $1.109 \mathrm{E}+01$ & $3.866 \mathrm{E}-01$ & $2.199 \mathrm{E}+01$ & $9.848 \mathrm{E}+04$ \\
\hline $8.305 \mathrm{E}+03$ & $6.426 \mathrm{E}+01$ & $1.09 \mathrm{E}+01$ & $9.330 \mathrm{E}-01$ & $5.96 \mathrm{E}-02$ & $1.107 \mathrm{E}+01$ & $1.643 \mathrm{E}+00$ & $2.199 \mathrm{E}+01$ & $9.848 \mathrm{E}+04$ \\
\hline $1.004 \mathrm{E}+04$ & $9.444 \mathrm{E}+01$ & $1.02 \mathrm{E}+01$ & $1.339 \mathrm{E}+00$ & $6.19 \mathrm{E}-02$ & $1.105 \mathrm{E}+01$ & $2.569 \mathrm{E}+00$ & $2.196 \mathrm{E}+01$ & $9.848 \mathrm{E}+04$ \\
\hline $1.202 \mathrm{E}+04$ & $1.360 \mathrm{E}+02$ & $9.90 \mathrm{E}+00$ & $1.864 \mathrm{E}+00$ & $6.33 \mathrm{E}-02$ & $1.102 \mathrm{E}+01$ & $3.919 \mathrm{E}+00$ & $2.195 \mathrm{E}+01$ & $9.850 \mathrm{E}+04$ \\
\hline $1.406 \mathrm{E}+04$ & $1.865 \mathrm{E}+02$ & $1.05 \mathrm{E}+01$ & $2.556 \mathrm{E}+00$ & $7.02 \mathrm{E}-02$ & $1.097 \mathrm{E}+01$ & $5.908 \mathrm{E}+00$ & $2.194 \mathrm{E}+01$ & $9.850 \mathrm{E}+04$ \\
\hline $1.602 \mathrm{E}+04$ & $2.458 \mathrm{E}+02$ & $9.11 \mathrm{E}+00$ & $3.257 \mathrm{E}+00$ & $7.57 \mathrm{E}-02$ & $1.092 \mathrm{E}+01$ & $8.267 \mathrm{E}+00$ & $2.192 \mathrm{E}+01$ & $9.849 \mathrm{E}+04$ \\
\hline $1.796 \mathrm{E}+04$ & $3.086 \mathrm{E}+02$ & $8.86 \mathrm{E}+00$ & $4.082 \mathrm{E}+00$ & $8.58 \mathrm{E}-02$ & $1.086 \mathrm{E}+01$ & $1.138 \mathrm{E}+01$ & $2.197 \mathrm{E}+01$ & $9.850 \mathrm{E}+04$ \\
\hline $1.991 \mathrm{E}+04$ & $3.810 \mathrm{E}+02$ & $9.01 \mathrm{E}+00$ & $5.077 \mathrm{E}+00$ & $1.27 \mathrm{E}-01$ & $1.076 \mathrm{E}+01$ & $1.563 \mathrm{E}+01$ & $2.194 \mathrm{E}+01$ & $9.849 \mathrm{E}+04$ \\
\hline $2.175 \mathrm{E}+04$ & $4.526 \mathrm{E}+02$ & $9.15 \mathrm{E}+00$ & $6.240 \mathrm{E}+00$ & $1.20 \mathrm{E}-01$ & $1.064 \mathrm{E}+01$ & $2.107 \mathrm{E}+01$ & $2.195 \mathrm{E}+01$ & $9.850 \mathrm{E}+04$ \\
\hline
\end{tabular}

Table 57: Graupner 4.7 x 4.7 Static Calculated Values (Prop 3 Test 3)

\begin{tabular}{|c|c|c|c|c|c|c|c|}
\hline$n(\mathrm{RPM})$ & $\rho\left(\mathrm{kg} / \mathrm{m}^{\wedge} 3\right)$ & $P_{\mathrm{P}}(\mathrm{W})$ & $\Delta P_{\mathrm{P}}(\mathrm{W})$ & $P_{\mathrm{e}}(\mathrm{W})$ & $\Delta P_{\mathrm{e}}(\mathrm{W})$ & $C_{\mathrm{T}}$ & $\Delta C_{\mathrm{T}}$ \\
\hline $4.159 \mathrm{E}+03$ & $1.162 \mathrm{E}+00$ & $9.903 \mathrm{E}-01$ & $2.54 \mathrm{E}-01$ & $4.288 \mathrm{E}+00$ & $6.74 \mathrm{E}-02$ & $1.305 \mathrm{E}-01$ & $8.98 \mathrm{E}-02$ \\
\hline $8.305 \mathrm{E}+03$ & $1.162 \mathrm{E}+00$ & $7.957 \mathrm{E}+00$ & $5.08 \mathrm{E}-01$ & $1.818 \mathrm{E}+01$ & $2.14 \mathrm{E}-01$ & $1.351 \mathrm{E}-01$ & $2.28 \mathrm{E}-02$ \\
\hline $1.004 \mathrm{E}+04$ & $1.162 \mathrm{E}+00$ & $1.380 \mathrm{E}+01$ & $6.38 \mathrm{E}-01$ & $2.837 \mathrm{E}+01$ & $3.20 \mathrm{E}-01$ & $1.359 \mathrm{E}-01$ & $1.47 \mathrm{E}-02$ \\
\hline $1.202 \mathrm{E}+04$ & $1.163 \mathrm{E}+00$ & $2.300 \mathrm{E}+01$ & $7.81 \mathrm{E}-01$ & $4.317 \mathrm{E}+01$ & $4.78 \mathrm{E}-01$ & $1.364 \mathrm{E}-01$ & $9.94 \mathrm{E}-03$ \\
\hline $1.406 \mathrm{E}+04$ & $1.163 \mathrm{E}+00$ & $3.690 \mathrm{E}+01$ & $1.01 \mathrm{E}+00$ & $6.483 \mathrm{E}+01$ & $7.40 \mathrm{E}-01$ & $1.368 \mathrm{E}-01$ & $7.67 \mathrm{E}-03$ \\
\hline $1.602 \mathrm{E}+04$ & $1.163 \mathrm{E}+00$ & $5.358 \mathrm{E}+01$ & $1.24 \mathrm{E}+00$ & $9.030 \mathrm{E}+01$ & $1.02 \mathrm{E}+00$ & $1.389 \mathrm{E}-01$ & $5.14 \mathrm{E}-03$ \\
\hline $1.796 \mathrm{E}+04$ & $1.163 \mathrm{E}+00$ & $7.528 \mathrm{E}+01$ & $1.58 \mathrm{E}+00$ & $1.235 \mathrm{E}+02$ & $1.35 \mathrm{E}+00$ & $1.387 \mathrm{E}-01$ & $3.98 \mathrm{E}-03$ \\
\hline $1.991 \mathrm{E}+04$ & $1.163 \mathrm{E}+00$ & $1.038 \mathrm{E}+02$ & $2.59 \mathrm{E}+00$ & $1.682 \mathrm{E}+02$ & $1.81 \mathrm{E}+00$ & $1.392 \mathrm{E}-01$ & $3.29 \mathrm{E}-03$ \\
\hline $2.175 \mathrm{E}+04$ & $1.163 \mathrm{E}+00$ & $1.394 \mathrm{E}+02$ & $2.69 \mathrm{E}+00$ & $2.241 \mathrm{E}+02$ & $2.37 \mathrm{E}+00$ & $1.386 \mathrm{E}-01$ & $2.80 \mathrm{E}-03$ \\
\hline$C_{\mathrm{P}}$ & $\Delta C_{\mathrm{P}}$ & $C_{\mathrm{Q}}$ & $\Delta C_{\mathrm{Q}}$ & $\eta_{\mathrm{T}}$ & $\Delta \eta_{\mathrm{T}}$ & $R e_{0.75}$ & $\Delta R e_{0.75}$ \\
\hline $1.015 \mathrm{E}-01$ & $2.60 \mathrm{E}-02$ & $1.615 \mathrm{E}-02$ & $4.14 \mathrm{E}-03$ & $2.309 \mathrm{E}-01$ & $5.92 \mathrm{E}-02$ & $1.126 \mathrm{E}+04$ & $1.63 \mathrm{E}+01$ \\
\hline $1.024 \mathrm{E}-01$ & $6.54 \mathrm{E}-03$ & $1.630 \mathrm{E}-02$ & $1.04 \mathrm{E}-03$ & $4.377 \mathrm{E}-01$ & $2.84 \mathrm{E}-02$ & $2.248 \mathrm{E}+04$ & $2.88 \mathrm{E}+01$ \\
\hline $1.006 \mathrm{E}-01$ & $4.66 \mathrm{E}-03$ & $1.601 \mathrm{E}-02$ & $7.41 \mathrm{E}-04$ & $4.863 \mathrm{E}-01$ & $2.32 \mathrm{E}-02$ & $2.717 \mathrm{E}+04$ & $3.46 \mathrm{E}+01$ \\
\hline $9.764 \mathrm{E}-02$ & $3.32 \mathrm{E}-03$ & $1.554 \mathrm{E}-02$ & $5.28 \mathrm{E}-04$ & $5.328 \mathrm{E}-01$ & $1.90 \mathrm{E}-02$ & $3.255 \mathrm{E}+04$ & $4.07 \mathrm{E}+01$ \\
\hline $9.787 \mathrm{E}-02$ & $2.69 \mathrm{E}-03$ & $1.558 \mathrm{E}-02$ & $4.28 \mathrm{E}-04$ & $5.691 \mathrm{E}-01$ & $1.69 \mathrm{E}-02$ & $3.808 \mathrm{E}+04$ & $4.71 \mathrm{E}+01$ \\
\hline $9.610 \mathrm{E}-02$ & $2.23 \mathrm{E}-03$ & $1.529 \mathrm{E}-02$ & $3.55 \mathrm{E}-04$ & $5.933 \mathrm{E}-01$ & $1.53 \mathrm{E}-02$ & $4.339 \mathrm{E}+04$ & $5.31 \mathrm{E}+01$ \\
\hline $9.580 \mathrm{E}-02$ & $2.02 \mathrm{E}-03$ & $1.525 \mathrm{E}-02$ & $3.21 \mathrm{E}-04$ & $6.094 \mathrm{E}-01$ & $1.44 \mathrm{E}-02$ & $4.863 \mathrm{E}+04$ & $5.93 \mathrm{E}+01$ \\
\hline $9.689 \mathrm{E}-02$ & $2.42 \mathrm{E}-03$ & $1.542 \mathrm{E}-02$ & $3.85 \mathrm{E}-04$ & $6.172 \mathrm{E}-01$ & $1.68 \mathrm{E}-02$ & $5.393 \mathrm{E}+04$ & $6.60 \mathrm{E}+01$ \\
\hline $9.979 \mathrm{E}-02$ & $1.93 \mathrm{E}-03$ & $1.615 \mathrm{E}-02$ & $3.07 \mathrm{E}-04$ & $6.219 \mathrm{E}-01$ & $1.37 \mathrm{E}-02$ & $5.892 \mathrm{E}+04$ & $7.33 \mathrm{E}+01$ \\
\hline
\end{tabular}




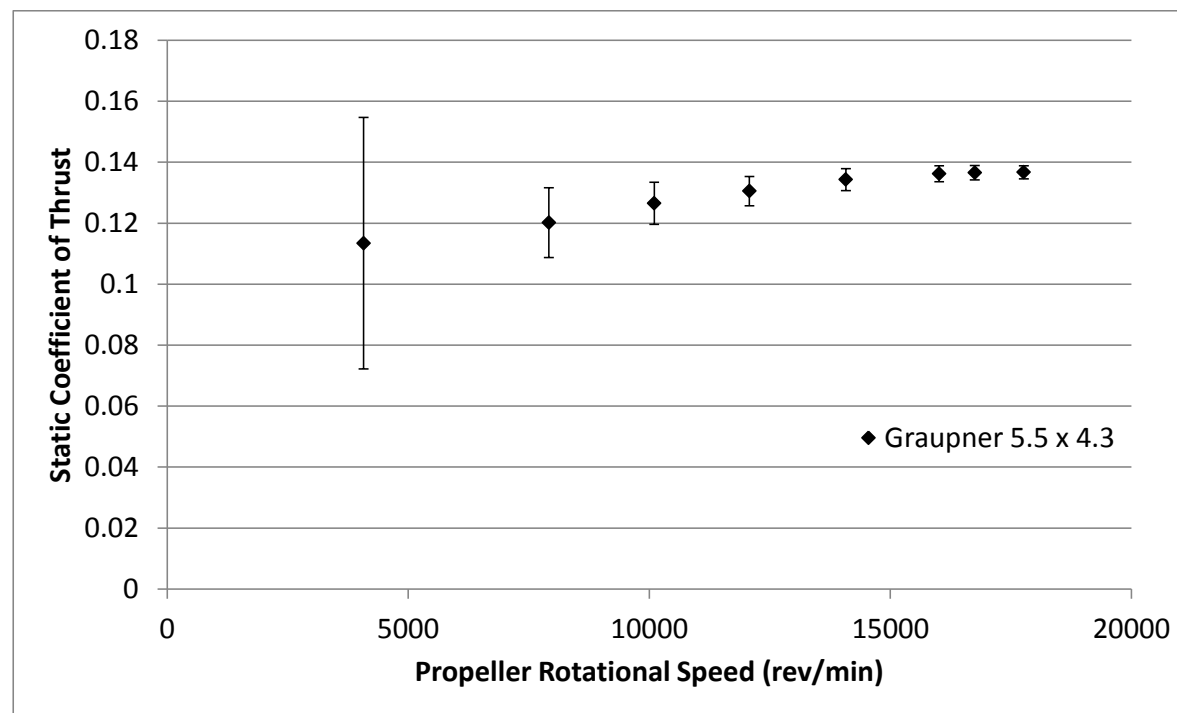

(a)

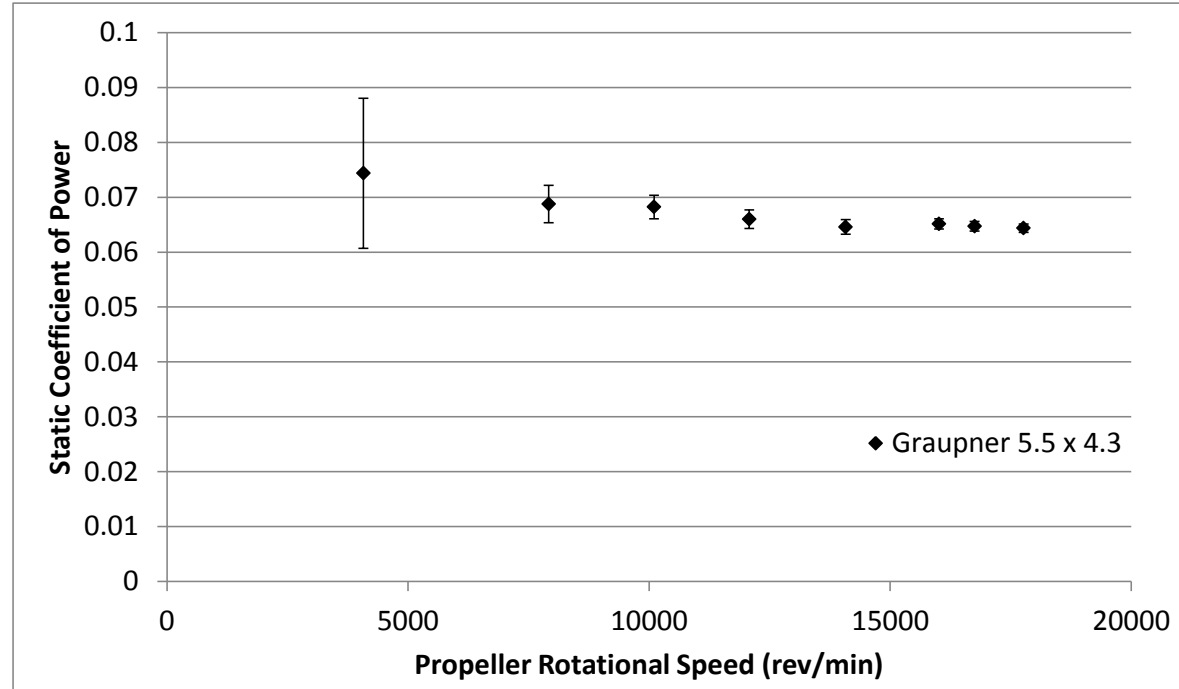

(b)

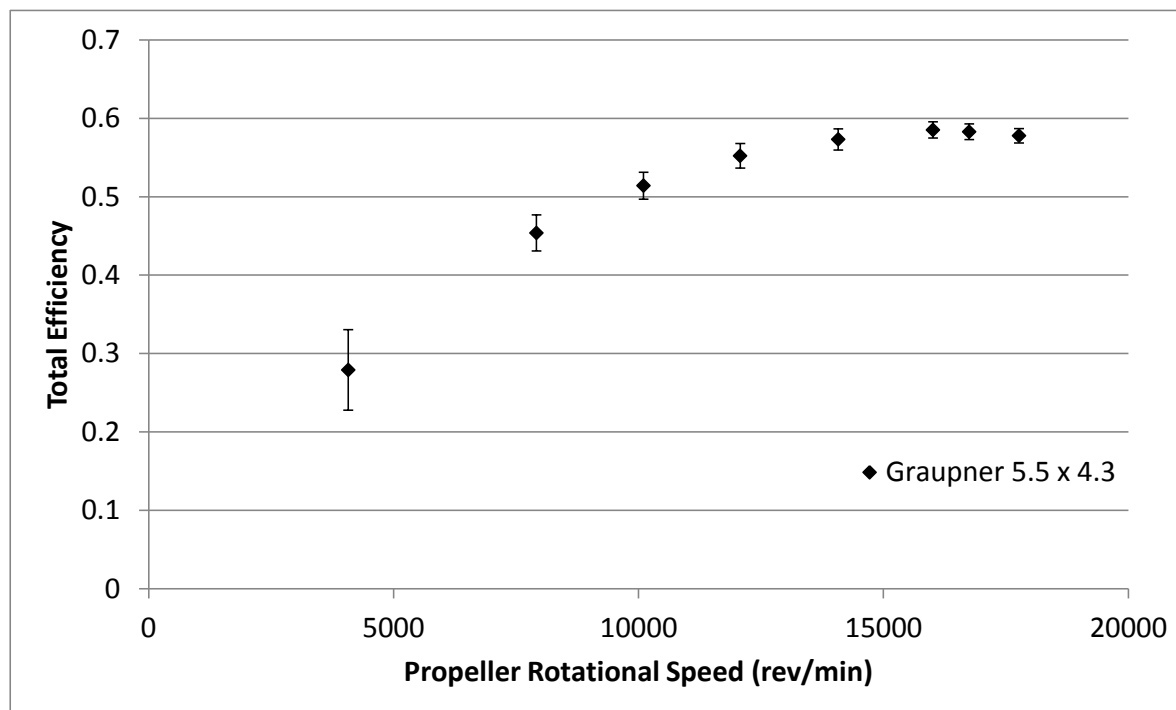

(c)

Figure 68: Graupner 5.5 x 4.3 Static Test Results: (a) Coefficient of Thrust, (b) Coefficient of Power, (c) Total Efficiency. 
Table 58: Graupner 5.5 x 4.3 Static Measured Values

\begin{tabular}{|c|c|c|c|c|c|c|c|c|}
\hline$n(\mathrm{RPM})$ & $T(\mathrm{~g})$ & $\Delta T(\mathrm{~g})$ & $Q(\mathrm{~g}-\mathrm{m})$ & $\Delta Q(\mathrm{~g}-\mathrm{m})$ & $V(\mathrm{~V})$ & $I(\mathrm{~A})$ & $T_{\text {atm }}\left({ }^{\circ} \mathrm{C}\right)$ & $P_{\text {atm }}(\mathrm{Pa})$ \\
\hline $4.075 \mathrm{E}+03$ & $2.463 \mathrm{E}+01$ & $8.96 \mathrm{E}+00$ & $3.626 \mathrm{E}-01$ & $6.66 \mathrm{E}-02$ & $1.108 \mathrm{E}+01$ & $4.910 \mathrm{E}-01$ & $2.175 \mathrm{E}+01$ & $9.857 \mathrm{E}+04$ \\
\hline $7.918 \mathrm{E}+03$ & $9.858 \mathrm{E}+01$ & $9.39 \mathrm{E}+00$ & $1.267 \mathrm{E}+00$ & $6.27 \mathrm{E}-02$ & $1.104 \mathrm{E}+01$ & $2.055 \mathrm{E}+00$ & $2.160 \mathrm{E}+01$ & $9.856 \mathrm{E}+04$ \\
\hline $1.010 \mathrm{E}+04$ & $1.690 \mathrm{E}+02$ & $9.20 \mathrm{E}+00$ & $2.047 \mathrm{E}+00$ & $6.46 \mathrm{E}-02$ & $1.101 \mathrm{E}+01$ & $3.754 \mathrm{E}+00$ & $2.155 \mathrm{E}+01$ & $9.857 \mathrm{E}+04$ \\
\hline $1.208 \mathrm{E}+04$ & $2.491 \mathrm{E}+02$ & $9.18 \mathrm{E}+00$ & $2.830 \mathrm{E}+00$ & $7.30 \mathrm{E}-02$ & $1.096 \mathrm{E}+01$ & $5.799 \mathrm{E}+00$ & $2.157 \mathrm{E}+01$ & $9.857 \mathrm{E}+04$ \\
\hline $1.408 \mathrm{E}+04$ & $3.484 \mathrm{E}+02$ & $9.36 \mathrm{E}+00$ & $3.763 \mathrm{E}+00$ & $7.73 \mathrm{E}-02$ & $1.090 \mathrm{E}+01$ & $8.712 \mathrm{E}+00$ & $2.152 \mathrm{E}+01$ & $9.857 \mathrm{E}+04$ \\
\hline $1.601 \mathrm{E}+04$ & $4.572 \mathrm{E}+02$ & $8.82 \mathrm{E}+00$ & $4.911 \mathrm{E}+00$ & $6.95 \mathrm{E}-02$ & $1.080 \mathrm{E}+01$ & $1.277 \mathrm{E}+01$ & $2.154 \mathrm{E}+01$ & $9.858 \mathrm{E}+04$ \\
\hline $1.675 \mathrm{E}+04$ & $5.017 \mathrm{E}+02$ & $8.67 \mathrm{E}+00$ & $5.341 \mathrm{E}+00$ & $7.14 \mathrm{E}-02$ & $1.076 \mathrm{E}+01$ & $1.465 \mathrm{E}+01$ & $2.143 \mathrm{E}+01$ & $9.859 \mathrm{E}+04$ \\
\hline $1.777 \mathrm{E}+04$ & $5.650 \mathrm{E}+02$ & $8.72 \mathrm{E}+00$ & $5.976 \mathrm{E}+00$ & $7.07 \mathrm{E}-02$ & $1.069 \mathrm{E}+01$ & $1.765 \mathrm{E}+01$ & $2.149 \mathrm{E}+01$ & $9.858 \mathrm{E}+04$ \\
\hline
\end{tabular}

Table 59: Graupner 5.5 x 4.3 Static Calculated Values

\begin{tabular}{|c|c|c|c|c|c|c|c|}
\hline$n(\mathrm{RPM})$ & $\rho\left(\mathrm{kg} / \mathrm{m}^{\wedge} 3\right)$ & $P_{\mathrm{P}}(\mathrm{W})$ & $\Delta P_{\mathrm{P}}(\mathrm{W})$ & $P_{\mathrm{e}}(\mathrm{W})$ & $\Delta P_{\mathrm{e}}(\mathrm{W})$ & $C_{\mathrm{T}}$ & $\Delta C_{\mathrm{T}}$ \\
\hline $4.075 \mathrm{E}+03$ & $1.164 \mathrm{E}+00$ & $1.518 \mathrm{E}+00$ & $2.79 \mathrm{E}-01$ & $5.439 \mathrm{E}+00$ & $8.15 \mathrm{E}-02$ & $1.134 \mathrm{E}-01$ & $4.13 \mathrm{E}-02$ \\
\hline $7.918 \mathrm{E}+03$ & $1.165 \mathrm{E}+00$ & $1.030 \mathrm{E}+01$ & $5.10 \mathrm{E}-01$ & $2.269 \mathrm{E}+01$ & $2.66 \mathrm{E}-01$ & $1.202 \mathrm{E}-01$ & $1.15 \mathrm{E}-02$ \\
\hline $1.010 \mathrm{E}+04$ & $1.165 \mathrm{E}+00$ & $2.124 \mathrm{E}+01$ & $6.71 \mathrm{E}-01$ & $4.132 \mathrm{E}+01$ & $4.63 \mathrm{E}-01$ & $1.265 \mathrm{E}-01$ & $6.89 \mathrm{E}-03$ \\
\hline $1.208 \mathrm{E}+04$ & $1.165 \mathrm{E}+00$ & $3.510 \mathrm{E}+01$ & $9.05 \mathrm{E}-01$ & $6.356 \mathrm{E}+01$ & $7.23 \mathrm{E}-01$ & $1.305 \mathrm{E}-01$ & $4.81 \mathrm{E}-03$ \\
\hline $1.408 \mathrm{E}+04$ & $1.165 \mathrm{E}+00$ & $5.440 \mathrm{E}+01$ & $1.12 \mathrm{E}+00$ & $9.492 \mathrm{E}+01$ & $1.06 \mathrm{E}+00$ & $1.343 \mathrm{E}-01$ & $3.61 \mathrm{E}-03$ \\
\hline $1.601 \mathrm{E}+04$ & $1.165 \mathrm{E}+00$ & $8.077 \mathrm{E}+01$ & $1.14 \mathrm{E}+00$ & $1.380 \mathrm{E}+02$ & $1.50 \mathrm{E}+00$ & $1.362 \mathrm{E}-01$ & $2.63 \mathrm{E}-03$ \\
\hline $1.675 \mathrm{E}+04$ & $1.166 \mathrm{E}+00$ & $9.189 \mathrm{E}+01$ & $1.23 \mathrm{E}+00$ & $1.577 \mathrm{E}+02$ & $1.70 \mathrm{E}+00$ & $1.365 \mathrm{E}-01$ & $2.36 \mathrm{E}-03$ \\
\hline $1.777 \mathrm{E}+04$ & $1.166 \mathrm{E}+00$ & $1.091 \mathrm{E}+02$ & $1.29 \mathrm{E}+00$ & $1.888 \mathrm{E}+02$ & $2.02 \mathrm{E}+00$ & $1.367 \mathrm{E}-01$ & $2.11 \mathrm{E}-03$ \\
\hline$C_{\mathrm{P}}$ & $\Delta C_{\mathrm{P}}$ & $C_{\mathrm{Q}}$ & $\Delta C_{\mathrm{Q}}$ & $\eta_{\mathrm{T}}$ & $\Delta \eta_{\mathrm{T}}$ & $R e_{0.75}$ & $\Delta R e_{0.75}$ \\
\hline $7.436 \mathrm{E}-02$ & $1.37 \mathrm{E}-02$ & $1.184 \mathrm{E}-02$ & $2.17 \mathrm{E}-03$ & $2.790 \mathrm{E}-01$ & $5.14 \mathrm{E}-02$ & $1.520 \mathrm{E}+04$ & $1.91 \mathrm{E}+01$ \\
\hline $6.876 \mathrm{E}-02$ & $3.41 \mathrm{E}-03$ & $1.094 \mathrm{E}-02$ & $5.42 \mathrm{E}-04$ & $4.538 \mathrm{E}-01$ & $2.31 \mathrm{E}-02$ & $2.956 \mathrm{E}+04$ & $4.16 \mathrm{E}+01$ \\
\hline $6.823 \mathrm{E}-02$ & $2.16 \mathrm{E}-03$ & $1.086 \mathrm{E}-02$ & $3.43 \mathrm{E}-04$ & $5.140 \mathrm{E}-01$ & $1.72 \mathrm{E}-02$ & $3.773 \mathrm{E}+04$ & $4.21 \mathrm{E}+01$ \\
\hline $6.601 \mathrm{E}-02$ & $1.71 \mathrm{E}-03$ & $1.051 \mathrm{E}-02$ & $2.71 \mathrm{E}-04$ & $5.522 \mathrm{E}-01$ & $1.56 \mathrm{E}-02$ & $4.510 \mathrm{E}+04$ & $5.45 \mathrm{E}+01$ \\
\hline $6.459 \mathrm{E}-02$ & $1.33 \mathrm{E}-03$ & $1.028 \mathrm{E}-02$ & $2.11 \mathrm{E}-04$ & $5.731 \mathrm{E}-01$ & $1.34 \mathrm{E}-02$ & $5.259 \mathrm{E}+04$ & $6.27 \mathrm{E}+01$ \\
\hline $6.515 \mathrm{E}-02$ & $9.24 \mathrm{E}-04$ & $1.037 \mathrm{E}-02$ & $1.47 \mathrm{E}-04$ & $5.852 \mathrm{E}-01$ & $1.04 \mathrm{E}-02$ & $5.982 \mathrm{E}+04$ & $6.43 \mathrm{E}+01$ \\
\hline $6.471 \mathrm{E}-02$ & $8.68 \mathrm{E}-04$ & $1.030 \mathrm{E}-02$ & $1.38 \mathrm{E}-04$ & $5.829 \mathrm{E}-01$ & $1.00 \mathrm{E}-02$ & $6.262 \mathrm{E}+04$ & $6.74 \mathrm{E}+01$ \\
\hline $6.438 \mathrm{E}-02$ & $7.66 \mathrm{E}-04$ & $1.025 \mathrm{E}-02$ & $1.22 \mathrm{E}-04$ & $5.777 \mathrm{E}-01$ & $9.22 \mathrm{E}-03$ & $6.639 \mathrm{E}+04$ & $7.32 \mathrm{E}+01$ \\
\hline
\end{tabular}




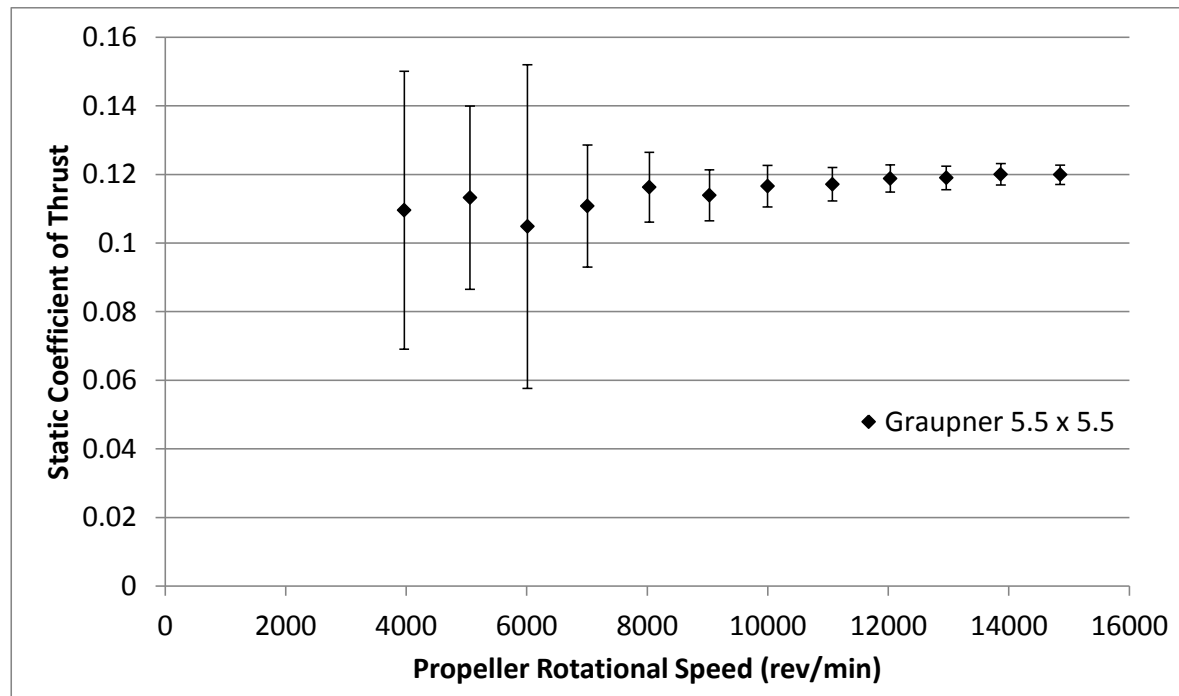

(a)

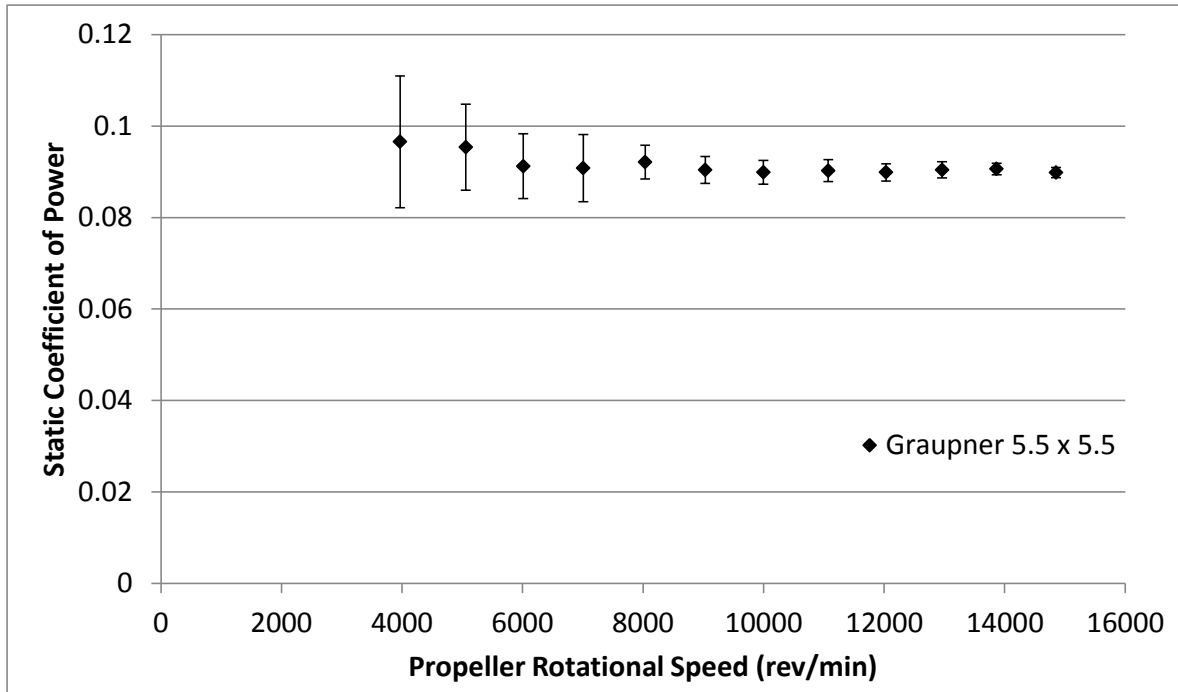

(b)

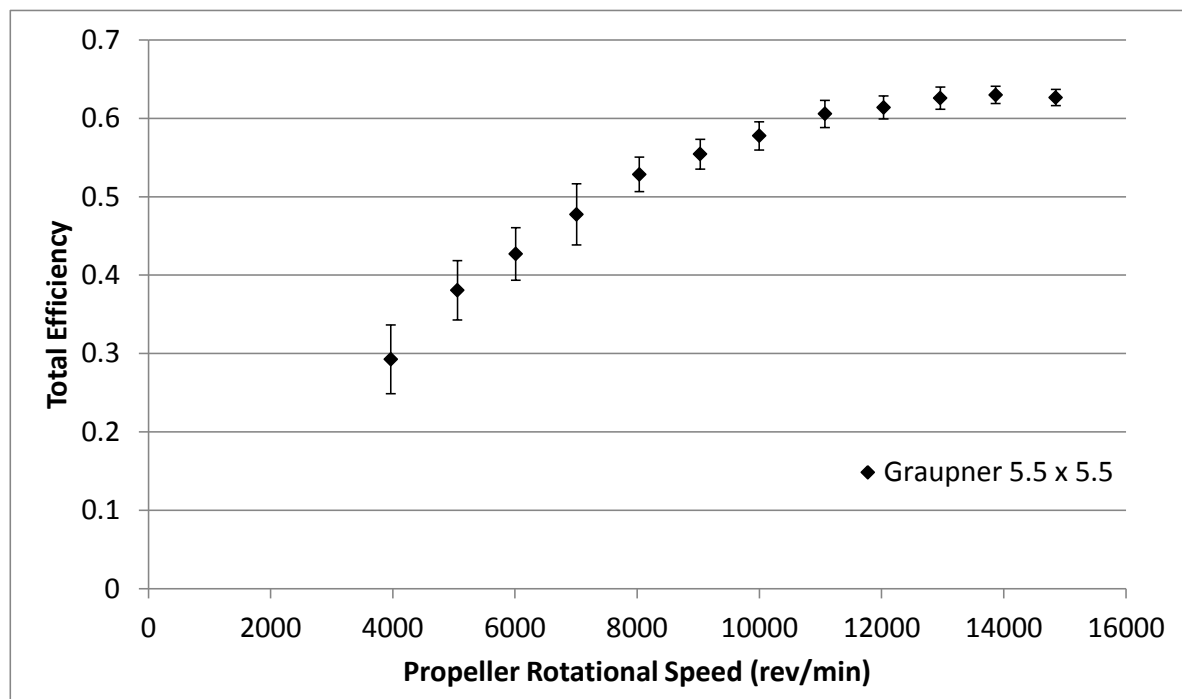

(c)

Figure 69: Graupner 5.5 x 5.5 Static Test Results: (a) Coefficient of Thrust, (b) Coefficient of Power, (c) Total Efficiency. 
Table 60: Graupner 5.5 x 5.5 Static Measured Values

\begin{tabular}{|c|c|c|c|c|c|c|c|c|}
\hline$n(\mathrm{RPM})$ & $T(\mathrm{~g})$ & $\Delta T(\mathrm{~g})$ & $Q(\mathrm{~g}-\mathrm{m})$ & $\Delta Q(\mathrm{~g}-\mathrm{m})$ & $V(\mathrm{~V})$ & $I(\mathrm{~A})$ & $T_{\text {atm }}\left({ }^{\circ} \mathrm{C}\right)$ & $P_{\text {atm }}(\mathrm{Pa})$ \\
\hline $3.967 \mathrm{E}+03$ & $2.173 \mathrm{E}+01$ & $8.04 \mathrm{E}+00$ & $4.269 \mathrm{E}-01$ & $6.37 \mathrm{E}-02$ & $1.110 \mathrm{E}+01$ & $5.356 \mathrm{E}-01$ & $2.375 \mathrm{E}+01$ & $9.856 \mathrm{E}+04$ \\
\hline $5.058 \mathrm{E}+03$ & $3.650 \mathrm{E}+01$ & $8.61 \mathrm{E}+00$ & $6.854 \mathrm{E}-01$ & $6.76 \mathrm{E}-02$ & $1.109 \mathrm{E}+01$ & $8.433 \mathrm{E}-01$ & $2.374 \mathrm{E}+01$ & $9.855 \mathrm{E}+04$ \\
\hline $6.012 \mathrm{E}+03$ & $4.775 \mathrm{E}+01$ & $2.15 \mathrm{E}+01$ & $9.262 \mathrm{E}-01$ & $7.20 \mathrm{E}-02$ & $1.108 \mathrm{E}+01$ & $1.208 \mathrm{E}+00$ & $2.369 \mathrm{E}+01$ & $9.855 \mathrm{E}+04$ \\
\hline $7.008 \mathrm{E}+03$ & $6.855 \mathrm{E}+01$ & $1.10 \mathrm{E}+01$ & $1.253 \mathrm{E}+00$ & $1.01 \mathrm{E}-01$ & $1.107 \mathrm{E}+01$ & $1.706 \mathrm{E}+00$ & $2.373 \mathrm{E}+01$ & $9.856 \mathrm{E}+04$ \\
\hline $8.035 \mathrm{E}+03$ & $9.460 \mathrm{E}+01$ & $8.27 \mathrm{E}+00$ & $1.671 \mathrm{E}+00$ & $6.67 \mathrm{E}-02$ & $1.105 \mathrm{E}+01$ & $2.361 \mathrm{E}+00$ & $2.374 \mathrm{E}+01$ & $9.857 \mathrm{E}+04$ \\
\hline $9.033 \mathrm{E}+03$ & $1.171 \mathrm{E}+02$ & $7.62 \mathrm{E}+00$ & $2.072 \mathrm{E}+00$ & $6.73 \mathrm{E}-02$ & $1.103 \mathrm{E}+01$ & $3.144 \mathrm{E}+00$ & $2.373 \mathrm{E}+01$ & $9.858 \mathrm{E}+04$ \\
\hline $9.997 \mathrm{E}+03$ & $1.469 \mathrm{E}+02$ & $7.66 \mathrm{E}+00$ & $2.525 \mathrm{E}+00$ & $7.33 \mathrm{E}-02$ & $1.101 \mathrm{E}+01$ & $4.078 \mathrm{E}+00$ & $2.374 \mathrm{E}+01$ & $9.859 \mathrm{E}+04$ \\
\hline $1.107 \mathrm{E}+04$ & $1.811 \mathrm{E}+02$ & $7.51 \mathrm{E}+00$ & $3.111 \mathrm{E}+00$ & $8.19 \mathrm{E}-02$ & $1.097 \mathrm{E}+01$ & $5.324 \mathrm{E}+00$ & $2.371 \mathrm{E}+01$ & $9.860 \mathrm{E}+04$ \\
\hline $1.203 \mathrm{E}+04$ & $2.170 \mathrm{E}+02$ & $7.25 \mathrm{E}+00$ & $3.658 \mathrm{E}+00$ & $7.70 \mathrm{E}-02$ & $1.093 \mathrm{E}+01$ & $6.738 \mathrm{E}+00$ & $2.370 \mathrm{E}+01$ & $9.861 \mathrm{E}+04$ \\
\hline $1.296 \mathrm{E}+04$ & $2.522 \mathrm{E}+02$ & $7.29 \mathrm{E}+00$ & $4.272 \mathrm{E}+00$ & $8.34 \mathrm{E}-02$ & $1.089 \mathrm{E}+01$ & $8.347 \mathrm{E}+00$ & $2.367 \mathrm{E}+01$ & $9.862 \mathrm{E}+04$ \\
\hline $1.387 \mathrm{E}+04$ & $2.912 \mathrm{E}+02$ & $7.54 \mathrm{E}+00$ & $4.900 \mathrm{E}+00$ & $6.68 \mathrm{E}-02$ & $1.084 \mathrm{E}+01$ & $1.022 \mathrm{E}+01$ & $2.364 \mathrm{E}+01$ & $9.862 \mathrm{E}+04$ \\
\hline $1.485 \mathrm{E}+04$ & $3.337 \mathrm{E}+02$ & $7.91 \mathrm{E}+00$ & $5.572 \mathrm{E}+00$ & $6.93 \mathrm{E}-02$ & $1.077 \mathrm{E}+01$ & $1.260 \mathrm{E}+01$ & $2.364 \mathrm{E}+01$ & $9.863 \mathrm{E}+04$ \\
\hline
\end{tabular}

Table 61: Graupner 5.5 x 5.5 Static Calculated Values

\begin{tabular}{|c|c|c|c|c|c|c|c|}
\hline$n(\mathrm{RPM})$ & $\rho\left(\mathrm{kg} / \mathrm{m}^{\wedge} 3\right)$ & $P_{\mathrm{P}}(\mathrm{W})$ & $\Delta P_{\mathrm{P}}(\mathrm{W})$ & $P_{\mathrm{e}}(\mathrm{W})$ & $\Delta P_{\mathrm{e}}(\mathrm{W})$ & $C_{\mathrm{T}}$ & $\Delta C_{\mathrm{T}}$ \\
\hline $3.967 \mathrm{E}+03$ & $1.156 \mathrm{E}+00$ & $1.739 \mathrm{E}+00$ & $2.59 \mathrm{E}-01$ & $5.946 \mathrm{E}+00$ & $8.55 \mathrm{E}-02$ & 1.096E-01 & $4.05 \mathrm{E}-02$ \\
\hline $5.058 \mathrm{E}+03$ & $1.156 \mathrm{E}+00$ & $3.560 \mathrm{E}+00$ & $3.51 \mathrm{E}-01$ & $9.354 \mathrm{E}+00$ & $1.20 \mathrm{E}-01$ & $E-01$ & $2.67 \mathrm{E}-02$ \\
\hline $6.012 \mathrm{E}+03$ & $1.157 \mathrm{E}+00$ & $5.719 \mathrm{E}+00$ & 4.45E-01 & $1.339 \mathrm{E}+01$ & $1.63 \mathrm{E}-01$ & 1.048E-01 & $4.72 \mathrm{E}-02$ \\
\hline $7.008 \mathrm{E}+03$ & $1.156 \mathrm{E}+00$ & $9.017 \mathrm{E}+00$ & $7.28 \mathrm{E}-01$ & $1.888 \mathrm{E}+01$ & $2.22 \mathrm{E}-01$ & $1.108 \mathrm{E}-01$ & $1.78 \mathrm{E}-02$ \\
\hline $8.035 E+03$ & $1.157 \mathrm{E}+00$ & $1.379 \mathrm{E}+01$ & $5.51 \mathrm{E}-01$ & $2.609 \mathrm{E}+01$ & $2.98 \mathrm{E}-01$ & $1.163 \mathrm{E}-01$ & $1.02 \mathrm{E}-02$ \\
\hline $9.033 \mathrm{E}+03$ & $1.157 \mathrm{E}+00$ & $1.922 \mathrm{E}+01$ & $6.24 \mathrm{E}-01$ & $3.468 \mathrm{E}+01$ & $4.02 \mathrm{E}-01$ & $1.139 \mathrm{E}-01$ & $7.42 \mathrm{E}-03$ \\
\hline $9.997 \mathrm{E}+03$ & $1.157 \mathrm{E}+00$ & $2.592 \mathrm{E}+01$ & $7.53 \mathrm{E}-01$ & $4.488 \mathrm{E}+01$ & $5.27 \mathrm{E}-01$ & $1.166 \mathrm{E}-01$ & $6.08 \mathrm{E}-03$ \\
\hline $1.107 \mathrm{E}+04$ & $1.157 \mathrm{E}+00$ & $3.537 \mathrm{E}+01$ & $9.31 \mathrm{E}-01$ & $5.841 \mathrm{E}+01$ & $6.70 \mathrm{E}-01$ & $1.171 \mathrm{E}-01$ & $4.86 \mathrm{E}-03$ \\
\hline $1.203 \mathrm{E}+04$ & $1.157 \mathrm{E}+00$ & $4.521 \mathrm{E}+01$ & $9.52 \mathrm{E}-01$ & $7.365 \mathrm{E}+01$ & $8.20 \mathrm{E}-01$ & $1.188 \mathrm{E}-01$ & $3.97 \mathrm{E}-03$ \\
\hline $1.296 \mathrm{E}+04$ & $1.157 \mathrm{E}+00$ & $5.687 \mathrm{E}+01$ & $1.11 \mathrm{E}+00$ & $9.088 \mathrm{E}+01$ & $1.00 \mathrm{E}+00$ & 1.190E-01 & $3.44 \mathrm{E}-03$ \\
\hline $1.387 \mathrm{E}+04$ & $1.158 \mathrm{E}+00$ & $6.978 \mathrm{E}+01$ & $9.51 \mathrm{E}-01$ & $1.108 \mathrm{E}+02$ & $1.20 \mathrm{E}+00$ & $1.200 \mathrm{E}-01$ & $3.11 \mathrm{E}-03$ \\
\hline $1.485 \mathrm{E}+04$ & $1.158 \mathrm{E}+00$ & $8.500 \mathrm{E}+01$ & $1.06 \mathrm{E}+00$ & $1.357 \mathrm{E}+02$ & $1.47 \mathrm{E}+00$ & 1.199E-01 & $2.84 \mathrm{E}-03$ \\
\hline$C_{\mathrm{P}}$ & $\Delta C_{\mathrm{P}}$ & $C_{\mathrm{Q}}$ & $\Delta C_{\mathrm{Q}}$ & $\eta_{\mathrm{T}}$ & $\Delta \eta_{\mathrm{T}}$ & $R e_{0.75}$ & $\Delta R e_{0.75}$ \\
\hline $9.657 \mathrm{E}-02$ & $1.44 \mathrm{E}-02$ & $1.537 \mathrm{E}-02$ & $2.29 \mathrm{E}-03$ & $2.925 \mathrm{E}-01$ & $4.38 \mathrm{E}-02$ & $1.367 \mathrm{E}+04$ & $1.76 \mathrm{E}+01$ \\
\hline $9.539 \mathrm{E}-02$ & $9.42 \mathrm{E}-03$ & $1.518 \mathrm{E}-02$ & $1.50 \mathrm{E}-03$ & $3.806 \mathrm{E}-01$ & $3.79 \mathrm{E}-02$ & $1.743 \mathrm{E}+04$ & $2.26 \mathrm{E}+01$ \\
\hline $9.122 \mathrm{E}-02$ & 7.09E-03 & $1.452 \mathrm{E}-02$ & $1.13 \mathrm{E}-03$ & 4.270E-01 & $3.36 \mathrm{E}-02$ & $2.072 \mathrm{E}+04$ & $2.73 \mathrm{E}+01$ \\
\hline 9.082E-02 & 7.34E-03 & $1.445 \mathrm{E}-02$ & $1.17 \mathrm{E}-03$ & 4.775E-01 & $3.90 \mathrm{E}-02$ & $2.415 \mathrm{E}+04$ & $3.09 \mathrm{E}+01$ \\
\hline $9.212 \mathrm{E}-02$ & $3.68 \mathrm{E}-03$ & $1.466 \mathrm{E}-02$ & $5.86 \mathrm{E}-04$ & $5.285 \mathrm{E}-01$ & $2.20 \mathrm{E}-02$ & $2.769 \mathrm{E}+04$ & $3.45 \mathrm{E}+01$ \\
\hline $9.041 \mathrm{E}-02$ & $2.94 \mathrm{E}-03$ & $1.439 \mathrm{E}-02$ & $4.67 \mathrm{E}-04$ & $5.543 \mathrm{E}-01$ & $1.91 \mathrm{E}-02$ & $3.113 \mathrm{E}+04$ & $3.62 \mathrm{E}+01$ \\
\hline 8.991E-02 & $2.61 \mathrm{E}-03$ & $1.431 \mathrm{E}-02$ & $4.16 \mathrm{E}-04$ & 5.776E-01 & $1.81 \mathrm{E}-02$ & $3.446 \mathrm{E}+04$ & $4.03 \mathrm{E}+01$ \\
\hline $9.026 \mathrm{E}-02$ & $2.38 \mathrm{E}-03$ & $1.437 \mathrm{E}-02$ & $3.78 \mathrm{E}-04$ & $6.056 \mathrm{E}-01$ & $1.74 \mathrm{E}-02$ & $3.818 \mathrm{E}+04$ & $4.40 \mathrm{E}+01$ \\
\hline 8.988E-02 & $1.90 \mathrm{E}-03$ & $1.537 \mathrm{E}-02$ & $3.01 \mathrm{E}-04$ & 6.139E-01 & $1.46 \mathrm{E}-02$ & $4.149 \mathrm{E}+04$ & $4.81 \mathrm{E}+01$ \\
\hline $9.043 \mathrm{E}-02$ & $1.77 \mathrm{E}-03$ & $1.439 \mathrm{E}-02$ & $2.81 \mathrm{E}-04$ & $6.258 \mathrm{E}-01$ & $1.40 \mathrm{E}-02$ & $4.471 \mathrm{E}+04$ & $5.17 \mathrm{E}+01$ \\
\hline $9.061 \mathrm{E}-02$ & $1.24 \mathrm{E}-03$ & $1.442 \mathrm{E}-02$ & $1.97 \mathrm{E}-04$ & 6.299E-01 & $1.10 \mathrm{E}-02$ & $4.784 \mathrm{E}+04$ & $5.51 \mathrm{E}+01$ \\
\hline 8.983E-02 & $1.12 \mathrm{E}-03$ & $1.430 \mathrm{E}-02$ & $1.78 \mathrm{E}-04$ & $6.265 \mathrm{E}-01$ & $1.03 \mathrm{E}-02$ & $5.124 \mathrm{E}+04$ & $5.92 \mathrm{E}+01$ \\
\hline
\end{tabular}




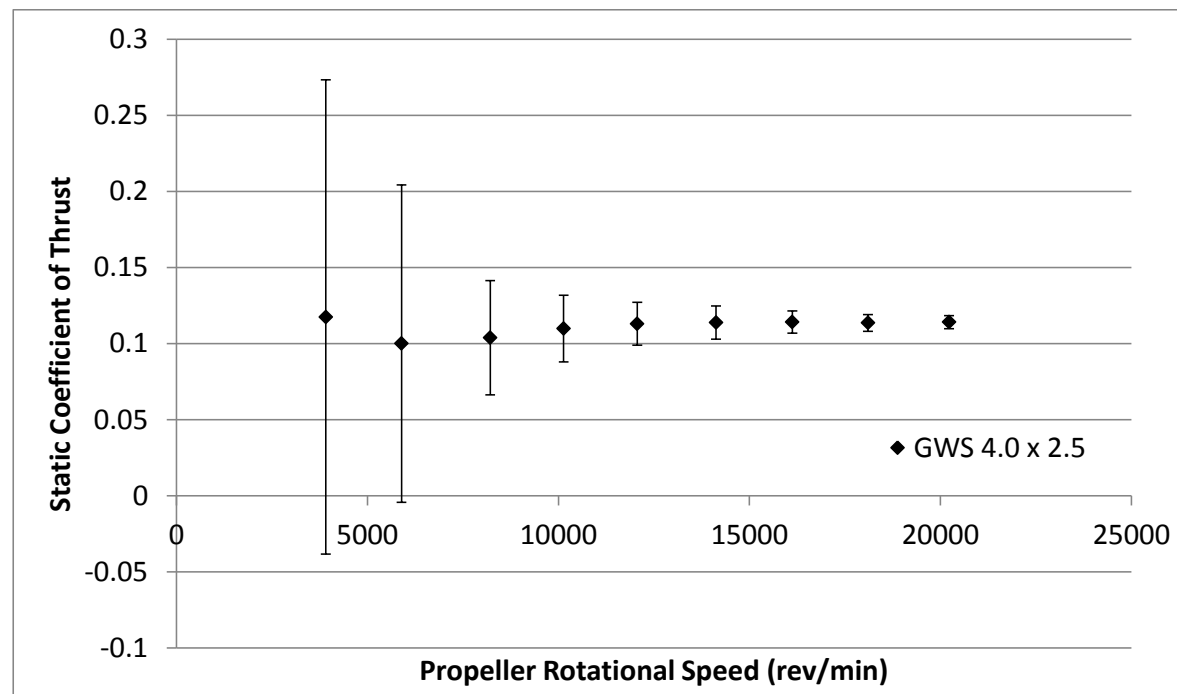

(a)

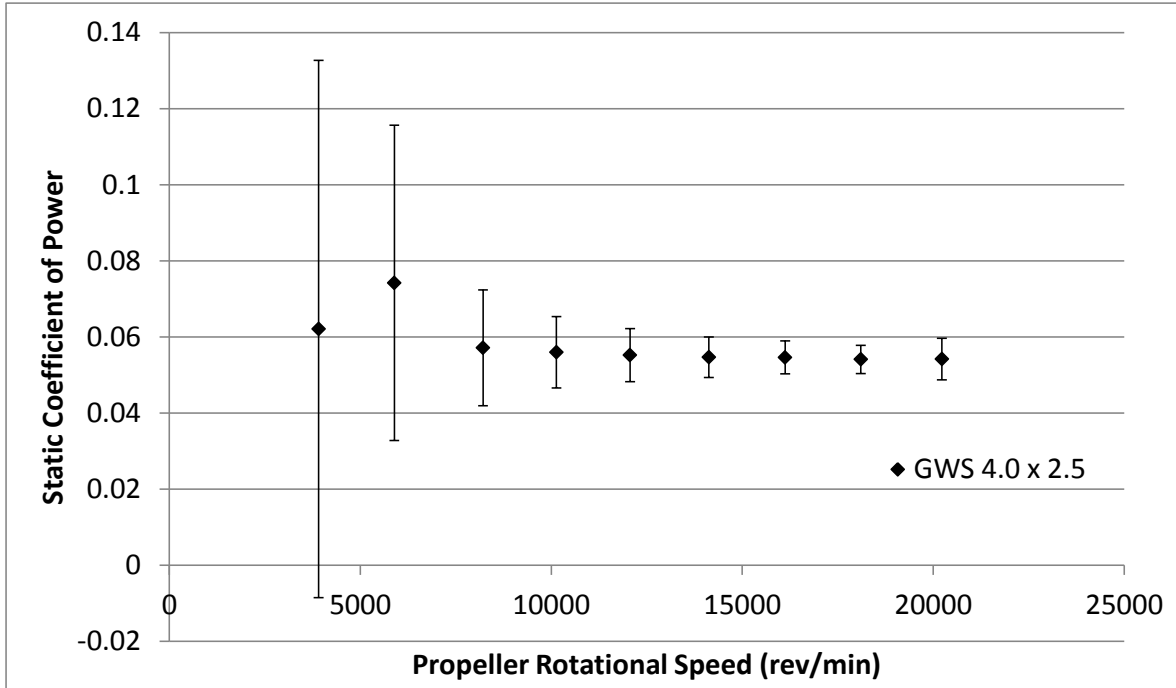

(b)

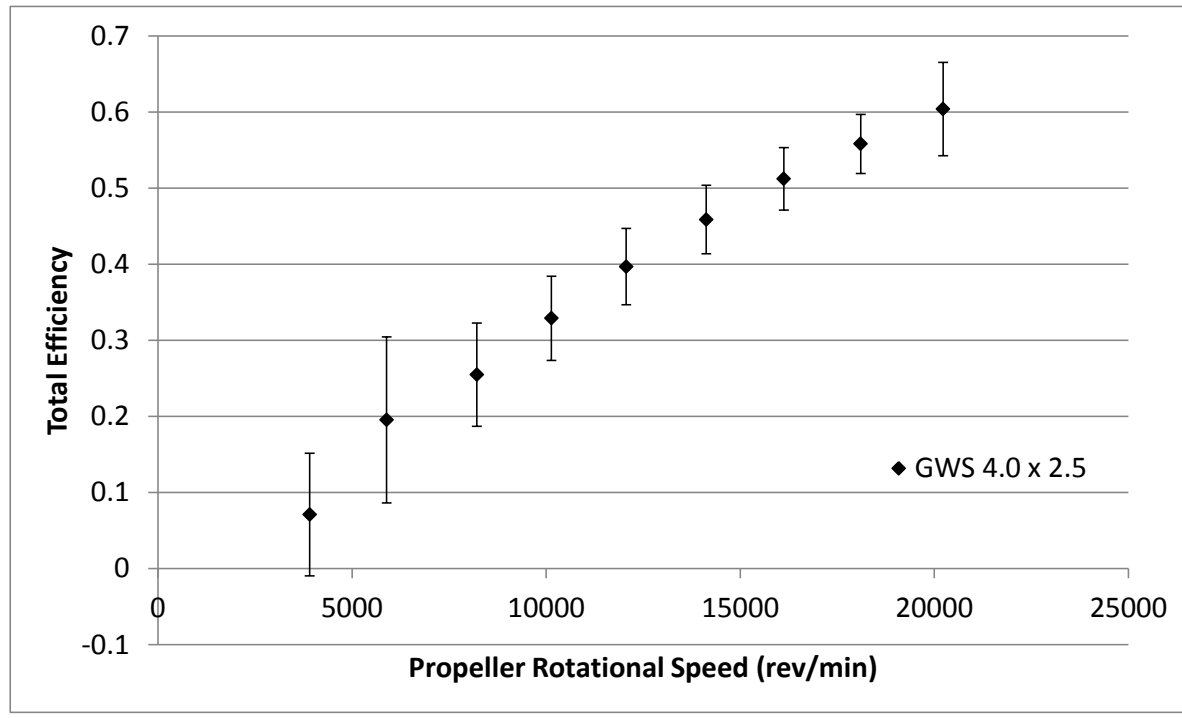

(c)

Figure 70: GWS 4.0 x 2.5 Static Test Results: (a) Coefficient of Thrust, (b) Coefficient of Power, (c) Total Efficiency. 
Table 62: GWS 4.0 x 2.5 Static Measured Values

\begin{tabular}{|c|c|c|c|c|c|c|c|c|}
\hline$n(\mathrm{RPM})$ & $T(\mathrm{~g})$ & $\Delta T(\mathrm{~g})$ & $Q(\mathrm{~g}-\mathrm{m})$ & $\Delta Q(\mathrm{~g}-\mathrm{m})$ & $V(\mathrm{~V})$ & $I(\mathrm{~A})$ & $T_{\mathrm{atm}}\left({ }^{\circ} \mathrm{C}\right)$ & $P_{\mathrm{atm}}(\mathrm{Pa})$ \\
\hline $3.908 \mathrm{E}+03$ & $6.300 \mathrm{E}+00$ & $8.36 \mathrm{E}+00$ & $5.384 \mathrm{E}-02$ & $6.12 \mathrm{E}-02$ & $1.111 \mathrm{E}+01$ & $2.742 \mathrm{E}-01$ & $2.210 \mathrm{E}+01$ & $9.875 \mathrm{E}+04$ \\
\hline $5.890 \mathrm{E}+03$ & $1.218 \mathrm{E}+01$ & $1.27 \mathrm{E}+01$ & $1.461 \mathrm{E}-01$ & $8.16 \mathrm{E}-02$ & $1.110 \mathrm{E}+01$ & $4.076 \mathrm{E}-01$ & $2.216 \mathrm{E}+01$ & $9.875 \mathrm{E}+04$ \\
\hline $8.214 \mathrm{E}+03$ & $2.459 \mathrm{E}+01$ & $8.90 \mathrm{E}+00$ & $2.188 \mathrm{E}-01$ & $5.82 \mathrm{E}-02$ & $1.110 \mathrm{E}+01$ & $6.531 \mathrm{E}-01$ & $2.211 \mathrm{E}+01$ & $9.874 \mathrm{E}+04$ \\
\hline $1.014 \mathrm{E}+04$ & $3.960 \mathrm{E}+01$ & $7.92 \mathrm{E}+00$ & $3.262 \mathrm{E}-01$ & $5.47 \mathrm{E}-02$ & $1.109 \mathrm{E}+01$ & $9.310 \mathrm{E}-01$ & $2.217 \mathrm{E}+01$ & $9.871 \mathrm{E}+04$ \\
\hline $1.206 \mathrm{E}+04$ & $5.770 \mathrm{E}+01$ & $7.19 \mathrm{E}+00$ & $4.557 \mathrm{E}-01$ & $5.75 \mathrm{E}-02$ & $1.108 \mathrm{E}+01$ & $1.284 \mathrm{E}+00$ & $2.212 \mathrm{E}+01$ & $9.870 \mathrm{E}+04$ \\
\hline $1.413 \mathrm{E}+04$ & $7.968 \mathrm{E}+01$ & $7.64 \mathrm{E}+00$ & $6.187 \mathrm{E}-01$ & $6.03 \mathrm{E}-02$ & $1.107 \mathrm{E}+01$ & $1.768 \mathrm{E}+00$ & $2.207 \mathrm{E}+01$ & $9.869 \mathrm{E}+04$ \\
\hline $1.612 \mathrm{E}+04$ & $1.042 \mathrm{E}+02$ & $6.65 \mathrm{E}+00$ & $8.053 \mathrm{E}-01$ & $6.39 \mathrm{E}-02$ & $1.105 \mathrm{E}+01$ & $2.357 \mathrm{E}+00$ & $2.206 \mathrm{E}+01$ & $9.867 \mathrm{E}+04$ \\
\hline $1.811 \mathrm{E}+04$ & $1.307 \mathrm{E}+02$ & $6.32 \mathrm{E}+00$ & $1.005 \mathrm{E}+00$ & $6.90 \mathrm{E}-02$ & $1.103 \mathrm{E}+01$ & $3.036 \mathrm{E}+00$ & $2.208 \mathrm{E}+01$ & $9.867 \mathrm{E}+04$ \\
\hline $2.023 \mathrm{E}+04$ & $1.638 \mathrm{E}+02$ & $6.19 \mathrm{E}+00$ & $1.258 \mathrm{E}+00$ & $1.27 \mathrm{E}-01$ & $1.101 \mathrm{E}+01$ & $3.930 \mathrm{E}+00$ & $2.209 \mathrm{E}+01$ & $9.866 \mathrm{E}+04$ \\
\hline
\end{tabular}

Table 63: GWS 4.0 x 2.5 Static Calculated Values

\begin{tabular}{|r|c|c|c|c|c|c|c|}
\hline$n(\mathrm{RPM})$ & $\rho\left(\mathrm{kg} / \mathrm{m}^{\wedge} 3\right)$ & $P_{\mathrm{P}}(\mathrm{W})$ & $\Delta P_{\mathrm{P}}(\mathrm{W})$ & $P_{\mathrm{e}}(\mathrm{W})$ & $\Delta P_{\mathrm{e}}(\mathrm{W})$ & $C_{\mathrm{T}}$ & $\Delta C_{\mathrm{T}}$ \\
\hline $3.908 \mathrm{E}+03$ & $1.165 \mathrm{E}+00$ & $2.161 \mathrm{E}-01$ & $2.46 \mathrm{E}-01$ & $3.046 \mathrm{E}+00$ & $5.20 \mathrm{E}-02$ & $1.175 \mathrm{E}-01$ & $1.56 \mathrm{E}-01$ \\
\hline $5.890 \mathrm{E}+03$ & $1.165 \mathrm{E}+00$ & $8.837 \mathrm{E}-01$ & $4.93 \mathrm{E}-01$ & $4.526 \mathrm{E}+00$ & $6.59 \mathrm{E}-02$ & $1.000 \mathrm{E}-01$ & $1.04 \mathrm{E}-01$ \\
\hline $8.214 \mathrm{E}+03$ & $1.165 \mathrm{E}+00$ & $1.846 \mathrm{E}+00$ & $4.91 \mathrm{E}-01$ & $7.248 \mathrm{E}+00$ & $9.56 \mathrm{E}-02$ & $1.038 \mathrm{E}-01$ & $3.76 \mathrm{E}-02$ \\
\hline $1.014 \mathrm{E}+04$ & $1.164 \mathrm{E}+00$ & $3.396 \mathrm{E}+00$ & $5.69 \mathrm{E}-01$ & $1.032 \mathrm{E}+01$ & $1.27 \mathrm{E}-01$ & $1.098 \mathrm{E}-01$ & $2.20 \mathrm{E}-02$ \\
\hline $1.206 \mathrm{E}+04$ & $1.164 \mathrm{E}+00$ & $5.645 \mathrm{E}+00$ & $7.12 \mathrm{E}-01$ & $1.422 \mathrm{E}+01$ & $1.68 \mathrm{E}-01$ & $1.130 \mathrm{E}-01$ & $1.41 \mathrm{E}-02$ \\
\hline $1.413 \mathrm{E}+04$ & $1.164 \mathrm{E}+00$ & $8.975 \mathrm{E}+00$ & $8.75 \mathrm{E}-01$ & $1.957 \mathrm{E}+01$ & $2.27 \mathrm{E}-01$ & $1.138 \mathrm{E}-01$ & $1.09 \mathrm{E}-02$ \\
\hline $1.612 \mathrm{E}+04$ & $1.164 \mathrm{E}+00$ & $1.334 \mathrm{E}+01$ & $1.06 \mathrm{E}+00$ & $2.604 \mathrm{E}+01$ & $2.98 \mathrm{E}-01$ & $1.142 \mathrm{E}-01$ & $7.29 \mathrm{E}-03$ \\
\hline $1.811 \mathrm{E}+04$ & $1.164 \mathrm{E}+00$ & $1.869 \mathrm{E}+01$ & $1.28 \mathrm{E}+00$ & $3.349 \mathrm{E}+01$ & $3.77 \mathrm{E}-01$ & $1.136 \mathrm{E}-01$ & $5.50 \mathrm{E}-03$ \\
\hline $2.023 \mathrm{E}+04$ & $1.164 \mathrm{E}+00$ & $2.613 \mathrm{E}+01$ & $2.63 \mathrm{E}+00$ & $4.326 \mathrm{E}+01$ & $5.20 \mathrm{E}-01$ & $1.141 \mathrm{E}-01$ & $4.31 \mathrm{E}-03$ \\
\hline$C_{\mathrm{P}}$ & $\Delta C_{\mathrm{P}}$ & $C_{\mathrm{Q}}$ & $\Delta C_{\mathrm{Q}}$ & $\eta_{\mathrm{T}}$ & $\Delta \eta_{\mathrm{T}}$ & $R e_{0.75}$ & $\Delta R e_{0.75}$ \\
\hline $6.212 \mathrm{E}-02$ & $7.06 \mathrm{E}-02$ & $9.887 \mathrm{E}-03$ & $1.12 \mathrm{E}-02$ & $7.094 \mathrm{E}-02$ & $8.07 \mathrm{E}-02$ & 12351.07 & $1.45 \mathrm{E}+01$ \\
\hline $7.421 \mathrm{E}-02$ & $4.14 \mathrm{E}-02$ & $1.181 \mathrm{E}-02$ & $6.60 \mathrm{E}-03$ & $1.952 \mathrm{E}-01$ & $1.09 \mathrm{E}-01$ & 18609.94 & $2.21 \mathrm{E}+01$ \\
\hline $5.716 \mathrm{E}-02$ & $1.52 \mathrm{E}-02$ & $9.097 \mathrm{E}-03$ & $2.42 \mathrm{E}-03$ & $2.547 \mathrm{E}-01$ & $6.79 \mathrm{E}-02$ & 25957.43 & $2.88 \mathrm{E}+01$ \\
\hline $5.598 \mathrm{E}-02$ & $9.38 \mathrm{E}-03$ & $8.910 \mathrm{E}-03$ & $1.49 \mathrm{E}-03$ & $3.289 \mathrm{E}-01$ & $5.53 \mathrm{E}-02$ & 32013.46 & $3.28 \mathrm{E}+01$ \\
\hline $5.521 \mathrm{E}-02$ & $6.97 \mathrm{E}-03$ & $8.787 \mathrm{E}-03$ & $1.11 \mathrm{E}-03$ & $3.969 \mathrm{E}-01$ & $5.03 \mathrm{E}-02$ & 38103.15 & $3.95 \mathrm{E}+01$ \\
\hline $5.466 \mathrm{E}-02$ & $5.33 \mathrm{E}-03$ & $8.699 \mathrm{E}-03$ & $8.48 \mathrm{E}-04$ & $4.587 \mathrm{E}-01$ & $4.50 \mathrm{E}-02$ & 44626.57 & $4.45 \mathrm{E}+01$ \\
\hline $5.461 \mathrm{E}-02$ & $4.33 \mathrm{E}-03$ & $8.691 \mathrm{E}-03$ & $6.89 \mathrm{E}-04$ & $5.121 \mathrm{E}-01$ & $4.10 \mathrm{E}-02$ & 50937.13 & $4.98 \mathrm{E}+01$ \\
\hline $5.408 \mathrm{E}-02$ & $3.71 \mathrm{E}-03$ & $8.608 \mathrm{E}-03$ & $5.91 \mathrm{E}-04$ & $5.582 \mathrm{E}-01$ & $3.88 \mathrm{E}-02$ & 57184.55 & $5.53 \mathrm{E}+01$ \\
\hline $5.420 \mathrm{E}-02$ & $5.46 \mathrm{E}-03$ & $9.887 \mathrm{E}-03$ & $8.69 \mathrm{E}-04$ & $6.039 \mathrm{E}-01$ & $6.12 \mathrm{E}-02$ & 63883.67 & $6.30 \mathrm{E}+01$ \\
\hline
\end{tabular}




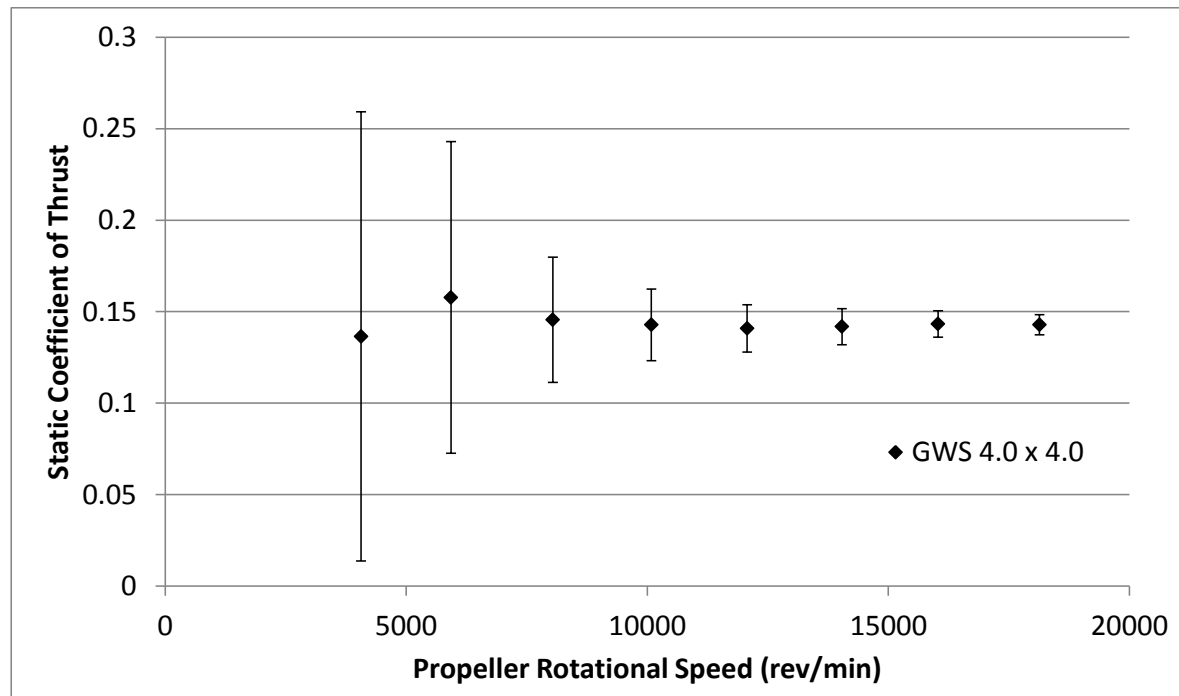

(a)

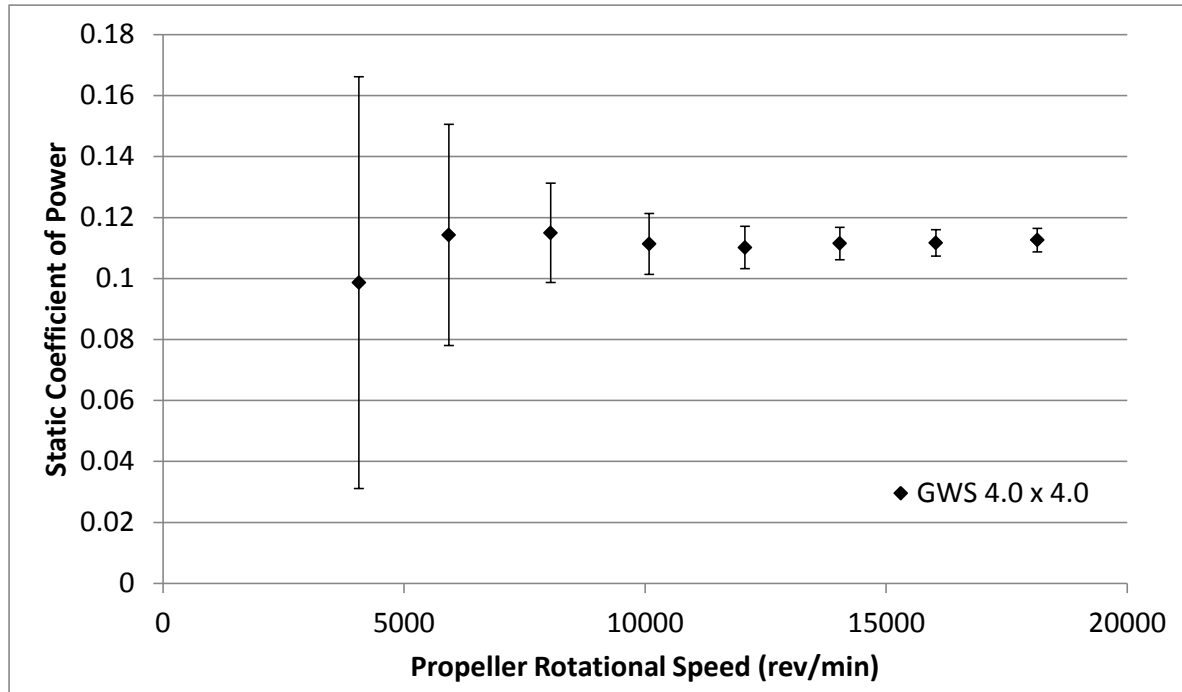

(b)

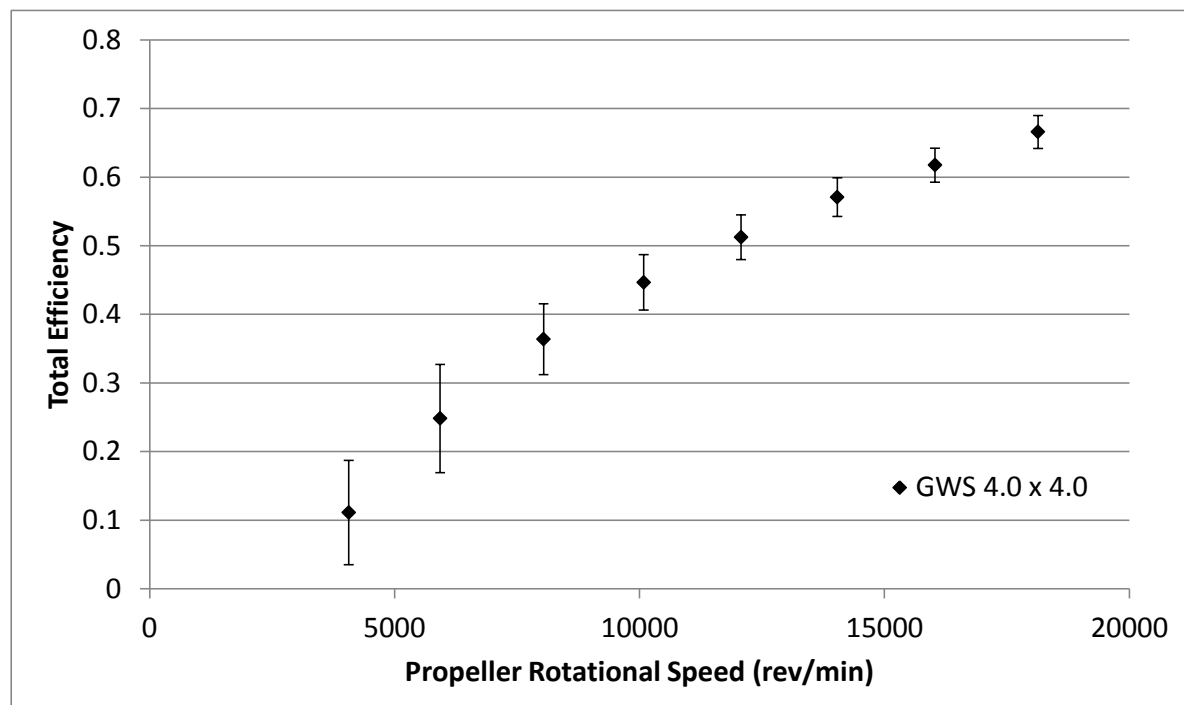

(c)

Figure 71: GWS 4.0 x 4.0 Static Test Results: (a) Coefficient of Thrust, (b) Coefficient of Power, (c) Total Efficiency. 
Table 64: GWS 4.0 x 4.0 Static Measured Values

\begin{tabular}{|c|c|c|c|c|c|c|c|c|}
\hline$n(\mathrm{RPM})$ & $T(\mathrm{~g})$ & $\Delta T(\mathrm{~g})$ & $Q(\mathrm{~g}-\mathrm{m})$ & $\Delta Q(\mathrm{~g}-\mathrm{m})$ & $V(\mathrm{~V})$ & $I(\mathrm{~A})$ & $T_{\text {atm }}\left({ }^{\circ} \mathrm{C}\right)$ & $P_{\text {atm }}(\mathrm{Pa})$ \\
\hline $4.064 \mathrm{E}+03$ & $8.028 \mathrm{E}+00$ & $7.22 \mathrm{E}+00$ & $9.421 \mathrm{E}-02$ & $6.45 \mathrm{E}-02$ & $1.111 \mathrm{E}+01$ & $3.188 \mathrm{E}-01$ & $2.217 \mathrm{E}+01$ & $9.850 \mathrm{E}+04$ \\
\hline $5.930 \mathrm{E}+03$ & $1.976 \mathrm{E}+01$ & $1.07 \mathrm{E}+01$ & $2.323 \mathrm{E}-01$ & $7.38 \mathrm{E}-02$ & $1.110 \mathrm{E}+01$ & $5.135 \mathrm{E}-01$ & $2.212 \mathrm{E}+01$ & $9.850 \mathrm{E}+04$ \\
\hline $8.042 \mathrm{E}+03$ & $3.352 \mathrm{E}+01$ & $7.87 \mathrm{E}+00$ & $4.297 \mathrm{E}-01$ & $6.08 \mathrm{E}-02$ & $1.109 \mathrm{E}+01$ & $8.797 \mathrm{E}-01$ & $2.222 \mathrm{E}+01$ & $9.850 \mathrm{E}+04$ \\
\hline $1.009 \mathrm{E}+04$ & $5.168 \mathrm{E}+01$ & $7.10 \mathrm{E}+00$ & $6.541 \mathrm{E}-01$ & $5.86 \mathrm{E}-02$ & $1.108 \mathrm{E}+01$ & $1.369 \mathrm{E}+00$ & $2.244 \mathrm{E}+01$ & $9.850 \mathrm{E}+04$ \\
\hline $1.207 \mathrm{E}+04$ & $7.303 \mathrm{E}+01$ & $6.69 \mathrm{E}+00$ & $9.272 \mathrm{E}-01$ & $5.83 \mathrm{E}-02$ & $1.106 \mathrm{E}+01$ & $2.028 \mathrm{E}+00$ & $2.251 \mathrm{E}+01$ & $9.850 \mathrm{E}+04$ \\
\hline $1.404 \mathrm{E}+04$ & $9.937 \mathrm{E}+01$ & $6.93 \mathrm{E}+00$ & $1.268 \mathrm{E}+00$ & $6.08 \mathrm{E}-02$ & $1.104 \mathrm{E}+01$ & $2.902 \mathrm{E}+00$ & $2.268 \mathrm{E}+01$ & $9.851 \mathrm{E}+04$ \\
\hline $1.603 \mathrm{E}+04$ & $1.309 \mathrm{E}+02$ & $6.57 \mathrm{E}+00$ & $1.656 \mathrm{E}+00$ & $6.43 \mathrm{E}-02$ & $1.100 \mathrm{E}+01$ & $4.014 \mathrm{E}+00$ & $2.271 \mathrm{E}+01$ & $9.851 \mathrm{E}+04$ \\
\hline $1.814 \mathrm{E}+04$ & $1.670 \mathrm{E}+02$ & $6.44 \mathrm{E}+00$ & $2.137 \mathrm{E}+00$ & $7.33 \mathrm{E}-02$ & $1.096 \mathrm{E}+01$ & $5.452 \mathrm{E}+00$ & $2.282 \mathrm{E}+01$ & $9.853 \mathrm{E}+04$ \\
\hline
\end{tabular}

Table 65: GWS 4.0 x 4.0 Static Calculated Values

\begin{tabular}{|c|c|c|c|c|c|c|c|}
\hline$n(\mathrm{RPM})$ & $\rho\left(\mathrm{kg} / \mathrm{m}^{\wedge} 3\right)$ & $P_{\mathrm{P}}(\mathrm{W})$ & $\Delta P_{\mathrm{P}}(\mathrm{W})$ & $P_{\mathrm{e}}(\mathrm{W})$ & $\Delta P_{\mathrm{e}}(\mathrm{W})$ & $C_{\mathrm{T}}$ & $\Delta C_{\mathrm{T}}$ \\
\hline $4.064 \mathrm{E}+03$ & $1.162 \mathrm{E}+00$ & $3.932 \mathrm{E}-01$ & $2.69 \mathrm{E}-01$ & $3.541 \mathrm{E}+00$ & $5.71 \mathrm{E}-02$ & $1.365 \mathrm{E}-01$ & $1.23 \mathrm{E}-01$ \\
\hline $5.930 \mathrm{E}+03$ & $1.162 \mathrm{E}+00$ & $1.415 \mathrm{E}+00$ & $4.49 \mathrm{E}-01$ & $5.701 \mathrm{E}+00$ & $7.96 \mathrm{E}-02$ & $1.577 \mathrm{E}-01$ & $8.52 \mathrm{E}-02$ \\
\hline $8.042 \mathrm{E}+03$ & $1.162 \mathrm{E}+00$ & $3.549 \mathrm{E}+00$ & $5.02 \mathrm{E}-01$ & $9.757 \mathrm{E}+00$ & $1.23 \mathrm{E}-01$ & $1.456 \mathrm{E}-01$ & $3.42 \mathrm{E}-02$ \\
\hline $1.009 \mathrm{E}+04$ & $1.161 \mathrm{E}+00$ & $6.775 \mathrm{E}+00$ & $6.07 \mathrm{E}-01$ & $1.517 \mathrm{E}+01$ & $1.79 \mathrm{E}-01$ & $1.428 \mathrm{E}-01$ & $1.96 \mathrm{E}-02$ \\
\hline $1.207 \mathrm{E}+04$ & $1.161 \mathrm{E}+00$ & $1.150 \mathrm{E}+01$ & $7.23 \mathrm{E}-01$ & $2.243 \mathrm{E}+01$ & $2.54 \mathrm{E}-01$ & $1.408 \mathrm{E}-01$ & $1.29 \mathrm{E}-02$ \\
\hline $1.404 \mathrm{E}+04$ & $1.160 \mathrm{E}+00$ & $1.828 \mathrm{E}+01$ & $8.77 \mathrm{E}-01$ & $3.203 \mathrm{E}+01$ & $3.57 \mathrm{E}-01$ & $1.418 \mathrm{E}-01$ & $9.89 \mathrm{E}-03$ \\
\hline $1.603 \mathrm{E}+04$ & $1.160 \mathrm{E}+00$ & $2.727 \mathrm{E}+01$ & $1.06 \mathrm{E}+00$ & $4.417 \mathrm{E}+01$ & $4.95 \mathrm{E}-01$ & $1.433 \mathrm{E}-01$ & $7.19 \mathrm{E}-03$ \\
\hline $1.814 \mathrm{E}+04$ & $1.160 \mathrm{E}+00$ & $3.980 \mathrm{E}+01$ & $1.37 \mathrm{E}+00$ & $5.977 \mathrm{E}+01$ & $6.72 \mathrm{E}-01$ & $1.428 \mathrm{E}-01$ & $5.51 \mathrm{E}-03$ \\
\hline$C_{\mathrm{P}}$ & $\Delta C_{\mathrm{P}}$ & $C_{\mathrm{Q}}$ & $\Delta C_{\mathrm{Q}}$ & $\eta_{\mathrm{T}}$ & $\Delta \eta_{\mathrm{T}}$ & $R e_{0.75}$ & $\Delta R e_{0.75}$ \\
\hline $9.866 \mathrm{E}-02$ & $6.75 \mathrm{E}-02$ & $1.570 \mathrm{E}-02$ & $1.08 \mathrm{E}-02$ & $1.111 \mathrm{E}-01$ & $7.61 \mathrm{E}-02$ & $1.084 \mathrm{E}+04$ & $1.46 \mathrm{E}+01$ \\
\hline $1.143 \mathrm{E}-01$ & $3.63 \mathrm{E}-02$ & $1.819 \mathrm{E}-02$ & $5.77 \mathrm{E}-03$ & $2.482 \mathrm{E}-01$ & $7.89 \mathrm{E}-02$ & $1.582 \mathrm{E}+04$ & $2.07 \mathrm{E}+01$ \\
\hline $1.150 \mathrm{E}-01$ & $1.63 \mathrm{E}-02$ & $1.830 \mathrm{E}-02$ & $2.59 \mathrm{E}-03$ & $3.637 \mathrm{E}-01$ & $5.17 \mathrm{E}-02$ & $2.144 \mathrm{E}+04$ & $2.75 \mathrm{E}+01$ \\
\hline $1.113 \mathrm{E}-01$ & $9.98 \mathrm{E}-03$ & $1.772 \mathrm{E}-02$ & $1.59 \mathrm{E}-03$ & $4.466 \mathrm{E}-01$ & $4.04 \mathrm{E}-02$ & $2.685 \mathrm{E}+04$ & $3.04 \mathrm{E}+01$ \\
\hline $1.102 \mathrm{E}-01$ & $6.93 \mathrm{E}-03$ & $1.753 \mathrm{E}-02$ & $1.10 \mathrm{E}-03$ & $5.124 \mathrm{E}-01$ & $3.27 \mathrm{E}-02$ & $3.213 \mathrm{E}+04$ & $3.66 \mathrm{E}+01$ \\
\hline $1.115 \mathrm{E}-01$ & $5.35 \mathrm{E}-03$ & $1.774 \mathrm{E}-02$ & $8.51 \mathrm{E}-04$ & $5.709 \mathrm{E}-01$ & $2.81 \mathrm{E}-02$ & $3.733 \mathrm{E}+04$ & $4.16 \mathrm{E}+01$ \\
\hline $1.117 \mathrm{E}-01$ & $4.33 \mathrm{E}-03$ & $1.777 \mathrm{E}-02$ & $6.90 \mathrm{E}-04$ & $6.174 \mathrm{E}-01$ & $2.49 \mathrm{E}-02$ & $4.263 \mathrm{E}+04$ & $4.75 \mathrm{E}+01$ \\
\hline $1.126 \mathrm{E}-01$ & $3.87 \mathrm{E}-03$ & $1.792 \mathrm{E}-02$ & $6.15 \mathrm{E}-04$ & $6.659 \mathrm{E}-01$ & $2.40 \mathrm{E}-02$ & $4.819 \mathrm{E}+04$ & $5.32 \mathrm{E}+01$ \\
\hline
\end{tabular}




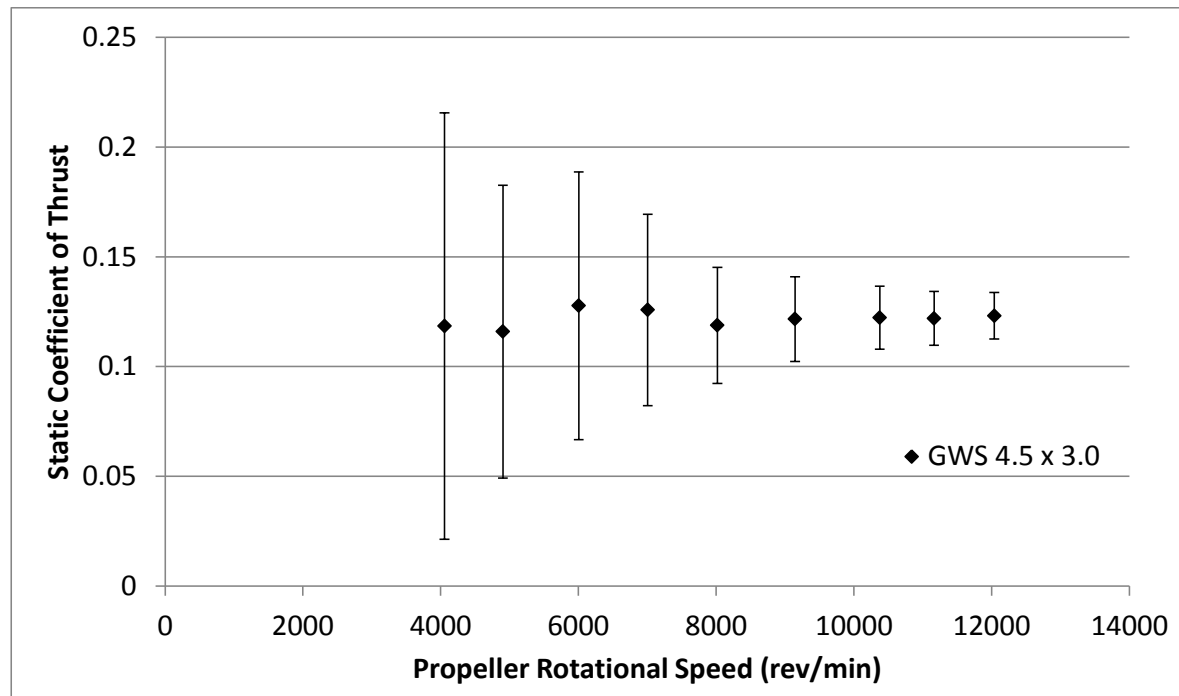

(a)

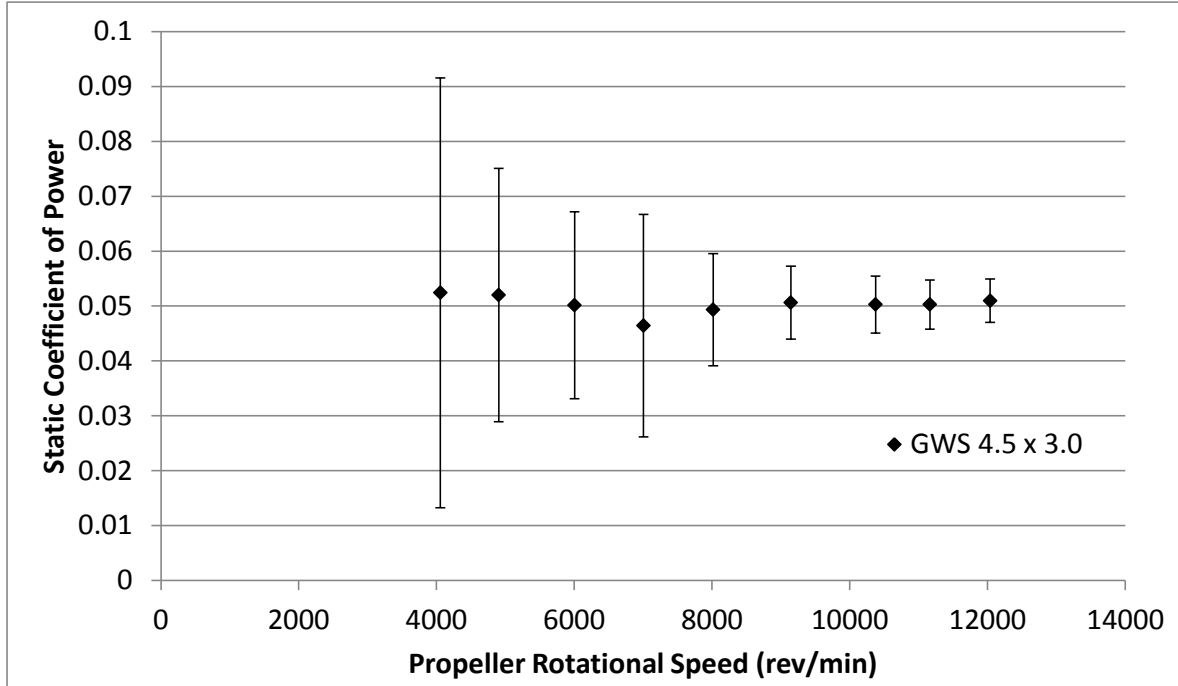

(b)

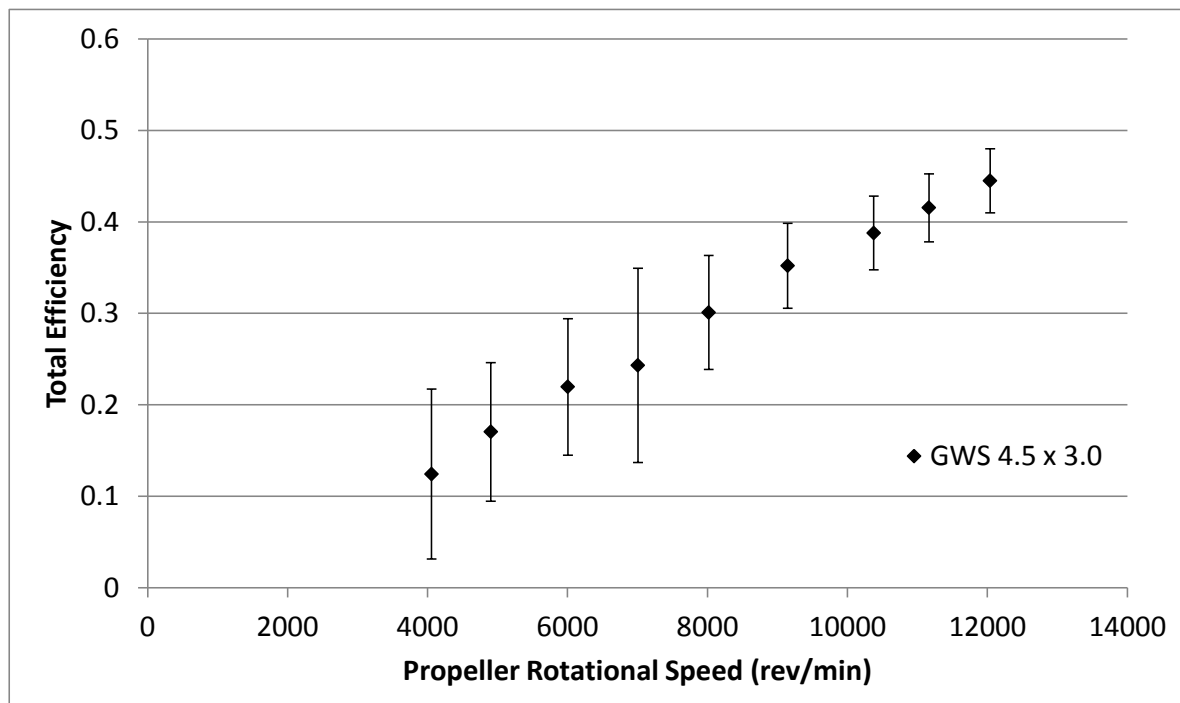

(c)

Figure 72: GWS 4.5 x 3.0 Static Test Results: (a) Coefficient of Thrust, (b) Coefficient of Power, (c) Total Efficiency. 
Table 66: GWS 4.5 x 3.0 Static Measured Values

\begin{tabular}{|c|c|c|c|c|c|c|c|c|}
\hline$n(\mathrm{RPM})$ & $T(\mathrm{~g})$ & $\Delta T(\mathrm{~g})$ & $Q(\mathrm{~g}-\mathrm{m})$ & $\Delta Q(\mathrm{~g}-\mathrm{m})$ & $V(\mathrm{~V})$ & $I(\mathrm{~A})$ & $T_{\text {atm }}\left({ }^{\circ} \mathrm{C}\right)$ & $P_{\text {atm }}(\mathrm{Pa})$ \\
\hline $4.057 \mathrm{E}+03$ & $1.099 \mathrm{E}+01$ & $9.01 \mathrm{E}+00$ & $8.846 \mathrm{E}-02$ & $6.61 \mathrm{E}-02$ & $1.108 \mathrm{E}+01$ & $2.676 \mathrm{E}-01$ & $2.066 \mathrm{E}+01$ & $9.838 \mathrm{E}+04$ \\
\hline $4.904 \mathrm{E}+03$ & $1.571 \mathrm{E}+01$ & $9.05 \mathrm{E}+00$ & $1.282 \mathrm{E}-01$ & $5.69 \mathrm{E}-02$ & $1.108 \mathrm{E}+01$ & $3.420 \mathrm{E}-01$ & $2.067 \mathrm{E}+01$ & $9.838 \mathrm{E}+04$ \\
\hline $6.006 \mathrm{E}+03$ & $2.596 \mathrm{E}+01$ & $1.24 \mathrm{E}+01$ & $1.855 \mathrm{E}-01$ & $6.29 \mathrm{E}-02$ & $1.108 \mathrm{E}+01$ & $4.701 \mathrm{E}-01$ & $2.069 \mathrm{E}+01$ & $9.839 \mathrm{E}+04$ \\
\hline $7.007 \mathrm{E}+03$ & $3.480 \mathrm{E}+01$ & $1.21 \mathrm{E}+01$ & $2.337 \mathrm{E}-01$ & $1.02 \mathrm{E}-01$ & $1.108 \mathrm{E}+01$ & $6.245 \mathrm{E}-01$ & $2.071 \mathrm{E}+01$ & $9.838 \mathrm{E}+04$ \\
\hline $8.018 \mathrm{E}+03$ & $4.302 \mathrm{E}+01$ & $9.57 \mathrm{E}+00$ & $3.250 \mathrm{E}-01$ & $6.73 \mathrm{E}-02$ & $1.107 \mathrm{E}+01$ & $8.032 \mathrm{E}-01$ & $2.072 \mathrm{E}+01$ & $9.837 \mathrm{E}+04$ \\
\hline $9.147 \mathrm{E}+03$ & $5.733 \mathrm{E}+01$ & $9.09 \mathrm{E}+00$ & $4.340 \mathrm{E}-01$ & $5.70 \mathrm{E}-02$ & $1.107 \mathrm{E}+01$ & $1.046 \mathrm{E}+00$ & $2.071 \mathrm{E}+01$ & $9.837 \mathrm{E}+04$ \\
\hline $1.038 \mathrm{E}+04$ & $7.417 \mathrm{E}+01$ & $8.72 \mathrm{E}+00$ & $5.549 \mathrm{E}-01$ & $5.74 \mathrm{E}-02$ & $1.106 \mathrm{E}+01$ & $1.379 \mathrm{E}+00$ & $2.070 \mathrm{E}+01$ & $9.837 \mathrm{E}+04$ \\
\hline $1.117 \mathrm{E}+04$ & $8.568 \mathrm{E}+01$ & $8.63 \mathrm{E}+00$ & $6.424 \mathrm{E}-01$ & $5.70 \mathrm{E}-02$ & $1.105 \mathrm{E}+01$ & $1.604 \mathrm{E}+00$ & $2.072 \mathrm{E}+01$ & $9.837 \mathrm{E}+04$ \\
\hline $1.204 \mathrm{E}+04$ & $1.006 \mathrm{E}+02$ & $8.66 \mathrm{E}+00$ & $7.573 \mathrm{E}-01$ & $5.89 \mathrm{E}-02$ & $1.105 \mathrm{E}+01$ & $1.905 \mathrm{E}+00$ & $2.073 \mathrm{E}+01$ & $9.837 \mathrm{E}+04$ \\
\hline
\end{tabular}

Table 67: GWS 4.5 x 3.0 Static Calculated Values

\begin{tabular}{|c|c|c|c|c|c|c|c|}
\hline$n(\mathrm{RPM})$ & $\rho\left(\mathrm{kg} / \mathrm{m}^{\wedge} 3\right)$ & $P_{\mathrm{P}}(\mathrm{W})$ & $\Delta P_{\mathrm{P}}(\mathrm{W})$ & $P_{\mathrm{e}}(\mathrm{W})$ & $\Delta P_{\mathrm{e}}(\mathrm{W})$ & $C_{\mathrm{T}}$ & $\Delta C_{\mathrm{T}}$ \\
\hline $4.057 \mathrm{E}+03$ & $1.167 \mathrm{E}+00$ & $3.685 \mathrm{E}-01$ & $2.75 \mathrm{E}-01$ & $2.966 \mathrm{E}+00$ & $5.38 \mathrm{E}-02$ & $1.185 \mathrm{E}-01$ & $9.71 \mathrm{E}-02$ \\
\hline $4.904 \mathrm{E}+03$ & $1.166 \mathrm{E}+00$ & $6.458 \mathrm{E}-01$ & $2.87 \mathrm{E}-01$ & $3.790 \mathrm{E}+00$ & $6.07 \mathrm{E}-02$ & 1.159E-01 & $6.67 \mathrm{E}-02$ \\
\hline $6.006 \mathrm{E}+03$ & $1.166 \mathrm{E}+00$ & $1.144 \mathrm{E}+00$ & $3.88 \mathrm{E}-01$ & $5.208 \mathrm{E}+00$ & 7.33E-02 & $1.277 \mathrm{E}-01$ & $6.10 \mathrm{E}-02$ \\
\hline $7.007 \mathrm{E}+03$ & $1.166 \mathrm{E}+00$ & $1.682 \mathrm{E}+00$ & 7.34E-01 & $6.917 \mathrm{E}+00$ & $9.21 \mathrm{E}-02$ & $1.258 \mathrm{E}-01$ & $4.36 \mathrm{E}-02$ \\
\hline $8.018 \mathrm{E}+03$ & $1.166 \mathrm{E}+00$ & $2.676 \mathrm{E}+00$ & $5.54 \mathrm{E}-01$ & $8.893 \mathrm{E}+00$ & $1.14 \mathrm{E}-01$ & $1.187 \mathrm{E}-01$ & $2.64 \mathrm{E}-02$ \\
\hline $9.147 \mathrm{E}+03$ & $1.166 \mathrm{E}+00$ & $4.077 \mathrm{E}+00$ & $5.36 \mathrm{E}-01$ & $1.158 \mathrm{E}+01$ & $1.40 \mathrm{E}-01$ & $1.216 \mathrm{E}-01$ & $1.93 \mathrm{E}-02$ \\
\hline $1.038 \mathrm{E}+04$ & $1.166 \mathrm{E}+00$ & $5.913 \mathrm{E}+00$ & $6.12 \mathrm{E}-01$ & $1.525 \mathrm{E}+01$ & $1.82 \mathrm{E}-01$ & $1.222 \mathrm{E}-01$ & $1.44 \mathrm{E}-02$ \\
\hline $1.117 \mathrm{E}+04$ & $1.166 \mathrm{E}+00$ & $7.366 \mathrm{E}+00$ & $6.53 \mathrm{E}-01$ & $1.773 \mathrm{E}+01$ & $2.05 \mathrm{E}-01$ & $1.219 \mathrm{E}-01$ & $1.23 \mathrm{E}-02$ \\
\hline $1.204 \mathrm{E}+04$ & $1.166 \mathrm{E}+00$ & $9.363 \mathrm{E}+00$ & $7.28 \mathrm{E}-01$ & $2.105 \mathrm{E}+01$ & $2.41 \mathrm{E}-01$ & $1.231 \mathrm{E}-01$ & $1.06 \mathrm{E}-02$ \\
\hline$C_{\mathrm{P}}$ & $\Delta C_{\mathrm{P}}$ & $C_{\mathrm{Q}}$ & $\Delta C_{\mathrm{Q}}$ & $\eta_{\mathrm{T}}$ & $\Delta \eta_{\mathrm{T}}$ & $\operatorname{Re}_{0.75}$ & $\Delta R e_{0.75}$ \\
\hline $5.241 \mathrm{E}-02$ & $3.92 \mathrm{E}-02$ & 8.342E-03 & $6.23 \mathrm{E}-03$ & $1.243 \mathrm{E}-01$ & $9.29 \mathrm{E}-02$ & $1.272 \mathrm{E}+04$ & $2.13 E+01$ \\
\hline $5.199 \mathrm{E}-02$ & $2.31 \mathrm{E}-02$ & $8.274 \mathrm{E}-03$ & $3.67 \mathrm{E}-03$ & $1.704 \mathrm{E}-01$ & $7.56 \mathrm{E}-02$ & $1.538 \mathrm{E}+04$ & $2.06 \mathrm{E}+01$ \\
\hline $5.015 \mathrm{E}-02$ & $1.70 \mathrm{E}-02$ & 7.982E-03 & $2.71 \mathrm{E}-03$ & $2.196 \mathrm{E}-01$ & $7.46 \mathrm{E}-02$ & $1.883 \mathrm{E}+04$ & $2.58 \mathrm{E}+01$ \\
\hline 4.643E-02 & $2.03 \mathrm{E}-02$ & 7.389E-03 & $3.22 \mathrm{E}-03$ & $2.431 \mathrm{E}-01$ & $1.06 \mathrm{E}-01$ & $2.197 \mathrm{E}+04$ & $2.86 \mathrm{E}+01$ \\
\hline 4.932E-02 & $1.02 \mathrm{E}-02$ & 7.849E-03 & $1.63 \mathrm{E}-03$ & $3.010 \mathrm{E}-01$ & $6.25 \mathrm{E}-02$ & $2.513 \mathrm{E}+04$ & $3.31 \mathrm{E}+01$ \\
\hline $5.060 \mathrm{E}-02$ & $6.65 \mathrm{E}-03$ & $8.053 \mathrm{E}-03$ & $1.06 \mathrm{E}-03$ & $3.521 \mathrm{E}-01$ & 4.65E-02 & $2.867 \mathrm{E}+04$ & $3.29 \mathrm{E}+01$ \\
\hline $5.027 \mathrm{E}-02$ & $5.20 \mathrm{E}-03$ & 8.000E-03 & $8.27 \mathrm{E}-04$ & 3.878E-01 & $4.04 \mathrm{E}-02$ & $3.253 \mathrm{E}+04$ & $3.64 \mathrm{E}+01$ \\
\hline $5.026 \mathrm{E}-02$ & $4.46 \mathrm{E}-03$ & 7.999E-03 & $7.10 \mathrm{E}-04$ & 4.154E-01 & $3.72 \mathrm{E}-02$ & $3.500 \mathrm{E}+04$ & $3.91 \mathrm{E}+01$ \\
\hline $5.097 \mathrm{E}-02$ & $3.97 \mathrm{E}-03$ & 8.342E-03 & $6.31 \mathrm{E}-04$ & 4.448E-01 & $3.50 \mathrm{E}-02$ & $3.773 \mathrm{E}+04$ & $4.29 \mathrm{E}+01$ \\
\hline
\end{tabular}




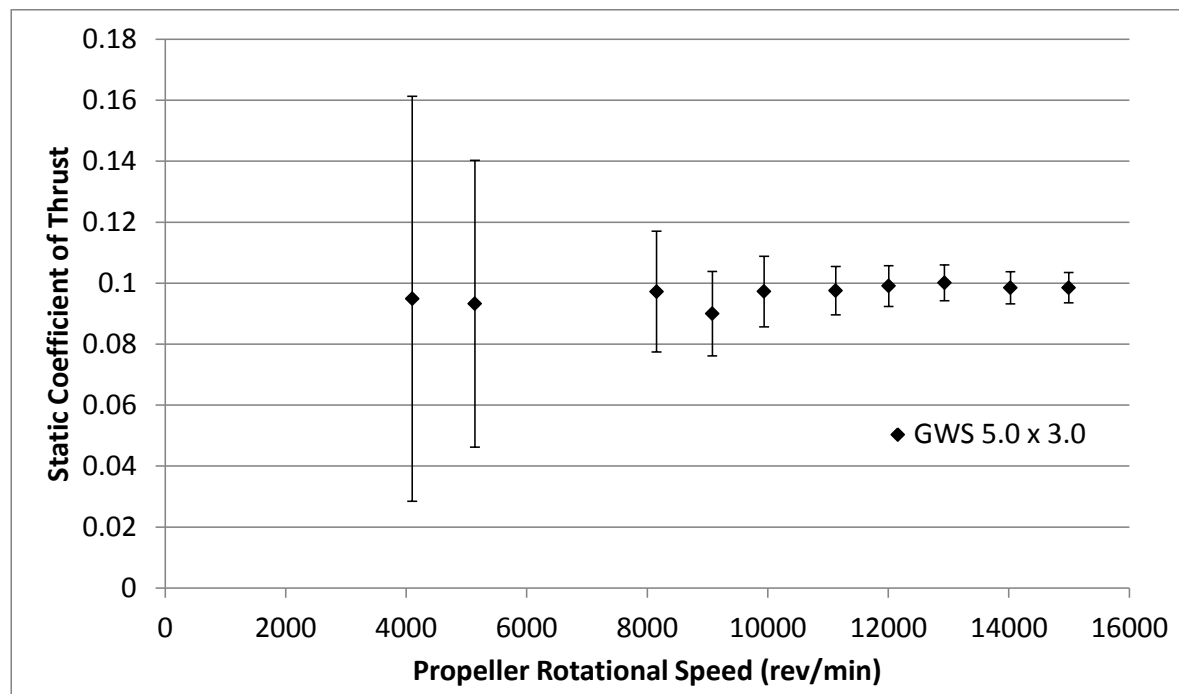

(a)

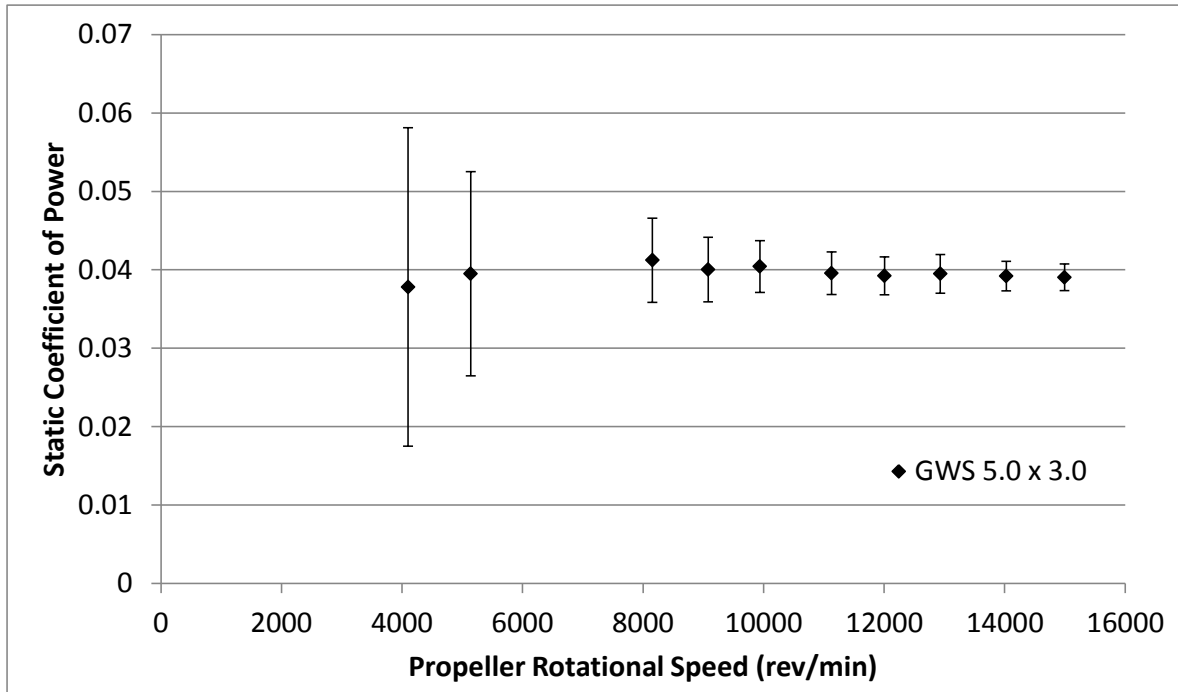

(b)

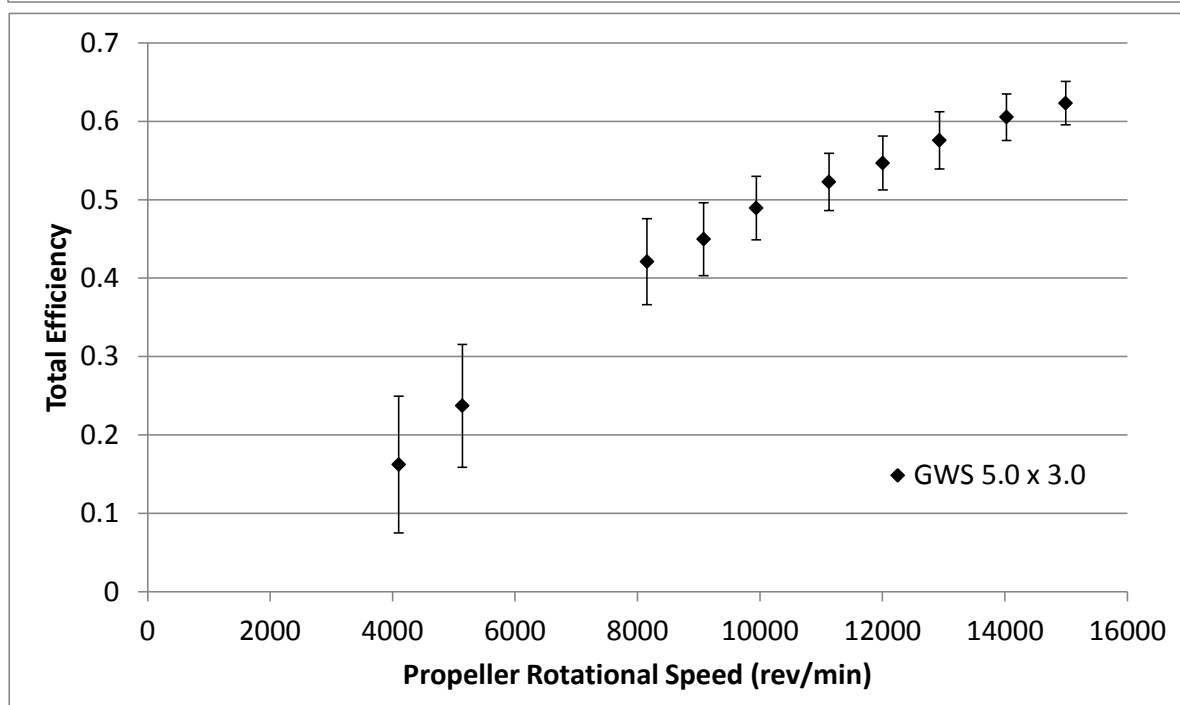

(c)

Figure 73: GWS 5.0 x 3.0 Static Test Results: (a) Coefficient of Thrust, (b) Coefficient of Power, (c) Total Efficiency. 
Table 68: GWS 5.0 x 3.0 Static Measured Values

\begin{tabular}{|c|c|c|c|c|c|c|c|c|}
\hline$n(\mathrm{RPM})$ & $T(\mathrm{~g})$ & $\Delta T(\mathrm{~g})$ & $Q(\mathrm{~g}-\mathrm{m})$ & $\Delta Q(\mathrm{~g}-\mathrm{m})$ & $V(\mathrm{~V})$ & $I(\mathrm{~A})$ & $T_{\text {atm }}\left({ }^{\circ} \mathrm{C}\right)$ & $P_{\text {atm }}(\mathrm{Pa})$ \\
\hline $4.100 \mathrm{E}+03$ & $1.376 \mathrm{E}+01$ & $9.63 \mathrm{E}+00$ & $1.110 \mathrm{E}-01$ & $5.96 \mathrm{E}-02$ & $1.111 \mathrm{E}+01$ & $2.594 \mathrm{E}-01$ & $2.072 \mathrm{E}+01$ & $9.807 \mathrm{E}+04$ \\
\hline $5.140 \mathrm{E}+03$ & $2.127 \mathrm{E}+01$ & $1.07 \mathrm{E}+01$ & $1.823 \mathrm{E}-01$ & $6.01 \mathrm{E}-02$ & $1.110 \mathrm{E}+01$ & $3.655 \mathrm{E}-01$ & $2.070 \mathrm{E}+01$ & $9.808 \mathrm{E}+04$ \\
\hline $8.154 \mathrm{E}+03$ & $5.580 \mathrm{E}+01$ & $1.14 \mathrm{E}+01$ & $4.790 \mathrm{E}-01$ & $6.22 \mathrm{E}-02$ & $1.109 \mathrm{E}+01$ & $8.589 \mathrm{E}-01$ & $2.072 \mathrm{E}+01$ & $9.809 \mathrm{E}+04$ \\
\hline $9.080 \mathrm{E}+03$ & $6.405 \mathrm{E}+01$ & $9.83 \mathrm{E}+00$ & $5.768 \mathrm{E}-01$ & $5.93 \mathrm{E}-02$ & $1.109 \mathrm{E}+01$ & $1.079 \mathrm{E}+00$ & $2.070 \mathrm{E}+01$ & $9.810 \mathrm{E}+04$ \\
\hline $9.939 \mathrm{E}+03$ & $8.297 \mathrm{E}+01$ & $9.89 \mathrm{E}+00$ & $6.983 \mathrm{E}-01$ & $5.72 \mathrm{E}-02$ & $1.108 \mathrm{E}+01$ & $1.315 \mathrm{E}+00$ & $2.064 \mathrm{E}+01$ & $9.812 \mathrm{E}+04$ \\
\hline $1.113 \mathrm{E}+04$ & $1.045 \mathrm{E}+02$ & $8.49 \mathrm{E}+00$ & $8.585 \mathrm{E}-01$ & $5.91 \mathrm{E}-02$ & $1.107 \mathrm{E}+01$ & $1.696 \mathrm{E}+00$ & $2.038 \mathrm{E}+01$ & $9.822 \mathrm{E}+04$ \\
\hline $1.201 \mathrm{E}+04$ & $1.236 \mathrm{E}+02$ & $8.37 \mathrm{E}+00$ & $9.911 \mathrm{E}-01$ & $6.12 \mathrm{E}-02$ & $1.106 \mathrm{E}+01$ & $2.020 \mathrm{E}+00$ & $2.045 \mathrm{E}+01$ & $9.826 \mathrm{E}+04$ \\
\hline $1.293 \mathrm{E}+04$ & $1.450 \mathrm{E}+02$ & $8.48 \mathrm{E}+00$ & $1.158 \mathrm{E}+00$ & $7.21 \mathrm{E}-02$ & $1.105 \mathrm{E}+01$ & $2.417 \mathrm{E}+00$ & $2.034 \mathrm{E}+01$ & $9.829 \mathrm{E}+04$ \\
\hline $1.403 \mathrm{E}+04$ & $1.678 \mathrm{E}+02$ & $8.99 \mathrm{E}+00$ & $1.353 \mathrm{E}+00$ & $6.47 \mathrm{E}-02$ & $1.104 \mathrm{E}+01$ & $2.916 \mathrm{E}+00$ & $2.030 \mathrm{E}+01$ & $9.831 \mathrm{E}+04$ \\
\hline $1.499 \mathrm{E}+04$ & $1.919 \mathrm{E}+02$ & $9.71 \mathrm{E}+00$ & $1.540 \mathrm{E}+00$ & $6.65 \mathrm{E}-02$ & $1.103 \mathrm{E}+01$ & $3.451 \mathrm{E}+00$ & $2.027 \mathrm{E}+01$ & $9.832 \mathrm{E}+04$ \\
\hline
\end{tabular}

Table 69: GWS 5.0 x 3.0 Static Calculated Values

\begin{tabular}{|c|c|c|c|c|c|c|c|}
\hline$n(\mathrm{RPM})$ & $\rho\left(\mathrm{kg} / \mathrm{m}^{\wedge} 3\right)$ & $P_{\mathrm{P}}(\mathrm{W})$ & $\Delta P_{\mathrm{P}}(\mathrm{W})$ & $P_{\mathrm{e}}(\mathrm{W})$ & $\Delta P_{\mathrm{e}}(\mathrm{W})$ & $C_{\mathrm{T}}$ & $\Delta C_{\mathrm{T}}$ \\
\hline $4.100 \mathrm{E}+03$ & $1.163 \mathrm{E}+00$ & $4.674 \mathrm{E}-01$ & $2.51 \mathrm{E}-01$ & $2.881 \mathrm{E}+00$ & $5.09 \mathrm{E}-02$ & $9.489 \mathrm{E}-02$ & $6.64 \mathrm{E}-02$ \\
\hline $5.140 \mathrm{E}+03$ & $1.163 \mathrm{E}+00$ & $9.624 \mathrm{E}-01$ & $3.17 \mathrm{E}-01$ & $4.059 \mathrm{E}+00$ & 6.19E-02 & $9.328 \mathrm{E}-02$ & $4.70 \mathrm{E}-02$ \\
\hline $8.154 \mathrm{E}+03$ & $1.163 \mathrm{E}+00$ & $4.011 \mathrm{E}+00$ & $5.21 \mathrm{E}-01$ & $9.527 \mathrm{E}+00$ & $1.21 \mathrm{E}-01$ & $9.723 \mathrm{E}-02$ & $1.98 \mathrm{E}-02$ \\
\hline $9.080 \mathrm{E}+03$ & $1.163 \mathrm{E}+00$ & $5.379 \mathrm{E}+00$ & $5.53 \mathrm{E}-01$ & $1.196 \mathrm{E}+01$ & $1.46 \mathrm{E}-01$ & $9.000 \mathrm{E}-02$ & $1.38 \mathrm{E}-02$ \\
\hline $9.939 \mathrm{E}+03$ & $1.163 \mathrm{E}+00$ & $7.127 \mathrm{E}+00$ & $5.84 \mathrm{E}-01$ & $1.457 \mathrm{E}+01$ & $1.73 \mathrm{E}-01$ & $9.725 \mathrm{E}-02$ & $1.16 \mathrm{E}-02$ \\
\hline $1.113 \mathrm{E}+04$ & $1.166 \mathrm{E}+00$ & $9.811 \mathrm{E}+00$ & $6.75 \mathrm{E}-01$ & $1.877 \mathrm{E}+01$ & $2.17 \mathrm{E}-01$ & $9.756 \mathrm{E}-02$ & 7.92E-03 \\
\hline $1.201 \mathrm{E}+04$ & $1.166 \mathrm{E}+00$ & $1.222 \mathrm{E}+01$ & $7.55 \mathrm{E}-01$ & $2.235 \mathrm{E}+01$ & $2.55 \mathrm{E}-01$ & $9.905 \mathrm{E}-02$ & $6.71 \mathrm{E}-03$ \\
\hline $1.293 \mathrm{E}+04$ & $1.167 \mathrm{E}+00$ & $1.538 \mathrm{E}+01$ & $9.58 \mathrm{E}-01$ & $2.671 \mathrm{E}+01$ & 3.07E-01 & $1.001 \mathrm{E}-01$ & $5.85 \mathrm{E}-03$ \\
\hline $1.403 \mathrm{E}+04$ & $1.167 \mathrm{E}+00$ & $1.949 \mathrm{E}+01$ & $9.32 \mathrm{E}-01$ & $3.220 \mathrm{E}+01$ & $3.60 \mathrm{E}-01$ & 9.846E-02 & $5.28 \mathrm{E}-03$ \\
\hline $1.499 \mathrm{E}+04$ & $1.167 \mathrm{E}+00$ & $2.372 \mathrm{E}+01$ & $1.02 \mathrm{E}+00$ & $3.805 \mathrm{E}+01$ & 4.25E-01 & $9.852 \mathrm{E}-02$ & 4.99E-03 \\
\hline$C_{\mathrm{P}}$ & $\Delta C_{\mathrm{P}}$ & $C_{\mathrm{Q}}$ & $\Delta C_{\mathrm{Q}}$ & $\eta_{\mathrm{T}}$ & $\Delta \eta_{\mathrm{T}}$ & $R e_{0.75}$ & $\Delta R e_{0.75}$ \\
\hline $3.780 \mathrm{E}-02$ & $2.03 \mathrm{E}-02$ & $6.016 \mathrm{E}-03$ & $3.23 \mathrm{E}-03$ & $1.622 \mathrm{E}-01$ & $8.72 \mathrm{E}-02$ & $1.632 \mathrm{E}+04$ & $2.02 \mathrm{E}+01$ \\
\hline $3.950 \mathrm{E}-02$ & $1.30 \mathrm{E}-02$ & $6.286 \mathrm{E}-03$ & $2.07 \mathrm{E}-03$ & $2.371 \mathrm{E}-01$ & $7.83 \mathrm{E}-02$ & $2.047 \mathrm{E}+04$ & $2.56 \mathrm{E}+01$ \\
\hline $4.122 \mathrm{E}-02$ & $5.36 \mathrm{E}-03$ & $6.560 \mathrm{E}-03$ & $8.53 \mathrm{E}-04$ & $4.210 \mathrm{E}-01$ & $5.50 \mathrm{E}-02$ & $3.247 \mathrm{E}+04$ & $4.08 \mathrm{E}+01$ \\
\hline $4.003 \mathrm{E}-02$ & $4.12 \mathrm{E}-03$ & $6.371 \mathrm{E}-03$ & $6.55 \mathrm{E}-04$ & $4.497 \mathrm{E}-01$ & $4.66 \mathrm{E}-02$ & $3.617 \mathrm{E}+04$ & $3.74 \mathrm{E}+01$ \\
\hline $4.042 \mathrm{E}-02$ & $3.31 \mathrm{E}-03$ & $6.433 \mathrm{E}-03$ & $5.27 \mathrm{E}-04$ & $4.892 \mathrm{E}-01$ & $4.05 \mathrm{E}-02$ & $3.961 \mathrm{E}+04$ & $4.01 \mathrm{E}+01$ \\
\hline 3.957E-02 & $2.72 \mathrm{E}-03$ & $6.298 \mathrm{E}-03$ & 4.34E-04 & $5.226 \mathrm{E}-01$ & $3.65 \mathrm{E}-02$ & $4.446 \mathrm{E}+04$ & $4.43 \mathrm{E}+01$ \\
\hline $3.923 \mathrm{E}-02$ & $2.42 \mathrm{E}-03$ & $6.244 \mathrm{E}-03$ & $3.86 \mathrm{E}-04$ & $5.468 \mathrm{E}-01$ & $3.43 \mathrm{E}-02$ & $4.798 \mathrm{E}+04$ & $5.02 \mathrm{E}+01$ \\
\hline $3.949 \mathrm{E}-02$ & $2.46 \mathrm{E}-03$ & $6.285 \mathrm{E}-03$ & $3.91 \mathrm{E}-04$ & $5.758 \mathrm{E}-01$ & 3.64E-02 & $5.173 \mathrm{E}+04$ & $5.53 \mathrm{E}+01$ \\
\hline $3.920 \mathrm{E}-02$ & $1.88 \mathrm{E}-03$ & $6.016 \mathrm{E}-03$ & $2.99 \mathrm{E}-04$ & $6.053 \mathrm{E}-01$ & $2.97 \mathrm{E}-02$ & $5.613 \mathrm{E}+04$ & $6.00 \mathrm{E}+01$ \\
\hline $3.905 \mathrm{E}-02$ & $1.69 \mathrm{E}-03$ & $6.215 \mathrm{E}-03$ & $2.68 \mathrm{E}-04$ & $6.232 \mathrm{E}-01$ & $2.78 \mathrm{E}-02$ & $6.001 \mathrm{E}+04$ & $5.80 \mathrm{E}+01$ \\
\hline
\end{tabular}




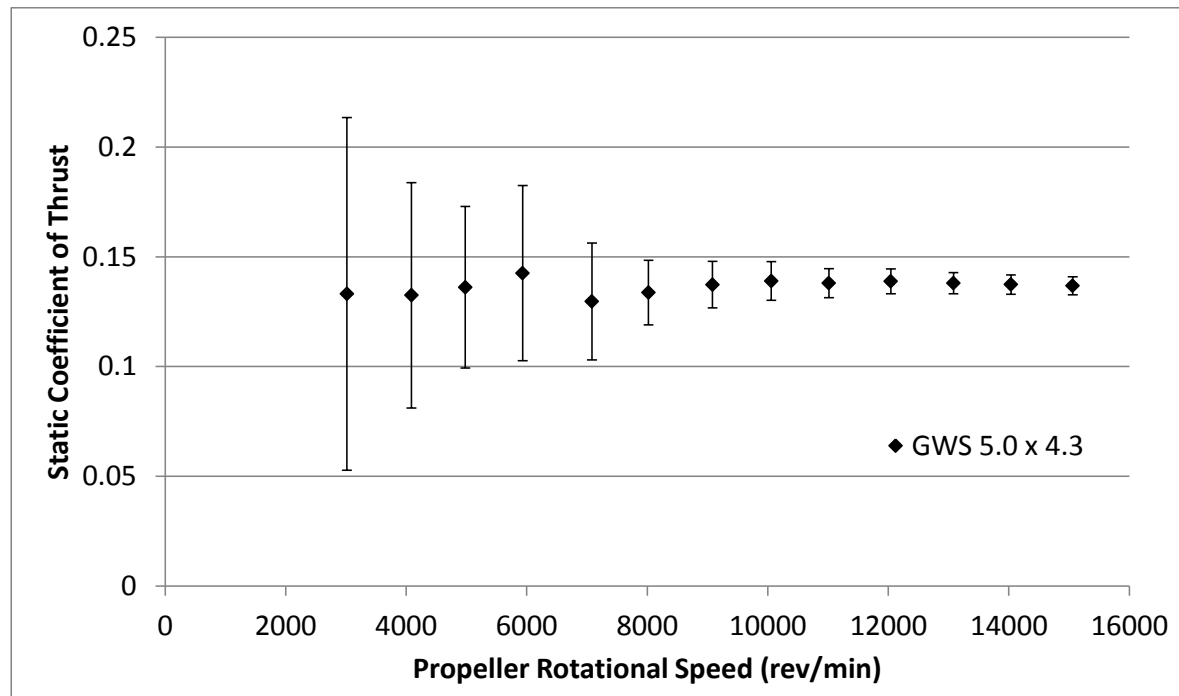

(a)

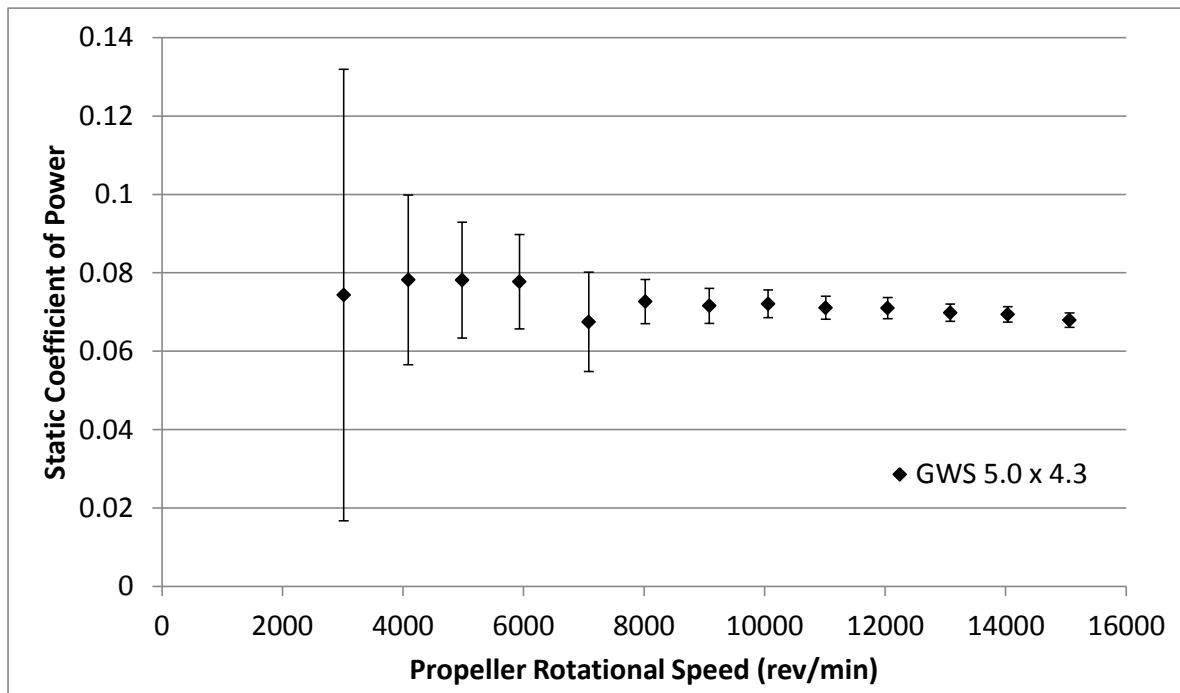

(b)

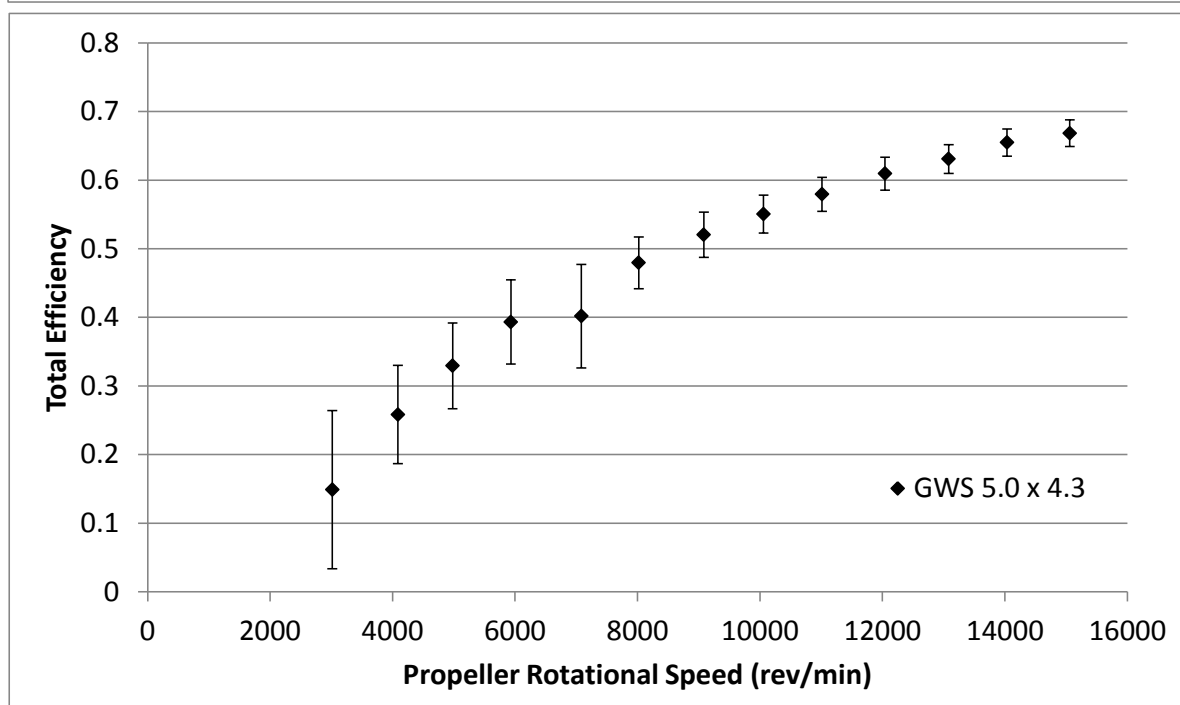

(c)

Figure 74: GWS 5.0 x 4.3 Static Test Results: (a) Coefficient of Thrust, (b) Coefficient of Power, (c) Total Efficiency. 
Table 70: GWS 5.0 x 4.3 Static Measured Values

\begin{tabular}{|c|c|c|c|c|c|c|c|c|}
\hline$n(R P M)$ & $T(g)$ & $\Delta T(g)$ & $Q(g-m)$ & $\Delta Q(\mathrm{~g}-\mathrm{m})$ & $V(\mathrm{~V})$ & $I(\mathrm{~A})$ & $T_{\text {atm }}\left({ }^{\circ} \mathrm{C}\right)$ & $P_{\text {atm }}(\mathrm{Pa})$ \\
\hline $3.016 \mathrm{E}+03$ & $1.034 \mathrm{E}+01$ & $6.25 \mathrm{E}+00$ & $1.167 \mathrm{E}-01$ & $9.04 \mathrm{E}-02$ & $1.111 \mathrm{E}+01$ & $2.188 \mathrm{E}-01$ & $2.239 \mathrm{E}+01$ & $9.864 \mathrm{E}+04$ \\
\hline $4.087 \mathrm{E}+03$ & $1.891 \mathrm{E}+01$ & $7.33 \mathrm{E}+00$ & $2.255 \mathrm{E}-01$ & $6.24 \mathrm{E}-02$ & $1.110 \mathrm{E}+01$ & $3.298 \mathrm{E}-01$ & $2.239 \mathrm{E}+01$ & $9.864 \mathrm{E}+04$ \\
\hline $4.981 \mathrm{E}+03$ & $2.885 \mathrm{E}+01$ & $7.79 \mathrm{E}+00$ & $3.344 \mathrm{E}-01$ & $6.32 \mathrm{E}-02$ & $1.110 \mathrm{E}+01$ & $4.678 \mathrm{E}-01$ & $2.244 \mathrm{E}+01$ & $9.864 \mathrm{E}+04$ \\
\hline $5.932 \mathrm{E}+03$ & $4.286 \mathrm{E}+01$ & $1.20 \mathrm{E}+01$ & $4.722 \mathrm{E}-01$ & $7.32 \mathrm{E}-02$ & $1.110 \mathrm{E}+01$ & $6.592 \mathrm{E}-01$ & $2.243 \mathrm{E}+01$ & $9.864 \mathrm{E}+04$ \\
\hline $7.083 \mathrm{E}+03$ & $5.556 \mathrm{E}+01$ & $1.14 \mathrm{E}+01$ & $5.843 \mathrm{E}-01$ & $1.09 \mathrm{E}-01$ & $1.109 \mathrm{E}+01$ & $9.539 \mathrm{E}-01$ & $2.246 \mathrm{E}+01$ & $9.863 \mathrm{E}+04$ \\
\hline $8.020 \mathrm{E}+03$ & $7.348 \mathrm{E}+01$ & $8.09 \mathrm{E}+00$ & $8.066 \mathrm{E}-01$ & $6.26 \mathrm{E}-02$ & $1.108 \mathrm{E}+01$ & $1.250 \mathrm{E}+00$ & $2.245 \mathrm{E}+01$ & $9.861 \mathrm{E}+04$ \\
\hline $9.083 \mathrm{E}+03$ & $9.674 \mathrm{E}+01$ & $7.48 \mathrm{E}+00$ & $1.019 \mathrm{E}+00$ & $6.36 \mathrm{E}-02$ & $1.107 \mathrm{E}+01$ & $1.649 \mathrm{E}+00$ & $2.251 \mathrm{E}+01$ & $9.861 \mathrm{E}+04$ \\
\hline $1.006 \mathrm{E}+04$ & $1.202 \mathrm{E}+02$ & $7.65 \mathrm{E}+00$ & $1.259 \mathrm{E}+00$ & $6.18 \mathrm{E}-02$ & $1.106 \mathrm{E}+01$ & $2.137 \mathrm{E}+00$ & $2.227 \mathrm{E}+01$ & $9.862 \mathrm{E}+04$ \\
\hline $1.101 \mathrm{E}+04$ & $1.431 \mathrm{E}+02$ & $6.82 \mathrm{E}+00$ & $1.489 \mathrm{E}+00$ & $6.13 \mathrm{E}-02$ & $1.105 \mathrm{E}+01$ & $2.631 \mathrm{E}+00$ & $2.233 \mathrm{E}+01$ & $9.861 \mathrm{E}+04$ \\
\hline $1.204 \mathrm{E}+04$ & $1.721 \mathrm{E}+02$ & $7.01 \mathrm{E}+00$ & $1.778 \mathrm{E}+00$ & $6.71 \mathrm{E}-02$ & $1.103 \mathrm{E}+01$ & $3.270 \mathrm{E}+00$ & $2.228 \mathrm{E}+01$ & $9.860 \mathrm{E}+04$ \\
\hline $1.308 \mathrm{E}+04$ & $2.018 \mathrm{E}+02$ & $7.06 \mathrm{E}+00$ & $2.062 \mathrm{E}+00$ & $6.50 \mathrm{E}-02$ & $1.101 \mathrm{E}+01$ & $3.989 \mathrm{E}+00$ & $2.226 \mathrm{E}+01$ & $9.860 \mathrm{E}+04$ \\
\hline $1.404 \mathrm{E}+04$ & $2.313 \mathrm{E}+02$ & $7.40 \mathrm{E}+00$ & $2.361 \mathrm{E}+00$ & $6.61 \mathrm{E}-02$ & $1.099 \mathrm{E}+01$ & $4.728 \mathrm{E}+00$ & $2.226 \mathrm{E}+01$ & $9.861 \mathrm{E}+04$ \\
\hline $1.506 \mathrm{E}+04$ & $2.651 \mathrm{E}+02$ & $7.97 \mathrm{E}+00$ & $2.659 \mathrm{E}+00$ & $7.14 \mathrm{E}-02$ & $1.097 \mathrm{E}+01$ & $5.609 \mathrm{E}+00$ & $2.230 \mathrm{E}+01$ & $9.862 \mathrm{E}+04$ \\
\hline
\end{tabular}


Table 71: GWS 5.0 x 4.3 Static Calculated Values

\begin{tabular}{|c|c|c|c|c|c|c|c|}
\hline$n$ & ) & & J) & & $\Delta P_{\mathrm{e}}(\mathrm{W})$ & $\mathrm{T}$ & $\Delta C_{\mathrm{T}}$ \\
\hline & & & & & & & \\
\hline-03 & CTUO & & 1 & & & 1 & 02 \\
\hline & $1.162 \mathrm{E}+00$ & & 1 & & & 1 & -02 \\
\hline+03 & $1.162 \mathrm{E}+00$ & +00 & $4.46 \mathrm{E}-01$ & & & 1.4 & -02 \\
\hline-03 & & & 7.9 & & & 01 & -02 \\
\hline$E+03$ & $1.162 \mathrm{E}+00$ & +00 & 1 & +01 & & 37E-01 & $7 \mathrm{E}-02$ \\
\hline & $\mathrm{E}+00$ & +00 & 5 & & & 1 & 2 \\
\hline & & & 6.3 & & & 01 & -03 \\
\hline+04 & $53 E+00$ & & 1 & & & & E-03 \\
\hline+04 & $\mathrm{E}+00$ & & 1 & & & & \\
\hline & $1.163 \mathrm{E}+00$ & $2.771 \mathrm{E}+01$ & 1 & $\mathrm{E}+01$ & 1 & & 3 \\
\hline $1.404 \mathrm{E}+04$ & $1.163 \mathrm{E}+00$ & & 9.5 & & & 01 & $E-03$ \\
\hline & & & & & & & \\
\hline $\mathrm{CP}$ & $\Delta C_{\mathrm{P}}$ & & & $\eta_{\mathrm{T}}$ & & & $\Delta R e_{0.75}$ \\
\hline & & & & & & & \\
\hline $7.820 \mathrm{E}-02$ & $6 E-02$ & $.245 \mathrm{E}-02$ & $3.44 \mathrm{E}-03$ & 2.58 & 1.16 & $1.636 \mathrm{E}+04$ & $1.96 \mathrm{E}+01$ \\
\hline $7.810 \mathrm{E}-02$ & $1.48 \mathrm{E}-02$ & $1.243 \mathrm{E}-02$ & $2.35 \mathrm{E}-03$ & $3.294 \mathrm{E}-01$ & $6.25 \mathrm{E}-02$ & $1.993 \mathrm{E}+04$ & $2.39 \mathrm{E}+01$ \\
\hline 7.774E-02 & $1.21 \mathrm{E}-02$ & $1.237 \mathrm{E}-02$ & $1.92 \mathrm{E}-03$ & $3.933 \mathrm{E}-01$ & $6.12 \mathrm{E}-02$ & $2.374 \mathrm{E}+04$ & $2.73 \mathrm{E}+01$ \\
\hline 49E-02 & 509 & & & & & $833 \mathrm{E}+04$ & $4.16 \mathrm{E}+01$ \\
\hline 7.267E-02 & $5.65 \mathrm{E}-03$ & $.157 \mathrm{E}-02$ & 8.98E-04 & 4.795E-01 & $3.77 \mathrm{E}-02$ & $3.208 \mathrm{E}+04$ & $4.31 \mathrm{E}+01$ \\
\hline $7.157 \mathrm{E}-02$ & $4.47 \mathrm{E}-03$ & $1.139 \mathrm{E}-02$ & $7.11 \mathrm{E}-04$ & 5.204E-01 & $3.30 \mathrm{E}-02$ & $3.632 \mathrm{E}+04$ & $3.67 \mathrm{E}+01$ \\
\hline 7.207E-02 & $3.54 \mathrm{E}-\mathrm{C}$ & $1.1+12$ & $5.63 \mathrm{E}$ & $5.504 \mathrm{E}$ & $2.77 \mathrm{E}$ & $4.028 \mathrm{E}+04$ & $4.04 \mathrm{E}+01$ \\
\hline $7.108 \mathrm{E}-02$ & $2.93 \mathrm{E}-03$ & $1.183 \mathrm{E}-02$ & $4.66 \mathrm{E}-04$ & 5.794E-01 & $2.48 \mathrm{E}-02$ & $4.409 \mathrm{E}+04$ & $4.43 \mathrm{E}+01$ \\
\hline $7.100 \mathrm{E}-02$ & $2.68 \mathrm{E}-03$ & $1.130 \mathrm{E}-02$ & $4.27 \mathrm{E}-04$ & $6.095 \mathrm{E}-01$ & $2.40 \mathrm{E}-02$ & $4.821 \mathrm{E}+04$ & $5.27 \mathrm{E}+01$ \\
\hline $6.980 \mathrm{E}-02$ & $2.20 \mathrm{E}-03$ & $1.111 \mathrm{E}-02$ & $3.50 \mathrm{E}-04$ & $6.308 \mathrm{E}-01$ & $2.11 \mathrm{E}-02$ & $5.238 \mathrm{E}+04$ & $5.75 \mathrm{E}+01$ \\
\hline $6.941 \mathrm{E}-02$ & $1.95 \mathrm{E}-03$ & $1.105 \mathrm{E}-02$ & $3.10 \mathrm{E}-04$ & $6.548 \mathrm{E}-01$ & $1.98 \mathrm{E}-02$ & $5.621 \mathrm{E}+04$ & $6.10 \mathrm{E}+01$ \\
\hline $6.790 \mathrm{E}-02$ & $1.82 \mathrm{E}-03$ & $1.081 \mathrm{E}-02$ & $2.90 \mathrm{E}-04$ & $6.684 \mathrm{E}-01$ & $1.95 \mathrm{E}-02$ & $6.030 \mathrm{E}+04$ & $5.75 \mathrm{E}+01$ \\
\hline
\end{tabular}




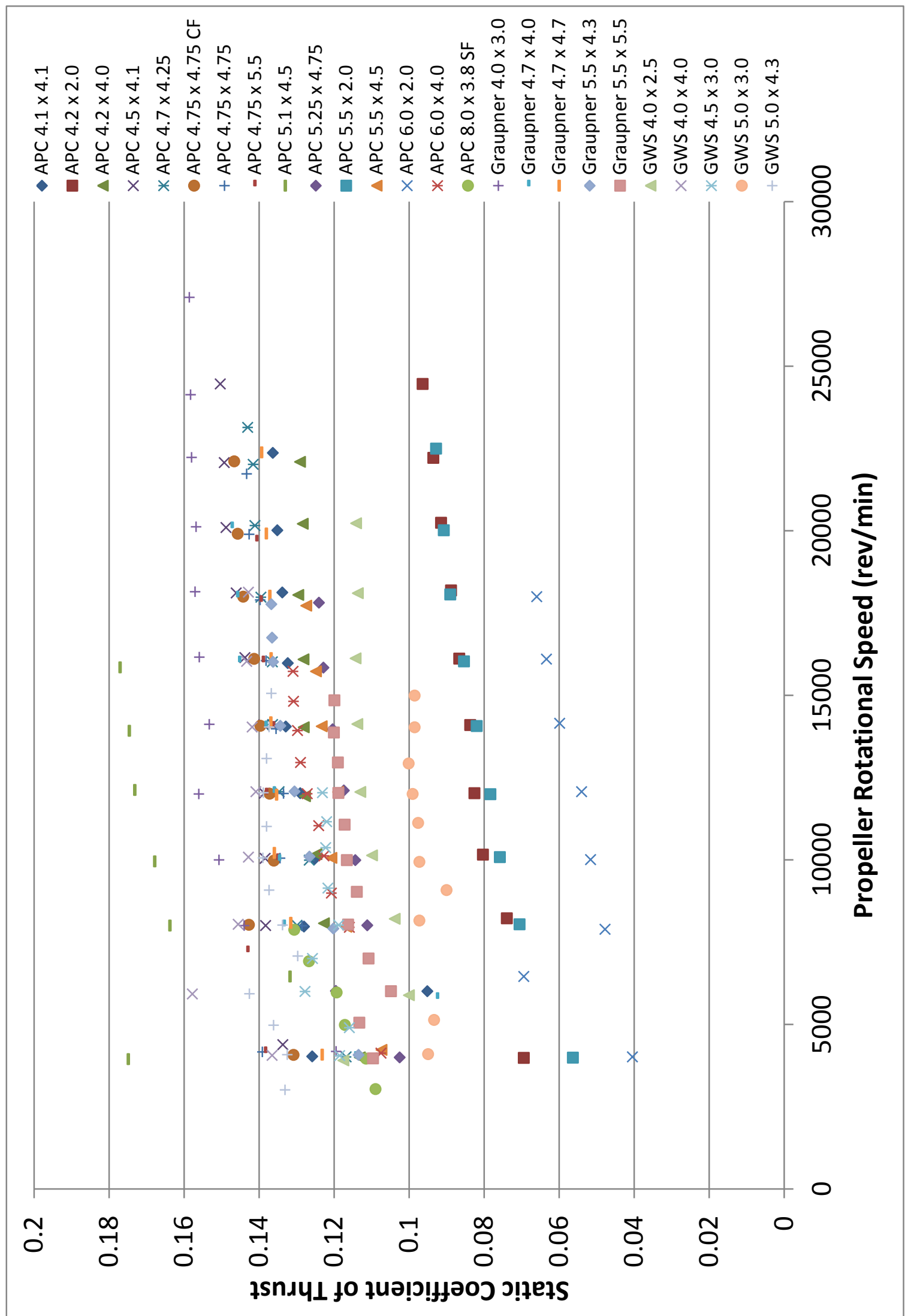

Figure 75: Summary of All Static Coefficients of Thrust 


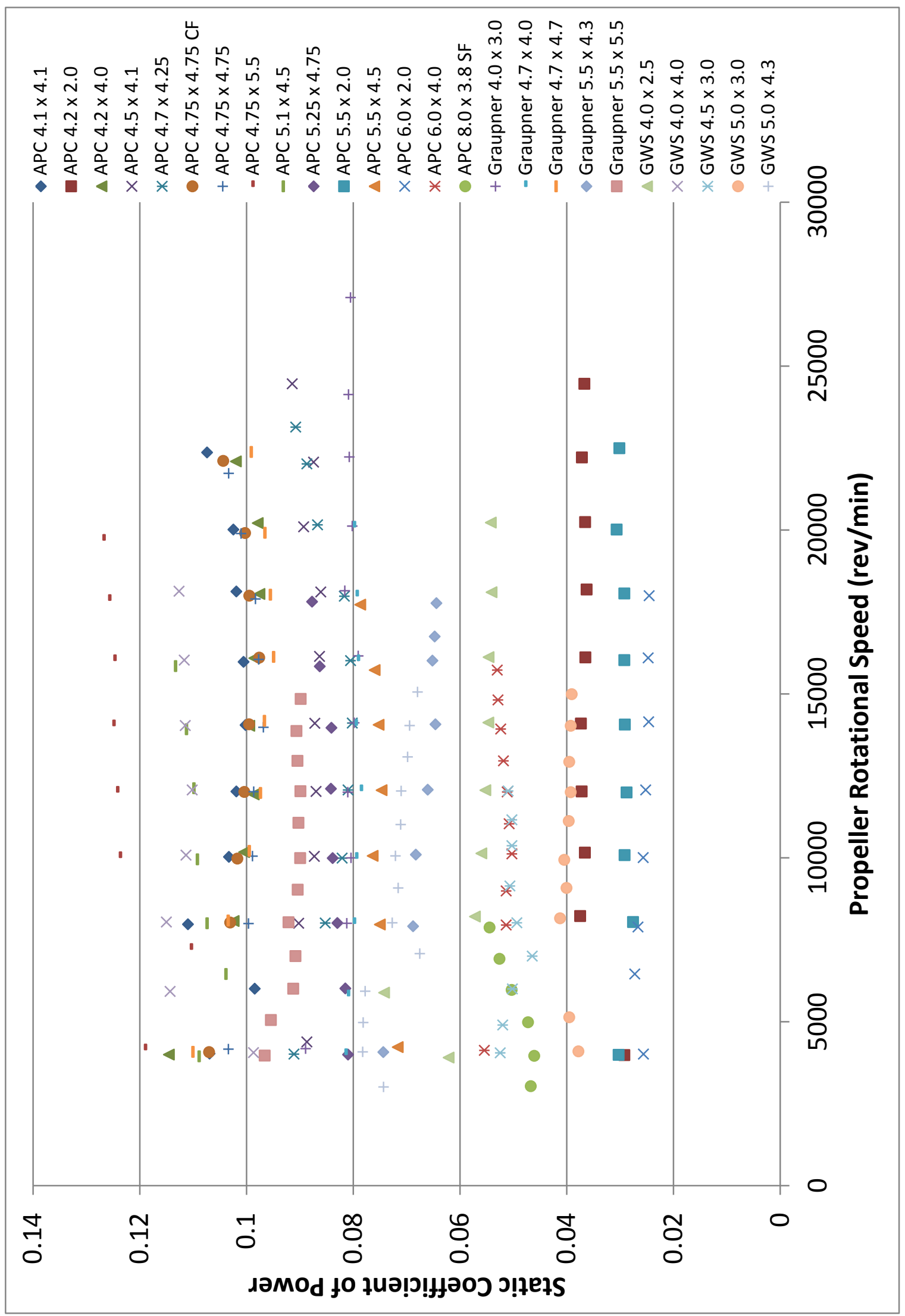

Figure 76: Summary of All Static Coefficients of Power 


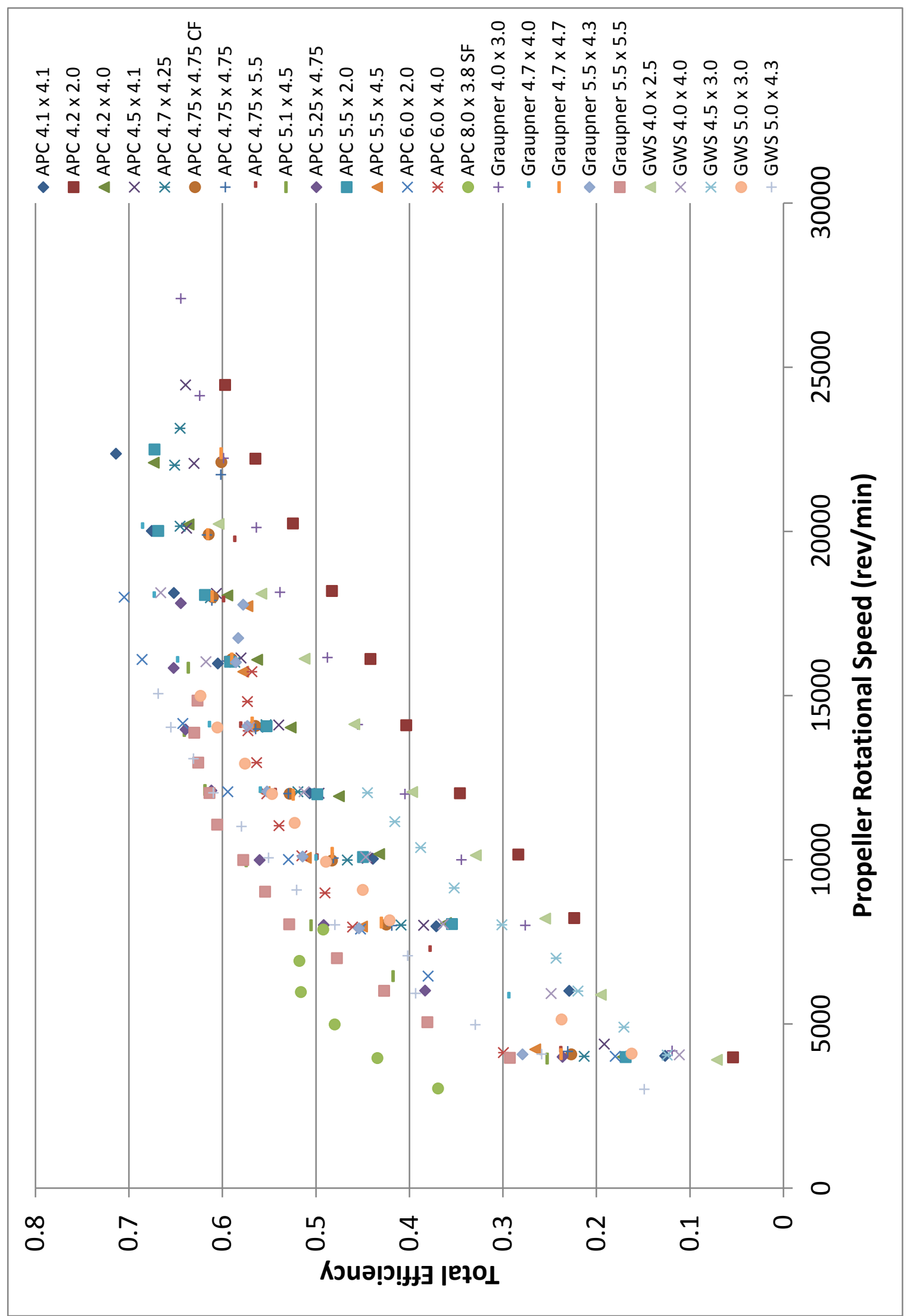

Figure 77: Summary of All Static Total Efficiencies 


\section{APPENDIX G: SUMMARY OF DYNAMIC TEST DATA}

The following pages present the data collected during the dynamic tests. This data includes measured data and calculated values and their associated uncertainties. 


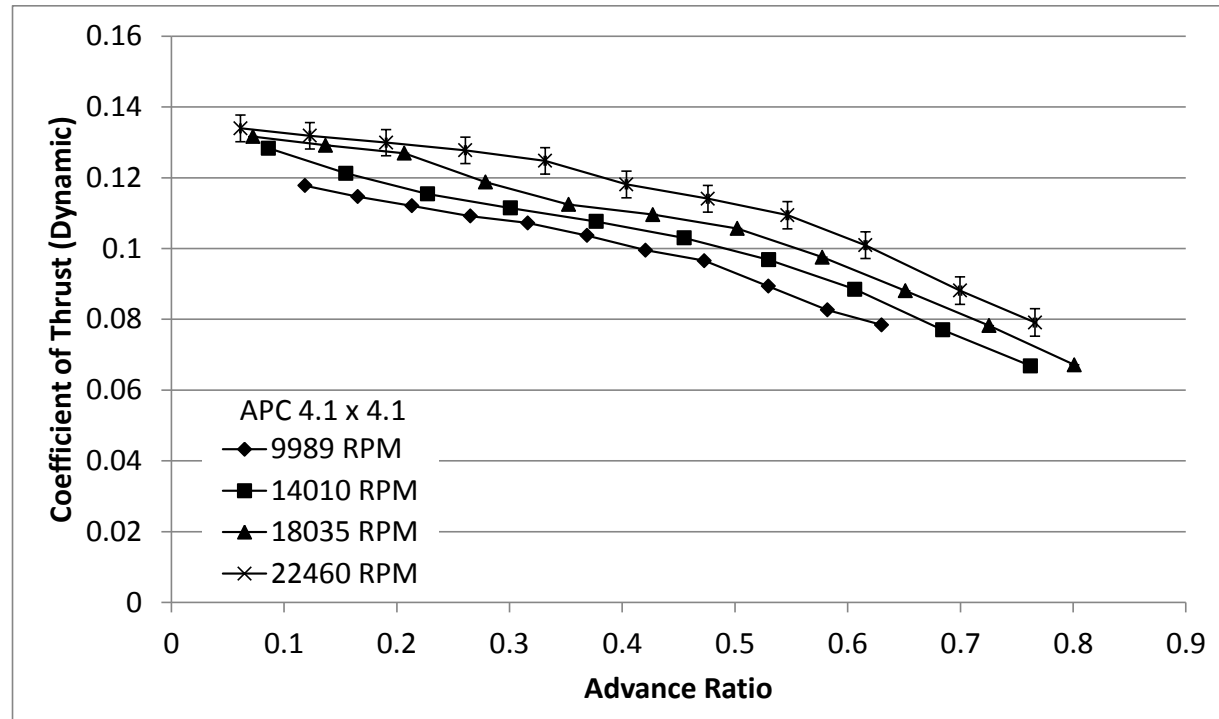

(a)

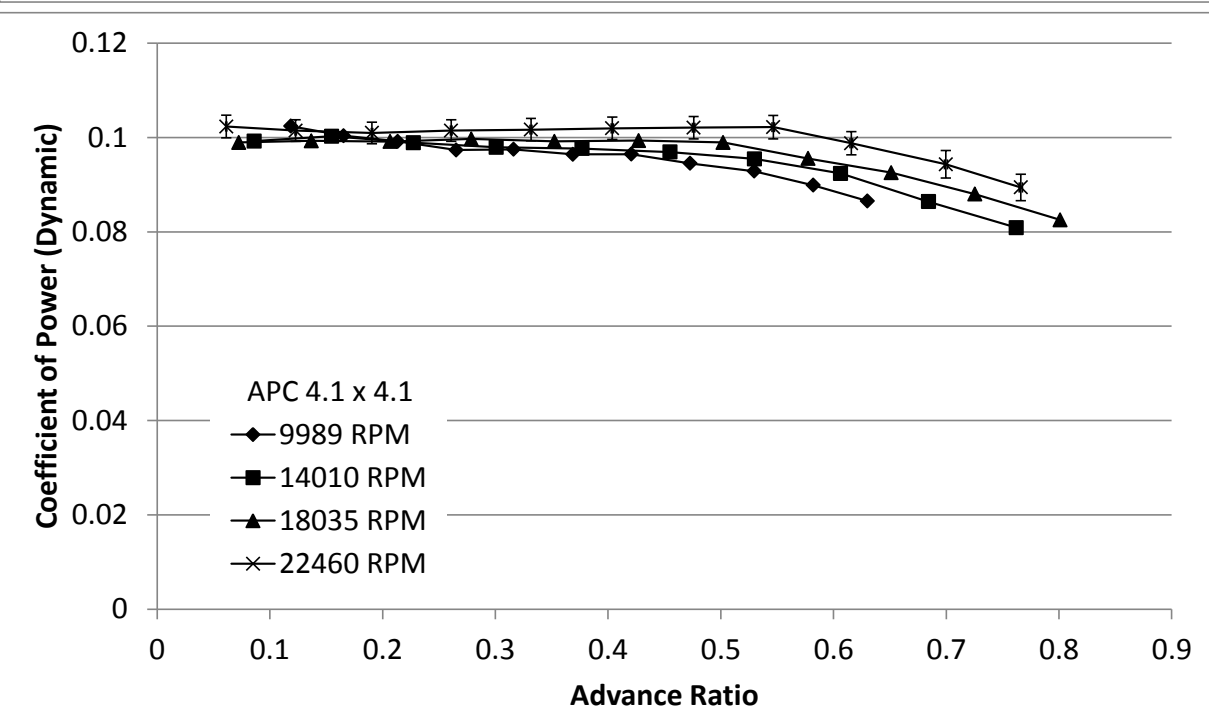

(b)

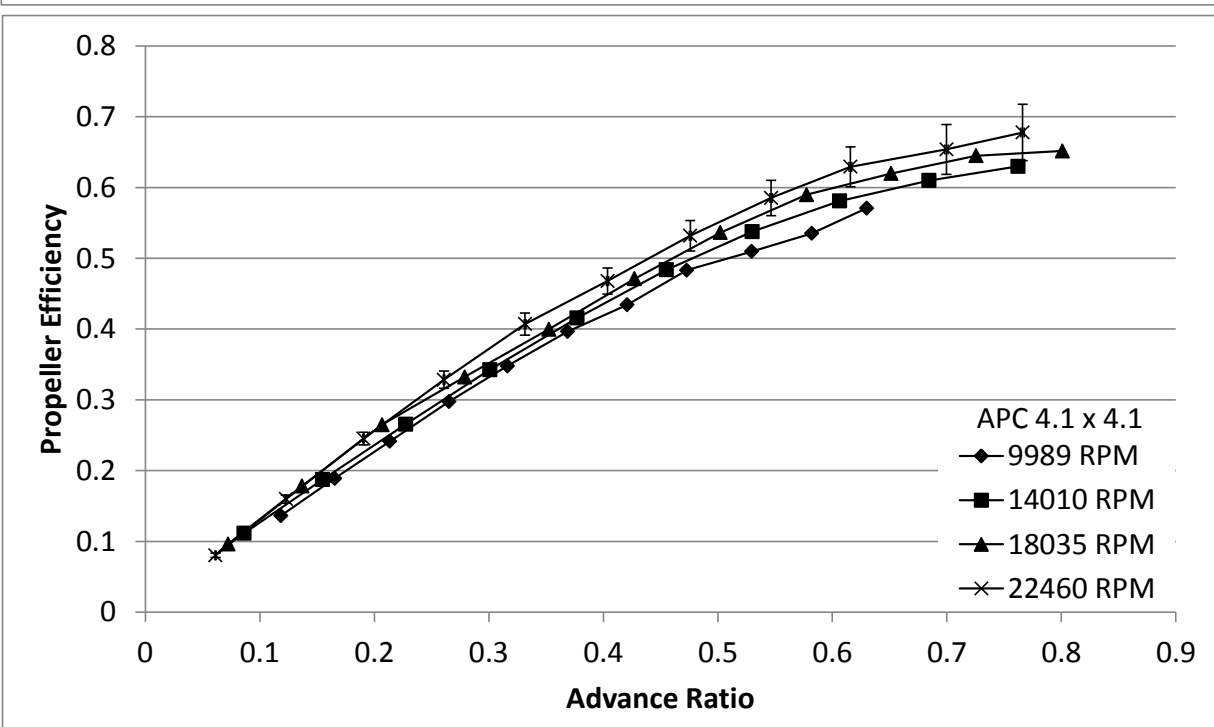

(c) 


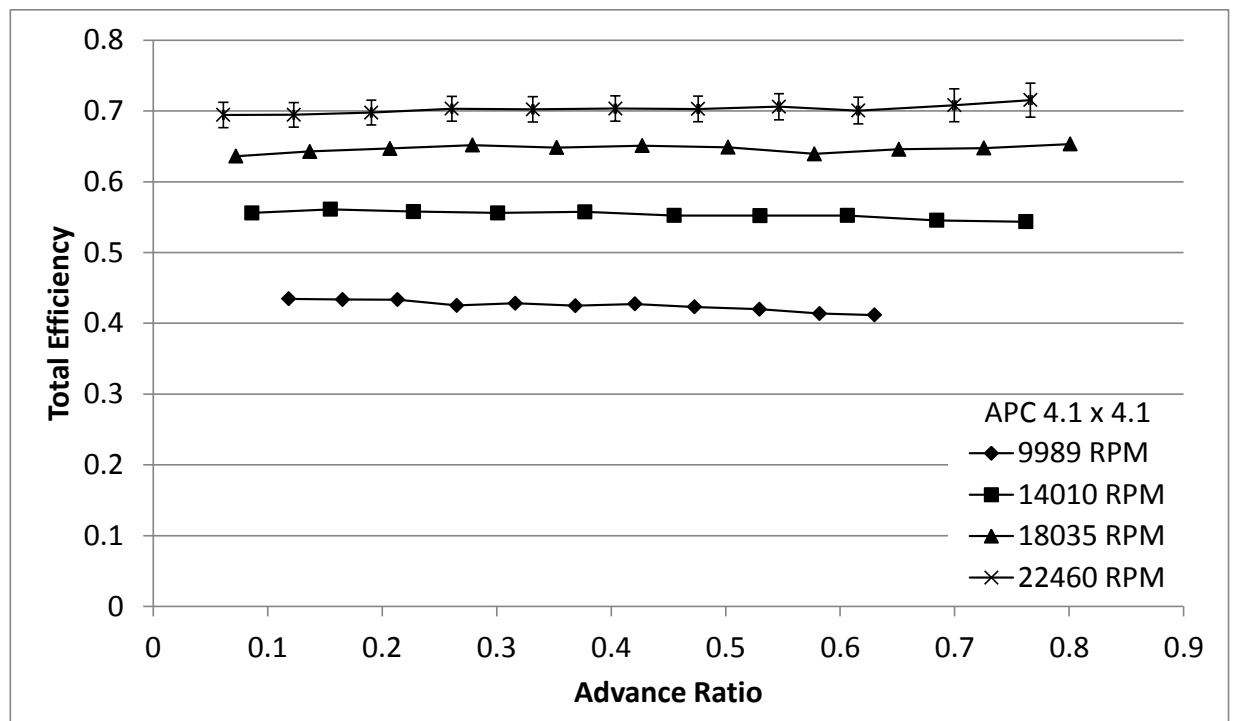

(d)

Figure 78: APC 4.1 x 4.1 Dynamic Test Results: (a) Coefficient of Thrust, (b) Coefficient of Power, (c) Propeller Efficiency, (d) Total Efficiency.

Table 72: APC 4.1 x 4.1 Dynamic Measured Values - 9989 RPM

\begin{tabular}{|c|c|c|c|c|c|c|c|}
\hline$n(\mathrm{RPM})$ & $Q(\mathrm{~g}-\mathrm{m})$ & $\Delta Q(\mathrm{~g}-\mathrm{m})$ & $V(\mathrm{~V})$ & $I(\mathrm{~A})$ & $T_{\text {atm }}\left({ }^{\circ} \mathrm{C}\right)$ & $P_{\text {atm }}(\mathrm{Pa})$ & $P_{\text {diff }}(\mathrm{Pa})$ \\
\hline $9.943 \mathrm{E}+03$ & $6.366 \mathrm{E}-01$ & $5.63 \mathrm{E}-02$ & $1.108 \mathrm{E}+01$ & $1.350 \mathrm{E}+00$ & $2.182 \mathrm{E}+01$ & $9.872 \mathrm{E}+04$ & $2.541 \mathrm{E}+00$ \\
\hline $9.994 \mathrm{E}+03$ & $6.305 \mathrm{E}-01$ & $5.56 \mathrm{E}-02$ & $1.108 \mathrm{E}+01$ & $1.347 \mathrm{E}+00$ & $2.178 \mathrm{E}+01$ & $9.872 \mathrm{E}+04$ & $4.925 \mathrm{E}+00$ \\
\hline $1.003 \mathrm{E}+04$ & $6.270 \mathrm{E}-01$ & $5.63 \mathrm{E}-02$ & $1.108 \mathrm{E}+01$ & $1.344 \mathrm{E}+00$ & $2.182 \mathrm{E}+01$ & $9.872 \mathrm{E}+04$ & $8.186 \mathrm{E}+00$ \\
\hline $9.950 \mathrm{E}+03$ & $6.059 \mathrm{E}-01$ & $5.69 \mathrm{E}-02$ & $1.108 \mathrm{E}+01$ & $1.314 \mathrm{E}+00$ & $2.183 \mathrm{E}+01$ & $9.871 \mathrm{E}+04$ & $1.237 \mathrm{E}+01$ \\
\hline $9.963 \mathrm{E}+03$ & $6.083 \mathrm{E}-01$ & $5.76 \mathrm{E}-02$ & $1.108 \mathrm{E}+01$ & $1.311 \mathrm{E}+00$ & $2.185 \mathrm{E}+01$ & $9.872 \mathrm{E}+04$ & $1.754 \mathrm{E}+01$ \\
\hline $9.972 \mathrm{E}+03$ & $6.029 \mathrm{E}-01$ & $5.73 \mathrm{E}-02$ & $1.108 \mathrm{E}+01$ & $1.312 \mathrm{E}+00$ & $2.188 \mathrm{E}+01$ & $9.871 \mathrm{E}+04$ & $2.382 \mathrm{E}+01$ \\
\hline $9.994 \mathrm{E}+03$ & $6.054 \mathrm{E}-01$ & $5.58 \mathrm{E}-02$ & $1.108 \mathrm{E}+01$ & $1.312 \mathrm{E}+00$ & $2.192 \mathrm{E}+01$ & $9.871 \mathrm{E}+04$ & $3.107 \mathrm{E}+01$ \\
\hline $1.002 \mathrm{E}+04$ & $5.960 \mathrm{E}-01$ & $5.58 \mathrm{E}-02$ & $1.108 \mathrm{E}+01$ & $1.308 \mathrm{E}+00$ & $2.205 \mathrm{E}+01$ & $9.870 \mathrm{E}+04$ & $3.936 \mathrm{E}+01$ \\
\hline $9.984 \mathrm{E}+03$ & $5.813 \mathrm{E}-01$ & $5.59 \mathrm{E}-02$ & $1.108 \mathrm{E}+01$ & $1.281 \mathrm{E}+00$ & $2.215 \mathrm{E}+01$ & $9.871 \mathrm{E}+04$ & $4.891 \mathrm{E}+01$ \\
\hline $9.970 \mathrm{E}+03$ & $5.609 \mathrm{E}-01$ & $5.70 \mathrm{E}-02$ & $1.108 \mathrm{E}+01$ & $1.252 \mathrm{E}+00$ & $2.225 \mathrm{E}+01$ & $9.871 \mathrm{E}+04$ & $5.880 \mathrm{E}+01$ \\
\hline $1.006 \mathrm{E}+04$ & $5.494 \mathrm{E}-01$ & $5.95 \mathrm{E}-02$ & $1.108 \mathrm{E}+01$ & $1.244 \mathrm{E}+00$ & $2.228 \mathrm{E}+01$ & $9.870 \mathrm{E}+04$ & $7.006 \mathrm{E}+01$ \\
\hline
\end{tabular}


Table 73: APC 4.1 x 4.1 Dynamic Calculated Values - 9989 RPM

\begin{tabular}{|c|c|c|c|c|c|c|c|}
\hline$n(\mathrm{RPM})$ & $V_{\infty}^{\prime}(\mathrm{m} / \mathrm{s})$ & $\Delta V_{\infty}^{\prime}(\mathrm{m} / \mathrm{s})$ & $T^{\prime}(\mathrm{g})$ & $\Delta T^{\prime}(\mathrm{g})$ & $V_{\mathrm{t}}(\mathrm{m} / \mathrm{s})$ & $P_{\mathrm{P}}(\mathrm{W})$ & $\Delta P_{\mathrm{P}}(\mathrm{W})$ \\
\hline $9.943 \mathrm{E}+03$ & $2.036 \mathrm{E}+00$ & $1.23 \mathrm{E}-02$ & $4.438 \mathrm{E}+01$ & $7.20 \mathrm{E}+00$ & $4.053 \mathrm{E}+01$ & $6.501 \mathrm{E}+00$ & $5.75 \mathrm{E}-01$ \\
\hline $9.994 \mathrm{E}+03$ & $2.856 \mathrm{E}+00$ & $1.23 \mathrm{E}-02$ & $4.365 \mathrm{E}+01$ & $7.18 \mathrm{E}+00$ & $4.078 \mathrm{E}+01$ & $6.470 \mathrm{E}+00$ & $5.71 \mathrm{E}-01$ \\
\hline $1.003 \mathrm{E}+04$ & $3.699 \mathrm{E}+00$ & $1.26 \mathrm{E}-02$ & $4.295 \mathrm{E}+01$ & $7.17 \mathrm{E}+00$ & $4.098 \mathrm{E}+01$ & $6.456 \mathrm{E}+00$ & $5.79 \mathrm{E}-01$ \\
\hline $9.950 \mathrm{E}+03$ & $4.560 \mathrm{E}+00$ & $1.35 \mathrm{E}-02$ & $4.120 \mathrm{E}+01$ & $7.19 \mathrm{E}+00$ & $4.076 \mathrm{E}+01$ & $6.191 \mathrm{E}+00$ & $5.82 \mathrm{E}-01$ \\
\hline $9.963 \mathrm{E}+03$ & $5.442 \mathrm{E}+00$ & $1.46 \mathrm{E}-02$ & $4.056 \mathrm{E}+01$ & $7.17 \mathrm{E}+00$ & $4.092 \mathrm{E}+01$ & $6.224 \mathrm{E}+00$ & $5.89 \mathrm{E}-01$ \\
\hline $9.972 \mathrm{E}+03$ & $6.352 \mathrm{E}+00$ & $1.57 \mathrm{E}-02$ & $3.929 \mathrm{E}+01$ & $7.17 \mathrm{E}+00$ & $4.109 \mathrm{E}+01$ & $6.174 \mathrm{E}+00$ & $5.87 \mathrm{E}-01$ \\
\hline $9.994 \mathrm{E}+03$ & $7.265 \mathrm{E}+00$ & $1.66 \mathrm{E}-02$ & $3.787 \mathrm{E}+01$ & $7.18 \mathrm{E}+00$ & $4.132 \mathrm{E}+01$ & $6.214 \mathrm{E}+00$ & $5.72 \mathrm{E}-01$ \\
\hline $1.002 \mathrm{E}+04$ & $8.186 \mathrm{E}+00$ & $1.78 \mathrm{E}-02$ & $3.692 \mathrm{E}+01$ & $7.19 \mathrm{E}+00$ & $4.160 \mathrm{E}+01$ & $6.134 \mathrm{E}+00$ & $5.75 \mathrm{E}-01$ \\
\hline $9.984 \mathrm{E}+03$ & $9.135 \mathrm{E}+00$ & $1.97 \mathrm{E}-02$ & $3.391 \mathrm{E}+01$ & $7.20 \mathrm{E}+00$ & $4.165 \mathrm{E}+01$ & $5.959 \mathrm{E}+00$ & $5.73 \mathrm{E}-01$ \\
\hline $9.970 \mathrm{E}+03$ & $1.002 \mathrm{E}+01$ & $2.10 \mathrm{E}-02$ & $3.127 \mathrm{E}+01$ & $7.21 \mathrm{E}+00$ & $4.180 \mathrm{E}+01$ & $5.743 \mathrm{E}+00$ & $5.83 \mathrm{E}-01$ \\
\hline $1.006 \mathrm{E}+04$ & $1.095 \mathrm{E}+01$ & $2.25 \mathrm{E}-02$ & $3.017 \mathrm{E}+01$ & $7.18 \mathrm{E}+00$ & $4.238 \mathrm{E}+01$ & $5.674 \mathrm{E}+00$ & $6.15 \mathrm{E}-01$ \\
\hline$P_{\mathrm{e}}(\mathrm{W})$ & $\Delta P_{\mathrm{e}}(\mathrm{W})$ & $C_{\mathrm{T}}$ & $\Delta C_{\mathrm{T}}$ & $C_{\mathrm{P}}$ & $\Delta C_{\mathrm{P}}$ & $C_{\mathrm{Q}}$ & $\Delta C_{\mathrm{Q}}$ \\
\hline $1.496 \mathrm{E}+01$ & $1.79 \mathrm{E}-01$ & $1.178 \mathrm{E}-01$ & $1.91 \mathrm{E}-02$ & $1.024 \mathrm{E}-01$ & $9.06 \mathrm{E}-03$ & $1.630 \mathrm{E}-02$ & $1.44 \mathrm{E}-03$ \\
\hline $1.492 \mathrm{E}+01$ & $1.78 \mathrm{E}-01$ & $1.147 \mathrm{E}-01$ & $1.89 \mathrm{E}-02$ & $1.004 \mathrm{E}-01$ & $8.86 \mathrm{E}-03$ & $1.598 \mathrm{E}-02$ & $1.41 \mathrm{E}-03$ \\
\hline $1.490 \mathrm{E}+01$ & $1.77 \mathrm{E}-01$ & $1.121 \mathrm{E}-01$ & $1.87 \mathrm{E}-02$ & $9.916 \mathrm{E}-02$ & $8.90 \mathrm{E}-03$ & $1.578 \mathrm{E}-02$ & $1.42 \mathrm{E}-03$ \\
\hline $1.456 \mathrm{E}+01$ & $1.74 \mathrm{E}-01$ & $1.092 \mathrm{E}-01$ & $1.90 \mathrm{E}-02$ & $9.735 \mathrm{E}-02$ & $9.15 \mathrm{E}-03$ & $1.549 \mathrm{E}-02$ & $1.46 \mathrm{E}-03$ \\
\hline $1.453 \mathrm{E}+01$ & $1.74 \mathrm{E}-01$ & $1.072 \mathrm{E}-01$ & $1.90 \mathrm{E}-02$ & $9.748 \mathrm{E}-02$ & $9.23 \mathrm{E}-03$ & $1.551 \mathrm{E}-02$ & $1.47 \mathrm{E}-03$ \\
\hline $1.453 \mathrm{E}+01$ & $1.74 \mathrm{E}-01$ & $1.037 \mathrm{E}-01$ & $1.89 \mathrm{E}-02$ & $9.644 \mathrm{E}-02$ & $9.17 \mathrm{E}-03$ & $1.535 \mathrm{E}-02$ & $1.46 \mathrm{E}-03$ \\
\hline $1.454 \mathrm{E}+01$ & $1.76 \mathrm{E}-01$ & $9.952 \mathrm{E}-02$ & $1.89 \mathrm{E}-02$ & $9.645 \mathrm{E}-02$ & $8.88 \mathrm{E}-03$ & $1.535 \mathrm{E}-02$ & $1.41 \mathrm{E}-03$ \\
\hline $1.449 \mathrm{E}+01$ & $1.74 \mathrm{E}-01$ & $9.655 \mathrm{E}-02$ & $1.88 \mathrm{E}-02$ & $9.450 \mathrm{E}-02$ & $8.85 \mathrm{E}-03$ & $1.504 \mathrm{E}-02$ & $1.41 \mathrm{E}-03$ \\
\hline $1.419 \mathrm{E}+01$ & $1.70 \mathrm{E}-01$ & 8.936E-02 & $1.90 \mathrm{E}-02$ & $9.286 \mathrm{E}-02$ & $8.93 \mathrm{E}-03$ & $1.478 \mathrm{E}-02$ & $1.42 \mathrm{E}-03$ \\
\hline $1.388 \mathrm{E}+01$ & $1.67 \mathrm{E}-01$ & $8.266 \mathrm{E}-02$ & $1.91 \mathrm{E}-02$ & 8.989E-02 & $9.13 \mathrm{E}-03$ & $1.431 \mathrm{E}-02$ & $1.45 \mathrm{E}-03$ \\
\hline $1.379 \mathrm{E}+01$ & $1.67 \mathrm{E}-01$ & $7.840 \mathrm{E}-02$ & $1.87 \mathrm{E}-02$ & 8.653E-02 & $9.37 \mathrm{E}-03$ & $1.377 \mathrm{E}-02$ & $1.49 \mathrm{E}-03$ \\
\hline$\rho\left(\mathrm{kg} / \mathrm{m}^{\wedge} 3\right)$ & $\eta_{\mathrm{T}}$ & $\Delta \eta_{\mathrm{T}}$ & $J$ & $\Delta J$ & $\eta_{\mathrm{P}}$ & $\Delta \eta_{\mathrm{P}}$ & $R e_{0.75}$ \\
\hline $1.166 \mathrm{E}+00$ & $4.346 \mathrm{E}-01$ & $3.88 \mathrm{E}-02$ & $1.185 \mathrm{E}-01$ & $7.16 \mathrm{E}-04$ & $1.363 \mathrm{E}-01$ & $2.52 \mathrm{E}-02$ & $2.104 \mathrm{E}+04$ \\
\hline $1.166 \mathrm{E}+00$ & 4.337E-01 & $3.86 \mathrm{E}-02$ & $1.654 \mathrm{E}-01$ & $7.14 \mathrm{E}-04$ & $1.890 \mathrm{E}-01$ & $3.53 \mathrm{E}-02$ & $2.118 \mathrm{E}+04$ \\
\hline $1.166 \mathrm{E}+00$ & 4.334E-01 & $3.92 \mathrm{E}-02$ & $2.135 \mathrm{E}-01$ & $7.28 \mathrm{E}-04$ & $2.413 \mathrm{E}-01$ & $4.58 \mathrm{E}-02$ & $2.128 \mathrm{E}+04$ \\
\hline $1.166 \mathrm{E}+00$ & $4.251 \mathrm{E}-01$ & 4.03E-02 & $2.653 \mathrm{E}-01$ & $7.88 \mathrm{E}-04$ & $2.975 \mathrm{E}-01$ & $5.90 \mathrm{E}-02$ & $2.116 \mathrm{E}+04$ \\
\hline $1.166 \mathrm{E}+00$ & $4.283 \mathrm{E}-01$ & 4.09E-02 & $3.162 \mathrm{E}-01$ & $8.56 \mathrm{E}-04$ & $3.478 \mathrm{E}-01$ & $6.98 \mathrm{E}-02$ & $2.124 \mathrm{E}+04$ \\
\hline $1.166 \mathrm{E}+00$ & $4.248 \mathrm{E}-01$ & 4.07E-02 & $3.687 \mathrm{E}-01$ & $9.22 \mathrm{E}-04$ & 3.964E-01 & $8.16 \mathrm{E}-02$ & $2.133 \mathrm{E}+04$ \\
\hline $1.165 \mathrm{E}+00$ & 4.273E-01 & $3.97 \mathrm{E}-02$ & $4.208 \mathrm{E}-01$ & $9.73 \mathrm{E}-04$ & 4.342E-01 & $9.16 \mathrm{E}-02$ & $2.144 \mathrm{E}+04$ \\
\hline $1.165 \mathrm{E}+00$ & $4.232 \mathrm{E}-01$ & $4.00 \mathrm{E}-02$ & 4.729E-01 & $1.04 \mathrm{E}-03$ & 4.831E-01 & $1.04 \mathrm{E}-01$ & $2.157 \mathrm{E}+04$ \\
\hline $1.164 \mathrm{E}+00$ & 4.199E-01 & 4.07E-02 & $5.296 \mathrm{E}-01$ & $1.16 \mathrm{E}-03$ & $5.097 \mathrm{E}-01$ & 1.19E-01 & $2.158 \mathrm{E}+04$ \\
\hline $1.164 \mathrm{E}+00$ & $4.138 \mathrm{E}-01$ & $4.23 \mathrm{E}-02$ & $5.820 \mathrm{E}-01$ & $1.24 \mathrm{E}-03$ & $5.352 \mathrm{E}-01$ & $1.35 \mathrm{E}-01$ & $2.165 \mathrm{E}+04$ \\
\hline $1.164 \mathrm{E}+00$ & $4.116 \mathrm{E}-01$ & 4.49E-02 & $6.301 \mathrm{E}-01$ & $1.31 \mathrm{E}-03$ & 5.709E-01 & $1.49 \mathrm{E}-01$ & $2.194 \mathrm{E}+04$ \\
\hline
\end{tabular}


Table 74: APC 4.1 x 4.1 Dynamic Measured Values - 14010 RPM

\begin{tabular}{|c|c|c|c|c|c|c|c|}
\hline$n(\mathrm{RPM})$ & $Q(\mathrm{~g}-\mathrm{m})$ & $\Delta Q(\mathrm{~g}-\mathrm{m})$ & $V(\mathrm{~V})$ & $I(\mathrm{~A})$ & $T_{\text {atm }}\left({ }^{\circ} \mathrm{C}\right)$ & $P_{\text {atm }}(\mathrm{Pa})$ & $P_{\text {diff }}(\mathrm{Pa})$ \\
\hline $1.407 \mathrm{E}+04$ & $1.234 \mathrm{E}+00$ & $5.90 \mathrm{E}-02$ & $1.104 \mathrm{E}+01$ & $2.906 \mathrm{E}+00$ & $2.198 \mathrm{E}+01$ & $9.871 \mathrm{E}+04$ & $2.749 \mathrm{E}+00$ \\
\hline $1.401 \mathrm{E}+04$ & $1.236 \mathrm{E}+00$ & $5.67 \mathrm{E}-02$ & $1.104 \mathrm{E}+01$ & $2.871 \mathrm{E}+00$ & $2.191 \mathrm{E}+01$ & $9.870 \mathrm{E}+04$ & $8.516 \mathrm{E}+00$ \\
\hline $1.397 \mathrm{E}+04$ & $1.213 \mathrm{E}+00$ & $5.70 \mathrm{E}-02$ & $1.104 \mathrm{E}+01$ & $2.827 \mathrm{E}+00$ & $2.200 \mathrm{E}+01$ & $9.869 \mathrm{E}+04$ & $1.800 \mathrm{E}+01$ \\
\hline $1.401 \mathrm{E}+04$ & $1.206 \mathrm{E}+00$ & $5.64 \mathrm{E}-02$ & $1.104 \mathrm{E}+01$ & $2.827 \mathrm{E}+00$ & $2.210 \mathrm{E}+01$ & $9.868 \mathrm{E}+04$ & $3.139 \mathrm{E}+01$ \\
\hline $1.403 \mathrm{E}+04$ & $1.206 \mathrm{E}+00$ & $5.60 \mathrm{E}-02$ & $1.104 \mathrm{E}+01$ & $2.824 \mathrm{E}+00$ & $2.224 \mathrm{E}+01$ & $9.867 \mathrm{E}+04$ & $4.918 \mathrm{E}+01$ \\
\hline $1.395 \mathrm{E}+04$ & $1.184 \mathrm{E}+00$ & $5.63 \mathrm{E}-02$ & $1.104 \mathrm{E}+01$ & $2.781 \mathrm{E}+00$ & $2.231 \mathrm{E}+01$ & $9.866 \mathrm{E}+04$ & $7.067 \mathrm{E}+01$ \\
\hline $1.402 \mathrm{E}+04$ & $1.177 \mathrm{E}+00$ & $5.67 \mathrm{E}-02$ & $1.104 \mathrm{E}+01$ & $2.781 \mathrm{E}+00$ & $2.241 \mathrm{E}+01$ & $9.866 \mathrm{E}+04$ & $9.655 \mathrm{E}+01$ \\
\hline $1.403 \mathrm{E}+04$ & $1.140 \mathrm{E}+00$ & $5.73 \mathrm{E}-02$ & $1.104 \mathrm{E}+01$ & $2.693 \mathrm{E}+00$ & $2.246 \mathrm{E}+01$ & $9.865 \mathrm{E}+04$ & $1.262 \mathrm{E}+02$ \\
\hline $1.401 \mathrm{E}+04$ & $1.063 \mathrm{E}+00$ & $5.76 \mathrm{E}-02$ & $1.105 \mathrm{E}+01$ & $2.539 \mathrm{E}+00$ & $2.249 \mathrm{E}+01$ & $9.864 \mathrm{E}+04$ & $1.601 \mathrm{E}+02$ \\
\hline $1.399 \mathrm{E}+04$ & $9.923 \mathrm{E}-01$ & $5.82 \mathrm{E}-02$ & $1.105 \mathrm{E}+01$ & $2.374 \mathrm{E}+00$ & $2.253 \mathrm{E}+01$ & $9.863 \mathrm{E}+04$ & $1.978 \mathrm{E}+02$ \\
\hline
\end{tabular}


Table 75: APC 4.1 x 4.1 Dynamic Calculated Values - 14010 RPM

\begin{tabular}{|c|c|c|c|c|c|c|c|}
\hline$n(\mathrm{RPM})$ & $V_{\infty}^{\prime}(\mathrm{m} / \mathrm{s})$ & $\Delta V_{\infty}^{\prime}(\mathrm{m} / \mathrm{s})$ & $T^{\prime}(\mathrm{g})$ & $\Delta T^{\prime}(\mathrm{g})$ & $V_{\mathrm{t}}(\mathrm{m} / \mathrm{s})$ & $P_{\mathrm{P}}(\mathrm{W})$ & $\Delta P_{\mathrm{P}}(\mathrm{W})$ \\
\hline $1.407 \mathrm{E}+04$ & $2.094 \mathrm{E}+00$ & $1.42 \mathrm{E}-02$ & $9.676 \mathrm{E}+01$ & $7.05 \mathrm{E}+00$ & $5.730 \mathrm{E}+01$ & $1.783 \mathrm{E}+01$ & $8.52 \mathrm{E}-01$ \\
\hline $1.401 \mathrm{E}+04$ & $3.750 \mathrm{E}+00$ & $1.46 \mathrm{E}-02$ & $9.066 \mathrm{E}+01$ & $7.04 \mathrm{E}+00$ & $5.714 \mathrm{E}+01$ & $1.778 \mathrm{E}+01$ & $8.16 \mathrm{E}-01$ \\
\hline $1.397 \mathrm{E}+04$ & $5.490 \mathrm{E}+00$ & $1.63 \mathrm{E}-02$ & $8.583 \mathrm{E}+01$ & $7.01 \mathrm{E}+00$ & $5.714 \mathrm{E}+01$ & $1.741 \mathrm{E}+01$ & $8.18 \mathrm{E}-01$ \\
\hline $1.401 \mathrm{E}+04$ & $7.279 \mathrm{E}+00$ & $1.81 \mathrm{E}-02$ & $8.324 \mathrm{E}+01$ & $7.01 \mathrm{E}+00$ & $5.748 \mathrm{E}+01$ & $1.735 \mathrm{E}+01$ & $8.12 \mathrm{E}-01$ \\
\hline $1.403 \mathrm{E}+04$ & $9.136 \mathrm{E}+00$ & $2.08 \mathrm{E}-02$ & $8.060 \mathrm{E}+01$ & $6.99 \mathrm{E}+00$ & $5.783 \mathrm{E}+01$ & $1.738 \mathrm{E}+01$ & 8.07E-01 \\
\hline $1.395 \mathrm{E}+04$ & $1.097 \mathrm{E}+01$ & $2.36 \mathrm{E}-02$ & $7.626 \mathrm{E}+01$ & $7.01 \mathrm{E}+00$ & $5.785 \mathrm{E}+01$ & $1.696 \mathrm{E}+01$ & 8.07E-01 \\
\hline $1.402 \mathrm{E}+04$ & $1.284 \mathrm{E}+01$ & $2.65 \mathrm{E}-02$ & $7.238 \mathrm{E}+01$ & $7.05 \mathrm{E}+00$ & $5.850 \mathrm{E}+01$ & $1.695 \mathrm{E}+01$ & 8.17E-01 \\
\hline $1.403 \mathrm{E}+04$ & $1.470 \mathrm{E}+01$ & $2.96 \mathrm{E}-02$ & $6.619 \mathrm{E}+01$ & $7.03 \mathrm{E}+00$ & $5.897 \mathrm{E}+01$ & $1.643 \mathrm{E}+01$ & $8.26 \mathrm{E}-01$ \\
\hline $1.401 \mathrm{E}+04$ & $1.656 \mathrm{E}+01$ & $3.25 \mathrm{E}-02$ & $5.745 \mathrm{E}+01$ & $7.03 \mathrm{E}+00$ & $5.939 \mathrm{E}+01$ & $1.530 \mathrm{E}+01$ & 8.29E-01 \\
\hline $1.399 \mathrm{E}+04$ & $1.842 \mathrm{E}+01$ & $3.53 \mathrm{E}-02$ & $4.971 \mathrm{E}+01$ & $7.05 \mathrm{E}+00$ & $5.986 \mathrm{E}+01$ & $1.426 \mathrm{E}+01$ & $8.36 \mathrm{E}-01$ \\
\hline$P_{\mathrm{e}}(\mathrm{W})$ & $\Delta P_{\mathrm{e}}(\mathrm{W})$ & $C_{\mathrm{T}}$ & $\Delta C_{\mathrm{T}}$ & $C_{\mathrm{P}}$ & $\Delta C_{\mathrm{P}}$ & $C_{\mathrm{Q}}$ & $\Delta C_{\mathrm{Q}}$ \\
\hline $3.208 \mathrm{E}+01$ & $3.57 \mathrm{E}-01$ & $1.283 \mathrm{E}-01$ & $9.36 \mathrm{E}-03$ & $9.924 \mathrm{E}-02$ & $4.75 \mathrm{E}-03$ & $1.579 \mathrm{E}-02$ & $7.55 \mathrm{E}-04$ \\
\hline $3.169 \mathrm{E}+01$ & $3.53 \mathrm{E}-01$ & $1.213 \mathrm{E}-01$ & $9.42 \mathrm{E}-03$ & $1.002 \mathrm{E}-01$ & 4.61E-03 & $1.595 \mathrm{E}-02$ & $7.33 \mathrm{E}-04$ \\
\hline $3.121 \mathrm{E}+01$ & $3.48 \mathrm{E}-01$ & $1.154 \mathrm{E}-01$ & $9.43 \mathrm{E}-03$ & $9.888 \mathrm{E}-02$ & $4.65 \mathrm{E}-03$ & $1.574 \mathrm{E}-02$ & 7.39E-04 \\
\hline $3.121 \mathrm{E}+01$ & $3.47 \mathrm{E}-01$ & $1.115 \mathrm{E}-01$ & $9.39 \mathrm{E}-03$ & $9.790 \mathrm{E}-02$ & $4.59 \mathrm{E}-03$ & $1.558 \mathrm{E}-02$ & 7.29E-04 \\
\hline $3.118 \mathrm{E}+01$ & $3.46 \mathrm{E}-01$ & $1.076 \mathrm{E}-01$ & $9.34 \mathrm{E}-03$ & $9.765 \mathrm{E}-02$ & $4.54 \mathrm{E}-03$ & $1.554 \mathrm{E}-02$ & $7.22 \mathrm{E}-04$ \\
\hline $3.071 \mathrm{E}+01$ & $3.41 \mathrm{E}-01$ & $1.030 \mathrm{E}-01$ & $9.47 \mathrm{E}-03$ & $9.690 \mathrm{E}-02$ & $4.61 \mathrm{E}-03$ & $1.542 \mathrm{E}-02$ & 7.34E-04 \\
\hline $3.070 \mathrm{E}+01$ & $3.42 \mathrm{E}-01$ & $9.683 \mathrm{E}-02$ & $9.43 \mathrm{E}-03$ & $9.545 \mathrm{E}-02$ & 4.61E-03 & $1.519 \mathrm{E}-02$ & 7.33E-04 \\
\hline $2.974 \mathrm{E}+01$ & $3.33 \mathrm{E}-01$ & $8.847 \mathrm{E}-02$ & $9.40 \mathrm{E}-03$ & $9.236 \mathrm{E}-02$ & $4.65 \mathrm{E}-03$ & $1.470 \mathrm{E}-02$ & 7.39E-04 \\
\hline $2.805 \mathrm{E}+01$ & $3.14 \mathrm{E}-01$ & $7.700 \mathrm{E}-02$ & $9.43 \mathrm{E}-03$ & 8.639E-02 & $4.68 \mathrm{E}-03$ & $1.375 \mathrm{E}-02$ & $7.45 \mathrm{E}-04$ \\
\hline $2.623 \mathrm{E}+01$ & $2.96 \mathrm{E}-01$ & $6.684 \mathrm{E}-02$ & $9.47 \mathrm{E}-03$ & 8.088E-02 & $4.75 \mathrm{E}-03$ & $1.287 \mathrm{E}-02$ & 7.55E-04 \\
\hline$\rho\left(\mathrm{kg} / \mathrm{m}^{\wedge} 3\right)$ & $\eta_{\mathrm{T}}$ & $\Delta \eta_{\mathrm{T}}$ & $J$ & $\Delta J$ & $\eta_{\mathrm{P}}$ & $\Delta \eta_{\mathrm{P}}$ & $R e_{0.75}$ \\
\hline $1.165 \mathrm{E}+00$ & 5.559E-01 & $2.73 \mathrm{E}-02$ & 8.617E-02 & $5.85 \mathrm{E}-04$ & $1.114 \mathrm{E}-01$ & $9.75 \mathrm{E}-03$ & $2.973 \mathrm{E}+04$ \\
\hline $1.165 \mathrm{E}+00$ & $5.611 \mathrm{E}-01$ & $2.65 \mathrm{E}-02$ & $1.549 \mathrm{E}-01$ & $6.10 \mathrm{E}-04$ & $1.874 \mathrm{E}-01$ & $1.69 \mathrm{E}-02$ & $2.965 \mathrm{E}+04$ \\
\hline $1.165 \mathrm{E}+00$ & $5.578 \mathrm{E}-01$ & $2.69 \mathrm{E}-02$ & $2.274 \mathrm{E}-01$ & $6.86 \mathrm{E}-04$ & $2.655 \mathrm{E}-01$ & $2.50 \mathrm{E}-02$ & $2.964 \mathrm{E}+04$ \\
\hline $1.164 \mathrm{E}+00$ & $5.560 \mathrm{E}-01$ & $2.67 \mathrm{E}-02$ & $3.008 \mathrm{E}-01$ & 7.69E-04 & $3.425 \mathrm{E}-01$ & $3.30 \mathrm{E}-02$ & $2.979 \mathrm{E}+04$ \\
\hline $1.164 \mathrm{E}+00$ & 5.574E-01 & $2.66 \mathrm{E}-02$ & $3.770 \mathrm{E}-01$ & $8.84 \mathrm{E}-04$ & 4.155E-01 & 4.09E-02 & $2.994 \mathrm{E}+04$ \\
\hline $1.163 \mathrm{E}+00$ & $5.524 \mathrm{E}-01$ & $2.70 \mathrm{E}-02$ & 4.551E-01 & $1.01 \mathrm{E}-03$ & 4.837E-01 & $5.01 \mathrm{E}-02$ & $2.993 \mathrm{E}+04$ \\
\hline $1.163 \mathrm{E}+00$ & $5.521 \mathrm{E}-01$ & $2.73 \mathrm{E}-02$ & 5.301E-01 & $1.14 \mathrm{E}-03$ & 5.377E-01 & $5.84 \mathrm{E}-02$ & $3.026 \mathrm{E}+04$ \\
\hline $1.163 \mathrm{E}+00$ & $5.523 \mathrm{E}-01$ & 2.84E-02 & $6.064 \mathrm{E}-01$ & $1.27 \mathrm{E}-03$ & $5.808 \mathrm{E}-01$ & $6.83 \mathrm{E}-02$ & $3.049 \mathrm{E}+04$ \\
\hline $1.162 \mathrm{E}+00$ & 5.454E-01 & $3.02 \mathrm{E}-02$ & $6.843 \mathrm{E}-01$ & $1.40 \mathrm{E}-03$ & $6.099 \mathrm{E}-01$ & $8.17 \mathrm{E}-02$ & $3.069 \mathrm{E}+04$ \\
\hline $1.162 \mathrm{E}+00$ & $5.435 \mathrm{E}-01$ & $3.25 \mathrm{E}-02$ & 7.622E-01 & $1.53 \mathrm{E}-03$ & $6.299 \mathrm{E}-01$ & $9.66 \mathrm{E}-02$ & $3.092 \mathrm{E}+04$ \\
\hline
\end{tabular}


Table 76: APC 4.1 x 4.1 Dynamic Measured Values - 18035 RPM

\begin{tabular}{|c|c|c|c|c|c|c|c|}
\hline$n(\mathrm{RPM})$ & $Q(\mathrm{~g}-\mathrm{m})$ & $\Delta Q(\mathrm{~g}-\mathrm{m})$ & $V(\mathrm{~V})$ & $I(\mathrm{~A})$ & $T_{\text {atm }}\left({ }^{\circ} \mathrm{C}\right)$ & $P_{\text {atm }}(\mathrm{Pa})$ & $P_{\text {diff }}(\mathrm{Pa})$ \\
\hline $1.798 \mathrm{E}+04$ & $2.007 \mathrm{E}+00$ & $6.45 \mathrm{E}-02$ & $1.097 \mathrm{E}+01$ & $5.311 \mathrm{E}+00$ & $2.211 \mathrm{E}+01$ & $9.857 \mathrm{E}+04$ & $3.215 \mathrm{E}+00$ \\
\hline $1.802 \mathrm{E}+04$ & $2.022 \mathrm{E}+00$ & $6.38 \mathrm{E}-02$ & $1.097 \mathrm{E}+01$ & $5.307 \mathrm{E}+00$ & $2.204 \mathrm{E}+01$ & $9.857 \mathrm{E}+04$ & $1.104 \mathrm{E}+01$ \\
\hline $1.804 \mathrm{E}+04$ & $2.023 \mathrm{E}+00$ & $6.58 \mathrm{E}-02$ & $1.097 \mathrm{E}+01$ & $5.278 \mathrm{E}+00$ & $2.222 \mathrm{E}+01$ & $9.857 \mathrm{E}+04$ & $2.485 \mathrm{E}+01$ \\
\hline $1.805 \mathrm{E}+04$ & $2.035 \mathrm{E}+00$ & $6.60 \mathrm{E}-02$ & $1.097 \mathrm{E}+01$ & $5.274 \mathrm{E}+00$ & $2.235 \mathrm{E}+01$ & $9.856 \mathrm{E}+04$ & $4.480 \mathrm{E}+01$ \\
\hline $1.805 \mathrm{E}+04$ & $2.024 \mathrm{E}+00$ & $6.61 \mathrm{E}-02$ & $1.097 \mathrm{E}+01$ & $5.274 \mathrm{E}+00$ & $2.246 \mathrm{E}+01$ & $9.855 \mathrm{E}+04$ & $7.113 \mathrm{E}+01$ \\
\hline $1.806 \mathrm{E}+04$ & $2.029 \mathrm{E}+00$ & $6.62 \mathrm{E}-02$ & $1.097 \mathrm{E}+01$ & $5.268 \mathrm{E}+00$ & $2.255 \mathrm{E}+01$ & $9.855 \mathrm{E}+04$ & $1.042 \mathrm{E}+02$ \\
\hline $1.806 \mathrm{E}+04$ & $2.019 \mathrm{E}+00$ & $6.59 \mathrm{E}-02$ & $1.097 \mathrm{E}+01$ & $5.259 \mathrm{E}+00$ & $2.262 \mathrm{E}+01$ & $9.855 \mathrm{E}+04$ & $1.436 \mathrm{E}+02$ \\
\hline $1.802 \mathrm{E}+04$ & $1.943 \mathrm{E}+00$ & $6.32 \mathrm{E}-02$ & $1.098 \mathrm{E}+01$ & $5.124 \mathrm{E}+00$ & $2.264 \mathrm{E}+01$ & $9.855 \mathrm{E}+04$ & $1.889 \mathrm{E}+02$ \\
\hline $1.805 \mathrm{E}+04$ & $1.886 \mathrm{E}+00$ & $6.23 \mathrm{E}-02$ & $1.098 \mathrm{E}+01$ & $4.928 \mathrm{E}+00$ & $2.273 \mathrm{E}+01$ & $9.855 \mathrm{E}+04$ & $2.403 \mathrm{E}+02$ \\
\hline $1.804 \mathrm{E}+04$ & $1.791 \mathrm{E}+00$ & $6.17 \mathrm{E}-02$ & $1.099 \mathrm{E}+01$ & $4.663 \mathrm{E}+00$ & $2.277 \mathrm{E}+01$ & $9.854 \mathrm{E}+04$ & $2.975 \mathrm{E}+02$ \\
\hline $1.802 \mathrm{E}+04$ & $1.676 \mathrm{E}+00$ & $6.09 \mathrm{E}-02$ & $1.100 \mathrm{E}+01$ & $4.316 \mathrm{E}+00$ & $2.284 \mathrm{E}+01$ & $9.853 \mathrm{E}+04$ & $3.614 \mathrm{E}+02$ \\
\hline
\end{tabular}


Table 77: APC 4.1 x 4.1 Dynamic Calculated Values - 18035 RPM

\begin{tabular}{|c|c|c|c|c|c|c|c|}
\hline$n(\mathrm{RPM})$ & $V_{\infty}^{\prime}(\mathrm{m} / \mathrm{s})$ & $\Delta V_{\infty}^{\prime}(\mathrm{m} / \mathrm{s})$ & $T^{\prime}(\mathrm{g})$ & $\Delta T^{\prime}(\mathrm{g})$ & $V_{\mathrm{t}}(\mathrm{m} / \mathrm{s})$ & $P_{\mathrm{P}}(\mathrm{W})$ & $\Delta P_{\mathrm{P}}(\mathrm{W})$ \\
\hline $1.798 \mathrm{E}+04$ & $2.250 \mathrm{E}+00$ & $1.65 \mathrm{E}-02$ & $1.619 \mathrm{E}+02$ & $7.49 \mathrm{E}+00$ & $7.323 \mathrm{E}+01$ & $3.706 \mathrm{E}+01$ & $1.19 \mathrm{E}+00$ \\
\hline $1.802 \mathrm{E}+04$ & $4.259 \mathrm{E}+00$ & $1.74 \mathrm{E}-02$ & $1.595 \mathrm{E}+02$ & $7.52 \mathrm{E}+00$ & $7.347 \mathrm{E}+01$ & $3.742 \mathrm{E}+01$ & $1.18 \mathrm{E}+00$ \\
\hline $1.804 \mathrm{E}+04$ & $6.443 \mathrm{E}+00$ & $1.96 \mathrm{E}-02$ & $1.570 \mathrm{E}+02$ & $7.80 \mathrm{E}+00$ & $7.371 \mathrm{E}+01$ & $3.747 \mathrm{E}+01$ & $1.22 \mathrm{E}+00$ \\
\hline $1.805 \mathrm{E}+04$ & $8.695 \mathrm{E}+00$ & $2.25 \mathrm{E}-02$ & $1.470 \mathrm{E}+02$ & $7.19 \mathrm{E}+00$ & $7.398 \mathrm{E}+01$ & $3.771 \mathrm{E}+01$ & $1.22 \mathrm{E}+00$ \\
\hline $1.805 \mathrm{E}+04$ & $1.099 \mathrm{E}+01$ & $2.57 \mathrm{E}-02$ & $1.391 \mathrm{E}+02$ & $7.08 \mathrm{E}+00$ & $7.429 \mathrm{E}+01$ & $3.751 \mathrm{E}+01$ & $1.22 \mathrm{E}+00$ \\
\hline $1.806 \mathrm{E}+04$ & $1.333 \mathrm{E}+01$ & $2.91 \mathrm{E}-02$ & $1.357 \mathrm{E}+02$ & $7.16 \mathrm{E}+00$ & $7.471 \mathrm{E}+01$ & $3.763 \mathrm{E}+01$ & $1.23 \mathrm{E}+00$ \\
\hline $1.806 \mathrm{E}+04$ & $1.566 \mathrm{E}+01$ & $3.22 \mathrm{E}-02$ & $1.307 \mathrm{E}+02$ & $7.25 \mathrm{E}+00$ & $7.514 \mathrm{E}+01$ & $3.744 \mathrm{E}+01$ & $1.22 \mathrm{E}+00$ \\
\hline $1.802 \mathrm{E}+04$ & $1.799 \mathrm{E}+01$ & $3.54 \mathrm{E}-02$ & $1.202 \mathrm{E}+02$ & $6.94 \mathrm{E}+00$ & $7.554 \mathrm{E}+01$ & $3.596 \mathrm{E}+01$ & $1.17 \mathrm{E}+00$ \\
\hline $1.805 \mathrm{E}+04$ & $2.030 \mathrm{E}+01$ & $3.89 \mathrm{E}-02$ & $1.088 \mathrm{E}+02$ & $6.96 \mathrm{E}+00$ & $7.621 \mathrm{E}+01$ & $3.495 \mathrm{E}+01$ & $1.15 \mathrm{E}+00$ \\
\hline $1.804 \mathrm{E}+04$ & $2.261 \mathrm{E}+01$ & $4.31 \mathrm{E}-02$ & $9.653 \mathrm{E}+01$ & $6.96 \mathrm{E}+00$ & $7.683 \mathrm{E}+01$ & $3.318 \mathrm{E}+01$ & $1.14 \mathrm{E}+00$ \\
\hline $1.802 \mathrm{E}+04$ & $2.493 \mathrm{E}+01$ & $4.69 \mathrm{E}-02$ & $8.264 \mathrm{E}+01$ & $6.97 \mathrm{E}+00$ & $7.746 \mathrm{E}+01$ & $3.101 \mathrm{E}+01$ & $1.13 \mathrm{E}+00$ \\
\hline$P_{\mathrm{e}}(\mathrm{W})$ & $\Delta P_{\mathrm{e}}(\mathrm{W})$ & $C_{\mathrm{T}}$ & $\Delta C_{\mathrm{T}}$ & $C_{\mathrm{P}}$ & $\Delta C_{\mathrm{P}}$ & $C_{\mathrm{Q}}$ & $\Delta C_{\mathrm{Q}}$ \\
\hline $5.827 \mathrm{E}+01$ & $6.49 \mathrm{E}-01$ & $1.316 \mathrm{E}-01$ & $6.09 \mathrm{E}-03$ & 9.894E-02 & $3.18 \mathrm{E}-03$ & $1.575 \mathrm{E}-02$ & $5.06 \mathrm{E}-04$ \\
\hline $5.822 \mathrm{E}+01$ & $6.55 \mathrm{E}-01$ & $1.292 \mathrm{E}-01$ & $6.09 \mathrm{E}-03$ & $9.928 \mathrm{E}-02$ & $3.14 \mathrm{E}-03$ & $1.580 \mathrm{E}-02$ & 4.99E-04 \\
\hline $5.791 \mathrm{E}+01$ & $6.47 \mathrm{E}-01$ & $1.269 \mathrm{E}-01$ & $6.31 \mathrm{E}-03$ & 9.914E-02 & $3.23 \mathrm{E}-03$ & $1.578 \mathrm{E}-02$ & $5.13 \mathrm{E}-04$ \\
\hline $5.786 \mathrm{E}+01$ & $6.47 \mathrm{E}-01$ & $1.188 \mathrm{E}-01$ & $5.81 \mathrm{E}-03$ & $9.968 \mathrm{E}-02$ & $3.23 \mathrm{E}-03$ & $1.586 \mathrm{E}-02$ & $5.14 \mathrm{E}-04$ \\
\hline $5.786 \mathrm{E}+01$ & $6.51 \mathrm{E}-01$ & $1.125 \mathrm{E}-01$ & $5.73 \mathrm{E}-03$ & $9.917 \mathrm{E}-02$ & $3.24 \mathrm{E}-03$ & $1.578 \mathrm{E}-02$ & $5.16 \mathrm{E}-04$ \\
\hline $5.780 \mathrm{E}+01$ & $6.48 \mathrm{E}-01$ & $1.096 \mathrm{E}-01$ & $5.78 \mathrm{E}-03$ & $9.935 \mathrm{E}-02$ & $3.24 \mathrm{E}-03$ & $1.581 \mathrm{E}-02$ & $5.16 \mathrm{E}-04$ \\
\hline $5.770 \mathrm{E}+01$ & $6.48 \mathrm{E}-01$ & $1.057 \mathrm{E}-01$ & $5.86 \mathrm{E}-03$ & 9.894E-02 & $3.23 \mathrm{E}-03$ & $1.575 \mathrm{E}-02$ & $5.14 \mathrm{E}-04$ \\
\hline $5.624 \mathrm{E}+01$ & $6.31 \mathrm{E}-01$ & $9.755 \mathrm{E}-02$ & $5.63 \mathrm{E}-03$ & $9.553 \mathrm{E}-02$ & $3.11 \mathrm{E}-03$ & $1.520 \mathrm{E}-02$ & $4.95 \mathrm{E}-04$ \\
\hline $5.412 \mathrm{E}+01$ & $6.04 \mathrm{E}-01$ & $8.806 \mathrm{E}-02$ & $5.63 \mathrm{E}-03$ & $9.254 \mathrm{E}-02$ & $3.06 \mathrm{E}-03$ & $1.473 \mathrm{E}-02$ & 4.87E-04 \\
\hline $5.124 \mathrm{E}+01$ & $5.73 \mathrm{E}-01$ & $7.822 \mathrm{E}-02$ & 5.64E-03 & 8.799E-02 & $3.03 \mathrm{E}-03$ & $1.400 \mathrm{E}-02$ & 4.82E-04 \\
\hline $4.746 \mathrm{E}+01$ & $5.29 \mathrm{E}-01$ & $6.714 \mathrm{E}-02$ & $5.66 \mathrm{E}-03$ & $8.253 \mathrm{E}-02$ & $3.00 \mathrm{E}-03$ & $1.314 \mathrm{E}-02$ & 4.78E-04 \\
\hline$\rho\left(\mathrm{kg} / \mathrm{m}^{\wedge} 3\right)$ & $\eta_{\mathrm{T}}$ & $\Delta \eta_{\mathrm{T}}$ & $J$ & $\Delta J$ & $\eta_{\mathrm{P}}$ & $\Delta \eta_{\mathrm{P}}$ & $R e_{0.75}$ \\
\hline $1.163 \mathrm{E}+00$ & $6.361 \mathrm{E}-01$ & $2.16 \mathrm{E}-02$ & $7.243 \mathrm{E}-02$ & $5.31 \mathrm{E}-04$ & $9.637 \mathrm{E}-02$ & $5.48 \mathrm{E}-03$ & $3.791 \mathrm{E}+04$ \\
\hline $1.163 \mathrm{E}+00$ & $6.428 \mathrm{E}-01$ & $2.15 \mathrm{E}-02$ & $1.368 \mathrm{E}-01$ & $5.64 \mathrm{E}-04$ & $1.780 \mathrm{E}-01$ & $1.01 \mathrm{E}-02$ & $3.805 \mathrm{E}+04$ \\
\hline $1.163 \mathrm{E}+00$ & $6.471 \mathrm{E}-01$ & $2.22 \mathrm{E}-02$ & $2.068 \mathrm{E}-01$ & $6.37 \mathrm{E}-04$ & $2.647 \mathrm{E}-01$ & $1.57 \mathrm{E}-02$ & $3.813 \mathrm{E}+04$ \\
\hline $1.162 \mathrm{E}+00$ & $6.518 \mathrm{E}-01$ & $2.24 \mathrm{E}-02$ & $2.789 \mathrm{E}-01$ & 7.33E-04 & $3.323 \mathrm{E}-01$ & $1.95 \mathrm{E}-02$ & $3.823 \mathrm{E}+04$ \\
\hline $1.161 \mathrm{E}+00$ & $6.483 \mathrm{E}-01$ & $2.24 \mathrm{E}-02$ & $3.524 \mathrm{E}-01$ & $8.41 \mathrm{E}-04$ & $3.997 \mathrm{E}-01$ & $2.42 \mathrm{E}-02$ & $3.837 \mathrm{E}+04$ \\
\hline $1.161 \mathrm{E}+00$ & $6.509 \mathrm{E}-01$ & $2.24 \mathrm{E}-02$ & 4.272E-01 & 9.52E-04 & 4.712E-01 & $2.93 \mathrm{E}-02$ & $3.856 \mathrm{E}+04$ \\
\hline $1.161 \mathrm{E}+00$ & $6.488 \mathrm{E}-01$ & $2.24 \mathrm{E}-02$ & $5.021 \mathrm{E}-01$ & $1.06 \mathrm{E}-03$ & 5.362E-01 & $3.45 \mathrm{E}-02$ & $3.877 \mathrm{E}+04$ \\
\hline $1.161 \mathrm{E}+00$ & 6.394E-01 & $2.20 \mathrm{E}-02$ & $5.776 \mathrm{E}-01$ & $1.17 \mathrm{E}-03$ & $5.898 \mathrm{E}-01$ & $3.91 \mathrm{E}-02$ & $3.897 \mathrm{E}+04$ \\
\hline $1.160 \mathrm{E}+00$ & $6.459 \mathrm{E}-01$ & $2.25 \mathrm{E}-02$ & $6.513 \mathrm{E}-01$ & $1.28 \mathrm{E}-03$ & $6.197 \mathrm{E}-01$ & $4.46 \mathrm{E}-02$ & $3.930 \mathrm{E}+04$ \\
\hline $1.160 \mathrm{E}+00$ & $6.476 \mathrm{E}-01$ & $2.34 \mathrm{E}-02$ & 7.254E-01 & $1.42 \mathrm{E}-03$ & $6.449 \mathrm{E}-01$ & $5.16 \mathrm{E}-02$ & $3.960 \mathrm{E}+04$ \\
\hline $1.160 \mathrm{E}+00$ & $6.533 \mathrm{E}-01$ & $2.48 \mathrm{E}-02$ & 8.010E-01 & $1.55 \mathrm{E}-03$ & $6.516 \mathrm{E}-01$ & $5.99 \mathrm{E}-02$ & $3.991 \mathrm{E}+04$ \\
\hline
\end{tabular}


Table 78: APC 4.1 x 4.1 Dynamic Measured Values - 22460 RPM

\begin{tabular}{|c|c|c|c|c|c|c|c|}
\hline$n(\mathrm{RPM})$ & $Q(\mathrm{~g}-\mathrm{m})$ & $\Delta Q(\mathrm{~g}-\mathrm{m})$ & $V(\mathrm{~V})$ & $I(\mathrm{~A})$ & $T_{\text {atm }}\left({ }^{\circ} \mathrm{C}\right)$ & $P_{\text {atm }}(\mathrm{Pa})$ & $P_{\text {diff }}(\mathrm{Pa})$ \\
\hline $2.243 \mathrm{E}+04$ & $3.225 \mathrm{E}+00$ & $7.57 \mathrm{E}-02$ & $1.084 \mathrm{E}+01$ & $9.861 \mathrm{E}+00$ & $2.223 \mathrm{E}+01$ & $9.852 \mathrm{E}+04$ & $3.641 \mathrm{E}+00$ \\
\hline $2.248 \mathrm{E}+04$ & $3.214 \mathrm{E}+00$ & $7.29 \mathrm{E}-02$ & $1.084 \mathrm{E}+01$ & $9.849 \mathrm{E}+00$ & $2.205 \mathrm{E}+01$ & $9.853 \mathrm{E}+04$ & $1.393 \mathrm{E}+01$ \\
\hline $2.251 \mathrm{E}+04$ & $3.206 \mathrm{E}+00$ & $7.25 \mathrm{E}-02$ & $1.085 \mathrm{E}+01$ & $9.793 \mathrm{E}+00$ & $2.218 \mathrm{E}+01$ & $9.852 \mathrm{E}+04$ & $3.295 \mathrm{E}+01$ \\
\hline $2.252 \mathrm{E}+04$ & $3.223 \mathrm{E}+00$ & $7.27 \mathrm{E}-02$ & $1.085 \mathrm{E}+01$ & $9.772 \mathrm{E}+00$ & $2.243 \mathrm{E}+01$ & $9.851 \mathrm{E}+04$ & $6.114 \mathrm{E}+01$ \\
\hline $2.251 \mathrm{E}+04$ & $3.225 \mathrm{E}+00$ & $7.58 \mathrm{E}-02$ & $1.085 \mathrm{E}+01$ & $9.790 \mathrm{E}+00$ & $2.253 \mathrm{E}+01$ & $9.851 \mathrm{E}+04$ & $9.813 \mathrm{E}+01$ \\
\hline $2.251 \mathrm{E}+04$ & $3.232 \mathrm{E}+00$ & $7.55 \mathrm{E}-02$ & $1.085 \mathrm{E}+01$ & $9.794 \mathrm{E}+00$ & $2.262 \mathrm{E}+01$ & $9.850 \mathrm{E}+04$ & $1.447 \mathrm{E}+02$ \\
\hline $2.250 \mathrm{E}+04$ & $3.233 \mathrm{E}+00$ & $7.55 \mathrm{E}-02$ & $1.084 \mathrm{E}+01$ & $9.803 \mathrm{E}+00$ & $2.270 \mathrm{E}+01$ & $9.849 \mathrm{E}+04$ & $2.004 \mathrm{E}+02$ \\
\hline $2.252 \mathrm{E}+04$ & $3.239 \mathrm{E}+00$ & $7.82 \mathrm{E}-02$ & $1.085 \mathrm{E}+01$ & $9.780 \mathrm{E}+00$ & $2.278 \mathrm{E}+01$ & $9.848 \mathrm{E}+04$ & $2.640 \mathrm{E}+02$ \\
\hline $2.259 \mathrm{E}+04$ & $3.151 \mathrm{E}+00$ & $7.85 \mathrm{E}-02$ & $1.085 \mathrm{E}+01$ & $9.616 \mathrm{E}+00$ & $2.289 \mathrm{E}+01$ & $9.848 \mathrm{E}+04$ & $3.366 \mathrm{E}+02$ \\
\hline $2.217 \mathrm{E}+04$ & $2.896 \mathrm{E}+00$ & $8.94 \mathrm{E}-02$ & $1.088 \mathrm{E}+01$ & $8.557 \mathrm{E}+00$ & $2.293 \mathrm{E}+01$ & $9.847 \mathrm{E}+04$ & $4.177 \mathrm{E}+02$ \\
\hline $2.232 \mathrm{E}+04$ & $2.782 \mathrm{E}+00$ & $8.76 \mathrm{E}-02$ & $1.089 \mathrm{E}+01$ & $8.185 \mathrm{E}+00$ & $2.300 \mathrm{E}+01$ & $9.846 \mathrm{E}+04$ & $5.071 \mathrm{E}+02$ \\
\hline
\end{tabular}


Table 79: APC 4.1 x 4.1 Dynamic Calculated Values - 22460 RPM

\begin{tabular}{|c|c|c|c|c|c|c|c|}
\hline$n(\mathrm{RPM})$ & $V_{\infty}^{\prime}(\mathrm{m} / \mathrm{s})$ & $\Delta V_{\infty}^{\prime}(\mathrm{m} / \mathrm{s})$ & $T^{\prime}(\mathrm{g})$ & $\Delta T^{\prime}(\mathrm{g})$ & $V_{\mathrm{t}}(\mathrm{m} / \mathrm{s})$ & $P_{\mathrm{P}}(\mathrm{W})$ & $\Delta P_{\mathrm{P}}(\mathrm{W})$ \\
\hline $2.243 \mathrm{E}+04$ & $2.376 \mathrm{E}+00$ & $1.96 \mathrm{E}-02$ & $2.559 \mathrm{E}+02$ & $7.18 \mathrm{E}+00$ & $9.131 \mathrm{E}+01$ & $7.426 \mathrm{E}+01$ & $1.74 \mathrm{E}+00$ \\
\hline $2.248 \mathrm{E}+04$ & $4.770 \mathrm{E}+00$ & $2.09 \mathrm{E}-02$ & $2.532 \mathrm{E}+02$ & $7.14 \mathrm{E}+00$ & $9.161 \mathrm{E}+01$ & $7.418 \mathrm{E}+01$ & $1.68 \mathrm{E}+00$ \\
\hline $2.251 \mathrm{E}+04$ & $7.410 \mathrm{E}+00$ & 2.37E-02 & $2.501 \mathrm{E}+02$ & $7.17 \mathrm{E}+00$ & $9.192 \mathrm{E}+01$ & $7.411 \mathrm{E}+01$ & $1.68 \mathrm{E}+00$ \\
\hline $2.252 \mathrm{E}+04$ & $1.015 \mathrm{E}+01$ & 2.84E-02 & $2.460 \mathrm{E}+02$ & $7.20 \mathrm{E}+00$ & $9.224 \mathrm{E}+01$ & $7.454 \mathrm{E}+01$ & $1.68 \mathrm{E}+00$ \\
\hline $2.251 \mathrm{E}+04$ & $1.290 \mathrm{E}+01$ & $3.16 \mathrm{E}-02$ & $2.400 \mathrm{E}+02$ & $7.19 \mathrm{E}+00$ & $9.255 \mathrm{E}+01$ & $7.457 \mathrm{E}+01$ & $1.75 \mathrm{E}+00$ \\
\hline $2.251 \mathrm{E}+04$ & $1.570 \mathrm{E}+01$ & $3.46 \mathrm{E}-02$ & $2.270 \mathrm{E}+02$ & $7.24 \mathrm{E}+00$ & $9.296 \mathrm{E}+01$ & $7.472 \mathrm{E}+01$ & $1.74 \mathrm{E}+00$ \\
\hline $2.250 \mathrm{E}+04$ & $1.850 \mathrm{E}+01$ & $3.79 \mathrm{E}-02$ & $2.191 \mathrm{E}+02$ & $7.27 \mathrm{E}+00$ & $9.345 \mathrm{E}+01$ & $7.472 \mathrm{E}+01$ & $1.75 \mathrm{E}+00$ \\
\hline $2.252 \mathrm{E}+04$ & $2.126 \mathrm{E}+01$ & $4.21 \mathrm{E}-02$ & $2.103 \mathrm{E}+02$ & $7.35 \mathrm{E}+00$ & $9.408 \mathrm{E}+01$ & $7.490 \mathrm{E}+01$ & $1.81 \mathrm{E}+00$ \\
\hline $2.259 \mathrm{E}+04$ & $2.403 \mathrm{E}+01$ & 4.64E-02 & $1.952 \mathrm{E}+02$ & $7.27 \mathrm{E}+00$ & $9.504 \mathrm{E}+01$ & $7.310 \mathrm{E}+01$ & $1.82 \mathrm{E}+00$ \\
\hline $2.217 \mathrm{E}+04$ & $2.679 \mathrm{E}+01$ & $5.14 \mathrm{E}-02$ & $1.640 \mathrm{E}+02$ & $7.24 \mathrm{E}+00$ & $9.413 \mathrm{E}+01$ & $6.593 \mathrm{E}+01$ & $2.03 \mathrm{E}+00$ \\
\hline $2.232 \mathrm{E}+04$ & $2.954 \mathrm{E}+01$ & $5.56 \mathrm{E}-02$ & $1.492 \mathrm{E}+02$ & $7.36 \mathrm{E}+00$ & $9.554 \mathrm{E}+01$ & $6.378 \mathrm{E}+01$ & $2.01 \mathrm{E}+00$ \\
\hline$P_{\mathrm{e}}(\mathrm{W})$ & $\Delta P_{\mathrm{e}}(\mathrm{W})$ & $C_{\mathrm{T}}$ & $\Delta C_{\mathrm{T}}$ & $C_{\mathrm{P}}$ & $\Delta C_{\mathrm{P}}$ & $C_{\mathrm{Q}}$ & $\Delta C_{\mathrm{Q}}$ \\
\hline $1.069 \mathrm{E}+02$ & $1.13 \mathrm{E}+00$ & $1.340 \mathrm{E}-01$ & $3.76 \mathrm{E}-03$ & $1.023 \mathrm{E}-01$ & $2.40 \mathrm{E}-03$ & $1.628 \mathrm{E}-02$ & 3.82E-04 \\
\hline $1.068 \mathrm{E}+02$ & $1.11 \mathrm{E}+00$ & $1.319 \mathrm{E}-01$ & $3.72 \mathrm{E}-03$ & $1.015 \mathrm{E}-01$ & $2.30 \mathrm{E}-03$ & $1.615 \mathrm{E}-02$ & $3.67 \mathrm{E}-04$ \\
\hline $1.062 \mathrm{E}+02$ & $1.10 \mathrm{E}+00$ & $1.299 \mathrm{E}-01$ & $3.73 \mathrm{E}-03$ & $1.010 \mathrm{E}-01$ & $2.28 \mathrm{E}-03$ & $1.607 \mathrm{E}-02$ & $3.63 \mathrm{E}-04$ \\
\hline $1.060 \mathrm{E}+02$ & $1.10 \mathrm{E}+00$ & $1.278 \mathrm{E}-01$ & $3.74 \mathrm{E}-03$ & $1.015 \mathrm{E}-01$ & $2.29 \mathrm{E}-03$ & $1.615 \mathrm{E}-02$ & $3.65 \mathrm{E}-04$ \\
\hline $1.062 \mathrm{E}+02$ & $1.10 \mathrm{E}+00$ & $1.248 \mathrm{E}-01$ & $3.74 \mathrm{E}-03$ & $1.017 \mathrm{E}-01$ & $2.39 \mathrm{E}-03$ & $1.618 \mathrm{E}-02$ & $3.80 \mathrm{E}-04$ \\
\hline $1.062 \mathrm{E}+02$ & $1.10 \mathrm{E}+00$ & $1.181 \mathrm{E}-01$ & $3.77 \mathrm{E}-03$ & $1.019 \mathrm{E}-01$ & $2.38 \mathrm{E}-03$ & 1.622E-02 & $3.79 \mathrm{E}-04$ \\
\hline $1.063 \mathrm{E}+02$ & $1.11 \mathrm{E}+00$ & $1.141 \mathrm{E}-01$ & $3.79 \mathrm{E}-03$ & $1.021 \mathrm{E}-01$ & $2.39 \mathrm{E}-03$ & $1.625 \mathrm{E}-02$ & $3.80 \mathrm{E}-04$ \\
\hline $1.061 \mathrm{E}+02$ & $1.10 \mathrm{E}+00$ & $1.094 \mathrm{E}-01$ & $3.83 \mathrm{E}-03$ & $1.022 \mathrm{E}-01$ & $2.47 \mathrm{E}-03$ & $1.626 \mathrm{E}-02$ & $3.93 \mathrm{E}-04$ \\
\hline $1.043 \mathrm{E}+02$ & $1.09 \mathrm{E}+00$ & $1.009 \mathrm{E}-01$ & $3.76 \mathrm{E}-03$ & $9.878 \mathrm{E}-02$ & $2.46 \mathrm{E}-03$ & $1.572 \mathrm{E}-02$ & 3.92E-04 \\
\hline $9.310 \mathrm{E}+01$ & $1.05 \mathrm{E}+00$ & $8.813 \mathrm{E}-02$ & $3.89 \mathrm{E}-03$ & $9.430 \mathrm{E}-02$ & $2.91 \mathrm{E}-03$ & $1.501 \mathrm{E}-02$ & 4.63E-04 \\
\hline $8.914 \mathrm{E}+01$ & $1.04 \mathrm{E}+00$ & 7.910E-02 & $3.90 \mathrm{E}-03$ & 8.941E-02 & $2.82 \mathrm{E}-03$ & $1.423 \mathrm{E}-02$ & 4.48E-04 \\
\hline$\rho\left(\mathrm{kg} / \mathrm{m}^{\wedge} 3\right)$ & $\eta_{\mathrm{T}}$ & $\Delta \eta_{\mathrm{T}}$ & $J$ & $\Delta J$ & $\eta_{\mathrm{P}}$ & $\Delta \eta_{\mathrm{P}}$ & $R e_{0.75}$ \\
\hline $1.162 \mathrm{E}+00$ & $6.945 \mathrm{E}-01$ & $1.79 \mathrm{E}-02$ & $6.132 \mathrm{E}-02$ & $5.06 \mathrm{E}-04$ & $8.028 \mathrm{E}-02$ & $3.01 \mathrm{E}-03$ & $4.721 \mathrm{E}+04$ \\
\hline $1.163 \mathrm{E}+00$ & $6.946 \mathrm{E}-01$ & $1.73 \mathrm{E}-02$ & $1.229 \mathrm{E}-01$ & $5.39 \mathrm{E}-04$ & $1.597 \mathrm{E}-01$ & $5.82 \mathrm{E}-03$ & $4.742 \mathrm{E}+04$ \\
\hline $1.162 \mathrm{E}+00$ & $6.978 \mathrm{E}-01$ & $1.74 \mathrm{E}-02$ & $1.906 \mathrm{E}-01$ & $6.10 \mathrm{E}-04$ & $2.452 \mathrm{E}-01$ & $8.99 \mathrm{E}-03$ & $4.754 \mathrm{E}+04$ \\
\hline $1.161 \mathrm{E}+00$ & 7.033E-01 & $1.75 \mathrm{E}-02$ & $2.608 \mathrm{E}-01$ & 7.32E-04 & $3.285 \mathrm{E}-01$ & $1.22 \mathrm{E}-02$ & $4.762 \mathrm{E}+04$ \\
\hline $1.161 \mathrm{E}+00$ & $7.024 \mathrm{E}-01$ & $1.81 \mathrm{E}-02$ & $3.316 \mathrm{E}-01$ & $8.15 \mathrm{E}-04$ & 4.071E-01 & $1.55 \mathrm{E}-02$ & $4.775 \mathrm{E}+04$ \\
\hline $1.160 \mathrm{E}+00$ & 7.034E-01 & $1.80 \mathrm{E}-02$ & 4.037E-01 & 8.92E-04 & 4.678E-01 & $1.85 \mathrm{E}-02$ & $4.794 \mathrm{E}+04$ \\
\hline $1.160 \mathrm{E}+00$ & 7.029E-01 & $1.80 \mathrm{E}-02$ & 4.759E-01 & $9.77 \mathrm{E}-04$ & 5.319E-01 & $2.16 \mathrm{E}-02$ & $4.816 \mathrm{E}+04$ \\
\hline $1.159 \mathrm{E}+00$ & 7.061E-01 & $1.86 \mathrm{E}-02$ & $5.465 \mathrm{E}-01$ & $1.09 \mathrm{E}-03$ & 5.853E-01 & $2.49 \mathrm{E}-02$ & $4.846 \mathrm{E}+04$ \\
\hline $1.159 \mathrm{E}+00$ & $7.006 \mathrm{E}-01$ & $1.89 \mathrm{E}-02$ & $6.158 \mathrm{E}-01$ & $1.19 \mathrm{E}-03$ & $6.292 \mathrm{E}-01$ & $2.82 \mathrm{E}-02$ & $4.892 \mathrm{E}+04$ \\
\hline $1.159 \mathrm{E}+00$ & $7.081 \mathrm{E}-01$ & $2.33 \mathrm{E}-02$ & $6.997 \mathrm{E}-01$ & $1.35 \mathrm{E}-03$ & $6.538 \mathrm{E}-01$ & $3.52 \mathrm{E}-02$ & $4.844 \mathrm{E}+04$ \\
\hline $1.158 \mathrm{E}+00$ & $7.155 \mathrm{E}-01$ & $2.40 \mathrm{E}-02$ & 7.662E-01 & $1.46 \mathrm{E}-03$ & $6.778 \mathrm{E}-01$ & $3.97 \mathrm{E}-02$ & $4.914 \mathrm{E}+04$ \\
\hline
\end{tabular}




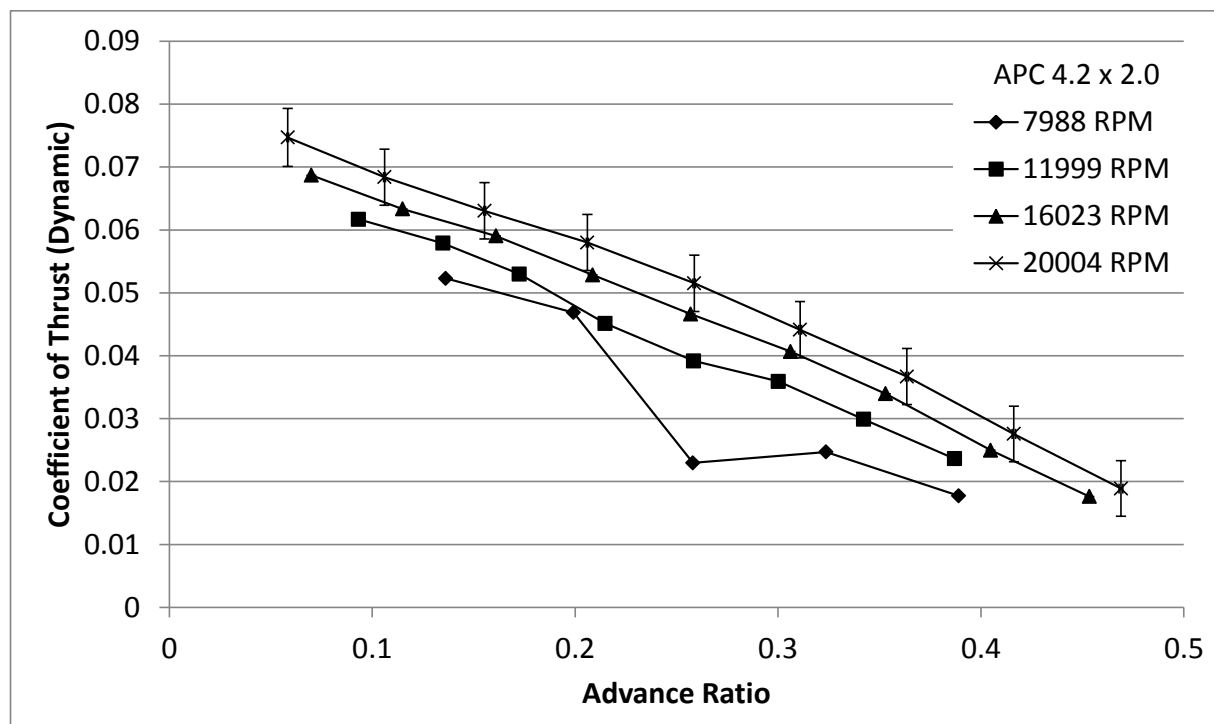

(a)
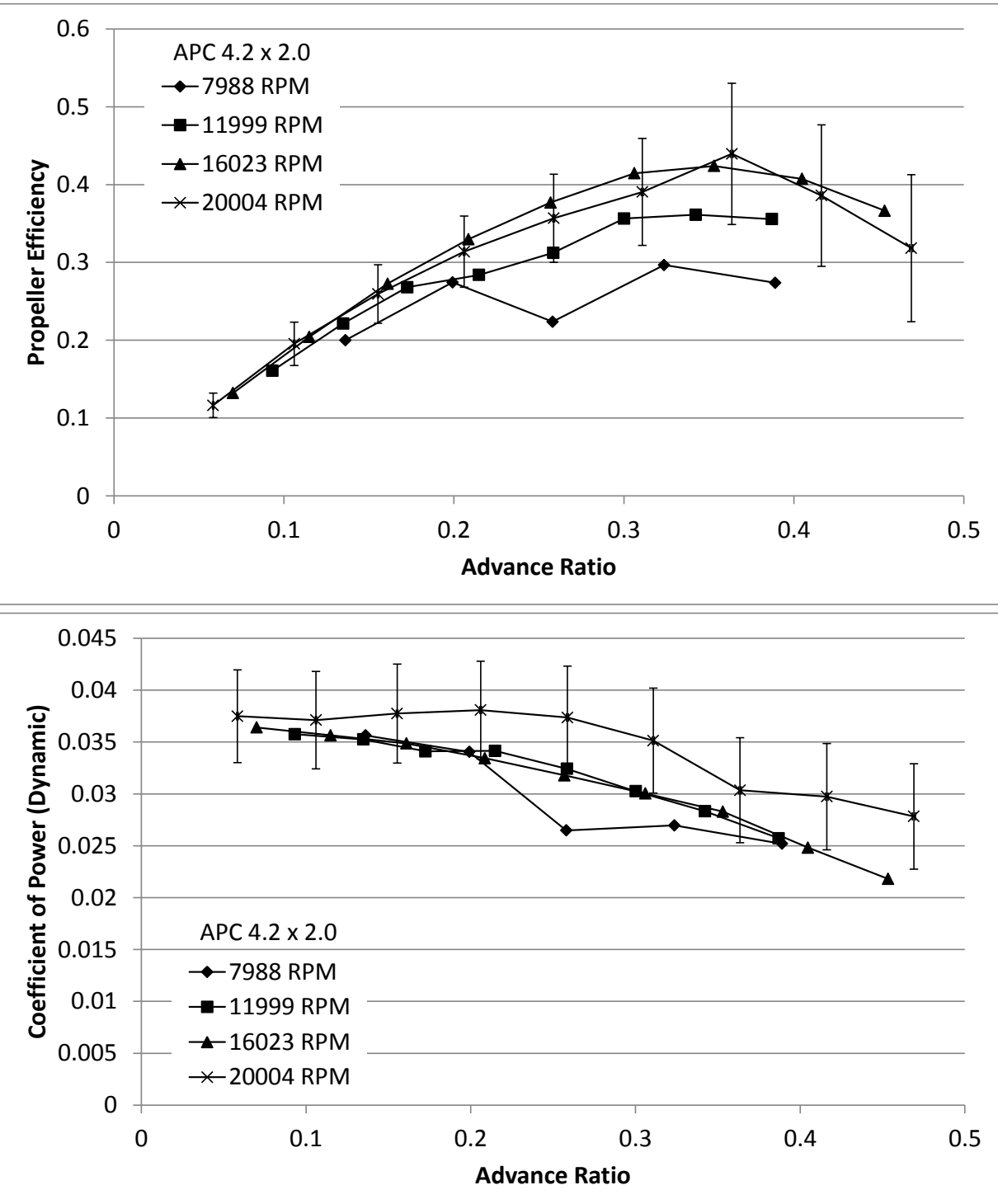

(b)

(c) 


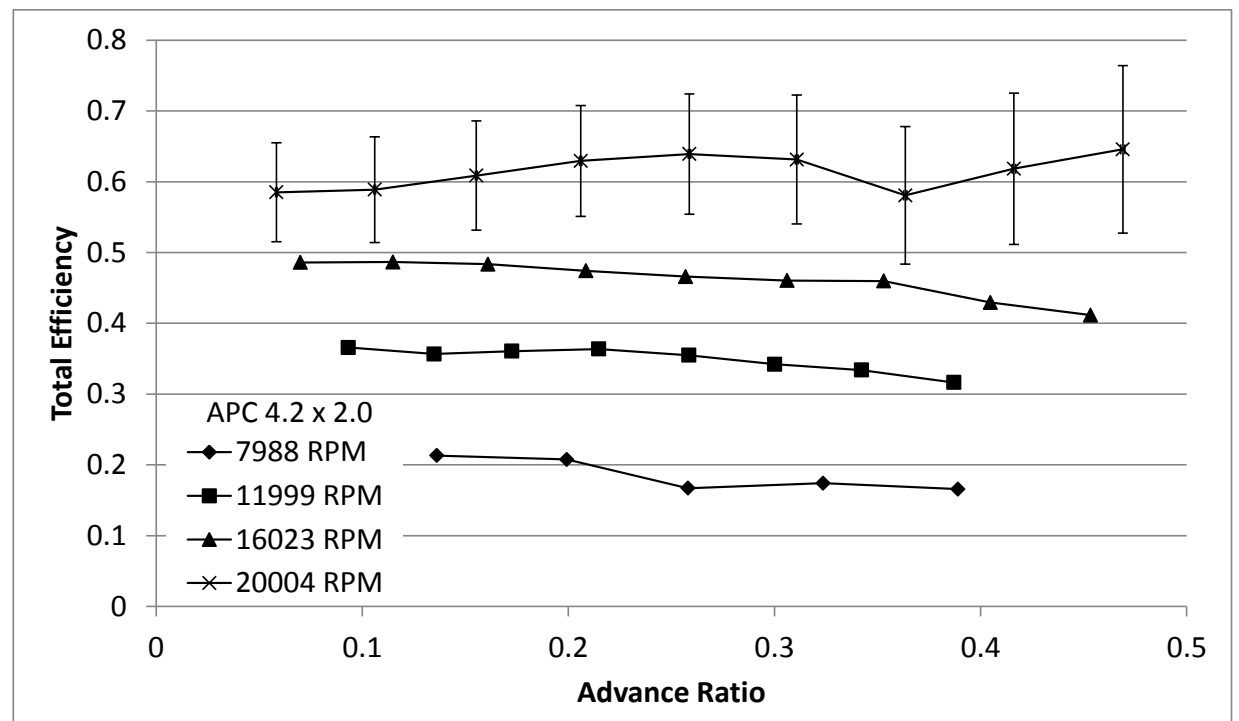

(d)

Figure 79: APC 4.2 x 2.0 Dynamic Test Results: (a) Coefficient of Thrust, (b) Coefficient of Power, (c) Propeller Efficiency, (d) Total Efficiency.

Table 80: APC 4.2 x 2.0 Dynamic Measured Values - 7988 RPM

\begin{tabular}{|c|c|c|c|c|c|c|c|}
\hline$n(\mathrm{RPM})$ & $Q(\mathrm{~g}-\mathrm{m})$ & $\Delta Q(\mathrm{~g}-\mathrm{m})$ & $V(\mathrm{~V})$ & $I(\mathrm{~A})$ & $T_{\text {atm }}\left({ }^{\circ} \mathrm{C}\right)$ & $P_{\text {atm }}(\mathrm{Pa})$ & $P_{\text {diff }}(\mathrm{Pa})$ \\
\hline $8.016 \mathrm{E}+03$ & $1.591 \mathrm{E}-01$ & $6.69 \mathrm{E}-02$ & $1.110 \mathrm{E}+01$ & $5.537 \mathrm{E}-01$ & $2.218 \mathrm{E}+01$ & $9.862 \mathrm{E}+04$ & $2.221 \mathrm{E}+00$ \\
\hline $7.951 \mathrm{E}+03$ & $1.495 \mathrm{E}-01$ & $6.80 \mathrm{E}-02$ & $1.110 \mathrm{E}+01$ & $5.298 \mathrm{E}-01$ & $2.220 \mathrm{E}+01$ & $9.860 \mathrm{E}+04$ & $4.616 \mathrm{E}+00$ \\
\hline $8.045 \mathrm{E}+03$ & $1.191 \mathrm{E}-01$ & $6.51 \mathrm{E}-02$ & $1.110 \mathrm{E}+01$ & $5.308 \mathrm{E}-01$ & $2.208 \mathrm{E}+01$ & $9.862 \mathrm{E}+04$ & $7.853 \mathrm{E}+00$ \\
\hline $7.981 \mathrm{E}+03$ & $1.193 \mathrm{E}-01$ & $6.65 \mathrm{E}-02$ & $1.110 \mathrm{E}+01$ & $5.061 \mathrm{E}-01$ & $2.223 \mathrm{E}+01$ & $9.861 \mathrm{E}+04$ & $1.213 \mathrm{E}+01$ \\
\hline $7.945 \mathrm{E}+03$ & $1.105 \mathrm{E}-01$ & $6.82 \mathrm{E}-02$ & $1.110 \mathrm{E}+01$ & $4.901 \mathrm{E}-01$ & $2.215 \mathrm{E}+01$ & $9.861 \mathrm{E}+04$ & $1.732 \mathrm{E}+01$ \\
\hline
\end{tabular}


Table 81: APC 4.2 x 2.0 Dynamic Calculated Values - 7988 RPM

\begin{tabular}{|c|c|c|c|c|c|c|c|}
\hline$n(\mathrm{RPM})$ & $V_{\infty}^{\prime}(\mathrm{m} / \mathrm{s})$ & $\Delta V_{\infty}^{\prime}(\mathrm{m} / \mathrm{s})$ & $T^{\prime}(\mathrm{g})$ & $\Delta T^{\prime}(\mathrm{g})$ & $V_{\mathrm{t}}(\mathrm{m} / \mathrm{s})$ & $P_{\mathrm{P}}(\mathrm{W})$ & $\Delta P_{\mathrm{P}}(\mathrm{W})$ \\
\hline $8.016 \mathrm{E}+03$ & $1.925 \mathrm{E}+00$ & $1.53 \mathrm{E}-02$ & $1.387 \mathrm{E}+01$ & $1.04 \mathrm{E}+01$ & $3.336 \mathrm{E}+01$ & $1.310 \mathrm{E}+00$ & $5.51 \mathrm{E}-01$ \\
\hline $7.951 \mathrm{E}+03$ & $2.793 \mathrm{E}+00$ & $1.63 \mathrm{E}-02$ & $1.223 \mathrm{E}+01$ & $1.05 \mathrm{E}+01$ & $3.315 \mathrm{E}+01$ & $1.221 \mathrm{E}+00$ & $5.55 \mathrm{E}-01$ \\
\hline $8.045 \mathrm{E}+03$ & $3.660 \mathrm{E}+00$ & $2.01 \mathrm{E}-02$ & $6.135 \mathrm{E}+00$ & $1.02 \mathrm{E}+01$ & $3.362 \mathrm{E}+01$ & $9.842 \mathrm{E}-01$ & $5.38 \mathrm{E}-01$ \\
\hline $7.981 \mathrm{E}+03$ & $4.554 \mathrm{E}+00$ & $1.98 \mathrm{E}-02$ & $6.493 \mathrm{E}+00$ & $1.04 \mathrm{E}+01$ & $3.347 \mathrm{E}+01$ & $9.777 \mathrm{E}-01$ & $5.45 \mathrm{E}-01$ \\
\hline $7.945 \mathrm{E}+03$ & $5.449 \mathrm{E}+00$ & $2.05 \mathrm{E}-02$ & $4.619 \mathrm{E}+00$ & $1.05 \mathrm{E}+01$ & $3.345 \mathrm{E}+01$ & $9.016 \mathrm{E}-01$ & $5.57 \mathrm{E}-01$ \\
\hline$P_{\mathrm{e}}(\mathrm{W})$ & $\Delta P_{\mathrm{e}}(\mathrm{W})$ & $C_{\mathrm{T}}$ & $\Delta C_{\mathrm{T}}$ & $C_{\mathrm{P}}$ & $\Delta C_{\mathrm{P}}$ & $C_{\mathrm{Q}}$ & $\Delta C_{\mathrm{Q}}$ \\
\hline $6.146 \mathrm{E}+00$ & $8.56 \mathrm{E}-02$ & $5.230 \mathrm{E}-02$ & $3.91 \mathrm{E}-02$ & $3.564 \mathrm{E}-02$ & $1.50 \mathrm{E}-02$ & $5.672 \mathrm{E}-03$ & $2.39 \mathrm{E}-03$ \\
\hline $5.881 \mathrm{E}+00$ & 8.29E-02 & 4.687E-02 & $4.02 \mathrm{E}-02$ & $3.405 \mathrm{E}-02$ & $1.55 \mathrm{E}-02$ & $5.419 \mathrm{E}-03$ & $2.46 \mathrm{E}-03$ \\
\hline $5.892 \mathrm{E}+00$ & 8.37E-02 & $2.296 \mathrm{E}-02$ & $3.82 \mathrm{E}-02$ & $2.648 \mathrm{E}-02$ & $1.45 \mathrm{E}-02$ & $4.215 \mathrm{E}-03$ & $2.30 \mathrm{E}-03$ \\
\hline $5.618 \mathrm{E}+00$ & 7.98E-02 & $2.471 \mathrm{E}-02$ & $3.96 \mathrm{E}-02$ & $2.696 \mathrm{E}-02$ & $1.50 \mathrm{E}-02$ & $4.291 \mathrm{E}-03$ & 2.39E-03 \\
\hline $5.441 \mathrm{E}+00$ & 7.72E-02 & $1.773 \mathrm{E}-02$ & $4.02 \mathrm{E}-02$ & $2.519 \mathrm{E}-02$ & $1.56 \mathrm{E}-02$ & $4.010 \mathrm{E}-03$ & $2.48 \mathrm{E}-03$ \\
\hline$\rho\left(\mathrm{kg} / \mathrm{m}^{\wedge} 3\right)$ & $\eta_{\mathrm{T}}$ & $\Delta \eta_{\mathrm{T}}$ & $J$ & $\Delta J$ & $\eta_{\mathrm{P}}$ & $\Delta \eta_{\mathrm{P}}$ & $\operatorname{Re}_{0.75}$ \\
\hline $1.163 \mathrm{E}+00$ & $2.132 \mathrm{E}-01$ & 8.97E-02 & $1.362 \mathrm{E}-01$ & $1.08 \mathrm{E}-03$ & $1.999 \mathrm{E}-01$ & $1.72 \mathrm{E}-01$ & $1.853 \mathrm{E}+04$ \\
\hline $1.163 \mathrm{E}+00$ & $2.076 \mathrm{E}-01$ & $9.45 \mathrm{E}-02$ & $1.992 \mathrm{E}-01$ & $1.17 \mathrm{E}-03$ & $2.743 \mathrm{E}-01$ & $2.66 \mathrm{E}-01$ & $1.840 \mathrm{E}+04$ \\
\hline $1.164 \mathrm{E}+00$ & $1.671 \mathrm{E}-01$ & $9.13 \mathrm{E}-02$ & $2.580 \mathrm{E}-01$ & $1.42 \mathrm{E}-03$ & $2.237 \mathrm{E}-01$ & 3.92E-01 & $1.868 \mathrm{E}+04$ \\
\hline $1.163 \mathrm{E}+00$ & $1.740 \mathrm{E}-01$ & $9.70 \mathrm{E}-02$ & $3.236 \mathrm{E}-01$ & $1.42 \mathrm{E}-03$ & $2.966 \mathrm{E}-01$ & $5.04 \mathrm{E}-01$ & $1.858 \mathrm{E}+04$ \\
\hline $1.163 \mathrm{E}+00$ & $1.657 \mathrm{E}-01$ & $1.02 \mathrm{E}-01$ & $3.890 \mathrm{E}-01$ & $1.48 \mathrm{E}-03$ & $2.737 \mathrm{E}-01$ & $6.43 \mathrm{E}-01$ & $1.858 \mathrm{E}+04$ \\
\hline
\end{tabular}


Table 82: APC 4.2 x 2.0 Dynamic Measured Values - 11999 RPM

\begin{tabular}{|c|c|c|c|c|c|c|c|}
\hline$n(\mathrm{RPM})$ & $Q(\mathrm{~g}-\mathrm{m})$ & $\Delta Q(\mathrm{~g}-\mathrm{m})$ & $V(\mathrm{~V})$ & $I(\mathrm{~A})$ & $T_{\text {atm }}\left({ }^{\circ} \mathrm{C}\right)$ & $P_{\text {atm }}(\mathrm{Pa})$ & $P_{\text {diff }}(\mathrm{Pa})$ \\
\hline $1.210 \mathrm{E}+04$ & $3.636 \mathrm{E}-01$ & $6.02 \mathrm{E}-02$ & $1.108 \mathrm{E}+01$ & $1.114 \mathrm{E}+00$ & $2.212 \mathrm{E}+01$ & $9.859 \mathrm{E}+04$ & $2.416 \mathrm{E}+00$ \\
\hline $1.183 \mathrm{E}+04$ & $3.426 \mathrm{E}-01$ & $5.96 \mathrm{E}-02$ & $1.109 \mathrm{E}+01$ & $1.053 \mathrm{E}+00$ & $2.208 \mathrm{E}+01$ & $9.859 \mathrm{E}+04$ & $4.746 \mathrm{E}+00$ \\
\hline $1.210 \mathrm{E}+04$ & $3.467 \mathrm{E}-01$ & $5.97 \mathrm{E}-02$ & $1.108 \mathrm{E}+01$ & $1.077 \mathrm{E}+00$ & $2.207 \mathrm{E}+01$ & $9.860 \mathrm{E}+04$ & $8.057 \mathrm{E}+00$ \\
\hline $1.199 \mathrm{E}+04$ & $3.409 \mathrm{E}-01$ & $5.94 \mathrm{E}-02$ & $1.109 \mathrm{E}+01$ & $1.041 \mathrm{E}+00$ & $2.218 \mathrm{E}+01$ & $9.859 \mathrm{E}+04$ & $1.217 \mathrm{E}+01$ \\
\hline $1.195 \mathrm{E}+04$ & $3.218 \mathrm{E}-01$ & $5.95 \mathrm{E}-02$ & $1.109 \mathrm{E}+01$ & $1.004 \mathrm{E}+00$ & $2.214 \mathrm{E}+01$ & $9.859 \mathrm{E}+04$ & $1.745 \mathrm{E}+01$ \\
\hline $1.200 \mathrm{E}+04$ & $3.024 \mathrm{E}-01$ & $5.92 \mathrm{E}-02$ & $1.109 \mathrm{E}+01$ & $9.818 \mathrm{E}-01$ & $2.233 \mathrm{E}+01$ & $9.860 \mathrm{E}+04$ & $2.363 \mathrm{E}+01$ \\
\hline $1.204 \mathrm{E}+04$ & $2.852 \mathrm{E}-01$ & $6.04 \mathrm{E}-02$ & $1.109 \mathrm{E}+01$ & $9.524 \mathrm{E}-01$ & $2.234 \mathrm{E}+01$ & $9.859 \mathrm{E}+04$ & $3.087 \mathrm{E}+01$ \\
\hline $1.199 \mathrm{E}+04$ & $2.567 \mathrm{E}-01$ & $5.92 \mathrm{E}-02$ & $1.109 \mathrm{E}+01$ & $9.007 \mathrm{E}-01$ & $2.237 \mathrm{E}+01$ & $9.859 \mathrm{E}+04$ & $3.905 \mathrm{E}+01$ \\
\hline
\end{tabular}


Table 83: APC 4.2 x 2.0 Dynamic Calculated Values - 11999 RPM

\begin{tabular}{|c|c|c|c|c|c|c|c|}
\hline$n(\mathrm{RPM})$ & $V_{\infty}^{\prime}(\mathrm{m} / \mathrm{s})$ & $\Delta V_{\infty}^{\prime}(\mathrm{m} / \mathrm{s})$ & $T^{\prime}(\mathrm{g})$ & $\Delta T^{\prime}(\mathrm{g})$ & $V_{\mathrm{t}}(\mathrm{m} / \mathrm{s})$ & $P_{\mathrm{P}}(\mathrm{W})$ & $\Delta P_{\mathrm{P}}(\mathrm{W})$ \\
\hline $1.210 \mathrm{E}+04$ & $1.990 \mathrm{E}+00$ & $1.22 \mathrm{E}-02$ & $3.725 \mathrm{E}+01$ & $8.53 \mathrm{E}+00$ & $5.030 \mathrm{E}+01$ & $4.517 \mathrm{E}+00$ & $7.48 \mathrm{E}-01$ \\
\hline $1.183 \mathrm{E}+04$ & $2.812 \mathrm{E}+00$ & $1.22 \mathrm{E}-02$ & $3.342 \mathrm{E}+01$ & $8.61 \mathrm{E}+00$ & $4.921 \mathrm{E}+01$ & $4.162 \mathrm{E}+00$ & $7.24 \mathrm{E}-01$ \\
\hline $1.210 \mathrm{E}+04$ & $3.680 \mathrm{E}+00$ & $1.28 \mathrm{E}-02$ & $3.199 \mathrm{E}+01$ & $8.51 \mathrm{E}+00$ & $5.038 \mathrm{E}+01$ & $4.307 \mathrm{E}+00$ & 7.42E-01 \\
\hline $1.199 \mathrm{E}+04$ & $4.540 \mathrm{E}+00$ & $1.39 \mathrm{E}-02$ & $2.677 \mathrm{E}+01$ & $8.59 \mathrm{E}+00$ & $5.001 \mathrm{E}+01$ & $4.197 \mathrm{E}+00$ & 7.31E-01 \\
\hline $1.195 \mathrm{E}+04$ & $5.447 \mathrm{E}+00$ & $1.52 \mathrm{E}-02$ & $2.310 \mathrm{E}+01$ & $8.56 \mathrm{E}+00$ & $4.995 \mathrm{E}+01$ & $3.950 \mathrm{E}+00$ & 7.31E-01 \\
\hline $1.200 \mathrm{E}+04$ & $6.349 \mathrm{E}+00$ & $1.61 \mathrm{E}-02$ & $2.132 \mathrm{E}+01$ & $8.55 \mathrm{E}+00$ & $5.024 \mathrm{E}+01$ & $3.725 \mathrm{E}+00$ & $7.29 \mathrm{E}-01$ \\
\hline $1.204 \mathrm{E}+04$ & $7.266 \mathrm{E}+00$ & $1.71 \mathrm{E}-02$ & $1.788 \mathrm{E}+01$ & $8.58 \mathrm{E}+00$ & $5.055 \mathrm{E}+01$ & $3.527 \mathrm{E}+00$ & 7.46E-01 \\
\hline $1.199 \mathrm{E}+04$ & $8.181 \mathrm{E}+00$ & $1.85 \mathrm{E}-02$ & $1.401 \mathrm{E}+01$ & $8.60 \mathrm{E}+00$ & $5.047 \mathrm{E}+01$ & $3.160 \mathrm{E}+00$ & 7.29E-01 \\
\hline$P_{\mathrm{e}}(\mathrm{W})$ & $\Delta P_{\mathrm{e}}(\mathrm{W})$ & $C_{\mathrm{T}}$ & $\Delta C_{\mathrm{T}}$ & $C_{\mathrm{P}}$ & $\Delta C_{\mathrm{P}}$ & $C_{\mathrm{Q}}$ & $\Delta C_{\mathrm{Q}}$ \\
\hline $1.235 \mathrm{E}+01$ & $1.50 \mathrm{E}-01$ & $6.168 \mathrm{E}-02$ & $1.41 \mathrm{E}-02$ & $3.576 \mathrm{E}-02$ & 5.92E-03 & $5.691 \mathrm{E}-03$ & $9.42 \mathrm{E}-04$ \\
\hline $1.167 \mathrm{E}+01$ & $1.41 \mathrm{E}-01$ & $5.789 \mathrm{E}-02$ & $1.49 \mathrm{E}-02$ & $3.525 \mathrm{E}-02$ & $6.14 \mathrm{E}-03$ & $5.610 \mathrm{E}-03$ & 9.77E-04 \\
\hline $1.194 \mathrm{E}+01$ & $1.47 \mathrm{E}-01$ & $5.298 \mathrm{E}-02$ & $1.41 \mathrm{E}-02$ & $3.410 \mathrm{E}-02$ & $5.87 \mathrm{E}-03$ & $5.427 \mathrm{E}-03$ & 9.35E-04 \\
\hline $1.154 \mathrm{E}+01$ & $1.41 \mathrm{E}-01$ & $4.513 \mathrm{E}-02$ & $1.45 \mathrm{E}-02$ & $3.414 \mathrm{E}-02$ & $5.94 \mathrm{E}-03$ & $5.434 \mathrm{E}-03$ & $9.46 \mathrm{E}-04$ \\
\hline $1.113 \mathrm{E}+01$ & $1.35 \mathrm{E}-01$ & $3.918 \mathrm{E}-02$ & $1.45 \mathrm{E}-02$ & $3.242 \mathrm{E}-02$ & $6.00 \mathrm{E}-03$ & $5.159 \mathrm{E}-03$ & $9.54 \mathrm{E}-04$ \\
\hline $1.089 \mathrm{E}+01$ & $1.34 \mathrm{E}-01$ & $3.592 \mathrm{E}-02$ & $1.44 \mathrm{E}-02$ & $3.025 \mathrm{E}-02$ & $5.92 \mathrm{E}-03$ & $4.815 \mathrm{E}-03$ & $9.42 \mathrm{E}-04$ \\
\hline $1.056 \mathrm{E}+01$ & $1.31 \mathrm{E}-01$ & $2.990 \mathrm{E}-02$ & $1.44 \mathrm{E}-02$ & $2.833 \mathrm{E}-02$ & 5.99E-03 & $4.508 \mathrm{E}-03$ & $9.54 \mathrm{E}-04$ \\
\hline $9.989 \mathrm{E}+00$ & $1.25 \mathrm{E}-01$ & $2.363 \mathrm{E}-02$ & $1.45 \mathrm{E}-02$ & $2.572 \mathrm{E}-02$ & 5.93E-03 & $4.094 \mathrm{E}-03$ & $9.44 \mathrm{E}-04$ \\
\hline$\rho\left(\mathrm{kg} / \mathrm{m}^{\wedge} 3\right)$ & $\eta_{\mathrm{T}}$ & $\Delta \eta_{\mathrm{T}}$ & $J$ & $\Delta J$ & $\eta_{\mathrm{P}}$ & $\Delta \eta_{\mathrm{P}}$ & $R e_{0.75}$ \\
\hline $1.163 \mathrm{E}+00$ & $3.658 \mathrm{E}-01$ & 6.07E-02 & $9.328 \mathrm{E}-02$ & 5.74E-04 & $1.609 \mathrm{E}-01$ & $4.55 \mathrm{E}-02$ & $2.793 \mathrm{E}+04$ \\
\hline $1.163 \mathrm{E}+00$ & $3.566 \mathrm{E}-01$ & $6.22 \mathrm{E}-02$ & $1.348 \mathrm{E}-01$ & 5.89E-04 & $2.214 \mathrm{E}-01$ & $6.89 \mathrm{E}-02$ & $2.734 \mathrm{E}+04$ \\
\hline $1.163 \mathrm{E}+00$ & $3.607 \mathrm{E}-01$ & $6.23 \mathrm{E}-02$ & $1.725 \mathrm{E}-01$ & $6.05 \mathrm{E}-04$ & $2.681 \mathrm{E}-01$ & 8.50E-02 & $2.799 \mathrm{E}+04$ \\
\hline $1.163 \mathrm{E}+00$ & $3.637 \mathrm{E}-01$ & $6.35 \mathrm{E}-02$ & $2.148 \mathrm{E}-01$ & $6.61 \mathrm{E}-04$ & $2.839 \mathrm{E}-01$ & $1.04 \mathrm{E}-01$ & $2.777 \mathrm{E}+04$ \\
\hline $1.163 \mathrm{E}+00$ & $3.548 \mathrm{E}-01$ & $6.58 \mathrm{E}-02$ & $2.584 \mathrm{E}-01$ & $7.25 \mathrm{E}-04$ & $3.124 \mathrm{E}-01$ & $1.29 \mathrm{E}-01$ & $2.774 \mathrm{E}+04$ \\
\hline $1.162 \mathrm{E}+00$ & $3.422 \mathrm{E}-01$ & $6.71 \mathrm{E}-02$ & $3.002 \mathrm{E}-01$ & $7.66 \mathrm{E}-04$ & $3.564 \mathrm{E}-01$ & $1.59 \mathrm{E}-01$ & $2.787 \mathrm{E}+04$ \\
\hline $1.162 \mathrm{E}+00$ & $3.340 \mathrm{E}-01$ & $7.08 \mathrm{E}-02$ & $3.422 \mathrm{E}-01$ & $8.15 \mathrm{E}-04$ & 3.612E-01 & $1.89 \mathrm{E}-01$ & $2.804 \mathrm{E}+04$ \\
\hline $1.162 \mathrm{E}+00$ & $3.164 \mathrm{E}-01$ & 7.31E-02 & $3.870 \mathrm{E}-01$ & 8.83E-04 & $3.556 \mathrm{E}-01$ & 2.33E-01 & $2.799 \mathrm{E}+04$ \\
\hline
\end{tabular}


Table 84: APC 4.2 x 2.0 Dynamic Measured Values - 16023 RPM

\begin{tabular}{|c|c|c|c|c|c|c|c|}
\hline$n(\mathrm{RPM})$ & $Q(\mathrm{~g}-\mathrm{m})$ & $\Delta Q(\mathrm{~g}-\mathrm{m})$ & $V(\mathrm{~V})$ & $I(\mathrm{~A})$ & $T_{\text {atm }}\left({ }^{\circ} \mathrm{C}\right)$ & $P_{\text {atm }}(\mathrm{Pa})$ & $P_{\text {diff }}(\mathrm{Pa})$ \\
\hline $1.607 \mathrm{E}+04$ & $6.531 \mathrm{E}-01$ & $6.90 \mathrm{E}-02$ & $1.106 \mathrm{E}+01$ & $2.005 \mathrm{E}+00$ & $2.209 \mathrm{E}+01$ & $9.855 \mathrm{E}+04$ & $2.452 \mathrm{E}+00$ \\
\hline $1.603 \mathrm{E}+04$ & $6.364 \mathrm{E}-01$ & $6.81 \mathrm{E}-02$ & $1.106 \mathrm{E}+01$ & $1.947 \mathrm{E}+00$ & $2.196 \mathrm{E}+01$ & $9.855 \mathrm{E}+04$ & $6.385 \mathrm{E}+00$ \\
\hline $1.602 \mathrm{E}+04$ & $6.217 \mathrm{E}-01$ & $6.81 \mathrm{E}-02$ & $1.106 \mathrm{E}+01$ & $1.913 \mathrm{E}+00$ & $2.201 \mathrm{E}+01$ & $9.855 \mathrm{E}+04$ & $1.235 \mathrm{E}+01$ \\
\hline $1.599 \mathrm{E}+04$ & $5.937 \mathrm{E}-01$ & $6.87 \mathrm{E}-02$ & $1.106 \mathrm{E}+01$ & $1.858 \mathrm{E}+00$ & $2.212 \mathrm{E}+01$ & $9.855 \mathrm{E}+04$ & $2.047 \mathrm{E}+01$ \\
\hline $1.598 \mathrm{E}+04$ & $5.636 \mathrm{E}-01$ & $6.79 \mathrm{E}-02$ & $1.106 \mathrm{E}+01$ & $1.794 \mathrm{E}+00$ & $2.225 \mathrm{E}+01$ & $9.854 \mathrm{E}+04$ & $3.084 \mathrm{E}+01$ \\
\hline $1.601 \mathrm{E}+04$ & $5.344 \mathrm{E}-01$ & $6.80 \mathrm{E}-02$ & $1.107 \mathrm{E}+01$ & $1.725 \mathrm{E}+00$ & $2.233 \mathrm{E}+01$ & $9.853 \mathrm{E}+04$ & $4.376 \mathrm{E}+01$ \\
\hline $1.609 \mathrm{E}+04$ & $5.082 \mathrm{E}-01$ & $6.92 \mathrm{E}-02$ & $1.107 \mathrm{E}+01$ & $1.650 \mathrm{E}+00$ & $2.242 \mathrm{E}+01$ & $9.853 \mathrm{E}+04$ & $5.862 \mathrm{E}+01$ \\
\hline $1.599 \mathrm{E}+04$ & $4.402 \mathrm{E}-01$ & $6.77 \mathrm{E}-02$ & $1.107 \mathrm{E}+01$ & $1.520 \mathrm{E}+00$ & $2.246 \mathrm{E}+01$ & $9.853 \mathrm{E}+04$ & $7.591 \mathrm{E}+01$ \\
\hline $1.602 \mathrm{E}+04$ & $3.879 \mathrm{E}-01$ & $6.75 \mathrm{E}-02$ & $1.108 \mathrm{E}+01$ & $1.400 \mathrm{E}+00$ & $2.256 \mathrm{E}+01$ & $9.853 \mathrm{E}+04$ & $9.538 \mathrm{E}+01$ \\
\hline
\end{tabular}


Table 85: APC 4.2 x 2.0 Dynamic Calculated Values - 16023 RPM

\begin{tabular}{|c|c|c|c|c|c|c|c|}
\hline$n(\mathrm{RPM})$ & $V_{\infty}^{\prime}(\mathrm{m} / \mathrm{s})$ & $\Delta V_{\infty}^{\prime}(\mathrm{m} / \mathrm{s})$ & $T^{\prime}(\mathrm{g})$ & $\Delta T^{\prime}(\mathrm{g})$ & $V_{\mathrm{t}}(\mathrm{m} / \mathrm{s})$ & $P_{\mathrm{P}}(\mathrm{W})$ & $\Delta P_{\mathrm{P}}(\mathrm{W})$ \\
\hline $1.607 \mathrm{E}+04$ & $1.984 \mathrm{E}+00$ & $1.31 \mathrm{E}-02$ & $7.320 \mathrm{E}+01$ & $7.90 \mathrm{E}+00$ & $6.679 \mathrm{E}+01$ & $1.078 \mathrm{E}+01$ & $1.14 \mathrm{E}+00$ \\
\hline $1.603 \mathrm{E}+04$ & $3.249 \mathrm{E}+00$ & $1.33 \mathrm{E}-02$ & $6.716 \mathrm{E}+01$ & $7.97 \mathrm{E}+00$ & $6.667 \mathrm{E}+01$ & $1.048 \mathrm{E}+01$ & $1.12 \mathrm{E}+00$ \\
\hline $1.602 \mathrm{E}+04$ & $4.548 \mathrm{E}+00$ & $1.41 \mathrm{E}-02$ & $6.254 \mathrm{E}+01$ & $8.06 \mathrm{E}+00$ & $6.671 \mathrm{E}+01$ & $1.023 \mathrm{E}+01$ & $1.12 \mathrm{E}+00$ \\
\hline $1.599 \mathrm{E}+04$ & $5.881 \mathrm{E}+00$ & $1.58 \mathrm{E}-02$ & $5.575 \mathrm{E}+01$ & $8.20 \mathrm{E}+00$ & $6.669 \mathrm{E}+01$ & $9.749 \mathrm{E}+00$ & $1.13 \mathrm{E}+00$ \\
\hline $1.598 \mathrm{E}+04$ & $7.239 \mathrm{E}+00$ & $1.73 \mathrm{E}-02$ & $4.913 \mathrm{E}+01$ & $8.33 \mathrm{E}+00$ & $6.679 \mathrm{E}+01$ & $9.250 \mathrm{E}+00$ & $1.11 \mathrm{E}+00$ \\
\hline $1.601 \mathrm{E}+04$ & $8.641 \mathrm{E}+00$ & $1.91 \mathrm{E}-02$ & $4.299 \mathrm{E}+01$ & $8.25 \mathrm{E}+00$ & $6.707 \mathrm{E}+01$ & $8.788 \mathrm{E}+00$ & $1.12 \mathrm{E}+00$ \\
\hline $1.609 \mathrm{E}+04$ & $1.002 \mathrm{E}+01$ & $2.11 \mathrm{E}-02$ & $3.624 \mathrm{E}+01$ & $8.04 \mathrm{E}+00$ & $6.759 \mathrm{E}+01$ & $8.397 \mathrm{E}+00$ & $1.14 \mathrm{E}+00$ \\
\hline $1.599 \mathrm{E}+04$ & $1.141 \mathrm{E}+01$ & $2.37 \mathrm{E}-02$ & $2.632 \mathrm{E}+01$ & $8.40 \mathrm{E}+00$ & $6.740 \mathrm{E}+01$ & $7.229 \mathrm{E}+00$ & $1.11 \mathrm{E}+00$ \\
\hline $1.602 \mathrm{E}+04$ & $1.281 \mathrm{E}+01$ & $2.60 \mathrm{E}-02$ & $1.861 \mathrm{E}+01$ & $8.39 \mathrm{E}+00$ & $6.776 \mathrm{E}+01$ & $6.380 \mathrm{E}+00$ & $1.11 \mathrm{E}+00$ \\
\hline$P_{\mathrm{e}}(\mathrm{W})$ & $\Delta P_{\mathrm{e}}(\mathrm{W})$ & $C_{\mathrm{T}}$ & $\Delta C_{\mathrm{T}}$ & $C_{\mathrm{P}}$ & $\Delta C_{\mathrm{P}}$ & $C_{\mathrm{Q}}$ & $\Delta C_{\mathrm{Q}}$ \\
\hline $2.218 \mathrm{E}+01$ & $2.57 \mathrm{E}-01$ & $6.870 \mathrm{E}-02$ & 7.42E-03 & $3.640 \mathrm{E}-02$ & $3.84 \mathrm{E}-03$ & $5.794 \mathrm{E}-03$ & $6.12 \mathrm{E}-04$ \\
\hline $2.153 \mathrm{E}+01$ & $2.50 \mathrm{E}-01$ & $6.334 \mathrm{E}-02$ & $7.52 \mathrm{E}-03$ & $3.565 \mathrm{E}-02$ & $3.82 \mathrm{E}-03$ & $5.674 \mathrm{E}-03$ & $6.07 \mathrm{E}-04$ \\
\hline $2.116 \mathrm{E}+01$ & $2.43 \mathrm{E}-01$ & $5.904 \mathrm{E}-02$ & $7.61 \mathrm{E}-03$ & $3.486 \mathrm{E}-02$ & $3.82 \mathrm{E}-03$ & $5.549 \mathrm{E}-03$ & $6.08 \mathrm{E}-04$ \\
\hline $2.056 \mathrm{E}+01$ & 2.37E-01 & $5.286 \mathrm{E}-02$ & 7.78E-03 & 3.344E-02 & $3.87 \mathrm{E}-03$ & $5.321 \mathrm{E}-03$ & $6.16 \mathrm{E}-04$ \\
\hline $1.985 \mathrm{E}+01$ & $2.29 \mathrm{E}-01$ & 4.664E-02 & $7.90 \mathrm{E}-03$ & $3.178 \mathrm{E}-02$ & $3.83 \mathrm{E}-03$ & $5.058 \mathrm{E}-03$ & $6.10 \mathrm{E}-04$ \\
\hline $1.909 \mathrm{E}+01$ & $2.22 \mathrm{E}-01$ & $4.069 \mathrm{E}-02$ & $7.81 \mathrm{E}-03$ & $3.004 \mathrm{E}-02$ & $3.82 \mathrm{E}-03$ & $4.781 \mathrm{E}-03$ & $6.08 \mathrm{E}-04$ \\
\hline $1.827 \mathrm{E}+01$ & $2.14 \mathrm{E}-01$ & $3.398 \mathrm{E}-02$ & 7.54E-03 & $2.830 \mathrm{E}-02$ & $3.85 \mathrm{E}-03$ & $4.503 \mathrm{E}-03$ & $6.13 \mathrm{E}-04$ \\
\hline $1.684 \mathrm{E}+01$ & $1.97 \mathrm{E}-01$ & $2.498 \mathrm{E}-02$ & 7.97E-03 & $2.482 \mathrm{E}-02$ & $3.82 \mathrm{E}-03$ & $3.950 \mathrm{E}-03$ & $6.08 \mathrm{E}-04$ \\
\hline $1.550 \mathrm{E}+01$ & $1.83 \mathrm{E}-01$ & $1.762 \mathrm{E}-02$ & $7.95 \mathrm{E}-03$ & $2.181 \mathrm{E}-02$ & $3.79 \mathrm{E}-03$ & $3.471 \mathrm{E}-03$ & $6.04 \mathrm{E}-04$ \\
\hline$\rho\left(\mathrm{kg} / \mathrm{m}^{\wedge} 3\right)$ & $\eta_{\mathrm{T}}$ & $\Delta \eta_{\mathrm{T}}$ & $J$ & $\Delta J$ & $\eta_{\mathrm{P}}$ & $\Delta \eta_{\mathrm{P}}$ & $R e_{0.75}$ \\
\hline $1.163 \mathrm{E}+00$ & $4.860 \mathrm{E}-01$ & $5.16 \mathrm{E}-02$ & $7.003 \mathrm{E}-02$ & 4.64E-04 & $1.322 \mathrm{E}-01$ & $2.00 \mathrm{E}-02$ & $3.709 \mathrm{E}+04$ \\
\hline $1.163 \mathrm{E}+00$ & $4.865 \mathrm{E}-01$ & 5.24E-02 & $1.150 \mathrm{E}-01$ & 4.71E-04 & $2.043 \mathrm{E}-01$ & $3.27 \mathrm{E}-02$ & $3.705 \mathrm{E}+04$ \\
\hline $1.163 \mathrm{E}+00$ & 4.834E-01 & 5.33E-02 & $1.610 \mathrm{E}-01$ & $5.01 \mathrm{E}-04$ & $2.727 \mathrm{E}-01$ & 4.61E-02 & $3.706 \mathrm{E}+04$ \\
\hline $1.163 \mathrm{E}+00$ & $4.742 \mathrm{E}-01$ & $5.52 \mathrm{E}-02$ & $2.086 \mathrm{E}-01$ & $5.63 \mathrm{E}-04$ & $3.298 \mathrm{E}-01$ & $6.18 \mathrm{E}-02$ & $3.702 \mathrm{E}+04$ \\
\hline $1.162 \mathrm{E}+00$ & $4.661 \mathrm{E}-01$ & $5.64 \mathrm{E}-02$ & $2.569 \mathrm{E}-01$ & $6.15 \mathrm{E}-04$ & $3.770 \mathrm{E}-01$ & $7.84 \mathrm{E}-02$ & $3.705 E+04$ \\
\hline $1.162 \mathrm{E}+00$ & 4.604E-01 & $5.88 \mathrm{E}-02$ & 3.061E-01 & $6.80 \mathrm{E}-04$ & 4.146E-01 & $9.54 \mathrm{E}-02$ & $3.719 \mathrm{E}+04$ \\
\hline $1.161 \mathrm{E}+00$ & $4.597 \mathrm{E}-01$ & $6.28 \mathrm{E}-02$ & $3.530 \mathrm{E}-01$ & $7.48 \mathrm{E}-04$ & $4.239 \mathrm{E}-01$ & $1.10 \mathrm{E}-01$ & $3.745 \mathrm{E}+04$ \\
\hline $1.161 \mathrm{E}+00$ & $4.294 \mathrm{E}-01$ & $6.62 \mathrm{E}-02$ & $4.048 \mathrm{E}-01$ & $8.47 \mathrm{E}-04$ & $4.074 \mathrm{E}-01$ & $1.44 \mathrm{E}-01$ & $3.734 \mathrm{E}+04$ \\
\hline $1.161 \mathrm{E}+00$ & $4.116 \mathrm{E}-01$ & 7.17E-02 & 4.534E-01 & $9.28 \mathrm{E}-04$ & 3.664E-01 & $1.77 \mathrm{E}-01$ & $3.751 \mathrm{E}+04$ \\
\hline
\end{tabular}


Table 86: APC 4.2 x 2.0 Dynamic Measured Values - 20004 RPM

\begin{tabular}{|c|c|c|c|c|c|c|c|}
\hline$n(\mathrm{RPM})$ & $Q(\mathrm{~g}-\mathrm{m})$ & $\Delta Q(\mathrm{~g}-\mathrm{m})$ & $V(\mathrm{~V})$ & $I(\mathrm{~A})$ & $T_{\text {atm }}\left({ }^{\circ} \mathrm{C}\right)$ & $P_{\text {atm }}(\mathrm{Pa})$ & $P_{\text {diff }}(\mathrm{Pa})$ \\
\hline $2.004 \mathrm{E}+04$ & $1.045 \mathrm{E}+00$ & $1.25 \mathrm{E}-01$ & $1.102 \mathrm{E}+01$ & $3.336 \mathrm{E}+00$ & $2.206 \mathrm{E}+01$ & $9.851 \mathrm{E}+04$ & $2.693 \mathrm{E}+00$ \\
\hline $2.000 \mathrm{E}+04$ & $1.031 \mathrm{E}+00$ & $1.30 \mathrm{E}-01$ & $1.102 \mathrm{E}+01$ & $3.262 \mathrm{E}+00$ & $2.194 \mathrm{E}+01$ & $9.852 \mathrm{E}+04$ & $8.502 \mathrm{E}+00$ \\
\hline $2.000 \mathrm{E}+04$ & $1.048 \mathrm{E}+00$ & $1.32 \mathrm{E}-01$ & $1.103 \mathrm{E}+01$ & $3.207 \mathrm{E}+00$ & $2.206 \mathrm{E}+01$ & $9.851 \mathrm{E}+04$ & $1.794 \mathrm{E}+01$ \\
\hline $2.001 \mathrm{E}+04$ & $1.058 \mathrm{E}+00$ & $1.31 \mathrm{E}-01$ & $1.103 \mathrm{E}+01$ & $3.133 \mathrm{E}+00$ & $2.226 \mathrm{E}+01$ & $9.851 \mathrm{E}+04$ & $3.129 \mathrm{E}+01$ \\
\hline $1.999 \mathrm{E}+04$ & $1.036 \mathrm{E}+00$ & $1.37 \mathrm{E}-01$ & $1.103 \mathrm{E}+01$ & $3.015 \mathrm{E}+00$ & $2.240 \mathrm{E}+01$ & $9.850 \mathrm{E}+04$ & $4.893 \mathrm{E}+01$ \\
\hline $1.999 \mathrm{E}+04$ & $9.731 \mathrm{E}-01$ & $1.40 \mathrm{E}-01$ & $1.104 \mathrm{E}+01$ & $2.866 \mathrm{E}+00$ & $2.254 \mathrm{E}+01$ & $9.850 \mathrm{E}+04$ & $7.029 \mathrm{E}+01$ \\
\hline $2.000 \mathrm{E}+04$ & $8.413 \mathrm{E}-01$ & $1.40 \mathrm{E}-01$ & $1.104 \mathrm{E}+01$ & $2.695 \mathrm{E}+00$ & $2.258 \mathrm{E}+01$ & $9.850 \mathrm{E}+04$ & $9.593 \mathrm{E}+01$ \\
\hline $2.001 \mathrm{E}+04$ & $8.248 \mathrm{E}-01$ & $1.42 \mathrm{E}-01$ & $1.105 \mathrm{E}+01$ & $2.481 \mathrm{E}+00$ & $2.262 \mathrm{E}+01$ & $9.851 \mathrm{E}+04$ & $1.255 \mathrm{E}+02$ \\
\hline $2.000 \mathrm{E}+04$ & $7.718 \mathrm{E}-01$ & $1.41 \mathrm{E}-01$ & $1.105 \mathrm{E}+01$ & $2.221 \mathrm{E}+00$ & $2.257 \mathrm{E}+01$ & $9.851 \mathrm{E}+04$ & $1.591 \mathrm{E}+02$ \\
\hline
\end{tabular}


Table 87: APC 4.2 x 2.0 Dynamic Calculated Values - 20004 RPM

\begin{tabular}{|c|c|c|c|c|c|c|c|}
\hline$n(\mathrm{RPM})$ & $V_{\infty}^{\prime}(\mathrm{m} / \mathrm{s})$ & $\Delta V_{\infty}^{\prime}(\mathrm{m} / \mathrm{s})$ & $T^{\prime}(\mathrm{g})$ & $\Delta T^{\prime}(\mathrm{g})$ & $V_{\mathrm{t}}(\mathrm{m} / \mathrm{s})$ & $P_{\mathrm{P}}(\mathrm{W})$ & $\Delta P_{\mathrm{P}}(\mathrm{W})$ \\
\hline $2.004 \mathrm{E}+04$ & $2.062 \mathrm{E}+00$ & $1.50 \mathrm{E}-02$ & $1.237 \mathrm{E}+02$ & $7.61 \mathrm{E}+00$ & $8.327 \mathrm{E}+01$ & $2.151 \mathrm{E}+01$ & $2.56 \mathrm{E}+00$ \\
\hline $2.000 \mathrm{E}+04$ & $3.739 \mathrm{E}+00$ & $1.50 \mathrm{E}-02$ & $1.129 \mathrm{E}+02$ & $7.39 \mathrm{E}+00$ & $8.316 \mathrm{E}+01$ & $2.118 \mathrm{E}+01$ & $2.68 \mathrm{E}+00$ \\
\hline $2.000 \mathrm{E}+04$ & $5.478 \mathrm{E}+00$ & $1.67 \mathrm{E}-02$ & $1.040 \mathrm{E}+02$ & $7.38 \mathrm{E}+00$ & $8.326 \mathrm{E}+01$ & $2.153 \mathrm{E}+01$ & $2.72 \mathrm{E}+00$ \\
\hline $2.001 \mathrm{E}+04$ & $7.269 \mathrm{E}+00$ & $1.87 \mathrm{E}-02$ & $9.574 \mathrm{E}+01$ & $7.36 \mathrm{E}+00$ & $8.346 \mathrm{E}+01$ & $2.175 \mathrm{E}+01$ & $2.69 \mathrm{E}+00$ \\
\hline $1.999 \mathrm{E}+04$ & $9.120 \mathrm{E}+00$ & $2.13 \mathrm{E}-02$ & $8.482 \mathrm{E}+01$ & $7.38 \mathrm{E}+00$ & $8.355 \mathrm{E}+01$ & $2.126 \mathrm{E}+01$ & $2.82 \mathrm{E}+00$ \\
\hline $1.999 \mathrm{E}+04$ & $1.096 \mathrm{E}+01$ & 2.34E-02 & $7.260 \mathrm{E}+01$ & $7.37 \mathrm{E}+00$ & $8.376 \mathrm{E}+01$ & $1.997 \mathrm{E}+01$ & $2.87 \mathrm{E}+00$ \\
\hline $2.000 \mathrm{E}+04$ & $1.282 \mathrm{E}+01$ & $2.65 \mathrm{E}-02$ & $6.041 \mathrm{E}+01$ & $7.35 \mathrm{E}+00$ & $8.407 \mathrm{E}+01$ & $1.728 \mathrm{E}+01$ & $2.88 \mathrm{E}+00$ \\
\hline $2.001 \mathrm{E}+04$ & $1.468 \mathrm{E}+01$ & 2.92E-02 & $4.543 \mathrm{E}+01$ & $7.29 \mathrm{E}+00$ & $8.439 \mathrm{E}+01$ & $1.695 \mathrm{E}+01$ & $2.92 \mathrm{E}+00$ \\
\hline $2.000 \mathrm{E}+04$ & $1.654 \mathrm{E}+01$ & $3.25 \mathrm{E}-02$ & $3.111 \mathrm{E}+01$ & $7.28 \mathrm{E}+00$ & $8.472 \mathrm{E}+01$ & $1.585 \mathrm{E}+01$ & $2.90 \mathrm{E}+00$ \\
\hline$P_{\mathrm{e}}(\mathrm{W})$ & $\Delta P_{\mathrm{e}}(\mathrm{W})$ & $C_{\mathrm{T}}$ & $\Delta C_{\mathrm{T}}$ & $C_{\mathrm{P}}$ & $\Delta C_{\mathrm{P}}$ & $C_{\mathrm{Q}}$ & $\Delta C_{\mathrm{Q}}$ \\
\hline $3.677 \mathrm{E}+01$ & 4.22E-01 & $7.470 \mathrm{E}-02$ & $4.59 \mathrm{E}-03$ & 3.749E-02 & 4.47E-03 & $5.967 \mathrm{E}-03$ & $7.11 \mathrm{E}-04$ \\
\hline $3.596 \mathrm{E}+01$ & $4.08 \mathrm{E}-01$ & $6.839 \mathrm{E}-02$ & $4.48 \mathrm{E}-03$ & $3.711 \mathrm{E}-02$ & $4.69 \mathrm{E}-03$ & $5.907 \mathrm{E}-03$ & 7.47E-04 \\
\hline $3.536 \mathrm{E}+01$ & $3.98 \mathrm{E}-01$ & $6.304 \mathrm{E}-02$ & $4.47 \mathrm{E}-03$ & $3.774 \mathrm{E}-02$ & $4.76 \mathrm{E}-03$ & $6.007 \mathrm{E}-03$ & $7.58 \mathrm{E}-04$ \\
\hline $3.456 \mathrm{E}+01$ & $3.92 \mathrm{E}-01$ & $5.800 \mathrm{E}-02$ & 4.46E-03 & $3.808 \mathrm{E}-02$ & 4.72E-03 & $6.061 \mathrm{E}-03$ & $7.51 \mathrm{E}-04$ \\
\hline $3.326 \mathrm{E}+01$ & $3.75 \mathrm{E}-01$ & $5.152 \mathrm{E}-02$ & $4.48 \mathrm{E}-03$ & $3.736 \mathrm{E}-02$ & $4.95 \mathrm{E}-03$ & $5.946 \mathrm{E}-03$ & $7.88 \mathrm{E}-04$ \\
\hline $3.163 \mathrm{E}+01$ & $3.58 \mathrm{E}-01$ & 4.414E-02 & $4.48 \mathrm{E}-03$ & $3.513 \mathrm{E}-02$ & $5.05 \mathrm{E}-03$ & $5.592 \mathrm{E}-03$ & $8.04 \mathrm{E}-04$ \\
\hline $2.975 \mathrm{E}+01$ & $3.40 \mathrm{E}-01$ & $3.669 \mathrm{E}-02$ & $4.46 \mathrm{E}-03$ & $3.035 \mathrm{E}-02$ & $5.06 \mathrm{E}-03$ & $4.830 \mathrm{E}-03$ & 8.05E-04 \\
\hline $2.740 \mathrm{E}+01$ & $3.10 \mathrm{E}-01$ & $2.757 \mathrm{E}-02$ & $4.42 \mathrm{E}-03$ & $2.974 \mathrm{E}-02$ & $5.13 \mathrm{E}-03$ & $4.733 \mathrm{E}-03$ & $8.16 \mathrm{E}-04$ \\
\hline $2.455 \mathrm{E}+01$ & $2.81 \mathrm{E}-01$ & $1.889 \mathrm{E}-02$ & $4.42 \mathrm{E}-03$ & $2.783 \mathrm{E}-02$ & $5.08 \mathrm{E}-03$ & $4.429 \mathrm{E}-03$ & $8.09 \mathrm{E}-04$ \\
\hline$\rho\left(\mathrm{kg} / \mathrm{m}^{\wedge} 3\right)$ & $\eta_{\mathrm{T}}$ & $\Delta \eta_{\mathrm{T}}$ & $J$ & $\Delta J$ & $\eta_{\mathrm{P}}$ & $\Delta \eta_{\mathrm{P}}$ & $R e_{0.75}$ \\
\hline $1.163 \mathrm{E}+00$ & $5.852 \mathrm{E}-01$ & $7.00 \mathrm{E}-02$ & $5.837 \mathrm{E}-02$ & $4.25 \mathrm{E}-04$ & $1.163 \mathrm{E}-01$ & $1.56 \mathrm{E}-02$ & $4.623 \mathrm{E}+04$ \\
\hline $1.163 \mathrm{E}+00$ & $5.889 \mathrm{E}-01$ & 7.47E-02 & $1.061 \mathrm{E}-01$ & $4.29 \mathrm{E}-04$ & $1.954 \mathrm{E}-01$ & $2.78 \mathrm{E}-02$ & $4.620 \mathrm{E}+04$ \\
\hline $1.163 \mathrm{E}+00$ & $6.088 \mathrm{E}-01$ & 7.72E-02 & $1.554 \mathrm{E}-01$ & $4.78 \mathrm{E}-04$ & $2.595 \mathrm{E}-01$ & $3.76 \mathrm{E}-02$ & $4.622 \mathrm{E}+04$ \\
\hline $1.162 \mathrm{E}+00$ & $6.296 \mathrm{E}-01$ & 7.83E-02 & $2.060 \mathrm{E}-01$ & $5.35 \mathrm{E}-04$ & $3.137 \mathrm{E}-01$ & $4.58 \mathrm{E}-02$ & $4.628 \mathrm{E}+04$ \\
\hline $1.161 \mathrm{E}+00$ & $6.392 \mathrm{E}-01$ & $8.51 \mathrm{E}-02$ & $2.587 \mathrm{E}-01$ & $6.13 \mathrm{E}-04$ & $3.568 \mathrm{E}-01$ & $5.66 \mathrm{E}-02$ & $4.629 \mathrm{E}+04$ \\
\hline $1.160 \mathrm{E}+00$ & $6.315 \mathrm{E}-01$ & $9.11 \mathrm{E}-02$ & $3.108 \mathrm{E}-01$ & $6.75 \mathrm{E}-04$ & $3.905 \mathrm{E}-01$ & $6.87 \mathrm{E}-02$ & $4.636 \mathrm{E}+04$ \\
\hline $1.160 \mathrm{E}+00$ & $5.808 \mathrm{E}-01$ & $9.71 \mathrm{E}-02$ & $3.635 \mathrm{E}-01$ & 7.64E-04 & 4.395E-01 & $9.07 \mathrm{E}-02$ & $4.652 \mathrm{E}+04$ \\
\hline $1.160 \mathrm{E}+00$ & $6.184 \mathrm{E}-01$ & $1.07 \mathrm{E}-01$ & $4.162 \mathrm{E}-01$ & $8.42 \mathrm{E}-04$ & $3.859 \mathrm{E}-01$ & $9.09 \mathrm{E}-02$ & $4.670 \mathrm{E}+04$ \\
\hline $1.161 \mathrm{E}+00$ & $6.459 \mathrm{E}-01$ & $1.18 \mathrm{E}-01$ & $4.690 \mathrm{E}-01$ & $9.39 \mathrm{E}-04$ & $3.183 \mathrm{E}-01$ & $9.45 \mathrm{E}-02$ & $4.689 \mathrm{E}+04$ \\
\hline
\end{tabular}




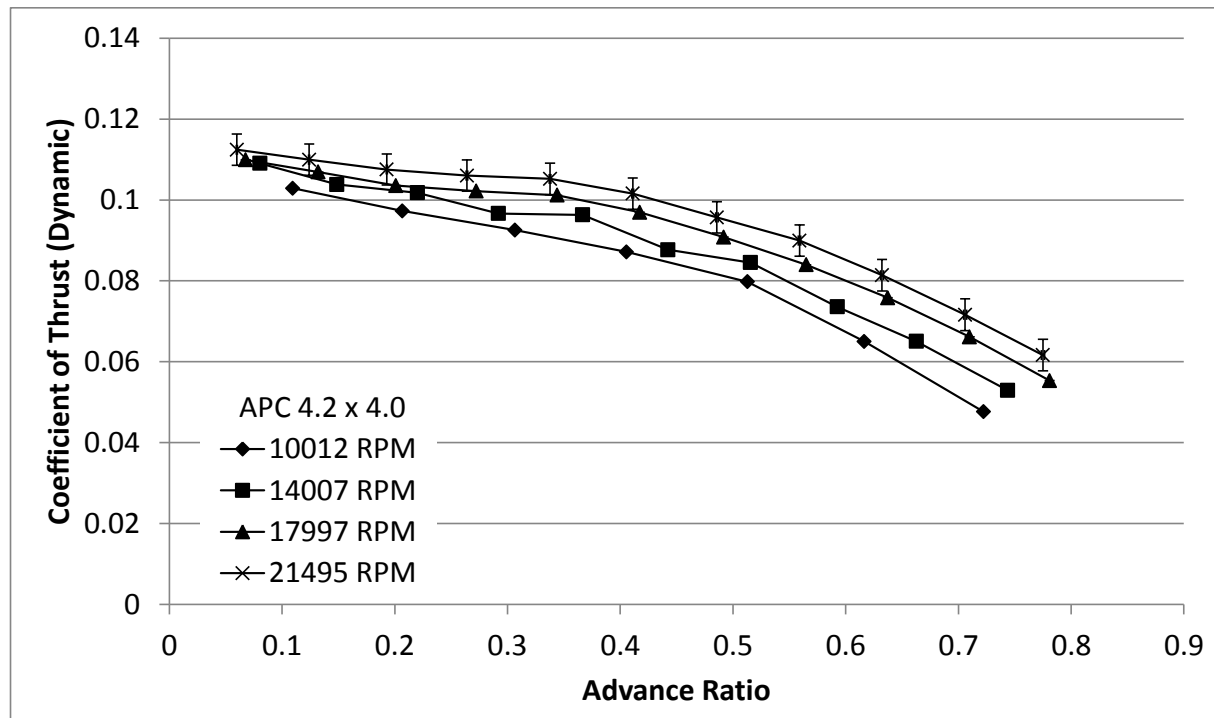

(a)

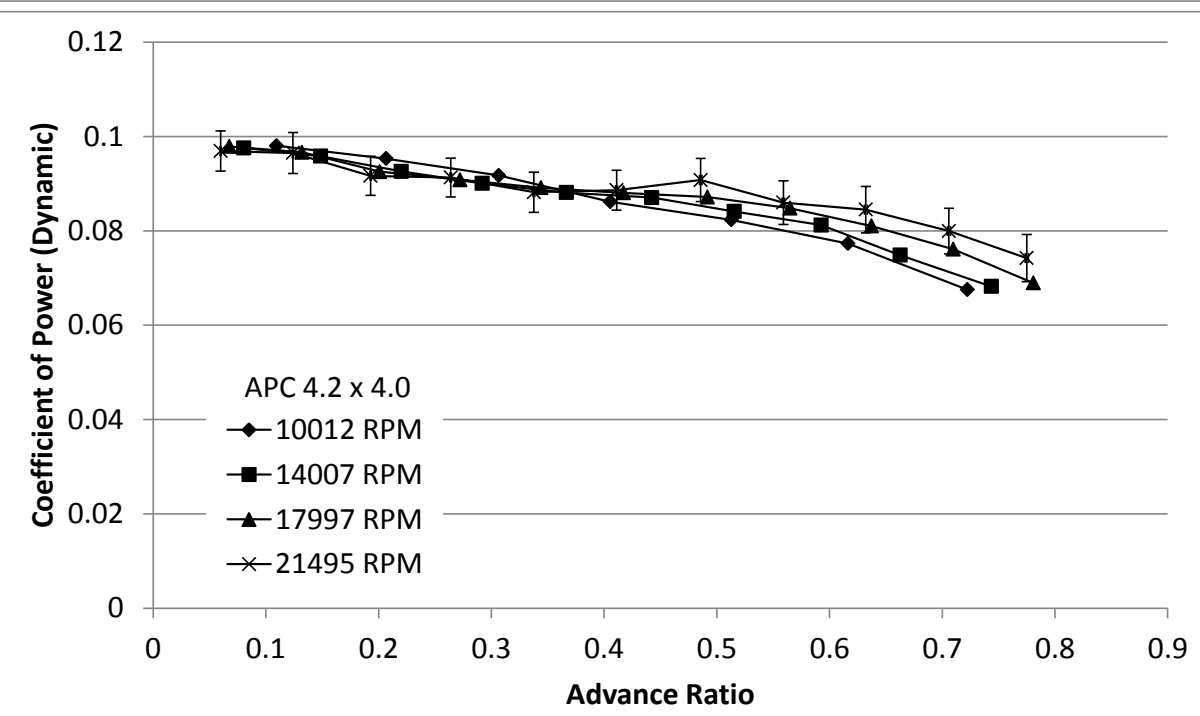

(b)

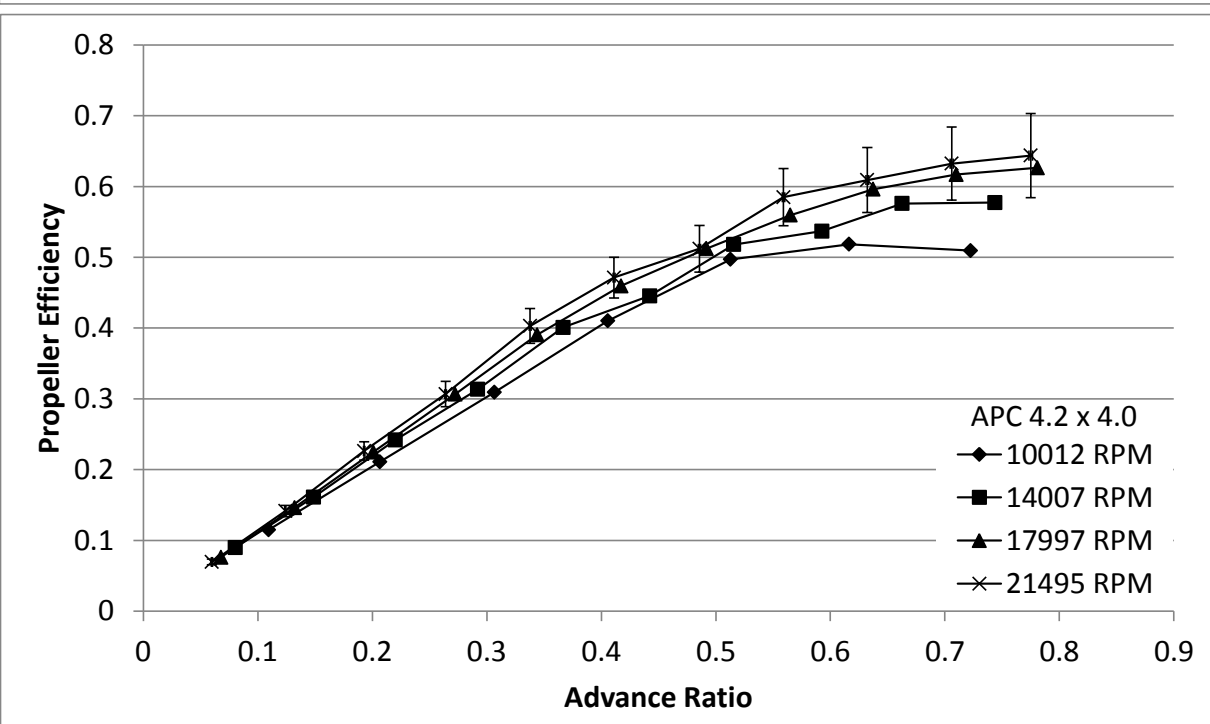

(c) 


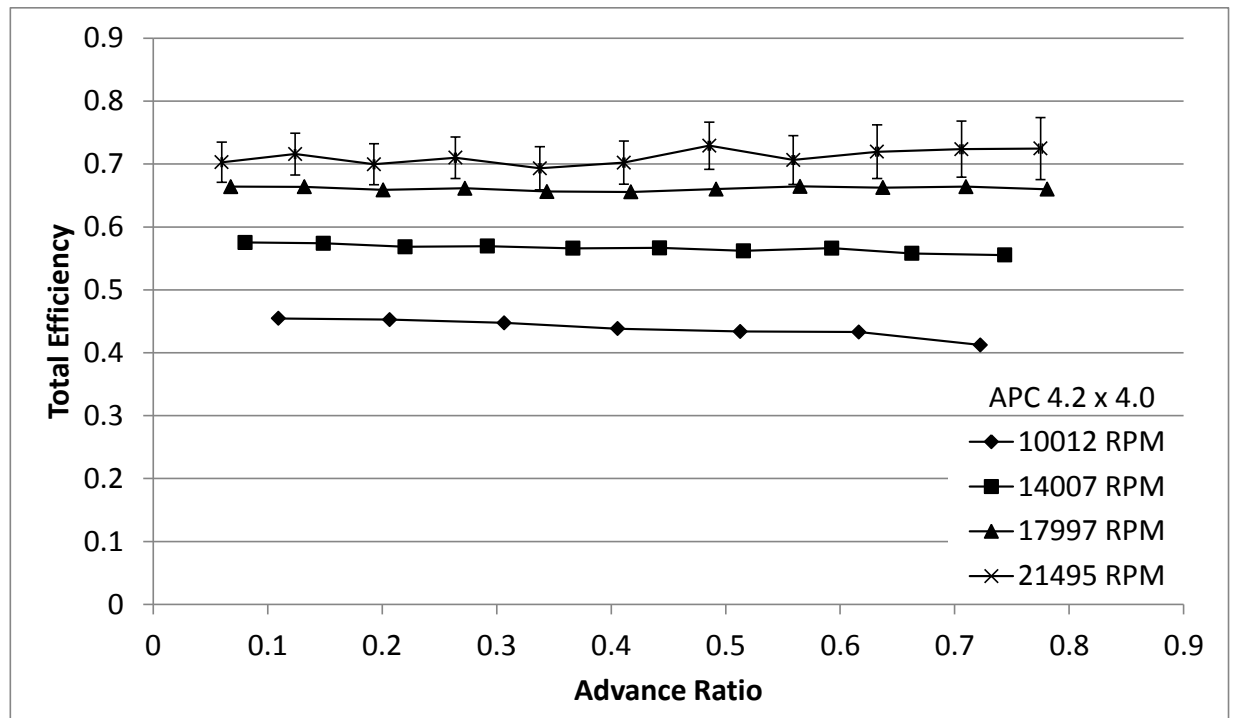

(d)

Figure 80: APC 4.2 x 4.0 Dynamic Test Results: (a) Coefficient of Thrust, (b) Coefficient of Power, (c) Propeller Efficiency, (d) Total Efficiency.

Table 88: APC 4.2 x 4.0 Dynamic Measured Values - 10012 RPM

\begin{tabular}{|c|c|c|c|c|c|c|c|}
\hline$n(\mathrm{RPM})$ & $Q(\mathrm{~g}-\mathrm{m})$ & $\Delta Q(\mathrm{~g}-\mathrm{m})$ & $V(\mathrm{~V})$ & $I(\mathrm{~A})$ & $T_{\text {atm }}\left({ }^{\circ} \mathrm{C}\right)$ & $P_{\text {atm }}(\mathrm{Pa})$ & $P_{\text {diff }}(\mathrm{Pa})$ \\
\hline $9.996 \mathrm{E}+03$ & $6.999 \mathrm{E}-01$ & $5.64 \mathrm{E}-02$ & $1.108 \mathrm{E}+01$ & $1.427 \mathrm{E}+00$ & $2.193 \mathrm{E}+01$ & $9.878 \mathrm{E}+04$ & $2.316 \mathrm{E}+00$ \\
\hline $9.980 \mathrm{E}+03$ & $6.784 \mathrm{E}-01$ & $5.64 \mathrm{E}-02$ & $1.108 \mathrm{E}+01$ & $1.387 \mathrm{E}+00$ & $2.185 \mathrm{E}+01$ & $9.878 \mathrm{E}+04$ & $7.998 \mathrm{E}+00$ \\
\hline $9.978 \mathrm{E}+03$ & $6.525 \mathrm{E}-01$ & $5.67 \mathrm{E}-02$ & $1.108 \mathrm{E}+01$ & $1.349 \mathrm{E}+00$ & $2.195 \mathrm{E}+01$ & $9.878 \mathrm{E}+04$ & $1.740 \mathrm{E}+01$ \\
\hline $1.009 \mathrm{E}+04$ & $6.271 \mathrm{E}-01$ & $5.83 \mathrm{E}-02$ & $1.108 \mathrm{E}+01$ & $1.339 \mathrm{E}+00$ & $2.207 \mathrm{E}+01$ & $9.878 \mathrm{E}+04$ & $3.101 \mathrm{E}+01$ \\
\hline $1.002 \mathrm{E}+04$ & $5.899 \mathrm{E}-01$ & $5.56 \mathrm{E}-02$ & $1.108 \mathrm{E}+01$ & $1.263 \mathrm{E}+00$ & $2.222 \mathrm{E}+01$ & $9.877 \mathrm{E}+04$ & $4.864 \mathrm{E}+01$ \\
\hline $1.002 \mathrm{E}+04$ & $5.537 \mathrm{E}-01$ & $5.57 \mathrm{E}-02$ & $1.108 \mathrm{E}+01$ & $1.187 \mathrm{E}+00$ & $2.232 \mathrm{E}+01$ & $9.877 \mathrm{E}+04$ & $7.004 \mathrm{E}+01$ \\
\hline $9.999 \mathrm{E}+03$ & $4.817 \mathrm{E}-01$ & $5.54 \mathrm{E}-02$ & $1.109 \mathrm{E}+01$ & $1.082 \mathrm{E}+00$ & $2.236 \mathrm{E}+01$ & $9.877 \mathrm{E}+04$ & $9.563 \mathrm{E}+01$ \\
\hline
\end{tabular}


Table 89: APC 4.2 x 4.0 Dynamic Calculated Values - 10012 RPM

\begin{tabular}{|c|c|c|c|c|c|c|c|}
\hline$n(\mathrm{RPM})$ & $V_{\infty}^{\prime}(\mathrm{m} / \mathrm{s})$ & $\Delta V_{\infty}^{\prime}(\mathrm{m} / \mathrm{s})$ & $T^{\prime}(\mathrm{g})$ & $\Delta T^{\prime}(\mathrm{g})$ & $V_{\mathrm{t}}(\mathrm{m} / \mathrm{s})$ & $P_{\mathrm{P}}(\mathrm{W})$ & $\Delta P_{\mathrm{P}}(\mathrm{W})$ \\
\hline $9.996 \mathrm{E}+03$ & $1.940 \mathrm{E}+00$ & $1.20 \mathrm{E}-02$ & $4.341 \mathrm{E}+01$ & $7.81 \mathrm{E}+00$ & $4.179 \mathrm{E}+01$ & $7.185 \mathrm{E}+00$ & $5.79 \mathrm{E}-01$ \\
\hline $9.980 \mathrm{E}+03$ & $3.655 \mathrm{E}+00$ & $1.27 \mathrm{E}-02$ & $4.093 \mathrm{E}+01$ & $7.83 \mathrm{E}+00$ & $4.184 \mathrm{E}+01$ & $6.953 \mathrm{E}+00$ & $5.78 \mathrm{E}-01$ \\
\hline $9.978 \mathrm{E}+03$ & $5.420 \mathrm{E}+00$ & $1.48 \mathrm{E}-02$ & $3.890 \mathrm{E}+01$ & $7.80 \mathrm{E}+00$ & $4.202 \mathrm{E}+01$ & $6.686 \mathrm{E}+00$ & $5.81 \mathrm{E}-01$ \\
\hline $1.009 \mathrm{E}+04$ & $7.256 \mathrm{E}+00$ & $1.67 \mathrm{E}-02$ & $3.748 \mathrm{E}+01$ & $7.84 \mathrm{E}+00$ & $4.278 \mathrm{E}+01$ & $6.501 \mathrm{E}+00$ & $6.05 \mathrm{E}-01$ \\
\hline $1.002 \mathrm{E}+04$ & $9.107 \mathrm{E}+00$ & $1.92 \mathrm{E}-02$ & $3.378 \mathrm{E}+01$ & $7.85 \mathrm{E}+00$ & $4.282 \mathrm{E}+01$ & $6.070 \mathrm{E}+00$ & $5.72 \mathrm{E}-01$ \\
\hline $1.002 \mathrm{E}+04$ & $1.094 \mathrm{E}+01$ & $2.25 \mathrm{E}-02$ & $2.751 \mathrm{E}+01$ & $7.86 \mathrm{E}+00$ & $4.325 \mathrm{E}+01$ & $5.697 \mathrm{E}+00$ & $5.73 \mathrm{E}-01$ \\
\hline $9.999 \mathrm{E}+03$ & $1.280 \mathrm{E}+01$ & $2.60 \mathrm{E}-02$ & $2.008 \mathrm{E}+01$ & $7.85 \mathrm{E}+00$ & $4.367 \mathrm{E}+01$ & $4.946 \mathrm{E}+00$ & $5.69 \mathrm{E}-01$ \\
\hline$P_{\mathrm{e}}(\mathrm{W})$ & $\Delta P_{\mathrm{e}}(\mathrm{W})$ & $C_{\mathrm{T}}$ & $\Delta C_{\mathrm{T}}$ & $C_{\mathrm{P}}$ & $\Delta C_{\mathrm{P}}$ & $C_{\mathrm{Q}}$ & $\Delta C_{\mathrm{Q}}$ \\
\hline $1.580 \mathrm{E}+01$ & $1.86 \mathrm{E}-01$ & $1.029 \mathrm{E}-01$ & $1.85 \mathrm{E}-02$ & $9.803 \mathrm{E}-02$ & $7.90 \mathrm{E}-03$ & $1.560 \mathrm{E}-02$ & $1.26 \mathrm{E}-03$ \\
\hline $1.537 \mathrm{E}+01$ & $1.80 \mathrm{E}-01$ & $9.729 \mathrm{E}-02$ & $1.86 \mathrm{E}-02$ & $9.530 \mathrm{E}-02$ & $7.92 \mathrm{E}-03$ & $1.517 \mathrm{E}-02$ & $1.26 \mathrm{E}-03$ \\
\hline $1.494 \mathrm{E}+01$ & $1.75 \mathrm{E}-01$ & $9.254 \mathrm{E}-02$ & $1.86 \mathrm{E}-02$ & $9.173 \mathrm{E}-02$ & $7.97 \mathrm{E}-03$ & $1.460 \mathrm{E}-02$ & $1.27 \mathrm{E}-03$ \\
\hline $1.483 \mathrm{E}+01$ & $1.76 \mathrm{E}-01$ & $8.715 \mathrm{E}-02$ & $1.82 \mathrm{E}-02$ & $8.618 \mathrm{E}-02$ & $8.02 \mathrm{E}-03$ & $1.372 \mathrm{E}-02$ & $1.28 \mathrm{E}-03$ \\
\hline $1.399 \mathrm{E}+01$ & $1.66 \mathrm{E}-01$ & $7.978 \mathrm{E}-02$ & $1.85 \mathrm{E}-02$ & $8.232 \mathrm{E}-02$ & 7.77E-03 & $1.310 \mathrm{E}-02$ & $1.24 \mathrm{E}-03$ \\
\hline $1.316 \mathrm{E}+01$ & $1.59 \mathrm{E}-01$ & $6.501 \mathrm{E}-02$ & $1.86 \mathrm{E}-02$ & $7.731 \mathrm{E}-02$ & $7.78 \mathrm{E}-03$ & $1.230 \mathrm{E}-02$ & $1.24 \mathrm{E}-03$ \\
\hline $1.200 \mathrm{E}+01$ & $1.45 \mathrm{E}-01$ & $4.763 \mathrm{E}-02$ & $1.86 \mathrm{E}-02$ & $6.754 \mathrm{E}-02$ & $7.77 \mathrm{E}-03$ & $1.075 \mathrm{E}-02$ & $1.24 \mathrm{E}-03$ \\
\hline$\rho\left(\mathrm{kg} / \mathrm{m}^{\wedge} 3\right)$ & $\eta_{\mathrm{T}}$ & $\Delta \eta_{\mathrm{T}}$ & $J$ & $\Delta J$ & $\eta_{\mathrm{P}}$ & $\Delta \eta_{\mathrm{P}}$ & $R e_{0.75}$ \\
\hline $1.166 \mathrm{E}+00$ & $4.547 \mathrm{E}-01$ & $3.70 \mathrm{E}-02$ & $1.095 \mathrm{E}-01$ & $6.78 \mathrm{E}-04$ & $1.149 \mathrm{E}-01$ & $2.27 \mathrm{E}-02$ & $2.352 \mathrm{E}+04$ \\
\hline $1.166 \mathrm{E}+00$ & $4.525 \mathrm{E}-01$ & $3.80 \mathrm{E}-02$ & $2.066 \mathrm{E}-01$ & $7.20 \mathrm{E}-04$ & $2.110 \mathrm{E}-01$ & 4.40E-02 & $2.356 \mathrm{E}+04$ \\
\hline $1.166 \mathrm{E}+00$ & $4.475 \mathrm{E}-01$ & $3.92 \mathrm{E}-02$ & $3.066 \mathrm{E}-01$ & $8.42 \mathrm{E}-04$ & $3.092 \mathrm{E}-01$ & $6.76 \mathrm{E}-02$ & $2.365 \mathrm{E}+04$ \\
\hline $1.166 \mathrm{E}+00$ & $4.383 \mathrm{E}-01$ & $4.11 \mathrm{E}-02$ & $4.056 \mathrm{E}-01$ & $9.43 \mathrm{E}-04$ & $4.102 \mathrm{E}-01$ & $9.40 \mathrm{E}-02$ & $2.406 \mathrm{E}+04$ \\
\hline $1.165 \mathrm{E}+00$ & $4.338 \mathrm{E}-01$ & $4.12 \mathrm{E}-02$ & $5.129 \mathrm{E}-01$ & $1.10 \mathrm{E}-03$ & $4.970 \mathrm{E}-01$ & $1.25 \mathrm{E}-01$ & $2.406 \mathrm{E}+04$ \\
\hline $1.164 \mathrm{E}+00$ & $4.329 \mathrm{E}-01$ & 4.39E-02 & $6.164 \mathrm{E}-01$ & $1.29 \mathrm{E}-03$ & $5.183 \mathrm{E}-01$ & $1.57 \mathrm{E}-01$ & $2.428 \mathrm{E}+04$ \\
\hline $1.164 \mathrm{E}+00$ & $4.124 \mathrm{E}-01$ & 4.77E-02 & $7.224 \mathrm{E}-01$ & $1.49 \mathrm{E}-03$ & $5.095 \mathrm{E}-01$ & $2.08 \mathrm{E}-01$ & $2.452 \mathrm{E}+04$ \\
\hline
\end{tabular}


Table 90: APC 4.2 x 4.0 Dynamic Measured Values - 14007 RPM

\begin{tabular}{|c|c|c|c|c|c|c|c|}
\hline$n(\mathrm{RPM})$ & $Q(\mathrm{~g}-\mathrm{m})$ & $\Delta Q(\mathrm{~g}-\mathrm{m})$ & $V(\mathrm{~V})$ & $I(\mathrm{~A})$ & $T_{\text {atm }}\left({ }^{\circ} \mathrm{C}\right)$ & $P_{\text {atm }}(\mathrm{Pa})$ & $P_{\text {diff }}(\mathrm{Pa})$ \\
\hline $1.401 \mathrm{E}+04$ & $1.368 \mathrm{E}+00$ & $5.97 \mathrm{E}-02$ & $1.103 \mathrm{E}+01$ & $3.104 \mathrm{E}+00$ & $2.207 \mathrm{E}+01$ & $9.876 \mathrm{E}+04$ & $2.508 \mathrm{E}+00$ \\
\hline $1.402 \mathrm{E}+04$ & $1.344 \mathrm{E}+00$ & $5.89 \mathrm{E}-02$ & $1.103 \mathrm{E}+01$ & $3.056 \mathrm{E}+00$ & $2.200 \mathrm{E}+01$ & $9.875 \mathrm{E}+04$ & $8.268 \mathrm{E}+00$ \\
\hline $1.397 \mathrm{E}+04$ & $1.291 \mathrm{E}+00$ & $5.82 \mathrm{E}-02$ & $1.103 \mathrm{E}+01$ & $2.954 \mathrm{E}+00$ & $2.197 \mathrm{E}+01$ & $9.874 \mathrm{E}+04$ & $1.776 \mathrm{E}+01$ \\
\hline $1.401 \mathrm{E}+04$ & $1.263 \mathrm{E}+00$ & $5.80 \mathrm{E}-02$ & $1.104 \mathrm{E}+01$ & $2.893 \mathrm{E}+00$ & $2.193 \mathrm{E}+01$ & $9.874 \mathrm{E}+04$ & $3.120 \mathrm{E}+01$ \\
\hline $1.401 \mathrm{E}+04$ & $1.234 \mathrm{E}+00$ & $5.77 \mathrm{E}-02$ & $1.104 \mathrm{E}+01$ & $2.843 \mathrm{E}+00$ & $2.226 \mathrm{E}+01$ & $9.874 \mathrm{E}+04$ & $4.888 \mathrm{E}+01$ \\
\hline $1.399 \mathrm{E}+04$ & $1.216 \mathrm{E}+00$ & $5.75 \mathrm{E}-02$ & $1.104 \mathrm{E}+01$ & $2.794 \mathrm{E}+00$ & $2.231 \mathrm{E}+01$ & $9.873 \mathrm{E}+04$ & $7.069 \mathrm{E}+01$ \\
\hline $1.402 \mathrm{E}+04$ & $1.180 \mathrm{E}+00$ & $5.76 \mathrm{E}-02$ & $1.104 \mathrm{E}+01$ & $2.741 \mathrm{E}+00$ & $2.228 \mathrm{E}+01$ & $9.874 \mathrm{E}+04$ & $9.630 \mathrm{E}+01$ \\
\hline $1.397 \mathrm{E}+04$ & $1.131 \mathrm{E}+00$ & $5.76 \mathrm{E}-02$ & $1.104 \mathrm{E}+01$ & $2.595 \mathrm{E}+00$ & $2.229 \mathrm{E}+01$ & $9.874 \mathrm{E}+04$ & $1.260 \mathrm{E}+02$ \\
\hline $1.408 \mathrm{E}+04$ & $1.059 \mathrm{E}+00$ & $5.79 \mathrm{E}-02$ & $1.105 \mathrm{E}+01$ & $2.485 \mathrm{E}+00$ & $2.228 \mathrm{E}+01$ & $9.873 \mathrm{E}+04$ & $1.599 \mathrm{E}+02$ \\
\hline $1.397 \mathrm{E}+04$ & $9.486 \mathrm{E}-01$ & $5.78 \mathrm{E}-02$ & $1.105 \mathrm{E}+01$ & $2.216 \mathrm{E}+00$ & $2.235 \mathrm{E}+01$ & $9.873 \mathrm{E}+04$ & $1.976 \mathrm{E}+02$ \\
\hline
\end{tabular}


Table 91: APC 4.2 x 4.0 Dynamic Calculated Values - 14007 RPM

\begin{tabular}{|c|c|c|c|c|c|c|c|}
\hline$n(\mathrm{RPM})$ & $V_{\infty}^{\prime}(\mathrm{m} / \mathrm{s})$ & $\Delta V_{\infty}^{\prime}(\mathrm{m} / \mathrm{s})$ & $T^{\prime}(\mathrm{g})$ & $\Delta T^{\prime}(\mathrm{g})$ & $V_{\mathrm{t}}(\mathrm{m} / \mathrm{s})$ & $P_{\mathrm{P}}(\mathrm{W})$ & $\Delta P_{\mathrm{P}}(\mathrm{W})$ \\
\hline $1.401 \mathrm{E}+04$ & $1.997 \mathrm{E}+00$ & $1.35 \mathrm{E}-02$ & $9.037 \mathrm{E}+01$ & $7.77 \mathrm{E}+00$ & $5.856 \mathrm{E}+01$ & $1.969 \mathrm{E}+01$ & $8.59 \mathrm{E}-01$ \\
\hline $1.402 \mathrm{E}+04$ & $3.693 \mathrm{E}+00$ & $1.41 \mathrm{E}-02$ & $8.608 \mathrm{E}+01$ & $7.75 \mathrm{E}+00$ & $5.865 \mathrm{E}+01$ & $1.935 \mathrm{E}+01$ & $8.48 \mathrm{E}-01$ \\
\hline $1.397 \mathrm{E}+04$ & $5.451 \mathrm{E}+00$ & $1.58 \mathrm{E}-02$ & $8.387 \mathrm{E}+01$ & $7.73 \mathrm{E}+00$ & $5.861 \mathrm{E}+01$ & $1.853 \mathrm{E}+01$ & 8.35E-01 \\
\hline $1.401 \mathrm{E}+04$ & $7.253 \mathrm{E}+00$ & $1.80 \mathrm{E}-02$ & $8.010 \mathrm{E}+01$ & $7.75 \mathrm{E}+00$ & $5.897 \mathrm{E}+01$ & $1.818 \mathrm{E}+01$ & 8.34E-01 \\
\hline $1.401 \mathrm{E}+04$ & $9.104 \mathrm{E}+00$ & $2.05 \mathrm{E}-02$ & $7.974 \mathrm{E}+01$ & $7.73 \mathrm{E}+00$ & $5.922 \mathrm{E}+01$ & $1.776 \mathrm{E}+01$ & $8.31 \mathrm{E}-01$ \\
\hline $1.399 \mathrm{E}+04$ & $1.097 \mathrm{E}+01$ & $2.36 \mathrm{E}-02$ & $7.236 \mathrm{E}+01$ & $7.73 \mathrm{E}+00$ & $5.946 \mathrm{E}+01$ & $1.748 \mathrm{E}+01$ & $8.26 \mathrm{E}-01$ \\
\hline $1.402 \mathrm{E}+04$ & $1.282 \mathrm{E}+01$ & 2.64E-02 & $7.007 \mathrm{E}+01$ & $7.78 \mathrm{E}+00$ & $5.996 \mathrm{E}+01$ & $1.700 \mathrm{E}+01$ & $8.29 \mathrm{E}-01$ \\
\hline $1.397 \mathrm{E}+04$ & $1.467 \mathrm{E}+01$ & $2.96 \mathrm{E}-02$ & $6.055 \mathrm{E}+01$ & $7.73 \mathrm{E}+00$ & $6.016 \mathrm{E}+01$ & $1.622 \mathrm{E}+01$ & 8.26E-01 \\
\hline $1.408 \mathrm{E}+04$ & $1.654 \mathrm{E}+01$ & $3.26 \mathrm{E}-02$ & $5.439 \mathrm{E}+01$ & $7.78 \mathrm{E}+00$ & $6.110 \mathrm{E}+01$ & $1.532 \mathrm{E}+01$ & $8.38 \mathrm{E}-01$ \\
\hline $1.397 \mathrm{E}+04$ & $1.840 \mathrm{E}+01$ & $3.54 \mathrm{E}-02$ & $4.352 \mathrm{E}+01$ & $7.74 \mathrm{E}+00$ & $6.116 \mathrm{E}+01$ & $1.361 \mathrm{E}+01$ & $8.29 \mathrm{E}-01$ \\
\hline$P_{\mathrm{e}}(\mathrm{W})$ & $\Delta P_{\mathrm{e}}(\mathrm{W})$ & $C_{\mathrm{T}}$ & $\Delta C_{\mathrm{T}}$ & $C_{\mathrm{P}}$ & $\Delta C_{\mathrm{P}}$ & $C_{\mathrm{Q}}$ & $\Delta C_{\mathrm{Q}}$ \\
\hline $3.423 \mathrm{E}+01$ & $3.85 \mathrm{E}-01$ & 1.091E-01 & 9.37E-03 & $9.756 \mathrm{E}-02$ & $4.26 \mathrm{E}-03$ & $1.553 \mathrm{E}-02$ & $6.78 \mathrm{E}-04$ \\
\hline $3.371 \mathrm{E}+01$ & $3.78 \mathrm{E}-01$ & $1.038 \mathrm{E}-01$ & $9.35 \mathrm{E}-03$ & $9.581 \mathrm{E}-02$ & $4.20 \mathrm{E}-03$ & $1.525 \mathrm{E}-02$ & $6.69 \mathrm{E}-04$ \\
\hline $3.259 \mathrm{E}+01$ & $3.66 \mathrm{E}-01$ & $1.018 \mathrm{E}-01$ & 9.39E-03 & $9.260 \mathrm{E}-02$ & $4.18 \mathrm{E}-03$ & $1.474 \mathrm{E}-02$ & $6.64 \mathrm{E}-04$ \\
\hline $3.193 \mathrm{E}+01$ & $3.57 \mathrm{E}-01$ & $9.664 \mathrm{E}-02$ & $9.35 \mathrm{E}-03$ & $9.006 \mathrm{E}-02$ & $4.13 \mathrm{E}-03$ & $1.433 \mathrm{E}-02$ & $6.58 \mathrm{E}-04$ \\
\hline $3.138 \mathrm{E}+01$ & $3.51 \mathrm{E}-01$ & $9.633 \mathrm{E}-02$ & $9.34 \mathrm{E}-03$ & $8.810 \mathrm{E}-02$ & 4.12E-03 & $1.402 \mathrm{E}-02$ & $6.56 \mathrm{E}-04$ \\
\hline $3.084 \mathrm{E}+01$ & $3.46 \mathrm{E}-01$ & $8.767 \mathrm{E}-02$ & $9.36 \mathrm{E}-03$ & $8.706 \mathrm{E}-02$ & $4.12 \mathrm{E}-03$ & $1.386 \mathrm{E}-02$ & $6.55 \mathrm{E}-04$ \\
\hline $3.026 \mathrm{E}+01$ & 3.37E-01 & $8.450 \mathrm{E}-02$ & $9.38 \mathrm{E}-03$ & $8.412 \mathrm{E}-02$ & $4.10 \mathrm{E}-03$ & $1.339 \mathrm{E}-02$ & $6.53 \mathrm{E}-04$ \\
\hline $2.866 \mathrm{E}+01$ & $3.21 \mathrm{E}-01$ & $7.358 \mathrm{E}-02$ & $9.40 \mathrm{E}-03$ & $8.121 \mathrm{E}-02$ & $4.14 \mathrm{E}-03$ & $1.292 \mathrm{E}-02$ & $6.58 \mathrm{E}-04$ \\
\hline $2.746 \mathrm{E}+01$ & $3.07 \mathrm{E}-01$ & $6.505 \mathrm{E}-02$ & $9.30 \mathrm{E}-03$ & $7.483 \mathrm{E}-02$ & 4.09E-03 & $1.191 \mathrm{E}-02$ & $6.51 \mathrm{E}-04$ \\
\hline $2.450 \mathrm{E}+01$ & $2.78 \mathrm{E}-01$ & $5.295 \mathrm{E}-02$ & $9.42 \mathrm{E}-03$ & $6.820 \mathrm{E}-02$ & $4.16 \mathrm{E}-03$ & $1.086 \mathrm{E}-02$ & $6.61 \mathrm{E}-04$ \\
\hline$\rho\left(\mathrm{kg} / \mathrm{m}^{\wedge} 3\right)$ & $\eta_{\mathrm{T}}$ & $\Delta \eta_{\mathrm{T}}$ & $J$ & $\Delta J$ & $\eta_{\mathrm{P}}$ & $\Delta \eta_{\mathrm{P}}$ & $R e_{0.75}$ \\
\hline $1.165 \mathrm{E}+00$ & 5.752E-01 & $2.59 \mathrm{E}-02$ & 8.043E-02 & $5.44 \mathrm{E}-04$ & 8.990E-02 & $8.69 \mathrm{E}-03$ & $3.293 \mathrm{E}+04$ \\
\hline $1.166 \mathrm{E}+00$ & $5.739 \mathrm{E}-01$ & $2.60 \mathrm{E}-02$ & $1.487 \mathrm{E}-01$ & $5.69 \mathrm{E}-04$ & $1.612 \mathrm{E}-01$ & $1.61 \mathrm{E}-02$ & $3.299 \mathrm{E}+04$ \\
\hline $1.166 \mathrm{E}+00$ & $5.685 \mathrm{E}-01$ & $2.64 \mathrm{E}-02$ & $2.201 \mathrm{E}-01$ & $6.42 \mathrm{E}-04$ & $2.420 \mathrm{E}-01$ & $2.48 \mathrm{E}-02$ & $3.297 \mathrm{E}+04$ \\
\hline $1.166 \mathrm{E}+00$ & 5.694E-01 & 2.69E-02 & $2.921 \mathrm{E}-01$ & $7.30 \mathrm{E}-04$ & $3.134 \mathrm{E}-01$ & $3.36 \mathrm{E}-02$ & $3.318 \mathrm{E}+04$ \\
\hline $1.164 \mathrm{E}+00$ & $5.660 \mathrm{E}-01$ & $2.72 \mathrm{E}-02$ & $3.666 \mathrm{E}-01$ & 8.33E-04 & 4.008E-01 & 4.32E-02 & $3.326 \mathrm{E}+04$ \\
\hline $1.164 \mathrm{E}+00$ & $5.667 \mathrm{E}-01$ & $2.75 \mathrm{E}-02$ & $4.423 \mathrm{E}-01$ & $9.62 \mathrm{E}-04$ & 4.454E-01 & $5.20 \mathrm{E}-02$ & $3.338 \mathrm{E}+04$ \\
\hline $1.164 \mathrm{E}+00$ & 5.619E-01 & $2.81 \mathrm{E}-02$ & $5.156 \mathrm{E}-01$ & $1.07 \mathrm{E}-03$ & $5.180 \mathrm{E}-01$ & $6.28 \mathrm{E}-02$ & $3.366 \mathrm{E}+04$ \\
\hline $1.164 \mathrm{E}+00$ & 5.662E-01 & $2.95 \mathrm{E}-02$ & $5.926 \mathrm{E}-01$ & $1.21 \mathrm{E}-03$ & 5.370E-01 & $7.38 \mathrm{E}-02$ & $3.378 \mathrm{E}+04$ \\
\hline $1.164 \mathrm{E}+00$ & $5.579 \mathrm{E}-01$ & $3.11 \mathrm{E}-02$ & $6.627 \mathrm{E}-01$ & $1.32 \mathrm{E}-03$ & $5.760 \mathrm{E}-01$ & $8.82 \mathrm{E}-02$ & $3.430 \mathrm{E}+04$ \\
\hline $1.164 \mathrm{E}+00$ & $5.553 \mathrm{E}-01$ & $3.44 \mathrm{E}-02$ & 7.437E-01 & $1.44 \mathrm{E}-03$ & 5.773E-01 & $1.09 \mathrm{E}-01$ & $3.432 \mathrm{E}+04$ \\
\hline
\end{tabular}


Table 92: APC 4.2 x 4.0 Dynamic Measured Values - 17997 RPM

\begin{tabular}{|c|c|c|c|c|c|c|c|}
\hline$n(\mathrm{RPM})$ & $Q(\mathrm{~g}-\mathrm{m})$ & $\Delta Q(\mathrm{~g}-\mathrm{m})$ & $V(\mathrm{~V})$ & $I(\mathrm{~A})$ & $T_{\text {atm }}\left({ }^{\circ} \mathrm{C}\right)$ & $P_{\text {atm }}(\mathrm{Pa})$ & $P_{\text {diff }}(\mathrm{Pa})$ \\
\hline $1.805 \mathrm{E}+04$ & $2.266 \mathrm{E}+00$ & $7.31 \mathrm{E}-02$ & $1.096 \mathrm{E}+01$ & $5.772 \mathrm{E}+00$ & $2.278 \mathrm{E}+01$ & $9.851 \mathrm{E}+04$ & $2.981 \mathrm{E}+00$ \\
\hline $1.801 \mathrm{E}+04$ & $2.227 \mathrm{E}+00$ & $6.80 \mathrm{E}-02$ & $1.096 \mathrm{E}+01$ & $5.660 \mathrm{E}+00$ & $2.278 \mathrm{E}+01$ & $9.852 \mathrm{E}+04$ & $1.078 \mathrm{E}+01$ \\
\hline $1.800 \mathrm{E}+04$ & $2.131 \mathrm{E}+00$ & $6.79 \mathrm{E}-02$ & $1.097 \mathrm{E}+01$ & $5.452 \mathrm{E}+00$ & $2.289 \mathrm{E}+01$ & $9.852 \mathrm{E}+04$ & $2.449 \mathrm{E}+01$ \\
\hline $1.796 \mathrm{E}+04$ & $2.081 \mathrm{E}+00$ & $6.68 \mathrm{E}-02$ & $1.097 \mathrm{E}+01$ & $5.293 \mathrm{E}+00$ & $2.302 \mathrm{E}+01$ & $9.852 \mathrm{E}+04$ & $4.441 \mathrm{E}+01$ \\
\hline $1.799 \mathrm{E}+04$ & $2.048 \mathrm{E}+00$ & $6.72 \mathrm{E}-02$ & $1.097 \mathrm{E}+01$ & $5.258 \mathrm{E}+00$ & $2.313 \mathrm{E}+01$ & $9.852 \mathrm{E}+04$ & $7.072 \mathrm{E}+01$ \\
\hline $1.802 \mathrm{E}+04$ & $2.030 \mathrm{E}+00$ & $6.75 \mathrm{E}-02$ & $1.097 \mathrm{E}+01$ & $5.221 \mathrm{E}+00$ & $2.315 \mathrm{E}+01$ & $9.851 \mathrm{E}+04$ & $1.040 \mathrm{E}+02$ \\
\hline $1.797 \mathrm{E}+04$ & $1.998 \mathrm{E}+00$ & $6.62 \mathrm{E}-02$ & $1.098 \mathrm{E}+01$ & $5.090 \mathrm{E}+00$ & $2.323 \mathrm{E}+01$ & $9.851 \mathrm{E}+04$ & $1.432 \mathrm{E}+02$ \\
\hline $1.796 \mathrm{E}+04$ & $1.941 \mathrm{E}+00$ & $6.54 \mathrm{E}-02$ & $1.098 \mathrm{E}+01$ & $4.907 \mathrm{E}+00$ & $2.329 \mathrm{E}+01$ & $9.851 \mathrm{E}+04$ & $1.884 \mathrm{E}+02$ \\
\hline $1.798 \mathrm{E}+04$ & $1.858 \mathrm{E}+00$ & $6.53 \mathrm{E}-02$ & $1.099 \mathrm{E}+01$ & $4.715 \mathrm{E}+00$ & $2.336 \mathrm{E}+01$ & $9.851 \mathrm{E}+04$ & $2.398 \mathrm{E}+02$ \\
\hline $1.799 \mathrm{E}+04$ & $1.746 \mathrm{E}+00$ & $6.38 \mathrm{E}-02$ & $1.099 \mathrm{E}+01$ & $4.418 \mathrm{E}+00$ & $2.343 \mathrm{E}+01$ & $9.851 \mathrm{E}+04$ & $2.973 \mathrm{E}+02$ \\
\hline $1.803 \mathrm{E}+04$ & $1.589 \mathrm{E}+00$ & $6.38 \mathrm{E}-02$ & $1.100 \mathrm{E}+01$ & $4.050 \mathrm{E}+00$ & $2.335 \mathrm{E}+01$ & $9.850 \mathrm{E}+04$ & $3.610 \mathrm{E}+02$ \\
\hline
\end{tabular}


Table 93: APC 4.2 x 4.0 Dynamic Calculated Values - 17997 RPM

\begin{tabular}{|c|c|c|c|c|c|c|c|}
\hline$n(\mathrm{RPM})$ & $V_{\infty}^{\prime}(\mathrm{m} / \mathrm{s})$ & $\Delta V_{\infty}^{\prime}(\mathrm{m} / \mathrm{s})$ & $T^{\prime}(\mathrm{g})$ & $\Delta T^{\prime}(\mathrm{g})$ & $V_{\mathrm{t}}(\mathrm{m} / \mathrm{s})$ & $P_{\mathrm{P}}(\mathrm{W})$ & $\Delta P_{\mathrm{P}}(\mathrm{W})$ \\
\hline $1.805 \mathrm{E}+04$ & $2.167 \mathrm{E}+00$ & $1.57 \mathrm{E}-02$ & $1.504 \mathrm{E}+02$ & $7.25 \mathrm{E}+00$ & $7.540 \mathrm{E}+01$ & $4.199 \mathrm{E}+01$ & $1.35 \mathrm{E}+00$ \\
\hline $1.801 \mathrm{E}+04$ & $4.216 \mathrm{E}+00$ & $1.67 \mathrm{E}-02$ & $1.456 \mathrm{E}+02$ & $7.18 \mathrm{E}+00$ & $7.532 \mathrm{E}+01$ & $4.118 \mathrm{E}+01$ & $1.26 \mathrm{E}+00$ \\
\hline $1.800 \mathrm{E}+04$ & $6.409 \mathrm{E}+00$ & $1.87 \mathrm{E}-02$ & $1.409 \mathrm{E}+02$ & $7.14 \mathrm{E}+00$ & $7.546 \mathrm{E}+01$ & $3.940 \mathrm{E}+01$ & $1.26 \mathrm{E}+00$ \\
\hline $1.796 \mathrm{E}+04$ & $8.670 \mathrm{E}+00$ & $2.19 \mathrm{E}-02$ & $1.384 \mathrm{E}+02$ & $7.16 \mathrm{E}+00$ & $7.552 \mathrm{E}+01$ & $3.840 \mathrm{E}+01$ & $1.23 \mathrm{E}+00$ \\
\hline $1.799 \mathrm{E}+04$ & $1.097 \mathrm{E}+01$ & $2.57 \mathrm{E}-02$ & $1.374 \mathrm{E}+02$ & $7.18 \mathrm{E}+00$ & $7.595 \mathrm{E}+01$ & $3.785 \mathrm{E}+01$ & $1.24 \mathrm{E}+00$ \\
\hline $1.802 \mathrm{E}+04$ & $1.333 \mathrm{E}+01$ & $2.87 \mathrm{E}-02$ & $1.321 \mathrm{E}+02$ & $7.21 \mathrm{E}+00$ & $7.643 \mathrm{E}+01$ & $3.756 \mathrm{E}+01$ & $1.25 \mathrm{E}+00$ \\
\hline $1.797 \mathrm{E}+04$ & $1.566 \mathrm{E}+01$ & $3.19 \mathrm{E}-02$ & $1.230 \mathrm{E}+02$ & $7.10 \mathrm{E}+00$ & $7.668 \mathrm{E}+01$ & $3.688 \mathrm{E}+01$ & $1.22 \mathrm{E}+00$ \\
\hline $1.796 \mathrm{E}+04$ & $1.799 \mathrm{E}+01$ & $3.55 \mathrm{E}-02$ & $1.136 \mathrm{E}+02$ & $7.08 \mathrm{E}+00$ & $7.714 \mathrm{E}+01$ & $3.580 \mathrm{E}+01$ & $1.21 \mathrm{E}+00$ \\
\hline $1.798 \mathrm{E}+04$ & $2.031 \mathrm{E}+01$ & $3.86 \mathrm{E}-02$ & $1.027 \mathrm{E}+02$ & $7.09 \mathrm{E}+00$ & $7.780 \mathrm{E}+01$ & $3.431 \mathrm{E}+01$ & $1.21 \mathrm{E}+00$ \\
\hline $1.799 \mathrm{E}+04$ & $2.263 \mathrm{E}+01$ & $4.30 \mathrm{E}-02$ & $8.965 \mathrm{E}+01$ & $7.09 \mathrm{E}+00$ & $7.845 \mathrm{E}+01$ & $3.225 \mathrm{E}+01$ & $1.18 \mathrm{E}+00$ \\
\hline $1.803 \mathrm{E}+04$ & $2.494 \mathrm{E}+01$ & $4.70 \mathrm{E}-02$ & $7.533 \mathrm{E}+01$ & $7.11 \mathrm{E}+00$ & $7.930 \mathrm{E}+01$ & $2.941 \mathrm{E}+01$ & $1.18 \mathrm{E}+00$ \\
\hline$P_{\mathrm{e}}(\mathrm{W})$ & $\Delta P_{\mathrm{e}}(\mathrm{W})$ & $C_{\mathrm{T}}$ & $\Delta C_{\mathrm{T}}$ & $C_{\mathrm{P}}$ & $\Delta C_{\mathrm{P}}$ & $C_{\mathrm{Q}}$ & $\Delta C_{\mathrm{Q}}$ \\
\hline $6.324 \mathrm{E}+01$ & 7.07E-01 & $1.099 \mathrm{E}-01$ & $5.30 \mathrm{E}-03$ & $9.789 \mathrm{E}-02$ & $3.16 \mathrm{E}-03$ & $1.558 \mathrm{E}-02$ & $5.03 \mathrm{E}-04$ \\
\hline $6.203 \mathrm{E}+01$ & $6.88 \mathrm{E}-01$ & $1.069 \mathrm{E}-01$ & $5.28 \mathrm{E}-03$ & $9.665 \mathrm{E}-02$ & $2.95 \mathrm{E}-03$ & $1.538 \mathrm{E}-02$ & $4.70 \mathrm{E}-04$ \\
\hline $5.978 \mathrm{E}+01$ & $6.67 \mathrm{E}-01$ & $1.035 \mathrm{E}-01$ & $5.25 \mathrm{E}-03$ & $9.254 \mathrm{E}-02$ & $2.95 \mathrm{E}-03$ & $1.473 \mathrm{E}-02$ & $4.70 \mathrm{E}-04$ \\
\hline $5.806 \mathrm{E}+01$ & $6.47 \mathrm{E}-01$ & $1.022 \mathrm{E}-01$ & $5.29 \mathrm{E}-03$ & $9.084 \mathrm{E}-02$ & $2.92 \mathrm{E}-03$ & $1.446 \mathrm{E}-02$ & $4.64 \mathrm{E}-04$ \\
\hline $5.768 \mathrm{E}+01$ & $6.45 \mathrm{E}-01$ & $1.012 \mathrm{E}-01$ & 5.29E-03 & 8.913E-02 & 2.92E-03 & 1.419E-02 & $4.65 \mathrm{E}-04$ \\
\hline $5.728 \mathrm{E}+01$ & $6.40 \mathrm{E}-01$ & $9.697 \mathrm{E}-02$ & 5.29E-03 & 8.806E-02 & $2.93 \mathrm{E}-03$ & $1.402 \mathrm{E}-02$ & $4.66 \mathrm{E}-04$ \\
\hline $5.586 \mathrm{E}+01$ & $6.26 \mathrm{E}-01$ & $9.082 \mathrm{E}-02$ & $5.24 \mathrm{E}-03$ & $8.717 \mathrm{E}-02$ & $2.89 \mathrm{E}-03$ & $1.387 \mathrm{E}-02$ & $4.60 \mathrm{E}-04$ \\
\hline $5.389 \mathrm{E}+01$ & $6.00 \mathrm{E}-01$ & 8.398E-02 & $5.24 \mathrm{E}-03$ & $8.480 \mathrm{E}-02$ & $2.86 \mathrm{E}-03$ & $1.350 \mathrm{E}-02$ & $4.55 \mathrm{E}-04$ \\
\hline $5.180 \mathrm{E}+01$ & 5.79E-01 & $7.580 \mathrm{E}-02$ & $5.23 \mathrm{E}-03$ & 8.103E-02 & $2.85 \mathrm{E}-03$ & $1.290 \mathrm{E}-02$ & $4.53 \mathrm{E}-04$ \\
\hline $4.857 \mathrm{E}+01$ & $5.39 \mathrm{E}-01$ & $6.614 \mathrm{E}-02$ & $5.23 \mathrm{E}-03$ & $7.611 \mathrm{E}-02$ & $2.78 \mathrm{E}-03$ & $1.211 \mathrm{E}-02$ & $4.43 \mathrm{E}-04$ \\
\hline $4.457 \mathrm{E}+01$ & 4.97E-01 & $5.532 \mathrm{E}-02$ & $5.22 \mathrm{E}-03$ & $6.896 \mathrm{E}-02$ & 2.77E-03 & $1.098 \mathrm{E}-02$ & $4.41 \mathrm{E}-04$ \\
\hline$\rho\left(\mathrm{kg} / \mathrm{m}^{\wedge} 3\right)$ & $\eta_{\mathrm{T}}$ & $\Delta \eta_{\mathrm{T}}$ & $J$ & $\Delta J$ & $\eta_{\mathrm{P}}$ & $\Delta \eta_{\mathrm{P}}$ & $R e_{0.75}$ \\
\hline $1.160 \mathrm{E}+00$ & $6.640 \mathrm{E}-01$ & $2.27 \mathrm{E}-02$ & $6.776 \mathrm{E}-02$ & 4.93E-04 & $7.610 \mathrm{E}-02$ & $4.45 \mathrm{E}-03$ & $4.211 \mathrm{E}+04$ \\
\hline $1.160 \mathrm{E}+00$ & 6.639E-01 & $2.16 \mathrm{E}-02$ & $1.321 \mathrm{E}-01$ & $5.23 \mathrm{E}-04$ & $1.461 \mathrm{E}-01$ & $8.50 \mathrm{E}-03$ & 4.207E+04 \\
\hline $1.159 \mathrm{E}+00$ & $6.590 \mathrm{E}-01$ & $2.23 \mathrm{E}-02$ & 2.009E-01 & $5.88 \mathrm{E}-04$ & $2.248 \mathrm{E}-01$ & $1.35 \mathrm{E}-02$ & $4.212 \mathrm{E}+04$ \\
\hline $1.159 \mathrm{E}+00$ & $6.614 \mathrm{E}-01$ & $2.25 \mathrm{E}-02$ & $2.723 \mathrm{E}-01$ & $6.91 \mathrm{E}-04$ & $3.064 \mathrm{E}-01$ & $1.87 \mathrm{E}-02$ & $4.212 \mathrm{E}+04$ \\
\hline $1.158 \mathrm{E}+00$ & $6.563 \mathrm{E}-01$ & $2.27 \mathrm{E}-02$ & $3.440 \mathrm{E}-01$ & $8.11 \mathrm{E}-04$ & $3.905 \mathrm{E}-01$ & $2.41 \mathrm{E}-02$ & $4.233 \mathrm{E}+04$ \\
\hline $1.158 \mathrm{E}+00$ & $6.558 \mathrm{E}-01$ & $2.30 \mathrm{E}-02$ & 4.172E-01 & $9.04 \mathrm{E}-04$ & 4.595E-01 & $2.94 \mathrm{E}-02$ & 4.259E+04 \\
\hline $1.158 \mathrm{E}+00$ & $6.603 \mathrm{E}-01$ & $2.31 \mathrm{E}-02$ & $4.917 \mathrm{E}-01$ & $1.01 \mathrm{E}-03$ & $5.123 \mathrm{E}-01$ & $3.41 \mathrm{E}-02$ & $4.271 \mathrm{E}+04$ \\
\hline $1.158 \mathrm{E}+00$ & $6.644 \mathrm{E}-01$ & $2.36 \mathrm{E}-02$ & $5.650 \mathrm{E}-01$ & $1.12 \mathrm{E}-03$ & $5.596 \mathrm{E}-01$ & $3.97 \mathrm{E}-02$ & $4.295 \mathrm{E}+04$ \\
\hline $1.157 \mathrm{E}+00$ & $6.624 \mathrm{E}-01$ & $2.44 \mathrm{E}-02$ & 6.373E-01 & $1.22 \mathrm{E}-03$ & 5.962E-01 & 4.62E-02 & 4.330E+04 \\
\hline $1.157 \mathrm{E}+00$ & $6.640 \mathrm{E}-01$ & $2.53 \mathrm{E}-02$ & 7.099E-01 & $1.36 \mathrm{E}-03$ & $6.170 \mathrm{E}-01$ & 5.38E-02 & $4.365 \mathrm{E}+04$ \\
\hline $1.157 \mathrm{E}+00$ & $6.599 \mathrm{E}-01$ & $2.75 \mathrm{E}-02$ & 7.809E-01 & $1.48 \mathrm{E}-03$ & $6.265 \mathrm{E}-01$ & $6.43 \mathrm{E}-02$ & 4.414E+04 \\
\hline
\end{tabular}


Table 94: APC 4.2 x 4.0 Dynamic Measured Values - 21495 RPM

\begin{tabular}{|c|c|c|c|c|c|c|c|}
\hline$n(\mathrm{RPM})$ & $Q(\mathrm{~g}-\mathrm{m})$ & $\Delta Q(\mathrm{~g}-\mathrm{m})$ & $V(\mathrm{~V})$ & $I(\mathrm{~A})$ & $T_{\text {atm }}\left({ }^{\circ} \mathrm{C}\right)$ & $P_{\text {atm }}(\mathrm{Pa})$ & $P_{\text {diff }}(\mathrm{Pa})$ \\
\hline $2.158 \mathrm{E}+04$ & $3.217 \mathrm{E}+00$ & $1.41 \mathrm{E}-01$ & $1.086 \mathrm{E}+01$ & $9.342 \mathrm{E}+00$ & $2.217 \mathrm{E}+01$ & $9.862 \mathrm{E}+04$ & $3.385 \mathrm{E}+00$ \\
\hline $2.142 \mathrm{E}+04$ & $3.156 \mathrm{E}+00$ & $1.42 \mathrm{E}-01$ & $1.087 \mathrm{E}+01$ & $8.922 \mathrm{E}+00$ & $2.218 \mathrm{E}+01$ & $9.863 \mathrm{E}+04$ & $1.355 \mathrm{E}+01$ \\
\hline $2.151 \mathrm{E}+04$ & $3.022 \mathrm{E}+00$ & $1.37 \mathrm{E}-01$ & $1.087 \mathrm{E}+01$ & $8.776 \mathrm{E}+00$ & $2.236 \mathrm{E}+01$ & $9.864 \mathrm{E}+04$ & $3.240 \mathrm{E}+01$ \\
\hline $2.156 \mathrm{E}+04$ & $3.024 \mathrm{E}+00$ & $1.37 \mathrm{E}-01$ & $1.088 \mathrm{E}+01$ & $8.668 \mathrm{E}+00$ & $2.233 \mathrm{E}+01$ & $9.864 \mathrm{E}+04$ & $6.042 \mathrm{E}+01$ \\
\hline $2.152 \mathrm{E}+04$ & $2.908 \mathrm{E}+00$ & $1.41 \mathrm{E}-01$ & $1.088 \mathrm{E}+01$ & $8.517 \mathrm{E}+00$ & $2.243 \mathrm{E}+01$ & $9.864 \mathrm{E}+04$ & $9.788 \mathrm{E}+01$ \\
\hline $2.153 \mathrm{E}+04$ & $2.926 \mathrm{E}+00$ & $1.40 \mathrm{E}-01$ & $1.088 \mathrm{E}+01$ & $8.468 \mathrm{E}+00$ & $2.251 \mathrm{E}+01$ & $9.864 \mathrm{E}+04$ & $1.447 \mathrm{E}+02$ \\
\hline $2.147 \mathrm{E}+04$ & $2.980 \mathrm{E}+00$ & $1.50 \mathrm{E}-01$ & $1.089 \mathrm{E}+01$ & $8.275 \mathrm{E}+00$ & $2.249 \mathrm{E}+01$ & $9.864 \mathrm{E}+04$ & $2.002 \mathrm{E}+02$ \\
\hline $2.145 \mathrm{E}+04$ & $2.816 \mathrm{E}+00$ & $1.52 \mathrm{E}-01$ & $1.089 \mathrm{E}+01$ & $8.060 \mathrm{E}+00$ & $2.268 \mathrm{E}+01$ & $9.864 \mathrm{E}+04$ & $2.640 \mathrm{E}+02$ \\
\hline $2.145 \mathrm{E}+04$ & $2.766 \mathrm{E}+00$ & $1.61 \mathrm{E}-01$ & $1.090 \mathrm{E}+01$ & $7.768 \mathrm{E}+00$ & $2.279 \mathrm{E}+01$ & $9.864 \mathrm{E}+04$ & $3.370 \mathrm{E}+02$ \\
\hline $2.143 \mathrm{E}+04$ & $2.610 \mathrm{E}+00$ & $1.58 \mathrm{E}-01$ & $1.092 \mathrm{E}+01$ & $7.272 \mathrm{E}+00$ & $2.289 \mathrm{E}+01$ & $9.864 \mathrm{E}+04$ & $4.185 \mathrm{E}+02$ \\
\hline $2.153 \mathrm{E}+04$ & $2.446 \mathrm{E}+00$ & $1.64 \mathrm{E}-01$ & $1.093 \mathrm{E}+01$ & $6.830 \mathrm{E}+00$ & $2.298 \mathrm{E}+01$ & $9.864 \mathrm{E}+04$ & $5.086 \mathrm{E}+02$ \\
\hline
\end{tabular}


Table 95: APC 4.2 x 4.0 Dynamic Calculated Values - 21495 RPM

\begin{tabular}{|c|c|c|c|c|c|c|c|}
\hline$n(\mathrm{RPM})$ & $V_{\infty}^{\prime}(\mathrm{m} / \mathrm{s})$ & $\Delta V_{\infty}^{\prime}(\mathrm{m} / \mathrm{s})$ & $T^{\prime}(\mathrm{g})$ & $\Delta T^{\prime}(\mathrm{g})$ & $V_{\mathrm{t}}(\mathrm{m} / \mathrm{s})$ & $P_{\mathrm{P}}(\mathrm{W})$ & $\Delta P_{\mathrm{P}}(\mathrm{W})$ \\
\hline $2.158 \mathrm{E}+04$ & $2.291 \mathrm{E}+00$ & $1.81 \mathrm{E}-02$ & $2.205 \mathrm{E}+02$ & $7.59 \mathrm{E}+00$ & $9.015 \mathrm{E}+01$ & $7.130 \mathrm{E}+01$ & $3.12 \mathrm{E}+00$ \\
\hline $2.142 \mathrm{E}+04$ & $4.709 \mathrm{E}+00$ & $1.91 \mathrm{E}-02$ & $2.125 \mathrm{E}+02$ & $7.51 \mathrm{E}+00$ & $8.957 \mathrm{E}+01$ & $6.941 \mathrm{E}+01$ & $3.12 \mathrm{E}+00$ \\
\hline $2.151 \mathrm{E}+04$ & $7.354 \mathrm{E}+00$ & $2.19 \mathrm{E}-02$ & $2.095 \mathrm{E}+02$ & $7.49 \mathrm{E}+00$ & $9.014 \mathrm{E}+01$ & $6.676 \mathrm{E}+01$ & $3.03 \mathrm{E}+00$ \\
\hline $2.156 \mathrm{E}+04$ & $1.009 \mathrm{E}+01$ & 2.67E-02 & $2.075 \mathrm{E}+02$ & $7.60 \mathrm{E}+00$ & $9.060 \mathrm{E}+01$ & $6.694 \mathrm{E}+01$ & $3.03 \mathrm{E}+00$ \\
\hline $2.152 \mathrm{E}+04$ & $1.288 \mathrm{E}+01$ & $3.05 \mathrm{E}-02$ & $2.050 \mathrm{E}+02$ & $7.60 \mathrm{E}+00$ & $9.077 \mathrm{E}+01$ & $6.425 \mathrm{E}+01$ & $3.11 \mathrm{E}+00$ \\
\hline $2.153 \mathrm{E}+04$ & $1.569 \mathrm{E}+01$ & $3.36 \mathrm{E}-02$ & $1.982 \mathrm{E}+02$ & $7.49 \mathrm{E}+00$ & $9.129 \mathrm{E}+01$ & $6.471 \mathrm{E}+01$ & $3.09 \mathrm{E}+00$ \\
\hline $2.147 \mathrm{E}+04$ & $1.848 \mathrm{E}+01$ & $3.73 \mathrm{E}-02$ & $1.856 \mathrm{E}+02$ & $7.49 \mathrm{E}+00$ & $9.154 \mathrm{E}+01$ & $6.569 \mathrm{E}+01$ & $3.30 \mathrm{E}+00$ \\
\hline $2.145 \mathrm{E}+04$ & $2.125 \mathrm{E}+01$ & $4.16 \mathrm{E}-02$ & $1.740 \mathrm{E}+02$ & $7.50 \mathrm{E}+00$ & $9.206 \mathrm{E}+01$ & $6.201 \mathrm{E}+01$ & $3.34 \mathrm{E}+00$ \\
\hline $2.145 \mathrm{E}+04$ & $2.403 \mathrm{E}+01$ & 4.64E-02 & $1.575 \mathrm{E}+02$ & $7.54 \mathrm{E}+00$ & $9.275 \mathrm{E}+01$ & $6.094 \mathrm{E}+01$ & $3.54 \mathrm{E}+00$ \\
\hline $2.143 \mathrm{E}+04$ & $2.680 \mathrm{E}+01$ & $5.14 \mathrm{E}-02$ & $1.382 \mathrm{E}+02$ & $7.55 \mathrm{E}+00$ & $9.341 \mathrm{E}+01$ & $5.744 \mathrm{E}+01$ & $3.48 \mathrm{E}+00$ \\
\hline $2.153 \mathrm{E}+04$ & $2.957 \mathrm{E}+01$ & $5.53 \mathrm{E}-02$ & $1.201 \mathrm{E}+02$ & $7.56 \mathrm{E}+00$ & $9.465 \mathrm{E}+01$ & $5.408 \mathrm{E}+01$ & $3.64 \mathrm{E}+00$ \\
\hline$P_{\mathrm{e}}(\mathrm{W})$ & $\Delta P_{\mathrm{e}}(\mathrm{W})$ & $C_{\mathrm{T}}$ & $\Delta C_{\mathrm{T}}$ & $C_{\mathrm{P}}$ & $\Delta C_{\mathrm{P}}$ & $C_{\mathrm{Q}}$ & $\Delta C_{\mathrm{Q}}$ \\
\hline $1.014 \mathrm{E}+02$ & $1.23 \mathrm{E}+00$ & $1.124 \mathrm{E}-01$ & $3.87 \mathrm{E}-03$ & $9.692 \mathrm{E}-02$ & $4.24 \mathrm{E}-03$ & $1.543 \mathrm{E}-02$ & $6.75 \mathrm{E}-04$ \\
\hline $9.697 \mathrm{E}+01$ & $1.02 \mathrm{E}+00$ & $1.100 \mathrm{E}-01$ & $3.89 \mathrm{E}-03$ & $9.650 \mathrm{E}-02$ & $4.34 \mathrm{E}-03$ & $1.536 \mathrm{E}-02$ & $6.90 \mathrm{E}-04$ \\
\hline $9.543 \mathrm{E}+01$ & $1.00 \mathrm{E}+00$ & $1.075 \mathrm{E}-01$ & $3.85 \mathrm{E}-03$ & $9.166 \mathrm{E}-02$ & $4.16 \mathrm{E}-03$ & $1.459 \mathrm{E}-02$ & $6.62 \mathrm{E}-04$ \\
\hline $9.427 \mathrm{E}+01$ & $9.85 \mathrm{E}-01$ & $1.060 \mathrm{E}-01$ & $3.88 \mathrm{E}-03$ & $9.130 \mathrm{E}-02$ & $4.13 \mathrm{E}-03$ & $1.453 \mathrm{E}-02$ & $6.57 \mathrm{E}-04$ \\
\hline $9.267 \mathrm{E}+01$ & $9.70 \mathrm{E}-01$ & $1.052 \mathrm{E}-01$ & $3.90 \mathrm{E}-03$ & $8.818 \mathrm{E}-02$ & $4.27 \mathrm{E}-03$ & $1.403 \mathrm{E}-02$ & $6.79 \mathrm{E}-04$ \\
\hline $9.215 \mathrm{E}+01$ & $9.65 \mathrm{E}-01$ & $1.016 \mathrm{E}-01$ & $3.84 \mathrm{E}-03$ & 8.861E-02 & $4.23 \mathrm{E}-03$ & $1.410 \mathrm{E}-02$ & $6.74 \mathrm{E}-04$ \\
\hline $9.010 \mathrm{E}+01$ & $9.76 \mathrm{E}-01$ & $9.570 \mathrm{E}-02$ & $3.86 \mathrm{E}-03$ & $9.077 \mathrm{E}-02$ & $4.56 \mathrm{E}-03$ & $1.445 \mathrm{E}-02$ & 7.25E-04 \\
\hline $8.779 \mathrm{E}+01$ & $9.46 \mathrm{E}-01$ & $8.996 \mathrm{E}-02$ & $3.88 \mathrm{E}-03$ & $8.600 \mathrm{E}-02$ & $4.64 \mathrm{E}-03$ & $1.369 \mathrm{E}-02$ & $7.38 \mathrm{E}-04$ \\
\hline $8.468 \mathrm{E}+01$ & 9.19E-01 & $8.141 \mathrm{E}-02$ & $3.90 \mathrm{E}-03$ & $8.450 \mathrm{E}-02$ & $4.91 \mathrm{E}-03$ & $1.345 \mathrm{E}-02$ & $7.81 \mathrm{E}-04$ \\
\hline $7.938 \mathrm{E}+01$ & 8.68E-01 & $7.162 \mathrm{E}-02$ & 3.91E-03 & 7.995E-02 & $4.85 \mathrm{E}-03$ & $1.272 \mathrm{E}-02$ & 7.72E-04 \\
\hline $7.464 \mathrm{E}+01$ & $7.90 \mathrm{E}-01$ & $6.165 \mathrm{E}-02$ & $3.88 \mathrm{E}-03$ & 7.422E-02 & $4.99 \mathrm{E}-03$ & $1.181 \mathrm{E}-02$ & 7.94E-04 \\
\hline$\rho\left(\mathrm{kg} / \mathrm{m}^{\wedge} 3\right)$ & $\eta_{\mathrm{T}}$ & $\Delta \eta_{\mathrm{T}}$ & $J$ & $\Delta J$ & $\eta_{\mathrm{P}}$ & $\Delta \eta_{\mathrm{P}}$ & $R e_{0.75}$ \\
\hline $1.163 \mathrm{E}+00$ & $7.029 \mathrm{E}-01$ & $3.19 \mathrm{E}-02$ & $5.990 \mathrm{E}-02$ & $4.74 \mathrm{E}-04$ & $6.948 \mathrm{E}-02$ & $3.91 \mathrm{E}-03$ & $5.059 \mathrm{E}+04$ \\
\hline $1.163 \mathrm{E}+00$ & $7.158 \mathrm{E}-01$ & $3.30 \mathrm{E}-02$ & $1.241 \mathrm{E}-01$ & $5.04 \mathrm{E}-04$ & $1.414 \mathrm{E}-01$ & $8.10 \mathrm{E}-03$ & $5.026 \mathrm{E}+04$ \\
\hline $1.163 \mathrm{E}+00$ & $6.996 \mathrm{E}-01$ & $3.26 \mathrm{E}-02$ & $1.929 \mathrm{E}-01$ & $5.76 \mathrm{E}-04$ & $2.263 \mathrm{E}-01$ & $1.31 \mathrm{E}-02$ & $5.053 \mathrm{E}+04$ \\
\hline $1.163 \mathrm{E}+00$ & 7.101E-01 & $3.30 \mathrm{E}-02$ & $2.640 \mathrm{E}-01$ & 7.01E-04 & $3.067 \mathrm{E}-01$ & $1.79 \mathrm{E}-02$ & $5.080 \mathrm{E}+04$ \\
\hline $1.163 \mathrm{E}+00$ & $6.933 \mathrm{E}-01$ & $3.43 \mathrm{E}-02$ & $3.378 \mathrm{E}-01$ & $8.02 \mathrm{E}-04$ & $4.030 \mathrm{E}-01$ & $2.46 \mathrm{E}-02$ & $5.087 \mathrm{E}+04$ \\
\hline $1.162 \mathrm{E}+00$ & $7.022 \mathrm{E}-01$ & $3.43 \mathrm{E}-02$ & 4.111E-01 & $8.83 \mathrm{E}-04$ & 4.713E-01 & $2.87 \mathrm{E}-02$ & $5.114 \mathrm{E}+04$ \\
\hline $1.162 \mathrm{E}+00$ & 7.292E-01 & $3.74 \mathrm{E}-02$ & $4.858 \mathrm{E}-01$ & $9.84 \mathrm{E}-04$ & $5.121 \mathrm{E}-01$ & $3.30 \mathrm{E}-02$ & $5.128 \mathrm{E}+04$ \\
\hline $1.162 \mathrm{E}+00$ & 7.064E-01 & $3.88 \mathrm{E}-02$ & $5.591 \mathrm{E}-01$ & $1.10 \mathrm{E}-03$ & $5.848 \mathrm{E}-01$ & $4.04 \mathrm{E}-02$ & $5.151 \mathrm{E}+04$ \\
\hline $1.161 \mathrm{E}+00$ & $7.196 \mathrm{E}-01$ & $4.25 \mathrm{E}-02$ & $6.322 \mathrm{E}-01$ & $1.23 \mathrm{E}-03$ & $6.091 \mathrm{E}-01$ & $4.58 \mathrm{E}-02$ & $5.187 \mathrm{E}+04$ \\
\hline $1.161 \mathrm{E}+00$ & $7.236 \mathrm{E}-01$ & $4.46 \mathrm{E}-02$ & 7.059E-01 & $1.36 \mathrm{E}-03$ & $6.323 \mathrm{E}-01$ & $5.16 \mathrm{E}-02$ & $5.220 \mathrm{E}+04$ \\
\hline $1.160 \mathrm{E}+00$ & 7.247E-01 & $4.93 \mathrm{E}-02$ & $7.750 \mathrm{E}-01$ & $1.46 \mathrm{E}-03$ & $6.437 \mathrm{E}-01$ & $5.93 \mathrm{E}-02$ & $5.287 \mathrm{E}+04$ \\
\hline
\end{tabular}




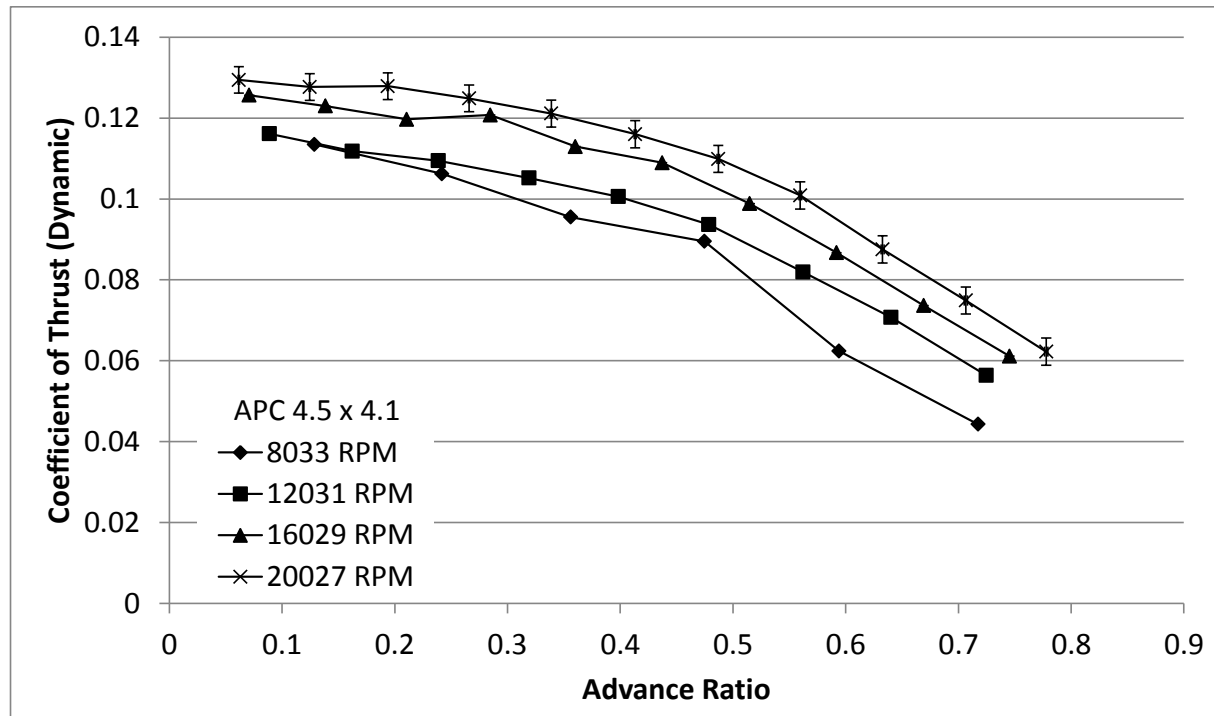

(a)

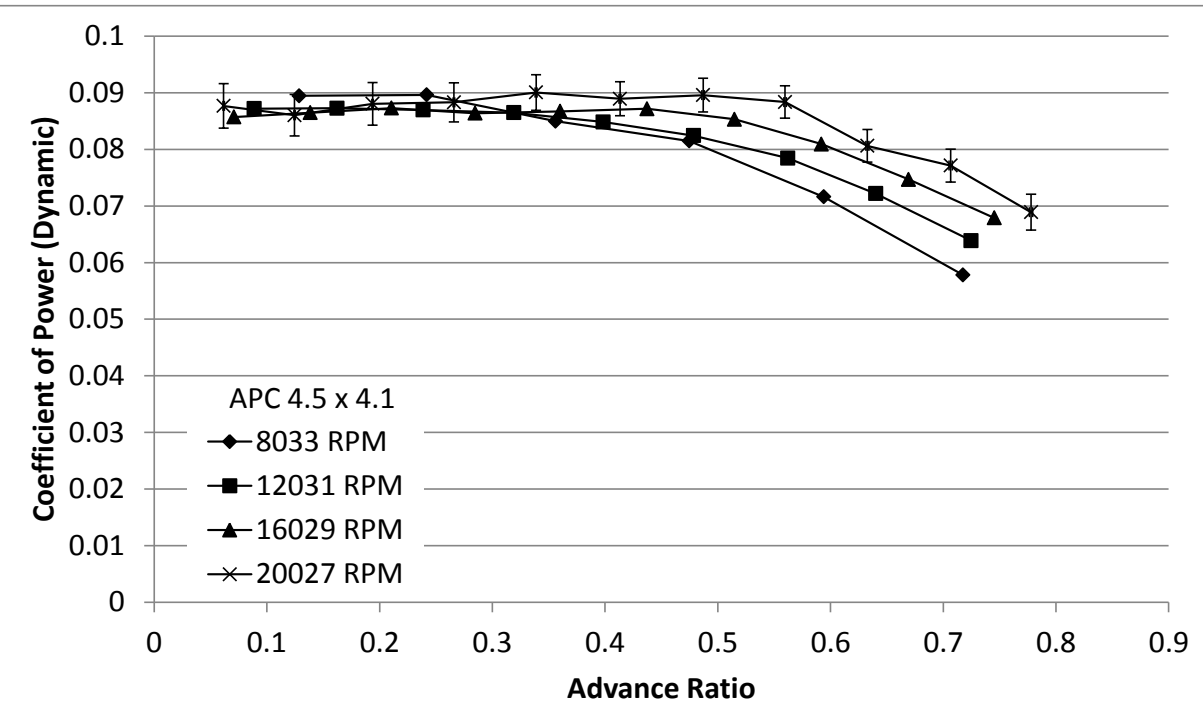

(b)

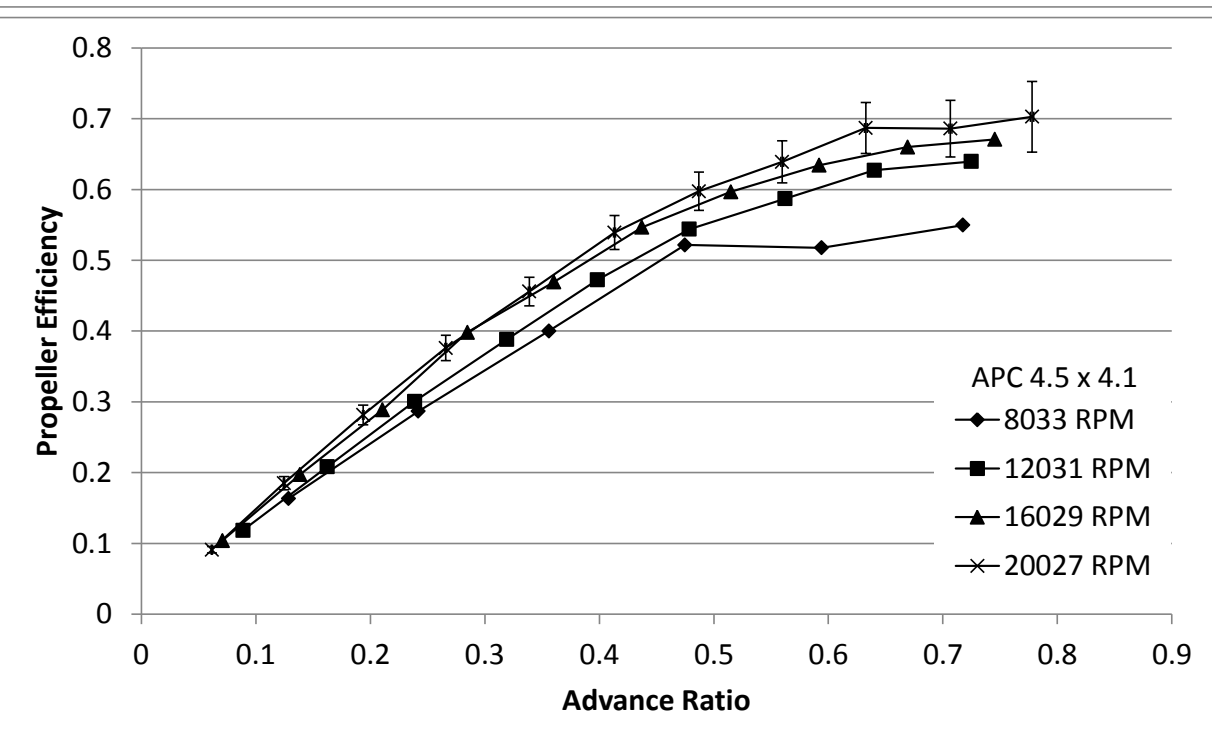

(c) 


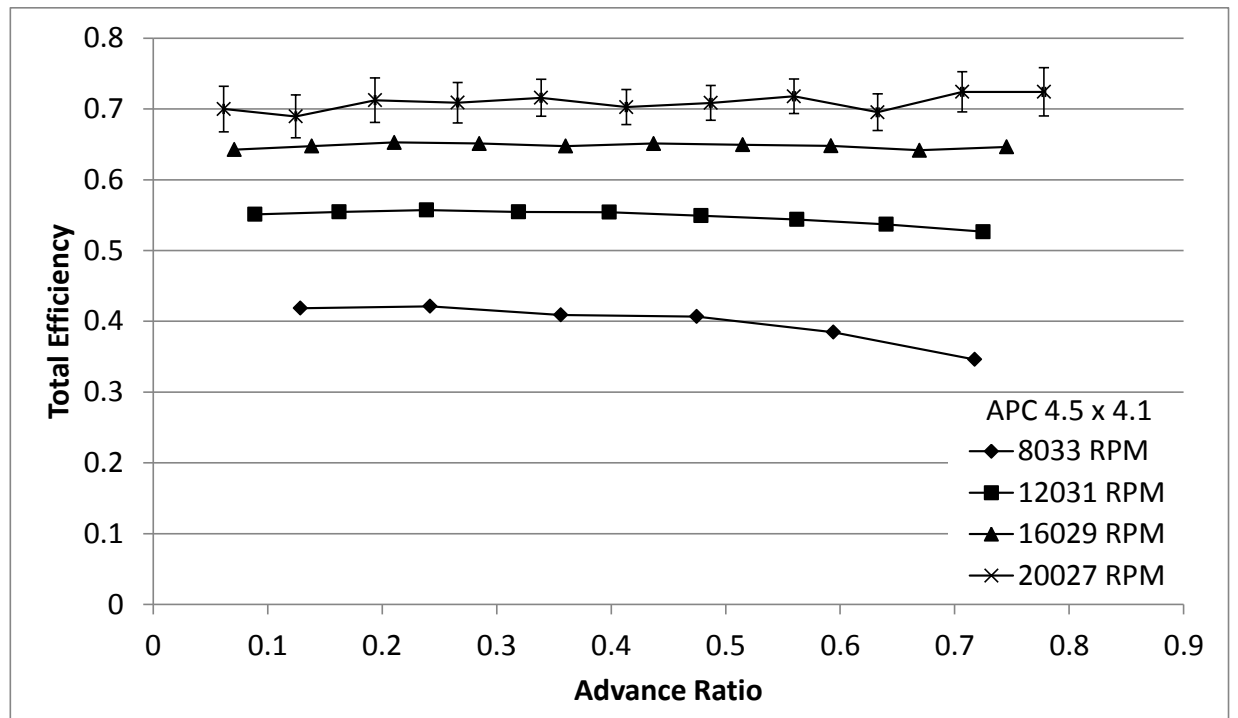

(d)

Figure 81: APC 4.5 x 4.1 Dynamic Test Results: (a) Coefficient of Thrust, (b) Coefficient of Power, (c) Propeller Efficiency, (d) Total Efficiency.

Table 96: APC 4.5 x 4.1 Dynamic Measured Values - 8033 RPM

\begin{tabular}{|c|c|c|c|c|c|c|c|}
\hline$n(\mathrm{RPM})$ & $Q(\mathrm{~g}-\mathrm{m})$ & $\Delta Q(\mathrm{~g}-\mathrm{m})$ & $V(\mathrm{~V})$ & $I(\mathrm{~A})$ & $T_{\text {atm }}\left({ }^{\circ} \mathrm{C}\right)$ & $P_{\text {atm }}(\mathrm{Pa})$ & $P_{\text {diff }}(\mathrm{Pa})$ \\
\hline $8.064 \mathrm{E}+03$ & $5.840 \mathrm{E}-01$ & $6.97 \mathrm{E}-02$ & $1.109 \mathrm{E}+01$ & $1.042 \mathrm{E}+00$ & $2.196 \mathrm{E}+01$ & $9.849 \mathrm{E}+04$ & $2.379 \mathrm{E}+00$ \\
\hline $7.975 \mathrm{E}+03$ & $5.724 \mathrm{E}-01$ & $7.27 \mathrm{E}-02$ & $1.109 \mathrm{E}+01$ & $1.004 \mathrm{E}+00$ & $2.182 \mathrm{E}+01$ & $9.850 \mathrm{E}+04$ & $8.005 \mathrm{E}+00$ \\
\hline $8.030 \mathrm{E}+03$ & $5.501 \mathrm{E}-01$ & $7.22 \mathrm{E}-02$ & $1.109 \mathrm{E}+01$ & $1.000 \mathrm{E}+00$ & $2.196 \mathrm{E}+01$ & $9.849 \mathrm{E}+04$ & $1.737 \mathrm{E}+01$ \\
\hline $8.042 \mathrm{E}+03$ & $5.287 \mathrm{E}-01$ & $7.36 \mathrm{E}-02$ & $1.109 \mathrm{E}+01$ & $9.682 \mathrm{E}-01$ & $2.210 \mathrm{E}+01$ & $9.850 \mathrm{E}+04$ & $3.081 \mathrm{E}+01$ \\
\hline $8.070 \mathrm{E}+03$ & $4.679 \mathrm{E}-01$ & $7.11 \mathrm{E}-02$ & $1.109 \mathrm{E}+01$ & $9.092 \mathrm{E}-01$ & $2.215 \mathrm{E}+01$ & $9.849 \mathrm{E}+04$ & $4.834 \mathrm{E}+01$ \\
\hline $8.021 \mathrm{E}+03$ & $3.731 \mathrm{E}-01$ & $7.42 \mathrm{E}-02$ & $1.109 \mathrm{E}+01$ & $8.004 \mathrm{E}-01$ & $2.227 \mathrm{E}+01$ & $9.850 \mathrm{E}+04$ & $6.946 \mathrm{E}+01$ \\
\hline
\end{tabular}


Table 97: APC 4.5 x 4.1 Dynamic Calculated Values - 8033 RPM

\begin{tabular}{|c|c|c|c|c|c|c|c|}
\hline$n(\mathrm{RPM})$ & $V_{\infty}^{\prime}(\mathrm{m} / \mathrm{s})$ & $\Delta V_{\infty}^{\prime}(\mathrm{m} / \mathrm{s})$ & $T^{\prime}(\mathrm{g})$ & $\Delta T^{\prime}(\mathrm{g})$ & $V_{\mathrm{t}}(\mathrm{m} / \mathrm{s})$ & $P_{\mathrm{P}}(\mathrm{W})$ & $\Delta P_{\mathrm{P}}(\mathrm{W})$ \\
\hline $8.064 \mathrm{E}+03$ & $1.968 \mathrm{E}+00$ & $1.22 \mathrm{E}-02$ & $4.088 \mathrm{E}+01$ & $9.34 \mathrm{E}+00$ & $3.612 \mathrm{E}+01$ & $4.836 \mathrm{E}+00$ & $5.77 \mathrm{E}-01$ \\
\hline $7.975 \mathrm{E}+03$ & $3.661 \mathrm{E}+00$ & $1.29 \mathrm{E}-02$ & $3.744 \mathrm{E}+01$ & $9.31 \mathrm{E}+00$ & $3.586 \mathrm{E}+01$ & $4.688 \mathrm{E}+00$ & $5.95 \mathrm{E}-01$ \\
\hline $8.030 \mathrm{E}+03$ & $5.425 \mathrm{E}+00$ & $1.51 \mathrm{E}-02$ & $3.411 \mathrm{E}+01$ & $9.41 \mathrm{E}+00$ & $3.632 \mathrm{E}+01$ & $4.536 \mathrm{E}+00$ & $5.95 \mathrm{E}-01$ \\
\hline $8.042 \mathrm{E}+03$ & $7.247 \mathrm{E}+00$ & $1.68 \mathrm{E}-02$ & $3.206 \mathrm{E}+01$ & $9.26 \mathrm{E}+00$ & $3.669 \mathrm{E}+01$ & $4.366 \mathrm{E}+00$ & $6.08 \mathrm{E}-01$ \\
\hline $8.070 \mathrm{E}+03$ & $9.098 \mathrm{E}+00$ & $2.00 \mathrm{E}-02$ & $2.250 \mathrm{E}+01$ & $9.40 \mathrm{E}+00$ & $3.722 \mathrm{E}+01$ & $3.878 \mathrm{E}+00$ & $5.89 \mathrm{E}-01$ \\
\hline $8.021 \mathrm{E}+03$ & $1.092 \mathrm{E}+01$ & $2.28 \mathrm{E}-02$ & $1.578 \mathrm{E}+01$ & $9.34 \mathrm{E}+00$ & $3.750 \mathrm{E}+01$ & $3.074 \mathrm{E}+00$ & $6.12 \mathrm{E}-01$ \\
\hline$P_{\mathrm{e}}(\mathrm{W})$ & $\Delta P_{\mathrm{e}}(\mathrm{W})$ & $C_{\mathrm{T}}$ & $\Delta C_{\mathrm{T}}$ & $C_{\mathrm{P}}$ & $\Delta C_{\mathrm{P}}$ & $C_{\mathrm{Q}}$ & $\Delta C_{\mathrm{Q}}$ \\
\hline $1.155 \mathrm{E}+01$ & $1.44 \mathrm{E}-01$ & $1.135 \mathrm{E}-01$ & $2.59 \mathrm{E}-02$ & $8.946 \mathrm{E}-02$ & $1.07 \mathrm{E}-02$ & $1.424 \mathrm{E}-02$ & $1.70 \mathrm{E}-03$ \\
\hline $1.113 \mathrm{E}+01$ & $1.39 \mathrm{E}-01$ & $1.062 \mathrm{E}-01$ & 2.64E-02 & $8.961 \mathrm{E}-02$ & $1.14 \mathrm{E}-02$ & $1.426 \mathrm{E}-02$ & $1.81 \mathrm{E}-03$ \\
\hline $1.109 \mathrm{E}+01$ & $1.37 \mathrm{E}-01$ & $9.552 \mathrm{E}-02$ & $2.63 \mathrm{E}-02$ & $8.498 \mathrm{E}-02$ & $1.12 \mathrm{E}-02$ & $1.353 \mathrm{E}-02$ & $1.77 \mathrm{E}-03$ \\
\hline $1.074 \mathrm{E}+01$ & $1.33 \mathrm{E}-01$ & 8.953E-02 & $2.59 \mathrm{E}-02$ & $8.148 \mathrm{E}-02$ & $1.14 \mathrm{E}-02$ & $1.297 \mathrm{E}-02$ & $1.81 \mathrm{E}-03$ \\
\hline $1.008 \mathrm{E}+01$ & $1.27 \mathrm{E}-01$ & $6.241 \mathrm{E}-02$ & $2.61 \mathrm{E}-02$ & 7.162E-02 & $1.09 \mathrm{E}-02$ & $1.140 \mathrm{E}-02$ & $1.73 \mathrm{E}-03$ \\
\hline $8.879 \mathrm{E}+00$ & $1.14 \mathrm{E}-01$ & $4.432 \mathrm{E}-02$ & $2.62 \mathrm{E}-02$ & $5.783 \mathrm{E}-02$ & $1.15 \mathrm{E}-02$ & $9.204 \mathrm{E}-03$ & $1.83 \mathrm{E}-03$ \\
\hline$\rho\left(\mathrm{kg} / \mathrm{m}^{\wedge} 3\right)$ & $\eta_{\mathrm{T}}$ & $\Delta \eta_{\mathrm{T}}$ & $J$ & $\Delta J$ & $\eta_{\mathrm{P}}$ & $\Delta \eta_{\mathrm{P}}$ & $R e_{0.75}$ \\
\hline $1.163 \mathrm{E}+00$ & 4.186E-01 & $5.02 \mathrm{E}-02$ & $1.286 \mathrm{E}-01$ & $8.08 \mathrm{E}-04$ & $1.632 \mathrm{E}-01$ & $4.21 \mathrm{E}-02$ & $1.988 \mathrm{E}+04$ \\
\hline $1.163 \mathrm{E}+00$ & $4.211 \mathrm{E}-01$ & 5.37E-02 & $2.419 \mathrm{E}-01$ & 8.83E-04 & $2.868 \mathrm{E}-01$ & 8.01E-02 & $1.975 \mathrm{E}+04$ \\
\hline $1.163 \mathrm{E}+00$ & $4.090 \mathrm{E}-01$ & 5.39E-02 & $3.560 \mathrm{E}-01$ & $1.05 \mathrm{E}-03$ & $4.001 \mathrm{E}-01$ & $1.22 \mathrm{E}-01$ & $1.999 \mathrm{E}+04$ \\
\hline $1.162 \mathrm{E}+00$ & 4.067E-01 & $5.69 \mathrm{E}-02$ & 4.748E-01 & $1.20 \mathrm{E}-03$ & $5.217 \mathrm{E}-01$ & $1.67 \mathrm{E}-01$ & $2.017 \mathrm{E}+04$ \\
\hline $1.162 \mathrm{E}+00$ & $3.846 \mathrm{E}-01$ & $5.86 \mathrm{E}-02$ & $5.940 \mathrm{E}-01$ & $1.43 \mathrm{E}-03$ & $5.176 \mathrm{E}-01$ & $2.30 \mathrm{E}-01$ & $2.046 \mathrm{E}+04$ \\
\hline $1.161 \mathrm{E}+00$ & $3.462 \mathrm{E}-01$ & $6.90 \mathrm{E}-02$ & 7.174E-01 & $1.65 \mathrm{E}-03$ & $5.497 \mathrm{E}-01$ & $3.43 \mathrm{E}-01$ & $2.060 \mathrm{E}+04$ \\
\hline
\end{tabular}


Table 98: APC 4.5 x 4.1 Dynamic Measured Values - 12031 RPM

\begin{tabular}{|c|c|c|c|c|c|c|c|}
\hline$n(\mathrm{RPM})$ & $Q(\mathrm{~g}-\mathrm{m})$ & $\Delta Q(\mathrm{~g}-\mathrm{m})$ & $V(\mathrm{~V})$ & $I(\mathrm{~A})$ & $T_{\text {atm }}\left({ }^{\circ} \mathrm{C}\right)$ & $P_{\text {atm }}(\mathrm{Pa})$ & $P_{\text {diff }}(\mathrm{Pa})$ \\
\hline $1.205 \mathrm{E}+04$ & $1.271 \mathrm{E}+00$ & $6.05 \mathrm{E}-02$ & $1.104 \mathrm{E}+01$ & $2.584 \mathrm{E}+00$ & $2.208 \mathrm{E}+01$ & $9.849 \mathrm{E}+04$ & $2.603 \mathrm{E}+00$ \\
\hline $1.201 \mathrm{E}+04$ & $1.264 \mathrm{E}+00$ & $5.77 \mathrm{E}-02$ & $1.105 \mathrm{E}+01$ & $2.544 \mathrm{E}+00$ & $2.207 \mathrm{E}+01$ & $9.849 \mathrm{E}+04$ & $8.313 \mathrm{E}+00$ \\
\hline $1.204 \mathrm{E}+04$ & $1.265 \mathrm{E}+00$ & $6.10 \mathrm{E}-02$ & $1.105 \mathrm{E}+01$ & $2.540 \mathrm{E}+00$ & $2.213 \mathrm{E}+01$ & $9.850 \mathrm{E}+04$ & $1.777 \mathrm{E}+01$ \\
\hline $1.198 \mathrm{E}+04$ & $1.245 \mathrm{E}+00$ & $5.69 \mathrm{E}-02$ & $1.105 \mathrm{E}+01$ & $2.499 \mathrm{E}+00$ & $2.222 \mathrm{E}+01$ & $9.850 \mathrm{E}+04$ & $3.115 \mathrm{E}+01$ \\
\hline $1.204 \mathrm{E}+04$ & $1.234 \mathrm{E}+00$ & $6.08 \mathrm{E}-02$ & $1.105 \mathrm{E}+01$ & $2.492 \mathrm{E}+00$ & $2.226 \mathrm{E}+01$ & $9.849 \mathrm{E}+04$ & $4.880 \mathrm{E}+01$ \\
\hline $1.205 \mathrm{E}+04$ & $1.200 \mathrm{E}+00$ & $5.92 \mathrm{E}-02$ & $1.105 \mathrm{E}+01$ & $2.447 \mathrm{E}+00$ & $2.228 \mathrm{E}+01$ & $9.850 \mathrm{E}+04$ & $7.023 \mathrm{E}+01$ \\
\hline $1.201 \mathrm{E}+04$ & $1.134 \mathrm{E}+00$ & $5.68 \mathrm{E}-02$ & $1.105 \mathrm{E}+01$ & $2.326 \mathrm{E}+00$ & $2.242 \mathrm{E}+01$ & $9.850 \mathrm{E}+04$ & $9.594 \mathrm{E}+01$ \\
\hline $1.207 \mathrm{E}+04$ & $1.055 \mathrm{E}+00$ & $5.75 \mathrm{E}-02$ & $1.106 \mathrm{E}+01$ & $2.202 \mathrm{E}+00$ & $2.244 \mathrm{E}+01$ & $9.850 \mathrm{E}+04$ & $1.255 \mathrm{E}+02$ \\
\hline $1.202 \mathrm{E}+04$ & $9.255 \mathrm{E}-01$ & $5.80 \mathrm{E}-02$ & $1.106 \mathrm{E}+01$ & $1.962 \mathrm{E}+00$ & $2.248 \mathrm{E}+01$ & $9.850 \mathrm{E}+04$ & $1.593 \mathrm{E}+02$ \\
\hline
\end{tabular}


Table 99: APC 4.5 x 4.1 Dynamic Calculated Values - 12031 RPM

\begin{tabular}{|c|c|c|c|c|c|c|c|}
\hline$n(\mathrm{RPM})$ & $V_{\infty}^{\prime}(\mathrm{m} / \mathrm{s})$ & $\Delta V_{\infty}^{\prime}(\mathrm{m} / \mathrm{s})$ & $T^{\prime}(\mathrm{g})$ & $\Delta T^{\prime}(\mathrm{g})$ & $V_{\mathrm{t}}(\mathrm{m} / \mathrm{s})$ & $P_{\mathrm{P}}(\mathrm{W})$ & $\Delta P_{\mathrm{P}}(\mathrm{W})$ \\
\hline $1.205 \mathrm{E}+04$ & $2.033 \mathrm{E}+00$ & $1.31 \mathrm{E}-02$ & $9.340 \mathrm{E}+01$ & $8.06 \mathrm{E}+00$ & $5.395 \mathrm{E}+01$ & $1.573 \mathrm{E}+01$ & $7.48 \mathrm{E}-01$ \\
\hline $1.201 \mathrm{E}+04$ & $3.703 \mathrm{E}+00$ & $1.35 \mathrm{E}-02$ & $8.935 \mathrm{E}+01$ & $8.10 \mathrm{E}+00$ & $5.386 \mathrm{E}+01$ & $1.559 \mathrm{E}+01$ & $7.11 \mathrm{E}-01$ \\
\hline $1.204 \mathrm{E}+04$ & $5.455 \mathrm{E}+00$ & $1.55 \mathrm{E}-02$ & $8.785 \mathrm{E}+01$ & $8.07 \mathrm{E}+00$ & $5.413 \mathrm{E}+01$ & $1.564 \mathrm{E}+01$ & $7.54 \mathrm{E}-01$ \\
\hline $1.198 \mathrm{E}+04$ & $7.255 \mathrm{E}+00$ & $1.75 \mathrm{E}-02$ & $8.356 \mathrm{E}+01$ & $8.08 \mathrm{E}+00$ & $5.407 \mathrm{E}+01$ & $1.532 \mathrm{E}+01$ & $6.99 \mathrm{E}-01$ \\
\hline $1.204 \mathrm{E}+04$ & $9.105 \mathrm{E}+00$ & $2.02 \mathrm{E}-02$ & $8.072 \mathrm{E}+01$ & $8.08 \mathrm{E}+00$ & $5.463 \mathrm{E}+01$ & $1.526 \mathrm{E}+01$ & $7.52 \mathrm{E}-01$ \\
\hline $1.205 \mathrm{E}+04$ & $1.094 \mathrm{E}+01$ & $2.31 \mathrm{E}-02$ & $7.526 \mathrm{E}+01$ & $8.11 \mathrm{E}+00$ & $5.499 \mathrm{E}+01$ & $1.485 \mathrm{E}+01$ & 7.33E-01 \\
\hline $1.201 \mathrm{E}+04$ & $1.281 \mathrm{E}+01$ & $2.59 \mathrm{E}-02$ & $6.534 \mathrm{E}+01$ & $8.10 \mathrm{E}+00$ & $5.521 \mathrm{E}+01$ & $1.398 \mathrm{E}+01$ & $7.00 \mathrm{E}-01$ \\
\hline $1.207 \mathrm{E}+04$ & $1.467 \mathrm{E}+01$ & $2.90 \mathrm{E}-02$ & $5.702 \mathrm{E}+01$ & $8.07 \mathrm{E}+00$ & $5.595 \mathrm{E}+01$ & $1.308 \mathrm{E}+01$ & $7.13 \mathrm{E}-01$ \\
\hline $1.202 \mathrm{E}+04$ & $1.654 \mathrm{E}+01$ & $3.20 \mathrm{E}-02$ & $4.507 \mathrm{E}+01$ & $8.07 \mathrm{E}+00$ & $5.626 \mathrm{E}+01$ & $1.143 \mathrm{E}+01$ & 7.17E-01 \\
\hline$P_{\mathrm{e}}(\mathrm{W})$ & $\Delta P_{\mathrm{e}}(\mathrm{W})$ & $C_{\mathrm{T}}$ & $\Delta C_{\mathrm{T}}$ & $C_{\mathrm{P}}$ & $\Delta C_{\mathrm{P}}$ & $C_{\mathrm{Q}}$ & $\Delta C_{\mathrm{Q}}$ \\
\hline $2.854 \mathrm{E}+01$ & $3.23 \mathrm{E}-01$ & $1.161 \mathrm{E}-01$ & $1.00 \mathrm{E}-02$ & $8.718 \mathrm{E}-02$ & $4.15 \mathrm{E}-03$ & $1.387 \mathrm{E}-02$ & $6.60 \mathrm{E}-04$ \\
\hline $2.811 \mathrm{E}+01$ & $3.16 \mathrm{E}-01$ & $1.118 \mathrm{E}-01$ & $1.01 \mathrm{E}-02$ & $8.726 \mathrm{E}-02$ & $3.99 \mathrm{E}-03$ & $1.389 \mathrm{E}-02$ & $6.34 \mathrm{E}-04$ \\
\hline $2.806 \mathrm{E}+01$ & $3.16 \mathrm{E}-01$ & $1.095 \mathrm{E}-01$ & $1.01 \mathrm{E}-02$ & 8.695E-02 & $4.20 \mathrm{E}-03$ & $1.384 \mathrm{E}-02$ & $6.68 \mathrm{E}-04$ \\
\hline $2.761 \mathrm{E}+01$ & $3.10 \mathrm{E}-01$ & $1.052 \mathrm{E}-01$ & $1.02 \mathrm{E}-02$ & $8.648 \mathrm{E}-02$ & $3.95 \mathrm{E}-03$ & $1.376 \mathrm{E}-02$ & $6.29 \mathrm{E}-04$ \\
\hline $2.753 \mathrm{E}+01$ & $3.09 \mathrm{E}-01$ & $1.006 \mathrm{E}-01$ & $1.01 \mathrm{E}-02$ & $8.484 \mathrm{E}-02$ & $4.18 \mathrm{E}-03$ & $1.350 \mathrm{E}-02$ & $6.65 \mathrm{E}-04$ \\
\hline $2.703 \mathrm{E}+01$ & $3.04 \mathrm{E}-01$ & $9.369 \mathrm{E}-02$ & $1.01 \mathrm{E}-02$ & $8.242 \mathrm{E}-02$ & $4.07 \mathrm{E}-03$ & $1.312 \mathrm{E}-02$ & $6.48 \mathrm{E}-04$ \\
\hline $2.571 \mathrm{E}+01$ & $2.87 \mathrm{E}-01$ & $8.194 \mathrm{E}-02$ & $1.02 \mathrm{E}-02$ & $7.846 \mathrm{E}-02$ & $3.93 \mathrm{E}-03$ & $1.249 \mathrm{E}-02$ & $6.26 \mathrm{E}-04$ \\
\hline $2.435 \mathrm{E}+01$ & $2.77 \mathrm{E}-01$ & 7.074E-02 & $1.00 \mathrm{E}-02$ & $7.218 \mathrm{E}-02$ & $3.94 \mathrm{E}-03$ & 1.149E-02 & $6.27 \mathrm{E}-04$ \\
\hline $2.171 \mathrm{E}+01$ & 2.47E-01 & $5.637 \mathrm{E}-02$ & $1.01 \mathrm{E}-02$ & $6.388 \mathrm{E}-02$ & $4.01 \mathrm{E}-03$ & $1.017 \mathrm{E}-02$ & $6.38 \mathrm{E}-04$ \\
\hline$\rho\left(\mathrm{kg} / \mathrm{m}^{\wedge} 3\right)$ & $\eta_{\mathrm{T}}$ & $\Delta \eta_{\mathrm{T}}$ & $J$ & $\Delta J$ & $\eta_{\mathrm{P}}$ & $\Delta \eta_{\mathrm{P}}$ & $R e_{0.75}$ \\
\hline $1.162 \mathrm{E}+00$ & $5.512 \mathrm{E}-01$ & $2.70 \mathrm{E}-02$ & $8.885 \mathrm{E}-02$ & $5.75 \mathrm{E}-04$ & $1.183 \mathrm{E}-01$ & $1.17 \mathrm{E}-02$ & $2.966 \mathrm{E}+04$ \\
\hline $1.162 \mathrm{E}+00$ & $5.547 \mathrm{E}-01$ & $2.61 \mathrm{E}-02$ & $1.624 \mathrm{E}-01$ & $6.03 \mathrm{E}-04$ & $2.081 \mathrm{E}-01$ & $2.11 \mathrm{E}-02$ & $2.962 \mathrm{E}+04$ \\
\hline $1.162 \mathrm{E}+00$ & $5.573 \mathrm{E}-01$ & $2.76 \mathrm{E}-02$ & $2.387 \mathrm{E}-01$ & $6.95 \mathrm{E}-04$ & $3.005 \mathrm{E}-01$ & $3.12 \mathrm{E}-02$ & $2.976 \mathrm{E}+04$ \\
\hline $1.162 \mathrm{E}+00$ & $5.547 \mathrm{E}-01$ & $2.61 \mathrm{E}-02$ & $3.191 \mathrm{E}-01$ & $7.74 \mathrm{E}-04$ & $3.881 \mathrm{E}-01$ & $4.15 \mathrm{E}-02$ & $2.971 \mathrm{E}+04$ \\
\hline $1.162 \mathrm{E}+00$ & $5.542 \mathrm{E}-01$ & $2.80 \mathrm{E}-02$ & $3.983 \mathrm{E}-01$ & $9.22 \mathrm{E}-04$ & 4.723E-01 & $5.27 \mathrm{E}-02$ & $3.001 \mathrm{E}+04$ \\
\hline $1.161 \mathrm{E}+00$ & $5.493 \mathrm{E}-01$ & $2.78 \mathrm{E}-02$ & $4.785 \mathrm{E}-01$ & $1.06 \mathrm{E}-03$ & $5.439 \mathrm{E}-01$ & $6.45 \mathrm{E}-02$ & $3.020 \mathrm{E}+04$ \\
\hline $1.161 \mathrm{E}+00$ & 5.439E-01 & $2.79 \mathrm{E}-02$ & $5.621 \mathrm{E}-01$ & $1.19 \mathrm{E}-03$ & $5.871 \mathrm{E}-01$ & $7.85 \mathrm{E}-02$ & $3.030 \mathrm{E}+04$ \\
\hline $1.161 \mathrm{E}+00$ & 5.371E-01 & $2.99 \mathrm{E}-02$ & $6.401 \mathrm{E}-01$ & $1.33 \mathrm{E}-03$ & $6.273 \mathrm{E}-01$ & $9.52 \mathrm{E}-02$ & $3.070 \mathrm{E}+04$ \\
\hline $1.161 \mathrm{E}+00$ & $5.265 \mathrm{E}-01$ & $3.36 \mathrm{E}-02$ & $7.248 \mathrm{E}-01$ & $1.48 \mathrm{E}-03$ & $6.396 \mathrm{E}-01$ & $1.21 \mathrm{E}-01$ & $3.087 \mathrm{E}+04$ \\
\hline
\end{tabular}


Table 100: APC 4.5 x 4.1 Dynamic Measured Values - 16029 RPM

\begin{tabular}{|c|c|c|c|c|c|c|c|}
\hline$n(\mathrm{RPM})$ & $Q(\mathrm{~g}-\mathrm{m})$ & $\Delta Q(\mathrm{~g}-\mathrm{m})$ & $V(\mathrm{~V})$ & $I(\mathrm{~A})$ & $T_{\text {atm }}\left({ }^{\circ} \mathrm{C}\right)$ & $P_{\text {atm }}(\mathrm{Pa})$ & $P_{\text {diff }}(\mathrm{Pa})$ \\
\hline $1.610 \mathrm{E}+04$ & $2.231 \mathrm{E}+00$ & $7.05 \mathrm{E}-02$ & $1.097 \mathrm{E}+01$ & $5.230 \mathrm{E}+00$ & $2.210 \mathrm{E}+01$ & $9.850 \mathrm{E}+04$ & $3.033 \mathrm{E}+00$ \\
\hline $1.604 \mathrm{E}+04$ & $2.234 \mathrm{E}+00$ & $6.73 \mathrm{E}-02$ & $1.097 \mathrm{E}+01$ & $5.179 \mathrm{E}+00$ & $2.207 \mathrm{E}+01$ & $9.849 \mathrm{E}+04$ & $1.090 \mathrm{E}+01$ \\
\hline $1.604 \mathrm{E}+04$ & $2.253 \mathrm{E}+00$ & $6.70 \mathrm{E}-02$ & $1.097 \mathrm{E}+01$ & $5.181 \mathrm{E}+00$ & $2.218 \mathrm{E}+01$ & $9.849 \mathrm{E}+04$ & $2.470 \mathrm{E}+01$ \\
\hline $1.606 \mathrm{E}+04$ & $2.235 \mathrm{E}+00$ & $6.82 \mathrm{E}-02$ & $1.098 \mathrm{E}+01$ & $5.158 \mathrm{E}+00$ & $2.229 \mathrm{E}+01$ & $9.849 \mathrm{E}+04$ & $4.481 \mathrm{E}+01$ \\
\hline $1.604 \mathrm{E}+04$ & $2.236 \mathrm{E}+00$ & $6.75 \mathrm{E}-02$ & $1.097 \mathrm{E}+01$ & $5.182 \mathrm{E}+00$ & $2.231 \mathrm{E}+01$ & $9.849 \mathrm{E}+04$ & $7.101 \mathrm{E}+01$ \\
\hline $1.604 \mathrm{E}+04$ & $2.248 \mathrm{E}+00$ & $6.68 \mathrm{E}-02$ & $1.097 \mathrm{E}+01$ & $5.181 \mathrm{E}+00$ & $2.242 \mathrm{E}+01$ & $9.850 \mathrm{E}+04$ & $1.042 \mathrm{E}+02$ \\
\hline $1.601 \mathrm{E}+04$ & $2.191 \mathrm{E}+00$ & $6.45 \mathrm{E}-02$ & $1.098 \mathrm{E}+01$ & $5.052 \mathrm{E}+00$ & $2.247 \mathrm{E}+01$ & $9.850 \mathrm{E}+04$ & $1.434 \mathrm{E}+02$ \\
\hline $1.599 \mathrm{E}+04$ & $2.075 \mathrm{E}+00$ & $6.33 \mathrm{E}-02$ & $1.099 \mathrm{E}+01$ & $4.788 \mathrm{E}+00$ & $2.248 \mathrm{E}+01$ & $9.850 \mathrm{E}+04$ & $1.887 \mathrm{E}+02$ \\
\hline $1.598 \mathrm{E}+04$ & $1.911 \mathrm{E}+00$ & $6.37 \mathrm{E}-02$ & $1.099 \mathrm{E}+01$ & $4.447 \mathrm{E}+00$ & $2.256 \mathrm{E}+01$ & $9.850 \mathrm{E}+04$ & $2.402 \mathrm{E}+02$ \\
\hline $1.598 \mathrm{E}+04$ & $1.737 \mathrm{E}+00$ & $6.37 \mathrm{E}-02$ & $1.101 \mathrm{E}+01$ & $4.005 \mathrm{E}+00$ & $2.261 \mathrm{E}+01$ & $9.850 \mathrm{E}+04$ & $2.974 \mathrm{E}+02$ \\
\hline
\end{tabular}


Table 101: APC 4.5 x 4.1 Dynamic Calculated Values - 16029 RPM

\begin{tabular}{|c|c|c|c|c|c|c|c|}
\hline$n(\mathrm{RPM})$ & $V_{\infty}^{\prime}(\mathrm{m} / \mathrm{s})$ & $\Delta V_{\infty}^{\prime}(\mathrm{m} / \mathrm{s})$ & $T^{\prime}(\mathrm{g})$ & $\Delta T^{\prime}(\mathrm{g})$ & $V_{\mathrm{t}}(\mathrm{m} / \mathrm{s})$ & $P_{\mathrm{P}}(\mathrm{W})$ & $\Delta P_{\mathrm{P}}(\mathrm{W})$ \\
\hline $1.610 \mathrm{E}+04$ & $2.167 \mathrm{E}+00$ & $1.60 \mathrm{E}-02$ & $1.804 \mathrm{E}+02$ & $7.60 \mathrm{E}+00$ & $7.206 \mathrm{E}+01$ & $3.689 \mathrm{E}+01$ & $1.17 \mathrm{E}+00$ \\
\hline $1.604 \mathrm{E}+04$ & $4.218 \mathrm{E}+00$ & $1.67 \mathrm{E}-02$ & $1.753 \mathrm{E}+02$ & $7.71 \mathrm{E}+00$ & $7.188 \mathrm{E}+01$ & $3.680 \mathrm{E}+01$ & $1.11 \mathrm{E}+00$ \\
\hline $1.604 \mathrm{E}+04$ & $6.413 \mathrm{E}+00$ & $1.88 \mathrm{E}-02$ & $1.705 \mathrm{E}+02$ & $7.81 \mathrm{E}+00$ & $7.203 \mathrm{E}+01$ & $3.711 \mathrm{E}+01$ & $1.10 \mathrm{E}+00$ \\
\hline $1.606 \mathrm{E}+04$ & $8.682 \mathrm{E}+00$ & $2.21 \mathrm{E}-02$ & $1.724 \mathrm{E}+02$ & $7.87 \mathrm{E}+00$ & $7.236 \mathrm{E}+01$ & $3.687 \mathrm{E}+01$ & $1.13 \mathrm{E}+00$ \\
\hline $1.604 \mathrm{E}+04$ & $1.097 \mathrm{E}+01$ & $2.57 \mathrm{E}-02$ & $1.607 \mathrm{E}+02$ & $8.00 \mathrm{E}+00$ & $7.256 \mathrm{E}+01$ & $3.683 \mathrm{E}+01$ & $1.11 \mathrm{E}+00$ \\
\hline $1.604 \mathrm{E}+04$ & $1.331 \mathrm{E}+01$ & $2.86 \mathrm{E}-02$ & $1.550 \mathrm{E}+02$ & $8.04 \mathrm{E}+00$ & $7.297 \mathrm{E}+01$ & $3.703 \mathrm{E}+01$ & $1.10 \mathrm{E}+00$ \\
\hline $1.601 \mathrm{E}+04$ & $1.565 \mathrm{E}+01$ & 3.19E-02 & $1.401 \mathrm{E}+02$ & $8.25 \mathrm{E}+00$ & $7.329 \mathrm{E}+01$ & $3.603 \mathrm{E}+01$ & $1.06 \mathrm{E}+00$ \\
\hline $1.599 \mathrm{E}+04$ & $1.797 \mathrm{E}+01$ & $3.46 \mathrm{E}-02$ & $1.227 \mathrm{E}+02$ & $8.31 \mathrm{E}+00$ & $7.376 \mathrm{E}+01$ & $3.408 \mathrm{E}+01$ & $1.04 \mathrm{E}+00$ \\
\hline $1.598 \mathrm{E}+04$ & $2.030 \mathrm{E}+01$ & $3.85 \mathrm{E}-02$ & $1.040 \mathrm{E}+02$ & $8.31 \mathrm{E}+00$ & $7.431 \mathrm{E}+01$ & $3.137 \mathrm{E}+01$ & $1.05 \mathrm{E}+00$ \\
\hline $1.598 \mathrm{E}+04$ & $2.260 \mathrm{E}+01$ & $4.25 \mathrm{E}-02$ & $8.626 \mathrm{E}+01$ & $8.32 \mathrm{E}+00$ & $7.495 \mathrm{E}+01$ & $2.850 \mathrm{E}+01$ & $1.05 \mathrm{E}+00$ \\
\hline$P_{\mathrm{e}}(\mathrm{W})$ & $\Delta P_{\mathrm{e}}(\mathrm{W})$ & $C_{\mathrm{T}}$ & $\Delta C_{\mathrm{T}}$ & $C_{\mathrm{P}}$ & $\Delta C_{\mathrm{P}}$ & $C_{\mathrm{Q}}$ & $\Delta C_{\mathrm{Q}}$ \\
\hline $5.739 \mathrm{E}+01$ & $6.57 \mathrm{E}-01$ & $1.257 \mathrm{E}-01$ & 5.29E-03 & 8.572E-02 & $2.71 \mathrm{E}-03$ & $1.364 \mathrm{E}-02$ & 4.32E-04 \\
\hline $5.684 \mathrm{E}+01$ & $6.47 \mathrm{E}-01$ & $1.230 \mathrm{E}-01$ & $5.41 \mathrm{E}-03$ & 8.647E-02 & $2.60 \mathrm{E}-03$ & $1.376 \mathrm{E}-02$ & $4.15 \mathrm{E}-04$ \\
\hline $5.686 \mathrm{E}+01$ & $6.47 \mathrm{E}-01$ & $1.197 \mathrm{E}-01$ & $5.48 \mathrm{E}-03$ & $8.728 \mathrm{E}-02$ & $2.60 \mathrm{E}-03$ & $1.389 \mathrm{E}-02$ & 4.13E-04 \\
\hline $5.661 \mathrm{E}+01$ & $6.47 \mathrm{E}-01$ & $1.208 \mathrm{E}-01$ & $5.51 \mathrm{E}-03$ & $8.638 \mathrm{E}-02$ & $2.64 \mathrm{E}-03$ & $1.375 \mathrm{E}-02$ & $4.20 \mathrm{E}-04$ \\
\hline $5.688 \mathrm{E}+01$ & $6.49 \mathrm{E}-01$ & $1.130 \mathrm{E}-01$ & $5.62 \mathrm{E}-03$ & 8.672E-02 & $2.62 \mathrm{E}-03$ & $1.380 \mathrm{E}-02$ & $4.16 \mathrm{E}-04$ \\
\hline $5.686 \mathrm{E}+01$ & $6.47 \mathrm{E}-01$ & $1.089 \mathrm{E}-01$ & $5.65 \mathrm{E}-03$ & $8.716 \mathrm{E}-02$ & $2.59 \mathrm{E}-03$ & $1.387 \mathrm{E}-02$ & $4.12 \mathrm{E}-04$ \\
\hline $5.546 \mathrm{E}+01$ & $6.31 \mathrm{E}-01$ & 9.887E-02 & $5.82 \mathrm{E}-03$ & $8.531 \mathrm{E}-02$ & $2.51 \mathrm{E}-03$ & $1.358 \mathrm{E}-02$ & $4.00 \mathrm{E}-04$ \\
\hline $5.259 \mathrm{E}+01$ & $5.92 \mathrm{E}-01$ & $8.671 \mathrm{E}-02$ & $5.88 \mathrm{E}-03$ & 8.092E-02 & $2.47 \mathrm{E}-03$ & $1.288 \mathrm{E}-02$ & $3.93 \mathrm{E}-04$ \\
\hline $4.889 \mathrm{E}+01$ & $5.53 \mathrm{E}-01$ & $7.366 \mathrm{E}-02$ & $5.89 \mathrm{E}-03$ & $7.469 \mathrm{E}-02$ & $2.49 \mathrm{E}-03$ & $1.189 \mathrm{E}-02$ & $3.96 \mathrm{E}-04$ \\
\hline $4.409 \mathrm{E}+01$ & $4.91 \mathrm{E}-01$ & $6.113 \mathrm{E}-02$ & $5.90 \mathrm{E}-03$ & $6.790 \mathrm{E}-02$ & $2.49 \mathrm{E}-03$ & $1.081 \mathrm{E}-02$ & 3.97E-04 \\
\hline$\rho\left(\mathrm{kg} / \mathrm{m}^{\wedge} 3\right)$ & $\eta_{\mathrm{T}}$ & $\Delta \eta_{\mathrm{T}}$ & $J$ & $\Delta J$ & $\eta_{\mathrm{P}}$ & $\Delta \eta_{\mathrm{P}}$ & $R e_{0.75}$ \\
\hline $1.162 \mathrm{E}+00$ & $6.427 \mathrm{E}-01$ & $2.16 \mathrm{E}-02$ & 7.091E-02 & $5.22 \mathrm{E}-04$ & $1.040 \mathrm{E}-01$ & $5.53 \mathrm{E}-03$ & $3.962 \mathrm{E}+04$ \\
\hline $1.162 \mathrm{E}+00$ & $6.475 \mathrm{E}-01$ & $2.08 \mathrm{E}-02$ & $1.385 \mathrm{E}-01$ & $5.49 \mathrm{E}-04$ & $1.970 \mathrm{E}-01$ & $1.05 \mathrm{E}-02$ & $3.953 \mathrm{E}+04$ \\
\hline $1.162 \mathrm{E}+00$ & $6.527 \mathrm{E}-01$ & $2.08 \mathrm{E}-02$ & $2.106 \mathrm{E}-01$ & $6.20 \mathrm{E}-04$ & $2.889 \mathrm{E}-01$ & $1.58 \mathrm{E}-02$ & $3.959 \mathrm{E}+04$ \\
\hline $1.161 \mathrm{E}+00$ & $6.512 \mathrm{E}-01$ & $2.12 \mathrm{E}-02$ & $2.848 \mathrm{E}-01$ & 7.27E-04 & $3.981 \mathrm{E}-01$ & $2.19 \mathrm{E}-02$ & $3.974 \mathrm{E}+04$ \\
\hline $1.161 \mathrm{E}+00$ & $6.475 \mathrm{E}-01$ & $2.09 \mathrm{E}-02$ & $3.603 \mathrm{E}-01$ & $8.49 \mathrm{E}-04$ & 4.693E-01 & $2.73 \mathrm{E}-02$ & $3.984 \mathrm{E}+04$ \\
\hline $1.161 \mathrm{E}+00$ & $6.513 \mathrm{E}-01$ & $2.07 \mathrm{E}-02$ & $4.372 \mathrm{E}-01$ & $9.45 \mathrm{E}-04$ & $5.465 \mathrm{E}-01$ & $3.27 \mathrm{E}-02$ & $4.004 \mathrm{E}+04$ \\
\hline $1.161 \mathrm{E}+00$ & $6.496 \mathrm{E}-01$ & $2.05 \mathrm{E}-02$ & $5.149 \mathrm{E}-01$ & $1.06 \mathrm{E}-03$ & $5.967 \mathrm{E}-01$ & $3.93 \mathrm{E}-02$ & $4.021 \mathrm{E}+04$ \\
\hline $1.161 \mathrm{E}+00$ & $6.479 \mathrm{E}-01$ & $2.11 \mathrm{E}-02$ & 5.919E-01 & $1.15 \mathrm{E}-03$ & $6.343 \mathrm{E}-01$ & 4.72E-02 & $4.046 \mathrm{E}+04$ \\
\hline $1.160 \mathrm{E}+00$ & $6.416 \mathrm{E}-01$ & $2.26 \mathrm{E}-02$ & $6.692 \mathrm{E}-01$ & $1.28 \mathrm{E}-03$ & $6.600 \mathrm{E}-01$ & $5.72 \mathrm{E}-02$ & $4.075 \mathrm{E}+04$ \\
\hline $1.160 \mathrm{E}+00$ & $6.464 \mathrm{E}-01$ & $2.48 \mathrm{E}-02$ & 7.453E-01 & $1.41 \mathrm{E}-03$ & $6.709 \mathrm{E}-01$ & $6.93 \mathrm{E}-02$ & $4.109 \mathrm{E}+04$ \\
\hline
\end{tabular}


Table 102: APC 4.5 x 4.1 Dynamic Measured Values - 20027 RPM

\begin{tabular}{|c|c|c|c|c|c|c|c|}
\hline$n(\mathrm{RPM})$ & $Q(\mathrm{~g}-\mathrm{m})$ & $\Delta Q(\mathrm{~g}-\mathrm{m})$ & $V(\mathrm{~V})$ & $I(\mathrm{~A})$ & $T_{\text {atm }}\left({ }^{\circ} \mathrm{C}\right)$ & $P_{\text {atm }}(\mathrm{Pa})$ & $P_{\text {diff }}(\mathrm{Pa})$ \\
\hline $2.011 \mathrm{E}+04$ & $3.553 \mathrm{E}+00$ & $1.59 \mathrm{E}-01$ & $1.085 \mathrm{E}+01$ & $9.659 \mathrm{E}+00$ & $2.227 \mathrm{E}+01$ & $9.845 \mathrm{E}+04$ & $3.636 \mathrm{E}+00$ \\
\hline $2.004 \mathrm{E}+04$ & $3.462 \mathrm{E}+00$ & $1.48 \mathrm{E}-01$ & $1.086 \mathrm{E}+01$ & $9.518 \mathrm{E}+00$ & $2.235 \mathrm{E}+01$ & $9.845 \mathrm{E}+04$ & $1.384 \mathrm{E}+01$ \\
\hline $2.008 \mathrm{E}+04$ & $3.558 \mathrm{E}+00$ & $1.52 \mathrm{E}-01$ & $1.086 \mathrm{E}+01$ & $9.487 \mathrm{E}+00$ & $2.244 \mathrm{E}+01$ & $9.845 \mathrm{E}+04$ & $3.287 \mathrm{E}+01$ \\
\hline $2.004 \mathrm{E}+04$ & $3.553 \mathrm{E}+00$ & $1.39 \mathrm{E}-01$ & $1.085 \mathrm{E}+01$ & $9.503 \mathrm{E}+00$ & $2.259 \mathrm{E}+01$ & $9.845 \mathrm{E}+04$ & $6.093 \mathrm{E}+01$ \\
\hline $2.002 \mathrm{E}+04$ & $3.611 \mathrm{E}+00$ & $1.26 \mathrm{E}-01$ & $1.085 \mathrm{E}+01$ & $9.554 \mathrm{E}+00$ & $2.261 \mathrm{E}+01$ & $9.844 \mathrm{E}+04$ & $9.792 \mathrm{E}+01$ \\
\hline $2.000 \mathrm{E}+04$ & $3.559 \mathrm{E}+00$ & $1.20 \mathrm{E}-01$ & $1.085 \mathrm{E}+01$ & $9.583 \mathrm{E}+00$ & $2.271 \mathrm{E}+01$ & $9.844 \mathrm{E}+04$ & $1.446 \mathrm{E}+02$ \\
\hline $2.000 \mathrm{E}+04$ & $3.586 \mathrm{E}+00$ & $1.19 \mathrm{E}-01$ & $1.085 \mathrm{E}+01$ & $9.577 \mathrm{E}+00$ & $2.276 \mathrm{E}+01$ & $9.844 \mathrm{E}+04$ & $2.002 \mathrm{E}+02$ \\
\hline $2.000 \mathrm{E}+04$ & $3.537 \mathrm{E}+00$ & $1.14 \mathrm{E}-01$ & $1.086 \mathrm{E}+01$ & $9.318 \mathrm{E}+00$ & $2.279 \mathrm{E}+01$ & $9.844 \mathrm{E}+04$ & $2.638 \mathrm{E}+02$ \\
\hline $2.001 \mathrm{E}+04$ & $3.228 \mathrm{E}+00$ & $1.15 \mathrm{E}-01$ & $1.088 \mathrm{E}+01$ & $8.770 \mathrm{E}+00$ & $2.288 \mathrm{E}+01$ & $9.845 \mathrm{E}+04$ & $3.364 \mathrm{E}+02$ \\
\hline $1.998 \mathrm{E}+04$ & $3.081 \mathrm{E}+00$ & $1.16 \mathrm{E}-01$ & $1.090 \mathrm{E}+01$ & $8.013 \mathrm{E}+00$ & $2.294 \mathrm{E}+01$ & $9.844 \mathrm{E}+04$ & $4.178 \mathrm{E}+02$ \\
\hline $2.002 \mathrm{E}+04$ & $2.761 \mathrm{E}+00$ & $1.27 \mathrm{E}-01$ & $1.092 \mathrm{E}+01$ & $7.178 \mathrm{E}+00$ & $2.303 \mathrm{E}+01$ & $9.844 \mathrm{E}+04$ & $5.074 \mathrm{E}+02$ \\
\hline
\end{tabular}


Table 103: APC 4.5 x 4.1 Dynamic Calculated Values - 20027 RPM

\begin{tabular}{|c|c|c|c|c|c|c|c|}
\hline$n(\mathrm{RPM})$ & $V_{\infty}^{\prime}(\mathrm{m} / \mathrm{s})$ & $\Delta V_{\infty}^{\prime}(\mathrm{m} / \mathrm{s})$ & $T^{\prime}(\mathrm{g})$ & $\Delta T^{\prime}(\mathrm{g})$ & $V_{\mathrm{t}}(\mathrm{m} / \mathrm{s})$ & $P_{\mathrm{P}}(\mathrm{W})$ & $\Delta P_{\mathrm{P}}(\mathrm{W})$ \\
\hline $2.011 \mathrm{E}+04$ & $2.354 \mathrm{E}+00$ & $1.88 \mathrm{E}-02$ & $2.895 \mathrm{E}+02$ & $7.30 \mathrm{E}+00$ & $8.996 \mathrm{E}+01$ & $7.338 \mathrm{E}+01$ & $3.29 \mathrm{E}+00$ \\
\hline $2.004 \mathrm{E}+04$ & $4.738 \mathrm{E}+00$ & $2.01 \mathrm{E}-02$ & $2.836 \mathrm{E}+02$ & $7.33 \mathrm{E}+00$ & $8.975 \mathrm{E}+01$ & $7.125 \mathrm{E}+01$ & $3.05 \mathrm{E}+00$ \\
\hline $2.008 \mathrm{E}+04$ & $7.386 \mathrm{E}+00$ & $2.30 \mathrm{E}-02$ & $2.851 \mathrm{E}+02$ & $7.31 \mathrm{E}+00$ & $9.012 \mathrm{E}+01$ & $7.338 \mathrm{E}+01$ & $3.13 \mathrm{E}+00$ \\
\hline $2.004 \mathrm{E}+04$ & $1.012 \mathrm{E}+01$ & $2.74 \mathrm{E}-02$ & $2.772 \mathrm{E}+02$ & $7.36 \mathrm{E}+00$ & $9.022 \mathrm{E}+01$ & $7.313 \mathrm{E}+01$ & $2.85 \mathrm{E}+00$ \\
\hline $2.002 \mathrm{E}+04$ & $1.287 \mathrm{E}+01$ & $3.11 \mathrm{E}-02$ & $2.680 \mathrm{E}+02$ & $7.35 \mathrm{E}+00$ & $9.044 \mathrm{E}+01$ & $7.423 \mathrm{E}+01$ & $2.60 \mathrm{E}+00$ \\
\hline $2.000 \mathrm{E}+04$ & $1.568 \mathrm{E}+01$ & 3.37E-02 & $2.562 \mathrm{E}+02$ & $7.40 \mathrm{E}+00$ & $9.080 \mathrm{E}+01$ & $7.308 \mathrm{E}+01$ & $2.46 \mathrm{E}+00$ \\
\hline $2.000 \mathrm{E}+04$ & $1.849 \mathrm{E}+01$ & $3.68 \mathrm{E}-02$ & $2.427 \mathrm{E}+02$ & $7.37 \mathrm{E}+00$ & $9.135 \mathrm{E}+01$ & $7.365 \mathrm{E}+01$ & $2.45 \mathrm{E}+00$ \\
\hline $2.000 \mathrm{E}+04$ & $2.125 \mathrm{E}+01$ & $4.10 \mathrm{E}-02$ & $2.228 \mathrm{E}+02$ & $7.45 \mathrm{E}+00$ & $9.195 \mathrm{E}+01$ & $7.266 \mathrm{E}+01$ & $2.35 \mathrm{E}+00$ \\
\hline $2.001 \mathrm{E}+04$ & $2.403 \mathrm{E}+01$ & $4.59 \mathrm{E}-02$ & $1.934 \mathrm{E}+02$ & $7.38 \mathrm{E}+00$ & $9.265 \mathrm{E}+01$ & $6.633 \mathrm{E}+01$ & $2.37 \mathrm{E}+00$ \\
\hline $1.998 \mathrm{E}+04$ & $2.680 \mathrm{E}+01$ & $5.10 \mathrm{E}-02$ & $1.651 \mathrm{E}+02$ & $7.38 \mathrm{E}+00$ & $9.331 \mathrm{E}+01$ & $6.324 \mathrm{E}+01$ & $2.38 \mathrm{E}+00$ \\
\hline $2.002 \mathrm{E}+04$ & $2.956 \mathrm{E}+01$ & $5.56 \mathrm{E}-02$ & $1.376 \mathrm{E}+02$ & $7.44 \mathrm{E}+00$ & $9.429 \mathrm{E}+01$ & $5.676 \mathrm{E}+01$ & $2.60 \mathrm{E}+00$ \\
\hline$P_{\mathrm{e}}(\mathrm{W})$ & $\Delta P_{\mathrm{e}}(\mathrm{W})$ & $C_{\mathrm{T}}$ & $\Delta C_{\mathrm{T}}$ & $C_{\mathrm{P}}$ & $\Delta C_{\mathrm{P}}$ & $C_{\mathrm{Q}}$ & $\Delta C_{\mathrm{Q}}$ \\
\hline $1.048 \mathrm{E}+02$ & $1.13 \mathrm{E}+00$ & $1.294 \mathrm{E}-01$ & $3.27 \mathrm{E}-03$ & $8.767 \mathrm{E}-02$ & $3.93 \mathrm{E}-03$ & $1.395 \mathrm{E}-02$ & $6.25 \mathrm{E}-04$ \\
\hline $1.033 \mathrm{E}+02$ & $1.09 \mathrm{E}+00$ & $1.277 \mathrm{E}-01$ & $3.30 \mathrm{E}-03$ & 8.603E-02 & $3.68 \mathrm{E}-03$ & $1.369 \mathrm{E}-02$ & $5.85 \mathrm{E}-04$ \\
\hline $1.030 \mathrm{E}+02$ & $1.09 \mathrm{E}+00$ & $1.279 \mathrm{E}-01$ & $3.28 \mathrm{E}-03$ & $8.805 \mathrm{E}-02$ & $3.75 \mathrm{E}-03$ & $1.401 \mathrm{E}-02$ & $5.97 \mathrm{E}-04$ \\
\hline $1.032 \mathrm{E}+02$ & $1.09 \mathrm{E}+00$ & $1.249 \mathrm{E}-01$ & $3.32 \mathrm{E}-03$ & 8.830E-02 & $3.45 \mathrm{E}-03$ & $1.405 \mathrm{E}-02$ & $5.48 \mathrm{E}-04$ \\
\hline $1.037 \mathrm{E}+02$ & $1.09 \mathrm{E}+00$ & $1.211 \mathrm{E}-01$ & $3.32 \mathrm{E}-03$ & $9.004 \mathrm{E}-02$ & $3.15 \mathrm{E}-03$ & $1.433 \mathrm{E}-02$ & $5.01 \mathrm{E}-04$ \\
\hline $1.040 \mathrm{E}+02$ & $1.09 \mathrm{E}+00$ & $1.160 \mathrm{E}-01$ & $3.35 \mathrm{E}-03$ & 8.892E-02 & $3.00 \mathrm{E}-03$ & $1.415 \mathrm{E}-02$ & 4.77E-04 \\
\hline $1.039 \mathrm{E}+02$ & $1.09 \mathrm{E}+00$ & $1.099 \mathrm{E}-01$ & $3.34 \mathrm{E}-03$ & 8.957E-02 & $2.98 \mathrm{E}-03$ & $1.426 \mathrm{E}-02$ & 4.74E-04 \\
\hline $1.012 \mathrm{E}+02$ & $1.07 \mathrm{E}+00$ & $1.009 \mathrm{E}-01$ & $3.37 \mathrm{E}-03$ & $8.835 \mathrm{E}-02$ & $2.86 \mathrm{E}-03$ & $1.406 \mathrm{E}-02$ & $4.55 \mathrm{E}-04$ \\
\hline $9.538 \mathrm{E}+01$ & $1.01 \mathrm{E}+00$ & $8.754 \mathrm{E}-02$ & $3.34 \mathrm{E}-03$ & 8.062E-02 & $2.88 \mathrm{E}-03$ & $1.283 \mathrm{E}-02$ & $4.59 \mathrm{E}-04$ \\
\hline $8.731 \mathrm{E}+01$ & 9.29E-01 & $7.490 \mathrm{E}-02$ & $3.35 \mathrm{E}-03$ & 7.715E-02 & $2.90 \mathrm{E}-03$ & $1.228 \mathrm{E}-02$ & 4.62E-04 \\
\hline $7.838 \mathrm{E}+01$ & 8.39E-01 & $6.226 \mathrm{E}-02$ & $3.36 \mathrm{E}-03$ & $6.891 \mathrm{E}-02$ & $3.16 \mathrm{E}-03$ & 1.097E-02 & 5.03E-04 \\
\hline$\rho\left(\mathrm{kg} / \mathrm{m}^{\wedge} 3\right)$ & $\eta_{\mathrm{T}}$ & $\Delta \eta_{\mathrm{T}}$ & $J$ & $\Delta J$ & $\eta_{\mathrm{P}}$ & $\Delta \eta_{\mathrm{P}}$ & $R e_{0.75}$ \\
\hline $1.161 \mathrm{E}+00$ & $7.000 \mathrm{E}-01$ & $3.22 \mathrm{E}-02$ & $6.167 \mathrm{E}-02$ & 4.93E-04 & $9.105 \mathrm{E}-02$ & $4.74 \mathrm{E}-03$ & $4.939 \mathrm{E}+04$ \\
\hline $1.161 \mathrm{E}+00$ & $6.896 \mathrm{E}-01$ & $3.04 \mathrm{E}-02$ & $1.246 \mathrm{E}-01$ & $5.30 \mathrm{E}-04$ & $1.849 \mathrm{E}-01$ & $9.27 \mathrm{E}-03$ & $4.925 \mathrm{E}+04$ \\
\hline $1.160 \mathrm{E}+00$ & $7.125 \mathrm{E}-01$ & $3.13 \mathrm{E}-02$ & $1.938 \mathrm{E}-01$ & $6.10 \mathrm{E}-04$ & $2.815 \mathrm{E}-01$ & $1.40 \mathrm{E}-02$ & $4.943 \mathrm{E}+04$ \\
\hline $1.160 \mathrm{E}+00$ & $7.090 \mathrm{E}-01$ & $2.86 \mathrm{E}-02$ & $2.659 \mathrm{E}-01$ & $7.30 \mathrm{E}-04$ & 3.761E-01 & $1.78 \mathrm{E}-02$ & $4.944 \mathrm{E}+04$ \\
\hline $1.159 \mathrm{E}+00$ & 7.159E-01 & $2.61 \mathrm{E}-02$ & $3.389 \mathrm{E}-01$ & 8.33E-04 & $4.558 \mathrm{E}-01$ & $2.03 \mathrm{E}-02$ & $4.955 \mathrm{E}+04$ \\
\hline $1.159 \mathrm{E}+00$ & $7.027 \mathrm{E}-01$ & $2.48 \mathrm{E}-02$ & 4.133E-01 & $9.08 \mathrm{E}-04$ & 5.392E-01 & $2.40 \mathrm{E}-02$ & $4.972 \mathrm{E}+04$ \\
\hline $1.159 \mathrm{E}+00$ & $7.086 \mathrm{E}-01$ & $2.47 \mathrm{E}-02$ & 4.870E-01 & $9.94 \mathrm{E}-04$ & $5.975 \mathrm{E}-01$ & $2.69 \mathrm{E}-02$ & $5.000 \mathrm{E}+04$ \\
\hline $1.159 \mathrm{E}+00$ & $7.180 \mathrm{E}-01$ & $2.44 \mathrm{E}-02$ & $5.597 \mathrm{E}-01$ & $1.11 \mathrm{E}-03$ & $6.390 \mathrm{E}-01$ & $2.98 \mathrm{E}-02$ & $5.032 \mathrm{E}+04$ \\
\hline $1.159 \mathrm{E}+00$ & $6.954 \mathrm{E}-01$ & $2.59 \mathrm{E}-02$ & $6.327 \mathrm{E}-01$ & $1.24 \mathrm{E}-03$ & $6.870 \mathrm{E}-01$ & $3.60 \mathrm{E}-02$ & $5.068 \mathrm{E}+04$ \\
\hline $1.158 \mathrm{E}+00$ & $7.243 \mathrm{E}-01$ & $2.83 \mathrm{E}-02$ & $7.066 \mathrm{E}-01$ & $1.38 \mathrm{E}-03$ & $6.860 \mathrm{E}-01$ & $4.01 \mathrm{E}-02$ & $5.102 \mathrm{E}+04$ \\
\hline $1.158 \mathrm{E}+00$ & 7.242E-01 & $3.41 \mathrm{E}-02$ & 7.779E-01 & $1.50 \mathrm{E}-03$ & $7.028 \mathrm{E}-01$ & $4.98 \mathrm{E}-02$ & $5.153 \mathrm{E}+04$ \\
\hline
\end{tabular}




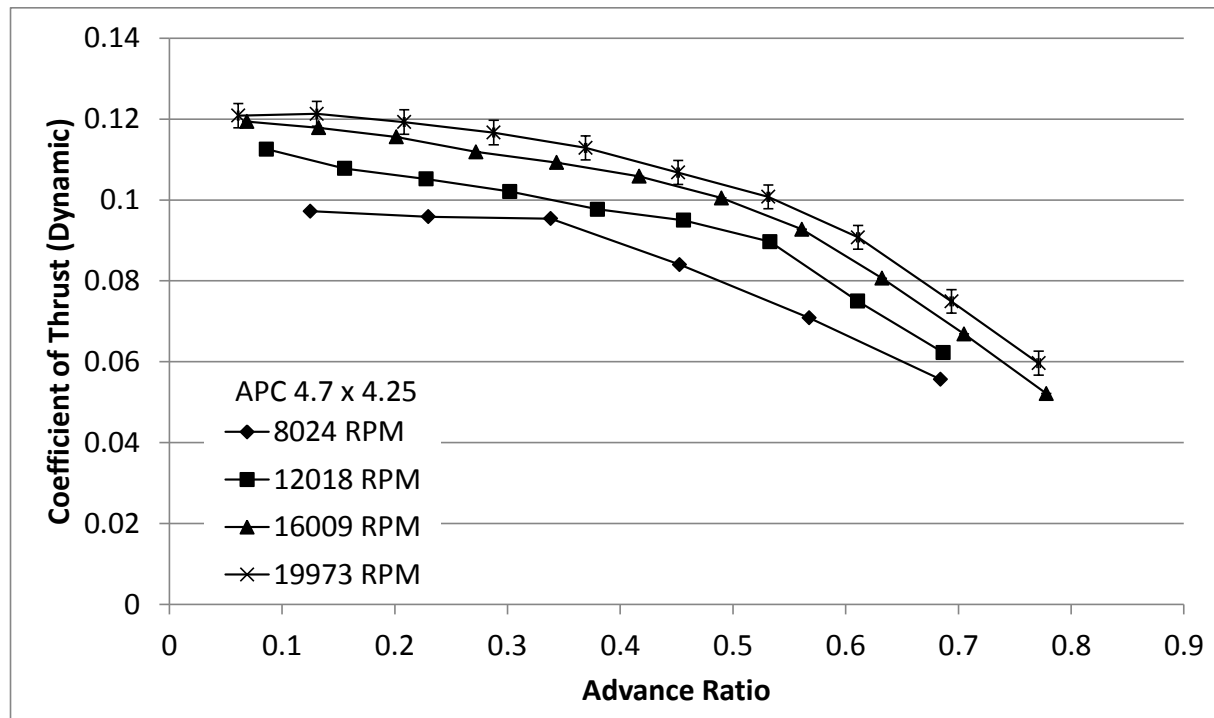

(a)

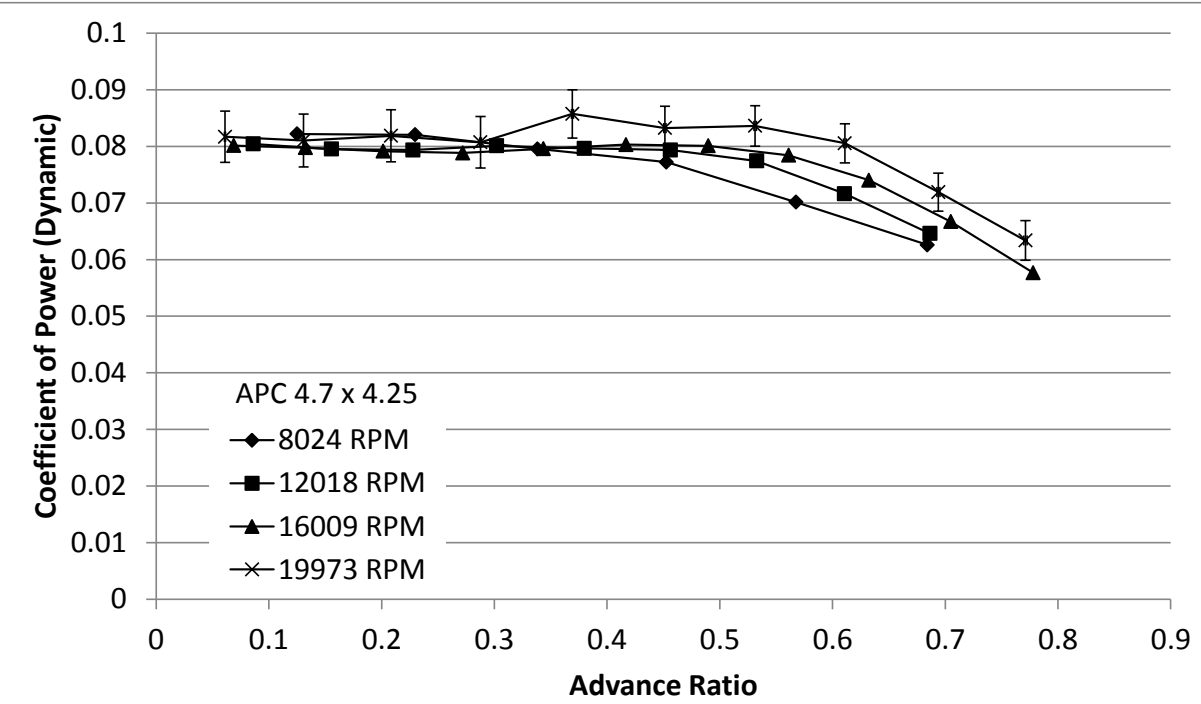

(b)

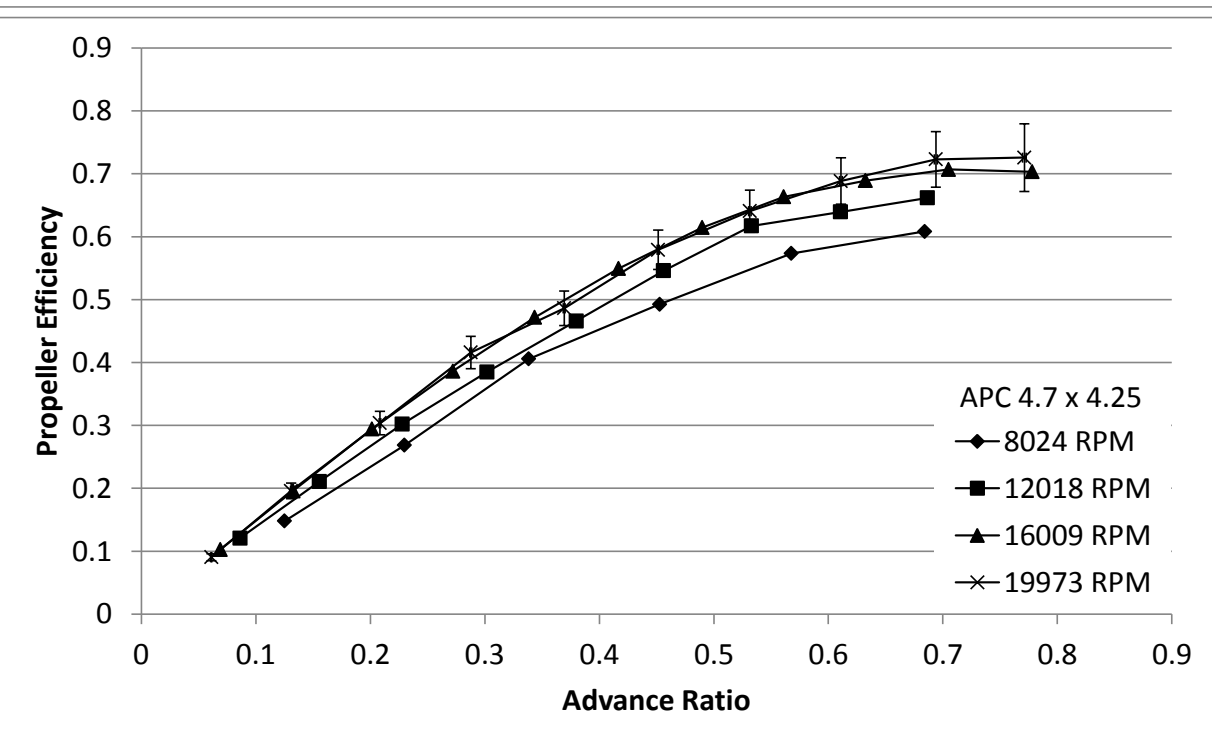

(c) 


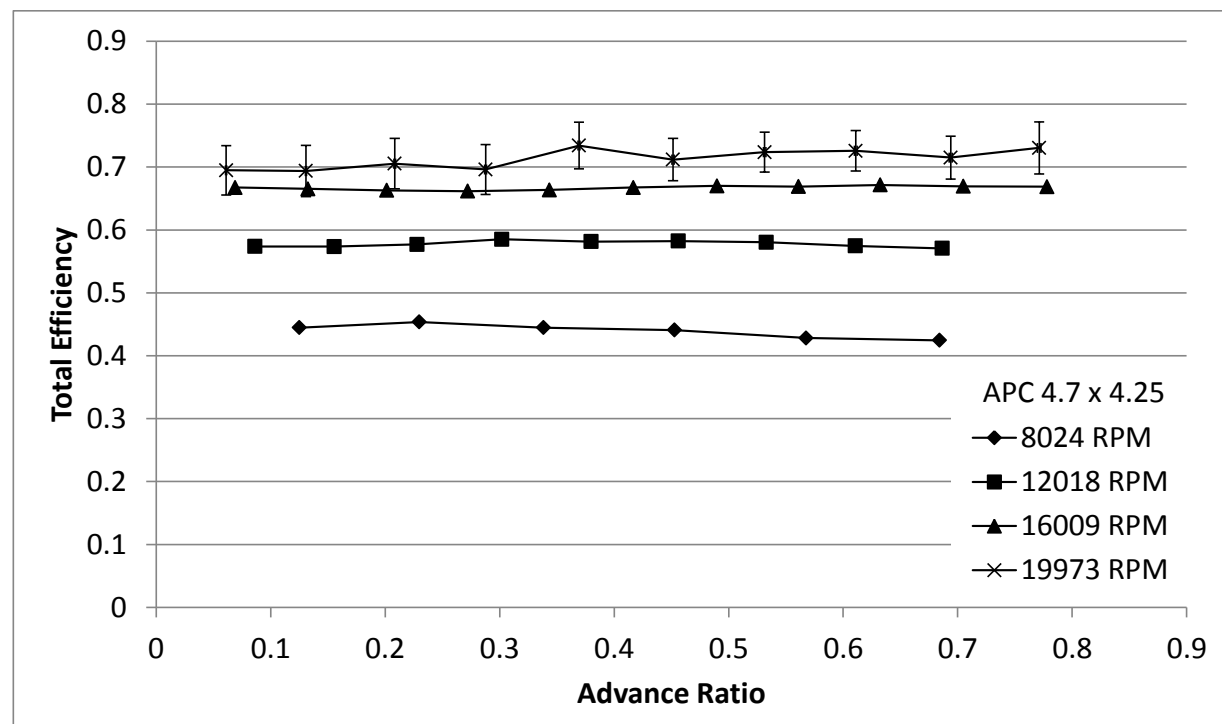

(d)

Figure 82: APC 4.7 x 4.25 Dynamic Test Results: (a) Coefficient of Thrust, (b)

Coefficient of Power, (c) Propeller Efficiency, (d) Total Efficiency.

Table 104: APC 4.7 x 4.25 Dynamic Measured Values - 8024 RPM

\begin{tabular}{|c|c|c|c|c|c|c|c|}
\hline$n(\mathrm{RPM})$ & $Q(\mathrm{~g}-\mathrm{m})$ & $\Delta Q(\mathrm{~g}-\mathrm{m})$ & $V(\mathrm{~V})$ & $I(\mathrm{~A})$ & $T_{\text {atm }}\left({ }^{\circ} \mathrm{C}\right)$ & $P_{\text {atm }}(\mathrm{Pa})$ & $P_{\text {diff }}(\mathrm{Pa})$ \\
\hline $8.040 \mathrm{E}+03$ & $6.973 \mathrm{E}-01$ & $7.24 \mathrm{E}-02$ & $1.108 \mathrm{E}+01$ & $1.168 \mathrm{E}+00$ & $2.223 \mathrm{E}+01$ & $9.839 \mathrm{E}+04$ & $2.498 \mathrm{E}+00$ \\
\hline $8.041 \mathrm{E}+03$ & $6.976 \mathrm{E}-01$ & $7.48 \mathrm{E}-02$ & $1.108 \mathrm{E}+01$ & $1.145 \mathrm{E}+00$ & $2.165 \mathrm{E}+01$ & $9.839 \mathrm{E}+04$ & $8.204 \mathrm{E}+00$ \\
\hline $8.038 \mathrm{E}+03$ & $6.768 \mathrm{E}-01$ & $7.78 \mathrm{E}-02$ & $1.108 \mathrm{E}+01$ & $1.133 \mathrm{E}+00$ & $2.127 \mathrm{E}+01$ & $9.840 \mathrm{E}+04$ & $1.760 \mathrm{E}+01$ \\
\hline $8.021 \mathrm{E}+03$ & $6.540 \mathrm{E}-01$ & $8.29 \mathrm{E}-02$ & $1.108 \mathrm{E}+01$ & $1.103 \mathrm{E}+00$ & $2.140 \mathrm{E}+01$ & $9.840 \mathrm{E}+04$ & $3.113 \mathrm{E}+01$ \\
\hline $8.018 \mathrm{E}+03$ & $5.930 \mathrm{E}-01$ & $8.54 \mathrm{E}-02$ & $1.109 \mathrm{E}+01$ & $1.029 \mathrm{E}+00$ & $2.163 \mathrm{E}+01$ & $9.840 \mathrm{E}+04$ & $4.867 \mathrm{E}+01$ \\
\hline $7.984 \mathrm{E}+03$ & $5.241 \mathrm{E}-01$ & $8.84 \mathrm{E}-02$ & $1.109 \mathrm{E}+01$ & $9.128 \mathrm{E}-01$ & $2.194 \mathrm{E}+01$ & $9.840 \mathrm{E}+04$ & $6.982 \mathrm{E}+01$ \\
\hline
\end{tabular}


Table 105: APC 4.7 x 4.25 Dynamic Calculated Values - 8024 RPM

\begin{tabular}{|c|c|c|c|c|c|c|c|}
\hline$n(\mathrm{RPM})$ & $V_{\infty}^{\prime}(\mathrm{m} / \mathrm{s})$ & $\Delta V_{\infty}^{\prime}(\mathrm{m} / \mathrm{s})$ & $T^{\prime}(\mathrm{g})$ & $\Delta T^{\prime}(\mathrm{g})$ & $V_{\mathrm{t}}(\mathrm{m} / \mathrm{s})$ & $P_{\mathrm{P}}(\mathrm{W})$ & $\Delta P_{\mathrm{P}}(\mathrm{W})$ \\
\hline $8.040 \mathrm{E}+03$ & $2.016 \mathrm{E}+00$ & $1.39 \mathrm{E}-02$ & $4.312 \mathrm{E}+01$ & $1.25 \mathrm{E}+01$ & $3.801 \mathrm{E}+01$ & $5.757 \mathrm{E}+00$ & $5.98 \mathrm{E}-01$ \\
\hline $8.041 \mathrm{E}+03$ & $3.702 \mathrm{E}+00$ & $1.47 \mathrm{E}-02$ & $4.261 \mathrm{E}+01$ & $1.27 \mathrm{E}+01$ & $3.814 \mathrm{E}+01$ & $5.761 \mathrm{E}+00$ & $6.18 \mathrm{E}-01$ \\
\hline $8.038 \mathrm{E}+03$ & $5.449 \mathrm{E}+00$ & $1.64 \mathrm{E}-02$ & $4.243 \mathrm{E}+01$ & $1.24 \mathrm{E}+01$ & $3.833 \mathrm{E}+01$ & $5.587 \mathrm{E}+00$ & $6.42 \mathrm{E}-01$ \\
\hline $8.021 \mathrm{E}+03$ & $7.274 \mathrm{E}+00$ & $1.86 \mathrm{E}-02$ & $3.720 \mathrm{E}+01$ & $1.26 \mathrm{E}+01$ & $3.856 \mathrm{E}+01$ & $5.387 \mathrm{E}+00$ & $6.83 \mathrm{E}-01$ \\
\hline $8.018 \mathrm{E}+03$ & $9.118 \mathrm{E}+00$ & $2.17 \mathrm{E}-02$ & $3.131 \mathrm{E}+01$ & $1.24 \mathrm{E}+01$ & $3.893 \mathrm{E}+01$ & $4.883 \mathrm{E}+00$ & 7.03E-01 \\
\hline 7.984E+03 & $1.094 \mathrm{E}+01$ & $2.40 \mathrm{E}-02$ & $2.436 \mathrm{E}+01$ & $1.25 \mathrm{E}+01$ & $3.924 \mathrm{E}+01$ & $4.297 \mathrm{E}+00$ & 7.24E-01 \\
\hline$P_{\mathrm{e}}(\mathrm{W})$ & $\Delta P_{\mathrm{e}}(\mathrm{W})$ & $C_{\mathrm{T}}$ & $\Delta C_{\mathrm{T}}$ & $C_{\mathrm{P}}$ & $\Delta C_{\mathrm{P}}$ & $C_{\mathrm{Q}}$ & $\Delta C_{\mathrm{Q}}$ \\
\hline $1.295 \mathrm{E}+01$ & $1.57 \mathrm{E}-01$ & $9.722 \mathrm{E}-02$ & $2.83 \mathrm{E}-02$ & $8.218 \mathrm{E}-02$ & 8.54E-03 & $1.308 \mathrm{E}-02$ & $1.36 \mathrm{E}-03$ \\
\hline $1.270 \mathrm{E}+01$ & $1.55 \mathrm{E}-01$ & $9.585 \mathrm{E}-02$ & $2.85 \mathrm{E}-02$ & $8.204 \mathrm{E}-02$ & 8.80E-03 & $1.306 \mathrm{E}-02$ & $1.40 \mathrm{E}-03$ \\
\hline $1.256 \mathrm{E}+01$ & $1.54 \mathrm{E}-01$ & $9.540 \mathrm{E}-02$ & $2.79 \mathrm{E}-02$ & 7.956E-02 & $9.15 \mathrm{E}-03$ & $1.266 \mathrm{E}-02$ & $1.46 \mathrm{E}-03$ \\
\hline $1.222 \mathrm{E}+01$ & $1.50 \mathrm{E}-01$ & 8.403E-02 & $2.85 \mathrm{E}-02$ & 7.722E-02 & 9.79E-03 & $1.229 \mathrm{E}-02$ & $1.56 \mathrm{E}-03$ \\
\hline $1.140 \mathrm{E}+01$ & $1.42 \mathrm{E}-01$ & 7.084E-02 & $2.81 \mathrm{E}-02$ & 7.013E-02 & $1.01 \mathrm{E}-02$ & $1.116 \mathrm{E}-02$ & $1.61 \mathrm{E}-03$ \\
\hline $1.012 \mathrm{E}+01$ & $1.28 \mathrm{E}-01$ & $5.564 \mathrm{E}-02$ & $2.86 \mathrm{E}-02$ & $6.258 \mathrm{E}-02$ & $1.05 \mathrm{E}-02$ & $9.960 \mathrm{E}-03$ & $1.68 \mathrm{E}-03$ \\
\hline$\rho\left(\mathrm{kg} / \mathrm{m}^{\wedge} 3\right)$ & $\eta_{\mathrm{T}}$ & $\Delta \eta_{\mathrm{T}}$ & $J$ & $\Delta J$ & $\eta_{\mathrm{P}}$ & $\Delta \eta_{\mathrm{P}}$ & $R e_{0.75}$ \\
\hline $1.160 \mathrm{E}+00$ & 4.447E-01 & $4.65 \mathrm{E}-02$ & $1.252 \mathrm{E}-01$ & $8.63 \mathrm{E}-04$ & $1.481 \mathrm{E}-01$ & $4.58 \mathrm{E}-02$ & $2.040 \mathrm{E}+04$ \\
\hline $1.163 \mathrm{E}+00$ & $4.538 \mathrm{E}-01$ & $4.90 \mathrm{E}-02$ & $2.298 \mathrm{E}-01$ & $9.14 \mathrm{E}-04$ & $2.685 \mathrm{E}-01$ & 8.49E-02 & $2.054 \mathrm{E}+04$ \\
\hline $1.164 \mathrm{E}+00$ & $4.448 \mathrm{E}-01$ & $5.14 \mathrm{E}-02$ & $3.384 \mathrm{E}-01$ & $1.03 \mathrm{E}-03$ & $4.058 \mathrm{E}-01$ & $1.28 \mathrm{E}-01$ & $2.069 \mathrm{E}+04$ \\
\hline $1.164 \mathrm{E}+00$ & $4.408 \mathrm{E}-01$ & $5.62 \mathrm{E}-02$ & 4.527E-01 & $1.17 \mathrm{E}-03$ & $4.926 \mathrm{E}-01$ & $1.78 \mathrm{E}-01$ & $2.080 \mathrm{E}+04$ \\
\hline $1.163 \mathrm{E}+00$ & 4.282E-01 & $6.19 \mathrm{E}-02$ & $5.677 \mathrm{E}-01$ & $1.37 \mathrm{E}-03$ & $5.734 \mathrm{E}-01$ & $2.42 \mathrm{E}-01$ & $2.097 \mathrm{E}+04$ \\
\hline $1.162 \mathrm{E}+00$ & $4.245 \mathrm{E}-01$ & 7.18E-02 & $6.841 \mathrm{E}-01$ & $1.53 \mathrm{E}-03$ & $6.083 \mathrm{E}-01$ & $3.29 \mathrm{E}-01$ & $2.110 \mathrm{E}+04$ \\
\hline
\end{tabular}


Table 106: APC 4.7 x 4.25 Dynamic Measured Values - 12018 RPM

\begin{tabular}{|c|c|c|c|c|c|c|c|}
\hline$n(\mathrm{RPM})$ & $Q(\mathrm{~g}-\mathrm{m})$ & $\Delta Q(\mathrm{~g}-\mathrm{m})$ & $V(\mathrm{~V})$ & $I(\mathrm{~A})$ & $T_{\text {atm }}\left({ }^{\circ} \mathrm{C}\right)$ & $P_{\text {atm }}(\mathrm{Pa})$ & $P_{\text {diff }}(\mathrm{Pa})$ \\
\hline $1.202 \mathrm{E}+04$ & $1.528 \mathrm{E}+00$ & $6.83 \mathrm{E}-02$ & $1.103 \mathrm{E}+01$ & $2.978 \mathrm{E}+00$ & $2.170 \mathrm{E}+01$ & $9.840 \mathrm{E}+04$ & $2.748 \mathrm{E}+00$ \\
\hline $1.200 \mathrm{E}+04$ & $1.506 \mathrm{E}+00$ & $6.60 \mathrm{E}-02$ & $1.103 \mathrm{E}+01$ & $2.932 \mathrm{E}+00$ & $2.161 \mathrm{E}+01$ & $9.840 \mathrm{E}+04$ & $8.535 \mathrm{E}+00$ \\
\hline $1.203 \mathrm{E}+04$ & $1.508 \mathrm{E}+00$ & $6.80 \mathrm{E}-02$ & $1.103 \mathrm{E}+01$ & $2.925 \mathrm{E}+00$ & $2.192 \mathrm{E}+01$ & $9.840 \mathrm{E}+04$ & $1.808 \mathrm{E}+01$ \\
\hline $1.204 \mathrm{E}+04$ & $1.525 \mathrm{E}+00$ & $6.96 \mathrm{E}-02$ & $1.103 \mathrm{E}+01$ & $2.921 \mathrm{E}+00$ & $2.219 \mathrm{E}+01$ & $9.840 \mathrm{E}+04$ & $3.149 \mathrm{E}+01$ \\
\hline $1.199 \mathrm{E}+04$ & $1.501 \mathrm{E}+00$ & $6.91 \mathrm{E}-02$ & $1.104 \mathrm{E}+01$ & $2.881 \mathrm{E}+00$ & $2.242 \mathrm{E}+01$ & $9.840 \mathrm{E}+04$ & $4.904 \mathrm{E}+01$ \\
\hline $1.201 \mathrm{E}+04$ & $1.500 \mathrm{E}+00$ & $6.56 \mathrm{E}-02$ & $1.104 \mathrm{E}+01$ & $2.878 \mathrm{E}+00$ & $2.261 \mathrm{E}+01$ & $9.841 \mathrm{E}+04$ & $7.062 \mathrm{E}+01$ \\
\hline $1.202 \mathrm{E}+04$ & $1.467 \mathrm{E}+00$ & $6.71 \mathrm{E}-02$ & $1.104 \mathrm{E}+01$ & $2.828 \mathrm{E}+00$ & $2.274 \mathrm{E}+01$ & $9.841 \mathrm{E}+04$ & $9.630 \mathrm{E}+01$ \\
\hline $1.201 \mathrm{E}+04$ & $1.355 \mathrm{E}+00$ & $6.50 \mathrm{E}-02$ & $1.104 \mathrm{E}+01$ & $2.633 \mathrm{E}+00$ & $2.274 \mathrm{E}+01$ & $9.840 \mathrm{E}+04$ & $1.259 \mathrm{E}+02$ \\
\hline $1.204 \mathrm{E}+04$ & $1.227 \mathrm{E}+00$ & $6.72 \mathrm{E}-02$ & $1.105 \mathrm{E}+01$ & $2.407 \mathrm{E}+00$ & $2.282 \mathrm{E}+01$ & $9.840 \mathrm{E}+04$ & $1.595 \mathrm{E}+02$ \\
\hline
\end{tabular}


Table 107: APC 4.7 x 4.25 Dynamic Calculated Values - 12018 RPM

\begin{tabular}{|c|c|c|c|c|c|c|c|}
\hline$n(\mathrm{RPM})$ & $V_{\infty}^{\prime}(\mathrm{m} / \mathrm{s})$ & $\Delta V_{\infty}^{\prime}(\mathrm{m} / \mathrm{s})$ & $T^{\prime}(\mathrm{g})$ & $\Delta T^{\prime}(\mathrm{g})$ & $V_{\mathrm{t}}(\mathrm{m} / \mathrm{s})$ & $P_{\mathrm{P}}(\mathrm{W})$ & $\Delta P_{\mathrm{P}}(\mathrm{W})$ \\
\hline $1.202 \mathrm{E}+04$ & $2.077 \mathrm{E}+00$ & $1.37 \mathrm{E}-02$ & $1.118 \mathrm{E}+02$ & $9.65 \mathrm{E}+00$ & $5.678 \mathrm{E}+01$ & $1.885 \mathrm{E}+01$ & $8.43 \mathrm{E}-01$ \\
\hline $1.200 \mathrm{E}+04$ & $3.739 \mathrm{E}+00$ & $1.43 \mathrm{E}-02$ & $1.067 \mathrm{E}+02$ & $9.72 \mathrm{E}+00$ & $5.677 \mathrm{E}+01$ & $1.856 \mathrm{E}+01$ & 8.13E-01 \\
\hline $1.203 \mathrm{E}+04$ & $5.492 \mathrm{E}+00$ & $1.61 \mathrm{E}-02$ & $1.045 \mathrm{E}+02$ & $9.70 \mathrm{E}+00$ & $5.704 \mathrm{E}+01$ & $1.862 \mathrm{E}+01$ & 8.39E-01 \\
\hline $1.204 \mathrm{E}+04$ & $7.286 \mathrm{E}+00$ & $1.83 \mathrm{E}-02$ & $1.016 \mathrm{E}+02$ & $9.67 \mathrm{E}+00$ & $5.732 \mathrm{E}+01$ & $1.886 \mathrm{E}+01$ & $8.61 \mathrm{E}-01$ \\
\hline $1.199 \mathrm{E}+04$ & $9.124 \mathrm{E}+00$ & $2.15 \mathrm{E}-02$ & $9.627 \mathrm{E}+01$ & $9.75 \mathrm{E}+00$ & $5.732 \mathrm{E}+01$ & $1.848 \mathrm{E}+01$ & $8.51 \mathrm{E}-01$ \\
\hline $1.201 \mathrm{E}+04$ & $1.097 \mathrm{E}+01$ & $2.40 \mathrm{E}-02$ & $9.386 \mathrm{E}+01$ & $9.74 \mathrm{E}+00$ & $5.774 \mathrm{E}+01$ & $1.850 \mathrm{E}+01$ & $8.09 \mathrm{E}-01$ \\
\hline $1.202 \mathrm{E}+04$ & $1.283 \mathrm{E}+01$ & $2.68 \mathrm{E}-02$ & $8.884 \mathrm{E}+01$ & $9.71 \mathrm{E}+00$ & $5.820 \mathrm{E}+01$ & $1.812 \mathrm{E}+01$ & $8.29 \mathrm{E}-01$ \\
\hline $1.201 \mathrm{E}+04$ & $1.470 \mathrm{E}+01$ & $2.95 \mathrm{E}-02$ & $7.412 \mathrm{E}+01$ & $9.69 \mathrm{E}+00$ & $5.858 \mathrm{E}+01$ & $1.671 \mathrm{E}+01$ & $8.02 \mathrm{E}-01$ \\
\hline $1.204 \mathrm{E}+04$ & $1.656 \mathrm{E}+01$ & $3.26 \mathrm{E}-02$ & $6.184 \mathrm{E}+01$ & $9.70 \mathrm{E}+00$ & $5.920 \mathrm{E}+01$ & $1.517 \mathrm{E}+01$ & $8.30 \mathrm{E}-01$ \\
\hline$P_{\mathrm{e}}(\mathrm{W})$ & $\Delta P_{\mathrm{e}}(\mathrm{W})$ & $C_{\mathrm{T}}$ & $\Delta C_{\mathrm{T}}$ & $C_{\mathrm{P}}$ & $\Delta C_{\mathrm{P}}$ & $C_{\mathrm{Q}}$ & $\Delta C_{\mathrm{Q}}$ \\
\hline $3.286 \mathrm{E}+01$ & 3.67E-01 & $1.126 \mathrm{E}-01$ & $9.72 \mathrm{E}-03$ & $8.041 \mathrm{E}-02$ & $3.59 \mathrm{E}-03$ & $1.280 \mathrm{E}-02$ & $5.72 \mathrm{E}-04$ \\
\hline $3.235 \mathrm{E}+01$ & $3.62 \mathrm{E}-01$ & $1.078 \mathrm{E}-01$ & $9.82 \mathrm{E}-03$ & 7.952E-02 & $3.49 \mathrm{E}-03$ & $1.266 \mathrm{E}-02$ & $5.55 \mathrm{E}-04$ \\
\hline $3.228 \mathrm{E}+01$ & $3.62 \mathrm{E}-01$ & $1.052 \mathrm{E}-01$ & $9.76 \mathrm{E}-03$ & $7.935 \mathrm{E}-02$ & $3.58 \mathrm{E}-03$ & $1.263 \mathrm{E}-02$ & $5.69 \mathrm{E}-04$ \\
\hline $3.223 \mathrm{E}+01$ & $3.64 \mathrm{E}-01$ & $1.021 \mathrm{E}-01$ & 9.72E-03 & 8.009E-02 & $3.66 \mathrm{E}-03$ & $1.275 \mathrm{E}-02$ & $5.82 \mathrm{E}-04$ \\
\hline $3.179 \mathrm{E}+01$ & $3.56 \mathrm{E}-01$ & $9.768 \mathrm{E}-02$ & $9.89 \mathrm{E}-03$ & 7.964E-02 & 3.67E-03 & $1.267 \mathrm{E}-02$ & $5.83 \mathrm{E}-04$ \\
\hline $3.176 \mathrm{E}+01$ & $3.58 \mathrm{E}-01$ & $9.499 \mathrm{E}-02$ & $9.86 \mathrm{E}-03$ & $7.935 \mathrm{E}-02$ & $3.47 \mathrm{E}-03$ & $1.263 \mathrm{E}-02$ & $5.53 \mathrm{E}-04$ \\
\hline $3.122 \mathrm{E}+01$ & $3.51 \mathrm{E}-01$ & 8.969E-02 & $9.81 \mathrm{E}-03$ & 7.743E-02 & $3.54 \mathrm{E}-03$ & $1.232 \mathrm{E}-02$ & 5.64E-04 \\
\hline $2.907 \mathrm{E}+01$ & $3.27 \mathrm{E}-01$ & 7.499E-02 & $9.81 \mathrm{E}-03$ & 7.164E-02 & $3.44 \mathrm{E}-03$ & $1.140 \mathrm{E}-02$ & $5.47 \mathrm{E}-04$ \\
\hline $2.659 \mathrm{E}+01$ & $3.00 \mathrm{E}-01$ & $6.229 \mathrm{E}-02$ & $9.77 \mathrm{E}-03$ & $6.461 \mathrm{E}-02$ & $3.54 \mathrm{E}-03$ & $1.028 \mathrm{E}-02$ & $5.63 \mathrm{E}-04$ \\
\hline$\rho\left(\mathrm{kg} / \mathrm{m}^{\wedge} 3\right)$ & $\eta_{\mathrm{T}}$ & $\Delta \eta_{\mathrm{T}}$ & $J$ & $\Delta J$ & $\eta_{\mathrm{P}}$ & $\Delta \eta_{\mathrm{P}}$ & $R e_{0.75}$ \\
\hline $1.163 \mathrm{E}+00$ & $5.738 \mathrm{E}-01$ & $2.64 \mathrm{E}-02$ & $8.628 \mathrm{E}-02$ & $5.70 \mathrm{E}-04$ & $1.208 \mathrm{E}-01$ & $1.18 \mathrm{E}-02$ & $3.057 \mathrm{E}+04$ \\
\hline $1.163 \mathrm{E}+00$ & $5.735 \mathrm{E}-01$ & $2.59 \mathrm{E}-02$ & $1.556 \mathrm{E}-01$ & $5.95 \mathrm{E}-04$ & $2.109 \mathrm{E}-01$ & $2.13 \mathrm{E}-02$ & $3.058 \mathrm{E}+04$ \\
\hline $1.162 \mathrm{E}+00$ & 5.770E-01 & $2.68 \mathrm{E}-02$ & 2.279E-01 & $6.71 \mathrm{E}-04$ & $3.021 \mathrm{E}-01$ & $3.12 \mathrm{E}-02$ & $3.067 \mathrm{E}+04$ \\
\hline $1.161 \mathrm{E}+00$ & $5.852 \mathrm{E}-01$ & $2.75 \mathrm{E}-02$ & $3.020 \mathrm{E}-01$ & $7.62 \mathrm{E}-04$ & $3.849 \mathrm{E}-01$ & $4.06 \mathrm{E}-02$ & $3.077 \mathrm{E}+04$ \\
\hline $1.160 \mathrm{E}+00$ & 5.815E-01 & $2.75 \mathrm{E}-02$ & 3.799E-01 & $9.03 \mathrm{E}-04$ & 4.660E-01 & $5.19 \mathrm{E}-02$ & $3.073 \mathrm{E}+04$ \\
\hline $1.159 \mathrm{E}+00$ & 5.824E-01 & 2.63E-02 & 4.561E-01 & $1.01 \mathrm{E}-03$ & $5.460 \mathrm{E}-01$ & $6.15 \mathrm{E}-02$ & $3.092 \mathrm{E}+04$ \\
\hline $1.159 \mathrm{E}+00$ & 5.804E-01 & $2.73 \mathrm{E}-02$ & $5.328 \mathrm{E}-01$ & $1.12 \mathrm{E}-03$ & $6.171 \mathrm{E}-01$ & 7.31E-02 & $3.115 \mathrm{E}+04$ \\
\hline $1.159 \mathrm{E}+00$ & $5.747 \mathrm{E}-01$ & $2.83 \mathrm{E}-02$ & $6.107 \mathrm{E}-01$ & $1.24 \mathrm{E}-03$ & $6.393 \mathrm{E}-01$ & $8.91 \mathrm{E}-02$ & $3.135 \mathrm{E}+04$ \\
\hline $1.158 \mathrm{E}+00$ & $5.707 \mathrm{E}-01$ & $3.19 \mathrm{E}-02$ & $6.865 \mathrm{E}-01$ & $1.37 \mathrm{E}-03$ & $6.617 \mathrm{E}-01$ & $1.10 \mathrm{E}-01$ & $3.167 \mathrm{E}+04$ \\
\hline
\end{tabular}


Table 108: APC 4.7 x 4.25 Dynamic Measured Values - 16009 RPM

\begin{tabular}{|c|c|c|c|c|c|c|c|}
\hline$n(\mathrm{RPM})$ & $Q(\mathrm{~g}-\mathrm{m})$ & $\Delta Q(\mathrm{~g}-\mathrm{m})$ & $V(\mathrm{~V})$ & $I(\mathrm{~A})$ & $T_{\text {atm }}\left({ }^{\circ} \mathrm{C}\right)$ & $P_{\text {atm }}(\mathrm{Pa})$ & $P_{\text {diff }}(\mathrm{Pa})$ \\
\hline $1.617 \mathrm{E}+04$ & $2.750 \mathrm{E}+00$ & $1.17 \mathrm{E}-01$ & $1.094 \mathrm{E}+01$ & $6.254 \mathrm{E}+00$ & $2.241 \mathrm{E}+01$ & $9.841 \mathrm{E}+04$ & $3.260 \mathrm{E}+00$ \\
\hline $1.604 \mathrm{E}+04$ & $2.692 \mathrm{E}+00$ & $8.02 \mathrm{E}-02$ & $1.095 \mathrm{E}+01$ & $6.086 \mathrm{E}+00$ & $2.240 \mathrm{E}+01$ & $9.841 \mathrm{E}+04$ & $1.117 \mathrm{E}+01$ \\
\hline $1.597 \mathrm{E}+04$ & $2.648 \mathrm{E}+00$ & $7.88 \mathrm{E}-02$ & $1.095 \mathrm{E}+01$ & $5.982 \mathrm{E}+00$ & $2.256 \mathrm{E}+01$ & $9.841 \mathrm{E}+04$ & $2.501 \mathrm{E}+01$ \\
\hline $1.598 \mathrm{E}+04$ & $2.638 \mathrm{E}+00$ & $7.84 \mathrm{E}-02$ & $1.095 \mathrm{E}+01$ & $5.975 \mathrm{E}+00$ & $2.273 \mathrm{E}+01$ & $9.841 \mathrm{E}+04$ & $4.510 \mathrm{E}+01$ \\
\hline $1.596 \mathrm{E}+04$ & $2.658 \mathrm{E}+00$ & $7.82 \mathrm{E}-02$ & $1.095 \mathrm{E}+01$ & $5.997 \mathrm{E}+00$ & $2.279 \mathrm{E}+01$ & $9.842 \mathrm{E}+04$ & $7.130 \mathrm{E}+01$ \\
\hline $1.596 \mathrm{E}+04$ & $2.680 \mathrm{E}+00$ & $7.79 \mathrm{E}-02$ & $1.095 \mathrm{E}+01$ & $6.008 \mathrm{E}+00$ & $2.286 \mathrm{E}+01$ & $9.842 \mathrm{E}+04$ & $1.043 \mathrm{E}+02$ \\
\hline $1.597 \mathrm{E}+04$ & $2.677 \mathrm{E}+00$ & $7.71 \mathrm{E}-02$ & $1.095 \mathrm{E}+01$ & $5.984 \mathrm{E}+00$ & $2.286 \mathrm{E}+01$ & $9.842 \mathrm{E}+04$ & $1.438 \mathrm{E}+02$ \\
\hline $1.601 \mathrm{E}+04$ & $2.635 \mathrm{E}+00$ & $7.57 \mathrm{E}-02$ & $1.095 \mathrm{E}+01$ & $5.913 \mathrm{E}+00$ & $2.296 \mathrm{E}+01$ & $9.842 \mathrm{E}+04$ & $1.892 \mathrm{E}+02$ \\
\hline $1.601 \mathrm{E}+04$ & $2.485 \mathrm{E}+00$ & $7.61 \mathrm{E}-02$ & $1.096 \mathrm{E}+01$ & $5.550 \mathrm{E}+00$ & $2.298 \mathrm{E}+01$ & $9.842 \mathrm{E}+04$ & $2.397 \mathrm{E}+02$ \\
\hline $1.602 \mathrm{E}+04$ & $2.241 \mathrm{E}+00$ & $7.51 \mathrm{E}-02$ & $1.098 \mathrm{E}+01$ & $5.017 \mathrm{E}+00$ & $2.309 \mathrm{E}+01$ & $9.842 \mathrm{E}+04$ & $2.976 \mathrm{E}+02$ \\
\hline $1.600 \mathrm{E}+04$ & $1.933 \mathrm{E}+00$ & $7.48 \mathrm{E}-02$ & $1.100 \mathrm{E}+01$ & $4.319 \mathrm{E}+00$ & $2.312 \mathrm{E}+01$ & $9.843 \mathrm{E}+04$ & $3.609 \mathrm{E}+02$ \\
\hline
\end{tabular}


Table 109: APC 4.7 x 4.25 Dynamic Calculated Values - 16009 RPM

\begin{tabular}{|c|c|c|c|c|c|c|c|}
\hline$n(\mathrm{RPM})$ & $V_{\infty}^{\prime}(\mathrm{m} / \mathrm{s})$ & $\Delta V_{\infty}^{\prime}(\mathrm{m} / \mathrm{s})$ & $T^{\prime}(\mathrm{g})$ & $\Delta T^{\prime}(\mathrm{g})$ & $V_{\mathrm{t}}(\mathrm{m} / \mathrm{s})$ & $P_{\mathrm{P}}(\mathrm{W})$ & $\Delta P_{\mathrm{P}}(\mathrm{W})$ \\
\hline $1.617 \mathrm{E}+04$ & $2.236 \mathrm{E}+00$ & $1.64 \mathrm{E}-02$ & $2.142 \mathrm{E}+02$ & $8.15 \mathrm{E}+00$ & $7.638 \mathrm{E}+01$ & $4.568 \mathrm{E}+01$ & $1.94 \mathrm{E}+00$ \\
\hline $1.604 \mathrm{E}+04$ & $4.257 \mathrm{E}+00$ & $1.74 \mathrm{E}-02$ & $2.079 \mathrm{E}+02$ & $8.66 \mathrm{E}+00$ & $7.583 \mathrm{E}+01$ & $4.434 \mathrm{E}+01$ & $1.32 \mathrm{E}+00$ \\
\hline $1.597 \mathrm{E}+04$ & $6.444 \mathrm{E}+00$ & $1.96 \mathrm{E}-02$ & $2.022 \mathrm{E}+02$ & $9.20 \mathrm{E}+00$ & $7.568 \mathrm{E}+01$ & $4.343 \mathrm{E}+01$ & $1.29 \mathrm{E}+00$ \\
\hline $1.598 \mathrm{E}+04$ & $8.708 \mathrm{E}+00$ & $2.29 \mathrm{E}-02$ & $1.958 \mathrm{E}+02$ & $9.25 \mathrm{E}+00$ & $7.594 \mathrm{E}+01$ & $4.329 \mathrm{E}+01$ & $1.29 \mathrm{E}+00$ \\
\hline $1.596 \mathrm{E}+04$ & $1.099 \mathrm{E}+01$ & $2.70 \mathrm{E}-02$ & $1.907 \mathrm{E}+02$ & $9.73 \mathrm{E}+00$ & $7.616 \mathrm{E}+01$ & 4.357E+01 & $1.28 \mathrm{E}+00$ \\
\hline $1.596 \mathrm{E}+04$ & $1.332 \mathrm{E}+01$ & $2.97 \mathrm{E}-02$ & $1.846 \mathrm{E}+02$ & $9.89 \mathrm{E}+00$ & $7.651 \mathrm{E}+01$ & $4.392 \mathrm{E}+01$ & $1.28 \mathrm{E}+00$ \\
\hline $1.597 \mathrm{E}+04$ & $1.567 \mathrm{E}+01$ & $3.23 \mathrm{E}-02$ & $1.756 \mathrm{E}+02$ & $1.01 \mathrm{E}+01$ & $7.701 \mathrm{E}+01$ & $4.390 \mathrm{E}+01$ & $1.26 \mathrm{E}+00$ \\
\hline $1.601 \mathrm{E}+04$ & $1.800 \mathrm{E}+01$ & $3.58 \mathrm{E}-02$ & $1.628 \mathrm{E}+02$ & $9.99 \mathrm{E}+00$ & $7.771 \mathrm{E}+01$ & $4.333 \mathrm{E}+01$ & $1.24 \mathrm{E}+00$ \\
\hline $1.601 \mathrm{E}+04$ & $2.028 \mathrm{E}+01$ & $3.94 \mathrm{E}-02$ & $1.416 \mathrm{E}+02$ & $1.00 \mathrm{E}+01$ & $7.826 \mathrm{E}+01$ & $4.086 \mathrm{E}+01$ & $1.25 \mathrm{E}+00$ \\
\hline $1.602 \mathrm{E}+04$ & $2.262 \mathrm{E}+01$ & 4.33E-02 & $1.174 \mathrm{E}+02$ & $9.92 \mathrm{E}+00$ & $7.894 \mathrm{E}+01$ & $3.686 \mathrm{E}+01$ & $1.24 \mathrm{E}+00$ \\
\hline $1.600 \mathrm{E}+04$ & $2.493 \mathrm{E}+01$ & 4.73E-02 & $9.135 \mathrm{E}+01$ & $9.90 \mathrm{E}+00$ & $7.954 \mathrm{E}+01$ & $3.176 \mathrm{E}+01$ & $1.23 \mathrm{E}+00$ \\
\hline$P_{\mathrm{e}}(\mathrm{W})$ & $\Delta P_{\mathrm{e}}(\mathrm{W})$ & $C_{\mathrm{T}}$ & $\Delta C_{\mathrm{T}}$ & $C_{\mathrm{P}}$ & $\Delta C_{\mathrm{P}}$ & $C_{\mathrm{Q}}$ & $\Delta C_{\mathrm{Q}}$ \\
\hline $6.844 \mathrm{E}+01$ & $8.05 \mathrm{E}-01$ & $1.194 \mathrm{E}-01$ & $4.54 \mathrm{E}-03$ & $8.014 \mathrm{E}-02$ & $3.40 \mathrm{E}-03$ & $1.275 \mathrm{E}-02$ & $5.42 \mathrm{E}-04$ \\
\hline $6.663 \mathrm{E}+01$ & $7.55 \mathrm{E}-01$ & $1.179 \mathrm{E}-01$ & 4.91E-03 & 7.975E-02 & $2.38 \mathrm{E}-03$ & $1.269 \mathrm{E}-02$ & $3.78 \mathrm{E}-04$ \\
\hline $6.551 \mathrm{E}+01$ & 7.31E-01 & $1.156 \mathrm{E}-01$ & $5.26 \mathrm{E}-03$ & 7.913E-02 & $2.36 \mathrm{E}-03$ & $1.259 \mathrm{E}-02$ & $3.75 \mathrm{E}-04$ \\
\hline $6.543 \mathrm{E}+01$ & 7.34E-01 & $1.119 \mathrm{E}-01$ & $5.29 \mathrm{E}-03$ & $7.883 \mathrm{E}-02$ & $2.34 \mathrm{E}-03$ & $1.255 \mathrm{E}-02$ & $3.73 \mathrm{E}-04$ \\
\hline $6.567 \mathrm{E}+01$ & 7.36E-01 & $1.092 \mathrm{E}-01$ & $5.58 \mathrm{E}-03$ & 7.957E-02 & 2.34E-03 & $1.266 \mathrm{E}-02$ & $3.73 \mathrm{E}-04$ \\
\hline $6.579 \mathrm{E}+01$ & 7.35E-01 & $1.059 \mathrm{E}-01$ & $5.67 \mathrm{E}-03$ & 8.031E-02 & $2.34 \mathrm{E}-03$ & $1.278 \mathrm{E}-02$ & $3.72 \mathrm{E}-04$ \\
\hline $6.553 \mathrm{E}+01$ & $7.34 \mathrm{E}-01$ & $1.005 \mathrm{E}-01$ & $5.79 \mathrm{E}-03$ & $8.010 \mathrm{E}-02$ & $2.31 \mathrm{E}-03$ & $1.275 \mathrm{E}-02$ & $3.67 \mathrm{E}-04$ \\
\hline $6.477 \mathrm{E}+01$ & 7.36E-01 & $9.275 \mathrm{E}-02$ & $5.69 \mathrm{E}-03$ & 7.843E-02 & $2.25 \mathrm{E}-03$ & $1.248 \mathrm{E}-02$ & $3.59 \mathrm{E}-04$ \\
\hline $6.084 \mathrm{E}+01$ & $6.93 \mathrm{E}-01$ & $8.066 \mathrm{E}-02$ & $5.72 \mathrm{E}-03$ & 7.401E-02 & $2.27 \mathrm{E}-03$ & $1.178 \mathrm{E}-02$ & $3.61 \mathrm{E}-04$ \\
\hline $5.507 \mathrm{E}+01$ & $6.24 \mathrm{E}-01$ & $6.687 \mathrm{E}-02$ & $5.65 \mathrm{E}-03$ & $6.669 \mathrm{E}-02$ & $2.24 \mathrm{E}-03$ & $1.061 \mathrm{E}-02$ & $3.56 \mathrm{E}-04$ \\
\hline $4.749 \mathrm{E}+01$ & $5.30 \mathrm{E}-01$ & $5.214 \mathrm{E}-02$ & $5.65 \mathrm{E}-03$ & $5.767 \mathrm{E}-02$ & $2.23 \mathrm{E}-03$ & $9.179 \mathrm{E}-03$ & $3.55 \mathrm{E}-04$ \\
\hline$\rho\left(\mathrm{kg} / \mathrm{m}^{\wedge} 3\right)$ & $\eta_{\mathrm{T}}$ & $\Delta \eta_{\mathrm{T}}$ & $J$ & $\Delta J$ & $\eta_{\mathrm{P}}$ & $\Delta \eta_{\mathrm{P}}$ & $R e_{0.75}$ \\
\hline $1.160 \mathrm{E}+00$ & $6.674 \mathrm{E}-01$ & $2.94 \mathrm{E}-02$ & $6.900 \mathrm{E}-02$ & $5.06 \mathrm{E}-04$ & $1.028 \mathrm{E}-01$ & $5.91 \mathrm{E}-03$ & $4.096 \mathrm{E}+04$ \\
\hline $1.160 \mathrm{E}+00$ & 6.654E-01 & $2.12 \mathrm{E}-02$ & $1.325 \mathrm{E}-01$ & $5.42 \mathrm{E}-04$ & $1.958 \mathrm{E}-01$ & $1.01 \mathrm{E}-02$ & $4.067 \mathrm{E}+04$ \\
\hline $1.159 \mathrm{E}+00$ & $6.629 \mathrm{E}-01$ & $2.11 \mathrm{E}-02$ & $2.014 \mathrm{E}-01$ & $6.14 \mathrm{E}-04$ & $2.942 \mathrm{E}-01$ & $1.60 \mathrm{E}-02$ & $4.055 \mathrm{E}+04$ \\
\hline $1.159 \mathrm{E}+00$ & $6.616 \mathrm{E}-01$ & $2.10 \mathrm{E}-02$ & $2.720 \mathrm{E}-01$ & $7.18 \mathrm{E}-04$ & $3.861 \mathrm{E}-01$ & $2.16 \mathrm{E}-02$ & $4.064 \mathrm{E}+04$ \\
\hline $1.158 \mathrm{E}+00$ & $6.635 \mathrm{E}-01$ & $2.09 \mathrm{E}-02$ & $3.435 \mathrm{E}-01$ & $8.46 \mathrm{E}-04$ & 4.716E-01 & $2.78 \mathrm{E}-02$ & $4.075 \mathrm{E}+04$ \\
\hline $1.158 \mathrm{E}+00$ & $6.675 \mathrm{E}-01$ & $2.08 \mathrm{E}-02$ & 4.168E-01 & 9.33E-04 & 5.494E-01 & $3.35 \mathrm{E}-02$ & $4.092 \mathrm{E}+04$ \\
\hline $1.158 \mathrm{E}+00$ & $6.699 \mathrm{E}-01$ & $2.07 \mathrm{E}-02$ & $4.898 \mathrm{E}-01$ & $1.02 \mathrm{E}-03$ & $6.146 \mathrm{E}-01$ & $3.96 \mathrm{E}-02$ & $4.119 \mathrm{E}+04$ \\
\hline $1.158 \mathrm{E}+00$ & $6.690 \mathrm{E}-01$ & $2.07 \mathrm{E}-02$ & 5.611E-01 & $1.12 \mathrm{E}-03$ & $6.634 \mathrm{E}-01$ & $4.50 \mathrm{E}-02$ & $4.154 \mathrm{E}+04$ \\
\hline $1.158 \mathrm{E}+00$ & $6.716 \mathrm{E}-01$ & 2.19E-02 & 6.323E-01 & $1.24 \mathrm{E}-03$ & $6.891 \mathrm{E}-01$ & 5.32E-02 & $4.183 \mathrm{E}+04$ \\
\hline $1.157 \mathrm{E}+00$ & $6.693 \mathrm{E}-01$ & $2.37 \mathrm{E}-02$ & 7.050E-01 & $1.36 \mathrm{E}-03$ & $7.068 \mathrm{E}-01$ & $6.43 \mathrm{E}-02$ & $4.216 \mathrm{E}+04$ \\
\hline $1.157 \mathrm{E}+00$ & $6.687 \mathrm{E}-01$ & 2.69E-02 & 7.779E-01 & $1.48 \mathrm{E}-03$ & 7.033E-01 & $8.10 \mathrm{E}-02$ & $4.248 \mathrm{E}+04$ \\
\hline
\end{tabular}


Table 110: APC 4.7 x 4.25 Dynamic Measured Values - 19973 RPM

\begin{tabular}{|c|c|c|c|c|c|c|c|}
\hline$n(\mathrm{RPM})$ & $Q(\mathrm{~g}-\mathrm{m})$ & $\Delta Q(\mathrm{~g}-\mathrm{m})$ & $V(\mathrm{~V})$ & $I(\mathrm{~A})$ & $T_{\text {atm }}\left({ }^{\circ} \mathrm{C}\right)$ & $P_{\text {atm }}(\mathrm{Pa})$ & $P_{\text {diff }}(\mathrm{Pa})$ \\
\hline $1.991 \mathrm{E}+04$ & $4.246 \mathrm{E}+00$ & $2.35 \mathrm{E}-01$ & $1.079 \mathrm{E}+01$ & $1.157 \mathrm{E}+01$ & $2.268 \mathrm{E}+01$ & $9.843 \mathrm{E}+04$ & $3.941 \mathrm{E}+00$ \\
\hline $1.996 \mathrm{E}+04$ & $4.231 \mathrm{E}+00$ & $2.44 \mathrm{E}-01$ & $1.079 \mathrm{E}+01$ & $1.158 \mathrm{E}+01$ & $2.267 \mathrm{E}+01$ & $9.844 \mathrm{E}+04$ & $1.687 \mathrm{E}+01$ \\
\hline $1.998 \mathrm{E}+04$ & $4.281 \mathrm{E}+00$ & $2.39 \mathrm{E}-01$ & $1.079 \mathrm{E}+01$ & $1.154 \mathrm{E}+01$ & $2.289 \mathrm{E}+01$ & $9.844 \mathrm{E}+04$ & $4.181 \mathrm{E}+01$ \\
\hline $1.998 \mathrm{E}+04$ & $4.222 \mathrm{E}+00$ & $2.38 \mathrm{E}-01$ & $1.080 \mathrm{E}+01$ & $1.153 \mathrm{E}+01$ & $2.293 \mathrm{E}+01$ & $9.845 \mathrm{E}+04$ & $7.878 \mathrm{E}+01$ \\
\hline $1.996 \mathrm{E}+04$ & $4.473 \mathrm{E}+00$ & $2.21 \mathrm{E}-01$ & $1.079 \mathrm{E}+01$ & $1.157 \mathrm{E}+01$ & $2.302 \mathrm{E}+01$ & $9.845 \mathrm{E}+04$ & $1.285 \mathrm{E}+02$ \\
\hline $1.995 \mathrm{E}+04$ & $4.339 \mathrm{E}+00$ & $2.01 \mathrm{E}-01$ & $1.079 \mathrm{E}+01$ & $1.157 \mathrm{E}+01$ & $2.308 \mathrm{E}+01$ & $9.845 \mathrm{E}+04$ & $1.910 \mathrm{E}+02$ \\
\hline $1.999 \mathrm{E}+04$ & $4.376 \mathrm{E}+00$ & $1.88 \mathrm{E}-01$ & $1.080 \mathrm{E}+01$ & $1.150 \mathrm{E}+01$ & $2.314 \mathrm{E}+01$ & $9.846 \mathrm{E}+04$ & $2.648 \mathrm{E}+02$ \\
\hline $2.003 \mathrm{E}+04$ & $4.230 \mathrm{E}+00$ & $1.81 \mathrm{E}-01$ & $1.081 \mathrm{E}+01$ & $1.109 \mathrm{E}+01$ & $2.320 \mathrm{E}+01$ & $9.845 \mathrm{E}+04$ & $3.504 \mathrm{E}+02$ \\
\hline $1.995 \mathrm{E}+04$ & $3.747 \mathrm{E}+00$ & $1.75 \mathrm{E}-01$ & $1.084 \mathrm{E}+01$ & $9.903 \mathrm{E}+00$ & $2.331 \mathrm{E}+01$ & $9.846 \mathrm{E}+04$ & $4.472 \mathrm{E}+02$ \\
\hline $2.003 \mathrm{E}+04$ & $3.326 \mathrm{E}+00$ & $1.84 \mathrm{E}-01$ & $1.088 \mathrm{E}+01$ & $8.610 \mathrm{E}+00$ & $2.343 \mathrm{E}+01$ & $9.846 \mathrm{E}+04$ & $5.556 \mathrm{E}+02$ \\
\hline
\end{tabular}


Table 111: APC 4.7 x 4.25 Dynamic Calculated Values - 19973 RPM

\begin{tabular}{|c|c|c|c|c|c|c|c|}
\hline$n(\mathrm{RPM})$ & $V_{\infty}^{\prime}(\mathrm{m} / \mathrm{s})$ & $\Delta V_{\infty}^{\prime}(\mathrm{m} / \mathrm{s})$ & $T^{\prime}(\mathrm{g})$ & $\Delta T^{\prime}(\mathrm{g})$ & $V_{\mathrm{t}}(\mathrm{m} / \mathrm{s})$ & $P_{\mathrm{P}}(\mathrm{W})$ & $\Delta P_{\mathrm{P}}(\mathrm{W})$ \\
\hline $1.991 \mathrm{E}+04$ & $2.440 \mathrm{E}+00$ & 1.92E-02 & $3.283 \mathrm{E}+02$ & $8.12 \mathrm{E}+00$ & $9.402 \mathrm{E}+01$ & $8.681 \mathrm{E}+01$ & $4.81 \mathrm{E}+00$ \\
\hline $1.996 \mathrm{E}+04$ & $5.230 \mathrm{E}+00$ & $2.10 \mathrm{E}-02$ & $3.311 \mathrm{E}+02$ & $8.31 \mathrm{E}+00$ & $9.435 \mathrm{E}+01$ & $8.670 \mathrm{E}+01$ & $5.00 \mathrm{E}+00$ \\
\hline $1.998 \mathrm{E}+04$ & $8.339 \mathrm{E}+00$ & $2.51 \mathrm{E}-02$ & $3.260 \mathrm{E}+02$ & $8.24 \mathrm{E}+00$ & $9.468 \mathrm{E}+01$ & $8.783 \mathrm{E}+01$ & $4.91 \mathrm{E}+00$ \\
\hline $1.998 \mathrm{E}+04$ & $1.152 \mathrm{E}+01$ & $3.05 \mathrm{E}-02$ & $3.190 \mathrm{E}+02$ & $8.26 \mathrm{E}+00$ & $9.502 \mathrm{E}+01$ & $8.662 \mathrm{E}+01$ & $4.87 \mathrm{E}+00$ \\
\hline $1.996 \mathrm{E}+04$ & $1.476 \mathrm{E}+01$ & $3.41 \mathrm{E}-02$ & $3.079 \mathrm{E}+02$ & $8.16 \mathrm{E}+00$ & $9.537 \mathrm{E}+01$ & $9.167 \mathrm{E}+01$ & $4.54 \mathrm{E}+00$ \\
\hline $1.995 \mathrm{E}+04$ & $1.804 \mathrm{E}+01$ & $3.76 \mathrm{E}-02$ & $2.910 \mathrm{E}+02$ & $8.08 \mathrm{E}+00$ & $9.590 \mathrm{E}+01$ & $8.892 \mathrm{E}+01$ & $4.11 \mathrm{E}+00$ \\
\hline $1.999 \mathrm{E}+04$ & $2.128 \mathrm{E}+01$ & 4.19E-02 & $2.758 \mathrm{E}+02$ & $8.04 \mathrm{E}+00$ & $9.675 \mathrm{E}+01$ & $8.985 \mathrm{E}+01$ & $3.85 \mathrm{E}+00$ \\
\hline $2.003 \mathrm{E}+04$ & $2.452 \mathrm{E}+01$ & 4.69E-02 & $2.492 \mathrm{E}+02$ & $8.03 \mathrm{E}+00$ & $9.768 \mathrm{E}+01$ & $8.700 \mathrm{E}+01$ & $3.73 \mathrm{E}+00$ \\
\hline $1.995 \mathrm{E}+04$ & $2.773 \mathrm{E}+01$ & $5.29 \mathrm{E}-02$ & $2.040 \mathrm{E}+02$ & $7.94 \mathrm{E}+00$ & $9.818 \mathrm{E}+01$ & $7.676 \mathrm{E}+01$ & $3.58 \mathrm{E}+00$ \\
\hline $2.003 \mathrm{E}+04$ & $3.094 \mathrm{E}+01$ & $5.74 \mathrm{E}-02$ & $1.636 \mathrm{E}+02$ & $8.10 \mathrm{E}+00$ & $9.948 \mathrm{E}+01$ & $6.841 \mathrm{E}+01$ & $3.79 \mathrm{E}+00$ \\
\hline$P_{\mathrm{e}}(\mathrm{W})$ & $\Delta P_{\mathrm{e}}(\mathrm{W})$ & $C_{\mathrm{T}}$ & $\Delta C_{\mathrm{T}}$ & $C_{\mathrm{P}}$ & $\Delta C_{\mathrm{P}}$ & $C_{\mathrm{Q}}$ & $\Delta C_{\mathrm{Q}}$ \\
\hline $1.249 \mathrm{E}+02$ & $1.36 \mathrm{E}+00$ & $1.209 \mathrm{E}-01$ & $2.99 \mathrm{E}-03$ & $8.170 \mathrm{E}-02$ & $4.53 \mathrm{E}-03$ & $1.300 \mathrm{E}-02$ & $7.20 \mathrm{E}-04$ \\
\hline $1.250 \mathrm{E}+02$ & $1.30 \mathrm{E}+00$ & $1.213 \mathrm{E}-01$ & $3.05 \mathrm{E}-03$ & $8.102 \mathrm{E}-02$ & $4.68 \mathrm{E}-03$ & $1.290 \mathrm{E}-02$ & $7.44 \mathrm{E}-04$ \\
\hline $1.245 \mathrm{E}+02$ & $1.30 \mathrm{E}+00$ & $1.193 \mathrm{E}-01$ & $3.02 \mathrm{E}-03$ & $8.186 \mathrm{E}-02$ & $4.58 \mathrm{E}-03$ & $1.303 \mathrm{E}-02$ & $7.28 \mathrm{E}-04$ \\
\hline $1.244 \mathrm{E}+02$ & $1.30 \mathrm{E}+00$ & $1.167 \mathrm{E}-01$ & 3.02E-03 & $8.072 \mathrm{E}-02$ & 4.54E-03 & $1.285 \mathrm{E}-02$ & 7.23E-04 \\
\hline $1.249 \mathrm{E}+02$ & $1.30 \mathrm{E}+00$ & $1.129 \mathrm{E}-01$ & $2.99 \mathrm{E}-03$ & $8.573 \mathrm{E}-02$ & $4.25 \mathrm{E}-03$ & $1.364 \mathrm{E}-02$ & $6.76 \mathrm{E}-04$ \\
\hline $1.249 \mathrm{E}+02$ & $1.30 \mathrm{E}+00$ & $1.068 \mathrm{E}-01$ & $2.97 \mathrm{E}-03$ & $8.323 \mathrm{E}-02$ & $3.85 \mathrm{E}-03$ & $1.325 \mathrm{E}-02$ & $6.12 \mathrm{E}-04$ \\
\hline $1.242 \mathrm{E}+02$ & $1.29 \mathrm{E}+00$ & $1.008 \mathrm{E}-01$ & 2.94E-03 & $8.361 \mathrm{E}-02$ & $3.59 \mathrm{E}-03$ & $1.331 \mathrm{E}-02$ & $5.71 \mathrm{E}-04$ \\
\hline $1.199 \mathrm{E}+02$ & $1.26 \mathrm{E}+00$ & $9.076 \mathrm{E}-02$ & 2.93E-03 & $8.054 \mathrm{E}-02$ & $3.46 \mathrm{E}-03$ & $1.282 \mathrm{E}-02$ & $5.50 \mathrm{E}-04$ \\
\hline $1.074 \mathrm{E}+02$ & $1.13 \mathrm{E}+00$ & 7.493E-02 & 2.92E-03 & $7.192 \mathrm{E}-02$ & $3.36 \mathrm{E}-03$ & $1.145 \mathrm{E}-02$ & $5.34 \mathrm{E}-04$ \\
\hline $9.366 \mathrm{E}+01$ & $1.01 \mathrm{E}+00$ & $5.964 \mathrm{E}-02$ & $2.95 \mathrm{E}-03$ & $6.338 \mathrm{E}-02$ & $3.51 \mathrm{E}-03$ & $1.009 \mathrm{E}-02$ & $5.59 \mathrm{E}-04$ \\
\hline$\rho\left(\mathrm{kg} / \mathrm{m}^{\wedge} 3\right)$ & $\eta_{\mathrm{T}}$ & $\Delta \eta_{\mathrm{T}}$ & $J$ & $\Delta J$ & $\eta_{\mathrm{P}}$ & $\Delta \eta_{\mathrm{P}}$ & $R e_{0.75}$ \\
\hline $1.159 \mathrm{E}+00$ & $6.949 \mathrm{E}-01$ & $3.92 \mathrm{E}-02$ & $6.118 \mathrm{E}-02$ & $4.82 \mathrm{E}-04$ & $9.050 \mathrm{E}-02$ & $5.54 \mathrm{E}-03$ & $5.035 \mathrm{E}+04$ \\
\hline $1.159 \mathrm{E}+00$ & $6.938 \mathrm{E}-01$ & 4.07E-02 & $1.308 \mathrm{E}-01$ & $5.27 \mathrm{E}-04$ & $1.959 \mathrm{E}-01$ & $1.24 \mathrm{E}-02$ & $5.053 \mathrm{E}+04$ \\
\hline $1.158 \mathrm{E}+00$ & 7.053E-01 & 4.01E-02 & $2.084 \mathrm{E}-01$ & $6.31 \mathrm{E}-04$ & $3.036 \mathrm{E}-01$ & $1.86 \mathrm{E}-02$ & $5.064 \mathrm{E}+04$ \\
\hline $1.158 \mathrm{E}+00$ & $6.961 \mathrm{E}-01$ & $3.98 \mathrm{E}-02$ & $2.877 \mathrm{E}-01$ & 7.65E-04 & 4.159E-01 & $2.58 \mathrm{E}-02$ & $5.081 \mathrm{E}+04$ \\
\hline $1.158 \mathrm{E}+00$ & $7.342 \mathrm{E}-01$ & $3.72 \mathrm{E}-02$ & $3.693 \mathrm{E}-01$ & 8.59E-04 & $4.862 \mathrm{E}-01$ & $2.73 \mathrm{E}-02$ & $5.097 \mathrm{E}+04$ \\
\hline $1.158 \mathrm{E}+00$ & $7.118 \mathrm{E}-01$ & $3.37 \mathrm{E}-02$ & $4.514 \mathrm{E}-01$ & $9.48 \mathrm{E}-04$ & $5.791 \mathrm{E}-01$ & $3.13 \mathrm{E}-02$ & $5.125 \mathrm{E}+04$ \\
\hline $1.158 \mathrm{E}+00$ & 7.237E-01 & $3.19 \mathrm{E}-02$ & $5.314 \mathrm{E}-01$ & $1.06 \mathrm{E}-03$ & $6.405 \mathrm{E}-01$ & $3.32 \mathrm{E}-02$ & $5.168 \mathrm{E}+04$ \\
\hline $1.157 \mathrm{E}+00$ & $7.258 \mathrm{E}-01$ & $3.21 \mathrm{E}-02$ & $6.110 \mathrm{E}-01$ & $1.18 \mathrm{E}-03$ & $6.885 \mathrm{E}-01$ & $3.70 \mathrm{E}-02$ & $5.216 \mathrm{E}+04$ \\
\hline $1.157 \mathrm{E}+00$ & $7.150 \mathrm{E}-01$ & $3.42 \mathrm{E}-02$ & $6.939 \mathrm{E}-01$ & $1.34 \mathrm{E}-03$ & $7.229 \mathrm{E}-01$ & $4.40 \mathrm{E}-02$ & $5.239 \mathrm{E}+04$ \\
\hline $1.157 \mathrm{E}+00$ & 7.304E-01 & 4.12E-02 & $7.712 \mathrm{E}-01$ & $1.45 \mathrm{E}-03$ & $7.257 \mathrm{E}-01$ & $5.39 \mathrm{E}-02$ & $5.305 \mathrm{E}+04$ \\
\hline
\end{tabular}




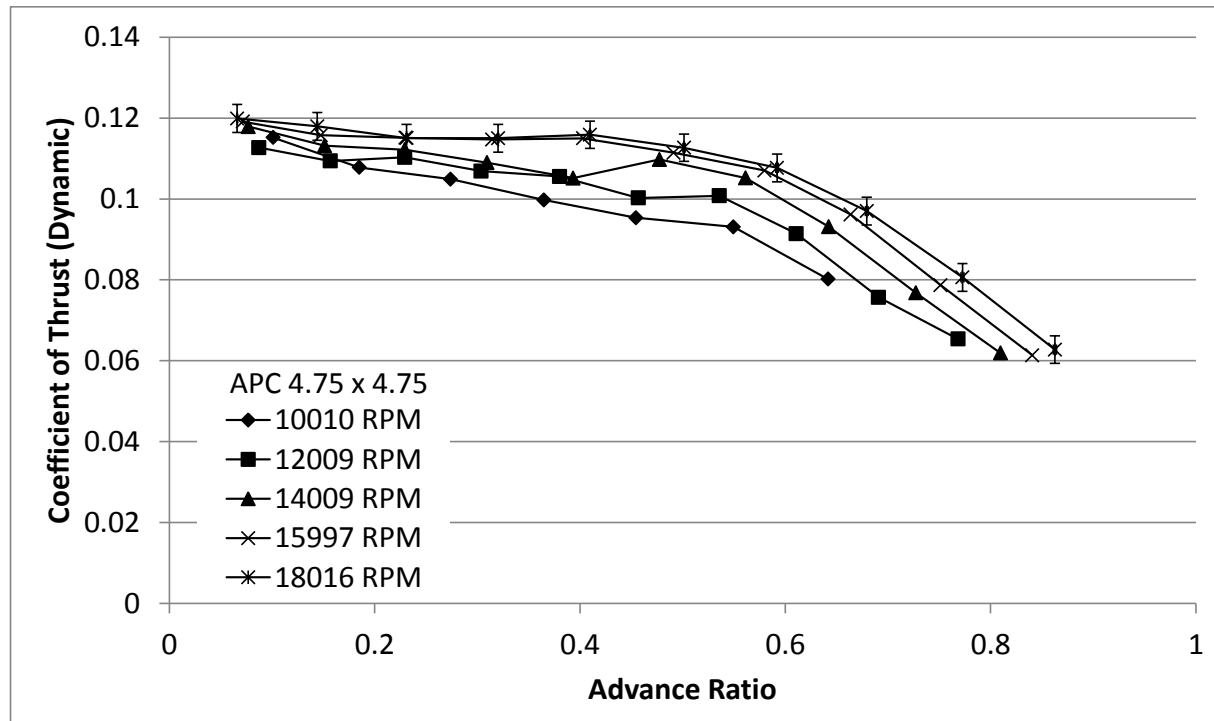

(a)

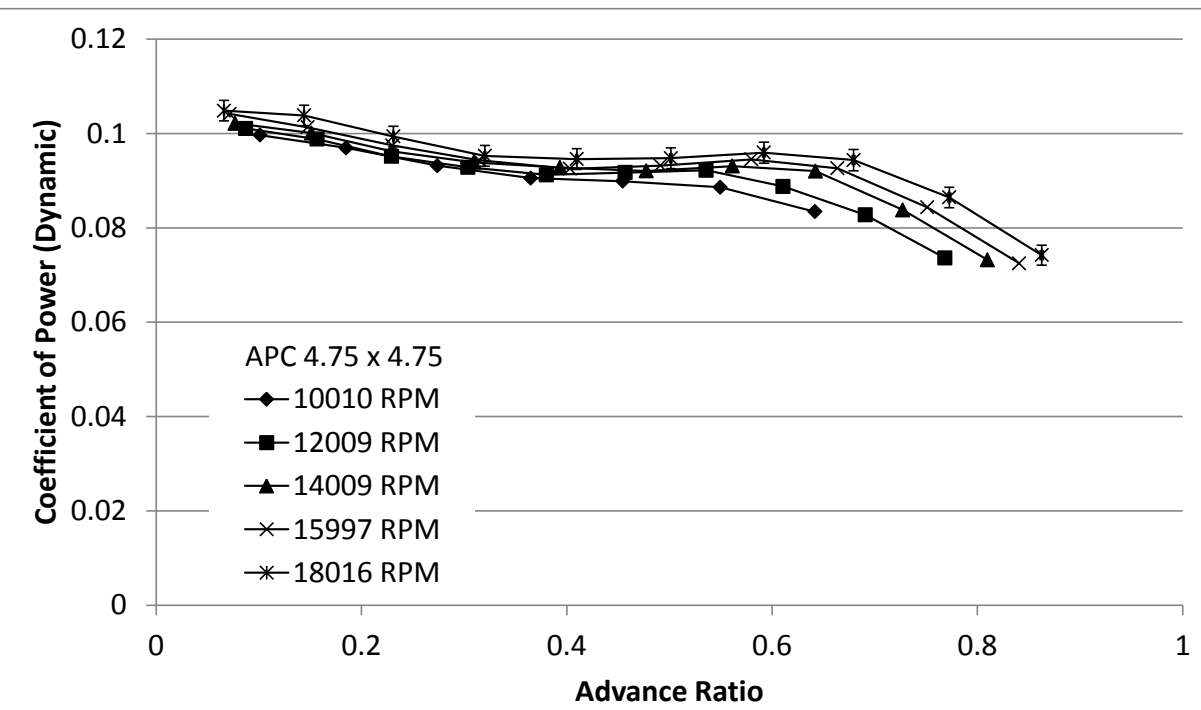

(b)

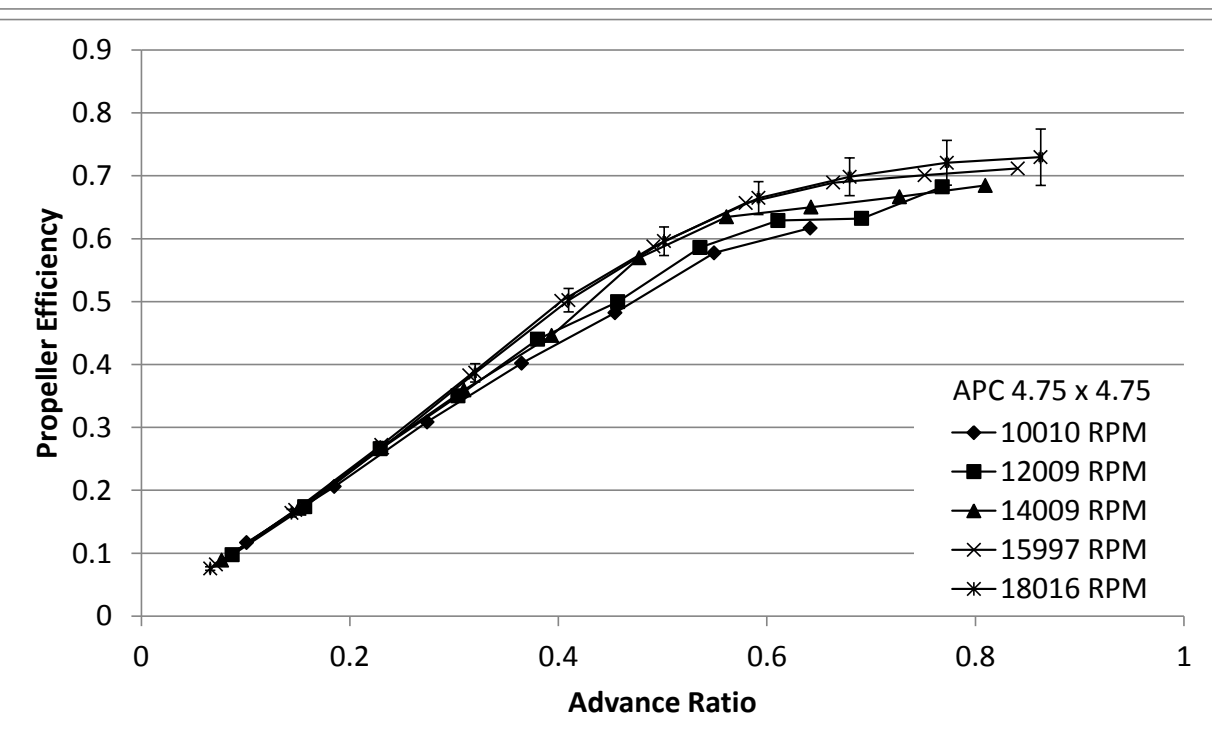

(c) 


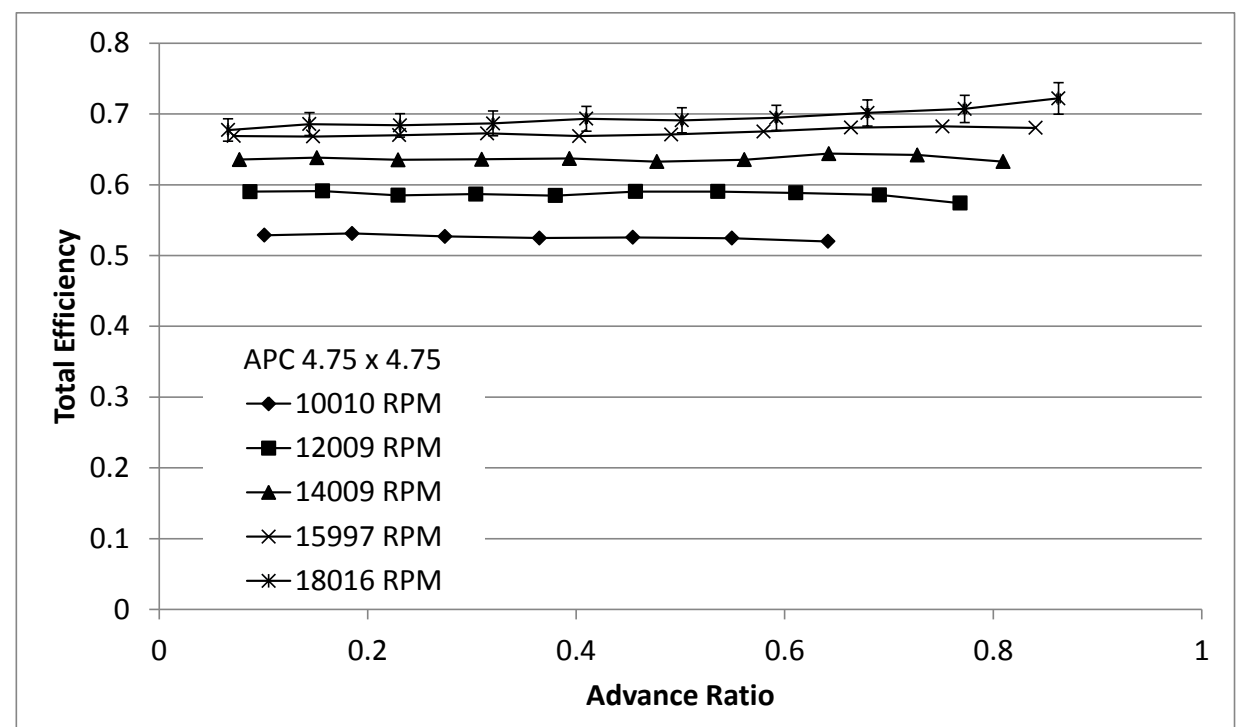

(d)

Figure 83: APC 4.75 x 4.75 Dynamic Test Results: (a) Coefficient of Thrust, (b)

Coefficient of Power, (c) Propeller Efficiency, (d) Total Efficiency.

Table 112: APC 4.75 x 4.75 Dynamic Measured Values - 10010 RPM

\begin{tabular}{|c|c|c|c|c|c|c|c|}
\hline$n(\mathrm{RPM})$ & $Q(\mathrm{~g}-\mathrm{m})$ & $\Delta Q(\mathrm{~g}-\mathrm{m})$ & $V(\mathrm{~V})$ & $I(\mathrm{~A})$ & $T_{\text {atm }}\left({ }^{\circ} \mathrm{C}\right)$ & $P_{\text {atm }}(\mathrm{Pa})$ & $P_{\text {diff }}(\mathrm{Pa})$ \\
\hline $9.997 \mathrm{E}+03$ & $1.299 \mathrm{E}+00$ & $6.18 \mathrm{E}-02$ & $1.105 \mathrm{E}+01$ & $2.283 \mathrm{E}+00$ & $2.220 \mathrm{E}+01$ & $9.914 \mathrm{E}+04$ & $2.577 \mathrm{E}+00$ \\
\hline $1.003 \mathrm{E}+04$ & $1.273 \mathrm{E}+00$ & $6.32 \mathrm{E}-02$ & $1.105 \mathrm{E}+01$ & $2.233 \mathrm{E}+00$ & $2.215 \mathrm{E}+01$ & $9.914 \mathrm{E}+04$ & $8.381 \mathrm{E}+00$ \\
\hline $9.994 \mathrm{E}+03$ & $1.214 \mathrm{E}+00$ & $6.01 \mathrm{E}-02$ & $1.105 \mathrm{E}+01$ & $2.138 \mathrm{E}+00$ & $2.225 \mathrm{E}+01$ & $9.914 \mathrm{E}+04$ & $1.795 \mathrm{E}+01$ \\
\hline $1.000 \mathrm{E}+04$ & $1.180 \mathrm{E}+00$ & $6.02 \mathrm{E}-02$ & $1.106 \mathrm{E}+01$ & $2.090 \mathrm{E}+00$ & $2.237 \mathrm{E}+01$ & $9.914 \mathrm{E}+04$ & $3.157 \mathrm{E}+01$ \\
\hline $1.003 \mathrm{E}+04$ & $1.179 \mathrm{E}+00$ & $6.17 \mathrm{E}-02$ & $1.106 \mathrm{E}+01$ & $2.089 \mathrm{E}+00$ & $2.236 \mathrm{E}+01$ & $9.915 \mathrm{E}+04$ & $4.906 \mathrm{E}+01$ \\
\hline $1.000 \mathrm{E}+04$ & $1.156 \mathrm{E}+00$ & $5.98 \mathrm{E}-02$ & $1.106 \mathrm{E}+01$ & $2.046 \mathrm{E}+00$ & $2.230 \mathrm{E}+01$ & $9.914 \mathrm{E}+04$ & $7.108 \mathrm{E}+01$ \\
\hline $1.002 \mathrm{E}+04$ & $1.092 \mathrm{E}+00$ & $6.06 \mathrm{E}-02$ & $1.106 \mathrm{E}+01$ & $1.954 \mathrm{E}+00$ & $2.228 \mathrm{E}+01$ & $9.914 \mathrm{E}+04$ & $9.704 \mathrm{E}+01$ \\
\hline
\end{tabular}


Table 113: APC 4.75 x 4.75 Dynamic Calculated Values - 10010 RPM

\begin{tabular}{|c|c|c|c|c|c|c|c|}
\hline$n(\mathrm{RPM})$ & $V_{\infty}^{\prime}(\mathrm{m} / \mathrm{s})$ & $\Delta V_{\infty}^{\prime}(\mathrm{m} / \mathrm{s})$ & $T^{\prime}(\mathrm{g})$ & $\Delta T^{\prime}(\mathrm{g})$ & $V_{\mathrm{t}}(\mathrm{m} / \mathrm{s})$ & $P_{\mathrm{P}}(\mathrm{W})$ & $\Delta P_{\mathrm{P}}(\mathrm{W})$ \\
\hline $9.997 \mathrm{E}+03$ & $2.019 \mathrm{E}+00$ & $1.29 \mathrm{E}-02$ & $7.873 \mathrm{E}+01$ & $9.56 \mathrm{E}+00$ & $4.710 \mathrm{E}+01$ & $1.334 \mathrm{E}+01$ & 6.34E-01 \\
\hline $1.003 \mathrm{E}+04$ & $3.711 \mathrm{E}+00$ & $1.36 \mathrm{E}-02$ & $7.418 \mathrm{E}+01$ & $9.52 \mathrm{E}+00$ & $4.736 \mathrm{E}+01$ & $1.311 \mathrm{E}+01$ & $6.52 \mathrm{E}-01$ \\
\hline $9.994 \mathrm{E}+03$ & $5.472 \mathrm{E}+00$ & $1.56 \mathrm{E}-02$ & $7.164 \mathrm{E}+01$ & $9.54 \mathrm{E}+00$ & $4.736 \mathrm{E}+01$ & $1.246 \mathrm{E}+01$ & $6.17 \mathrm{E}-01$ \\
\hline $1.000 \mathrm{E}+04$ & $7.289 \mathrm{E}+00$ & $1.83 \mathrm{E}-02$ & $6.818 \mathrm{E}+01$ & $9.62 \mathrm{E}+00$ & $4.763 \mathrm{E}+01$ & $1.212 \mathrm{E}+01$ & $6.18 \mathrm{E}-01$ \\
\hline $1.003 \mathrm{E}+04$ & $9.107 \mathrm{E}+00$ & $2.06 \mathrm{E}-02$ & $6.557 \mathrm{E}+01$ & $9.57 \mathrm{E}+00$ & $4.808 \mathrm{E}+01$ & $1.214 \mathrm{E}+01$ & $6.36 \mathrm{E}-01$ \\
\hline $1.000 \mathrm{E}+04$ & $1.098 \mathrm{E}+01$ & 2.33E-02 & $6.363 \mathrm{E}+01$ & $9.60 \mathrm{E}+00$ & $4.834 \mathrm{E}+01$ & $1.187 \mathrm{E}+01$ & $6.14 \mathrm{E}-01$ \\
\hline $1.002 \mathrm{E}+04$ & $1.285 \mathrm{E}+01$ & $2.65 \mathrm{E}-02$ & $5.503 \mathrm{E}+01$ & $9.53 \mathrm{E}+00$ & $4.888 \mathrm{E}+01$ & $1.124 \mathrm{E}+01$ & $6.23 \mathrm{E}-01$ \\
\hline$P_{\mathrm{e}}(\mathrm{W})$ & $\Delta P_{\mathrm{e}}(\mathrm{W})$ & $C_{\mathrm{T}}$ & $\Delta C_{\mathrm{T}}$ & $C_{\mathrm{P}}$ & $\Delta C_{\mathrm{P}}$ & $C_{\mathrm{Q}}$ & $\Delta C_{\mathrm{Q}}$ \\
\hline $2.523 \mathrm{E}+01$ & $2.88 \mathrm{E}-01$ & $1.152 \mathrm{E}-01$ & $1.40 \mathrm{E}-02$ & $9.967 \mathrm{E}-02$ & 4.74E-03 & $1.586 \mathrm{E}-02$ & 7.54E-04 \\
\hline $2.468 \mathrm{E}+01$ & $2.82 \mathrm{E}-01$ & $1.078 \mathrm{E}-01$ & $1.38 \mathrm{E}-02$ & $9.695 \mathrm{E}-02$ & 4.82E-03 & $1.543 \mathrm{E}-02$ & 7.67E-04 \\
\hline $2.364 \mathrm{E}+01$ & $2.71 \mathrm{E}-01$ & $1.049 \mathrm{E}-01$ & $1.40 \mathrm{E}-02$ & 9.317E-02 & $4.62 \mathrm{E}-03$ & $1.483 \mathrm{E}-02$ & 7.35E-04 \\
\hline $2.311 \mathrm{E}+01$ & $2.66 \mathrm{E}-01$ & 9.974E-02 & $1.41 \mathrm{E}-02$ & $9.052 \mathrm{E}-02$ & 4.62E-03 & $1.441 \mathrm{E}-02$ & 7.34E-04 \\
\hline $2.310 \mathrm{E}+01$ & $2.67 \mathrm{E}-01$ & 9.537E-02 & $1.39 \mathrm{E}-02$ & 8.989E-02 & $4.71 \mathrm{E}-03$ & $1.431 \mathrm{E}-02$ & 7.49E-04 \\
\hline $2.263 \mathrm{E}+01$ & $2.61 \mathrm{E}-01$ & $9.308 \mathrm{E}-02$ & $1.40 \mathrm{E}-02$ & $8.860 \mathrm{E}-02$ & $4.59 \mathrm{E}-03$ & $1.410 \mathrm{E}-02$ & 7.30E-04 \\
\hline $2.162 \mathrm{E}+01$ & $2.52 \mathrm{E}-01$ & 8.019E-02 & $1.39 \mathrm{E}-02$ & 8.342E-02 & 4.63E-03 & $1.328 \mathrm{E}-02$ & 7.36E-04 \\
\hline$\rho\left(\mathrm{kg} / \mathrm{m}^{\wedge} 3\right)$ & $\eta_{\mathrm{T}}$ & $\Delta \eta_{\mathrm{T}}$ & $J$ & $\Delta J$ & $\eta_{\mathrm{P}}$ & $\Delta \eta_{\mathrm{P}}$ & $R e_{0.75}$ \\
\hline $1.169 \mathrm{E}+00$ & $5.287 \mathrm{E}-01$ & $2.59 \mathrm{E}-02$ & $1.011 \mathrm{E}-01$ & $6.45 \mathrm{E}-04$ & $1.168 \mathrm{E}-01$ & $1.53 \mathrm{E}-02$ & $2.478 \mathrm{E}+04$ \\
\hline $1.170 \mathrm{E}+00$ & $5.313 \mathrm{E}-01$ & $2.71 \mathrm{E}-02$ & $1.852 \mathrm{E}-01$ & $6.83 \mathrm{E}-04$ & $2.059 \mathrm{E}-01$ & $2.83 \mathrm{E}-02$ & $2.492 \mathrm{E}+04$ \\
\hline $1.169 \mathrm{E}+00$ & $5.270 \mathrm{E}-01$ & $2.68 \mathrm{E}-02$ & $2.741 \mathrm{E}-01$ & $7.89 \mathrm{E}-04$ & $3.086 \mathrm{E}-01$ & $4.39 \mathrm{E}-02$ & $2.491 \mathrm{E}+04$ \\
\hline $1.169 \mathrm{E}+00$ & $5.247 \mathrm{E}-01$ & 2.74E-02 & 3.648E-01 & $9.25 \mathrm{E}-04$ & $4.020 \mathrm{E}-01$ & $6.03 \mathrm{E}-02$ & $2.504 \mathrm{E}+04$ \\
\hline $1.169 \mathrm{E}+00$ & $5.257 \mathrm{E}-01$ & 2.82E-02 & $4.545 \mathrm{E}-01$ & $1.04 \mathrm{E}-03$ & $4.822 \mathrm{E}-01$ & $7.48 \mathrm{E}-02$ & $2.527 \mathrm{E}+04$ \\
\hline $1.169 \mathrm{E}+00$ & $5.244 \mathrm{E}-01$ & $2.78 \mathrm{E}-02$ & $5.495 \mathrm{E}-01$ & $1.18 \mathrm{E}-03$ & $5.773 \mathrm{E}-01$ & $9.21 \mathrm{E}-02$ & $2.541 \mathrm{E}+04$ \\
\hline $1.169 \mathrm{E}+00$ & $5.199 \mathrm{E}-01$ & $2.95 \mathrm{E}-02$ & 6.417E-01 & $1.34 \mathrm{E}-03$ & $6.169 \mathrm{E}-01$ & $1.12 \mathrm{E}-01$ & $2.570 \mathrm{E}+04$ \\
\hline
\end{tabular}


Table 114: APC 4.75 x 4.75 Dynamic Measured Values - 12009 RPM

\begin{tabular}{|c|c|c|c|c|c|c|c|}
\hline$n(\mathrm{RPM})$ & $Q(\mathrm{~g}-\mathrm{m})$ & $\Delta Q(\mathrm{~g}-\mathrm{m})$ & $V(\mathrm{~V})$ & $I(\mathrm{~A})$ & $T_{\text {atm }}\left({ }^{\circ} \mathrm{C}\right)$ & $P_{\text {atm }}(\mathrm{Pa})$ & $P_{\text {diff }}(\mathrm{Pa})$ \\
\hline $1.202 \mathrm{E}+04$ & $1.902 \mathrm{E}+00$ & $7.07 \mathrm{E}-02$ & $1.101 \mathrm{E}+01$ & $3.613 \mathrm{E}+00$ & $2.257 \mathrm{E}+01$ & $9.905 \mathrm{E}+04$ & $2.804 \mathrm{E}+00$ \\
\hline $1.197 \mathrm{E}+04$ & $1.844 \mathrm{E}+00$ & $6.46 \mathrm{E}-02$ & $1.102 \mathrm{E}+01$ & $3.482 \mathrm{E}+00$ & $2.247 \mathrm{E}+01$ & $9.904 \mathrm{E}+04$ & $8.617 \mathrm{E}+00$ \\
\hline $1.200 \mathrm{E}+04$ & $1.782 \mathrm{E}+00$ & $6.64 \mathrm{E}-02$ & $1.102 \mathrm{E}+01$ & $3.406 \mathrm{E}+00$ & $2.263 \mathrm{E}+01$ & $9.904 \mathrm{E}+04$ & $1.818 \mathrm{E}+01$ \\
\hline $1.202 \mathrm{E}+04$ & $1.745 \mathrm{E}+00$ & $6.67 \mathrm{E}-02$ & $1.102 \mathrm{E}+01$ & $3.330 \mathrm{E}+00$ & $2.267 \mathrm{E}+01$ & $9.905 \mathrm{E}+04$ & $3.174 \mathrm{E}+01$ \\
\hline $1.200 \mathrm{E}+04$ & $1.709 \mathrm{E}+00$ & $6.40 \mathrm{E}-02$ & $1.102 \mathrm{E}+01$ & $3.267 \mathrm{E}+00$ & $2.278 \mathrm{E}+01$ & $9.904 \mathrm{E}+04$ & $4.922 \mathrm{E}+01$ \\
\hline $1.202 \mathrm{E}+04$ & $1.722 \mathrm{E}+00$ & $6.47 \mathrm{E}-02$ & $1.102 \mathrm{E}+01$ & $3.265 \mathrm{E}+00$ & $2.291 \mathrm{E}+01$ & $9.906 \mathrm{E}+04$ & $7.100 \mathrm{E}+01$ \\
\hline $1.200 \mathrm{E}+04$ & $1.725 \mathrm{E}+00$ & $6.46 \mathrm{E}-02$ & $1.102 \mathrm{E}+01$ & $3.266 \mathrm{E}+00$ & $2.295 \mathrm{E}+01$ & $9.905 \mathrm{E}+04$ & $9.709 \mathrm{E}+01$ \\
\hline $1.206 \mathrm{E}+04$ & $1.677 \mathrm{E}+00$ & $6.66 \mathrm{E}-02$ & $1.102 \mathrm{E}+01$ & $3.201 \mathrm{E}+00$ & $2.298 \mathrm{E}+01$ & $9.905 \mathrm{E}+04$ & $1.270 \mathrm{E}+02$ \\
\hline $1.200 \mathrm{E}+04$ & $1.550 \mathrm{E}+00$ & $6.49 \mathrm{E}-02$ & $1.103 \mathrm{E}+01$ & $2.957 \mathrm{E}+00$ & $2.301 \mathrm{E}+01$ & $9.906 \mathrm{E}+04$ & $1.608 \mathrm{E}+02$ \\
\hline $1.201 \mathrm{E}+04$ & $1.380 \mathrm{E}+00$ & $6.41 \mathrm{E}-02$ & $1.104 \mathrm{E}+01$ & $2.685 \mathrm{E}+00$ & $2.303 \mathrm{E}+01$ & $9.905 \mathrm{E}+04$ & $1.987 \mathrm{E}+02$ \\
\hline
\end{tabular}


Table 115: APC 4.75 x 4.75 Dynamic Calculated Values - 12009 RPM

\begin{tabular}{|c|c|c|c|c|c|c|c|}
\hline$n(\mathrm{RPM})$ & $V_{\infty}^{\prime}(\mathrm{m} / \mathrm{s})$ & $\Delta V_{\infty}^{\prime}(\mathrm{m} / \mathrm{s})$ & $T^{\prime}(\mathrm{g})$ & $\Delta T^{\prime}(\mathrm{g})$ & $V_{\mathrm{t}}(\mathrm{m} / \mathrm{s})$ & $P_{\mathrm{P}}(\mathrm{W})$ & $\Delta P_{\mathrm{P}}(\mathrm{W})$ \\
\hline $1.202 \mathrm{E}+04$ & $2.096 \mathrm{E}+00$ & $1.33 \mathrm{E}-02$ & $1.112 \mathrm{E}+02$ & $9.13 \mathrm{E}+00$ & $5.664 \mathrm{E}+01$ & $2.348 \mathrm{E}+01$ & $8.73 \mathrm{E}-01$ \\
\hline $1.197 \mathrm{E}+04$ & $3.751 \mathrm{E}+00$ & $1.39 \mathrm{E}-02$ & $1.070 \mathrm{E}+02$ & $9.17 \mathrm{E}+00$ & $5.649 \mathrm{E}+01$ & $2.268 \mathrm{E}+01$ & 7.95E-01 \\
\hline $1.200 \mathrm{E}+04$ & $5.495 \mathrm{E}+00$ & $1.55 \mathrm{E}-02$ & $1.083 \mathrm{E}+02$ & $9.16 \mathrm{E}+00$ & $5.674 \mathrm{E}+01$ & $2.195 \mathrm{E}+01$ & $8.18 \mathrm{E}-01$ \\
\hline $1.202 \mathrm{E}+04$ & $7.295 \mathrm{E}+00$ & $1.76 \mathrm{E}-02$ & $1.053 \mathrm{E}+02$ & $9.17 \mathrm{E}+00$ & $5.704 \mathrm{E}+01$ & $2.153 \mathrm{E}+01$ & $8.23 \mathrm{E}-01$ \\
\hline $1.200 \mathrm{E}+04$ & $9.113 \mathrm{E}+00$ & 2.09E-02 & $1.037 \mathrm{E}+02$ & $9.19 \mathrm{E}+00$ & $5.722 \mathrm{E}+01$ & $2.106 \mathrm{E}+01$ & $7.89 \mathrm{E}-01$ \\
\hline $1.202 \mathrm{E}+04$ & $1.097 \mathrm{E}+01$ & $2.33 \mathrm{E}-02$ & $9.866 \mathrm{E}+01$ & $9.18 \mathrm{E}+00$ & $5.761 \mathrm{E}+01$ & $2.124 \mathrm{E}+01$ & $7.98 \mathrm{E}-01$ \\
\hline $1.200 \mathrm{E}+04$ & $1.285 \mathrm{E}+01$ & $2.64 \mathrm{E}-02$ & $9.886 \mathrm{E}+01$ & $9.19 \mathrm{E}+00$ & $5.791 \mathrm{E}+01$ & $2.125 \mathrm{E}+01$ & 7.96E-01 \\
\hline $1.206 \mathrm{E}+04$ & $1.471 \mathrm{E}+01$ & $2.91 \mathrm{E}-02$ & $9.053 \mathrm{E}+01$ & $9.15 \mathrm{E}+00$ & $5.862 \mathrm{E}+01$ & $2.077 \mathrm{E}+01$ & $8.24 \mathrm{E}-01$ \\
\hline $1.200 \mathrm{E}+04$ & $1.657 \mathrm{E}+01$ & $3.19 \mathrm{E}-02$ & $7.430 \mathrm{E}+01$ & $9.17 \mathrm{E}+00$ & $5.888 \mathrm{E}+01$ & $1.910 \mathrm{E}+01$ & $8.00 \mathrm{E}-01$ \\
\hline $1.201 \mathrm{E}+04$ & $1.843 \mathrm{E}+01$ & $3.51 \mathrm{E}-02$ & $6.424 \mathrm{E}+01$ & $9.24 \mathrm{E}+00$ & $5.946 \mathrm{E}+01$ & $1.702 \mathrm{E}+01$ & $7.91 \mathrm{E}-01$ \\
\hline$P_{\mathrm{e}}(\mathrm{W})$ & $\Delta P_{\mathrm{e}}(\mathrm{W})$ & $C_{\mathrm{T}}$ & $\Delta C_{\mathrm{T}}$ & $C_{\mathrm{P}}$ & $\Delta C_{\mathrm{P}}$ & $C_{\mathrm{Q}}$ & $\Delta C_{\mathrm{Q}}$ \\
\hline $3.978 \mathrm{E}+01$ & $4.47 \mathrm{E}-01$ & $1.127 \mathrm{E}-01$ & $9.26 \mathrm{E}-03$ & $1.010 \mathrm{E}-01$ & $3.76 \mathrm{E}-03$ & $1.608 \mathrm{E}-02$ & $5.98 \mathrm{E}-04$ \\
\hline $3.836 \mathrm{E}+01$ & $4.28 \mathrm{E}-01$ & $1.094 \mathrm{E}-01$ & $9.37 \mathrm{E}-03$ & $9.879 \mathrm{E}-02$ & $3.46 \mathrm{E}-03$ & $1.572 \mathrm{E}-02$ & $5.51 \mathrm{E}-04$ \\
\hline $3.752 \mathrm{E}+01$ & 4.18E-01 & 1.103E-01 & $9.33 \mathrm{E}-03$ & $9.513 \mathrm{E}-02$ & $3.55 \mathrm{E}-03$ & $1.514 \mathrm{E}-02$ & $5.64 \mathrm{E}-04$ \\
\hline $3.670 \mathrm{E}+01$ & 4.10E-01 & $1.069 \mathrm{E}-01$ & $9.31 \mathrm{E}-03$ & $9.282 \mathrm{E}-02$ & $3.55 \mathrm{E}-03$ & $1.477 \mathrm{E}-02$ & $5.65 \mathrm{E}-04$ \\
\hline $3.601 \mathrm{E}+01$ & $4.02 \mathrm{E}-01$ & $1.056 \mathrm{E}-01$ & $9.36 \mathrm{E}-03$ & $9.122 \mathrm{E}-02$ & $3.42 \mathrm{E}-03$ & $1.452 \mathrm{E}-02$ & $5.44 \mathrm{E}-04$ \\
\hline $3.599 \mathrm{E}+01$ & 4.00E-01 & $1.003 \mathrm{E}-01$ & $9.33 \mathrm{E}-03$ & $9.172 \mathrm{E}-02$ & $3.45 \mathrm{E}-03$ & $1.460 \mathrm{E}-02$ & 5.49E-04 \\
\hline $3.600 \mathrm{E}+01$ & $4.01 \mathrm{E}-01$ & $1.008 \mathrm{E}-01$ & 9.37E-03 & $9.219 \mathrm{E}-02$ & $3.46 \mathrm{E}-03$ & $1.467 \mathrm{E}-02$ & $5.50 \mathrm{E}-04$ \\
\hline $3.529 \mathrm{E}+01$ & $3.97 \mathrm{E}-01$ & $9.141 \mathrm{E}-02$ & $9.24 \mathrm{E}-03$ & $8.877 \mathrm{E}-02$ & $3.52 \mathrm{E}-03$ & $1.413 \mathrm{E}-02$ & $5.61 \mathrm{E}-04$ \\
\hline $3.262 \mathrm{E}+01$ & 3.67E-01 & 7.567E-02 & 9.34E-03 & $8.274 \mathrm{E}-02$ & $3.47 \mathrm{E}-03$ & $1.317 \mathrm{E}-02$ & $5.52 \mathrm{E}-04$ \\
\hline $2.964 \mathrm{E}+01$ & $3.32 \mathrm{E}-01$ & $6.539 \mathrm{E}-02$ & $9.41 \mathrm{E}-03$ & $7.361 \mathrm{E}-02$ & $3.42 \mathrm{E}-03$ & $1.172 \mathrm{E}-02$ & $5.45 \mathrm{E}-04$ \\
\hline$\rho\left(\mathrm{kg} / \mathrm{m}^{\wedge} 3\right)$ & $\eta_{\mathrm{T}}$ & $\Delta \eta_{\mathrm{T}}$ & $J$ & $\Delta J$ & $\eta_{\mathrm{P}}$ & $\Delta \eta_{\mathrm{P}}$ & $R e_{0.75}$ \\
\hline $1.167 \mathrm{E}+00$ & 5.903E-01 & $2.29 \mathrm{E}-02$ & $8.726 \mathrm{E}-02$ & $5.54 \mathrm{E}-04$ & $9.731 \mathrm{E}-02$ & $8.80 \mathrm{E}-03$ & $2.970 \mathrm{E}+04$ \\
\hline $1.167 \mathrm{E}+00$ & 5.912E-01 & $2.17 \mathrm{E}-02$ & $1.568 \mathrm{E}-01$ & $5.85 \mathrm{E}-04$ & $1.736 \mathrm{E}-01$ & $1.61 \mathrm{E}-02$ & $2.964 \mathrm{E}+04$ \\
\hline $1.167 \mathrm{E}+00$ & $5.850 \mathrm{E}-01$ & $2.27 \mathrm{E}-02$ & 2.293E-01 & $6.50 \mathrm{E}-04$ & $2.659 \mathrm{E}-01$ & $2.46 \mathrm{E}-02$ & $2.974 \mathrm{E}+04$ \\
\hline $1.166 \mathrm{E}+00$ & $5.867 \mathrm{E}-01$ & $2.34 \mathrm{E}-02$ & $3.038 \mathrm{E}-01$ & $7.42 \mathrm{E}-04$ & 3.499E-01 & 3.33E-02 & $2.990 \mathrm{E}+04$ \\
\hline $1.166 \mathrm{E}+00$ & $5.847 \mathrm{E}-01$ & $2.29 \mathrm{E}-02$ & $3.801 \mathrm{E}-01$ & 8.84E-04 & $4.399 \mathrm{E}-01$ & $4.23 \mathrm{E}-02$ & $2.997 \mathrm{E}+04$ \\
\hline $1.166 \mathrm{E}+00$ & $5.903 \mathrm{E}-01$ & $2.31 \mathrm{E}-02$ & $4.570 \mathrm{E}-01$ & $9.88 \mathrm{E}-04$ & $4.996 \mathrm{E}-01$ & $5.02 \mathrm{E}-02$ & $3.016 \mathrm{E}+04$ \\
\hline $1.165 \mathrm{E}+00$ & 5.903E-01 & $2.31 \mathrm{E}-02$ & 5.359E-01 & $1.12 \mathrm{E}-03$ & 5.859E-01 & $5.88 \mathrm{E}-02$ & $3.031 \mathrm{E}+04$ \\
\hline $1.165 \mathrm{E}+00$ & $5.885 \mathrm{E}-01$ & $2.43 \mathrm{E}-02$ & $6.108 \mathrm{E}-01$ & $1.23 \mathrm{E}-03$ & $6.289 \mathrm{E}-01$ & $6.83 \mathrm{E}-02$ & $3.067 \mathrm{E}+04$ \\
\hline $1.165 \mathrm{E}+00$ & $5.857 \mathrm{E}-01$ & $2.54 \mathrm{E}-02$ & $6.910 \mathrm{E}-01$ & $1.35 \mathrm{E}-03$ & $6.320 \mathrm{E}-01$ & $8.24 \mathrm{E}-02$ & $3.080 \mathrm{E}+04$ \\
\hline $1.165 \mathrm{E}+00$ & 5.741E-01 & $2.74 \mathrm{E}-02$ & 7.684E-01 & $1.49 \mathrm{E}-03$ & $6.825 \mathrm{E}-01$ & $1.03 \mathrm{E}-01$ & $3.110 \mathrm{E}+04$ \\
\hline
\end{tabular}


Table 116: APC 4.75 x 4.75 Dynamic Measured Values - 14009 RPM

\begin{tabular}{|c|c|c|c|c|c|c|c|}
\hline$n(\mathrm{RPM})$ & $Q(\mathrm{~g}-\mathrm{m})$ & $\Delta Q(\mathrm{~g}-\mathrm{m})$ & $V(\mathrm{~V})$ & $I(\mathrm{~A})$ & $T_{\text {atm }}\left({ }^{\circ} \mathrm{C}\right)$ & $P_{\text {atm }}(\mathrm{Pa})$ & $P_{\text {diff }}(\mathrm{Pa})$ \\
\hline $1.405 \mathrm{E}+04$ & $2.632 \mathrm{E}+00$ & $7.79 \mathrm{E}-02$ & $1.096 \mathrm{E}+01$ & $5.453 \mathrm{E}+00$ & $2.205 \mathrm{E}+01$ & $9.911 \mathrm{E}+04$ & $3.033 \mathrm{E}+00$ \\
\hline $1.397 \mathrm{E}+04$ & $2.549 \mathrm{E}+00$ & $7.17 \mathrm{E}-02$ & $1.096 \mathrm{E}+01$ & $5.224 \mathrm{E}+00$ & $2.197 \mathrm{E}+01$ & $9.911 \mathrm{E}+04$ & $1.098 \mathrm{E}+01$ \\
\hline $1.405 \mathrm{E}+04$ & $2.479 \mathrm{E}+00$ & $7.21 \mathrm{E}-02$ & $1.097 \mathrm{E}+01$ & $5.134 \mathrm{E}+00$ & $2.208 \mathrm{E}+01$ & $9.912 \mathrm{E}+04$ & $2.505 \mathrm{E}+01$ \\
\hline $1.403 \mathrm{E}+04$ & $2.411 \mathrm{E}+00$ & $7.00 \mathrm{E}-02$ & $1.097 \mathrm{E}+01$ & $4.980 \mathrm{E}+00$ & $2.217 \mathrm{E}+01$ & $9.911 \mathrm{E}+04$ & $4.499 \mathrm{E}+01$ \\
\hline $1.399 \mathrm{E}+04$ & $2.370 \mathrm{E}+00$ & $7.15 \mathrm{E}-02$ & $1.097 \mathrm{E}+01$ & $4.870 \mathrm{E}+00$ & $2.225 \mathrm{E}+01$ & $9.911 \mathrm{E}+04$ & $7.186 \mathrm{E}+01$ \\
\hline $1.400 \mathrm{E}+04$ & $2.352 \mathrm{E}+00$ & $7.21 \mathrm{E}-02$ & $1.097 \mathrm{E}+01$ & $4.867 \mathrm{E}+00$ & $2.229 \mathrm{E}+01$ & $9.911 \mathrm{E}+04$ & $1.055 \mathrm{E}+02$ \\
\hline $1.398 \mathrm{E}+04$ & $2.371 \mathrm{E}+00$ & $6.98 \mathrm{E}-02$ & $1.097 \mathrm{E}+01$ & $4.882 \mathrm{E}+00$ & $2.229 \mathrm{E}+01$ & $9.911 \mathrm{E}+04$ & $1.450 \mathrm{E}+02$ \\
\hline $1.404 \mathrm{E}+04$ & $2.365 \mathrm{E}+00$ & $7.08 \mathrm{E}-02$ & $1.098 \mathrm{E}+01$ & $4.825 \mathrm{E}+00$ & $2.231 \mathrm{E}+01$ & $9.911 \mathrm{E}+04$ & $1.910 \mathrm{E}+02$ \\
\hline $1.398 \mathrm{E}+04$ & $2.135 \mathrm{E}+00$ & $6.97 \mathrm{E}-02$ & $1.099 \mathrm{E}+01$ & $4.344 \mathrm{E}+00$ & $2.232 \mathrm{E}+01$ & $9.911 \mathrm{E}+04$ & $2.423 \mathrm{E}+02$ \\
\hline $1.400 \mathrm{E}+04$ & $1.869 \mathrm{E}+00$ & $6.94 \mathrm{E}-02$ & $1.100 \mathrm{E}+01$ & $3.858 \mathrm{E}+00$ & $2.236 \mathrm{E}+01$ & $9.910 \mathrm{E}+04$ & $3.004 \mathrm{E}+02$ \\
\hline
\end{tabular}


Table 117: APC 4.75 x 4.75 Dynamic Calculated Values - 14009 RPM

\begin{tabular}{|c|c|c|c|c|c|c|c|}
\hline$n(\mathrm{RPM})$ & $V_{\infty}^{\prime}(\mathrm{m} / \mathrm{s})$ & $\Delta V_{\infty}^{\prime}(\mathrm{m} / \mathrm{s})$ & $T^{\prime}(\mathrm{g})$ & $\Delta T^{\prime}(\mathrm{g})$ & $V_{\mathrm{t}}(\mathrm{m} / \mathrm{s})$ & $P_{\mathrm{P}}(\mathrm{W})$ & $\Delta P_{\mathrm{P}}(\mathrm{W})$ \\
\hline $1.405 \mathrm{E}+04$ & $2.162 \mathrm{E}+00$ & $1.51 \mathrm{E}-02$ & $1.592 \mathrm{E}+02$ & $9.26 \mathrm{E}+00$ & $6.618 \mathrm{E}+01$ & $3.797 \mathrm{E}+01$ & $1.12 \mathrm{E}+00$ \\
\hline $1.397 \mathrm{E}+04$ & $4.224 \mathrm{E}+00$ & $1.58 \mathrm{E}-02$ & $1.511 \mathrm{E}+02$ & $9.28 \mathrm{E}+00$ & $6.589 \mathrm{E}+01$ & $3.656 \mathrm{E}+01$ & $1.03 \mathrm{E}+00$ \\
\hline $1.405 \mathrm{E}+04$ & $6.440 \mathrm{E}+00$ & $1.87 \mathrm{E}-02$ & $1.515 \mathrm{E}+02$ & $9.30 \mathrm{E}+00$ & $6.646 \mathrm{E}+01$ & $3.577 \mathrm{E}+01$ & $1.04 \mathrm{E}+00$ \\
\hline $1.403 \mathrm{E}+04$ & $8.676 \mathrm{E}+00$ & $2.23 \mathrm{E}-02$ & $1.467 \mathrm{E}+02$ & $9.35 \mathrm{E}+00$ & $6.662 \mathrm{E}+01$ & $3.475 \mathrm{E}+01$ & $1.01 \mathrm{E}+00$ \\
\hline $1.399 \mathrm{E}+04$ & $1.100 \mathrm{E}+01$ & $2.56 \mathrm{E}-02$ & $1.407 \mathrm{E}+02$ & $9.31 \mathrm{E}+00$ & $6.678 \mathrm{E}+01$ & $3.405 \mathrm{E}+01$ & $1.03 \mathrm{E}+00$ \\
\hline $1.400 \mathrm{E}+04$ & $1.335 \mathrm{E}+01$ & $2.93 \mathrm{E}-02$ & $1.470 \mathrm{E}+02$ & $9.32 \mathrm{E}+00$ & $6.723 \mathrm{E}+01$ & $3.380 \mathrm{E}+01$ & $1.04 \mathrm{E}+00$ \\
\hline $1.398 \mathrm{E}+04$ & $1.568 \mathrm{E}+01$ & $3.22 \mathrm{E}-02$ & $1.405 \mathrm{E}+02$ & $9.33 \mathrm{E}+00$ & $6.764 \mathrm{E}+01$ & $3.404 \mathrm{E}+01$ & $1.00 \mathrm{E}+00$ \\
\hline $1.404 \mathrm{E}+04$ & $1.802 \mathrm{E}+01$ & $3.54 \mathrm{E}-02$ & $1.255 \mathrm{E}+02$ & $9.30 \mathrm{E}+00$ & $6.851 \mathrm{E}+01$ & $3.411 \mathrm{E}+01$ & $1.02 \mathrm{E}+00$ \\
\hline $1.398 \mathrm{E}+04$ & $2.032 \mathrm{E}+01$ & 3.92E-02 & $1.026 \mathrm{E}+02$ & $9.27 \mathrm{E}+00$ & $6.888 \mathrm{E}+01$ & $3.066 \mathrm{E}+01$ & $1.00 \mathrm{E}+00$ \\
\hline $1.400 \mathrm{E}+04$ & $2.264 \mathrm{E}+01$ & $4.31 \mathrm{E}-02$ & $8.283 \mathrm{E}+01$ & $9.33 \mathrm{E}+00$ & $6.966 \mathrm{E}+01$ & $2.687 \mathrm{E}+01$ & $9.97 \mathrm{E}-01$ \\
\hline$P_{\mathrm{e}}(\mathrm{W})$ & $\Delta P_{\mathrm{e}}(\mathrm{W})$ & $C_{\mathrm{T}}$ & $\Delta C_{\mathrm{T}}$ & $C_{\mathrm{P}}$ & $\Delta C_{\mathrm{P}}$ & $C_{\mathrm{Q}}$ & $\Delta C_{\mathrm{Q}}$ \\
\hline $5.975 \mathrm{E}+01$ & $6.81 \mathrm{E}-01$ & $1.179 \mathrm{E}-01$ & $6.85 \mathrm{E}-03$ & $1.021 \mathrm{E}-01$ & $3.03 \mathrm{E}-03$ & $1.626 \mathrm{E}-02$ & $4.81 \mathrm{E}-04$ \\
\hline $5.728 \mathrm{E}+01$ & $6.48 \mathrm{E}-01$ & $1.132 \mathrm{E}-01$ & $6.95 \mathrm{E}-03$ & $1.001 \mathrm{E}-01$ & $2.82 \mathrm{E}-03$ & $1.593 \mathrm{E}-02$ & $4.48 \mathrm{E}-04$ \\
\hline $5.631 \mathrm{E}+01$ & $6.37 \mathrm{E}-01$ & $1.122 \mathrm{E}-01$ & $6.89 \mathrm{E}-03$ & $9.618 \mathrm{E}-02$ & $2.80 \mathrm{E}-03$ & $1.531 \mathrm{E}-02$ & $4.46 \mathrm{E}-04$ \\
\hline $5.463 \mathrm{E}+01$ & $6.18 \mathrm{E}-01$ & $1.090 \mathrm{E}-01$ & $6.95 \mathrm{E}-03$ & $9.388 \mathrm{E}-02$ & $2.73 \mathrm{E}-03$ & $1.494 \mathrm{E}-02$ & $4.34 \mathrm{E}-04$ \\
\hline $5.345 \mathrm{E}+01$ & $6.01 \mathrm{E}-01$ & $1.052 \mathrm{E}-01$ & $6.96 \mathrm{E}-03$ & $9.280 \mathrm{E}-02$ & $2.80 \mathrm{E}-03$ & $1.477 \mathrm{E}-02$ & $4.46 \mathrm{E}-04$ \\
\hline $5.342 \mathrm{E}+01$ & $6.01 \mathrm{E}-01$ & $1.098 \mathrm{E}-01$ & $6.96 \mathrm{E}-03$ & $9.204 \mathrm{E}-02$ & $2.82 \mathrm{E}-03$ & $1.465 \mathrm{E}-02$ & $4.49 \mathrm{E}-04$ \\
\hline $5.357 \mathrm{E}+01$ & $6.04 \mathrm{E}-01$ & $1.052 \mathrm{E}-01$ & $6.98 \mathrm{E}-03$ & $9.308 \mathrm{E}-02$ & $2.74 \mathrm{E}-03$ & $1.481 \mathrm{E}-02$ & 4.37E-04 \\
\hline $5.296 \mathrm{E}+01$ & $6.00 \mathrm{E}-01$ & $9.314 \mathrm{E}-02$ & $6.90 \mathrm{E}-03$ & $9.202 \mathrm{E}-02$ & $2.75 \mathrm{E}-03$ & $1.464 \mathrm{E}-02$ & $4.38 \mathrm{E}-04$ \\
\hline $4.774 \mathrm{E}+01$ & $5.37 \mathrm{E}-01$ & $7.679 \mathrm{E}-02$ & $6.94 \mathrm{E}-03$ & $8.379 \mathrm{E}-02$ & $2.74 \mathrm{E}-03$ & $1.334 \mathrm{E}-02$ & $4.35 \mathrm{E}-04$ \\
\hline $4.246 \mathrm{E}+01$ & $4.71 \mathrm{E}-01$ & $6.190 \mathrm{E}-02$ & $6.97 \mathrm{E}-03$ & $7.322 \mathrm{E}-02$ & $2.72 \mathrm{E}-03$ & $1.165 \mathrm{E}-02$ & $4.33 \mathrm{E}-04$ \\
\hline$\rho\left(\mathrm{kg} / \mathrm{m}^{\wedge} 3\right)$ & $\eta_{\mathrm{T}}$ & $\Delta \eta_{\mathrm{T}}$ & $J$ & $\Delta J$ & $\eta_{\mathrm{P}}$ & $\Delta \eta_{\mathrm{P}}$ & $R e_{0.75}$ \\
\hline $1.170 \mathrm{E}+00$ & $6.355 \mathrm{E}-01$ & $2.02 \mathrm{E}-02$ & $7.701 \mathrm{E}-02$ & $5.40 \mathrm{E}-04$ & $8.889 \mathrm{E}-02$ & $5.83 \mathrm{E}-03$ & $3.484 \mathrm{E}+04$ \\
\hline $1.170 \mathrm{E}+00$ & $6.383 \mathrm{E}-01$ & $1.93 \mathrm{E}-02$ & $1.514 \mathrm{E}-01$ & $5.70 \mathrm{E}-04$ & $1.711 \mathrm{E}-01$ & $1.16 \mathrm{E}-02$ & $3.470 \mathrm{E}+04$ \\
\hline $1.170 \mathrm{E}+00$ & $6.353 \mathrm{E}-01$ & $1.98 \mathrm{E}-02$ & $2.294 \mathrm{E}-01$ & 6.69E-04 & $2.676 \mathrm{E}-01$ & $1.82 \mathrm{E}-02$ & $3.498 \mathrm{E}+04$ \\
\hline $1.169 \mathrm{E}+00$ & $6.360 \mathrm{E}-01$ & $1.98 \mathrm{E}-02$ & $3.095 \mathrm{E}-01$ & $8.02 \mathrm{E}-04$ & $3.594 \mathrm{E}-01$ & $2.52 \mathrm{E}-02$ & $3.505 \mathrm{E}+04$ \\
\hline $1.169 \mathrm{E}+00$ & $6.371 \mathrm{E}-01$ & $2.05 \mathrm{E}-02$ & $3.935 \mathrm{E}-01$ & $9.26 \mathrm{E}-04$ & 4.459E-01 & $3.25 \mathrm{E}-02$ & $3.511 \mathrm{E}+04$ \\
\hline $1.169 \mathrm{E}+00$ & $6.328 \mathrm{E}-01$ & $2.07 \mathrm{E}-02$ & 4.774E-01 & $1.06 \mathrm{E}-03$ & $5.694 \mathrm{E}-01$ & 4.01E-02 & $3.534 \mathrm{E}+04$ \\
\hline $1.169 \mathrm{E}+00$ & $6.354 \mathrm{E}-01$ & $2.00 \mathrm{E}-02$ & $5.615 \mathrm{E}-01$ & $1.17 \mathrm{E}-03$ & $6.346 \mathrm{E}-01$ & 4.61E-02 & $3.556 \mathrm{E}+04$ \\
\hline $1.169 \mathrm{E}+00$ & $6.440 \mathrm{E}-01$ & $2.06 \mathrm{E}-02$ & $6.423 \mathrm{E}-01$ & $1.28 \mathrm{E}-03$ & $6.502 \mathrm{E}-01$ & $5.20 \mathrm{E}-02$ & $3.601 \mathrm{E}+04$ \\
\hline $1.168 \mathrm{E}+00$ & $6.422 \mathrm{E}-01$ & $2.22 \mathrm{E}-02$ & $7.273 \mathrm{E}-01$ & $1.42 \mathrm{E}-03$ & $6.665 \mathrm{E}-01$ & $6.40 \mathrm{E}-02$ & $3.620 \mathrm{E}+04$ \\
\hline $1.168 \mathrm{E}+00$ & $6.328 \mathrm{E}-01$ & $2.45 \mathrm{E}-02$ & 8.097E-01 & $1.56 \mathrm{E}-03$ & $6.845 \mathrm{E}-01$ & $8.12 \mathrm{E}-02$ & $3.660 \mathrm{E}+04$ \\
\hline
\end{tabular}


Table 118: APC 4.75 x 4.75 Dynamic Measured Values - 15997 RPM

\begin{tabular}{|c|c|c|c|c|c|c|c|}
\hline$n(\mathrm{RPM})$ & $Q(\mathrm{~g}-\mathrm{m})$ & $\Delta Q(\mathrm{~g}-\mathrm{m})$ & $V(\mathrm{~V})$ & $I(\mathrm{~A})$ & $T_{\text {atm }}\left({ }^{\circ} \mathrm{C}\right)$ & $P_{\text {atm }}(\mathrm{Pa})$ & $P_{\text {diff }}(\mathrm{Pa})$ \\
\hline $1.598 \mathrm{E}+04$ & $3.459 \mathrm{E}+00$ & $7.97 \mathrm{E}-02$ & $1.089 \mathrm{E}+01$ & $7.791 \mathrm{E}+00$ & $2.263 \mathrm{E}+01$ & $9.905 \mathrm{E}+04$ & $3.413 \mathrm{E}+00$ \\
\hline $1.599 \mathrm{E}+04$ & $3.373 \mathrm{E}+00$ & $7.73 \mathrm{E}-02$ & $1.089 \mathrm{E}+01$ & $7.610 \mathrm{E}+00$ & $2.254 \mathrm{E}+01$ & $9.904 \mathrm{E}+04$ & $1.368 \mathrm{E}+01$ \\
\hline $1.602 \mathrm{E}+04$ & $3.255 \mathrm{E}+00$ & $7.79 \mathrm{E}-02$ & $1.090 \mathrm{E}+01$ & $7.330 \mathrm{E}+00$ & $2.261 \mathrm{E}+01$ & $9.904 \mathrm{E}+04$ & $3.273 \mathrm{E}+01$ \\
\hline $1.603 \mathrm{E}+04$ & $3.154 \mathrm{E}+00$ & $7.93 \mathrm{E}-02$ & $1.091 \mathrm{E}+01$ & $7.075 \mathrm{E}+00$ & $2.278 \mathrm{E}+01$ & $9.903 \mathrm{E}+04$ & $6.055 \mathrm{E}+01$ \\
\hline $1.599 \mathrm{E}+04$ & $3.074 \mathrm{E}+00$ & $7.66 \mathrm{E}-02$ & $1.091 \mathrm{E}+01$ & $6.914 \mathrm{E}+00$ & $2.298 \mathrm{E}+01$ & $9.903 \mathrm{E}+04$ & $9.814 \mathrm{E}+01$ \\
\hline $1.598 \mathrm{E}+04$ & $3.094 \mathrm{E}+00$ & $7.77 \mathrm{E}-02$ & $1.091 \mathrm{E}+01$ & $6.931 \mathrm{E}+00$ & $2.299 \mathrm{E}+01$ & $9.903 \mathrm{E}+04$ & $1.451 \mathrm{E}+02$ \\
\hline $1.596 \mathrm{E}+04$ & $3.130 \mathrm{E}+00$ & $7.78 \mathrm{E}-02$ & $1.091 \mathrm{E}+01$ & $6.962 \mathrm{E}+00$ & $2.303 \mathrm{E}+01$ & $9.903 \mathrm{E}+04$ & $2.010 \mathrm{E}+02$ \\
\hline $1.603 \mathrm{E}+04$ & $3.092 \mathrm{E}+00$ & $8.00 \mathrm{E}-02$ & $1.092 \mathrm{E}+01$ & $6.847 \mathrm{E}+00$ & $2.311 \mathrm{E}+01$ & $9.903 \mathrm{E}+04$ & $2.649 \mathrm{E}+02$ \\
\hline $1.602 \mathrm{E}+04$ & $2.811 \mathrm{E}+00$ & $7.78 \mathrm{E}-02$ & $1.094 \mathrm{E}+01$ & $6.196 \mathrm{E}+00$ & $2.318 \mathrm{E}+01$ & $9.904 \mathrm{E}+04$ & $3.381 \mathrm{E}+02$ \\
\hline $1.597 \mathrm{E}+04$ & $2.401 \mathrm{E}+00$ & $7.78 \mathrm{E}-02$ & $1.096 \mathrm{E}+01$ & $5.279 \mathrm{E}+00$ & $2.328 \mathrm{E}+01$ & $9.905 \mathrm{E}+04$ & $4.201 \mathrm{E}+02$ \\
\hline
\end{tabular}


Table 119: APC 4.75 x 4.75 Dynamic Calculated Values - 15997 RPM

\begin{tabular}{|c|c|c|c|c|c|c|c|}
\hline$n(\mathrm{RPM})$ & $V_{\infty}^{\prime}(\mathrm{m} / \mathrm{s})$ & $\Delta V_{\infty}^{\prime}(\mathrm{m} / \mathrm{s})$ & $T^{\prime}(\mathrm{g})$ & $\Delta T^{\prime}(\mathrm{g})$ & $V_{\mathrm{t}}(\mathrm{m} / \mathrm{s})$ & $P_{\mathrm{P}}(\mathrm{W})$ & $\Delta P_{\mathrm{P}}(\mathrm{W})$ \\
\hline $1.598 \mathrm{E}+04$ & $2.287 \mathrm{E}+00$ & $1.60 \mathrm{E}-02$ & $2.074 \mathrm{E}+02$ & $8.35 \mathrm{E}+00$ & $7.523 \mathrm{E}+01$ & $5.675 \mathrm{E}+01$ & $1.31 \mathrm{E}+00$ \\
\hline $1.599 \mathrm{E}+04$ & $4.716 \mathrm{E}+00$ & $1.73 \mathrm{E}-02$ & $2.021 \mathrm{E}+02$ & $8.38 \mathrm{E}+00$ & $7.543 \mathrm{E}+01$ & $5.540 \mathrm{E}+01$ & $1.27 \mathrm{E}+00$ \\
\hline $1.602 \mathrm{E}+04$ & $7.370 \mathrm{E}+00$ & 2.03E-02 & $2.016 \mathrm{E}+02$ & $8.33 \mathrm{E}+00$ & $7.577 \mathrm{E}+01$ & $5.355 \mathrm{E}+01$ & $1.28 \mathrm{E}+00$ \\
\hline $1.603 \mathrm{E}+04$ & $1.008 \mathrm{E}+01$ & $2.48 \mathrm{E}-02$ & $2.010 \mathrm{E}+02$ & $8.33 \mathrm{E}+00$ & $7.614 \mathrm{E}+01$ & $5.192 \mathrm{E}+01$ & $1.30 \mathrm{E}+00$ \\
\hline $1.599 \mathrm{E}+04$ & $1.287 \mathrm{E}+01$ & $2.90 \mathrm{E}-02$ & $2.003 \mathrm{E}+02$ & $8.54 \mathrm{E}+00$ & $7.635 \mathrm{E}+01$ & $5.047 \mathrm{E}+01$ & $1.26 \mathrm{E}+00$ \\
\hline $1.598 \mathrm{E}+04$ & $1.569 \mathrm{E}+01$ & $3.19 \mathrm{E}-02$ & $1.939 \mathrm{E}+02$ & $8.65 \mathrm{E}+00$ & $7.684 \mathrm{E}+01$ & $5.077 \mathrm{E}+01$ & $1.27 \mathrm{E}+00$ \\
\hline $1.596 \mathrm{E}+04$ & $1.849 \mathrm{E}+01$ & $3.60 \mathrm{E}-02$ & $1.858 \mathrm{E}+02$ & $8.80 \mathrm{E}+00$ & $7.738 \mathrm{E}+01$ & $5.131 \mathrm{E}+01$ & $1.27 \mathrm{E}+00$ \\
\hline $1.603 \mathrm{E}+04$ & $2.126 \mathrm{E}+01$ & 4.04E-02 & $1.683 \mathrm{E}+02$ & $8.77 \mathrm{E}+00$ & $7.839 \mathrm{E}+01$ & $5.090 \mathrm{E}+01$ & $1.32 \mathrm{E}+00$ \\
\hline $1.602 \mathrm{E}+04$ & $2.404 \mathrm{E}+01$ & $4.55 \mathrm{E}-02$ & $1.375 \mathrm{E}+02$ & $8.91 \mathrm{E}+00$ & $7.914 \mathrm{E}+01$ & $4.625 \mathrm{E}+01$ & $1.28 \mathrm{E}+00$ \\
\hline $1.597 \mathrm{E}+04$ & $2.683 \mathrm{E}+01$ & $5.06 \mathrm{E}-02$ & $1.065 \mathrm{E}+02$ & $9.28 \mathrm{E}+00$ & $7.982 \mathrm{E}+01$ & $3.938 \mathrm{E}+01$ & $1.28 \mathrm{E}+00$ \\
\hline$P_{\mathrm{e}}(\mathrm{W})$ & $\Delta P_{\mathrm{e}}(\mathrm{W})$ & $C_{\mathrm{T}}$ & $\Delta C_{\mathrm{T}}$ & $C_{\mathrm{P}}$ & $\Delta C_{\mathrm{P}}$ & $C_{\mathrm{Q}}$ & $\Delta C_{\mathrm{Q}}$ \\
\hline $8.484 \mathrm{E}+01$ & $9.37 \mathrm{E}-01$ & $1.191 \mathrm{E}-01$ & 4.79E-03 & $1.041 \mathrm{E}-01$ & $2.40 \mathrm{E}-03$ & $1.657 \mathrm{E}-02$ & $3.82 \mathrm{E}-04$ \\
\hline $8.290 \mathrm{E}+01$ & $9.11 \mathrm{E}-01$ & $1.158 \mathrm{E}-01$ & $4.80 \mathrm{E}-03$ & $1.013 \mathrm{E}-01$ & $2.32 \mathrm{E}-03$ & $1.612 \mathrm{E}-02$ & $3.70 \mathrm{E}-04$ \\
\hline $7.992 \mathrm{E}+01$ & 8.81E-01 & $1.151 \mathrm{E}-01$ & 4.76E-03 & $9.746 \mathrm{E}-02$ & 2.33E-03 & $1.551 \mathrm{E}-02$ & $3.71 \mathrm{E}-04$ \\
\hline $7.719 \mathrm{E}+01$ & $8.56 \mathrm{E}-01$ & $1.147 \mathrm{E}-01$ & $4.75 \mathrm{E}-03$ & $9.433 \mathrm{E}-02$ & $2.37 \mathrm{E}-03$ & $1.501 \mathrm{E}-02$ & $3.77 \mathrm{E}-04$ \\
\hline $7.546 \mathrm{E}+01$ & $8.30 \mathrm{E}-01$ & $1.150 \mathrm{E}-01$ & 4.91E-03 & $9.251 \mathrm{E}-02$ & $2.31 \mathrm{E}-03$ & $1.472 \mathrm{E}-02$ & $3.67 \mathrm{E}-04$ \\
\hline $7.565 \mathrm{E}+01$ & 8.37E-01 & $1.114 \mathrm{E}-01$ & 4.97E-03 & $9.320 \mathrm{E}-02$ & 2.34E-03 & $1.483 \mathrm{E}-02$ & $3.73 \mathrm{E}-04$ \\
\hline $7.598 \mathrm{E}+01$ & $8.35 \mathrm{E}-01$ & $1.070 \mathrm{E}-01$ & $5.07 \mathrm{E}-03$ & $9.452 \mathrm{E}-02$ & $2.35 \mathrm{E}-03$ & $1.504 \mathrm{E}-02$ & $3.74 \mathrm{E}-04$ \\
\hline $7.475 \mathrm{E}+01$ & $8.28 \mathrm{E}-01$ & $9.616 \mathrm{E}-02$ & $5.01 \mathrm{E}-03$ & $9.262 \mathrm{E}-02$ & $2.40 \mathrm{E}-03$ & $1.474 \mathrm{E}-02$ & $3.82 \mathrm{E}-04$ \\
\hline $6.776 \mathrm{E}+01$ & $7.61 \mathrm{E}-01$ & 7.869E-02 & $5.10 \mathrm{E}-03$ & $8.434 \mathrm{E}-02$ & 2.34E-03 & $1.342 \mathrm{E}-02$ & $3.72 \mathrm{E}-04$ \\
\hline $5.787 \mathrm{E}+01$ & $6.48 \mathrm{E}-01$ & $6.134 \mathrm{E}-02$ & 5.34E-03 & $7.247 \mathrm{E}-02$ & $2.35 \mathrm{E}-03$ & $1.153 \mathrm{E}-02$ & $3.74 \mathrm{E}-04$ \\
\hline$\rho\left(\mathrm{kg} / \mathrm{m}^{\wedge} 3\right)$ & $\eta_{\mathrm{T}}$ & $\Delta \eta_{\mathrm{T}}$ & $J$ & $\Delta J$ & $\eta_{\mathrm{P}}$ & $\Delta \eta_{\mathrm{P}}$ & $R e_{0.75}$ \\
\hline $1.167 \mathrm{E}+00$ & $6.689 \mathrm{E}-01$ & $1.71 \mathrm{E}-02$ & $7.165 \mathrm{E}-02$ & $5.02 \mathrm{E}-04$ & $8.197 \mathrm{E}-02$ & $3.84 \mathrm{E}-03$ & $3.944 \mathrm{E}+04$ \\
\hline $1.167 \mathrm{E}+00$ & $6.682 \mathrm{E}-01$ & $1.70 \mathrm{E}-02$ & $1.476 \mathrm{E}-01$ & $5.42 \mathrm{E}-04$ & $1.687 \mathrm{E}-01$ & $8.02 \mathrm{E}-03$ & $3.956 \mathrm{E}+04$ \\
\hline $1.166 \mathrm{E}+00$ & $6.701 \mathrm{E}-01$ & $1.77 \mathrm{E}-02$ & $2.303 \mathrm{E}-01$ & $6.35 \mathrm{E}-04$ & $2.720 \mathrm{E}-01$ & $1.30 \mathrm{E}-02$ & $3.972 \mathrm{E}+04$ \\
\hline $1.166 \mathrm{E}+00$ & $6.726 \mathrm{E}-01$ & $1.85 \mathrm{E}-02$ & $3.147 \mathrm{E}-01$ & 7.77E-04 & $3.826 \mathrm{E}-01$ & $1.86 \mathrm{E}-02$ & $3.987 \mathrm{E}+04$ \\
\hline $1.165 \mathrm{E}+00$ & $6.688 \mathrm{E}-01$ & $1.82 \mathrm{E}-02$ & $4.031 \mathrm{E}-01$ & $9.12 \mathrm{E}-04$ & $5.010 \mathrm{E}-01$ & $2.48 \mathrm{E}-02$ & $3.994 \mathrm{E}+04$ \\
\hline $1.165 \mathrm{E}+00$ & $6.711 \mathrm{E}-01$ & $1.84 \mathrm{E}-02$ & $4.914 \mathrm{E}-01$ & $1.01 \mathrm{E}-03$ & $5.875 \mathrm{E}-01$ & $3.01 \mathrm{E}-02$ & $4.019 \mathrm{E}+04$ \\
\hline $1.165 \mathrm{E}+00$ & $6.753 \mathrm{E}-01$ & $1.83 \mathrm{E}-02$ & $5.799 \mathrm{E}-01$ & $1.14 \mathrm{E}-03$ & $6.567 \mathrm{E}-01$ & $3.52 \mathrm{E}-02$ & $4.047 \mathrm{E}+04$ \\
\hline $1.164 \mathrm{E}+00$ & $6.808 \mathrm{E}-01$ & $1.92 \mathrm{E}-02$ & $6.638 \mathrm{E}-01$ & $1.27 \mathrm{E}-03$ & $6.891 \mathrm{E}-01$ & 4.01E-02 & $4.097 \mathrm{E}+04$ \\
\hline $1.164 \mathrm{E}+00$ & $6.825 \mathrm{E}-01$ & $2.04 \mathrm{E}-02$ & $7.513 \mathrm{E}-01$ & $1.43 \mathrm{E}-03$ & $7.009 \mathrm{E}-01$ & $4.94 \mathrm{E}-02$ & $4.135 \mathrm{E}+04$ \\
\hline $1.164 \mathrm{E}+00$ & $6.805 \mathrm{E}-01$ & $2.33 \mathrm{E}-02$ & 8.407E-01 & $1.60 \mathrm{E}-03$ & $7.116 \mathrm{E}-01$ & $6.62 \mathrm{E}-02$ & $4.169 \mathrm{E}+04$ \\
\hline
\end{tabular}


Table 120: APC 4.75 x 4.75 Dynamic Measured Values - 18016 RPM

\begin{tabular}{|c|c|c|c|c|c|c|c|}
\hline$n(\mathrm{RPM})$ & $Q(\mathrm{~g}-\mathrm{m})$ & $\Delta Q(\mathrm{~g}-\mathrm{m})$ & $V(\mathrm{~V})$ & $I(\mathrm{~A})$ & $T_{\text {atm }}\left({ }^{\circ} \mathrm{C}\right)$ & $P_{\text {atm }}(\mathrm{Pa})$ & $P_{\text {diff }}(\mathrm{Pa})$ \\
\hline $1.796 \mathrm{E}+04$ & $4.410 \mathrm{E}+00$ & $9.18 \mathrm{E}-02$ & $1.079 \mathrm{E}+01$ & $1.113 \mathrm{E}+01$ & $2.207 \mathrm{E}+01$ & $9.908 \mathrm{E}+04$ & $3.718 \mathrm{E}+00$ \\
\hline $1.804 \mathrm{E}+04$ & $4.408 \mathrm{E}+00$ & $9.30 \mathrm{E}-02$ & $1.079 \mathrm{E}+01$ & $1.104 \mathrm{E}+01$ & $2.198 \mathrm{E}+01$ & $9.909 \mathrm{E}+04$ & $1.668 \mathrm{E}+01$ \\
\hline $1.803 \mathrm{E}+04$ & $4.212 \mathrm{E}+00$ & $9.24 \mathrm{E}-02$ & $1.081 \mathrm{E}+01$ & $1.055 \mathrm{E}+01$ & $2.211 \mathrm{E}+01$ & $9.908 \mathrm{E}+04$ & $4.184 \mathrm{E}+01$ \\
\hline $1.800 \mathrm{E}+04$ & $4.021 \mathrm{E}+00$ & $9.30 \mathrm{E}-02$ & $1.082 \mathrm{E}+01$ & $9.999 \mathrm{E}+00$ & $2.227 \mathrm{E}+01$ & $9.907 \mathrm{E}+04$ & $7.919 \mathrm{E}+01$ \\
\hline $1.805 \mathrm{E}+04$ & $4.013 \mathrm{E}+00$ & $9.29 \mathrm{E}-02$ & $1.083 \mathrm{E}+01$ & $9.909 \mathrm{E}+00$ & $2.234 \mathrm{E}+01$ & $9.907 \mathrm{E}+04$ & $1.295 \mathrm{E}+02$ \\
\hline $1.803 \mathrm{E}+04$ & $4.013 \mathrm{E}+00$ & $9.32 \mathrm{E}-02$ & $1.083 \mathrm{E}+01$ & $9.931 \mathrm{E}+00$ & $2.242 \mathrm{E}+01$ & $9.906 \mathrm{E}+04$ & $1.927 \mathrm{E}+02$ \\
\hline $1.801 \mathrm{E}+04$ & $4.053 \mathrm{E}+00$ & $9.37 \mathrm{E}-02$ & $1.082 \mathrm{E}+01$ & $9.971 \mathrm{E}+00$ & $2.244 \mathrm{E}+01$ & $9.905 \mathrm{E}+04$ & $2.673 \mathrm{E}+02$ \\
\hline $1.808 \mathrm{E}+04$ & $4.018 \mathrm{E}+00$ & $9.65 \mathrm{E}-02$ & $1.083 \mathrm{E}+01$ & $9.821 \mathrm{E}+00$ & $2.244 \mathrm{E}+01$ & $9.904 \mathrm{E}+04$ & $3.538 \mathrm{E}+02$ \\
\hline $1.799 \mathrm{E}+04$ & $3.643 \mathrm{E}+00$ & $9.11 \mathrm{E}-02$ & $1.086 \mathrm{E}+01$ & $8.765 \mathrm{E}+00$ & $2.258 \mathrm{E}+01$ & $9.904 \mathrm{E}+04$ & $4.521 \mathrm{E}+02$ \\
\hline $1.798 \mathrm{E}+04$ & $3.119 \mathrm{E}+00$ & $8.97 \mathrm{E}-02$ & $1.090 \mathrm{E}+01$ & $7.314 \mathrm{E}+00$ & $2.276 \mathrm{E}+01$ & $9.903 \mathrm{E}+04$ & $5.612 \mathrm{E}+02$ \\
\hline
\end{tabular}


Table 121: APC 4.75 x 4.75 Dynamic Calculated Values - 18016 RPM

\begin{tabular}{|c|c|c|c|c|c|c|c|}
\hline$n(\mathrm{RPM})$ & $V_{\infty}^{\prime}(\mathrm{m} / \mathrm{s})$ & $\Delta V_{\infty}^{\prime}(\mathrm{m} / \mathrm{s})$ & $T^{\prime}(\mathrm{g})$ & $\Delta T^{\prime}(\mathrm{g})$ & $V_{\mathrm{t}}(\mathrm{m} / \mathrm{s})$ & $P_{\mathrm{P}}(\mathrm{W})$ & $\Delta P_{\mathrm{P}}(\mathrm{W})$ \\
\hline $1.796 \mathrm{E}+04$ & $2.372 \mathrm{E}+00$ & $1.77 \mathrm{E}-02$ & $2.643 \mathrm{E}+02$ & 7.64E+00 & $8.456 \mathrm{E}+01$ & $8.132 \mathrm{E}+01$ & $1.69 \mathrm{E}+00$ \\
\hline $1.804 \mathrm{E}+04$ & $5.196 \mathrm{E}+00$ & $1.95 \mathrm{E}-02$ & $2.626 \mathrm{E}+02$ & $7.64 \mathrm{E}+00$ & $8.507 \mathrm{E}+01$ & $8.167 \mathrm{E}+01$ & $1.72 \mathrm{E}+00$ \\
\hline $1.803 \mathrm{E}+04$ & $8.325 \mathrm{E}+00$ & $2.38 \mathrm{E}-02$ & $2.557 \mathrm{E}+02$ & $7.57 \mathrm{E}+00$ & $8.527 \mathrm{E}+01$ & $7.797 \mathrm{E}+01$ & $1.71 \mathrm{E}+00$ \\
\hline $1.800 \mathrm{E}+04$ & $1.152 \mathrm{E}+01$ & $2.94 \mathrm{E}-02$ & $2.545 \mathrm{E}+02$ & $7.60 \mathrm{E}+00$ & $8.549 \mathrm{E}+01$ & $7.431 \mathrm{E}+01$ & $1.72 \mathrm{E}+00$ \\
\hline $1.805 \mathrm{E}+04$ & $1.477 \mathrm{E}+01$ & 3.34E-02 & $2.577 \mathrm{E}+02$ & $7.52 \mathrm{E}+00$ & $8.622 \mathrm{E}+01$ & $7.437 \mathrm{E}+01$ & $1.72 \mathrm{E}+00$ \\
\hline $1.803 \mathrm{E}+04$ & $1.806 \mathrm{E}+01$ & $3.72 \mathrm{E}-02$ & $2.501 \mathrm{E}+02$ & $7.52 \mathrm{E}+00$ & $8.677 \mathrm{E}+01$ & $7.430 \mathrm{E}+01$ & $1.73 \mathrm{E}+00$ \\
\hline $1.801 \mathrm{E}+04$ & $2.130 \mathrm{E}+01$ & 4.17E-02 & $2.385 \mathrm{E}+02$ & $7.58 \mathrm{E}+00$ & $8.742 \mathrm{E}+01$ & $7.497 \mathrm{E}+01$ & $1.73 \mathrm{E}+00$ \\
\hline $1.808 \mathrm{E}+04$ & $2.454 \mathrm{E}+01$ & 4.68E-02 & $2.165 \mathrm{E}+02$ & $7.66 \mathrm{E}+00$ & $8.858 \mathrm{E}+01$ & $7.460 \mathrm{E}+01$ & $1.79 \mathrm{E}+00$ \\
\hline $1.799 \mathrm{E}+04$ & $2.778 \mathrm{E}+01$ & $5.23 \mathrm{E}-02$ & $1.781 \mathrm{E}+02$ & $7.59 \mathrm{E}+00$ & $8.914 \mathrm{E}+01$ & $6.732 \mathrm{E}+01$ & $1.68 \mathrm{E}+00$ \\
\hline $1.798 \mathrm{E}+04$ & $3.098 \mathrm{E}+01$ & $5.75 \mathrm{E}-02$ & $1.382 \mathrm{E}+02$ & $7.52 \mathrm{E}+00$ & $9.011 \mathrm{E}+01$ & $5.758 \mathrm{E}+01$ & $1.66 \mathrm{E}+00$ \\
\hline$P_{\mathrm{e}}(\mathrm{W})$ & $\Delta P_{\mathrm{e}}(\mathrm{W})$ & $C_{\mathrm{T}}$ & $\Delta C_{\mathrm{T}}$ & $C_{\mathrm{P}}$ & $\Delta C_{\mathrm{P}}$ & $C_{\mathrm{Q}}$ & $\Delta C_{\mathrm{Q}}$ \\
\hline $1.201 \mathrm{E}+02$ & $1.28 \mathrm{E}+00$ & $1.199 \mathrm{E}-01$ & $3.47 \mathrm{E}-03$ & $1.048 \mathrm{E}-01$ & $2.19 \mathrm{E}-03$ & $1.669 \mathrm{E}-02$ & $3.48 \mathrm{E}-04$ \\
\hline $1.191 \mathrm{E}+02$ & $1.25 \mathrm{E}+00$ & $1.180 \mathrm{E}-01$ & $3.43 \mathrm{E}-03$ & $1.038 \mathrm{E}-01$ & $2.19 \mathrm{E}-03$ & $1.652 \mathrm{E}-02$ & $3.49 \mathrm{E}-04$ \\
\hline $1.140 \mathrm{E}+02$ & $1.20 \mathrm{E}+00$ & $1.151 \mathrm{E}-01$ & $3.41 \mathrm{E}-03$ & $9.936 \mathrm{E}-02$ & $2.18 \mathrm{E}-03$ & $1.581 \mathrm{E}-02$ & $3.47 \mathrm{E}-04$ \\
\hline $1.082 \mathrm{E}+02$ & $1.15 \mathrm{E}+00$ & $1.150 \mathrm{E}-01$ & $3.43 \mathrm{E}-03$ & $9.525 \mathrm{E}-02$ & $2.20 \mathrm{E}-03$ & $1.516 \mathrm{E}-02$ & $3.51 \mathrm{E}-04$ \\
\hline $1.073 \mathrm{E}+02$ & $1.14 \mathrm{E}+00$ & $1.159 \mathrm{E}-01$ & $3.38 \mathrm{E}-03$ & $9.457 \mathrm{E}-02$ & $2.19 \mathrm{E}-03$ & $1.505 \mathrm{E}-02$ & $3.49 \mathrm{E}-04$ \\
\hline $1.075 \mathrm{E}+02$ & $1.15 \mathrm{E}+00$ & $1.127 \mathrm{E}-01$ & $3.39 \mathrm{E}-03$ & $9.477 \mathrm{E}-02$ & $2.20 \mathrm{E}-03$ & $1.508 \mathrm{E}-02$ & $3.51 \mathrm{E}-04$ \\
\hline $1.079 \mathrm{E}+02$ & $1.15 \mathrm{E}+00$ & $1.077 \mathrm{E}-01$ & $3.42 \mathrm{E}-03$ & $9.593 \mathrm{E}-02$ & $2.22 \mathrm{E}-03$ & $1.527 \mathrm{E}-02$ & $3.53 \mathrm{E}-04$ \\
\hline $1.063 \mathrm{E}+02$ & $1.14 \mathrm{E}+00$ & $9.701 \mathrm{E}-02$ & $3.43 \mathrm{E}-03$ & $9.437 \mathrm{E}-02$ & $2.27 \mathrm{E}-03$ & $1.502 \mathrm{E}-02$ & $3.61 \mathrm{E}-04$ \\
\hline $9.518 \mathrm{E}+01$ & $1.02 \mathrm{E}+00$ & $8.063 \mathrm{E}-02$ & $3.44 \mathrm{E}-03$ & 8.645E-02 & $2.16 \mathrm{E}-03$ & $1.376 \mathrm{E}-02$ & $3.44 \mathrm{E}-04$ \\
\hline $7.974 \mathrm{E}+01$ & 8.79E-01 & $6.275 \mathrm{E}-02$ & $3.41 \mathrm{E}-03$ & $7.421 \mathrm{E}-02$ & $2.13 \mathrm{E}-03$ & $1.181 \mathrm{E}-02$ & $3.40 \mathrm{E}-04$ \\
\hline$\rho\left(\mathrm{kg} / \mathrm{m}^{\wedge} 3\right)$ & $\eta_{\mathrm{T}}$ & $\Delta \eta_{\mathrm{T}}$ & $J$ & $\Delta J$ & $\eta_{\mathrm{P}}$ & $\Delta \eta_{\mathrm{P}}$ & $R e_{0.75}$ \\
\hline $1.169 \mathrm{E}+00$ & $6.773 \mathrm{E}-01$ & $1.59 \mathrm{E}-02$ & $6.613 \mathrm{E}-02$ & 4.92E-04 & $7.563 \mathrm{E}-02$ & $2.75 \mathrm{E}-03$ & $4.450 \mathrm{E}+04$ \\
\hline $1.170 \mathrm{E}+00$ & $6.858 \mathrm{E}-01$ & $1.62 \mathrm{E}-02$ & $1.442 \mathrm{E}-01$ & $5.41 \mathrm{E}-04$ & $1.638 \mathrm{E}-01$ & $5.92 \mathrm{E}-03$ & $4.479 \mathrm{E}+04$ \\
\hline $1.169 \mathrm{E}+00$ & $6.839 \mathrm{E}-01$ & $1.66 \mathrm{E}-02$ & $2.311 \mathrm{E}-01$ & $6.64 \mathrm{E}-04$ & $2.677 \mathrm{E}-01$ & $9.90 \mathrm{E}-03$ & $4.486 \mathrm{E}+04$ \\
\hline $1.168 \mathrm{E}+00$ & $6.867 \mathrm{E}-01$ & $1.75 \mathrm{E}-02$ & 3.203E-01 & 8.20E-04 & $3.868 \mathrm{E}-01$ & $1.46 \mathrm{E}-02$ & $4.493 \mathrm{E}+04$ \\
\hline $1.168 \mathrm{E}+00$ & $6.933 \mathrm{E}-01$ & $1.77 \mathrm{E}-02$ & $4.098 \mathrm{E}-01$ & $9.32 \mathrm{E}-04$ & $5.021 \mathrm{E}-01$ & $1.87 \mathrm{E}-02$ & $4.529 \mathrm{E}+04$ \\
\hline $1.168 \mathrm{E}+00$ & $6.911 \mathrm{E}-01$ & $1.77 \mathrm{E}-02$ & $5.014 \mathrm{E}-01$ & $1.04 \mathrm{E}-03$ & 5.961E-01 & $2.27 \mathrm{E}-02$ & $4.555 \mathrm{E}+04$ \\
\hline $1.167 \mathrm{E}+00$ & $6.947 \mathrm{E}-01$ & $1.77 \mathrm{E}-02$ & $5.920 \mathrm{E}-01$ & $1.17 \mathrm{E}-03$ & $6.646 \mathrm{E}-01$ & $2.62 \mathrm{E}-02$ & $4.588 \mathrm{E}+04$ \\
\hline $1.167 \mathrm{E}+00$ & 7.015E-01 & $1.84 \mathrm{E}-02$ & 6.794E-01 & $1.31 \mathrm{E}-03$ & 6.984E-01 & $2.99 \mathrm{E}-02$ & $4.649 \mathrm{E}+04$ \\
\hline $1.167 \mathrm{E}+00$ & $7.073 \mathrm{E}-01$ & $1.92 \mathrm{E}-02$ & 7.727E-01 & $1.47 \mathrm{E}-03$ & 7.207E-01 & $3.56 \mathrm{E}-02$ & $4.674 \mathrm{E}+04$ \\
\hline $1.166 \mathrm{E}+00$ & $7.220 \mathrm{E}-01$ & $2.22 \mathrm{E}-02$ & 8.627E-01 & $1.61 \mathrm{E}-03$ & $7.295 \mathrm{E}-01$ & 4.49E-02 & $4.720 \mathrm{E}+04$ \\
\hline
\end{tabular}




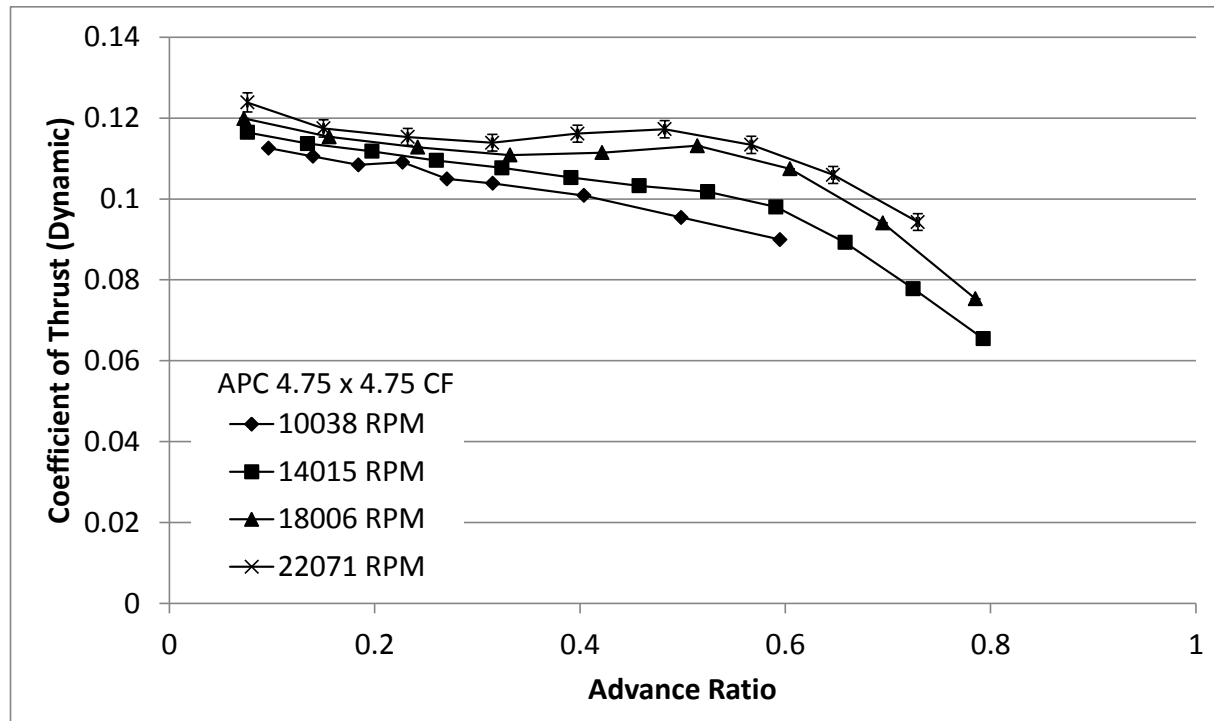

(a)

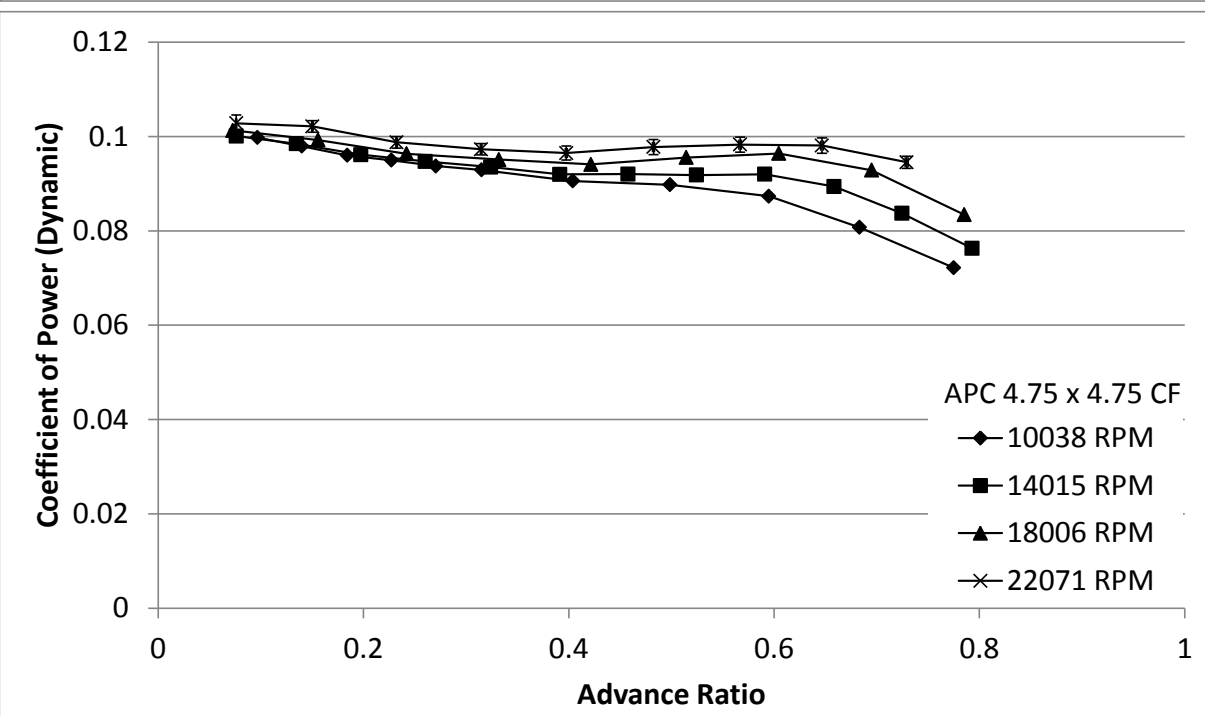

(b)

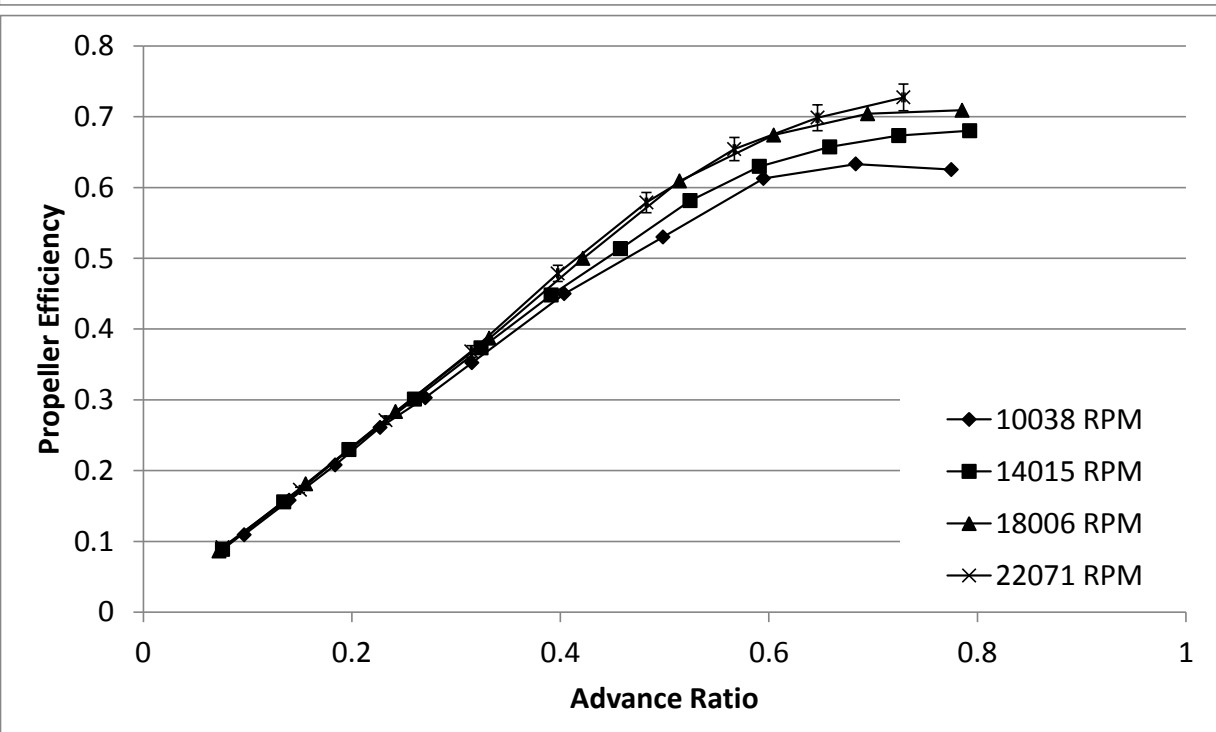

(c) 


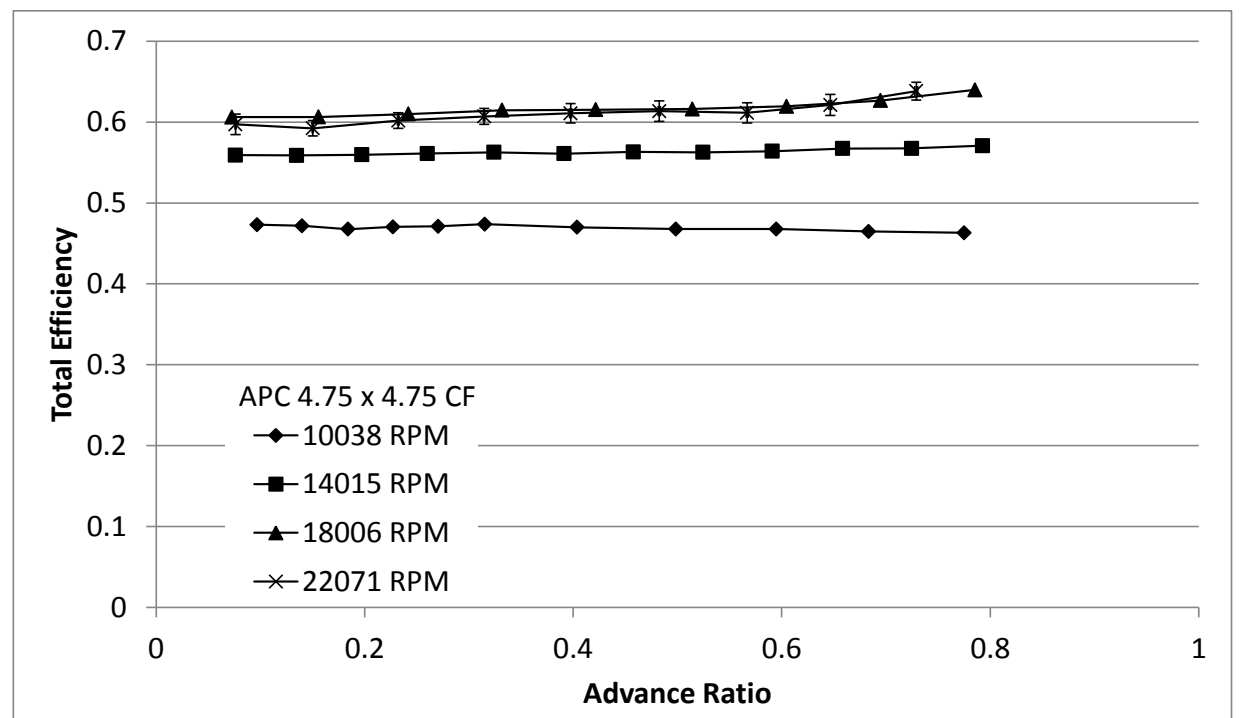

(d)

Figure 84: APC 4.75 x 4.75 Carbon Fiber Dynamic Test Results: (a) Coefficient of Thrust, (b) Coefficient of Power, (c) Propeller Efficiency, (d) Total Efficiency.

Table 122: APC 4.75 x 4.75 Carbon Fiber Dynamic Measured Values - 10038 RPM

\begin{tabular}{|c|c|c|c|c|c|c|c|}
\hline$n(\mathrm{RPM})$ & $Q(\mathrm{~g}-\mathrm{m})$ & $\Delta Q(\mathrm{~g}-\mathrm{m})$ & $V(\mathrm{~V})$ & $I(\mathrm{~A})$ & $T_{\text {atm }}\left({ }^{\circ} \mathrm{C}\right)$ & $P_{\text {atm }}(\mathrm{Pa})$ & $P_{\text {diff }}(\mathrm{Pa})$ \\
\hline $1.003 \mathrm{E}+04$ & $1.300 \mathrm{E}+00$ & $6.13 \mathrm{E}-02$ & $1.102 \mathrm{E}+01$ & $2.569 \mathrm{E}+00$ & $1.945 \mathrm{E}+01$ & $9.743 \mathrm{E}+04$ & $2.367 \mathrm{E}+00$ \\
\hline $1.007 \mathrm{E}+04$ & $1.287 \mathrm{E}+00$ & $6.60 \mathrm{E}-02$ & $1.103 \mathrm{E}+01$ & $2.559 \mathrm{E}+00$ & $1.944 \mathrm{E}+01$ & $9.743 \mathrm{E}+04$ & $4.867 \mathrm{E}+00$ \\
\hline $9.971 \mathrm{E}+03$ & $1.235 \mathrm{E}+00$ & $6.12 \mathrm{E}-02$ & $1.103 \mathrm{E}+01$ & $2.452 \mathrm{E}+00$ & $1.943 \mathrm{E}+01$ & $9.743 \mathrm{E}+04$ & $8.127 \mathrm{E}+00$ \\
\hline $1.002 \mathrm{E}+04$ & $1.234 \mathrm{E}+00$ & $5.91 \mathrm{E}-02$ & $1.103 \mathrm{E}+01$ & $2.448 \mathrm{E}+00$ & $1.938 \mathrm{E}+01$ & $9.744 \mathrm{E}+04$ & $1.239 \mathrm{E}+01$ \\
\hline $1.004 \mathrm{E}+04$ & $1.224 \mathrm{E}+00$ & $6.19 \mathrm{E}-02$ & $1.103 \mathrm{E}+01$ & $2.430 \mathrm{E}+00$ & $1.944 \mathrm{E}+01$ & $9.744 \mathrm{E}+04$ & $1.753 \mathrm{E}+01$ \\
\hline $1.007 \mathrm{E}+04$ & $1.220 \mathrm{E}+00$ & $6.57 \mathrm{E}-02$ & $1.103 \mathrm{E}+01$ & $2.415 \mathrm{E}+00$ & $1.962 \mathrm{E}+01$ & $9.744 \mathrm{E}+04$ & $2.382 \mathrm{E}+01$ \\
\hline $1.007 \mathrm{E}+04$ & $1.186 \mathrm{E}+00$ & $6.49 \mathrm{E}-02$ & $1.103 \mathrm{E}+01$ & $2.365 \mathrm{E}+00$ & $2.002 \mathrm{E}+01$ & $9.744 \mathrm{E}+04$ & $3.876 \mathrm{E}+01$ \\
\hline $1.004 \mathrm{E}+04$ & $1.168 \mathrm{E}+00$ & $6.07 \mathrm{E}-02$ & $1.103 \mathrm{E}+01$ & $2.333 \mathrm{E}+00$ & $2.002 \mathrm{E}+01$ & $9.743 \mathrm{E}+04$ & $5.849 \mathrm{E}+01$ \\
\hline $9.981 \mathrm{E}+03$ & $1.122 \mathrm{E}+00$ & $5.89 \mathrm{E}-02$ & $1.103 \mathrm{E}+01$ & $2.229 \mathrm{E}+00$ & $2.041 \mathrm{E}+01$ & $9.744 \mathrm{E}+04$ & $8.197 \mathrm{E}+01$ \\
\hline $1.005 \mathrm{E}+04$ & $1.051 \mathrm{E}+00$ & $6.15 \mathrm{E}-02$ & $1.104 \mathrm{E}+01$ & $2.113 \mathrm{E}+00$ & $2.045 \mathrm{E}+01$ & $9.743 \mathrm{E}+04$ & $1.093 \mathrm{E}+02$ \\
\hline $1.007 \mathrm{E}+04$ & $9.434 \mathrm{E}-01$ & $6.55 \mathrm{E}-02$ & $1.104 \mathrm{E}+01$ & $1.907 \mathrm{E}+00$ & $2.052 \mathrm{E}+01$ & $9.742 \mathrm{E}+04$ & $1.409 \mathrm{E}+02$ \\
\hline
\end{tabular}


Table 123: APC 4.75 x 4.75 Carbon Fiber Dynamic Calculated Values - 10038 RPM

\begin{tabular}{|c|c|c|c|c|c|c|c|}
\hline$n(\mathrm{RPM})$ & $V_{\infty}^{\prime}(\mathrm{m} / \mathrm{s})$ & $\Delta V_{\infty}^{\prime}(\mathrm{m} / \mathrm{s})$ & $T^{\prime}(\mathrm{g})$ & $\Delta T^{\prime}(\mathrm{g})$ & $V_{\mathrm{t}}(\mathrm{m} / \mathrm{s})$ & $P_{\mathrm{P}}(\mathrm{W})$ & $\Delta P_{\mathrm{P}}(\mathrm{W})$ \\
\hline $1.003 \mathrm{E}+04$ & $1.940 \mathrm{E}+00$ & $1.28 \mathrm{E}-02$ & $7.689 \mathrm{E}+01$ & $6.76 \mathrm{E}+00$ & $4.727 \mathrm{E}+01$ & $1.340 \mathrm{E}+01$ & $6.32 \mathrm{E}-01$ \\
\hline $1.007 \mathrm{E}+04$ & $2.818 \mathrm{E}+00$ & $1.28 \mathrm{E}-02$ & $7.612 \mathrm{E}+01$ & $6.84 \mathrm{E}+00$ & $4.751 \mathrm{E}+01$ & $1.331 \mathrm{E}+01$ & $6.83 \mathrm{E}-01$ \\
\hline $9.971 \mathrm{E}+03$ & $3.668 \mathrm{E}+00$ & $1.31 \mathrm{E}-02$ & $7.313 \mathrm{E}+01$ & $6.72 \mathrm{E}+00$ & $4.708 \mathrm{E}+01$ & $1.265 \mathrm{E}+01$ & $6.27 \mathrm{E}-01$ \\
\hline $1.002 \mathrm{E}+04$ & $4.547 \mathrm{E}+00$ & $1.38 \mathrm{E}-02$ & $7.431 \mathrm{E}+01$ & $6.76 \mathrm{E}+00$ & $4.738 \mathrm{E}+01$ & $1.270 \mathrm{E}+01$ & $6.09 \mathrm{E}-01$ \\
\hline $1.004 \mathrm{E}+04$ & $5.428 \mathrm{E}+00$ & $1.52 \mathrm{E}-02$ & $7.184 \mathrm{E}+01$ & $6.76 \mathrm{E}+00$ & $4.759 \mathrm{E}+01$ & $1.263 \mathrm{E}+01$ & $6.38 \mathrm{E}-01$ \\
\hline $1.007 \mathrm{E}+04$ & $6.343 \mathrm{E}+00$ & $1.63 \mathrm{E}-02$ & $7.145 \mathrm{E}+01$ & $6.79 \mathrm{E}+00$ & $4.784 \mathrm{E}+01$ & $1.262 \mathrm{E}+01$ & $6.79 \mathrm{E}-01$ \\
\hline $1.007 \mathrm{E}+04$ & $8.123 \mathrm{E}+00$ & $1.97 \mathrm{E}-02$ & $6.920 \mathrm{E}+01$ & $6.84 \mathrm{E}+00$ & $4.808 \mathrm{E}+01$ & $1.226 \mathrm{E}+01$ & $6.71 \mathrm{E}-01$ \\
\hline $1.004 \mathrm{E}+04$ & $9.999 \mathrm{E}+00$ & $2.12 \mathrm{E}-02$ & $6.509 \mathrm{E}+01$ & $6.80 \mathrm{E}+00$ & $4.829 \mathrm{E}+01$ & $1.204 \mathrm{E}+01$ & $6.25 \mathrm{E}-01$ \\
\hline $9.981 \mathrm{E}+03$ & $1.186 \mathrm{E}+01$ & $2.43 \mathrm{E}-02$ & $6.059 \mathrm{E}+01$ & $6.77 \mathrm{E}+00$ & $4.845 \mathrm{E}+01$ & $1.150 \mathrm{E}+01$ & $6.03 \mathrm{E}-01$ \\
\hline $1.005 \mathrm{E}+04$ & $1.371 \mathrm{E}+01$ & $2.75 \mathrm{E}-02$ & $5.103 \mathrm{E}+01$ & $6.84 \mathrm{E}+00$ & $4.923 \mathrm{E}+01$ & $1.084 \mathrm{E}+01$ & $6.35 \mathrm{E}-01$ \\
\hline $1.007 \mathrm{E}+04$ & $1.559 \mathrm{E}+01$ & $3.04 \mathrm{E}-02$ & $3.990 \mathrm{E}+01$ & $6.88 \mathrm{E}+00$ & $4.989 \mathrm{E}+01$ & $9.754 \mathrm{E}+00$ & $6.77 \mathrm{E}-01$ \\
\hline$P_{\mathrm{e}}(\mathrm{W})$ & $\Delta P_{\mathrm{e}}(\mathrm{W})$ & $C_{\mathrm{T}}$ & $\Delta C_{\mathrm{T}}$ & $C_{\mathrm{P}}$ & $\Delta C_{\mathrm{P}}$ & $C_{\mathrm{Q}}$ & $\Delta C_{\mathrm{Q}}$ \\
\hline $2.832 \mathrm{E}+01$ & $3.40 \mathrm{E}-01$ & $1.126 \mathrm{E}-01$ & $9.89 \mathrm{E}-03$ & $9.977 \mathrm{E}-02$ & $4.71 \mathrm{E}-03$ & $1.588 \mathrm{E}-02$ & $7.49 \mathrm{E}-04$ \\
\hline $2.822 \mathrm{E}+01$ & 3.37E-01 & $1.105 \mathrm{E}-01$ & 9.94E-03 & $9.795 \mathrm{E}-02$ & $5.03 \mathrm{E}-03$ & $1.559 \mathrm{E}-02$ & $8.00 \mathrm{E}-04$ \\
\hline $2.704 \mathrm{E}+01$ & $3.24 \mathrm{E}-01$ & $1.084 \mathrm{E}-01$ & $9.97 \mathrm{E}-03$ & $9.599 \mathrm{E}-02$ & $4.76 \mathrm{E}-03$ & $1.528 \mathrm{E}-02$ & $7.57 \mathrm{E}-04$ \\
\hline $2.699 \mathrm{E}+01$ & $3.24 \mathrm{E}-01$ & $1.091 \mathrm{E}-01$ & $9.92 \mathrm{E}-03$ & $9.496 \mathrm{E}-02$ & $4.55 \mathrm{E}-03$ & $1.511 \mathrm{E}-02$ & $7.24 \mathrm{E}-04$ \\
\hline $2.680 \mathrm{E}+01$ & $3.20 \mathrm{E}-01$ & $1.050 \mathrm{E}-01$ & $9.87 \mathrm{E}-03$ & $9.373 \mathrm{E}-02$ & 4.74E-03 & 1.492E-02 & $7.54 \mathrm{E}-04$ \\
\hline $2.664 \mathrm{E}+01$ & $3.20 \mathrm{E}-01$ & $1.038 \mathrm{E}-01$ & $9.87 \mathrm{E}-03$ & $9.291 \mathrm{E}-02$ & $5.00 \mathrm{E}-03$ & $1.479 \mathrm{E}-02$ & $7.96 \mathrm{E}-04$ \\
\hline $2.608 \mathrm{E}+01$ & $3.19 \mathrm{E}-01$ & $1.009 \mathrm{E}-01$ & $9.97 \mathrm{E}-03$ & $9.057 \mathrm{E}-02$ & $4.96 \mathrm{E}-03$ & $1.441 \mathrm{E}-02$ & $7.90 \mathrm{E}-04$ \\
\hline $2.574 \mathrm{E}+01$ & 3.07E-01 & $9.543 \mathrm{E}-02$ & $9.97 \mathrm{E}-03$ & 8.976E-02 & 4.66E-03 & $1.429 \mathrm{E}-02$ & $7.42 \mathrm{E}-04$ \\
\hline $2.459 \mathrm{E}+01$ & $2.96 \mathrm{E}-01$ & $8.996 \mathrm{E}-02$ & $1.01 \mathrm{E}-02$ & $8.735 \mathrm{E}-02$ & $4.58 \mathrm{E}-03$ & $1.390 \mathrm{E}-02$ & $7.29 \mathrm{E}-04$ \\
\hline $2.332 \mathrm{E}+01$ & $2.79 \mathrm{E}-01$ & $7.480 \mathrm{E}-02$ & $1.00 \mathrm{E}-02$ & $8.073 \mathrm{E}-02$ & $4.73 \mathrm{E}-03$ & $1.285 \mathrm{E}-02$ & $7.52 \mathrm{E}-04$ \\
\hline $2.106 \mathrm{E}+01$ & $2.56 \mathrm{E}-01$ & $5.825 \mathrm{E}-02$ & $1.00 \mathrm{E}-02$ & $7.219 \mathrm{E}-02$ & $5.01 \mathrm{E}-03$ & 1.149E-02 & $7.98 \mathrm{E}-04$ \\
\hline$\rho\left(\mathrm{kg} / \mathrm{m}^{\wedge} 3\right)$ & $\eta_{\mathrm{T}}$ & $\Delta \eta_{\mathrm{T}}$ & $J$ & $\Delta J$ & $\eta_{\mathrm{P}}$ & $\Delta \eta_{\mathrm{P}}$ & $R e_{0.75}$ \\
\hline $1.160 \mathrm{E}+00$ & $4.730 \mathrm{E}-01$ & $2.30 \mathrm{E}-02$ & $9.678 \mathrm{E}-02$ & $6.39 \mathrm{E}-04$ & $1.092 \mathrm{E}-01$ & $1.09 \mathrm{E}-02$ & $2.485 \mathrm{E}+04$ \\
\hline $1.160 \mathrm{E}+00$ & 4.718E-01 & $2.49 \mathrm{E}-02$ & $1.400 \mathrm{E}-01$ & $6.37 \mathrm{E}-04$ & $1.580 \mathrm{E}-01$ & $1.64 \mathrm{E}-02$ & $2.497 \mathrm{E}+04$ \\
\hline $1.160 \mathrm{E}+00$ & 4.677E-01 & $2.38 \mathrm{E}-02$ & $1.842 \mathrm{E}-01$ & $6.59 \mathrm{E}-04$ & $2.080 \mathrm{E}-01$ & $2.17 \mathrm{E}-02$ & $2.475 \mathrm{E}+04$ \\
\hline $1.160 \mathrm{E}+00$ & 4.705E-01 & $2.32 \mathrm{E}-02$ & $2.272 \mathrm{E}-01$ & $6.96 \mathrm{E}-04$ & $2.610 \mathrm{E}-01$ & $2.68 \mathrm{E}-02$ & $2.492 \mathrm{E}+04$ \\
\hline $1.160 \mathrm{E}+00$ & 4.712E-01 & $2.45 \mathrm{E}-02$ & 2.705E-01 & 7.64E-04 & $3.029 \mathrm{E}-01$ & $3.24 \mathrm{E}-02$ & $2.502 \mathrm{E}+04$ \\
\hline $1.159 \mathrm{E}+00$ & 4.737E-01 & 2.61E-02 & 3.152E-01 & $8.16 \mathrm{E}-04$ & $3.523 \mathrm{E}-01$ & $3.85 \mathrm{E}-02$ & $2.512 \mathrm{E}+04$ \\
\hline $1.158 \mathrm{E}+00$ & 4.699E-01 & $2.64 \mathrm{E}-02$ & 4.039E-01 & $9.94 \mathrm{E}-04$ & 4.497E-01 & $5.08 \mathrm{E}-02$ & $2.518 \mathrm{E}+04$ \\
\hline $1.158 \mathrm{E}+00$ & $4.678 \mathrm{E}-01$ & $2.49 \mathrm{E}-02$ & 4.987E-01 & $1.07 \mathrm{E}-03$ & 5.301E-01 & $6.18 \mathrm{E}-02$ & $2.530 \mathrm{E}+04$ \\
\hline $1.156 \mathrm{E}+00$ & 4.678E-01 & $2.52 \mathrm{E}-02$ & 5.949E-01 & $1.23 \mathrm{E}-03$ & $6.127 \mathrm{E}-01$ & 7.57E-02 & $2.532 \mathrm{E}+04$ \\
\hline $1.156 \mathrm{E}+00$ & 4.648E-01 & $2.78 \mathrm{E}-02$ & $6.833 \mathrm{E}-01$ & $1.39 \mathrm{E}-03$ & 6.332E-01 & $9.26 \mathrm{E}-02$ & $2.572 \mathrm{E}+04$ \\
\hline $1.156 \mathrm{E}+00$ & 4.631E-01 & $3.26 \mathrm{E}-02$ & $7.750 \mathrm{E}-01$ & $1.53 \mathrm{E}-03$ & $6.253 \mathrm{E}-01$ & $1.16 \mathrm{E}-01$ & $2.605 E+04$ \\
\hline
\end{tabular}


Table 124: APC 4.75 x 4.75 Carbon Fiber Dynamic Measured Values - 14015 RPM

\begin{tabular}{|c|c|c|c|c|c|c|c|}
\hline$n(\mathrm{RPM})$ & $Q(\mathrm{~g}-\mathrm{m})$ & $\Delta Q(\mathrm{~g}-\mathrm{m})$ & $V(\mathrm{~V})$ & $I(\mathrm{~A})$ & $T_{\text {atm }}\left({ }^{\circ} \mathrm{C}\right)$ & $P_{\text {atm }}(\mathrm{Pa})$ & $P_{\text {diff }}(\mathrm{Pa})$ \\
\hline $1.401 \mathrm{E}+04$ & $2.547 \mathrm{E}+00$ & $6.95 \mathrm{E}-02$ & $1.094 \mathrm{E}+01$ & $5.992 \mathrm{E}+00$ & $1.906 \mathrm{E}+01$ & $9.746 \mathrm{E}+04$ & $2.925 \mathrm{E}+00$ \\
\hline $1.405 \mathrm{E}+04$ & $2.518 \mathrm{E}+00$ & $7.06 \mathrm{E}-02$ & $1.094 \mathrm{E}+01$ & $5.943 \mathrm{E}+00$ & $1.915 \mathrm{E}+01$ & $9.746 \mathrm{E}+04$ & $8.799 \mathrm{E}+00$ \\
\hline $1.400 \mathrm{E}+04$ & $2.442 \mathrm{E}+00$ & $6.91 \mathrm{E}-02$ & $1.094 \mathrm{E}+01$ & $5.733 \mathrm{E}+00$ & $1.911 \mathrm{E}+01$ & $9.746 \mathrm{E}+04$ & $1.838 \mathrm{E}+01$ \\
\hline $1.399 \mathrm{E}+04$ & $2.400 \mathrm{E}+00$ & $7.54 \mathrm{E}-02$ & $1.095 \mathrm{E}+01$ & $5.615 \mathrm{E}+00$ & $1.960 \mathrm{E}+01$ & $9.746 \mathrm{E}+04$ & $3.152 \mathrm{E}+01$ \\
\hline $1.405 \mathrm{E}+04$ & $2.385 \mathrm{E}+00$ & $7.25 \mathrm{E}-02$ & $1.095 \mathrm{E}+01$ & $5.586 \mathrm{E}+00$ & $1.978 \mathrm{E}+01$ & $9.746 \mathrm{E}+04$ & $4.894 \mathrm{E}+01$ \\
\hline $1.400 \mathrm{E}+04$ & $2.332 \mathrm{E}+00$ & $6.35 \mathrm{E}-02$ & $1.095 \mathrm{E}+01$ & $5.458 \mathrm{E}+00$ & $1.957 \mathrm{E}+01$ & $9.746 \mathrm{E}+04$ & $7.061 \mathrm{E}+01$ \\
\hline $1.402 \mathrm{E}+04$ & $2.338 \mathrm{E}+00$ & $6.35 \mathrm{E}-02$ & $1.095 \mathrm{E}+01$ & $5.457 \mathrm{E}+00$ & $2.001 \mathrm{E}+01$ & $9.747 \mathrm{E}+04$ & $9.639 \mathrm{E}+01$ \\
\hline $1.402 \mathrm{E}+04$ & $2.331 \mathrm{E}+00$ & $6.31 \mathrm{E}-02$ & $1.095 \mathrm{E}+01$ & $5.446 \mathrm{E}+00$ & $2.020 \mathrm{E}+01$ & $9.746 \mathrm{E}+04$ & $1.262 \mathrm{E}+02$ \\
\hline $1.403 \mathrm{E}+04$ & $2.337 \mathrm{E}+00$ & $6.23 \mathrm{E}-02$ & $1.095 \mathrm{E}+01$ & $5.451 \mathrm{E}+00$ & $2.051 \mathrm{E}+01$ & $9.746 \mathrm{E}+04$ & $1.600 \mathrm{E}+02$ \\
\hline $1.402 \mathrm{E}+04$ & $2.265 \mathrm{E}+00$ & $6.23 \mathrm{E}-02$ & $1.096 \mathrm{E}+01$ & $5.246 \mathrm{E}+00$ & $2.055 \mathrm{E}+01$ & $9.746 \mathrm{E}+04$ & $1.977 \mathrm{E}+02$ \\
\hline $1.401 \mathrm{E}+04$ & $2.116 \mathrm{E}+00$ & $6.29 \mathrm{E}-02$ & $1.096 \mathrm{E}+01$ & $4.892 \mathrm{E}+00$ & $2.101 \mathrm{E}+01$ & $9.745 \mathrm{E}+04$ & $2.385 \mathrm{E}+02$ \\
\hline $1.399 \mathrm{E}+04$ & $1.922 \mathrm{E}+00$ & $6.17 \mathrm{E}-02$ & $1.098 \mathrm{E}+01$ & $4.406 \mathrm{E}+00$ & $2.125 \mathrm{E}+01$ & $9.745 \mathrm{E}+04$ & $2.841 \mathrm{E}+02$ \\
\hline
\end{tabular}


Table 125: APC 4.75 x 4.75 Carbon Fiber Dynamic Calculated Values - 14015 RPM

\begin{tabular}{|c|c|c|c|c|c|c|c|}
\hline$n(\mathrm{RPM})$ & $V_{\infty}^{\prime}(\mathrm{m} / \mathrm{s})$ & $\Delta V_{\infty}^{\prime}(\mathrm{m} / \mathrm{s})$ & $T^{\prime}(\mathrm{g})$ & $\Delta T^{\prime}(\mathrm{g})$ & $V_{\mathrm{t}}(\mathrm{m} / \mathrm{s})$ & $P_{\mathrm{P}}(\mathrm{W})$ & $\Delta P_{\mathrm{P}}(\mathrm{W})$ \\
\hline $1.401 \mathrm{E}+04$ & $2.129 \mathrm{E}+00$ & $1.49 \mathrm{E}-02$ & $1.554 \mathrm{E}+02$ & $6.80 \mathrm{E}+00$ & $6.599 \mathrm{E}+01$ & $3.664 \mathrm{E}+01$ & $1.00 \mathrm{E}+00$ \\
\hline $1.405 \mathrm{E}+04$ & $3.781 \mathrm{E}+00$ & $1.56 \mathrm{E}-02$ & $1.524 \mathrm{E}+02$ & $6.73 \mathrm{E}+00$ & $6.623 \mathrm{E}+01$ & $3.633 \mathrm{E}+01$ & $1.02 \mathrm{E}+00$ \\
\hline $1.400 \mathrm{E}+04$ & $5.520 \mathrm{E}+00$ & $1.73 \mathrm{E}-02$ & $1.489 \mathrm{E}+02$ & $6.72 \mathrm{E}+00$ & $6.613 \mathrm{E}+01$ & $3.511 \mathrm{E}+01$ & $9.93 \mathrm{E}-01$ \\
\hline $1.399 \mathrm{E}+04$ & $7.272 \mathrm{E}+00$ & $1.96 \mathrm{E}-02$ & $1.455 \mathrm{E}+02$ & $6.69 \mathrm{E}+00$ & $6.627 \mathrm{E}+01$ & $3.449 \mathrm{E}+01$ & $1.08 \mathrm{E}+00$ \\
\hline $1.405 \mathrm{E}+04$ & $9.095 \mathrm{E}+00$ & 2.22E-02 & $1.440 \mathrm{E}+02$ & $6.64 \mathrm{E}+00$ & $6.673 \mathrm{E}+01$ & $3.440 \mathrm{E}+01$ & $1.05 \mathrm{E}+00$ \\
\hline $1.400 \mathrm{E}+04$ & $1.095 \mathrm{E}+01$ & $2.50 \mathrm{E}-02$ & $1.400 \mathrm{E}+02$ & $6.70 \mathrm{E}+00$ & $6.680 \mathrm{E}+01$ & $3.353 \mathrm{E}+01$ & $9.13 \mathrm{E}-01$ \\
\hline $1.402 \mathrm{E}+04$ & $1.282 \mathrm{E}+01$ & $2.75 \mathrm{E}-02$ & $1.375 \mathrm{E}+02$ & $6.77 \mathrm{E}+00$ & $6.723 \mathrm{E}+01$ & $3.366 \mathrm{E}+01$ & $9.15 \mathrm{E}-01$ \\
\hline $1.402 \mathrm{E}+04$ & $1.469 \mathrm{E}+01$ & $3.02 \mathrm{E}-02$ & $1.354 \mathrm{E}+02$ & $6.80 \mathrm{E}+00$ & $6.761 \mathrm{E}+01$ & $3.355 \mathrm{E}+01$ & $9.09 \mathrm{E}-01$ \\
\hline $1.403 \mathrm{E}+04$ & $1.657 \mathrm{E}+01$ & $3.29 \mathrm{E}-02$ & $1.305 \mathrm{E}+02$ & $6.80 \mathrm{E}+00$ & $6.810 \mathrm{E}+01$ & $3.367 \mathrm{E}+01$ & $8.98 \mathrm{E}-01$ \\
\hline $1.402 \mathrm{E}+04$ & $1.844 \mathrm{E}+01$ & $3.56 \mathrm{E}-02$ & $1.185 \mathrm{E}+02$ & $6.75 \mathrm{E}+00$ & $6.850 \mathrm{E}+01$ & $3.260 \mathrm{E}+01$ & $8.97 \mathrm{E}-01$ \\
\hline $1.401 \mathrm{E}+04$ & $2.028 \mathrm{E}+01$ & $3.87 \mathrm{E}-02$ & $1.031 \mathrm{E}+02$ & $6.76 \mathrm{E}+00$ & $6.900 \mathrm{E}+01$ & $3.044 \mathrm{E}+01$ & $9.05 \mathrm{E}-01$ \\
\hline $1.399 \mathrm{E}+04$ & $2.216 \mathrm{E}+01$ & $4.22 \mathrm{E}-02$ & $8.641 \mathrm{E}+01$ & $6.74 \mathrm{E}+00$ & $6.948 \mathrm{E}+01$ & $2.761 \mathrm{E}+01$ & $8.87 \mathrm{E}-01$ \\
\hline$P_{\mathrm{e}}(\mathrm{W})$ & $\Delta P_{\mathrm{e}}(\mathrm{W})$ & $C_{\mathrm{T}}$ & $\Delta C_{\mathrm{T}}$ & $C_{\mathrm{P}}$ & $\Delta C_{\mathrm{P}}$ & $C_{\mathrm{Q}}$ & $\Delta C_{\mathrm{Q}}$ \\
\hline $6.554 \mathrm{E}+01$ & 7.69E-01 & $1.165 \mathrm{E}-01$ & $5.10 \mathrm{E}-03$ & $1.001 \mathrm{E}-01$ & $2.73 \mathrm{E}-03$ & $1.593 \mathrm{E}-02$ & 4.35E-04 \\
\hline $6.501 \mathrm{E}+01$ & $7.61 \mathrm{E}-01$ & $1.137 \mathrm{E}-01$ & $5.02 \mathrm{E}-03$ & $9.847 \mathrm{E}-02$ & $2.76 \mathrm{E}-03$ & $1.567 \mathrm{E}-02$ & $4.40 \mathrm{E}-04$ \\
\hline $6.274 \mathrm{E}+01$ & $7.28 \mathrm{E}-01$ & $1.118 \mathrm{E}-01$ & $5.05 \mathrm{E}-03$ & $9.615 \mathrm{E}-02$ & $2.72 \mathrm{E}-03$ & $1.530 \mathrm{E}-02$ & $4.33 \mathrm{E}-04$ \\
\hline $6.146 \mathrm{E}+01$ & $7.32 \mathrm{E}-01$ & $1.095 \mathrm{E}-01$ & $5.04 \mathrm{E}-03$ & $9.472 \mathrm{E}-02$ & $2.98 \mathrm{E}-03$ & $1.507 \mathrm{E}-02$ & 4.74E-04 \\
\hline $6.115 \mathrm{E}+01$ & $7.28 \mathrm{E}-01$ & $1.077 \mathrm{E}-01$ & $4.96 \mathrm{E}-03$ & $9.350 \mathrm{E}-02$ & $2.84 \mathrm{E}-03$ & $1.488 \mathrm{E}-02$ & $4.52 \mathrm{E}-04$ \\
\hline $5.978 \mathrm{E}+01$ & $6.91 \mathrm{E}-01$ & $1.053 \mathrm{E}-01$ & $5.04 \mathrm{E}-03$ & $9.196 \mathrm{E}-02$ & $2.50 \mathrm{E}-03$ & $1.464 \mathrm{E}-02$ & $3.99 \mathrm{E}-04$ \\
\hline $5.976 \mathrm{E}+01$ & $6.92 \mathrm{E}-01$ & $1.033 \mathrm{E}-01$ & $5.08 \mathrm{E}-03$ & $9.203 \mathrm{E}-02$ & $2.50 \mathrm{E}-03$ & $1.465 \mathrm{E}-02$ & $3.98 \mathrm{E}-04$ \\
\hline $5.964 \mathrm{E}+01$ & $6.84 \mathrm{E}-01$ & $1.018 \mathrm{E}-01$ & $5.11 \mathrm{E}-03$ & $9.184 \mathrm{E}-02$ & $2.49 \mathrm{E}-03$ & $1.462 \mathrm{E}-02$ & $3.96 \mathrm{E}-04$ \\
\hline $5.970 \mathrm{E}+01$ & $6.91 \mathrm{E}-01$ & $9.803 \mathrm{E}-02$ & $5.11 \mathrm{E}-03$ & $9.199 \mathrm{E}-02$ & $2.46 \mathrm{E}-03$ & $1.464 \mathrm{E}-02$ & $3.91 \mathrm{E}-04$ \\
\hline $5.747 \mathrm{E}+01$ & $6.61 \mathrm{E}-01$ & $8.925 \mathrm{E}-02$ & $5.09 \mathrm{E}-03$ & $8.938 \mathrm{E}-02$ & $2.46 \mathrm{E}-03$ & $1.423 \mathrm{E}-02$ & $3.92 \mathrm{E}-04$ \\
\hline $5.364 \mathrm{E}+01$ & $6.17 \mathrm{E}-01$ & $7.780 \mathrm{E}-02$ & $5.10 \mathrm{E}-03$ & $8.372 \mathrm{E}-02$ & $2.49 \mathrm{E}-03$ & $1.332 \mathrm{E}-02$ & $3.96 \mathrm{E}-04$ \\
\hline $4.837 \mathrm{E}+01$ & $5.64 \mathrm{E}-01$ & $6.547 \mathrm{E}-02$ & $5.11 \mathrm{E}-03$ & $7.631 \mathrm{E}-02$ & $2.45 \mathrm{E}-03$ & $1.215 \mathrm{E}-02$ & $3.90 \mathrm{E}-04$ \\
\hline$\rho\left(\mathrm{kg} / \mathrm{m}^{\wedge} 3\right)$ & $\eta_{\mathrm{T}}$ & $\Delta \eta_{\mathrm{T}}$ & $J$ & $\Delta J$ & $\eta_{\mathrm{P}}$ & $\Delta \eta_{\mathrm{P}}$ & $R e_{0.75}$ \\
\hline $1.162 \mathrm{E}+00$ & $5.591 \mathrm{E}-01$ & $1.66 \mathrm{E}-02$ & $7.607 \mathrm{E}-02$ & $5.31 \mathrm{E}-04$ & $8.853 \mathrm{E}-02$ & $4.61 \mathrm{E}-03$ & $3.478 \mathrm{E}+04$ \\
\hline $1.162 \mathrm{E}+00$ & $5.588 \mathrm{E}-01$ & $1.70 \mathrm{E}-02$ & $1.347 \mathrm{E}-01$ & $5.58 \mathrm{E}-04$ & $1.556 \mathrm{E}-01$ & $8.17 \mathrm{E}-03$ & $3.489 \mathrm{E}+04$ \\
\hline $1.162 \mathrm{E}+00$ & $5.597 \mathrm{E}-01$ & $1.71 \mathrm{E}-02$ & $1.974 \mathrm{E}-01$ & $6.20 \mathrm{E}-04$ & $2.295 \mathrm{E}-01$ & $1.22 \mathrm{E}-02$ & $3.484 \mathrm{E}+04$ \\
\hline $1.160 \mathrm{E}+00$ & $5.612 \mathrm{E}-01$ & $1.89 \mathrm{E}-02$ & $2.601 \mathrm{E}-01$ & $7.05 \mathrm{E}-04$ & $3.008 \mathrm{E}-01$ & $1.68 \mathrm{E}-02$ & $3.481 \mathrm{E}+04$ \\
\hline $1.159 \mathrm{E}+00$ & $5.626 \mathrm{E}-01$ & $1.84 \mathrm{E}-02$ & $3.241 \mathrm{E}-01$ & $7.96 \mathrm{E}-04$ & $3.733 \mathrm{E}-01$ & $2.06 \mathrm{E}-02$ & $3.502 \mathrm{E}+04$ \\
\hline $1.160 \mathrm{E}+00$ & $5.610 \mathrm{E}-01$ & $1.66 \mathrm{E}-02$ & 3.914E-01 & 8.99E-04 & 4.482E-01 & $2.47 \mathrm{E}-02$ & $3.510 \mathrm{E}+04$ \\
\hline $1.158 \mathrm{E}+00$ & $5.632 \mathrm{E}-01$ & $1.66 \mathrm{E}-02$ & $4.577 \mathrm{E}-01$ & $9.87 \mathrm{E}-04$ & $5.135 \mathrm{E}-01$ & $2.89 \mathrm{E}-02$ & $3.523 \mathrm{E}+04$ \\
\hline $1.157 \mathrm{E}+00$ & $5.626 \mathrm{E}-01$ & $1.66 \mathrm{E}-02$ & $5.245 \mathrm{E}-01$ & $1.09 \mathrm{E}-03$ & $5.813 \mathrm{E}-01$ & $3.32 \mathrm{E}-02$ & $3.539 \mathrm{E}+04$ \\
\hline $1.156 \mathrm{E}+00$ & $5.640 \mathrm{E}-01$ & $1.64 \mathrm{E}-02$ & $5.909 \mathrm{E}-01$ & $1.18 \mathrm{E}-03$ & $6.297 \mathrm{E}-01$ & $3.69 \mathrm{E}-02$ & $3.558 \mathrm{E}+04$ \\
\hline $1.156 \mathrm{E}+00$ & $5.672 \mathrm{E}-01$ & $1.69 \mathrm{E}-02$ & $6.584 \mathrm{E}-01$ & $1.28 \mathrm{E}-03$ & $6.574 \mathrm{E}-01$ & $4.16 \mathrm{E}-02$ & $3.578 \mathrm{E}+04$ \\
\hline $1.154 \mathrm{E}+00$ & $5.675 \mathrm{E}-01$ & $1.81 \mathrm{E}-02$ & $7.246 \mathrm{E}-01$ & $1.40 \mathrm{E}-03$ & $6.734 \mathrm{E}-01$ & $4.85 \mathrm{E}-02$ & $3.593 \mathrm{E}+04$ \\
\hline $1.153 \mathrm{E}+00$ & $5.708 \mathrm{E}-01$ & $1.95 \mathrm{E}-02$ & $7.928 \mathrm{E}-01$ & $1.53 \mathrm{E}-03$ & $6.802 \mathrm{E}-01$ & $5.74 \mathrm{E}-02$ & $3.614 \mathrm{E}+04$ \\
\hline
\end{tabular}


Table 126: APC 4.75 x 4.75 Carbon Fiber Dynamic Measured Values - 18006 RPM

\begin{tabular}{|c|c|c|c|c|c|c|c|}
\hline$n(\mathrm{RPM})$ & $Q(\mathrm{~g}-\mathrm{m})$ & $\Delta Q(\mathrm{~g}-\mathrm{m})$ & $V(\mathrm{~V})$ & $I(\mathrm{~A})$ & $T_{\text {atm }}\left({ }^{\circ} \mathrm{C}\right)$ & $P_{\text {atm }}(\mathrm{Pa})$ & $P_{\text {diff }}(\mathrm{Pa})$ \\
\hline $1.798 \mathrm{E}+04$ & $4.231 \mathrm{E}+00$ & $6.87 \mathrm{E}-02$ & $1.078 \mathrm{E}+01$ & $1.195 \mathrm{E}+01$ & $1.987 \mathrm{E}+01$ & $9.745 \mathrm{E}+04$ & $4.420 \mathrm{E}+00$ \\
\hline $1.797 \mathrm{E}+04$ & $4.144 \mathrm{E}+00$ & $7.29 \mathrm{E}-02$ & $1.079 \mathrm{E}+01$ & $1.169 \mathrm{E}+01$ & $1.987 \mathrm{E}+01$ & $9.744 \mathrm{E}+04$ & $1.905 \mathrm{E}+01$ \\
\hline $1.805 \mathrm{E}+04$ & $4.055 \mathrm{E}+00$ & $6.80 \mathrm{E}-02$ & $1.080 \mathrm{E}+01$ & $1.141 \mathrm{E}+01$ & $1.997 \mathrm{E}+01$ & $9.743 \mathrm{E}+04$ & $4.545 \mathrm{E}+01$ \\
\hline $1.799 \mathrm{E}+04$ & $3.974 \mathrm{E}+00$ & $6.82 \mathrm{E}-02$ & $1.081 \mathrm{E}+01$ & $1.105 \mathrm{E}+01$ & $2.020 \mathrm{E}+01$ & $9.744 \mathrm{E}+04$ & $8.405 \mathrm{E}+01$ \\
\hline $1.805 \mathrm{E}+04$ & $3.951 \mathrm{E}+00$ & $7.07 \mathrm{E}-02$ & $1.081 \mathrm{E}+01$ & $1.101 \mathrm{E}+01$ & $2.055 \mathrm{E}+01$ & $9.743 \mathrm{E}+04$ & $1.356 \mathrm{E}+02$ \\
\hline $1.801 \mathrm{E}+04$ & $3.989 \mathrm{E}+00$ & $7.23 \mathrm{E}-02$ & $1.080 \mathrm{E}+01$ & $1.108 \mathrm{E}+01$ & $2.101 \mathrm{E}+01$ & $9.743 \mathrm{E}+04$ & $1.998 \mathrm{E}+02$ \\
\hline $1.801 \mathrm{E}+04$ & $4.021 \mathrm{E}+00$ & $7.56 \mathrm{E}-02$ & $1.080 \mathrm{E}+01$ & $1.111 \mathrm{E}+01$ & $2.127 \mathrm{E}+01$ & $9.742 \mathrm{E}+04$ & $2.752 \mathrm{E}+02$ \\
\hline $1.801 \mathrm{E}+04$ & $3.869 \mathrm{E}+00$ & $7.11 \mathrm{E}-02$ & $1.082 \mathrm{E}+01$ & $1.055 \mathrm{E}+01$ & $2.140 \mathrm{E}+01$ & $9.742 \mathrm{E}+04$ & $3.622 \mathrm{E}+02$ \\
\hline $1.799 \mathrm{E}+04$ & $3.468 \mathrm{E}+00$ & $7.04 \mathrm{E}-02$ & $1.085 \mathrm{E}+01$ & $9.225 \mathrm{E}+00$ & $2.155 \mathrm{E}+01$ & $9.742 \mathrm{E}+04$ & $4.605 \mathrm{E}+02$ \\
\hline
\end{tabular}


Table 127: APC 4.75 x 4.75 Carbon Fiber Dynamic Calculated Values - 18006 RPM

\begin{tabular}{|c|c|c|c|c|c|c|c|}
\hline$n(\mathrm{RPM})$ & $V_{\infty}^{\prime}(\mathrm{m} / \mathrm{s})$ & $\Delta V_{\infty}^{\prime}(\mathrm{m} / \mathrm{s})$ & $T^{\prime}(\mathrm{g})$ & $\Delta T^{\prime}(\mathrm{g})$ & $V_{\mathrm{t}}(\mathrm{m} / \mathrm{s})$ & $P_{\mathrm{P}}(\mathrm{W})$ & $\Delta P_{\mathrm{P}}(\mathrm{W})$ \\
\hline $1.798 \mathrm{E}+04$ & $2.613 \mathrm{E}+00$ & $1.82 \mathrm{E}-02$ & $2.625 \mathrm{E}+02$ & $6.92 \mathrm{E}+00$ & $8.466 \mathrm{E}+01$ & $7.810 \mathrm{E}+01$ & $1.27 \mathrm{E}+00$ \\
\hline $1.797 \mathrm{E}+04$ & $5.593 \mathrm{E}+00$ & $1.95 \mathrm{E}-02$ & $2.524 \mathrm{E}+02$ & $6.72 \mathrm{E}+00$ & $8.478 \mathrm{E}+01$ & $7.648 \mathrm{E}+01$ & $1.35 \mathrm{E}+00$ \\
\hline $1.805 \mathrm{E}+04$ & $8.727 \mathrm{E}+00$ & $2.43 \mathrm{E}-02$ & $2.488 \mathrm{E}+02$ & $6.63 \mathrm{E}+00$ & $8.541 \mathrm{E}+01$ & $7.516 \mathrm{E}+01$ & $1.26 \mathrm{E}+00$ \\
\hline $1.799 \mathrm{E}+04$ & $1.193 \mathrm{E}+01$ & 2.91E-02 & $2.428 \mathrm{E}+02$ & $6.63 \mathrm{E}+00$ & $8.553 \mathrm{E}+01$ & $7.343 \mathrm{E}+01$ & $1.26 \mathrm{E}+00$ \\
\hline $1.805 \mathrm{E}+04$ & $1.520 \mathrm{E}+01$ & 3.32E-02 & $2.454 \mathrm{E}+02$ & $6.64 \mathrm{E}+00$ & $8.630 \mathrm{E}+01$ & $7.323 \mathrm{E}+01$ & $1.31 \mathrm{E}+00$ \\
\hline $1.801 \mathrm{E}+04$ & $1.851 \mathrm{E}+01$ & $3.75 \mathrm{E}-02$ & $2.476 \mathrm{E}+02$ & $6.72 \mathrm{E}+00$ & $8.677 \mathrm{E}+01$ & $7.378 \mathrm{E}+01$ & $1.34 \mathrm{E}+00$ \\
\hline $1.801 \mathrm{E}+04$ & $2.176 \mathrm{E}+01$ & $4.23 \mathrm{E}-02$ & $2.350 \mathrm{E}+02$ & $6.75 \mathrm{E}+00$ & $8.752 \mathrm{E}+01$ & $7.438 \mathrm{E}+01$ & $1.40 \mathrm{E}+00$ \\
\hline $1.801 \mathrm{E}+04$ & $2.500 \mathrm{E}+01$ & 4.81E-02 & $2.055 \mathrm{E}+02$ & $6.71 \mathrm{E}+00$ & $8.836 \mathrm{E}+01$ & $7.155 \mathrm{E}+01$ & $1.32 \mathrm{E}+00$ \\
\hline $1.799 \mathrm{E}+04$ & $2.822 \mathrm{E}+01$ & $5.34 \mathrm{E}-02$ & $1.641 \mathrm{E}+02$ & $6.66 \mathrm{E}+00$ & $8.925 \mathrm{E}+01$ & $6.406 \mathrm{E}+01$ & $1.30 \mathrm{E}+00$ \\
\hline$P_{\mathrm{e}}(\mathrm{W})$ & $\Delta P_{\mathrm{e}}(\mathrm{W})$ & $C_{\mathrm{T}}$ & $\Delta C_{\mathrm{T}}$ & $C_{\mathrm{P}}$ & $\Delta C_{\mathrm{P}}$ & $C_{\mathrm{Q}}$ & $\Delta C_{\mathrm{Q}}$ \\
\hline $1.288 \mathrm{E}+02$ & $1.41 \mathrm{E}+00$ & $1.199 \mathrm{E}-01$ & $3.16 \mathrm{E}-03$ & $1.013 \mathrm{E}-01$ & $1.65 \mathrm{E}-03$ & $1.612 \mathrm{E}-02$ & $2.62 \mathrm{E}-04$ \\
\hline $1.261 \mathrm{E}+02$ & $1.41 \mathrm{E}+00$ & $1.153 \mathrm{E}-01$ & $3.07 \mathrm{E}-03$ & $9.926 \mathrm{E}-02$ & $1.75 \mathrm{E}-03$ & $1.580 \mathrm{E}-02$ & $2.78 \mathrm{E}-04$ \\
\hline $1.232 \mathrm{E}+02$ & $1.35 \mathrm{E}+00$ & $1.128 \mathrm{E}-01$ & $3.01 \mathrm{E}-03$ & $9.634 \mathrm{E}-02$ & $1.62 \mathrm{E}-03$ & $1.533 \mathrm{E}-02$ & $2.57 \mathrm{E}-04$ \\
\hline $1.195 \mathrm{E}+02$ & $1.32 \mathrm{E}+00$ & $1.109 \mathrm{E}-01$ & $3.03 \mathrm{E}-03$ & $9.510 \mathrm{E}-02$ & $1.63 \mathrm{E}-03$ & $1.514 \mathrm{E}-02$ & $2.60 \mathrm{E}-04$ \\
\hline $1.190 \mathrm{E}+02$ & $1.31 \mathrm{E}+00$ & $1.115 \mathrm{E}-01$ & $3.02 \mathrm{E}-03$ & $9.408 \mathrm{E}-02$ & $1.68 \mathrm{E}-03$ & $1.497 \mathrm{E}-02$ & $2.68 \mathrm{E}-04$ \\
\hline $1.197 \mathrm{E}+02$ & $1.33 \mathrm{E}+00$ & $1.132 \mathrm{E}-01$ & $3.07 \mathrm{E}-03$ & $9.553 \mathrm{E}-02$ & $1.73 \mathrm{E}-03$ & $1.520 \mathrm{E}-02$ & $2.76 \mathrm{E}-04$ \\
\hline $1.201 \mathrm{E}+02$ & $1.34 \mathrm{E}+00$ & $1.075 \mathrm{E}-01$ & $3.09 \mathrm{E}-03$ & $9.639 \mathrm{E}-02$ & $1.81 \mathrm{E}-03$ & $1.534 \mathrm{E}-02$ & $2.89 \mathrm{E}-04$ \\
\hline $1.142 \mathrm{E}+02$ & $1.26 \mathrm{E}+00$ & 9.409E-02 & $3.07 \mathrm{E}-03$ & $9.285 \mathrm{E}-02$ & $1.71 \mathrm{E}-03$ & $1.478 \mathrm{E}-02$ & $2.72 \mathrm{E}-04$ \\
\hline $1.001 \mathrm{E}+02$ & $1.12 \mathrm{E}+00$ & $7.531 \mathrm{E}-02$ & $3.06 \mathrm{E}-03$ & $8.342 \mathrm{E}-02$ & $1.70 \mathrm{E}-03$ & $1.328 \mathrm{E}-02$ & $2.70 \mathrm{E}-04$ \\
\hline$\rho\left(\mathrm{kg} / \mathrm{m}^{\wedge} 3\right)$ & $\eta_{\mathrm{T}}$ & $\Delta \eta_{\mathrm{T}}$ & $J$ & $\Delta J$ & $\eta_{\mathrm{P}}$ & $\Delta \eta_{\mathrm{P}}$ & $R e_{0.75}$ \\
\hline $1.159 \mathrm{E}+00$ & $6.062 \mathrm{E}-01$ & $1.19 \mathrm{E}-02$ & $7.276 \mathrm{E}-02$ & $5.07 \mathrm{E}-04$ & $8.613 \mathrm{E}-02$ & $2.74 \mathrm{E}-03$ & $4.439 \mathrm{E}+04$ \\
\hline $1.158 \mathrm{E}+00$ & $6.064 \mathrm{E}-01$ & $1.26 \mathrm{E}-02$ & $1.558 \mathrm{E}-01$ & $5.45 \mathrm{E}-04$ & $1.810 \mathrm{E}-01$ & $5.82 \mathrm{E}-03$ & $4.446 \mathrm{E}+04$ \\
\hline $1.158 \mathrm{E}+00$ & $6.099 \mathrm{E}-01$ & $1.22 \mathrm{E}-02$ & $2.420 \mathrm{E}-01$ & $6.77 \mathrm{E}-04$ & $2.833 \mathrm{E}-01$ & $8.96 \mathrm{E}-03$ & 4.475E+04 \\
\hline $1.157 \mathrm{E}+00$ & $6.147 \mathrm{E}-01$ & $1.25 \mathrm{E}-02$ & $3.319 \mathrm{E}-01$ & $8.13 \mathrm{E}-04$ & $3.869 \mathrm{E}-01$ & $1.25 \mathrm{E}-02$ & $4.476 \mathrm{E}+04$ \\
\hline $1.156 \mathrm{E}+00$ & $6.153 \mathrm{E}-01$ & $1.29 \mathrm{E}-02$ & 4.217E-01 & $9.25 \mathrm{E}-04$ & 4.997E-01 & $1.62 \mathrm{E}-02$ & $4.507 \mathrm{E}+04$ \\
\hline $1.154 \mathrm{E}+00$ & $6.161 \mathrm{E}-01$ & $1.31 \mathrm{E}-02$ & 5.143E-01 & $1.05 \mathrm{E}-03$ & $6.092 \mathrm{E}-01$ & $1.99 \mathrm{E}-02$ & $4.518 \mathrm{E}+04$ \\
\hline $1.153 \mathrm{E}+00$ & $6.195 \mathrm{E}-01$ & $1.35 \mathrm{E}-02$ & $6.047 \mathrm{E}-01$ & $1.18 \mathrm{E}-03$ & $6.742 \mathrm{E}-01$ & $2.32 \mathrm{E}-02$ & $4.550 \mathrm{E}+04$ \\
\hline $1.152 \mathrm{E}+00$ & $6.266 \mathrm{E}-01$ & $1.34 \mathrm{E}-02$ & $6.950 \mathrm{E}-01$ & $1.34 \mathrm{E}-03$ & $7.042 \mathrm{E}-01$ & $2.64 \mathrm{E}-02$ & $4.590 \mathrm{E}+04$ \\
\hline $1.152 \mathrm{E}+00$ & $6.398 \mathrm{E}-01$ & $1.48 \mathrm{E}-02$ & 7.854E-01 & $1.50 \mathrm{E}-03$ & $7.091 \mathrm{E}-01$ & $3.22 \mathrm{E}-02$ & $4.632 \mathrm{E}+04$ \\
\hline
\end{tabular}


Table 128: APC 4.75 x 4.75 Carbon Fiber Dynamic Measured Values - 22071 RPM

\begin{tabular}{|c|c|c|c|c|c|c|c|}
\hline$n(\mathrm{RPM})$ & $Q(\mathrm{~g}-\mathrm{m})$ & $\Delta Q(\mathrm{~g}-\mathrm{m})$ & $V(\mathrm{~V})$ & $I(\mathrm{~A})$ & $T_{\text {atm }}\left({ }^{\circ} \mathrm{C}\right)$ & $P_{\text {atm }}(\mathrm{Pa})$ & $P_{\text {diff }}(\mathrm{Pa})$ \\
\hline $2.173 \mathrm{E}+04$ & $6.299 \mathrm{E}+00$ & $1.07 \mathrm{E}-01$ & $1.050 \mathrm{E}+01$ & $2.240 \mathrm{E}+01$ & $1.853 \mathrm{E}+01$ & $9.741 \mathrm{E}+04$ & $7.079 \mathrm{E}+00$ \\
\hline $2.208 \mathrm{E}+04$ & $6.456 \mathrm{E}+00$ & $7.71 \mathrm{E}-02$ & $1.046 \mathrm{E}+01$ & $2.363 \mathrm{E}+01$ & $1.900 \mathrm{E}+01$ & $9.740 \mathrm{E}+04$ & $2.690 \mathrm{E}+01$ \\
\hline $2.190 \mathrm{E}+04$ & $6.136 \mathrm{E}+00$ & $7.52 \mathrm{E}-02$ & $1.051 \mathrm{E}+01$ & $2.182 \mathrm{E}+01$ & $1.922 \mathrm{E}+01$ & $9.741 \mathrm{E}+04$ & $6.192 \mathrm{E}+01$ \\
\hline $2.204 \mathrm{E}+04$ & $6.109 \mathrm{E}+00$ & $7.51 \mathrm{E}-02$ & $1.052 \mathrm{E}+01$ & $2.166 \mathrm{E}+01$ & $1.979 \mathrm{E}+01$ & $9.741 \mathrm{E}+04$ & $1.137 \mathrm{E}+02$ \\
\hline $2.216 \mathrm{E}+04$ & $6.111 \mathrm{E}+00$ & $9.43 \mathrm{E}-02$ & $1.052 \mathrm{E}+01$ & $2.165 \mathrm{E}+01$ & $2.046 \mathrm{E}+01$ & $9.741 \mathrm{E}+04$ & $1.821 \mathrm{E}+02$ \\
\hline $2.213 \mathrm{E}+04$ & $6.164 \mathrm{E}+00$ & $1.01 \mathrm{E}-01$ & $1.051 \mathrm{E}+01$ & $2.172 \mathrm{E}+01$ & $2.094 \mathrm{E}+01$ & $9.741 \mathrm{E}+04$ & $2.660 \mathrm{E}+02$ \\
\hline $2.212 \mathrm{E}+04$ & $6.184 \mathrm{E}+00$ & $1.02 \mathrm{E}-01$ & $1.051 \mathrm{E}+01$ & $2.186 \mathrm{E}+01$ & $2.119 \mathrm{E}+01$ & $9.739 \mathrm{E}+04$ & $3.654 \mathrm{E}+02$ \\
\hline $2.223 \mathrm{E}+04$ & $6.231 \mathrm{E}+00$ & $1.04 \mathrm{E}-01$ & $1.051 \mathrm{E}+01$ & $2.180 \mathrm{E}+01$ & $2.134 \mathrm{E}+01$ & $9.739 \mathrm{E}+04$ & $4.789 \mathrm{E}+02$ \\
\hline $2.224 \mathrm{E}+04$ & $6.003 \mathrm{E}+00$ & $8.25 \mathrm{E}-02$ & $1.055 \mathrm{E}+01$ & $2.036 \mathrm{E}+01$ & $2.155 \mathrm{E}+01$ & $9.739 \mathrm{E}+04$ & $6.074 \mathrm{E}+02$ \\
\hline
\end{tabular}


Table 129: APC 4.75 x 4.75 Carbon Fiber Dynamic Calculated Values - 22071 RPM

\begin{tabular}{|c|c|c|c|c|c|c|c|}
\hline$n(\mathrm{RPM})$ & $V_{\infty}^{\prime}(\mathrm{m} / \mathrm{s})$ & $\Delta V_{\infty}^{\prime}(\mathrm{m} / \mathrm{s})$ & $T^{\prime}(\mathrm{g})$ & $\Delta T^{\prime}(\mathrm{g})$ & $V_{\mathrm{t}}(\mathrm{m} / \mathrm{s})$ & $P_{\mathrm{P}}(\mathrm{W})$ & $\Delta P_{\mathrm{P}}(\mathrm{W})$ \\
\hline $2.173 \mathrm{E}+04$ & $3.305 \mathrm{E}+00$ & $2.18 \mathrm{E}-02$ & $3.979 \mathrm{E}+02$ & $7.51 \mathrm{E}+00$ & $1.023 \mathrm{E}+02$ & $1.405 \mathrm{E}+02$ & $2.38 \mathrm{E}+00$ \\
\hline $2.208 \mathrm{E}+04$ & $6.630 \mathrm{E}+00$ & $2.36 \mathrm{E}-02$ & $3.891 \mathrm{E}+02$ & $6.99 \mathrm{E}+00$ & $1.042 \mathrm{E}+02$ & $1.464 \mathrm{E}+02$ & $1.75 \mathrm{E}+00$ \\
\hline $2.190 \mathrm{E}+04$ & $1.016 \mathrm{E}+01$ & $2.90 \mathrm{E}-02$ & $3.756 \mathrm{E}+02$ & $6.85 \mathrm{E}+00$ & $1.036 \mathrm{E}+02$ & $1.380 \mathrm{E}+02$ & $1.69 \mathrm{E}+00$ \\
\hline $2.204 \mathrm{E}+04$ & $1.386 \mathrm{E}+01$ & $3.44 \mathrm{E}-02$ & $3.749 \mathrm{E}+02$ & $6.79 \mathrm{E}+00$ & $1.047 \mathrm{E}+02$ & $1.383 \mathrm{E}+02$ & $1.70 \mathrm{E}+00$ \\
\hline $2.216 \mathrm{E}+04$ & $1.760 \mathrm{E}+01$ & $3.79 \mathrm{E}-02$ & $3.856 \mathrm{E}+02$ & $6.94 \mathrm{E}+00$ & $1.058 \mathrm{E}+02$ & $1.391 \mathrm{E}+02$ & $2.15 \mathrm{E}+00$ \\
\hline $2.213 \mathrm{E}+04$ & $2.134 \mathrm{E}+01$ & $4.30 \mathrm{E}-02$ & $3.875 \mathrm{E}+02$ & $6.96 \mathrm{E}+00$ & $1.063 \mathrm{E}+02$ & $1.401 \mathrm{E}+02$ & $2.31 \mathrm{E}+00$ \\
\hline $2.212 \mathrm{E}+04$ & $2.505 \mathrm{E}+01$ & $4.82 \mathrm{E}-02$ & $3.740 \mathrm{E}+02$ & $7.01 \mathrm{E}+00$ & $1.071 \mathrm{E}+02$ & $1.405 \mathrm{E}+02$ & $2.31 \mathrm{E}+00$ \\
\hline $2.223 \mathrm{E}+04$ & $2.872 \mathrm{E}+01$ & $5.50 \mathrm{E}-02$ & $3.528 \mathrm{E}+02$ & $7.00 \mathrm{E}+00$ & $1.085 \mathrm{E}+02$ & $1.423 \mathrm{E}+02$ & $2.38 \mathrm{E}+00$ \\
\hline $2.224 \mathrm{E}+04$ & $3.239 \mathrm{E}+01$ & $6.06 \mathrm{E}-02$ & $3.139 \mathrm{E}+02$ & $6.95 \mathrm{E}+00$ & $1.096 \mathrm{E}+02$ & $1.371 \mathrm{E}+02$ & $1.88 \mathrm{E}+00$ \\
\hline$P_{\mathrm{e}}(\mathrm{W})$ & $\Delta P_{\mathrm{e}}(\mathrm{W})$ & $C_{\mathrm{T}}$ & $\Delta C_{\mathrm{T}}$ & $C_{\mathrm{P}}$ & $\Delta C_{\mathrm{P}}$ & $C_{\mathrm{Q}}$ & $\Delta C_{\mathrm{Q}}$ \\
\hline $2.353 \mathrm{E}+02$ & $2.95 \mathrm{E}+00$ & $1.239 \mathrm{E}-01$ & $2.34 \mathrm{E}-03$ & $1.028 \mathrm{E}-01$ & $1.75 \mathrm{E}-03$ & $1.636 \mathrm{E}-02$ & $2.78 \mathrm{E}-04$ \\
\hline $2.472 \mathrm{E}+02$ & $2.70 \mathrm{E}+00$ & $1.175 \mathrm{E}-01$ & $2.11 \mathrm{E}-03$ & $1.021 \mathrm{E}-01$ & $1.22 \mathrm{E}-03$ & $1.626 \mathrm{E}-02$ & $1.95 \mathrm{E}-04$ \\
\hline $2.294 \mathrm{E}+02$ & $2.40 \mathrm{E}+00$ & $1.153 \mathrm{E}-01$ & $2.10 \mathrm{E}-03$ & $9.876 \mathrm{E}-02$ & $1.21 \mathrm{E}-03$ & $1.572 \mathrm{E}-02$ & $1.93 \mathrm{E}-04$ \\
\hline $2.278 \mathrm{E}+02$ & $2.37 \mathrm{E}+00$ & 1.139E-01 & $2.07 \mathrm{E}-03$ & 9.729E-02 & $1.20 \mathrm{E}-03$ & $1.548 \mathrm{E}-02$ & $1.91 \mathrm{E}-04$ \\
\hline $2.277 \mathrm{E}+02$ & $2.79 \mathrm{E}+00$ & $1.162 \mathrm{E}-01$ & $2.09 \mathrm{E}-03$ & $9.650 \mathrm{E}-02$ & $1.50 \mathrm{E}-03$ & $1.536 \mathrm{E}-02$ & $2.38 \mathrm{E}-04$ \\
\hline $2.283 \mathrm{E}+02$ & $2.78 \mathrm{E}+00$ & $1.173 \mathrm{E}-01$ & $2.11 \mathrm{E}-03$ & $9.777 \mathrm{E}-02$ & $1.62 \mathrm{E}-03$ & $1.556 \mathrm{E}-02$ & $2.57 \mathrm{E}-04$ \\
\hline $2.297 \mathrm{E}+02$ & $2.86 \mathrm{E}+00$ & $1.134 \mathrm{E}-01$ & $2.13 \mathrm{E}-03$ & $9.827 \mathrm{E}-02$ & $1.62 \mathrm{E}-03$ & $1.564 \mathrm{E}-02$ & $2.58 \mathrm{E}-04$ \\
\hline $2.290 \mathrm{E}+02$ & $2.87 \mathrm{E}+00$ & $1.059 \mathrm{E}-01$ & $2.11 \mathrm{E}-03$ & $9.807 \mathrm{E}-02$ & $1.65 \mathrm{E}-03$ & $1.561 \mathrm{E}-02$ & $2.62 \mathrm{E}-04$ \\
\hline $2.148 \mathrm{E}+02$ & $2.24 \mathrm{E}+00$ & $9.430 \mathrm{E}-02$ & $2.09 \mathrm{E}-03$ & $9.453 \mathrm{E}-02$ & $1.30 \mathrm{E}-03$ & $1.504 \mathrm{E}-02$ & $2.07 \mathrm{E}-04$ \\
\hline$\rho\left(\mathrm{kg} / \mathrm{m}^{\wedge} 3\right)$ & $\eta_{\mathrm{T}}$ & $\Delta \eta_{\mathrm{T}}$ & $J$ & $\Delta J$ & $\eta_{\mathrm{P}}$ & $\Delta \eta_{\mathrm{P}}$ & $R e_{0.75}$ \\
\hline $1.163 \mathrm{E}+00$ & $5.972 \mathrm{E}-01$ & $1.26 \mathrm{E}-02$ & $7.615 \mathrm{E}-02$ & $5.04 \mathrm{E}-04$ & $9.176 \mathrm{E}-02$ & $2.41 \mathrm{E}-03$ & $5.407 \mathrm{E}+04$ \\
\hline $1.161 \mathrm{E}+00$ & 5.924E-01 & $9.59 \mathrm{E}-03$ & $1.503 \mathrm{E}-01$ & $5.36 \mathrm{E}-04$ & $1.728 \mathrm{E}-01$ & $3.79 \mathrm{E}-03$ & $5.489 \mathrm{E}+04$ \\
\hline $1.161 \mathrm{E}+00$ & $6.018 \mathrm{E}-01$ & $9.69 \mathrm{E}-03$ & 2.323E-01 & $6.65 \mathrm{E}-04$ & 2.712E-01 & $6.01 \mathrm{E}-03$ & $5.452 \mathrm{E}+04$ \\
\hline $1.158 \mathrm{E}+00$ & $6.070 \mathrm{E}-01$ & $9.78 \mathrm{E}-03$ & $3.147 \mathrm{E}-01$ & $7.83 \mathrm{E}-04$ & $3.684 \mathrm{E}-01$ & $8.13 \mathrm{E}-03$ & $5.490 \mathrm{E}+04$ \\
\hline $1.156 \mathrm{E}+00$ & $6.109 \mathrm{E}-01$ & $1.20 \mathrm{E}-02$ & $3.976 \mathrm{E}-01$ & $8.76 \mathrm{E}-04$ & $4.786 \mathrm{E}-01$ & $1.14 \mathrm{E}-02$ & $5.526 \mathrm{E}+04$ \\
\hline $1.154 \mathrm{E}+00$ & $6.136 \mathrm{E}-01$ & $1.26 \mathrm{E}-02$ & 4.826E-01 & 9.94E-04 & $5.788 \mathrm{E}-01$ & $1.42 \mathrm{E}-02$ & $5.538 \mathrm{E}+04$ \\
\hline $1.153 \mathrm{E}+00$ & $6.114 \mathrm{E}-01$ & $1.26 \mathrm{E}-02$ & $5.669 \mathrm{E}-01$ & $1.12 \mathrm{E}-03$ & $6.542 \mathrm{E}-01$ & $1.64 \mathrm{E}-02$ & $5.569 \mathrm{E}+04$ \\
\hline $1.152 \mathrm{E}+00$ & $6.212 \mathrm{E}-01$ & $1.30 \mathrm{E}-02$ & $6.467 \mathrm{E}-01$ & $1.27 \mathrm{E}-03$ & $6.986 \mathrm{E}-01$ & $1.82 \mathrm{E}-02$ & $5.638 \mathrm{E}+04$ \\
\hline $1.151 \mathrm{E}+00$ & $6.383 \mathrm{E}-01$ & $1.10 \mathrm{E}-02$ & 7.291E-01 & $1.37 \mathrm{E}-03$ & $7.273 \mathrm{E}-01$ & $1.90 \mathrm{E}-02$ & $5.685 \mathrm{E}+04$ \\
\hline
\end{tabular}




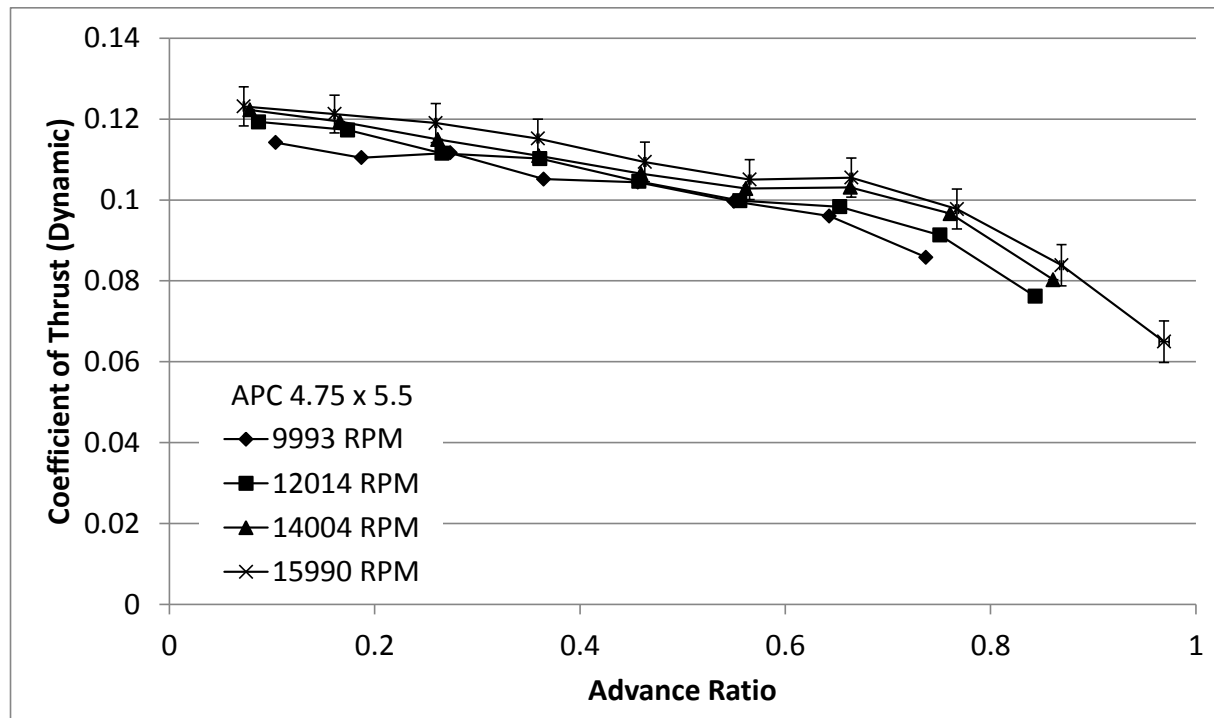

(a)

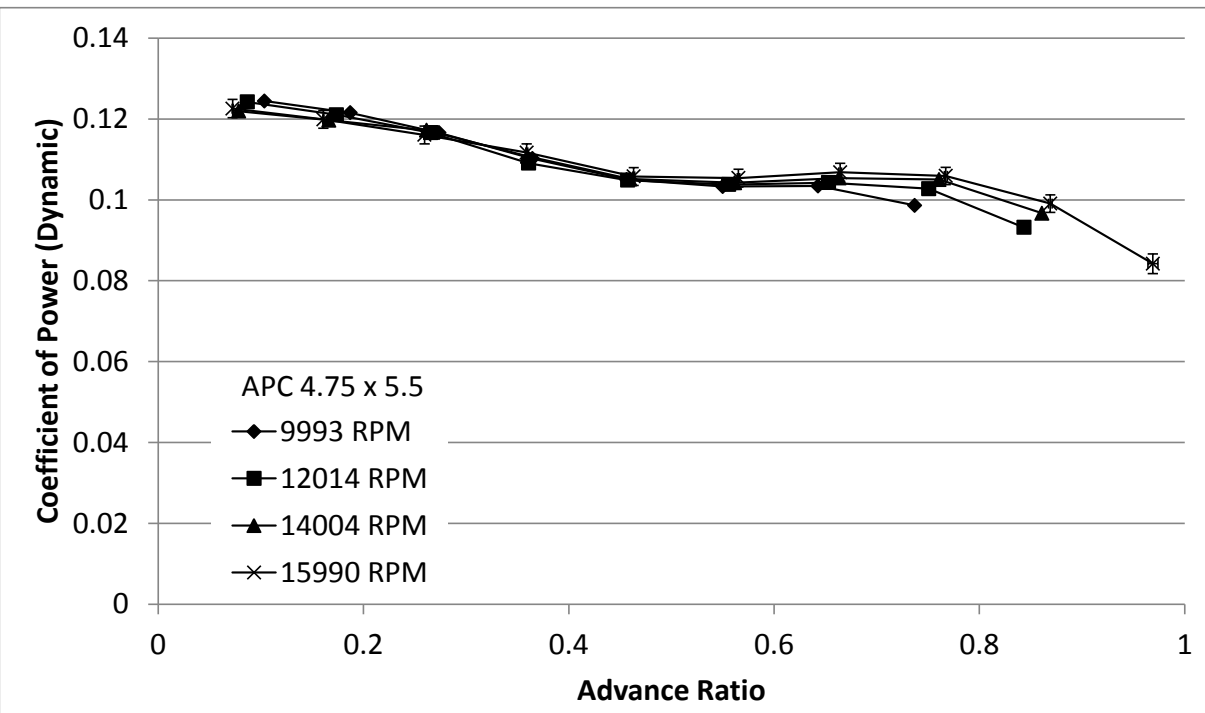

(b)

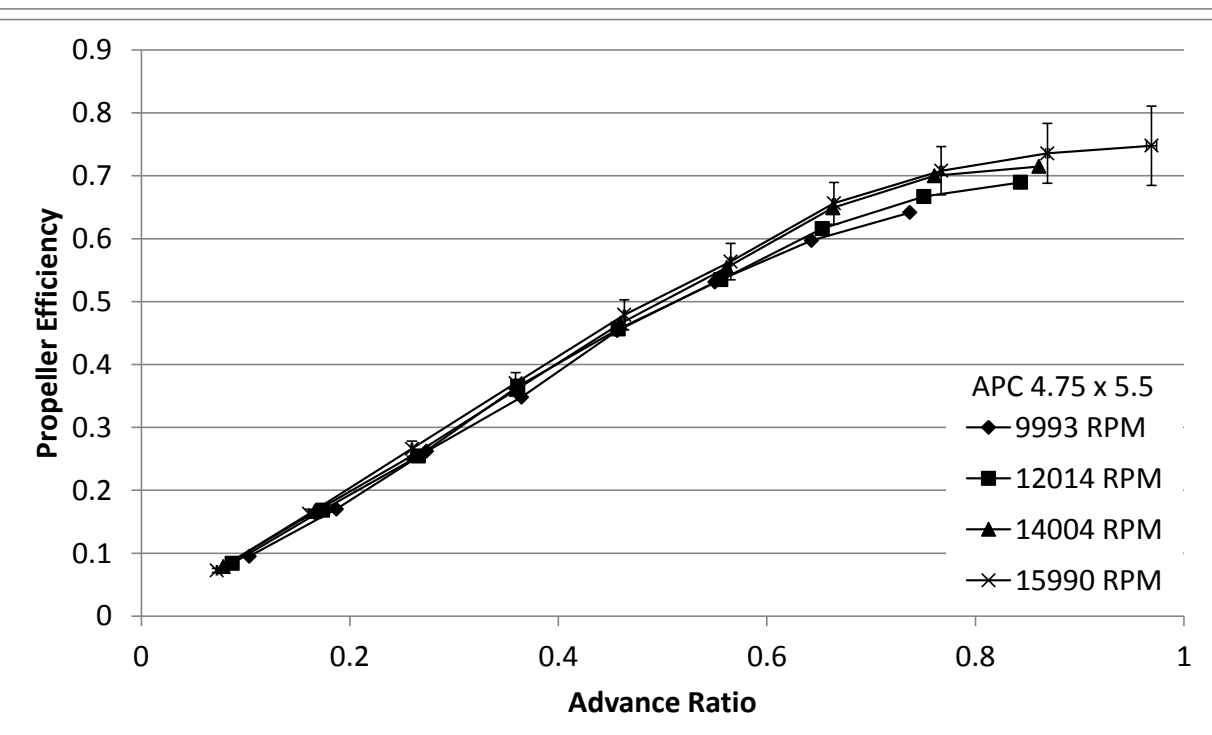

(c) 


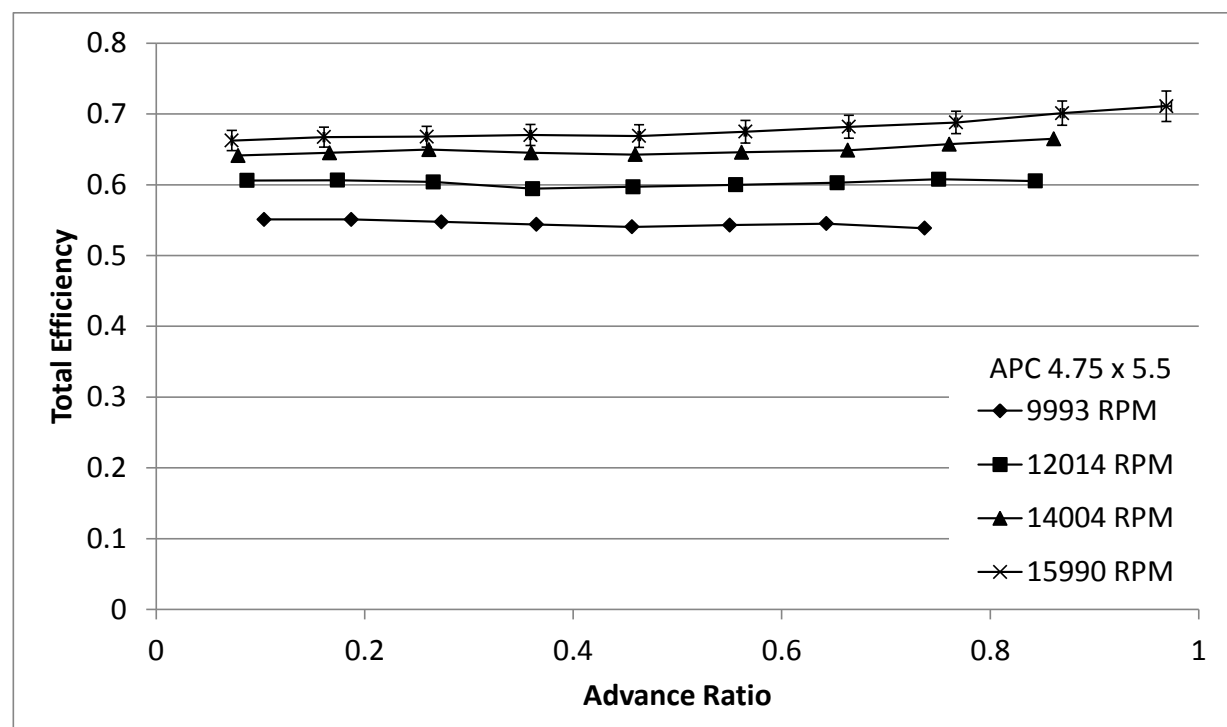

(d)

Figure 85: APC 4.75 x 5.5 Dynamic Test Results: (a) Coefficient of Thrust, (b)

Coefficient of Power, (c) Propeller Efficiency, (d) Total Efficiency.

Table 130: APC 4.75 x 5.5 Dynamic Measured Values - 9993 RPM

\begin{tabular}{|c|c|c|c|c|c|c|c|}
\hline$n(\mathrm{RPM})$ & $Q(\mathrm{~g}-\mathrm{m})$ & $\Delta Q(\mathrm{~g}-\mathrm{m})$ & $V(\mathrm{~V})$ & $I(\mathrm{~A})$ & $T_{\mathrm{atm}}\left({ }^{\circ} \mathrm{C}\right)$ & $P_{\text {atm }}(\mathrm{Pa})$ & $P_{\text {diff }}(\mathrm{Pa})$ \\
\hline $9.981 \mathrm{E}+03$ & $1.616 \mathrm{E}+00$ & $6.06 \mathrm{E}-02$ & $1.104 \mathrm{E}+01$ & $2.724 \mathrm{E}+00$ & $2.268 \mathrm{E}+01$ & $9.905 \mathrm{E}+04$ & $2.688 \mathrm{E}+00$ \\
\hline $9.986 \mathrm{E}+03$ & $1.581 \mathrm{E}+00$ & $6.20 \mathrm{E}-02$ & $1.104 \mathrm{E}+01$ & $2.666 \mathrm{E}+00$ & $2.257 \mathrm{E}+01$ & $9.906 \mathrm{E}+04$ & $8.474 \mathrm{E}+00$ \\
\hline $1.002 \mathrm{E}+04$ & $1.526 \mathrm{E}+00$ & $6.09 \mathrm{E}-02$ & $1.104 \mathrm{E}+01$ & $2.597 \mathrm{E}+00$ & $2.269 \mathrm{E}+01$ & $9.906 \mathrm{E}+04$ & $1.797 \mathrm{E}+01$ \\
\hline $9.996 \mathrm{E}+03$ & $1.436 \mathrm{E}+00$ & $6.09 \mathrm{E}-02$ & $1.105 \mathrm{E}+01$ & $2.453 \mathrm{E}+00$ & $2.274 \mathrm{E}+01$ & $9.905 \mathrm{E}+04$ & $3.151 \mathrm{E}+01$ \\
\hline $9.994 \mathrm{E}+03$ & $1.367 \mathrm{E}+00$ & $6.08 \mathrm{E}-02$ & $1.105 \mathrm{E}+01$ & $2.350 \mathrm{E}+00$ & $2.285 \mathrm{E}+01$ & $9.906 \mathrm{E}+04$ & $4.908 \mathrm{E}+01$ \\
\hline $9.991 \mathrm{E}+03$ & $1.343 \mathrm{E}+00$ & $6.06 \mathrm{E}-02$ & $1.105 \mathrm{E}+01$ & $2.297 \mathrm{E}+00$ & $2.294 \mathrm{E}+01$ & $9.906 \mathrm{E}+04$ & $7.100 \mathrm{E}+01$ \\
\hline $9.996 \mathrm{E}+03$ & $1.346 \mathrm{E}+00$ & $6.01 \mathrm{E}-02$ & $1.105 \mathrm{E}+01$ & $2.294 \mathrm{E}+00$ & $2.299 \mathrm{E}+01$ & $9.906 \mathrm{E}+04$ & $9.680 \mathrm{E}+01$ \\
\hline $9.985 \mathrm{E}+03$ & $1.281 \mathrm{E}+00$ & $6.17 \mathrm{E}-02$ & $1.105 \mathrm{E}+01$ & $2.206 \mathrm{E}+00$ & $2.305 \mathrm{E}+01$ & $9.905 \mathrm{E}+04$ & $1.267 \mathrm{E}+02$ \\
\hline
\end{tabular}


Table 131: APC 4.75 x 5.5 Dynamic Calculated Values - 9993 RPM

\begin{tabular}{|c|c|c|c|c|c|c|c|}
\hline$n(\mathrm{RPM})$ & $V_{\infty}^{\prime}(\mathrm{m} / \mathrm{s})$ & $\Delta V_{\infty}^{\prime}(\mathrm{m} / \mathrm{s})$ & $T^{\prime}(\mathrm{g})$ & $\Delta T^{\prime}(\mathrm{g})$ & $V_{\mathrm{t}}(\mathrm{m} / \mathrm{s})$ & $P_{\mathrm{P}}(\mathrm{W})$ & $\Delta P_{\mathrm{P}}(\mathrm{W})$ \\
\hline $9.981 \mathrm{E}+03$ & $2.067 \mathrm{E}+00$ & $1.30 \mathrm{E}-02$ & $7.773 \mathrm{E}+01$ & $9.07 \mathrm{E}+00$ & $4.705 \mathrm{E}+01$ & $1.657 \mathrm{E}+01$ & $6.21 \mathrm{E}-01$ \\
\hline $9.986 \mathrm{E}+03$ & $3.735 \mathrm{E}+00$ & $1.37 \mathrm{E}-02$ & $7.529 \mathrm{E}+01$ & $9.15 \mathrm{E}+00$ & $4.717 \mathrm{E}+01$ & $1.622 \mathrm{E}+01$ & $6.35 \mathrm{E}-01$ \\
\hline $1.002 \mathrm{E}+04$ & $5.480 \mathrm{E}+00$ & $1.57 \mathrm{E}-02$ & $7.656 \mathrm{E}+01$ & $9.13 \mathrm{E}+00$ & $4.749 \mathrm{E}+01$ & $1.570 \mathrm{E}+01$ & $6.26 \mathrm{E}-01$ \\
\hline $9.996 \mathrm{E}+03$ & $7.287 \mathrm{E}+00$ & 1.79E-02 & $7.179 \mathrm{E}+01$ & $9.07 \mathrm{E}+00$ & $4.763 \mathrm{E}+01$ & $1.474 \mathrm{E}+01$ & $6.25 \mathrm{E}-01$ \\
\hline $9.994 \mathrm{E}+03$ & $9.118 \mathrm{E}+00$ & $2.12 \mathrm{E}-02$ & $7.120 \mathrm{E}+01$ & $9.12 \mathrm{E}+00$ & $4.794 \mathrm{E}+01$ & $1.404 \mathrm{E}+01$ & $6.24 \mathrm{E}-01$ \\
\hline $9.991 \mathrm{E}+03$ & $1.099 \mathrm{E}+01$ & 2.33E-02 & $6.793 \mathrm{E}+01$ & $9.19 \mathrm{E}+00$ & $4.832 \mathrm{E}+01$ & $1.378 \mathrm{E}+01$ & $6.21 \mathrm{E}-01$ \\
\hline $9.996 \mathrm{E}+03$ & $1.284 \mathrm{E}+01$ & $2.66 \mathrm{E}-02$ & $6.550 \mathrm{E}+01$ & $9.12 \mathrm{E}+00$ & $4.879 \mathrm{E}+01$ & $1.382 \mathrm{E}+01$ & $6.17 \mathrm{E}-01$ \\
\hline $9.985 \mathrm{E}+03$ & $1.471 \mathrm{E}+01$ & $2.95 \mathrm{E}-02$ & $5.839 \mathrm{E}+01$ & $9.13 \mathrm{E}+00$ & $4.927 \mathrm{E}+01$ & $1.313 \mathrm{E}+01$ & $6.32 \mathrm{E}-01$ \\
\hline$P_{\mathrm{e}}(\mathrm{W})$ & $\Delta P_{\mathrm{e}}(\mathrm{W})$ & $C_{\mathrm{T}}$ & $\Delta C_{\mathrm{T}}$ & $C_{\mathrm{P}}$ & $\Delta C_{\mathrm{P}}$ & $C_{\mathrm{Q}}$ & $\Delta C_{\mathrm{Q}}$ \\
\hline $3.007 \mathrm{E}+01$ & $3.39 \mathrm{E}-01$ & $1.142 \mathrm{E}-01$ & $1.33 \mathrm{E}-02$ & $1.244 \mathrm{E}-01$ & $4.66 \mathrm{E}-03$ & $1.980 \mathrm{E}-02$ & $7.42 \mathrm{E}-04$ \\
\hline $2.943 \mathrm{E}+01$ & 3.32E-01 & $1.104 \mathrm{E}-01$ & $1.34 \mathrm{E}-02$ & $1.215 \mathrm{E}-01$ & $4.76 \mathrm{E}-03$ & $1.934 \mathrm{E}-02$ & $7.58 \mathrm{E}-04$ \\
\hline $2.868 \mathrm{E}+01$ & $3.26 \mathrm{E}-01$ & $1.117 \mathrm{E}-01$ & $1.33 \mathrm{E}-02$ & $1.166 \mathrm{E}-01$ & 4.65E-03 & $1.856 \mathrm{E}-02$ & $7.40 \mathrm{E}-04$ \\
\hline $2.709 \mathrm{E}+01$ & $3.08 \mathrm{E}-01$ & $1.052 \mathrm{E}-01$ & $1.33 \mathrm{E}-02$ & 1.102E-01 & $4.68 \mathrm{E}-03$ & $1.754 \mathrm{E}-02$ & $7.44 \mathrm{E}-04$ \\
\hline $2.597 \mathrm{E}+01$ & $2.97 \mathrm{E}-01$ & $1.044 \mathrm{E}-01$ & $1.34 \mathrm{E}-02$ & $1.050 \mathrm{E}-01$ & $4.67 \mathrm{E}-03$ & $1.672 \mathrm{E}-02$ & 7.43E-04 \\
\hline $2.538 \mathrm{E}+01$ & $2.90 \mathrm{E}-01$ & $9.967 \mathrm{E}-02$ & $1.35 \mathrm{E}-02$ & $1.033 \mathrm{E}-01$ & 4.66E-03 & $1.644 \mathrm{E}-02$ & $7.41 \mathrm{E}-04$ \\
\hline $2.535 \mathrm{E}+01$ & $2.91 \mathrm{E}-01$ & 9.604E-02 & $1.34 \mathrm{E}-02$ & $1.034 \mathrm{E}-01$ & $4.62 \mathrm{E}-03$ & $1.646 \mathrm{E}-02$ & $7.35 \mathrm{E}-04$ \\
\hline $2.438 \mathrm{E}+01$ & $2.78 \mathrm{E}-01$ & $8.582 \mathrm{E}-02$ & $1.34 \mathrm{E}-02$ & $9.861 \mathrm{E}-02$ & 4.75E-03 & $1.570 \mathrm{E}-02$ & $7.56 \mathrm{E}-04$ \\
\hline$\rho\left(\mathrm{kg} / \mathrm{m}^{\wedge} 3\right)$ & $\eta_{\mathrm{T}}$ & $\Delta \eta_{\mathrm{T}}$ & $J$ & $\Delta J$ & $\eta_{\mathrm{P}}$ & $\Delta \eta_{\mathrm{P}}$ & $R e_{0.75}$ \\
\hline $1.166 \mathrm{E}+00$ & $5.509 \mathrm{E}-01$ & $2.16 \mathrm{E}-02$ & $1.036 \mathrm{E}-01$ & $6.54 \mathrm{E}-04$ & $9.510 \mathrm{E}-02$ & $1.17 \mathrm{E}-02$ & $2.442 \mathrm{E}+04$ \\
\hline $1.167 \mathrm{E}+00$ & $5.510 \mathrm{E}-01$ & $2.25 \mathrm{E}-02$ & $1.872 \mathrm{E}-01$ & 6.91E-04 & $1.701 \mathrm{E}-01$ & $2.17 \mathrm{E}-02$ & $2.450 \mathrm{E}+04$ \\
\hline $1.166 \mathrm{E}+00$ & $5.475 \mathrm{E}-01$ & $2.27 \mathrm{E}-02$ & $2.737 \mathrm{E}-01$ & 7.93E-04 & $2.620 \mathrm{E}-01$ & $3.30 \mathrm{E}-02$ & $2.465 \mathrm{E}+04$ \\
\hline $1.166 \mathrm{E}+00$ & $5.439 \mathrm{E}-01$ & 2.39E-02 & $3.648 \mathrm{E}-01$ & $9.07 \mathrm{E}-04$ & $3.481 \mathrm{E}-01$ & 4.64E-02 & $2.471 \mathrm{E}+04$ \\
\hline $1.166 \mathrm{E}+00$ & $5.405 \mathrm{E}-01$ & $2.48 \mathrm{E}-02$ & $4.565 \mathrm{E}-01$ & $1.07 \mathrm{E}-03$ & $4.536 \mathrm{E}-01$ & $6.15 \mathrm{E}-02$ & $2.486 \mathrm{E}+04$ \\
\hline $1.165 \mathrm{E}+00$ & $5.431 \mathrm{E}-01$ & $2.53 \mathrm{E}-02$ & $5.502 \mathrm{E}-01$ & $1.18 \mathrm{E}-03$ & $5.309 \mathrm{E}-01$ & $7.57 \mathrm{E}-02$ & $2.504 \mathrm{E}+04$ \\
\hline $1.165 \mathrm{E}+00$ & $5.450 \mathrm{E}-01$ & $2.51 \mathrm{E}-02$ & $6.429 \mathrm{E}-01$ & $1.35 \mathrm{E}-03$ & $5.971 \mathrm{E}-01$ & $8.73 \mathrm{E}-02$ & $2.528 \mathrm{E}+04$ \\
\hline $1.165 \mathrm{E}+00$ & $5.386 \mathrm{E}-01$ & $2.67 \mathrm{E}-02$ & $7.371 \mathrm{E}-01$ & $1.50 \mathrm{E}-03$ & $6.415 \mathrm{E}-01$ & $1.05 \mathrm{E}-01$ & $2.552 \mathrm{E}+04$ \\
\hline
\end{tabular}


Table 132: APC 4.75 x 5.5 Dynamic Measured Values - 12014 RPM

\begin{tabular}{|c|c|c|c|c|c|c|c|}
\hline$n(\mathrm{RPM})$ & $Q(\mathrm{~g}-\mathrm{m})$ & $\Delta Q(\mathrm{~g}-\mathrm{m})$ & $V(\mathrm{~V})$ & $I(\mathrm{~A})$ & $T_{\text {atm }}\left({ }^{\circ} \mathrm{C}\right)$ & $P_{\text {atm }}(\mathrm{Pa})$ & $P_{\text {diff }}(\mathrm{Pa})$ \\
\hline $1.202 \mathrm{E}+04$ & $2.341 \mathrm{E}+00$ & $7.84 \mathrm{E}-02$ & $1.099 \mathrm{E}+01$ & $4.339 \mathrm{E}+00$ & $2.270 \mathrm{E}+01$ & $9.906 \mathrm{E}+04$ & $2.803 \mathrm{E}+00$ \\
\hline $1.202 \mathrm{E}+04$ & $2.282 \mathrm{E}+00$ & $7.75 \mathrm{E}-02$ & $1.099 \mathrm{E}+01$ & $4.227 \mathrm{E}+00$ & $2.254 \mathrm{E}+01$ & $9.906 \mathrm{E}+04$ & $1.065 \mathrm{E}+01$ \\
\hline $1.203 \mathrm{E}+04$ & $2.203 \mathrm{E}+00$ & $7.59 \mathrm{E}-02$ & $1.100 \mathrm{E}+01$ & $4.100 \mathrm{E}+00$ & $2.256 \mathrm{E}+01$ & $9.906 \mathrm{E}+04$ & $2.450 \mathrm{E}+01$ \\
\hline $1.203 \mathrm{E}+04$ & $2.057 \mathrm{E}+00$ & $7.43 \mathrm{E}-02$ & $1.100 \mathrm{E}+01$ & $3.884 \mathrm{E}+00$ & $2.266 \mathrm{E}+01$ & $9.907 \mathrm{E}+04$ & $4.475 \mathrm{E}+01$ \\
\hline $1.201 \mathrm{E}+04$ & $1.972 \mathrm{E}+00$ & $6.61 \mathrm{E}-02$ & $1.101 \mathrm{E}+01$ & $3.702 \mathrm{E}+00$ & $2.284 \mathrm{E}+01$ & $9.907 \mathrm{E}+04$ & $7.121 \mathrm{E}+01$ \\
\hline $1.199 \mathrm{E}+04$ & $1.946 \mathrm{E}+00$ & $6.69 \mathrm{E}-02$ & $1.101 \mathrm{E}+01$ & $3.627 \mathrm{E}+00$ & $2.291 \mathrm{E}+01$ & $9.907 \mathrm{E}+04$ & $1.045 \mathrm{E}+02$ \\
\hline $1.199 \mathrm{E}+04$ & $1.954 \mathrm{E}+00$ & $6.68 \mathrm{E}-02$ & $1.101 \mathrm{E}+01$ & $3.626 \mathrm{E}+00$ & $2.295 \mathrm{E}+01$ & $9.908 \mathrm{E}+04$ & $1.439 \mathrm{E}+02$ \\
\hline $1.199 \mathrm{E}+04$ & $1.926 \mathrm{E}+00$ & $6.59 \mathrm{E}-02$ & $1.101 \mathrm{E}+01$ & $3.543 \mathrm{E}+00$ & $2.300 \mathrm{E}+01$ & $9.908 \mathrm{E}+04$ & $1.896 \mathrm{E}+02$ \\
\hline $1.204 \mathrm{E}+04$ & $1.760 \mathrm{E}+00$ & $6.92 \mathrm{E}-02$ & $1.102 \mathrm{E}+01$ & $3.261 \mathrm{E}+00$ & $2.301 \mathrm{E}+01$ & $9.908 \mathrm{E}+04$ & $2.409 \mathrm{E}+02$ \\
\hline
\end{tabular}


Table 133: APC 4.75 x 5.5 Dynamic Calculated Values - 12014 RPM

\begin{tabular}{|c|c|c|c|c|c|c|c|}
\hline$n(\mathrm{RPM})$ & $V_{\infty}^{\prime}(\mathrm{m} / \mathrm{s})$ & $\Delta V_{\infty}^{\prime}(\mathrm{m} / \mathrm{s})$ & $T^{\prime}(\mathrm{g})$ & $\Delta T^{\prime}(\mathrm{g})$ & $V_{\mathrm{t}}(\mathrm{m} / \mathrm{s})$ & $P_{\mathrm{P}}(\mathrm{W})$ & $\Delta P_{\mathrm{P}}(\mathrm{W})$ \\
\hline $1.202 \mathrm{E}+04$ & $2.093 \mathrm{E}+00$ & $1.39 \mathrm{E}-02$ & $1.178 \mathrm{E}+02$ & $8.47 \mathrm{E}+00$ & $5.665 \mathrm{E}+01$ & $2.890 \mathrm{E}+01$ & $9.69 \mathrm{E}-01$ \\
\hline $1.202 \mathrm{E}+04$ & $4.177 \mathrm{E}+00$ & $1.50 \mathrm{E}-02$ & $1.159 \mathrm{E}+02$ & $8.52 \mathrm{E}+00$ & $5.676 \mathrm{E}+01$ & $2.817 \mathrm{E}+01$ & $9.58 \mathrm{E}-01$ \\
\hline $1.203 \mathrm{E}+04$ & $6.393 \mathrm{E}+00$ & $1.74 \mathrm{E}-02$ & $1.104 \mathrm{E}+02$ & $8.47 \mathrm{E}+00$ & $5.703 \mathrm{E}+01$ & $2.723 \mathrm{E}+01$ & $9.39 \mathrm{E}-01$ \\
\hline $1.203 \mathrm{E}+04$ & $8.679 \mathrm{E}+00$ & $2.12 \mathrm{E}-02$ & $1.090 \mathrm{E}+02$ & $8.48 \mathrm{E}+00$ & $5.729 \mathrm{E}+01$ & $2.541 \mathrm{E}+01$ & $9.18 \mathrm{E}-01$ \\
\hline $1.201 \mathrm{E}+04$ & $1.098 \mathrm{E}+01$ & $2.45 \mathrm{E}-02$ & $1.031 \mathrm{E}+02$ & $8.47 \mathrm{E}+00$ & $5.762 \mathrm{E}+01$ & $2.433 \mathrm{E}+01$ & $8.16 \mathrm{E}-01$ \\
\hline $1.199 \mathrm{E}+04$ & $1.333 \mathrm{E}+01$ & $2.80 \mathrm{E}-02$ & $9.805 \mathrm{E}+01$ & $8.47 \mathrm{E}+00$ & $5.803 \mathrm{E}+01$ & $2.396 \mathrm{E}+01$ & $8.24 \mathrm{E}-01$ \\
\hline 1.199E+04 & $1.566 \mathrm{E}+01$ & $3.14 \mathrm{E}-02$ & $9.655 \mathrm{E}+01$ & $8.45 \mathrm{E}+00$ & $5.861 \mathrm{E}+01$ & $2.407 \mathrm{E}+01$ & $8.23 \mathrm{E}-01$ \\
\hline $1.199 \mathrm{E}+04$ & $1.799 \mathrm{E}+01$ & $3.53 \mathrm{E}-02$ & $8.965 \mathrm{E}+01$ & $8.47 \mathrm{E}+00$ & $5.927 \mathrm{E}+01$ & $2.371 \mathrm{E}+01$ & $8.11 \mathrm{E}-01$ \\
\hline $1.204 \mathrm{E}+04$ & $2.029 \mathrm{E}+01$ & $3.88 \mathrm{E}-02$ & $7.538 \mathrm{E}+01$ & $8.46 \mathrm{E}+00$ & $6.022 \mathrm{E}+01$ & $2.176 \mathrm{E}+01$ & $8.56 \mathrm{E}-01$ \\
\hline$P_{\mathrm{e}}(\mathrm{W})$ & $\Delta P_{\mathrm{e}}(\mathrm{W})$ & $C_{\mathrm{T}}$ & $\Delta C_{\mathrm{T}}$ & $C_{\mathrm{P}}$ & $\Delta C_{\mathrm{P}}$ & $C_{\mathrm{Q}}$ & $\Delta C_{\mathrm{Q}}$ \\
\hline $4.769 \mathrm{E}+01$ & $5.45 \mathrm{E}-01$ & 1.193E-01 & $8.59 \mathrm{E}-03$ & $1.242 \mathrm{E}-01$ & 4.19E-03 & $1.977 \mathrm{E}-02$ & $6.64 \mathrm{E}-04$ \\
\hline $4.646 \mathrm{E}+01$ & 5.34E-01 & $1.173 \mathrm{E}-01$ & $8.62 \mathrm{E}-03$ & $1.210 \mathrm{E}-01$ & $4.14 \mathrm{E}-03$ & $1.926 \mathrm{E}-02$ & $6.56 \mathrm{E}-04$ \\
\hline $4.509 \mathrm{E}+01$ & $5.14 \mathrm{E}-01$ & $1.115 \mathrm{E}-01$ & $8.56 \mathrm{E}-03$ & $1.166 \mathrm{E}-01$ & 4.04E-03 & $1.856 \mathrm{E}-02$ & $6.41 \mathrm{E}-04$ \\
\hline $4.274 \mathrm{E}+01$ & $4.81 \mathrm{E}-01$ & $1.102 \mathrm{E}-01$ & $8.58 \mathrm{E}-03$ & $1.091 \mathrm{E}-01$ & $3.96 \mathrm{E}-03$ & $1.736 \mathrm{E}-02$ & $6.28 \mathrm{E}-04$ \\
\hline $4.075 \mathrm{E}+01$ & $4.59 \mathrm{E}-01$ & $1.046 \mathrm{E}-01$ & 8.59E-03 & $1.049 \mathrm{E}-01$ & $3.54 \mathrm{E}-03$ & $1.669 \mathrm{E}-02$ & 5.61E-04 \\
\hline $3.994 \mathrm{E}+01$ & $4.47 \mathrm{E}-01$ & $9.984 \mathrm{E}-02$ & $8.63 \mathrm{E}-03$ & $1.038 \mathrm{E}-01$ & $3.58 \mathrm{E}-03$ & $1.652 \mathrm{E}-02$ & $5.69 \mathrm{E}-04$ \\
\hline $3.992 \mathrm{E}+01$ & $4.48 \mathrm{E}-01$ & $9.832 \mathrm{E}-02$ & $8.61 \mathrm{E}-03$ & 1.043E-01 & $3.57 \mathrm{E}-03$ & $1.660 \mathrm{E}-02$ & $5.68 \mathrm{E}-04$ \\
\hline $3.902 \mathrm{E}+01$ & 4.37E-01 & $9.132 \mathrm{E}-02$ & 8.63E-03 & $1.028 \mathrm{E}-01$ & $3.52 \mathrm{E}-03$ & $1.636 \mathrm{E}-02$ & $5.60 \mathrm{E}-04$ \\
\hline $3.594 \mathrm{E}+01$ & 4.01E-01 & 7.619E-02 & $8.55 \mathrm{E}-03$ & $9.319 \mathrm{E}-02$ & $3.68 \mathrm{E}-03$ & $1.483 \mathrm{E}-02$ & $5.84 \mathrm{E}-04$ \\
\hline$\rho\left(\mathrm{kg} / \mathrm{m}^{\wedge} 3\right)$ & $\eta_{\mathrm{T}}$ & $\Delta \eta_{\mathrm{T}}$ & $J$ & $\Delta J$ & $\eta_{\mathrm{P}}$ & $\Delta \eta_{\mathrm{P}}$ & $R e_{0.75}$ \\
\hline $1.166 \mathrm{E}+00$ & $6.060 \mathrm{E}-01$ & $2.15 \mathrm{E}-02$ & $8.711 \mathrm{E}-02$ & $5.88 \mathrm{E}-04$ & $8.368 \mathrm{E}-02$ & $6.67 \mathrm{E}-03$ & $2.940 \mathrm{E}+04$ \\
\hline $1.167 \mathrm{E}+00$ & $6.064 \mathrm{E}-01$ & $2.18 \mathrm{E}-02$ & $1.738 \mathrm{E}-01$ & $6.61 \mathrm{E}-04$ & $1.685 \mathrm{E}-01$ & $1.37 \mathrm{E}-02$ & $2.949 \mathrm{E}+04$ \\
\hline $1.167 \mathrm{E}+00$ & $6.039 \mathrm{E}-01$ & 2.19E-02 & $2.658 \mathrm{E}-01$ & 7.93E-04 & $2.542 \mathrm{E}-01$ & $2.14 \mathrm{E}-02$ & $2.963 \mathrm{E}+04$ \\
\hline $1.167 \mathrm{E}+00$ & $5.944 \mathrm{E}-01$ & $2.25 \mathrm{E}-02$ & $3.611 \mathrm{E}-01$ & $9.85 \mathrm{E}-04$ & $3.650 \mathrm{E}-01$ & $3.14 \mathrm{E}-02$ & $2.974 \mathrm{E}+04$ \\
\hline $1.166 \mathrm{E}+00$ & $5.971 \mathrm{E}-01$ & $2.11 \mathrm{E}-02$ & $4.574 \mathrm{E}-01$ & $1.16 \mathrm{E}-03$ & $4.562 \mathrm{E}-01$ & 4.05E-02 & $2.988 \mathrm{E}+04$ \\
\hline $1.166 \mathrm{E}+00$ & $6.000 \mathrm{E}-01$ & $2.17 \mathrm{E}-02$ & $5.559 \mathrm{E}-01$ & $1.21 \mathrm{E}-03$ & $5.347 \mathrm{E}-01$ & $4.98 \mathrm{E}-02$ & $3.008 \mathrm{E}+04$ \\
\hline $1.166 \mathrm{E}+00$ & $6.029 \mathrm{E}-01$ & $2.17 \mathrm{E}-02$ & $6.533 \mathrm{E}-01$ & $1.37 \mathrm{E}-03$ & $6.160 \mathrm{E}-01$ & $5.79 \mathrm{E}-02$ & $3.038 \mathrm{E}+04$ \\
\hline $1.166 \mathrm{E}+00$ & $6.078 \mathrm{E}-01$ & $2.19 \mathrm{E}-02$ & $7.507 \mathrm{E}-01$ & $1.58 \mathrm{E}-03$ & $6.671 \mathrm{E}-01$ & $6.71 \mathrm{E}-02$ & $3.071 \mathrm{E}+04$ \\
\hline $1.165 \mathrm{E}+00$ & $6.054 \mathrm{E}-01$ & $2.47 \mathrm{E}-02$ & $8.435 \mathrm{E}-01$ & $1.92 \mathrm{E}-03$ & $6.895 \mathrm{E}-01$ & $8.21 \mathrm{E}-02$ & $3.120 \mathrm{E}+04$ \\
\hline
\end{tabular}


Table 134: APC 4.75 x 5.5 Dynamic Measured Values - 14004 RPM

\begin{tabular}{|c|c|c|c|c|c|c|c|}
\hline$n(\mathrm{RPM})$ & $Q(\mathrm{~g}-\mathrm{m})$ & $\Delta Q(\mathrm{~g}-\mathrm{m})$ & $V(\mathrm{~V})$ & $I(\mathrm{~A})$ & $T_{\text {atm }}\left({ }^{\circ} \mathrm{C}\right)$ & $P_{\text {atm }}(\mathrm{Pa})$ & $P_{\text {diff }}(\mathrm{Pa})$ \\
\hline $1.403 \mathrm{E}+04$ & $3.145 \mathrm{E}+00$ & $7.40 \mathrm{E}-02$ & $1.093 \mathrm{E}+01$ & $6.469 \mathrm{E}+00$ & $2.195 \mathrm{E}+01$ & $9.921 \mathrm{E}+04$ & $3.156 \mathrm{E}+00$ \\
\hline $1.405 \mathrm{E}+04$ & $3.093 \mathrm{E}+00$ & $7.26 \mathrm{E}-02$ & $1.093 \mathrm{E}+01$ & $6.328 \mathrm{E}+00$ & $2.193 \mathrm{E}+01$ & $9.921 \mathrm{E}+04$ & $1.340 \mathrm{E}+01$ \\
\hline $1.404 \mathrm{E}+04$ & $3.022 \mathrm{E}+00$ & $7.22 \mathrm{E}-02$ & $1.094 \mathrm{E}+01$ & $6.131 \mathrm{E}+00$ & $2.198 \mathrm{E}+01$ & $9.919 \mathrm{E}+04$ & $3.244 \mathrm{E}+01$ \\
\hline $1.399 \mathrm{E}+04$ & $2.835 \mathrm{E}+00$ & $7.10 \mathrm{E}-02$ & $1.095 \mathrm{E}+01$ & $5.766 \mathrm{E}+00$ & $2.208 \mathrm{E}+01$ & $9.918 \mathrm{E}+04$ & $6.033 \mathrm{E}+01$ \\
\hline $1.403 \mathrm{E}+04$ & $2.707 \mathrm{E}+00$ & $7.06 \mathrm{E}-02$ & $1.095 \mathrm{E}+01$ & $5.540 \mathrm{E}+00$ & $2.214 \mathrm{E}+01$ & $9.918 \mathrm{E}+04$ & $9.836 \mathrm{E}+01$ \\
\hline $1.397 \mathrm{E}+04$ & $2.662 \mathrm{E}+00$ & $7.13 \mathrm{E}-02$ & $1.096 \mathrm{E}+01$ & $5.398 \mathrm{E}+00$ & $2.212 \mathrm{E}+01$ & $9.918 \mathrm{E}+04$ & $1.453 \mathrm{E}+02$ \\
\hline $1.395 \mathrm{E}+04$ & $2.685 \mathrm{E}+00$ & $7.45 \mathrm{E}-02$ & $1.096 \mathrm{E}+01$ & $5.412 \mathrm{E}+00$ & $2.211 \mathrm{E}+01$ & $9.919 \mathrm{E}+04$ & $2.017 \mathrm{E}+02$ \\
\hline $1.399 \mathrm{E}+04$ & $2.689 \mathrm{E}+00$ & $7.34 \mathrm{E}-02$ & $1.096 \mathrm{E}+01$ & $5.365 \mathrm{E}+00$ & $2.222 \mathrm{E}+01$ & $9.918 \mathrm{E}+04$ & $2.662 \mathrm{E}+02$ \\
\hline $1.398 \mathrm{E}+04$ & $2.472 \mathrm{E}+00$ & $7.01 \mathrm{E}-02$ & $1.097 \mathrm{E}+01$ & $4.864 \mathrm{E}+00$ & $2.233 \mathrm{E}+01$ & $9.918 \mathrm{E}+04$ & $3.398 \mathrm{E}+02$ \\
\hline
\end{tabular}


Table 135: APC 4.75 x 5.5 Dynamic Calculated Values - 14004 RPM

\begin{tabular}{|c|c|c|c|c|c|c|c|}
\hline$n(\mathrm{RPM})$ & $V_{\infty}^{\prime}(\mathrm{m} / \mathrm{s})$ & $\Delta V_{\infty}^{\prime}(\mathrm{m} / \mathrm{s})$ & $T^{\prime}(\mathrm{g})$ & $\Delta T^{\prime}(\mathrm{g})$ & $V_{\mathrm{t}}(\mathrm{m} / \mathrm{s})$ & $P_{\mathrm{P}}(\mathrm{W})$ & $\Delta P_{\mathrm{P}}(\mathrm{W})$ \\
\hline $1.403 \mathrm{E}+04$ & $2.204 \mathrm{E}+00$ & $1.51 \mathrm{E}-02$ & $1.652 \mathrm{E}+02$ & $9.05 \mathrm{E}+00$ & $6.613 \mathrm{E}+01$ & $4.533 \mathrm{E}+01$ & $1.07 \mathrm{E}+00$ \\
\hline $1.405 \mathrm{E}+04$ & $4.672 \mathrm{E}+00$ & $1.64 \mathrm{E}-02$ & $1.616 \mathrm{E}+02$ & $8.97 \mathrm{E}+00$ & $6.632 \mathrm{E}+01$ & $4.463 \mathrm{E}+01$ & $1.05 \mathrm{E}+00$ \\
\hline $1.404 \mathrm{E}+04$ & $7.341 \mathrm{E}+00$ & $1.96 \mathrm{E}-02$ & $1.553 \mathrm{E}+02$ & $8.95 \mathrm{E}+00$ & $6.650 \mathrm{E}+01$ & $4.356 \mathrm{E}+01$ & $1.04 \mathrm{E}+00$ \\
\hline $1.399 \mathrm{E}+04$ & $1.006 \mathrm{E}+01$ & $2.43 \mathrm{E}-02$ & $1.488 \mathrm{E}+02$ & $8.97 \mathrm{E}+00$ & $6.662 \mathrm{E}+01$ & $4.072 \mathrm{E}+01$ & $1.02 \mathrm{E}+00$ \\
\hline $1.403 \mathrm{E}+04$ & $1.288 \mathrm{E}+01$ & $2.82 \mathrm{E}-02$ & $1.436 \mathrm{E}+02$ & $9.11 \mathrm{E}+00$ & $6.730 \mathrm{E}+01$ & $3.899 \mathrm{E}+01$ & $1.02 \mathrm{E}+00$ \\
\hline $1.397 \mathrm{E}+04$ & $1.569 \mathrm{E}+01$ & $3.21 \mathrm{E}-02$ & $1.377 \mathrm{E}+02$ & $9.11 \mathrm{E}+00$ & $6.765 \mathrm{E}+01$ & $3.821 \mathrm{E}+01$ & $1.02 \mathrm{E}+00$ \\
\hline $1.395 \mathrm{E}+04$ & $1.850 \mathrm{E}+01$ & $3.61 \mathrm{E}-02$ & $1.377 \mathrm{E}+02$ & $9.13 \mathrm{E}+00$ & $6.827 \mathrm{E}+01$ & $3.848 \mathrm{E}+01$ & $1.07 \mathrm{E}+00$ \\
\hline $1.399 \mathrm{E}+04$ & $2.128 \mathrm{E}+01$ & 4.08E-02 & $1.296 \mathrm{E}+02$ & $9.05 \mathrm{E}+00$ & $6.924 \mathrm{E}+01$ & $3.865 \mathrm{E}+01$ & $1.06 \mathrm{E}+00$ \\
\hline $1.398 \mathrm{E}+04$ & $2.406 \mathrm{E}+01$ & $4.56 \mathrm{E}-02$ & $1.075 \mathrm{E}+02$ & $9.07 \mathrm{E}+00$ & $7.011 \mathrm{E}+01$ & $3.550 \mathrm{E}+01$ & $1.01 \mathrm{E}+00$ \\
\hline$P_{\mathrm{e}}(\mathrm{W})$ & $\Delta P_{\mathrm{e}}(\mathrm{W})$ & $C_{\mathrm{T}}$ & $\Delta C_{\mathrm{T}}$ & $C_{\mathrm{P}}$ & $\Delta C_{\mathrm{P}}$ & $C_{\mathrm{Q}}$ & $\Delta C_{\mathrm{Q}}$ \\
\hline $7.068 \mathrm{E}+01$ & $7.93 \mathrm{E}-01$ & $1.223 \mathrm{E}-01$ & $6.71 \mathrm{E}-03$ & $1.219 \mathrm{E}-01$ & $2.91 \mathrm{E}-03$ & $1.941 \mathrm{E}-02$ & $4.59 \mathrm{E}-04$ \\
\hline $6.917 \mathrm{E}+01$ & 7.73E-01 & $1.194 \mathrm{E}-01$ & $6.63 \mathrm{E}-03$ & $1.197 \mathrm{E}-01$ & $2.85 \mathrm{E}-03$ & $1.905 \mathrm{E}-02$ & 4.50E-04 \\
\hline $6.704 \mathrm{E}+01$ & $7.54 \mathrm{E}-01$ & $1.150 \mathrm{E}-01$ & $6.63 \mathrm{E}-03$ & $1.172 \mathrm{E}-01$ & $2.84 \mathrm{E}-03$ & $1.865 \mathrm{E}-02$ & $4.48 \mathrm{E}-04$ \\
\hline $6.312 \mathrm{E}+01$ & $7.10 \mathrm{E}-01$ & $1.109 \mathrm{E}-01$ & $6.70 \mathrm{E}-03$ & $1.108 \mathrm{E}-01$ & $2.81 \mathrm{E}-03$ & $1.763 \mathrm{E}-02$ & 4.44E-04 \\
\hline $6.068 \mathrm{E}+01$ & $6.83 \mathrm{E}-01$ & $1.065 \mathrm{E}-01$ & $6.76 \mathrm{E}-03$ & $1.052 \mathrm{E}-01$ & $2.78 \mathrm{E}-03$ & $1.674 \mathrm{E}-02$ & 4.39E-04 \\
\hline $5.915 \mathrm{E}+01$ & $6.68 \mathrm{E}-01$ & $1.028 \mathrm{E}-01$ & $6.81 \mathrm{E}-03$ & $1.042 \mathrm{E}-01$ & $2.82 \mathrm{E}-03$ & $1.659 \mathrm{E}-02$ & $4.46 \mathrm{E}-04$ \\
\hline $5.930 \mathrm{E}+01$ & $6.67 \mathrm{E}-01$ & $1.031 \mathrm{E}-01$ & $6.85 \mathrm{E}-03$ & $1.054 \mathrm{E}-01$ & $2.96 \mathrm{E}-03$ & $1.677 \mathrm{E}-02$ & $4.67 \mathrm{E}-04$ \\
\hline $5.880 \mathrm{E}+01$ & $6.68 \mathrm{E}-01$ & $9.664 \mathrm{E}-02$ & $6.75 \mathrm{E}-03$ & $1.050 \mathrm{E}-01$ & $2.90 \mathrm{E}-03$ & $1.672 \mathrm{E}-02$ & $4.58 \mathrm{E}-04$ \\
\hline $5.338 \mathrm{E}+01$ & $6.01 \mathrm{E}-01$ & $8.030 \mathrm{E}-02$ & $6.78 \mathrm{E}-03$ & $9.670 \mathrm{E}-02$ & $2.77 \mathrm{E}-03$ & $1.539 \mathrm{E}-02$ & $4.38 \mathrm{E}-04$ \\
\hline$\rho\left(\mathrm{kg} / \mathrm{m}^{\wedge} 3\right)$ & $\eta_{\mathrm{T}}$ & $\Delta \eta_{\mathrm{T}}$ & $J$ & $\Delta J$ & $\eta_{\mathrm{P}}$ & $\Delta \eta_{\mathrm{P}}$ & $R e_{0.75}$ \\
\hline $1.171 \mathrm{E}+00$ & $6.413 \mathrm{E}-01$ & $1.67 \mathrm{E}-02$ & 7.857E-02 & $5.49 \mathrm{E}-04$ & $7.878 \mathrm{E}-02$ & $4.74 \mathrm{E}-03$ & $3.453 \mathrm{E}+04$ \\
\hline $1.171 \mathrm{E}+00$ & $6.452 \mathrm{E}-01$ & $1.68 \mathrm{E}-02$ & $1.664 \mathrm{E}-01$ & $6.21 \mathrm{E}-04$ & $1.659 \mathrm{E}-01$ & $1.00 \mathrm{E}-02$ & $3.463 \mathrm{E}+04$ \\
\hline $1.171 \mathrm{E}+00$ & $6.497 \mathrm{E}-01$ & $1.72 \mathrm{E}-02$ & 2.617E-01 & 7.72E-04 & $2.567 \mathrm{E}-01$ & $1.61 \mathrm{E}-02$ & $3.471 \mathrm{E}+04$ \\
\hline $1.170 \mathrm{E}+00$ & $6.450 \mathrm{E}-01$ & $1.77 \mathrm{E}-02$ & $3.599 \mathrm{E}-01$ & $9.84 \mathrm{E}-04$ & $3.605 \mathrm{E}-01$ & $2.36 \mathrm{E}-02$ & $3.475 \mathrm{E}+04$ \\
\hline $1.170 \mathrm{E}+00$ & $6.426 \mathrm{E}-01$ & $1.83 \mathrm{E}-02$ & $4.595 \mathrm{E}-01$ & $1.16 \mathrm{E}-03$ & 4.654E-01 & $3.20 \mathrm{E}-02$ & $3.509 \mathrm{E}+04$ \\
\hline $1.170 \mathrm{E}+00$ & $6.460 \mathrm{E}-01$ & $1.88 \mathrm{E}-02$ & 5.617E-01 & $1.36 \mathrm{E}-03$ & 5.543E-01 & $3.97 \mathrm{E}-02$ & $3.528 \mathrm{E}+04$ \\
\hline $1.170 \mathrm{E}+00$ & $6.488 \mathrm{E}-01$ & $1.94 \mathrm{E}-02$ & $6.634 \mathrm{E}-01$ & $1.55 \mathrm{E}-03$ & $6.491 \mathrm{E}-01$ & $4.68 \mathrm{E}-02$ & $3.560 \mathrm{E}+04$ \\
\hline $1.170 \mathrm{E}+00$ & $6.573 \mathrm{E}-01$ & $1.94 \mathrm{E}-02$ & $7.608 \mathrm{E}-01$ & $1.76 \mathrm{E}-03$ & $7.000 \mathrm{E}-01$ & $5.26 \mathrm{E}-02$ & $3.608 \mathrm{E}+04$ \\
\hline $1.169 \mathrm{E}+00$ & $6.650 \mathrm{E}-01$ & $2.03 \mathrm{E}-02$ & 8.610E-01 & $1.97 \mathrm{E}-03$ & 7.149E-01 & $6.38 \mathrm{E}-02$ & $3.651 \mathrm{E}+04$ \\
\hline
\end{tabular}


Table 136: APC 4.75 x 5.5 Dynamic Measured Values - 15990 RPM

\begin{tabular}{|c|c|c|c|c|c|c|c|}
\hline$n(\mathrm{RPM})$ & $Q(\mathrm{~g}-\mathrm{m})$ & $\Delta Q(\mathrm{~g}-\mathrm{m})$ & $V(\mathrm{~V})$ & $I(\mathrm{~A})$ & $T_{\text {atm }}\left({ }^{\circ} \mathrm{C}\right)$ & $P_{\text {atm }}(\mathrm{Pa})$ & $P_{\text {diff }}(\mathrm{Pa})$ \\
\hline $1.594 \mathrm{E}+04$ & $4.073 \mathrm{E}+00$ & $7.57 \mathrm{E}-02$ & $1.084 \mathrm{E}+01$ & $9.282 \mathrm{E}+00$ & $2.209 \mathrm{E}+01$ & $9.918 \mathrm{E}+04$ & $3.495 \mathrm{E}+00$ \\
\hline $1.603 \mathrm{E}+04$ & $4.032 \mathrm{E}+00$ & $7.37 \mathrm{E}-02$ & $1.084 \mathrm{E}+01$ & $9.170 \mathrm{E}+00$ & $2.203 \mathrm{E}+01$ & $9.917 \mathrm{E}+04$ & $1.636 \mathrm{E}+01$ \\
\hline $1.598 \mathrm{E}+04$ & $3.875 \mathrm{E}+00$ & $7.34 \mathrm{E}-02$ & $1.086 \mathrm{E}+01$ & $8.767 \mathrm{E}+00$ & $2.211 \mathrm{E}+01$ & $9.917 \mathrm{E}+04$ & $4.136 \mathrm{E}+01$ \\
\hline $1.602 \mathrm{E}+04$ & $3.748 \mathrm{E}+00$ & $7.20 \mathrm{E}-02$ & $1.087 \mathrm{E}+01$ & $8.465 \mathrm{E}+00$ & $2.225 \mathrm{E}+01$ & $9.917 \mathrm{E}+04$ & $7.875 \mathrm{E}+01$ \\
\hline $1.595 \mathrm{E}+04$ & $3.519 \mathrm{E}+00$ & $7.37 \mathrm{E}-02$ & $1.088 \mathrm{E}+01$ & $7.919 \mathrm{E}+00$ & $2.223 \mathrm{E}+01$ & $9.916 \mathrm{E}+04$ & $1.292 \mathrm{E}+02$ \\
\hline $1.597 \mathrm{E}+04$ & $3.514 \mathrm{E}+00$ & $7.38 \mathrm{E}-02$ & $1.088 \mathrm{E}+01$ & $7.843 \mathrm{E}+00$ & $2.226 \mathrm{E}+01$ & $9.915 \mathrm{E}+04$ & $1.922 \mathrm{E}+02$ \\
\hline $1.604 \mathrm{E}+04$ & $3.591 \mathrm{E}+00$ & $7.55 \mathrm{E}-02$ & $1.088 \mathrm{E}+01$ & $7.971 \mathrm{E}+00$ & $2.232 \mathrm{E}+01$ & $9.915 \mathrm{E}+04$ & $2.670 \mathrm{E}+02$ \\
\hline $1.600 \mathrm{E}+04$ & $3.544 \mathrm{E}+00$ & $7.18 \mathrm{E}-02$ & $1.089 \mathrm{E}+01$ & $7.776 \mathrm{E}+00$ & $2.236 \mathrm{E}+01$ & $9.915 \mathrm{E}+04$ & $3.538 \mathrm{E}+02$ \\
\hline $1.599 \mathrm{E}+04$ & $3.309 \mathrm{E}+00$ & $7.28 \mathrm{E}-02$ & $1.091 \mathrm{E}+01$ & $7.105 \mathrm{E}+00$ & $2.241 \mathrm{E}+01$ & $9.915 \mathrm{E}+04$ & $4.524 \mathrm{E}+02$ \\
\hline $1.599 \mathrm{E}+04$ & $2.811 \mathrm{E}+00$ & $7.48 \mathrm{E}-02$ & $1.094 \mathrm{E}+01$ & $5.933 \mathrm{E}+00$ & $2.255 \mathrm{E}+01$ & $9.914 \mathrm{E}+04$ & $5.614 \mathrm{E}+02$ \\
\hline
\end{tabular}


Table 137: APC 4.75 x 5.5 Dynamic Calculated Values - 15990 RPM

\begin{tabular}{|c|c|c|c|c|c|c|c|}
\hline$n(\mathrm{RPM})$ & $V_{\infty}^{\prime}(\mathrm{m} / \mathrm{s})$ & $\Delta V_{\infty}^{\prime}(\mathrm{m} / \mathrm{s})$ & $T^{\prime}(\mathrm{g})$ & $\Delta T^{\prime}(\mathrm{g})$ & $V_{\mathrm{t}}(\mathrm{m} / \mathrm{s})$ & $P_{\mathrm{P}}(\mathrm{W})$ & $\Delta P_{\mathrm{P}}(\mathrm{W})$ \\
\hline $1.594 \mathrm{E}+04$ & $2.310 \mathrm{E}+00$ & $1.62 \mathrm{E}-02$ & $2.144 \mathrm{E}+02$ & $8.41 \mathrm{E}+00$ & $7.509 \mathrm{E}+01$ & $6.667 \mathrm{E}+01$ & $1.24 \mathrm{E}+00$ \\
\hline $1.603 \mathrm{E}+04$ & $5.158 \mathrm{E}+00$ & $1.82 \mathrm{E}-02$ & $2.136 \mathrm{E}+02$ & $8.24 \mathrm{E}+00$ & $7.566 \mathrm{E}+01$ & $6.637 \mathrm{E}+01$ & $1.21 \mathrm{E}+00$ \\
\hline $1.598 \mathrm{E}+04$ & $8.287 \mathrm{E}+00$ & 2.24E-02 & $2.082 \mathrm{E}+02$ & $8.47 \mathrm{E}+00$ & $7.569 \mathrm{E}+01$ & $6.358 \mathrm{E}+01$ & $1.20 \mathrm{E}+00$ \\
\hline $1.602 \mathrm{E}+04$ & $1.150 \mathrm{E}+01$ & 2.79E-02 & $2.025 \mathrm{E}+02$ & $8.34 \mathrm{E}+00$ & $7.631 \mathrm{E}+01$ & $6.166 \mathrm{E}+01$ & $1.18 \mathrm{E}+00$ \\
\hline $1.595 \mathrm{E}+04$ & $1.477 \mathrm{E}+01$ & $3.20 \mathrm{E}-02$ & $1.907 \mathrm{E}+02$ & $8.55 \mathrm{E}+00$ & $7.655 \mathrm{E}+01$ & $5.765 \mathrm{E}+01$ & $1.21 \mathrm{E}+00$ \\
\hline $1.597 \mathrm{E}+04$ & $1.805 \mathrm{E}+01$ & $3.61 \mathrm{E}-02$ & $1.835 \mathrm{E}+02$ & $8.60 \mathrm{E}+00$ & $7.735 \mathrm{E}+01$ & $5.763 \mathrm{E}+01$ & $1.21 \mathrm{E}+00$ \\
\hline $1.604 \mathrm{E}+04$ & $2.130 \mathrm{E}+01$ & $4.08 \mathrm{E}-02$ & $1.859 \mathrm{E}+02$ & $8.54 \mathrm{E}+00$ & $7.847 \mathrm{E}+01$ & $5.914 \mathrm{E}+01$ & $1.24 \mathrm{E}+00$ \\
\hline $1.600 \mathrm{E}+04$ & $2.454 \mathrm{E}+01$ & $4.67 \mathrm{E}-02$ & $1.714 \mathrm{E}+02$ & $8.64 \mathrm{E}+00$ & $7.925 \mathrm{E}+01$ & $5.825 \mathrm{E}+01$ & $1.18 \mathrm{E}+00$ \\
\hline $1.599 \mathrm{E}+04$ & $2.777 \mathrm{E}+01$ & $5.23 \mathrm{E}-02$ & $1.468 \mathrm{E}+02$ & $8.94 \mathrm{E}+00$ & $8.025 \mathrm{E}+01$ & $5.434 \mathrm{E}+01$ & $1.20 \mathrm{E}+00$ \\
\hline $1.599 \mathrm{E}+04$ & $3.097 \mathrm{E}+01$ & $1.04 \mathrm{E}-01$ & $1.137 \mathrm{E}+02$ & $8.95 \mathrm{E}+00$ & $8.142 \mathrm{E}+01$ & $4.616 \mathrm{E}+01$ & $1.24 \mathrm{E}+00$ \\
\hline$P_{\mathrm{e}}(\mathrm{W})$ & $\Delta P_{\mathrm{e}}(\mathrm{W})$ & $C_{\mathrm{T}}$ & $\Delta C_{\mathrm{T}}$ & $C_{\mathrm{P}}$ & $\Delta C_{\mathrm{P}}$ & $C_{\mathrm{Q}}$ & $\Delta C_{\mathrm{Q}}$ \\
\hline $1.006 \mathrm{E}+02$ & $1.09 \mathrm{E}+00$ & $1.231 \mathrm{E}-01$ & 4.83E-03 & $1.226 \mathrm{E}-01$ & $2.28 \mathrm{E}-03$ & $1.951 \mathrm{E}-02$ & $3.63 \mathrm{E}-04$ \\
\hline $9.945 \mathrm{E}+01$ & $1.08 \mathrm{E}+00$ & $1.213 \mathrm{E}-01$ & $4.68 \mathrm{E}-03$ & 1.199E-01 & $2.19 \mathrm{E}-03$ & $1.908 \mathrm{E}-02$ & $3.49 \mathrm{E}-04$ \\
\hline $9.518 \mathrm{E}+01$ & $1.03 \mathrm{E}+00$ & $1.190 \mathrm{E}-01$ & 4.84E-03 & $1.161 \mathrm{E}-01$ & $2.20 \mathrm{E}-03$ & $1.847 \mathrm{E}-02$ & $3.50 \mathrm{E}-04$ \\
\hline $9.198 \mathrm{E}+01$ & $1.00 \mathrm{E}+00$ & $1.152 \mathrm{E}-01$ & $4.75 \mathrm{E}-03$ & $1.117 \mathrm{E}-01$ & $2.15 \mathrm{E}-03$ & $1.778 \mathrm{E}-02$ & $3.42 \mathrm{E}-04$ \\
\hline $8.618 \mathrm{E}+01$ & $9.74 \mathrm{E}-01$ & $1.094 \mathrm{E}-01$ & 4.91E-03 & $1.058 \mathrm{E}-01$ & $2.22 \mathrm{E}-03$ & $1.684 \mathrm{E}-02$ & $3.53 \mathrm{E}-04$ \\
\hline $8.537 \mathrm{E}+01$ & $9.30 \mathrm{E}-01$ & $1.051 \mathrm{E}-01$ & 4.92E-03 & $1.054 \mathrm{E}-01$ & $2.21 \mathrm{E}-03$ & $1.677 \mathrm{E}-02$ & $3.52 \mathrm{E}-04$ \\
\hline $8.673 \mathrm{E}+01$ & $9.51 \mathrm{E}-01$ & $1.055 \mathrm{E}-01$ & $4.85 \mathrm{E}-03$ & $1.068 \mathrm{E}-01$ & $2.25 \mathrm{E}-03$ & $1.700 \mathrm{E}-02$ & $3.57 \mathrm{E}-04$ \\
\hline $8.465 \mathrm{E}+01$ & $9.24 \mathrm{E}-01$ & $9.775 \mathrm{E}-02$ & 4.93E-03 & $1.059 \mathrm{E}-01$ & $2.15 \mathrm{E}-03$ & $1.686 \mathrm{E}-02$ & $3.42 \mathrm{E}-04$ \\
\hline $7.749 \mathrm{E}+01$ & $8.55 \mathrm{E}-01$ & $8.388 \mathrm{E}-02$ & $5.11 \mathrm{E}-03$ & 9.909E-02 & $2.18 \mathrm{E}-03$ & $1.577 \mathrm{E}-02$ & $3.47 \mathrm{E}-04$ \\
\hline $6.492 \mathrm{E}+01$ & $8.96 \mathrm{E}-01$ & $6.498 \mathrm{E}-02$ & $5.14 \mathrm{E}-03$ & $8.421 \mathrm{E}-02$ & $2.45 \mathrm{E}-03$ & $1.340 \mathrm{E}-02$ & $3.70 \mathrm{E}-04$ \\
\hline$\rho\left(\mathrm{kg} / \mathrm{m}^{\wedge} 3\right)$ & $\eta_{\mathrm{T}}$ & $\Delta \eta_{\mathrm{T}}$ & $J$ & $\Delta J$ & $\eta_{\mathrm{P}}$ & $\Delta \eta_{\mathrm{P}}$ & $R e_{0.75}$ \\
\hline $1.170 \mathrm{E}+00$ & $6.625 \mathrm{E}-01$ & $1.42 \mathrm{E}-02$ & $7.251 \mathrm{E}-02$ & $5.10 \mathrm{E}-04$ & 7.284E-02 & $3.20 \mathrm{E}-03$ & $3.916 \mathrm{E}+04$ \\
\hline $1.170 \mathrm{E}+00$ & $6.674 \mathrm{E}-01$ & $1.42 \mathrm{E}-02$ & $1.610 \mathrm{E}-01$ & $5.69 \mathrm{E}-04$ & $1.628 \mathrm{E}-01$ & $6.98 \mathrm{E}-03$ & $3.947 \mathrm{E}+04$ \\
\hline $1.170 \mathrm{E}+00$ & $6.679 \mathrm{E}-01$ & $1.46 \mathrm{E}-02$ & $2.596 \mathrm{E}-01$ & $7.05 \mathrm{E}-04$ & $2.662 \mathrm{E}-01$ & $1.20 \mathrm{E}-02$ & $3.947 \mathrm{E}+04$ \\
\hline $1.169 \mathrm{E}+00$ & $6.703 \mathrm{E}-01$ & $1.48 \mathrm{E}-02$ & $3.590 \mathrm{E}-01$ & 8.74E-04 & $3.703 \mathrm{E}-01$ & $1.69 \mathrm{E}-02$ & $3.975 \mathrm{E}+04$ \\
\hline $1.170 \mathrm{E}+00$ & $6.689 \mathrm{E}-01$ & $1.59 \mathrm{E}-02$ & 4.633E-01 & $1.02 \mathrm{E}-03$ & 4.791E-01 & 2.37E-02 & $3.989 \mathrm{E}+04$ \\
\hline $1.169 \mathrm{E}+00$ & $6.751 \mathrm{E}-01$ & $1.60 \mathrm{E}-02$ & $5.653 \mathrm{E}-01$ & $1.14 \mathrm{E}-03$ & $5.636 \mathrm{E}-01$ & $2.90 \mathrm{E}-02$ & $4.029 \mathrm{E}+04$ \\
\hline $1.169 \mathrm{E}+00$ & 6.819E-01 & $1.62 \mathrm{E}-02$ & $6.644 \mathrm{E}-01$ & $1.28 \mathrm{E}-03$ & $6.564 \mathrm{E}-01$ & $3.32 \mathrm{E}-02$ & $4.086 \mathrm{E}+04$ \\
\hline $1.169 \mathrm{E}+00$ & $6.881 \mathrm{E}-01$ & $1.58 \mathrm{E}-02$ & $7.672 \mathrm{E}-01$ & $1.47 \mathrm{E}-03$ & 7.081E-01 & $3.85 \mathrm{E}-02$ & $4.126 \mathrm{E}+04$ \\
\hline $1.169 \mathrm{E}+00$ & 7.012E-01 & $1.73 \mathrm{E}-02$ & $8.691 \mathrm{E}-01$ & $1.65 \mathrm{E}-03$ & 7.357E-01 & 4.77E-02 & $4.176 \mathrm{E}+04$ \\
\hline $1.168 \mathrm{E}+00$ & 7.111E-01 & $2.15 \mathrm{E}-02$ & $9.690 \mathrm{E}-01$ & $4.85 \mathrm{E}-03$ & $7.478 \mathrm{E}-01$ & $6.31 \mathrm{E}-02$ & $4.233 \mathrm{E}+04$ \\
\hline
\end{tabular}




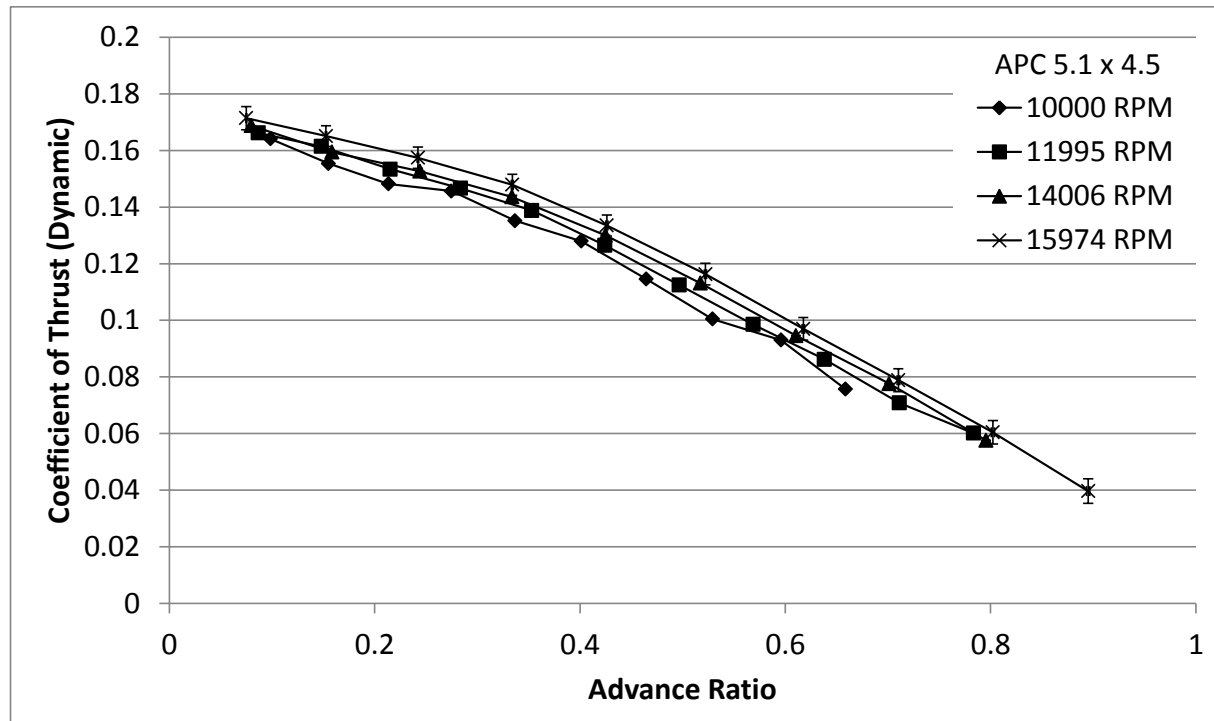

(a)

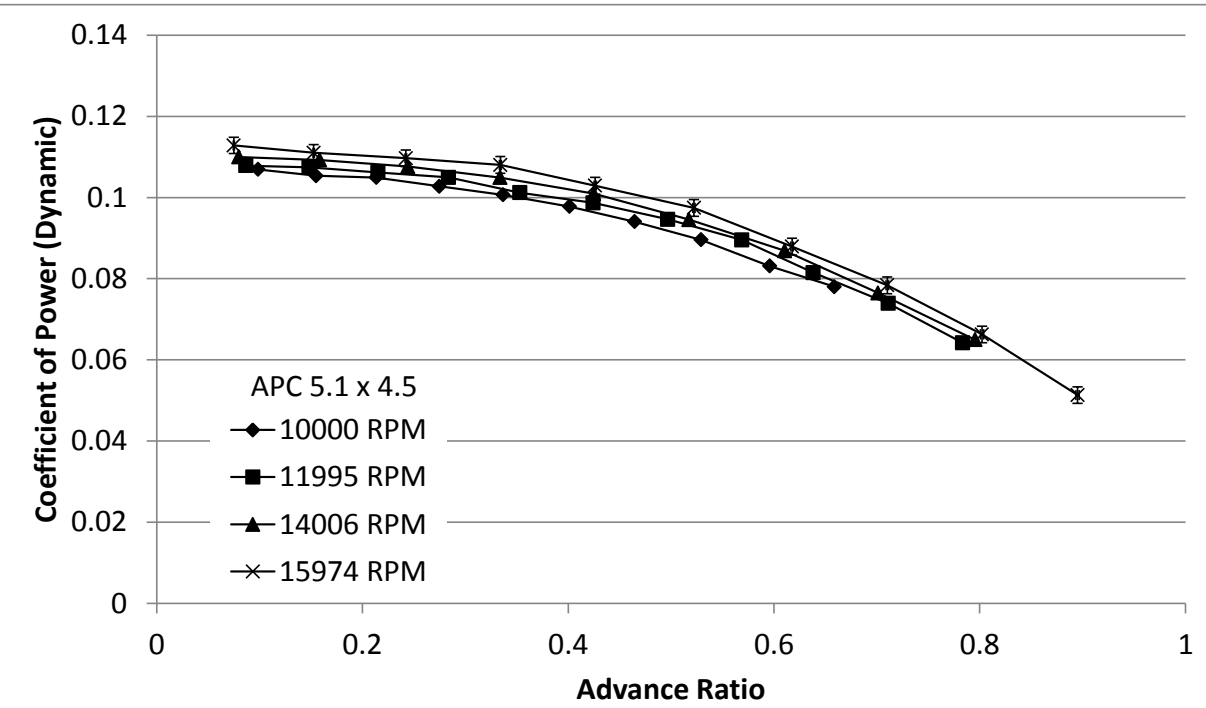

(b)

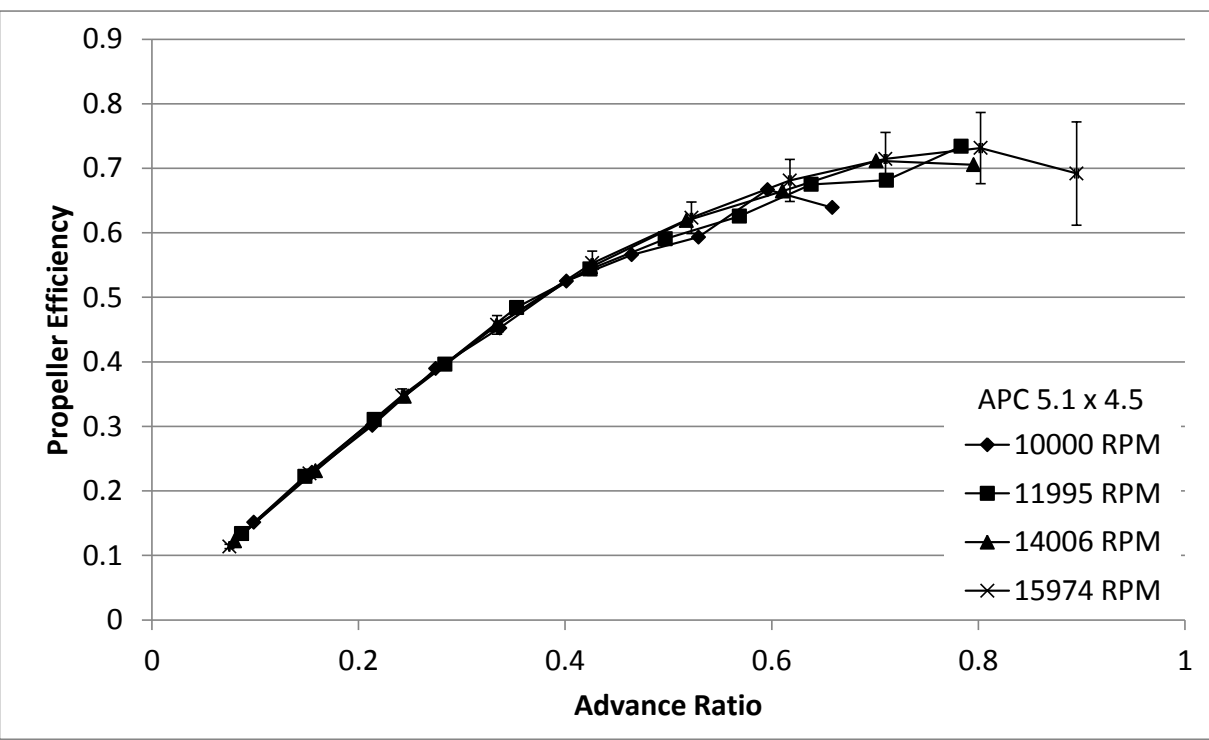

(c) 


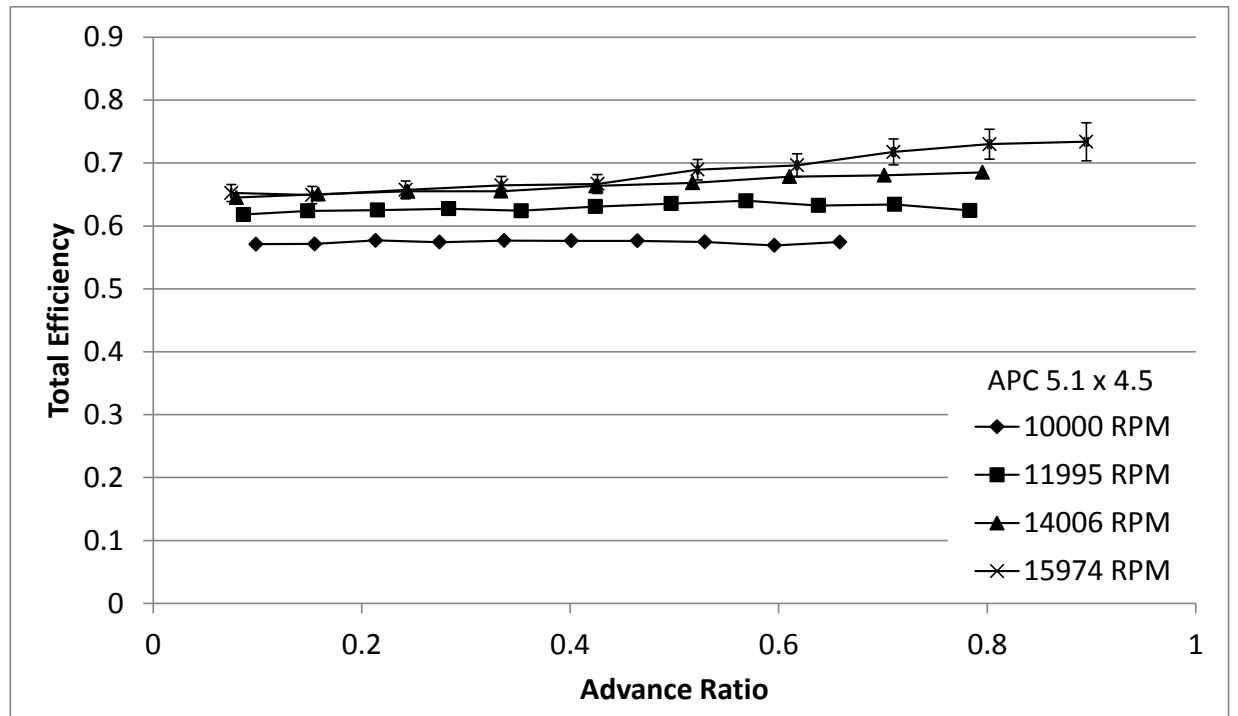

(d)

Figure 86: APC 5.1 x 4.5 Dynamic Test Results: (a) Coefficient of Thrust, (b) Coefficient of Power, (c) Propeller Efficiency, (d) Total Efficiency.

Table 138: APC 5.1 x 4.5 Dynamic Measured Values - 10000 RPM

\begin{tabular}{|c|c|c|c|c|c|c|c|}
\hline$n(\mathrm{RPM})$ & $Q(\mathrm{~g}-\mathrm{m})$ & $\Delta Q(\mathrm{~g}-\mathrm{m})$ & $V(\mathrm{~V})$ & $I(\mathrm{~A})$ & $T_{\text {atm }}\left({ }^{\circ} \mathrm{C}\right)$ & $P_{\text {atm }}(\mathrm{Pa})$ & $P_{\text {diff }}(\mathrm{Pa})$ \\
\hline $9.962 \mathrm{E}+03$ & $2.003 \mathrm{E}+00$ & $6.70 \mathrm{E}-02$ & $1.102 \mathrm{E}+01$ & $3.255 \mathrm{E}+00$ & $2.374 \mathrm{E}+01$ & $9.836 \mathrm{E}+04$ & $2.901 \mathrm{E}+00$ \\
\hline $1.000 \mathrm{E}+04$ & $1.990 \mathrm{E}+00$ & $6.66 \mathrm{E}-02$ & $1.102 \mathrm{E}+01$ & $3.245 \mathrm{E}+00$ & $2.379 \mathrm{E}+01$ & $9.837 \mathrm{E}+04$ & $6.914 \mathrm{E}+00$ \\
\hline $1.003 \mathrm{E}+04$ & $1.992 \mathrm{E}+00$ & $7.44 \mathrm{E}-02$ & $1.103 \mathrm{E}+01$ & $3.227 \mathrm{E}+00$ & $2.389 \mathrm{E}+01$ & $9.837 \mathrm{E}+04$ & $1.291 \mathrm{E}+01$ \\
\hline $1.001 \mathrm{E}+04$ & $1.941 \mathrm{E}+00$ & $6.74 \mathrm{E}-02$ & $1.103 \mathrm{E}+01$ & $3.151 \mathrm{E}+00$ & $2.390 \mathrm{E}+01$ & $9.836 \mathrm{E}+04$ & $2.102 \mathrm{E}+01$ \\
\hline $1.001 \mathrm{E}+04$ & $1.903 \mathrm{E}+00$ & $6.75 \mathrm{E}-02$ & $1.103 \mathrm{E}+01$ & $3.076 \mathrm{E}+00$ & $2.388 \mathrm{E}+01$ & $9.836 \mathrm{E}+04$ & $3.132 \mathrm{E}+01$ \\
\hline $9.985 \mathrm{E}+03$ & $1.838 \mathrm{E}+00$ & $6.67 \mathrm{E}-02$ & $1.103 \mathrm{E}+01$ & $2.965 \mathrm{E}+00$ & $2.401 \mathrm{E}+01$ & $9.836 \mathrm{E}+04$ & $4.392 \mathrm{E}+01$ \\
\hline $1.003 \mathrm{E}+04$ & $1.784 \mathrm{E}+00$ & $6.97 \mathrm{E}-02$ & $1.103 \mathrm{E}+01$ & $2.890 \mathrm{E}+00$ & $2.407 \mathrm{E}+01$ & $9.836 \mathrm{E}+04$ & $5.911 \mathrm{E}+01$ \\
\hline $9.999 \mathrm{E}+03$ & $1.689 \mathrm{E}+00$ & $6.64 \mathrm{E}-02$ & $1.104 \mathrm{E}+01$ & $2.734 \mathrm{E}+00$ & $2.410 \mathrm{E}+01$ & $9.836 \mathrm{E}+04$ & $7.595 \mathrm{E}+01$ \\
\hline $9.972 \mathrm{E}+03$ & $1.558 \mathrm{E}+00$ & $6.65 \mathrm{E}-02$ & $1.104 \mathrm{E}+01$ & $2.539 \mathrm{E}+00$ & $2.418 \mathrm{E}+01$ & $9.838 \mathrm{E}+04$ & $9.552 \mathrm{E}+01$ \\
\hline $1.000 \mathrm{E}+04$ & $1.472 \mathrm{E}+00$ & $6.57 \mathrm{E}-02$ & $1.105 \mathrm{E}+01$ & $2.383 \mathrm{E}+00$ & $2.418 \mathrm{E}+01$ & $9.840 \mathrm{E}+04$ & $1.172 \mathrm{E}+02$ \\
\hline
\end{tabular}


Table 139: APC 5.1 x 4.5 Dynamic Calculated Values - 10000 RPM

\begin{tabular}{|c|c|c|c|c|c|c|c|}
\hline$n(\mathrm{RPM})$ & $V_{\infty}^{\prime}(\mathrm{m} / \mathrm{s})$ & $\Delta V_{\infty}^{\prime}(\mathrm{m} / \mathrm{s})$ & $T^{\prime}(\mathrm{g})$ & $\Delta T^{\prime}(\mathrm{g})$ & $V_{\mathrm{t}}(\mathrm{m} / \mathrm{s})$ & $P_{\mathrm{P}}(\mathrm{W})$ & $\Delta P_{\mathrm{P}}(\mathrm{W})$ \\
\hline $9.962 \mathrm{E}+03$ & $2.121 \mathrm{E}+00$ & $1.45 \mathrm{E}-02$ & $1.493 \mathrm{E}+02$ & $1.25 \mathrm{E}+01$ & $5.066 \mathrm{E}+01$ & $2.049 \mathrm{E}+01$ & $6.85 \mathrm{E}-01$ \\
\hline $1.000 \mathrm{E}+04$ & $3.345 \mathrm{E}+00$ & $1.51 \mathrm{E}-02$ & $1.425 \mathrm{E}+02$ & $1.25 \mathrm{E}+01$ & $5.093 \mathrm{E}+01$ & $2.044 \mathrm{E}+01$ & $6.84 \mathrm{E}-01$ \\
\hline $1.003 \mathrm{E}+04$ & $4.619 \mathrm{E}+00$ & $1.59 \mathrm{E}-02$ & $1.366 \mathrm{E}+02$ & $1.28 \mathrm{E}+01$ & $5.118 \mathrm{E}+01$ & $2.052 \mathrm{E}+01$ & 7.67E-01 \\
\hline $1.001 \mathrm{E}+04$ & $5.931 \mathrm{E}+00$ & $1.73 \mathrm{E}-02$ & $1.336 \mathrm{E}+02$ & $1.25 \mathrm{E}+01$ & $5.119 \mathrm{E}+01$ & $1.995 \mathrm{E}+01$ & $6.92 \mathrm{E}-01$ \\
\hline $1.001 \mathrm{E}+04$ & $7.272 \mathrm{E}+00$ & $1.90 \mathrm{E}-02$ & $1.241 \mathrm{E}+02$ & $1.25 \mathrm{E}+01$ & $5.138 \mathrm{E}+01$ & $1.957 \mathrm{E}+01$ & $6.94 \mathrm{E}-01$ \\
\hline $9.985 \mathrm{E}+03$ & $8.640 \mathrm{E}+00$ & 2.12E-02 & $1.168 \mathrm{E}+02$ & $1.23 \mathrm{E}+01$ & $5.147 \mathrm{E}+01$ & $1.885 \mathrm{E}+01$ & $6.84 \mathrm{E}-01$ \\
\hline $1.003 \mathrm{E}+04$ & $1.005 \mathrm{E}+01$ & $2.34 \mathrm{E}-02$ & $1.056 \mathrm{E}+02$ & $1.25 \mathrm{E}+01$ & $5.194 \mathrm{E}+01$ & $1.838 \mathrm{E}+01$ & $7.18 \mathrm{E}-01$ \\
\hline $9.999 \mathrm{E}+03$ & $1.141 \mathrm{E}+01$ & $2.51 \mathrm{E}-02$ & $9.195 \mathrm{E}+01$ & $1.25 \mathrm{E}+01$ & $5.207 \mathrm{E}+01$ & $1.734 \mathrm{E}+01$ & $6.82 \mathrm{E}-01$ \\
\hline $9.972 \mathrm{E}+03$ & $1.282 \mathrm{E}+01$ & $2.70 \mathrm{E}-02$ & $8.466 \mathrm{E}+01$ & $1.25 \mathrm{E}+01$ & $5.226 \mathrm{E}+01$ & $1.596 \mathrm{E}+01$ & $6.81 \mathrm{E}-01$ \\
\hline $1.000 \mathrm{E}+04$ & $1.421 \mathrm{E}+01$ & $2.90 \mathrm{E}-02$ & $6.934 \mathrm{E}+01$ & $1.25 \mathrm{E}+01$ & $5.278 \mathrm{E}+01$ & $1.513 \mathrm{E}+01$ & $6.75 \mathrm{E}-01$ \\
\hline$P_{\mathrm{e}}(\mathrm{W})$ & $\Delta P_{\mathrm{e}}(\mathrm{W})$ & $C_{\mathrm{T}}$ & $\Delta C_{\mathrm{T}}$ & $C_{\mathrm{P}}$ & $\Delta C_{\mathrm{P}}$ & $C_{\mathrm{Q}}$ & $\Delta C_{\mathrm{Q}}$ \\
\hline $3.589 \mathrm{E}+01$ & $4.08 \mathrm{E}-01$ & $1.641 \mathrm{E}-01$ & $1.37 \mathrm{E}-02$ & $1.069 \mathrm{E}-01$ & $3.58 \mathrm{E}-03$ & 1.702E-02 & $5.69 \mathrm{E}-04$ \\
\hline $3.577 \mathrm{E}+01$ & $4.06 \mathrm{E}-01$ & $1.554 \mathrm{E}-01$ & $1.36 \mathrm{E}-02$ & $1.054 \mathrm{E}-01$ & $3.53 \mathrm{E}-03$ & $1.677 \mathrm{E}-02$ & $5.61 \mathrm{E}-04$ \\
\hline $3.558 \mathrm{E}+01$ & 4.04E-01 & $1.482 \mathrm{E}-01$ & $1.39 \mathrm{E}-02$ & $1.049 \mathrm{E}-01$ & $3.92 \mathrm{E}-03$ & $1.670 \mathrm{E}-02$ & $6.24 \mathrm{E}-04$ \\
\hline $3.474 \mathrm{E}+01$ & $3.95 \mathrm{E}-01$ & $1.457 \mathrm{E}-01$ & $1.37 \mathrm{E}-02$ & $1.028 \mathrm{E}-01$ & $3.57 \mathrm{E}-03$ & $1.635 \mathrm{E}-02$ & $5.68 \mathrm{E}-04$ \\
\hline $3.392 \mathrm{E}+01$ & $3.84 \mathrm{E}-01$ & $1.352 \mathrm{E}-01$ & $1.37 \mathrm{E}-02$ & $1.006 \mathrm{E}-01$ & $3.57 \mathrm{E}-03$ & $1.602 \mathrm{E}-02$ & $5.68 \mathrm{E}-04$ \\
\hline $3.271 \mathrm{E}+01$ & 3.67E-01 & $1.280 \mathrm{E}-01$ & $1.35 \mathrm{E}-02$ & $9.778 \mathrm{E}-02$ & $3.55 \mathrm{E}-03$ & $1.556 \mathrm{E}-02$ & $5.65 \mathrm{E}-04$ \\
\hline $3.189 \mathrm{E}+01$ & $3.60 \mathrm{E}-01$ & $1.146 \mathrm{E}-01$ & $1.35 \mathrm{E}-02$ & $9.407 \mathrm{E}-02$ & $3.67 \mathrm{E}-03$ & 1.497E-02 & $5.85 \mathrm{E}-04$ \\
\hline $3.018 \mathrm{E}+01$ & $3.40 \mathrm{E}-01$ & $1.004 \mathrm{E}-01$ & $1.36 \mathrm{E}-02$ & 8.959E-02 & $3.52 \mathrm{E}-03$ & $1.426 \mathrm{E}-02$ & $5.61 \mathrm{E}-04$ \\
\hline $2.805 \mathrm{E}+01$ & $3.17 \mathrm{E}-01$ & $9.302 \mathrm{E}-02$ & $1.37 \mathrm{E}-02$ & $8.313 \mathrm{E}-02$ & $3.55 \mathrm{E}-03$ & $1.323 \mathrm{E}-02$ & $5.64 \mathrm{E}-04$ \\
\hline $2.633 \mathrm{E}+01$ & $3.00 \mathrm{E}-01$ & 7.567E-02 & $1.36 \mathrm{E}-02$ & 7.802E-02 & $3.48 \mathrm{E}-03$ & $1.242 \mathrm{E}-02$ & $5.54 \mathrm{E}-04$ \\
\hline$\rho\left(\mathrm{kg} / \mathrm{m}^{\wedge} 3\right)$ & $\eta_{\mathrm{T}}$ & $\Delta \eta_{\mathrm{T}}$ & $J$ & $\Delta J$ & $\eta_{\mathrm{P}}$ & $\Delta \eta_{\mathrm{P}}$ & $R e_{0.75}$ \\
\hline $1.154 \mathrm{E}+00$ & $5.711 \mathrm{E}-01$ & $2.02 \mathrm{E}-02$ & $9.870 \mathrm{E}-02$ & $6.74 \mathrm{E}-04$ & $1.515 \mathrm{E}-01$ & $1.37 \mathrm{E}-02$ & $4.876 \mathrm{E}+04$ \\
\hline $1.154 \mathrm{E}+00$ & $5.713 \mathrm{E}-01$ & $2.02 \mathrm{E}-02$ & $1.551 \mathrm{E}-01$ & 7.03E-04 & $2.287 \mathrm{E}-01$ & $2.14 \mathrm{E}-02$ & $4.901 \mathrm{E}+04$ \\
\hline $1.154 \mathrm{E}+00$ & $5.769 \mathrm{E}-01$ & $2.25 \mathrm{E}-02$ & $2.135 \mathrm{E}-01$ & 7.37E-04 & $3.016 \mathrm{E}-01$ & $3.04 \mathrm{E}-02$ & $4.922 \mathrm{E}+04$ \\
\hline $1.154 \mathrm{E}+00$ & $5.742 \mathrm{E}-01$ & $2.10 \mathrm{E}-02$ & $2.748 \mathrm{E}-01$ & $8.07 \mathrm{E}-04$ & $3.895 \mathrm{E}-01$ & $3.90 \mathrm{E}-02$ & $4.922 \mathrm{E}+04$ \\
\hline $1.154 \mathrm{E}+00$ & 5.767E-01 & $2.15 \mathrm{E}-02$ & $3.368 \mathrm{E}-01$ & $8.88 \mathrm{E}-04$ & 4.524E-01 & $4.85 \mathrm{E}-02$ & $4.941 \mathrm{E}+04$ \\
\hline $1.153 \mathrm{E}+00$ & $5.763 \mathrm{E}-01$ & $2.19 \mathrm{E}-02$ & $4.012 \mathrm{E}-01$ & $9.93 \mathrm{E}-04$ & $5.251 \mathrm{E}-01$ & $5.86 \mathrm{E}-02$ & $4.945 \mathrm{E}+04$ \\
\hline $1.153 \mathrm{E}+00$ & $5.764 \mathrm{E}-01$ & $2.34 \mathrm{E}-02$ & 4.645E-01 & $1.09 \mathrm{E}-03$ & $5.659 \mathrm{E}-01$ & $7.04 \mathrm{E}-02$ & $4.990 \mathrm{E}+04$ \\
\hline $1.153 \mathrm{E}+00$ & $5.746 \mathrm{E}-01$ & $2.35 \mathrm{E}-02$ & 5.292E-01 & $1.17 \mathrm{E}-03$ & $5.933 \mathrm{E}-01$ & 8.37E-02 & $5.001 \mathrm{E}+04$ \\
\hline $1.153 \mathrm{E}+00$ & $5.690 \mathrm{E}-01$ & $2.51 \mathrm{E}-02$ & 5.959E-01 & $1.27 \mathrm{E}-03$ & $6.668 \mathrm{E}-01$ & $1.02 \mathrm{E}-01$ & $5.018 \mathrm{E}+04$ \\
\hline $1.153 \mathrm{E}+00$ & $5.744 \mathrm{E}-01$ & $2.65 \mathrm{E}-02$ & $6.588 \mathrm{E}-01$ & $1.37 \mathrm{E}-03$ & $6.390 \mathrm{E}-01$ & $1.18 \mathrm{E}-01$ & $5.069 \mathrm{E}+04$ \\
\hline
\end{tabular}


Table 140: APC 5.1 x 4.5 Dynamic Measured Values - 11995 RPM

\begin{tabular}{|c|c|c|c|c|c|c|c|}
\hline$n(\mathrm{RPM})$ & $Q(\mathrm{~g}-\mathrm{m})$ & $\Delta Q(\mathrm{~g}-\mathrm{m})$ & $V(\mathrm{~V})$ & $I(\mathrm{~A})$ & $T_{\text {atm }}\left({ }^{\circ} \mathrm{C}\right)$ & $P_{\text {atm }}(\mathrm{Pa})$ & $P_{\text {diff }}(\mathrm{Pa})$ \\
\hline $1.197 \mathrm{E}+04$ & $2.916 \mathrm{E}+00$ & $8.17 \mathrm{E}-02$ & $1.097 \mathrm{E}+01$ & $5.288 \mathrm{E}+00$ & $2.380 \mathrm{E}+01$ & $9.840 \mathrm{E}+04$ & $3.284 \mathrm{E}+00$ \\
\hline $1.201 \mathrm{E}+04$ & $2.924 \mathrm{E}+00$ & $8.12 \mathrm{E}-02$ & $1.097 \mathrm{E}+01$ & $5.268 \mathrm{E}+00$ & $2.383 \mathrm{E}+01$ & $9.839 \mathrm{E}+04$ & $9.136 \mathrm{E}+00$ \\
\hline $1.197 \mathrm{E}+04$ & $2.871 \mathrm{E}+00$ & $8.04 \mathrm{E}-02$ & $1.097 \mathrm{E}+01$ & $5.144 \mathrm{E}+00$ & $2.388 \mathrm{E}+01$ & $9.838 \mathrm{E}+04$ & $1.867 \mathrm{E}+01$ \\
\hline $1.198 \mathrm{E}+04$ & $2.843 \mathrm{E}+00$ & $8.95 \mathrm{E}-02$ & $1.097 \mathrm{E}+01$ & $5.083 \mathrm{E}+00$ & $2.405 \mathrm{E}+01$ & $9.840 \mathrm{E}+04$ & $3.207 \mathrm{E}+01$ \\
\hline $1.199 \mathrm{E}+04$ & $2.746 \mathrm{E}+00$ & $8.95 \mathrm{E}-02$ & $1.098 \mathrm{E}+01$ & $4.937 \mathrm{E}+00$ & $2.406 \mathrm{E}+01$ & $9.840 \mathrm{E}+04$ & $4.934 \mathrm{E}+01$ \\
\hline $1.200 \mathrm{E}+04$ & $2.679 \mathrm{E}+00$ & $8.67 \mathrm{E}-02$ & $1.098 \mathrm{E}+01$ & $4.765 \mathrm{E}+00$ & $2.409 \mathrm{E}+01$ & $9.842 \mathrm{E}+04$ & $7.078 \mathrm{E}+01$ \\
\hline $1.197 \mathrm{E}+04$ & $2.558 \mathrm{E}+00$ & $7.68 \mathrm{E}-02$ & $1.099 \mathrm{E}+01$ & $4.504 \mathrm{E}+00$ & $2.416 \mathrm{E}+01$ & $9.842 \mathrm{E}+04$ & $9.623 \mathrm{E}+01$ \\
\hline $1.199 \mathrm{E}+04$ & $2.427 \mathrm{E}+00$ & $9.00 \mathrm{E}-02$ & $1.100 \mathrm{E}+01$ & $4.245 \mathrm{E}+00$ & $2.431 \mathrm{E}+01$ & $9.842 \mathrm{E}+04$ & $1.259 \mathrm{E}+02$ \\
\hline $1.204 \mathrm{E}+04$ & $2.226 \mathrm{E}+00$ & $8.81 \mathrm{E}-02$ & $1.100 \mathrm{E}+01$ & $3.953 \mathrm{E}+00$ & $2.442 \mathrm{E}+01$ & $9.842 \mathrm{E}+04$ & $1.594 \mathrm{E}+02$ \\
\hline $1.201 \mathrm{E}+04$ & $2.009 \mathrm{E}+00$ & $7.30 \mathrm{E}-02$ & $1.102 \mathrm{E}+01$ & $3.548 \mathrm{E}+00$ & $2.448 \mathrm{E}+01$ & $9.841 \mathrm{E}+04$ & $1.966 \mathrm{E}+02$ \\
\hline $1.201 \mathrm{E}+04$ & $1.745 \mathrm{E}+00$ & $7.37 \mathrm{E}-02$ & $1.103 \mathrm{E}+01$ & $3.125 \mathrm{E}+00$ & $2.447 \mathrm{E}+01$ & $9.841 \mathrm{E}+04$ & $2.381 \mathrm{E}+02$ \\
\hline
\end{tabular}


Table 141: APC 5.1 x 4.5 Dynamic Calculated Values - 11995 RPM

\begin{tabular}{|c|c|c|c|c|c|c|c|}
\hline$n(\mathrm{RPM})$ & $V_{\infty}^{\prime}(\mathrm{m} / \mathrm{s})$ & $\Delta V_{\infty}^{\prime}(\mathrm{m} / \mathrm{s})$ & $T^{\prime}(\mathrm{g})$ & $\Delta T^{\prime}(\mathrm{g})$ & $V_{\mathrm{t}}(\mathrm{m} / \mathrm{s})$ & $P_{\mathrm{P}}(\mathrm{W})$ & $\Delta P_{\mathrm{P}}(\mathrm{W})$ \\
\hline $1.197 \mathrm{E}+04$ & $2.238 \mathrm{E}+00$ & $1.59 \mathrm{E}-02$ & $2.182 \mathrm{E}+02$ & $1.04 \mathrm{E}+01$ & $6.085 \mathrm{E}+01$ & $3.584 \mathrm{E}+01$ & $1.00 \mathrm{E}+00$ \\
\hline $1.201 \mathrm{E}+04$ & $3.836 \mathrm{E}+00$ & $1.68 \mathrm{E}-02$ & $2.133 \mathrm{E}+02$ & $1.06 \mathrm{E}+01$ & $6.113 \mathrm{E}+01$ & $3.605 \mathrm{E}+01$ & $1.00 \mathrm{E}+00$ \\
\hline $1.197 \mathrm{E}+04$ & $5.554 \mathrm{E}+00$ & $1.78 \mathrm{E}-02$ & $2.013 \mathrm{E}+02$ & $1.06 \mathrm{E}+01$ & $6.106 \mathrm{E}+01$ & $3.529 \mathrm{E}+01$ & $9.89 \mathrm{E}-01$ \\
\hline $1.198 \mathrm{E}+04$ & $7.330 \mathrm{E}+00$ & $2.01 \mathrm{E}-02$ & $1.929 \mathrm{E}+02$ & $1.05 \mathrm{E}+01$ & $6.133 \mathrm{E}+01$ & $3.499 \mathrm{E}+01$ & $1.10 \mathrm{E}+00$ \\
\hline $1.199 \mathrm{E}+04$ & $9.133 \mathrm{E}+00$ & $2.26 \mathrm{E}-02$ & $1.829 \mathrm{E}+02$ & $1.05 \mathrm{E}+01$ & $6.162 \mathrm{E}+01$ & $3.382 \mathrm{E}+01$ & $1.10 \mathrm{E}+00$ \\
\hline $1.200 \mathrm{E}+04$ & $1.097 \mathrm{E}+01$ & $2.62 \mathrm{E}-02$ & $1.668 \mathrm{E}+02$ & $1.05 \mathrm{E}+01$ & $6.193 \mathrm{E}+01$ & $3.300 \mathrm{E}+01$ & $1.07 \mathrm{E}+00$ \\
\hline $1.197 \mathrm{E}+04$ & $1.283 \mathrm{E}+01$ & $2.80 \mathrm{E}-02$ & $1.476 \mathrm{E}+02$ & $1.04 \mathrm{E}+01$ & $6.217 \mathrm{E}+01$ & $3.145 \mathrm{E}+01$ & $9.44 \mathrm{E}-01$ \\
\hline $1.199 \mathrm{E}+04$ & $1.471 \mathrm{E}+01$ & $3.04 \mathrm{E}-02$ & $1.296 \mathrm{E}+02$ & $1.04 \mathrm{E}+01$ & $6.267 \mathrm{E}+01$ & $2.988 \mathrm{E}+01$ & $1.11 \mathrm{E}+00$ \\
\hline $1.204 \mathrm{E}+04$ & $1.657 \mathrm{E}+01$ & $3.32 \mathrm{E}-02$ & $1.143 \mathrm{E}+02$ & $1.04 \mathrm{E}+01$ & $6.337 \mathrm{E}+01$ & $2.752 \mathrm{E}+01$ & $1.09 \mathrm{E}+00$ \\
\hline $1.201 \mathrm{E}+04$ & $1.843 \mathrm{E}+01$ & $3.57 \mathrm{E}-02$ & $9.349 \mathrm{E}+01$ & $1.04 \mathrm{E}+01$ & $6.376 \mathrm{E}+01$ & $2.479 \mathrm{E}+01$ & $9.01 \mathrm{E}-01$ \\
\hline $1.201 \mathrm{E}+04$ & $2.029 \mathrm{E}+01$ & $3.89 \mathrm{E}-02$ & $7.938 \mathrm{E}+01$ & $1.04 \mathrm{E}+01$ & $6.431 \mathrm{E}+01$ & $2.152 \mathrm{E}+01$ & $9.09 \mathrm{E}-01$ \\
\hline$P_{\mathrm{e}}(\mathrm{W})$ & $\Delta P_{\mathrm{e}}(\mathrm{W})$ & $C_{\mathrm{T}}$ & $\Delta C_{\mathrm{T}}$ & $C_{\mathrm{P}}$ & $\Delta C_{\mathrm{P}}$ & $C_{\mathrm{Q}}$ & $\Delta C_{\mathrm{Q}}$ \\
\hline $5.799 \mathrm{E}+01$ & $6.66 \mathrm{E}-01$ & $1.662 \mathrm{E}-01$ & $7.93 \mathrm{E}-03$ & $1.078 \mathrm{E}-01$ & $3.02 \mathrm{E}-03$ & $1.716 \mathrm{E}-02$ & $4.81 \mathrm{E}-04$ \\
\hline $5.778 \mathrm{E}+01$ & $6.58 \mathrm{E}-01$ & $1.614 \mathrm{E}-01$ & $8.00 \mathrm{E}-03$ & $1.074 \mathrm{E}-01$ & $2.99 \mathrm{E}-03$ & $1.710 \mathrm{E}-02$ & $4.75 \mathrm{E}-04$ \\
\hline $5.644 \mathrm{E}+01$ & $6.44 \mathrm{E}-01$ & $1.534 \mathrm{E}-01$ & 8.06E-03 & $1.062 \mathrm{E}-01$ & $2.98 \mathrm{E}-03$ & $1.690 \mathrm{E}-02$ & 4.74E-04 \\
\hline $5.577 \mathrm{E}+01$ & $6.44 \mathrm{E}-01$ & $1.467 \mathrm{E}-01$ & $8.00 \mathrm{E}-03$ & 1.049E-01 & $3.31 \mathrm{E}-03$ & $1.670 \mathrm{E}-02$ & $5.26 \mathrm{E}-04$ \\
\hline $5.419 \mathrm{E}+01$ & $6.22 \mathrm{E}-01$ & $1.388 \mathrm{E}-01$ & $7.98 \mathrm{E}-03$ & $1.012 \mathrm{E}-01$ & $3.30 \mathrm{E}-03$ & $1.610 \mathrm{E}-02$ & $5.25 \mathrm{E}-04$ \\
\hline $5.232 \mathrm{E}+01$ & $6.01 \mathrm{E}-01$ & $1.265 \mathrm{E}-01$ & $7.96 \mathrm{E}-03$ & $9.868 \mathrm{E}-02$ & $3.20 \mathrm{E}-03$ & $1.571 \mathrm{E}-02$ & $5.09 \mathrm{E}-04$ \\
\hline $4.949 \mathrm{E}+01$ & $5.65 \mathrm{E}-01$ & $1.125 \mathrm{E}-01$ & 7.93E-03 & $9.461 \mathrm{E}-02$ & $2.84 \mathrm{E}-03$ & $1.506 \mathrm{E}-02$ & 4.52E-04 \\
\hline $4.667 \mathrm{E}+01$ & $5.30 \mathrm{E}-01$ & $9.851 \mathrm{E}-02$ & $7.93 \mathrm{E}-03$ & $8.955 \mathrm{E}-02$ & $3.32 \mathrm{E}-03$ & $1.425 \mathrm{E}-02$ & $5.28 \mathrm{E}-04$ \\
\hline $4.350 \mathrm{E}+01$ & $4.95 \mathrm{E}-01$ & $8.618 \mathrm{E}-02$ & $7.88 \mathrm{E}-03$ & 8.149E-02 & $3.23 \mathrm{E}-03$ & $1.297 \mathrm{E}-02$ & $5.14 \mathrm{E}-04$ \\
\hline $3.908 \mathrm{E}+01$ & 4.39E-01 & $7.082 \mathrm{E}-02$ & 7.91E-03 & 7.391E-02 & $2.69 \mathrm{E}-03$ & $1.176 \mathrm{E}-02$ & $4.28 \mathrm{E}-04$ \\
\hline $3.446 \mathrm{E}+01$ & $3.86 \mathrm{E}-01$ & $6.016 \mathrm{E}-02$ & 7.89E-03 & $6.422 \mathrm{E}-02$ & $2.71 \mathrm{E}-03$ & $1.022 \mathrm{E}-02$ & 4.32E-04 \\
\hline$\rho\left(\mathrm{kg} / \mathrm{m}^{\wedge} 3\right)$ & $\eta_{\mathrm{T}}$ & $\Delta \eta_{\mathrm{T}}$ & $J$ & $\Delta J$ & $\eta_{\mathrm{P}}$ & $\Delta \eta_{\mathrm{P}}$ & $R e_{0.75}$ \\
\hline $1.154 \mathrm{E}+00$ & $6.181 \mathrm{E}-01$ & $1.87 \mathrm{E}-02$ & $8.672 \mathrm{E}-02$ & $6.16 \mathrm{E}-04$ & $1.336 \mathrm{E}-01$ & $7.46 \mathrm{E}-03$ & $5.857 \mathrm{E}+04$ \\
\hline $1.154 \mathrm{E}+00$ & $6.240 \mathrm{E}-01$ & $1.87 \mathrm{E}-02$ & $1.481 \mathrm{E}-01$ & $6.50 \mathrm{E}-04$ & $2.226 \mathrm{E}-01$ & $1.27 \mathrm{E}-02$ & $5.882 \mathrm{E}+04$ \\
\hline $1.154 \mathrm{E}+00$ & $6.252 \mathrm{E}-01$ & $1.89 \mathrm{E}-02$ & $2.152 \mathrm{E}-01$ & $6.94 \mathrm{E}-04$ & $3.107 \mathrm{E}-01$ & $1.85 \mathrm{E}-02$ & $5.874 \mathrm{E}+04$ \\
\hline $1.153 \mathrm{E}+00$ & $6.274 \mathrm{E}-01$ & $2.10 \mathrm{E}-02$ & $2.836 \mathrm{E}-01$ & $7.81 \mathrm{E}-04$ & 3.964E-01 & $2.50 \mathrm{E}-02$ & $5.894 \mathrm{E}+04$ \\
\hline $1.153 \mathrm{E}+00$ & $6.241 \mathrm{E}-01$ & $2.16 \mathrm{E}-02$ & $3.531 \mathrm{E}-01$ & $8.82 \mathrm{E}-04$ & $4.843 \mathrm{E}-01$ & $3.20 \mathrm{E}-02$ & $5.922 \mathrm{E}+04$ \\
\hline $1.153 \mathrm{E}+00$ & $6.308 \mathrm{E}-01$ & $2.17 \mathrm{E}-02$ & 4.242E-01 & $1.02 \mathrm{E}-03$ & $5.438 \mathrm{E}-01$ & $3.85 \mathrm{E}-02$ & $5.952 \mathrm{E}+04$ \\
\hline $1.153 \mathrm{E}+00$ & $6.354 \mathrm{E}-01$ & $2.04 \mathrm{E}-02$ & 4.969E-01 & $1.09 \mathrm{E}-03$ & 5.907E-01 & $4.53 \mathrm{E}-02$ & $5.972 \mathrm{E}+04$ \\
\hline $1.153 \mathrm{E}+00$ & $6.401 \mathrm{E}-01$ & $2.48 \mathrm{E}-02$ & $5.687 \mathrm{E}-01$ & $1.19 \mathrm{E}-03$ & $6.257 \mathrm{E}-01$ & $5.55 \mathrm{E}-02$ & $6.015 \mathrm{E}+04$ \\
\hline $1.152 \mathrm{E}+00$ & $6.326 \mathrm{E}-01$ & 2.61E-02 & $6.382 \mathrm{E}-01$ & $1.29 \mathrm{E}-03$ & $6.750 \mathrm{E}-01$ & $6.72 \mathrm{E}-02$ & $6.079 \mathrm{E}+04$ \\
\hline $1.152 \mathrm{E}+00$ & $6.343 \mathrm{E}-01$ & $2.41 \mathrm{E}-02$ & 7.112E-01 & $1.40 \mathrm{E}-03$ & $6.815 \mathrm{E}-01$ & $8.01 \mathrm{E}-02$ & $6.113 \mathrm{E}+04$ \\
\hline $1.152 \mathrm{E}+00$ & $6.245 \mathrm{E}-01$ & $2.73 \mathrm{E}-02$ & 7.834E-01 & $1.52 \mathrm{E}-03$ & 7.339E-01 & $1.01 \mathrm{E}-01$ & $6.166 \mathrm{E}+04$ \\
\hline
\end{tabular}


Table 142: APC 5.1 x 4.5 Dynamic Measured Values - 14006 RPM

\begin{tabular}{|c|c|c|c|c|c|c|c|}
\hline$n(\mathrm{RPM})$ & $Q(\mathrm{~g}-\mathrm{m})$ & $\Delta Q(\mathrm{~g}-\mathrm{m})$ & $V(\mathrm{~V})$ & $I(\mathrm{~A})$ & $T_{\text {atm }}\left({ }^{\circ} \mathrm{C}\right)$ & $P_{\text {atm }}(\mathrm{Pa})$ & $P_{\text {diff }}(\mathrm{Pa})$ \\
\hline $1.392 \mathrm{E}+04$ & $4.031 \mathrm{E}+00$ & $8.04 \mathrm{E}-02$ & $1.088 \mathrm{E}+01$ & $8.209 \mathrm{E}+00$ & $2.343 \mathrm{E}+01$ & $9.843 \mathrm{E}+04$ & $3.827 \mathrm{E}+00$ \\
\hline $1.398 \mathrm{E}+04$ & $4.035 \mathrm{E}+00$ & $8.05 \mathrm{E}-02$ & $1.088 \mathrm{E}+01$ & $8.179 \mathrm{E}+00$ & $2.349 \mathrm{E}+01$ & $9.843 \mathrm{E}+04$ & $1.410 \mathrm{E}+01$ \\
\hline $1.404 \mathrm{E}+04$ & $4.005 \mathrm{E}+00$ & $8.27 \mathrm{E}-02$ & $1.089 \mathrm{E}+01$ & $8.096 \mathrm{E}+00$ & $2.378 \mathrm{E}+01$ & $9.842 \mathrm{E}+04$ & $3.291 \mathrm{E}+01$ \\
\hline $1.404 \mathrm{E}+04$ & $3.901 \mathrm{E}+00$ & $8.47 \mathrm{E}-02$ & $1.089 \mathrm{E}+01$ & $7.879 \mathrm{E}+00$ & $2.403 \mathrm{E}+01$ & $9.841 \mathrm{E}+04$ & $6.055 \mathrm{E}+01$ \\
\hline $1.403 \mathrm{E}+04$ & $3.747 \mathrm{E}+00$ & $8.32 \mathrm{E}-02$ & $1.090 \mathrm{E}+01$ & $7.459 \mathrm{E}+00$ & $2.416 \mathrm{E}+01$ & $9.839 \mathrm{E}+04$ & $9.704 \mathrm{E}+01$ \\
\hline $1.403 \mathrm{E}+04$ & $3.508 \mathrm{E}+00$ & $8.34 \mathrm{E}-02$ & $1.092 \mathrm{E}+01$ & $6.925 \mathrm{E}+00$ & $2.431 \mathrm{E}+01$ & $9.838 \mathrm{E}+04$ & $1.431 \mathrm{E}+02$ \\
\hline $1.401 \mathrm{E}+04$ & $3.214 \mathrm{E}+00$ & $8.31 \mathrm{E}-02$ & $1.094 \mathrm{E}+01$ & $6.233 \mathrm{E}+00$ & $2.442 \mathrm{E}+01$ & $9.838 \mathrm{E}+04$ & $1.977 \mathrm{E}+02$ \\
\hline $1.402 \mathrm{E}+04$ & $2.830 \mathrm{E}+00$ & $8.22 \mathrm{E}-02$ & $1.096 \mathrm{E}+01$ & $5.462 \mathrm{E}+00$ & $2.444 \mathrm{E}+01$ & $9.837 \mathrm{E}+04$ & $2.603 \mathrm{E}+02$ \\
\hline $1.398 \mathrm{E}+04$ & $2.391 \mathrm{E}+00$ & $8.05 \mathrm{E}-02$ & $1.099 \mathrm{E}+01$ & $4.561 \mathrm{E}+00$ & $2.459 \mathrm{E}+01$ & $9.837 \mathrm{E}+04$ & $3.324 \mathrm{E}+02$ \\
\hline
\end{tabular}


Table 143: APC 5.1 x 4.5 Dynamic Calculated Values - 14006 RPM

\begin{tabular}{|c|c|c|c|c|c|c|c|}
\hline$n(\mathrm{RPM})$ & $V_{\infty}^{\prime}(\mathrm{m} / \mathrm{s})$ & $\Delta V_{\infty}^{\prime}(\mathrm{m} / \mathrm{s})$ & $T^{\prime}(\mathrm{g})$ & $\Delta T^{\prime}(\mathrm{g})$ & $V_{\mathrm{t}}(\mathrm{m} / \mathrm{s})$ & $P_{\mathrm{P}}(\mathrm{W})$ & $\Delta P_{\mathrm{P}}(\mathrm{W})$ \\
\hline $1.392 \mathrm{E}+04$ & $2.400 \mathrm{E}+00$ & $1.77 \mathrm{E}-02$ & $3.003 \mathrm{E}+02$ & $1.13 \mathrm{E}+01$ & $7.078 \mathrm{E}+01$ & $5.764 \mathrm{E}+01$ & $1.15 \mathrm{E}+00$ \\
\hline $1.398 \mathrm{E}+04$ & $4.774 \mathrm{E}+00$ & $1.90 \mathrm{E}-02$ & $2.859 \mathrm{E}+02$ & $1.14 \mathrm{E}+01$ & $7.117 \mathrm{E}+01$ & $5.791 \mathrm{E}+01$ & $1.16 \mathrm{E}+00$ \\
\hline $1.404 \mathrm{E}+04$ & $7.394 \mathrm{E}+00$ & $2.17 \mathrm{E}-02$ & $2.759 \mathrm{E}+02$ & $1.15 \mathrm{E}+01$ & $7.170 \mathrm{E}+01$ & $5.773 \mathrm{E}+01$ & $1.19 \mathrm{E}+00$ \\
\hline $1.404 \mathrm{E}+04$ & $1.011 \mathrm{E}+01$ & 2.67E-02 & $2.592 \mathrm{E}+02$ & $1.14 \mathrm{E}+01$ & $7.204 \mathrm{E}+01$ & $5.623 \mathrm{E}+01$ & $1.22 \mathrm{E}+00$ \\
\hline $1.403 \mathrm{E}+04$ & $1.285 \mathrm{E}+01$ & $3.01 \mathrm{E}-02$ & $2.343 \mathrm{E}+02$ & $1.14 \mathrm{E}+01$ & $7.243 \mathrm{E}+01$ & $5.398 \mathrm{E}+01$ & $1.20 \mathrm{E}+00$ \\
\hline $1.403 \mathrm{E}+04$ & $1.566 \mathrm{E}+01$ & $3.29 \mathrm{E}-02$ & $2.038 \mathrm{E}+02$ & $1.13 \mathrm{E}+01$ & $7.300 \mathrm{E}+01$ & $5.056 \mathrm{E}+01$ & $1.20 \mathrm{E}+00$ \\
\hline $1.401 \mathrm{E}+04$ & $1.845 \mathrm{E}+01$ & $3.63 \mathrm{E}-02$ & $1.699 \mathrm{E}+02$ & $1.14 \mathrm{E}+01$ & $7.356 \mathrm{E}+01$ & $4.626 \mathrm{E}+01$ & $1.20 \mathrm{E}+00$ \\
\hline $1.402 \mathrm{E}+04$ & $2.120 \mathrm{E}+01$ & $4.11 \mathrm{E}-02$ & $1.395 \mathrm{E}+02$ & $1.13 \mathrm{E}+01$ & $7.431 \mathrm{E}+01$ & $4.075 \mathrm{E}+01$ & $1.18 \mathrm{E}+00$ \\
\hline $1.398 \mathrm{E}+04$ & $2.399 \mathrm{E}+01$ & $4.52 \mathrm{E}-02$ & $1.030 \mathrm{E}+02$ & $1.13 \mathrm{E}+01$ & $7.499 \mathrm{E}+01$ & $3.434 \mathrm{E}+01$ & $1.16 \mathrm{E}+00$ \\
\hline$P_{\mathrm{e}}(\mathrm{W})$ & $\Delta P_{\mathrm{e}}(\mathrm{W})$ & $C_{\mathrm{T}}$ & $\Delta C_{\mathrm{T}}$ & $C_{\mathrm{P}}$ & $\Delta C_{\mathrm{P}}$ & $C_{\mathrm{Q}}$ & $\Delta C_{\mathrm{Q}}$ \\
\hline $8.934 \mathrm{E}+01$ & $9.75 \mathrm{E}-01$ & $1.687 \mathrm{E}-01$ & $6.37 \mathrm{E}-03$ & $1.100 \mathrm{E}-01$ & $2.20 \mathrm{E}-03$ & $1.750 \mathrm{E}-02$ & $3.49 \mathrm{E}-04$ \\
\hline $8.902 \mathrm{E}+01$ & $9.69 \mathrm{E}-01$ & $1.594 \mathrm{E}-01$ & $6.34 \mathrm{E}-03$ & $1.093 \mathrm{E}-01$ & $2.18 \mathrm{E}-03$ & $1.739 \mathrm{E}-02$ & $3.47 \mathrm{E}-04$ \\
\hline $8.813 \mathrm{E}+01$ & $9.57 \mathrm{E}-01$ & $1.527 \mathrm{E}-01$ & $6.34 \mathrm{E}-03$ & $1.076 \mathrm{E}-01$ & $2.23 \mathrm{E}-03$ & $1.713 \mathrm{E}-02$ & $3.54 \mathrm{E}-04$ \\
\hline $8.582 \mathrm{E}+01$ & $9.33 \mathrm{E}-01$ & $1.436 \mathrm{E}-01$ & $6.31 \mathrm{E}-03$ & $1.049 \mathrm{E}-01$ & $2.28 \mathrm{E}-03$ & $1.670 \mathrm{E}-02$ & $3.63 \mathrm{E}-04$ \\
\hline $8.134 \mathrm{E}+01$ & $8.85 \mathrm{E}-01$ & $1.301 \mathrm{E}-01$ & $6.34 \mathrm{E}-03$ & $1.010 \mathrm{E}-01$ & $2.24 \mathrm{E}-03$ & $1.607 \mathrm{E}-02$ & $3.57 \mathrm{E}-04$ \\
\hline $7.562 \mathrm{E}+01$ & $8.42 \mathrm{E}-01$ & $1.131 \mathrm{E}-01$ & $6.29 \mathrm{E}-03$ & $9.454 \mathrm{E}-02$ & $2.25 \mathrm{E}-03$ & $1.505 \mathrm{E}-02$ & $3.58 \mathrm{E}-04$ \\
\hline $6.819 \mathrm{E}+01$ & $7.61 \mathrm{E}-01$ & $9.459 \mathrm{E}-02$ & $6.33 \mathrm{E}-03$ & $8.687 \mathrm{E}-02$ & $2.25 \mathrm{E}-03$ & $1.383 \mathrm{E}-02$ & $3.58 \mathrm{E}-04$ \\
\hline $5.987 \mathrm{E}+01$ & $6.74 \mathrm{E}-01$ & $7.760 \mathrm{E}-02$ & $6.28 \mathrm{E}-03$ & 7.647E-02 & $2.22 \mathrm{E}-03$ & $1.217 \mathrm{E}-02$ & $3.53 \mathrm{E}-04$ \\
\hline $5.011 \mathrm{E}+01$ & $5.61 \mathrm{E}-01$ & $5.762 \mathrm{E}-02$ & $6.31 \mathrm{E}-03$ & 6.497E-02 & $2.19 \mathrm{E}-03$ & $1.034 \mathrm{E}-02$ & $3.48 \mathrm{E}-04$ \\
\hline$\rho\left(\mathrm{kg} / \mathrm{m}^{\wedge} 3\right)$ & $\eta_{\mathrm{T}}$ & $\Delta \eta_{\mathrm{T}}$ & $J$ & $\Delta J$ & $\eta_{\mathrm{P}}$ & $\Delta \eta_{\mathrm{P}}$ & $R e_{0.75}$ \\
\hline $1.156 \mathrm{E}+00$ & $6.452 \mathrm{E}-01$ & $1.47 \mathrm{E}-02$ & 7.994E-02 & $5.91 \mathrm{E}-04$ & $1.226 \mathrm{E}-01$ & $5.31 \mathrm{E}-03$ & $6.830 \mathrm{E}+04$ \\
\hline $1.156 \mathrm{E}+00$ & $6.506 \mathrm{E}-01$ & $1.48 \mathrm{E}-02$ & $1.584 \mathrm{E}-01$ & $6.32 \mathrm{E}-04$ & $2.311 \mathrm{E}-01$ & $1.03 \mathrm{E}-02$ & $6.865 \mathrm{E}+04$ \\
\hline $1.155 \mathrm{E}+00$ & $6.551 \mathrm{E}-01$ & $1.53 \mathrm{E}-02$ & $2.443 \mathrm{E}-01$ & $7.21 \mathrm{E}-04$ & $3.465 \mathrm{E}-01$ & $1.61 \mathrm{E}-02$ & $6.904 \mathrm{E}+04$ \\
\hline $1.154 \mathrm{E}+00$ & $6.553 \mathrm{E}-01$ & $1.59 \mathrm{E}-02$ & $3.338 \mathrm{E}-01$ & $8.86 \mathrm{E}-04$ & $4.568 \mathrm{E}-01$ & $2.24 \mathrm{E}-02$ & $6.925 \mathrm{E}+04$ \\
\hline $1.153 \mathrm{E}+00$ & $6.637 \mathrm{E}-01$ & $1.64 \mathrm{E}-02$ & $4.248 \mathrm{E}-01$ & $1.00 \mathrm{E}-03$ & $5.471 \mathrm{E}-01$ & $2.94 \mathrm{E}-02$ & $6.956 \mathrm{E}+04$ \\
\hline $1.152 \mathrm{E}+00$ & $6.686 \mathrm{E}-01$ & $1.75 \mathrm{E}-02$ & $5.174 \mathrm{E}-01$ & $1.10 \mathrm{E}-03$ & $6.190 \mathrm{E}-01$ & $3.74 \mathrm{E}-02$ & $7.004 \mathrm{E}+04$ \\
\hline $1.152 \mathrm{E}+00$ & $6.784 \mathrm{E}-01$ & $1.91 \mathrm{E}-02$ & $6.104 \mathrm{E}-01$ & $1.21 \mathrm{E}-03$ & $6.646 \mathrm{E}-01$ & $4.77 \mathrm{E}-02$ & $7.052 \mathrm{E}+04$ \\
\hline $1.152 \mathrm{E}+00$ & $6.806 \mathrm{E}-01$ & $2.12 \mathrm{E}-02$ & 7.012E-01 & $1.37 \mathrm{E}-03$ & $7.116 \mathrm{E}-01$ & $6.12 \mathrm{E}-02$ & $7.124 \mathrm{E}+04$ \\
\hline $1.151 \mathrm{E}+00$ & $6.853 \mathrm{E}-01$ & $2.43 \mathrm{E}-02$ & $7.955 \mathrm{E}-01$ & $1.51 \mathrm{E}-03$ & $7.055 \mathrm{E}-01$ & $8.09 \mathrm{E}-02$ & $7.182 \mathrm{E}+04$ \\
\hline
\end{tabular}


Table 144: APC 5.1 x 4.5 Dynamic Measured Values - 15974 RPM

\begin{tabular}{|c|c|c|c|c|c|c|c|}
\hline$n(\mathrm{RPM})$ & $Q(\mathrm{~g}-\mathrm{m})$ & $\Delta Q(\mathrm{~g}-\mathrm{m})$ & $V(\mathrm{~V})$ & $I(\mathrm{~A})$ & $T_{\text {atm }}\left({ }^{\circ} \mathrm{C}\right)$ & $P_{\text {atm }}(\mathrm{Pa})$ & $P_{\text {diff }}(\mathrm{Pa})$ \\
\hline $1.584 \mathrm{E}+04$ & $5.342 \mathrm{E}+00$ & $9.43 \mathrm{E}-02$ & $1.076 \mathrm{E}+01$ & $1.238 \mathrm{E}+01$ & $2.376 \mathrm{E}+01$ & $9.836 \mathrm{E}+04$ & $4.393 \mathrm{E}+00$ \\
\hline $1.600 \mathrm{E}+04$ & $5.362 \mathrm{E}+00$ & $9.84 \mathrm{E}-02$ & $1.075 \mathrm{E}+01$ & $1.262 \mathrm{E}+01$ & $2.373 \mathrm{E}+01$ & $9.835 \mathrm{E}+04$ & $1.719 \mathrm{E}+01$ \\
\hline $1.597 \mathrm{E}+04$ & $5.270 \mathrm{E}+00$ & $9.89 \mathrm{E}-02$ & $1.076 \mathrm{E}+01$ & $1.221 \mathrm{E}+01$ & $2.413 \mathrm{E}+01$ & $9.833 \mathrm{E}+04$ & $4.181 \mathrm{E}+01$ \\
\hline $1.599 \mathrm{E}+04$ & $5.202 \mathrm{E}+00$ & $9.86 \mathrm{E}-02$ & $1.077 \mathrm{E}+01$ & $1.194 \mathrm{E}+01$ & $2.429 \mathrm{E}+01$ & $9.833 \mathrm{E}+04$ & $7.862 \mathrm{E}+01$ \\
\hline $1.603 \mathrm{E}+04$ & $4.982 \mathrm{E}+00$ & $9.96 \mathrm{E}-02$ & $1.079 \mathrm{E}+01$ & $1.141 \mathrm{E}+01$ & $2.441 \mathrm{E}+01$ & $9.833 \mathrm{E}+04$ & $1.275 \mathrm{E}+02$ \\
\hline $1.599 \mathrm{E}+04$ & $4.692 \mathrm{E}+00$ & $9.86 \mathrm{E}-02$ & $1.082 \mathrm{E}+01$ & $1.033 \mathrm{E}+01$ & $2.448 \mathrm{E}+01$ & $9.833 \mathrm{E}+04$ & $1.892 \mathrm{E}+02$ \\
\hline $1.594 \mathrm{E}+04$ & $4.203 \mathrm{E}+00$ & $9.92 \mathrm{E}-02$ & $1.086 \mathrm{E}+01$ & $9.098 \mathrm{E}+00$ & $2.453 \mathrm{E}+01$ & $9.832 \mathrm{E}+04$ & $2.616 \mathrm{E}+02$ \\
\hline $1.597 \mathrm{E}+04$ & $3.763 \mathrm{E}+00$ & $9.95 \mathrm{E}-02$ & $1.089 \mathrm{E}+01$ & $7.897 \mathrm{E}+00$ & $2.460 \mathrm{E}+01$ & $9.832 \mathrm{E}+04$ & $3.463 \mathrm{E}+02$ \\
\hline $1.600 \mathrm{E}+04$ & $3.191 \mathrm{E}+00$ & $9.80 \mathrm{E}-02$ & $1.093 \mathrm{E}+01$ & $6.573 \mathrm{E}+00$ & $2.480 \mathrm{E}+01$ & $9.831 \mathrm{E}+04$ & $4.423 \mathrm{E}+02$ \\
\hline $1.601 \mathrm{E}+04$ & $2.472 \mathrm{E}+00$ & $9.86 \mathrm{E}-02$ & $1.097 \mathrm{E}+01$ & $5.047 \mathrm{E}+00$ & $2.489 \mathrm{E}+01$ & $9.831 \mathrm{E}+04$ & $5.498 \mathrm{E}+02$ \\
\hline
\end{tabular}


Table 145: APC 5.1 x 4.5 Dynamic Calculated Values - 15974 RPM

\begin{tabular}{|c|c|c|c|c|c|c|c|}
\hline$n(\mathrm{RPM})$ & $V_{\infty}^{\prime}(\mathrm{m} / \mathrm{s})$ & $\Delta V_{\infty}^{\prime}(\mathrm{m} / \mathrm{s})$ & $T^{\prime}(\mathrm{g})$ & $\Delta T^{\prime}(\mathrm{g})$ & $V_{\mathrm{t}}(\mathrm{m} / \mathrm{s})$ & $P_{\mathrm{P}}(\mathrm{W})$ & $\Delta P_{\mathrm{P}}(\mathrm{W})$ \\
\hline $1.584 \mathrm{E}+04$ & $2.561 \mathrm{E}+00$ & $1.95 \mathrm{E}-02$ & $3.941 \mathrm{E}+02$ & $9.37 \mathrm{E}+00$ & $8.052 \mathrm{E}+01$ & $8.689 \mathrm{E}+01$ & $1.53 \mathrm{E}+00$ \\
\hline $1.600 \mathrm{E}+04$ & $5.265 \mathrm{E}+00$ & $2.14 \mathrm{E}-02$ & $3.871 \mathrm{E}+02$ & $8.54 \mathrm{E}+00$ & $8.145 \mathrm{E}+01$ & $8.807 \mathrm{E}+01$ & $1.62 \mathrm{E}+00$ \\
\hline $1.597 \mathrm{E}+04$ & $8.339 \mathrm{E}+00$ & $2.49 \mathrm{E}-02$ & $3.672 \mathrm{E}+02$ & $8.75 \mathrm{E}+00$ & $8.155 \mathrm{E}+01$ & $8.640 \mathrm{E}+01$ & $1.62 \mathrm{E}+00$ \\
\hline $1.599 \mathrm{E}+04$ & $1.152 \mathrm{E}+01$ & $3.02 \mathrm{E}-02$ & $3.458 \mathrm{E}+02$ & $8.70 \mathrm{E}+00$ & $8.205 \mathrm{E}+01$ & $8.542 \mathrm{E}+01$ & $1.62 \mathrm{E}+00$ \\
\hline $1.603 \mathrm{E}+04$ & $1.474 \mathrm{E}+01$ & $3.40 \mathrm{E}-02$ & $3.138 \mathrm{E}+02$ & $8.70 \mathrm{E}+00$ & $8.279 \mathrm{E}+01$ & $8.204 \mathrm{E}+01$ & $1.64 \mathrm{E}+00$ \\
\hline $1.599 \mathrm{E}+04$ & $1.801 \mathrm{E}+01$ & $3.68 \mathrm{E}-02$ & $2.719 \mathrm{E}+02$ & $8.92 \mathrm{E}+00$ & $8.323 \mathrm{E}+01$ & $7.705 \mathrm{E}+01$ & $1.62 \mathrm{E}+00$ \\
\hline $1.594 \mathrm{E}+04$ & $2.123 \mathrm{E}+01$ & 4.14E-02 & $2.251 \mathrm{E}+02$ & $9.32 \mathrm{E}+00$ & $8.372 \mathrm{E}+01$ & $6.879 \mathrm{E}+01$ & $1.62 \mathrm{E}+00$ \\
\hline $1.597 \mathrm{E}+04$ & $2.447 \mathrm{E}+01$ & 4.65E-02 & $1.838 \mathrm{E}+02$ & $9.44 \mathrm{E}+00$ & $8.477 \mathrm{E}+01$ & $6.172 \mathrm{E}+01$ & $1.63 \mathrm{E}+00$ \\
\hline $1.600 \mathrm{E}+04$ & $2.769 \mathrm{E}+01$ & $5.18 \mathrm{E}-02$ & $1.413 \mathrm{E}+02$ & $9.71 \mathrm{E}+00$ & $8.590 \mathrm{E}+01$ & $5.245 \mathrm{E}+01$ & $1.61 \mathrm{E}+00$ \\
\hline $1.601 \mathrm{E}+04$ & $3.090 \mathrm{E}+01$ & $5.69 \mathrm{E}-02$ & $9.278 \mathrm{E}+01$ & $1.01 \mathrm{E}+01$ & $8.701 \mathrm{E}+01$ & $4.064 \mathrm{E}+01$ & $1.62 \mathrm{E}+00$ \\
\hline$P_{\mathrm{e}}(\mathrm{W})$ & $\Delta P_{\mathrm{e}}(\mathrm{W})$ & $C_{\mathrm{T}}$ & $\Delta C_{\mathrm{T}}$ & $C_{\mathrm{P}}$ & $\Delta C_{\mathrm{P}}$ & $C_{\mathrm{Q}}$ & $\Delta C_{\mathrm{Q}}$ \\
\hline $1.332 \mathrm{E}+02$ & $1.42 \mathrm{E}+00$ & $1.714 \mathrm{E}-01$ & $4.08 \mathrm{E}-03$ & $1.128 \mathrm{E}-01$ & $1.99 \mathrm{E}-03$ & $1.796 \mathrm{E}-02$ & $3.17 \mathrm{E}-04$ \\
\hline $1.357 \mathrm{E}+02$ & $1.43 \mathrm{E}+00$ & $1.651 \mathrm{E}-01$ & $3.64 \mathrm{E}-03$ & $1.110 \mathrm{E}-01$ & $2.04 \mathrm{E}-03$ & $1.767 \mathrm{E}-02$ & $3.24 \mathrm{E}-04$ \\
\hline $1.314 \mathrm{E}+02$ & $1.39 \mathrm{E}+00$ & $1.574 \mathrm{E}-01$ & $3.75 \mathrm{E}-03$ & $1.097 \mathrm{E}-01$ & $2.06 \mathrm{E}-03$ & $1.746 \mathrm{E}-02$ & $3.28 \mathrm{E}-04$ \\
\hline $1.286 \mathrm{E}+02$ & $1.36 \mathrm{E}+00$ & $1.479 \mathrm{E}-01$ & $3.72 \mathrm{E}-03$ & $1.080 \mathrm{E}-01$ & $2.05 \mathrm{E}-03$ & 1.719E-02 & $3.26 \mathrm{E}-04$ \\
\hline $1.231 \mathrm{E}+02$ & $1.31 \mathrm{E}+00$ & $1.335 \mathrm{E}-01$ & $3.70 \mathrm{E}-03$ & $1.029 \mathrm{E}-01$ & $2.06 \mathrm{E}-03$ & $1.638 \mathrm{E}-02$ & $3.28 \mathrm{E}-04$ \\
\hline $1.117 \mathrm{E}+02$ & $1.19 \mathrm{E}+00$ & $1.163 \mathrm{E}-01$ & $3.82 \mathrm{E}-03$ & $9.745 \mathrm{E}-02$ & $2.05 \mathrm{E}-03$ & $1.551 \mathrm{E}-02$ & $3.26 \mathrm{E}-04$ \\
\hline $9.877 \mathrm{E}+01$ & $1.07 \mathrm{E}+00$ & $9.696 \mathrm{E}-02$ & $4.02 \mathrm{E}-03$ & 8.792E-02 & $2.08 \mathrm{E}-03$ & $1.399 \mathrm{E}-02$ & $3.30 \mathrm{E}-04$ \\
\hline $8.601 \mathrm{E}+01$ & $9.42 \mathrm{E}-01$ & $7.886 \mathrm{E}-02$ & $4.05 \mathrm{E}-03$ & $7.838 \mathrm{E}-02$ & $2.07 \mathrm{E}-03$ & $1.248 \mathrm{E}-02$ & $3.30 \mathrm{E}-04$ \\
\hline $7.185 \mathrm{E}+01$ & $7.97 \mathrm{E}-01$ & $6.043 \mathrm{E}-02$ & $4.15 \mathrm{E}-03$ & $6.628 \mathrm{E}-02$ & $2.04 \mathrm{E}-03$ & $1.055 \mathrm{E}-02$ & $3.24 \mathrm{E}-04$ \\
\hline $5.538 \mathrm{E}+01$ & $6.24 \mathrm{E}-01$ & $3.968 \mathrm{E}-02$ & 4.30E-03 & 5.133E-02 & $2.05 \mathrm{E}-03$ & $8.170 \mathrm{E}-03$ & $3.26 \mathrm{E}-04$ \\
\hline$\rho\left(\mathrm{kg} / \mathrm{m}^{\wedge} 3\right)$ & $\eta_{\mathrm{T}}$ & $\Delta \eta_{\mathrm{T}}$ & $J$ & $\Delta J$ & $\eta_{\mathrm{P}}$ & $\Delta \eta_{\mathrm{P}}$ & $R e_{0.75}$ \\
\hline $1.154 \mathrm{E}+00$ & $6.523 \mathrm{E}-01$ & $1.35 \mathrm{E}-02$ & 7.497E-02 & $5.71 \mathrm{E}-04$ & $1.139 \mathrm{E}-01$ & $3.48 \mathrm{E}-03$ & $7.748 \mathrm{E}+04$ \\
\hline $1.154 \mathrm{E}+00$ & $6.492 \mathrm{E}-01$ & $1.37 \mathrm{E}-02$ & $1.526 \mathrm{E}-01$ & $6.20 \mathrm{E}-04$ & $2.270 \mathrm{E}-01$ & $6.58 \mathrm{E}-03$ & $7.838 \mathrm{E}+04$ \\
\hline $1.152 \mathrm{E}+00$ & $6.573 \mathrm{E}-01$ & $1.42 \mathrm{E}-02$ & $2.422 \mathrm{E}-01$ & $7.26 \mathrm{E}-04$ & $3.476 \mathrm{E}-01$ & $1.06 \mathrm{E}-02$ & $7.829 \mathrm{E}+04$ \\
\hline $1.152 \mathrm{E}+00$ & $6.644 \mathrm{E}-01$ & $1.44 \mathrm{E}-02$ & $3.341 \mathrm{E}-01$ & 8.79E-04 & $4.573 \mathrm{E}-01$ & $1.45 \mathrm{E}-02$ & $7.869 \mathrm{E}+04$ \\
\hline $1.151 \mathrm{E}+00$ & $6.667 \mathrm{E}-01$ & $1.51 \mathrm{E}-02$ & 4.262E-01 & $9.89 \mathrm{E}-04$ & $5.529 \mathrm{E}-01$ & $1.89 \mathrm{E}-02$ & $7.934 \mathrm{E}+04$ \\
\hline $1.151 \mathrm{E}+00$ & $6.896 \mathrm{E}-01$ & $1.63 \mathrm{E}-02$ & $5.223 \mathrm{E}-01$ & $1.07 \mathrm{E}-03$ & $6.234 \mathrm{E}-01$ & $2.43 \mathrm{E}-02$ & $7.973 \mathrm{E}+04$ \\
\hline $1.151 \mathrm{E}+00$ & $6.964 \mathrm{E}-01$ & $1.81 \mathrm{E}-02$ & $6.176 \mathrm{E}-01$ & $1.21 \mathrm{E}-03$ & $6.811 \mathrm{E}-01$ & $3.25 \mathrm{E}-02$ & $8.016 \mathrm{E}+04$ \\
\hline $1.150 \mathrm{E}+00$ & $7.176 \mathrm{E}-01$ & $2.05 \mathrm{E}-02$ & 7.102E-01 & $1.36 \mathrm{E}-03$ & $7.145 \mathrm{E}-01$ & 4.13E-02 & $8.114 \mathrm{E}+04$ \\
\hline $1.149 \mathrm{E}+00$ & $7.300 \mathrm{E}-01$ & $2.38 \mathrm{E}-02$ & $8.023 \mathrm{E}-01$ & $1.51 \mathrm{E}-03$ & 7.314E-01 & $5.51 \mathrm{E}-02$ & $8.211 \mathrm{E}+04$ \\
\hline $1.149 \mathrm{E}+00$ & 7.338E-01 & 3.04E-02 & 8.951E-01 & $1.66 \mathrm{E}-03$ & 6.919E-01 & $8.00 \mathrm{E}-02$ & $8.313 \mathrm{E}+04$ \\
\hline
\end{tabular}




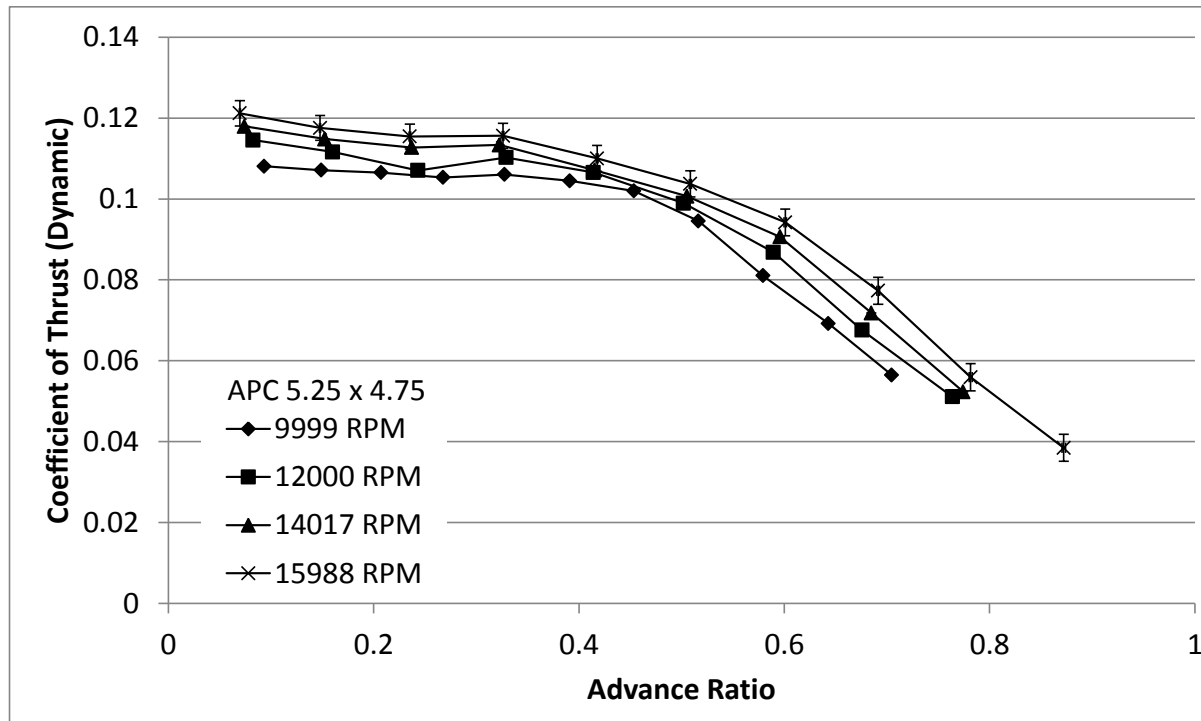

(a)

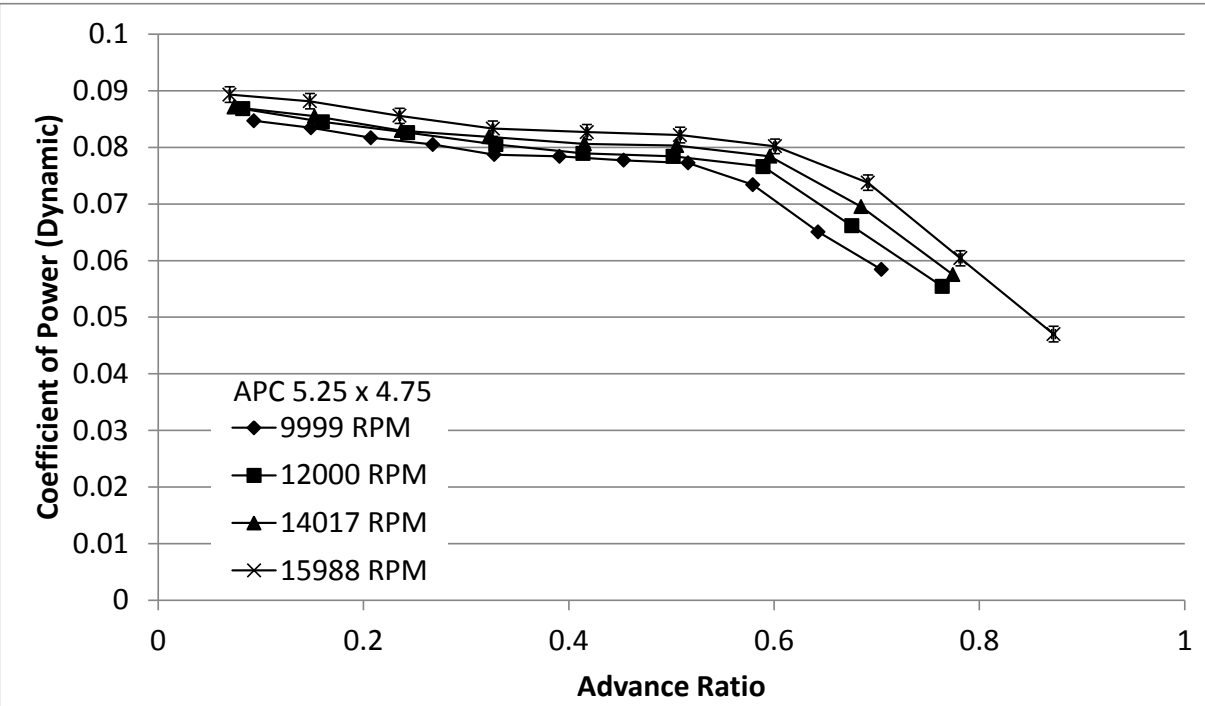

(b)

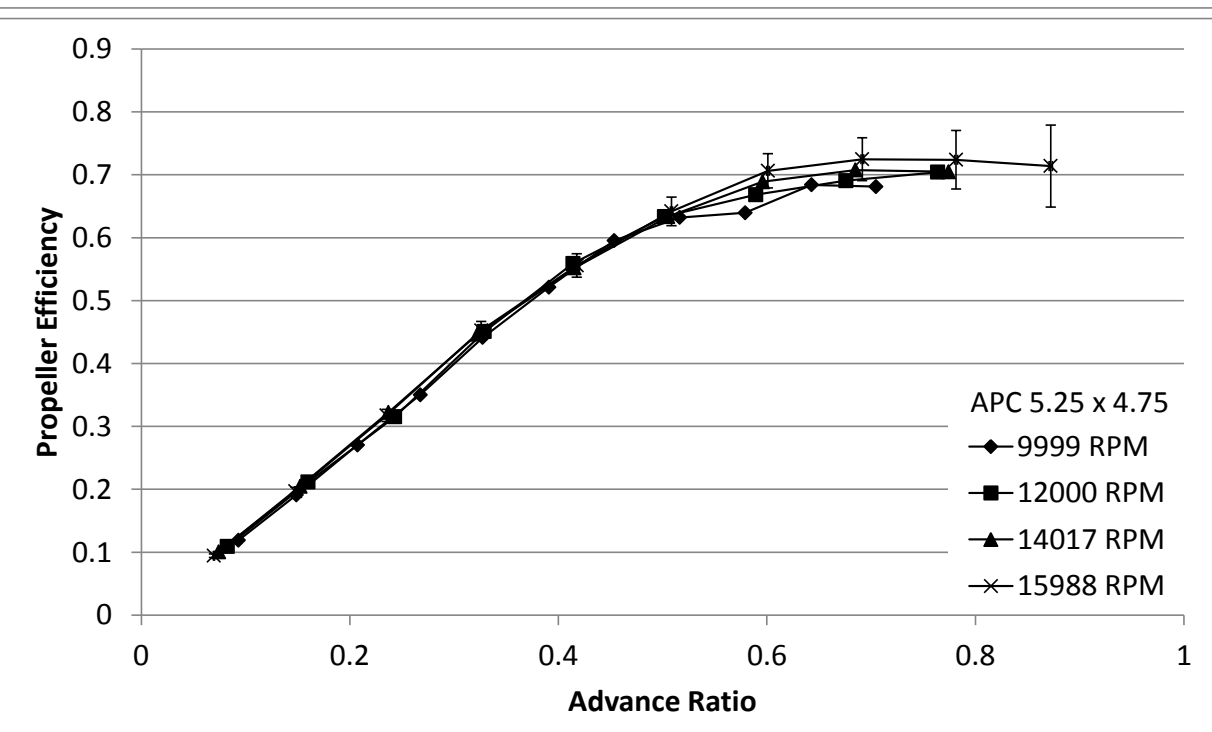

(c) 


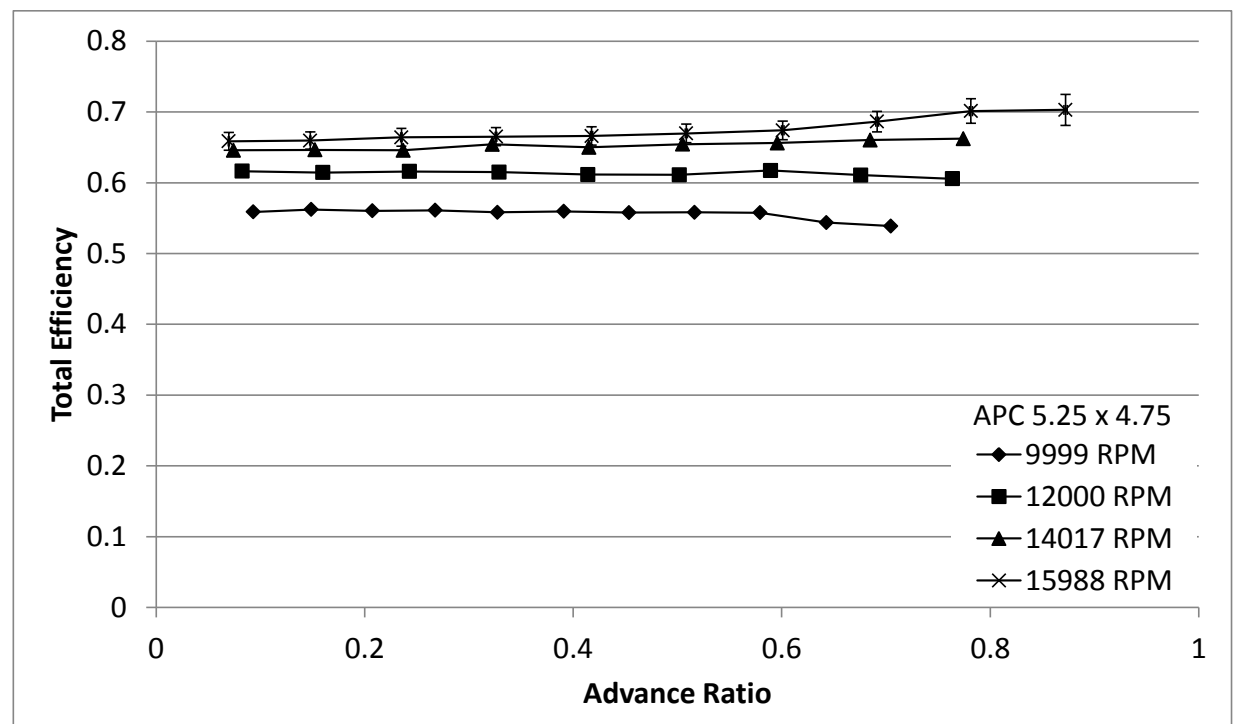

(d)

Figure 87: APC 5.25 x 4.75 Dynamic Test Results: (a) Coefficient of Thrust, (b)

Coefficient of Power, (c) Propeller Efficiency, (d) Total Efficiency.

Table 146: APC 5.25 x 4.75 Dynamic Measured Values - 9999 RPM

\begin{tabular}{|c|c|c|c|c|c|c|c|}
\hline$n(\mathrm{RPM})$ & $Q(\mathrm{~g}-\mathrm{m})$ & $\Delta Q(\mathrm{~g}-\mathrm{m})$ & $V(\mathrm{~V})$ & $I(\mathrm{~A})$ & $T_{\text {atm }}\left({ }^{\circ} \mathrm{C}\right)$ & $P_{\text {atm }}(\mathrm{Pa})$ & $P_{\text {diff }}(\mathrm{Pa})$ \\
\hline $9.999 \mathrm{E}+03$ & $1.813 \mathrm{E}+00$ & $5.98 \mathrm{E}-02$ & $1.103 \mathrm{E}+01$ & $3.020 \mathrm{E}+00$ & $2.390 \mathrm{E}+01$ & $9.833 \mathrm{E}+04$ & $2.715 \mathrm{E}+00$ \\
\hline $9.996 \mathrm{E}+03$ & $1.787 \mathrm{E}+00$ & $5.98 \mathrm{E}-02$ & $1.103 \mathrm{E}+01$ & $2.957 \mathrm{E}+00$ & $2.379 \mathrm{E}+01$ & $9.834 \mathrm{E}+04$ & $6.647 \mathrm{E}+00$ \\
\hline $9.989 \mathrm{E}+03$ & $1.746 \mathrm{E}+00$ & $5.96 \mathrm{E}-02$ & $1.103 \mathrm{E}+01$ & $2.897 \mathrm{E}+00$ & $2.402 \mathrm{E}+01$ & $9.838 \mathrm{E}+04$ & $1.264 \mathrm{E}+01$ \\
\hline $9.983 \mathrm{E}+03$ & $1.719 \mathrm{E}+00$ & $5.98 \mathrm{E}-02$ & $1.104 \mathrm{E}+01$ & $2.846 \mathrm{E}+00$ & $2.395 \mathrm{E}+01$ & $9.841 \mathrm{E}+04$ & $2.080 \mathrm{E}+01$ \\
\hline $1.002 \mathrm{E}+04$ & $1.694 \mathrm{E}+00$ & $6.08 \mathrm{E}-02$ & $1.104 \mathrm{E}+01$ & $2.829 \mathrm{E}+00$ & $2.401 \mathrm{E}+01$ & $9.842 \mathrm{E}+04$ & $3.118 \mathrm{E}+01$ \\
\hline $9.992 \mathrm{E}+03$ & $1.676 \mathrm{E}+00$ & $5.95 \mathrm{E}-02$ & $1.104 \mathrm{E}+01$ & $2.785 \mathrm{E}+00$ & $2.414 \mathrm{E}+01$ & $9.844 \mathrm{E}+04$ & $4.392 \mathrm{E}+01$ \\
\hline $1.001 \mathrm{E}+04$ & $1.666 \mathrm{E}+00$ & $5.91 \mathrm{E}-02$ & $1.104 \mathrm{E}+01$ & $2.780 \mathrm{E}+00$ & $2.424 \mathrm{E}+01$ & $9.844 \mathrm{E}+04$ & $5.904 \mathrm{E}+01$ \\
\hline $9.979 \mathrm{E}+03$ & $1.646 \mathrm{E}+00$ & $5.96 \mathrm{E}-02$ & $1.104 \mathrm{E}+01$ & $2.738 \mathrm{E}+00$ & $2.423 \mathrm{E}+01$ & $9.841 \mathrm{E}+04$ & $7.579 \mathrm{E}+01$ \\
\hline $1.001 \mathrm{E}+04$ & $1.574 \mathrm{E}+00$ & $5.95 \mathrm{E}-02$ & $1.104 \mathrm{E}+01$ & $2.628 \mathrm{E}+00$ & $2.427 \mathrm{E}+01$ & $9.840 \mathrm{E}+04$ & $9.565 \mathrm{E}+01$ \\
\hline $9.999 \mathrm{E}+03$ & $1.394 \mathrm{E}+00$ & $6.03 \mathrm{E}-02$ & $1.105 \mathrm{E}+01$ & $2.382 \mathrm{E}+00$ & $2.389 \mathrm{E}+01$ & $9.840 \mathrm{E}+04$ & $1.175 \mathrm{E}+02$ \\
\hline $1.002 \mathrm{E}+04$ & $1.257 \mathrm{E}+00$ & $5.99 \mathrm{E}-02$ & $1.106 \mathrm{E}+01$ & $2.171 \mathrm{E}+00$ & $2.375 \mathrm{E}+01$ & $9.840 \mathrm{E}+04$ & $1.415 \mathrm{E}+02$ \\
\hline
\end{tabular}


Table 147: APC 5.25 x 4.75 Dynamic Calculated Values - 9999 RPM

\begin{tabular}{|c|c|c|c|c|c|c|c|}
\hline$n(\mathrm{RPM})$ & $V_{\infty}^{\prime}(\mathrm{m} / \mathrm{s})$ & $\Delta V_{\infty}^{\prime}(\mathrm{m} / \mathrm{s})$ & $T^{\prime}(\mathrm{g})$ & $\Delta T^{\prime}(\mathrm{g})$ & $V_{\mathrm{t}}(\mathrm{m} / \mathrm{s})$ & $P_{\mathrm{P}}(\mathrm{W})$ & $\Delta P_{\mathrm{P}}(\mathrm{W})$ \\
\hline $9.999 \mathrm{E}+03$ & $2.064 \mathrm{E}+00$ & $1.30 \mathrm{E}-02$ & $1.096 \mathrm{E}+02$ & $9.20 \mathrm{E}+00$ & $5.216 \mathrm{E}+01$ & $1.862 \mathrm{E}+01$ & $6.14 \mathrm{E}-01$ \\
\hline $9.996 \mathrm{E}+03$ & $3.291 \mathrm{E}+00$ & $1.36 \mathrm{E}-02$ & $1.086 \mathrm{E}+02$ & $9.09 \mathrm{E}+00$ & $5.220 \mathrm{E}+01$ & $1.834 \mathrm{E}+01$ & $6.14 \mathrm{E}-01$ \\
\hline $9.989 \mathrm{E}+03$ & $4.582 \mathrm{E}+00$ & $1.47 \mathrm{E}-02$ & $1.078 \mathrm{E}+02$ & $9.17 \mathrm{E}+00$ & $5.226 \mathrm{E}+01$ & $1.791 \mathrm{E}+01$ & $6.12 \mathrm{E}-01$ \\
\hline $9.983 \mathrm{E}+03$ & $5.910 \mathrm{E}+00$ & $1.64 \mathrm{E}-02$ & $1.065 \mathrm{E}+02$ & $9.10 \mathrm{E}+00$ & $5.236 \mathrm{E}+01$ & $1.763 \mathrm{E}+01$ & $6.13 \mathrm{E}-01$ \\
\hline $1.002 \mathrm{E}+04$ & $7.262 \mathrm{E}+00$ & $1.81 \mathrm{E}-02$ & $1.081 \mathrm{E}+02$ & $9.27 \mathrm{E}+00$ & $5.274 \mathrm{E}+01$ & $1.743 \mathrm{E}+01$ & $6.25 \mathrm{E}-01$ \\
\hline $9.992 \mathrm{E}+03$ & $8.642 \mathrm{E}+00$ & $2.04 \mathrm{E}-02$ & $1.058 \mathrm{E}+02$ & $9.19 \mathrm{E}+00$ & $5.279 \mathrm{E}+01$ & $1.720 \mathrm{E}+01$ & $6.10 \mathrm{E}-01$ \\
\hline $1.001 \mathrm{E}+04$ & $1.004 \mathrm{E}+01$ & $2.27 \mathrm{E}-02$ & $1.035 \mathrm{E}+02$ & $9.20 \mathrm{E}+00$ & $5.311 \mathrm{E}+01$ & $1.712 \mathrm{E}+01$ & $6.07 \mathrm{E}-01$ \\
\hline $9.979 \mathrm{E}+03$ & $1.140 \mathrm{E}+01$ & $2.48 \mathrm{E}-02$ & $9.541 \mathrm{E}+01$ & $9.21 \mathrm{E}+00$ & $5.324 \mathrm{E}+01$ & $1.687 \mathrm{E}+01$ & $6.10 \mathrm{E}-01$ \\
\hline $1.001 \mathrm{E}+04$ & $1.283 \mathrm{E}+01$ & $2.66 \mathrm{E}-02$ & $8.229 \mathrm{E}+01$ & $9.21 \mathrm{E}+00$ & $5.372 \mathrm{E}+01$ & $1.618 \mathrm{E}+01$ & $6.12 \mathrm{E}-01$ \\
\hline $9.999 \mathrm{E}+03$ & $1.422 \mathrm{E}+01$ & $2.84 \mathrm{E}-02$ & $7.022 \mathrm{E}+01$ & $9.24 \mathrm{E}+00$ & $5.402 \mathrm{E}+01$ & $1.431 \mathrm{E}+01$ & $6.20 \mathrm{E}-01$ \\
\hline $1.002 \mathrm{E}+04$ & $1.562 \mathrm{E}+01$ & $3.09 \mathrm{E}-02$ & $5.752 \mathrm{E}+01$ & $9.20 \mathrm{E}+00$ & $5.450 \mathrm{E}+01$ & $1.293 \mathrm{E}+01$ & $6.16 \mathrm{E}-01$ \\
\hline$P_{\mathrm{e}}(\mathrm{W})$ & $\Delta P_{\mathrm{e}}(\mathrm{W})$ & $C_{\mathrm{T}}$ & $\Delta C_{\mathrm{T}}$ & $C_{\mathrm{P}}$ & $\Delta C_{\mathrm{P}}$ & $C_{\mathrm{Q}}$ & $\Delta C_{\mathrm{Q}}$ \\
\hline $3.332 \mathrm{E}+01$ & $3.74 \mathrm{E}-01$ & $1.081 \mathrm{E}-01$ & $9.08 \mathrm{E}-03$ & 8.469E-02 & $2.80 \mathrm{E}-03$ & $1.348 \mathrm{E}-02$ & $4.45 \mathrm{E}-04$ \\
\hline $3.262 \mathrm{E}+01$ & $3.66 \mathrm{E}-01$ & $1.071 \mathrm{E}-01$ & 8.97E-03 & $8.345 \mathrm{E}-02$ & $2.79 \mathrm{E}-03$ & $1.328 \mathrm{E}-02$ & $4.45 \mathrm{E}-04$ \\
\hline $3.196 \mathrm{E}+01$ & $3.61 \mathrm{E}-01$ & $1.065 \mathrm{E}-01$ & $9.06 \mathrm{E}-03$ & $8.169 \mathrm{E}-02$ & $2.79 \mathrm{E}-03$ & $1.300 \mathrm{E}-02$ & 4.44E-04 \\
\hline $3.141 \mathrm{E}+01$ & $3.53 \mathrm{E}-01$ & $1.053 \mathrm{E}-01$ & $9.01 \mathrm{E}-03$ & $8.050 \mathrm{E}-02$ & $2.80 \mathrm{E}-03$ & $1.281 \mathrm{E}-02$ & $4.45 \mathrm{E}-04$ \\
\hline $3.123 \mathrm{E}+01$ & $3.53 \mathrm{E}-01$ & $1.061 \mathrm{E}-01$ & $9.10 \mathrm{E}-03$ & $7.870 \mathrm{E}-02$ & $2.82 \mathrm{E}-03$ & $1.253 \mathrm{E}-02$ & 4.49E-04 \\
\hline $3.074 \mathrm{E}+01$ & $3.46 \mathrm{E}-01$ & $1.045 \mathrm{E}-01$ & $9.08 \mathrm{E}-03$ & 7.839E-02 & $2.78 \mathrm{E}-03$ & $1.248 \mathrm{E}-02$ & $4.43 \mathrm{E}-04$ \\
\hline $3.069 \mathrm{E}+01$ & $3.46 \mathrm{E}-01$ & $1.020 \mathrm{E}-01$ & $9.06 \mathrm{E}-03$ & 7.771E-02 & $2.76 \mathrm{E}-03$ & $1.237 \mathrm{E}-02$ & 4.38E-04 \\
\hline $3.022 \mathrm{E}+01$ & $3.40 \mathrm{E}-01$ & $9.455 \mathrm{E}-02$ & $9.13 \mathrm{E}-03$ & 7.724E-02 & $2.80 \mathrm{E}-03$ & $1.229 \mathrm{E}-02$ & 4.45E-04 \\
\hline $2.902 \mathrm{E}+01$ & $3.30 \mathrm{E}-01$ & $8.106 \mathrm{E}-02$ & $9.07 \mathrm{E}-03$ & $7.341 \mathrm{E}-02$ & $2.78 \mathrm{E}-03$ & $1.168 \mathrm{E}-02$ & 4.42E-04 \\
\hline $2.632 \mathrm{E}+01$ & $3.01 \mathrm{E}-01$ & $6.922 \mathrm{E}-02$ & $9.11 \mathrm{E}-03$ & $6.506 \mathrm{E}-02$ & $2.82 \mathrm{E}-03$ & $1.035 \mathrm{E}-02$ & 4.48E-04 \\
\hline $2.400 \mathrm{E}+01$ & $2.77 \mathrm{E}-01$ & $5.646 \mathrm{E}-02$ & 9.03E-03 & $5.841 \mathrm{E}-02$ & $2.78 \mathrm{E}-03$ & $9.296 \mathrm{E}-03$ & 4.43E-04 \\
\hline$\rho\left(\mathrm{kg} / \mathrm{m}^{\wedge} 3\right)$ & $\eta_{\mathrm{T}}$ & $\Delta \eta_{\mathrm{T}}$ & $J$ & $\Delta J$ & $\eta_{\mathrm{P}}$ & $\Delta \eta_{\mathrm{P}}$ & $R e_{0.75}$ \\
\hline $1.153 \mathrm{E}+00$ & $5.589 \mathrm{E}-01$ & $1.95 \mathrm{E}-02$ & $9.330 \mathrm{E}-02$ & $5.87 \mathrm{E}-04$ & $1.191 \mathrm{E}-01$ & $1.08 \mathrm{E}-02$ & $3.066 \mathrm{E}+04$ \\
\hline $1.154 \mathrm{E}+00$ & $5.622 \mathrm{E}-01$ & $1.98 \mathrm{E}-02$ & $1.488 \mathrm{E}-01$ & $6.15 \mathrm{E}-04$ & $1.911 \mathrm{E}-01$ & $1.73 \mathrm{E}-02$ & $3.071 \mathrm{E}+04$ \\
\hline $1.153 \mathrm{E}+00$ & $5.603 \mathrm{E}-01$ & $2.02 \mathrm{E}-02$ & $2.074 \mathrm{E}-01$ & $6.68 \mathrm{E}-04$ & $2.704 \mathrm{E}-01$ & $2.48 \mathrm{E}-02$ & $3.072 \mathrm{E}+04$ \\
\hline $1.154 \mathrm{E}+00$ & 5.611E-01 & $2.05 \mathrm{E}-02$ & $2.676 \mathrm{E}-01$ & 7.49E-04 & $3.502 \mathrm{E}-01$ & $3.23 \mathrm{E}-02$ & $3.080 \mathrm{E}+04$ \\
\hline $1.154 \mathrm{E}+00$ & $5.583 \mathrm{E}-01$ & $2.10 \mathrm{E}-02$ & $3.275 \mathrm{E}-01$ & $8.26 \mathrm{E}-04$ & 4.414E-01 & $4.11 \mathrm{E}-02$ & $3.101 \mathrm{E}+04$ \\
\hline $1.153 \mathrm{E}+00$ & $5.596 \mathrm{E}-01$ & $2.08 \mathrm{E}-02$ & $3.910 \mathrm{E}-01$ & 9.32E-04 & $5.212 \mathrm{E}-01$ & 4.89E-02 & $3.102 \mathrm{E}+04$ \\
\hline $1.153 \mathrm{E}+00$ & $5.579 \mathrm{E}-01$ & $2.08 \mathrm{E}-02$ & $4.536 \mathrm{E}-01$ & $1.04 \mathrm{E}-03$ & 5.954E-01 & $5.70 \mathrm{E}-02$ & $3.119 \mathrm{E}+04$ \\
\hline $1.153 \mathrm{E}+00$ & $5.583 \mathrm{E}-01$ & 2.12E-02 & $5.163 \mathrm{E}-01$ & $1.14 \mathrm{E}-03$ & $6.321 \mathrm{E}-01$ & $6.52 \mathrm{E}-02$ & $3.126 \mathrm{E}+04$ \\
\hline $1.153 \mathrm{E}+00$ & $5.576 \mathrm{E}-01$ & $2.20 \mathrm{E}-02$ & $5.792 \mathrm{E}-01$ & $1.21 \mathrm{E}-03$ & $6.396 \mathrm{E}-01$ & 7.56E-02 & $3.153 \mathrm{E}+04$ \\
\hline $1.154 \mathrm{E}+00$ & $5.438 \mathrm{E}-01$ & $2.43 \mathrm{E}-02$ & $6.429 \mathrm{E}-01$ & $1.30 \mathrm{E}-03$ & $6.840 \mathrm{E}-01$ & $9.47 \mathrm{E}-02$ & $3.178 \mathrm{E}+04$ \\
\hline $1.155 \mathrm{E}+00$ & 5.389E-01 & 2.64E-02 & $7.047 \mathrm{E}-01$ & $1.42 \mathrm{E}-03$ & $6.812 \mathrm{E}-01$ & $1.14 \mathrm{E}-01$ & $3.209 \mathrm{E}+04$ \\
\hline
\end{tabular}


Table 148: APC 5.25 x 4.75 Dynamic Measured Values - 12000 RPM

\begin{tabular}{|c|c|c|c|c|c|c|c|}
\hline$n(\mathrm{RPM})$ & $Q(\mathrm{~g}-\mathrm{m})$ & $\Delta Q(\mathrm{~g}-\mathrm{m})$ & $V(\mathrm{~V})$ & $I(\mathrm{~A})$ & $T_{\text {atm }}\left({ }^{\circ} \mathrm{C}\right)$ & $P_{\text {atm }}(\mathrm{Pa})$ & $P_{\text {diff }}(\mathrm{Pa})$ \\
\hline $1.200 \mathrm{E}+04$ & $2.689 \mathrm{E}+00$ & $7.46 \mathrm{E}-02$ & $1.098 \mathrm{E}+01$ & $4.898 \mathrm{E}+00$ & $2.288 \mathrm{E}+01$ & $9.839 \mathrm{E}+04$ & $3.128 \mathrm{E}+00$ \\
\hline $1.199 \mathrm{E}+04$ & $2.617 \mathrm{E}+00$ & $7.97 \mathrm{E}-02$ & $1.098 \mathrm{E}+01$ & $4.776 \mathrm{E}+00$ & $2.249 \mathrm{E}+01$ & $9.839 \mathrm{E}+04$ & $1.108 \mathrm{E}+01$ \\
\hline $1.198 \mathrm{E}+04$ & $2.553 \mathrm{E}+00$ & $7.11 \mathrm{E}-02$ & $1.099 \mathrm{E}+01$ & $4.641 \mathrm{E}+00$ & $2.244 \mathrm{E}+01$ & $9.839 \mathrm{E}+04$ & $2.495 \mathrm{E}+01$ \\
\hline $1.196 \mathrm{E}+04$ & $2.481 \mathrm{E}+00$ & $7.10 \mathrm{E}-02$ & $1.099 \mathrm{E}+01$ & $4.510 \mathrm{E}+00$ & $2.246 \mathrm{E}+01$ & $9.840 \mathrm{E}+04$ & $4.508 \mathrm{E}+01$ \\
\hline $1.199 \mathrm{E}+04$ & $2.444 \mathrm{E}+00$ & $7.81 \mathrm{E}-02$ & $1.099 \mathrm{E}+01$ & $4.479 \mathrm{E}+00$ & $2.253 \mathrm{E}+01$ & $9.841 \mathrm{E}+04$ & $7.123 \mathrm{E}+01$ \\
\hline $1.201 \mathrm{E}+04$ & $2.433 \mathrm{E}+00$ & $7.11 \mathrm{E}-02$ & $1.099 \mathrm{E}+01$ & $4.466 \mathrm{E}+00$ & $2.259 \mathrm{E}+01$ & $9.842 \mathrm{E}+04$ & $1.043 \mathrm{E}+02$ \\
\hline $1.202 \mathrm{E}+04$ & $2.381 \mathrm{E}+00$ & $7.42 \mathrm{E}-02$ & $1.100 \mathrm{E}+01$ & $4.327 \mathrm{E}+00$ & $2.261 \mathrm{E}+01$ & $9.842 \mathrm{E}+04$ & $1.436 \mathrm{E}+02$ \\
\hline $1.203 \mathrm{E}+04$ & $2.060 \mathrm{E}+00$ & $7.10 \mathrm{E}-02$ & $1.101 \mathrm{E}+01$ & $3.783 \mathrm{E}+00$ & $2.255 \mathrm{E}+01$ & $9.841 \mathrm{E}+04$ & $1.886 \mathrm{E}+02$ \\
\hline $1.201 \mathrm{E}+04$ & $1.722 \mathrm{E}+00$ & $6.42 \mathrm{E}-02$ & $1.103 \mathrm{E}+01$ & $3.182 \mathrm{E}+00$ & $2.254 \mathrm{E}+01$ & $9.841 \mathrm{E}+04$ & $2.397 \mathrm{E}+02$ \\
\hline
\end{tabular}


Table 149: APC 5.25 x 4.75 Dynamic Calculated Values - 12000 RPM

\begin{tabular}{|c|c|c|c|c|c|c|c|}
\hline$n(\mathrm{RPM})$ & $V_{\infty}^{\prime}(\mathrm{m} / \mathrm{s})$ & $\Delta V_{\infty}^{\prime}(\mathrm{m} / \mathrm{s})$ & $T^{\prime}(\mathrm{g})$ & $\Delta T^{\prime}(\mathrm{g})$ & $V_{\mathrm{t}}(\mathrm{m} / \mathrm{s})$ & $P_{\mathrm{P}}(\mathrm{W})$ & $\Delta P_{\mathrm{P}}(\mathrm{W})$ \\
\hline $1.200 \mathrm{E}+04$ & $2.193 \mathrm{E}+00$ & $1.48 \mathrm{E}-02$ & $1.680 \mathrm{E}+02$ & $8.47 \mathrm{E}+00$ & $6.260 \mathrm{E}+01$ & $3.314 \mathrm{E}+01$ & $9.20 \mathrm{E}-01$ \\
\hline $1.199 \mathrm{E}+04$ & $4.246 \mathrm{E}+00$ & $1.59 \mathrm{E}-02$ & $1.637 \mathrm{E}+02$ & $8.42 \mathrm{E}+00$ & $6.265 \mathrm{E}+01$ & $3.223 \mathrm{E}+01$ & $9.82 \mathrm{E}-01$ \\
\hline $1.198 \mathrm{E}+04$ & $6.444 \mathrm{E}+00$ & $1.81 \mathrm{E}-02$ & $1.566 \mathrm{E}+02$ & $8.40 \mathrm{E}+00$ & $6.277 \mathrm{E}+01$ & $3.140 \mathrm{E}+01$ & $8.75 \mathrm{E}-01$ \\
\hline $1.196 \mathrm{E}+04$ & $8.707 \mathrm{E}+00$ & $2.16 \mathrm{E}-02$ & $1.609 \mathrm{E}+02$ & $8.37 \mathrm{E}+00$ & $6.296 \mathrm{E}+01$ & $3.049 \mathrm{E}+01$ & 8.73E-01 \\
\hline $1.199 \mathrm{E}+04$ & $1.098 \mathrm{E}+01$ & $2.58 \mathrm{E}-02$ & $1.562 \mathrm{E}+02$ & $8.43 \mathrm{E}+00$ & $6.346 \mathrm{E}+01$ & $3.010 \mathrm{E}+01$ & $9.62 \mathrm{E}-01$ \\
\hline $1.201 \mathrm{E}+04$ & $1.333 \mathrm{E}+01$ & $2.85 \mathrm{E}-02$ & $1.454 \mathrm{E}+02$ & $8.45 \mathrm{E}+00$ & $6.398 \mathrm{E}+01$ & $3.000 \mathrm{E}+01$ & 8.77E-01 \\
\hline $1.202 \mathrm{E}+04$ & $1.567 \mathrm{E}+01$ & $3.16 \mathrm{E}-02$ & $1.278 \mathrm{E}+02$ & $8.51 \mathrm{E}+00$ & $6.455 \mathrm{E}+01$ & $2.937 \mathrm{E}+01$ & $9.15 \mathrm{E}-01$ \\
\hline $1.203 \mathrm{E}+04$ & $1.798 \mathrm{E}+01$ & $3.50 \mathrm{E}-02$ & $9.966 \mathrm{E}+01$ & $8.55 \mathrm{E}+00$ & $6.522 \mathrm{E}+01$ & $2.545 \mathrm{E}+01$ & 8.77E-01 \\
\hline $1.201 \mathrm{E}+04$ & $2.030 \mathrm{E}+01$ & $3.86 \mathrm{E}-02$ & $7.517 \mathrm{E}+01$ & $8.47 \mathrm{E}+00$ & $6.582 \mathrm{E}+01$ & $2.125 \mathrm{E}+01$ & 7.93E-01 \\
\hline$P_{\mathrm{e}}(\mathrm{W})$ & $\Delta P_{\mathrm{e}}(\mathrm{W})$ & $C_{\mathrm{T}}$ & $\Delta C_{\mathrm{T}}$ & $C_{\mathrm{P}}$ & $\Delta C_{\mathrm{P}}$ & $C_{\mathrm{Q}}$ & $\Delta C_{\mathrm{Q}}$ \\
\hline $5.377 \mathrm{E}+01$ & $6.24 \mathrm{E}-01$ & $1.146 \mathrm{E}-01$ & $5.78 \mathrm{E}-03$ & $8.680 \mathrm{E}-02$ & $2.41 \mathrm{E}-03$ & $1.382 \mathrm{E}-02$ & $3.84 \mathrm{E}-04$ \\
\hline $5.246 \mathrm{E}+01$ & $6.06 \mathrm{E}-01$ & $1.116 \mathrm{E}-01$ & $5.74 \mathrm{E}-03$ & $8.450 \mathrm{E}-02$ & $2.58 \mathrm{E}-03$ & $1.345 \mathrm{E}-02$ & 4.10E-04 \\
\hline $5.099 \mathrm{E}+01$ & $5.82 \mathrm{E}-01$ & $1.071 \mathrm{E}-01$ & $5.74 \mathrm{E}-03$ & $8.259 \mathrm{E}-02$ & $2.30 \mathrm{E}-03$ & $1.315 \mathrm{E}-02$ & $3.66 \mathrm{E}-04$ \\
\hline $4.957 \mathrm{E}+01$ & $5.70 \mathrm{E}-01$ & $1.103 \mathrm{E}-01$ & $5.74 \mathrm{E}-03$ & $8.049 \mathrm{E}-02$ & $2.31 \mathrm{E}-03$ & $1.281 \mathrm{E}-02$ & $3.67 \mathrm{E}-04$ \\
\hline $4.923 \mathrm{E}+01$ & $5.67 \mathrm{E}-01$ & $1.066 \mathrm{E}-01$ & $5.75 \mathrm{E}-03$ & $7.892 \mathrm{E}-02$ & $2.52 \mathrm{E}-03$ & $1.256 \mathrm{E}-02$ & $4.02 \mathrm{E}-04$ \\
\hline $4.908 \mathrm{E}+01$ & $5.62 \mathrm{E}-01$ & 9.897E-02 & $5.75 \mathrm{E}-03$ & $7.840 \mathrm{E}-02$ & $2.29 \mathrm{E}-03$ & $1.248 \mathrm{E}-02$ & $3.65 \mathrm{E}-04$ \\
\hline $4.758 \mathrm{E}+01$ & $5.45 \mathrm{E}-01$ & $8.683 \mathrm{E}-02$ & $5.79 \mathrm{E}-03$ & 7.659E-02 & $2.39 \mathrm{E}-03$ & $1.219 \mathrm{E}-02$ & $3.80 \mathrm{E}-04$ \\
\hline $4.166 \mathrm{E}+01$ & $4.71 \mathrm{E}-01$ & $6.758 \mathrm{E}-02$ & $5.80 \mathrm{E}-03$ & $6.614 \mathrm{E}-02$ & $2.28 \mathrm{E}-03$ & $1.053 \mathrm{E}-02$ & $3.63 \mathrm{E}-04$ \\
\hline $3.509 \mathrm{E}+01$ & $3.96 \mathrm{E}-01$ & $5.110 \mathrm{E}-02$ & $5.76 \mathrm{E}-03$ & $5.543 \mathrm{E}-02$ & $2.07 \mathrm{E}-03$ & $8.822 \mathrm{E}-03$ & $3.29 \mathrm{E}-04$ \\
\hline$\rho\left(\mathrm{kg} / \mathrm{m}^{\wedge} 3\right)$ & $\eta_{\mathrm{T}}$ & $\Delta \eta_{\mathrm{T}}$ & $J$ & $\Delta J$ & $\eta_{\mathrm{P}}$ & $\Delta \eta_{\mathrm{P}}$ & $R e_{0.75}$ \\
\hline $1.158 \mathrm{E}+00$ & $6.164 \mathrm{E}-01$ & $1.85 \mathrm{E}-02$ & $8.258 \mathrm{E}-02$ & $5.57 \mathrm{E}-04$ & $1.090 \mathrm{E}-01$ & $6.32 \mathrm{E}-03$ & $3.704 \mathrm{E}+04$ \\
\hline $1.159 \mathrm{E}+00$ & $6.145 \mathrm{E}-01$ & $2.00 \mathrm{E}-02$ & $1.601 \mathrm{E}-01$ & $6.02 \mathrm{E}-04$ & $2.115 \mathrm{E}-01$ & $1.27 \mathrm{E}-02$ & $3.716 \mathrm{E}+04$ \\
\hline $1.160 \mathrm{E}+00$ & $6.159 \mathrm{E}-01$ & $1.85 \mathrm{E}-02$ & $2.431 \mathrm{E}-01$ & $6.86 \mathrm{E}-04$ & $3.152 \mathrm{E}-01$ & $1.91 \mathrm{E}-02$ & $3.724 \mathrm{E}+04$ \\
\hline $1.160 \mathrm{E}+00$ & $6.150 \mathrm{E}-01$ & $1.90 \mathrm{E}-02$ & $3.290 \mathrm{E}-01$ & $8.20 \mathrm{E}-04$ & $4.506 \mathrm{E}-01$ & $2.68 \mathrm{E}-02$ & $3.736 \mathrm{E}+04$ \\
\hline $1.159 \mathrm{E}+00$ & $6.115 \mathrm{E}-01$ & $2.08 \mathrm{E}-02$ & $4.140 \mathrm{E}-01$ & $9.80 \mathrm{E}-04$ & $5.591 \mathrm{E}-01$ & $3.51 \mathrm{E}-02$ & $3.764 \mathrm{E}+04$ \\
\hline $1.159 \mathrm{E}+00$ & $6.112 \mathrm{E}-01$ & $1.92 \mathrm{E}-02$ & $5.018 \mathrm{E}-01$ & $1.09 \mathrm{E}-03$ & $6.335 \mathrm{E}-01$ & 4.13E-02 & $3.794 \mathrm{E}+04$ \\
\hline $1.159 \mathrm{E}+00$ & $6.173 \mathrm{E}-01$ & $2.05 \mathrm{E}-02$ & $5.895 \mathrm{E}-01$ & $1.21 \mathrm{E}-03$ & $6.683 \mathrm{E}-01$ & $4.92 \mathrm{E}-02$ & $3.827 \mathrm{E}+04$ \\
\hline $1.159 \mathrm{E}+00$ & $6.109 \mathrm{E}-01$ & $2.22 \mathrm{E}-02$ & $6.758 \mathrm{E}-01$ & $1.34 \mathrm{E}-03$ & $6.906 \mathrm{E}-01$ & $6.39 \mathrm{E}-02$ & $3.868 \mathrm{E}+04$ \\
\hline $1.159 \mathrm{E}+00$ & $6.056 \mathrm{E}-01$ & $2.36 \mathrm{E}-02$ & $7.638 \mathrm{E}-01$ & $1.48 \mathrm{E}-03$ & $7.041 \mathrm{E}-01$ & $8.36 \mathrm{E}-02$ & $3.904 \mathrm{E}+04$ \\
\hline
\end{tabular}


Table 150: APC 5.25 x 4.75 Dynamic Measured Values - 14017 RPM

\begin{tabular}{|c|c|c|c|c|c|c|c|}
\hline$n(\mathrm{RPM})$ & $Q(\mathrm{~g}-\mathrm{m})$ & $\Delta Q(\mathrm{~g}-\mathrm{m})$ & $V(\mathrm{~V})$ & $I(\mathrm{~A})$ & $T_{\text {atm }}\left({ }^{\circ} \mathrm{C}\right)$ & $P_{\text {atm }}(\mathrm{Pa})$ & $P_{\text {diff }}(\mathrm{Pa})$ \\
\hline $1.398 \mathrm{E}+04$ & $3.673 \mathrm{E}+00$ & $7.18 \mathrm{E}-02$ & $1.091 \mathrm{E}+01$ & $7.483 \mathrm{E}+00$ & $2.152 \mathrm{E}+01$ & $9.832 \mathrm{E}+04$ & $3.486 \mathrm{E}+00$ \\
\hline $1.398 \mathrm{E}+04$ & $3.607 \mathrm{E}+00$ & $6.92 \mathrm{E}-02$ & $1.091 \mathrm{E}+01$ & $7.341 \mathrm{E}+00$ & $2.146 \mathrm{E}+01$ & $9.833 \mathrm{E}+04$ & $1.374 \mathrm{E}+01$ \\
\hline $1.405 \mathrm{E}+04$ & $3.534 \mathrm{E}+00$ & $7.29 \mathrm{E}-02$ & $1.091 \mathrm{E}+01$ & $7.232 \mathrm{E}+00$ & $2.160 \mathrm{E}+01$ & $9.833 \mathrm{E}+04$ & $3.278 \mathrm{E}+01$ \\
\hline $1.411 \mathrm{E}+04$ & $3.515 \mathrm{E}+00$ & $7.36 \mathrm{E}-02$ & $1.092 \mathrm{E}+01$ & $7.133 \mathrm{E}+00$ & $2.177 \mathrm{E}+01$ & $9.832 \mathrm{E}+04$ & $6.043 \mathrm{E}+01$ \\
\hline $1.399 \mathrm{E}+04$ & $3.404 \mathrm{E}+00$ & $7.10 \mathrm{E}-02$ & $1.092 \mathrm{E}+01$ & $6.891 \mathrm{E}+00$ & $2.178 \mathrm{E}+01$ & $9.830 \mathrm{E}+04$ & $9.773 \mathrm{E}+01$ \\
\hline $1.402 \mathrm{E}+04$ & $3.403 \mathrm{E}+00$ & $7.21 \mathrm{E}-02$ & $1.092 \mathrm{E}+01$ & $6.854 \mathrm{E}+00$ & $2.193 \mathrm{E}+01$ & $9.830 \mathrm{E}+04$ & $1.443 \mathrm{E}+02$ \\
\hline $1.401 \mathrm{E}+04$ & $3.316 \mathrm{E}+00$ & $7.21 \mathrm{E}-02$ & $1.093 \mathrm{E}+01$ & $6.652 \mathrm{E}+00$ & $2.199 \mathrm{E}+01$ & $9.831 \mathrm{E}+04$ & $1.997 \mathrm{E}+02$ \\
\hline $1.401 \mathrm{E}+04$ & $2.938 \mathrm{E}+00$ & $7.36 \mathrm{E}-02$ & $1.095 \mathrm{E}+01$ & $5.844 \mathrm{E}+00$ & $2.210 \mathrm{E}+01$ & $9.831 \mathrm{E}+04$ & $2.628 \mathrm{E}+02$ \\
\hline $1.401 \mathrm{E}+04$ & $2.430 \mathrm{E}+00$ & $7.27 \mathrm{E}-02$ & $1.098 \mathrm{E}+01$ & $4.805 \mathrm{E}+00$ & $2.216 \mathrm{E}+01$ & $9.830 \mathrm{E}+04$ & $3.349 \mathrm{E}+02$ \\
\hline
\end{tabular}


Table 151: APC 5.25 x 4.75 Dynamic Calculated Values - 14017 RPM

\begin{tabular}{|c|c|c|c|c|c|c|c|}
\hline$n(\mathrm{RPM})$ & $V_{\infty}^{\prime}(\mathrm{m} / \mathrm{s})$ & $\Delta V_{\infty}^{\prime}(\mathrm{m} / \mathrm{s})$ & $T^{\prime}(\mathrm{g})$ & $\Delta T^{\prime}(\mathrm{g})$ & $V_{\mathrm{t}}(\mathrm{m} / \mathrm{s})$ & $P_{\mathrm{P}}(\mathrm{W})$ & $\Delta P_{\mathrm{P}}(\mathrm{W})$ \\
\hline $1.398 \mathrm{E}+04$ & $2.293 \mathrm{E}+00$ & $1.60 \mathrm{E}-02$ & $2.354 \mathrm{E}+02$ & $9.08 \mathrm{E}+00$ & $7.288 \mathrm{E}+01$ & $5.271 \mathrm{E}+01$ & $1.03 \mathrm{E}+00$ \\
\hline $1.398 \mathrm{E}+04$ & $4.712 \mathrm{E}+00$ & $1.75 \mathrm{E}-02$ & $2.293 \mathrm{E}+02$ & $9.07 \mathrm{E}+00$ & $7.300 \mathrm{E}+01$ & $5.178 \mathrm{E}+01$ & 9.93E-01 \\
\hline $1.405 \mathrm{E}+04$ & $7.370 \mathrm{E}+00$ & $2.26 \mathrm{E}-02$ & $2.273 \mathrm{E}+02$ & $9.09 \mathrm{E}+00$ & $7.359 \mathrm{E}+01$ & $5.098 \mathrm{E}+01$ & $1.05 \mathrm{E}+00$ \\
\hline $1.411 \mathrm{E}+04$ & $1.007 \mathrm{E}+01$ & $2.59 \mathrm{E}-02$ & $2.304 \mathrm{E}+02$ & $9.14 \mathrm{E}+00$ & $7.422 \mathrm{E}+01$ & $5.094 \mathrm{E}+01$ & $1.07 \mathrm{E}+00$ \\
\hline $1.399 \mathrm{E}+04$ & $1.286 \mathrm{E}+01$ & $2.98 \mathrm{E}-02$ & $2.142 \mathrm{E}+02$ & $9.07 \mathrm{E}+00$ & $7.406 \mathrm{E}+01$ & $4.892 \mathrm{E}+01$ & $1.02 \mathrm{E}+00$ \\
\hline $1.402 \mathrm{E}+04$ & $1.567 \mathrm{E}+01$ & $3.26 \mathrm{E}-02$ & $2.020 \mathrm{E}+02$ & $9.09 \mathrm{E}+00$ & $7.474 \mathrm{E}+01$ & $4.900 \mathrm{E}+01$ & $1.04 \mathrm{E}+00$ \\
\hline $1.401 \mathrm{E}+04$ & $1.847 \mathrm{E}+01$ & $3.62 \mathrm{E}-02$ & $1.815 \mathrm{E}+02$ & $9.10 \mathrm{E}+00$ & $7.531 \mathrm{E}+01$ & $4.771 \mathrm{E}+01$ & $1.04 \mathrm{E}+00$ \\
\hline $1.401 \mathrm{E}+04$ & $2.122 \mathrm{E}+01$ & 4.08E-02 & $1.437 \mathrm{E}+02$ & $9.11 \mathrm{E}+00$ & $7.603 \mathrm{E}+01$ & $4.226 \mathrm{E}+01$ & $1.06 \mathrm{E}+00$ \\
\hline $1.401 \mathrm{E}+04$ & $2.399 \mathrm{E}+01$ & $4.55 \mathrm{E}-02$ & $1.047 \mathrm{E}+02$ & $9.07 \mathrm{E}+00$ & $7.684 \mathrm{E}+01$ & $3.494 \mathrm{E}+01$ & $1.05 \mathrm{E}+00$ \\
\hline$P_{\mathrm{e}}(\mathrm{W})$ & $\Delta P_{\mathrm{e}}(\mathrm{W})$ & $C_{\mathrm{T}}$ & $\Delta C_{\mathrm{T}}$ & $C_{\mathrm{P}}$ & $\Delta C_{\mathrm{P}}$ & $C_{\mathrm{Q}}$ & $\Delta C_{\mathrm{Q}}$ \\
\hline $8.161 \mathrm{E}+01$ & 8.94E-01 & $1.180 \mathrm{E}-01$ & $4.55 \mathrm{E}-03$ & $8.710 \mathrm{E}-02$ & $1.71 \mathrm{E}-03$ & $1.386 \mathrm{E}-02$ & $2.71 \mathrm{E}-04$ \\
\hline $8.009 \mathrm{E}+01$ & 8.71E-01 & $1.148 \mathrm{E}-01$ & $4.54 \mathrm{E}-03$ & $8.550 \mathrm{E}-02$ & $1.64 \mathrm{E}-03$ & $1.361 \mathrm{E}-02$ & $2.61 \mathrm{E}-04$ \\
\hline $7.892 \mathrm{E}+01$ & $8.70 \mathrm{E}-01$ & $1.127 \mathrm{E}-01$ & $4.51 \mathrm{E}-03$ & $8.295 \mathrm{E}-02$ & $1.71 \mathrm{E}-03$ & $1.320 \mathrm{E}-02$ & $2.72 \mathrm{E}-04$ \\
\hline $7.786 \mathrm{E}+01$ & $8.56 \mathrm{E}-01$ & $1.134 \mathrm{E}-01$ & $4.50 \mathrm{E}-03$ & $8.187 \mathrm{E}-02$ & $1.72 \mathrm{E}-03$ & $1.303 \mathrm{E}-02$ & $2.73 \mathrm{E}-04$ \\
\hline $7.526 \mathrm{E}+01$ & $8.25 \mathrm{E}-01$ & $1.071 \mathrm{E}-01$ & $4.54 \mathrm{E}-03$ & $8.062 \mathrm{E}-02$ & $1.69 \mathrm{E}-03$ & $1.283 \mathrm{E}-02$ & $2.68 \mathrm{E}-04$ \\
\hline $7.487 \mathrm{E}+01$ & $8.22 \mathrm{E}-01$ & $1.007 \mathrm{E}-01$ & $4.53 \mathrm{E}-03$ & $8.030 \mathrm{E}-02$ & $1.70 \mathrm{E}-03$ & $1.278 \mathrm{E}-02$ & $2.71 \mathrm{E}-04$ \\
\hline $7.270 \mathrm{E}+01$ & $8.01 \mathrm{E}-01$ & $9.065 \mathrm{E}-02$ & $4.54 \mathrm{E}-03$ & $7.841 \mathrm{E}-02$ & $1.71 \mathrm{E}-03$ & $1.248 \mathrm{E}-02$ & $2.72 \mathrm{E}-04$ \\
\hline $6.401 \mathrm{E}+01$ & $7.18 \mathrm{E}-01$ & $7.180 \mathrm{E}-02$ & $4.55 \mathrm{E}-03$ & $6.951 \mathrm{E}-02$ & $1.74 \mathrm{E}-03$ & $1.106 \mathrm{E}-02$ & $2.77 \mathrm{E}-04$ \\
\hline $5.277 \mathrm{E}+01$ & $5.92 \mathrm{E}-01$ & $5.234 \mathrm{E}-02$ & $4.54 \mathrm{E}-03$ & $5.751 \mathrm{E}-02$ & $1.72 \mathrm{E}-03$ & $9.154 \mathrm{E}-03$ & $2.74 \mathrm{E}-04$ \\
\hline$\rho\left(\mathrm{kg} / \mathrm{m}^{\wedge} 3\right)$ & $\eta_{\mathrm{T}}$ & $\Delta \eta_{\mathrm{T}}$ & $J$ & $\Delta J$ & $\eta_{\mathrm{P}}$ & $\Delta \eta_{\mathrm{P}}$ & $R e_{0.75}$ \\
\hline $1.162 \mathrm{E}+00$ & $6.459 \mathrm{E}-01$ & $1.45 \mathrm{E}-02$ & $7.417 \mathrm{E}-02$ & $5.18 \mathrm{E}-04$ & $1.004 \mathrm{E}-01$ & $4.40 \mathrm{E}-03$ & $4.345 \mathrm{E}+04$ \\
\hline $1.163 \mathrm{E}+00$ & $6.465 \mathrm{E}-01$ & $1.43 \mathrm{E}-02$ & $1.524 \mathrm{E}-01$ & 5.69E-04 & $2.047 \mathrm{E}-01$ & $9.03 \mathrm{E}-03$ & $4.354 \mathrm{E}+04$ \\
\hline $1.162 \mathrm{E}+00$ & $6.460 \mathrm{E}-01$ & $1.51 \mathrm{E}-02$ & $2.371 \mathrm{E}-01$ & 7.34E-04 & $3.222 \mathrm{E}-01$ & $1.45 \mathrm{E}-02$ & $4.386 \mathrm{E}+04$ \\
\hline $1.161 \mathrm{E}+00$ & $6.542 \mathrm{E}-01$ & $1.55 \mathrm{E}-02$ & $3.226 \mathrm{E}-01$ & $8.41 \mathrm{E}-04$ & 4.467E-01 & $2.01 \mathrm{E}-02$ & $4.419 \mathrm{E}+04$ \\
\hline $1.161 \mathrm{E}+00$ & $6.501 \mathrm{E}-01$ & $1.53 \mathrm{E}-02$ & 4.153E-01 & $9.78 \mathrm{E}-04$ & $5.520 \mathrm{E}-01$ & $2.61 \mathrm{E}-02$ & $4.408 \mathrm{E}+04$ \\
\hline $1.161 \mathrm{E}+00$ & $6.544 \mathrm{E}-01$ & $1.56 \mathrm{E}-02$ & 5.052E-01 & $1.07 \mathrm{E}-03$ & $6.334 \mathrm{E}-01$ & $3.16 \mathrm{E}-02$ & $4.444 \mathrm{E}+04$ \\
\hline $1.160 \mathrm{E}+00$ & $6.562 \mathrm{E}-01$ & $1.60 \mathrm{E}-02$ & $5.958 \mathrm{E}-01$ & $1.19 \mathrm{E}-03$ & $6.888 \mathrm{E}-01$ & $3.77 \mathrm{E}-02$ & 4.477E+04 \\
\hline $1.160 \mathrm{E}+00$ & $6.603 \mathrm{E}-01$ & $1.81 \mathrm{E}-02$ & $6.849 \mathrm{E}-01$ & $1.34 \mathrm{E}-03$ & $7.075 \mathrm{E}-01$ & $4.83 \mathrm{E}-02$ & $4.517 \mathrm{E}+04$ \\
\hline $1.160 \mathrm{E}+00$ & $6.622 \mathrm{E}-01$ & $2.12 \mathrm{E}-02$ & 7.743E-01 & $1.50 \mathrm{E}-03$ & $7.047 \mathrm{E}-01$ & $6.46 \mathrm{E}-02$ & $4.562 \mathrm{E}+04$ \\
\hline
\end{tabular}


Table 152: APC 5.25 x 4.75 Dynamic Measured Values - 15988 RPM

\begin{tabular}{|c|c|c|c|c|c|c|c|}
\hline$n(\mathrm{RPM})$ & $Q(\mathrm{~g}-\mathrm{m})$ & $\Delta Q(\mathrm{~g}-\mathrm{m})$ & $V(\mathrm{~V})$ & $I(\mathrm{~A})$ & $T_{\text {atm }}\left({ }^{\circ} \mathrm{C}\right)$ & $P_{\text {atm }}(\mathrm{Pa})$ & $P_{\text {diff }}(\mathrm{Pa})$ \\
\hline $1.592 \mathrm{E}+04$ & $4.883 \mathrm{E}+00$ & $7.62 \mathrm{E}-02$ & $1.080 \mathrm{E}+01$ & $1.122 \mathrm{E}+01$ & $2.160 \mathrm{E}+01$ & $9.832 \mathrm{E}+04$ & $4.033 \mathrm{E}+00$ \\
\hline $1.598 \mathrm{E}+04$ & $4.856 \mathrm{E}+00$ & $7.42 \mathrm{E}-02$ & $1.080 \mathrm{E}+01$ & $1.119 \mathrm{E}+01$ & $2.156 \mathrm{E}+01$ & $9.833 \mathrm{E}+04$ & $1.694 \mathrm{E}+01$ \\
\hline $1.600 \mathrm{E}+04$ & $4.725 \mathrm{E}+00$ & $7.36 \mathrm{E}-02$ & $1.081 \mathrm{E}+01$ & $1.082 \mathrm{E}+01$ & $2.173 \mathrm{E}+01$ & $9.833 \mathrm{E}+04$ & $4.188 \mathrm{E}+01$ \\
\hline $1.596 \mathrm{E}+04$ & $4.575 \mathrm{E}+00$ & $7.35 \mathrm{E}-02$ & $1.082 \mathrm{E}+01$ & $1.042 \mathrm{E}+01$ & $2.182 \mathrm{E}+01$ & $9.832 \mathrm{E}+04$ & $7.898 \mathrm{E}+01$ \\
\hline $1.598 \mathrm{E}+04$ & $4.553 \mathrm{E}+00$ & $7.33 \mathrm{E}-02$ & $1.082 \mathrm{E}+01$ & $1.037 \mathrm{E}+01$ & $2.195 \mathrm{E}+01$ & $9.831 \mathrm{E}+04$ & $1.289 \mathrm{E}+02$ \\
\hline $1.602 \mathrm{E}+04$ & $4.543 \mathrm{E}+00$ & $7.53 \mathrm{E}-02$ & $1.082 \mathrm{E}+01$ & $1.031 \mathrm{E}+01$ & $2.205 \mathrm{E}+01$ & $9.830 \mathrm{E}+04$ & $1.909 \mathrm{E}+02$ \\
\hline $1.599 \mathrm{E}+04$ & $4.414 \mathrm{E}+00$ & $7.19 \mathrm{E}-02$ & $1.083 \mathrm{E}+01$ & $9.925 \mathrm{E}+00$ & $2.216 \mathrm{E}+01$ & $9.830 \mathrm{E}+04$ & $2.645 \mathrm{E}+02$ \\
\hline $1.601 \mathrm{E}+04$ & $4.068 \mathrm{E}+00$ & $7.42 \mathrm{E}-02$ & $1.086 \mathrm{E}+01$ & $8.971 \mathrm{E}+00$ & $2.233 \mathrm{E}+01$ & $9.830 \mathrm{E}+04$ & $3.498 \mathrm{E}+02$ \\
\hline $1.602 \mathrm{E}+04$ & $3.335 \mathrm{E}+00$ & $7.36 \mathrm{E}-02$ & $1.091 \mathrm{E}+01$ & $7.168 \mathrm{E}+00$ & $2.242 \mathrm{E}+01$ & $9.830 \mathrm{E}+04$ & $4.460 \mathrm{E}+02$ \\
\hline $1.601 \mathrm{E}+04$ & $2.592 \mathrm{E}+00$ & $7.56 \mathrm{E}-02$ & $1.096 \mathrm{E}+01$ & $5.531 \mathrm{E}+00$ & $2.252 \mathrm{E}+01$ & $9.830 \mathrm{E}+04$ & $5.539 \mathrm{E}+02$ \\
\hline
\end{tabular}


Table 153: APC 5.25 x 4.75 Dynamic Calculated Values - 15988 RPM

\begin{tabular}{|c|c|c|c|c|c|c|c|}
\hline$n(\mathrm{RPM})$ & $V_{\infty}^{\prime}(\mathrm{m} / \mathrm{s})$ & $\Delta V_{\infty}^{\prime}(\mathrm{m} / \mathrm{s})$ & $T^{\prime}(\mathrm{g})$ & $\Delta T^{\prime}(\mathrm{g})$ & $V_{\mathrm{t}}(\mathrm{m} / \mathrm{s})$ & $P_{\mathrm{P}}(\mathrm{W})$ & $\Delta P_{\mathrm{P}}(\mathrm{W})$ \\
\hline $1.592 \mathrm{E}+04$ & $2.454 \mathrm{E}+00$ & $1.75 \mathrm{E}-02$ & $3.136 \mathrm{E}+02$ & $8.11 \mathrm{E}+00$ & $8.298 \mathrm{E}+01$ & $7.980 \mathrm{E}+01$ & $1.25 \mathrm{E}+00$ \\
\hline $1.598 \mathrm{E}+04$ & $5.225 \mathrm{E}+00$ & $1.95 \mathrm{E}-02$ & $3.066 \mathrm{E}+02$ & $8.02 \mathrm{E}+00$ & $8.343 \mathrm{E}+01$ & $7.967 \mathrm{E}+01$ & $1.22 \mathrm{E}+00$ \\
\hline $1.600 \mathrm{E}+04$ & $8.329 \mathrm{E}+00$ & $2.38 \mathrm{E}-02$ & $3.018 \mathrm{E}+02$ & $8.01 \mathrm{E}+00$ & $8.381 \mathrm{E}+01$ & $7.764 \mathrm{E}+01$ & $1.21 \mathrm{E}+00$ \\
\hline $1.596 \mathrm{E}+04$ & $1.151 \mathrm{E}+01$ & $2.95 \mathrm{E}-02$ & $3.006 \mathrm{E}+02$ & $8.11 \mathrm{E}+00$ & $8.397 \mathrm{E}+01$ & $7.499 \mathrm{E}+01$ & $1.21 \mathrm{E}+00$ \\
\hline $1.598 \mathrm{E}+04$ & $1.477 \mathrm{E}+01$ & $3.35 \mathrm{E}-02$ & $2.868 \mathrm{E}+02$ & $8.37 \mathrm{E}+00$ & $8.460 \mathrm{E}+01$ & $7.473 \mathrm{E}+01$ & $1.20 \mathrm{E}+00$ \\
\hline $1.602 \mathrm{E}+04$ & $1.802 \mathrm{E}+01$ & $3.69 \mathrm{E}-02$ & $2.715 \mathrm{E}+02$ & $8.45 \mathrm{E}+00$ & $8.542 \mathrm{E}+01$ & $7.475 \mathrm{E}+01$ & $1.24 \mathrm{E}+00$ \\
\hline $1.599 \mathrm{E}+04$ & $2.126 \mathrm{E}+01$ & 4.12E-02 & $2.456 \mathrm{E}+02$ & $8.61 \mathrm{E}+00$ & $8.600 \mathrm{E}+01$ & $7.249 \mathrm{E}+01$ & $1.18 \mathrm{E}+00$ \\
\hline $1.601 \mathrm{E}+04$ & $2.449 \mathrm{E}+01$ & $4.66 \mathrm{E}-02$ & $2.018 \mathrm{E}+02$ & $8.72 \mathrm{E}+00$ & $8.696 \mathrm{E}+01$ & $6.689 \mathrm{E}+01$ & $1.22 \mathrm{E}+00$ \\
\hline $1.602 \mathrm{E}+04$ & $2.769 \mathrm{E}+01$ & $5.20 \mathrm{E}-02$ & $1.462 \mathrm{E}+02$ & $8.81 \mathrm{E}+00$ & $8.796 \mathrm{E}+01$ & $5.486 \mathrm{E}+01$ & $1.21 \mathrm{E}+00$ \\
\hline $1.601 \mathrm{E}+04$ & $3.089 \mathrm{E}+01$ & $5.71 \mathrm{E}-02$ & $1.004 \mathrm{E}+02$ & $8.68 \mathrm{E}+00$ & $8.898 \mathrm{E}+01$ & $4.262 \mathrm{E}+01$ & $1.24 \mathrm{E}+00$ \\
\hline$P_{\mathrm{e}}(\mathrm{W})$ & $\Delta P_{\mathrm{e}}(\mathrm{W})$ & $C_{\mathrm{T}}$ & $\Delta C_{\mathrm{T}}$ & $C_{\mathrm{P}}$ & $\Delta C_{\mathrm{P}}$ & $C_{\mathrm{Q}}$ & $\Delta C_{\mathrm{Q}}$ \\
\hline $1.212 \mathrm{E}+02$ & $1.32 \mathrm{E}+00$ & $1.212 \mathrm{E}-01$ & $3.14 \mathrm{E}-03$ & 8.932E-02 & $1.40 \mathrm{E}-03$ & $1.422 \mathrm{E}-02$ & $2.22 \mathrm{E}-04$ \\
\hline $1.208 \mathrm{E}+02$ & $1.30 \mathrm{E}+00$ & $1.176 \mathrm{E}-01$ & $3.08 \mathrm{E}-03$ & $8.815 \mathrm{E}-02$ & $1.35 \mathrm{E}-03$ & $1.403 \mathrm{E}-02$ & $2.14 \mathrm{E}-04$ \\
\hline $1.169 \mathrm{E}+02$ & $1.25 \mathrm{E}+00$ & $1.155 \mathrm{E}-01$ & $3.06 \mathrm{E}-03$ & $8.556 \mathrm{E}-02$ & $1.34 \mathrm{E}-03$ & $1.362 \mathrm{E}-02$ & $2.12 \mathrm{E}-04$ \\
\hline $1.127 \mathrm{E}+02$ & $1.21 \mathrm{E}+00$ & $1.156 \mathrm{E}-01$ & $3.12 \mathrm{E}-03$ & $8.330 \mathrm{E}-02$ & $1.34 \mathrm{E}-03$ & $1.326 \mathrm{E}-02$ & $2.13 \mathrm{E}-04$ \\
\hline $1.122 \mathrm{E}+02$ & $1.20 \mathrm{E}+00$ & $1.100 \mathrm{E}-01$ & $3.21 \mathrm{E}-03$ & $8.270 \mathrm{E}-02$ & $1.33 \mathrm{E}-03$ & $1.316 \mathrm{E}-02$ & 2.12E-04 \\
\hline $1.116 \mathrm{E}+02$ & $1.20 \mathrm{E}+00$ & $1.037 \mathrm{E}-01$ & $3.23 \mathrm{E}-03$ & $8.217 \mathrm{E}-02$ & $1.36 \mathrm{E}-03$ & $1.308 \mathrm{E}-02$ & $2.17 \mathrm{E}-04$ \\
\hline $1.075 \mathrm{E}+02$ & $1.16 \mathrm{E}+00$ & $9.423 \mathrm{E}-02$ & $3.30 \mathrm{E}-03$ & $8.018 \mathrm{E}-02$ & $1.31 \mathrm{E}-03$ & $1.276 \mathrm{E}-02$ & $2.08 \mathrm{E}-04$ \\
\hline $9.744 \mathrm{E}+01$ & $1.06 \mathrm{E}+00$ & $7.730 \mathrm{E}-02$ & $3.34 \mathrm{E}-03$ & 7.376E-02 & $1.35 \mathrm{E}-03$ & $1.174 \mathrm{E}-02$ & $2.14 \mathrm{E}-04$ \\
\hline $7.823 \mathrm{E}+01$ & $8.69 \mathrm{E}-01$ & $5.595 \mathrm{E}-02$ & $3.37 \mathrm{E}-03$ & $6.041 \mathrm{E}-02$ & $1.33 \mathrm{E}-03$ & $9.614 \mathrm{E}-03$ & $2.12 \mathrm{E}-04$ \\
\hline $6.062 \mathrm{E}+01$ & $6.85 \mathrm{E}-01$ & $3.848 \mathrm{E}-02$ & $3.33 \mathrm{E}-03$ & 4.702E-02 & $1.37 \mathrm{E}-03$ & $7.483 \mathrm{E}-03$ & $2.18 \mathrm{E}-04$ \\
\hline$\rho\left(\mathrm{kg} / \mathrm{m}^{\wedge} 3\right)$ & $\eta_{\mathrm{T}}$ & $\Delta \eta_{\mathrm{T}}$ & $J$ & $\Delta J$ & $\eta_{\mathrm{P}}$ & $\Delta \eta_{\mathrm{P}}$ & $R e_{0.75}$ \\
\hline $1.162 \mathrm{E}+00$ & $6.586 \mathrm{E}-01$ & $1.25 \mathrm{E}-02$ & $6.970 \mathrm{E}-02$ & $4.98 \mathrm{E}-04$ & $9.457 \mathrm{E}-02$ & $2.94 \mathrm{E}-03$ & $4.945 \mathrm{E}+04$ \\
\hline $1.162 \mathrm{E}+00$ & $6.597 \mathrm{E}-01$ & $1.23 \mathrm{E}-02$ & $1.479 \mathrm{E}-01$ & $5.53 \mathrm{E}-04$ & $1.972 \mathrm{E}-01$ & $6.02 \mathrm{E}-03$ & $4.973 \mathrm{E}+04$ \\
\hline $1.162 \mathrm{E}+00$ & $6.642 \mathrm{E}-01$ & $1.26 \mathrm{E}-02$ & 2.353E-01 & $6.75 \mathrm{E}-04$ & $3.175 \mathrm{E}-01$ & $9.82 \mathrm{E}-03$ & $4.990 \mathrm{E}+04$ \\
\hline $1.161 \mathrm{E}+00$ & $6.651 \mathrm{E}-01$ & $1.28 \mathrm{E}-02$ & $3.260 \mathrm{E}-01$ & $8.40 \mathrm{E}-04$ & $4.526 \mathrm{E}-01$ & $1.43 \mathrm{E}-02$ & $4.997 \mathrm{E}+04$ \\
\hline $1.161 \mathrm{E}+00$ & $6.661 \mathrm{E}-01$ & $1.29 \mathrm{E}-02$ & 4.177E-01 & $9.52 \mathrm{E}-04$ & $5.558 \mathrm{E}-01$ & $1.86 \mathrm{E}-02$ & $5.030 \mathrm{E}+04$ \\
\hline $1.160 \mathrm{E}+00$ & $6.698 \mathrm{E}-01$ & $1.32 \mathrm{E}-02$ & $5.085 \mathrm{E}-01$ & $1.05 \mathrm{E}-03$ & $6.418 \mathrm{E}-01$ & $2.27 \mathrm{E}-02$ & $5.075 \mathrm{E}+04$ \\
\hline $1.160 \mathrm{E}+00$ & $6.741 \mathrm{E}-01$ & $1.32 \mathrm{E}-02$ & $6.010 \mathrm{E}-01$ & $1.17 \mathrm{E}-03$ & 7.064E-01 & $2.73 \mathrm{E}-02$ & $5.107 \mathrm{E}+04$ \\
\hline $1.159 \mathrm{E}+00$ & 6.864E-01 & $1.46 \mathrm{E}-02$ & $6.915 \mathrm{E}-01$ & $1.32 \mathrm{E}-03$ & $7.248 \mathrm{E}-01$ & $3.40 \mathrm{E}-02$ & $5.158 \mathrm{E}+04$ \\
\hline $1.159 \mathrm{E}+00$ & 7.013E-01 & $1.73 \mathrm{E}-02$ & $7.815 \mathrm{E}-01$ & $1.48 \mathrm{E}-03$ & $7.238 \mathrm{E}-01$ & 4.65E-02 & $5.215 \mathrm{E}+04$ \\
\hline $1.158 \mathrm{E}+00$ & 7.030E-01 & $2.20 \mathrm{E}-02$ & 8.723E-01 & $1.62 \mathrm{E}-03$ & 7.139E-01 & $6.52 \mathrm{E}-02$ & $5.272 \mathrm{E}+04$ \\
\hline
\end{tabular}




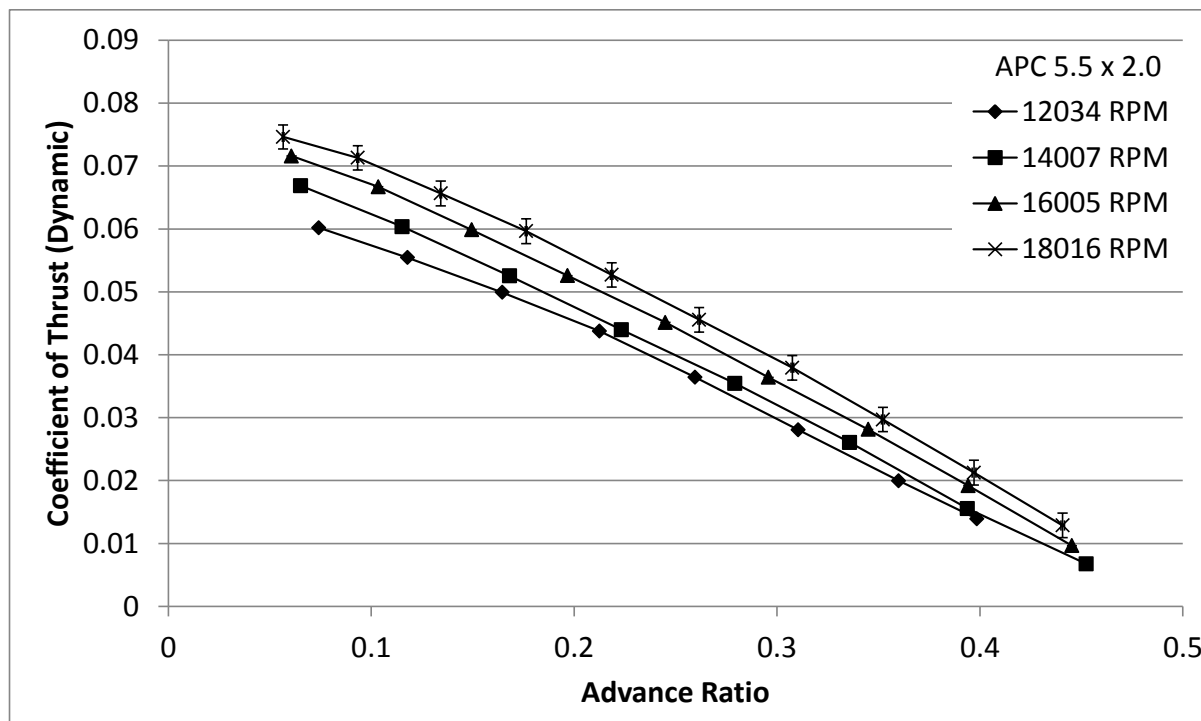

(a)

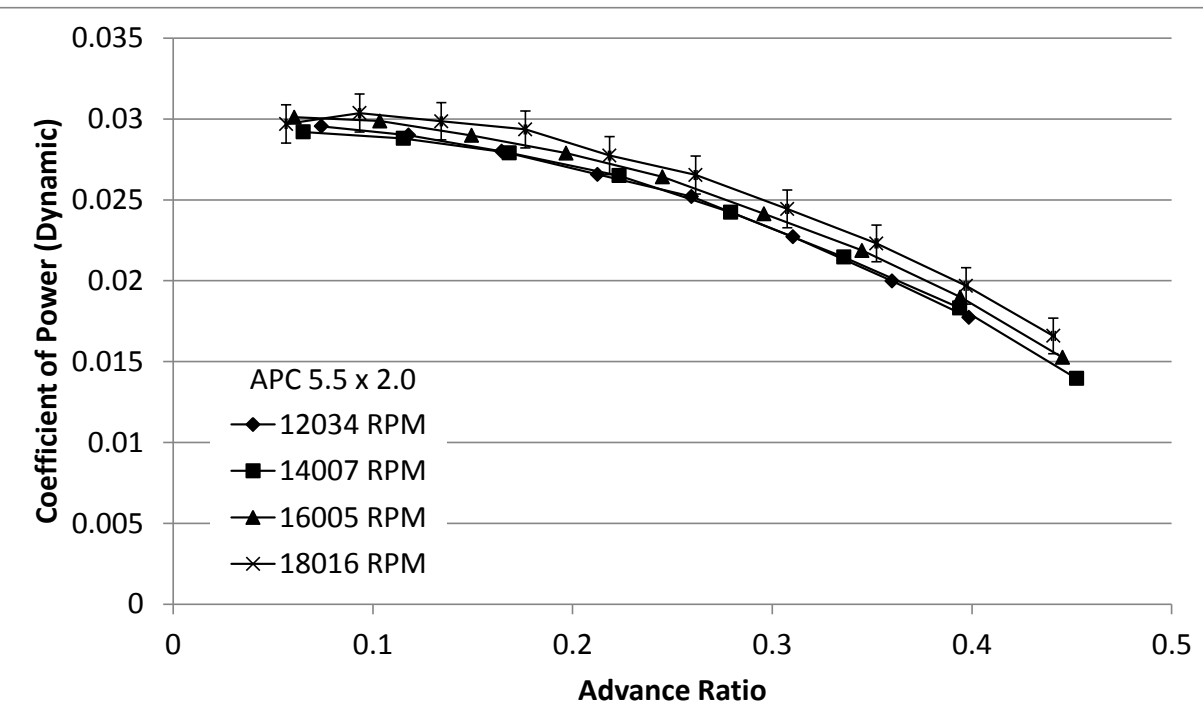

(b)

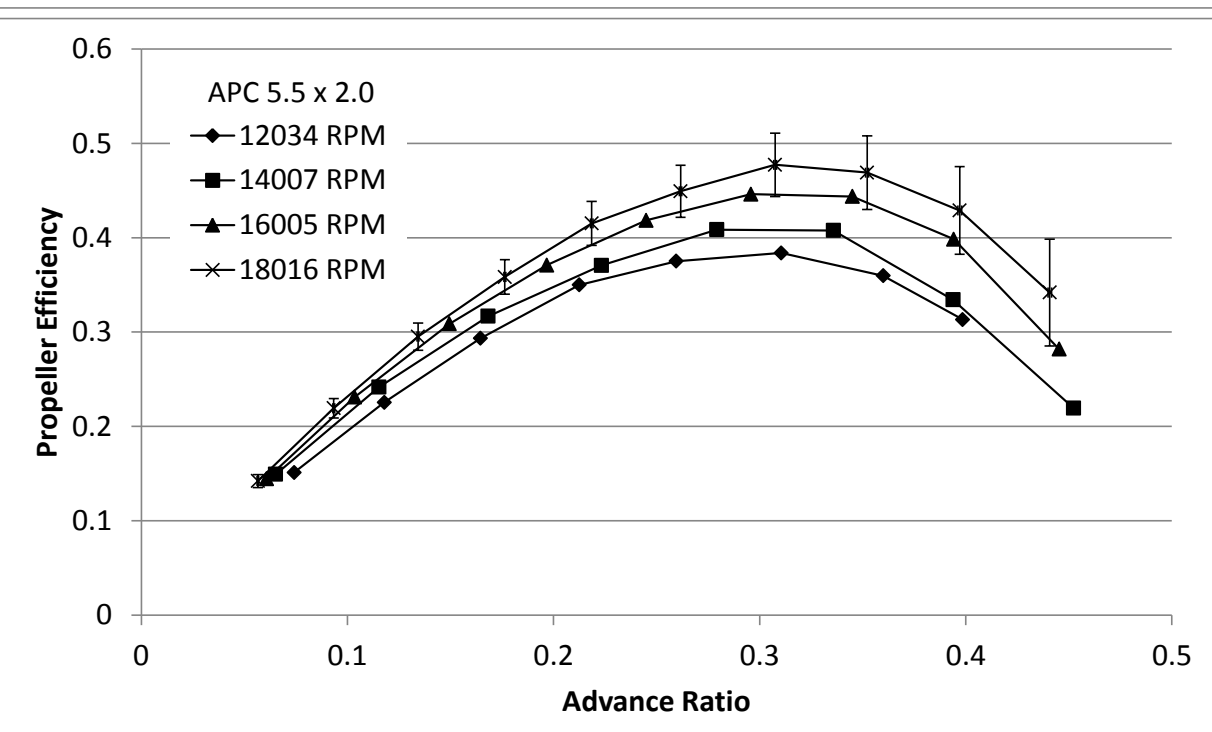

(c) 


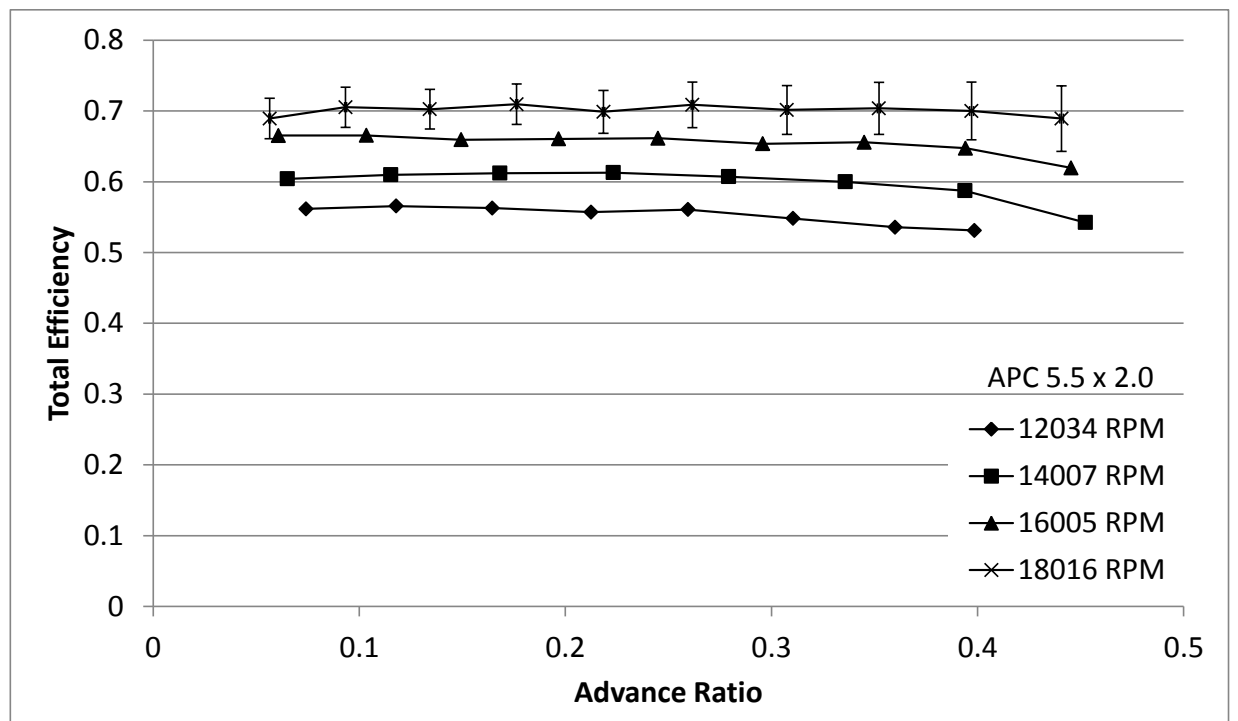

(d)

Figure 88: APC 5.5 x 2.0 Dynamic Test Results: (a) Coefficient of Thrust, (b) Coefficient of Power, (c) Propeller Efficiency, (d) Total Efficiency.

Table 154: APC 5.5 x 2.0 Dynamic Measured Values - 12034 RPM

\begin{tabular}{|c|c|c|c|c|c|c|c|}
\hline$n(\mathrm{RPM})$ & $Q(\mathrm{~g}-\mathrm{m})$ & $\Delta Q(\mathrm{~g}-\mathrm{m})$ & $V(\mathrm{~V})$ & $I(\mathrm{~A})$ & $T_{\mathrm{atm}}\left({ }^{\circ} \mathrm{C}\right)$ & $P_{\text {atm }}(\mathrm{Pa})$ & $P_{\text {diff }}(\mathrm{Pa})$ \\
\hline $1.194 \mathrm{E}+04$ & $1.156 \mathrm{E}+00$ & $6.21 \mathrm{E}-02$ & $1.105 \mathrm{E}+01$ & $2.285 \mathrm{E}+00$ & $2.275 \mathrm{E}+01$ & $9.868 \mathrm{E}+04$ & $2.724 \mathrm{E}+00$ \\
\hline $1.202 \mathrm{E}+04$ & $1.150 \mathrm{E}+00$ & $6.23 \mathrm{E}-02$ & $1.105 \mathrm{E}+01$ & $2.272 \mathrm{E}+00$ & $2.273 \mathrm{E}+01$ & $9.868 \mathrm{E}+04$ & $6.683 \mathrm{E}+00$ \\
\hline $1.199 \mathrm{E}+04$ & $1.103 \mathrm{E}+00$ & $6.20 \mathrm{E}-02$ & $1.105 \mathrm{E}+01$ & $2.183 \mathrm{E}+00$ & $2.275 \mathrm{E}+01$ & $9.867 \mathrm{E}+04$ & $1.266 \mathrm{E}+01$ \\
\hline $1.197 \mathrm{E}+04$ & $1.043 \mathrm{E}+00$ & $6.16 \mathrm{E}-02$ & $1.106 \mathrm{E}+01$ & $2.081 \mathrm{E}+00$ & $2.288 \mathrm{E}+01$ & $9.867 \mathrm{E}+04$ & $2.077 \mathrm{E}+01$ \\
\hline $1.204 \mathrm{E}+04$ & $1.001 \mathrm{E}+00$ & $6.25 \mathrm{E}-02$ & $1.106 \mathrm{E}+01$ & $1.996 \mathrm{E}+00$ & $2.309 \mathrm{E}+01$ & $9.867 \mathrm{E}+04$ & $3.107 \mathrm{E}+01$ \\
\hline $1.198 \mathrm{E}+04$ & $8.925 \mathrm{E}-01$ & $6.17 \mathrm{E}-02$ & $1.106 \mathrm{E}+01$ & $1.810 \mathrm{E}+00$ & $2.308 \mathrm{E}+01$ & $9.866 \mathrm{E}+04$ & $4.370 \mathrm{E}+01$ \\
\hline $1.199 \mathrm{E}+04$ & $7.862 \mathrm{E}-01$ & $6.15 \mathrm{E}-02$ & $1.107 \mathrm{E}+01$ & $1.632 \mathrm{E}+00$ & $2.317 \mathrm{E}+01$ & $9.866 \mathrm{E}+04$ & $5.855 \mathrm{E}+01$ \\
\hline $1.234 \mathrm{E}+04$ & $7.390 \mathrm{E}-01$ & $6.22 \mathrm{E}-02$ & $1.107 \mathrm{E}+01$ & $1.593 \mathrm{E}+00$ & $2.319 \mathrm{E}+01$ & $9.865 \mathrm{E}+04$ & $7.583 \mathrm{E}+01$ \\
\hline
\end{tabular}


Table 155: APC 5.5 x 2.0 Dynamic Calculated Values - 12034 RPM

\begin{tabular}{|c|c|c|c|c|c|c|c|}
\hline$n(\mathrm{RPM})$ & $V_{\infty}^{\prime}(\mathrm{m} / \mathrm{s})$ & $\Delta V_{\infty}^{\prime}(\mathrm{m} / \mathrm{s})$ & $T^{\prime}(\mathrm{g})$ & $\Delta T^{\prime}(\mathrm{g})$ & $V_{\mathrm{t}}(\mathrm{m} / \mathrm{s})$ & $P_{\mathrm{P}}(\mathrm{W})$ & $\Delta P_{\mathrm{P}}(\mathrm{W})$ \\
\hline $1.194 \mathrm{E}+04$ & $2.056 \mathrm{E}+00$ & $1.30 \mathrm{E}-02$ & $1.063 \mathrm{E}+02$ & $9.20 \mathrm{E}+00$ & $6.535 \mathrm{E}+01$ & $1.418 \mathrm{E}+01$ & $7.62 \mathrm{E}-01$ \\
\hline $1.202 \mathrm{E}+04$ & $3.290 \mathrm{E}+00$ & $1.33 \mathrm{E}-02$ & $9.919 \mathrm{E}+01$ & $9.09 \mathrm{E}+00$ & $6.584 \mathrm{E}+01$ & $1.420 \mathrm{E}+01$ & 7.69E-01 \\
\hline $1.199 \mathrm{E}+04$ & $4.578 \mathrm{E}+00$ & $1.45 \mathrm{E}-02$ & $8.872 \mathrm{E}+01$ & $9.15 \mathrm{E}+00$ & $6.571 \mathrm{E}+01$ & $1.358 \mathrm{E}+01$ & 7.64E-01 \\
\hline $1.197 \mathrm{E}+04$ & $5.901 \mathrm{E}+00$ & $1.61 \mathrm{E}-02$ & $7.752 \mathrm{E}+01$ & $9.12 \mathrm{E}+00$ & $6.571 \mathrm{E}+01$ & $1.282 \mathrm{E}+01$ & $7.57 \mathrm{E}-01$ \\
\hline $1.204 \mathrm{E}+04$ & $7.252 \mathrm{E}+00$ & $1.77 \mathrm{E}-02$ & $6.526 \mathrm{E}+01$ & $9.14 \mathrm{E}+00$ & $6.624 \mathrm{E}+01$ & $1.238 \mathrm{E}+01$ & 7.72E-01 \\
\hline $1.198 \mathrm{E}+04$ & $8.629 \mathrm{E}+00$ & $1.99 \mathrm{E}-02$ & $4.978 \mathrm{E}+01$ & $9.14 \mathrm{E}+00$ & $6.607 \mathrm{E}+01$ & $1.098 \mathrm{E}+01$ & $7.59 \mathrm{E}-01$ \\
\hline $1.199 \mathrm{E}+04$ & $1.001 \mathrm{E}+01$ & $2.16 \mathrm{E}-02$ & $3.544 \mathrm{E}+01$ & $9.16 \mathrm{E}+00$ & $6.631 \mathrm{E}+01$ & $9.678 \mathrm{E}+00$ & $7.57 \mathrm{E}-01$ \\
\hline $1.234 \mathrm{E}+04$ & $1.141 \mathrm{E}+01$ & $2.40 \mathrm{E}-02$ & $2.620 \mathrm{E}+01$ & $9.05 \mathrm{E}+00$ & $6.844 \mathrm{E}+01$ & $9.365 \mathrm{E}+00$ & $7.88 \mathrm{E}-01$ \\
\hline$P_{\mathrm{e}}(\mathrm{W})$ & $\Delta P_{\mathrm{e}}(\mathrm{W})$ & $C_{\mathrm{T}}$ & $\Delta C_{\mathrm{T}}$ & $C_{\mathrm{P}}$ & $\Delta C_{\mathrm{P}}$ & $C_{\mathrm{Q}}$ & $\Delta C_{\mathrm{Q}}$ \\
\hline $2.525 \mathrm{E}+01$ & $2.87 \mathrm{E}-01$ & $6.020 \mathrm{E}-02$ & $5.21 \mathrm{E}-03$ & $2.955 \mathrm{E}-02$ & $1.59 \mathrm{E}-03$ & $4.703 \mathrm{E}-03$ & $2.53 \mathrm{E}-04$ \\
\hline $2.510 \mathrm{E}+01$ & $2.84 \mathrm{E}-01$ & $5.544 \mathrm{E}-02$ & $5.08 \mathrm{E}-03$ & $2.900 \mathrm{E}-02$ & $1.57 \mathrm{E}-03$ & $4.615 \mathrm{E}-03$ & $2.50 \mathrm{E}-04$ \\
\hline $2.413 \mathrm{E}+01$ & $2.74 \mathrm{E}-01$ & 4.991E-02 & $5.15 \mathrm{E}-03$ & $2.800 \mathrm{E}-02$ & $1.58 \mathrm{E}-03$ & $4.456 \mathrm{E}-03$ & $2.51 \mathrm{E}-04$ \\
\hline $2.301 \mathrm{E}+01$ & $2.62 \mathrm{E}-01$ & 4.377E-02 & $5.15 \mathrm{E}-03$ & $2.657 \mathrm{E}-02$ & $1.57 \mathrm{E}-03$ & 4.229E-03 & $2.50 \mathrm{E}-04$ \\
\hline $2.207 \mathrm{E}+01$ & $2.51 \mathrm{E}-01$ & 3.643E-02 & $5.10 \mathrm{E}-03$ & $2.521 \mathrm{E}-02$ & $1.57 \mathrm{E}-03$ & $4.012 \mathrm{E}-03$ & $2.50 \mathrm{E}-04$ \\
\hline $2.003 \mathrm{E}+01$ & $2.31 \mathrm{E}-01$ & $2.808 \mathrm{E}-02$ & $5.15 \mathrm{E}-03$ & $2.271 \mathrm{E}-02$ & $1.57 \mathrm{E}-03$ & $3.615 \mathrm{E}-03$ & $2.50 \mathrm{E}-04$ \\
\hline $1.806 \mathrm{E}+01$ & $2.12 \mathrm{E}-01$ & $1.997 \mathrm{E}-02$ & $5.16 \mathrm{E}-03$ & 1.999E-02 & $1.56 \mathrm{E}-03$ & $3.181 \mathrm{E}-03$ & $2.49 \mathrm{E}-04$ \\
\hline $1.763 \mathrm{E}+01$ & $2.08 \mathrm{E}-01$ & $1.393 \mathrm{E}-02$ & $4.81 \mathrm{E}-03$ & $1.773 \mathrm{E}-02$ & $1.49 \mathrm{E}-03$ & $2.822 \mathrm{E}-03$ & $2.38 \mathrm{E}-04$ \\
\hline$\rho\left(\mathrm{kg} / \mathrm{m}^{\wedge} 3\right)$ & $\eta_{\mathrm{T}}$ & $\Delta \eta_{\mathrm{T}}$ & $J$ & $\Delta J$ & $\eta_{\mathrm{P}}$ & $\Delta \eta_{\mathrm{P}}$ & $R e_{0.75}$ \\
\hline $1.162 \mathrm{E}+00$ & $5.617 \mathrm{E}-01$ & $3.08 \mathrm{E}-02$ & $7.418 \mathrm{E}-02$ & $4.68 \mathrm{E}-04$ & $1.511 \mathrm{E}-01$ & $1.54 \mathrm{E}-02$ & $4.086 \mathrm{E}+04$ \\
\hline $1.162 \mathrm{E}+00$ & $5.656 \mathrm{E}-01$ & $3.13 \mathrm{E}-02$ & $1.179 \mathrm{E}-01$ & $4.81 \mathrm{E}-04$ & $2.254 \mathrm{E}-01$ & $2.40 \mathrm{E}-02$ & $4.116 \mathrm{E}+04$ \\
\hline $1.162 \mathrm{E}+00$ & $5.628 \mathrm{E}-01$ & $3.23 \mathrm{E}-02$ & $1.645 \mathrm{E}-01$ & $5.22 \mathrm{E}-04$ & $2.933 \mathrm{E}-01$ & $3.45 \mathrm{E}-02$ & $4.108 \mathrm{E}+04$ \\
\hline $1.161 \mathrm{E}+00$ & $5.571 \mathrm{E}-01$ & $3.35 \mathrm{E}-02$ & $2.125 \mathrm{E}-01$ & $5.82 \mathrm{E}-04$ & $3.499 \mathrm{E}-01$ & 4.61E-02 & $4.105 \mathrm{E}+04$ \\
\hline $1.160 \mathrm{E}+00$ & $5.607 \mathrm{E}-01$ & $3.56 \mathrm{E}-02$ & $2.595 \mathrm{E}-01$ & $6.44 \mathrm{E}-04$ & $3.751 \mathrm{E}-01$ & $5.75 \mathrm{E}-02$ & $4.133 \mathrm{E}+04$ \\
\hline $1.160 \mathrm{E}+00$ & $5.482 \mathrm{E}-01$ & $3.84 \mathrm{E}-02$ & $3.104 \mathrm{E}-01$ & $7.22 \mathrm{E}-04$ & $3.837 \mathrm{E}-01$ & $7.53 \mathrm{E}-02$ & $4.122 \mathrm{E}+04$ \\
\hline $1.160 \mathrm{E}+00$ & $5.358 \mathrm{E}-01$ & $4.24 \mathrm{E}-02$ & $3.599 \mathrm{E}-01$ & $7.81 \mathrm{E}-04$ & $3.596 \mathrm{E}-01$ & $9.71 \mathrm{E}-02$ & $4.134 \mathrm{E}+04$ \\
\hline $1.160 \mathrm{E}+00$ & $5.311 \mathrm{E}-01$ & $4.52 \mathrm{E}-02$ & $3.985 \mathrm{E}-01$ & 8.57E-04 & $3.131 \mathrm{E}-01$ & $1.11 \mathrm{E}-01$ & $4.266 \mathrm{E}+04$ \\
\hline
\end{tabular}


Table 156: APC 5.5 x 2.0 Dynamic Measured Values - 14007 RPM

\begin{tabular}{|c|c|c|c|c|c|c|c|}
\hline$n(\mathrm{RPM})$ & $Q(\mathrm{~g}-\mathrm{m})$ & $\Delta Q(\mathrm{~g}-\mathrm{m})$ & $V(\mathrm{~V})$ & $I(\mathrm{~A})$ & $T_{\text {atm }}\left({ }^{\circ} \mathrm{C}\right)$ & $P_{\text {atm }}(\mathrm{Pa})$ & $P_{\text {diff }}(\mathrm{Pa})$ \\
\hline $1.394 \mathrm{E}+04$ & $1.559 \mathrm{E}+00$ & $6.56 \mathrm{E}-02$ & $1.102 \mathrm{E}+01$ & $3.352 \mathrm{E}+00$ & $2.198 \mathrm{E}+01$ & $9.856 \mathrm{E}+04$ & $2.930 \mathrm{E}+00$ \\
\hline $1.403 \mathrm{E}+04$ & $1.558 \mathrm{E}+00$ & $6.65 \mathrm{E}-02$ & $1.102 \mathrm{E}+01$ & $3.342 \mathrm{E}+00$ & $2.186 \mathrm{E}+01$ & $9.856 \mathrm{E}+04$ & $8.761 \mathrm{E}+00$ \\
\hline $1.404 \mathrm{E}+04$ & $1.511 \mathrm{E}+00$ & $6.60 \mathrm{E}-02$ & $1.102 \mathrm{E}+01$ & $3.229 \mathrm{E}+00$ & $2.203 \mathrm{E}+01$ & $9.855 \mathrm{E}+04$ & $1.820 \mathrm{E}+01$ \\
\hline $1.403 \mathrm{E}+04$ & $1.430 \mathrm{E}+00$ & $6.54 \mathrm{E}-02$ & $1.103 \mathrm{E}+01$ & $3.049 \mathrm{E}+00$ & $2.234 \mathrm{E}+01$ & $9.855 \mathrm{E}+04$ & $3.149 \mathrm{E}+01$ \\
\hline $1.399 \mathrm{E}+04$ & $1.298 \mathrm{E}+00$ & $6.49 \mathrm{E}-02$ & $1.104 \mathrm{E}+01$ & $2.784 \mathrm{E}+00$ & $2.309 \mathrm{E}+01$ & $9.855 \mathrm{E}+04$ & $4.842 \mathrm{E}+01$ \\
\hline $1.404 \mathrm{E}+04$ & $1.158 \mathrm{E}+00$ & $6.52 \mathrm{E}-02$ & $1.104 \mathrm{E}+01$ & $2.521 \mathrm{E}+00$ & $2.311 \mathrm{E}+01$ & $9.854 \mathrm{E}+04$ & $7.006 \mathrm{E}+01$ \\
\hline $1.400 \mathrm{E}+04$ & $9.828 \mathrm{E}-01$ & $6.50 \mathrm{E}-02$ & $1.105 \mathrm{E}+01$ & $2.177 \mathrm{E}+00$ & $2.314 \mathrm{E}+01$ & $9.854 \mathrm{E}+04$ & $9.537 \mathrm{E}+01$ \\
\hline $1.396 \mathrm{E}+04$ & $7.438 \mathrm{E}-01$ & $6.50 \mathrm{E}-02$ & $1.106 \mathrm{E}+01$ & $1.777 \mathrm{E}+00$ & $2.334 \mathrm{E}+01$ & $9.854 \mathrm{E}+04$ & $1.246 \mathrm{E}+02$ \\
\hline
\end{tabular}


Table 157: APC 5.5 x 2.0 Dynamic Calculated Values - 14007 RPM

\begin{tabular}{|c|c|c|c|c|c|c|c|}
\hline$n(\mathrm{RPM})$ & $V_{\infty}^{\prime}(\mathrm{m} / \mathrm{s})$ & $\Delta V_{\infty}^{\prime}(\mathrm{m} / \mathrm{s})$ & $T^{\prime}(\mathrm{g})$ & $\Delta T^{\prime}(\mathrm{g})$ & $V_{\mathrm{t}}(\mathrm{m} / \mathrm{s})$ & $P_{\mathrm{P}}(\mathrm{W})$ & $\Delta P_{\mathrm{P}}(\mathrm{W})$ \\
\hline $1.394 \mathrm{E}+04$ & $2.109 \mathrm{E}+00$ & $1.42 \mathrm{E}-02$ & $1.610 \mathrm{E}+02$ & $9.70 \mathrm{E}+00$ & $7.627 \mathrm{E}+01$ & $2.232 \mathrm{E}+01$ & $9.39 \mathrm{E}-01$ \\
\hline $1.403 \mathrm{E}+04$ & $3.755 \mathrm{E}+00$ & $1.48 \mathrm{E}-02$ & $1.473 \mathrm{E}+02$ & $9.88 \mathrm{E}+00$ & $7.684 \mathrm{E}+01$ & $2.246 \mathrm{E}+01$ & $9.59 \mathrm{E}-01$ \\
\hline $1.404 \mathrm{E}+04$ & $5.484 \mathrm{E}+00$ & $1.65 \mathrm{E}-02$ & $1.283 \mathrm{E}+02$ & $9.95 \mathrm{E}+00$ & $7.699 \mathrm{E}+01$ & $2.179 \mathrm{E}+01$ & $9.52 \mathrm{E}-01$ \\
\hline $1.403 \mathrm{E}+04$ & $7.270 \mathrm{E}+00$ & $1.86 \mathrm{E}-02$ & $1.071 \mathrm{E}+02$ & $9.72 \mathrm{E}+00$ & $7.706 \mathrm{E}+01$ & $2.061 \mathrm{E}+01$ & $9.43 \mathrm{E}-01$ \\
\hline $1.399 \mathrm{E}+04$ & $9.069 \mathrm{E}+00$ & $2.15 \mathrm{E}-02$ & $8.568 \mathrm{E}+01$ & $9.75 \mathrm{E}+00$ & $7.706 \mathrm{E}+01$ & $1.865 \mathrm{E}+01$ & $9.33 \mathrm{E}-01$ \\
\hline $1.404 \mathrm{E}+04$ & $1.095 \mathrm{E}+01$ & 2.33E-02 & $6.343 \mathrm{E}+01$ & $9.65 \mathrm{E}+00$ & $7.758 \mathrm{E}+01$ & $1.670 \mathrm{E}+01$ & $9.41 \mathrm{E}-01$ \\
\hline $1.400 \mathrm{E}+04$ & $1.280 \mathrm{E}+01$ & 2.63E-02 & $3.763 \mathrm{E}+01$ & $9.73 \mathrm{E}+00$ & $7.765 \mathrm{E}+01$ & $1.413 \mathrm{E}+01$ & $9.35 \mathrm{E}-01$ \\
\hline $1.396 \mathrm{E}+04$ & $1.466 \mathrm{E}+01$ & $2.97 \mathrm{E}-02$ & $1.627 \mathrm{E}+01$ & $9.77 \mathrm{E}+00$ & $7.775 \mathrm{E}+01$ & $1.067 \mathrm{E}+01$ & $9.33 \mathrm{E}-01$ \\
\hline$P_{\mathrm{e}}(\mathrm{W})$ & $\Delta P_{\mathrm{e}}(\mathrm{W})$ & $C_{\mathrm{T}}$ & $\Delta C_{\mathrm{T}}$ & $C_{\mathrm{P}}$ & $\Delta C_{\mathrm{P}}$ & $C_{\mathrm{Q}}$ & $\Delta C_{\mathrm{Q}}$ \\
\hline $3.694 \mathrm{E}+01$ & $4.10 \mathrm{E}-01$ & $6.686 \mathrm{E}-02$ & $4.03 \mathrm{E}-03$ & $2.920 \mathrm{E}-02$ & $1.23 \mathrm{E}-03$ & $4.647 \mathrm{E}-03$ & $1.96 \mathrm{E}-04$ \\
\hline $3.682 \mathrm{E}+01$ & $4.10 \mathrm{E}-01$ & $6.035 \mathrm{E}-02$ & $4.05 \mathrm{E}-03$ & $2.880 \mathrm{E}-02$ & $1.23 \mathrm{E}-03$ & $4.584 \mathrm{E}-03$ & $1.96 \mathrm{E}-04$ \\
\hline $3.559 \mathrm{E}+01$ & $3.97 \mathrm{E}-01$ & $5.253 \mathrm{E}-02$ & $4.07 \mathrm{E}-03$ & $2.791 \mathrm{E}-02$ & $1.22 \mathrm{E}-03$ & 4.442E-03 & $1.94 \mathrm{E}-04$ \\
\hline $3.362 \mathrm{E}+01$ & $3.75 \mathrm{E}-01$ & $4.398 \mathrm{E}-02$ & 3.99E-03 & $2.650 \mathrm{E}-02$ & $1.21 \mathrm{E}-03$ & $4.218 \mathrm{E}-03$ & $1.93 \mathrm{E}-04$ \\
\hline $3.072 \mathrm{E}+01$ & $3.41 \mathrm{E}-01$ & $3.545 \mathrm{E}-02$ & $4.03 \mathrm{E}-03$ & $2.423 \mathrm{E}-02$ & $1.21 \mathrm{E}-03$ & $3.856 \mathrm{E}-03$ & $1.93 \mathrm{E}-04$ \\
\hline $2.784 \mathrm{E}+01$ & $3.15 \mathrm{E}-01$ & $2.606 \mathrm{E}-02$ & $3.96 \mathrm{E}-03$ & $2.146 \mathrm{E}-02$ & $1.21 \mathrm{E}-03$ & $3.416 \mathrm{E}-03$ & $1.92 \mathrm{E}-04$ \\
\hline $2.407 \mathrm{E}+01$ & $2.73 \mathrm{E}-01$ & $1.555 \mathrm{E}-02$ & $4.02 \mathrm{E}-03$ & $1.832 \mathrm{E}-02$ & $1.21 \mathrm{E}-03$ & $2.916 \mathrm{E}-03$ & $1.93 \mathrm{E}-04$ \\
\hline $1.966 \mathrm{E}+01$ & $2.27 \mathrm{E}-01$ & $6.768 \mathrm{E}-03$ & $4.06 \mathrm{E}-03$ & $1.396 \mathrm{E}-02$ & $1.22 \mathrm{E}-03$ & $2.222 \mathrm{E}-03$ & $1.94 \mathrm{E}-04$ \\
\hline$\rho\left(\mathrm{kg} / \mathrm{m}^{\wedge} 3\right)$ & $\eta_{\mathrm{T}}$ & $\Delta \eta_{\mathrm{T}}$ & $J$ & $\Delta J$ & $\eta_{\mathrm{P}}$ & $\Delta \eta_{\mathrm{P}}$ & $R e_{0.75}$ \\
\hline $1.163 \mathrm{E}+00$ & $6.042 \mathrm{E}-01$ & $2.63 \mathrm{E}-02$ & $6.519 \mathrm{E}-02$ & $4.41 \mathrm{E}-04$ & $1.493 \mathrm{E}-01$ & $1.10 \mathrm{E}-02$ & $4.785 \mathrm{E}+04$ \\
\hline $1.164 \mathrm{E}+00$ & $6.099 \mathrm{E}-01$ & $2.69 \mathrm{E}-02$ & $1.153 \mathrm{E}-01$ & $4.56 \mathrm{E}-04$ & $2.416 \mathrm{E}-01$ & $1.92 \mathrm{E}-02$ & $4.823 \mathrm{E}+04$ \\
\hline $1.163 \mathrm{E}+00$ & $6.121 \mathrm{E}-01$ & $2.76 \mathrm{E}-02$ & $1.683 \mathrm{E}-01$ & $5.10 \mathrm{E}-04$ & $3.167 \mathrm{E}-01$ & $2.82 \mathrm{E}-02$ & $4.828 \mathrm{E}+04$ \\
\hline $1.162 \mathrm{E}+00$ & $6.129 \mathrm{E}-01$ & $2.89 \mathrm{E}-02$ & $2.233 \mathrm{E}-01$ & $5.78 \mathrm{E}-04$ & $3.706 \mathrm{E}-01$ & 3.77E-02 & $4.823 \mathrm{E}+04$ \\
\hline $1.159 \mathrm{E}+00$ & $6.072 \mathrm{E}-01$ & $3.11 \mathrm{E}-02$ & $2.792 \mathrm{E}-01$ & $6.73 \mathrm{E}-04$ & $4.085 \mathrm{E}-01$ & $5.08 \mathrm{E}-02$ & $4.802 \mathrm{E}+04$ \\
\hline $1.159 \mathrm{E}+00$ & $5.999 \mathrm{E}-01$ & $3.45 \mathrm{E}-02$ & $3.358 \mathrm{E}-01$ & $7.29 \mathrm{E}-04$ & $4.077 \mathrm{E}-01$ & $6.61 \mathrm{E}-02$ & $4.833 \mathrm{E}+04$ \\
\hline $1.159 \mathrm{E}+00$ & 5.873E-01 & 3.94E-02 & $3.938 \mathrm{E}-01$ & $8.27 \mathrm{E}-04$ & $3.342 \mathrm{E}-01$ & 8.92E-02 & $4.837 \mathrm{E}+04$ \\
\hline $1.158 \mathrm{E}+00$ & $5.425 \mathrm{E}-01$ & $4.78 \mathrm{E}-02$ & $4.524 \mathrm{E}-01$ & $9.38 \mathrm{E}-04$ & $2.193 \mathrm{E}-01$ & $1.33 \mathrm{E}-01$ & $4.837 \mathrm{E}+04$ \\
\hline
\end{tabular}


Table 158: APC 5.5 x 2.0 Dynamic Measured Values - 16005 RPM

\begin{tabular}{|c|c|c|c|c|c|c|c|}
\hline$n(\mathrm{RPM})$ & $Q(\mathrm{~g}-\mathrm{m})$ & $\Delta Q(\mathrm{~g}-\mathrm{m})$ & $V(\mathrm{~V})$ & $I(\mathrm{~A})$ & $T_{\text {atm }}\left({ }^{\circ} \mathrm{C}\right)$ & $P_{\text {atm }}(\mathrm{Pa})$ & $P_{\text {diff }}(\mathrm{Pa})$ \\
\hline $1.601 \mathrm{E}+04$ & $2.116 \mathrm{E}+00$ & $8.06 \mathrm{E}-02$ & $1.098 \mathrm{E}+01$ & $4.762 \mathrm{E}+00$ & $2.206 \mathrm{E}+01$ & $9.847 \mathrm{E}+04$ & $3.395 \mathrm{E}+00$ \\
\hline $1.599 \mathrm{E}+04$ & $2.094 \mathrm{E}+00$ & $7.84 \mathrm{E}-02$ & $1.098 \mathrm{E}+01$ & $4.704 \mathrm{E}+00$ & $2.205 \mathrm{E}+01$ & $9.847 \mathrm{E}+04$ & $9.250 \mathrm{E}+00$ \\
\hline $1.598 \mathrm{E}+04$ & $2.028 \mathrm{E}+00$ & $7.86 \mathrm{E}-02$ & $1.098 \mathrm{E}+01$ & $4.595 \mathrm{E}+00$ & $2.220 \mathrm{E}+01$ & $9.847 \mathrm{E}+04$ & $1.875 \mathrm{E}+01$ \\
\hline $1.602 \mathrm{E}+04$ & $1.961 \mathrm{E}+00$ & $7.94 \mathrm{E}-02$ & $1.099 \mathrm{E}+01$ & $4.445 \mathrm{E}+00$ & $2.226 \mathrm{E}+01$ & $9.846 \mathrm{E}+04$ & $3.211 \mathrm{E}+01$ \\
\hline $1.600 \mathrm{E}+04$ & $1.853 \mathrm{E}+00$ & $7.77 \mathrm{E}-02$ & $1.099 \mathrm{E}+01$ & $4.185 \mathrm{E}+00$ & $2.230 \mathrm{E}+01$ & $9.847 \mathrm{E}+04$ & $4.912 \mathrm{E}+01$ \\
\hline $1.597 \mathrm{E}+04$ & $1.686 \mathrm{E}+00$ & $7.69 \mathrm{E}-02$ & $1.100 \mathrm{E}+01$ & $3.845 \mathrm{E}+00$ & $2.237 \mathrm{E}+01$ & $9.846 \mathrm{E}+04$ & $7.081 \mathrm{E}+01$ \\
\hline $1.603 \mathrm{E}+04$ & $1.539 \mathrm{E}+00$ & $7.77 \mathrm{E}-02$ & $1.101 \mathrm{E}+01$ & $3.507 \mathrm{E}+00$ & $2.240 \mathrm{E}+01$ & $9.846 \mathrm{E}+04$ & $9.646 \mathrm{E}+01$ \\
\hline $1.605 \mathrm{E}+04$ & $1.340 \mathrm{E}+00$ & $7.72 \mathrm{E}-02$ & $1.103 \mathrm{E}+01$ & $3.095 \mathrm{E}+00$ & $2.244 \mathrm{E}+01$ & $9.847 \mathrm{E}+04$ & $1.258 \mathrm{E}+02$ \\
\hline $1.601 \mathrm{E}+04$ & $1.072 \mathrm{E}+00$ & $7.59 \mathrm{E}-02$ & $1.104 \mathrm{E}+01$ & $2.577 \mathrm{E}+00$ & $2.247 \mathrm{E}+01$ & $9.846 \mathrm{E}+04$ & $1.593 \mathrm{E}+02$ \\
\hline
\end{tabular}


Table 159: APC 5.5 x 2.0 Dynamic Calculated Values - 16005 RPM

\begin{tabular}{|c|c|c|c|c|c|c|c|}
\hline$n(\mathrm{RPM})$ & $V_{\infty}^{\prime}(\mathrm{m} / \mathrm{s})$ & $\Delta V_{\infty}^{\prime}(\mathrm{m} / \mathrm{s})$ & $T^{\prime}(\mathrm{g})$ & $\Delta T^{\prime}(\mathrm{g})$ & $V_{\mathrm{t}}(\mathrm{m} / \mathrm{s})$ & $P_{\mathrm{P}}(\mathrm{W})$ & $\Delta P_{\mathrm{P}}(\mathrm{W})$ \\
\hline $1.601 \mathrm{E}+04$ & $2.257 \mathrm{E}+00$ & $1.56 \mathrm{E}-02$ & $2.270 \mathrm{E}+02$ & $7.98 \mathrm{E}+00$ & $8.756 \mathrm{E}+01$ & $3.478 \mathrm{E}+01$ & $1.33 \mathrm{E}+00$ \\
\hline $1.599 \mathrm{E}+04$ & $3.838 \mathrm{E}+00$ & $1.62 \mathrm{E}-02$ & $2.110 \mathrm{E}+02$ & $8.35 \mathrm{E}+00$ & $8.750 \mathrm{E}+01$ & $3.437 \mathrm{E}+01$ & $1.29 \mathrm{E}+00$ \\
\hline $1.598 \mathrm{E}+04$ & $5.544 \mathrm{E}+00$ & $1.74 \mathrm{E}-02$ & $1.890 \mathrm{E}+02$ & $8.81 \mathrm{E}+00$ & $8.755 \mathrm{E}+01$ & $3.328 \mathrm{E}+01$ & $1.29 \mathrm{E}+00$ \\
\hline $1.602 \mathrm{E}+04$ & $7.315 \mathrm{E}+00$ & $1.92 \mathrm{E}-02$ & $1.668 \mathrm{E}+02$ & $8.29 \mathrm{E}+00$ & $8.790 \mathrm{E}+01$ & $3.226 \mathrm{E}+01$ & $1.31 \mathrm{E}+00$ \\
\hline $1.600 \mathrm{E}+04$ & $9.095 \mathrm{E}+00$ & $2.24 \mathrm{E}-02$ & $1.428 \mathrm{E}+02$ & $8.07 \mathrm{E}+00$ & $8.794 \mathrm{E}+01$ & $3.043 \mathrm{E}+01$ & $1.28 \mathrm{E}+00$ \\
\hline $1.597 \mathrm{E}+04$ & $1.096 \mathrm{E}+01$ & $2.41 \mathrm{E}-02$ & $1.148 \mathrm{E}+02$ & $8.20 \mathrm{E}+00$ & $8.802 \mathrm{E}+01$ & $2.766 \mathrm{E}+01$ & $1.26 \mathrm{E}+00$ \\
\hline $1.603 \mathrm{E}+04$ & $1.283 \mathrm{E}+01$ & $2.65 \mathrm{E}-02$ & $8.932 \mathrm{E}+01$ & $8.16 \mathrm{E}+00$ & $8.857 \mathrm{E}+01$ & $2.533 \mathrm{E}+01$ & $1.28 \mathrm{E}+00$ \\
\hline $1.605 \mathrm{E}+04$ & $1.469 \mathrm{E}+01$ & $2.94 \mathrm{E}-02$ & $6.112 \mathrm{E}+01$ & $8.23 \mathrm{E}+00$ & $8.900 \mathrm{E}+01$ & $2.209 \mathrm{E}+01$ & $1.27 \mathrm{E}+00$ \\
\hline $1.601 \mathrm{E}+04$ & $1.655 \mathrm{E}+01$ & $3.22 \mathrm{E}-02$ & $3.062 \mathrm{E}+01$ & $8.32 \mathrm{E}+00$ & $8.912 \mathrm{E}+01$ & $1.763 \mathrm{E}+01$ & $1.25 \mathrm{E}+00$ \\
\hline$P_{\mathrm{e}}(\mathrm{W})$ & $\Delta P_{\mathrm{e}}(\mathrm{W})$ & $C_{\mathrm{T}}$ & $\Delta C_{\mathrm{T}}$ & $C_{\mathrm{P}}$ & $\Delta C_{\mathrm{P}}$ & $C_{\mathrm{Q}}$ & $\Delta C_{\mathrm{Q}}$ \\
\hline $5.227 \mathrm{E}+01$ & $5.91 \mathrm{E}-01$ & $7.160 \mathrm{E}-02$ & $2.52 \mathrm{E}-03$ & $3.011 \mathrm{E}-02$ & $1.15 \mathrm{E}-03$ & 4.792E-03 & $1.83 \mathrm{E}-04$ \\
\hline $5.165 \mathrm{E}+01$ & $5.81 \mathrm{E}-01$ & $6.671 \mathrm{E}-02$ & $2.64 \mathrm{E}-03$ & $2.987 \mathrm{E}-02$ & $1.12 \mathrm{E}-03$ & $4.754 \mathrm{E}-03$ & $1.78 \mathrm{E}-04$ \\
\hline $5.046 \mathrm{E}+01$ & $5.64 \mathrm{E}-01$ & $5.987 \mathrm{E}-02$ & 2.79E-03 & 2.899E-02 & $1.12 \mathrm{E}-03$ & $4.613 \mathrm{E}-03$ & $1.79 \mathrm{E}-04$ \\
\hline $4.884 \mathrm{E}+01$ & $5.54 \mathrm{E}-01$ & $5.258 \mathrm{E}-02$ & $2.61 \mathrm{E}-03$ & $2.789 \mathrm{E}-02$ & $1.13 \mathrm{E}-03$ & $4.439 \mathrm{E}-03$ & $1.80 \mathrm{E}-04$ \\
\hline $4.601 \mathrm{E}+01$ & $5.17 \mathrm{E}-01$ & 4.513E-02 & $2.55 \mathrm{E}-03$ & 2.642E-02 & $1.11 \mathrm{E}-03$ & 4.205E-03 & $1.76 \mathrm{E}-04$ \\
\hline $4.231 \mathrm{E}+01$ & $4.73 \mathrm{E}-01$ & $3.640 \mathrm{E}-02$ & $2.60 \mathrm{E}-03$ & $2.414 \mathrm{E}-02$ & $1.10 \mathrm{E}-03$ & $3.841 \mathrm{E}-03$ & $1.75 \mathrm{E}-04$ \\
\hline $3.863 \mathrm{E}+01$ & $4.30 \mathrm{E}-01$ & $2.814 \mathrm{E}-02$ & $2.57 \mathrm{E}-03$ & $2.187 \mathrm{E}-02$ & $1.10 \mathrm{E}-03$ & $3.481 \mathrm{E}-03$ & $1.76 \mathrm{E}-04$ \\
\hline $3.413 \mathrm{E}+01$ & 4.37E-01 & $1.919 \mathrm{E}-02$ & $2.58 \mathrm{E}-03$ & $1.899 \mathrm{E}-02$ & $1.10 \mathrm{E}-03$ & 3.022E-03 & $1.74 \mathrm{E}-04$ \\
\hline $2.845 \mathrm{E}+01$ & $3.24 \mathrm{E}-01$ & $9.662 \mathrm{E}-03$ & 2.62E-03 & $1.526 \mathrm{E}-02$ & $1.08 \mathrm{E}-03$ & $2.429 \mathrm{E}-03$ & $1.72 \mathrm{E}-04$ \\
\hline$\rho\left(\mathrm{kg} / \mathrm{m}^{\wedge} 3\right)$ & $\eta_{\mathrm{T}}$ & $\Delta \eta_{\mathrm{T}}$ & $J$ & $\Delta J$ & $\eta_{\mathrm{P}}$ & $\Delta \eta_{\mathrm{P}}$ & $R e_{0.75}$ \\
\hline $1.162 \mathrm{E}+00$ & $6.653 \mathrm{E}-01$ & $2.65 \mathrm{E}-02$ & $6.075 \mathrm{E}-02$ & $4.20 \mathrm{E}-04$ & $1.444 \mathrm{E}-01$ & $7.56 \mathrm{E}-03$ & $5.485 \mathrm{E}+04$ \\
\hline $1.162 \mathrm{E}+00$ & $6.654 \mathrm{E}-01$ & $2.60 \mathrm{E}-02$ & $1.035 \mathrm{E}-01$ & 4.37E-04 & $2.310 \mathrm{E}-01$ & $1.26 \mathrm{E}-02$ & $5.482 \mathrm{E}+04$ \\
\hline $1.161 \mathrm{E}+00$ & $6.595 \mathrm{E}-01$ & $2.66 \mathrm{E}-02$ & $1.495 \mathrm{E}-01$ & $4.72 \mathrm{E}-04$ & $3.088 \mathrm{E}-01$ & $1.87 \mathrm{E}-02$ & $5.479 \mathrm{E}+04$ \\
\hline $1.161 \mathrm{E}+00$ & $6.605 \mathrm{E}-01$ & $2.78 \mathrm{E}-02$ & $1.968 \mathrm{E}-01$ & $5.18 \mathrm{E}-04$ & $3.709 \mathrm{E}-01$ & $2.38 \mathrm{E}-02$ & $5.499 \mathrm{E}+04$ \\
\hline $1.161 \mathrm{E}+00$ & $6.615 \mathrm{E}-01$ & $2.87 \mathrm{E}-02$ & $2.450 \mathrm{E}-01$ & $6.05 \mathrm{E}-04$ & 4.184E-01 & $2.95 \mathrm{E}-02$ & $5.501 \mathrm{E}+04$ \\
\hline $1.161 \mathrm{E}+00$ & $6.537 \mathrm{E}-01$ & $3.07 \mathrm{E}-02$ & $2.958 \mathrm{E}-01$ & $6.53 \mathrm{E}-04$ & 4.461E-01 & $3.78 \mathrm{E}-02$ & $5.503 \mathrm{E}+04$ \\
\hline $1.161 \mathrm{E}+00$ & $6.557 \mathrm{E}-01$ & $3.39 \mathrm{E}-02$ & $3.450 \mathrm{E}-01$ & $7.16 \mathrm{E}-04$ & 4.437E-01 & $4.63 \mathrm{E}-02$ & $5.537 \mathrm{E}+04$ \\
\hline $1.160 \mathrm{E}+00$ & $6.473 \mathrm{E}-01$ & $3.82 \mathrm{E}-02$ & 3.942E-01 & $8.47 \mathrm{E}-04$ & $3.984 \mathrm{E}-01$ & $5.84 \mathrm{E}-02$ & $5.563 \mathrm{E}+04$ \\
\hline $1.160 \mathrm{E}+00$ & $6.197 \mathrm{E}-01$ & $4.44 \mathrm{E}-02$ & $4.453 \mathrm{E}-01$ & $8.71 \mathrm{E}-04$ & $2.819 \mathrm{E}-01$ & $7.91 \mathrm{E}-02$ & $5.569 \mathrm{E}+04$ \\
\hline
\end{tabular}


Table 160: APC 5.5 x 2.0 Dynamic Measured Values - 18016 RPM

\begin{tabular}{|c|c|c|c|c|c|c|c|}
\hline$n(\mathrm{RPM})$ & $Q(\mathrm{~g}-\mathrm{m})$ & $\Delta Q(\mathrm{~g}-\mathrm{m})$ & $V(\mathrm{~V})$ & $I(\mathrm{~A})$ & $T_{\text {atm }}\left({ }^{\circ} \mathrm{C}\right)$ & $P_{\text {atm }}(\mathrm{Pa})$ & $P_{\text {diff }}(\mathrm{Pa})$ \\
\hline $1.807 \mathrm{E}+04$ & $2.658 \mathrm{E}+00$ & $1.06 \mathrm{E}-01$ & $1.092 \mathrm{E}+01$ & $6.548 \mathrm{E}+00$ & $2.204 \mathrm{E}+01$ & $9.846 \mathrm{E}+04$ & $3.798 \mathrm{E}+00$ \\
\hline $1.807 \mathrm{E}+04$ & $2.718 \mathrm{E}+00$ & $1.05 \mathrm{E}-01$ & $1.092 \mathrm{E}+01$ & $6.543 \mathrm{E}+00$ & $2.204 \mathrm{E}+01$ & $9.847 \mathrm{E}+04$ & $9.734 \mathrm{E}+00$ \\
\hline $1.799 \mathrm{E}+04$ & $2.646 \mathrm{E}+00$ & $1.02 \mathrm{E}-01$ & $1.093 \mathrm{E}+01$ & $6.367 \mathrm{E}+00$ & $2.245 \mathrm{E}+01$ & $9.847 \mathrm{E}+04$ & $1.932 \mathrm{E}+01$ \\
\hline $1.798 \mathrm{E}+04$ & $2.597 \mathrm{E}+00$ & $1.01 \mathrm{E}-01$ & $1.094 \mathrm{E}+01$ & $6.179 \mathrm{E}+00$ & $2.270 \mathrm{E}+01$ & $9.847 \mathrm{E}+04$ & $3.271 \mathrm{E}+01$ \\
\hline $1.807 \mathrm{E}+04$ & $2.478 \mathrm{E}+00$ & $1.04 \mathrm{E}-01$ & $1.094 \mathrm{E}+01$ & $6.015 \mathrm{E}+00$ & $2.280 \mathrm{E}+01$ & $9.847 \mathrm{E}+04$ & $5.011 \mathrm{E}+01$ \\
\hline $1.805 \mathrm{E}+04$ & $2.365 \mathrm{E}+00$ & $1.04 \mathrm{E}-01$ & $1.095 \mathrm{E}+01$ & $5.649 \mathrm{E}+00$ & $2.285 \mathrm{E}+01$ & $9.847 \mathrm{E}+04$ & $7.108 \mathrm{E}+01$ \\
\hline $1.798 \mathrm{E}+04$ & $2.160 \mathrm{E}+00$ & $1.04 \mathrm{E}-01$ & $1.096 \mathrm{E}+01$ & $5.187 \mathrm{E}+00$ & $2.302 \mathrm{E}+01$ & $9.847 \mathrm{E}+04$ & $9.676 \mathrm{E}+01$ \\
\hline $1.798 \mathrm{E}+04$ & $1.971 \mathrm{E}+00$ & $1.01 \mathrm{E}-01$ & $1.098 \mathrm{E}+01$ & $4.709 \mathrm{E}+00$ & $2.323 \mathrm{E}+01$ & $9.847 \mathrm{E}+04$ & $1.262 \mathrm{E}+02$ \\
\hline $1.798 \mathrm{E}+04$ & $1.737 \mathrm{E}+00$ & $9.94 \mathrm{E}-02$ & $1.099 \mathrm{E}+01$ & $4.167 \mathrm{E}+00$ & $2.343 \mathrm{E}+01$ & $9.847 \mathrm{E}+04$ & $1.597 \mathrm{E}+02$ \\
\hline $1.800 \mathrm{E}+04$ & $1.467 \mathrm{E}+00$ & $9.73 \mathrm{E}-02$ & $1.101 \mathrm{E}+01$ & $3.573 \mathrm{E}+00$ & $2.344 \mathrm{E}+01$ & $9.847 \mathrm{E}+04$ & $1.966 \mathrm{E}+02$ \\
\hline
\end{tabular}


Table 161: APC 5.5 x 2.0 Dynamic Calculated Values - 18016 RPM

\begin{tabular}{|c|c|c|c|c|c|c|c|}
\hline$n(\mathrm{RPM})$ & $V_{\infty}^{\prime}(\mathrm{m} / \mathrm{s})$ & $\Delta V_{\infty}^{\prime}(\mathrm{m} / \mathrm{s})$ & $T^{\prime}(\mathrm{g})$ & $\Delta T^{\prime}(\mathrm{g})$ & $V_{\mathrm{t}}(\mathrm{m} / \mathrm{s})$ & $P_{\mathrm{P}}(\mathrm{W})$ & $\Delta P_{\mathrm{P}}(\mathrm{W})$ \\
\hline $1.807 \mathrm{E}+04$ & $2.371 \mathrm{E}+00$ & $1.70 \mathrm{E}-02$ & $3.014 \mathrm{E}+02$ & $7.73 \mathrm{E}+00$ & $9.882 \mathrm{E}+01$ & $4.932 \mathrm{E}+01$ & $1.97 \mathrm{E}+00$ \\
\hline $1.807 \mathrm{E}+04$ & $3.915 \mathrm{E}+00$ & $1.77 \mathrm{E}-02$ & $2.880 \mathrm{E}+02$ & $7.72 \mathrm{E}+00$ & $9.887 \mathrm{E}+01$ & $5.043 \mathrm{E}+01$ & $1.95 \mathrm{E}+00$ \\
\hline $1.799 \mathrm{E}+04$ & $5.605 \mathrm{E}+00$ & $1.87 \mathrm{E}-02$ & $2.626 \mathrm{E}+02$ & $7.85 \mathrm{E}+00$ & $9.853 \mathrm{E}+01$ & $4.889 \mathrm{E}+01$ & $1.88 \mathrm{E}+00$ \\
\hline $1.798 \mathrm{E}+04$ & $7.360 \mathrm{E}+00$ & $2.06 \mathrm{E}-02$ & $2.381 \mathrm{E}+02$ & $7.85 \mathrm{E}+00$ & $9.860 \mathrm{E}+01$ & $4.795 \mathrm{E}+01$ & $1.87 \mathrm{E}+00$ \\
\hline $1.807 \mathrm{E}+04$ & $9.164 \mathrm{E}+00$ & 2.33E-02 & $2.124 \mathrm{E}+02$ & $7.83 \mathrm{E}+00$ & $9.924 \mathrm{E}+01$ & $4.598 \mathrm{E}+01$ & $1.94 \mathrm{E}+00$ \\
\hline $1.805 \mathrm{E}+04$ & $1.096 \mathrm{E}+01$ & $2.62 \mathrm{E}-02$ & $1.833 \mathrm{E}+02$ & $7.83 \mathrm{E}+00$ & $9.932 \mathrm{E}+01$ & $4.384 \mathrm{E}+01$ & $1.93 \mathrm{E}+00$ \\
\hline $1.798 \mathrm{E}+04$ & $1.283 \mathrm{E}+01$ & $2.76 \mathrm{E}-02$ & $1.513 \mathrm{E}+02$ & $7.79 \mathrm{E}+00$ & $9.916 \mathrm{E}+01$ & $3.989 \mathrm{E}+01$ & $1.91 \mathrm{E}+00$ \\
\hline $1.798 \mathrm{E}+04$ & $1.470 \mathrm{E}+01$ & $3.00 \mathrm{E}-02$ & $1.184 \mathrm{E}+02$ & $7.75 \mathrm{E}+00$ & $9.943 \mathrm{E}+01$ & $3.639 \mathrm{E}+01$ & $1.86 \mathrm{E}+00$ \\
\hline $1.798 \mathrm{E}+04$ & $1.657 \mathrm{E}+01$ & $3.84 \mathrm{E}-02$ & $8.465 \mathrm{E}+01$ & $7.80 \mathrm{E}+00$ & $9.970 \mathrm{E}+01$ & $3.207 \mathrm{E}+01$ & $1.84 \mathrm{E}+00$ \\
\hline $1.800 \mathrm{E}+04$ & $1.841 \mathrm{E}+01$ & $3.53 \mathrm{E}-02$ & $5.135 \mathrm{E}+01$ & $7.79 \mathrm{E}+00$ & $1.001 \mathrm{E}+02$ & $2.712 \mathrm{E}+01$ & $1.80 \mathrm{E}+00$ \\
\hline$P_{\mathrm{e}}(\mathrm{W})$ & $\Delta P_{\mathrm{e}}(\mathrm{W})$ & $C_{\mathrm{T}}$ & $\Delta C_{\mathrm{T}}$ & $C_{\mathrm{P}}$ & $\Delta C_{\mathrm{P}}$ & $C_{\mathrm{Q}}$ & $\Delta C_{\mathrm{Q}}$ \\
\hline $7.154 \mathrm{E}+01$ & 7.99E-01 & 7.462E-02 & $1.91 \mathrm{E}-03$ & $2.970 \mathrm{E}-02$ & $1.19 \mathrm{E}-03$ & $4.726 \mathrm{E}-03$ & $1.89 \mathrm{E}-04$ \\
\hline $7.149 \mathrm{E}+01$ & 8.02E-01 & $7.130 \mathrm{E}-02$ & $1.91 \mathrm{E}-03$ & $3.036 \mathrm{E}-02$ & $1.17 \mathrm{E}-03$ & 4.833E-03 & $1.87 \mathrm{E}-04$ \\
\hline $6.959 \mathrm{E}+01$ & 7.63E-01 & $6.565 \mathrm{E}-02$ & $1.96 \mathrm{E}-03$ & $2.986 \mathrm{E}-02$ & $1.15 \mathrm{E}-03$ & $4.752 \mathrm{E}-03$ & $1.83 \mathrm{E}-04$ \\
\hline $6.757 \mathrm{E}+01$ & 7.46E-01 & $5.965 \mathrm{E}-02$ & $1.97 \mathrm{E}-03$ & $2.935 \mathrm{E}-02$ & $1.14 \mathrm{E}-03$ & 4.672E-03 & $1.82 \mathrm{E}-04$ \\
\hline $6.581 \mathrm{E}+01$ & 7.37E-01 & $5.269 \mathrm{E}-02$ & $1.94 \mathrm{E}-03$ & $2.774 \mathrm{E}-02$ & $1.17 \mathrm{E}-03$ & 4.414E-03 & $1.86 \mathrm{E}-04$ \\
\hline $6.186 \mathrm{E}+01$ & $6.88 \mathrm{E}-01$ & $4.557 \mathrm{E}-02$ & $1.95 \mathrm{E}-03$ & $2.654 \mathrm{E}-02$ & $1.17 \mathrm{E}-03$ & $4.224 \mathrm{E}-03$ & $1.86 \mathrm{E}-04$ \\
\hline $5.687 \mathrm{E}+01$ & $6.39 \mathrm{E}-01$ & 3.793E-02 & $1.95 \mathrm{E}-03$ & 2.444E-02 & $1.17 \mathrm{E}-03$ & $3.890 \mathrm{E}-03$ & $1.86 \mathrm{E}-04$ \\
\hline $5.171 \mathrm{E}+01$ & $5.75 \mathrm{E}-01$ & $2.970 \mathrm{E}-02$ & $1.94 \mathrm{E}-03$ & $2.231 \mathrm{E}-02$ & $1.14 \mathrm{E}-03$ & $3.550 \mathrm{E}-03$ & $1.81 \mathrm{E}-04$ \\
\hline $4.581 \mathrm{E}+01$ & $5.12 \mathrm{E}-01$ & $2.126 \mathrm{E}-02$ & $1.96 \mathrm{E}-03$ & $1.969 \mathrm{E}-02$ & $1.13 \mathrm{E}-03$ & $3.133 \mathrm{E}-03$ & $1.79 \mathrm{E}-04$ \\
\hline $3.934 \mathrm{E}+01$ & 4.38E-01 & $1.287 \mathrm{E}-02$ & $1.95 \mathrm{E}-03$ & $1.659 \mathrm{E}-02$ & $1.10 \mathrm{E}-03$ & $2.641 \mathrm{E}-03$ & $1.75 \mathrm{E}-04$ \\
\hline$\rho\left(\mathrm{kg} / \mathrm{m}^{\wedge} 3\right)$ & $\eta_{\mathrm{T}}$ & $\Delta \eta_{\mathrm{T}}$ & $J$ & $\Delta J$ & $\eta_{\mathrm{P}}$ & $\Delta \eta_{\mathrm{P}}$ & $R e_{0.75}$ \\
\hline $1.162 \mathrm{E}+00$ & $6.894 \mathrm{E}-01$ & $2.86 \mathrm{E}-02$ & $5.656 \mathrm{E}-02$ & 4.07E-04 & $1.421 \mathrm{E}-01$ & $6.83 \mathrm{E}-03$ & $6.191 \mathrm{E}+04$ \\
\hline $1.162 \mathrm{E}+00$ & $7.054 \mathrm{E}-01$ & $2.84 \mathrm{E}-02$ & $9.337 \mathrm{E}-02$ & 4.24E-04 & $2.192 \mathrm{E}-01$ & $1.04 \mathrm{E}-02$ & $6.194 \mathrm{E}+04$ \\
\hline $1.160 \mathrm{E}+00$ & $7.025 \mathrm{E}-01$ & $2.81 \mathrm{E}-02$ & $1.343 \mathrm{E}-01$ & $4.49 \mathrm{E}-04$ & $2.952 \mathrm{E}-01$ & $1.44 \mathrm{E}-02$ & $6.158 \mathrm{E}+04$ \\
\hline $1.160 \mathrm{E}+00$ & $7.096 \mathrm{E}-01$ & $2.87 \mathrm{E}-02$ & $1.764 \mathrm{E}-01$ & $4.96 \mathrm{E}-04$ & $3.584 \mathrm{E}-01$ & $1.83 \mathrm{E}-02$ & $6.153 \mathrm{E}+04$ \\
\hline $1.159 \mathrm{E}+00$ & $6.988 \mathrm{E}-01$ & $3.04 \mathrm{E}-02$ & $2.185 \mathrm{E}-01$ & $5.62 \mathrm{E}-04$ & 4.151E-01 & $2.33 \mathrm{E}-02$ & $6.190 \mathrm{E}+04$ \\
\hline $1.159 \mathrm{E}+00$ & 7.087E-01 & $3.22 \mathrm{E}-02$ & $2.616 \mathrm{E}-01$ & $6.33 \mathrm{E}-04$ & 4.492E-01 & $2.76 \mathrm{E}-02$ & $6.192 \mathrm{E}+04$ \\
\hline $1.158 \mathrm{E}+00$ & 7.014E-01 & $3.45 \mathrm{E}-02$ & $3.075 \mathrm{E}-01$ & $6.65 \mathrm{E}-04$ & $4.772 \mathrm{E}-01$ & $3.36 \mathrm{E}-02$ & $6.177 \mathrm{E}+04$ \\
\hline $1.157 \mathrm{E}+00$ & $7.038 \mathrm{E}-01$ & $3.68 \mathrm{E}-02$ & $3.522 \mathrm{E}-01$ & 7.22E-04 & 4.690E-01 & $3.89 \mathrm{E}-02$ & $6.185 \mathrm{E}+04$ \\
\hline $1.157 \mathrm{E}+00$ & $7.000 \mathrm{E}-01$ & $4.08 \mathrm{E}-02$ & $3.970 \mathrm{E}-01$ & $9.24 \mathrm{E}-04$ & $4.289 \mathrm{E}-01$ & $4.66 \mathrm{E}-02$ & $6.195 \mathrm{E}+04$ \\
\hline $1.157 \mathrm{E}+00$ & $6.893 \mathrm{E}-01$ & 4.63E-02 & 4.407E-01 & 8.53E-04 & $3.419 \mathrm{E}-01$ & $5.66 \mathrm{E}-02$ & $6.221 \mathrm{E}+04$ \\
\hline
\end{tabular}




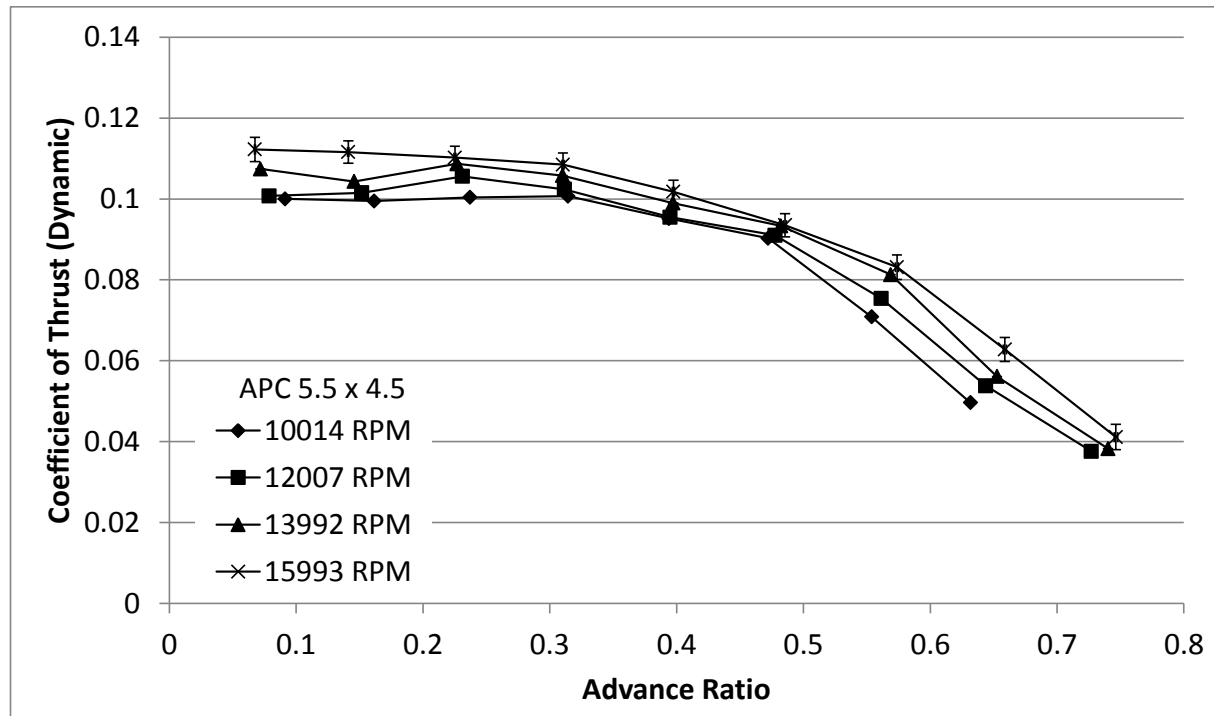

(a)

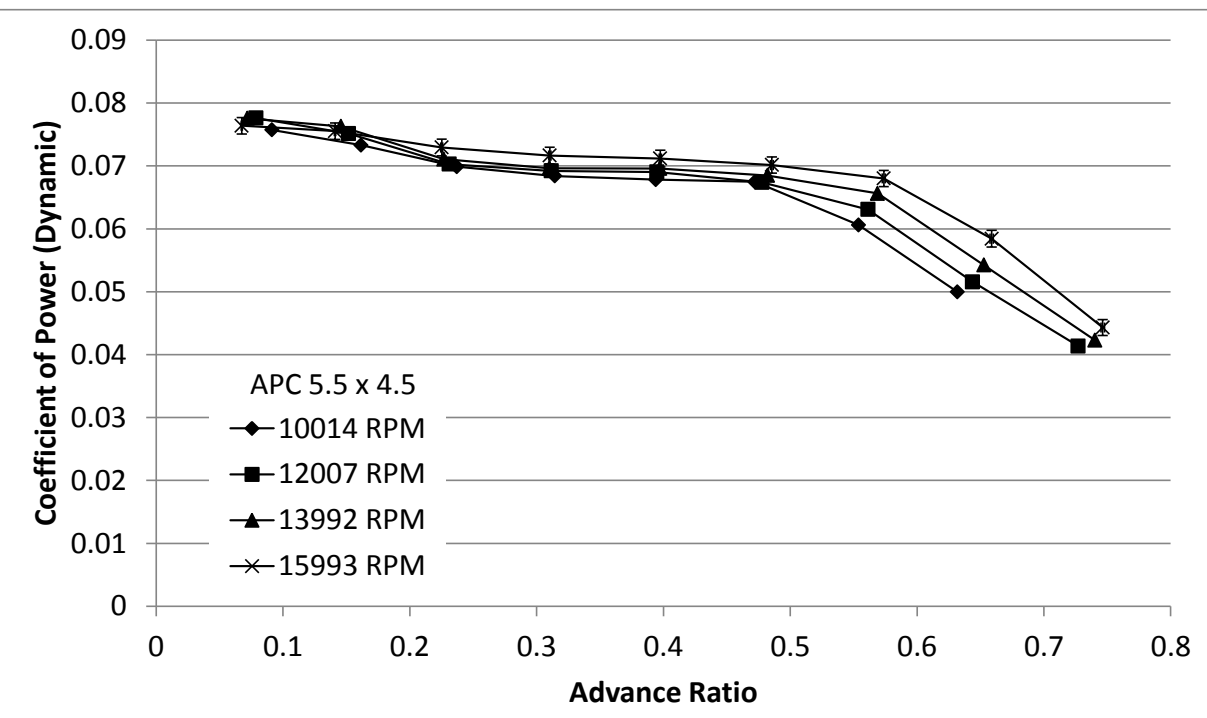

(b)

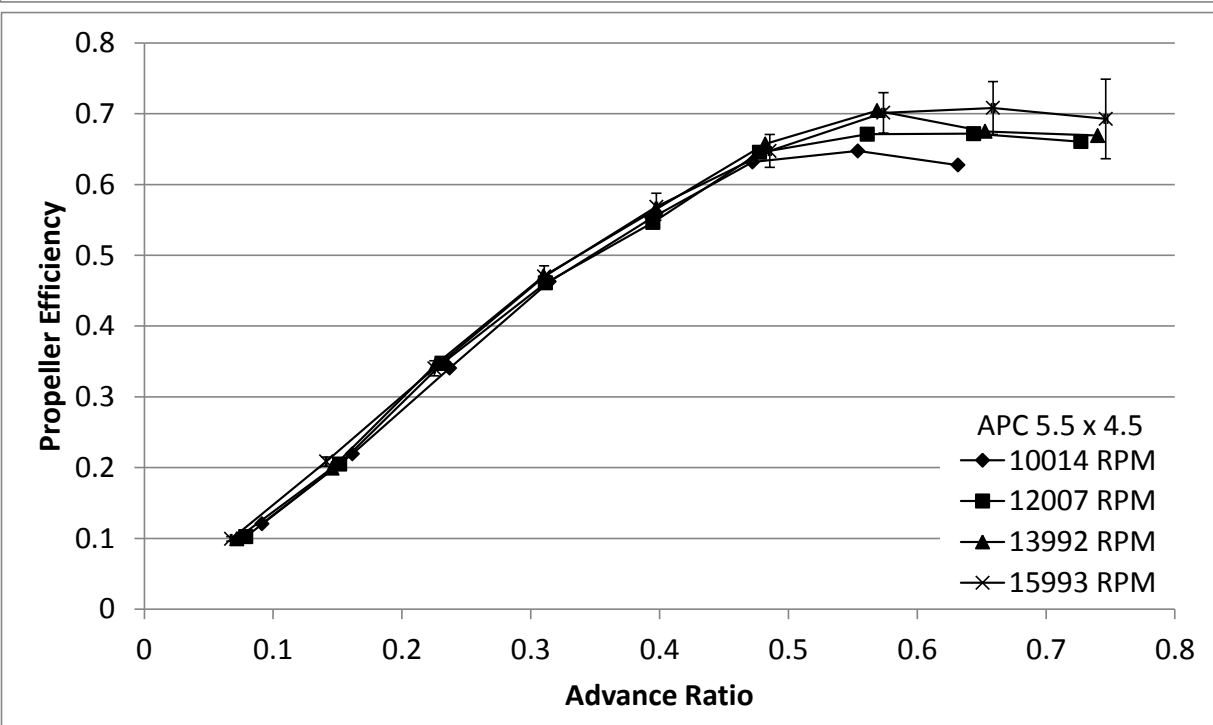

(c) 


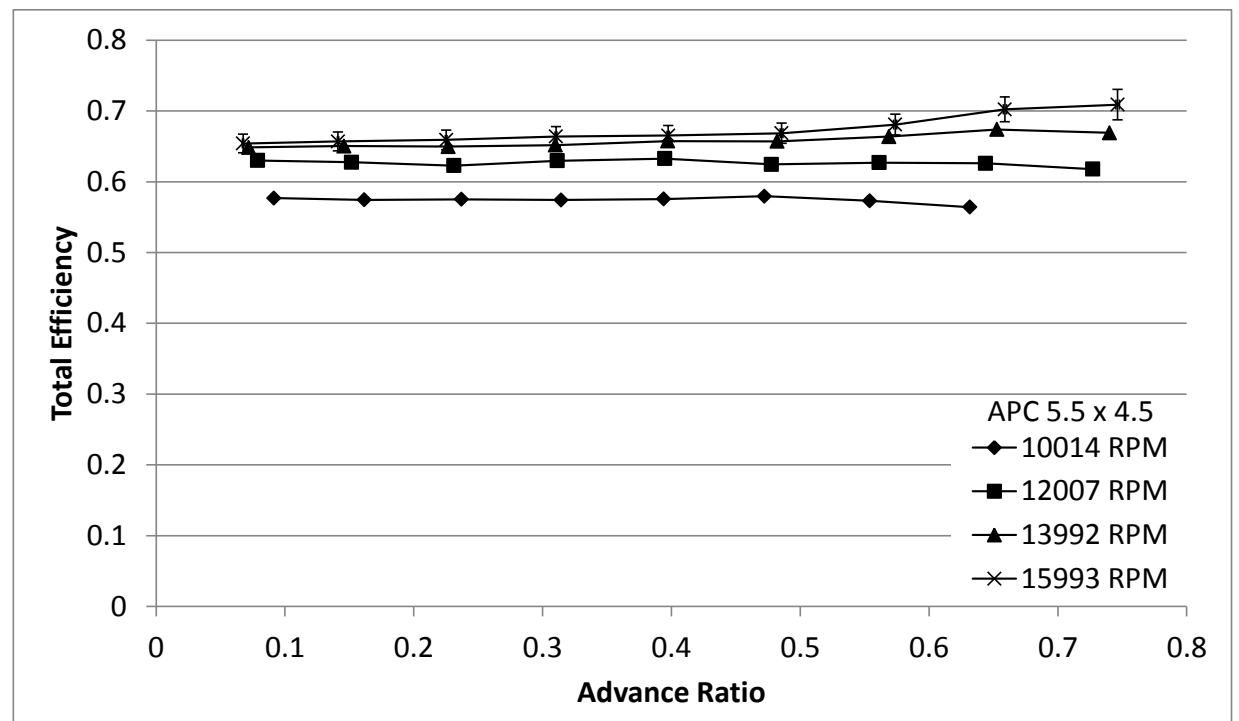

(d)

Figure 89: APC 5.5 x 4.5 Dynamic Test Results: (a) Coefficient of Thrust, (b) Coefficient of Power, (c) Propeller Efficiency, (d) Total Efficiency.

Table 162: APC 5.5 x 4.5 Dynamic Measured Values - 10014 RPM

\begin{tabular}{|c|c|c|c|c|c|c|c|}
\hline$n(\mathrm{RPM})$ & $Q(\mathrm{~g}-\mathrm{m})$ & $\Delta Q(\mathrm{~g}-\mathrm{m})$ & $V(\mathrm{~V})$ & $I(\mathrm{~A})$ & $T_{\mathrm{atm}}\left({ }^{\circ} \mathrm{C}\right)$ & $P_{\text {atm }}(\mathrm{Pa})$ & $P_{\text {diff }}(\mathrm{Pa})$ \\
\hline $1.003 \mathrm{E}+04$ & $2.114 \mathrm{E}+00$ & $7.36 \mathrm{E}-02$ & $1.102 \mathrm{E}+01$ & $3.426 \mathrm{E}+00$ & $2.222 \mathrm{E}+01$ & $9.909 \mathrm{E}+04$ & $2.955 \mathrm{E}+00$ \\
\hline $1.003 \mathrm{E}+04$ & $2.047 \mathrm{E}+00$ & $7.38 \mathrm{E}-02$ & $1.102 \mathrm{E}+01$ & $3.332 \mathrm{E}+00$ & $2.222 \mathrm{E}+01$ & $9.909 \mathrm{E}+04$ & $8.798 \mathrm{E}+00$ \\
\hline $1.002 \mathrm{E}+04$ & $1.945 \mathrm{E}+00$ & $6.63 \mathrm{E}-02$ & $1.103 \mathrm{E}+01$ & $3.155 \mathrm{E}+00$ & $2.227 \mathrm{E}+01$ & $9.910 \mathrm{E}+04$ & $1.849 \mathrm{E}+01$ \\
\hline $1.001 \mathrm{E}+04$ & $1.901 \mathrm{E}+00$ & $6.47 \mathrm{E}-02$ & $1.103 \mathrm{E}+01$ & $3.086 \mathrm{E}+00$ & $2.237 \mathrm{E}+01$ & $9.909 \mathrm{E}+04$ & $3.212 \mathrm{E}+01$ \\
\hline $9.988 \mathrm{E}+03$ & $1.875 \mathrm{E}+00$ & $6.35 \mathrm{E}-02$ & $1.103 \mathrm{E}+01$ & $3.030 \mathrm{E}+00$ & $2.251 \mathrm{E}+01$ & $9.909 \mathrm{E}+04$ & $4.979 \mathrm{E}+01$ \\
\hline $1.001 \mathrm{E}+04$ & $1.873 \mathrm{E}+00$ & $6.40 \mathrm{E}-02$ & $1.103 \mathrm{E}+01$ & $3.012 \mathrm{E}+00$ & $2.258 \mathrm{E}+01$ & $9.908 \mathrm{E}+04$ & $7.144 \mathrm{E}+01$ \\
\hline $9.992 \mathrm{E}+03$ & $1.676 \mathrm{E}+00$ & $6.32 \mathrm{E}-02$ & $1.104 \mathrm{E}+01$ & $2.719 \mathrm{E}+00$ & $2.259 \mathrm{E}+01$ & $9.908 \mathrm{E}+04$ & $9.743 \mathrm{E}+01$ \\
\hline $1.003 \mathrm{E}+04$ & $1.392 \mathrm{E}+00$ & $6.47 \mathrm{E}-02$ & $1.105 \mathrm{E}+01$ & $2.299 \mathrm{E}+00$ & $2.257 \mathrm{E}+01$ & $9.908 \mathrm{E}+04$ & $1.271 \mathrm{E}+02$ \\
\hline
\end{tabular}


Table 163: APC 5.5 x 4.5 Dynamic Calculated Values - 10014 RPM

\begin{tabular}{|c|c|c|c|c|c|c|c|}
\hline$n(\mathrm{RPM})$ & $V_{\infty}^{\prime}(\mathrm{m} / \mathrm{s})$ & $\Delta V_{\infty}^{\prime}(\mathrm{m} / \mathrm{s})$ & $T^{\prime}(\mathrm{g})$ & $\Delta T^{\prime}(\mathrm{g})$ & $V_{\mathrm{t}}(\mathrm{m} / \mathrm{s})$ & $P_{\mathrm{P}}(\mathrm{W})$ & $\Delta P_{\mathrm{P}}(\mathrm{W})$ \\
\hline $1.003 \mathrm{E}+04$ & $2.130 \mathrm{E}+00$ & $1.36 \mathrm{E}-02$ & $1.258 \mathrm{E}+02$ & $1.18 \mathrm{E}+01$ & $5.496 \mathrm{E}+01$ & $2.178 \mathrm{E}+01$ & $7.58 \mathrm{E}-01$ \\
\hline $1.003 \mathrm{E}+04$ & $3.766 \mathrm{E}+00$ & $1.45 \mathrm{E}-02$ & $1.252 \mathrm{E}+02$ & $1.19 \mathrm{E}+01$ & $5.505 \mathrm{E}+01$ & $2.109 \mathrm{E}+01$ & $7.61 \mathrm{E}-01$ \\
\hline $1.002 \mathrm{E}+04$ & $5.516 \mathrm{E}+00$ & $1.66 \mathrm{E}-02$ & $1.259 \mathrm{E}+02$ & $1.20 \mathrm{E}+01$ & $5.511 \mathrm{E}+01$ & $2.001 \mathrm{E}+01$ & $6.83 \mathrm{E}-01$ \\
\hline $1.001 \mathrm{E}+04$ & $7.314 \mathrm{E}+00$ & $1.89 \mathrm{E}-02$ & $1.261 \mathrm{E}+02$ & $1.18 \mathrm{E}+01$ & $5.530 \mathrm{E}+01$ & $1.955 \mathrm{E}+01$ & $6.65 \mathrm{E}-01$ \\
\hline $9.988 \mathrm{E}+03$ & $9.144 \mathrm{E}+00$ & $2.20 \mathrm{E}-02$ & $1.186 \mathrm{E}+02$ & $1.20 \mathrm{E}+01$ & $5.544 \mathrm{E}+01$ & $1.923 \mathrm{E}+01$ & $6.51 \mathrm{E}-01$ \\
\hline $1.001 \mathrm{E}+04$ & $1.098 \mathrm{E}+01$ & $2.45 \mathrm{E}-02$ & $1.130 \mathrm{E}+02$ & $1.19 \mathrm{E}+01$ & $5.589 \mathrm{E}+01$ & $1.926 \mathrm{E}+01$ & $6.58 \mathrm{E}-01$ \\
\hline $9.992 \mathrm{E}+03$ & $1.286 \mathrm{E}+01$ & $2.72 \mathrm{E}-02$ & $8.832 \mathrm{E}+01$ & $1.18 \mathrm{E}+01$ & $5.620 \mathrm{E}+01$ & $1.720 \mathrm{E}+01$ & $6.49 \mathrm{E}-01$ \\
\hline $1.003 \mathrm{E}+04$ & $1.472 \mathrm{E}+01$ & $3.00 \mathrm{E}-02$ & $6.232 \mathrm{E}+01$ & $1.18 \mathrm{E}+01$ & $5.683 \mathrm{E}+01$ & $1.433 \mathrm{E}+01$ & $6.66 \mathrm{E}-01$ \\
\hline$P_{\mathrm{e}}(\mathrm{W})$ & $\Delta P_{\mathrm{e}}(\mathrm{W})$ & $C_{\mathrm{T}}$ & $\Delta C_{\mathrm{T}}$ & $C_{\mathrm{P}}$ & $\Delta C_{\mathrm{P}}$ & $C_{\mathrm{Q}}$ & $\Delta C_{\mathrm{Q}}$ \\
\hline $3.775 \mathrm{E}+01$ & $4.33 \mathrm{E}-01$ & $1.000 \mathrm{E}-01$ & $9.41 \mathrm{E}-03$ & $7.572 \mathrm{E}-02$ & $2.64 \mathrm{E}-03$ & $1.205 \mathrm{E}-02$ & $4.19 \mathrm{E}-04$ \\
\hline $3.672 \mathrm{E}+01$ & $4.22 \mathrm{E}-01$ & $9.950 \mathrm{E}-02$ & $9.49 \mathrm{E}-03$ & $7.331 \mathrm{E}-02$ & $2.64 \mathrm{E}-03$ & $1.167 \mathrm{E}-02$ & $4.21 \mathrm{E}-04$ \\
\hline $3.479 \mathrm{E}+01$ & $3.96 \mathrm{E}-01$ & $1.004 \mathrm{E}-01$ & $9.54 \mathrm{E}-03$ & $6.989 \mathrm{E}-02$ & $2.39 \mathrm{E}-03$ & $1.112 \mathrm{E}-02$ & $3.80 \mathrm{E}-04$ \\
\hline $3.404 \mathrm{E}+01$ & $3.88 \mathrm{E}-01$ & $1.007 \mathrm{E}-01$ & 9.39E-03 & $6.840 \mathrm{E}-02$ & $2.33 \mathrm{E}-03$ & 1.089E-02 & $3.71 \mathrm{E}-04$ \\
\hline $3.342 \mathrm{E}+01$ & $3.76 \mathrm{E}-01$ & $9.515 \mathrm{E}-02$ & $9.60 \mathrm{E}-03$ & $6.782 \mathrm{E}-02$ & $2.30 \mathrm{E}-03$ & $1.079 \mathrm{E}-02$ & $3.66 \mathrm{E}-04$ \\
\hline $3.323 \mathrm{E}+01$ & $3.78 \mathrm{E}-01$ & $9.027 \mathrm{E}-02$ & $9.48 \mathrm{E}-03$ & $6.747 \mathrm{E}-02$ & $2.31 \mathrm{E}-03$ & $1.074 \mathrm{E}-02$ & $3.67 \mathrm{E}-04$ \\
\hline $3.002 \mathrm{E}+01$ & $3.41 \mathrm{E}-01$ & $7.084 \mathrm{E}-02$ & $9.50 \mathrm{E}-03$ & $6.060 \mathrm{E}-02$ & $2.29 \mathrm{E}-03$ & $9.645 \mathrm{E}-03$ & 3.64E-04 \\
\hline $2.541 \mathrm{E}+01$ & $2.92 \mathrm{E}-01$ & $4.965 \mathrm{E}-02$ & $9.42 \mathrm{E}-03$ & 4.999E-02 & $2.32 \mathrm{E}-03$ & $7.956 \mathrm{E}-03$ & $3.70 \mathrm{E}-04$ \\
\hline$\rho\left(\mathrm{kg} / \mathrm{m}^{\wedge} 3\right)$ & $\eta_{\mathrm{T}}$ & $\Delta \eta_{\mathrm{T}}$ & $J$ & $\Delta J$ & $\eta_{\mathrm{P}}$ & $\Delta \eta_{\mathrm{P}}$ & $R e_{0.75}$ \\
\hline $1.169 \mathrm{E}+00$ & $5.770 \mathrm{E}-01$ & $2.11 \mathrm{E}-02$ & $9.139 \mathrm{E}-02$ & $5.84 \mathrm{E}-04$ & $1.207 \mathrm{E}-01$ & $1.21 \mathrm{E}-02$ & $3.373 \mathrm{E}+04$ \\
\hline $1.169 \mathrm{E}+00$ & 5.744E-01 & $2.17 \mathrm{E}-02$ & $1.615 \mathrm{E}-01$ & $6.25 \mathrm{E}-04$ & $2.193 \mathrm{E}-01$ & $2.24 \mathrm{E}-02$ & $3.379 \mathrm{E}+04$ \\
\hline $1.169 \mathrm{E}+00$ & $5.752 \mathrm{E}-01$ & $2.07 \mathrm{E}-02$ & $2.370 \mathrm{E}-01$ & $7.19 \mathrm{E}-04$ & $3.404 \mathrm{E}-01$ & $3.44 \mathrm{E}-02$ & $3.382 \mathrm{E}+04$ \\
\hline $1.168 \mathrm{E}+00$ & 5.743E-01 & $2.06 \mathrm{E}-02$ & $3.144 \mathrm{E}-01$ & $8.18 \mathrm{E}-04$ & $4.628 \mathrm{E}-01$ & $4.60 \mathrm{E}-02$ & $3.391 \mathrm{E}+04$ \\
\hline $1.168 \mathrm{E}+00$ & $5.755 \mathrm{E}-01$ & $2.05 \mathrm{E}-02$ & $3.940 \mathrm{E}-01$ & $9.56 \mathrm{E}-04$ & $5.528 \mathrm{E}-01$ & $5.88 \mathrm{E}-02$ & $3.397 \mathrm{E}+04$ \\
\hline $1.167 \mathrm{E}+00$ & $5.796 \mathrm{E}-01$ & $2.09 \mathrm{E}-02$ & $4.722 \mathrm{E}-01$ & $1.07 \mathrm{E}-03$ & $6.318 \mathrm{E}-01$ & $6.98 \mathrm{E}-02$ & $3.423 \mathrm{E}+04$ \\
\hline $1.167 \mathrm{E}+00$ & $5.731 \mathrm{E}-01$ & $2.26 \mathrm{E}-02$ & $5.539 \mathrm{E}-01$ & $1.19 \mathrm{E}-03$ & $6.474 \mathrm{E}-01$ & $9.02 \mathrm{E}-02$ & $3.441 \mathrm{E}+04$ \\
\hline $1.167 \mathrm{E}+00$ & $5.642 \mathrm{E}-01$ & $2.70 \mathrm{E}-02$ & $6.317 \mathrm{E}-01$ & $1.31 \mathrm{E}-03$ & $6.275 \mathrm{E}-01$ & $1.23 \mathrm{E}-01$ & $3.480 \mathrm{E}+04$ \\
\hline
\end{tabular}


Table 164: APC 5.5 x 4.5 Dynamic Measured Values - 12007 RPM

\begin{tabular}{|c|c|c|c|c|c|c|c|}
\hline$n(\mathrm{RPM})$ & $Q(\mathrm{~g}-\mathrm{m})$ & $\Delta Q(\mathrm{~g}-\mathrm{m})$ & $V(\mathrm{~V})$ & $I(\mathrm{~A})$ & $T_{\text {atm }}\left({ }^{\circ} \mathrm{C}\right)$ & $P_{\text {atm }}(\mathrm{Pa})$ & $P_{\text {diff }}(\mathrm{Pa})$ \\
\hline $1.197 \mathrm{E}+04$ & $3.086 \mathrm{E}+00$ & $8.37 \mathrm{E}-02$ & $1.096 \mathrm{E}+01$ & $5.493 \mathrm{E}+00$ & $2.228 \mathrm{E}+01$ & $9.911 \mathrm{E}+04$ & $3.183 \mathrm{E}+00$ \\
\hline $1.201 \mathrm{E}+04$ & $3.009 \mathrm{E}+00$ & $8.20 \mathrm{E}-02$ & $1.096 \mathrm{E}+01$ & $5.397 \mathrm{E}+00$ & $2.225 \mathrm{E}+01$ & $9.910 \mathrm{E}+04$ & $1.118 \mathrm{E}+01$ \\
\hline $1.199 \mathrm{E}+04$ & $2.801 \mathrm{E}+00$ & $8.25 \mathrm{E}-02$ & $1.097 \mathrm{E}+01$ & $5.045 \mathrm{E}+00$ & $2.238 \mathrm{E}+01$ & $9.911 \mathrm{E}+04$ & $2.524 \mathrm{E}+01$ \\
\hline $1.204 \mathrm{E}+04$ & $2.780 \mathrm{E}+00$ & $9.32 \mathrm{E}-02$ & $1.097 \mathrm{E}+01$ & $4.975 \mathrm{E}+00$ & $2.255 \mathrm{E}+01$ & $9.911 \mathrm{E}+04$ & $4.560 \mathrm{E}+01$ \\
\hline $1.199 \mathrm{E}+04$ & $2.748 \mathrm{E}+00$ & $7.91 \mathrm{E}-02$ & $1.098 \mathrm{E}+01$ & $4.870 \mathrm{E}+00$ & $2.264 \mathrm{E}+01$ & $9.911 \mathrm{E}+04$ & $7.200 \mathrm{E}+01$ \\
\hline $1.202 \mathrm{E}+04$ & $2.696 \mathrm{E}+00$ & $8.82 \mathrm{E}-02$ & $1.098 \mathrm{E}+01$ & $4.852 \mathrm{E}+00$ & $2.266 \mathrm{E}+01$ & $9.910 \mathrm{E}+04$ & $1.053 \mathrm{E}+02$ \\
\hline $1.202 \mathrm{E}+04$ & $2.522 \mathrm{E}+00$ & $9.01 \mathrm{E}-02$ & $1.099 \mathrm{E}+01$ & $4.518 \mathrm{E}+00$ & $2.273 \mathrm{E}+01$ & $9.910 \mathrm{E}+04$ & $1.447 \mathrm{E}+02$ \\
\hline $1.203 \mathrm{E}+04$ & $2.067 \mathrm{E}+00$ & $8.07 \mathrm{E}-02$ & $1.101 \mathrm{E}+01$ & $3.705 \mathrm{E}+00$ & $2.272 \mathrm{E}+01$ & $9.910 \mathrm{E}+04$ & $1.900 \mathrm{E}+02$ \\
\hline $1.201 \mathrm{E}+04$ & $1.652 \mathrm{E}+00$ & $7.30 \mathrm{E}-02$ & $1.103 \mathrm{E}+01$ & $2.990 \mathrm{E}+00$ & $2.284 \mathrm{E}+01$ & $9.911 \mathrm{E}+04$ & $2.409 \mathrm{E}+02$ \\
\hline
\end{tabular}


Table 165: APC 5.5 x 4.5 Dynamic Calculated Values - 12007 RPM

\begin{tabular}{|c|c|c|c|c|c|c|c|}
\hline$n(\mathrm{RPM})$ & $V_{\infty}^{\prime}(\mathrm{m} / \mathrm{s})$ & $\Delta V_{\infty}^{\prime}(\mathrm{m} / \mathrm{s})$ & $T^{\prime}(\mathrm{g})$ & $\Delta T^{\prime}(\mathrm{g})$ & $V_{\mathrm{t}}(\mathrm{m} / \mathrm{s})$ & $P_{\mathrm{P}}(\mathrm{W})$ & $\Delta P_{\mathrm{P}}(\mathrm{W})$ \\
\hline $1.197 \mathrm{E}+04$ & $2.191 \mathrm{E}+00$ & $1.44 \mathrm{E}-02$ & $1.805 \mathrm{E}+02$ & $1.01 \mathrm{E}+01$ & $6.557 \mathrm{E}+01$ & $3.793 \mathrm{E}+01$ & $1.03 \mathrm{E}+00$ \\
\hline $1.201 \mathrm{E}+04$ & $4.234 \mathrm{E}+00$ & $1.58 \mathrm{E}-02$ & $1.832 \mathrm{E}+02$ & $1.02 \mathrm{E}+01$ & $6.590 \mathrm{E}+01$ & $3.712 \mathrm{E}+01$ & $1.01 \mathrm{E}+00$ \\
\hline 1.199E+04 & $6.439 \mathrm{E}+00$ & $1.87 \mathrm{E}-02$ & $1.896 \mathrm{E}+02$ & $1.03 \mathrm{E}+01$ & $6.594 \mathrm{E}+01$ & $3.448 \mathrm{E}+01$ & $1.02 \mathrm{E}+00$ \\
\hline $1.204 \mathrm{E}+04$ & $8.714 \mathrm{E}+00$ & $2.20 \mathrm{E}-02$ & $1.854 \mathrm{E}+02$ & $1.03 \mathrm{E}+01$ & $6.648 \mathrm{E}+01$ & $3.437 \mathrm{E}+01$ & $1.15 \mathrm{E}+00$ \\
\hline $1.199 \mathrm{E}+04$ & $1.100 \mathrm{E}+01$ & $2.59 \mathrm{E}-02$ & $1.713 \mathrm{E}+02$ & $1.03 \mathrm{E}+01$ & $6.653 \mathrm{E}+01$ & $3.382 \mathrm{E}+01$ & $9.74 \mathrm{E}-01$ \\
\hline $1.202 \mathrm{E}+04$ & $1.334 \mathrm{E}+01$ & $2.90 \mathrm{E}-02$ & $1.641 \mathrm{E}+02$ & $1.03 \mathrm{E}+01$ & $6.712 \mathrm{E}+01$ & $3.326 \mathrm{E}+01$ & $1.09 \mathrm{E}+00$ \\
\hline $1.202 \mathrm{E}+04$ & $1.567 \mathrm{E}+01$ & $3.17 \mathrm{E}-02$ & $1.359 \mathrm{E}+02$ & $1.02 \mathrm{E}+01$ & $6.762 \mathrm{E}+01$ & $3.112 \mathrm{E}+01$ & $1.11 \mathrm{E}+00$ \\
\hline $1.203 \mathrm{E}+04$ & $1.799 \mathrm{E}+01$ & $3.50 \mathrm{E}-02$ & $9.721 \mathrm{E}+01$ & $1.02 \mathrm{E}+01$ & $6.827 \mathrm{E}+01$ & $2.553 \mathrm{E}+01$ & $9.97 \mathrm{E}-01$ \\
\hline $1.201 \mathrm{E}+04$ & $2.029 \mathrm{E}+01$ & $3.89 \mathrm{E}-02$ & $6.767 \mathrm{E}+01$ & $1.03 \mathrm{E}+01$ & $6.882 \mathrm{E}+01$ & $2.038 \mathrm{E}+01$ & $9.00 \mathrm{E}-01$ \\
\hline$P_{\mathrm{e}}(\mathrm{W})$ & $\Delta P_{\mathrm{e}}(\mathrm{W})$ & $C_{\mathrm{T}}$ & $\Delta C_{\mathrm{T}}$ & $C_{\mathrm{P}}$ & $\Delta C_{\mathrm{P}}$ & $C_{\mathrm{Q}}$ & $\Delta C_{\mathrm{Q}}$ \\
\hline $6.019 \mathrm{E}+01$ & $7.03 \mathrm{E}-01$ & $1.008 \mathrm{E}-01$ & $5.65 \mathrm{E}-03$ & $7.762 \mathrm{E}-02$ & $2.11 \mathrm{E}-03$ & $1.235 \mathrm{E}-02$ & $3.35 \mathrm{E}-04$ \\
\hline $5.915 \mathrm{E}+01$ & $6.80 \mathrm{E}-01$ & $1.015 \mathrm{E}-01$ & $5.67 \mathrm{E}-03$ & $7.516 \mathrm{E}-02$ & $2.05 \mathrm{E}-03$ & $1.196 \mathrm{E}-02$ & $3.26 \mathrm{E}-04$ \\
\hline $5.535 \mathrm{E}+01$ & $6.38 \mathrm{E}-01$ & $1.056 \mathrm{E}-01$ & $5.74 \mathrm{E}-03$ & 7.029E-02 & $2.07 \mathrm{E}-03$ & 1.119E-02 & $3.30 \mathrm{E}-04$ \\
\hline $5.459 \mathrm{E}+01$ & $6.34 \mathrm{E}-01$ & $1.024 \mathrm{E}-01$ & $5.68 \mathrm{E}-03$ & $6.920 \mathrm{E}-02$ & $2.32 \mathrm{E}-03$ & $1.101 \mathrm{E}-02$ & $3.69 \mathrm{E}-04$ \\
\hline $5.345 \mathrm{E}+01$ & $6.15 \mathrm{E}-01$ & $9.550 \mathrm{E}-02$ & $5.73 \mathrm{E}-03$ & $6.903 \mathrm{E}-02$ & $1.99 \mathrm{E}-03$ & 1.099E-02 & $3.16 \mathrm{E}-04$ \\
\hline $5.326 \mathrm{E}+01$ & $6.17 \mathrm{E}-01$ & $9.103 \mathrm{E}-02$ & $5.69 \mathrm{E}-03$ & $6.739 \mathrm{E}-02$ & $2.21 \mathrm{E}-03$ & $1.073 \mathrm{E}-02$ & $3.51 \mathrm{E}-04$ \\
\hline $4.964 \mathrm{E}+01$ & $5.69 \mathrm{E}-01$ & $7.540 \mathrm{E}-02$ & $5.67 \mathrm{E}-03$ & $6.307 \mathrm{E}-02$ & $2.25 \mathrm{E}-03$ & $1.004 \mathrm{E}-02$ & $3.59 \mathrm{E}-04$ \\
\hline $4.079 \mathrm{E}+01$ & 4.61E-01 & 5.381E-02 & $5.67 \mathrm{E}-03$ & 5.157E-02 & $2.01 \mathrm{E}-03$ & $8.208 \mathrm{E}-03$ & $3.20 \mathrm{E}-04$ \\
\hline $3.298 \mathrm{E}+01$ & $3.71 \mathrm{E}-01$ & $3.758 \mathrm{E}-02$ & $5.71 \mathrm{E}-03$ & 4.137E-02 & $1.83 \mathrm{E}-03$ & $6.584 \mathrm{E}-03$ & 2.91E-04 \\
\hline$\rho\left(\mathrm{kg} / \mathrm{m}^{\wedge} 3\right)$ & $\eta_{\mathrm{T}}$ & $\Delta \eta_{\mathrm{T}}$ & $J$ & $\Delta J$ & $\eta_{\mathrm{P}}$ & $\Delta \eta_{\mathrm{P}}$ & $R e_{0.75}$ \\
\hline $1.169 \mathrm{E}+00$ & $6.301 \mathrm{E}-01$ & $1.86 \mathrm{E}-02$ & $7.878 \mathrm{E}-02$ & $5.19 \mathrm{E}-04$ & $1.023 \mathrm{E}-01$ & $6.41 \mathrm{E}-03$ & $4.024 \mathrm{E}+04$ \\
\hline $1.169 \mathrm{E}+00$ & $6.276 \mathrm{E}-01$ & $1.86 \mathrm{E}-02$ & $1.517 \mathrm{E}-01$ & $5.69 \mathrm{E}-04$ & $2.049 \mathrm{E}-01$ & $1.28 \mathrm{E}-02$ & $4.045 \mathrm{E}+04$ \\
\hline $1.168 \mathrm{E}+00$ & $6.229 \mathrm{E}-01$ & $1.97 \mathrm{E}-02$ & $2.312 \mathrm{E}-01$ & $6.75 \mathrm{E}-04$ & $3.473 \mathrm{E}-01$ & $2.15 \mathrm{E}-02$ & $4.044 \mathrm{E}+04$ \\
\hline $1.168 \mathrm{E}+00$ & $6.296 \mathrm{E}-01$ & $2.23 \mathrm{E}-02$ & $3.115 \mathrm{E}-01$ & $7.94 \mathrm{E}-04$ & 4.609E-01 & $2.99 \mathrm{E}-02$ & $4.073 \mathrm{E}+04$ \\
\hline $1.167 \mathrm{E}+00$ & $6.328 \mathrm{E}-01$ & $1.96 \mathrm{E}-02$ & 3.949E-01 & $9.38 \mathrm{E}-04$ & $5.463 \mathrm{E}-01$ & 3.64E-02 & $4.074 \mathrm{E}+04$ \\
\hline $1.167 \mathrm{E}+00$ & $6.245 \mathrm{E}-01$ & $2.17 \mathrm{E}-02$ & 4.777E-01 & $1.05 \mathrm{E}-03$ & $6.452 \mathrm{E}-01$ & $4.55 \mathrm{E}-02$ & $4.109 E+04$ \\
\hline $1.167 \mathrm{E}+00$ & $6.270 \mathrm{E}-01$ & $2.35 \mathrm{E}-02$ & $5.614 \mathrm{E}-01$ & $1.15 \mathrm{E}-03$ & $6.711 \mathrm{E}-01$ & $5.59 \mathrm{E}-02$ & $4.138 \mathrm{E}+04$ \\
\hline $1.167 \mathrm{E}+00$ & $6.261 \mathrm{E}-01$ & $2.54 \mathrm{E}-02$ & $6.438 \mathrm{E}-01$ & $1.27 \mathrm{E}-03$ & $6.717 \mathrm{E}-01$ & $7.55 \mathrm{E}-02$ & $4.178 \mathrm{E}+04$ \\
\hline $1.166 \mathrm{E}+00$ & $6.179 \mathrm{E}-01$ & $2.82 \mathrm{E}-02$ & $7.270 \mathrm{E}-01$ & $1.42 \mathrm{E}-03$ & $6.606 \mathrm{E}-01$ & $1.05 \mathrm{E}-01$ & $4.209 \mathrm{E}+04$ \\
\hline
\end{tabular}


Table 166: APC 5.5 x 4.5 Dynamic Measured Values - 13992 RPM

\begin{tabular}{|c|c|c|c|c|c|c|c|}
\hline$n(\mathrm{RPM})$ & $Q(\mathrm{~g}-\mathrm{m})$ & $\Delta Q(\mathrm{~g}-\mathrm{m})$ & $V(\mathrm{~V})$ & $I(\mathrm{~A})$ & $T_{\text {atm }}\left({ }^{\circ} \mathrm{C}\right)$ & $P_{\text {atm }}(\mathrm{Pa})$ & $P_{\text {diff }}(\mathrm{Pa})$ \\
\hline $1.392 \mathrm{E}+04$ & $4.166 \mathrm{E}+00$ & $7.80 \mathrm{E}-02$ & $1.087 \mathrm{E}+01$ & $8.446 \mathrm{E}+00$ & $2.246 \mathrm{E}+01$ & $9.909 \mathrm{E}+04$ & $3.632 \mathrm{E}+00$ \\
\hline $1.399 \mathrm{E}+04$ & $4.139 \mathrm{E}+00$ & $7.89 \mathrm{E}-02$ & $1.087 \mathrm{E}+01$ & $8.406 \mathrm{E}+00$ & $2.233 \mathrm{E}+01$ & $9.909 \mathrm{E}+04$ & $1.403 \mathrm{E}+01$ \\
\hline $1.403 \mathrm{E}+04$ & $3.880 \mathrm{E}+00$ & $8.06 \mathrm{E}-02$ & $1.089 \mathrm{E}+01$ & $7.905 \mathrm{E}+00$ & $2.253 \mathrm{E}+01$ & $9.909 \mathrm{E}+04$ & $3.333 \mathrm{E}+01$ \\
\hline $1.402 \mathrm{E}+04$ & $3.790 \mathrm{E}+00$ & $7.97 \mathrm{E}-02$ & $1.089 \mathrm{E}+01$ & $7.683 \mathrm{E}+00$ & $2.266 \mathrm{E}+01$ & $9.908 \mathrm{E}+04$ & $6.123 \mathrm{E}+01$ \\
\hline $1.397 \mathrm{E}+04$ & $3.762 \mathrm{E}+00$ & $7.95 \mathrm{E}-02$ & $1.090 \mathrm{E}+01$ & $7.533 \mathrm{E}+00$ & $2.278 \mathrm{E}+01$ & $9.907 \mathrm{E}+04$ & $9.891 \mathrm{E}+01$ \\
\hline $1.401 \mathrm{E}+04$ & $3.722 \mathrm{E}+00$ & $7.97 \mathrm{E}-02$ & $1.090 \mathrm{E}+01$ & $7.478 \mathrm{E}+00$ & $2.280 \mathrm{E}+01$ & $9.906 \mathrm{E}+04$ & $1.458 \mathrm{E}+02$ \\
\hline $1.400 \mathrm{E}+04$ & $3.558 \mathrm{E}+00$ & $8.16 \mathrm{E}-02$ & $1.091 \mathrm{E}+01$ & $7.061 \mathrm{E}+00$ & $2.285 \mathrm{E}+01$ & $9.906 \mathrm{E}+04$ & $2.015 \mathrm{E}+02$ \\
\hline $1.402 \mathrm{E}+04$ & $2.948 \mathrm{E}+00$ & $8.09 \mathrm{E}-02$ & $1.095 \mathrm{E}+01$ & $5.752 \mathrm{E}+00$ & $2.293 \mathrm{E}+01$ & $9.906 \mathrm{E}+04$ & $2.648 \mathrm{E}+02$ \\
\hline $1.397 \mathrm{E}+04$ & $2.285 \mathrm{E}+00$ & $7.91 \mathrm{E}-02$ & $1.099 \mathrm{E}+01$ & $4.461 \mathrm{E}+00$ & $2.300 \mathrm{E}+01$ & $9.905 \mathrm{E}+04$ & $3.376 \mathrm{E}+02$ \\
\hline
\end{tabular}


Table 167: APC 5.5 x 4.5 Dynamic Calculated Values - 13992 RPM

\begin{tabular}{|c|c|c|c|c|c|c|c|}
\hline$n(\mathrm{RPM})$ & $V_{\infty}^{\prime}(\mathrm{m} / \mathrm{s})$ & $\Delta V_{\infty}^{\prime}(\mathrm{m} / \mathrm{s})$ & $T^{\prime}(\mathrm{g})$ & $\Delta T^{\prime}(\mathrm{g})$ & $V_{\mathrm{t}}(\mathrm{m} / \mathrm{s})$ & $P_{\mathrm{P}}(\mathrm{W})$ & $\Delta P_{\mathrm{P}}(\mathrm{W})$ \\
\hline $1.392 \mathrm{E}+04$ & $2.322 \mathrm{E}+00$ & $1.60 \mathrm{E}-02$ & $2.600 \mathrm{E}+02$ & $1.08 \mathrm{E}+01$ & $7.624 \mathrm{E}+01$ & $5.955 \mathrm{E}+01$ & $1.12 \mathrm{E}+00$ \\
\hline $1.399 \mathrm{E}+04$ & $4.736 \mathrm{E}+00$ & $1.76 \mathrm{E}-02$ & $2.551 \mathrm{E}+02$ & $1.09 \mathrm{E}+01$ & $7.672 \mathrm{E}+01$ & $5.945 \mathrm{E}+01$ & $1.13 \mathrm{E}+00$ \\
\hline $1.403 \mathrm{E}+04$ & $7.396 \mathrm{E}+00$ & $2.11 \mathrm{E}-02$ & $2.674 \mathrm{E}+02$ & $1.10 \mathrm{E}+01$ & $7.719 \mathrm{E}+01$ & $5.592 \mathrm{E}+01$ & $1.16 \mathrm{E}+00$ \\
\hline $1.402 \mathrm{E}+04$ & $1.010 \mathrm{E}+01$ & 2.63E-02 & $2.594 \mathrm{E}+02$ & $1.09 \mathrm{E}+01$ & $7.740 \mathrm{E}+01$ & $5.455 \mathrm{E}+01$ & $1.15 \mathrm{E}+00$ \\
\hline $1.397 \mathrm{E}+04$ & $1.289 \mathrm{E}+01$ & 2.99E-02 & $2.411 \mathrm{E}+02$ & $1.08 \mathrm{E}+01$ & $7.757 \mathrm{E}+01$ & $5.398 \mathrm{E}+01$ & $1.14 \mathrm{E}+00$ \\
\hline $1.401 \mathrm{E}+04$ & $1.570 \mathrm{E}+01$ & $3.32 \mathrm{E}-02$ & $2.287 \mathrm{E}+02$ & $1.09 \mathrm{E}+01$ & $7.831 \mathrm{E}+01$ & $5.356 \mathrm{E}+01$ & $1.15 \mathrm{E}+00$ \\
\hline $1.400 \mathrm{E}+04$ & $1.850 \mathrm{E}+01$ & $3.66 \mathrm{E}-02$ & $1.986 \mathrm{E}+02$ & $1.08 \mathrm{E}+01$ & $7.883 \mathrm{E}+01$ & $5.115 \mathrm{E}+01$ & $1.17 \mathrm{E}+00$ \\
\hline $1.402 \mathrm{E}+04$ & $2.125 \mathrm{E}+01$ & $4.09 \mathrm{E}-02$ & $1.374 \mathrm{E}+02$ & $1.08 \mathrm{E}+01$ & $7.963 \mathrm{E}+01$ & $4.244 \mathrm{E}+01$ & $1.16 \mathrm{E}+00$ \\
\hline $1.397 \mathrm{E}+04$ & $2.403 \mathrm{E}+01$ & 4.57E-02 & $9.311 \mathrm{E}+01$ & $1.08 \mathrm{E}+01$ & $8.019 \mathrm{E}+01$ & $3.280 \mathrm{E}+01$ & $1.13 \mathrm{E}+00$ \\
\hline$P_{\mathrm{e}}(\mathrm{W})$ & $\Delta P_{\mathrm{e}}(\mathrm{W})$ & $C_{\mathrm{T}}$ & $\Delta C_{\mathrm{T}}$ & $C_{\mathrm{P}}$ & $\Delta C_{\mathrm{P}}$ & $C_{\mathrm{Q}}$ & $\Delta C_{\mathrm{Q}}$ \\
\hline $9.181 \mathrm{E}+01$ & $1.01 \mathrm{E}+00$ & $1.074 \mathrm{E}-01$ & $4.46 \mathrm{E}-03$ & $7.756 \mathrm{E}-02$ & $1.46 \mathrm{E}-03$ & $1.234 \mathrm{E}-02$ & $2.32 \mathrm{E}-04$ \\
\hline $9.139 \mathrm{E}+01$ & $9.93 \mathrm{E}-01$ & $1.043 \mathrm{E}-01$ & $4.45 \mathrm{E}-03$ & $7.630 \mathrm{E}-02$ & $1.46 \mathrm{E}-03$ & $1.214 \mathrm{E}-02$ & $2.32 \mathrm{E}-04$ \\
\hline $8.606 \mathrm{E}+01$ & $9.40 \mathrm{E}-01$ & $1.087 \mathrm{E}-01$ & 4.49E-03 & 7.107E-02 & $1.48 \mathrm{E}-03$ & $1.131 \mathrm{E}-02$ & $2.35 \mathrm{E}-04$ \\
\hline $8.370 \mathrm{E}+01$ & $9.18 \mathrm{E}-01$ & $1.058 \mathrm{E}-01$ & $4.44 \mathrm{E}-03$ & $6.965 \mathrm{E}-02$ & $1.47 \mathrm{E}-03$ & $1.109 \mathrm{E}-02$ & $2.33 \mathrm{E}-04$ \\
\hline $8.209 \mathrm{E}+01$ & $9.00 \mathrm{E}-01$ & $9.898 \mathrm{E}-02$ & $4.45 \mathrm{E}-03$ & $6.959 \mathrm{E}-02$ & $1.47 \mathrm{E}-03$ & $1.108 \mathrm{E}-02$ & $2.34 \mathrm{E}-04$ \\
\hline $8.150 \mathrm{E}+01$ & $8.91 \mathrm{E}-01$ & $9.334 \mathrm{E}-02$ & $4.44 \mathrm{E}-03$ & $6.847 \mathrm{E}-02$ & $1.47 \mathrm{E}-03$ & $1.090 \mathrm{E}-02$ & $2.33 \mathrm{E}-04$ \\
\hline $7.705 \mathrm{E}+01$ & $8.48 \mathrm{E}-01$ & $8.127 \mathrm{E}-02$ & $4.42 \mathrm{E}-03$ & $6.563 \mathrm{E}-02$ & $1.51 \mathrm{E}-03$ & $1.045 \mathrm{E}-02$ & $2.40 \mathrm{E}-04$ \\
\hline $6.298 \mathrm{E}+01$ & 7.09E-01 & $5.610 \mathrm{E}-02$ & $4.41 \mathrm{E}-03$ & $5.424 \mathrm{E}-02$ & $1.49 \mathrm{E}-03$ & $8.632 \mathrm{E}-03$ & $2.37 \mathrm{E}-04$ \\
\hline $4.902 \mathrm{E}+01$ & $5.51 \mathrm{E}-01$ & $3.825 \mathrm{E}-02$ & 4.43E-03 & $4.231 \mathrm{E}-02$ & $1.46 \mathrm{E}-03$ & $6.734 \mathrm{E}-03$ & $2.33 \mathrm{E}-04$ \\
\hline$\rho\left(\mathrm{kg} / \mathrm{m}^{\wedge} 3\right)$ & $\eta_{\mathrm{T}}$ & $\Delta \eta_{\mathrm{T}}$ & $J$ & $\Delta J$ & $\eta_{\mathrm{P}}$ & $\Delta \eta_{\mathrm{P}}$ & $R e_{0.75}$ \\
\hline $1.168 \mathrm{E}+00$ & $6.486 \mathrm{E}-01$ & $1.41 \mathrm{E}-02$ & 7.181E-02 & 4.95E-04 & $9.945 \mathrm{E}-02$ & $4.58 \mathrm{E}-03$ & $4.673 \mathrm{E}+04$ \\
\hline $1.168 \mathrm{E}+00$ & $6.505 \mathrm{E}-01$ & $1.43 \mathrm{E}-02$ & $1.457 \mathrm{E}-01$ & $5.43 \mathrm{E}-04$ & $1.993 \mathrm{E}-01$ & $9.35 \mathrm{E}-03$ & $4.705 \mathrm{E}+04$ \\
\hline $1.167 \mathrm{E}+00$ & $6.498 \mathrm{E}-01$ & $1.53 \mathrm{E}-02$ & $2.268 \mathrm{E}-01$ & $6.50 \mathrm{E}-04$ & $3.468 \mathrm{E}-01$ & $1.61 \mathrm{E}-02$ & $4.729 \mathrm{E}+04$ \\
\hline $1.167 \mathrm{E}+00$ & $6.518 \mathrm{E}-01$ & $1.55 \mathrm{E}-02$ & $3.100 \mathrm{E}-01$ & $8.12 \mathrm{E}-04$ & $4.708 \mathrm{E}-01$ & $2.21 \mathrm{E}-02$ & $4.737 \mathrm{E}+04$ \\
\hline $1.166 \mathrm{E}+00$ & $6.575 \mathrm{E}-01$ & $1.56 \mathrm{E}-02$ & $3.971 \mathrm{E}-01$ & $9.31 \mathrm{E}-04$ & $5.648 \mathrm{E}-01$ & $2.81 \mathrm{E}-02$ & $4.744 \mathrm{E}+04$ \\
\hline $1.166 \mathrm{E}+00$ & $6.572 \mathrm{E}-01$ & $1.58 \mathrm{E}-02$ & $4.821 \mathrm{E}-01$ & $1.03 \mathrm{E}-03$ & $6.572 \mathrm{E}-01$ & $3.43 \mathrm{E}-02$ & $4.788 \mathrm{E}+04$ \\
\hline $1.166 \mathrm{E}+00$ & $6.638 \mathrm{E}-01$ & $1.69 \mathrm{E}-02$ & $5.688 \mathrm{E}-01$ & $1.14 \mathrm{E}-03$ & $7.045 \mathrm{E}-01$ & $4.16 \mathrm{E}-02$ & $4.819 \mathrm{E}+04$ \\
\hline $1.166 \mathrm{E}+00$ & $6.738 \mathrm{E}-01$ & $2.00 \mathrm{E}-02$ & $6.526 \mathrm{E}-01$ & $1.27 \mathrm{E}-03$ & $6.750 \mathrm{E}-01$ & $5.63 \mathrm{E}-02$ & $4.865 \mathrm{E}+04$ \\
\hline $1.165 \mathrm{E}+00$ & $6.691 \mathrm{E}-01$ & $2.43 \mathrm{E}-02$ & $7.402 \mathrm{E}-01$ & $1.43 \mathrm{E}-03$ & $6.691 \mathrm{E}-01$ & $8.09 \mathrm{E}-02$ & $4.897 \mathrm{E}+04$ \\
\hline
\end{tabular}


Table 168: APC 5.5 x 4.5 Dynamic Measured Values - 15993 RPM

\begin{tabular}{|c|c|c|c|c|c|c|c|}
\hline$n(\mathrm{RPM})$ & $Q(\mathrm{~g}-\mathrm{m})$ & $\Delta Q(\mathrm{~g}-\mathrm{m})$ & $V(\mathrm{~V})$ & $I(\mathrm{~A})$ & $T_{\text {atm }}\left({ }^{\circ} \mathrm{C}\right)$ & $P_{\text {atm }}(\mathrm{Pa})$ & $P_{\text {diff }}(\mathrm{Pa})$ \\
\hline $1.591 \mathrm{E}+04$ & $5.359 \mathrm{E}+00$ & $9.16 \mathrm{E}-02$ & $1.075 \mathrm{E}+01$ & $1.246 \mathrm{E}+01$ & $2.258 \mathrm{E}+01$ & $9.903 \mathrm{E}+04$ & $4.241 \mathrm{E}+00$ \\
\hline $1.603 \mathrm{E}+04$ & $5.375 \mathrm{E}+00$ & $9.35 \mathrm{E}-02$ & $1.075 \mathrm{E}+01$ & $1.253 \mathrm{E}+01$ & $2.250 \mathrm{E}+01$ & $9.903 \mathrm{E}+04$ & $1.734 \mathrm{E}+01$ \\
\hline $1.598 \mathrm{E}+04$ & $5.155 \mathrm{E}+00$ & $9.35 \mathrm{E}-02$ & $1.076 \mathrm{E}+01$ & $1.192 \mathrm{E}+01$ & $2.268 \mathrm{E}+01$ & $9.903 \mathrm{E}+04$ & $4.257 \mathrm{E}+01$ \\
\hline $1.599 \mathrm{E}+04$ & $5.069 \mathrm{E}+00$ & $9.25 \mathrm{E}-02$ & $1.077 \mathrm{E}+01$ & $1.164 \mathrm{E}+01$ & $2.282 \mathrm{E}+01$ & $9.904 \mathrm{E}+04$ & $7.983 \mathrm{E}+01$ \\
\hline $1.601 \mathrm{E}+04$ & $5.051 \mathrm{E}+00$ & $9.39 \mathrm{E}-02$ & $1.077 \mathrm{E}+01$ & $1.159 \mathrm{E}+01$ & $2.286 \mathrm{E}+01$ & $9.904 \mathrm{E}+04$ & $1.301 \mathrm{E}+02$ \\
\hline $1.601 \mathrm{E}+04$ & $4.975 \mathrm{E}+00$ & $9.26 \mathrm{E}-02$ & $1.078 \mathrm{E}+01$ & $1.135 \mathrm{E}+01$ & $2.289 \mathrm{E}+01$ & $9.905 \mathrm{E}+04$ & $1.929 \mathrm{E}+02$ \\
\hline $1.597 \mathrm{E}+04$ & $4.802 \mathrm{E}+00$ & $9.08 \mathrm{E}-02$ & $1.080 \mathrm{E}+01$ & $1.071 \mathrm{E}+01$ & $2.287 \mathrm{E}+01$ & $9.905 \mathrm{E}+04$ & $2.670 \mathrm{E}+02$ \\
\hline $1.602 \mathrm{E}+04$ & $4.150 \mathrm{E}+00$ & $9.40 \mathrm{E}-02$ & $1.085 \mathrm{E}+01$ & $8.957 \mathrm{E}+00$ & $2.305 \mathrm{E}+01$ & $9.905 \mathrm{E}+04$ & $3.528 \mathrm{E}+02$ \\
\hline $1.600 \mathrm{E}+04$ & $3.138 \mathrm{E}+00$ & $8.91 \mathrm{E}-02$ & $1.092 \mathrm{E}+01$ & $6.658 \mathrm{E}+00$ & $2.313 \mathrm{E}+01$ & $9.905 \mathrm{E}+04$ & $4.501 \mathrm{E}+02$ \\
\hline
\end{tabular}


Table 169: APC 5.5 x 4.5 Dynamic Calculated Values - 15993 RPM

\begin{tabular}{|c|c|c|c|c|c|c|c|}
\hline$n(\mathrm{RPM})$ & $V_{\infty}^{\prime}(\mathrm{m} / \mathrm{s})$ & $\Delta V_{\infty}^{\prime}(\mathrm{m} / \mathrm{s})$ & $T^{\prime}(\mathrm{g})$ & $\Delta T^{\prime}(\mathrm{g})$ & $V_{\mathrm{t}}(\mathrm{m} / \mathrm{s})$ & $P_{\mathrm{P}}(\mathrm{W})$ & $\Delta P_{\mathrm{P}}(\mathrm{W})$ \\
\hline $1.591 \mathrm{E}+04$ & $2.495 \mathrm{E}+00$ & $1.78 \mathrm{E}-02$ & $3.549 \mathrm{E}+02$ & $9.43 \mathrm{E}+00$ & $8.717 \mathrm{E}+01$ & $8.758 \mathrm{E}+01$ & $1.50 \mathrm{E}+00$ \\
\hline $1.603 \mathrm{E}+04$ & $5.256 \mathrm{E}+00$ & $2.00 \mathrm{E}-02$ & $3.579 \mathrm{E}+02$ & $8.87 \mathrm{E}+00$ & $8.791 \mathrm{E}+01$ & $8.847 \mathrm{E}+01$ & $1.54 \mathrm{E}+00$ \\
\hline $1.598 \mathrm{E}+04$ & $8.359 \mathrm{E}+00$ & $2.43 \mathrm{E}-02$ & $3.511 \mathrm{E}+02$ & $9.08 \mathrm{E}+00$ & $8.787 \mathrm{E}+01$ & $8.458 \mathrm{E}+01$ & $1.53 \mathrm{E}+00$ \\
\hline $1.599 \mathrm{E}+04$ & $1.153 \mathrm{E}+01$ & 3.04E-02 & $3.460 \mathrm{E}+02$ & $9.23 \mathrm{E}+00$ & $8.830 \mathrm{E}+01$ & $8.325 \mathrm{E}+01$ & $1.52 \mathrm{E}+00$ \\
\hline $1.601 \mathrm{E}+04$ & $1.479 \mathrm{E}+01$ & $3.32 \mathrm{E}-02$ & $3.256 \mathrm{E}+02$ & $9.08 \mathrm{E}+00$ & $8.891 \mathrm{E}+01$ & $8.307 \mathrm{E}+01$ & $1.54 \mathrm{E}+00$ \\
\hline $1.601 \mathrm{E}+04$ & $1.806 \mathrm{E}+01$ & $3.67 \mathrm{E}-02$ & $2.990 \mathrm{E}+02$ & $9.17 \mathrm{E}+00$ & $8.949 \mathrm{E}+01$ & $8.179 \mathrm{E}+01$ & $1.52 \mathrm{E}+00$ \\
\hline $1.597 \mathrm{E}+04$ & $2.129 \mathrm{E}+01$ & $4.16 \mathrm{E}-02$ & $2.645 \mathrm{E}+02$ & $9.57 \mathrm{E}+00$ & $9.000 \mathrm{E}+01$ & $7.876 \mathrm{E}+01$ & $1.49 \mathrm{E}+00$ \\
\hline $1.602 \mathrm{E}+04$ & $2.453 \mathrm{E}+01$ & $4.63 \mathrm{E}-02$ & $2.010 \mathrm{E}+02$ & $9.53 \mathrm{E}+00$ & $9.110 \mathrm{E}+01$ & $6.829 \mathrm{E}+01$ & $1.55 \mathrm{E}+00$ \\
\hline $1.600 \mathrm{E}+04$ & $2.775 \mathrm{E}+01$ & $5.20 \mathrm{E}-02$ & $1.313 \mathrm{E}+02$ & $9.98 \mathrm{E}+00$ & $9.191 \mathrm{E}+01$ & $5.157 \mathrm{E}+01$ & $1.47 \mathrm{E}+00$ \\
\hline$P_{\mathrm{e}}(\mathrm{W})$ & $\Delta P_{\mathrm{e}}(\mathrm{W})$ & $C_{\mathrm{T}}$ & $\Delta C_{\mathrm{T}}$ & $C_{\mathrm{P}}$ & $\Delta C_{\mathrm{P}}$ & $C_{\mathrm{Q}}$ & $\Delta C_{\mathrm{Q}}$ \\
\hline $1.339 \mathrm{E}+02$ & $1.43 \mathrm{E}+00$ & $1.123 \mathrm{E}-01$ & $2.98 \mathrm{E}-03$ & $7.639 \mathrm{E}-02$ & $1.31 \mathrm{E}-03$ & $1.216 \mathrm{E}-02$ & $2.08 \mathrm{E}-04$ \\
\hline $1.347 \mathrm{E}+02$ & $1.42 \mathrm{E}+00$ & $1.116 \mathrm{E}-01$ & $2.77 \mathrm{E}-03$ & $7.553 \mathrm{E}-02$ & $1.32 \mathrm{E}-03$ & $1.202 \mathrm{E}-02$ & $2.09 \mathrm{E}-04$ \\
\hline $1.283 \mathrm{E}+02$ & $1.36 \mathrm{E}+00$ & $1.102 \mathrm{E}-01$ & $2.85 \mathrm{E}-03$ & 7.293E-02 & $1.32 \mathrm{E}-03$ & $1.161 \mathrm{E}-02$ & $2.11 \mathrm{E}-04$ \\
\hline $1.254 \mathrm{E}+02$ & $1.33 \mathrm{E}+00$ & $1.085 \mathrm{E}-01$ & $2.90 \mathrm{E}-03$ & $7.164 \mathrm{E}-02$ & $1.31 \mathrm{E}-03$ & $1.140 \mathrm{E}-02$ & $2.08 \mathrm{E}-04$ \\
\hline $1.249 \mathrm{E}+02$ & $1.33 \mathrm{E}+00$ & $1.018 \mathrm{E}-01$ & $2.84 \mathrm{E}-03$ & $7.118 \mathrm{E}-02$ & $1.32 \mathrm{E}-03$ & $1.133 \mathrm{E}-02$ & $2.11 \mathrm{E}-04$ \\
\hline $1.223 \mathrm{E}+02$ & $1.30 \mathrm{E}+00$ & $9.353 \mathrm{E}-02$ & $2.87 \mathrm{E}-03$ & 7.014E-02 & $1.31 \mathrm{E}-03$ & $1.116 \mathrm{E}-02$ & $2.08 \mathrm{E}-04$ \\
\hline $1.157 \mathrm{E}+02$ & $1.23 \mathrm{E}+00$ & $8.314 \mathrm{E}-02$ & $3.01 \mathrm{E}-03$ & $6.802 \mathrm{E}-02$ & $1.29 \mathrm{E}-03$ & $1.082 \mathrm{E}-02$ & $2.05 \mathrm{E}-04$ \\
\hline $9.722 \mathrm{E}+01$ & $1.06 \mathrm{E}+00$ & $6.280 \mathrm{E}-02$ & $2.98 \mathrm{E}-03$ & 5.843E-02 & $1.32 \mathrm{E}-03$ & $9.300 \mathrm{E}-03$ & $2.11 \mathrm{E}-04$ \\
\hline $7.273 \mathrm{E}+01$ & $8.02 \mathrm{E}-01$ & 4.113E-02 & $3.13 \mathrm{E}-03$ & $4.431 \mathrm{E}-02$ & $1.26 \mathrm{E}-03$ & 7.053E-03 & $2.00 \mathrm{E}-04$ \\
\hline$\rho\left(\mathrm{kg} / \mathrm{m}^{\wedge} 3\right)$ & $\eta_{\mathrm{T}}$ & $\Delta \eta_{\mathrm{T}}$ & $J$ & $\Delta J$ & $\eta_{\mathrm{P}}$ & $\Delta \eta_{\mathrm{P}}$ & $R e_{0.75}$ \\
\hline $1.167 \mathrm{E}+00$ & $6.541 \mathrm{E}-01$ & $1.32 \mathrm{E}-02$ & $6.748 \mathrm{E}-02$ & $4.80 \mathrm{E}-04$ & $9.916 \mathrm{E}-02$ & $3.21 \mathrm{E}-03$ & $5.335 \mathrm{E}+04$ \\
\hline $1.167 \mathrm{E}+00$ & $6.570 \mathrm{E}-01$ & $1.34 \mathrm{E}-02$ & $1.411 \mathrm{E}-01$ & $5.38 \mathrm{E}-04$ & $2.085 \mathrm{E}-01$ & $6.37 \mathrm{E}-03$ & $5.383 \mathrm{E}+04$ \\
\hline $1.166 \mathrm{E}+00$ & $6.592 \mathrm{E}-01$ & $1.38 \mathrm{E}-02$ & $2.252 \mathrm{E}-01$ & $6.57 \mathrm{E}-04$ & $3.403 \mathrm{E}-01$ & $1.08 \mathrm{E}-02$ & $5.375 \mathrm{E}+04$ \\
\hline $1.166 \mathrm{E}+00$ & $6.639 \mathrm{E}-01$ & $1.40 \mathrm{E}-02$ & $3.104 \mathrm{E}-01$ & $8.22 \mathrm{E}-04$ & $4.700 \mathrm{E}-01$ & $1.53 \mathrm{E}-02$ & $5.397 \mathrm{E}+04$ \\
\hline $1.166 \mathrm{E}+00$ & $6.653 \mathrm{E}-01$ & $1.42 \mathrm{E}-02$ & $3.975 \mathrm{E}-01$ & 8.97E-04 & $5.685 \mathrm{E}-01$ & $1.91 \mathrm{E}-02$ & $5.434 \mathrm{E}+04$ \\
\hline $1.166 \mathrm{E}+00$ & $6.686 \mathrm{E}-01$ & $1.43 \mathrm{E}-02$ & $4.855 \mathrm{E}-01$ & $9.92 \mathrm{E}-04$ & $6.474 \mathrm{E}-01$ & $2.33 \mathrm{E}-02$ & $5.469 \mathrm{E}+04$ \\
\hline $1.166 \mathrm{E}+00$ & $6.808 \mathrm{E}-01$ & $1.48 \mathrm{E}-02$ & 5.737E-01 & $1.13 \mathrm{E}-03$ & $7.013 \mathrm{E}-01$ & $2.87 \mathrm{E}-02$ & $5.500 \mathrm{E}+04$ \\
\hline $1.165 \mathrm{E}+00$ & 7.024E-01 & $1.76 \mathrm{E}-02$ & $6.588 \mathrm{E}-01$ & $1.25 \mathrm{E}-03$ & 7.081E-01 & $3.72 \mathrm{E}-02$ & $5.561 \mathrm{E}+04$ \\
\hline $1.165 \mathrm{E}+00$ & $7.090 \mathrm{E}-01$ & $2.16 \mathrm{E}-02$ & 7.464E-01 & $1.41 \mathrm{E}-03$ & $6.927 \mathrm{E}-01$ & $5.63 \mathrm{E}-02$ & $5.608 \mathrm{E}+04$ \\
\hline
\end{tabular}




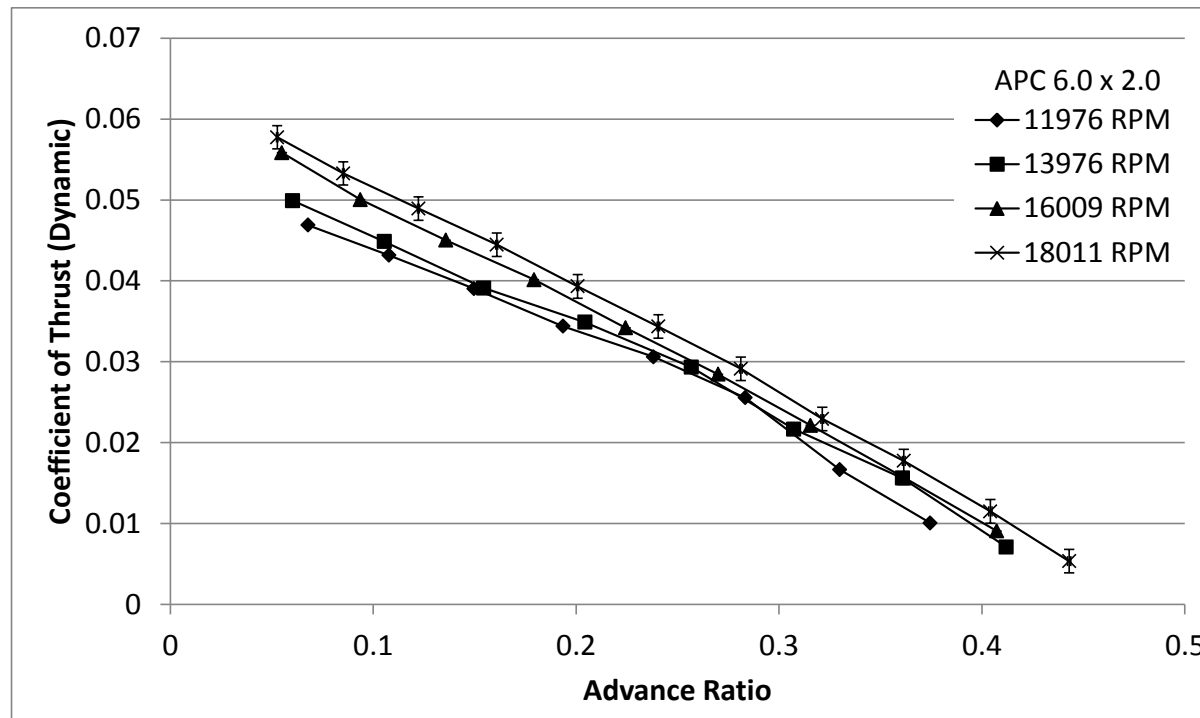

(a)

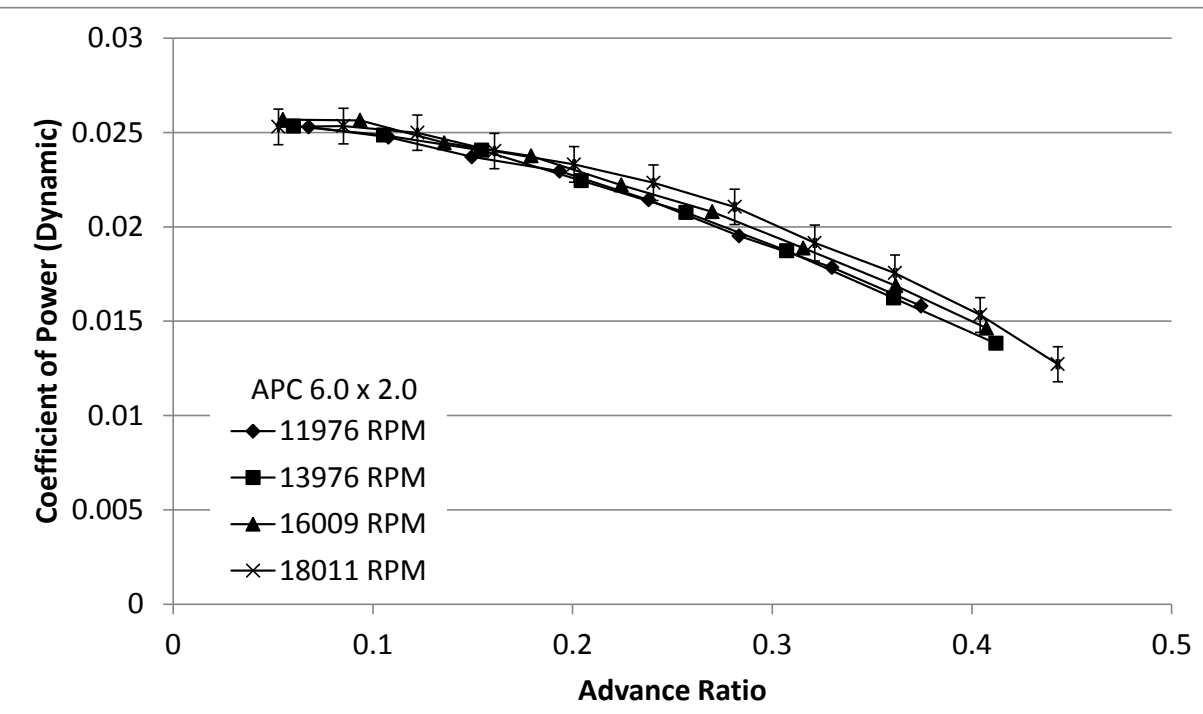

(b)

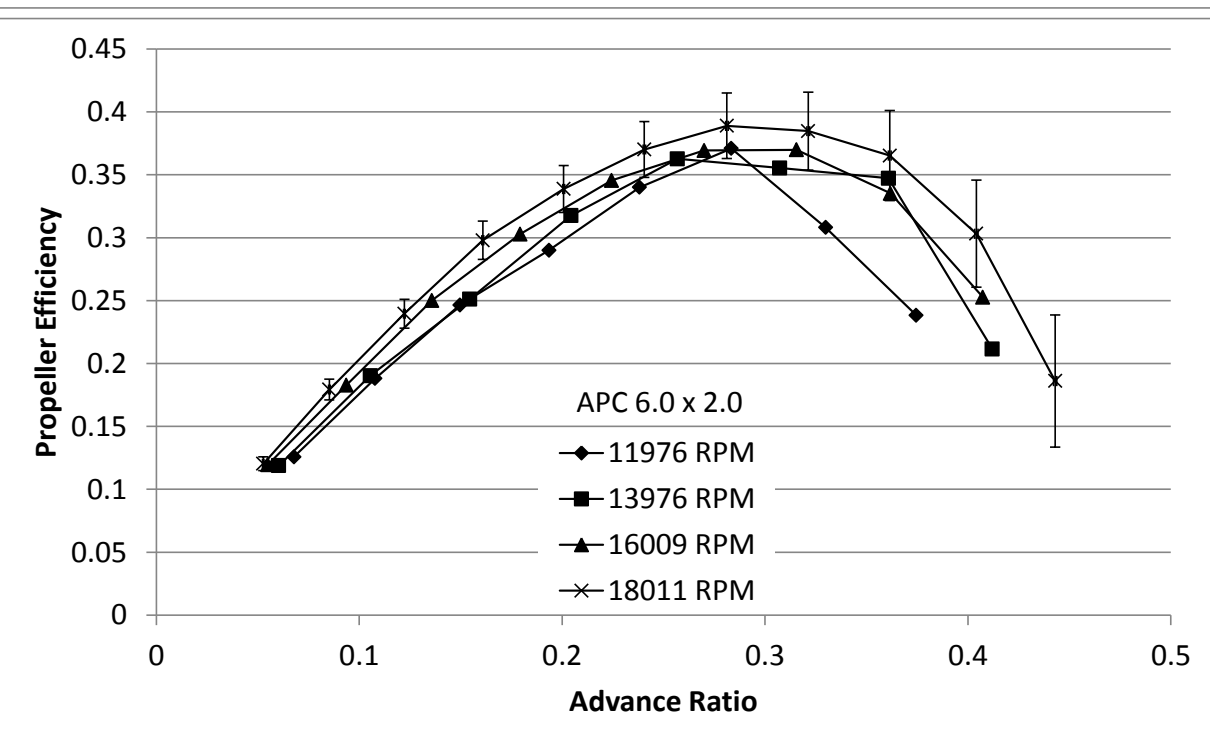

(c) 


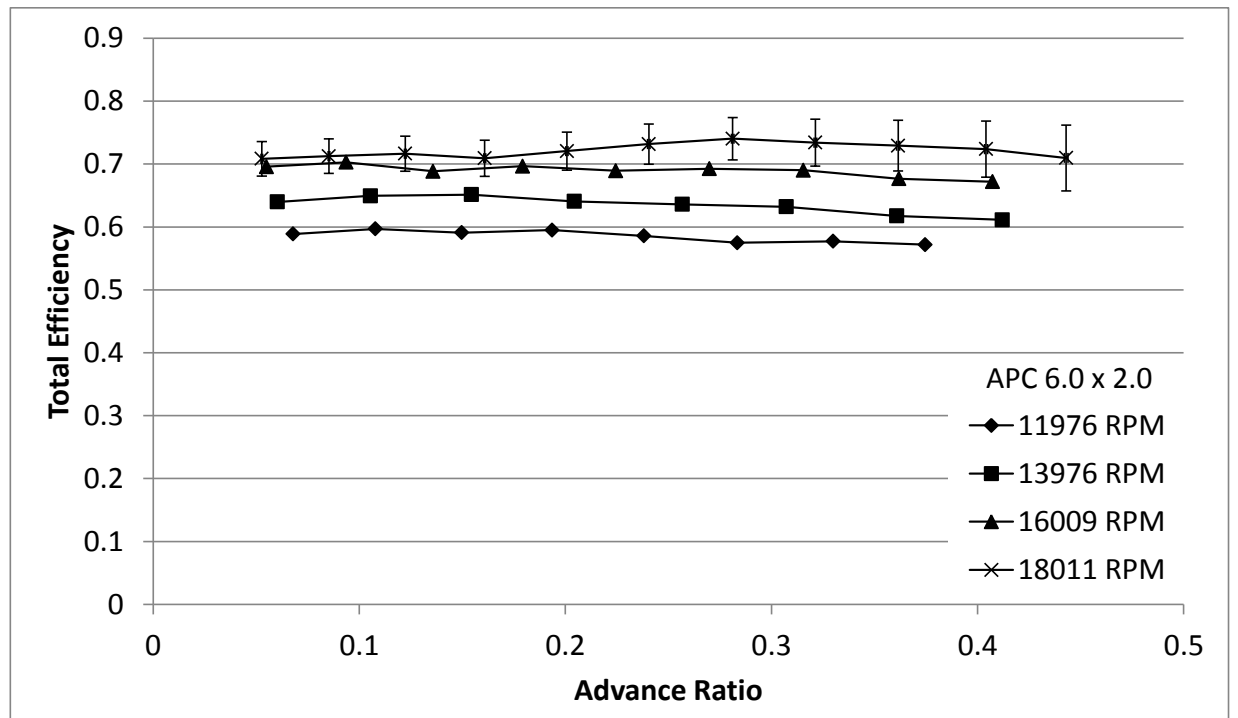

(d)

Figure 90: APC 6.0 x 2.0 Dynamic Test Results: (a) Coefficient of Thrust, (b) Coefficient of Power, (c) Propeller Efficiency, (d) Total Efficiency.

Table 170: APC 6.0 x 2.0 Dynamic Measured Values - 11976 RPM

\begin{tabular}{|c|c|c|c|c|c|c|c|}
\hline$n(\mathrm{RPM})$ & $Q(\mathrm{~g}-\mathrm{m})$ & $\Delta Q(\mathrm{~g}-\mathrm{m})$ & $V(\mathrm{~V})$ & $I(\mathrm{~A})$ & $T_{\mathrm{atm}}\left({ }^{\circ} \mathrm{C}\right)$ & $P_{\text {atm }}(\mathrm{Pa})$ & $P_{\text {diff }}(\mathrm{Pa})$ \\
\hline $1.187 \mathrm{E}+04$ & $1.520 \mathrm{E}+00$ & $7.04 \mathrm{E}-02$ & $1.103 \mathrm{E}+01$ & $2.854 \mathrm{E}+00$ & $2.305 \mathrm{E}+01$ & $9.847 \mathrm{E}+04$ & $2.721 \mathrm{E}+00$ \\
\hline $1.198 \mathrm{E}+04$ & $1.512 \mathrm{E}+00$ & $7.16 \mathrm{E}-02$ & $1.103 \mathrm{E}+01$ & $2.824 \mathrm{E}+00$ & $2.317 \mathrm{E}+01$ & $9.847 \mathrm{E}+04$ & $6.661 \mathrm{E}+00$ \\
\hline $1.202 \mathrm{E}+04$ & $1.460 \mathrm{E}+00$ & $7.54 \mathrm{E}-02$ & $1.104 \mathrm{E}+01$ & $2.764 \mathrm{E}+00$ & $2.322 \mathrm{E}+01$ & $9.845 \mathrm{E}+04$ & $1.262 \mathrm{E}+01$ \\
\hline $1.198 \mathrm{E}+04$ & $1.404 \mathrm{E}+00$ & $7.19 \mathrm{E}-02$ & $1.104 \mathrm{E}+01$ & $2.630 \mathrm{E}+00$ & $2.320 \mathrm{E}+01$ & $9.844 \mathrm{E}+04$ & $2.065 \mathrm{E}+01$ \\
\hline $1.199 \mathrm{E}+04$ & $1.312 \mathrm{E}+00$ & $7.33 \mathrm{E}-02$ & $1.104 \mathrm{E}+01$ & $2.497 \mathrm{E}+00$ & $2.333 \mathrm{E}+01$ & $9.844 \mathrm{E}+04$ & $3.100 \mathrm{E}+01$ \\
\hline $1.200 \mathrm{E}+04$ & $1.196 \mathrm{E}+00$ & $7.10 \mathrm{E}-02$ & $1.105 \mathrm{E}+01$ & $2.321 \mathrm{E}+00$ & $2.339 \mathrm{E}+01$ & $9.844 \mathrm{E}+04$ & $4.366 \mathrm{E}+01$ \\
\hline $1.196 \mathrm{E}+04$ & $1.086 \mathrm{E}+00$ & $7.09 \mathrm{E}-02$ & $1.106 \mathrm{E}+01$ & $2.090 \mathrm{E}+00$ & $2.342 \mathrm{E}+01$ & $9.844 \mathrm{E}+04$ & $5.834 \mathrm{E}+01$ \\
\hline $1.200 \mathrm{E}+04$ & $9.686 \mathrm{E}-01$ & $7.13 \mathrm{E}-02$ & $1.106 \mathrm{E}+01$ & $1.888 \mathrm{E}+00$ & $2.352 \mathrm{E}+01$ & $9.844 \mathrm{E}+04$ & $7.544 \mathrm{E}+01$ \\
\hline
\end{tabular}


Table 171: APC 6.0 x 2.0 Dynamic Calculated Values - 11976 RPM

\begin{tabular}{|c|c|c|c|c|c|c|c|}
\hline$n(\mathrm{RPM})$ & $V_{\infty}^{\prime}(\mathrm{m} / \mathrm{s})$ & $\Delta V_{\infty}^{\prime}(\mathrm{m} / \mathrm{s})$ & $T^{\prime}(\mathrm{g})$ & $\Delta T^{\prime}(\mathrm{g})$ & $V_{\mathrm{t}}(\mathrm{m} / \mathrm{s})$ & $P_{\mathrm{P}}(\mathrm{W})$ & $\Delta P_{\mathrm{P}}(\mathrm{W})$ \\
\hline $1.187 \mathrm{E}+04$ & $2.043 \mathrm{E}+00$ & $1.29 \mathrm{E}-02$ & $1.164 \mathrm{E}+02$ & $1.06 \mathrm{E}+01$ & $7.099 \mathrm{E}+01$ & $1.854 \mathrm{E}+01$ & $8.58 \mathrm{E}-01$ \\
\hline $1.198 \mathrm{E}+04$ & $3.276 \mathrm{E}+00$ & $1.35 \mathrm{E}-02$ & $1.089 \mathrm{E}+02$ & $1.06 \mathrm{E}+01$ & $7.165 \mathrm{E}+01$ & $1.860 \mathrm{E}+01$ & $8.80 \mathrm{E}-01$ \\
\hline $1.202 \mathrm{E}+04$ & $4.565 \mathrm{E}+00$ & $1.44 \mathrm{E}-02$ & $9.927 \mathrm{E}+01$ & $1.06 \mathrm{E}+01$ & $7.201 \mathrm{E}+01$ & $1.803 \mathrm{E}+01$ & $9.31 \mathrm{E}-01$ \\
\hline $1.198 \mathrm{E}+04$ & $5.883 \mathrm{E}+00$ & $1.64 \mathrm{E}-02$ & $8.683 \mathrm{E}+01$ & $1.06 \mathrm{E}+01$ & $7.186 \mathrm{E}+01$ & $1.727 \mathrm{E}+01$ & $8.85 \mathrm{E}-01$ \\
\hline $1.199 \mathrm{E}+04$ & $7.243 \mathrm{E}+00$ & $1.78 \mathrm{E}-02$ & $7.733 \mathrm{E}+01$ & $1.06 \mathrm{E}+01$ & $7.203 \mathrm{E}+01$ & $1.615 \mathrm{E}+01$ & $9.03 \mathrm{E}-01$ \\
\hline $1.200 \mathrm{E}+04$ & $8.626 \mathrm{E}+00$ & $2.00 \mathrm{E}-02$ & $6.465 \mathrm{E}+01$ & $1.06 \mathrm{E}+01$ & $7.224 \mathrm{E}+01$ & $1.474 \mathrm{E}+01$ & $8.75 \mathrm{E}-01$ \\
\hline $1.196 \mathrm{E}+04$ & $1.001 \mathrm{E}+01$ & $2.24 \mathrm{E}-02$ & $4.186 \mathrm{E}+01$ & $1.05 \mathrm{E}+01$ & $7.215 \mathrm{E}+01$ & $1.333 \mathrm{E}+01$ & $8.71 \mathrm{E}-01$ \\
\hline $1.200 \mathrm{E}+04$ & $1.140 \mathrm{E}+01$ & $2.42 \mathrm{E}-02$ & $2.545 \mathrm{E}+01$ & $1.06 \mathrm{E}+01$ & $7.263 \mathrm{E}+01$ & $1.194 \mathrm{E}+01$ & $8.79 \mathrm{E}-01$ \\
\hline$P_{\mathrm{e}}(\mathrm{W})$ & $\Delta P_{\mathrm{e}}(\mathrm{W})$ & $C_{\mathrm{T}}$ & $\Delta C_{\mathrm{T}}$ & $C_{\mathrm{P}}$ & $\Delta C_{\mathrm{P}}$ & $C_{\mathrm{Q}}$ & $\Delta C_{\mathrm{Q}}$ \\
\hline $3.149 \mathrm{E}+01$ & $3.57 \mathrm{E}-01$ & $4.690 \mathrm{E}-02$ & $4.29 \mathrm{E}-03$ & $2.530 \mathrm{E}-02$ & $1.17 \mathrm{E}-03$ & $4.026 \mathrm{E}-03$ & $1.86 \mathrm{E}-04$ \\
\hline $3.116 \mathrm{E}+01$ & $3.50 \mathrm{E}-01$ & $4.316 \mathrm{E}-02$ & $4.21 \mathrm{E}-03$ & $2.475 \mathrm{E}-02$ & $1.17 \mathrm{E}-03$ & $3.939 \mathrm{E}-03$ & $1.86 \mathrm{E}-04$ \\
\hline $3.050 \mathrm{E}+01$ & $3.43 \mathrm{E}-01$ & $3.904 \mathrm{E}-02$ & $4.17 \mathrm{E}-03$ & $2.371 \mathrm{E}-02$ & $1.22 \mathrm{E}-03$ & $3.773 \mathrm{E}-03$ & $1.95 \mathrm{E}-04$ \\
\hline $2.903 \mathrm{E}+01$ & $3.28 \mathrm{E}-01$ & $3.439 \mathrm{E}-02$ & $4.21 \mathrm{E}-03$ & $2.295 \mathrm{E}-02$ & $1.18 \mathrm{E}-03$ & $3.653 \mathrm{E}-03$ & $1.87 \mathrm{E}-04$ \\
\hline $2.757 \mathrm{E}+01$ & $3.11 \mathrm{E}-01$ & $3.060 \mathrm{E}-02$ & $4.18 \mathrm{E}-03$ & $2.143 \mathrm{E}-02$ & $1.20 \mathrm{E}-03$ & $3.410 \mathrm{E}-03$ & $1.91 \mathrm{E}-04$ \\
\hline $2.565 \mathrm{E}+01$ & $2.90 \mathrm{E}-01$ & $2.555 \mathrm{E}-02$ & 4.19E-03 & $1.952 \mathrm{E}-02$ & $1.16 \mathrm{E}-03$ & $3.106 \mathrm{E}-03$ & $1.84 \mathrm{E}-04$ \\
\hline $2.310 \mathrm{E}+01$ & $2.66 \mathrm{E}-01$ & $1.667 \mathrm{E}-02$ & 4.19E-03 & $1.785 \mathrm{E}-02$ & $1.17 \mathrm{E}-03$ & $2.841 \mathrm{E}-03$ & $1.86 \mathrm{E}-04$ \\
\hline $2.088 \mathrm{E}+01$ & $2.41 \mathrm{E}-01$ & $1.006 \mathrm{E}-02$ & $4.19 \mathrm{E}-03$ & $1.580 \mathrm{E}-02$ & $1.16 \mathrm{E}-03$ & $2.515 \mathrm{E}-03$ & $1.85 \mathrm{E}-04$ \\
\hline$\rho\left(\mathrm{kg} / \mathrm{m}^{\wedge} 3\right)$ & $\eta_{\mathrm{T}}$ & $\Delta \eta_{\mathrm{T}}$ & $J$ & $\Delta J$ & $\eta_{\mathrm{P}}$ & $\Delta \eta_{\mathrm{P}}$ & $R e_{0.75}$ \\
\hline $1.158 \mathrm{E}+00$ & $5.887 \mathrm{E}-01$ & $2.81 \mathrm{E}-02$ & $6.784 \mathrm{E}-02$ & $4.30 \mathrm{E}-04$ & $1.258 \mathrm{E}-01$ & $1.29 \mathrm{E}-02$ & $3.997 \mathrm{E}+04$ \\
\hline $1.158 \mathrm{E}+00$ & $5.970 \mathrm{E}-01$ & $2.90 \mathrm{E}-02$ & $1.078 \mathrm{E}-01$ & $4.45 \mathrm{E}-04$ & $1.880 \mathrm{E}-01$ & $2.04 \mathrm{E}-02$ & $4.032 \mathrm{E}+04$ \\
\hline $1.157 \mathrm{E}+00$ & 5.910E-01 & $3.12 \mathrm{E}-02$ & $1.497 \mathrm{E}-01$ & $4.76 \mathrm{E}-04$ & $2.465 \mathrm{E}-01$ & $2.93 \mathrm{E}-02$ & $4.050 \mathrm{E}+04$ \\
\hline $1.157 \mathrm{E}+00$ & 5.949E-01 & $3.12 \mathrm{E}-02$ & $1.935 \mathrm{E}-01$ & $5.42 \mathrm{E}-04$ & $2.900 \mathrm{E}-01$ & $3.85 \mathrm{E}-02$ & $4.041 \mathrm{E}+04$ \\
\hline $1.157 \mathrm{E}+00$ & $5.858 \mathrm{E}-01$ & $3.34 \mathrm{E}-02$ & $2.381 \mathrm{E}-01$ & $5.90 \mathrm{E}-04$ & $3.401 \mathrm{E}-01$ & $5.02 \mathrm{E}-02$ & $4.048 \mathrm{E}+04$ \\
\hline $1.156 \mathrm{E}+00$ & $5.749 \mathrm{E}-01$ & $3.47 \mathrm{E}-02$ & $2.834 \mathrm{E}-01$ & $6.64 \mathrm{E}-04$ & $3.709 \mathrm{E}-01$ & $6.47 \mathrm{E}-02$ & $4.058 \mathrm{E}+04$ \\
\hline $1.156 \mathrm{E}+00$ & 5.772E-01 & $3.83 \mathrm{E}-02$ & $3.299 \mathrm{E}-01$ & $7.45 \mathrm{E}-04$ & $3.081 \mathrm{E}-01$ & $8.00 \mathrm{E}-02$ & $4.053 \mathrm{E}+04$ \\
\hline $1.156 \mathrm{E}+00$ & $5.718 \mathrm{E}-01$ & $4.26 \mathrm{E}-02$ & $3.745 \mathrm{E}-01$ & 8.04E-04 & $2.383 \mathrm{E}-01$ & $1.01 \mathrm{E}-01$ & $4.077 \mathrm{E}+04$ \\
\hline
\end{tabular}


Table 172: APC 6.0 x 2.0 Dynamic Measured Values - 13976 RPM

\begin{tabular}{|c|c|c|c|c|c|c|c|}
\hline$n(\mathrm{RPM})$ & $Q(\mathrm{~g}-\mathrm{m})$ & $\Delta Q(\mathrm{~g}-\mathrm{m})$ & $V(\mathrm{~V})$ & $I(\mathrm{~A})$ & $T_{\text {atm }}\left({ }^{\circ} \mathrm{C}\right)$ & $P_{\text {atm }}(\mathrm{Pa})$ & $P_{\text {diff }}(\mathrm{Pa})$ \\
\hline $1.381 \mathrm{E}+04$ & $2.058 \mathrm{E}+00$ & $7.58 \mathrm{E}-02$ & $1.100 \mathrm{E}+01$ & $4.147 \mathrm{E}+00$ & $2.325 \mathrm{E}+01$ & $9.843 \mathrm{E}+04$ & $2.961 \mathrm{E}+00$ \\
\hline $1.398 \mathrm{E}+04$ & $2.069 \mathrm{E}+00$ & $7.89 \mathrm{E}-02$ & $1.100 \mathrm{E}+01$ & $4.159 \mathrm{E}+00$ & $2.337 \mathrm{E}+01$ & $9.843 \mathrm{E}+04$ & $8.704 \mathrm{E}+00$ \\
\hline $1.397 \mathrm{E}+04$ & $1.998 \mathrm{E}+00$ & $7.86 \mathrm{E}-02$ & $1.101 \mathrm{E}+01$ & $3.999 \mathrm{E}+00$ & $2.339 \mathrm{E}+01$ & $9.842 \mathrm{E}+04$ & $1.811 \mathrm{E}+01$ \\
\hline $1.402 \mathrm{E}+04$ & $1.876 \mathrm{E}+00$ & $7.80 \mathrm{E}-02$ & $1.101 \mathrm{E}+01$ & $3.830 \mathrm{E}+00$ & $2.352 \mathrm{E}+01$ & $9.841 \mathrm{E}+04$ & $3.140 \mathrm{E}+01$ \\
\hline $1.398 \mathrm{E}+04$ & $1.725 \mathrm{E}+00$ & $7.62 \mathrm{E}-02$ & $1.102 \mathrm{E}+01$ & $3.533 \mathrm{E}+00$ & $2.358 \mathrm{E}+01$ & $9.841 \mathrm{E}+04$ & $4.879 \mathrm{E}+01$ \\
\hline $1.404 \mathrm{E}+04$ & $1.570 \mathrm{E}+00$ & $7.63 \mathrm{E}-02$ & $1.103 \mathrm{E}+01$ & $3.247 \mathrm{E}+00$ & $2.360 \mathrm{E}+01$ & $9.840 \mathrm{E}+04$ & $6.993 \mathrm{E}+01$ \\
\hline $1.399 \mathrm{E}+04$ & $1.349 \mathrm{E}+00$ & $7.59 \mathrm{E}-02$ & $1.104 \mathrm{E}+01$ & $2.844 \mathrm{E}+00$ & $2.367 \mathrm{E}+01$ & $9.840 \mathrm{E}+04$ & $9.525 \mathrm{E}+01$ \\
\hline $1.402 \mathrm{E}+04$ & $1.156 \mathrm{E}+00$ & $7.64 \mathrm{E}-02$ & $1.105 \mathrm{E}+01$ & $2.464 \mathrm{E}+00$ & $2.378 \mathrm{E}+01$ & $9.841 \mathrm{E}+04$ & $1.243 \mathrm{E}+02$ \\
\hline
\end{tabular}


Table 173: APC 6.0 x 2.0 Dynamic Calculated Values - 13976 RPM

\begin{tabular}{|c|c|c|c|c|c|c|c|}
\hline$n(\mathrm{RPM})$ & $V_{\infty}^{\prime}(\mathrm{m} / \mathrm{s})$ & $\Delta V_{\infty}^{\prime}(\mathrm{m} / \mathrm{s})$ & $T^{\prime}(\mathrm{g})$ & $\Delta T^{\prime}(\mathrm{g})$ & $V_{\mathrm{t}}(\mathrm{m} / \mathrm{s})$ & $P_{\mathrm{P}}(\mathrm{W})$ & $\Delta P_{\mathrm{P}}(\mathrm{W})$ \\
\hline $1.381 \mathrm{E}+04$ & $2.112 \mathrm{E}+00$ & $1.39 \mathrm{E}-02$ & $1.674 \mathrm{E}+02$ & $1.14 \mathrm{E}+01$ & $8.257 \mathrm{E}+01$ & $2.919 \mathrm{E}+01$ & $1.08 \mathrm{E}+00$ \\
\hline $1.398 \mathrm{E}+04$ & $3.740 \mathrm{E}+00$ & $1.47 \mathrm{E}-02$ & $1.541 \mathrm{E}+02$ & $1.16 \mathrm{E}+01$ & $8.366 \mathrm{E}+01$ & $2.971 \mathrm{E}+01$ & $1.13 \mathrm{E}+00$ \\
\hline $1.397 \mathrm{E}+04$ & $5.475 \mathrm{E}+00$ & $1.63 \mathrm{E}-02$ & $1.341 \mathrm{E}+02$ & $1.16 \mathrm{E}+01$ & $8.367 \mathrm{E}+01$ & $2.866 \mathrm{E}+01$ & $1.13 \mathrm{E}+00$ \\
\hline $1.402 \mathrm{E}+04$ & $7.266 \mathrm{E}+00$ & $1.85 \mathrm{E}-02$ & $1.204 \mathrm{E}+02$ & $1.15 \mathrm{E}+01$ & $8.409 \mathrm{E}+01$ & $2.701 \mathrm{E}+01$ & $1.12 \mathrm{E}+00$ \\
\hline $1.398 \mathrm{E}+04$ & $9.104 \mathrm{E}+00$ & 2.19E-02 & $1.005 \mathrm{E}+02$ & $1.14 \mathrm{E}+01$ & $8.402 \mathrm{E}+01$ & $2.475 \mathrm{E}+01$ & $1.09 \mathrm{E}+00$ \\
\hline $1.404 \mathrm{E}+04$ & $1.094 \mathrm{E}+01$ & $2.40 \mathrm{E}-02$ & $7.496 \mathrm{E}+01$ & $1.15 \mathrm{E}+01$ & $8.462 \mathrm{E}+01$ & $2.263 \mathrm{E}+01$ & $1.10 \mathrm{E}+00$ \\
\hline 1.399E+04 & $1.280 \mathrm{E}+01$ & $2.68 \mathrm{E}-02$ & $5.362 \mathrm{E}+01$ & $1.15 \mathrm{E}+01$ & $8.457 \mathrm{E}+01$ & $1.938 \mathrm{E}+01$ & $1.09 \mathrm{E}+00$ \\
\hline $1.402 \mathrm{E}+04$ & $1.466 \mathrm{E}+01$ & 2.99E-02 & $2.450 \mathrm{E}+01$ & $1.15 \mathrm{E}+01$ & $8.509 \mathrm{E}+01$ & $1.664 \mathrm{E}+01$ & $1.10 \mathrm{E}+00$ \\
\hline$P_{\mathrm{e}}(\mathrm{W})$ & $\Delta P_{\mathrm{e}}(\mathrm{W})$ & $C_{\mathrm{T}}$ & $\Delta C_{\mathrm{T}}$ & $C_{\mathrm{P}}$ & $\Delta C_{\mathrm{P}}$ & $C_{\mathrm{Q}}$ & $\Delta C_{\mathrm{Q}}$ \\
\hline $4.562 \mathrm{E}+01$ & $5.13 \mathrm{E}-01$ & 4.992E-02 & $3.41 \mathrm{E}-03$ & $2.534 \mathrm{E}-02$ & $9.34 \mathrm{E}-04$ & 4.032E-03 & $1.49 \mathrm{E}-04$ \\
\hline $4.575 \mathrm{E}+01$ & $5.12 \mathrm{E}-01$ & $4.486 \mathrm{E}-02$ & $3.38 \mathrm{E}-03$ & $2.485 \mathrm{E}-02$ & $9.49 \mathrm{E}-04$ & $3.956 \mathrm{E}-03$ & $1.51 \mathrm{E}-04$ \\
\hline $4.401 \mathrm{E}+01$ & 4.89E-01 & $3.911 \mathrm{E}-02$ & $3.38 \mathrm{E}-03$ & $2.406 \mathrm{E}-02$ & $9.46 \mathrm{E}-04$ & $3.829 \mathrm{E}-03$ & $1.51 \mathrm{E}-04$ \\
\hline $4.217 \mathrm{E}+01$ & $4.70 \mathrm{E}-01$ & $3.489 \mathrm{E}-02$ & $3.33 \mathrm{E}-03$ & $2.245 \mathrm{E}-02$ & 9.34E-04 & $3.573 \mathrm{E}-03$ & $1.49 \mathrm{E}-04$ \\
\hline $3.893 \mathrm{E}+01$ & $4.30 \mathrm{E}-01$ & $2.932 \mathrm{E}-02$ & $3.33 \mathrm{E}-03$ & $2.076 \mathrm{E}-02$ & $9.17 \mathrm{E}-04$ & $3.305 \mathrm{E}-03$ & $1.46 \mathrm{E}-04$ \\
\hline $3.580 \mathrm{E}+01$ & $4.00 \mathrm{E}-01$ & $2.166 \mathrm{E}-02$ & 3.33E-03 & $1.873 \mathrm{E}-02$ & $9.11 \mathrm{E}-04$ & $2.981 \mathrm{E}-03$ & $1.45 \mathrm{E}-04$ \\
\hline $3.139 \mathrm{E}+01$ & $3.53 \mathrm{E}-01$ & $1.562 \mathrm{E}-02$ & $3.34 \mathrm{E}-03$ & $1.623 \mathrm{E}-02$ & $9.13 \mathrm{E}-04$ & $2.582 \mathrm{E}-03$ & $1.45 \mathrm{E}-04$ \\
\hline $2.722 \mathrm{E}+01$ & $3.09 \mathrm{E}-01$ & 7.100E-03 & $3.34 \mathrm{E}-03$ & $1.382 \mathrm{E}-02$ & $9.14 \mathrm{E}-04$ & $2.200 \mathrm{E}-03$ & $1.45 \mathrm{E}-04$ \\
\hline$\rho\left(\mathrm{kg} / \mathrm{m}^{\wedge} 3\right)$ & $\eta_{\mathrm{T}}$ & $\Delta \eta_{\mathrm{T}}$ & $J$ & $\Delta J$ & $\eta_{\mathrm{P}}$ & $\Delta \eta_{\mathrm{P}}$ & $\operatorname{Re}_{0.75}$ \\
\hline $1.157 \mathrm{E}+00$ & 6.397E-01 & $2.47 \mathrm{E}-02$ & $6.029 \mathrm{E}-02$ & $3.98 \mathrm{E}-04$ & $1.188 \mathrm{E}-01$ & $9.26 \mathrm{E}-03$ & $4.642 \mathrm{E}+04$ \\
\hline $1.156 \mathrm{E}+00$ & 6.494E-01 & $2.58 \mathrm{E}-02$ & $1.054 \mathrm{E}-01$ & 4.15E-04 & $1.903 \mathrm{E}-01$ & $1.61 \mathrm{E}-02$ & $4.700 \mathrm{E}+04$ \\
\hline $1.156 \mathrm{E}+00$ & $6.513 \mathrm{E}-01$ & $2.66 \mathrm{E}-02$ & $1.545 \mathrm{E}-01$ & 4.62E-04 & $2.512 \mathrm{E}-01$ & $2.38 \mathrm{E}-02$ & $4.699 \mathrm{E}+04$ \\
\hline $1.156 \mathrm{E}+00$ & $6.406 \mathrm{E}-01$ & $2.76 \mathrm{E}-02$ & $2.043 \mathrm{E}-01$ & $5.22 \mathrm{E}-04$ & $3.175 \mathrm{E}-01$ & $3.31 \mathrm{E}-02$ & $4.719 \mathrm{E}+04$ \\
\hline $1.155 \mathrm{E}+00$ & $6.359 \mathrm{E}-01$ & $2.89 \mathrm{E}-02$ & $2.568 \mathrm{E}-01$ & $6.21 \mathrm{E}-04$ & $3.626 \mathrm{E}-01$ & 4.42E-02 & $4.713 \mathrm{E}+04$ \\
\hline $1.155 \mathrm{E}+00$ & $6.321 \mathrm{E}-01$ & $3.15 \mathrm{E}-02$ & $3.072 \mathrm{E}-01$ & $6.77 \mathrm{E}-04$ & $3.553 \mathrm{E}-01$ & $5.74 \mathrm{E}-02$ & $4.746 \mathrm{E}+04$ \\
\hline $1.155 \mathrm{E}+00$ & $6.176 \mathrm{E}-01$ & $3.54 \mathrm{E}-02$ & $3.608 \mathrm{E}-01$ & $7.60 \mathrm{E}-04$ & $3.473 \mathrm{E}-01$ & 7.67E-02 & $4.741 \mathrm{E}+04$ \\
\hline $1.155 \mathrm{E}+00$ & $6.113 \mathrm{E}-01$ & $4.10 \mathrm{E}-02$ & 4.120E-01 & $8.46 \mathrm{E}-04$ & $2.116 \mathrm{E}-01$ & $1.00 \mathrm{E}-01$ & $4.767 \mathrm{E}+04$ \\
\hline
\end{tabular}


Table 174: APC 6.0 x 2.0 Dynamic Measured Values - 16009 RPM

\begin{tabular}{|c|c|c|c|c|c|c|c|}
\hline$n(\mathrm{RPM})$ & $Q(\mathrm{~g}-\mathrm{m})$ & $\Delta Q(\mathrm{~g}-\mathrm{m})$ & $V(\mathrm{~V})$ & $I(\mathrm{~A})$ & $T_{\text {atm }}\left({ }^{\circ} \mathrm{C}\right)$ & $P_{\text {atm }}(\mathrm{Pa})$ & $P_{\text {diff }}(\mathrm{Pa})$ \\
\hline $1.604 \mathrm{E}+04$ & $2.810 \mathrm{E}+00$ & $9.73 \mathrm{E}-02$ & $1.095 \mathrm{E}+01$ & $6.077 \mathrm{E}+00$ & $2.341 \mathrm{E}+01$ & $9.841 \mathrm{E}+04$ & $3.390 \mathrm{E}+00$ \\
\hline $1.602 \mathrm{E}+04$ & $2.799 \mathrm{E}+00$ & $9.70 \mathrm{E}-02$ & $1.095 \mathrm{E}+01$ & $5.985 \mathrm{E}+00$ & $2.356 \mathrm{E}+01$ & $9.841 \mathrm{E}+04$ & $9.137 \mathrm{E}+00$ \\
\hline $1.601 \mathrm{E}+04$ & $2.663 \mathrm{E}+00$ & $9.72 \mathrm{E}-02$ & $1.095 \mathrm{E}+01$ & $5.805 \mathrm{E}+00$ & $2.361 \mathrm{E}+01$ & $9.840 \mathrm{E}+04$ & $1.857 \mathrm{E}+01$ \\
\hline $1.603 \mathrm{E}+04$ & $2.596 \mathrm{E}+00$ & $9.81 \mathrm{E}-02$ & $1.096 \mathrm{E}+01$ & $5.598 \mathrm{E}+00$ & $2.364 \mathrm{E}+01$ & $9.840 \mathrm{E}+04$ & $3.187 \mathrm{E}+01$ \\
\hline $1.602 \mathrm{E}+04$ & $2.421 \mathrm{E}+00$ & $9.67 \mathrm{E}-02$ & $1.097 \mathrm{E}+01$ & $5.265 \mathrm{E}+00$ & $2.370 \mathrm{E}+01$ & $9.840 \mathrm{E}+04$ & $4.922 \mathrm{E}+01$ \\
\hline $1.597 \mathrm{E}+04$ & $2.255 \mathrm{E}+00$ & $9.61 \mathrm{E}-02$ & $1.098 \mathrm{E}+01$ & $4.863 \mathrm{E}+00$ & $2.372 \mathrm{E}+01$ & $9.840 \mathrm{E}+04$ & $7.025 \mathrm{E}+01$ \\
\hline $1.601 \mathrm{E}+04$ & $2.055 \mathrm{E}+00$ & $9.73 \mathrm{E}-02$ & $1.099 \mathrm{E}+01$ & $4.453 \mathrm{E}+00$ & $2.384 \mathrm{E}+01$ & $9.839 \mathrm{E}+04$ & $9.577 \mathrm{E}+01$ \\
\hline $1.599 \mathrm{E}+04$ & $1.833 \mathrm{E}+00$ & $9.48 \mathrm{E}-02$ & $1.100 \mathrm{E}+01$ & $4.043 \mathrm{E}+00$ & $2.392 \mathrm{E}+01$ & $9.839 \mathrm{E}+04$ & $1.251 \mathrm{E}+02$ \\
\hline $1.599 \mathrm{E}+04$ & $1.589 \mathrm{E}+00$ & $9.62 \mathrm{E}-02$ & $1.102 \mathrm{E}+01$ & $3.526 \mathrm{E}+00$ & $2.403 \mathrm{E}+01$ & $9.840 \mathrm{E}+04$ & $1.579 \mathrm{E}+02$ \\
\hline
\end{tabular}


Table 175: APC 6.0 x 2.0 Dynamic Calculated Values - 16009 RPM

\begin{tabular}{|c|c|c|c|c|c|c|c|}
\hline$n(\mathrm{RPM})$ & $V_{\infty}^{\prime}(\mathrm{m} / \mathrm{s})$ & $\Delta V_{\infty}^{\prime}(\mathrm{m} / \mathrm{s})$ & $T^{\prime}(\mathrm{g})$ & $\Delta T^{\prime}(\mathrm{g})$ & $V_{\mathrm{t}}(\mathrm{m} / \mathrm{s})$ & $P_{\mathrm{P}}(\mathrm{W})$ & $\Delta P_{\mathrm{P}}(\mathrm{W})$ \\
\hline $1.604 \mathrm{E}+04$ & $2.236 \mathrm{E}+00$ & $1.49 \mathrm{E}-02$ & $2.522 \mathrm{E}+02$ & $8.83 \mathrm{E}+00$ & $9.586 \mathrm{E}+01$ & $4.628 \mathrm{E}+01$ & $1.60 \mathrm{E}+00$ \\
\hline $1.602 \mathrm{E}+04$ & $3.806 \mathrm{E}+00$ & $1.57 \mathrm{E}-02$ & $2.255 \mathrm{E}+02$ & $8.85 \mathrm{E}+00$ & $9.582 \mathrm{E}+01$ & $4.605 \mathrm{E}+01$ & $1.60 \mathrm{E}+00$ \\
\hline $1.601 \mathrm{E}+04$ & $5.514 \mathrm{E}+00$ & $1.70 \mathrm{E}-02$ & $2.025 \mathrm{E}+02$ & $8.96 \mathrm{E}+00$ & $9.582 \mathrm{E}+01$ & $4.378 \mathrm{E}+01$ & $1.60 \mathrm{E}+00$ \\
\hline $1.603 \mathrm{E}+04$ & $7.291 \mathrm{E}+00$ & $1.89 \mathrm{E}-02$ & $1.811 \mathrm{E}+02$ & $8.83 \mathrm{E}+00$ & $9.610 \mathrm{E}+01$ & $4.274 \mathrm{E}+01$ & $1.62 \mathrm{E}+00$ \\
\hline $1.602 \mathrm{E}+04$ & $9.117 \mathrm{E}+00$ & $2.22 \mathrm{E}-02$ & $1.539 \mathrm{E}+02$ & $8.92 \mathrm{E}+00$ & $9.614 \mathrm{E}+01$ & $3.982 \mathrm{E}+01$ & $1.59 \mathrm{E}+00$ \\
\hline $1.597 \mathrm{E}+04$ & $1.094 \mathrm{E}+01$ & $2.44 \mathrm{E}-02$ & $1.273 \mathrm{E}+02$ & $9.19 \mathrm{E}+00$ & $9.608 \mathrm{E}+01$ & $3.698 \mathrm{E}+01$ & $1.58 \mathrm{E}+00$ \\
\hline $1.601 \mathrm{E}+04$ & $1.281 \mathrm{E}+01$ & 2.69E-02 & $9.946 \mathrm{E}+01$ & $9.05 \mathrm{E}+00$ & $9.653 \mathrm{E}+01$ & $3.379 \mathrm{E}+01$ & $1.60 \mathrm{E}+00$ \\
\hline $1.599 \mathrm{E}+04$ & $1.468 \mathrm{E}+01$ & $2.94 \mathrm{E}-02$ & $7.011 \mathrm{E}+01$ & $9.24 \mathrm{E}+00$ & $9.669 \mathrm{E}+01$ & $3.010 \mathrm{E}+01$ & $1.56 \mathrm{E}+00$ \\
\hline $1.599 \mathrm{E}+04$ & $1.652 \mathrm{E}+01$ & $3.24 \mathrm{E}-02$ & $4.070 \mathrm{E}+01$ & $9.23 \mathrm{E}+00$ & $9.700 \mathrm{E}+01$ & $2.610 \mathrm{E}+01$ & $1.58 \mathrm{E}+00$ \\
\hline$P_{\mathrm{e}}(\mathrm{W})$ & $\Delta P_{\mathrm{e}}(\mathrm{W})$ & $C_{\mathrm{T}}$ & $\Delta C_{\mathrm{T}}$ & $C_{\mathrm{P}}$ & $\Delta C_{\mathrm{P}}$ & $C_{\mathrm{Q}}$ & $\Delta C_{\mathrm{Q}}$ \\
\hline $6.652 \mathrm{E}+01$ & $7.55 \mathrm{E}-01$ & $5.584 \mathrm{E}-02$ & $1.96 \mathrm{E}-03$ & $2.569 \mathrm{E}-02$ & $8.90 \mathrm{E}-04$ & $4.088 \mathrm{E}-03$ & $1.42 \mathrm{E}-04$ \\
\hline $6.553 \mathrm{E}+01$ & 7.37E-01 & $5.004 \mathrm{E}-02$ & $1.96 \mathrm{E}-03$ & $2.564 \mathrm{E}-02$ & $8.89 \mathrm{E}-04$ & $4.081 \mathrm{E}-03$ & $1.41 \mathrm{E}-04$ \\
\hline $6.359 \mathrm{E}+01$ & 7.19E-01 & $4.502 \mathrm{E}-02$ & $1.99 \mathrm{E}-03$ & $2.445 \mathrm{E}-02$ & $8.92 \mathrm{E}-04$ & $3.891 \mathrm{E}-03$ & $1.42 \mathrm{E}-04$ \\
\hline $6.135 \mathrm{E}+01$ & $6.95 \mathrm{E}-01$ & $4.013 \mathrm{E}-02$ & $1.96 \mathrm{E}-03$ & $2.376 \mathrm{E}-02$ & 8.98E-04 & $3.781 \mathrm{E}-03$ & $1.43 \mathrm{E}-04$ \\
\hline $5.776 \mathrm{E}+01$ & $6.54 \mathrm{E}-01$ & $3.419 \mathrm{E}-02$ & $1.98 \mathrm{E}-03$ & $2.221 \mathrm{E}-02$ & 8.87E-04 & $3.535 \mathrm{E}-03$ & $1.41 \mathrm{E}-04$ \\
\hline $5.340 \mathrm{E}+01$ & $6.00 \mathrm{E}-01$ & $2.845 \mathrm{E}-02$ & $2.05 \mathrm{E}-03$ & 2.079E-02 & $8.87 \mathrm{E}-04$ & $3.310 \mathrm{E}-03$ & $1.41 \mathrm{E}-04$ \\
\hline $4.895 \mathrm{E}+01$ & $5.54 \mathrm{E}-01$ & $2.213 \mathrm{E}-02$ & $2.01 \mathrm{E}-03$ & $1.888 \mathrm{E}-02$ & $8.94 \mathrm{E}-04$ & $3.004 \mathrm{E}-03$ & $1.42 \mathrm{E}-04$ \\
\hline $4.448 \mathrm{E}+01$ & 4.95E-01 & $1.564 \mathrm{E}-02$ & $2.06 \mathrm{E}-03$ & $1.688 \mathrm{E}-02$ & $8.73 \mathrm{E}-04$ & $2.686 \mathrm{E}-03$ & $1.39 \mathrm{E}-04$ \\
\hline $3.885 \mathrm{E}+01$ & $4.31 \mathrm{E}-01$ & $9.079 \mathrm{E}-03$ & $2.06 \mathrm{E}-03$ & $1.463 \mathrm{E}-02$ & $8.86 \mathrm{E}-04$ & $2.329 \mathrm{E}-03$ & $1.41 \mathrm{E}-04$ \\
\hline$\rho\left(\mathrm{kg} / \mathrm{m}^{\wedge} 3\right)$ & $\eta_{\mathrm{T}}$ & $\Delta \eta_{\mathrm{T}}$ & $J$ & $\Delta J$ & $\eta_{\mathrm{P}}$ & $\Delta \eta_{\mathrm{P}}$ & $R e_{0.75}$ \\
\hline $1.156 \mathrm{E}+00$ & $6.958 \mathrm{E}-01$ & $2.53 \mathrm{E}-02$ & $5.498 \mathrm{E}-02$ & 3.67E-04 & $1.195 \mathrm{E}-01$ & $5.94 \mathrm{E}-03$ & $5.383 \mathrm{E}+04$ \\
\hline $1.155 \mathrm{E}+00$ & $7.028 \mathrm{E}-01$ & $2.56 \mathrm{E}-02$ & $9.365 \mathrm{E}-02$ & $3.86 \mathrm{E}-04$ & $1.828 \mathrm{E}-01$ & $9.60 \mathrm{E}-03$ & $5.376 \mathrm{E}+04$ \\
\hline $1.155 \mathrm{E}+00$ & $6.885 \mathrm{E}-01$ & $2.63 \mathrm{E}-02$ & $1.358 \mathrm{E}-01$ & $4.19 \mathrm{E}-04$ & $2.501 \mathrm{E}-01$ & $1.44 \mathrm{E}-02$ & $5.374 \mathrm{E}+04$ \\
\hline $1.155 \mathrm{E}+00$ & $6.967 \mathrm{E}-01$ & $2.75 \mathrm{E}-02$ & $1.793 \mathrm{E}-01$ & $4.67 \mathrm{E}-04$ & $3.029 \mathrm{E}-01$ & $1.87 \mathrm{E}-02$ & $5.388 \mathrm{E}+04$ \\
\hline $1.155 \mathrm{E}+00$ & $6.894 \mathrm{E}-01$ & $2.86 \mathrm{E}-02$ & 2.244E-01 & $5.50 \mathrm{E}-04$ & $3.455 \mathrm{E}-01$ & $2.43 \mathrm{E}-02$ & $5.389 \mathrm{E}+04$ \\
\hline $1.155 \mathrm{E}+00$ & $6.925 \mathrm{E}-01$ & $3.05 \mathrm{E}-02$ & $2.700 \mathrm{E}-01$ & $6.05 \mathrm{E}-04$ & $3.693 \mathrm{E}-01$ & $3.10 \mathrm{E}-02$ & $5.385 \mathrm{E}+04$ \\
\hline $1.154 \mathrm{E}+00$ & $6.903 \mathrm{E}-01$ & $3.36 \mathrm{E}-02$ & $3.155 \mathrm{E}-01$ & $6.65 \mathrm{E}-04$ & $3.699 \mathrm{E}-01$ & $3.80 \mathrm{E}-02$ & $5.406 \mathrm{E}+04$ \\
\hline $1.154 \mathrm{E}+00$ & $6.767 \mathrm{E}-01$ & $3.58 \mathrm{E}-02$ & $3.618 \mathrm{E}-01$ & $7.29 \mathrm{E}-04$ & $3.352 \mathrm{E}-01$ & $4.74 \mathrm{E}-02$ & $5.412 \mathrm{E}+04$ \\
\hline $1.153 \mathrm{E}+00$ & $6.719 \mathrm{E}-01$ & $4.13 \mathrm{E}-02$ & $4.073 \mathrm{E}-01$ & $8.03 \mathrm{E}-04$ & $2.527 \mathrm{E}-01$ & $5.93 \mathrm{E}-02$ & $5.426 \mathrm{E}+04$ \\
\hline
\end{tabular}


Table 176: APC 6.0 x 2.0 Dynamic Measured Values - 18011 RPM

\begin{tabular}{|c|c|c|c|c|c|c|c|}
\hline$n(\mathrm{RPM})$ & $Q(\mathrm{~g}-\mathrm{m})$ & $\Delta Q(\mathrm{~g}-\mathrm{m})$ & $V(\mathrm{~V})$ & $I(\mathrm{~A})$ & $T_{\text {atm }}\left({ }^{\circ} \mathrm{C}\right)$ & $P_{\text {atm }}(\mathrm{Pa})$ & $P_{\text {diff }}(\mathrm{Pa})$ \\
\hline $1.799 \mathrm{E}+04$ & $3.482 \mathrm{E}+00$ & $1.29 \mathrm{E}-01$ & $1.088 \mathrm{E}+01$ & $8.349 \mathrm{E}+00$ & $2.355 \mathrm{E}+01$ & $9.839 \mathrm{E}+04$ & $3.957 \mathrm{E}+00$ \\
\hline $1.804 \mathrm{E}+04$ & $3.504 \mathrm{E}+00$ & $1.30 \mathrm{E}-01$ & $1.088 \mathrm{E}+01$ & $8.377 \mathrm{E}+00$ & $2.374 \mathrm{E}+01$ & $9.839 \mathrm{E}+04$ & $9.713 \mathrm{E}+00$ \\
\hline $1.802 \mathrm{E}+04$ & $3.447 \mathrm{E}+00$ & $1.29 \mathrm{E}-01$ & $1.088 \mathrm{E}+01$ & $8.180 \mathrm{E}+00$ & $2.367 \mathrm{E}+01$ & $9.839 \mathrm{E}+04$ & $1.926 \mathrm{E}+01$ \\
\hline $1.802 \mathrm{E}+04$ & $3.314 \mathrm{E}+00$ & $1.30 \mathrm{E}-01$ & $1.089 \mathrm{E}+01$ & $7.941 \mathrm{E}+00$ & $2.377 \mathrm{E}+01$ & $9.839 \mathrm{E}+04$ & $3.267 \mathrm{E}+01$ \\
\hline $1.799 \mathrm{E}+04$ & $3.203 \mathrm{E}+00$ & $1.30 \mathrm{E}-01$ & $1.090 \mathrm{E}+01$ & $7.533 \mathrm{E}+00$ & $2.393 \mathrm{E}+01$ & $9.839 \mathrm{E}+04$ & $4.999 \mathrm{E}+01$ \\
\hline $1.798 \mathrm{E}+04$ & $3.065 \mathrm{E}+00$ & $1.29 \mathrm{E}-01$ & $1.092 \mathrm{E}+01$ & $7.086 \mathrm{E}+00$ & $2.392 \mathrm{E}+01$ & $9.838 \mathrm{E}+04$ & $7.102 \mathrm{E}+01$ \\
\hline $1.800 \mathrm{E}+04$ & $2.896 \mathrm{E}+00$ & $1.28 \mathrm{E}-01$ & $1.093 \mathrm{E}+01$ & $6.616 \mathrm{E}+00$ & $2.396 \mathrm{E}+01$ & $9.838 \mathrm{E}+04$ & $9.658 \mathrm{E}+01$ \\
\hline $1.804 \mathrm{E}+04$ & $2.644 \mathrm{E}+00$ & $1.31 \mathrm{E}-01$ & $1.094 \mathrm{E}+01$ & $6.098 \mathrm{E}+00$ & $2.396 \mathrm{E}+01$ & $9.838 \mathrm{E}+04$ & $1.260 \mathrm{E}+02$ \\
\hline $1.805 \mathrm{E}+04$ & $2.428 \mathrm{E}+00$ & $1.32 \mathrm{E}-01$ & $1.096 \mathrm{E}+01$ & $5.635 \mathrm{E}+00$ & $2.419 \mathrm{E}+01$ & $9.838 \mathrm{E}+04$ & $1.591 \mathrm{E}+02$ \\
\hline $1.797 \mathrm{E}+04$ & $2.099 \mathrm{E}+00$ & $1.27 \mathrm{E}-01$ & $1.098 \mathrm{E}+01$ & $4.876 \mathrm{E}+00$ & $2.422 \mathrm{E}+01$ & $9.838 \mathrm{E}+04$ & $1.963 \mathrm{E}+02$ \\
\hline $1.802 \mathrm{E}+04$ & $1.752 \mathrm{E}+00$ & $1.28 \mathrm{E}-01$ & $1.100 \mathrm{E}+01$ & $4.153 \mathrm{E}+00$ & $2.426 \mathrm{E}+01$ & $9.838 \mathrm{E}+04$ & $2.366 \mathrm{E}+02$ \\
\hline
\end{tabular}


Table 177: APC 6.0 x 2.0 Dynamic Calculated Values - 18011 RPM

\begin{tabular}{|c|c|c|c|c|c|c|c|}
\hline$n(\mathrm{RPM})$ & $V_{\infty}^{\prime}(\mathrm{m} / \mathrm{s})$ & $\Delta V_{\infty}^{\prime}(\mathrm{m} / \mathrm{s})$ & $T^{\prime}(\mathrm{g})$ & $\Delta T^{\prime}(\mathrm{g})$ & $V_{\mathrm{t}}(\mathrm{m} / \mathrm{s})$ & $P_{\mathrm{P}}(\mathrm{W})$ & $\Delta P_{\mathrm{P}}(\mathrm{W})$ \\
\hline $1.799 \mathrm{E}+04$ & $2.406 \mathrm{E}+00$ & $1.62 \mathrm{E}-02$ & $3.282 \mathrm{E}+02$ & $8.21 \mathrm{E}+00$ & $1.076 \mathrm{E}+02$ & $6.434 \mathrm{E}+01$ & $2.39 \mathrm{E}+00$ \\
\hline $1.804 \mathrm{E}+04$ & $3.902 \mathrm{E}+00$ & $1.74 \mathrm{E}-02$ & $3.042 \mathrm{E}+02$ & $8.27 \mathrm{E}+00$ & $1.079 \mathrm{E}+02$ & $6.493 \mathrm{E}+01$ & $2.41 \mathrm{E}+00$ \\
\hline $1.802 \mathrm{E}+04$ & $5.590 \mathrm{E}+00$ & $1.83 \mathrm{E}-02$ & $2.788 \mathrm{E}+02$ & $8.31 \mathrm{E}+00$ & $1.078 \mathrm{E}+02$ & $6.378 \mathrm{E}+01$ & $2.38 \mathrm{E}+00$ \\
\hline $1.802 \mathrm{E}+04$ & $7.355 \mathrm{E}+00$ & $2.01 \mathrm{E}-02$ & $2.534 \mathrm{E}+02$ & $8.31 \mathrm{E}+00$ & $1.080 \mathrm{E}+02$ & $6.133 \mathrm{E}+01$ & $2.40 \mathrm{E}+00$ \\
\hline $1.799 \mathrm{E}+04$ & $9.161 \mathrm{E}+00$ & $2.32 \mathrm{E}-02$ & $2.231 \mathrm{E}+02$ & $8.31 \mathrm{E}+00$ & $1.079 \mathrm{E}+02$ & $5.918 \mathrm{E}+01$ & $2.40 \mathrm{E}+00$ \\
\hline $1.798 \mathrm{E}+04$ & $1.097 \mathrm{E}+01$ & $2.60 \mathrm{E}-02$ & $1.947 \mathrm{E}+02$ & $8.29 \mathrm{E}+00$ & $1.080 \mathrm{E}+02$ & $5.660 \mathrm{E}+01$ & $2.39 \mathrm{E}+00$ \\
\hline $1.800 \mathrm{E}+04$ & $1.284 \mathrm{E}+01$ & $2.79 \mathrm{E}-02$ & $1.654 \mathrm{E}+02$ & $8.32 \mathrm{E}+00$ & $1.083 \mathrm{E}+02$ & $5.353 \mathrm{E}+01$ & $2.37 \mathrm{E}+00$ \\
\hline $1.804 \mathrm{E}+04$ & $1.470 \mathrm{E}+01$ & $3.01 \mathrm{E}-02$ & $1.307 \mathrm{E}+02$ & $8.27 \mathrm{E}+00$ & $1.088 \mathrm{E}+02$ & $4.899 \mathrm{E}+01$ & $2.43 \mathrm{E}+00$ \\
\hline $1.805 \mathrm{E}+04$ & $1.655 \mathrm{E}+01$ & $3.26 \mathrm{E}-02$ & $1.013 \mathrm{E}+02$ & $8.27 \mathrm{E}+00$ & $1.092 \mathrm{E}+02$ & $4.502 \mathrm{E}+01$ & $2.44 \mathrm{E}+00$ \\
\hline $1.797 \mathrm{E}+04$ & $1.842 \mathrm{E}+01$ & $3.51 \mathrm{E}-02$ & $6.502 \mathrm{E}+01$ & $8.24 \mathrm{E}+00$ & $1.090 \mathrm{E}+02$ & $3.874 \mathrm{E}+01$ & $2.34 \mathrm{E}+00$ \\
\hline $1.802 \mathrm{E}+04$ & $2.025 \mathrm{E}+01$ & $3.78 \mathrm{E}-02$ & $3.039 \mathrm{E}+01$ & $8.29 \mathrm{E}+00$ & $1.096 \mathrm{E}+02$ & $3.242 \mathrm{E}+01$ & $2.37 \mathrm{E}+00$ \\
\hline$P_{\mathrm{e}}(\mathrm{W})$ & $\Delta P_{\mathrm{e}}(\mathrm{W})$ & $C_{\mathrm{T}}$ & $\Delta C_{\mathrm{T}}$ & $C_{\mathrm{P}}$ & $\Delta C_{\mathrm{P}}$ & $C_{\mathrm{Q}}$ & $\Delta C_{\mathrm{Q}}$ \\
\hline $9.083 \mathrm{E}+01$ & $9.86 \mathrm{E}-01$ & $5.776 \mathrm{E}-02$ & $1.45 \mathrm{E}-03$ & $2.530 \mathrm{E}-02$ & $9.40 \mathrm{E}-04$ & $4.026 \mathrm{E}-03$ & $1.50 \mathrm{E}-04$ \\
\hline $9.113 \mathrm{E}+01$ & $9.77 \mathrm{E}-01$ & $5.329 \mathrm{E}-02$ & $1.45 \mathrm{E}-03$ & $2.534 \mathrm{E}-02$ & $9.40 \mathrm{E}-04$ & $4.033 \mathrm{E}-03$ & $1.50 \mathrm{E}-04$ \\
\hline $8.902 \mathrm{E}+01$ & $9.54 \mathrm{E}-01$ & 4.894E-02 & $1.46 \mathrm{E}-03$ & $2.499 \mathrm{E}-02$ & $9.32 \mathrm{E}-04$ & 3.977E-03 & $1.48 \mathrm{E}-04$ \\
\hline $8.648 \mathrm{E}+01$ & $9.30 \mathrm{E}-01$ & 4.447E-02 & $1.46 \mathrm{E}-03$ & $2.401 \mathrm{E}-02$ & $9.40 \mathrm{E}-04$ & $3.822 \mathrm{E}-03$ & $1.50 \mathrm{E}-04$ \\
\hline $8.213 \mathrm{E}+01$ & $8.86 \mathrm{E}-01$ & $3.933 \mathrm{E}-02$ & $1.47 \mathrm{E}-03$ & $2.331 \mathrm{E}-02$ & $9.47 \mathrm{E}-04$ & $3.710 \mathrm{E}-03$ & $1.51 \mathrm{E}-04$ \\
\hline $7.734 \mathrm{E}+01$ & 8.37E-01 & $3.436 \mathrm{E}-02$ & $1.46 \mathrm{E}-03$ & $2.233 \mathrm{E}-02$ & $9.43 \mathrm{E}-04$ & $3.554 \mathrm{E}-03$ & $1.50 \mathrm{E}-04$ \\
\hline $7.230 \mathrm{E}+01$ & 7.91E-01 & $2.913 \mathrm{E}-02$ & $1.46 \mathrm{E}-03$ & $2.106 \mathrm{E}-02$ & $9.31 \mathrm{E}-04$ & $3.352 \mathrm{E}-03$ & $1.48 \mathrm{E}-04$ \\
\hline $6.673 \mathrm{E}+01$ & $7.37 \mathrm{E}-01$ & $2.293 \mathrm{E}-02$ & $1.45 \mathrm{E}-03$ & $1.914 \mathrm{E}-02$ & $9.51 \mathrm{E}-04$ & $3.047 \mathrm{E}-03$ & $1.51 \mathrm{E}-04$ \\
\hline $6.174 \mathrm{E}+01$ & $6.97 \mathrm{E}-01$ & $1.774 \mathrm{E}-02$ & $1.45 \mathrm{E}-03$ & $1.756 \mathrm{E}-02$ & $9.51 \mathrm{E}-04$ & $2.794 \mathrm{E}-03$ & $1.51 \mathrm{E}-04$ \\
\hline $5.353 \mathrm{E}+01$ & $5.96 \mathrm{E}-01$ & $1.150 \mathrm{E}-02$ & $1.46 \mathrm{E}-03$ & $1.533 \mathrm{E}-02$ & $9.25 \mathrm{E}-04$ & $2.440 \mathrm{E}-03$ & $1.47 \mathrm{E}-04$ \\
\hline $4.568 \mathrm{E}+01$ & $5.10 \mathrm{E}-01$ & $5.345 \mathrm{E}-03$ & $1.46 \mathrm{E}-03$ & $1.272 \mathrm{E}-02$ & $9.28 \mathrm{E}-04$ & $2.025 \mathrm{E}-03$ & $1.48 \mathrm{E}-04$ \\
\hline$\rho\left(\mathrm{kg} / \mathrm{m}^{\wedge} 3\right)$ & $\eta_{\mathrm{T}}$ & $\Delta \eta_{\mathrm{T}}$ & $J$ & $\Delta J$ & $\eta_{\mathrm{P}}$ & $\Delta \eta_{\mathrm{P}}$ & $R e_{0.75}$ \\
\hline $1.155 \mathrm{E}+00$ & $7.084 \mathrm{E}-01$ & $2.74 \mathrm{E}-02$ & $5.271 \mathrm{E}-02$ & $3.56 \mathrm{E}-04$ & $1.203 \mathrm{E}-01$ & $5.45 \mathrm{E}-03$ & $6.033 \mathrm{E}+04$ \\
\hline $1.154 \mathrm{E}+00$ & $7.125 \mathrm{E}-01$ & $2.75 \mathrm{E}-02$ & $8.526 \mathrm{E}-02$ & $3.80 \mathrm{E}-04$ & $1.793 \mathrm{E}-01$ & $8.28 \mathrm{E}-03$ & $6.045 \mathrm{E}+04$ \\
\hline $1.155 \mathrm{E}+00$ & $7.165 \mathrm{E}-01$ & $2.78 \mathrm{E}-02$ & $1.223 \mathrm{E}-01$ & $4.02 \mathrm{E}-04$ & $2.396 \mathrm{E}-01$ & $1.15 \mathrm{E}-02$ & $6.044 \mathrm{E}+04$ \\
\hline $1.154 \mathrm{E}+00$ & 7.093E-01 & $2.88 \mathrm{E}-02$ & $1.609 \mathrm{E}-01$ & $4.41 \mathrm{E}-04$ & $2.980 \mathrm{E}-01$ & $1.52 \mathrm{E}-02$ & $6.048 \mathrm{E}+04$ \\
\hline $1.154 \mathrm{E}+00$ & $7.206 \mathrm{E}-01$ & $3.03 \mathrm{E}-02$ & $2.008 \mathrm{E}-01$ & $5.10 \mathrm{E}-04$ & $3.387 \mathrm{E}-01$ & $1.87 \mathrm{E}-02$ & $6.039 \mathrm{E}+04$ \\
\hline $1.154 \mathrm{E}+00$ & $7.318 \mathrm{E}-01$ & $3.19 \mathrm{E}-02$ & $2.405 \mathrm{E}-01$ & $5.72 \mathrm{E}-04$ & 3.701E-01 & $2.22 \mathrm{E}-02$ & $6.045 \mathrm{E}+04$ \\
\hline $1.154 \mathrm{E}+00$ & 7.403E-01 & $3.37 \mathrm{E}-02$ & 2.812E-01 & $6.13 \mathrm{E}-04$ & 3.889E-01 & $2.61 \mathrm{E}-02$ & $6.061 \mathrm{E}+04$ \\
\hline $1.153 \mathrm{E}+00$ & 7.340E-01 & $3.74 \mathrm{E}-02$ & $3.213 \mathrm{E}-01$ & $6.61 \mathrm{E}-04$ & $3.847 \mathrm{E}-01$ & $3.10 \mathrm{E}-02$ & $6.088 \mathrm{E}+04$ \\
\hline $1.153 \mathrm{E}+00$ & 7.291E-01 & 4.04E-02 & $3.615 \mathrm{E}-01$ & $7.18 \mathrm{E}-04$ & $3.652 \mathrm{E}-01$ & $3.58 \mathrm{E}-02$ & $6.100 \mathrm{E}+04$ \\
\hline $1.152 \mathrm{E}+00$ & 7.237E-01 & $4.44 \mathrm{E}-02$ & $4.041 \mathrm{E}-01$ & $7.75 \mathrm{E}-04$ & $3.032 \mathrm{E}-01$ & $4.25 \mathrm{E}-02$ & $6.087 \mathrm{E}+04$ \\
\hline $1.152 \mathrm{E}+00$ & 7.097E-01 & $5.24 \mathrm{E}-02$ & $4.430 \mathrm{E}-01$ & 8.33E-04 & $1.861 \mathrm{E}-01$ & $5.26 \mathrm{E}-02$ & $6.120 \mathrm{E}+04$ \\
\hline
\end{tabular}




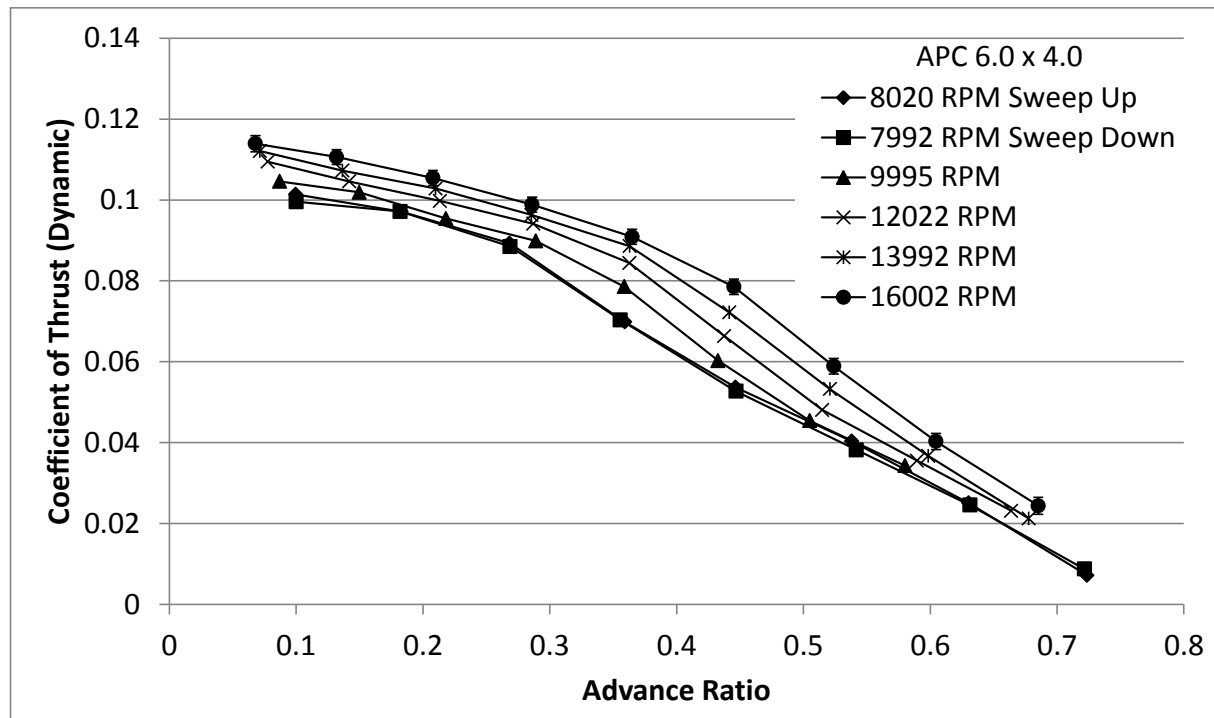

(a)

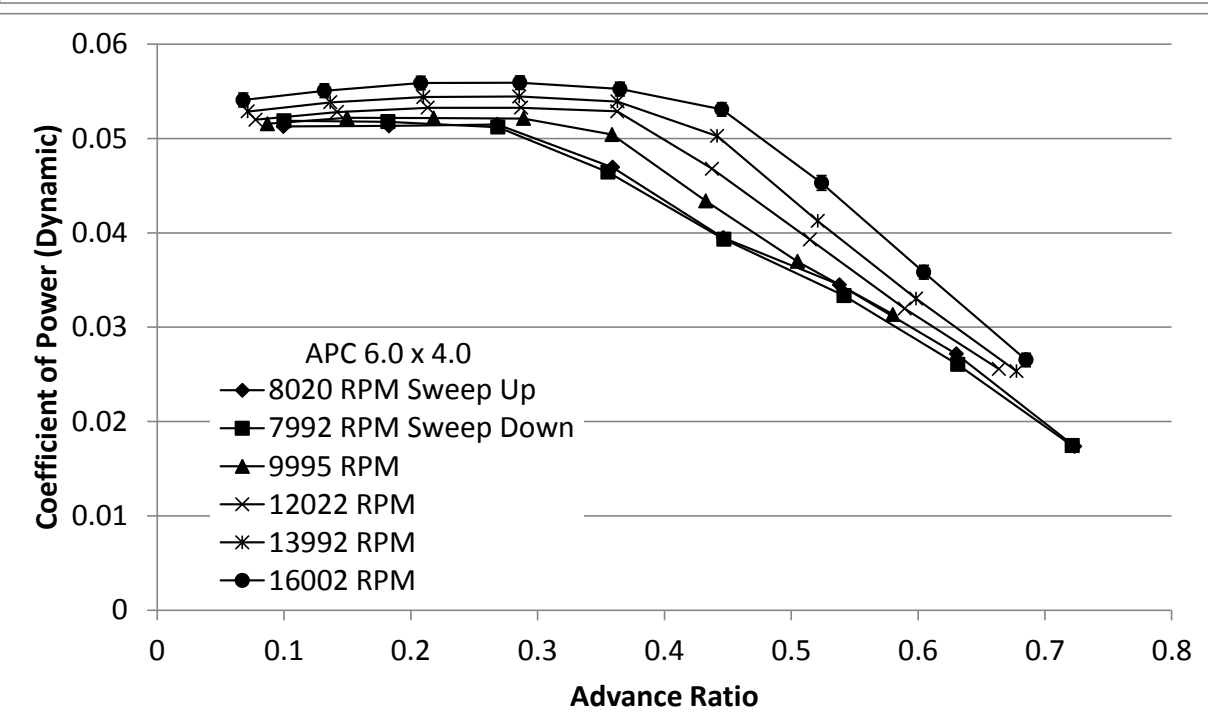

(b)

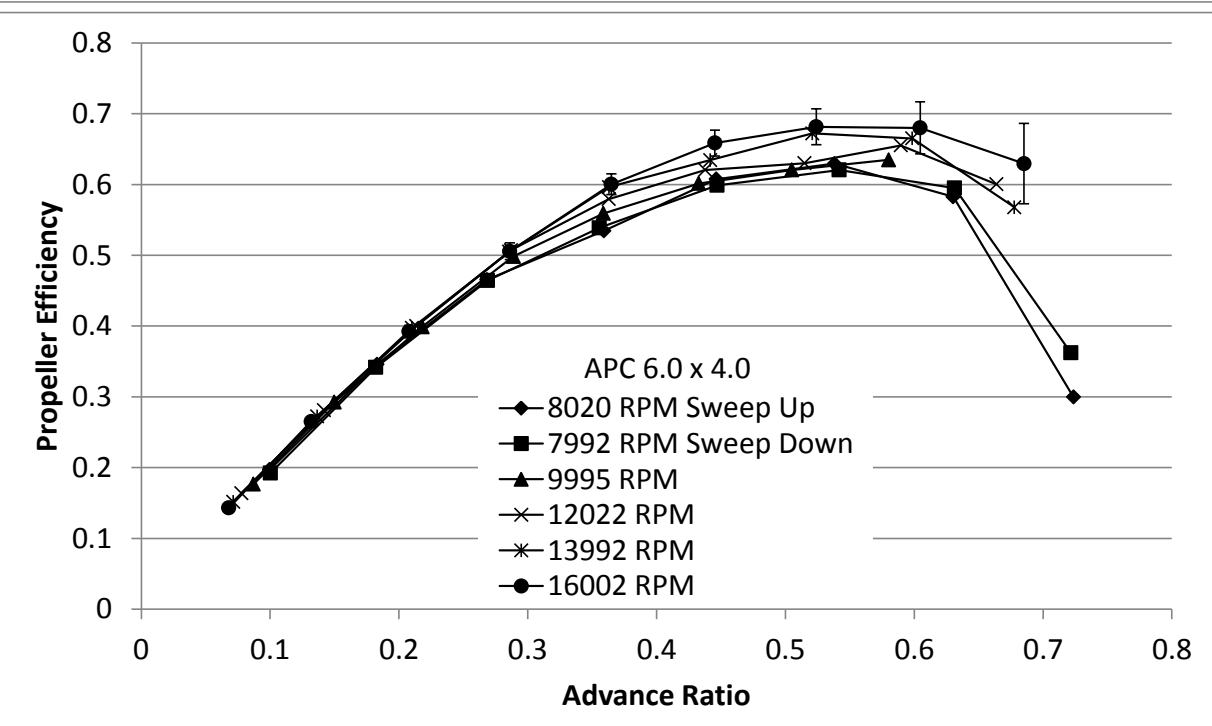

(c) 


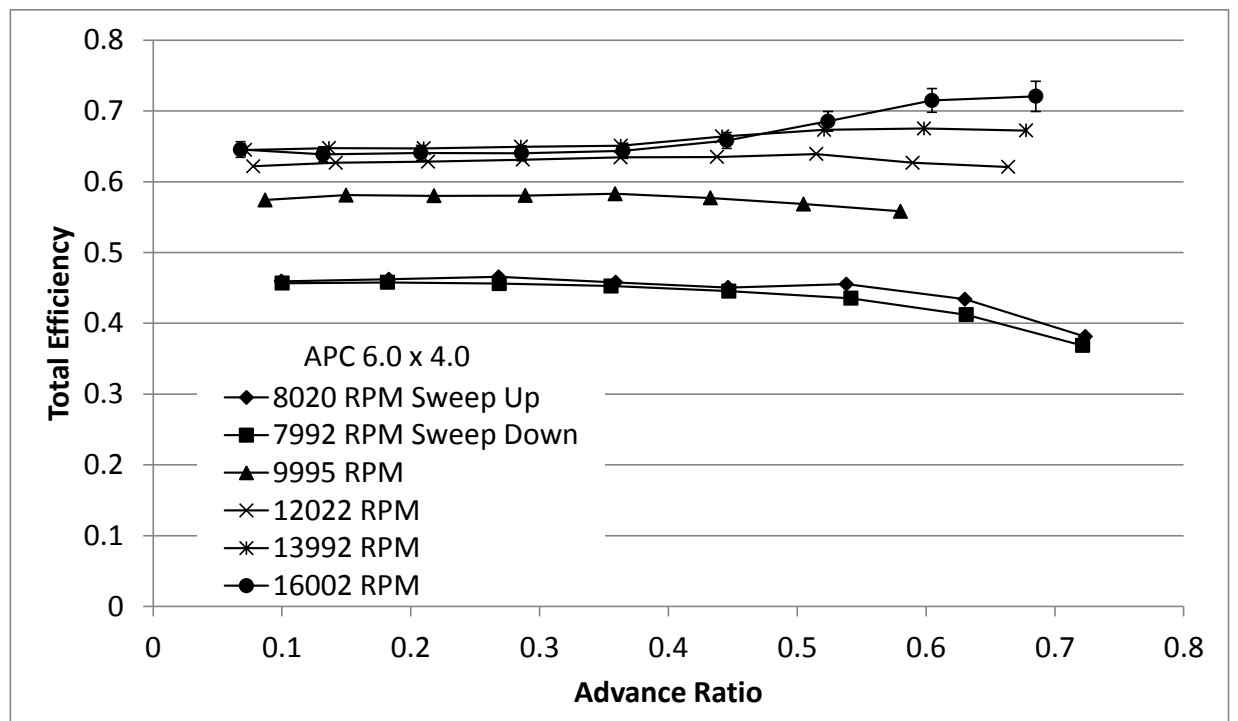

(d)

Figure 91: APC 6.0 x 4.0 Dynamic Test Results: (a) Coefficient of Thrust, (b) Coefficient of Power, (c) Propeller Efficiency, (d) Total Efficiency.

Table 178: APC 6.0 x 4.0 Dynamic Measured Values - 8020 RPM Sweep Up

\begin{tabular}{|c|c|c|c|c|c|c|c|}
\hline$n(\mathrm{RPM})$ & $Q(\mathrm{~g}-\mathrm{m})$ & $\Delta Q(\mathrm{~g}-\mathrm{m})$ & $V(\mathrm{~V})$ & $I(\mathrm{~A})$ & $T_{\text {atm }}\left({ }^{\circ} \mathrm{C}\right)$ & $P_{\text {atm }}(\mathrm{Pa})$ & $P_{\text {diff }}(\mathrm{Pa})$ \\
\hline $8.013 \mathrm{E}+03$ & $1.411 \mathrm{E}+00$ & $6.67 \mathrm{E}-02$ & $1.103 \mathrm{E}+01$ & $2.293 \mathrm{E}+00$ & $1.993 \mathrm{E}+01$ & $9.896 \mathrm{E}+04$ & $2.706 \mathrm{E}+00$ \\
\hline $8.019 \mathrm{E}+03$ & $1.415 \mathrm{E}+00$ & $6.68 \mathrm{E}-02$ & $1.103 \mathrm{E}+01$ & $2.287 \mathrm{E}+00$ & $1.979 \mathrm{E}+01$ & $9.895 \mathrm{E}+04$ & $8.624 \mathrm{E}+00$ \\
\hline $8.029 \mathrm{E}+03$ & $1.423 \mathrm{E}+00$ & $6.84 \mathrm{E}-02$ & $1.103 \mathrm{E}+01$ & $2.285 \mathrm{E}+00$ & $1.996 \mathrm{E}+01$ & $9.895 \mathrm{E}+04$ & $1.815 \mathrm{E}+01$ \\
\hline $7.991 \mathrm{E}+03$ & $1.284 \mathrm{E}+00$ & $6.66 \mathrm{E}-02$ & $1.103 \mathrm{E}+01$ & $2.085 \mathrm{E}+00$ & $2.018 \mathrm{E}+01$ & $9.894 \mathrm{E}+04$ & $3.169 \mathrm{E}+01$ \\
\hline $8.044 \mathrm{E}+03$ & $1.094 \mathrm{E}+00$ & $7.32 \mathrm{E}-02$ & $1.104 \mathrm{E}+01$ & $1.816 \mathrm{E}+00$ & $2.014 \mathrm{E}+01$ & $9.894 \mathrm{E}+04$ & $4.919 \mathrm{E}+01$ \\
\hline $8.032 \mathrm{E}+03$ & $9.531 \mathrm{E}-01$ & $7.02 \mathrm{E}-02$ & $1.105 \mathrm{E}+01$ & $1.563 \mathrm{E}+00$ & $2.017 \mathrm{E}+01$ & $9.893 \mathrm{E}+04$ & $7.085 \mathrm{E}+01$ \\
\hline $8.025 \mathrm{E}+03$ & $7.485 \mathrm{E}-01$ & $6.73 \mathrm{E}-02$ & $1.105 \mathrm{E}+01$ & $1.286 \mathrm{E}+00$ & $2.035 \mathrm{E}+01$ & $9.892 \mathrm{E}+04$ & $9.658 \mathrm{E}+01$ \\
\hline $8.008 \mathrm{E}+03$ & $4.761 \mathrm{E}-01$ & $6.54 \mathrm{E}-02$ & $1.106 \mathrm{E}+01$ & $9.285 \mathrm{E}-01$ & $2.054 \mathrm{E}+01$ & $9.892 \mathrm{E}+04$ & $1.264 \mathrm{E}+02$ \\
\hline
\end{tabular}


Table 179: APC 6.0 x 4.0 Dynamic Calculated Values - 8020 RPM Sweep Up

\begin{tabular}{|c|c|c|c|c|c|c|c|}
\hline$n(\mathrm{RPM})$ & $V_{\infty}^{\prime}(\mathrm{m} / \mathrm{s})$ & $\Delta V_{\infty}^{\prime}(\mathrm{m} / \mathrm{s})$ & $T^{\prime}(\mathrm{g})$ & $\Delta T^{\prime}(\mathrm{g})$ & $V_{\mathrm{t}}(\mathrm{m} / \mathrm{s})$ & $P_{\mathrm{P}}(\mathrm{W})$ & $\Delta P_{\mathrm{P}}(\mathrm{W})$ \\
\hline $8.013 \mathrm{E}+03$ & $2.022 \mathrm{E}+00$ & $1.25 \mathrm{E}-02$ & $1.154 \mathrm{E}+02$ & $7.43 \mathrm{E}+00$ & $4.784 \mathrm{E}+01$ & $1.161 \mathrm{E}+01$ & $5.49 \mathrm{E}-01$ \\
\hline $8.019 \mathrm{E}+03$ & $3.714 \mathrm{E}+00$ & $1.31 \mathrm{E}-02$ & $1.107 \mathrm{E}+02$ & $7.45 \mathrm{E}+00$ & $4.797 \mathrm{E}+01$ & $1.166 \mathrm{E}+01$ & $5.50 \mathrm{E}-01$ \\
\hline $8.029 \mathrm{E}+03$ & $5.454 \mathrm{E}+00$ & $1.51 \mathrm{E}-02$ & $1.021 \mathrm{E}+02$ & $7.47 \mathrm{E}+00$ & $4.820 \mathrm{E}+01$ & $1.173 \mathrm{E}+01$ & $5.64 \mathrm{E}-01$ \\
\hline $7.991 \mathrm{E}+03$ & $7.266 \mathrm{E}+00$ & $1.74 \mathrm{E}-02$ & $7.901 \mathrm{E}+01$ & $7.47 \mathrm{E}+00$ & $4.821 \mathrm{E}+01$ & $1.053 \mathrm{E}+01$ & $5.46 \mathrm{E}-01$ \\
\hline $8.044 \mathrm{E}+03$ & $9.091 \mathrm{E}+00$ & $1.94 \mathrm{E}-02$ & $6.158 \mathrm{E}+01$ & $7.41 \mathrm{E}+00$ & $4.884 \mathrm{E}+01$ & $9.037 \mathrm{E}+00$ & $6.04 \mathrm{E}-01$ \\
\hline $8.032 \mathrm{E}+03$ & $1.094 \mathrm{E}+01$ & $2.26 \mathrm{E}-02$ & $4.612 \mathrm{E}+01$ & $7.49 \mathrm{E}+00$ & $4.914 \mathrm{E}+01$ & $7.862 \mathrm{E}+00$ & 5.79E-01 \\
\hline $8.025 \mathrm{E}+03$ & $1.280 \mathrm{E}+01$ & $2.58 \mathrm{E}-02$ & $2.862 \mathrm{E}+01$ & $7.53 \mathrm{E}+00$ & $4.955 \mathrm{E}+01$ & $6.169 \mathrm{E}+00$ & 5.54E-01 \\
\hline $8.008 \mathrm{E}+03$ & $1.467 \mathrm{E}+01$ & $2.88 \mathrm{E}-02$ & $8.157 \mathrm{E}+00$ & $7.53 \mathrm{E}+00$ & $4.997 \mathrm{E}+01$ & $3.916 \mathrm{E}+00$ & $5.38 \mathrm{E}-01$ \\
\hline$P_{\mathrm{e}}(\mathrm{W})$ & $\Delta P_{\mathrm{e}}(\mathrm{W})$ & $C_{\mathrm{T}}$ & $\Delta C_{\mathrm{T}}$ & $C_{\mathrm{P}}$ & $\Delta C_{\mathrm{P}}$ & $C_{\mathrm{Q}}$ & $\Delta C_{\mathrm{Q}}$ \\
\hline $2.529 \mathrm{E}+01$ & $3.10 \mathrm{E}-01$ & $1.014 \mathrm{E}-01$ & $6.53 \mathrm{E}-03$ & $5.128 \mathrm{E}-02$ & $2.43 \mathrm{E}-03$ & $8.161 \mathrm{E}-03$ & $3.86 \mathrm{E}-04$ \\
\hline $2.522 \mathrm{E}+01$ & $3.08 \mathrm{E}-01$ & $9.709 \mathrm{E}-02$ & $6.53 \mathrm{E}-03$ & $5.133 \mathrm{E}-02$ & $2.42 \mathrm{E}-03$ & $8.170 \mathrm{E}-03$ & $3.85 \mathrm{E}-04$ \\
\hline $2.519 \mathrm{E}+01$ & $3.06 \mathrm{E}-01$ & $8.931 \mathrm{E}-02$ & $6.54 \mathrm{E}-03$ & $5.148 \mathrm{E}-02$ & $2.48 \mathrm{E}-03$ & $8.194 \mathrm{E}-03$ & 3.94E-04 \\
\hline $2.301 \mathrm{E}+01$ & $2.81 \mathrm{E}-01$ & $6.985 \mathrm{E}-02$ & $6.61 \mathrm{E}-03$ & $4.694 \mathrm{E}-02$ & $2.44 \mathrm{E}-03$ & $7.471 \mathrm{E}-03$ & $3.88 \mathrm{E}-04$ \\
\hline $2.005 \mathrm{E}+01$ & $2.45 \mathrm{E}-01$ & $5.372 \mathrm{E}-02$ & $6.47 \mathrm{E}-03$ & $3.947 \mathrm{E}-02$ & $2.64 \mathrm{E}-03$ & $6.283 \mathrm{E}-03$ & 4.20E-04 \\
\hline $1.727 \mathrm{E}+01$ & $2.17 \mathrm{E}-01$ & $4.036 \mathrm{E}-02$ & $6.55 \mathrm{E}-03$ & $3.450 \mathrm{E}-02$ & $2.54 \mathrm{E}-03$ & $5.491 \mathrm{E}-03$ & $4.05 \mathrm{E}-04$ \\
\hline $1.422 \mathrm{E}+01$ & $1.79 \mathrm{E}-01$ & $2.511 \mathrm{E}-02$ & $6.60 \mathrm{E}-03$ & $2.716 \mathrm{E}-02$ & $2.44 \mathrm{E}-03$ & $4.323 \mathrm{E}-03$ & $3.89 \mathrm{E}-04$ \\
\hline $1.027 \mathrm{E}+01$ & $1.35 \mathrm{E}-01$ & $7.191 \mathrm{E}-03$ & $6.64 \mathrm{E}-03$ & $1.736 \mathrm{E}-02$ & $2.39 \mathrm{E}-03$ & $2.763 \mathrm{E}-03$ & $3.80 \mathrm{E}-04$ \\
\hline$\rho\left(\mathrm{kg} / \mathrm{m}^{\wedge} 3\right)$ & $\eta_{\mathrm{T}}$ & $\Delta \eta_{\mathrm{T}}$ & $J$ & $\Delta J$ & $\eta_{\mathrm{P}}$ & $\Delta \eta_{\mathrm{P}}$ & $R e_{0.75}$ \\
\hline $1.176 \mathrm{E}+00$ & $4.593 \mathrm{E}-01$ & $2.24 \mathrm{E}-02$ & $9.968 \mathrm{E}-02$ & $6.18 \mathrm{E}-04$ & $1.971 \mathrm{E}-01$ & $1.58 \mathrm{E}-02$ & $3.224 \mathrm{E}+04$ \\
\hline $1.177 \mathrm{E}+00$ & $4.622 \mathrm{E}-01$ & $2.25 \mathrm{E}-02$ & $1.829 \mathrm{E}-01$ & $6.50 \mathrm{E}-04$ & $3.460 \mathrm{E}-01$ & $2.84 \mathrm{E}-02$ & $3.236 \mathrm{E}+04$ \\
\hline $1.176 \mathrm{E}+00$ & $4.656 \mathrm{E}-01$ & $2.31 \mathrm{E}-02$ & $2.683 \mathrm{E}-01$ & $7.51 \mathrm{E}-04$ & $4.655 \mathrm{E}-01$ & $4.08 \mathrm{E}-02$ & $3.248 \mathrm{E}+04$ \\
\hline $1.175 \mathrm{E}+00$ & $4.578 \mathrm{E}-01$ & $2.44 \mathrm{E}-02$ & $3.592 \mathrm{E}-01$ & $8.76 \mathrm{E}-04$ & $5.345 \mathrm{E}-01$ & $5.77 \mathrm{E}-02$ & $3.244 \mathrm{E}+04$ \\
\hline $1.175 \mathrm{E}+00$ & $4.506 \mathrm{E}-01$ & $3.06 \mathrm{E}-02$ & $4.464 \mathrm{E}-01$ & $9.73 \mathrm{E}-04$ & $6.075 \mathrm{E}-01$ & $8.37 \mathrm{E}-02$ & $3.287 \mathrm{E}+04$ \\
\hline $1.175 \mathrm{E}+00$ & $4.554 \mathrm{E}-01$ & $3.40 \mathrm{E}-02$ & $5.381 \mathrm{E}-01$ & $1.13 \mathrm{E}-03$ & $6.294 \mathrm{E}-01$ & $1.12 \mathrm{E}-01$ & $3.307 \mathrm{E}+04$ \\
\hline $1.174 \mathrm{E}+00$ & $4.340 \mathrm{E}-01$ & $3.94 \mathrm{E}-02$ & $6.302 \mathrm{E}-01$ & $1.30 \mathrm{E}-03$ & $5.826 \mathrm{E}-01$ & $1.62 \mathrm{E}-01$ & $3.330 \mathrm{E}+04$ \\
\hline $1.173 \mathrm{E}+00$ & $3.812 \mathrm{E}-01$ & $5.26 \mathrm{E}-02$ & 7.237E-01 & $1.45 \mathrm{E}-03$ & $2.997 \mathrm{E}-01$ & $2.80 \mathrm{E}-01$ & $3.354 \mathrm{E}+04$ \\
\hline
\end{tabular}


Table 180: APC 6.0 x 4.0 Dynamic Measured Values - 7992 RPM Sweep Down

\begin{tabular}{|c|c|c|c|c|c|c|c|}
\hline$n(\mathrm{RPM})$ & $Q(\mathrm{~g}-\mathrm{m})$ & $\Delta Q(\mathrm{~g}-\mathrm{m})$ & $V(\mathrm{~V})$ & $I(\mathrm{~A})$ & $T_{\text {atm }}\left({ }^{\circ} \mathrm{C}\right)$ & $P_{\text {atm }}(\mathrm{Pa})$ & $P_{\text {diff }}(\mathrm{Pa})$ \\
\hline $8.013 \mathrm{E}+03$ & $4.655 \mathrm{E}-01$ & $6.54 \mathrm{E}-02$ & $1.106 \mathrm{E}+01$ & $9.395 \mathrm{E}-01$ & $2.233 \mathrm{E}+01$ & $9.670 \mathrm{E}+04$ & $1.223 \mathrm{E}+02$ \\
\hline $7.994 \mathrm{E}+03$ & $6.910 \mathrm{E}-01$ & $6.53 \mathrm{E}-02$ & $1.105 \mathrm{E}+01$ & $1.246 \mathrm{E}+00$ & $2.257 \mathrm{E}+01$ & $9.671 \mathrm{E}+04$ & $9.330 \mathrm{E}+01$ \\
\hline $7.959 \mathrm{E}+03$ & $8.766 \mathrm{E}-01$ & $6.56 \mathrm{E}-02$ & $1.105 \mathrm{E}+01$ & $1.490 \mathrm{E}+00$ & $2.261 \mathrm{E}+01$ & $9.670 \mathrm{E}+04$ & $6.833 \mathrm{E}+01$ \\
\hline $8.032 \mathrm{E}+03$ & $1.053 \mathrm{E}+00$ & $6.77 \mathrm{E}-02$ & $1.104 \mathrm{E}+01$ & $1.767 \mathrm{E}+00$ & $2.255 \mathrm{E}+01$ & $9.670 \mathrm{E}+04$ & $4.762 \mathrm{E}+01$ \\
\hline $8.028 \mathrm{E}+03$ & $1.242 \mathrm{E}+00$ & $6.79 \mathrm{E}-02$ & $1.103 \mathrm{E}+01$ & $2.051 \mathrm{E}+00$ & $2.248 \mathrm{E}+01$ & $9.664 \mathrm{E}+04$ & $3.040 \mathrm{E}+01$ \\
\hline $7.984 \mathrm{E}+03$ & $1.354 \mathrm{E}+00$ & $6.56 \mathrm{E}-02$ & $1.103 \mathrm{E}+01$ & $2.207 \mathrm{E}+00$ & $2.264 \mathrm{E}+01$ & $9.660 \mathrm{E}+04$ & $1.742 \mathrm{E}+01$ \\
\hline $7.969 \mathrm{E}+03$ & $1.365 \mathrm{E}+00$ & $6.55 \mathrm{E}-02$ & $1.103 \mathrm{E}+01$ & $2.213 \mathrm{E}+00$ & $2.225 \mathrm{E}+01$ & $9.659 \mathrm{E}+04$ & $8.165 \mathrm{E}+00$ \\
\hline $7.956 \mathrm{E}+03$ & $1.363 \mathrm{E}+00$ & $6.57 \mathrm{E}-02$ & $1.103 \mathrm{E}+01$ & $2.211 \mathrm{E}+00$ & $2.221 \mathrm{E}+01$ & $9.656 \mathrm{E}+04$ & $2.602 \mathrm{E}+00$ \\
\hline
\end{tabular}


Table 181: APC 6.0 x 4.0 Dynamic Calculated Values - 7992 RPM Sweep Down

\begin{tabular}{|c|c|c|c|c|c|c|c|}
\hline$n(\mathrm{RPM})$ & $V_{\infty}^{\prime}(\mathrm{m} / \mathrm{s})$ & $\Delta V_{\infty}^{\prime}(\mathrm{m} / \mathrm{s})$ & $T^{\prime}(\mathrm{g})$ & $\Delta T^{\prime}(\mathrm{g})$ & $V_{\mathrm{t}}(\mathrm{m} / \mathrm{s})$ & $P_{\mathrm{P}}(\mathrm{W})$ & $\Delta P_{\mathrm{P}}(\mathrm{W})$ \\
\hline $8.013 \mathrm{E}+03$ & $1.464 \mathrm{E}+01$ & $2.90 \mathrm{E}-02$ & $9.671 \mathrm{E}+00$ & $7.52 \mathrm{E}+00$ & $4.999 \mathrm{E}+01$ & $3.831 \mathrm{E}+00$ & $5.38 \mathrm{E}-01$ \\
\hline $7.994 \mathrm{E}+03$ & $1.278 \mathrm{E}+01$ & $2.57 \mathrm{E}-02$ & $2.694 \mathrm{E}+01$ & $7.56 \mathrm{E}+00$ & $4.937 \mathrm{E}+01$ & $5.673 \mathrm{E}+00$ & $5.36 \mathrm{E}-01$ \\
\hline $7.959 \mathrm{E}+03$ & $1.092 \mathrm{E}+01$ & $2.26 \mathrm{E}-02$ & $4.154 \mathrm{E}+01$ & $7.48 \mathrm{E}+00$ & $4.871 \mathrm{E}+01$ & $7.165 \mathrm{E}+00$ & $5.36 \mathrm{E}-01$ \\
\hline $8.032 \mathrm{E}+03$ & $9.086 \mathrm{E}+00$ & $1.95 \mathrm{E}-02$ & $5.839 \mathrm{E}+01$ & $7.41 \mathrm{E}+00$ & $4.876 \mathrm{E}+01$ & $8.687 \mathrm{E}+00$ & $5.58 \mathrm{E}-01$ \\
\hline $8.028 \mathrm{E}+03$ & $7.227 \mathrm{E}+00$ & $1.70 \mathrm{E}-02$ & $7.785 \mathrm{E}+01$ & $7.38 \mathrm{E}+00$ & $4.843 \mathrm{E}+01$ & $1.024 \mathrm{E}+01$ & $5.60 \mathrm{E}-01$ \\
\hline $7.984 \mathrm{E}+03$ & $5.432 \mathrm{E}+00$ & $1.50 \mathrm{E}-02$ & $9.675 \mathrm{E}+01$ & $7.42 \mathrm{E}+00$ & $4.793 \mathrm{E}+01$ & $1.110 \mathrm{E}+01$ & $5.38 \mathrm{E}-01$ \\
\hline $7.969 \mathrm{E}+03$ & $3.672 \mathrm{E}+00$ & $1.31 \mathrm{E}-02$ & $1.060 \mathrm{E}+02$ & $7.43 \mathrm{E}+00$ & $4.768 \mathrm{E}+01$ & $1.117 \mathrm{E}+01$ & 5.36E-01 \\
\hline $7.956 \mathrm{E}+03$ & $2.017 \mathrm{E}+00$ & $1.23 \mathrm{E}-02$ & $1.082 \mathrm{E}+02$ & $7.43 \mathrm{E}+00$ & $4.750 \mathrm{E}+01$ & $1.113 \mathrm{E}+01$ & $5.37 \mathrm{E}-01$ \\
\hline$P_{\mathrm{e}}(\mathrm{W})$ & $\Delta P_{\mathrm{e}}(\mathrm{W})$ & $C_{\mathrm{T}}$ & $\Delta C_{\mathrm{T}}$ & $C_{\mathrm{P}}$ & $\Delta C_{\mathrm{P}}$ & $C_{\mathrm{Q}}$ & $\Delta C_{\mathrm{Q}}$ \\
\hline $1.039 \mathrm{E}+01$ & $1.34 \mathrm{E}-01$ & $8.762 \mathrm{E}-03$ & $6.82 \mathrm{E}-03$ & $1.745 \mathrm{E}-02$ & $2.45 \mathrm{E}-03$ & $2.777 \mathrm{E}-03$ & $3.90 \mathrm{E}-04$ \\
\hline $1.377 \mathrm{E}+01$ & $1.75 \mathrm{E}-01$ & $2.454 \mathrm{E}-02$ & $6.89 \mathrm{E}-03$ & $2.604 \mathrm{E}-02$ & $2.46 \mathrm{E}-03$ & $4.144 \mathrm{E}-03$ & $3.92 \mathrm{E}-04$ \\
\hline $1.646 \mathrm{E}+01$ & $2.05 \mathrm{E}-01$ & $3.819 \mathrm{E}-02$ & $6.87 \mathrm{E}-03$ & $3.333 \mathrm{E}-02$ & $2.50 \mathrm{E}-03$ & $5.305 \mathrm{E}-03$ & $3.97 \mathrm{E}-04$ \\
\hline $1.950 \mathrm{E}+01$ & $2.40 \mathrm{E}-01$ & $5.270 \mathrm{E}-02$ & $6.69 \mathrm{E}-03$ & $3.932 \mathrm{E}-02$ & $2.53 \mathrm{E}-03$ & $6.258 \mathrm{E}-03$ & $4.02 \mathrm{E}-04$ \\
\hline $2.262 \mathrm{E}+01$ & $2.76 \mathrm{E}-01$ & $7.036 \mathrm{E}-02$ & $6.67 \mathrm{E}-03$ & $4.643 \mathrm{E}-02$ & $2.54 \mathrm{E}-03$ & $7.389 \mathrm{E}-03$ & 4.04E-04 \\
\hline $2.433 \mathrm{E}+01$ & $2.95 \mathrm{E}-01$ & $8.848 \mathrm{E}-02$ & $6.79 \mathrm{E}-03$ & $5.121 \mathrm{E}-02$ & $2.48 \mathrm{E}-03$ & $8.150 \mathrm{E}-03$ & $3.95 \mathrm{E}-04$ \\
\hline $2.440 \mathrm{E}+01$ & $2.95 \mathrm{E}-01$ & $9.716 \mathrm{E}-02$ & $6.81 \mathrm{E}-03$ & $5.177 \mathrm{E}-02$ & $2.49 \mathrm{E}-03$ & $8.239 \mathrm{E}-03$ & $3.96 \mathrm{E}-04$ \\
\hline $2.438 \mathrm{E}+01$ & $2.94 \mathrm{E}-01$ & $9.956 \mathrm{E}-02$ & $6.83 \mathrm{E}-03$ & $5.187 \mathrm{E}-02$ & $2.50 \mathrm{E}-03$ & $8.255 \mathrm{E}-03$ & $3.98 \mathrm{E}-04$ \\
\hline$\rho\left(\mathrm{kg} / \mathrm{m}^{\wedge} 3\right)$ & $\eta_{\mathrm{T}}$ & $\Delta \eta_{\mathrm{T}}$ & $J$ & $\Delta J$ & $\eta_{\mathrm{P}}$ & $\Delta \eta_{\mathrm{P}}$ & $R e_{0.75}$ \\
\hline $1.140 \mathrm{E}+00$ & $3.686 \mathrm{E}-01$ & $5.20 \mathrm{E}-02$ & $7.216 \mathrm{E}-01$ & $1.46 \mathrm{E}-03$ & $3.624 \mathrm{E}-01$ & $2.86 \mathrm{E}-01$ & $3.246 \mathrm{E}+04$ \\
\hline $1.139 \mathrm{E}+00$ & $4.120 \mathrm{E}-01$ & $3.93 \mathrm{E}-02$ & $6.313 \mathrm{E}-01$ & $1.30 \mathrm{E}-03$ & 5.949E-01 & $1.76 \mathrm{E}-01$ & $3.201 \mathrm{E}+04$ \\
\hline $1.139 \mathrm{E}+00$ & 4.353E-01 & $3.30 \mathrm{E}-02$ & $5.417 \mathrm{E}-01$ & $1.15 \mathrm{E}-03$ & $6.206 \mathrm{E}-01$ & $1.21 \mathrm{E}-01$ & $3.158 \mathrm{E}+04$ \\
\hline $1.139 \mathrm{E}+00$ & $4.454 \mathrm{E}-01$ & $2.91 \mathrm{E}-02$ & $4.469 \mathrm{E}-01$ & $9.80 \mathrm{E}-04$ & $5.989 \mathrm{E}-01$ & $8.52 \mathrm{E}-02$ & $3.162 \mathrm{E}+04$ \\
\hline $1.139 \mathrm{E}+00$ & $4.526 \mathrm{E}-01$ & $2.54 \mathrm{E}-02$ & $3.556 \mathrm{E}-01$ & $8.48 \mathrm{E}-04$ & $5.389 \mathrm{E}-01$ & $5.90 \mathrm{E}-02$ & $3.139 \mathrm{E}+04$ \\
\hline $1.138 \mathrm{E}+00$ & $4.561 \mathrm{E}-01$ & $2.28 \mathrm{E}-02$ & $2.687 \mathrm{E}-01$ & $7.51 \mathrm{E}-04$ & $4.643 \mathrm{E}-01$ & $4.22 \mathrm{E}-02$ & $3.103 \mathrm{E}+04$ \\
\hline $1.139 \mathrm{E}+00$ & $4.577 \mathrm{E}-01$ & $2.27 \mathrm{E}-02$ & $1.820 \mathrm{E}-01$ & $6.54 \mathrm{E}-04$ & $3.416 \mathrm{E}-01$ & $2.91 \mathrm{E}-02$ & $3.093 \mathrm{E}+04$ \\
\hline $1.139 \mathrm{E}+00$ & $4.566 \mathrm{E}-01$ & $2.27 \mathrm{E}-02$ & $1.001 \mathrm{E}-01$ & $6.13 \mathrm{E}-04$ & $1.922 \mathrm{E}-01$ & $1.62 \mathrm{E}-02$ & $3.082 \mathrm{E}+04$ \\
\hline
\end{tabular}


Table 182: APC 6.0 x 4.0 Dynamic Measured Values - 9995 RPM

\begin{tabular}{|c|c|c|c|c|c|c|c|}
\hline$n(\mathrm{RPM})$ & $Q(\mathrm{~g}-\mathrm{m})$ & $\Delta Q(\mathrm{~g}-\mathrm{m})$ & $V(\mathrm{~V})$ & $I(\mathrm{~A})$ & $T_{\text {atm }}\left({ }^{\circ} \mathrm{C}\right)$ & $P_{\text {atm }}(\mathrm{Pa})$ & $P_{\text {diff }}(\mathrm{Pa})$ \\
\hline $9.932 \mathrm{E}+03$ & $2.145 \mathrm{E}+00$ & $6.40 \mathrm{E}-02$ & $1.102 \mathrm{E}+01$ & $3.457 \mathrm{E}+00$ & $2.195 \mathrm{E}+01$ & $9.799 \mathrm{E}+04$ & $3.180 \mathrm{E}+00$ \\
\hline $9.994 \mathrm{E}+03$ & $2.198 \mathrm{E}+00$ & $6.28 \mathrm{E}-02$ & $1.102 \mathrm{E}+01$ & $3.523 \mathrm{E}+00$ & $2.190 \mathrm{E}+01$ & $9.800 \mathrm{E}+04$ & $8.974 \mathrm{E}+00$ \\
\hline $9.993 \mathrm{E}+03$ & $2.196 \mathrm{E}+00$ & $6.32 \mathrm{E}-02$ & $1.102 \mathrm{E}+01$ & $3.526 \mathrm{E}+00$ & $2.199 \mathrm{E}+01$ & $9.799 \mathrm{E}+04$ & $1.850 \mathrm{E}+01$ \\
\hline $9.994 \mathrm{E}+03$ & $2.193 \mathrm{E}+00$ & $6.28 \mathrm{E}-02$ & $1.102 \mathrm{E}+01$ & $3.519 \mathrm{E}+00$ & $2.214 \mathrm{E}+01$ & $9.800 \mathrm{E}+04$ & $3.195 \mathrm{E}+01$ \\
\hline $1.002 \mathrm{E}+04$ & $2.131 \mathrm{E}+00$ & $6.45 \mathrm{E}-02$ & $1.102 \mathrm{E}+01$ & $3.413 \mathrm{E}+00$ & $2.226 \mathrm{E}+01$ & $9.799 \mathrm{E}+04$ & $4.896 \mathrm{E}+01$ \\
\hline $1.000 \mathrm{E}+04$ & $1.826 \mathrm{E}+00$ & $6.04 \mathrm{E}-02$ & $1.103 \mathrm{E}+01$ & $2.945 \mathrm{E}+00$ & $2.237 \mathrm{E}+01$ & $9.799 \mathrm{E}+04$ & $7.033 \mathrm{E}+01$ \\
\hline $1.003 \mathrm{E}+04$ & $1.566 \mathrm{E}+00$ & $6.18 \mathrm{E}-02$ & $1.104 \mathrm{E}+01$ & $2.570 \mathrm{E}+00$ & $2.244 \mathrm{E}+01$ & $9.800 \mathrm{E}+04$ & $9.583 \mathrm{E}+01$ \\
\hline $9.995 \mathrm{E}+03$ & $1.318 \mathrm{E}+00$ & $5.96 \mathrm{E}-02$ & $1.105 \mathrm{E}+01$ & $2.193 \mathrm{E}+00$ & $2.240 \mathrm{E}+01$ & $9.800 \mathrm{E}+04$ & $1.252 \mathrm{E}+02$ \\
\hline
\end{tabular}


Table 183: APC 6.0 x 4.0 Dynamic Calculated Values - 9995 RPM

\begin{tabular}{|c|c|c|c|c|c|c|c|}
\hline$n(\mathrm{RPM})$ & $V_{\infty}^{\prime}(\mathrm{m} / \mathrm{s})$ & $\Delta V_{\infty}^{\prime}(\mathrm{m} / \mathrm{s})$ & $T^{\prime}(\mathrm{g})$ & $\Delta T^{\prime}(\mathrm{g})$ & $V_{\mathrm{t}}(\mathrm{m} / \mathrm{s})$ & $P_{\mathrm{P}}(\mathrm{W})$ & $\Delta P_{\mathrm{P}}(\mathrm{W})$ \\
\hline $9.932 \mathrm{E}+03$ & $2.189 \mathrm{E}+00$ & $1.39 \mathrm{E}-02$ & $1.800 \mathrm{E}+02$ & $1.01 \mathrm{E}+01$ & $5.929 \mathrm{E}+01$ & $2.188 \mathrm{E}+01$ & $6.53 \mathrm{E}-01$ \\
\hline $9.994 \mathrm{E}+03$ & $3.788 \mathrm{E}+00$ & $1.50 \mathrm{E}-02$ & $1.775 \mathrm{E}+02$ & $1.01 \mathrm{E}+01$ & $5.973 \mathrm{E}+01$ & $2.255 \mathrm{E}+01$ & $6.45 \mathrm{E}-01$ \\
\hline $9.993 \mathrm{E}+03$ & $5.517 \mathrm{E}+00$ & $1.68 \mathrm{E}-02$ & $1.662 \mathrm{E}+02$ & $1.01 \mathrm{E}+01$ & $5.986 \mathrm{E}+01$ & $2.253 \mathrm{E}+01$ & $6.49 \mathrm{E}-01$ \\
\hline $9.994 \mathrm{E}+03$ & $7.309 \mathrm{E}+00$ & $1.89 \mathrm{E}-02$ & $1.565 \mathrm{E}+02$ & $1.00 \mathrm{E}+01$ & $6.006 \mathrm{E}+01$ & $2.251 \mathrm{E}+01$ & $6.45 \mathrm{E}-01$ \\
\hline $1.002 \mathrm{E}+04$ & $9.098 \mathrm{E}+00$ & $2.18 \mathrm{E}-02$ & $1.374 \mathrm{E}+02$ & $1.01 \mathrm{E}+01$ & $6.045 \mathrm{E}+01$ & $2.193 \mathrm{E}+01$ & $6.64 \mathrm{E}-01$ \\
\hline $1.000 \mathrm{E}+04$ & $1.095 \mathrm{E}+01$ & 2.39E-02 & $1.050 \mathrm{E}+02$ & $1.02 \mathrm{E}+01$ & $6.065 \mathrm{E}+01$ & $1.875 \mathrm{E}+01$ & $6.20 \mathrm{E}-01$ \\
\hline $1.003 \mathrm{E}+04$ & $1.282 \mathrm{E}+01$ & $2.65 \mathrm{E}-02$ & $7.960 \mathrm{E}+01$ & $1.01 \mathrm{E}+01$ & $6.120 \mathrm{E}+01$ & $1.613 \mathrm{E}+01$ & $6.37 \mathrm{E}-01$ \\
\hline $9.995 \mathrm{E}+03$ & $1.468 \mathrm{E}+01$ & $2.96 \mathrm{E}-02$ & $5.967 \mathrm{E}+01$ & $1.01 \mathrm{E}+01$ & $6.140 \mathrm{E}+01$ & $1.353 \mathrm{E}+01$ & $6.12 \mathrm{E}-01$ \\
\hline$P_{\mathrm{e}}(\mathrm{W})$ & $\Delta P_{\mathrm{e}}(\mathrm{W})$ & $C_{\mathrm{T}}$ & $\Delta C_{\mathrm{T}}$ & $C_{\mathrm{P}}$ & $\Delta C_{\mathrm{P}}$ & $C_{\mathrm{Q}}$ & $\Delta C_{\mathrm{Q}}$ \\
\hline $3.809 \mathrm{E}+01$ & 4.37E-01 & $1.046 \mathrm{E}-01$ & $5.86 \mathrm{E}-03$ & $5.156 \mathrm{E}-02$ & $1.54 \mathrm{E}-03$ & $8.206 \mathrm{E}-03$ & $2.45 \mathrm{E}-04$ \\
\hline $3.881 \mathrm{E}+01$ & $4.45 \mathrm{E}-01$ & $1.019 \mathrm{E}-01$ & $5.79 \mathrm{E}-03$ & $5.218 \mathrm{E}-02$ & $1.49 \mathrm{E}-03$ & $8.305 \mathrm{E}-03$ & 2.38E-04 \\
\hline $3.884 \mathrm{E}+01$ & 4.47E-01 & $9.543 \mathrm{E}-02$ & $5.82 \mathrm{E}-03$ & $5.216 \mathrm{E}-02$ & $1.50 \mathrm{E}-03$ & 8.301E-03 & 2.39E-04 \\
\hline $3.876 \mathrm{E}+01$ & 4.46E-01 & $8.988 \mathrm{E}-02$ & $5.76 \mathrm{E}-03$ & $5.211 \mathrm{E}-02$ & $1.49 \mathrm{E}-03$ & $8.293 \mathrm{E}-03$ & $2.38 \mathrm{E}-04$ \\
\hline $3.761 \mathrm{E}+01$ & 4.30E-01 & 7.857E-02 & $5.75 \mathrm{E}-03$ & $5.041 \mathrm{E}-02$ & $1.53 \mathrm{E}-03$ & $8.024 \mathrm{E}-03$ & $2.43 \mathrm{E}-04$ \\
\hline $3.249 \mathrm{E}+01$ & $3.68 \mathrm{E}-01$ & $6.029 \mathrm{E}-02$ & $5.85 \mathrm{E}-03$ & 4.337E-02 & $1.44 \mathrm{E}-03$ & $6.902 \mathrm{E}-03$ & $2.28 \mathrm{E}-04$ \\
\hline $2.838 \mathrm{E}+01$ & 3.22E-01 & $4.542 \mathrm{E}-02$ & $5.75 \mathrm{E}-03$ & $3.696 \mathrm{E}-02$ & $1.46 \mathrm{E}-03$ & $5.883 \mathrm{E}-03$ & 2.32E-04 \\
\hline $2.424 \mathrm{E}+01$ & $2.78 \mathrm{E}-01$ & $3.430 \mathrm{E}-02$ & $5.81 \mathrm{E}-03$ & $3.134 \mathrm{E}-02$ & $1.42 \mathrm{E}-03$ & $4.988 \mathrm{E}-03$ & $2.26 \mathrm{E}-04$ \\
\hline$\rho\left(\mathrm{kg} / \mathrm{m}^{\wedge} 3\right)$ & $\eta_{\mathrm{T}}$ & $\Delta \eta_{\mathrm{T}}$ & $J$ & $\Delta J$ & $\eta_{\mathrm{P}}$ & $\Delta \eta_{\mathrm{P}}$ & $\operatorname{Re}_{0.75}$ \\
\hline $1.157 \mathrm{E}+00$ & $5.742 \mathrm{E}-01$ & $1.84 \mathrm{E}-02$ & 8.706E-02 & $5.54 \mathrm{E}-04$ & $1.766 \mathrm{E}-01$ & $1.13 \mathrm{E}-02$ & $3.909 \mathrm{E}+04$ \\
\hline $1.157 \mathrm{E}+00$ & $5.811 \mathrm{E}-01$ & $1.79 \mathrm{E}-02$ & $1.497 \mathrm{E}-01$ & $5.96 \mathrm{E}-04$ & $2.924 \mathrm{E}-01$ & $1.86 \mathrm{E}-02$ & $3.940 \mathrm{E}+04$ \\
\hline $1.157 \mathrm{E}+00$ & $5.801 \mathrm{E}-01$ & $1.80 \mathrm{E}-02$ & $2.181 \mathrm{E}-01$ & $6.69 \mathrm{E}-04$ & $3.990 \mathrm{E}-01$ & 2.69E-02 & $3.946 \mathrm{E}+04$ \\
\hline $1.156 \mathrm{E}+00$ & $5.806 \mathrm{E}-01$ & $1.79 \mathrm{E}-02$ & $2.889 \mathrm{E}-01$ & $7.54 \mathrm{E}-04$ & $4.983 \mathrm{E}-01$ & $3.50 \mathrm{E}-02$ & $3.956 \mathrm{E}+04$ \\
\hline $1.156 \mathrm{E}+00$ & $5.830 \mathrm{E}-01$ & $1.89 \mathrm{E}-02$ & $3.587 \mathrm{E}-01$ & 8.67E-04 & $5.591 \mathrm{E}-01$ & $4.43 \mathrm{E}-02$ & $3.979 \mathrm{E}+04$ \\
\hline $1.155 \mathrm{E}+00$ & $5.772 \mathrm{E}-01$ & $2.02 \mathrm{E}-02$ & 4.327E-01 & $9.55 \mathrm{E}-04$ & $6.015 \mathrm{E}-01$ & 6.17E-02 & $3.989 \mathrm{E}+04$ \\
\hline $1.155 \mathrm{E}+00$ & $5.685 \mathrm{E}-01$ & $2.34 \mathrm{E}-02$ & $5.049 \mathrm{E}-01$ & $1.06 \mathrm{E}-03$ & $6.205 \mathrm{E}-01$ & $8.23 \mathrm{E}-02$ & $4.024 \mathrm{E}+04$ \\
\hline $1.155 \mathrm{E}+00$ & $5.582 \mathrm{E}-01$ & $2.60 \mathrm{E}-02$ & $5.802 \mathrm{E}-01$ & $1.19 \mathrm{E}-03$ & $6.348 \mathrm{E}-01$ & $1.11 \mathrm{E}-01$ & $4.038 \mathrm{E}+04$ \\
\hline
\end{tabular}


Table 184: APC 6.0 x 4.0 Dynamic Measured Values - 12022 RPM

\begin{tabular}{|c|c|c|c|c|c|c|c|}
\hline$n(\mathrm{RPM})$ & $Q(\mathrm{~g}-\mathrm{m})$ & $\Delta Q(\mathrm{~g}-\mathrm{m})$ & $V(\mathrm{~V})$ & $I(\mathrm{~A})$ & $T_{\text {atm }}\left({ }^{\circ} \mathrm{C}\right)$ & $P_{\text {atm }}(\mathrm{Pa})$ & $P_{\text {diff }}(\mathrm{Pa})$ \\
\hline $1.203 \mathrm{E}+04$ & $3.172 \mathrm{E}+00$ & $8.14 \mathrm{E}-02$ & $1.095 \mathrm{E}+01$ & $5.755 \mathrm{E}+00$ & $2.189 \mathrm{E}+01$ & $9.799 \mathrm{E}+04$ & $3.793 \mathrm{E}+00$ \\
\hline $1.203 \mathrm{E}+04$ & $3.221 \mathrm{E}+00$ & $8.39 \mathrm{E}-02$ & $1.095 \mathrm{E}+01$ & $5.796 \mathrm{E}+00$ & $2.181 \mathrm{E}+01$ & $9.799 \mathrm{E}+04$ & $1.174 \mathrm{E}+01$ \\
\hline $1.201 \mathrm{E}+04$ & $3.240 \mathrm{E}+00$ & $8.09 \mathrm{E}-02$ & $1.095 \mathrm{E}+01$ & $5.809 \mathrm{E}+00$ & $2.202 \mathrm{E}+01$ & $9.800 \mathrm{E}+04$ & $2.567 \mathrm{E}+01$ \\
\hline $1.202 \mathrm{E}+04$ & $3.244 \mathrm{E}+00$ & $8.29 \mathrm{E}-02$ & $1.095 \mathrm{E}+01$ & $5.795 \mathrm{E}+00$ & $2.216 \mathrm{E}+01$ & $9.799 \mathrm{E}+04$ & $4.569 \mathrm{E}+01$ \\
\hline $1.198 \mathrm{E}+04$ & $3.197 \mathrm{E}+00$ & $7.44 \mathrm{E}-02$ & $1.095 \mathrm{E}+01$ & $5.660 \mathrm{E}+00$ & $2.227 \mathrm{E}+01$ & $9.799 \mathrm{E}+04$ & $7.170 \mathrm{E}+01$ \\
\hline $1.203 \mathrm{E}+04$ & $2.850 \mathrm{E}+00$ & $8.11 \mathrm{E}-02$ & $1.097 \mathrm{E}+01$ & $5.054 \mathrm{E}+00$ & $2.237 \mathrm{E}+01$ & $9.799 \mathrm{E}+04$ & $1.042 \mathrm{E}+02$ \\
\hline $1.200 \mathrm{E}+04$ & $2.383 \mathrm{E}+00$ & $7.29 \mathrm{E}-02$ & $1.100 \mathrm{E}+01$ & $4.180 \mathrm{E}+00$ & $2.239 \mathrm{E}+01$ & $9.800 \mathrm{E}+04$ & $1.427 \mathrm{E}+02$ \\
\hline $1.203 \mathrm{E}+04$ & $1.948 \mathrm{E}+00$ & $7.40 \mathrm{E}-02$ & $1.102 \mathrm{E}+01$ & $3.485 \mathrm{E}+00$ & $2.246 \mathrm{E}+01$ & $9.800 \mathrm{E}+04$ & $1.873 \mathrm{E}+02$ \\
\hline $1.205 \mathrm{E}+04$ & $1.562 \mathrm{E}+00$ & $6.78 \mathrm{E}-02$ & $1.104 \mathrm{E}+01$ & $2.821 \mathrm{E}+00$ & $2.246 \mathrm{E}+01$ & $9.799 \mathrm{E}+04$ & $2.375 \mathrm{E}+02$ \\
\hline
\end{tabular}


Table 185: APC 6.0 x 4.0 Dynamic Calculated Values - 12022 RPM

\begin{tabular}{|c|c|c|c|c|c|c|c|}
\hline$n(\mathrm{RPM})$ & $V_{\infty}^{\prime}(\mathrm{m} / \mathrm{s})$ & $\Delta V_{\infty}^{\prime}(\mathrm{m} / \mathrm{s})$ & $T^{\prime}(\mathrm{g})$ & $\Delta T^{\prime}(\mathrm{g})$ & $V_{\mathrm{t}}(\mathrm{m} / \mathrm{s})$ & $P_{\mathrm{P}}(\mathrm{W})$ & $\Delta P_{\mathrm{P}}(\mathrm{W})$ \\
\hline $1.203 \mathrm{E}+04$ & $2.367 \mathrm{E}+00$ & $1.55 \mathrm{E}-02$ & $2.764 \mathrm{E}+02$ & $9.03 \mathrm{E}+00$ & $7.180 \mathrm{E}+01$ & $3.919 \mathrm{E}+01$ & $1.01 \mathrm{E}+00$ \\
\hline $1.203 \mathrm{E}+04$ & $4.319 \mathrm{E}+00$ & $1.67 \mathrm{E}-02$ & $2.639 \mathrm{E}+02$ & $9.05 \mathrm{E}+00$ & $7.187 \mathrm{E}+01$ & $3.978 \mathrm{E}+01$ & $1.04 \mathrm{E}+00$ \\
\hline $1.201 \mathrm{E}+04$ & $6.491 \mathrm{E}+00$ & $1.91 \mathrm{E}-02$ & $2.511 \mathrm{E}+02$ & $9.08 \mathrm{E}+00$ & $7.195 \mathrm{E}+01$ & $3.997 \mathrm{E}+01$ & $9.98 \mathrm{E}-01$ \\
\hline $1.202 \mathrm{E}+04$ & $8.735 \mathrm{E}+00$ & $2.28 \mathrm{E}-02$ & $2.371 \mathrm{E}+02$ & $9.12 \mathrm{E}+00$ & $7.225 \mathrm{E}+01$ & $4.006 \mathrm{E}+01$ & $1.02 \mathrm{E}+00$ \\
\hline $1.198 \mathrm{E}+04$ & $1.101 \mathrm{E}+01$ & $2.64 \mathrm{E}-02$ & $2.112 \mathrm{E}+02$ & $9.15 \mathrm{E}+00$ & $7.231 \mathrm{E}+01$ & $3.934 \mathrm{E}+01$ & $9.15 \mathrm{E}-01$ \\
\hline $1.203 E+04$ & $1.333 \mathrm{E}+01$ & $2.86 \mathrm{E}-02$ & $1.672 \mathrm{E}+02$ & $9.12 \mathrm{E}+00$ & $7.298 \mathrm{E}+01$ & $3.521 \mathrm{E}+01$ & $1.00 \mathrm{E}+00$ \\
\hline $1.200 \mathrm{E}+04$ & $1.565 \mathrm{E}+01$ & $3.15 \mathrm{E}-02$ & $1.207 \mathrm{E}+02$ & $9.10 \mathrm{E}+00$ & $7.330 \mathrm{E}+01$ & $2.938 \mathrm{E}+01$ & 8.99E-01 \\
\hline $1.203 \mathrm{E}+04$ & $1.796 \mathrm{E}+01$ & $3.53 \mathrm{E}-02$ & $8.953 \mathrm{E}+01$ & $9.11 \mathrm{E}+00$ & $7.398 \mathrm{E}+01$ & $2.407 \mathrm{E}+01$ & $9.14 \mathrm{E}-01$ \\
\hline $1.205 \mathrm{E}+04$ & $2.025 \mathrm{E}+01$ & $3.88 \mathrm{E}-02$ & $5.843 \mathrm{E}+01$ & $9.12 \mathrm{E}+00$ & $7.467 \mathrm{E}+01$ & $1.933 \mathrm{E}+01$ & 8.39E-01 \\
\hline$P_{\mathrm{e}}(\mathrm{W})$ & $\Delta P_{\mathrm{e}}(\mathrm{W})$ & $C_{\mathrm{T}}$ & $\Delta C_{\mathrm{T}}$ & $C_{\mathrm{P}}$ & $\Delta C_{\mathrm{P}}$ & $C_{\mathrm{Q}}$ & $\Delta C_{\mathrm{Q}}$ \\
\hline $6.303 \mathrm{E}+01$ & $7.22 \mathrm{E}-01$ & $1.095 \mathrm{E}-01$ & $3.58 \mathrm{E}-03$ & $5.198 \mathrm{E}-02$ & $1.34 \mathrm{E}-03$ & $8.274 \mathrm{E}-03$ & $2.13 \mathrm{E}-04$ \\
\hline $6.347 \mathrm{E}+01$ & $7.14 \mathrm{E}-01$ & $1.046 \mathrm{E}-01$ & $3.59 \mathrm{E}-03$ & $5.280 \mathrm{E}-02$ & $1.38 \mathrm{E}-03$ & $8.403 \mathrm{E}-03$ & $2.19 \mathrm{E}-04$ \\
\hline $6.361 \mathrm{E}+01$ & $7.21 \mathrm{E}-01$ & $9.976 \mathrm{E}-02$ & $3.61 \mathrm{E}-03$ & $5.325 \mathrm{E}-02$ & $1.33 \mathrm{E}-03$ & $8.476 \mathrm{E}-03$ & $2.12 \mathrm{E}-04$ \\
\hline $6.346 \mathrm{E}+01$ & $7.15 \mathrm{E}-01$ & $9.411 \mathrm{E}-02$ & $3.62 \mathrm{E}-03$ & $5.326 \mathrm{E}-02$ & $1.36 \mathrm{E}-03$ & $8.476 \mathrm{E}-03$ & $2.17 \mathrm{E}-04$ \\
\hline $6.201 \mathrm{E}+01$ & 7.04E-01 & 8.442E-02 & $3.66 \mathrm{E}-03$ & $5.287 \mathrm{E}-02$ & $1.23 \mathrm{E}-03$ & $8.415 \mathrm{E}-03$ & $1.96 \mathrm{E}-04$ \\
\hline $5.545 \mathrm{E}+01$ & $6.42 \mathrm{E}-01$ & $6.634 \mathrm{E}-02$ & $3.62 \mathrm{E}-03$ & $4.678 \mathrm{E}-02$ & $1.33 \mathrm{E}-03$ & $7.445 \mathrm{E}-03$ & $2.12 \mathrm{E}-04$ \\
\hline $4.597 \mathrm{E}+01$ & $5.27 \mathrm{E}-01$ & $4.807 \mathrm{E}-02$ & $3.62 \mathrm{E}-03$ & $3.928 \mathrm{E}-02$ & $1.20 \mathrm{E}-03$ & $6.252 \mathrm{E}-03$ & $1.91 \mathrm{E}-04$ \\
\hline $3.839 \mathrm{E}+01$ & $4.35 \mathrm{E}-01$ & $3.552 \mathrm{E}-02$ & $3.61 \mathrm{E}-03$ & $3.196 \mathrm{E}-02$ & $1.21 \mathrm{E}-03$ & 5.087E-03 & $1.93 \mathrm{E}-04$ \\
\hline $3.113 \mathrm{E}+01$ & $3.52 \mathrm{E}-01$ & $2.312 \mathrm{E}-02$ & $3.61 \mathrm{E}-03$ & $2.556 \mathrm{E}-02$ & $1.11 \mathrm{E}-03$ & $4.068 \mathrm{E}-03$ & $1.77 \mathrm{E}-04$ \\
\hline$\rho\left(\mathrm{kg} / \mathrm{m}^{\wedge} 3\right)$ & $\eta_{\mathrm{T}}$ & $\Delta \eta_{\mathrm{T}}$ & $J$ & $\Delta J$ & $\eta_{\mathrm{P}}$ & $\Delta \eta_{\mathrm{P}}$ & $R e_{0.75}$ \\
\hline $1.157 \mathrm{E}+00$ & $6.219 \mathrm{E}-01$ & $1.75 \mathrm{E}-02$ & 7.772E-02 & $5.09 \mathrm{E}-04$ & $1.637 \mathrm{E}-01$ & $6.89 \mathrm{E}-03$ & $4.736 \mathrm{E}+04$ \\
\hline $1.157 \mathrm{E}+00$ & $6.268 \mathrm{E}-01$ & $1.78 \mathrm{E}-02$ & $1.419 \mathrm{E}-01$ & $5.51 \mathrm{E}-04$ & $2.810 \mathrm{E}-01$ & $1.22 \mathrm{E}-02$ & $4.743 \mathrm{E}+04$ \\
\hline $1.157 \mathrm{E}+00$ & $6.284 \mathrm{E}-01$ & $1.72 \mathrm{E}-02$ & $2.134 \mathrm{E}-01$ & $6.33 \mathrm{E}-04$ & $3.998 \mathrm{E}-01$ & $1.76 \mathrm{E}-02$ & $4.743 \mathrm{E}+04$ \\
\hline $1.156 \mathrm{E}+00$ & $6.312 \mathrm{E}-01$ & $1.76 \mathrm{E}-02$ & $2.870 \mathrm{E}-01$ & $7.53 \mathrm{E}-04$ & $5.071 \mathrm{E}-01$ & $2.35 \mathrm{E}-02$ & $4.758 \mathrm{E}+04$ \\
\hline $1.156 \mathrm{E}+00$ & $6.344 \mathrm{E}-01$ & $1.64 \mathrm{E}-02$ & $3.628 \mathrm{E}-01$ & 8.77E-04 & 5.794E-01 & $2.85 \mathrm{E}-02$ & $4.759 \mathrm{E}+04$ \\
\hline $1.155 \mathrm{E}+00$ & $6.351 \mathrm{E}-01$ & $1.95 \mathrm{E}-02$ & $4.376 \mathrm{E}-01$ & $9.50 \mathrm{E}-04$ & $6.206 \mathrm{E}-01$ & $3.82 \mathrm{E}-02$ & $4.801 \mathrm{E}+04$ \\
\hline $1.155 \mathrm{E}+00$ & 6.392E-01 & $2.09 \mathrm{E}-02$ & $5.148 \mathrm{E}-01$ & $1.05 \mathrm{E}-03$ & $6.301 \mathrm{E}-01$ & $5.13 \mathrm{E}-02$ & $4.821 \mathrm{E}+04$ \\
\hline $1.155 \mathrm{E}+00$ & $6.269 \mathrm{E}-01$ & $2.48 \mathrm{E}-02$ & $5.896 \mathrm{E}-01$ & $1.18 \mathrm{E}-03$ & $6.552 \mathrm{E}-01$ & $7.12 \mathrm{E}-02$ & $4.864 \mathrm{E}+04$ \\
\hline $1.155 \mathrm{E}+00$ & $6.208 \mathrm{E}-01$ & $2.78 \mathrm{E}-02$ & $6.638 \mathrm{E}-01$ & $1.29 \mathrm{E}-03$ & $6.004 \mathrm{E}-01$ & $9.73 \mathrm{E}-02$ & $4.909 \mathrm{E}+04$ \\
\hline
\end{tabular}


Table 186: APC 6.0 x 4.0 Dynamic Measured Values - 13992 RPM

\begin{tabular}{|c|c|c|c|c|c|c|c|}
\hline$n(\mathrm{RPM})$ & $Q(\mathrm{~g}-\mathrm{m})$ & $\Delta Q(\mathrm{~g}-\mathrm{m})$ & $V(\mathrm{~V})$ & $I(\mathrm{~A})$ & $T_{\text {atm }}\left({ }^{\circ} \mathrm{C}\right)$ & $P_{\text {atm }}(\mathrm{Pa})$ & $P_{\text {diff }}(\mathrm{Pa})$ \\
\hline $1.392 \mathrm{E}+04$ & $4.322 \mathrm{E}+00$ & $7.09 \mathrm{E}-02$ & $1.086 \mathrm{E}+01$ & $8.822 \mathrm{E}+00$ & $2.184 \mathrm{E}+01$ & $9.800 \mathrm{E}+04$ & $4.360 \mathrm{E}+00$ \\
\hline $1.396 \mathrm{E}+04$ & $4.426 \mathrm{E}+00$ & $7.14 \mathrm{E}-02$ & $1.086 \mathrm{E}+01$ & $9.026 \mathrm{E}+00$ & $2.175 \mathrm{E}+01$ & $9.800 \mathrm{E}+04$ & $1.474 \mathrm{E}+01$ \\
\hline $1.399 \mathrm{E}+04$ & $4.491 \mathrm{E}+00$ & $7.32 \mathrm{E}-02$ & $1.085 \mathrm{E}+01$ & $9.188 \mathrm{E}+00$ & $2.200 \mathrm{E}+01$ & $9.799 \mathrm{E}+04$ & $3.382 \mathrm{E}+01$ \\
\hline $1.400 \mathrm{E}+04$ & $4.497 \mathrm{E}+00$ & $7.33 \mathrm{E}-02$ & $1.085 \mathrm{E}+01$ & $9.175 \mathrm{E}+00$ & $2.221 \mathrm{E}+01$ & $9.799 \mathrm{E}+04$ & $6.141 \mathrm{E}+01$ \\
\hline $1.404 \mathrm{E}+04$ & $4.474 \mathrm{E}+00$ & $7.57 \mathrm{E}-02$ & $1.085 \mathrm{E}+01$ & $9.131 \mathrm{E}+00$ & $2.230 \mathrm{E}+01$ & $9.799 \mathrm{E}+04$ & $9.859 \mathrm{E}+01$ \\
\hline $1.403 \mathrm{E}+04$ & $4.163 \mathrm{E}+00$ & $7.61 \mathrm{E}-02$ & $1.088 \mathrm{E}+01$ & $8.304 \mathrm{E}+00$ & $2.236 \mathrm{E}+01$ & $9.799 \mathrm{E}+04$ & $1.444 \mathrm{E}+02$ \\
\hline $1.400 \mathrm{E}+04$ & $3.406 \mathrm{E}+00$ & $7.52 \mathrm{E}-02$ & $1.093 \mathrm{E}+01$ & $6.658 \mathrm{E}+00$ & $2.240 \mathrm{E}+01$ & $9.799 \mathrm{E}+04$ & $1.989 \mathrm{E}+02$ \\
\hline $1.400 \mathrm{E}+04$ & $2.724 \mathrm{E}+00$ & $7.63 \mathrm{E}-02$ & $1.097 \mathrm{E}+01$ & $5.290 \mathrm{E}+00$ & $2.244 \mathrm{E}+01$ & $9.799 \mathrm{E}+04$ & $2.614 \mathrm{E}+02$ \\
\hline $1.398 \mathrm{E}+04$ & $2.083 \mathrm{E}+00$ & $7.14 \mathrm{E}-02$ & $1.100 \mathrm{E}+01$ & $4.042 \mathrm{E}+00$ & $2.254 \mathrm{E}+01$ & $9.799 \mathrm{E}+04$ & $3.329 \mathrm{E}+02$ \\
\hline
\end{tabular}


Table 187: APC 6.0 x 4.0 Dynamic Calculated Values - 13992 RPM

\begin{tabular}{|c|c|c|c|c|c|c|c|}
\hline$n(\mathrm{RPM})$ & $V_{\infty}^{\prime}(\mathrm{m} / \mathrm{s})$ & $\Delta V_{\infty}^{\prime}(\mathrm{m} / \mathrm{s})$ & $T^{\prime}(\mathrm{g})$ & $\Delta T^{\prime}(\mathrm{g})$ & $V_{\mathrm{t}}(\mathrm{m} / \mathrm{s})$ & $P_{\mathrm{P}}(\mathrm{W})$ & $\Delta P_{\mathrm{P}}(\mathrm{W})$ \\
\hline $1.392 \mathrm{E}+04$ & $2.518 \mathrm{E}+00$ & $1.76 \mathrm{E}-02$ & $3.792 \mathrm{E}+02$ & $9.67 \mathrm{E}+00$ & $8.309 \mathrm{E}+01$ & $6.179 \mathrm{E}+01$ & $1.01 \mathrm{E}+00$ \\
\hline $1.396 \mathrm{E}+04$ & $4.829 \mathrm{E}+00$ & $1.91 \mathrm{E}-02$ & $3.647 \mathrm{E}+02$ & $9.70 \mathrm{E}+00$ & $8.340 \mathrm{E}+01$ & $6.345 \mathrm{E}+01$ & $1.02 \mathrm{E}+00$ \\
\hline $1.399 \mathrm{E}+04$ & $7.443 \mathrm{E}+00$ & $2.22 \mathrm{E}-02$ & $3.512 \mathrm{E}+02$ & $9.61 \mathrm{E}+00$ & $8.381 \mathrm{E}+01$ & $6.454 \mathrm{E}+01$ & $1.05 \mathrm{E}+00$ \\
\hline $1.400 \mathrm{E}+04$ & $1.012 \mathrm{E}+01$ & $2.74 \mathrm{E}-02$ & $3.290 \mathrm{E}+02$ & $9.69 \mathrm{E}+00$ & $8.413 \mathrm{E}+01$ & $6.466 \mathrm{E}+01$ & $1.05 \mathrm{E}+00$ \\
\hline $1.404 \mathrm{E}+04$ & $1.290 \mathrm{E}+01$ & $3.01 \mathrm{E}-02$ & $3.041 \mathrm{E}+02$ & $9.73 \mathrm{E}+00$ & $8.472 \mathrm{E}+01$ & $6.450 \mathrm{E}+01$ & $1.09 \mathrm{E}+00$ \\
\hline $1.403 \mathrm{E}+04$ & $1.568 \mathrm{E}+01$ & $3.30 \mathrm{E}-02$ & $2.474 \mathrm{E}+02$ & $9.75 \mathrm{E}+00$ & $8.513 \mathrm{E}+01$ & $5.997 \mathrm{E}+01$ & $1.10 \mathrm{E}+00$ \\
\hline $1.400 \mathrm{E}+04$ & $1.847 \mathrm{E}+01$ & $3.63 \mathrm{E}-02$ & $1.818 \mathrm{E}+02$ & $9.75 \mathrm{E}+00$ & $8.554 \mathrm{E}+01$ & $4.897 \mathrm{E}+01$ & $1.08 \mathrm{E}+00$ \\
\hline $1.400 \mathrm{E}+04$ & $2.122 \mathrm{E}+01$ & $4.04 \mathrm{E}-02$ & $1.252 \mathrm{E}+02$ & $9.71 \mathrm{E}+00$ & $8.617 \mathrm{E}+01$ & $3.917 \mathrm{E}+01$ & $1.10 \mathrm{E}+00$ \\
\hline $1.398 \mathrm{E}+04$ & $2.398 \mathrm{E}+01$ & $4.51 \mathrm{E}-02$ & $7.217 \mathrm{E}+01$ & $9.68 \mathrm{E}+00$ & $8.676 \mathrm{E}+01$ & $2.990 \mathrm{E}+01$ & $1.02 \mathrm{E}+00$ \\
\hline$P_{\mathrm{e}}(\mathrm{W})$ & $\Delta P_{\mathrm{e}}(\mathrm{W})$ & $C_{\mathrm{T}}$ & $\Delta C_{\mathrm{T}}$ & $C_{\mathrm{P}}$ & $\Delta C_{\mathrm{P}}$ & $C_{\mathrm{Q}}$ & $\Delta C_{\mathrm{Q}}$ \\
\hline $9.582 \mathrm{E}+01$ & $1.05 \mathrm{E}+00$ & $1.121 \mathrm{E}-01$ & $2.86 \mathrm{E}-03$ & $5.285 \mathrm{E}-02$ & $8.69 \mathrm{E}-04$ & $8.412 \mathrm{E}-03$ & $1.38 \mathrm{E}-04$ \\
\hline $9.799 \mathrm{E}+01$ & $1.05 \mathrm{E}+00$ & $1.072 \mathrm{E}-01$ & $2.85 \mathrm{E}-03$ & $5.384 \mathrm{E}-02$ & $8.70 \mathrm{E}-04$ & $8.568 \mathrm{E}-03$ & $1.38 \mathrm{E}-04$ \\
\hline $9.970 \mathrm{E}+01$ & $1.08 \mathrm{E}+00$ & $1.028 \mathrm{E}-01$ & $2.81 \mathrm{E}-03$ & 5.439E-02 & $8.89 \mathrm{E}-04$ & $8.657 \mathrm{E}-03$ & $1.41 \mathrm{E}-04$ \\
\hline $9.956 \mathrm{E}+01$ & $1.08 \mathrm{E}+00$ & $9.630 \mathrm{E}-02$ & $2.84 \mathrm{E}-03$ & $5.445 \mathrm{E}-02$ & $8.90 \mathrm{E}-04$ & $8.666 \mathrm{E}-03$ & $1.41 \mathrm{E}-04$ \\
\hline $9.910 \mathrm{E}+01$ & $1.07 \mathrm{E}+00$ & $8.858 \mathrm{E}-02$ & $2.83 \mathrm{E}-03$ & $5.391 \mathrm{E}-02$ & $9.14 \mathrm{E}-04$ & $8.581 \mathrm{E}-03$ & $1.45 \mathrm{E}-04$ \\
\hline $9.033 \mathrm{E}+01$ & $9.98 \mathrm{E}-01$ & $7.219 \mathrm{E}-02$ & $2.85 \mathrm{E}-03$ & $5.026 \mathrm{E}-02$ & $9.21 \mathrm{E}-04$ & 7.999E-03 & $1.46 \mathrm{E}-04$ \\
\hline $7.274 \mathrm{E}+01$ & $8.04 \mathrm{E}-01$ & $5.323 \mathrm{E}-02$ & $2.86 \mathrm{E}-03$ & $4.126 \mathrm{E}-02$ & $9.12 \mathrm{E}-04$ & $6.567 \mathrm{E}-03$ & $1.45 \mathrm{E}-04$ \\
\hline $5.801 \mathrm{E}+01$ & $6.58 \mathrm{E}-01$ & $3.669 \mathrm{E}-02$ & $2.84 \mathrm{E}-03$ & $3.302 \mathrm{E}-02$ & $9.25 \mathrm{E}-04$ & $5.255 \mathrm{E}-03$ & $1.47 \mathrm{E}-04$ \\
\hline $4.447 \mathrm{E}+01$ & 4.94E-01 & $2.122 \mathrm{E}-02$ & $2.85 \mathrm{E}-03$ & $2.533 \mathrm{E}-02$ & $8.69 \mathrm{E}-04$ & 4.032E-03 & $1.38 \mathrm{E}-04$ \\
\hline$\rho\left(\mathrm{kg} / \mathrm{m}^{\wedge} 3\right)$ & $\eta_{\mathrm{T}}$ & $\Delta \eta_{\mathrm{T}}$ & $J$ & $\Delta J$ & $\eta_{\mathrm{P}}$ & $\Delta \eta_{\mathrm{P}}$ & $R e_{0.75}$ \\
\hline $1.157 \mathrm{E}+00$ & $6.449 \mathrm{E}-01$ & $1.27 \mathrm{E}-02$ & 7.143E-02 & 4.99E-04 & $1.515 \mathrm{E}-01$ & $4.72 \mathrm{E}-03$ & $5.483 \mathrm{E}+04$ \\
\hline $1.158 \mathrm{E}+00$ & $6.475 \mathrm{E}-01$ & $1.26 \mathrm{E}-02$ & $1.366 \mathrm{E}-01$ & $5.42 \mathrm{E}-04$ & $2.722 \mathrm{E}-01$ & $8.54 \mathrm{E}-03$ & $5.507 \mathrm{E}+04$ \\
\hline $1.157 \mathrm{E}+00$ & $6.473 \mathrm{E}-01$ & $1.27 \mathrm{E}-02$ & $2.101 \mathrm{E}-01$ & $6.29 \mathrm{E}-04$ & $3.972 \mathrm{E}-01$ & $1.27 \mathrm{E}-02$ & $5.525 \mathrm{E}+04$ \\
\hline $1.156 \mathrm{E}+00$ & $6.494 \mathrm{E}-01$ & $1.27 \mathrm{E}-02$ & $2.856 \mathrm{E}-01$ & 7.77E-04 & $5.051 \mathrm{E}-01$ & $1.71 \mathrm{E}-02$ & $5.539 \mathrm{E}+04$ \\
\hline $1.155 \mathrm{E}+00$ & $6.509 \mathrm{E}-01$ & $1.31 \mathrm{E}-02$ & $3.630 \mathrm{E}-01$ & $8.53 \mathrm{E}-04$ & 5.964E-01 & $2.16 \mathrm{E}-02$ & $5.575 \mathrm{E}+04$ \\
\hline $1.155 \mathrm{E}+00$ & $6.640 \mathrm{E}-01$ & $1.42 \mathrm{E}-02$ & $4.417 \mathrm{E}-01$ & 9.39E-04 & $6.344 \mathrm{E}-01$ & $2.76 \mathrm{E}-02$ & $5.600 \mathrm{E}+04$ \\
\hline $1.155 \mathrm{E}+00$ & $6.733 \mathrm{E}-01$ & $1.66 \mathrm{E}-02$ & $5.210 \mathrm{E}-01$ & $1.03 \mathrm{E}-03$ & $6.721 \mathrm{E}-01$ & $3.90 \mathrm{E}-02$ & $5.626 \mathrm{E}+04$ \\
\hline $1.155 \mathrm{E}+00$ & $6.753 \mathrm{E}-01$ & $2.04 \mathrm{E}-02$ & $5.985 \mathrm{E}-01$ & $1.15 \mathrm{E}-03$ & $6.652 \mathrm{E}-01$ & $5.48 \mathrm{E}-02$ & $5.666 \mathrm{E}+04$ \\
\hline $1.154 \mathrm{E}+00$ & $6.724 \mathrm{E}-01$ & $2.42 \mathrm{E}-02$ & $6.777 \mathrm{E}-01$ & $1.29 \mathrm{E}-03$ & $5.677 \mathrm{E}-01$ & $7.86 \mathrm{E}-02$ & $5.701 \mathrm{E}+04$ \\
\hline
\end{tabular}


Table 188: APC 6.0 x 4.0 Dynamic Measured Values - 16002 RPM

\begin{tabular}{|c|c|c|c|c|c|c|c|}
\hline$n(\mathrm{RPM})$ & $Q(\mathrm{~g}-\mathrm{m})$ & $\Delta Q(\mathrm{~g}-\mathrm{m})$ & $V(\mathrm{~V})$ & $I(\mathrm{~A})$ & $T_{\text {atm }}\left({ }^{\circ} \mathrm{C}\right)$ & $P_{\text {atm }}(\mathrm{Pa})$ & $P_{\text {diff }}(\mathrm{Pa})$ \\
\hline $1.583 \mathrm{E}+04$ & $5.771 \mathrm{E}+00$ & $7.82 \mathrm{E}-02$ & $1.072 \mathrm{E}+01$ & $1.355 \mathrm{E}+01$ & $2.194 \mathrm{E}+01$ & $9.898 \mathrm{E}+04$ & $5.195 \mathrm{E}+00$ \\
\hline $1.603 \mathrm{E}+04$ & $6.026 \mathrm{E}+00$ & $7.86 \mathrm{E}-02$ & $1.069 \mathrm{E}+01$ & $1.453 \mathrm{E}+01$ & $2.215 \mathrm{E}+01$ & $9.900 \mathrm{E}+04$ & $1.837 \mathrm{E}+01$ \\
\hline $1.599 \mathrm{E}+04$ & $6.078 \mathrm{E}+00$ & $7.74 \mathrm{E}-02$ & $1.069 \mathrm{E}+01$ & $1.458 \mathrm{E}+01$ & $2.231 \mathrm{E}+01$ & $9.900 \mathrm{E}+04$ & $4.367 \mathrm{E}+01$ \\
\hline $1.599 \mathrm{E}+04$ & $6.078 \mathrm{E}+00$ & $7.86 \mathrm{E}-02$ & $1.069 \mathrm{E}+01$ & $1.458 \mathrm{E}+01$ & $2.240 \mathrm{E}+01$ & $9.902 \mathrm{E}+04$ & $8.112 \mathrm{E}+01$ \\
\hline $1.604 \mathrm{E}+04$ & $6.050 \mathrm{E}+00$ & $7.96 \mathrm{E}-02$ & $1.069 \mathrm{E}+01$ & $1.449 \mathrm{E}+01$ & $2.239 \mathrm{E}+01$ & $9.904 \mathrm{E}+04$ & $1.315 \mathrm{E}+02$ \\
\hline $1.604 \mathrm{E}+04$ & $5.813 \mathrm{E}+00$ & $7.85 \mathrm{E}-02$ & $1.072 \mathrm{E}+01$ & $1.357 \mathrm{E}+01$ & $2.243 \mathrm{E}+01$ & $9.905 \mathrm{E}+04$ & $1.942 \mathrm{E}+02$ \\
\hline $1.607 \mathrm{E}+04$ & $4.972 \mathrm{E}+00$ & $8.66 \mathrm{E}-02$ & $1.080 \mathrm{E}+01$ & $1.109 \mathrm{E}+01$ & $2.250 \mathrm{E}+01$ & $9.905 \mathrm{E}+04$ & $2.678 \mathrm{E}+02$ \\
\hline $1.603 \mathrm{E}+04$ & $3.913 \mathrm{E}+00$ & $8.09 \mathrm{E}-02$ & $1.088 \mathrm{E}+01$ & $8.280 \mathrm{E}+00$ & $2.256 \mathrm{E}+01$ & $9.905 \mathrm{E}+04$ & $3.531 \mathrm{E}+02$ \\
\hline $1.600 \mathrm{E}+04$ & $2.888 \mathrm{E}+00$ & $7.85 \mathrm{E}-02$ & $1.094 \mathrm{E}+01$ & $6.016 \mathrm{E}+00$ & $2.262 \mathrm{E}+01$ & $9.905 \mathrm{E}+04$ & $4.507 \mathrm{E}+02$ \\
\hline
\end{tabular}


Table 189: APC 6.0 x 4.0 Dynamic Calculated Values - 16002 RPM

\begin{tabular}{|c|c|c|c|c|c|c|c|}
\hline$n(\mathrm{RPM})$ & $V_{\infty}^{\prime}(\mathrm{m} / \mathrm{s})$ & $\Delta V_{\infty}^{\prime}(\mathrm{m} / \mathrm{s})$ & $T^{\prime}(\mathrm{g})$ & $\Delta T^{\prime}(\mathrm{g})$ & $V_{\mathrm{t}}(\mathrm{m} / \mathrm{s})$ & $P_{\mathrm{P}}(\mathrm{W})$ & $\Delta P_{\mathrm{P}}(\mathrm{W})$ \\
\hline $1.583 \mathrm{E}+04$ & $2.721 \mathrm{E}+00$ & $1.92 \mathrm{E}-02$ & $5.030 \mathrm{E}+02$ & $8.84 \mathrm{E}+00$ & $9.446 \mathrm{E}+01$ & $9.381 \mathrm{E}+01$ & $1.27 \mathrm{E}+00$ \\
\hline $1.603 \mathrm{E}+04$ & $5.355 \mathrm{E}+00$ & $2.13 \mathrm{E}-02$ & $5.007 \mathrm{E}+02$ & $8.33 \mathrm{E}+00$ & $9.579 \mathrm{E}+01$ & $9.922 \mathrm{E}+01$ & $1.29 \mathrm{E}+00$ \\
\hline $1.599 \mathrm{E}+04$ & $8.413 \mathrm{E}+00$ & $2.56 \mathrm{E}-02$ & $4.745 \mathrm{E}+02$ & $8.41 \mathrm{E}+00$ & $9.575 \mathrm{E}+01$ & $9.980 \mathrm{E}+01$ & $1.27 \mathrm{E}+00$ \\
\hline $1.599 \mathrm{E}+04$ & $1.158 \mathrm{E}+01$ & $3.15 \mathrm{E}-02$ & $4.444 \mathrm{E}+02$ & $8.39 \mathrm{E}+00$ & $9.605 \mathrm{E}+01$ & $9.977 \mathrm{E}+01$ & $1.29 \mathrm{E}+00$ \\
\hline $1.604 \mathrm{E}+04$ & $1.482 \mathrm{E}+01$ & $3.43 \mathrm{E}-02$ & $4.118 \mathrm{E}+02$ & $8.41 \mathrm{E}+00$ & $9.684 \mathrm{E}+01$ & $9.968 \mathrm{E}+01$ & $1.31 \mathrm{E}+00$ \\
\hline $1.604 \mathrm{E}+04$ & $1.809 \mathrm{E}+01$ & $3.69 \mathrm{E}-02$ & $3.557 \mathrm{E}+02$ & $8.59 \mathrm{E}+00$ & $9.739 \mathrm{E}+01$ & $9.578 \mathrm{E}+01$ & $1.29 \mathrm{E}+00$ \\
\hline $1.607 \mathrm{E}+04$ & $2.131 \mathrm{E}+01$ & 4.12E-02 & $2.676 \mathrm{E}+02$ & $8.78 \mathrm{E}+00$ & $9.817 \mathrm{E}+01$ & $8.204 \mathrm{E}+01$ & $1.43 \mathrm{E}+00$ \\
\hline $1.603 \mathrm{E}+04$ & $2.453 \mathrm{E}+01$ & $4.68 \mathrm{E}-02$ & $1.821 \mathrm{E}+02$ & $9.10 \mathrm{E}+00$ & $9.869 \mathrm{E}+01$ & $6.440 \mathrm{E}+01$ & $1.33 \mathrm{E}+00$ \\
\hline $1.600 \mathrm{E}+04$ & $2.775 \mathrm{E}+01$ & $5.20 \mathrm{E}-02$ & $1.098 \mathrm{E}+02$ & $9.46 \mathrm{E}+00$ & $9.940 \mathrm{E}+01$ & $4.746 \mathrm{E}+01$ & $1.29 \mathrm{E}+00$ \\
\hline$P_{\mathrm{e}}(\mathrm{W})$ & $\Delta P_{\mathrm{e}}(\mathrm{W})$ & $C_{\mathrm{T}}$ & $\Delta C_{\mathrm{T}}$ & $C_{\mathrm{P}}$ & $\Delta C_{\mathrm{P}}$ & $C_{\mathrm{Q}}$ & $\Delta C_{\mathrm{Q}}$ \\
\hline $1.453 \mathrm{E}+02$ & $1.55 \mathrm{E}+00$ & $1.139 \mathrm{E}-01$ & $2.00 \mathrm{E}-03$ & $5.407 \mathrm{E}-02$ & $7.35 \mathrm{E}-04$ & $8.606 \mathrm{E}-03$ & $1.17 \mathrm{E}-04$ \\
\hline $1.554 \mathrm{E}+02$ & $1.64 \mathrm{E}+00$ & $1.106 \mathrm{E}-01$ & $1.84 \mathrm{E}-03$ & $5.506 \mathrm{E}-02$ & $7.19 \mathrm{E}-04$ & $8.764 \mathrm{E}-03$ & $1.14 \mathrm{E}-04$ \\
\hline $1.558 \mathrm{E}+02$ & $1.63 \mathrm{E}+00$ & $1.055 \mathrm{E}-01$ & $1.87 \mathrm{E}-03$ & $5.588 \mathrm{E}-02$ & 7.13E-04 & 8.893E-03 & $1.13 \mathrm{E}-04$ \\
\hline $1.558 \mathrm{E}+02$ & $1.63 \mathrm{E}+00$ & 9.884E-02 & $1.87 \mathrm{E}-03$ & $5.591 \mathrm{E}-02$ & $7.25 \mathrm{E}-04$ & $8.899 \mathrm{E}-03$ & $1.15 \mathrm{E}-04$ \\
\hline $1.549 \mathrm{E}+02$ & $1.63 \mathrm{E}+00$ & $9.089 \mathrm{E}-02$ & $1.86 \mathrm{E}-03$ & $5.524 \mathrm{E}-02$ & $7.28 \mathrm{E}-04$ & 8.792E-03 & $1.16 \mathrm{E}-04$ \\
\hline $1.455 \mathrm{E}+02$ & $1.53 \mathrm{E}+00$ & $7.852 \mathrm{E}-02$ & $1.90 \mathrm{E}-03$ & $5.308 \mathrm{E}-02$ & $7.18 \mathrm{E}-04$ & $8.448 \mathrm{E}-03$ & $1.14 \mathrm{E}-04$ \\
\hline $1.197 \mathrm{E}+02$ & $1.28 \mathrm{E}+00$ & $5.891 \mathrm{E}-02$ & $1.93 \mathrm{E}-03$ & $4.529 \mathrm{E}-02$ & $7.89 \mathrm{E}-04$ & $7.208 \mathrm{E}-03$ & $1.26 \mathrm{E}-04$ \\
\hline $9.008 \mathrm{E}+01$ & $9.88 \mathrm{E}-01$ & $4.030 \mathrm{E}-02$ & $2.01 \mathrm{E}-03$ & $3.583 \mathrm{E}-02$ & $7.41 \mathrm{E}-04$ & 5.702E-03 & $1.18 \mathrm{E}-04$ \\
\hline $6.585 \mathrm{E}+01$ & $7.45 \mathrm{E}-01$ & $2.438 \mathrm{E}-02$ & $2.10 \mathrm{E}-03$ & $2.653 \mathrm{E}-02$ & $7.21 \mathrm{E}-04$ & 4.222E-03 & $1.15 \mathrm{E}-04$ \\
\hline$\rho\left(\mathrm{kg} / \mathrm{m}^{\wedge} 3\right)$ & $\eta_{\mathrm{T}}$ & $\Delta \eta_{\mathrm{T}}$ & $J$ & $\Delta J$ & $\eta_{\mathrm{P}}$ & $\Delta \eta_{\mathrm{P}}$ & $R e_{0.75}$ \\
\hline $1.169 \mathrm{E}+00$ & $6.456 \mathrm{E}-01$ & $1.11 \mathrm{E}-02$ & $6.791 \mathrm{E}-02$ & $4.80 \mathrm{E}-04$ & $1.431 \mathrm{E}-01$ & $3.34 \mathrm{E}-03$ & $6.292 \mathrm{E}+04$ \\
\hline $1.168 \mathrm{E}+00$ & $6.386 \mathrm{E}-01$ & $1.07 \mathrm{E}-02$ & $1.319 \mathrm{E}-01$ & $5.26 \mathrm{E}-04$ & $2.651 \mathrm{E}-01$ & $5.71 \mathrm{E}-03$ & $6.374 \mathrm{E}+04$ \\
\hline $1.167 \mathrm{E}+00$ & $6.407 \mathrm{E}-01$ & $1.06 \mathrm{E}-02$ & $2.078 \mathrm{E}-01$ & $6.34 \mathrm{E}-04$ & $3.922 \mathrm{E}-01$ & $8.66 \mathrm{E}-03$ & $6.365 \mathrm{E}+04$ \\
\hline $1.167 \mathrm{E}+00$ & $6.403 \mathrm{E}-01$ & $1.06 \mathrm{E}-02$ & $2.861 \mathrm{E}-01$ & $7.82 \mathrm{E}-04$ & $5.057 \mathrm{E}-01$ & $1.17 \mathrm{E}-02$ & $6.383 \mathrm{E}+04$ \\
\hline $1.167 \mathrm{E}+00$ & $6.437 \mathrm{E}-01$ & $1.08 \mathrm{E}-02$ & 3.649E-01 & $8.48 \mathrm{E}-04$ & $6.004 \mathrm{E}-01$ & $1.47 \mathrm{E}-02$ & $6.437 \mathrm{E}+04$ \\
\hline $1.167 \mathrm{E}+00$ & $6.583 \mathrm{E}-01$ & $1.13 \mathrm{E}-02$ & $4.453 \mathrm{E}-01$ & $9.13 \mathrm{E}-04$ & $6.587 \mathrm{E}-01$ & $1.83 \mathrm{E}-02$ & $\overline{6.473 E+04}$ \\
\hline $1.167 \mathrm{E}+00$ & $6.855 \mathrm{E}-01$ & $1.40 \mathrm{E}-02$ & $5.239 \mathrm{E}-01$ & $1.02 \mathrm{E}-03$ & $6.816 \mathrm{E}-01$ & $2.54 \mathrm{E}-02$ & $6.522 \mathrm{E}+04$ \\
\hline $1.167 \mathrm{E}+00$ & 7.149E-01 & $1.67 \mathrm{E}-02$ & $6.046 \mathrm{E}-01$ & $1.16 \mathrm{E}-03$ & $6.800 \mathrm{E}-01$ & $3.68 \mathrm{E}-02$ & $6.554 \mathrm{E}+04$ \\
\hline $1.167 \mathrm{E}+00$ & $7.207 \mathrm{E}-01$ & $2.12 \mathrm{E}-02$ & $6.852 \mathrm{E}-01$ & $1.29 \mathrm{E}-03$ & $6.295 \mathrm{E}-01$ & $5.69 \mathrm{E}-02$ & $6.599 \mathrm{E}+04$ \\
\hline
\end{tabular}




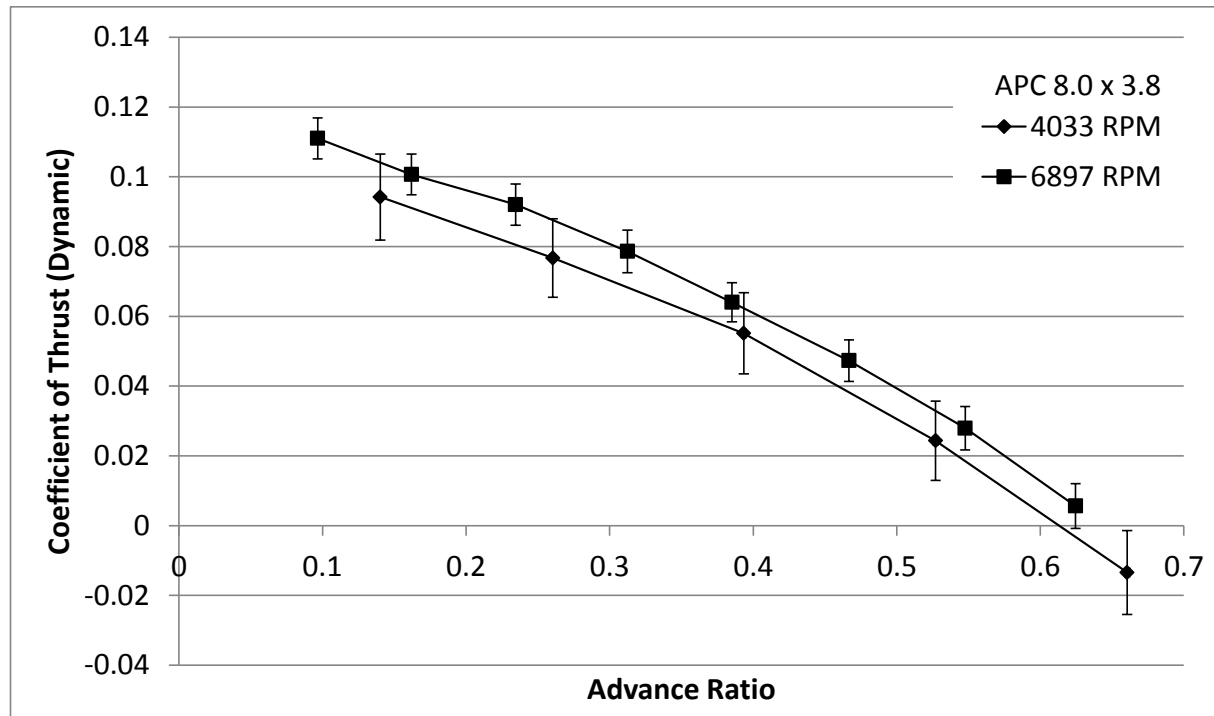

(a)

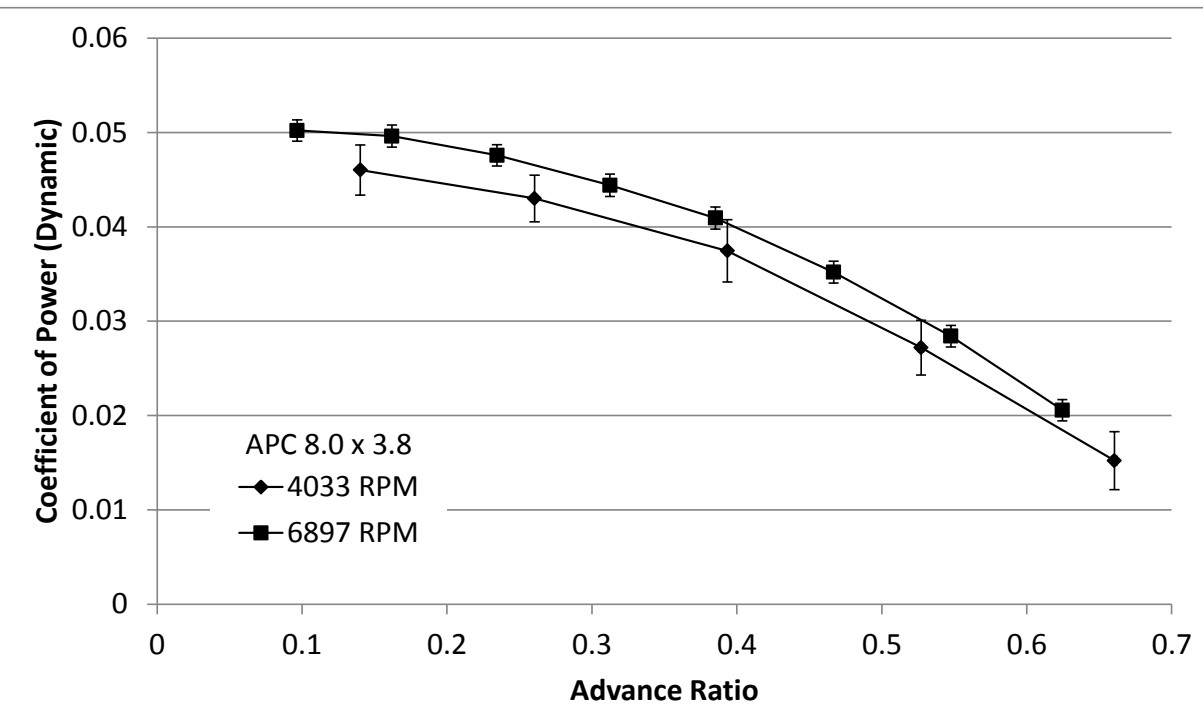

(b)

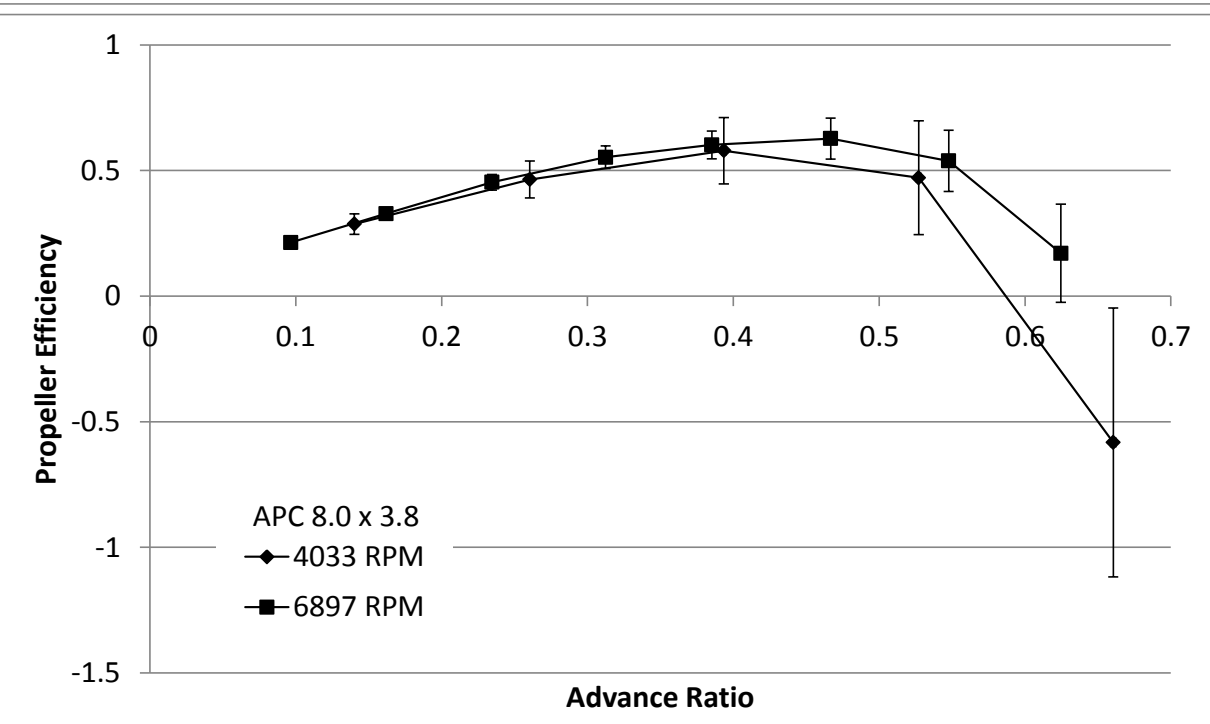

(c) 


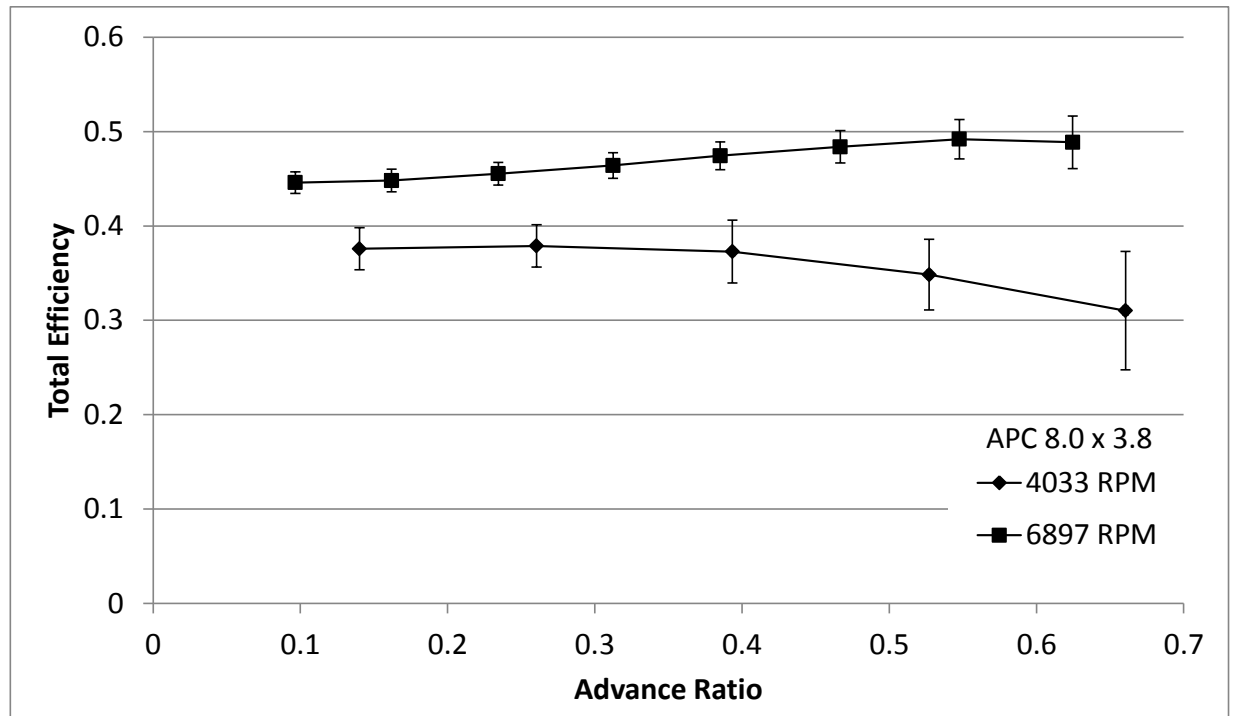

(d)

Figure 92: APC 8.0 x 3.8 Dynamic Test Results: (a) Coefficient of Thrust, (b) Coefficient of Power, (c) Propeller Efficiency, (d) Total Efficiency.

Table 190: APC 8.0 x 3.8 Dynamic Measured Values - 4033 RPM

\begin{tabular}{|c|c|c|c|c|c|c|c|}
\hline$n(\mathrm{RPM})$ & $Q(\mathrm{~g}-\mathrm{m})$ & $\Delta Q(\mathrm{~g}-\mathrm{m})$ & $V(\mathrm{~V})$ & $I(\mathrm{~A})$ & $T_{\text {atm }}\left({ }^{\circ} \mathrm{C}\right)$ & $P_{\text {atm }}(\mathrm{Pa})$ & $P_{\text {diff }}(\mathrm{Pa})$ \\
\hline $3.986 \mathrm{E}+03$ & $1.342 \mathrm{E}+00$ & $7.72 \mathrm{E}-02$ & $1.105 \mathrm{E}+01$ & $1.323 \mathrm{E}+00$ & $1.871 \mathrm{E}+01$ & $9.739 \mathrm{E}+04$ & $2.408 \mathrm{E}+00$ \\
\hline $4.077 \mathrm{E}+03$ & $1.313 \mathrm{E}+00$ & $7.54 \mathrm{E}-02$ & $1.105 \mathrm{E}+01$ & $1.313 \mathrm{E}+00$ & $1.873 \mathrm{E}+01$ & $9.738 \mathrm{E}+04$ & $8.037 \mathrm{E}+00$ \\
\hline $4.031 \mathrm{E}+03$ & $1.117 \mathrm{E}+00$ & $9.85 \mathrm{E}-02$ & $1.106 \mathrm{E}+01$ & $1.121 \mathrm{E}+00$ & $1.872 \mathrm{E}+01$ & $9.738 \mathrm{E}+04$ & $1.733 \mathrm{E}+01$ \\
\hline $4.046 \mathrm{E}+03$ & $8.165 \mathrm{E}-01$ & $8.70 \mathrm{E}-02$ & $1.106 \mathrm{E}+01$ & $8.802 \mathrm{E}-01$ & $1.903 \mathrm{E}+01$ & $9.738 \mathrm{E}+04$ & $3.068 \mathrm{E}+01$ \\
\hline $4.024 \mathrm{E}+03$ & $4.517 \mathrm{E}-01$ & $9.11 \mathrm{E}-02$ & $1.107 \mathrm{E}+01$ & $5.434 \mathrm{E}-01$ & $1.921 \mathrm{E}+01$ & $9.738 \mathrm{E}+04$ & $4.705 \mathrm{E}+01$ \\
\hline
\end{tabular}


Table 191: APC 8.0 x 3.8 Dynamic Calculated Values - 4033 RPM

\begin{tabular}{|c|c|c|c|c|c|c|c|}
\hline$n(\mathrm{RPM})$ & $V_{\infty}^{\prime}(\mathrm{m} / \mathrm{s})$ & $\Delta V_{\infty}^{\prime}(\mathrm{m} / \mathrm{s})$ & $T^{\prime}(\mathrm{g})$ & $\Delta T^{\prime}(\mathrm{g})$ & $V_{\mathrm{t}}(\mathrm{m} / \mathrm{s})$ & $P_{\mathrm{P}}(\mathrm{W})$ & $\Delta P_{\mathrm{P}}(\mathrm{W})$ \\
\hline $3.986 \mathrm{E}+03$ & $1.897 \mathrm{E}+00$ & $1.41 \mathrm{E}-02$ & $8.475 \mathrm{E}+01$ & $1.11 \mathrm{E}+01$ & $3.193 \mathrm{E}+01$ & $5.494 \mathrm{E}+00$ & $3.16 \mathrm{E}-01$ \\
\hline $4.077 \mathrm{E}+03$ & $3.604 \mathrm{E}+00$ & $1.50 \mathrm{E}-02$ & $7.222 \mathrm{E}+01$ & $1.06 \mathrm{E}+01$ & $3.281 \mathrm{E}+01$ & $5.496 \mathrm{E}+00$ & $3.16 \mathrm{E}-01$ \\
\hline $4.031 \mathrm{E}+03$ & $5.383 \mathrm{E}+00$ & $1.78 \mathrm{E}-02$ & $5.070 \mathrm{E}+01$ & $1.07 \mathrm{E}+01$ & $3.268 \mathrm{E}+01$ & $4.623 \mathrm{E}+00$ & $4.08 \mathrm{E}-01$ \\
\hline $4.046 \mathrm{E}+03$ & $7.238 \mathrm{E}+00$ & $2.06 \mathrm{E}-02$ & $2.253 \mathrm{E}+01$ & $1.05 \mathrm{E}+01$ & $3.316 \mathrm{E}+01$ & $3.393 \mathrm{E}+00$ & $3.61 \mathrm{E}-01$ \\
\hline $4.024 \mathrm{E}+03$ & $9.022 \mathrm{E}+00$ & $2.32 \mathrm{E}-02$ & $-1.229 \mathrm{E}+01$ & $1.10 \mathrm{E}+01$ & $3.343 \mathrm{E}+01$ & $1.867 \mathrm{E}+00$ & $3.77 \mathrm{E}-01$ \\
\hline$P_{\mathrm{e}}(\mathrm{W})$ & $\Delta P_{\mathrm{e}}(\mathrm{W})$ & $C_{\mathrm{T}}$ & $\Delta C_{\mathrm{T}}$ & $C_{\mathrm{P}}$ & $\Delta C_{\mathrm{P}}$ & $C_{\mathrm{Q}}$ & $\Delta C_{\mathrm{Q}}$ \\
\hline $1.462 \mathrm{E}+01$ & $1.96 \mathrm{E}-01$ & $9.418 \mathrm{E}-02$ & $1.23 \mathrm{E}-02$ & $4.602 \mathrm{E}-02$ & $2.65 \mathrm{E}-03$ & $7.325 \mathrm{E}-03$ & $4.22 \mathrm{E}-04$ \\
\hline $1.451 \mathrm{E}+01$ & $1.95 \mathrm{E}-01$ & $7.671 \mathrm{E}-02$ & $1.13 \mathrm{E}-02$ & $4.302 \mathrm{E}-02$ & $2.47 \mathrm{E}-03$ & $6.846 \mathrm{E}-03$ & $3.94 \mathrm{E}-04$ \\
\hline $1.240 \mathrm{E}+01$ & $1.68 \mathrm{E}-01$ & $5.510 \mathrm{E}-02$ & $1.16 \mathrm{E}-02$ & $3.745 \mathrm{E}-02$ & $3.30 \mathrm{E}-03$ & $5.960 \mathrm{E}-03$ & $5.26 \mathrm{E}-04$ \\
\hline $9.739 \mathrm{E}+00$ & $1.35 \mathrm{E}-01$ & $2.433 \mathrm{E}-02$ & $1.14 \mathrm{E}-02$ & $2.720 \mathrm{E}-02$ & $2.90 \mathrm{E}-03$ & $4.330 \mathrm{E}-03$ & $4.61 \mathrm{E}-04$ \\
\hline $6.017 \mathrm{E}+00$ & $9.08 \mathrm{E}-02$ & $-1.343 \mathrm{E}-02$ & $1.20 \mathrm{E}-02$ & $1.522 \mathrm{E}-02$ & $3.07 \mathrm{E}-03$ & $2.423 \mathrm{E}-03$ & $4.89 \mathrm{E}-04$ \\
\hline$\rho\left(\mathrm{kg} / m^{\wedge} 3\right)$ & $\eta_{\mathrm{T}}$ & $\Delta \eta_{\mathrm{T}}$ & $J$ & $\Delta J$ & $\eta_{\mathrm{P}}$ & $\Delta \eta_{\mathrm{P}}$ & $R e_{0.75}$ \\
\hline $1.162 \mathrm{E}+00$ & $3.758 \mathrm{E}-01$ & $2.22 \mathrm{E}-02$ & $1.402 \mathrm{E}-01$ & $1.05 \mathrm{E}-03$ & $2.869 \mathrm{E}-01$ & $4.11 \mathrm{E}-02$ & $4.278 \mathrm{E}+04$ \\
\hline $1.162 \mathrm{E}+00$ & $3.789 \mathrm{E}-01$ & $2.24 \mathrm{E}-02$ & $2.604 \mathrm{E}-01$ & $1.09 \mathrm{E}-03$ & $4.644 \mathrm{E}-01$ & $7.32 \mathrm{E}-02$ & $4.394 \mathrm{E}+04$ \\
\hline $1.162 \mathrm{E}+00$ & $3.728 \mathrm{E}-01$ & $3.33 \mathrm{E}-02$ & $3.935 \mathrm{E}-01$ & $1.32 \mathrm{E}-03$ & $5.789 \mathrm{E}-01$ & $1.33 \mathrm{E}-01$ & $4.378 \mathrm{E}+04$ \\
\hline $1.161 \mathrm{E}+00$ & $3.484 \mathrm{E}-01$ & $3.74 \mathrm{E}-02$ & $5.271 \mathrm{E}-01$ & $1.54 \mathrm{E}-03$ & $4.714 \mathrm{E}-01$ & $2.26 \mathrm{E}-01$ & $4.433 \mathrm{E}+04$ \\
\hline $1.160 \mathrm{E}+00$ & $3.102 \mathrm{E}-01$ & $6.28 \mathrm{E}-02$ & $6.605 \mathrm{E}-01$ & $1.75 \mathrm{E}-03$ & $-5.827 \mathrm{E}-01$ & $5.35 \mathrm{E}-01$ & $4.464 \mathrm{E}+04$ \\
\hline
\end{tabular}

Table 192: APC 8.0 x 3.8 Dynamic Measured Values - 6897 RPM

\begin{tabular}{|c|c|c|c|c|c|c|c|}
\hline$n(\mathrm{RPM})$ & $Q(\mathrm{~g}-\mathrm{m})$ & $\Delta Q(\mathrm{~g}-\mathrm{m})$ & $V(\mathrm{~V})$ & $I(\mathrm{~A})$ & $T_{\text {atm }}\left({ }^{\circ} \mathrm{C}\right)$ & $P_{\text {atm }}(\mathrm{Pa})$ & $P_{\text {diff }}(\mathrm{Pa})$ \\
\hline $6.913 \mathrm{E}+03$ & $4.399 \mathrm{E}+00$ & $9.90 \mathrm{E}-02$ & $1.093 \mathrm{E}+01$ & $6.409 \mathrm{E}+00$ & $1.902 \mathrm{E}+01$ & $9.739 \mathrm{E}+04$ & $3.729 \mathrm{E}+00$ \\
\hline $6.893 \mathrm{E}+03$ & $4.323 \mathrm{E}+00$ & $1.03 \mathrm{E}-01$ & $1.093 \mathrm{E}+01$ & $6.245 \mathrm{E}+00$ & $1.903 \mathrm{E}+01$ & $9.739 \mathrm{E}+04$ & $9.455 \mathrm{E}+00$ \\
\hline $6.914 \mathrm{E}+03$ & $4.172 \mathrm{E}+00$ & $9.77 \mathrm{E}-02$ & $1.094 \mathrm{E}+01$ & $5.945 \mathrm{E}+00$ & $1.896 \mathrm{E}+01$ & $9.740 \mathrm{E}+04$ & $1.904 \mathrm{E}+01$ \\
\hline $6.863 \mathrm{E}+03$ & $3.832 \mathrm{E}+00$ & $1.01 \mathrm{E}-01$ & $1.096 \mathrm{E}+01$ & $5.311 \mathrm{E}+00$ & $1.927 \mathrm{E}+01$ & $9.740 \mathrm{E}+04$ & $3.232 \mathrm{E}+01$ \\
\hline $6.932 \mathrm{E}+03$ & $3.601 \mathrm{E}+00$ & $1.03 \mathrm{E}-01$ & $1.097 \mathrm{E}+01$ & $4.927 \mathrm{E}+00$ & $1.958 \mathrm{E}+01$ & $9.740 \mathrm{E}+04$ & $4.920 \mathrm{E}+01$ \\
\hline $6.893 \mathrm{E}+03$ & $3.056 \mathrm{E}+00$ & $1.01 \mathrm{E}-01$ & $1.099 \mathrm{E}+01$ & $4.068 \mathrm{E}+00$ & $2.004 \mathrm{E}+01$ & $9.741 \mathrm{E}+04$ & $7.034 \mathrm{E}+01$ \\
\hline $6.869 \mathrm{E}+03$ & $2.450 \mathrm{E}+00$ & $9.89 \mathrm{E}-02$ & $1.101 \mathrm{E}+01$ & $3.190 \mathrm{E}+00$ & $2.017 \mathrm{E}+01$ & $9.741 \mathrm{E}+04$ & $9.519 \mathrm{E}+01$ \\
\hline $6.897 \mathrm{E}+03$ & $1.786 \mathrm{E}+00$ & $9.88 \mathrm{E}-02$ & $1.103 \mathrm{E}+01$ & $2.348 \mathrm{E}+00$ & $2.037 \mathrm{E}+01$ & $9.742 \mathrm{E}+04$ & $1.238 \mathrm{E}+02$ \\
\hline
\end{tabular}


Table 193: APC 8.0 x 3.8 Dynamic Calculated Values - 6897 RPM

\begin{tabular}{|c|c|c|c|c|c|c|c|}
\hline$n(\mathrm{RPM})$ & $V_{\infty}^{\prime}(\mathrm{m} / \mathrm{s})$ & $\Delta V_{\infty}^{\prime}(\mathrm{m} / \mathrm{s})$ & $T^{\prime}(\mathrm{g})$ & $\Delta T^{\prime}(\mathrm{g})$ & $V_{\mathrm{t}}(\mathrm{m} / \mathrm{s})$ & $P_{\mathrm{P}}(\mathrm{W})$ & $\Delta P_{\mathrm{P}}(\mathrm{W})$ \\
\hline $6.913 \mathrm{E}+03$ & $2.266 \mathrm{E}+00$ & $1.48 \mathrm{E}-02$ & $3.001 \mathrm{E}+02$ & $1.59 \mathrm{E}+01$ & $5.533 \mathrm{E}+01$ & $3.123 \mathrm{E}+01$ & 7.04E-01 \\
\hline $6.893 \mathrm{E}+03$ & $3.790 \mathrm{E}+00$ & $1.60 \mathrm{E}-02$ & $2.706 \mathrm{E}+02$ & $1.57 \mathrm{E}+01$ & $5.526 \mathrm{E}+01$ & $3.060 \mathrm{E}+01$ & 7.27E-01 \\
\hline $6.914 \mathrm{E}+03$ & $5.503 \mathrm{E}+00$ & $1.77 \mathrm{E}-02$ & $2.489 \mathrm{E}+02$ & $1.59 \mathrm{E}+01$ & $5.557 \mathrm{E}+01$ & $2.962 \mathrm{E}+01$ & $6.94 \mathrm{E}-01$ \\
\hline $6.863 \mathrm{E}+03$ & $7.278 \mathrm{E}+00$ & $2.03 \mathrm{E}-02$ & $2.093 \mathrm{E}+02$ & $1.62 \mathrm{E}+01$ & $5.537 \mathrm{E}+01$ & $2.701 \mathrm{E}+01$ & $7.15 \mathrm{E}-01$ \\
\hline $6.932 \mathrm{E}+03$ & $9.064 \mathrm{E}+00$ & $2.26 \mathrm{E}-02$ & $1.737 \mathrm{E}+02$ & $1.52 \mathrm{E}+01$ & $5.618 \mathrm{E}+01$ & $2.563 \mathrm{E}+01$ & 7.34E-01 \\
\hline $6.893 \mathrm{E}+03$ & $1.092 \mathrm{E}+01$ & $2.55 \mathrm{E}-02$ & $1.267 \mathrm{E}+02$ & $1.59 \mathrm{E}+01$ & $5.619 \mathrm{E}+01$ & $2.163 \mathrm{E}+01$ & $7.13 \mathrm{E}-01$ \\
\hline $6.869 \mathrm{E}+03$ & $1.277 \mathrm{E}+01$ & 2.79E-02 & 7.432E+01 & $1.66 \mathrm{E}+01$ & $5.640 \mathrm{E}+01$ & $1.728 \mathrm{E}+01$ & $6.98 \mathrm{E}-01$ \\
\hline $6.897 \mathrm{E}+03$ & $1.462 \mathrm{E}+01$ & $3.12 \mathrm{E}-02$ & $1.504 \mathrm{E}+01$ & $1.72 \mathrm{E}+01$ & $5.707 \mathrm{E}+01$ & $1.265 \mathrm{E}+01$ & $7.00 \mathrm{E}-01$ \\
\hline$P_{\mathrm{e}}(\mathrm{W})$ & $\Delta P_{\mathrm{e}}(\mathrm{W})$ & $C_{\mathrm{T}}$ & $\Delta C_{\mathrm{T}}$ & $C_{\mathrm{P}}$ & $\Delta C_{\mathrm{P}}$ & $C_{\mathrm{Q}}$ & $\Delta C_{\mathrm{Q}}$ \\
\hline $7.004 \mathrm{E}+01$ & 8.39E-01 & $1.110 \mathrm{E}-01$ & $5.88 \mathrm{E}-03$ & $5.021 \mathrm{E}-02$ & $1.14 \mathrm{E}-03$ & 7.991E-03 & $1.80 \mathrm{E}-04$ \\
\hline $6.829 \mathrm{E}+01$ & 8.51E-01 & $1.007 \mathrm{E}-01$ & $5.86 \mathrm{E}-03$ & 4.962E-02 & $1.18 \mathrm{E}-03$ & 7.897E-03 & $1.88 \mathrm{E}-04$ \\
\hline $6.505 \mathrm{E}+01$ & 7.83E-01 & $9.202 \mathrm{E}-02$ & $5.89 \mathrm{E}-03$ & $4.758 \mathrm{E}-02$ & $1.12 \mathrm{E}-03$ & $7.572 \mathrm{E}-03$ & $1.78 \mathrm{E}-04$ \\
\hline $5.819 \mathrm{E}+01$ & 7.09E-01 & $7.861 \mathrm{E}-02$ & $6.10 \mathrm{E}-03$ & $4.440 \mathrm{E}-02$ & $1.18 \mathrm{E}-03$ & 7.067E-03 & $1.87 \mathrm{E}-04$ \\
\hline $5.404 \mathrm{E}+01$ & $6.63 \mathrm{E}-01$ & $6.401 \mathrm{E}-02$ & $5.59 \mathrm{E}-03$ & 4.093E-02 & $1.17 \mathrm{E}-03$ & $6.515 \mathrm{E}-03$ & $1.87 \mathrm{E}-04$ \\
\hline $4.470 \mathrm{E}+01$ & 5.72E-01 & $4.730 \mathrm{E}-02$ & $5.94 \mathrm{E}-03$ & $3.519 \mathrm{E}-02$ & $1.16 \mathrm{E}-03$ & $5.601 \mathrm{E}-03$ & $1.85 \mathrm{E}-04$ \\
\hline $3.513 \mathrm{E}+01$ & 4.47E-01 & $2.794 \mathrm{E}-02$ & $6.23 \mathrm{E}-03$ & $2.842 \mathrm{E}-02$ & $1.15 \mathrm{E}-03$ & $4.523 \mathrm{E}-03$ & $1.83 \mathrm{E}-04$ \\
\hline $2.589 \mathrm{E}+01$ & $3.28 \mathrm{E}-01$ & $5.613 \mathrm{E}-03$ & $6.43 \mathrm{E}-03$ & $2.057 \mathrm{E}-02$ & $1.14 \mathrm{E}-03$ & $3.273 \mathrm{E}-03$ & $1.81 \mathrm{E}-04$ \\
\hline$\rho\left(\mathrm{kg} / \mathrm{m}^{\wedge} 3\right)$ & $\eta_{\mathrm{T}}$ & $\Delta \eta_{\mathrm{T}}$ & $J$ & $\Delta J$ & $\eta_{\mathrm{P}}$ & $\Delta \eta_{\mathrm{P}}$ & $\operatorname{Re}_{0.75}$ \\
\hline $1.161 \mathrm{E}+00$ & $4.459 \mathrm{E}-01$ & $1.14 \mathrm{E}-02$ & $9.660 \mathrm{E}-02$ & $6.35 \mathrm{E}-04$ & $2.136 \mathrm{E}-01$ & $1.24 \mathrm{E}-02$ & $7.399 \mathrm{E}+04$ \\
\hline $1.161 \mathrm{E}+00$ & $4.481 \mathrm{E}-01$ & $1.20 \mathrm{E}-02$ & $1.620 \mathrm{E}-01$ & $6.95 \mathrm{E}-04$ & $3.286 \mathrm{E}-01$ & $2.07 \mathrm{E}-02$ & $7.388 \mathrm{E}+04$ \\
\hline $1.162 \mathrm{E}+00$ & $4.554 \mathrm{E}-01$ & $1.20 \mathrm{E}-02$ & $2.345 \mathrm{E}-01$ & 7.69E-04 & $4.535 \mathrm{E}-01$ & $3.10 \mathrm{E}-02$ & $7.434 \mathrm{E}+04$ \\
\hline $1.160 \mathrm{E}+00$ & 4.641E-01 & $1.35 \mathrm{E}-02$ & $3.124 \mathrm{E}-01$ & 8.97E-04 & $5.531 \mathrm{E}-01$ & $4.54 \mathrm{E}-02$ & $7.393 \mathrm{E}+04$ \\
\hline $1.159 \mathrm{E}+00$ & 4.744E-01 & $1.48 \mathrm{E}-02$ & $3.852 \mathrm{E}-01$ & $9.96 \mathrm{E}-04$ & $6.024 \mathrm{E}-01$ & $5.54 \mathrm{E}-02$ & $7.488 \mathrm{E}+04$ \\
\hline $1.157 \mathrm{E}+00$ & $4.839 \mathrm{E}-01$ & $1.71 \mathrm{E}-02$ & 4.667E-01 & $1.14 \mathrm{E}-03$ & $6.273 \mathrm{E}-01$ & 8.14E-02 & $7.470 \mathrm{E}+04$ \\
\hline $1.157 \mathrm{E}+00$ & $4.920 \mathrm{E}-01$ & $2.08 \mathrm{E}-02$ & $5.477 \mathrm{E}-01$ & $1.25 \mathrm{E}-03$ & $5.385 \mathrm{E}-01$ & $1.22 \mathrm{E}-01$ & $7.491 \mathrm{E}+04$ \\
\hline $1.156 \mathrm{E}+00$ & $4.886 \mathrm{E}-01$ & 2.77E-02 & $6.246 \mathrm{E}-01$ & $1.40 \mathrm{E}-03$ & $1.705 \mathrm{E}-01$ & $1.95 \mathrm{E}-01$ & $7.571 \mathrm{E}+04$ \\
\hline
\end{tabular}




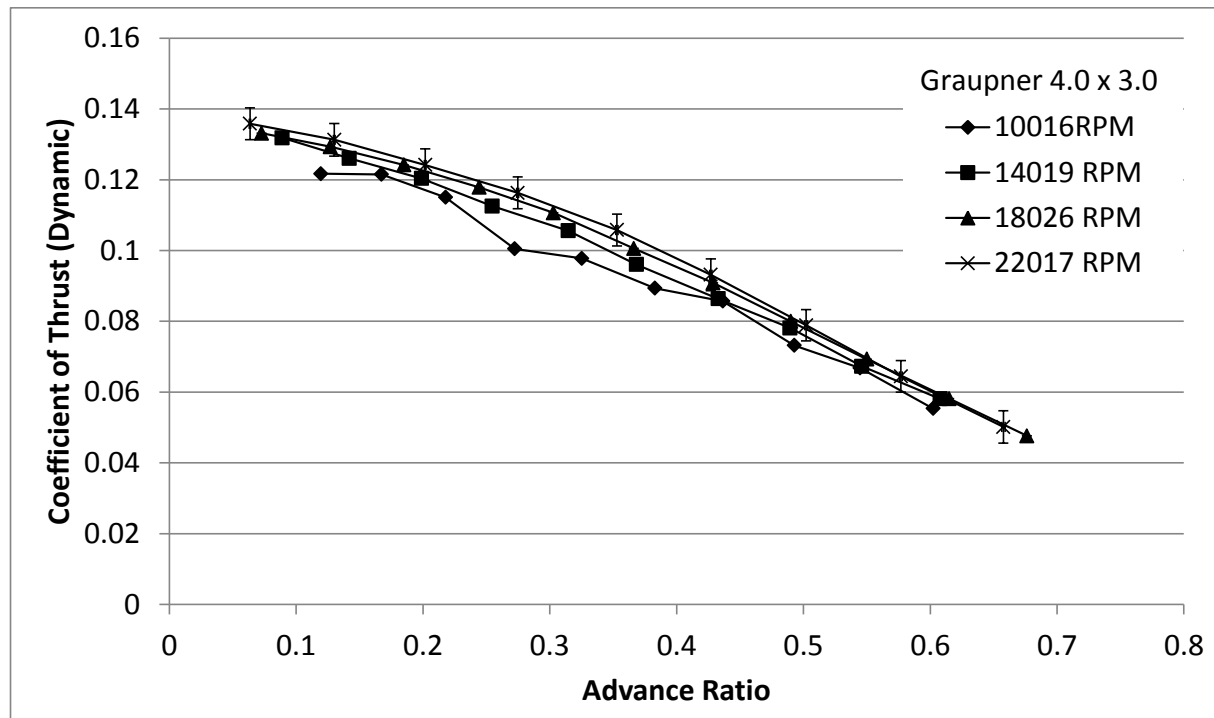

(a)

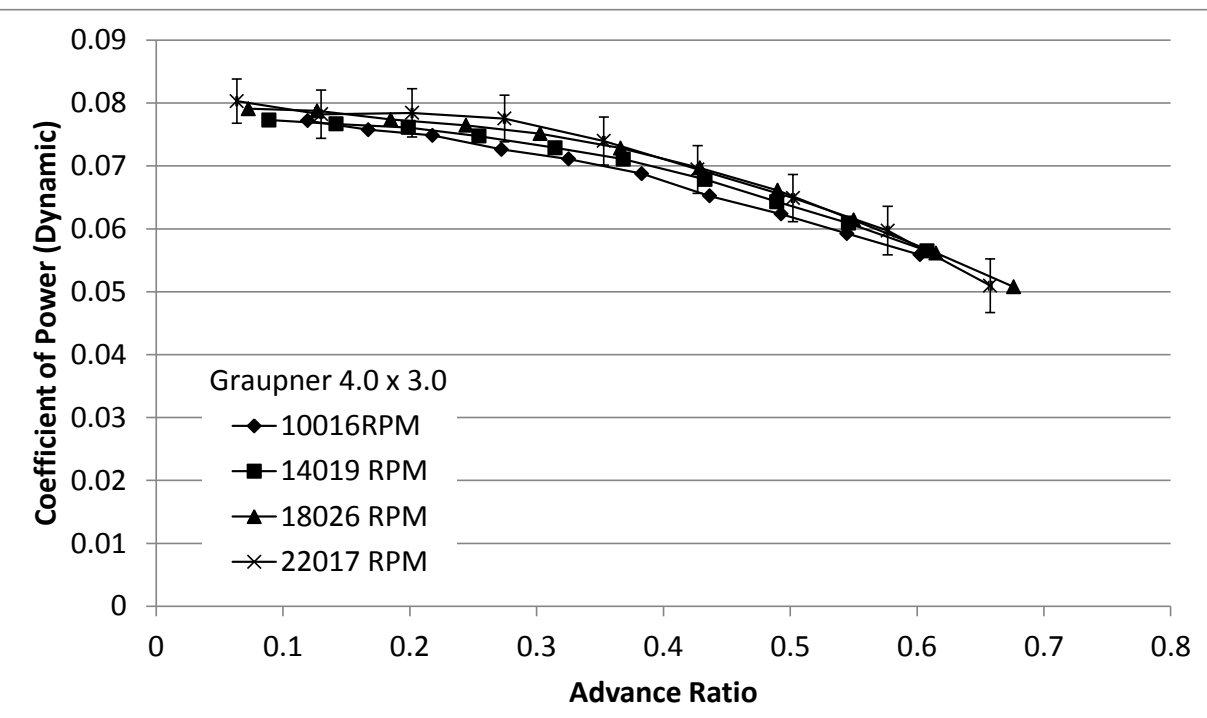

(b)

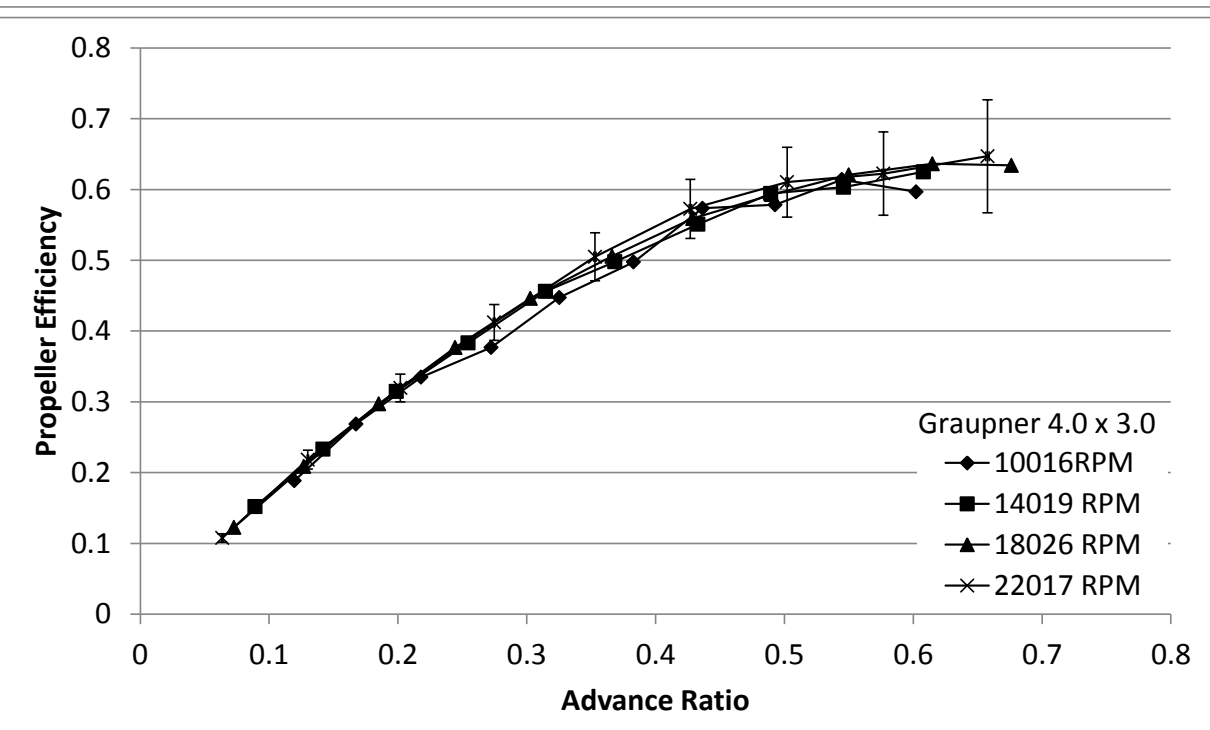

(c) 


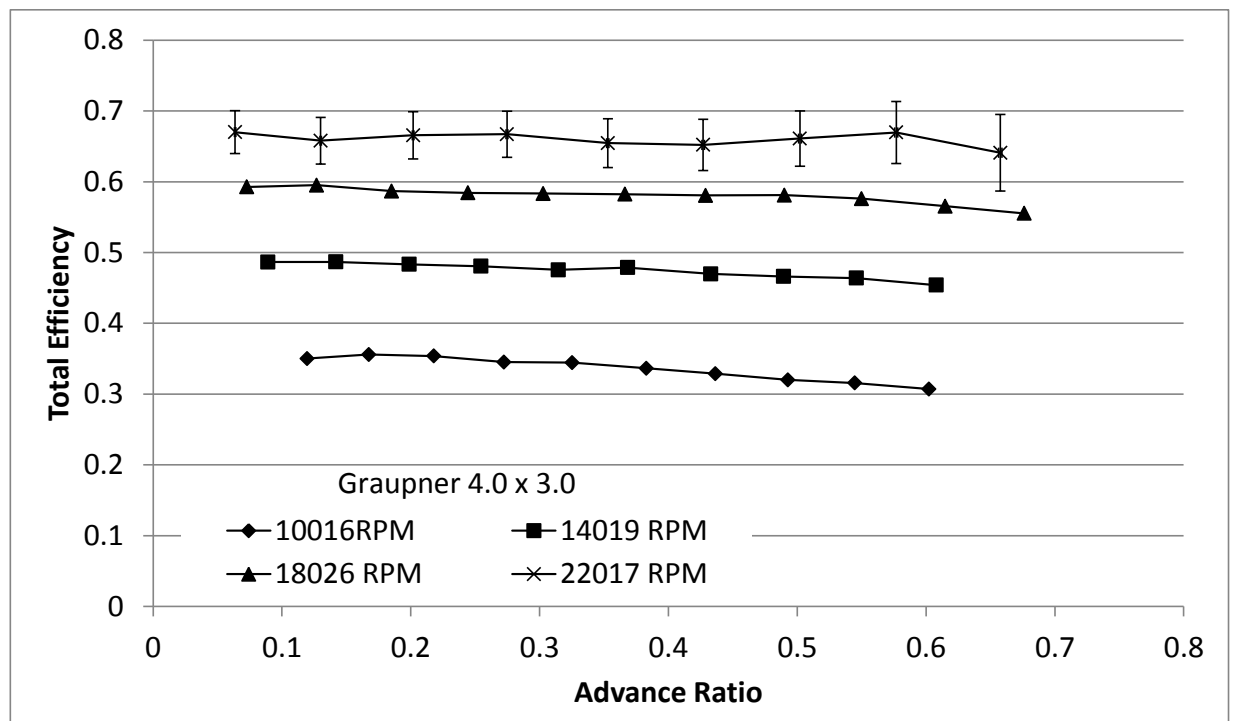

(d)

Figure 93: Graupner 4.0 x 3.0 Dynamic Test Results: (a) Coefficient of Thrust, (b) Coefficient of Power, (c) Propeller Efficiency, (d) Total Efficiency.

Table 194: Graupner 4.0 x 3.0 Dynamic Measured Values - 10016 RPM

\begin{tabular}{|c|c|c|c|c|c|c|c|}
\hline$n(\mathrm{RPM})$ & $Q(\mathrm{~g}-\mathrm{m})$ & $\Delta Q(\mathrm{~g}-\mathrm{m})$ & $V(\mathrm{~V})$ & $I(\mathrm{~A})$ & $T_{\text {atm }}\left({ }^{\circ} \mathrm{C}\right)$ & $P_{\text {atm }}(\mathrm{Pa})$ & $P_{\text {diff }}(\mathrm{Pa})$ \\
\hline $9.902 \mathrm{E}+03$ & $3.941 \mathrm{E}-01$ & $5.56 \mathrm{E}-02$ & $1.109 \mathrm{E}+01$ & $1.032 \mathrm{E}+00$ & $2.274 \mathrm{E}+01$ & $9.849 \mathrm{E}+04$ & $2.360 \mathrm{E}+00$ \\
\hline $1.011 \mathrm{E}+04$ & $4.032 \mathrm{E}-01$ & $5.74 \mathrm{E}-02$ & $1.109 \mathrm{E}+01$ & $1.061 \mathrm{E}+00$ & $2.258 \mathrm{E}+01$ & $9.850 \mathrm{E}+04$ & $4.760 \mathrm{E}+00$ \\
\hline $1.013 \mathrm{E}+04$ & $4.005 \mathrm{E}-01$ & $5.63 \mathrm{E}-02$ & $1.109 \mathrm{E}+01$ & $1.063 \mathrm{E}+00$ & $2.257 \mathrm{E}+01$ & $9.850 \mathrm{E}+04$ & $8.034 \mathrm{E}+00$ \\
\hline $1.002 \mathrm{E}+04$ & $3.800 \mathrm{E}-01$ & $5.54 \mathrm{E}-02$ & $1.109 \mathrm{E}+01$ & $1.022 \mathrm{E}+00$ & $2.260 \mathrm{E}+01$ & $9.851 \mathrm{E}+04$ & $1.220 \mathrm{E}+01$ \\
\hline $1.004 \mathrm{E}+04$ & $3.738 \mathrm{E}-01$ & $5.73 \mathrm{E}-02$ & $1.109 \mathrm{E}+01$ & $1.010 \mathrm{E}+00$ & $2.252 \mathrm{E}+01$ & $9.850 \mathrm{E}+04$ & $1.741 \mathrm{E}+01$ \\
\hline $9.976 \mathrm{E}+03$ & $3.569 \mathrm{E}-01$ & $5.58 \mathrm{E}-02$ & $1.109 \mathrm{E}+01$ & $9.801 \mathrm{E}-01$ & $2.231 \mathrm{E}+01$ & $9.851 \mathrm{E}+04$ & $2.374 \mathrm{E}+01$ \\
\hline $1.004 \mathrm{E}+04$ & $3.432 \mathrm{E}-01$ & $5.66 \mathrm{E}-02$ & $1.109 \mathrm{E}+01$ & $9.711 \mathrm{E}-01$ & $2.222 \mathrm{E}+01$ & $9.851 \mathrm{E}+04$ & $3.121 \mathrm{E}+01$ \\
\hline $9.937 \mathrm{E}+03$ & $3.212 \mathrm{E}-01$ & $5.51 \mathrm{E}-02$ & $1.109 \mathrm{E}+01$ & $9.234 \mathrm{E}-01$ & $2.223 \mathrm{E}+01$ & $9.852 \mathrm{E}+04$ & $3.888 \mathrm{E}+01$ \\
\hline $1.001 \mathrm{E}+04$ & $3.099 \mathrm{E}-01$ & $5.54 \mathrm{E}-02$ & $1.109 \mathrm{E}+01$ & $9.106 \mathrm{E}-01$ & $2.222 \mathrm{E}+01$ & $9.852 \mathrm{E}+04$ & $4.817 \mathrm{E}+01$ \\
\hline $9.984 \mathrm{E}+03$ & $2.906 \mathrm{E}-01$ & $5.44 \mathrm{E}-02$ & $1.109 \mathrm{E}+01$ & $8.748 \mathrm{E}-01$ & $2.224 \mathrm{E}+01$ & $9.852 \mathrm{E}+04$ & $5.845 \mathrm{E}+01$ \\
\hline
\end{tabular}


Table 195: Graupner 4.0 x 3.0 Dynamic Calculated Values - 10016 RPM

\begin{tabular}{|c|c|c|c|c|c|c|c|}
\hline$n(\mathrm{RPM})$ & $V_{\infty}^{\prime}(\mathrm{m} / \mathrm{s})$ & $\Delta V_{\infty}^{\prime}(\mathrm{m} / \mathrm{s})$ & $T^{\prime}(\mathrm{g})$ & $\Delta T^{\prime}(\mathrm{g})$ & $V_{\mathrm{t}}(\mathrm{m} / \mathrm{s})$ & $P_{\mathrm{P}}(\mathrm{W})$ & $\Delta P_{\mathrm{P}}(\mathrm{W})$ \\
\hline $9.902 \mathrm{E}+03$ & $1.970 \mathrm{E}+00$ & $1.23 \mathrm{E}-02$ & $3.907 \mathrm{E}+01$ & $8.52 \mathrm{E}+00$ & $3.890 \mathrm{E}+01$ & $4.007 \mathrm{E}+00$ & $5.65 \mathrm{E}-01$ \\
\hline $1.011 \mathrm{E}+04$ & $2.817 \mathrm{E}+00$ & $1.25 \mathrm{E}-02$ & $4.067 \mathrm{E}+01$ & $8.53 \mathrm{E}+00$ & $3.976 \mathrm{E}+01$ & $4.184 \mathrm{E}+00$ & $5.96 \mathrm{E}-01$ \\
\hline $1.013 \mathrm{E}+04$ & $3.677 \mathrm{E}+00$ & $1.30 \mathrm{E}-02$ & $3.870 \mathrm{E}+01$ & $8.56 \mathrm{E}+00$ & $3.994 \mathrm{E}+01$ & $4.168 \mathrm{E}+00$ & $5.86 \mathrm{E}-01$ \\
\hline $1.002 \mathrm{E}+04$ & $4.547 \mathrm{E}+00$ & $1.39 \mathrm{E}-02$ & $3.305 \mathrm{E}+01$ & $8.50 \mathrm{E}+00$ & $3.958 \mathrm{E}+01$ & $3.910 \mathrm{E}+00$ & $5.71 \mathrm{E}-01$ \\
\hline $1.004 \mathrm{E}+04$ & $5.440 \mathrm{E}+00$ & $1.50 \mathrm{E}-02$ & $3.232 \mathrm{E}+01$ & $8.52 \mathrm{E}+00$ & $3.979 \mathrm{E}+01$ & $3.856 \mathrm{E}+00$ & 5.92E-01 \\
\hline $9.976 \mathrm{E}+03$ & $6.362 \mathrm{E}+00$ & $1.63 \mathrm{E}-02$ & $2.916 \mathrm{E}+01$ & $8.53 \mathrm{E}+00$ & $3.966 \mathrm{E}+01$ & $3.656 \mathrm{E}+00$ & $5.72 \mathrm{E}-01$ \\
\hline $1.004 \mathrm{E}+04$ & $7.299 \mathrm{E}+00$ & $1.77 \mathrm{E}-02$ & $2.835 \mathrm{E}+01$ & $8.50 \mathrm{E}+00$ & $4.007 \mathrm{E}+01$ & $3.539 \mathrm{E}+00$ & $5.84 \mathrm{E}-01$ \\
\hline $9.937 \mathrm{E}+03$ & $8.156 \mathrm{E}+00$ & $1.89 \mathrm{E}-02$ & $2.371 \mathrm{E}+01$ & $8.53 \mathrm{E}+00$ & $3.984 \mathrm{E}+01$ & $3.278 \mathrm{E}+00$ & $5.62 \mathrm{E}-01$ \\
\hline $1.001 \mathrm{E}+04$ & $9.084 \mathrm{E}+00$ & $1.99 \mathrm{E}-02$ & $2.197 \mathrm{E}+01$ & $8.55 \mathrm{E}+00$ & $4.033 \mathrm{E}+01$ & $3.187 \mathrm{E}+00$ & $5.69 \mathrm{E}-01$ \\
\hline $9.984 \mathrm{E}+03$ & $1.001 \mathrm{E}+01$ & $2.13 \mathrm{E}-02$ & $1.811 \mathrm{E}+01$ & $8.55 \mathrm{E}+00$ & $4.043 \mathrm{E}+01$ & $2.979 \mathrm{E}+00$ & $5.57 \mathrm{E}-01$ \\
\hline$P_{\mathrm{e}}(\mathrm{W})$ & $\Delta P_{\mathrm{e}}(\mathrm{W})$ & $C_{\mathrm{T}}$ & $\Delta C_{\mathrm{T}}$ & $C_{\mathrm{P}}$ & $\Delta C_{\mathrm{P}}$ & $C_{\mathrm{Q}}$ & $\Delta C_{\mathrm{Q}}$ \\
\hline $1.145 \mathrm{E}+01$ & $1.43 \mathrm{E}-01$ & $1.217 \mathrm{E}-01$ & $2.65 \mathrm{E}-02$ & $7.718 \mathrm{E}-02$ & $1.09 \mathrm{E}-02$ & $1.228 \mathrm{E}-02$ & $1.73 \mathrm{E}-03$ \\
\hline $1.176 \mathrm{E}+01$ & $1.49 \mathrm{E}-01$ & $1.215 \mathrm{E}-01$ & $2.55 \mathrm{E}-02$ & $7.576 \mathrm{E}-02$ & $1.08 \mathrm{E}-02$ & $1.206 \mathrm{E}-02$ & $1.72 \mathrm{E}-03$ \\
\hline $1.178 \mathrm{E}+01$ & $1.47 \mathrm{E}-01$ & $1.150 \mathrm{E}-01$ & $2.54 \mathrm{E}-02$ & 7.484E-02 & $1.05 \mathrm{E}-02$ & 1.191E-02 & $1.67 \mathrm{E}-03$ \\
\hline $1.133 \mathrm{E}+01$ & $1.41 \mathrm{E}-01$ & $1.005 \mathrm{E}-01$ & $2.58 \mathrm{E}-02$ & $7.263 \mathrm{E}-02$ & $1.06 \mathrm{E}-02$ & $1.156 \mathrm{E}-02$ & $1.69 \mathrm{E}-03$ \\
\hline 1.119E+01 & $1.40 \mathrm{E}-01$ & 9.777E-02 & $2.58 \mathrm{E}-02$ & $7.110 \mathrm{E}-02$ & 1.09E-02 & $1.132 \mathrm{E}-02$ & $1.74 \mathrm{E}-03$ \\
\hline $1.087 \mathrm{E}+01$ & $1.37 \mathrm{E}-01$ & $8.934 \mathrm{E}-02$ & $2.61 \mathrm{E}-02$ & $6.877 \mathrm{E}-02$ & $1.08 \mathrm{E}-02$ & $1.095 \mathrm{E}-02$ & $1.71 \mathrm{E}-03$ \\
\hline $1.077 \mathrm{E}+01$ & $1.34 \mathrm{E}-01$ & $8.570 \mathrm{E}-02$ & $2.57 \mathrm{E}-02$ & $6.523 \mathrm{E}-02$ & $1.08 \mathrm{E}-02$ & $1.038 \mathrm{E}-02$ & $1.71 \mathrm{E}-03$ \\
\hline $1.024 \mathrm{E}+01$ & $1.30 \mathrm{E}-01$ & 7.319E-02 & $2.63 \mathrm{E}-02$ & $6.235 \mathrm{E}-02$ & $1.07 \mathrm{E}-02$ & $9.923 \mathrm{E}-03$ & $1.70 \mathrm{E}-03$ \\
\hline $1.010 \mathrm{E}+01$ & $1.29 \mathrm{E}-01$ & $6.679 \mathrm{E}-02$ & $2.60 \mathrm{E}-02$ & $5.924 \mathrm{E}-02$ & $1.06 \mathrm{E}-02$ & $9.429 \mathrm{E}-03$ & $1.68 \mathrm{E}-03$ \\
\hline $9.702 \mathrm{E}+00$ & $1.23 \mathrm{E}-01$ & $5.537 \mathrm{E}-02$ & $2.62 \mathrm{E}-02$ & $5.587 \mathrm{E}-02$ & $1.05 \mathrm{E}-02$ & $8.893 \mathrm{E}-03$ & $1.66 \mathrm{E}-03$ \\
\hline$\rho\left(\mathrm{kg} / \mathrm{m}^{\wedge} 3\right)$ & $\eta_{\mathrm{T}}$ & $\Delta \eta_{\mathrm{T}}$ & $J$ & $\Delta J$ & $\eta_{\mathrm{P}}$ & $\Delta \eta_{\mathrm{P}}$ & $R e_{0.75}$ \\
\hline $1.160 \mathrm{E}+00$ & $3.501 \mathrm{E}-01$ & $4.96 \mathrm{E}-02$ & $1.195 \mathrm{E}-01$ & $7.49 \mathrm{E}-04$ & $1.884 \mathrm{E}-01$ & $4.90 \mathrm{E}-02$ & $2.341 \mathrm{E}+04$ \\
\hline $1.160 \mathrm{E}+00$ & $3.558 \mathrm{E}-01$ & $5.09 \mathrm{E}-02$ & $1.674 \mathrm{E}-01$ & $7.45 \mathrm{E}-04$ & $2.685 \mathrm{E}-01$ & $6.81 \mathrm{E}-02$ & $2.395 \mathrm{E}+04$ \\
\hline $1.160 \mathrm{E}+00$ & $3.538 \mathrm{E}-01$ & $4.99 \mathrm{E}-02$ & $2.178 \mathrm{E}-01$ & 7.77E-04 & $3.348 \mathrm{E}-01$ & $8.77 \mathrm{E}-02$ & $2.406 \mathrm{E}+04$ \\
\hline $1.160 \mathrm{E}+00$ & $3.453 \mathrm{E}-01$ & $5.06 \mathrm{E}-02$ & $2.724 \mathrm{E}-01$ & $8.38 \mathrm{E}-04$ & $3.769 \mathrm{E}-01$ & $1.11 \mathrm{E}-01$ & $2.384 \mathrm{E}+04$ \\
\hline $1.161 \mathrm{E}+00$ & $3.444 \mathrm{E}-01$ & $5.30 \mathrm{E}-02$ & $3.252 \mathrm{E}-01$ & $9.08 \mathrm{E}-04$ & 4.472E-01 & $1.36 \mathrm{E}-01$ & $2.398 \mathrm{E}+04$ \\
\hline $1.161 \mathrm{E}+00$ & $3.365 \mathrm{E}-01$ & $5.28 \mathrm{E}-02$ & 3.829E-01 & $9.90 \mathrm{E}-04$ & 4.974E-01 & $1.65 \mathrm{E}-01$ & $2.393 \mathrm{E}+04$ \\
\hline $1.162 \mathrm{E}+00$ & $3.287 \mathrm{E}-01$ & $5.43 \mathrm{E}-02$ & $4.365 \mathrm{E}-01$ & $1.07 \mathrm{E}-03$ & $5.734 \mathrm{E}-01$ & $1.96 \mathrm{E}-01$ & $2.420 \mathrm{E}+04$ \\
\hline $1.162 \mathrm{E}+00$ & $3.201 \mathrm{E}-01$ & $5.51 \mathrm{E}-02$ & $4.928 \mathrm{E}-01$ & $1.16 \mathrm{E}-03$ & $5.785 \mathrm{E}-01$ & $2.31 \mathrm{E}-01$ & $2.405 \mathrm{E}+04$ \\
\hline $1.162 \mathrm{E}+00$ & $3.156 \mathrm{E}-01$ & $5.65 \mathrm{E}-02$ & $5.447 \mathrm{E}-01$ & $1.22 \mathrm{E}-03$ & $6.142 \mathrm{E}-01$ & $2.63 \mathrm{E}-01$ & $2.435 \mathrm{E}+04$ \\
\hline $1.162 \mathrm{E}+00$ & $3.071 \mathrm{E}-01$ & $5.76 \mathrm{E}-02$ & $6.022 \mathrm{E}-01$ & $1.30 \mathrm{E}-03$ & $5.968 \mathrm{E}-01$ & 3.03E-01 & $2.441 \mathrm{E}+04$ \\
\hline
\end{tabular}


Table 196: Graupner 4.0 x 3.0 Dynamic Measured Values - 14019 RPM

\begin{tabular}{|c|c|c|c|c|c|c|c|}
\hline$n(\mathrm{RPM})$ & $Q(\mathrm{~g}-\mathrm{m})$ & $\Delta Q(\mathrm{~g}-\mathrm{m})$ & $V(\mathrm{~V})$ & $I(\mathrm{~A})$ & $T_{\text {atm }}\left({ }^{\circ} \mathrm{C}\right)$ & $P_{\text {atm }}(\mathrm{Pa})$ & $P_{\text {diff }}(\mathrm{Pa})$ \\
\hline $1.405 \mathrm{E}+04$ & $7.965 \mathrm{E}-01$ & $5.90 \mathrm{E}-02$ & $1.105 \mathrm{E}+01$ & $2.136 \mathrm{E}+00$ & $2.191 \mathrm{E}+01$ & $9.852 \mathrm{E}+04$ & $2.696 \mathrm{E}+00$ \\
\hline $1.408 \mathrm{E}+04$ & $7.941 \mathrm{E}-01$ & $5.94 \mathrm{E}-02$ & $1.105 \mathrm{E}+01$ & $2.134 \mathrm{E}+00$ & $2.177 \mathrm{E}+01$ & $9.853 \mathrm{E}+04$ & $6.701 \mathrm{E}+00$ \\
\hline $1.395 \mathrm{E}+04$ & $7.741 \mathrm{E}-01$ & $5.87 \mathrm{E}-02$ & $1.106 \mathrm{E}+01$ & $2.075 \mathrm{E}+00$ & $2.179 \mathrm{E}+01$ & $9.853 \mathrm{E}+04$ & $1.276 \mathrm{E}+01$ \\
\hline $1.402 \mathrm{E}+04$ & $7.676 \mathrm{E}-01$ & $5.91 \mathrm{E}-02$ & $1.106 \mathrm{E}+01$ & $2.079 \mathrm{E}+00$ & $2.185 \mathrm{E}+01$ & $9.853 \mathrm{E}+04$ & $2.096 \mathrm{E}+01$ \\
\hline $1.394 \mathrm{E}+04$ & $7.394 \mathrm{E}-01$ & $5.87 \mathrm{E}-02$ & $1.106 \mathrm{E}+01$ & $2.013 \mathrm{E}+00$ & $2.196 \mathrm{E}+01$ & $9.853 \mathrm{E}+04$ & $3.149 \mathrm{E}+01$ \\
\hline $1.407 \mathrm{E}+04$ & $7.348 \mathrm{E}-01$ & $5.84 \mathrm{E}-02$ & $1.106 \mathrm{E}+01$ & $2.006 \mathrm{E}+00$ & $2.204 \mathrm{E}+01$ & $9.853 \mathrm{E}+04$ & $4.382 \mathrm{E}+01$ \\
\hline $1.392 \mathrm{E}+04$ & $6.867 \mathrm{E}-01$ & $5.83 \mathrm{E}-02$ & $1.106 \mathrm{E}+01$ & $1.889 \mathrm{E}+00$ & $2.207 \mathrm{E}+01$ & $9.853 \mathrm{E}+04$ & $5.903 \mathrm{E}+01$ \\
\hline $1.402 \mathrm{E}+04$ & $6.596 \mathrm{E}-01$ & $5.94 \mathrm{E}-02$ & $1.106 \mathrm{E}+01$ & $1.841 \mathrm{E}+00$ & $2.212 \mathrm{E}+01$ & $9.852 \mathrm{E}+04$ & $7.637 \mathrm{E}+01$ \\
\hline $1.410 \mathrm{E}+04$ & $6.316 \mathrm{E}-01$ & $5.96 \mathrm{E}-02$ & $1.106 \mathrm{E}+01$ & $1.781 \mathrm{E}+00$ & $2.213 \mathrm{E}+01$ & $9.853 \mathrm{E}+04$ & $9.595 \mathrm{E}+01$ \\
\hline $1.404 \mathrm{E}+04$ & $5.816 \mathrm{E}-01$ & $5.89 \mathrm{E}-02$ & $1.107 \mathrm{E}+01$ & $1.669 \mathrm{E}+00$ & $2.213 \mathrm{E}+01$ & $9.853 \mathrm{E}+04$ & $1.179 \mathrm{E}+02$ \\
\hline
\end{tabular}


Table 197: Graupner 4.0 x 3.0 Dynamic Calculated Values - 14019 RPM

\begin{tabular}{|c|c|c|c|c|c|c|c|}
\hline$n(\mathrm{RPM})$ & $V_{\infty}^{\prime}(\mathrm{m} / \mathrm{s})$ & $\Delta V_{\infty}^{\prime}(\mathrm{m} / \mathrm{s})$ & $T^{\prime}(\mathrm{g})$ & $\Delta T^{\prime}(\mathrm{g})$ & $V_{\mathrm{t}}(\mathrm{m} / \mathrm{s})$ & $P_{\mathrm{P}}(\mathrm{W})$ & $\Delta P_{\mathrm{P}}(\mathrm{W})$ \\
\hline $1.405 \mathrm{E}+04$ & $2.082 \mathrm{E}+00$ & $1.42 \mathrm{E}-02$ & $8.543 \mathrm{E}+01$ & $8.22 \mathrm{E}+00$ & $5.515 \mathrm{E}+01$ & $1.149 \mathrm{E}+01$ & $8.51 \mathrm{E}-01$ \\
\hline $1.408 \mathrm{E}+04$ & $3.325 \mathrm{E}+00$ & $1.43 \mathrm{E}-02$ & $8.211 \mathrm{E}+01$ & $8.25 \mathrm{E}+00$ & $5.534 \mathrm{E}+01$ & $1.148 \mathrm{E}+01$ & $8.58 \mathrm{E}-01$ \\
\hline $1.395 \mathrm{E}+04$ & $4.619 \mathrm{E}+00$ & $1.51 \mathrm{E}-02$ & $7.698 \mathrm{E}+01$ & $8.19 \mathrm{E}+00$ & $5.494 \mathrm{E}+01$ & $1.109 \mathrm{E}+01$ & $8.41 \mathrm{E}-01$ \\
\hline $1.402 \mathrm{E}+04$ & $5.943 \mathrm{E}+00$ & $1.66 \mathrm{E}-02$ & $7.269 \mathrm{E}+01$ & $8.22 \mathrm{E}+00$ & $5.533 \mathrm{E}+01$ & $1.105 \mathrm{E}+01$ & $8.51 \mathrm{E}-01$ \\
\hline $1.394 \mathrm{E}+04$ & $7.305 \mathrm{E}+00$ & $1.84 \mathrm{E}-02$ & $6.738 \mathrm{E}+01$ & $8.18 \mathrm{E}+00$ & $5.518 \mathrm{E}+01$ & $1.058 \mathrm{E}+01$ & $8.40 \mathrm{E}-01$ \\
\hline $1.407 \mathrm{E}+04$ & $8.632 \mathrm{E}+00$ & $2.02 \mathrm{E}-02$ & $6.244 \mathrm{E}+01$ & $8.25 \mathrm{E}+00$ & $5.589 \mathrm{E}+01$ & $1.062 \mathrm{E}+01$ & 8.44E-01 \\
\hline $1.392 \mathrm{E}+04$ & $1.003 \mathrm{E}+01$ & $2.14 \mathrm{E}-02$ & $5.495 \mathrm{E}+01$ & $8.13 \mathrm{E}+00$ & $5.554 \mathrm{E}+01$ & $9.817 \mathrm{E}+00$ & 8.33E-01 \\
\hline $1.402 \mathrm{E}+04$ & $1.143 \mathrm{E}+01$ & $2.37 \mathrm{E}-02$ & $5.037 \mathrm{E}+01$ & $8.27 \mathrm{E}+00$ & $5.618 \mathrm{E}+01$ & $9.495 \mathrm{E}+00$ & $8.56 \mathrm{E}-01$ \\
\hline $1.410 \mathrm{E}+04$ & $1.282 \mathrm{E}+01$ & $2.64 \mathrm{E}-02$ & $4.388 \mathrm{E}+01$ & $8.27 \mathrm{E}+00$ & $5.678 \mathrm{E}+01$ & $9.143 \mathrm{E}+00$ & 8.63E-01 \\
\hline $1.404 \mathrm{E}+04$ & $1.422 \mathrm{E}+01$ & $2.87 \mathrm{E}-02$ & $3.760 \mathrm{E}+01$ & $8.19 \mathrm{E}+00$ & $5.691 \mathrm{E}+01$ & $8.388 \mathrm{E}+00$ & $8.49 \mathrm{E}-01$ \\
\hline$P_{\mathrm{e}}(\mathrm{W})$ & $\Delta P_{\mathrm{e}}(\mathrm{W})$ & $C_{\mathrm{T}}$ & $\Delta C_{\mathrm{T}}$ & $C_{\mathrm{P}}$ & $\Delta C_{\mathrm{P}}$ & $C_{\mathrm{Q}}$ & $\Delta C_{\mathrm{Q}}$ \\
\hline $2.361 \mathrm{E}+01$ & $2.70 \mathrm{E}-01$ & $1.318 \mathrm{E}-01$ & $1.27 \mathrm{E}-02$ & $7.730 \mathrm{E}-02$ & $5.72 \mathrm{E}-03$ & $1.230 \mathrm{E}-02$ & $9.11 \mathrm{E}-04$ \\
\hline $2.359 \mathrm{E}+01$ & $2.70 \mathrm{E}-01$ & $1.261 \mathrm{E}-01$ & $1.27 \mathrm{E}-02$ & $7.667 \mathrm{E}-02$ & $5.73 \mathrm{E}-03$ & $1.220 \mathrm{E}-02$ & $9.12 \mathrm{E}-04$ \\
\hline $2.294 \mathrm{E}+01$ & $2.62 \mathrm{E}-01$ & $1.204 \mathrm{E}-01$ & $1.28 \mathrm{E}-02$ & 7.612E-02 & $5.78 \mathrm{E}-03$ & $1.211 \mathrm{E}-02$ & $9.19 \mathrm{E}-04$ \\
\hline $2.299 \mathrm{E}+01$ & 2.63E-01 & $1.126 \mathrm{E}-01$ & $1.27 \mathrm{E}-02$ & $7.475 \mathrm{E}-02$ & $5.76 \mathrm{E}-03$ & $1.190 \mathrm{E}-02$ & $9.16 \mathrm{E}-04$ \\
\hline $2.226 \mathrm{E}+01$ & $2.56 \mathrm{E}-01$ & $1.056 \mathrm{E}-01$ & $1.28 \mathrm{E}-02$ & $7.287 \mathrm{E}-02$ & $5.78 \mathrm{E}-03$ & $1.160 \mathrm{E}-02$ & $9.21 \mathrm{E}-04$ \\
\hline $2.218 \mathrm{E}+01$ & $2.55 \mathrm{E}-01$ & $9.605 \mathrm{E}-02$ & $1.27 \mathrm{E}-02$ & $7.108 \mathrm{E}-02$ & $5.65 \mathrm{E}-03$ & $1.131 \mathrm{E}-02$ & 8.99E-04 \\
\hline $2.090 \mathrm{E}+01$ & $2.40 \mathrm{E}-01$ & $8.636 \mathrm{E}-02$ & $1.28 \mathrm{E}-02$ & $6.787 \mathrm{E}-02$ & $5.76 \mathrm{E}-03$ & $1.080 \mathrm{E}-02$ & $9.17 \mathrm{E}-04$ \\
\hline $2.037 \mathrm{E}+01$ & $2.37 \mathrm{E}-01$ & $7.809 \mathrm{E}-02$ & $1.28 \mathrm{E}-02$ & $6.430 \mathrm{E}-02$ & $5.80 \mathrm{E}-03$ & $1.023 \mathrm{E}-02$ & $9.22 \mathrm{E}-04$ \\
\hline $1.971 \mathrm{E}+01$ & 2.33E-01 & $6.727 \mathrm{E}-02$ & $1.27 \mathrm{E}-02$ & $6.089 \mathrm{E}-02$ & $5.75 \mathrm{E}-03$ & $9.691 \mathrm{E}-03$ & $9.15 \mathrm{E}-04$ \\
\hline $1.848 \mathrm{E}+01$ & $2.18 \mathrm{E}-01$ & $5.808 \mathrm{E}-02$ & $1.26 \mathrm{E}-02$ & $5.650 \mathrm{E}-02$ & $5.72 \mathrm{E}-03$ & $8.992 \mathrm{E}-03$ & $9.10 \mathrm{E}-04$ \\
\hline$\rho\left(\mathrm{kg} / \mathrm{m}^{\wedge} 3\right)$ & $\eta_{\mathrm{T}}$ & $\Delta \eta_{\mathrm{T}}$ & $J$ & $\Delta J$ & $\eta_{\mathrm{P}}$ & $\Delta \eta_{\mathrm{P}}$ & $R e_{0.75}$ \\
\hline $1.163 \mathrm{E}+00$ & 4.867E-01 & $3.65 \mathrm{E}-02$ & 8.902E-02 & $6.09 \mathrm{E}-04$ & $1.518 \mathrm{E}-01$ & $1.85 \mathrm{E}-02$ & $3.337 \mathrm{E}+04$ \\
\hline $1.164 \mathrm{E}+00$ & $4.868 \mathrm{E}-01$ & $3.68 \mathrm{E}-02$ & $1.418 \mathrm{E}-01$ & $6.15 \mathrm{E}-04$ & 2.332E-01 & $2.92 \mathrm{E}-02$ & $3.351 \mathrm{E}+04$ \\
\hline $1.164 \mathrm{E}+00$ & 4.834E-01 & $3.71 \mathrm{E}-02$ & $1.988 \mathrm{E}-01$ & $6.60 \mathrm{E}-04$ & $3.144 \mathrm{E}-01$ & 4.11E-02 & $3.326 \mathrm{E}+04$ \\
\hline $1.164 \mathrm{E}+00$ & 4.807E-01 & $3.74 \mathrm{E}-02$ & $2.545 \mathrm{E}-01$ & $7.26 \mathrm{E}-04$ & 3.833E-01 & $5.25 \mathrm{E}-02$ & $3.349 \mathrm{E}+04$ \\
\hline $1.163 \mathrm{E}+00$ & $4.755 \mathrm{E}-01$ & $3.81 \mathrm{E}-02$ & $3.147 \mathrm{E}-01$ & $8.10 \mathrm{E}-04$ & $4.560 \mathrm{E}-01$ & $6.61 \mathrm{E}-02$ & $3.337 \mathrm{E}+04$ \\
\hline $1.163 \mathrm{E}+00$ & $4.788 \mathrm{E}-01$ & $3.84 \mathrm{E}-02$ & $3.684 \mathrm{E}-01$ & $8.85 \mathrm{E}-04$ & $4.978 \mathrm{E}-01$ & $7.68 \mathrm{E}-02$ & $3.378 \mathrm{E}+04$ \\
\hline $1.163 \mathrm{E}+00$ & 4.697E-01 & $4.02 \mathrm{E}-02$ & 4.328E-01 & $9.59 \mathrm{E}-04$ & $5.508 \mathrm{E}-01$ & $9.40 \mathrm{E}-02$ & $3.357 \mathrm{E}+04$ \\
\hline $1.162 \mathrm{E}+00$ & 4.662E-01 & 4.24E-02 & 4.894E-01 & $1.06 \mathrm{E}-03$ & 5.944E-01 & $1.11 \mathrm{E}-01$ & $3.395 \mathrm{E}+04$ \\
\hline $1.162 \mathrm{E}+00$ & $4.640 \mathrm{E}-01$ & $4.41 \mathrm{E}-02$ & $5.460 \mathrm{E}-01$ & $1.17 \mathrm{E}-03$ & $6.032 \mathrm{E}-01$ & $1.27 \mathrm{E}-01$ & $3.431 \mathrm{E}+04$ \\
\hline $1.162 \mathrm{E}+00$ & $4.540 \mathrm{E}-01$ & 4.63E-02 & $6.079 \mathrm{E}-01$ & $1.28 \mathrm{E}-03$ & $6.249 \mathrm{E}-01$ & $1.50 \mathrm{E}-01$ & $3.439 \mathrm{E}+04$ \\
\hline
\end{tabular}


Table 198: Graupner 4.0 x 3.0 Dynamic Measured Values -18026 RPM

\begin{tabular}{|c|c|c|c|c|c|c|c|}
\hline$n(\mathrm{RPM})$ & $Q(\mathrm{~g}-\mathrm{m})$ & $\Delta Q(\mathrm{~g}-\mathrm{m})$ & $V(\mathrm{~V})$ & $I(\mathrm{~A})$ & $T_{\text {atm }}\left({ }^{\circ} \mathrm{C}\right)$ & $P_{\text {atm }}(\mathrm{Pa})$ & $P_{\text {diff }}(\mathrm{Pa})$ \\
\hline $1.803 \mathrm{E}+04$ & $1.345 \mathrm{E}+00$ & $6.94 \mathrm{E}-02$ & $1.100 \mathrm{E}+01$ & $3.817 \mathrm{E}+00$ & $2.170 \mathrm{E}+01$ & $9.854 \mathrm{E}+04$ & $3.018 \mathrm{E}+00$ \\
\hline $1.807 \mathrm{E}+04$ & $1.345 \mathrm{E}+00$ & $6.88 \mathrm{E}-02$ & $1.100 \mathrm{E}+01$ & $3.812 \mathrm{E}+00$ & $2.163 \mathrm{E}+01$ & $9.854 \mathrm{E}+04$ & $8.909 \mathrm{E}+00$ \\
\hline $1.799 \mathrm{E}+04$ & $1.309 \mathrm{E}+00$ & $6.75 \mathrm{E}-02$ & $1.101 \mathrm{E}+01$ & $3.745 \mathrm{E}+00$ & $2.171 \mathrm{E}+01$ & $9.855 \mathrm{E}+04$ & $1.845 \mathrm{E}+01$ \\
\hline $1.802 \mathrm{E}+04$ & $1.298 \mathrm{E}+00$ & $6.79 \mathrm{E}-02$ & $1.101 \mathrm{E}+01$ & $3.734 \mathrm{E}+00$ & $2.188 \mathrm{E}+01$ & $9.855 \mathrm{E}+04$ & $3.200 \mathrm{E}+01$ \\
\hline $1.808 \mathrm{E}+04$ & $1.283 \mathrm{E}+00$ & $6.88 \mathrm{E}-02$ & $1.101 \mathrm{E}+01$ & $3.708 \mathrm{E}+00$ & $2.198 \mathrm{E}+01$ & $9.855 \mathrm{E}+04$ & $4.915 \mathrm{E}+01$ \\
\hline $1.801 \mathrm{E}+04$ & $1.234 \mathrm{E}+00$ & $6.77 \mathrm{E}-02$ & $1.101 \mathrm{E}+01$ & $3.557 \mathrm{E}+00$ & $2.203 \mathrm{E}+01$ & $9.855 \mathrm{E}+04$ & $7.098 \mathrm{E}+01$ \\
\hline $1.798 \mathrm{E}+04$ & $1.176 \mathrm{E}+00$ & $6.71 \mathrm{E}-02$ & $1.102 \mathrm{E}+01$ & $3.395 \mathrm{E}+00$ & $2.211 \mathrm{E}+01$ & $9.856 \mathrm{E}+04$ & $9.671 \mathrm{E}+01$ \\
\hline $1.802 \mathrm{E}+04$ & $1.121 \mathrm{E}+00$ & $6.75 \mathrm{E}-02$ & $1.102 \mathrm{E}+01$ & $3.239 \mathrm{E}+00$ & $2.220 \mathrm{E}+01$ & $9.856 \mathrm{E}+04$ & $1.266 \mathrm{E}+02$ \\
\hline $1.809 \mathrm{E}+04$ & $1.048 \mathrm{E}+00$ & $6.85 \mathrm{E}-02$ & $1.103 \mathrm{E}+01$ & $3.065 \mathrm{E}+00$ & $2.226 \mathrm{E}+01$ & $9.856 \mathrm{E}+04$ & $1.603 \mathrm{E}+02$ \\
\hline $1.799 \mathrm{E}+04$ & $9.491 \mathrm{E}-01$ & $6.70 \mathrm{E}-02$ & $1.103 \mathrm{E}+01$ & $2.810 \mathrm{E}+00$ & $2.225 \mathrm{E}+01$ & $9.856 \mathrm{E}+04$ & $1.980 \mathrm{E}+02$ \\
\hline $1.800 \mathrm{E}+04$ & $8.578 \mathrm{E}-01$ & $6.66 \mathrm{E}-02$ & $1.104 \mathrm{E}+01$ & $2.586 \mathrm{E}+00$ & $2.237 \mathrm{E}+01$ & $9.856 \mathrm{E}+04$ & $2.392 \mathrm{E}+02$ \\
\hline
\end{tabular}


Table 199: Graupner 4.0 x 3.0 Dynamic Calculated Values - 18026 RPM

\begin{tabular}{|c|c|c|c|c|c|c|c|}
\hline$n(\mathrm{RPM})$ & $V_{\infty}^{\prime}(\mathrm{m} / \mathrm{s})$ & $\Delta V_{\infty}^{\prime}(\mathrm{m} / \mathrm{s})$ & $T^{\prime}(\mathrm{g})$ & $\Delta T^{\prime}(\mathrm{g})$ & $V_{\mathrm{t}}(\mathrm{m} / \mathrm{s})$ & $P_{\mathrm{P}}(\mathrm{W})$ & $\Delta P_{\mathrm{P}}(\mathrm{W})$ \\
\hline $1.803 \mathrm{E}+04$ & $2.186 \mathrm{E}+00$ & $1.64 \mathrm{E}-02$ & $1.423 \mathrm{E}+02$ & $7.09 \mathrm{E}+00$ & $7.079 \mathrm{E}+01$ & $2.490 \mathrm{E}+01$ & $1.29 \mathrm{E}+00$ \\
\hline $1.807 \mathrm{E}+04$ & $3.823 \mathrm{E}+00$ & $1.69 \mathrm{E}-02$ & $1.389 \mathrm{E}+02$ & $7.14 \mathrm{E}+00$ & $7.102 \mathrm{E}+01$ & $2.497 \mathrm{E}+01$ & $1.28 \mathrm{E}+00$ \\
\hline $1.799 \mathrm{E}+04$ & $5.545 \mathrm{E}+00$ & $1.82 \mathrm{E}-02$ & $1.321 \mathrm{E}+02$ & $7.13 \mathrm{E}+00$ & $7.081 \mathrm{E}+01$ & $2.419 \mathrm{E}+01$ & $1.25 \mathrm{E}+00$ \\
\hline $1.802 \mathrm{E}+04$ & $7.337 \mathrm{E}+00$ & $2.03 \mathrm{E}-02$ & $1.258 \mathrm{E}+02$ & $7.09 \mathrm{E}+00$ & $7.110 \mathrm{E}+01$ & $2.402 \mathrm{E}+01$ & $1.26 \mathrm{E}+00$ \\
\hline $1.808 \mathrm{E}+04$ & $9.119 \mathrm{E}+00$ & $2.27 \mathrm{E}-02$ & $1.188 \mathrm{E}+02$ & $7.07 \mathrm{E}+00$ & $7.152 \mathrm{E}+01$ & $2.381 \mathrm{E}+01$ & $1.28 \mathrm{E}+00$ \\
\hline $1.801 \mathrm{E}+04$ & $1.098 \mathrm{E}+01$ & $2.48 \mathrm{E}-02$ & $1.071 \mathrm{E}+02$ & $7.07 \mathrm{E}+00$ & $7.151 \mathrm{E}+01$ & $2.282 \mathrm{E}+01$ & $1.25 \mathrm{E}+00$ \\
\hline $1.798 \mathrm{E}+04$ & $1.284 \mathrm{E}+01$ & $2.76 \mathrm{E}-02$ & $9.641 \mathrm{E}+01$ & $7.04 \mathrm{E}+00$ & $7.171 \mathrm{E}+01$ & $2.172 \mathrm{E}+01$ & $1.24 \mathrm{E}+00$ \\
\hline $1.802 \mathrm{E}+04$ & $1.471 \mathrm{E}+01$ & $3.01 \mathrm{E}-02$ & $8.526 \mathrm{E}+01$ & $7.06 \mathrm{E}+00$ & $7.223 \mathrm{E}+01$ & $2.075 \mathrm{E}+01$ & $1.25 \mathrm{E}+00$ \\
\hline $1.809 \mathrm{E}+04$ & $1.657 \mathrm{E}+01$ & $3.29 \mathrm{E}-02$ & $7.440 \mathrm{E}+01$ & $7.07 \mathrm{E}+00$ & $7.288 \mathrm{E}+01$ & $1.948 \mathrm{E}+01$ & $1.27 \mathrm{E}+00$ \\
\hline $1.799 \mathrm{E}+04$ & $1.843 \mathrm{E}+01$ & $3.60 \mathrm{E}-02$ & $6.176 \mathrm{E}+01$ & $7.06 \mathrm{E}+00$ & $7.297 \mathrm{E}+01$ & $1.754 \mathrm{E}+01$ & $1.24 \mathrm{E}+00$ \\
\hline $1.800 \mathrm{E}+04$ & $2.027 \mathrm{E}+01$ & $3.87 \mathrm{E}-02$ & $5.058 \mathrm{E}+01$ & $7.06 \mathrm{E}+00$ & $7.347 \mathrm{E}+01$ & $1.585 \mathrm{E}+01$ & $1.23 \mathrm{E}+00$ \\
\hline$P_{\mathrm{e}}(\mathrm{W})$ & $\Delta P_{\mathrm{e}}(\mathrm{W})$ & $C_{\mathrm{T}}$ & $\Delta C_{\mathrm{T}}$ & $C_{\mathrm{P}}$ & $\Delta C_{\mathrm{P}}$ & $C_{\mathrm{Q}}$ & $\Delta C_{\mathrm{Q}}$ \\
\hline $4.200 \mathrm{E}+01$ & $4.66 \mathrm{E}-01$ & $1.332 \mathrm{E}-01$ & $6.63 \mathrm{E}-03$ & $7.910 \mathrm{E}-02$ & $4.09 \mathrm{E}-03$ & $1.259 \mathrm{E}-02$ & $6.50 \mathrm{E}-04$ \\
\hline $4.195 \mathrm{E}+01$ & 4.67E-01 & $1.293 \mathrm{E}-01$ & $6.65 \mathrm{E}-03$ & $7.875 \mathrm{E}-02$ & $4.03 \mathrm{E}-03$ & $1.253 \mathrm{E}-02$ & $6.41 \mathrm{E}-04$ \\
\hline $4.123 \mathrm{E}+01$ & 4.54E-01 & $1.242 \mathrm{E}-01$ & $6.70 \mathrm{E}-03$ & $7.738 \mathrm{E}-02$ & 3.99E-03 & $1.232 \mathrm{E}-02$ & $6.35 \mathrm{E}-04$ \\
\hline $4.110 \mathrm{E}+01$ & $4.55 \mathrm{E}-01$ & $1.179 \mathrm{E}-01$ & $6.64 \mathrm{E}-03$ & $7.646 \mathrm{E}-02$ & $4.00 \mathrm{E}-03$ & $1.217 \mathrm{E}-02$ & $6.37 \mathrm{E}-04$ \\
\hline $4.082 \mathrm{E}+01$ & 4.54E-01 & $1.107 \mathrm{E}-01$ & $6.59 \mathrm{E}-03$ & 7.513E-02 & 4.03E-03 & $1.196 \mathrm{E}-02$ & $6.42 \mathrm{E}-04$ \\
\hline $3.918 \mathrm{E}+01$ & 4.33E-01 & $1.006 \mathrm{E}-01$ & $6.64 \mathrm{E}-03$ & 7.284E-02 & $4.00 \mathrm{E}-03$ & $1.159 \mathrm{E}-02$ & $6.36 \mathrm{E}-04$ \\
\hline $3.740 \mathrm{E}+01$ & $4.14 \mathrm{E}-01$ & $9.082 \mathrm{E}-02$ & $6.63 \mathrm{E}-03$ & $6.968 \mathrm{E}-02$ & $3.98 \mathrm{E}-03$ & $1.109 \mathrm{E}-02$ & $6.33 \mathrm{E}-04$ \\
\hline $3.570 \mathrm{E}+01$ & $3.96 \mathrm{E}-01$ & 7.997E-02 & $6.63 \mathrm{E}-03$ & $6.612 \mathrm{E}-02$ & $3.98 \mathrm{E}-03$ & $1.052 \mathrm{E}-02$ & $6.34 \mathrm{E}-04$ \\
\hline $3.380 \mathrm{E}+01$ & $3.80 \mathrm{E}-01$ & $6.929 \mathrm{E}-02$ & $6.58 \mathrm{E}-03$ & $6.140 \mathrm{E}-02$ & 4.01E-03 & $9.772 \mathrm{E}-03$ & $6.39 \mathrm{E}-04$ \\
\hline $3.101 \mathrm{E}+01$ & $3.50 \mathrm{E}-01$ & $5.812 \mathrm{E}-02$ & $6.64 \mathrm{E}-03$ & $5.616 \mathrm{E}-02$ & $3.96 \mathrm{E}-03$ & $8.939 \mathrm{E}-03$ & $6.31 \mathrm{E}-04$ \\
\hline $2.855 \mathrm{E}+01$ & $3.21 \mathrm{E}-01$ & $4.760 \mathrm{E}-02$ & $6.64 \mathrm{E}-03$ & $5.077 \mathrm{E}-02$ & $3.94 \mathrm{E}-03$ & 8.080E-03 & $6.27 \mathrm{E}-04$ \\
\hline$\rho\left(\mathrm{kg} / \mathrm{m}^{\wedge} 3\right)$ & $\eta_{\mathrm{T}}$ & $\Delta \eta_{\mathrm{T}}$ & $J$ & $\Delta J$ & $\eta_{\mathrm{P}}$ & $\Delta \eta_{\mathrm{P}}$ & $R e_{0.75}$ \\
\hline $1.164 \mathrm{E}+00$ & $5.928 \mathrm{E}-01$ & $3.13 \mathrm{E}-02$ & $7.278 \mathrm{E}-02$ & $5.47 \mathrm{E}-04$ & $1.225 \mathrm{E}-01$ & $8.84 \mathrm{E}-03$ & $4.289 \mathrm{E}+04$ \\
\hline $1.165 \mathrm{E}+00$ & 5.953E-01 & $3.12 \mathrm{E}-02$ & $1.270 \mathrm{E}-01$ & $5.63 \mathrm{E}-04$ & $2.086 \mathrm{E}-01$ & $1.52 \mathrm{E}-02$ & 4.305E+04 \\
\hline $1.164 \mathrm{E}+00$ & $5.867 \mathrm{E}-01$ & $3.09 \mathrm{E}-02$ & $1.851 \mathrm{E}-01$ & $6.10 \mathrm{E}-04$ & $2.970 \mathrm{E}-01$ & $2.22 \mathrm{E}-02$ & $4.290 \mathrm{E}+04$ \\
\hline $1.164 \mathrm{E}+00$ & 5.844E-01 & $3.13 \mathrm{E}-02$ & $2.445 \mathrm{E}-01$ & $6.83 \mathrm{E}-04$ & $3.768 \mathrm{E}-01$ & $2.90 \mathrm{E}-02$ & $4.303 \mathrm{E}+04$ \\
\hline $1.163 \mathrm{E}+00$ & 5.834E-01 & $3.20 \mathrm{E}-02$ & 3.029E-01 & 7.64E-04 & 4.461E-01 & $3.58 \mathrm{E}-02$ & $4.326 \mathrm{E}+04$ \\
\hline $1.163 \mathrm{E}+00$ & 5.824E-01 & $3.26 \mathrm{E}-02$ & 3.662E-01 & 8.38E-04 & $5.057 \mathrm{E}-01$ & 4.34E-02 & 4.324E+04 \\
\hline $1.163 \mathrm{E}+00$ & $5.808 \mathrm{E}-01$ & $3.38 \mathrm{E}-02$ & $4.288 \mathrm{E}-01$ & $9.25 \mathrm{E}-04$ & $5.589 \mathrm{E}-01$ & $5.18 \mathrm{E}-02$ & $4.335 \mathrm{E}+04$ \\
\hline $1.163 \mathrm{E}+00$ & $5.811 \mathrm{E}-01$ & $3.56 \mathrm{E}-02$ & 4.901E-01 & $1.03 \mathrm{E}-03$ & $5.927 \mathrm{E}-01$ & $6.07 \mathrm{E}-02$ & $4.364 \mathrm{E}+04$ \\
\hline $1.162 \mathrm{E}+00$ & 5.762E-01 & $3.82 \mathrm{E}-02$ & $5.500 \mathrm{E}-01$ & $1.12 \mathrm{E}-03$ & $6.207 \mathrm{E}-01$ & $7.16 \mathrm{E}-02$ & $4.402 \mathrm{E}+04$ \\
\hline $1.162 \mathrm{E}+00$ & $5.655 \mathrm{E}-01$ & 4.04E-02 & 6.149E-01 & $1.22 \mathrm{E}-03$ & 6.364E-01 & 8.55E-02 & $4.407 \mathrm{E}+04$ \\
\hline $1.162 \mathrm{E}+00$ & $5.553 \mathrm{E}-01$ & $4.36 \mathrm{E}-02$ & $6.762 \mathrm{E}-01$ & $1.31 \mathrm{E}-03$ & $6.341 \mathrm{E}-01$ & $1.01 \mathrm{E}-01$ & 4.434E+04 \\
\hline
\end{tabular}


Table 200: Graupner 4.0 x 3.0 Dynamic Measured Values - 22017 RPM

\begin{tabular}{|c|c|c|c|c|c|c|c|}
\hline$n(\mathrm{RPM})$ & $Q(\mathrm{~g}-\mathrm{m})$ & $\Delta Q(\mathrm{~g}-\mathrm{m})$ & $V(\mathrm{~V})$ & $I(\mathrm{~A})$ & $T_{\text {atm }}\left({ }^{\circ} \mathrm{C}\right)$ & $P_{\text {atm }}(\mathrm{Pa})$ & $P_{\text {diff }}(\mathrm{Pa})$ \\
\hline $2.212 \mathrm{E}+04$ & $2.053 \mathrm{E}+00$ & $8.99 \mathrm{E}-02$ & $1.093 \mathrm{E}+01$ & $6.367 \mathrm{E}+00$ & $2.173 \mathrm{E}+01$ & $9.857 \mathrm{E}+04$ & $3.511 \mathrm{E}+00$ \\
\hline $2.196 \mathrm{E}+04$ & $1.974 \mathrm{E}+00$ & $9.69 \mathrm{E}-02$ & $1.094 \mathrm{E}+01$ & $6.187 \mathrm{E}+00$ & $2.165 \mathrm{E}+01$ & $9.858 \mathrm{E}+04$ & $1.379 \mathrm{E}+01$ \\
\hline $2.201 \mathrm{E}+04$ & $1.985 \mathrm{E}+00$ & $9.71 \mathrm{E}-02$ & $1.094 \mathrm{E}+01$ & $6.162 \mathrm{E}+00$ & $2.182 \mathrm{E}+01$ & $9.858 \mathrm{E}+04$ & $3.273 \mathrm{E}+01$ \\
\hline $2.204 \mathrm{E}+04$ & $1.968 \mathrm{E}+00$ & $9.39 \mathrm{E}-02$ & $1.094 \mathrm{E}+01$ & $6.102 \mathrm{E}+00$ & $2.198 \mathrm{E}+01$ & $9.858 \mathrm{E}+04$ & $6.026 \mathrm{E}+01$ \\
\hline $2.191 \mathrm{E}+04$ & $1.855 \mathrm{E}+00$ & $9.60 \mathrm{E}-02$ & $1.095 \mathrm{E}+01$ & $5.827 \mathrm{E}+00$ & $2.204 \mathrm{E}+01$ & $9.858 \mathrm{E}+04$ & $9.776 \mathrm{E}+01$ \\
\hline $2.204 \mathrm{E}+04$ & $1.762 \mathrm{E}+00$ & $9.62 \mathrm{E}-02$ & $1.095 \mathrm{E}+01$ & $5.585 \mathrm{E}+00$ & $2.219 \mathrm{E}+01$ & $9.857 \mathrm{E}+04$ & $1.441 \mathrm{E}+02$ \\
\hline $2.207 \mathrm{E}+04$ & $1.650 \mathrm{E}+00$ & $9.57 \mathrm{E}-02$ & $1.097 \mathrm{E}+01$ & $5.157 \mathrm{E}+00$ & $2.224 \mathrm{E}+01$ & $9.858 \mathrm{E}+04$ & $1.992 \mathrm{E}+02$ \\
\hline $2.209 \mathrm{E}+04$ & $1.520 \mathrm{E}+00$ & $9.82 \mathrm{E}-02$ & $1.098 \mathrm{E}+01$ & $4.689 \mathrm{E}+00$ & $2.234 \mathrm{E}+01$ & $9.857 \mathrm{E}+04$ & $2.627 \mathrm{E}+02$ \\
\hline $2.191 \mathrm{E}+04$ & $1.277 \mathrm{E}+00$ & $1.07 \mathrm{E}-01$ & $1.100 \mathrm{E}+01$ & $4.075 \mathrm{E}+00$ & $2.240 \mathrm{E}+01$ & $9.858 \mathrm{E}+04$ & $3.355 \mathrm{E}+02$ \\
\hline
\end{tabular}


Table 201: Graupner 4.0 x 3.0 Dynamic Calculated Values - 22017 RPM

\begin{tabular}{|c|c|c|c|c|c|c|c|}
\hline$n(\mathrm{RPM})$ & $V_{\infty}^{\prime}(\mathrm{m} / \mathrm{s})$ & $\Delta V_{\infty}^{\prime}(\mathrm{m} / \mathrm{s})$ & $T^{\prime}(\mathrm{g})$ & $\Delta T^{\prime}(\mathrm{g})$ & $V_{\mathrm{t}}(\mathrm{m} / \mathrm{s})$ & $P_{\mathrm{P}}(\mathrm{W})$ & $\Delta P_{\mathrm{P}}(\mathrm{W})$ \\
\hline $2.212 \mathrm{E}+04$ & $2.342 \mathrm{E}+00$ & 1.89E-02 & $2.185 \mathrm{E}+02$ & $7.22 \mathrm{E}+00$ & $8.681 \mathrm{E}+01$ & $4.664 \mathrm{E}+01$ & $2.04 \mathrm{E}+00$ \\
\hline $2.196 \mathrm{E}+04$ & $4.758 \mathrm{E}+00$ & $1.99 \mathrm{E}-02$ & $2.083 \mathrm{E}+02$ & $7.28 \mathrm{E}+00$ & $8.631 \mathrm{E}+01$ & $4.452 \mathrm{E}+01$ & $2.19 \mathrm{E}+00$ \\
\hline $2.201 \mathrm{E}+04$ & $7.397 \mathrm{E}+00$ & $2.25 \mathrm{E}-02$ & $1.977 \mathrm{E}+02$ & $7.23 \mathrm{E}+00$ & $8.666 \mathrm{E}+01$ & $4.486 \mathrm{E}+01$ & $2.19 \mathrm{E}+00$ \\
\hline $2.204 \mathrm{E}+04$ & $1.008 \mathrm{E}+01$ & $2.67 \mathrm{E}-02$ & $1.856 \mathrm{E}+02$ & $7.18 \mathrm{E}+00$ & $8.707 \mathrm{E}+01$ & $4.453 \mathrm{E}+01$ & $2.13 \mathrm{E}+00$ \\
\hline $2.191 \mathrm{E}+04$ & $1.288 \mathrm{E}+01$ & $2.98 \mathrm{E}-02$ & $1.669 \mathrm{E}+02$ & $7.12 \mathrm{E}+00$ & $8.694 \mathrm{E}+01$ & $4.175 \mathrm{E}+01$ & $2.16 \mathrm{E}+00$ \\
\hline $2.204 \mathrm{E}+04$ & $1.567 \mathrm{E}+01$ & $3.29 \mathrm{E}-02$ & $1.486 \mathrm{E}+02$ & $7.14 \mathrm{E}+00$ & $8.791 \mathrm{E}+01$ & $3.989 \mathrm{E}+01$ & $2.18 \mathrm{E}+00$ \\
\hline $2.207 \mathrm{E}+04$ & $1.845 \mathrm{E}+01$ & $3.67 \mathrm{E}-02$ & $1.261 \mathrm{E}+02$ & $7.09 \mathrm{E}+00$ & $8.854 \mathrm{E}+01$ & $3.739 \mathrm{E}+01$ & $2.17 \mathrm{E}+00$ \\
\hline $2.209 \mathrm{E}+04$ & $2.122 \mathrm{E}+01$ & $4.10 \mathrm{E}-02$ & $1.031 \mathrm{E}+02$ & $7.14 \mathrm{E}+00$ & $8.922 \mathrm{E}+01$ & $3.447 \mathrm{E}+01$ & $2.23 \mathrm{E}+00$ \\
\hline $2.191 \mathrm{E}+04$ & $2.400 \mathrm{E}+01$ & $4.55 \mathrm{E}-02$ & $7.897 \mathrm{E}+01$ & $7.19 \mathrm{E}+00$ & $8.926 \mathrm{E}+01$ & $2.872 \mathrm{E}+01$ & $2.40 \mathrm{E}+00$ \\
\hline$P_{\mathrm{e}}(\mathrm{W})$ & $\Delta P_{\mathrm{e}}(\mathrm{W})$ & $C_{\mathrm{T}}$ & $\Delta C_{\mathrm{T}}$ & $C_{\mathrm{P}}$ & $\Delta C_{\mathrm{P}}$ & $C_{\mathrm{Q}}$ & $\Delta C_{\mathrm{Q}}$ \\
\hline $6.960 \mathrm{E}+01$ & $7.53 \mathrm{E}-01$ & $1.358 \mathrm{E}-01$ & 4.49E-03 & $8.029 \mathrm{E}-02$ & $3.52 \mathrm{E}-03$ & $1.278 \mathrm{E}-02$ & $5.60 \mathrm{E}-04$ \\
\hline $6.766 \mathrm{E}+01$ & $7.31 \mathrm{E}-01$ & $1.313 \mathrm{E}-01$ & $4.59 \mathrm{E}-03$ & $7.822 \mathrm{E}-02$ & $3.84 \mathrm{E}-03$ & $1.245 \mathrm{E}-02$ & $6.11 \mathrm{E}-04$ \\
\hline $6.739 \mathrm{E}+01$ & $7.26 \mathrm{E}-01$ & $1.242 \mathrm{E}-01$ & $4.54 \mathrm{E}-03$ & 7.842E-02 & $3.84 \mathrm{E}-03$ & $1.248 \mathrm{E}-02$ & $6.11 \mathrm{E}-04$ \\
\hline $6.675 \mathrm{E}+01$ & $7.23 \mathrm{E}-01$ & $1.163 \mathrm{E}-01$ & $4.50 \mathrm{E}-03$ & 7.753E-02 & $3.70 \mathrm{E}-03$ & $1.234 \mathrm{E}-02$ & 5.89E-04 \\
\hline $6.378 \mathrm{E}+01$ & $6.81 \mathrm{E}-01$ & $1.058 \mathrm{E}-01$ & $4.52 \mathrm{E}-03$ & $7.396 \mathrm{E}-02$ & $3.83 \mathrm{E}-03$ & $1.177 \mathrm{E}-02$ & $6.09 \mathrm{E}-04$ \\
\hline $6.117 \mathrm{E}+01$ & $6.77 \mathrm{E}-01$ & $9.315 \mathrm{E}-02$ & $4.48 \mathrm{E}-03$ & $6.945 \mathrm{E}-02$ & $3.79 \mathrm{E}-03$ & $1.105 \mathrm{E}-02$ & $6.03 \mathrm{E}-04$ \\
\hline $5.655 \mathrm{E}+01$ & $6.21 \mathrm{E}-01$ & $7.888 \mathrm{E}-02$ & 4.44E-03 & $6.489 \mathrm{E}-02$ & $3.77 \mathrm{E}-03$ & $1.033 \mathrm{E}-02$ & 5.99E-04 \\
\hline $5.148 \mathrm{E}+01$ & $5.69 \mathrm{E}-01$ & $6.443 \mathrm{E}-02$ & $4.46 \mathrm{E}-03$ & $5.971 \mathrm{E}-02$ & $3.86 \mathrm{E}-03$ & $9.503 \mathrm{E}-03$ & $6.14 \mathrm{E}-04$ \\
\hline $4.482 \mathrm{E}+01$ & $4.98 \mathrm{E}-01$ & $5.013 \mathrm{E}-02$ & $4.56 \mathrm{E}-03$ & $5.096 \mathrm{E}-02$ & $4.26 \mathrm{E}-03$ & $8.110 \mathrm{E}-03$ & $6.78 \mathrm{E}-04$ \\
\hline$\rho\left(\mathrm{kg} / \mathrm{m}^{\wedge} 3\right)$ & $\eta_{\mathrm{T}}$ & $\Delta \eta_{\mathrm{T}}$ & $J$ & $\Delta J$ & $\eta_{\mathrm{P}}$ & $\Delta \eta_{\mathrm{P}}$ & $R e_{0.75}$ \\
\hline $1.165 \mathrm{E}+00$ & $6.701 \mathrm{E}-01$ & $3.02 \mathrm{E}-02$ & $6.359 \mathrm{E}-02$ & $5.12 \mathrm{E}-04$ & $1.076 \mathrm{E}-01$ & $5.97 \mathrm{E}-03$ & $5.260 \mathrm{E}+04$ \\
\hline $1.165 \mathrm{E}+00$ & $6.580 \mathrm{E}-01$ & $3.31 \mathrm{E}-02$ & $1.301 \mathrm{E}-01$ & $5.45 \mathrm{E}-04$ & $2.183 \mathrm{E}-01$ & $1.32 \mathrm{E}-02$ & $5.233 \mathrm{E}+04$ \\
\hline $1.164 \mathrm{E}+00$ & $6.657 \mathrm{E}-01$ & $3.33 \mathrm{E}-02$ & $2.018 \mathrm{E}-01$ & $6.16 \mathrm{E}-04$ & $3.197 \mathrm{E}-01$ & $1.95 \mathrm{E}-02$ & $5.248 \mathrm{E}+04$ \\
\hline $1.164 \mathrm{E}+00$ & $6.672 \mathrm{E}-01$ & $3.27 \mathrm{E}-02$ & $2.747 \mathrm{E}-01$ & $7.29 \mathrm{E}-04$ & $4.121 \mathrm{E}-01$ & $2.54 \mathrm{E}-02$ & $5.268 \mathrm{E}+04$ \\
\hline $1.163 \mathrm{E}+00$ & $6.546 \mathrm{E}-01$ & $3.46 \mathrm{E}-02$ & $3.530 \mathrm{E}-01$ & $8.18 \mathrm{E}-04$ & $5.049 \mathrm{E}-01$ & $3.39 \mathrm{E}-02$ & $5.259 \mathrm{E}+04$ \\
\hline $1.163 \mathrm{E}+00$ & $6.521 \mathrm{E}-01$ & $3.63 \mathrm{E}-02$ & $4.270 \mathrm{E}-01$ & $9.00 \mathrm{E}-04$ & $5.726 \mathrm{E}-01$ & $4.17 \mathrm{E}-02$ & $5.312 \mathrm{E}+04$ \\
\hline $1.163 \mathrm{E}+00$ & $6.611 \mathrm{E}-01$ & $3.90 \mathrm{E}-02$ & $5.021 \mathrm{E}-01$ & $1.00 \mathrm{E}-03$ & $6.104 \mathrm{E}-01$ & $4.93 \mathrm{E}-02$ & $5.349 \mathrm{E}+04$ \\
\hline $1.162 \mathrm{E}+00$ & $6.696 \mathrm{E}-01$ & $4.39 \mathrm{E}-02$ & $5.769 \mathrm{E}-01$ & $1.12 \mathrm{E}-03$ & $6.225 \mathrm{E}-01$ & $5.90 \mathrm{E}-02$ & $5.386 \mathrm{E}+04$ \\
\hline $1.162 \mathrm{E}+00$ & $6.409 \mathrm{E}-01$ & $5.40 \mathrm{E}-02$ & $6.577 \mathrm{E}-01$ & $1.25 \mathrm{E}-03$ & $6.470 \mathrm{E}-01$ & $8.00 \mathrm{E}-02$ & $5.387 \mathrm{E}+04$ \\
\hline
\end{tabular}




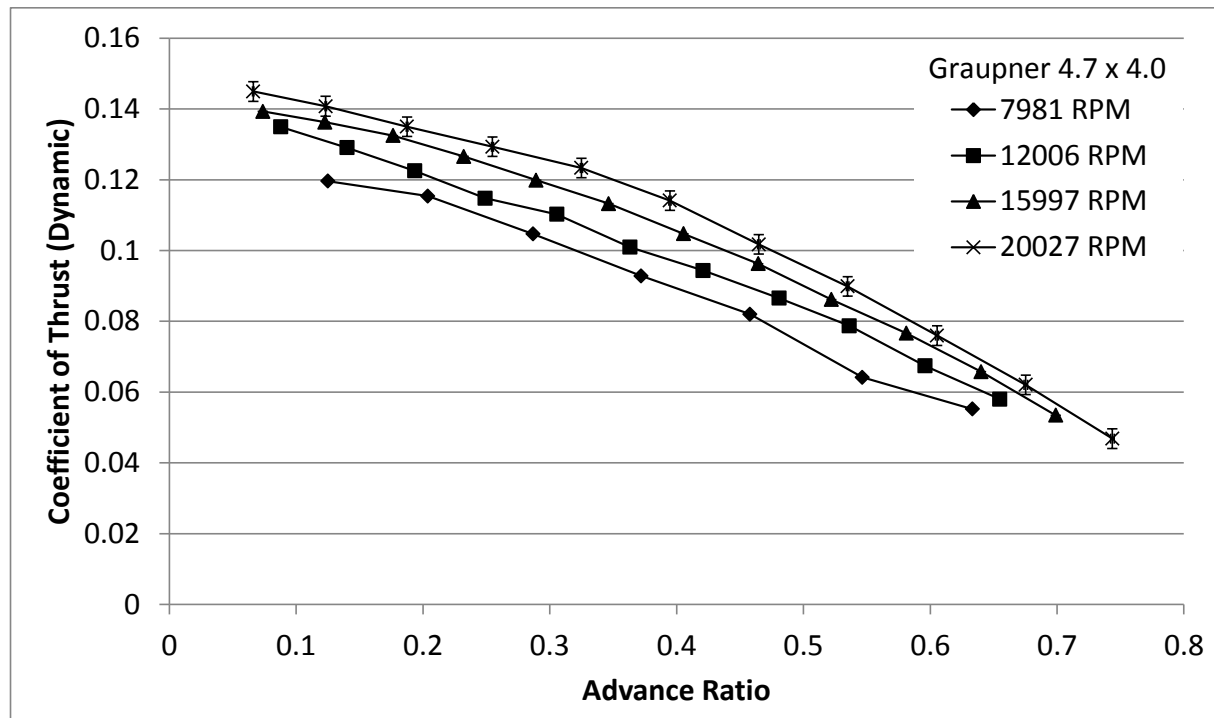

(a)
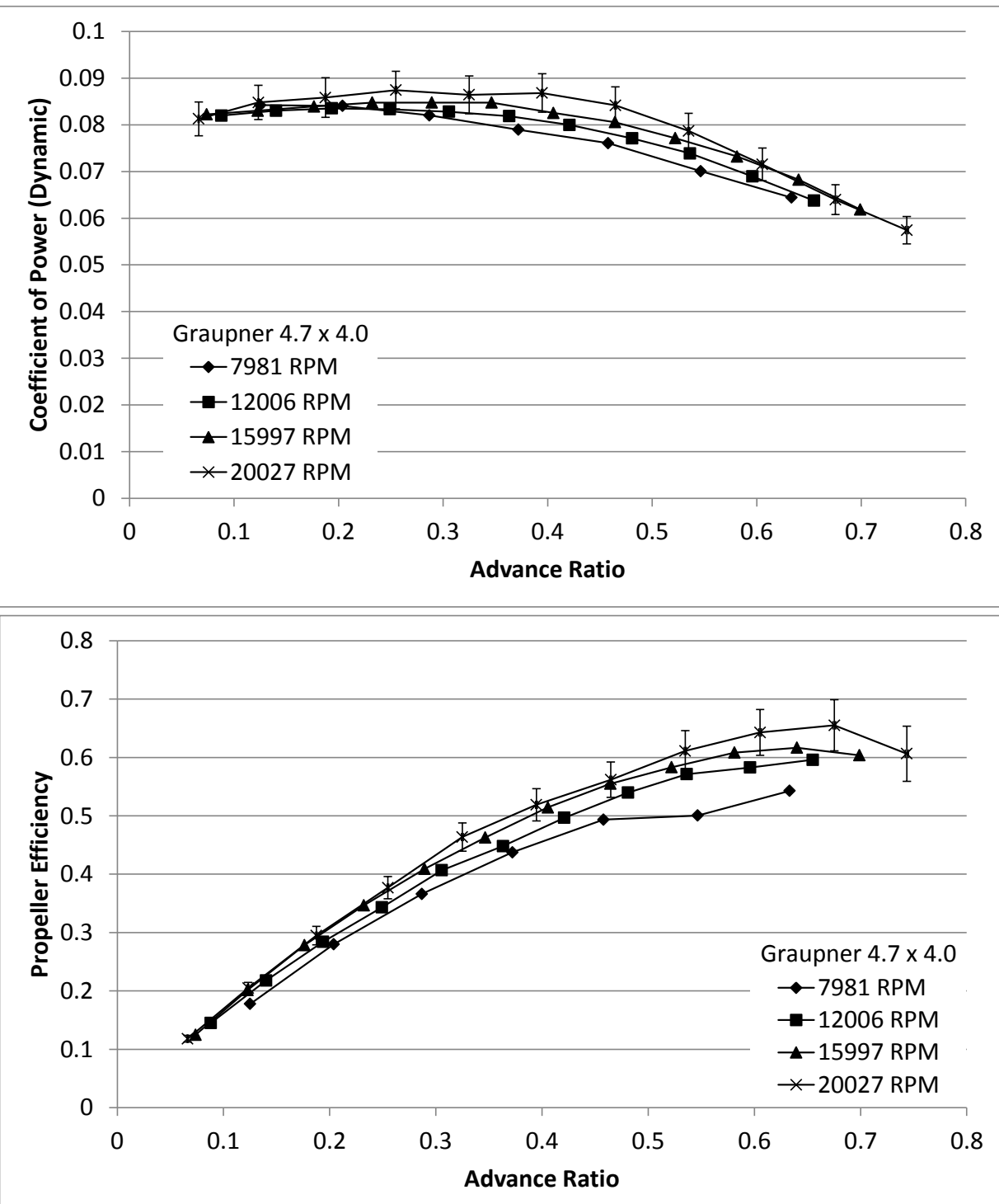

(b)

(c) 


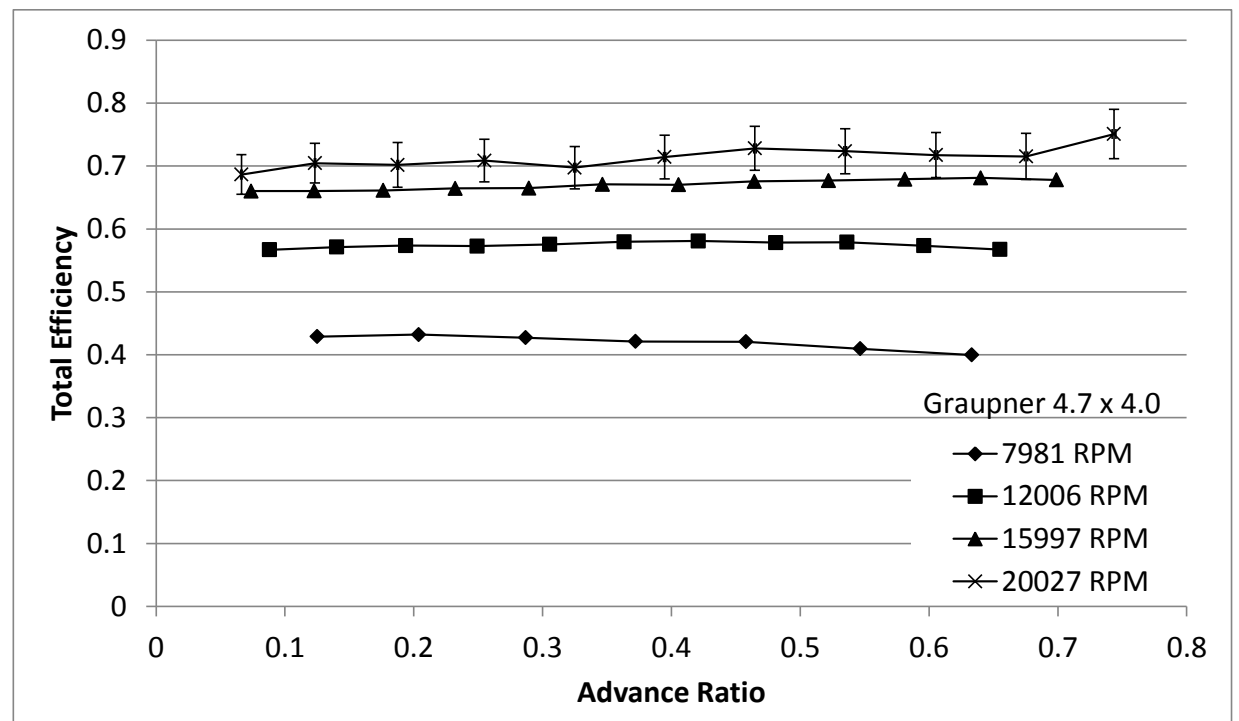

(d)

Figure 94: Graupner 4.7 x 4.0 Dynamic Test Results: (a) Coefficient of Thrust, (b) Coefficient of Power, (c) Propeller Efficiency, (d) Total Efficiency.

Table 202: Graupner 4.7 x 4.0 Dynamic Measured Values - 7981 RPM

\begin{tabular}{|c|c|c|c|c|c|c|c|}
\hline$n(\mathrm{RPM})$ & $Q(\mathrm{~g}-\mathrm{m})$ & $\Delta Q(\mathrm{~g}-\mathrm{m})$ & $V(\mathrm{~V})$ & $I(\mathrm{~A})$ & $T_{\text {atm }}\left({ }^{\circ} \mathrm{C}\right)$ & $P_{\text {atm }}(\mathrm{Pa})$ & $P_{\text {diff }}(\mathrm{Pa})$ \\
\hline $7.990 \mathrm{E}+03$ & $6.768 \mathrm{E}-01$ & $6.59 \mathrm{E}-02$ & $1.108 \mathrm{E}+01$ & $1.169 \mathrm{E}+00$ & $2.190 \mathrm{E}+01$ & $9.875 \mathrm{E}+04$ & $2.443 \mathrm{E}+00$ \\
\hline $8.014 \mathrm{E}+03$ & $6.797 \mathrm{E}-01$ & $6.50 \mathrm{E}-02$ & $1.108 \mathrm{E}+01$ & $1.168 \mathrm{E}+00$ & $2.186 \mathrm{E}+01$ & $9.874 \mathrm{E}+04$ & $6.359 \mathrm{E}+00$ \\
\hline $7.979 \mathrm{E}+03$ & $6.574 \mathrm{E}-01$ & $6.51 \mathrm{E}-02$ & $1.108 \mathrm{E}+01$ & $1.138 \mathrm{E}+00$ & $2.195 \mathrm{E}+01$ & $9.875 \mathrm{E}+04$ & $1.232 \mathrm{E}+01$ \\
\hline $7.967 \mathrm{E}+03$ & $6.308 \mathrm{E}-01$ & $6.53 \mathrm{E}-02$ & $1.108 \mathrm{E}+01$ & $1.106 \mathrm{E}+00$ & $2.198 \mathrm{E}+01$ & $9.875 \mathrm{E}+04$ & $2.050 \mathrm{E}+01$ \\
\hline $7.981 \mathrm{E}+03$ & $6.099 \mathrm{E}-01$ & $6.51 \mathrm{E}-02$ & $1.108 \mathrm{E}+01$ & $1.072 \mathrm{E}+00$ & $2.205 \mathrm{E}+01$ & $9.876 \mathrm{E}+04$ & $3.099 \mathrm{E}+01$ \\
\hline $7.970 \mathrm{E}+03$ & $5.601 \mathrm{E}-01$ & $6.52 \mathrm{E}-02$ & $1.109 \mathrm{E}+01$ & $1.010 \mathrm{E}+00$ & $2.206 \mathrm{E}+01$ & $9.876 \mathrm{E}+04$ & $4.383 \mathrm{E}+01$ \\
\hline $7.966 \mathrm{E}+03$ & $5.143 \mathrm{E}-01$ & $6.54 \mathrm{E}-02$ & $1.109 \mathrm{E}+01$ & $9.491 \mathrm{E}-01$ & $2.209 \mathrm{E}+01$ & $9.876 \mathrm{E}+04$ & $5.867 \mathrm{E}+01$ \\
\hline
\end{tabular}


Table 203: Graupner 4.7 x 4.0 Dynamic Calculated Values - 7981 RPM

\begin{tabular}{|c|c|c|c|c|c|c|c|}
\hline$n(\mathrm{RPM})$ & $V_{\infty}^{\prime}(\mathrm{m} / \mathrm{s})$ & $\Delta V_{\infty}^{\prime}(\mathrm{m} / \mathrm{s})$ & $T^{\prime}(\mathrm{g})$ & $\Delta T^{\prime}(\mathrm{g})$ & $V_{\mathrm{t}}(\mathrm{m} / \mathrm{s})$ & $P_{\mathrm{P}}(\mathrm{W})$ & $\Delta P_{\mathrm{P}}(\mathrm{W})$ \\
\hline $7.990 \mathrm{E}+03$ & $1.983 \mathrm{E}+00$ & $1.29 \mathrm{E}-02$ & $5.072 \mathrm{E}+01$ & $1.07 \mathrm{E}+01$ & $3.742 \mathrm{E}+01$ & $5.554 \mathrm{E}+00$ & $5.41 \mathrm{E}-01$ \\
\hline $8.014 \mathrm{E}+03$ & $3.242 \mathrm{E}+00$ & $1.33 \mathrm{E}-02$ & $4.923 \mathrm{E}+01$ & $1.07 \mathrm{E}+01$ & $3.762 \mathrm{E}+01$ & $5.594 \mathrm{E}+00$ & 5.35E-01 \\
\hline 7.979E+03 & $4.544 \mathrm{E}+00$ & $1.46 \mathrm{E}-02$ & $4.425 \mathrm{E}+01$ & $1.07 \mathrm{E}+01$ & $3.759 \mathrm{E}+01$ & $5.387 \mathrm{E}+00$ & 5.33E-01 \\
\hline $7.967 \mathrm{E}+03$ & $5.885 \mathrm{E}+00$ & $1.65 \mathrm{E}-02$ & $3.912 \mathrm{E}+01$ & $1.07 \mathrm{E}+01$ & $3.772 \mathrm{E}+01$ & $5.162 \mathrm{E}+00$ & 5.34E-01 \\
\hline $7.981 \mathrm{E}+03$ & $7.254 \mathrm{E}+00$ & $1.79 \mathrm{E}-02$ & $3.468 \mathrm{E}+01$ & $1.08 \mathrm{E}+01$ & $3.803 \mathrm{E}+01$ & $4.999 \mathrm{E}+00$ & 5.34E-01 \\
\hline $7.970 \mathrm{E}+03$ & $8.645 \mathrm{E}+00$ & $1.98 \mathrm{E}-02$ & $2.706 \mathrm{E}+01$ & $1.08 \mathrm{E}+01$ & $3.826 \mathrm{E}+01$ & $4.584 \mathrm{E}+00$ & 5.34E-01 \\
\hline $7.966 \mathrm{E}+03$ & $1.001 \mathrm{E}+01$ & $2.21 \mathrm{E}-02$ & $2.326 \mathrm{E}+01$ & $1.07 \mathrm{E}+01$ & $3.857 \mathrm{E}+01$ & $4.207 \mathrm{E}+00$ & $5.35 \mathrm{E}-01$ \\
\hline$P_{\mathrm{e}}(\mathrm{W})$ & $\Delta P_{\mathrm{e}}(\mathrm{W})$ & $C_{\mathrm{T}}$ & $\Delta C_{\mathrm{T}}$ & $C_{\mathrm{P}}$ & $\Delta C_{\mathrm{P}}$ & $C_{\mathrm{Q}}$ & $\Delta C_{\mathrm{Q}}$ \\
\hline $1.295 \mathrm{E}+01$ & $1.58 \mathrm{E}-01$ & $1.196 \mathrm{E}-01$ & $2.52 \mathrm{E}-02$ & $8.421 \mathrm{E}-02$ & $8.20 \mathrm{E}-03$ & $1.340 \mathrm{E}-02$ & $1.31 \mathrm{E}-03$ \\
\hline $1.294 \mathrm{E}+01$ & $1.56 \mathrm{E}-01$ & $1.154 \mathrm{E}-01$ & $2.50 \mathrm{E}-02$ & $8.404 \mathrm{E}-02$ & $8.04 \mathrm{E}-03$ & $1.337 \mathrm{E}-02$ & $1.28 \mathrm{E}-03$ \\
\hline $1.261 \mathrm{E}+01$ & $1.53 \mathrm{E}-01$ & $1.047 \mathrm{E}-01$ & $2.52 \mathrm{E}-02$ & $8.203 \mathrm{E}-02$ & $8.12 \mathrm{E}-03$ & $1.306 \mathrm{E}-02$ & $1.29 \mathrm{E}-03$ \\
\hline $1.226 \mathrm{E}+01$ & $1.50 \mathrm{E}-01$ & $9.280 \mathrm{E}-02$ & $2.55 \mathrm{E}-02$ & $7.895 \mathrm{E}-02$ & $8.17 \mathrm{E}-03$ & $1.257 \mathrm{E}-02$ & $1.30 \mathrm{E}-03$ \\
\hline $1.189 \mathrm{E}+01$ & $1.46 \mathrm{E}-01$ & $8.198 \mathrm{E}-02$ & $2.54 \mathrm{E}-02$ & $7.608 \mathrm{E}-02$ & $8.12 \mathrm{E}-03$ & $1.211 \mathrm{E}-02$ & $1.29 \mathrm{E}-03$ \\
\hline $1.119 \mathrm{E}+01$ & $1.39 \mathrm{E}-01$ & $6.416 \mathrm{E}-02$ & $2.55 \mathrm{E}-02$ & $7.006 \mathrm{E}-02$ & $8.16 \mathrm{E}-03$ & $1.115 \mathrm{E}-02$ & $1.30 \mathrm{E}-03$ \\
\hline $1.052 \mathrm{E}+01$ & $1.32 \mathrm{E}-01$ & $5.521 \mathrm{E}-02$ & $2.55 \mathrm{E}-02$ & $6.442 \mathrm{E}-02$ & $8.20 \mathrm{E}-03$ & $1.025 \mathrm{E}-02$ & $1.30 \mathrm{E}-03$ \\
\hline$\rho\left(\mathrm{kg} / \mathrm{m}^{\wedge} 3\right)$ & $\eta_{\mathrm{T}}$ & $\Delta \eta_{\mathrm{T}}$ & $J$ & $\Delta J$ & $\eta_{\mathrm{P}}$ & $\Delta \eta_{\mathrm{P}}$ & $\operatorname{Re}_{0.75}$ \\
\hline $1.166 \mathrm{E}+00$ & $4.289 \mathrm{E}-01$ & $4.21 \mathrm{E}-02$ & $1.251 \mathrm{E}-01$ & $8.14 \mathrm{E}-04$ & $1.776 \mathrm{E}-01$ & $4.13 \mathrm{E}-02$ & $2.267 \mathrm{E}+04$ \\
\hline $1.166 \mathrm{E}+00$ & $4.322 \mathrm{E}-01$ & $4.17 \mathrm{E}-02$ & $2.038 \mathrm{E}-01$ & $8.38 \mathrm{E}-04$ & $2.798 \mathrm{E}-01$ & $6.62 \mathrm{E}-02$ & $2.279 \mathrm{E}+04$ \\
\hline $1.166 \mathrm{E}+00$ & $4.272 \mathrm{E}-01$ & $4.26 \mathrm{E}-02$ & 2.869E-01 & $9.32 \mathrm{E}-04$ & $3.661 \mathrm{E}-01$ & $9.53 \mathrm{E}-02$ & $2.276 \mathrm{E}+04$ \\
\hline $1.166 \mathrm{E}+00$ & $4.211 \mathrm{E}-01$ & 4.39E-02 & $3.721 \mathrm{E}-01$ & $1.05 \mathrm{E}-03$ & $4.374 \mathrm{E}-01$ & $1.28 \mathrm{E}-01$ & $2.284 \mathrm{E}+04$ \\
\hline $1.165 \mathrm{E}+00$ & $4.206 \mathrm{E}-01$ & $4.52 \mathrm{E}-02$ & $4.579 \mathrm{E}-01$ & $1.15 \mathrm{E}-03$ & $4.934 \mathrm{E}-01$ & $1.62 \mathrm{E}-01$ & $2.301 \mathrm{E}+04$ \\
\hline $1.165 \mathrm{E}+00$ & $4.095 \mathrm{E}-01$ & 4.79E-02 & $5.465 \mathrm{E}-01$ & $1.27 \mathrm{E}-03$ & $5.004 \mathrm{E}-01$ & $2.07 \mathrm{E}-01$ & $2.316 \mathrm{E}+04$ \\
\hline $1.165 \mathrm{E}+00$ & $3.998 \mathrm{E}-01$ & $5.11 \mathrm{E}-02$ & $6.333 \mathrm{E}-01$ & $1.42 \mathrm{E}-03$ & $5.427 \mathrm{E}-01$ & $2.60 \mathrm{E}-01$ & $2.334 \mathrm{E}+04$ \\
\hline
\end{tabular}


Table 204: Graupner 4.7 x 4.0 Dynamic Measured Values - 12006 RPM

\begin{tabular}{|c|c|c|c|c|c|c|c|}
\hline$n(\mathrm{RPM})$ & $Q(\mathrm{~g}-\mathrm{m})$ & $\Delta Q(\mathrm{~g}-\mathrm{m})$ & $V(\mathrm{~V})$ & $I(\mathrm{~A})$ & $T_{\text {atm }}\left({ }^{\circ} \mathrm{C}\right)$ & $P_{\text {atm }}(\mathrm{Pa})$ & $P_{\text {diff }}(\mathrm{Pa})$ \\
\hline $1.197 \mathrm{E}+04$ & $1.479 \mathrm{E}+00$ & $6.20 \mathrm{E}-02$ & $1.103 \mathrm{E}+01$ & $2.907 \mathrm{E}+00$ & $2.196 \mathrm{E}+01$ & $9.877 \mathrm{E}+04$ & $2.804 \mathrm{E}+00$ \\
\hline $1.196 \mathrm{E}+04$ & $1.495 \mathrm{E}+00$ & $6.24 \mathrm{E}-02$ & $1.103 \mathrm{E}+01$ & $2.915 \mathrm{E}+00$ & $2.196 \mathrm{E}+01$ & $9.877 \mathrm{E}+04$ & $6.845 \mathrm{E}+00$ \\
\hline $1.201 \mathrm{E}+04$ & $1.517 \mathrm{E}+00$ & $6.19 \mathrm{E}-02$ & $1.103 \mathrm{E}+01$ & $2.958 \mathrm{E}+00$ & $2.205 \mathrm{E}+01$ & $9.877 \mathrm{E}+04$ & $1.293 \mathrm{E}+01$ \\
\hline $1.202 \mathrm{E}+04$ & $1.514 \mathrm{E}+00$ & $6.24 \mathrm{E}-02$ & $1.103 \mathrm{E}+01$ & $2.958 \mathrm{E}+00$ & $2.212 \mathrm{E}+01$ & $9.877 \mathrm{E}+04$ & $2.118 \mathrm{E}+01$ \\
\hline $1.201 \mathrm{E}+04$ & $1.503 \mathrm{E}+00$ & $6.19 \mathrm{E}-02$ & $1.103 \mathrm{E}+01$ & $2.920 \mathrm{E}+00$ & $2.223 \mathrm{E}+01$ & $9.878 \mathrm{E}+04$ & $3.160 \mathrm{E}+01$ \\
\hline $1.200 \mathrm{E}+04$ & $1.484 \mathrm{E}+00$ & $6.17 \mathrm{E}-02$ & $1.103 \mathrm{E}+01$ & $2.860 \mathrm{E}+00$ & $2.232 \mathrm{E}+01$ & $9.878 \mathrm{E}+04$ & $4.437 \mathrm{E}+01$ \\
\hline $1.204 \mathrm{E}+04$ & $1.457 \mathrm{E}+00$ & $6.44 \mathrm{E}-02$ & $1.103 \mathrm{E}+01$ & $2.810 \mathrm{E}+00$ & $2.239 \mathrm{E}+01$ & $9.878 \mathrm{E}+04$ & $5.968 \mathrm{E}+01$ \\
\hline $1.197 \mathrm{E}+04$ & $1.389 \mathrm{E}+00$ & $6.06 \mathrm{E}-02$ & $1.104 \mathrm{E}+01$ & $2.675 \mathrm{E}+00$ & $2.243 \mathrm{E}+01$ & $9.878 \mathrm{E}+04$ & $7.678 \mathrm{E}+01$ \\
\hline $1.205 \mathrm{E}+04$ & $1.349 \mathrm{E}+00$ & $6.30 \mathrm{E}-02$ & $1.104 \mathrm{E}+01$ & $2.612 \mathrm{E}+00$ & $2.245 \mathrm{E}+01$ & $9.878 \mathrm{E}+04$ & $9.653 \mathrm{E}+01$ \\
\hline $1.202 \mathrm{E}+04$ & $1.253 \mathrm{E}+00$ & $6.16 \mathrm{E}-02$ & $1.105 \mathrm{E}+01$ & $2.442 \mathrm{E}+00$ & $2.244 \mathrm{E}+01$ & $9.877 \mathrm{E}+04$ & $1.185 \mathrm{E}+02$ \\
\hline $1.201 \mathrm{E}+04$ & $1.156 \mathrm{E}+00$ & $6.07 \mathrm{E}-02$ & $1.105 \mathrm{E}+01$ & $2.276 \mathrm{E}+00$ & $2.253 \mathrm{E}+01$ & $9.878 \mathrm{E}+04$ & $1.426 \mathrm{E}+02$ \\
\hline
\end{tabular}


Table 205: Graupner 4.7 x 4.0 Dynamic Calculated Values - 12006 RPM

\begin{tabular}{|c|c|c|c|c|c|c|c|}
\hline$n(\mathrm{RPM})$ & $V_{\infty}^{\prime}(\mathrm{m} / \mathrm{s})$ & $\Delta V_{\infty}^{\prime}(\mathrm{m} / \mathrm{s})$ & $T^{\prime}(\mathrm{g})$ & $\Delta T^{\prime}(\mathrm{g})$ & $V_{\mathrm{t}}(\mathrm{m} / \mathrm{s})$ & $P_{\mathrm{P}}(\mathrm{W})$ & $\Delta P_{\mathrm{P}}(\mathrm{W})$ \\
\hline $1.197 \mathrm{E}+04$ & $2.090 \mathrm{E}+00$ & $1.43 \mathrm{E}-02$ & $1.284 \mathrm{E}+02$ & $8.65 \mathrm{E}+00$ & $5.602 \mathrm{E}+01$ & $1.818 \mathrm{E}+01$ & $7.62 \mathrm{E}-01$ \\
\hline $1.196 \mathrm{E}+04$ & $3.328 \mathrm{E}+00$ & $1.46 \mathrm{E}-02$ & $1.226 \mathrm{E}+02$ & $8.66 \mathrm{E}+00$ & $5.604 \mathrm{E}+01$ & $1.837 \mathrm{E}+01$ & $7.66 \mathrm{E}-01$ \\
\hline $1.201 \mathrm{E}+04$ & $4.617 \mathrm{E}+00$ & $1.55 \mathrm{E}-02$ & $1.174 \mathrm{E}+02$ & $8.63 \mathrm{E}+00$ & $5.636 \mathrm{E}+01$ & $1.871 \mathrm{E}+01$ & 7.63E-01 \\
\hline $1.202 \mathrm{E}+04$ & $5.942 \mathrm{E}+00$ & $1.71 \mathrm{E}-02$ & $1.100 \mathrm{E}+02$ & $8.61 \mathrm{E}+00$ & $5.651 \mathrm{E}+01$ & $1.869 \mathrm{E}+01$ & 7.70E-01 \\
\hline $1.201 \mathrm{E}+04$ & $7.284 \mathrm{E}+00$ & $1.83 \mathrm{E}-02$ & $1.055 \mathrm{E}+02$ & $8.60 \mathrm{E}+00$ & $5.664 \mathrm{E}+01$ & $1.853 \mathrm{E}+01$ & 7.63E-01 \\
\hline $1.200 \mathrm{E}+04$ & $8.656 \mathrm{E}+00$ & $2.01 \mathrm{E}-02$ & $9.652 \mathrm{E}+01$ & $8.61 \mathrm{E}+00$ & $5.681 \mathrm{E}+01$ & $1.829 \mathrm{E}+01$ & 7.60E-01 \\
\hline $1.204 \mathrm{E}+04$ & $1.006 \mathrm{E}+01$ & $2.25 \mathrm{E}-02$ & $9.069 \mathrm{E}+01$ & $8.62 \mathrm{E}+00$ & $5.719 \mathrm{E}+01$ & $1.801 \mathrm{E}+01$ & 7.96E-01 \\
\hline $1.197 \mathrm{E}+04$ & $1.143 \mathrm{E}+01$ & $2.47 \mathrm{E}-02$ & $8.225 \mathrm{E}+01$ & $8.65 \mathrm{E}+00$ & $5.714 \mathrm{E}+01$ & $1.707 \mathrm{E}+01$ & $7.45 \mathrm{E}-01$ \\
\hline $1.205 \mathrm{E}+04$ & $1.283 \mathrm{E}+01$ & $2.67 \mathrm{E}-02$ & $7.582 \mathrm{E}+01$ & $8.64 \mathrm{E}+00$ & $5.780 \mathrm{E}+01$ & $1.669 \mathrm{E}+01$ & $7.80 \mathrm{E}-01$ \\
\hline $1.202 \mathrm{E}+04$ & $1.422 \mathrm{E}+01$ & $2.86 \mathrm{E}-02$ & $6.464 \mathrm{E}+01$ & $8.65 \mathrm{E}+00$ & $5.801 \mathrm{E}+01$ & $1.547 \mathrm{E}+01$ & 7.61E-01 \\
\hline $1.201 \mathrm{E}+04$ & $1.562 \mathrm{E}+01$ & $3.09 \mathrm{E}-02$ & $5.551 \mathrm{E}+01$ & $8.66 \mathrm{E}+00$ & $5.832 \mathrm{E}+01$ & $1.427 \mathrm{E}+01$ & 7.49E-01 \\
\hline$P_{\mathrm{e}}(\mathrm{W})$ & $\Delta P_{\mathrm{e}}(\mathrm{W})$ & $C_{\mathrm{T}}$ & $\Delta C_{\mathrm{T}}$ & $C_{\mathrm{P}}$ & $\Delta C_{\mathrm{P}}$ & $C_{\mathrm{Q}}$ & $\Delta C_{\mathrm{Q}}$ \\
\hline $3.207 \mathrm{E}+01$ & $3.59 \mathrm{E}-01$ & $1.349 \mathrm{E}-01$ & $9.09 \mathrm{E}-03$ & $8.197 \mathrm{E}-02$ & $3.44 \mathrm{E}-03$ & $1.305 \mathrm{E}-02$ & $5.47 \mathrm{E}-04$ \\
\hline $3.215 \mathrm{E}+01$ & $3.59 \mathrm{E}-01$ & $1.291 \mathrm{E}-01$ & $9.12 \mathrm{E}-03$ & $8.302 \mathrm{E}-02$ & $3.46 \mathrm{E}-03$ & $1.321 \mathrm{E}-02$ & $5.51 \mathrm{E}-04$ \\
\hline $3.262 \mathrm{E}+01$ & $3.65 \mathrm{E}-01$ & $1.225 \mathrm{E}-01$ & $9.01 \mathrm{E}-03$ & 8.353E-02 & $3.41 \mathrm{E}-03$ & $1.329 \mathrm{E}-02$ & $5.42 \mathrm{E}-04$ \\
\hline $3.263 \mathrm{E}+01$ & $3.66 \mathrm{E}-01$ & $1.148 \mathrm{E}-01$ & $8.98 \mathrm{E}-03$ & 8.333E-02 & $3.43 \mathrm{E}-03$ & $1.326 \mathrm{E}-02$ & $5.46 \mathrm{E}-04$ \\
\hline $3.221 \mathrm{E}+01$ & 3.63E-01 & $1.102 \mathrm{E}-01$ & 8.99E-03 & 8.280E-02 & $3.41 \mathrm{E}-03$ & $1.318 \mathrm{E}-02$ & $5.43 \mathrm{E}-04$ \\
\hline $3.156 \mathrm{E}+01$ & $3.52 \mathrm{E}-01$ & $1.009 \mathrm{E}-01$ & $9.01 \mathrm{E}-03$ & $8.186 \mathrm{E}-02$ & $3.40 \mathrm{E}-03$ & $1.303 \mathrm{E}-02$ & $5.42 \mathrm{E}-04$ \\
\hline $3.101 \mathrm{E}+01$ & $3.48 \mathrm{E}-01$ & $9.436 \mathrm{E}-02$ & $8.97 \mathrm{E}-03$ & $7.998 \mathrm{E}-02$ & $3.53 \mathrm{E}-03$ & $1.273 \mathrm{E}-02$ & $5.62 \mathrm{E}-04$ \\
\hline $2.953 \mathrm{E}+01$ & $3.30 \mathrm{E}-01$ & 8.654E-02 & $9.10 \mathrm{E}-03$ & 7.709E-02 & $3.36 \mathrm{E}-03$ & $1.227 \mathrm{E}-02$ & $5.35 \mathrm{E}-04$ \\
\hline $2.883 \mathrm{E}+01$ & $3.23 \mathrm{E}-01$ & $7.871 \mathrm{E}-02$ & 8.97E-03 & $7.388 \mathrm{E}-02$ & $3.45 \mathrm{E}-03$ & $1.176 \mathrm{E}-02$ & $5.50 \mathrm{E}-04$ \\
\hline $2.698 \mathrm{E}+01$ & $3.03 \mathrm{E}-01$ & $6.741 \mathrm{E}-02$ & $9.02 \mathrm{E}-03$ & $6.894 \mathrm{E}-02$ & $3.39 \mathrm{E}-03$ & $1.097 \mathrm{E}-02$ & $5.39 \mathrm{E}-04$ \\
\hline $2.515 \mathrm{E}+01$ & $2.82 \mathrm{E}-01$ & $5.800 \mathrm{E}-02$ & $9.05 \mathrm{E}-03$ & $6.375 \mathrm{E}-02$ & $3.34 \mathrm{E}-03$ & $1.015 \mathrm{E}-02$ & $5.32 \mathrm{E}-04$ \\
\hline$\rho\left(\mathrm{kg} / \mathrm{m}^{\wedge} 3\right)$ & $\eta_{\mathrm{T}}$ & $\Delta \eta_{\mathrm{T}}$ & $J$ & $\Delta J$ & $\eta_{\mathrm{P}}$ & $\Delta \eta_{\mathrm{P}}$ & $R e_{0.75}$ \\
\hline $1.166 \mathrm{E}+00$ & $5.668 \mathrm{E}-01$ & $2.46 \mathrm{E}-02$ & $8.798 \mathrm{E}-02$ & $6.03 \mathrm{E}-04$ & $1.448 \mathrm{E}-01$ & $1.15 \mathrm{E}-02$ & $3.393 \mathrm{E}+04$ \\
\hline $1.166 \mathrm{E}+00$ & 5.712E-01 & $2.47 \mathrm{E}-02$ & 1.402E-01 & $6.16 \mathrm{E}-04$ & 2.179E-01 & $1.79 \mathrm{E}-02$ & $3.394 \mathrm{E}+04$ \\
\hline $1.166 \mathrm{E}+00$ & $5.735 \mathrm{E}-01$ & $2.43 \mathrm{E}-02$ & $1.937 \mathrm{E}-01$ & $6.53 \mathrm{E}-04$ & $2.841 \mathrm{E}-01$ & $2.39 \mathrm{E}-02$ & $3.412 \mathrm{E}+04$ \\
\hline $1.165 \mathrm{E}+00$ & $5.727 \mathrm{E}-01$ & $2.44 \mathrm{E}-02$ & $2.491 \mathrm{E}-01$ & $7.18 \mathrm{E}-04$ & $3.430 \mathrm{E}-01$ & $3.04 \mathrm{E}-02$ & $3.420 \mathrm{E}+04$ \\
\hline $1.165 \mathrm{E}+00$ & 5.754E-01 & $2.46 \mathrm{E}-02$ & $3.055 \mathrm{E}-01$ & $7.75 \mathrm{E}-04$ & 4.067E-01 & $3.72 \mathrm{E}-02$ & $3.425 \mathrm{E}+04$ \\
\hline $1.165 \mathrm{E}+00$ & $5.796 \mathrm{E}-01$ & $2.49 \mathrm{E}-02$ & 3.633E-01 & $8.52 \mathrm{E}-04$ & 4.480E-01 & 4.41E-02 & $3.433 \mathrm{E}+04$ \\
\hline $1.164 \mathrm{E}+00$ & $5.808 \mathrm{E}-01$ & $2.65 \mathrm{E}-02$ & $4.210 \mathrm{E}-01$ & $9.50 \mathrm{E}-04$ & 4.967E-01 & $5.21 \mathrm{E}-02$ & $3.455 \mathrm{E}+04$ \\
\hline $1.164 \mathrm{E}+00$ & $5.782 \mathrm{E}-01$ & $2.60 \mathrm{E}-02$ & 4.809E-01 & $1.05 \mathrm{E}-03$ & $5.398 \mathrm{E}-01$ & $6.15 \mathrm{E}-02$ & $3.451 \mathrm{E}+04$ \\
\hline $1.164 \mathrm{E}+00$ & 5.789E-01 & $2.78 \mathrm{E}-02$ & 5.362E-01 & $1.13 \mathrm{E}-03$ & 5.714E-01 & $7.04 \mathrm{E}-02$ & $3.491 \mathrm{E}+04$ \\
\hline $1.164 \mathrm{E}+00$ & 5.734E-01 & $2.89 \mathrm{E}-02$ & $5.960 \mathrm{E}-01$ & $1.21 \mathrm{E}-03$ & $5.828 \mathrm{E}-01$ & 8.31E-02 & $3.503 \mathrm{E}+04$ \\
\hline $1.164 \mathrm{E}+00$ & $5.673 \mathrm{E}-01$ & $3.04 \mathrm{E}-02$ & $6.550 \mathrm{E}-01$ & $1.31 \mathrm{E}-03$ & 5.959E-01 & $9.81 \mathrm{E}-02$ & $3.520 \mathrm{E}+04$ \\
\hline
\end{tabular}


Table 206: Graupner 4.7 x 4.0 Dynamic Measured Values - 15997 RPM

\begin{tabular}{|c|c|c|c|c|c|c|c|}
\hline$n(\mathrm{RPM})$ & $Q(\mathrm{~g}-\mathrm{m})$ & $\Delta Q(\mathrm{~g}-\mathrm{m})$ & $V(\mathrm{~V})$ & $I(\mathrm{~A})$ & $T_{\text {atm }}\left({ }^{\circ} \mathrm{C}\right)$ & $P_{\text {atm }}(\mathrm{Pa})$ & $P_{\text {diff }}(\mathrm{Pa})$ \\
\hline $1.601 \mathrm{E}+04$ & $2.655 \mathrm{E}+00$ & $7.36 \mathrm{E}-02$ & $1.094 \mathrm{E}+01$ & $6.044 \mathrm{E}+00$ & $2.198 \mathrm{E}+01$ & $9.877 \mathrm{E}+04$ & $3.600 \mathrm{E}+00$ \\
\hline $1.602 \mathrm{E}+04$ & $2.682 \mathrm{E}+00$ & $7.24 \mathrm{E}-02$ & $1.094 \mathrm{E}+01$ & $6.106 \mathrm{E}+00$ & $2.203 \mathrm{E}+01$ & $9.877 \mathrm{E}+04$ & $9.512 \mathrm{E}+00$ \\
\hline $1.599 \mathrm{E}+04$ & $2.701 \mathrm{E}+00$ & $7.15 \mathrm{E}-02$ & $1.094 \mathrm{E}+01$ & $6.135 \mathrm{E}+00$ & $2.220 \mathrm{E}+01$ & $9.878 \mathrm{E}+04$ & $1.914 \mathrm{E}+01$ \\
\hline $1.598 \mathrm{E}+04$ & $2.723 \mathrm{E}+00$ & $7.20 \mathrm{E}-02$ & $1.094 \mathrm{E}+01$ & $6.150 \mathrm{E}+00$ & $2.236 \mathrm{E}+01$ & $9.877 \mathrm{E}+04$ & $3.265 \mathrm{E}+01$ \\
\hline $1.599 \mathrm{E}+04$ & $2.723 \mathrm{E}+00$ & $7.20 \mathrm{E}-02$ & $1.094 \mathrm{E}+01$ & $6.147 \mathrm{E}+00$ & $2.246 \mathrm{E}+01$ & $9.877 \mathrm{E}+04$ & $5.028 \mathrm{E}+01$ \\
\hline $1.600 \mathrm{E}+04$ & $2.728 \mathrm{E}+00$ & $7.24 \mathrm{E}-02$ & $1.094 \mathrm{E}+01$ & $6.110 \mathrm{E}+00$ & $2.256 \mathrm{E}+01$ & $9.877 \mathrm{E}+04$ & $7.183 \mathrm{E}+01$ \\
\hline $1.599 \mathrm{E}+04$ & $2.651 \mathrm{E}+00$ & $7.25 \mathrm{E}-02$ & $1.094 \mathrm{E}+01$ & $5.935 \mathrm{E}+00$ & $2.261 \mathrm{E}+01$ & $9.877 \mathrm{E}+04$ & $9.778 \mathrm{E}+01$ \\
\hline $1.599 \mathrm{E}+04$ & $2.587 \mathrm{E}+00$ & $7.04 \mathrm{E}-02$ & $1.095 \mathrm{E}+01$ & $5.744 \mathrm{E}+00$ & $2.267 \mathrm{E}+01$ & $9.877 \mathrm{E}+04$ & $1.278 \mathrm{E}+02$ \\
\hline $1.603 \mathrm{E}+04$ & $2.488 \mathrm{E}+00$ & $7.32 \mathrm{E}-02$ & $1.096 \mathrm{E}+01$ & $5.523 \mathrm{E}+00$ & $2.274 \mathrm{E}+01$ & $9.877 \mathrm{E}+04$ & $1.618 \mathrm{E}+02$ \\
\hline $1.601 \mathrm{E}+04$ & $2.355 \mathrm{E}+00$ & $7.17 \mathrm{E}-02$ & $1.096 \mathrm{E}+01$ & $5.200 \mathrm{E}+00$ & $2.284 \mathrm{E}+01$ & $9.878 \mathrm{E}+04$ & $1.996 \mathrm{E}+02$ \\
\hline $1.598 \mathrm{E}+04$ & $2.187 \mathrm{E}+00$ & $7.08 \mathrm{E}-02$ & $1.098 \mathrm{E}+01$ & $4.800 \mathrm{E}+00$ & $2.294 \mathrm{E}+01$ & $9.878 \mathrm{E}+04$ & $2.407 \mathrm{E}+02$ \\
\hline $1.597 \mathrm{E}+04$ & $1.980 \mathrm{E}+00$ & $6.95 \mathrm{E}-02$ & $1.099 \mathrm{E}+01$ & $4.359 \mathrm{E}+00$ & $2.296 \mathrm{E}+01$ & $9.878 \mathrm{E}+04$ & $2.863 \mathrm{E}+02$ \\
\hline
\end{tabular}


Table 207: Graupner 4.7 x 4.0 Dynamic Calculated Values - 15997 RPM

\begin{tabular}{|c|c|c|c|c|c|c|c|}
\hline$n(\mathrm{RPM})$ & $V_{\infty}^{\prime}(\mathrm{m} / \mathrm{s})$ & $\Delta V_{\infty}^{\prime}(\mathrm{m} / \mathrm{s})$ & $T^{\prime}(\mathrm{g})$ & $\Delta T^{\prime}(\mathrm{g})$ & $V_{\mathrm{t}}(\mathrm{m} / \mathrm{s})$ & $P_{\mathrm{P}}(\mathrm{W})$ & $\Delta P_{\mathrm{P}}(\mathrm{W})$ \\
\hline $1.601 \mathrm{E}+04$ & $2.344 \mathrm{E}+00$ & $1.71 \mathrm{E}-02$ & $2.373 \mathrm{E}+02$ & $8.01 E+00$ & $7.493 \mathrm{E}+01$ & $4.366 \mathrm{E}+01$ & $1.21 \mathrm{E}+00$ \\
\hline $1.602 \mathrm{E}+04$ & $3.903 \mathrm{E}+00$ & $1.80 \mathrm{E}-02$ & $2.322 \mathrm{E}+02$ & $8.05 E+00$ & $7.501 \mathrm{E}+01$ & $4.411 \mathrm{E}+01$ & $1.19 \mathrm{E}+00$ \\
\hline $1.599 \mathrm{E}+04$ & $5.600 \mathrm{E}+00$ & $1.93 \mathrm{E}-02$ & $2.249 \mathrm{E}+02$ & $8.06 \mathrm{E}+00$ & $7.501 \mathrm{E}+01$ & $4.437 \mathrm{E}+01$ & $1.18 \mathrm{E}+00$ \\
\hline $1.598 \mathrm{E}+04$ & $7.364 \mathrm{E}+00$ & $2.13 \mathrm{E}-02$ & $2.145 \mathrm{E}+02$ & $8.13 \mathrm{E}+00$ & $7.511 \mathrm{E}+01$ & $4.470 \mathrm{E}+01$ & $1.18 \mathrm{E}+00$ \\
\hline $1.599 \mathrm{E}+04$ & $9.178 \mathrm{E}+00$ & $2.41 \mathrm{E}-02$ & $2.031 \mathrm{E}+02$ & $8.13 \mathrm{E}+00$ & $7.532 \mathrm{E}+01$ & $4.470 \mathrm{E}+01$ & $1.18 \mathrm{E}+00$ \\
\hline $1.600 \mathrm{E}+04$ & $1.100 \mathrm{E}+01$ & $2.68 \mathrm{E}-02$ & $1.923 \mathrm{E}+02$ & $8.19 \mathrm{E}+00$ & $7.565 \mathrm{E}+01$ & $4.483 \mathrm{E}+01$ & $1.19 \mathrm{E}+00$ \\
\hline $1.599 \mathrm{E}+04$ & $1.287 \mathrm{E}+01$ & $2.92 \mathrm{E}-02$ & $1.775 \mathrm{E}+02$ & $8.42 \mathrm{E}+00$ & $7.588 \mathrm{E}+01$ & $4.354 \mathrm{E}+01$ & $1.19 \mathrm{E}+00$ \\
\hline $1.599 \mathrm{E}+04$ & $1.474 \mathrm{E}+01$ & $3.14 \mathrm{E}-02$ & $1.631 \mathrm{E}+02$ & $8.55 \mathrm{E}+00$ & $7.622 \mathrm{E}+01$ & $4.249 \mathrm{E}+01$ & $1.16 \mathrm{E}+00$ \\
\hline $1.603 \mathrm{E}+04$ & $1.661 \mathrm{E}+01$ & $3.34 \mathrm{E}-02$ & $1.466 \mathrm{E}+02$ & $8.48 \mathrm{E}+00$ & $7.678 \mathrm{E}+01$ & $4.096 \mathrm{E}+01$ & $1.21 \mathrm{E}+00$ \\
\hline $1.601 \mathrm{E}+04$ & $1.847 \mathrm{E}+01$ & $3.61 \mathrm{E}-02$ & $1.300 \mathrm{E}+02$ & $8.55 \mathrm{E}+00$ & $7.711 \mathrm{E}+01$ & $3.871 \mathrm{E}+01$ & $1.18 \mathrm{E}+00$ \\
\hline $1.598 \mathrm{E}+04$ & $2.030 \mathrm{E}+01$ & $3.94 \mathrm{E}-02$ & $1.112 \mathrm{E}+02$ & $8.75 \mathrm{E}+00$ & $7.744 \mathrm{E}+01$ & $3.589 \mathrm{E}+01$ & $1.16 \mathrm{E}+00$ \\
\hline $1.597 \mathrm{E}+04$ & $2.216 \mathrm{E}+01$ & $4.26 \mathrm{E}-02$ & $9.025 \mathrm{E}+01$ & $8.91 \mathrm{E}+00$ & $7.791 \mathrm{E}+01$ & $3.248 \mathrm{E}+01$ & $1.14 \mathrm{E}+00$ \\
\hline$P_{\mathrm{e}}(\mathrm{W})$ & $\Delta P_{\mathrm{e}}(\mathrm{W})$ & $C_{\mathrm{T}}$ & $\Delta C_{\mathrm{T}}$ & $C_{\mathrm{P}}$ & $\Delta C_{\mathrm{P}}$ & $C_{\mathrm{Q}}$ & $\Delta C_{\mathrm{Q}}$ \\
\hline $6.613 \mathrm{E}+01$ & $7.43 \mathrm{E}-01$ & $1.393 \mathrm{E}-01$ & $4.70 \mathrm{E}-03$ & $8.223 \mathrm{E}-02$ & $2.28 \mathrm{E}-03$ & $1.309 \mathrm{E}-02$ & $3.63 \mathrm{E}-04$ \\
\hline $6.679 \mathrm{E}+01$ & $7.45 \mathrm{E}-01$ & $1.363 \mathrm{E}-01$ & $4.72 \mathrm{E}-03$ & $8.303 \mathrm{E}-02$ & $2.24 \mathrm{E}-03$ & $1.322 \mathrm{E}-02$ & $3.57 \mathrm{E}-04$ \\
\hline $6.711 \mathrm{E}+01$ & 7.44E-01 & $1.325 \mathrm{E}-01$ & $4.75 \mathrm{E}-03$ & 8.394E-02 & $2.22 \mathrm{E}-03$ & $1.336 \mathrm{E}-02$ & $3.54 \mathrm{E}-04$ \\
\hline $6.726 \mathrm{E}+01$ & 7.49E-01 & $1.266 \mathrm{E}-01$ & $4.80 \mathrm{E}-03$ & $8.477 \mathrm{E}-02$ & $2.24 \mathrm{E}-03$ & $1.349 \mathrm{E}-02$ & 3.57E-04 \\
\hline $6.723 \mathrm{E}+01$ & $7.45 \mathrm{E}-01$ & $1.199 \mathrm{E}-01$ & $4.80 \mathrm{E}-03$ & $8.477 \mathrm{E}-02$ & $2.24 \mathrm{E}-03$ & $1.349 \mathrm{E}-02$ & $3.57 \mathrm{E}-04$ \\
\hline $6.683 \mathrm{E}+01$ & $7.45 \mathrm{E}-01$ & $1.132 \mathrm{E}-01$ & $4.83 \mathrm{E}-03$ & $8.477 \mathrm{E}-02$ & $2.25 \mathrm{E}-03$ & $1.349 \mathrm{E}-02$ & $3.58 \mathrm{E}-04$ \\
\hline $6.495 \mathrm{E}+01$ & $7.24 \mathrm{E}-01$ & $1.047 \mathrm{E}-01$ & $4.97 \mathrm{E}-03$ & $8.255 \mathrm{E}-02$ & $2.26 \mathrm{E}-03$ & $1.314 \mathrm{E}-02$ & $3.60 \mathrm{E}-04$ \\
\hline $6.289 \mathrm{E}+01$ & $7.03 \mathrm{E}-01$ & $9.627 \mathrm{E}-02$ & $5.05 \mathrm{E}-03$ & $8.056 \mathrm{E}-02$ & $2.19 \mathrm{E}-03$ & $1.282 \mathrm{E}-02$ & $3.49 \mathrm{E}-04$ \\
\hline $6.050 \mathrm{E}+01$ & $6.82 \mathrm{E}-01$ & $8.615 \mathrm{E}-02$ & $4.98 \mathrm{E}-03$ & $7.713 \mathrm{E}-02$ & $2.27 \mathrm{E}-03$ & $1.228 \mathrm{E}-02$ & $3.61 \mathrm{E}-04$ \\
\hline $5.702 \mathrm{E}+01$ & $6.47 \mathrm{E}-01$ & $7.662 \mathrm{E}-02$ & $5.04 \mathrm{E}-03$ & $7.320 \mathrm{E}-02$ & $2.23 \mathrm{E}-03$ & $1.165 \mathrm{E}-02$ & $3.55 \mathrm{E}-04$ \\
\hline $5.268 \mathrm{E}+01$ & $5.87 \mathrm{E}-01$ & $6.576 \mathrm{E}-02$ & $5.17 \mathrm{E}-03$ & $6.825 \mathrm{E}-02$ & $2.21 \mathrm{E}-03$ & $1.086 \mathrm{E}-02$ & $3.52 \mathrm{E}-04$ \\
\hline $4.790 \mathrm{E}+01$ & 5.33E-01 & $5.344 \mathrm{E}-02$ & $5.27 \mathrm{E}-03$ & $6.186 \mathrm{E}-02$ & $2.17 \mathrm{E}-03$ & $9.846 \mathrm{E}-03$ & $3.46 \mathrm{E}-04$ \\
\hline$\rho\left(\mathrm{kg} / \mathrm{m}^{\wedge} 3\right)$ & $\eta_{\mathrm{T}}$ & $\Delta \eta_{\mathrm{T}}$ & $J$ & $\Delta J$ & $\eta_{\mathrm{P}}$ & $\Delta \eta_{\mathrm{P}}$ & $R e_{0.75}$ \\
\hline $1.166 \mathrm{E}+00$ & $6.602 \mathrm{E}-01$ & $1.98 \mathrm{E}-02$ & $7.376 \mathrm{E}-02$ & $5.39 \mathrm{E}-04$ & $1.250 \mathrm{E}-01$ & $5.54 \mathrm{E}-03$ & $4.538 \mathrm{E}+04$ \\
\hline $1.166 \mathrm{E}+00$ & $6.604 \mathrm{E}-01$ & $1.93 \mathrm{E}-02$ & $1.228 \mathrm{E}-01$ & $5.67 \mathrm{E}-04$ & $2.015 \mathrm{E}-01$ & $8.90 \mathrm{E}-03$ & $4.541 \mathrm{E}+04$ \\
\hline $1.165 \mathrm{E}+00$ & $6.612 \mathrm{E}-01$ & $1.90 \mathrm{E}-02$ & $1.764 \mathrm{E}-01$ & $6.10 \mathrm{E}-04$ & $2.784 \mathrm{E}-01$ & $1.24 \mathrm{E}-02$ & $4.537 \mathrm{E}+04$ \\
\hline $1.164 \mathrm{E}+00$ & $6.645 \mathrm{E}-01$ & $1.91 \mathrm{E}-02$ & $2.321 \mathrm{E}-01$ & $6.75 \mathrm{E}-04$ & $3.466 \mathrm{E}-01$ & $1.60 \mathrm{E}-02$ & $4.539 \mathrm{E}+04$ \\
\hline $1.164 \mathrm{E}+00$ & $6.648 \mathrm{E}-01$ & $1.91 \mathrm{E}-02$ & $2.893 \mathrm{E}-01$ & $7.61 \mathrm{E}-04$ & $4.091 \mathrm{E}-01$ & $1.97 \mathrm{E}-02$ & $4.548 \mathrm{E}+04$ \\
\hline $1.164 \mathrm{E}+00$ & $6.708 \mathrm{E}-01$ & $1.93 \mathrm{E}-02$ & $3.464 \mathrm{E}-01$ & $8.47 \mathrm{E}-04$ & $4.628 \mathrm{E}-01$ & $2.33 \mathrm{E}-02$ & $4.566 \mathrm{E}+04$ \\
\hline $1.163 \mathrm{E}+00$ & $6.703 \mathrm{E}-01$ & $1.98 \mathrm{E}-02$ & $4.055 \mathrm{E}-01$ & $9.24 \mathrm{E}-04$ & $5.145 \mathrm{E}-01$ & $2.82 \mathrm{E}-02$ & $4.578 \mathrm{E}+04$ \\
\hline $1.163 \mathrm{E}+00$ & $6.756 \mathrm{E}-01$ & $1.99 \mathrm{E}-02$ & 4.643E-01 & $9.95 \mathrm{E}-04$ & $5.549 \mathrm{E}-01$ & $3.28 \mathrm{E}-02$ & $4.597 \mathrm{E}+04$ \\
\hline $1.163 \mathrm{E}+00$ & $6.769 \mathrm{E}-01$ & $2.13 \mathrm{E}-02$ & $5.221 \mathrm{E}-01$ & $1.06 \mathrm{E}-03$ & $5.831 \mathrm{E}-01$ & $3.79 \mathrm{E}-02$ & $4.629 \mathrm{E}+04$ \\
\hline $1.163 \mathrm{E}+00$ & $6.789 \mathrm{E}-01$ & $2.21 \mathrm{E}-02$ & 5.812E-01 & $1.15 \mathrm{E}-03$ & $6.084 \mathrm{E}-01$ & $4.41 \mathrm{E}-02$ & $4.646 \mathrm{E}+04$ \\
\hline $1.162 \mathrm{E}+00$ & $6.812 \mathrm{E}-01$ & $2.33 \mathrm{E}-02$ & $6.401 \mathrm{E}-01$ & $1.25 \mathrm{E}-03$ & $6.168 \mathrm{E}-01$ & $5.25 \mathrm{E}-02$ & $4.663 \mathrm{E}+04$ \\
\hline $1.162 \mathrm{E}+00$ & $6.780 \mathrm{E}-01$ & $2.50 \mathrm{E}-02$ & $6.989 \mathrm{E}-01$ & $1.35 \mathrm{E}-03$ & $6.038 \mathrm{E}-01$ & $6.33 \mathrm{E}-02$ & $4.691 \mathrm{E}+04$ \\
\hline
\end{tabular}


Table 208: Graupner 4.7 x 4.0 Dynamic Measured Values - 20027 RPM

\begin{tabular}{|c|c|c|c|c|c|c|c|}
\hline$n(\mathrm{RPM})$ & $Q(\mathrm{~g}-\mathrm{m})$ & $\Delta Q(\mathrm{~g}-\mathrm{m})$ & $V(\mathrm{~V})$ & $I(\mathrm{~A})$ & $T_{\text {atm }}\left({ }^{\circ} \mathrm{C}\right)$ & $P_{\text {atm }}(\mathrm{Pa})$ & $P_{\text {diff }}(\mathrm{Pa})$ \\
\hline $1.996 \mathrm{E}+04$ & $4.069 \mathrm{E}+00$ & $1.82 \mathrm{E}-01$ & $1.078 \mathrm{E}+01$ & $1.126 \mathrm{E}+01$ & $2.232 \mathrm{E}+01$ & $9.873 \mathrm{E}+04$ & $4.567 \mathrm{E}+00$ \\
\hline $1.994 \mathrm{E}+04$ & $4.235 \mathrm{E}+00$ & $1.83 \mathrm{E}-01$ & $1.078 \mathrm{E}+01$ & $1.142 \mathrm{E}+01$ & $2.255 \mathrm{E}+01$ & $9.874 \mathrm{E}+04$ & $1.485 \mathrm{E}+01$ \\
\hline $2.010 \mathrm{E}+04$ & $4.351 \mathrm{E}+00$ & $2.15 \mathrm{E}-01$ & $1.076 \mathrm{E}+01$ & $1.189 \mathrm{E}+01$ & $2.276 \mathrm{E}+01$ & $9.873 \mathrm{E}+04$ & $3.396 \mathrm{E}+01$ \\
\hline $2.007 \mathrm{E}+04$ & $4.416 \mathrm{E}+00$ & $2.04 \mathrm{E}-01$ & $1.076 \mathrm{E}+01$ & $1.193 \mathrm{E}+01$ & $2.298 \mathrm{E}+01$ & $9.873 \mathrm{E}+04$ & $6.166 \mathrm{E}+01$ \\
\hline $2.005 \mathrm{E}+04$ & $4.356 \mathrm{E}+00$ & $2.05 \mathrm{E}-01$ & $1.076 \mathrm{E}+01$ & $1.195 \mathrm{E}+01$ & $2.311 \mathrm{E}+01$ & $9.873 \mathrm{E}+04$ & $9.924 \mathrm{E}+01$ \\
\hline $2.006 \mathrm{E}+04$ & $4.378 \mathrm{E}+00$ & $2.07 \mathrm{E}-01$ & $1.077 \mathrm{E}+01$ & $1.173 \mathrm{E}+01$ & $2.324 \mathrm{E}+01$ & $9.873 \mathrm{E}+04$ & $1.457 \mathrm{E}+02$ \\
\hline $2.007 \mathrm{E}+04$ & $4.250 \mathrm{E}+00$ & $1.99 \mathrm{E}-01$ & $1.079 \mathrm{E}+01$ & $1.116 \mathrm{E}+01$ & $2.320 \mathrm{E}+01$ & $9.872 \mathrm{E}+04$ & $2.014 \mathrm{E}+02$ \\
\hline $2.004 \mathrm{E}+04$ & $3.958 \mathrm{E}+00$ & $1.91 \mathrm{E}-01$ & $1.081 \mathrm{E}+01$ & $1.041 \mathrm{E}+01$ & $2.332 \mathrm{E}+01$ & $9.872 \mathrm{E}+04$ & $2.649 \mathrm{E}+02$ \\
\hline $2.000 \mathrm{E}+04$ & $3.584 \mathrm{E}+00$ & $1.76 \mathrm{E}-01$ & $1.084 \mathrm{E}+01$ & $9.467 \mathrm{E}+00$ & $2.333 \mathrm{E}+01$ & $9.872 \mathrm{E}+04$ & $3.371 \mathrm{E}+02$ \\
\hline $1.999 \mathrm{E}+04$ & $3.201 \mathrm{E}+00$ & $1.60 \mathrm{E}-01$ & $1.087 \mathrm{E}+01$ & $8.454 \mathrm{E}+00$ & $2.354 \mathrm{E}+01$ & $9.872 \mathrm{E}+04$ & $4.181 \mathrm{E}+02$ \\
\hline $2.002 \mathrm{E}+04$ & $2.883 \mathrm{E}+00$ & $1.47 \mathrm{E}-01$ & $1.090 \mathrm{E}+01$ & $7.240 \mathrm{E}+00$ & $2.354 \mathrm{E}+01$ & $9.872 \mathrm{E}+04$ & $5.076 \mathrm{E}+02$ \\
\hline
\end{tabular}


Table 209: Graupner 4.7 x 4.0 Dynamic Calculated Values - 20027 RPM

\begin{tabular}{|c|c|c|c|c|c|c|c|}
\hline$n(\mathrm{RPM})$ & $V_{\infty}^{\prime}(\mathrm{m} / \mathrm{s})$ & $\Delta V_{\infty}^{\prime}(\mathrm{m} / \mathrm{s})$ & $T^{\prime}(\mathrm{g})$ & $\Delta T^{\prime}(\mathrm{g})$ & $V_{\mathrm{t}}(\mathrm{m} / \mathrm{s})$ & $P_{\mathrm{P}}(\mathrm{W})$ & $\Delta P_{\mathrm{P}}(\mathrm{W})$ \\
\hline $1.996 \mathrm{E}+04$ & $2.622 \mathrm{E}+00$ & 2.07E-02 & $3.829 \mathrm{E}+02$ & $7.34 \mathrm{E}+00$ & $9.337 \mathrm{E}+01$ & $8.339 \mathrm{E}+01$ & $3.72 \mathrm{E}+00$ \\
\hline $1.994 \mathrm{E}+04$ & $4.879 \mathrm{E}+00$ & $2.21 \mathrm{E}-02$ & $3.709 \mathrm{E}+02$ & $7.44 \mathrm{E}+00$ & $9.338 \mathrm{E}+01$ & $8.672 \mathrm{E}+01$ & $3.76 \mathrm{E}+00$ \\
\hline $2.010 \mathrm{E}+04$ & $7.477 \mathrm{E}+00$ & $2.48 \mathrm{E}-02$ & $3.611 \mathrm{E}+02$ & $7.31 \mathrm{E}+00$ & $9.428 \mathrm{E}+01$ & $8.980 \mathrm{E}+01$ & $4.44 \mathrm{E}+00$ \\
\hline $2.007 \mathrm{E}+04$ & $1.015 \mathrm{E}+01$ & $2.92 \mathrm{E}-02$ & $3.446 \mathrm{E}+02$ & $7.28 \mathrm{E}+00$ & $9.439 \mathrm{E}+01$ & $9.100 \mathrm{E}+01$ & $4.21 \mathrm{E}+00$ \\
\hline $2.005 \mathrm{E}+04$ & $1.293 \mathrm{E}+01$ & $3.30 \mathrm{E}-02$ & $3.279 \mathrm{E}+02$ & $7.27 \mathrm{E}+00$ & $9.466 \mathrm{E}+01$ & $8.968 \mathrm{E}+01$ & $4.21 \mathrm{E}+00$ \\
\hline $2.006 \mathrm{E}+04$ & $1.572 \mathrm{E}+01$ & $3.52 \mathrm{E}-02$ & $3.036 \mathrm{E}+02$ & $7.32 \mathrm{E}+00$ & $9.513 \mathrm{E}+01$ & $9.019 \mathrm{E}+01$ & $4.27 \mathrm{E}+00$ \\
\hline $2.007 \mathrm{E}+04$ & $1.852 \mathrm{E}+01$ & $3.79 \mathrm{E}-02$ & $2.711 \mathrm{E}+02$ & $7.24 \mathrm{E}+00$ & $9.569 \mathrm{E}+01$ & $8.762 \mathrm{E}+01$ & $4.10 \mathrm{E}+00$ \\
\hline $2.004 \mathrm{E}+04$ & $2.128 \mathrm{E}+01$ & 4.15E-02 & $2.386 \mathrm{E}+02$ & $7.29 \mathrm{E}+00$ & $9.611 \mathrm{E}+01$ & $8.145 \mathrm{E}+01$ & $3.94 \mathrm{E}+00$ \\
\hline $2.000 \mathrm{E}+04$ & $2.403 \mathrm{E}+01$ & $4.58 \mathrm{E}-02$ & $2.008 \mathrm{E}+02$ & $7.29 \mathrm{E}+00$ & $9.658 \mathrm{E}+01$ & $7.362 \mathrm{E}+01$ & $3.61 \mathrm{E}+00$ \\
\hline $1.999 \mathrm{E}+04$ & $2.680 \mathrm{E}+01$ & $5.10 \mathrm{E}-02$ & $1.638 \mathrm{E}+02$ & $7.29 \mathrm{E}+00$ & $9.727 \mathrm{E}+01$ & $6.573 \mathrm{E}+01$ & $3.29 \mathrm{E}+00$ \\
\hline $2.002 \mathrm{E}+04$ & $2.955 \mathrm{E}+01$ & $5.51 \mathrm{E}-02$ & $1.240 \mathrm{E}+02$ & $7.35 \mathrm{E}+00$ & $9.820 \mathrm{E}+01$ & $5.928 \mathrm{E}+01$ & $3.02 \mathrm{E}+00$ \\
\hline$P_{\mathrm{e}}(\mathrm{W})$ & $\Delta P_{\mathrm{e}}(\mathrm{W})$ & $C_{\mathrm{T}}$ & $\Delta C_{\mathrm{T}}$ & $C_{\mathrm{P}}$ & $\Delta C_{\mathrm{P}}$ & $C_{\mathrm{Q}}$ & $\Delta C_{\mathrm{Q}}$ \\
\hline $1.215 \mathrm{E}+02$ & $1.29 \mathrm{E}+00$ & $1.450 \mathrm{E}-01$ & $2.78 \mathrm{E}-03$ & $8.128 \mathrm{E}-02$ & $3.63 \mathrm{E}-03$ & $1.294 \mathrm{E}-02$ & $5.78 \mathrm{E}-04$ \\
\hline $1.231 \mathrm{E}+02$ & $1.28 \mathrm{E}+00$ & $1.408 \mathrm{E}-01$ & $2.82 \mathrm{E}-03$ & $8.480 \mathrm{E}-02$ & $3.67 \mathrm{E}-03$ & $1.350 \mathrm{E}-02$ & $5.84 \mathrm{E}-04$ \\
\hline $1.280 \mathrm{E}+02$ & $1.47 \mathrm{E}+00$ & $1.350 \mathrm{E}-01$ & $2.74 \mathrm{E}-03$ & $8.583 \mathrm{E}-02$ & $4.25 \mathrm{E}-03$ & $1.366 \mathrm{E}-02$ & $6.76 \mathrm{E}-04$ \\
\hline $1.284 \mathrm{E}+02$ & $1.47 \mathrm{E}+00$ & $1.293 \mathrm{E}-01$ & $2.73 \mathrm{E}-03$ & $8.744 \mathrm{E}-02$ & $4.04 \mathrm{E}-03$ & $1.392 \mathrm{E}-02$ & $6.44 \mathrm{E}-04$ \\
\hline $1.286 \mathrm{E}+02$ & $1.48 \mathrm{E}+00$ & $1.233 \mathrm{E}-01$ & $2.74 \mathrm{E}-03$ & 8.643E-02 & $4.06 \mathrm{E}-03$ & $1.376 \mathrm{E}-02$ & $6.46 \mathrm{E}-04$ \\
\hline $1.263 \mathrm{E}+02$ & $1.53 \mathrm{E}+00$ & $1.141 \mathrm{E}-01$ & $2.75 \mathrm{E}-03$ & 8.681E-02 & $4.11 \mathrm{E}-03$ & $1.382 \mathrm{E}-02$ & $6.54 \mathrm{E}-04$ \\
\hline $1.203 \mathrm{E}+02$ & $1.26 \mathrm{E}+00$ & $1.018 \mathrm{E}-01$ & $2.72 \mathrm{E}-03$ & $8.417 \mathrm{E}-02$ & $3.94 \mathrm{E}-03$ & $1.340 \mathrm{E}-02$ & $6.27 \mathrm{E}-04$ \\
\hline $1.126 \mathrm{E}+02$ & $1.19 \mathrm{E}+00$ & 8.990E-02 & $2.75 \mathrm{E}-03$ & $7.868 \mathrm{E}-02$ & $3.80 \mathrm{E}-03$ & $1.252 \mathrm{E}-02$ & $6.05 \mathrm{E}-04$ \\
\hline $1.026 \mathrm{E}+02$ & $1.08 \mathrm{E}+00$ & $7.595 \mathrm{E}-02$ & $2.76 \mathrm{E}-03$ & 7.153E-02 & $3.50 \mathrm{E}-03$ & $1.138 \mathrm{E}-02$ & $5.58 \mathrm{E}-04$ \\
\hline $9.188 \mathrm{E}+01$ & $9.69 \mathrm{E}-01$ & $6.205 \mathrm{E}-02$ & $2.76 \mathrm{E}-03$ & $6.398 \mathrm{E}-02$ & $3.21 \mathrm{E}-03$ & $1.018 \mathrm{E}-02$ & $5.10 \mathrm{E}-04$ \\
\hline $7.894 \mathrm{E}+01$ & 8.93E-01 & $4.684 \mathrm{E}-02$ & $2.78 \mathrm{E}-03$ & $5.744 \mathrm{E}-02$ & 2.93E-03 & $9.142 \mathrm{E}-03$ & $4.66 \mathrm{E}-04$ \\
\hline$\rho\left(\mathrm{kg} / \mathrm{m}^{\wedge} 3\right)$ & $\eta_{\mathrm{T}}$ & $\Delta \eta_{\mathrm{T}}$ & $J$ & $\Delta J$ & $\eta_{\mathrm{P}}$ & $\Delta \eta_{\mathrm{P}}$ & $R e_{0.75}$ \\
\hline $1.164 \mathrm{E}+00$ & $6.865 \mathrm{E}-01$ & $3.15 \mathrm{E}-02$ & $6.620 \mathrm{E}-02$ & $5.23 \mathrm{E}-04$ & $1.181 \mathrm{E}-01$ & $5.81 \mathrm{E}-03$ & $5.640 \mathrm{E}+04$ \\
\hline $1.163 \mathrm{E}+00$ & $7.046 \mathrm{E}-01$ & $3.14 \mathrm{E}-02$ & $1.233 \mathrm{E}-01$ & $5.60 \mathrm{E}-04$ & $2.047 \mathrm{E}-01$ & $9.81 \mathrm{E}-03$ & $5.634 \mathrm{E}+04$ \\
\hline $1.162 \mathrm{E}+00$ & $7.018 \mathrm{E}-01$ & $3.57 \mathrm{E}-02$ & $1.875 \mathrm{E}-01$ & $6.24 \mathrm{E}-04$ & $2.948 \mathrm{E}-01$ & $1.58 \mathrm{E}-02$ & $5.681 \mathrm{E}+04$ \\
\hline $1.162 \mathrm{E}+00$ & $7.086 \mathrm{E}-01$ & $3.38 \mathrm{E}-02$ & $2.548 \mathrm{E}-01$ & 7.39E-04 & $3.769 \mathrm{E}-01$ & $1.92 \mathrm{E}-02$ & $5.680 \mathrm{E}+04$ \\
\hline $1.161 \mathrm{E}+00$ & $6.974 \mathrm{E}-01$ & 3.37E-02 & 3.249E-01 & $8.36 \mathrm{E}-04$ & 4.637E-01 & $2.41 \mathrm{E}-02$ & $5.691 \mathrm{E}+04$ \\
\hline $1.160 \mathrm{E}+00$ & 7.143E-01 & $3.49 \mathrm{E}-02$ & 3.947E-01 & $9.00 \mathrm{E}-04$ & 5.189E-01 & $2.76 \mathrm{E}-02$ & $5.715 \mathrm{E}+04$ \\
\hline $1.161 \mathrm{E}+00$ & $7.281 \mathrm{E}-01$ & $3.49 \mathrm{E}-02$ & $4.648 \mathrm{E}-01$ & $9.57 \mathrm{E}-04$ & 5.619E-01 & $3.03 \mathrm{E}-02$ & $5.750 \mathrm{E}+04$ \\
\hline $1.160 \mathrm{E}+00$ & $7.236 \mathrm{E}-01$ & $3.58 \mathrm{E}-02$ & 5.349E-01 & $1.05 \mathrm{E}-03$ & $6.112 \mathrm{E}-01$ & $3.50 \mathrm{E}-02$ & $5.771 \mathrm{E}+04$ \\
\hline $1.160 \mathrm{E}+00$ & $7.175 \mathrm{E}-01$ & $3.59 \mathrm{E}-02$ & $6.054 \mathrm{E}-01$ & $1.16 \mathrm{E}-03$ & $6.429 \mathrm{E}-01$ & $3.92 \mathrm{E}-02$ & $5.799 \mathrm{E}+04$ \\
\hline $1.159 \mathrm{E}+00$ & 7.154E-01 & $3.66 \mathrm{E}-02$ & $6.753 \mathrm{E}-01$ & $1.29 \mathrm{E}-03$ & $6.550 \mathrm{E}-01$ & 4.39E-02 & $5.833 \mathrm{E}+04$ \\
\hline $1.159 \mathrm{E}+00$ & $7.509 \mathrm{E}-01$ & $3.92 \mathrm{E}-02$ & $7.436 \mathrm{E}-01$ & $1.40 \mathrm{E}-03$ & $6.063 \mathrm{E}-01$ & 4.74E-02 & $5.889 \mathrm{E}+04$ \\
\hline
\end{tabular}




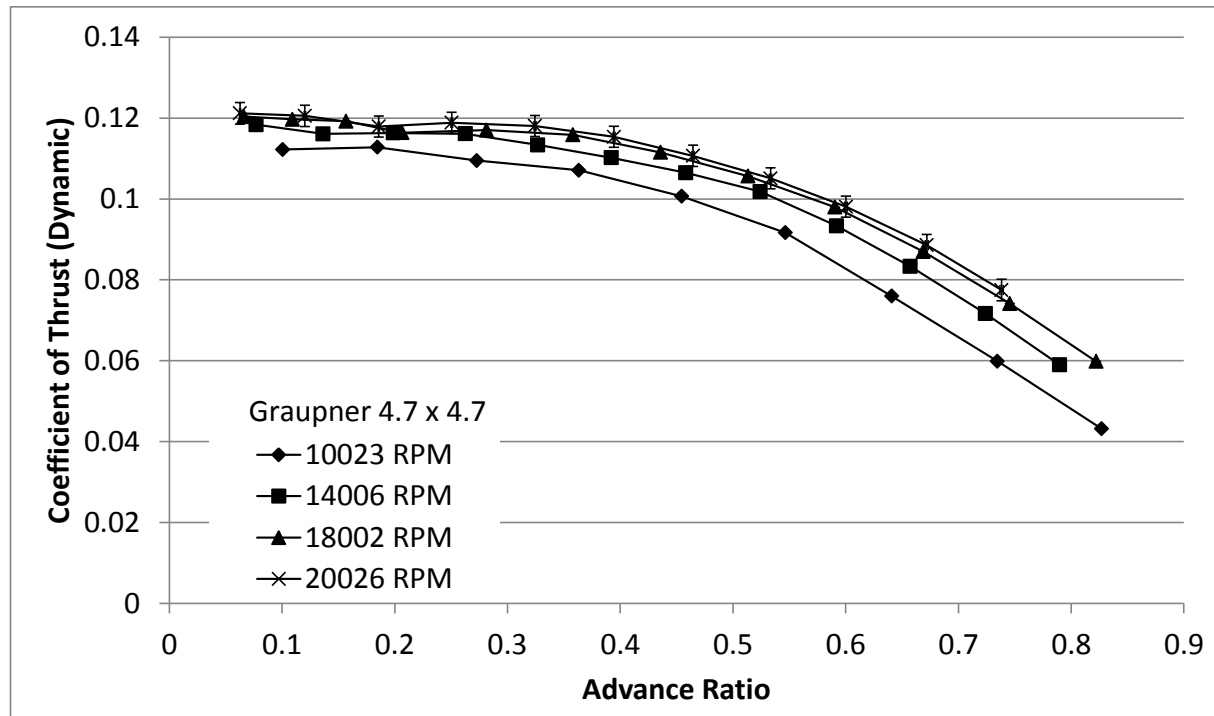

(a)

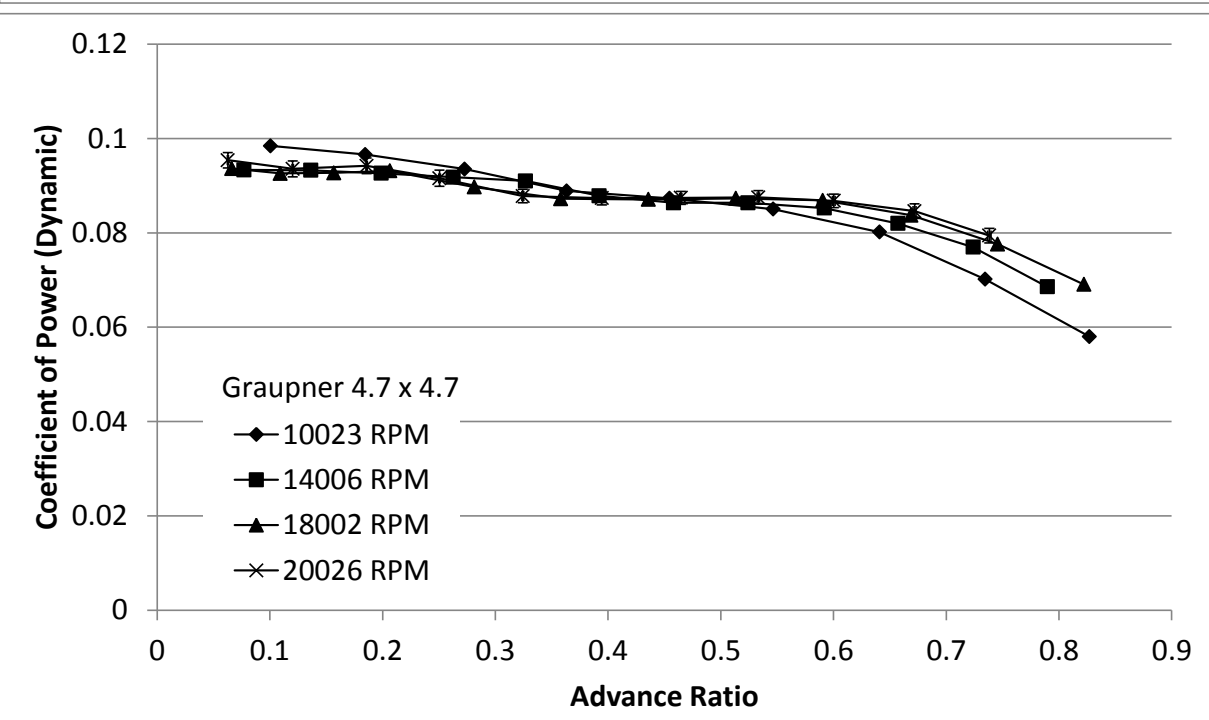

(b)

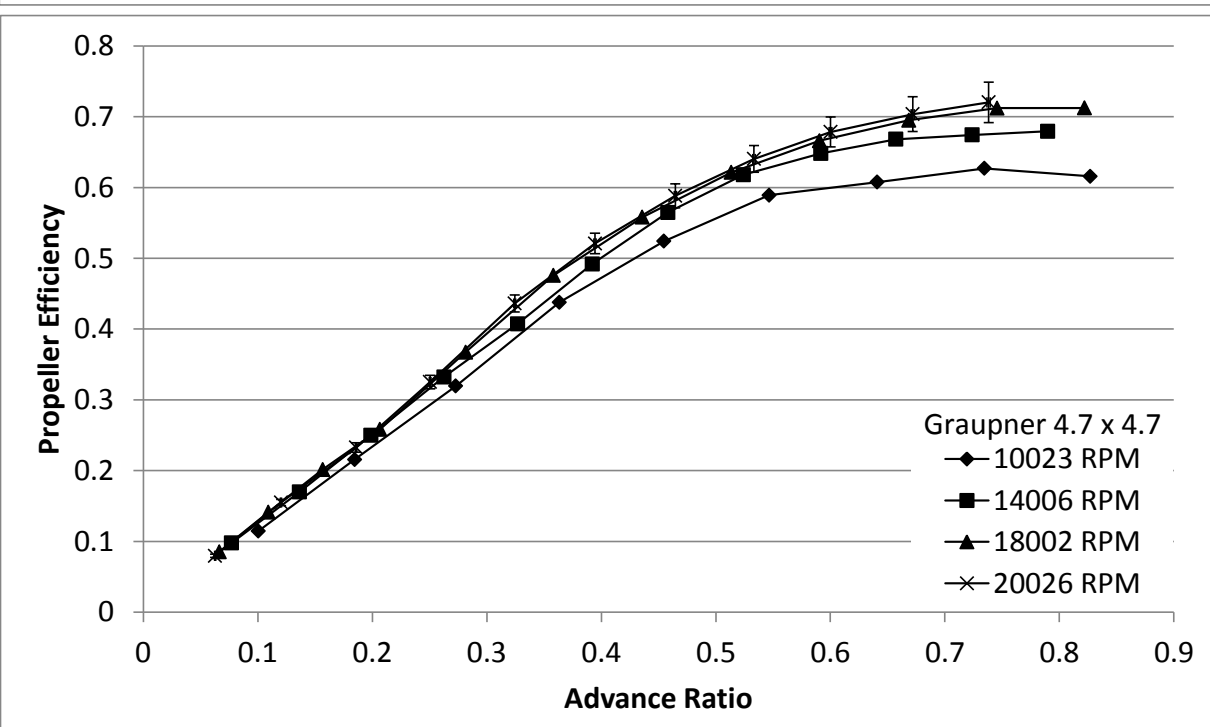

(c) 


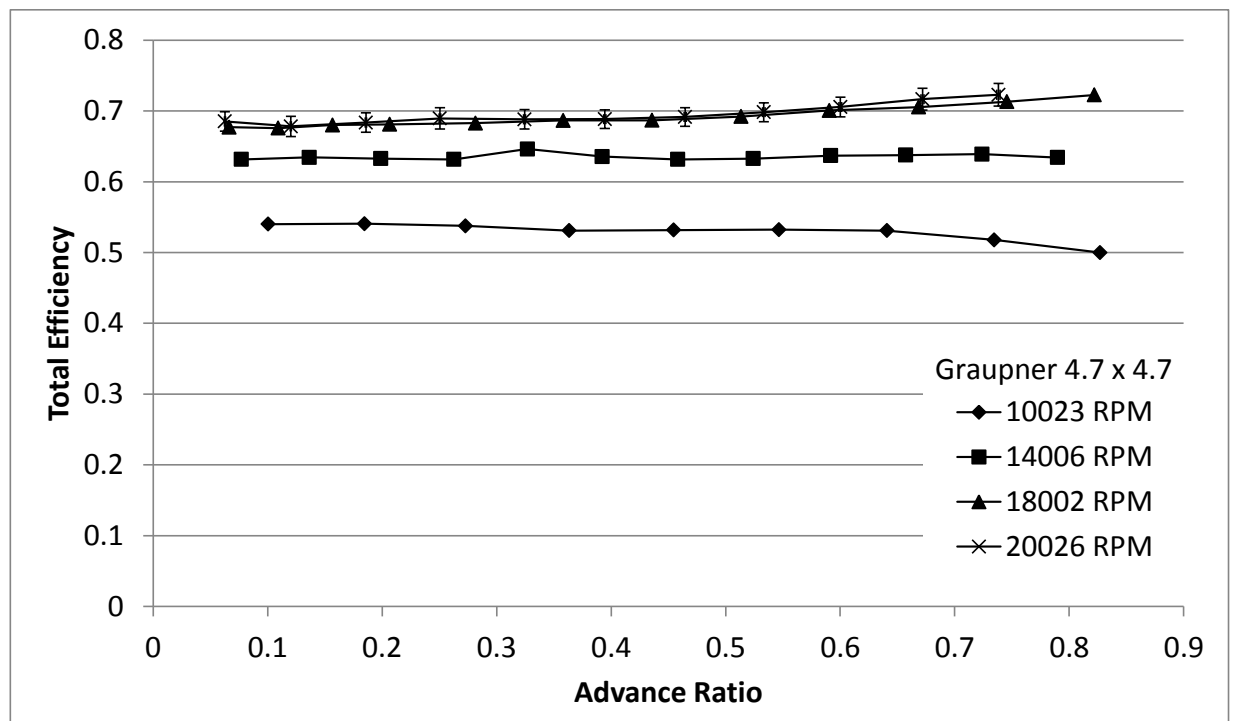

(d)

Figure 95: Graupner 4.7 x 4.7 Dynamic Test Results: (a) Coefficient of Thrust, (b) Coefficient of Power, (c) Propeller Efficiency, (d) Total Efficiency.

Table 210: Graupner 4.7 x 4.7 Dynamic Measured Values - 10023 RPM

\begin{tabular}{|c|c|c|c|c|c|c|c|}
\hline$n(\mathrm{RPM})$ & $Q(\mathrm{~g}-\mathrm{m})$ & $\Delta Q(\mathrm{~g}-\mathrm{m})$ & $V(\mathrm{~V})$ & $I(\mathrm{~A})$ & $T_{\text {atm }}\left({ }^{\circ} \mathrm{C}\right)$ & $P_{\text {atm }}(\mathrm{Pa})$ & $P_{\text {diff }}(\mathrm{Pa})$ \\
\hline $1.008 \mathrm{E}+04$ & $1.349 \mathrm{E}+00$ & $5.91 \mathrm{E}-02$ & $1.106 \mathrm{E}+01$ & $2.339 \mathrm{E}+00$ & $1.815 \mathrm{E}+01$ & $9.933 \mathrm{E}+04$ & $2.652 \mathrm{E}+00$ \\
\hline $1.007 \mathrm{E}+04$ & $1.323 \mathrm{E}+00$ & $5.94 \mathrm{E}-02$ & $1.106 \mathrm{E}+01$ & $2.289 \mathrm{E}+00$ & $1.805 \mathrm{E}+01$ & $9.934 \mathrm{E}+04$ & $8.609 \mathrm{E}+00$ \\
\hline $1.003 \mathrm{E}+04$ & $1.270 \mathrm{E}+00$ & $5.98 \mathrm{E}-02$ & $1.106 \mathrm{E}+01$ & $2.200 \mathrm{E}+00$ & $1.808 \mathrm{E}+01$ & $9.934 \mathrm{E}+04$ & $1.836 \mathrm{E}+01$ \\
\hline $1.001 \mathrm{E}+04$ & $1.202 \mathrm{E}+00$ & $5.70 \mathrm{E}-02$ & $1.106 \mathrm{E}+01$ & $2.104 \mathrm{E}+00$ & $1.820 \mathrm{E}+01$ & $9.935 \mathrm{E}+04$ & $3.216 \mathrm{E}+01$ \\
\hline $1.000 \mathrm{E}+04$ & $1.179 \mathrm{E}+00$ & $5.68 \mathrm{E}-02$ & $1.106 \mathrm{E}+01$ & $2.058 \mathrm{E}+00$ & $1.822 \mathrm{E}+01$ & $9.936 \mathrm{E}+04$ & $5.001 \mathrm{E}+01$ \\
\hline $1.003 \mathrm{E}+04$ & $1.153 \mathrm{E}+00$ & $5.82 \mathrm{E}-02$ & $1.106 \mathrm{E}+01$ & $2.017 \mathrm{E}+00$ & $1.825 \mathrm{E}+01$ & $9.937 \mathrm{E}+04$ & $7.234 \mathrm{E}+01$ \\
\hline $1.000 \mathrm{E}+04$ & $1.082 \mathrm{E}+00$ & $5.70 \mathrm{E}-02$ & $1.107 \mathrm{E}+01$ & $1.891 \mathrm{E}+00$ & $1.826 \mathrm{E}+01$ & $9.937 \mathrm{E}+04$ & $9.866 \mathrm{E}+01$ \\
\hline $9.990 \mathrm{E}+03$ & $9.451 \mathrm{E}-01$ & $5.75 \mathrm{E}-02$ & $1.107 \mathrm{E}+01$ & $1.691 \mathrm{E}+00$ & $1.823 \mathrm{E}+01$ & $9.938 \mathrm{E}+04$ & $1.291 \mathrm{E}+02$ \\
\hline $1.000 \mathrm{E}+04$ & $7.839 \mathrm{E}-01$ & $5.76 \mathrm{E}-02$ & $1.108 \mathrm{E}+01$ & $1.454 \mathrm{E}+00$ & $1.823 \mathrm{E}+01$ & $9.938 \mathrm{E}+04$ & $1.639 \mathrm{E}+02$ \\
\hline
\end{tabular}


Table 211: Graupner 4.7 x 4.7 Dynamic Calculated Values - 10023 RPM

\begin{tabular}{|c|c|c|c|c|c|c|c|}
\hline$n(\mathrm{RPM})$ & $V_{\infty}^{\prime}(\mathrm{m} / \mathrm{s})$ & $\Delta V_{\infty}^{\prime}(\mathrm{m} / \mathrm{s})$ & $T^{\prime}(\mathrm{g})$ & $\Delta T^{\prime}(\mathrm{g})$ & $V_{\mathrm{t}}(\mathrm{m} / \mathrm{s})$ & $P_{\mathrm{P}}(\mathrm{W})$ & $\Delta P_{\mathrm{P}}(\mathrm{W})$ \\
\hline $1.008 \mathrm{E}+04$ & $2.032 \mathrm{E}+00$ & $1.28 \mathrm{E}-02$ & $8.034 \mathrm{E}+01$ & $7.92 \mathrm{E}+00$ & $4.765 \mathrm{E}+01$ & $1.396 \mathrm{E}+01$ & $6.12 \mathrm{E}-01$ \\
\hline $1.007 \mathrm{E}+04$ & $3.728 \mathrm{E}+00$ & $1.33 \mathrm{E}-02$ & $8.070 \mathrm{E}+01$ & $7.91 \mathrm{E}+00$ & $4.773 \mathrm{E}+01$ & $1.369 \mathrm{E}+01$ & $6.15 \mathrm{E}-01$ \\
\hline $1.003 \mathrm{E}+04$ & $5.487 \mathrm{E}+00$ & $1.55 \mathrm{E}-02$ & $7.765 \mathrm{E}+01$ & $7.88 \mathrm{E}+00$ & $4.769 \mathrm{E}+01$ & $1.308 \mathrm{E}+01$ & $6.16 \mathrm{E}-01$ \\
\hline $1.001 \mathrm{E}+04$ & $7.293 \mathrm{E}+00$ & $1.81 \mathrm{E}-02$ & $7.564 \mathrm{E}+01$ & $7.91 \mathrm{E}+00$ & $4.784 \mathrm{E}+01$ & $1.235 \mathrm{E}+01$ & $5.86 \mathrm{E}-01$ \\
\hline $1.000 \mathrm{E}+04$ & $9.118 \mathrm{E}+00$ & $1.99 \mathrm{E}-02$ & $7.099 \mathrm{E}+01$ & $7.88 \mathrm{E}+00$ & $4.812 \mathrm{E}+01$ & $1.211 \mathrm{E}+01$ & $5.84 \mathrm{E}-01$ \\
\hline $1.003 \mathrm{E}+04$ & $1.099 \mathrm{E}+01$ & 2.27E-02 & $6.493 \mathrm{E}+01$ & $7.91 \mathrm{E}+00$ & $4.862 \mathrm{E}+01$ & $1.188 \mathrm{E}+01$ & $6.00 \mathrm{E}-01$ \\
\hline $1.000 \mathrm{E}+04$ & $1.285 \mathrm{E}+01$ & 2.61E-02 & $5.358 \mathrm{E}+01$ & $7.86 \mathrm{E}+00$ & $4.896 \mathrm{E}+01$ & $1.111 \mathrm{E}+01$ & $5.85 \mathrm{E}-01$ \\
\hline $9.990 \mathrm{E}+03$ & $1.471 \mathrm{E}+01$ & 2.89E-02 & $4.213 \mathrm{E}+01$ & $7.89 \mathrm{E}+00$ & $4.943 \mathrm{E}+01$ & $9.696 \mathrm{E}+00$ & $5.90 \mathrm{E}-01$ \\
\hline $1.000 \mathrm{E}+04$ & $1.659 \mathrm{E}+01$ & $3.23 \mathrm{E}-02$ & $3.049 \mathrm{E}+01$ & $7.88 \mathrm{E}+00$ & $5.009 \mathrm{E}+01$ & $8.054 \mathrm{E}+00$ & $5.92 \mathrm{E}-01$ \\
\hline$P_{\mathrm{e}}(\mathrm{W})$ & $\Delta P_{\mathrm{e}}(\mathrm{W})$ & $C_{\mathrm{T}}$ & $\Delta C_{\mathrm{T}}$ & $C_{\mathrm{P}}$ & $\Delta C_{\mathrm{P}}$ & $C_{\mathrm{Q}}$ & $\Delta C_{\mathrm{Q}}$ \\
\hline $2.585 \mathrm{E}+01$ & $2.95 \mathrm{E}-01$ & $1.122 \mathrm{E}-01$ & $1.11 \mathrm{E}-02$ & $9.843 \mathrm{E}-02$ & 4.32E-03 & $1.567 \mathrm{E}-02$ & $6.87 \mathrm{E}-04$ \\
\hline $2.531 \mathrm{E}+01$ & $2.89 \mathrm{E}-01$ & $1.128 \mathrm{E}-01$ & $1.11 \mathrm{E}-02$ & $9.658 \mathrm{E}-02$ & $4.34 \mathrm{E}-03$ & $1.537 \mathrm{E}-02$ & $6.91 \mathrm{E}-04$ \\
\hline $2.432 \mathrm{E}+01$ & $2.78 \mathrm{E}-01$ & $1.095 \mathrm{E}-01$ & $1.11 \mathrm{E}-02$ & $9.351 \mathrm{E}-02$ & $4.41 \mathrm{E}-03$ & $1.488 \mathrm{E}-02$ & $7.01 \mathrm{E}-04$ \\
\hline $2.327 \mathrm{E}+01$ & $2.66 \mathrm{E}-01$ & $1.071 \mathrm{E}-01$ & $1.12 \mathrm{E}-02$ & $8.889 \mathrm{E}-02$ & $4.22 \mathrm{E}-03$ & $1.415 \mathrm{E}-02$ & $6.71 \mathrm{E}-04$ \\
\hline $2.277 \mathrm{E}+01$ & $2.63 \mathrm{E}-01$ & $1.006 \mathrm{E}-01$ & $1.12 \mathrm{E}-02$ & $8.729 \mathrm{E}-02$ & $4.21 \mathrm{E}-03$ & $1.389 \mathrm{E}-02$ & $6.70 \mathrm{E}-04$ \\
\hline $2.231 \mathrm{E}+01$ & $2.57 \mathrm{E}-01$ & $9.164 \mathrm{E}-02$ & $1.12 \mathrm{E}-02$ & $8.501 \mathrm{E}-02$ & $4.29 \mathrm{E}-03$ & $1.353 \mathrm{E}-02$ & $6.83 \mathrm{E}-04$ \\
\hline $2.093 \mathrm{E}+01$ & $2.40 \mathrm{E}-01$ & 7.599E-02 & $1.12 \mathrm{E}-02$ & $8.015 \mathrm{E}-02$ & 4.22E-03 & $1.276 \mathrm{E}-02$ & $6.72 \mathrm{E}-04$ \\
\hline $1.872 \mathrm{E}+01$ & $2.18 \mathrm{E}-01$ & $5.987 \mathrm{E}-02$ & $1.12 \mathrm{E}-02$ & $7.015 \mathrm{E}-02$ & 4.27E-03 & $1.116 \mathrm{E}-02$ & $6.80 \mathrm{E}-04$ \\
\hline $1.611 \mathrm{E}+01$ & $1.92 \mathrm{E}-01$ & $4.320 \mathrm{E}-02$ & $1.12 \mathrm{E}-02$ & $5.801 \mathrm{E}-02$ & $4.27 \mathrm{E}-03$ & $9.232 \mathrm{E}-03$ & $6.79 \mathrm{E}-04$ \\
\hline$\rho\left(\mathrm{kg} / \mathrm{m}^{\wedge} 3\right)$ & $\eta_{\mathrm{T}}$ & $\Delta \eta_{\mathrm{T}}$ & $J$ & $\Delta J$ & $\eta_{\mathrm{P}}$ & $\Delta \eta_{\mathrm{P}}$ & $R e_{0.75}$ \\
\hline $1.188 \mathrm{E}+00$ & $5.400 \mathrm{E}-01$ & $2.45 \mathrm{E}-02$ & $1.006 \mathrm{E}-01$ & $6.37 \mathrm{E}-04$ & $1.147 \mathrm{E}-01$ & $1.24 \mathrm{E}-02$ & $2.818 \mathrm{E}+04$ \\
\hline $1.188 \mathrm{E}+00$ & $5.407 \mathrm{E}-01$ & $2.51 \mathrm{E}-02$ & $1.846 \mathrm{E}-01$ & $6.61 \mathrm{E}-04$ & $2.156 \mathrm{E}-01$ & $2.33 \mathrm{E}-02$ & $2.825 \mathrm{E}+04$ \\
\hline $1.188 \mathrm{E}+00$ & 5.377E-01 & $2.61 \mathrm{E}-02$ & 2.729E-01 & 7.76E-04 & $3.195 \mathrm{E}-01$ & $3.57 \mathrm{E}-02$ & $2.823 \mathrm{E}+04$ \\
\hline $1.188 \mathrm{E}+00$ & 5.309E-01 & $2.59 \mathrm{E}-02$ & $3.634 \mathrm{E}-01$ & $9.12 \mathrm{E}-04$ & $4.379 \mathrm{E}-01$ & $5.03 \mathrm{E}-02$ & $2.830 \mathrm{E}+04$ \\
\hline $1.188 \mathrm{E}+00$ & $5.318 \mathrm{E}-01$ & $2.64 \mathrm{E}-02$ & $4.546 \mathrm{E}-01$ & $1.01 \mathrm{E}-03$ & $5.242 \mathrm{E}-01$ & $6.34 \mathrm{E}-02$ & $2.846 \mathrm{E}+04$ \\
\hline $1.188 \mathrm{E}+00$ & $5.323 \mathrm{E}-01$ & $2.76 \mathrm{E}-02$ & $5.465 \mathrm{E}-01$ & $1.15 \mathrm{E}-03$ & $5.892 \mathrm{E}-01$ & 7.77E-02 & $2.875 \mathrm{E}+04$ \\
\hline $1.188 \mathrm{E}+00$ & $5.310 \mathrm{E}-01$ & $2.86 \mathrm{E}-02$ & $6.408 \mathrm{E}-01$ & $1.32 \mathrm{E}-03$ & $6.076 \mathrm{E}-01$ & $9.48 \mathrm{E}-02$ & $2.895 \mathrm{E}+04$ \\
\hline $1.188 \mathrm{E}+00$ & $5.178 \mathrm{E}-01$ & $3.21 \mathrm{E}-02$ & $7.346 \mathrm{E}-01$ & $1.46 \mathrm{E}-03$ & $6.270 \mathrm{E}-01$ & $1.23 \mathrm{E}-01$ & $2.924 \mathrm{E}+04$ \\
\hline $1.188 \mathrm{E}+00$ & 4.999E-01 & $3.72 \mathrm{E}-02$ & $8.270 \mathrm{E}-01$ & $1.63 \mathrm{E}-03$ & $6.159 \mathrm{E}-01$ & $1.66 \mathrm{E}-01$ & $2.963 \mathrm{E}+04$ \\
\hline
\end{tabular}


Table 212: Graupner 4.7 x 4.7 Dynamic Measured Values - 14006 RPM

\begin{tabular}{|c|c|c|c|c|c|c|c|}
\hline$n(\mathrm{RPM})$ & $Q(\mathrm{~g}-\mathrm{m})$ & $\Delta Q(\mathrm{~g}-\mathrm{m})$ & $V(\mathrm{~V})$ & $I(\mathrm{~A})$ & $T_{\text {atm }}\left({ }^{\circ} \mathrm{C}\right)$ & $P_{\text {atm }}(\mathrm{Pa})$ & $P_{\text {diff }}(\mathrm{Pa})$ \\
\hline $1.407 \mathrm{E}+04$ & $2.493 \mathrm{E}+00$ & $6.79 \mathrm{E}-02$ & $1.098 \mathrm{E}+01$ & $5.192 \mathrm{E}+00$ & $1.834 \mathrm{E}+01$ & $9.939 \mathrm{E}+04$ & $3.119 \mathrm{E}+00$ \\
\hline $1.393 \mathrm{E}+04$ & $2.445 \mathrm{E}+00$ & $6.57 \mathrm{E}-02$ & $1.099 \mathrm{E}+01$ & $5.018 \mathrm{E}+00$ & $1.833 \mathrm{E}+01$ & $9.940 \mathrm{E}+04$ & $9.152 \mathrm{E}+00$ \\
\hline $1.395 \mathrm{E}+04$ & $2.433 \mathrm{E}+00$ & $7.00 \mathrm{E}-02$ & $1.099 \mathrm{E}+01$ & $5.011 \mathrm{E}+00$ & $1.822 \mathrm{E}+01$ & $9.940 \mathrm{E}+04$ & $1.909 \mathrm{E}+01$ \\
\hline $1.397 \mathrm{E}+04$ & $2.418 \mathrm{E}+00$ & $6.60 \mathrm{E}-02$ & $1.099 \mathrm{E}+01$ & $4.996 \mathrm{E}+00$ & $1.828 \mathrm{E}+01$ & $9.941 \mathrm{E}+04$ & $3.306 \mathrm{E}+01$ \\
\hline $1.397 \mathrm{E}+04$ & $2.396 \mathrm{E}+00$ & $6.56 \mathrm{E}-02$ & $1.099 \mathrm{E}+01$ & $4.837 \mathrm{E}+00$ & $1.835 \mathrm{E}+01$ & $9.941 \mathrm{E}+04$ & $5.085 \mathrm{E}+01$ \\
\hline $1.401 \mathrm{E}+04$ & $2.327 \mathrm{E}+00$ & $6.48 \mathrm{E}-02$ & $1.099 \mathrm{E}+01$ & $4.792 \mathrm{E}+00$ & $1.838 \mathrm{E}+01$ & $9.942 \mathrm{E}+04$ & $7.327 \mathrm{E}+01$ \\
\hline $1.404 \mathrm{E}+04$ & $2.298 \mathrm{E}+00$ & $6.46 \mathrm{E}-02$ & $1.099 \mathrm{E}+01$ & $4.771 \mathrm{E}+00$ & $1.837 \mathrm{E}+01$ & $9.942 \mathrm{E}+04$ & $1.001 \mathrm{E}+02$ \\
\hline $1.404 \mathrm{E}+04$ & $2.300 \mathrm{E}+00$ & $6.62 \mathrm{E}-02$ & $1.099 \mathrm{E}+01$ & $4.768 \mathrm{E}+00$ & $1.840 \mathrm{E}+01$ & $9.943 \mathrm{E}+04$ & $1.308 \mathrm{E}+02$ \\
\hline $1.402 \mathrm{E}+04$ & $2.261 \mathrm{E}+00$ & $6.48 \mathrm{E}-02$ & $1.100 \mathrm{E}+01$ & $4.647 \mathrm{E}+00$ & $1.844 \mathrm{E}+01$ & $9.943 \mathrm{E}+04$ & $1.657 \mathrm{E}+02$ \\
\hline $1.404 \mathrm{E}+04$ & $2.183 \mathrm{E}+00$ & $6.48 \mathrm{E}-02$ & $1.100 \mathrm{E}+01$ & $4.488 \mathrm{E}+00$ & $1.847 \mathrm{E}+01$ & $9.943 \mathrm{E}+04$ & $2.047 \mathrm{E}+02$ \\
\hline $1.401 \mathrm{E}+04$ & $2.041 \mathrm{E}+00$ & $6.46 \mathrm{E}-02$ & $1.101 \mathrm{E}+01$ & $4.175 \mathrm{E}+00$ & $1.845 \mathrm{E}+01$ & $9.944 \mathrm{E}+04$ & $2.470 \mathrm{E}+02$ \\
\hline $1.402 \mathrm{E}+04$ & $1.820 \mathrm{E}+00$ & $6.28 \mathrm{E}-02$ & $1.102 \mathrm{E}+01$ & $3.750 \mathrm{E}+00$ & $1.851 \mathrm{E}+01$ & $9.945 \mathrm{E}+04$ & $2.939 \mathrm{E}+02$ \\
\hline
\end{tabular}


Table 213: Graupner 4.7 x 4.7 Dynamic Calculated Values - 14006 RPM

\begin{tabular}{|c|c|c|c|c|c|c|c|}
\hline$n(\mathrm{RPM})$ & $V_{\infty}^{\prime}(\mathrm{m} / \mathrm{s})$ & $\Delta V_{\infty}^{\prime}(\mathrm{m} / \mathrm{s})$ & $T^{\prime}(\mathrm{g})$ & $\Delta T^{\prime}(\mathrm{g})$ & $V_{\mathrm{t}}(\mathrm{m} / \mathrm{s})$ & $P_{\mathrm{P}}(\mathrm{W})$ & $\Delta P_{\mathrm{P}}(\mathrm{W})$ \\
\hline $1.407 \mathrm{E}+04$ & $2.174 \mathrm{E}+00$ & $1.49 \mathrm{E}-02$ & $1.651 \mathrm{E}+02$ & $7.81 \mathrm{E}+00$ & $6.649 \mathrm{E}+01$ & $3.602 \mathrm{E}+01$ & $9.81 \mathrm{E}-01$ \\
\hline $1.393 \mathrm{E}+04$ & $3.813 \mathrm{E}+00$ & $1.55 \mathrm{E}-02$ & $1.589 \mathrm{E}+02$ & $7.74 \mathrm{E}+00$ & $6.594 \mathrm{E}+01$ & $3.498 \mathrm{E}+01$ & $9.41 \mathrm{E}-01$ \\
\hline $1.395 \mathrm{E}+04$ & $5.560 \mathrm{E}+00$ & $1.73 \mathrm{E}-02$ & $1.596 \mathrm{E}+02$ & $7.72 \mathrm{E}+00$ & $6.612 \mathrm{E}+01$ & $3.485 \mathrm{E}+01$ & $1.00 \mathrm{E}+00$ \\
\hline $1.397 \mathrm{E}+04$ & $7.355 \mathrm{E}+00$ & $1.99 \mathrm{E}-02$ & $1.597 \mathrm{E}+02$ & $7.73 \mathrm{E}+00$ & $6.638 \mathrm{E}+01$ & $3.467 \mathrm{E}+01$ & $9.47 \mathrm{E}-01$ \\
\hline $1.397 \mathrm{E}+04$ & $9.155 \mathrm{E}+00$ & $2.24 \mathrm{E}-02$ & $1.559 \mathrm{E}+02$ & $7.71 \mathrm{E}+00$ & $6.661 \mathrm{E}+01$ & $3.437 \mathrm{E}+01$ & $9.41 \mathrm{E}-01$ \\
\hline $1.401 \mathrm{E}+04$ & $1.102 \mathrm{E}+01$ & $2.55 \mathrm{E}-02$ & $1.525 \mathrm{E}+02$ & $7.75 \mathrm{E}+00$ & $6.709 \mathrm{E}+01$ & $3.348 \mathrm{E}+01$ & $9.32 \mathrm{E}-01$ \\
\hline $1.404 \mathrm{E}+04$ & $1.289 \mathrm{E}+01$ & 2.77E-02 & $1.480 \mathrm{E}+02$ & $7.84 \mathrm{E}+00$ & $6.756 \mathrm{E}+01$ & $3.313 \mathrm{E}+01$ & 9.32E-01 \\
\hline $1.404 \mathrm{E}+04$ & $1.476 \mathrm{E}+01$ & $3.06 \mathrm{E}-02$ & $1.416 \mathrm{E}+02$ & $7.82 \mathrm{E}+00$ & $6.797 \mathrm{E}+01$ & $3.317 \mathrm{E}+01$ & $9.55 \mathrm{E}-01$ \\
\hline $1.402 \mathrm{E}+04$ & $1.664 \mathrm{E}+01$ & $3.30 \mathrm{E}-02$ & $1.293 \mathrm{E}+02$ & $7.76 \mathrm{E}+00$ & $6.828 \mathrm{E}+01$ & $3.255 \mathrm{E}+01$ & $9.33 \mathrm{E}-01$ \\
\hline $1.404 \mathrm{E}+04$ & $1.851 \mathrm{E}+01$ & $3.61 \mathrm{E}-02$ & $1.159 \mathrm{E}+02$ & $7.78 \mathrm{E}+00$ & $6.888 \mathrm{E}+01$ & $3.149 \mathrm{E}+01$ & $9.35 \mathrm{E}-01$ \\
\hline $1.401 \mathrm{E}+04$ & $2.034 \mathrm{E}+01$ & $3.89 \mathrm{E}-02$ & $9.926 \mathrm{E}+01$ & $7.75 \mathrm{E}+00$ & $6.926 \mathrm{E}+01$ & $2.937 \mathrm{E}+01$ & $9.30 \mathrm{E}-01$ \\
\hline $1.402 \mathrm{E}+04$ & $2.221 \mathrm{E}+01$ & $4.24 \mathrm{E}-02$ & $8.179 \mathrm{E}+01$ & $7.78 \mathrm{E}+00$ & $6.987 \mathrm{E}+01$ & $2.622 \mathrm{E}+01$ & $9.04 \mathrm{E}-01$ \\
\hline$P_{\mathrm{e}}(\mathrm{W})$ & $\Delta P_{\mathrm{e}}(\mathrm{W})$ & $C_{\mathrm{T}}$ & $\Delta C_{\mathrm{T}}$ & $C_{\mathrm{P}}$ & $\Delta C_{\mathrm{P}}$ & $C_{\mathrm{Q}}$ & $\Delta C_{\mathrm{Q}}$ \\
\hline $5.703 \mathrm{E}+01$ & $6.53 \mathrm{E}-01$ & $1.184 \mathrm{E}-01$ & $5.60 \mathrm{E}-03$ & $9.333 \mathrm{E}-02$ & $2.55 \mathrm{E}-03$ & $1.485 \mathrm{E}-02$ & $4.05 \mathrm{E}-04$ \\
\hline $5.514 \mathrm{E}+01$ & $6.20 \mathrm{E}-01$ & $1.161 \mathrm{E}-01$ & $5.66 \mathrm{E}-03$ & $9.326 \mathrm{E}-02$ & $2.51 \mathrm{E}-03$ & $1.484 \mathrm{E}-02$ & $3.99 \mathrm{E}-04$ \\
\hline $5.507 \mathrm{E}+01$ & $6.20 \mathrm{E}-01$ & $1.163 \mathrm{E}-01$ & $5.63 \mathrm{E}-03$ & $9.262 \mathrm{E}-02$ & 2.67E-03 & $1.474 \mathrm{E}-02$ & $4.24 \mathrm{E}-04$ \\
\hline $5.490 \mathrm{E}+01$ & $6.19 \mathrm{E}-01$ & 1.161E-01 & $5.62 \mathrm{E}-03$ & $9.179 \mathrm{E}-02$ & $2.51 \mathrm{E}-03$ & $1.461 \mathrm{E}-02$ & 3.99E-04 \\
\hline $5.317 \mathrm{E}+01$ & $6.02 \mathrm{E}-01$ & $1.133 \mathrm{E}-01$ & $5.61 \mathrm{E}-03$ & $9.099 \mathrm{E}-02$ & $2.49 \mathrm{E}-03$ & $1.448 \mathrm{E}-02$ & $3.97 \mathrm{E}-04$ \\
\hline $5.268 \mathrm{E}+01$ & $5.95 \mathrm{E}-01$ & $1.102 \mathrm{E}-01$ & $5.60 \mathrm{E}-03$ & $8.784 \mathrm{E}-02$ & $2.45 \mathrm{E}-03$ & $1.398 \mathrm{E}-02$ & $3.89 \mathrm{E}-04$ \\
\hline $5.246 \mathrm{E}+01$ & $5.92 \mathrm{E}-01$ & $1.065 \mathrm{E}-01$ & $5.64 \mathrm{E}-03$ & $8.637 \mathrm{E}-02$ & $2.43 \mathrm{E}-03$ & $1.375 \mathrm{E}-02$ & $3.87 \mathrm{E}-04$ \\
\hline $5.242 \mathrm{E}+01$ & $5.91 \mathrm{E}-01$ & $1.018 \mathrm{E}-01$ & $5.62 \mathrm{E}-03$ & $8.636 \mathrm{E}-02$ & $2.49 \mathrm{E}-03$ & $1.374 \mathrm{E}-02$ & $3.96 \mathrm{E}-04$ \\
\hline $5.110 \mathrm{E}+01$ & $5.77 \mathrm{E}-01$ & $9.336 \mathrm{E}-02$ & $5.60 \mathrm{E}-03$ & $8.525 \mathrm{E}-02$ & $2.45 \mathrm{E}-03$ & $1.357 \mathrm{E}-02$ & $3.89 \mathrm{E}-04$ \\
\hline $4.937 \mathrm{E}+01$ & $5.59 \mathrm{E}-01$ & $8.336 \mathrm{E}-02$ & $5.60 \mathrm{E}-03$ & $8.200 \mathrm{E}-02$ & $2.44 \mathrm{E}-03$ & $1.305 \mathrm{E}-02$ & $3.88 \mathrm{E}-04$ \\
\hline $4.597 \mathrm{E}+01$ & $5.15 \mathrm{E}-01$ & $7.168 \mathrm{E}-02$ & $5.60 \mathrm{E}-03$ & $7.696 \mathrm{E}-02$ & $2.44 \mathrm{E}-03$ & $1.225 \mathrm{E}-02$ & $3.88 \mathrm{E}-04$ \\
\hline $4.133 \mathrm{E}+01$ & $4.59 \mathrm{E}-01$ & $5.899 \mathrm{E}-02$ & $5.61 \mathrm{E}-03$ & $6.857 \mathrm{E}-02$ & $2.37 \mathrm{E}-03$ & $1.091 \mathrm{E}-02$ & $3.77 \mathrm{E}-04$ \\
\hline$\rho\left(\mathrm{kg} / \mathrm{m}^{\wedge} 3\right)$ & $\eta_{\mathrm{T}}$ & $\Delta \eta_{\mathrm{T}}$ & $J$ & $\Delta J$ & $\eta_{\mathrm{P}}$ & $\Delta \eta_{\mathrm{P}}$ & $R e_{0.75}$ \\
\hline $1.188 \mathrm{E}+00$ & $6.316 \mathrm{E}-01$ & $1.87 \mathrm{E}-02$ & $7.708 \mathrm{E}-02$ & $5.29 \mathrm{E}-04$ & $9.777 \mathrm{E}-02$ & $5.38 \mathrm{E}-03$ & $3.931 \mathrm{E}+04$ \\
\hline $1.188 \mathrm{E}+00$ & $6.344 \mathrm{E}-01$ & $1.85 \mathrm{E}-02$ & $1.365 \mathrm{E}-01$ & $5.58 \mathrm{E}-04$ & $1.698 \mathrm{E}-01$ & $9.48 \mathrm{E}-03$ & $3.899 \mathrm{E}+04$ \\
\hline $1.188 \mathrm{E}+00$ & $6.328 \mathrm{E}-01$ & $1.96 \mathrm{E}-02$ & $1.988 \mathrm{E}-01$ & $6.27 \mathrm{E}-04$ & $2.497 \mathrm{E}-01$ & $1.41 \mathrm{E}-02$ & $3.912 \mathrm{E}+04$ \\
\hline $1.188 \mathrm{E}+00$ & $6.316 \mathrm{E}-01$ & $1.87 \mathrm{E}-02$ & $2.626 \mathrm{E}-01$ & $7.23 \mathrm{E}-04$ & $3.323 \mathrm{E}-01$ & $1.85 \mathrm{E}-02$ & $3.927 \mathrm{E}+04$ \\
\hline $1.188 \mathrm{E}+00$ & $6.463 \mathrm{E}-01$ & $1.91 \mathrm{E}-02$ & $3.269 \mathrm{E}-01$ & $8.14 \mathrm{E}-04$ & $4.072 \mathrm{E}-01$ & $2.30 \mathrm{E}-02$ & $3.939 \mathrm{E}+04$ \\
\hline $1.188 \mathrm{E}+00$ & $6.355 \mathrm{E}-01$ & $1.91 \mathrm{E}-02$ & $3.921 \mathrm{E}-01$ & $9.28 \mathrm{E}-04$ & 4.919E-01 & $2.85 \mathrm{E}-02$ & $3.967 \mathrm{E}+04$ \\
\hline $1.188 \mathrm{E}+00$ & $6.316 \mathrm{E}-01$ & $1.91 \mathrm{E}-02$ & $4.580 \mathrm{E}-01$ & $1.01 \mathrm{E}-03$ & $5.648 \mathrm{E}-01$ & $3.39 \mathrm{E}-02$ & $3.995 \mathrm{E}+04$ \\
\hline $1.188 \mathrm{E}+00$ & $6.328 \mathrm{E}-01$ & $1.96 \mathrm{E}-02$ & $5.242 \mathrm{E}-01$ & $1.12 \mathrm{E}-03$ & $6.180 \mathrm{E}-01$ & $3.85 \mathrm{E}-02$ & $4.019 \mathrm{E}+04$ \\
\hline $1.188 \mathrm{E}+00$ & $6.369 \mathrm{E}-01$ & $1.96 \mathrm{E}-02$ & $5.918 \mathrm{E}-01$ & $1.21 \mathrm{E}-03$ & $6.481 \mathrm{E}-01$ & 4.31E-02 & $4.035 \mathrm{E}+04$ \\
\hline $1.188 \mathrm{E}+00$ & $6.378 \mathrm{E}-01$ & $2.03 \mathrm{E}-02$ & $6.572 \mathrm{E}-01$ & $1.32 \mathrm{E}-03$ & $6.681 \mathrm{E}-01$ & 4.91E-02 & $4.071 \mathrm{E}+04$ \\
\hline $1.188 \mathrm{E}+00$ & $6.390 \mathrm{E}-01$ & $2.15 \mathrm{E}-02$ & $7.240 \mathrm{E}-01$ & $1.43 \mathrm{E}-03$ & $6.742 \mathrm{E}-01$ & $5.68 \mathrm{E}-02$ & $4.094 \mathrm{E}+04$ \\
\hline $1.188 \mathrm{E}+00$ & $6.344 \mathrm{E}-01$ & $2.30 \mathrm{E}-02$ & $7.898 \mathrm{E}-01$ & $1.55 \mathrm{E}-03$ & 6.794E-01 & $6.87 \mathrm{E}-02$ & 4.129E+04 \\
\hline
\end{tabular}


Table 214: Graupner 4.7 x 4.7 Dynamic Measured Values - 18002 RPM

\begin{tabular}{|c|c|c|c|c|c|c|c|}
\hline$n(\mathrm{RPM})$ & $Q(\mathrm{~g}-\mathrm{m})$ & $\Delta Q(\mathrm{~g}-\mathrm{m})$ & $V(\mathrm{~V})$ & $I(\mathrm{~A})$ & $T_{\text {atm }}\left({ }^{\circ} \mathrm{C}\right)$ & $P_{\text {atm }}(\mathrm{Pa})$ & $P_{\text {diff }}(\mathrm{Pa})$ \\
\hline $1.798 \mathrm{E}+04$ & $4.089 \mathrm{E}+00$ & $6.94 \mathrm{E}-02$ & $1.086 \mathrm{E}+01$ & $1.028 \mathrm{E}+01$ & $1.841 \mathrm{E}+01$ & $9.946 \mathrm{E}+04$ & $3.845 \mathrm{E}+00$ \\
\hline $1.800 \mathrm{E}+04$ & $4.054 \mathrm{E}+00$ & $6.86 \mathrm{E}-02$ & $1.086 \mathrm{E}+01$ & $1.022 \mathrm{E}+01$ & $1.833 \mathrm{E}+01$ & $9.946 \mathrm{E}+04$ & $9.961 \mathrm{E}+00$ \\
\hline $1.802 \mathrm{E}+04$ & $4.066 \mathrm{E}+00$ & $6.63 \mathrm{E}-02$ & $1.086 \mathrm{E}+01$ & $1.019 \mathrm{E}+01$ & $1.839 \mathrm{E}+01$ & $9.946 \mathrm{E}+04$ & $2.004 \mathrm{E}+01$ \\
\hline $1.801 \mathrm{E}+04$ & $4.079 \mathrm{E}+00$ & $6.67 \mathrm{E}-02$ & $1.086 \mathrm{E}+01$ & $1.020 \mathrm{E}+01$ & $1.857 \mathrm{E}+01$ & $9.947 \mathrm{E}+04$ & $3.430 \mathrm{E}+01$ \\
\hline $1.799 \mathrm{E}+04$ & $3.918 \mathrm{E}+00$ & $6.73 \mathrm{E}-02$ & $1.087 \mathrm{E}+01$ & $9.753 \mathrm{E}+00$ & $1.852 \mathrm{E}+01$ & $9.947 \mathrm{E}+04$ & $6.287 \mathrm{E}+01$ \\
\hline $1.801 \mathrm{E}+04$ & $3.821 \mathrm{E}+00$ & $6.61 \mathrm{E}-02$ & $1.088 \mathrm{E}+01$ & $9.466 \mathrm{E}+00$ & $1.848 \mathrm{E}+01$ & $9.948 \mathrm{E}+04$ & $1.013 \mathrm{E}+02$ \\
\hline $1.802 \mathrm{E}+04$ & $3.817 \mathrm{E}+00$ & $6.59 \mathrm{E}-02$ & $1.088 \mathrm{E}+01$ & $9.451 \mathrm{E}+00$ & $1.851 \mathrm{E}+01$ & $9.948 \mathrm{E}+04$ & $1.494 \mathrm{E}+02$ \\
\hline $1.803 \mathrm{E}+04$ & $3.832 \mathrm{E}+00$ & $6.66 \mathrm{E}-02$ & $1.088 \mathrm{E}+01$ & $9.425 \mathrm{E}+00$ & $1.858 \mathrm{E}+01$ & $9.948 \mathrm{E}+04$ & $2.069 \mathrm{E}+02$ \\
\hline $1.801 \mathrm{E}+04$ & $3.804 \mathrm{E}+00$ & $6.63 \mathrm{E}-02$ & $1.088 \mathrm{E}+01$ & $9.226 \mathrm{E}+00$ & $1.861 \mathrm{E}+01$ & $9.949 \mathrm{E}+04$ & $2.724 \mathrm{E}+02$ \\
\hline $1.798 \mathrm{E}+04$ & $3.652 \mathrm{E}+00$ & $6.66 \mathrm{E}-02$ & $1.089 \mathrm{E}+01$ & $8.773 \mathrm{E}+00$ & $1.862 \mathrm{E}+01$ & $9.950 \mathrm{E}+04$ & $3.472 \mathrm{E}+02$ \\
\hline $1.798 \mathrm{E}+04$ & $3.386 \mathrm{E}+00$ & $6.88 \mathrm{E}-02$ & $1.091 \mathrm{E}+01$ & $8.033 \mathrm{E}+00$ & $1.867 \mathrm{E}+01$ & $9.949 \mathrm{E}+04$ & $4.310 \mathrm{E}+02$ \\
\hline $1.799 \mathrm{E}+04$ & $3.013 \mathrm{E}+00$ & $6.88 \mathrm{E}-02$ & $1.094 \mathrm{E}+01$ & $7.041 \mathrm{E}+00$ & $1.883 \mathrm{E}+01$ & $9.949 \mathrm{E}+04$ & $5.234 \mathrm{E}+02$ \\
\hline
\end{tabular}


Table 215: Graupner 4.7 x 4.7 Dynamic Calculated Values - 18002 RPM

\begin{tabular}{|c|c|c|c|c|c|c|c|}
\hline$n(\mathrm{RPM})$ & $V_{\infty}^{\prime}(\mathrm{m} / \mathrm{s})$ & $\Delta V_{\infty}^{\prime}(\mathrm{m} / \mathrm{s})$ & $T^{\prime}(\mathrm{g})$ & $\Delta T^{\prime}(\mathrm{g})$ & $V_{\mathrm{t}}(\mathrm{m} / \mathrm{s})$ & $P_{\mathrm{P}}(\mathrm{W})$ & $\Delta P_{\mathrm{P}}(\mathrm{W})$ \\
\hline $1.798 \mathrm{E}+04$ & $2.392 \mathrm{E}+00$ & $1.76 \mathrm{E}-02$ & $2.746 \mathrm{E}+02$ & $7.43 \mathrm{E}+00$ & $8.499 \mathrm{E}+01$ & $7.553 \mathrm{E}+01$ & $1.28 \mathrm{E}+00$ \\
\hline $1.800 \mathrm{E}+04$ & $3.945 \mathrm{E}+00$ & $1.87 \mathrm{E}-02$ & $2.737 \mathrm{E}+02$ & $7.39 \mathrm{E}+00$ & $8.514 \mathrm{E}+01$ & $7.496 \mathrm{E}+01$ & $1.27 \mathrm{E}+00$ \\
\hline $1.802 \mathrm{E}+04$ & $5.661 \mathrm{E}+00$ & $2.01 \mathrm{E}-02$ & $2.731 \mathrm{E}+02$ & $7.40 \mathrm{E}+00$ & $8.531 \mathrm{E}+01$ & $7.523 \mathrm{E}+01$ & $1.23 \mathrm{E}+00$ \\
\hline $1.801 \mathrm{E}+04$ & $7.460 \mathrm{E}+00$ & $2.24 \mathrm{E}-02$ & $2.662 \mathrm{E}+02$ & $7.35 \mathrm{E}+00$ & $8.541 \mathrm{E}+01$ & $7.544 \mathrm{E}+01$ & $1.23 \mathrm{E}+00$ \\
\hline $1.799 \mathrm{E}+04$ & $1.015 \mathrm{E}+01$ & $2.70 \mathrm{E}-02$ & $2.670 \mathrm{E}+02$ & $7.24 \mathrm{E}+00$ & $8.558 \mathrm{E}+01$ & $7.238 \mathrm{E}+01$ & $1.24 \mathrm{E}+00$ \\
\hline $1.801 \mathrm{E}+04$ & $1.293 \mathrm{E}+01$ & 3.07E-02 & $2.652 \mathrm{E}+02$ & $7.21 \mathrm{E}+00$ & $8.607 \mathrm{E}+01$ & $7.069 \mathrm{E}+01$ & $1.22 \mathrm{E}+00$ \\
\hline $1.802 \mathrm{E}+04$ & $1.574 \mathrm{E}+01$ & 3.39E-02 & $2.552 \mathrm{E}+02$ & $7.27 \mathrm{E}+00$ & $8.655 \mathrm{E}+01$ & $7.061 \mathrm{E}+01$ & $1.22 \mathrm{E}+00$ \\
\hline $1.803 \mathrm{E}+04$ & $1.856 \mathrm{E}+01$ & $3.72 \mathrm{E}-02$ & $2.422 \mathrm{E}+02$ & $7.25 \mathrm{E}+00$ & $8.718 \mathrm{E}+01$ & $7.096 \mathrm{E}+01$ & $1.23 \mathrm{E}+00$ \\
\hline $1.801 \mathrm{E}+04$ & $2.133 \mathrm{E}+01$ & $4.12 \mathrm{E}-02$ & $2.240 \mathrm{E}+02$ & $7.25 \mathrm{E}+00$ & $8.771 \mathrm{E}+01$ & $7.035 \mathrm{E}+01$ & $1.23 \mathrm{E}+00$ \\
\hline $1.798 \mathrm{E}+04$ & $2.410 \mathrm{E}+01$ & $4.60 \mathrm{E}-02$ & $1.982 \mathrm{E}+02$ & $7.36 \mathrm{E}+00$ & $8.827 \mathrm{E}+01$ & $6.742 \mathrm{E}+01$ & $1.23 \mathrm{E}+00$ \\
\hline $1.798 \mathrm{E}+04$ & $2.688 \mathrm{E}+01$ & $5.10 \mathrm{E}-02$ & $1.689 \mathrm{E}+02$ & $7.32 \mathrm{E}+00$ & $8.908 \mathrm{E}+01$ & $6.251 \mathrm{E}+01$ & $1.27 \mathrm{E}+00$ \\
\hline $1.799 \mathrm{E}+04$ & $2.965 \mathrm{E}+01$ & $5.46 \mathrm{E}-02$ & $1.364 \mathrm{E}+02$ & $7.39 \mathrm{E}+00$ & $8.999 \mathrm{E}+01$ & $5.566 \mathrm{E}+01$ & $1.27 \mathrm{E}+00$ \\
\hline$P_{\mathrm{e}}(\mathrm{W})$ & $\Delta P_{\mathrm{e}}(\mathrm{W})$ & $C_{\mathrm{T}}$ & $\Delta C_{\mathrm{T}}$ & $C_{\mathrm{P}}$ & $\Delta C_{\mathrm{P}}$ & $C_{\mathrm{Q}}$ & $\Delta C_{\mathrm{Q}}$ \\
\hline $1.116 \mathrm{E}+02$ & $1.21 \mathrm{E}+00$ & $1.204 \mathrm{E}-01$ & $3.26 \mathrm{E}-03$ & $9.363 \mathrm{E}-02$ & $1.59 \mathrm{E}-03$ & $1.490 \mathrm{E}-02$ & $2.53 \mathrm{E}-04$ \\
\hline $1.109 \mathrm{E}+02$ & $1.18 \mathrm{E}+00$ & $1.197 \mathrm{E}-01$ & $3.24 \mathrm{E}-03$ & $9.261 \mathrm{E}-02$ & $1.57 \mathrm{E}-03$ & $1.474 \mathrm{E}-02$ & $2.50 \mathrm{E}-04$ \\
\hline $1.106 \mathrm{E}+02$ & $1.18 \mathrm{E}+00$ & 1.192E-01 & $3.23 \mathrm{E}-03$ & $9.271 \mathrm{E}-02$ & $1.52 \mathrm{E}-03$ & $1.476 \mathrm{E}-02$ & $2.41 \mathrm{E}-04$ \\
\hline $1.107 \mathrm{E}+02$ & $1.18 \mathrm{E}+00$ & $1.164 \mathrm{E}-01$ & $3.22 \mathrm{E}-03$ & $9.315 \mathrm{E}-02$ & $1.53 \mathrm{E}-03$ & $1.482 \mathrm{E}-02$ & $2.43 \mathrm{E}-04$ \\
\hline $1.060 \mathrm{E}+02$ & $1.13 \mathrm{E}+00$ & $1.170 \mathrm{E}-01$ & $3.18 \mathrm{E}-03$ & $8.970 \mathrm{E}-02$ & $1.54 \mathrm{E}-03$ & $1.428 \mathrm{E}-02$ & $2.46 \mathrm{E}-04$ \\
\hline $1.030 \mathrm{E}+02$ & $1.11 \mathrm{E}+00$ & $1.159 \mathrm{E}-01$ & $3.15 \mathrm{E}-03$ & $8.721 \mathrm{E}-02$ & $1.51 \mathrm{E}-03$ & $1.388 \mathrm{E}-02$ & $2.40 \mathrm{E}-04$ \\
\hline $1.028 \mathrm{E}+02$ & $1.10 \mathrm{E}+00$ & $1.115 \mathrm{E}-01$ & $3.18 \mathrm{E}-03$ & $8.709 \mathrm{E}-02$ & $1.51 \mathrm{E}-03$ & $1.386 \mathrm{E}-02$ & $2.40 \mathrm{E}-04$ \\
\hline $1.025 \mathrm{E}+02$ & $1.10 \mathrm{E}+00$ & $1.057 \mathrm{E}-01$ & $3.16 \mathrm{E}-03$ & $8.729 \mathrm{E}-02$ & $1.52 \mathrm{E}-03$ & $1.389 \mathrm{E}-02$ & $2.42 \mathrm{E}-04$ \\
\hline $1.004 \mathrm{E}+02$ & $1.08 \mathrm{E}+00$ & $9.798 \mathrm{E}-02$ & $3.17 \mathrm{E}-03$ & $8.687 \mathrm{E}-02$ & $1.52 \mathrm{E}-03$ & $1.383 \mathrm{E}-02$ & $2.41 \mathrm{E}-04$ \\
\hline $9.557 \mathrm{E}+01$ & $1.03 \mathrm{E}+00$ & $8.702 \mathrm{E}-02$ & $3.23 \mathrm{E}-03$ & $8.372 \mathrm{E}-02$ & $1.53 \mathrm{E}-03$ & $1.332 \mathrm{E}-02$ & $2.43 \mathrm{E}-04$ \\
\hline $8.766 \mathrm{E}+01$ & $9.51 \mathrm{E}-01$ & 7.413E-02 & $3.21 \mathrm{E}-03$ & $7.761 \mathrm{E}-02$ & $1.58 \mathrm{E}-03$ & $1.235 \mathrm{E}-02$ & $2.51 \mathrm{E}-04$ \\
\hline $7.701 \mathrm{E}+01$ & $8.45 \mathrm{E}-01$ & $5.984 \mathrm{E}-02$ & $3.24 \mathrm{E}-03$ & $6.906 \mathrm{E}-02$ & $1.58 \mathrm{E}-03$ & $1.099 \mathrm{E}-02$ & $2.51 \mathrm{E}-04$ \\
\hline$\rho\left(\mathrm{kg} / \mathrm{m}^{\wedge} 3\right)$ & $\eta_{\mathrm{T}}$ & $\Delta \eta_{\mathrm{T}}$ & $J$ & $\Delta J$ & $\eta_{\mathrm{P}}$ & $\Delta \eta_{\mathrm{P}}$ & $R e_{0.75}$ \\
\hline $1.188 \mathrm{E}+00$ & $6.770 \mathrm{E}-01$ & $1.36 \mathrm{E}-02$ & $6.634 \mathrm{E}-02$ & $4.90 \mathrm{E}-04$ & $8.529 \mathrm{E}-02$ & $2.80 \mathrm{E}-03$ & $5.026 \mathrm{E}+04$ \\
\hline $1.189 \mathrm{E}+00$ & $6.758 \mathrm{E}-01$ & $1.35 \mathrm{E}-02$ & $1.093 \mathrm{E}-01$ & $5.20 \mathrm{E}-04$ & $1.412 \mathrm{E}-01$ & $4.56 \mathrm{E}-03$ & $5.037 \mathrm{E}+04$ \\
\hline $1.189 \mathrm{E}+00$ & $6.802 \mathrm{E}-01$ & $1.33 \mathrm{E}-02$ & $1.567 \mathrm{E}-01$ & $5.59 \mathrm{E}-04$ & $2.015 \mathrm{E}-01$ & $6.42 \mathrm{E}-03$ & $5.046 \mathrm{E}+04$ \\
\hline $1.188 \mathrm{E}+00$ & $6.812 \mathrm{E}-01$ & $1.33 \mathrm{E}-02$ & $2.065 \mathrm{E}-01$ & $6.25 \mathrm{E}-04$ & $2.581 \mathrm{E}-01$ & $8.33 \mathrm{E}-03$ & $5.047 \mathrm{E}+04$ \\
\hline $1.188 \mathrm{E}+00$ & $6.828 \mathrm{E}-01$ & $1.38 \mathrm{E}-02$ & $2.815 \mathrm{E}-01$ & $7.51 \mathrm{E}-04$ & $3.673 \mathrm{E}-01$ & $1.18 \mathrm{E}-02$ & $5.058 \mathrm{E}+04$ \\
\hline $1.188 \mathrm{E}+00$ & $6.866 \mathrm{E}-01$ & $1.40 \mathrm{E}-02$ & $3.581 \mathrm{E}-01$ & $8.60 \mathrm{E}-04$ & 4.757E-01 & $1.54 \mathrm{E}-02$ & $5.089 \mathrm{E}+04$ \\
\hline $1.188 \mathrm{E}+00$ & $6.869 \mathrm{E}-01$ & $1.40 \mathrm{E}-02$ & $4.358 \mathrm{E}-01$ & $9.55 \mathrm{E}-04$ & $5.580 \mathrm{E}-01$ & $1.86 \mathrm{E}-02$ & $5.116 \mathrm{E}+04$ \\
\hline $1.188 \mathrm{E}+00$ & $6.921 \mathrm{E}-01$ & $1.41 \mathrm{E}-02$ & $5.134 \mathrm{E}-01$ & $1.05 \mathrm{E}-03$ & $6.214 \mathrm{E}-01$ & $2.16 \mathrm{E}-02$ & $5.151 \mathrm{E}+04$ \\
\hline $1.188 \mathrm{E}+00$ & $7.008 \mathrm{E}-01$ & $1.44 \mathrm{E}-02$ & $5.905 \mathrm{E}-01$ & $1.16 \mathrm{E}-03$ & $6.660 \mathrm{E}-01$ & $2.45 \mathrm{E}-02$ & $5.182 \mathrm{E}+04$ \\
\hline $1.188 \mathrm{E}+00$ & $7.055 \mathrm{E}-01$ & $1.49 \mathrm{E}-02$ & $6.687 \mathrm{E}-01$ & $1.28 \mathrm{E}-03$ & $6.950 \mathrm{E}-01$ & $2.88 \mathrm{E}-02$ & $5.216 \mathrm{E}+04$ \\
\hline $1.188 \mathrm{E}+00$ & $7.131 \mathrm{E}-01$ & $1.64 \mathrm{E}-02$ & $7.456 \mathrm{E}-01$ & $1.42 \mathrm{E}-03$ & $7.121 \mathrm{E}-01$ & $3.41 \mathrm{E}-02$ & $5.262 \mathrm{E}+04$ \\
\hline $1.187 \mathrm{E}+00$ & 7.227E-01 & $1.83 \mathrm{E}-02$ & 8.221E-01 & $1.52 \mathrm{E}-03$ & 7.124E-01 & 4.19E-02 & $5.310 \mathrm{E}+04$ \\
\hline
\end{tabular}


Table 216: Graupner 4.7 x 4.7 Dynamic Measured Values - 20026 RPM

\begin{tabular}{|c|c|c|c|c|c|c|c|}
\hline$n(\mathrm{RPM})$ & $Q(\mathrm{~g}-\mathrm{m})$ & $\Delta Q(\mathrm{~g}-\mathrm{m})$ & $V(\mathrm{~V})$ & $I(\mathrm{~A})$ & $T_{\text {atm }}\left({ }^{\circ} \mathrm{C}\right)$ & $P_{\text {atm }}(\mathrm{Pa})$ & $P_{\text {diff }}(\mathrm{Pa})$ \\
\hline $2.003 \mathrm{E}+04$ & $5.162 \mathrm{E}+00$ & $8.81 \mathrm{E}-02$ & $1.075 \mathrm{E}+01$ & $1.441 \mathrm{E}+01$ & $1.875 \mathrm{E}+01$ & $9.949 \mathrm{E}+04$ & $4.282 \mathrm{E}+00$ \\
\hline $2.011 \mathrm{E}+04$ & $5.106 \mathrm{E}+00$ & $9.21 \mathrm{E}-02$ & $1.074 \mathrm{E}+01$ & $1.447 \mathrm{E}+01$ & $1.883 \mathrm{E}+01$ & $9.948 \mathrm{E}+04$ & $1.494 \mathrm{E}+01$ \\
\hline $2.013 \mathrm{E}+04$ & $5.146 \mathrm{E}+00$ & $8.90 \mathrm{E}-02$ & $1.074 \mathrm{E}+01$ & $1.448 \mathrm{E}+01$ & $1.885 \mathrm{E}+01$ & $9.948 \mathrm{E}+04$ & $3.481 \mathrm{E}+01$ \\
\hline $2.027 \mathrm{E}+04$ & $5.075 \mathrm{E}+00$ & $9.54 \mathrm{E}-02$ & $1.075 \mathrm{E}+01$ & $1.425 \mathrm{E}+01$ & $1.877 \mathrm{E}+01$ & $9.948 \mathrm{E}+04$ & $6.341 \mathrm{E}+01$ \\
\hline $1.992 \mathrm{E}+04$ & $4.699 \mathrm{E}+00$ & $7.69 \mathrm{E}-02$ & $1.078 \mathrm{E}+01$ & $1.295 \mathrm{E}+01$ & $1.877 \mathrm{E}+01$ & $9.948 \mathrm{E}+04$ & $1.019 \mathrm{E}+02$ \\
\hline $1.992 \mathrm{E}+04$ & $4.676 \mathrm{E}+00$ & $7.59 \mathrm{E}-02$ & $1.079 \mathrm{E}+01$ & $1.288 \mathrm{E}+01$ & $1.880 \mathrm{E}+01$ & $9.947 \mathrm{E}+04$ & $1.498 \mathrm{E}+02$ \\
\hline $1.993 \mathrm{E}+04$ & $4.685 \mathrm{E}+00$ & $7.46 \mathrm{E}-02$ & $1.079 \mathrm{E}+01$ & $1.286 \mathrm{E}+01$ & $1.884 \mathrm{E}+01$ & $9.949 \mathrm{E}+04$ & $2.073 \mathrm{E}+02$ \\
\hline $1.997 \mathrm{E}+04$ & $4.705 \mathrm{E}+00$ & $7.53 \mathrm{E}-02$ & $1.079 \mathrm{E}+01$ & $1.281 \mathrm{E}+01$ & $1.888 \mathrm{E}+01$ & $9.949 \mathrm{E}+04$ & $2.733 \mathrm{E}+02$ \\
\hline $2.003 \mathrm{E}+04$ & $4.697 \mathrm{E}+00$ & $7.77 \mathrm{E}-02$ & $1.079 \mathrm{E}+01$ & $1.269 \mathrm{E}+01$ & $1.889 \mathrm{E}+01$ & $9.949 \mathrm{E}+04$ & $3.478 \mathrm{E}+02$ \\
\hline $1.995 \mathrm{E}+04$ & $4.541 \mathrm{E}+00$ & $8.25 \mathrm{E}-02$ & $1.081 \mathrm{E}+01$ & $1.201 \mathrm{E}+01$ & $1.895 \mathrm{E}+01$ & $9.949 \mathrm{E}+04$ & $4.314 \mathrm{E}+02$ \\
\hline $2.003 \mathrm{E}+04$ & $4.293 \mathrm{E}+00$ & $8.28 \mathrm{E}-02$ & $1.083 \mathrm{E}+01$ & $1.128 \mathrm{E}+01$ & $1.903 \mathrm{E}+01$ & $9.948 \mathrm{E}+04$ & $5.237 \mathrm{E}+02$ \\
\hline
\end{tabular}


Table 217: Graupner 4.7 x 4.7 Dynamic Calculated Values - 20026 RPM

\begin{tabular}{|c|c|c|c|c|c|c|c|}
\hline$n(\mathrm{RPM})$ & $V_{\infty}^{\prime}(\mathrm{m} / \mathrm{s})$ & $\Delta V_{\infty}^{\prime}(\mathrm{m} / \mathrm{s})$ & $T^{\prime}(\mathrm{g})$ & $\Delta T^{\prime}(\mathrm{g})$ & $V_{\mathrm{t}}(\mathrm{m} / \mathrm{s})$ & $P_{\mathrm{P}}(\mathrm{W})$ & $\Delta P_{\mathrm{P}}(\mathrm{W})$ \\
\hline $2.003 \mathrm{E}+04$ & $2.516 \mathrm{E}+00$ & $1.92 \mathrm{E}-02$ & $3.424 \mathrm{E}+02$ & $7.50 \mathrm{E}+00$ & $9.463 \mathrm{E}+01$ & $1.061 \mathrm{E}+02$ & $1.81 \mathrm{E}+00$ \\
\hline $2.011 \mathrm{E}+04$ & $4.851 \mathrm{E}+00$ & $2.08 \mathrm{E}-02$ & $3.435 \mathrm{E}+02$ & $7.58 \mathrm{E}+00$ & $9.514 \mathrm{E}+01$ & $1.055 \mathrm{E}+02$ & $1.90 \mathrm{E}+00$ \\
\hline $2.013 \mathrm{E}+04$ & $7.499 \mathrm{E}+00$ & $2.36 \mathrm{E}-02$ & $3.364 \mathrm{E}+02$ & $7.46 \mathrm{E}+00$ & $9.537 \mathrm{E}+01$ & $1.064 \mathrm{E}+02$ & $1.84 \mathrm{E}+00$ \\
\hline $2.027 \mathrm{E}+04$ & $1.018 \mathrm{E}+01$ & $2.88 \mathrm{E}-02$ & $3.441 \mathrm{E}+02$ & $7.48 \mathrm{E}+00$ & $9.629 \mathrm{E}+01$ & $1.057 \mathrm{E}+02$ & $1.99 \mathrm{E}+00$ \\
\hline $1.992 \mathrm{E}+04$ & $1.296 \mathrm{E}+01$ & $3.23 \mathrm{E}-02$ & $3.300 \mathrm{E}+02$ & $7.25 \mathrm{E}+00$ & $9.498 \mathrm{E}+01$ & $9.612 \mathrm{E}+01$ & $1.57 \mathrm{E}+00$ \\
\hline $1.992 \mathrm{E}+04$ & $1.576 \mathrm{E}+01$ & $3.49 \mathrm{E}-02$ & $3.225 \mathrm{E}+02$ & $7.28 \mathrm{E}+00$ & $9.542 \mathrm{E}+01$ & $9.567 \mathrm{E}+01$ & $1.55 \mathrm{E}+00$ \\
\hline $1.993 \mathrm{E}+04$ & $1.857 \mathrm{E}+01$ & $3.83 \mathrm{E}-02$ & $3.098 \mathrm{E}+02$ & $7.29 \mathrm{E}+00$ & $9.598 \mathrm{E}+01$ & $9.591 \mathrm{E}+01$ & $1.53 \mathrm{E}+00$ \\
\hline $1.997 \mathrm{E}+04$ & $2.135 \mathrm{E}+01$ & 4.18E-02 & $2.950 \mathrm{E}+02$ & $7.30 \mathrm{E}+00$ & $9.671 \mathrm{E}+01$ & $9.648 \mathrm{E}+01$ & $1.54 \mathrm{E}+00$ \\
\hline $2.003 \mathrm{E}+04$ & $2.411 \mathrm{E}+01$ & $4.66 \mathrm{E}-02$ & $2.773 \mathrm{E}+02$ & $7.31 \mathrm{E}+00$ & $9.766 \mathrm{E}+01$ & $9.664 \mathrm{E}+01$ & $1.60 \mathrm{E}+00$ \\
\hline $1.995 \mathrm{E}+04$ & $2.688 \mathrm{E}+01$ & $5.15 \mathrm{E}-02$ & $2.484 \mathrm{E}+02$ & $7.38 \mathrm{E}+00$ & $9.802 \mathrm{E}+01$ & $9.306 \mathrm{E}+01$ & $1.69 \mathrm{E}+00$ \\
\hline $2.003 \mathrm{E}+04$ & $2.965 \mathrm{E}+01$ & $5.56 \mathrm{E}-02$ & $2.188 \mathrm{E}+02$ & $7.54 \mathrm{E}+00$ & $9.915 \mathrm{E}+01$ & $8.830 \mathrm{E}+01$ & $1.70 \mathrm{E}+00$ \\
\hline$P_{\mathrm{e}}(\mathrm{W})$ & $\Delta P_{\mathrm{e}}(\mathrm{W})$ & $C_{\mathrm{T}}$ & $\Delta C_{\mathrm{T}}$ & $C_{\mathrm{P}}$ & $\Delta C_{\mathrm{P}}$ & $C_{\mathrm{Q}}$ & $\Delta C_{\mathrm{Q}}$ \\
\hline $1.549 \mathrm{E}+02$ & $1.63 \mathrm{E}+00$ & $1.212 \mathrm{E}-01$ & $2.66 \mathrm{E}-03$ & $9.540 \mathrm{E}-02$ & $1.63 \mathrm{E}-03$ & $1.518 \mathrm{E}-02$ & $2.60 \mathrm{E}-04$ \\
\hline $1.555 \mathrm{E}+02$ & $1.69 \mathrm{E}+00$ & $1.205 \mathrm{E}-01$ & $2.66 \mathrm{E}-03$ & $9.356 \mathrm{E}-02$ & $1.69 \mathrm{E}-03$ & $1.489 \mathrm{E}-02$ & $2.69 \mathrm{E}-04$ \\
\hline $1.556 \mathrm{E}+02$ & $1.61 \mathrm{E}+00$ & $1.179 \mathrm{E}-01$ & $2.62 \mathrm{E}-03$ & $9.421 \mathrm{E}-02$ & $1.63 \mathrm{E}-03$ & $1.499 \mathrm{E}-02$ & $2.60 \mathrm{E}-04$ \\
\hline $1.532 \mathrm{E}+02$ & $1.67 \mathrm{E}+00$ & $1.189 \mathrm{E}-01$ & $2.59 \mathrm{E}-03$ & $9.156 \mathrm{E}-02$ & $1.73 \mathrm{E}-03$ & $1.457 \mathrm{E}-02$ & $2.74 \mathrm{E}-04$ \\
\hline $1.397 \mathrm{E}+02$ & $1.53 \mathrm{E}+00$ & $1.181 \mathrm{E}-01$ & $2.60 \mathrm{E}-03$ & 8.780E-02 & $1.44 \mathrm{E}-03$ & $1.397 \mathrm{E}-02$ & $2.29 \mathrm{E}-04$ \\
\hline $1.389 \mathrm{E}+02$ & $1.45 \mathrm{E}+00$ & $1.154 \mathrm{E}-01$ & $2.61 \mathrm{E}-03$ & $8.736 \mathrm{E}-02$ & $1.42 \mathrm{E}-03$ & $1.390 \mathrm{E}-02$ & $2.26 \mathrm{E}-04$ \\
\hline $1.387 \mathrm{E}+02$ & $1.44 \mathrm{E}+00$ & $1.107 \mathrm{E}-01$ & $2.61 \mathrm{E}-03$ & $8.741 \mathrm{E}-02$ & $1.40 \mathrm{E}-03$ & $1.391 \mathrm{E}-02$ & $2.22 \mathrm{E}-04$ \\
\hline $1.382 \mathrm{E}+02$ & $1.44 \mathrm{E}+00$ & $1.051 \mathrm{E}-01$ & $2.60 \mathrm{E}-03$ & 8.752E-02 & $1.41 \mathrm{E}-03$ & $1.393 \mathrm{E}-02$ & $2.23 \mathrm{E}-04$ \\
\hline $1.370 \mathrm{E}+02$ & $1.43 \mathrm{E}+00$ & $9.810 \mathrm{E}-02$ & $2.59 \mathrm{E}-03$ & $8.678 \mathrm{E}-02$ & $1.44 \mathrm{E}-03$ & $1.381 \mathrm{E}-02$ & $2.29 \mathrm{E}-04$ \\
\hline $1.298 \mathrm{E}+02$ & $1.47 \mathrm{E}+00$ & $8.859 \mathrm{E}-02$ & $2.64 \mathrm{E}-03$ & $8.459 \mathrm{E}-02$ & $1.54 \mathrm{E}-03$ & $1.346 \mathrm{E}-02$ & $2.45 \mathrm{E}-04$ \\
\hline $1.221 \mathrm{E}+02$ & $1.28 \mathrm{E}+00$ & 7.749E-02 & $2.67 \mathrm{E}-03$ & 7.942E-02 & $1.53 \mathrm{E}-03$ & $1.264 \mathrm{E}-02$ & $2.44 \mathrm{E}-04$ \\
\hline$\rho\left(\mathrm{kg} / \mathrm{m}^{\wedge} 3\right)$ & $\eta_{\mathrm{T}}$ & $\Delta \eta_{\mathrm{T}}$ & $J$ & $\Delta J$ & $\eta_{\mathrm{P}}$ & $\Delta \eta_{\mathrm{P}}$ & $R e_{0.75}$ \\
\hline $1.187 \mathrm{E}+00$ & $6.853 \mathrm{E}-01$ & $1.37 \mathrm{E}-02$ & $6.267 \mathrm{E}-02$ & 4.80E-04 & $7.961 \mathrm{E}-02$ & $2.30 \mathrm{E}-03$ & $5.586 \mathrm{E}+04$ \\
\hline $1.187 \mathrm{E}+00$ & $6.783 \mathrm{E}-01$ & $1.43 \mathrm{E}-02$ & 1.203E-01 & 5.19E-04 & $1.550 \mathrm{E}-01$ & $4.48 \mathrm{E}-03$ & $5.614 \mathrm{E}+04$ \\
\hline $1.187 \mathrm{E}+00$ & $6.837 \mathrm{E}-01$ & $1.38 \mathrm{E}-02$ & $1.858 \mathrm{E}-01$ & 5.89E-04 & $2.326 \mathrm{E}-01$ & $6.59 \mathrm{E}-03$ & $5.626 \mathrm{E}+04$ \\
\hline $1.187 \mathrm{E}+00$ & $6.895 \mathrm{E}-01$ & $1.50 \mathrm{E}-02$ & $2.505 \mathrm{E}-01$ & 7.19E-04 & $3.251 \mathrm{E}-01$ & $9.41 \mathrm{E}-03$ & $5.684 \mathrm{E}+04$ \\
\hline $1.187 \mathrm{E}+00$ & $6.883 \mathrm{E}-01$ & $1.36 \mathrm{E}-02$ & 3.244E-01 & 8.23E-04 & 4.362E-01 & $1.20 \mathrm{E}-02$ & $5.606 \mathrm{E}+04$ \\
\hline $1.187 \mathrm{E}+00$ & $6.885 \mathrm{E}-01$ & $1.33 \mathrm{E}-02$ & $3.945 \mathrm{E}-01$ & 8.88E-04 & 5.209E-01 & $1.45 \mathrm{E}-02$ & $5.630 \mathrm{E}+04$ \\
\hline $1.187 \mathrm{E}+00$ & $6.916 \mathrm{E}-01$ & $1.32 \mathrm{E}-02$ & $4.646 \mathrm{E}-01$ & $9.76 \mathrm{E}-04$ & $5.882 \mathrm{E}-01$ & $1.68 \mathrm{E}-02$ & $5.663 \mathrm{E}+04$ \\
\hline $1.187 \mathrm{E}+00$ & $6.983 \mathrm{E}-01$ & $1.33 \mathrm{E}-02$ & 5.333E-01 & $1.07 \mathrm{E}-03$ & $6.403 \mathrm{E}-01$ & $1.89 \mathrm{E}-02$ & $5.704 \mathrm{E}+04$ \\
\hline $1.187 \mathrm{E}+00$ & 7.057E-01 & $1.38 \mathrm{E}-02$ & $6.002 \mathrm{E}-01$ & $1.18 \mathrm{E}-03$ & $6.785 \mathrm{E}-01$ & $2.12 \mathrm{E}-02$ & $5.761 \mathrm{E}+04$ \\
\hline $1.187 \mathrm{E}+00$ & 7.167E-01 & $1.53 \mathrm{E}-02$ & 6.719E-01 & $1.32 \mathrm{E}-03$ & $7.036 \mathrm{E}-01$ & $2.46 \mathrm{E}-02$ & $5.780 \mathrm{E}+04$ \\
\hline $1.186 \mathrm{E}+00$ & $7.229 \mathrm{E}-01$ & $1.59 \mathrm{E}-02$ & 7.383E-01 & $1.41 \mathrm{E}-03$ & 7.203E-01 & $2.85 \mathrm{E}-02$ & $5.843 \mathrm{E}+04$ \\
\hline
\end{tabular}




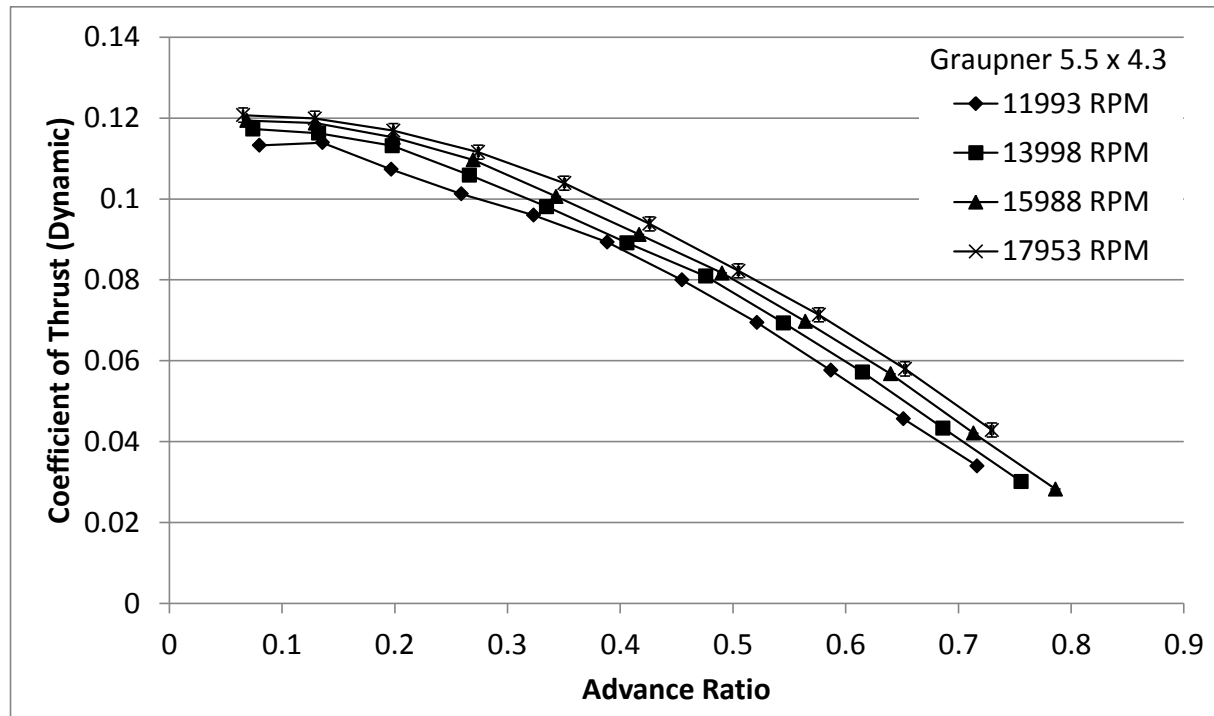

(a)

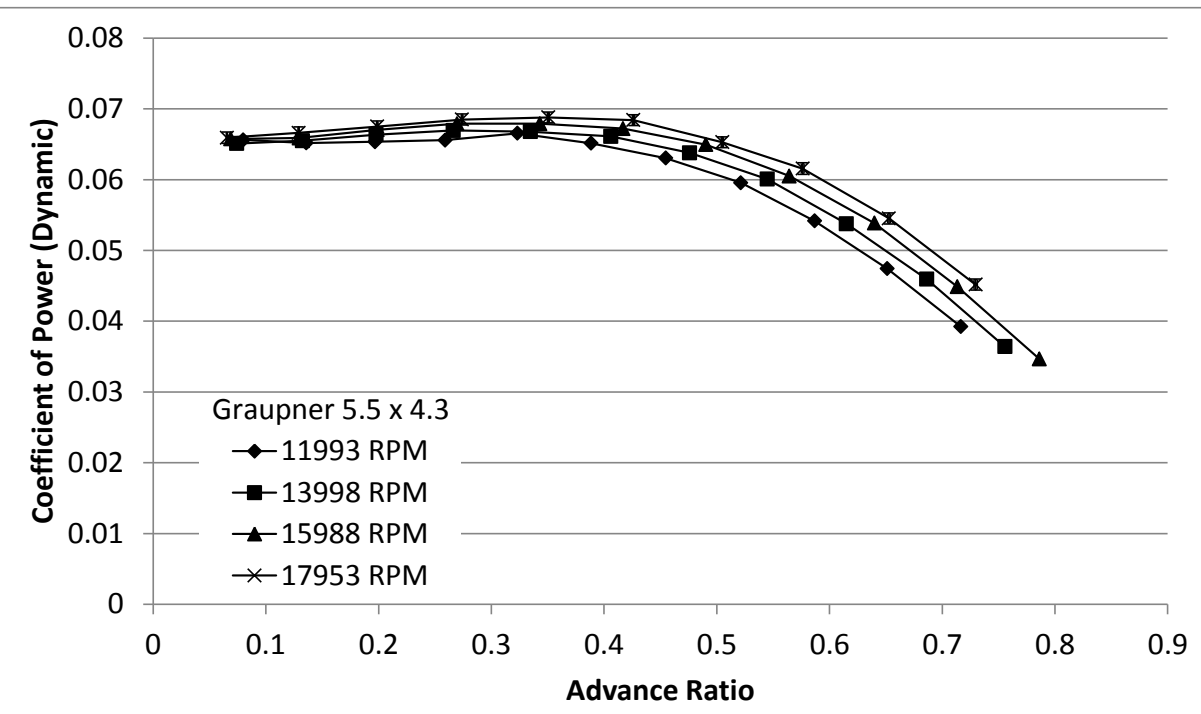

(b)

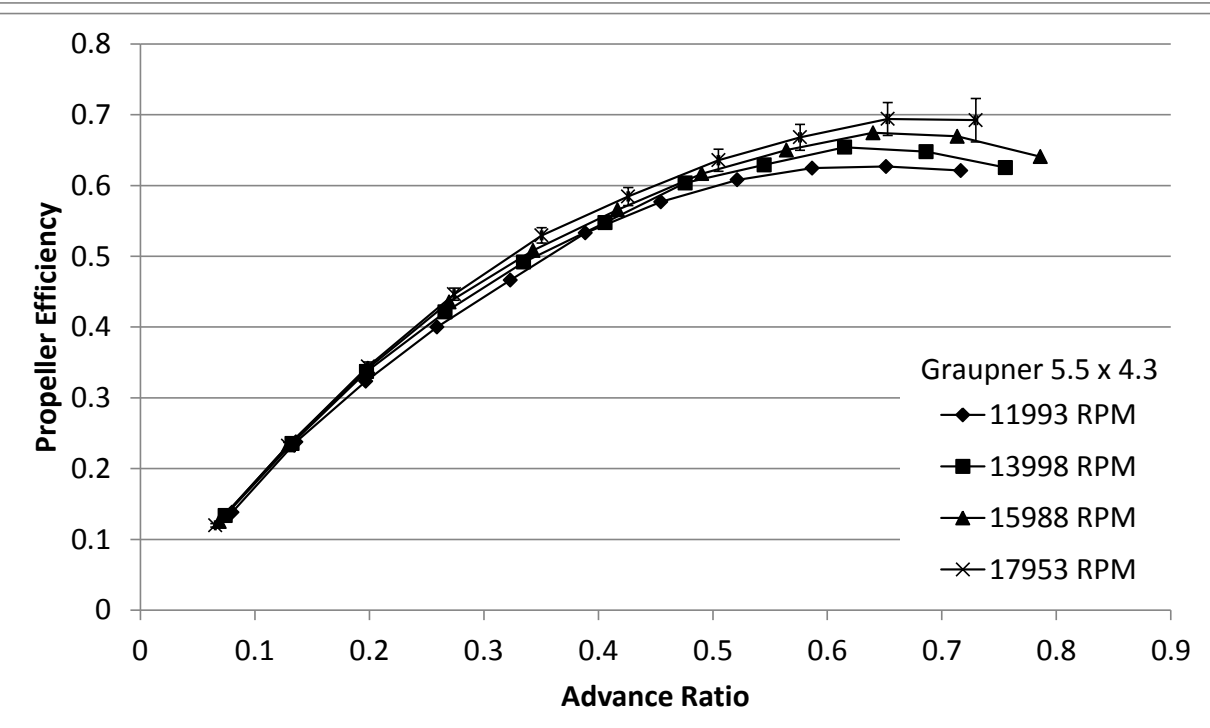

(c) 


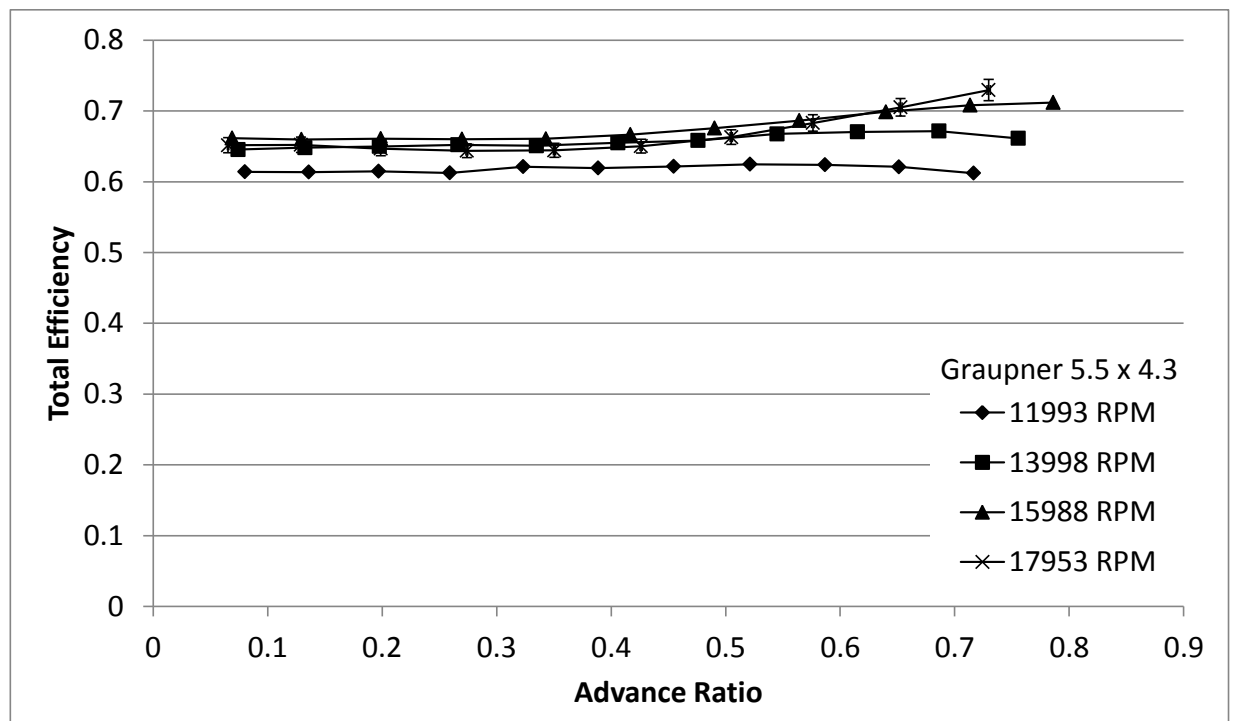

(d)

Figure 96: Graupner 5.5 x 4.3 Dynamic Test Results: (a) Coefficient of Thrust, (b) Coefficient of Power, (c) Propeller Efficiency, (d) Total Efficiency.

Table 218: Graupner 5.5 x 4.3 Dynamic Measured Values - 11993 RPM

\begin{tabular}{|c|c|c|c|c|c|c|c|}
\hline$n(\mathrm{RPM})$ & $Q(\mathrm{~g}-\mathrm{m})$ & $\Delta Q(\mathrm{~g}-\mathrm{m})$ & $V(\mathrm{~V})$ & $I(\mathrm{~A})$ & $T_{\text {atm }}\left({ }^{\circ} \mathrm{C}\right)$ & $P_{\text {atm }}(\mathrm{Pa})$ & $P_{\text {diff }}(\mathrm{Pa})$ \\
\hline $1.194 \mathrm{E}+04$ & $2.730 \mathrm{E}+00$ & $7.04 \mathrm{E}-02$ & $1.098 \mathrm{E}+01$ & $4.965 \mathrm{E}+00$ & $2.164 \mathrm{E}+01$ & $9.795 \mathrm{E}+04$ & $3.343 \mathrm{E}+00$ \\
\hline $1.196 \mathrm{E}+04$ & $2.724 \mathrm{E}+00$ & $7.64 \mathrm{E}-02$ & $1.098 \mathrm{E}+01$ & $4.969 \mathrm{E}+00$ & $2.158 \mathrm{E}+01$ & $9.796 \mathrm{E}+04$ & $9.156 \mathrm{E}+00$ \\
\hline $1.197 \mathrm{E}+04$ & $2.731 \mathrm{E}+00$ & $7.64 \mathrm{E}-02$ & $1.098 \mathrm{E}+01$ & $4.973 \mathrm{E}+00$ & $2.169 \mathrm{E}+01$ & $9.795 \mathrm{E}+04$ & $1.869 \mathrm{E}+01$ \\
\hline $1.202 \mathrm{E}+04$ & $2.766 \mathrm{E}+00$ & $8.41 \mathrm{E}-02$ & $1.097 \mathrm{E}+01$ & $5.081 \mathrm{E}+00$ & $2.175 \mathrm{E}+01$ & $9.796 \mathrm{E}+04$ & $3.217 \mathrm{E}+01$ \\
\hline $1.202 \mathrm{E}+04$ & $2.805 \mathrm{E}+00$ & $7.93 \mathrm{E}-02$ & $1.097 \mathrm{E}+01$ & $5.078 \mathrm{E}+00$ & $2.188 \mathrm{E}+01$ & $9.797 \mathrm{E}+04$ & $4.960 \mathrm{E}+01$ \\
\hline $1.199 \mathrm{E}+04$ & $2.731 \mathrm{E}+00$ & $7.79 \mathrm{E}-02$ & $1.098 \mathrm{E}+01$ & $4.945 \mathrm{E}+00$ & $2.195 \mathrm{E}+01$ & $9.797 \mathrm{E}+04$ & $7.079 \mathrm{E}+01$ \\
\hline $1.199 \mathrm{E}+04$ & $2.645 \mathrm{E}+00$ & $8.10 \mathrm{E}-02$ & $1.098 \mathrm{E}+01$ & $4.770 \mathrm{E}+00$ & $2.202 \mathrm{E}+01$ & $9.797 \mathrm{E}+04$ & $9.649 \mathrm{E}+01$ \\
\hline $1.199 \mathrm{E}+04$ & $2.497 \mathrm{E}+00$ & $7.95 \mathrm{E}-02$ & $1.099 \mathrm{E}+01$ & $4.479 \mathrm{E}+00$ & $2.208 \mathrm{E}+01$ & $9.797 \mathrm{E}+04$ & $1.263 \mathrm{E}+02$ \\
\hline $1.201 \mathrm{E}+04$ & $2.276 \mathrm{E}+00$ & $6.74 \mathrm{E}-02$ & $1.100 \mathrm{E}+01$ & $4.089 \mathrm{E}+00$ & $2.217 \mathrm{E}+01$ & $9.798 \mathrm{E}+04$ & $1.598 \mathrm{E}+02$ \\
\hline $1.203 \mathrm{E}+04$ & $2.001 \mathrm{E}+00$ & $7.06 \mathrm{E}-02$ & $1.101 \mathrm{E}+01$ & $3.614 \mathrm{E}+00$ & $2.220 \mathrm{E}+01$ & $9.798 \mathrm{E}+04$ & $1.973 \mathrm{E}+02$ \\
\hline $1.201 \mathrm{E}+04$ & $1.650 \mathrm{E}+00$ & $6.16 \mathrm{E}-02$ & $1.103 \mathrm{E}+01$ & $3.015 \mathrm{E}+00$ & $2.228 \mathrm{E}+01$ & $9.799 \mathrm{E}+04$ & $2.376 \mathrm{E}+02$ \\
\hline
\end{tabular}


Table 219: Graupner 5.5 x 4.3 Dynamic Calculated Values - 11993 RPM

\begin{tabular}{|c|c|c|c|c|c|c|c|}
\hline$n(\mathrm{RPM})$ & $V_{\infty}^{\prime}(\mathrm{m} / \mathrm{s})$ & $\Delta V_{\infty}^{\prime}(\mathrm{m} / \mathrm{s})$ & $T^{\prime}(\mathrm{g})$ & $\Delta T^{\prime}(\mathrm{g})$ & $V_{\mathrm{t}}(\mathrm{m} / \mathrm{s})$ & $P_{\mathrm{P}}(\mathrm{W})$ & $\Delta P_{\mathrm{P}}(\mathrm{W})$ \\
\hline $1.194 \mathrm{E}+04$ & $2.247 \mathrm{E}+00$ & $1.48 \mathrm{E}-02$ & $2.098 \mathrm{E}+02$ & $8.31 \mathrm{E}+00$ & $6.619 \mathrm{E}+01$ & $3.346 \mathrm{E}+01$ & $8.63 \mathrm{E}-01$ \\
\hline $1.196 \mathrm{E}+04$ & $3.823 \mathrm{E}+00$ & $1.58 \mathrm{E}-02$ & $2.120 \mathrm{E}+02$ & $8.13 \mathrm{E}+00$ & $6.641 \mathrm{E}+01$ & $3.347 \mathrm{E}+01$ & $9.38 \mathrm{E}-01$ \\
\hline $1.197 \mathrm{E}+04$ & $5.539 \mathrm{E}+00$ & $1.73 \mathrm{E}-02$ & $1.997 \mathrm{E}+02$ & $8.04 \mathrm{E}+00$ & $6.654 \mathrm{E}+01$ & $3.356 \mathrm{E}+01$ & $9.39 \mathrm{E}-01$ \\
\hline $1.202 \mathrm{E}+04$ & $7.323 \mathrm{E}+00$ & $1.94 \mathrm{E}-02$ & $1.902 \mathrm{E}+02$ & $8.07 \mathrm{E}+00$ & $6.702 \mathrm{E}+01$ & $3.415 \mathrm{E}+01$ & $1.04 \mathrm{E}+00$ \\
\hline $1.202 \mathrm{E}+04$ & $9.138 \mathrm{E}+00$ & $2.26 \mathrm{E}-02$ & $1.802 \mathrm{E}+02$ & $8.13 \mathrm{E}+00$ & $6.723 \mathrm{E}+01$ & $3.462 \mathrm{E}+01$ & $9.79 \mathrm{E}-01$ \\
\hline $1.199 \mathrm{E}+04$ & $1.095 \mathrm{E}+01$ & $2.57 \mathrm{E}-02$ & $1.667 \mathrm{E}+02$ & $8.15 \mathrm{E}+00$ & $6.733 \mathrm{E}+01$ & $3.361 \mathrm{E}+01$ & $9.59 \mathrm{E}-01$ \\
\hline $1.199 \mathrm{E}+04$ & $1.282 \mathrm{E}+01$ & $2.77 \mathrm{E}-02$ & $1.494 \mathrm{E}+02$ & $7.94 \mathrm{E}+00$ & $6.769 \mathrm{E}+01$ & $3.257 \mathrm{E}+01$ & 9.97E-01 \\
\hline 1.199E+04 & $1.470 \mathrm{E}+01$ & $3.01 \mathrm{E}-02$ & $1.296 \mathrm{E}+02$ & $7.80 \mathrm{E}+00$ & $6.806 \mathrm{E}+01$ & $3.075 \mathrm{E}+01$ & $9.79 \mathrm{E}-01$ \\
\hline $1.201 \mathrm{E}+04$ & $1.657 \mathrm{E}+01$ & $3.25 \mathrm{E}-02$ & $1.079 \mathrm{E}+02$ & $7.84 \mathrm{E}+00$ & $6.857 \mathrm{E}+01$ & $2.807 \mathrm{E}+01$ & 8.31E-01 \\
\hline $1.203 \mathrm{E}+04$ & $1.843 \mathrm{E}+01$ & $3.57 \mathrm{E}-02$ & $8.574 \mathrm{E}+01$ & $7.86 \mathrm{E}+00$ & $6.918 \mathrm{E}+01$ & $2.472 \mathrm{E}+01$ & 8.72E-01 \\
\hline $1.201 \mathrm{E}+04$ & $2.025 \mathrm{E}+01$ & $3.88 \mathrm{E}-02$ & $6.369 \mathrm{E}+01$ & $7.91 \mathrm{E}+00$ & $6.959 \mathrm{E}+01$ & $2.036 \mathrm{E}+01$ & 7.60E-01 \\
\hline$P_{\mathrm{e}}(\mathrm{W})$ & $\Delta P_{\mathrm{e}}(\mathrm{W})$ & $C_{\mathrm{T}}$ & $\Delta C_{\mathrm{T}}$ & $C_{\mathrm{P}}$ & $\Delta C_{\mathrm{P}}$ & $C_{\mathrm{Q}}$ & $\Delta C_{\mathrm{Q}}$ \\
\hline 5.449E+01 & $6.36 \mathrm{E}-01$ & $1.133 \mathrm{E}-01$ & 4.49E-03 & $6.562 \mathrm{E}-02$ & $1.69 \mathrm{E}-03$ & $1.044 \mathrm{E}-02$ & $2.70 \mathrm{E}-04$ \\
\hline $5.454 \mathrm{E}+01$ & $6.31 \mathrm{E}-01$ & $1.139 \mathrm{E}-01$ & 4.37E-03 & $6.518 \mathrm{E}-02$ & $1.83 \mathrm{E}-03$ & $1.037 \mathrm{E}-02$ & $2.91 \mathrm{E}-04$ \\
\hline $5.458 \mathrm{E}+01$ & $6.30 \mathrm{E}-01$ & $1.073 \mathrm{E}-01$ & $4.32 \mathrm{E}-03$ & $6.535 \mathrm{E}-02$ & $1.83 \mathrm{E}-03$ & $1.040 \mathrm{E}-02$ & $2.91 \mathrm{E}-04$ \\
\hline $5.575 \mathrm{E}+01$ & $6.45 \mathrm{E}-01$ & $1.013 \mathrm{E}-01$ & $4.30 \mathrm{E}-03$ & $6.558 \mathrm{E}-02$ & $2.00 \mathrm{E}-03$ & $1.044 \mathrm{E}-02$ & $3.18 \mathrm{E}-04$ \\
\hline $5.572 \mathrm{E}+01$ & $6.41 \mathrm{E}-01$ & $9.600 \mathrm{E}-02$ & 4.33E-03 & $6.655 \mathrm{E}-02$ & $1.88 \mathrm{E}-03$ & $1.059 \mathrm{E}-02$ & $3.00 \mathrm{E}-04$ \\
\hline $5.428 \mathrm{E}+01$ & $6.24 \mathrm{E}-01$ & 8.933E-02 & 4.37E-03 & $6.515 \mathrm{E}-02$ & $1.86 \mathrm{E}-03$ & $1.037 \mathrm{E}-02$ & $2.96 \mathrm{E}-04$ \\
\hline $5.239 \mathrm{E}+01$ & $6.02 \mathrm{E}-01$ & $7.999 \mathrm{E}-02$ & $4.25 \mathrm{E}-03$ & $6.305 \mathrm{E}-02$ & $1.93 \mathrm{E}-03$ & $1.003 \mathrm{E}-02$ & $3.07 \mathrm{E}-04$ \\
\hline 4.923E+01 & $5.65 \mathrm{E}-01$ & $6.943 \mathrm{E}-02$ & 4.18E-03 & $5.955 \mathrm{E}-02$ & $1.90 \mathrm{E}-03$ & $9.478 \mathrm{E}-03$ & $3.02 \mathrm{E}-04$ \\
\hline 4.499E+01 & $5.12 \mathrm{E}-01$ & $5.765 \mathrm{E}-02$ & 4.19E-03 & $5.416 \mathrm{E}-02$ & $1.60 \mathrm{E}-03$ & $8.621 \mathrm{E}-03$ & $2.55 \mathrm{E}-04$ \\
\hline $3.980 \mathrm{E}+01$ & $4.48 \mathrm{E}-01$ & $4.563 \mathrm{E}-02$ & $4.18 \mathrm{E}-03$ & $4.742 \mathrm{E}-02$ & $1.67 \mathrm{E}-03$ & $7.546 \mathrm{E}-03$ & $2.66 \mathrm{E}-04$ \\
\hline $3.326 \mathrm{E}+01$ & $3.73 \mathrm{E}-01$ & $3.401 \mathrm{E}-02$ & 4.22E-03 & $3.923 \mathrm{E}-02$ & $1.47 \mathrm{E}-03$ & $6.244 \mathrm{E}-03$ & $2.33 \mathrm{E}-04$ \\
\hline$\rho\left(\mathrm{kg} / \mathrm{m}^{\wedge} 3\right)$ & $\eta_{\mathrm{T}}$ & $\Delta \eta_{\mathrm{T}}$ & $J$ & $\Delta J$ & $\eta_{\mathrm{P}}$ & $\Delta \eta_{\mathrm{P}}$ & $\operatorname{Re}_{0.75}$ \\
\hline $1.158 \mathrm{E}+00$ & $6.141 \mathrm{E}-01$ & $1.74 \mathrm{E}-02$ & $8.003 \mathrm{E}-02$ & $5.28 \mathrm{E}-04$ & $1.381 \mathrm{E}-01$ & $6.59 \mathrm{E}-03$ & $4.430 \mathrm{E}+04$ \\
\hline $1.158 \mathrm{E}+00$ & $6.137 \mathrm{E}-01$ & $1.86 \mathrm{E}-02$ & $1.359 \mathrm{E}-01$ & $5.62 \mathrm{E}-04$ & $2.375 \mathrm{E}-01$ & $1.13 \mathrm{E}-02$ & $4.446 \mathrm{E}+04$ \\
\hline $1.157 \mathrm{E}+00$ & $6.149 \mathrm{E}-01$ & $1.86 \mathrm{E}-02$ & $1.968 \mathrm{E}-01$ & $6.16 \mathrm{E}-04$ & $3.232 \mathrm{E}-01$ & $1.59 \mathrm{E}-02$ & $4.452 \mathrm{E}+04$ \\
\hline $1.157 \mathrm{E}+00$ & $6.125 \mathrm{E}-01$ & $1.99 \mathrm{E}-02$ & $2.590 \mathrm{E}-01$ & $6.96 \mathrm{E}-04$ & $3.999 \mathrm{E}-01$ & $2.09 \mathrm{E}-02$ & $4.483 \mathrm{E}+04$ \\
\hline $1.157 \mathrm{E}+00$ & $6.213 \mathrm{E}-01$ & $1.90 \mathrm{E}-02$ & $3.233 \mathrm{E}-01$ & $8.10 \mathrm{E}-04$ & 4.663E-01 & 2.49E-02 & 4.494E+04 \\
\hline $1.157 \mathrm{E}+00$ & 6.193E-01 & $1.91 \mathrm{E}-02$ & $3.886 \mathrm{E}-01$ & $9.18 \mathrm{E}-04$ & $5.328 \mathrm{E}-01$ & $3.02 \mathrm{E}-02$ & 4.499E+04 \\
\hline $1.156 \mathrm{E}+00$ & $6.218 \mathrm{E}-01$ & $2.03 \mathrm{E}-02$ & $4.547 \mathrm{E}-01$ & $9.94 \mathrm{E}-04$ & $5.769 \mathrm{E}-01$ & $3.54 \mathrm{E}-02$ & $4.521 \mathrm{E}+04$ \\
\hline $1.156 \mathrm{E}+00$ & $6.247 \mathrm{E}-01$ & $2.11 \mathrm{E}-02$ & $5.214 \mathrm{E}-01$ & $1.08 \mathrm{E}-03$ & $6.079 \mathrm{E}-01$ & 4.14E-02 & $4.544 \mathrm{E}+04$ \\
\hline $1.156 \mathrm{E}+00$ & $6.239 \mathrm{E}-01$ & $1.98 \mathrm{E}-02$ & $5.867 \mathrm{E}-01$ & $1.18 \mathrm{E}-03$ & $6.245 \mathrm{E}-01$ & 4.90E-02 & $4.576 \mathrm{E}+04$ \\
\hline $1.156 \mathrm{E}+00$ & $6.212 \mathrm{E}-01$ & $2.30 \mathrm{E}-02$ & $6.513 \mathrm{E}-01$ & $1.29 \mathrm{E}-03$ & $6.268 \mathrm{E}-01$ & $6.16 \mathrm{E}-02$ & $4.616 \mathrm{E}+04$ \\
\hline $1.155 \mathrm{E}+00$ & $6.122 \mathrm{E}-01$ & $2.39 \mathrm{E}-02$ & $7.166 \mathrm{E}-01$ & $1.41 \mathrm{E}-03$ & $6.212 \mathrm{E}-01$ & $8.05 \mathrm{E}-02$ & $4.641 \mathrm{E}+04$ \\
\hline
\end{tabular}


Table 220: Graupner 5.5 x 4.3 Dynamic Measured Values - 13998 RPM

\begin{tabular}{|c|c|c|c|c|c|c|c|}
\hline$n(\mathrm{RPM})$ & $Q(\mathrm{~g}-\mathrm{m})$ & $\Delta Q(\mathrm{~g}-\mathrm{m})$ & $V(\mathrm{~V})$ & $I(\mathrm{~A})$ & $T_{\text {atm }}\left({ }^{\circ} \mathrm{C}\right)$ & $P_{\text {atm }}(\mathrm{Pa})$ & $P_{\text {diff }}(\mathrm{Pa})$ \\
\hline $1.395 \mathrm{E}+04$ & $3.697 \mathrm{E}+00$ & $6.74 \mathrm{E}-02$ & $1.090 \mathrm{E}+01$ & $7.527 \mathrm{E}+00$ & $2.191 \mathrm{E}+01$ & $9.799 \mathrm{E}+04$ & $3.976 \mathrm{E}+00$ \\
\hline $1.397 \mathrm{E}+04$ & $3.729 \mathrm{E}+00$ & $6.52 \mathrm{E}-02$ & $1.090 \mathrm{E}+01$ & $7.567 \mathrm{E}+00$ & $2.185 \mathrm{E}+01$ & $9.799 \mathrm{E}+04$ & $1.191 \mathrm{E}+01$ \\
\hline $1.401 \mathrm{E}+04$ & $3.799 \mathrm{E}+00$ & $6.55 \mathrm{E}-02$ & $1.090 \mathrm{E}+01$ & $7.715 \mathrm{E}+00$ & $2.205 \mathrm{E}+01$ & $9.799 \mathrm{E}+04$ & $2.587 \mathrm{E}+01$ \\
\hline $1.399 \mathrm{E}+04$ & $3.822 \mathrm{E}+00$ & $6.45 \mathrm{E}-02$ & $1.090 \mathrm{E}+01$ & $7.727 \mathrm{E}+00$ & $2.218 \mathrm{E}+01$ & $9.799 \mathrm{E}+04$ & $4.601 \mathrm{E}+01$ \\
\hline $1.400 \mathrm{E}+04$ & $3.812 \mathrm{E}+00$ & $6.58 \mathrm{E}-02$ & $1.090 \mathrm{E}+01$ & $7.724 \mathrm{E}+00$ & $2.230 \mathrm{E}+01$ & $9.799 \mathrm{E}+04$ & $7.198 \mathrm{E}+01$ \\
\hline $1.397 \mathrm{E}+04$ & $3.759 \mathrm{E}+00$ & $6.55 \mathrm{E}-02$ & $1.090 \mathrm{E}+01$ & $7.550 \mathrm{E}+00$ & $2.235 \mathrm{E}+01$ & $9.799 \mathrm{E}+04$ & $1.047 \mathrm{E}+02$ \\
\hline $1.400 \mathrm{E}+04$ & $3.644 \mathrm{E}+00$ & $6.74 \mathrm{E}-02$ & $1.091 \mathrm{E}+01$ & $7.294 \mathrm{E}+00$ & $2.237 \mathrm{E}+01$ & $9.799 \mathrm{E}+04$ & $1.438 \mathrm{E}+02$ \\
\hline $1.404 \mathrm{E}+04$ & $3.451 \mathrm{E}+00$ & $6.68 \mathrm{E}-02$ & $1.092 \mathrm{E}+01$ & $6.825 \mathrm{E}+00$ & $2.241 \mathrm{E}+01$ & $9.799 \mathrm{E}+04$ & $1.889 \mathrm{E}+02$ \\
\hline $1.402 \mathrm{E}+04$ & $3.079 \mathrm{E}+00$ & $6.82 \mathrm{E}-02$ & $1.095 \mathrm{E}+01$ & $6.039 \mathrm{E}+00$ & $2.250 \mathrm{E}+01$ & $9.800 \mathrm{E}+04$ & $2.391 \mathrm{E}+02$ \\
\hline $1.400 \mathrm{E}+04$ & $2.623 \mathrm{E}+00$ & $6.87 \mathrm{E}-02$ & $1.097 \mathrm{E}+01$ & $5.118 \mathrm{E}+00$ & $2.256 \mathrm{E}+01$ & $9.800 \mathrm{E}+04$ & $2.963 \mathrm{E}+02$ \\
\hline $1.402 \mathrm{E}+04$ & $2.084 \mathrm{E}+00$ & $6.58 \mathrm{E}-02$ & $1.100 \mathrm{E}+01$ & $4.123 \mathrm{E}+00$ & $2.262 \mathrm{E}+01$ & $9.801 \mathrm{E}+04$ & $3.593 \mathrm{E}+02$ \\
\hline
\end{tabular}


Table 221: Graupner 5.5 x 4.3 Dynamic Calculated Values - 13998 RPM

\begin{tabular}{|c|c|c|c|c|c|c|c|}
\hline$n(\mathrm{RPM})$ & $V_{\infty}^{\prime}(\mathrm{m} / \mathrm{s})$ & $\Delta V_{\infty}^{\prime}(\mathrm{m} / \mathrm{s})$ & $T^{\prime}(\mathrm{g})$ & $\Delta T^{\prime}(\mathrm{g})$ & $V_{\mathrm{t}}(\mathrm{m} / \mathrm{s})$ & $P_{\mathrm{P}}(\mathrm{W})$ & $\Delta P_{\mathrm{P}}(\mathrm{W})$ \\
\hline $1.395 \mathrm{E}+04$ & $2.435 \mathrm{E}+00$ & $1.64 \mathrm{E}-02$ & $2.967 \mathrm{E}+02$ & $8.33 \mathrm{E}+00$ & $7.735 \mathrm{E}+01$ & $5.297 \mathrm{E}+01$ & $9.65 \mathrm{E}-01$ \\
\hline $1.397 \mathrm{E}+04$ & $4.355 \mathrm{E}+00$ & $1.81 \mathrm{E}-02$ & $2.947 \mathrm{E}+02$ & $8.29 \mathrm{E}+00$ & $7.752 \mathrm{E}+01$ & $5.348 \mathrm{E}+01$ & $9.36 \mathrm{E}-01$ \\
\hline $1.401 \mathrm{E}+04$ & $6.515 \mathrm{E}+00$ & $2.02 \mathrm{E}-02$ & $2.884 \mathrm{E}+02$ & $8.35 \mathrm{E}+00$ & $7.791 \mathrm{E}+01$ & $5.465 \mathrm{E}+01$ & $9.42 \mathrm{E}-01$ \\
\hline $1.399 \mathrm{E}+04$ & $8.763 \mathrm{E}+00$ & 2.39E-02 & $2.691 \mathrm{E}+02$ & $8.24 \mathrm{E}+00$ & $7.804 \mathrm{E}+01$ & $5.491 \mathrm{E}+01$ & $9.27 \mathrm{E}-01$ \\
\hline $1.400 \mathrm{E}+04$ & $1.102 \mathrm{E}+01$ & $2.76 \mathrm{E}-02$ & $2.493 \mathrm{E}+02$ & $8.32 \mathrm{E}+00$ & $7.834 \mathrm{E}+01$ & $5.479 \mathrm{E}+01$ & $9.46 \mathrm{E}-01$ \\
\hline $1.397 \mathrm{E}+04$ & $1.334 \mathrm{E}+01$ & $3.02 \mathrm{E}-02$ & $2.257 \mathrm{E}+02$ & $8.28 \mathrm{E}+00$ & $7.855 \mathrm{E}+01$ & $5.393 \mathrm{E}+01$ & $9.40 \mathrm{E}-01$ \\
\hline $1.400 \mathrm{E}+04$ & $1.567 \mathrm{E}+01$ & $3.25 \mathrm{E}-02$ & $2.058 \mathrm{E}+02$ & $8.36 \mathrm{E}+00$ & $7.916 \mathrm{E}+01$ & $5.239 \mathrm{E}+01$ & $9.69 \mathrm{E}-01$ \\
\hline $1.404 \mathrm{E}+04$ & $1.800 \mathrm{E}+01$ & $3.52 \mathrm{E}-02$ & $1.774 \mathrm{E}+02$ & $8.33 \mathrm{E}+00$ & $7.988 \mathrm{E}+01$ & $4.978 \mathrm{E}+01$ & $9.63 \mathrm{E}-01$ \\
\hline $1.402 \mathrm{E}+04$ & $2.028 \mathrm{E}+01$ & $3.90 \mathrm{E}-02$ & $1.458 \mathrm{E}+02$ & $8.29 \mathrm{E}+00$ & $8.030 \mathrm{E}+01$ & $4.433 \mathrm{E}+01$ & $9.82 \mathrm{E}-01$ \\
\hline $1.400 \mathrm{E}+04$ & $2.260 \mathrm{E}+01$ & $4.32 \mathrm{E}-02$ & $1.102 \mathrm{E}+02$ & $8.30 \mathrm{E}+00$ & $8.083 \mathrm{E}+01$ & $3.771 \mathrm{E}+01$ & $9.88 \mathrm{E}-01$ \\
\hline $1.402 \mathrm{E}+04$ & $2.492 \mathrm{E}+01$ & $4.69 \mathrm{E}-02$ & $7.676 \mathrm{E}+01$ & $8.34 \mathrm{E}+00$ & $8.160 \mathrm{E}+01$ & $3.000 \mathrm{E}+01$ & $9.47 \mathrm{E}-01$ \\
\hline$P_{\mathrm{e}}(\mathrm{W})$ & $\Delta P_{\mathrm{e}}(\mathrm{W})$ & $C_{\mathrm{T}}$ & $\Delta C_{\mathrm{T}}$ & $C_{\mathrm{P}}$ & $\Delta C_{\mathrm{P}}$ & $C_{\mathrm{Q}}$ & $\Delta C_{\mathrm{Q}}$ \\
\hline $8.208 \mathrm{E}+01$ & 8.98E-01 & 1.173E-01 & $3.30 \mathrm{E}-03$ & $6.510 \mathrm{E}-02$ & $1.19 \mathrm{E}-03$ & $1.036 \mathrm{E}-02$ & $1.89 \mathrm{E}-04$ \\
\hline $8.251 \mathrm{E}+01$ & $9.00 \mathrm{E}-01$ & $1.163 \mathrm{E}-01$ & $3.27 \mathrm{E}-03$ & $6.550 \mathrm{E}-02$ & $1.15 \mathrm{E}-03$ & 1.042E-02 & $1.83 \mathrm{E}-04$ \\
\hline $8.408 \mathrm{E}+01$ & $9.18 \mathrm{E}-01$ & $1.131 \mathrm{E}-01$ & $3.28 \mathrm{E}-03$ & $6.637 \mathrm{E}-02$ & $1.15 \mathrm{E}-03$ & $1.056 \mathrm{E}-02$ & $1.82 \mathrm{E}-04$ \\
\hline $8.421 \mathrm{E}+01$ & $9.18 \mathrm{E}-01$ & $1.059 \mathrm{E}-01$ & $3.24 \mathrm{E}-03$ & $6.695 \mathrm{E}-02$ & $1.13 \mathrm{E}-03$ & $1.066 \mathrm{E}-02$ & $1.80 \mathrm{E}-04$ \\
\hline $8.418 \mathrm{E}+01$ & $9.19 \mathrm{E}-01$ & $9.809 \mathrm{E}-02$ & $3.28 \mathrm{E}-03$ & $6.677 \mathrm{E}-02$ & $1.16 \mathrm{E}-03$ & $1.063 \mathrm{E}-02$ & $1.84 \mathrm{E}-04$ \\
\hline $8.232 \mathrm{E}+01$ & 8.95E-01 & 8.915E-02 & $3.27 \mathrm{E}-03$ & $6.611 \mathrm{E}-02$ & $1.16 \mathrm{E}-03$ & $1.052 \mathrm{E}-02$ & $1.84 \mathrm{E}-04$ \\
\hline $7.958 \mathrm{E}+01$ & $8.70 \mathrm{E}-01$ & $8.091 \mathrm{E}-02$ & $3.29 \mathrm{E}-03$ & $6.380 \mathrm{E}-02$ & $1.18 \mathrm{E}-03$ & $1.015 \mathrm{E}-02$ & $1.88 \mathrm{E}-04$ \\
\hline $7.456 \mathrm{E}+01$ & $8.23 \mathrm{E}-01$ & $6.936 \mathrm{E}-02$ & $3.26 \mathrm{E}-03$ & $6.007 \mathrm{E}-02$ & $1.17 \mathrm{E}-03$ & $9.561 \mathrm{E}-03$ & $1.85 \mathrm{E}-04$ \\
\hline $6.611 \mathrm{E}+01$ & $7.38 \mathrm{E}-01$ & $5.718 \mathrm{E}-02$ & $3.25 \mathrm{E}-03$ & $5.377 \mathrm{E}-02$ & $1.19 \mathrm{E}-03$ & $8.558 \mathrm{E}-03$ & $1.90 \mathrm{E}-04$ \\
\hline $5.616 \mathrm{E}+01$ & $6.34 \mathrm{E}-01$ & 4.334E-02 & $3.27 \mathrm{E}-03$ & $4.593 \mathrm{E}-02$ & $1.21 \mathrm{E}-03$ & $7.310 \mathrm{E}-03$ & $1.92 \mathrm{E}-04$ \\
\hline $4.536 \mathrm{E}+01$ & $5.04 \mathrm{E}-01$ & $3.012 \mathrm{E}-02$ & $3.27 \mathrm{E}-03$ & $3.640 \mathrm{E}-02$ & $1.15 \mathrm{E}-03$ & $5.793 \mathrm{E}-03$ & $1.83 \mathrm{E}-04$ \\
\hline$\rho\left(\mathrm{kg} / \mathrm{m}^{\wedge} 3\right)$ & $\eta_{\mathrm{T}}$ & $\Delta \eta_{\mathrm{T}}$ & $J$ & $\Delta J$ & $\eta_{\mathrm{P}}$ & $\Delta \eta_{\mathrm{P}}$ & $R e_{0.75}$ \\
\hline $1.157 \mathrm{E}+00$ & $6.454 \mathrm{E}-01$ & $1.37 \mathrm{E}-02$ & 7.422E-02 & $5.00 \mathrm{E}-04$ & $1.337 \mathrm{E}-01$ & $4.57 \mathrm{E}-03$ & $5.171 \mathrm{E}+04$ \\
\hline $1.157 \mathrm{E}+00$ & $6.482 \mathrm{E}-01$ & $1.34 \mathrm{E}-02$ & $1.326 \mathrm{E}-01$ & $5.53 \mathrm{E}-04$ & $2.354 \mathrm{E}-01$ & 7.87E-03 & $5.184 \mathrm{E}+04$ \\
\hline $1.156 \mathrm{E}+00$ & $6.500 \mathrm{E}-01$ & $1.33 \mathrm{E}-02$ & $1.977 \mathrm{E}-01$ & $6.18 \mathrm{E}-04$ & $3.371 \mathrm{E}-01$ & $1.14 \mathrm{E}-02$ & $5.204 \mathrm{E}+04$ \\
\hline $1.156 \mathrm{E}+00$ & $6.521 \mathrm{E}-01$ & $1.31 \mathrm{E}-02$ & 2.663E-01 & 7.34E-04 & 4.212E-01 & $1.48 \mathrm{E}-02$ & $5.208 \mathrm{E}+04$ \\
\hline $1.155 \mathrm{E}+00$ & $6.509 \mathrm{E}-01$ & $1.33 \mathrm{E}-02$ & $3.348 \mathrm{E}-01$ & $8.50 \mathrm{E}-04$ & 4.917E-01 & $1.85 \mathrm{E}-02$ & $5.225 \mathrm{E}+04$ \\
\hline $1.155 \mathrm{E}+00$ & $6.550 \mathrm{E}-01$ & $1.35 \mathrm{E}-02$ & $4.060 \mathrm{E}-01$ & $9.34 \mathrm{E}-04$ & $5.475 \mathrm{E}-01$ & $2.23 \mathrm{E}-02$ & $5.237 \mathrm{E}+04$ \\
\hline $1.155 \mathrm{E}+00$ & $6.583 \mathrm{E}-01$ & $1.41 \mathrm{E}-02$ & $4.758 \mathrm{E}-01$ & $1.01 \mathrm{E}-03$ & $6.035 \mathrm{E}-01$ & $2.70 \mathrm{E}-02$ & $5.277 \mathrm{E}+04$ \\
\hline $1.155 \mathrm{E}+00$ & $6.676 \mathrm{E}-01$ & $1.49 \mathrm{E}-02$ & 5.449E-01 & $1.09 \mathrm{E}-03$ & $6.291 \mathrm{E}-01$ & $3.20 \mathrm{E}-02$ & $5.324 \mathrm{E}+04$ \\
\hline $1.155 \mathrm{E}+00$ & $6.705 \mathrm{E}-01$ & $1.66 \mathrm{E}-02$ & $6.151 \mathrm{E}-01$ & $1.21 \mathrm{E}-03$ & $6.541 \mathrm{E}-01$ & $4.00 \mathrm{E}-02$ & $5.350 \mathrm{E}+04$ \\
\hline $1.155 \mathrm{E}+00$ & $6.716 \mathrm{E}-01$ & $1.92 \mathrm{E}-02$ & $6.864 \mathrm{E}-01$ & $1.34 \mathrm{E}-03$ & $6.477 \mathrm{E}-01$ & $5.17 \mathrm{E}-02$ & $5.383 \mathrm{E}+04$ \\
\hline $1.154 \mathrm{E}+00$ & $6.614 \mathrm{E}-01$ & $2.21 \mathrm{E}-02$ & $7.556 \mathrm{E}-01$ & $1.46 \mathrm{E}-03$ & $6.252 \mathrm{E}-01$ & 7.07E-02 & $5.433 \mathrm{E}+04$ \\
\hline
\end{tabular}


Table 222: Graupner 5.5 x 4.3 Dynamic Measured Values - 15988 RPM

\begin{tabular}{|c|c|c|c|c|c|c|c|}
\hline$n(\mathrm{RPM})$ & $Q(\mathrm{~g}-\mathrm{m})$ & $\Delta Q(\mathrm{~g}-\mathrm{m})$ & $V(\mathrm{~V})$ & $I(\mathrm{~A})$ & $T_{\text {atm }}\left({ }^{\circ} \mathrm{C}\right)$ & $P_{\text {atm }}(\mathrm{Pa})$ & $P_{\text {diff }}(\mathrm{Pa})$ \\
\hline $1.598 \mathrm{E}+04$ & $4.897 \mathrm{E}+00$ & $6.60 \mathrm{E}-02$ & $1.080 \mathrm{E}+01$ & $1.125 \mathrm{E}+01$ & $2.205 \mathrm{E}+01$ & $9.801 \mathrm{E}+04$ & $4.560 \mathrm{E}+00$ \\
\hline $1.597 \mathrm{E}+04$ & $4.906 \mathrm{E}+00$ & $6.56 \mathrm{E}-02$ & $1.079 \mathrm{E}+01$ & $1.130 \mathrm{E}+01$ & $2.200 \mathrm{E}+01$ & $9.801 \mathrm{E}+04$ & $1.491 \mathrm{E}+01$ \\
\hline $1.599 \mathrm{E}+04$ & $4.998 \mathrm{E}+00$ & $6.42 \mathrm{E}-02$ & $1.079 \mathrm{E}+01$ & $1.151 \mathrm{E}+01$ & $2.220 \mathrm{E}+01$ & $9.801 \mathrm{E}+04$ & $3.404 \mathrm{E}+01$ \\
\hline $1.600 \mathrm{E}+04$ & $5.065 \mathrm{E}+00$ & $6.56 \mathrm{E}-02$ & $1.078 \mathrm{E}+01$ & $1.169 \mathrm{E}+01$ & $2.237 \mathrm{E}+01$ & $9.801 \mathrm{E}+04$ & $6.170 \mathrm{E}+01$ \\
\hline $1.599 \mathrm{E}+04$ & $5.060 \mathrm{E}+00$ & $6.65 \mathrm{E}-02$ & $1.078 \mathrm{E}+01$ & $1.167 \mathrm{E}+01$ & $2.247 \mathrm{E}+01$ & $9.801 \mathrm{E}+04$ & $9.864 \mathrm{E}+01$ \\
\hline $1.601 \mathrm{E}+04$ & $5.017 \mathrm{E}+00$ & $6.58 \mathrm{E}-02$ & $1.079 \mathrm{E}+01$ & $1.146 \mathrm{E}+01$ & $2.245 \mathrm{E}+01$ & $9.801 \mathrm{E}+04$ & $1.448 \mathrm{E}+02$ \\
\hline $1.603 \mathrm{E}+04$ & $4.862 \mathrm{E}+00$ & $6.62 \mathrm{E}-02$ & $1.080 \mathrm{E}+01$ & $1.096 \mathrm{E}+01$ & $2.253 \mathrm{E}+01$ & $9.800 \mathrm{E}+04$ & $2.000 \mathrm{E}+02$ \\
\hline $1.601 \mathrm{E}+04$ & $4.517 \mathrm{E}+00$ & $6.60 \mathrm{E}-02$ & $1.083 \mathrm{E}+01$ & $9.986 \mathrm{E}+00$ & $2.243 \mathrm{E}+01$ & $9.800 \mathrm{E}+04$ & $2.631 \mathrm{E}+02$ \\
\hline $1.596 \mathrm{E}+04$ & $3.993 \mathrm{E}+00$ & $6.73 \mathrm{E}-02$ & $1.087 \mathrm{E}+01$ & $8.613 \mathrm{E}+00$ & $2.262 \mathrm{E}+01$ & $9.800 \mathrm{E}+04$ & $3.350 \mathrm{E}+02$ \\
\hline $1.595 \mathrm{E}+04$ & $3.324 \mathrm{E}+00$ & $6.62 \mathrm{E}-02$ & $1.092 \mathrm{E}+01$ & $7.044 \mathrm{E}+00$ & $2.273 \mathrm{E}+01$ & $9.800 \mathrm{E}+04$ & $4.151 \mathrm{E}+02$ \\
\hline $1.597 \mathrm{E}+04$ & $2.573 \mathrm{E}+00$ & $6.76 \mathrm{E}-02$ & $1.096 \mathrm{E}+01$ & $5.404 \mathrm{E}+00$ & $2.277 \mathrm{E}+01$ & $9.801 \mathrm{E}+04$ & $5.040 \mathrm{E}+02$ \\
\hline
\end{tabular}


Table 223: Graupner 5.5 x 4.3 Dynamic Calculated Values - 15988 RPM

\begin{tabular}{|c|c|c|c|c|c|c|c|}
\hline$n(\mathrm{RPM})$ & $V_{\infty}^{\prime}(\mathrm{m} / \mathrm{s})$ & $\Delta V_{\infty}^{\prime}(\mathrm{m} / \mathrm{s})$ & $T^{\prime}(\mathrm{g})$ & $\Delta T^{\prime}(\mathrm{g})$ & $V_{\mathrm{t}}(\mathrm{m} / \mathrm{s})$ & $P_{\mathrm{P}}(\mathrm{W})$ & $\Delta P_{\mathrm{P}}(\mathrm{W})$ \\
\hline $1.598 \mathrm{E}+04$ & $2.592 \mathrm{E}+00$ & $1.85 \mathrm{E}-02$ & $3.959 \mathrm{E}+02$ & $7.44 \mathrm{E}+00$ & $8.858 \mathrm{E}+01$ & $8.034 \mathrm{E}+01$ & $1.08 \mathrm{E}+00$ \\
\hline $1.597 \mathrm{E}+04$ & $4.866 \mathrm{E}+00$ & $2.01 \mathrm{E}-02$ & $3.938 \mathrm{E}+02$ & $7.42 \mathrm{E}+00$ & $8.866 \mathrm{E}+01$ & $8.049 \mathrm{E}+01$ & $1.08 \mathrm{E}+00$ \\
\hline $1.599 \mathrm{E}+04$ & $7.473 \mathrm{E}+00$ & $2.31 \mathrm{E}-02$ & $3.825 \mathrm{E}+02$ & $7.39 \mathrm{E}+00$ & $8.893 \mathrm{E}+01$ & $8.207 \mathrm{E}+01$ & $1.05 \mathrm{E}+00$ \\
\hline $1.600 \mathrm{E}+04$ & $1.015 \mathrm{E}+01$ & $2.84 \mathrm{E}-02$ & $3.642 \mathrm{E}+02$ & $7.23 \mathrm{E}+00$ & $8.924 \mathrm{E}+01$ & $8.322 \mathrm{E}+01$ & $1.08 \mathrm{E}+00$ \\
\hline $1.599 \mathrm{E}+04$ & $1.290 \mathrm{E}+01$ & $3.20 \mathrm{E}-02$ & $3.339 \mathrm{E}+02$ & $7.34 \mathrm{E}+00$ & $8.957 \mathrm{E}+01$ & $8.312 \mathrm{E}+01$ & $1.09 \mathrm{E}+00$ \\
\hline $1.601 \mathrm{E}+04$ & $1.569 \mathrm{E}+01$ & $3.44 \mathrm{E}-02$ & $3.032 \mathrm{E}+02$ & $7.37 \mathrm{E}+00$ & $9.008 \mathrm{E}+01$ & $8.246 \mathrm{E}+01$ & $1.08 \mathrm{E}+00$ \\
\hline $1.603 \mathrm{E}+04$ & $1.849 \mathrm{E}+01$ & $3.73 \mathrm{E}-02$ & $2.723 \mathrm{E}+02$ & $7.41 \mathrm{E}+00$ & $9.076 \mathrm{E}+01$ & $8.005 \mathrm{E}+01$ & $1.09 \mathrm{E}+00$ \\
\hline $1.601 \mathrm{E}+04$ & $2.124 \mathrm{E}+01$ & $4.16 \mathrm{E}-02$ & $2.317 \mathrm{E}+02$ & $7.49 \mathrm{E}+00$ & $9.122 \mathrm{E}+01$ & $7.426 \mathrm{E}+01$ & $1.09 \mathrm{E}+00$ \\
\hline $1.596 \mathrm{E}+04$ & $2.401 \mathrm{E}+01$ & $4.56 \mathrm{E}-02$ & $1.874 \mathrm{E}+02$ & $7.84 \mathrm{E}+00$ & $9.164 \mathrm{E}+01$ & $6.544 \mathrm{E}+01$ & $1.10 \mathrm{E}+00$ \\
\hline $1.595 \mathrm{E}+04$ & $2.677 \mathrm{E}+01$ & $5.05 \mathrm{E}-02$ & $1.389 \mathrm{E}+02$ & $7.78 \mathrm{E}+00$ & $9.238 \mathrm{E}+01$ & $5.447 \mathrm{E}+01$ & $1.09 \mathrm{E}+00$ \\
\hline $1.597 \mathrm{E}+04$ & $2.952 \mathrm{E}+01$ & $5.46 \mathrm{E}-02$ & $9.336 \mathrm{E}+01$ & $7.79 \mathrm{E}+00$ & $9.330 \mathrm{E}+01$ & $4.219 \mathrm{E}+01$ & $1.11 \mathrm{E}+00$ \\
\hline$P_{\mathrm{e}}(\mathrm{W})$ & $\Delta P_{\mathrm{e}}(\mathrm{W})$ & $C_{\mathrm{T}}$ & $\Delta C_{\mathrm{T}}$ & $C_{\mathrm{P}}$ & $\Delta C_{\mathrm{P}}$ & $C_{\mathrm{Q}}$ & $\Delta C_{\mathrm{Q}}$ \\
\hline $1.214 \mathrm{E}+02$ & $1.30 \mathrm{E}+00$ & 1.194E-01 & $2.24 \mathrm{E}-03$ & $6.576 \mathrm{E}-02$ & $8.88 \mathrm{E}-04$ & $1.047 \mathrm{E}-02$ & $1.41 \mathrm{E}-04$ \\
\hline $1.220 \mathrm{E}+02$ & $1.30 \mathrm{E}+00$ & $1.188 \mathrm{E}-01$ & $2.24 \mathrm{E}-03$ & $6.589 \mathrm{E}-02$ & $8.83 \mathrm{E}-04$ & 1.049E-02 & $1.40 \mathrm{E}-04$ \\
\hline $1.242 \mathrm{E}+02$ & $1.32 \mathrm{E}+00$ & $1.152 \mathrm{E}-01$ & $2.23 \mathrm{E}-03$ & $6.704 \mathrm{E}-02$ & $8.63 \mathrm{E}-04$ & $1.067 \mathrm{E}-02$ & $1.37 \mathrm{E}-04$ \\
\hline $1.261 \mathrm{E}+02$ & $1.34 \mathrm{E}+00$ & $1.097 \mathrm{E}-01$ & $2.18 \mathrm{E}-03$ & $6.791 \mathrm{E}-02$ & 8.82E-04 & $1.081 \mathrm{E}-02$ & $1.40 \mathrm{E}-04$ \\
\hline $1.258 \mathrm{E}+02$ & $1.34 \mathrm{E}+00$ & $1.006 \mathrm{E}-01$ & $2.21 \mathrm{E}-03$ & $6.790 \mathrm{E}-02$ & 8.94E-04 & $1.081 \mathrm{E}-02$ & $1.42 \mathrm{E}-04$ \\
\hline $1.237 \mathrm{E}+02$ & $1.31 \mathrm{E}+00$ & $9.121 \mathrm{E}-02$ & 2.22E-03 & $6.721 \mathrm{E}-02$ & $8.83 \mathrm{E}-04$ & $1.070 \mathrm{E}-02$ & $1.40 \mathrm{E}-04$ \\
\hline $1.184 \mathrm{E}+02$ & $1.26 \mathrm{E}+00$ & $8.169 \mathrm{E}-02$ & $2.22 \mathrm{E}-03$ & $6.494 \mathrm{E}-02$ & $8.86 \mathrm{E}-04$ & $1.034 \mathrm{E}-02$ & $1.41 \mathrm{E}-04$ \\
\hline $1.082 \mathrm{E}+02$ & $1.17 \mathrm{E}+00$ & $6.971 \mathrm{E}-02$ & $2.25 \mathrm{E}-03$ & $6.051 \mathrm{E}-02$ & $8.86 \mathrm{E}-04$ & $9.631 \mathrm{E}-03$ & $1.41 \mathrm{E}-04$ \\
\hline $9.364 \mathrm{E}+01$ & $1.02 \mathrm{E}+00$ & $5.678 \mathrm{E}-02$ & $2.38 \mathrm{E}-03$ & $5.387 \mathrm{E}-02$ & 9.09E-04 & $8.573 \mathrm{E}-03$ & $1.45 \mathrm{E}-04$ \\
\hline $7.690 \mathrm{E}+01$ & $8.47 \mathrm{E}-01$ & $4.211 \mathrm{E}-02$ & $2.36 \mathrm{E}-03$ & $4.487 \mathrm{E}-02$ & 8.95E-04 & $7.141 \mathrm{E}-03$ & $1.42 \mathrm{E}-04$ \\
\hline $5.925 \mathrm{E}+01$ & $6.65 \mathrm{E}-01$ & $2.825 \mathrm{E}-02$ & $2.36 \mathrm{E}-03$ & $3.466 \mathrm{E}-02$ & $9.11 \mathrm{E}-04$ & $5.517 \mathrm{E}-03$ & $1.45 \mathrm{E}-04$ \\
\hline$\rho\left(\mathrm{kg} / \mathrm{m}^{\wedge} 3\right)$ & $\eta_{\mathrm{T}}$ & $\Delta \eta_{\mathrm{T}}$ & $J$ & $\Delta J$ & $\eta_{\mathrm{P}}$ & $\Delta \eta_{\mathrm{P}}$ & $R e_{0.75}$ \\
\hline $1.157 \mathrm{E}+00$ & $6.618 \mathrm{E}-01$ & $1.14 \mathrm{E}-02$ & $6.898 \mathrm{E}-02$ & $4.92 \mathrm{E}-04$ & $1.253 \mathrm{E}-01$ & $3.03 \mathrm{E}-03$ & $5.918 \mathrm{E}+04$ \\
\hline $1.157 \mathrm{E}+00$ & $6.597 \mathrm{E}-01$ & $1.13 \mathrm{E}-02$ & $1.295 \mathrm{E}-01$ & $5.35 \mathrm{E}-04$ & $2.335 \mathrm{E}-01$ & $5.49 \mathrm{E}-03$ & $5.925 \mathrm{E}+04$ \\
\hline $1.156 \mathrm{E}+00$ & $6.609 \mathrm{E}-01$ & $1.10 \mathrm{E}-02$ & $1.987 \mathrm{E}-01$ & $6.15 \mathrm{E}-04$ & $3.416 \mathrm{E}-01$ & $8.00 \mathrm{E}-03$ & $5.936 \mathrm{E}+04$ \\
\hline $1.155 \mathrm{E}+00$ & $6.601 \mathrm{E}-01$ & $1.11 \mathrm{E}-02$ & 2.697E-01 & 7.57E-04 & $4.356 \mathrm{E}-01$ & $1.04 \mathrm{E}-02$ & $5.950 \mathrm{E}+04$ \\
\hline $1.155 \mathrm{E}+00$ & $6.608 \mathrm{E}-01$ & $1.12 \mathrm{E}-02$ & $3.430 \mathrm{E}-01$ & $8.54 \mathrm{E}-04$ & $5.083 \mathrm{E}-01$ & $1.31 \mathrm{E}-02$ & $5.969 \mathrm{E}+04$ \\
\hline $1.155 \mathrm{E}+00$ & $6.668 \mathrm{E}-01$ & $1.12 \mathrm{E}-02$ & $4.168 \mathrm{E}-01$ & $9.19 \mathrm{E}-04$ & $5.656 \mathrm{E}-01$ & $1.57 \mathrm{E}-02$ & $6.004 \mathrm{E}+04$ \\
\hline $1.155 \mathrm{E}+00$ & $6.759 \mathrm{E}-01$ & $1.17 \mathrm{E}-02$ & 4.904E-01 & $9.94 \mathrm{E}-04$ & $6.168 \mathrm{E}-01$ & $1.88 \mathrm{E}-02$ & $6.045 \mathrm{E}+04$ \\
\hline $1.155 \mathrm{E}+00$ & $6.866 \mathrm{E}-01$ & $1.25 \mathrm{E}-02$ & 5.643E-01 & $1.11 \mathrm{E}-03$ & $6.501 \mathrm{E}-01$ & $2.31 \mathrm{E}-02$ & $6.080 \mathrm{E}+04$ \\
\hline $1.154 \mathrm{E}+00$ & $6.989 \mathrm{E}-01$ & $1.40 \mathrm{E}-02$ & $6.399 \mathrm{E}-01$ & $1.22 \mathrm{E}-03$ & $6.745 \mathrm{E}-01$ & $3.04 \mathrm{E}-02$ & $6.100 \mathrm{E}+04$ \\
\hline $1.154 \mathrm{E}+00$ & $7.083 \mathrm{E}-01$ & $1.61 \mathrm{E}-02$ & $7.135 \mathrm{E}-01$ & $1.35 \mathrm{E}-03$ & $6.695 \mathrm{E}-01$ & $3.98 \mathrm{E}-02$ & $6.146 \mathrm{E}+04$ \\
\hline $1.154 \mathrm{E}+00$ & $7.121 \mathrm{E}-01$ & 2.03E-02 & 7.861E-01 & $1.46 \mathrm{E}-03$ & $6.407 \mathrm{E}-01$ & $5.60 \mathrm{E}-02$ & $6.206 \mathrm{E}+04$ \\
\hline
\end{tabular}


Table 224: Graupner 5.5 x 4.3 Dynamic Measured Values - 17953 RPM

\begin{tabular}{|c|c|c|c|c|c|c|c|}
\hline$n(\mathrm{RPM})$ & $Q(\mathrm{~g}-\mathrm{m})$ & $\Delta Q(\mathrm{~g}-\mathrm{m})$ & $V(\mathrm{~V})$ & $I(\mathrm{~A})$ & $T_{\text {atm }}\left({ }^{\circ} \mathrm{C}\right)$ & $P_{\text {atm }}(\mathrm{Pa})$ & $P_{\text {diff }}(\mathrm{Pa})$ \\
\hline $1.780 \mathrm{E}+04$ & $6.089 \mathrm{E}+00$ & $7.34 \mathrm{E}-02$ & $1.065 \mathrm{E}+01$ & $1.604 \mathrm{E}+01$ & $2.212 \mathrm{E}+01$ & $9.801 \mathrm{E}+04$ & $5.143 \mathrm{E}+00$ \\
\hline $1.764 \mathrm{E}+04$ & $6.047 \mathrm{E}+00$ & $7.52 \mathrm{E}-02$ & $1.065 \mathrm{E}+01$ & $1.576 \mathrm{E}+01$ & $2.202 \mathrm{E}+01$ & $9.800 \mathrm{E}+04$ & $1.806 \mathrm{E}+01$ \\
\hline $1.805 \mathrm{E}+04$ & $6.411 \mathrm{E}+00$ & $7.28 \mathrm{E}-02$ & $1.060 \mathrm{E}+01$ & $1.733 \mathrm{E}+01$ & $2.225 \mathrm{E}+01$ & $9.800 \mathrm{E}+04$ & $4.340 \mathrm{E}+01$ \\
\hline $1.798 \mathrm{E}+04$ & $6.449 \mathrm{E}+00$ & $7.28 \mathrm{E}-02$ & $1.060 \mathrm{E}+01$ & $1.744 \mathrm{E}+01$ & $2.239 \mathrm{E}+01$ & $9.800 \mathrm{E}+04$ & $8.035 \mathrm{E}+01$ \\
\hline $1.796 \mathrm{E}+04$ & $6.465 \mathrm{E}+00$ & $7.25 \mathrm{E}-02$ & $1.060 \mathrm{E}+01$ & $1.746 \mathrm{E}+01$ & $2.247 \mathrm{E}+01$ & $9.800 \mathrm{E}+04$ & $1.298 \mathrm{E}+02$ \\
\hline $1.802 \mathrm{E}+04$ & $6.469 \mathrm{E}+00$ & $7.22 \mathrm{E}-02$ & $1.060 \mathrm{E}+01$ & $1.736 \mathrm{E}+01$ & $2.252 \mathrm{E}+01$ & $9.800 \mathrm{E}+04$ & $1.917 \mathrm{E}+02$ \\
\hline $1.792 \mathrm{E}+04$ & $6.104 \mathrm{E}+00$ & $7.15 \mathrm{E}-02$ & $1.065 \mathrm{E}+01$ & $1.591 \mathrm{E}+01$ & $2.258 \mathrm{E}+01$ & $9.800 \mathrm{E}+04$ & $2.647 \mathrm{E}+02$ \\
\hline $1.809 \mathrm{E}+04$ & $5.865 \mathrm{E}+00$ & $7.65 \mathrm{E}-02$ & $1.068 \mathrm{E}+01$ & $1.494 \mathrm{E}+01$ & $2.267 \mathrm{E}+01$ & $9.800 \mathrm{E}+04$ & $3.500 \mathrm{E}+02$ \\
\hline $1.806 \mathrm{E}+04$ & $5.175 \mathrm{E}+00$ & $7.28 \mathrm{E}-02$ & $1.075 \mathrm{E}+01$ & $1.266 \mathrm{E}+01$ & $2.277 \mathrm{E}+01$ & $9.800 \mathrm{E}+04$ & $4.462 \mathrm{E}+02$ \\
\hline $1.802 \mathrm{E}+04$ & $4.267 \mathrm{E}+00$ & $7.43 \mathrm{E}-02$ & $1.083 \mathrm{E}+01$ & $9.992 \mathrm{E}+00$ & $2.286 \mathrm{E}+01$ & $9.800 \mathrm{E}+04$ & $5.535 \mathrm{E}+02$ \\
\hline
\end{tabular}


Table 225: Graupner 5.5 x 4.3 Dynamic Calculated Values - 17953 RPM

\begin{tabular}{|c|c|c|c|c|c|c|c|}
\hline$n(\mathrm{RPM})$ & $V_{\infty}^{\prime}(\mathrm{m} / \mathrm{s})$ & $\Delta V_{\infty}^{\prime}(\mathrm{m} / \mathrm{s})$ & $T^{\prime}(\mathrm{g})$ & $\Delta T^{\prime}(\mathrm{g})$ & $V_{\mathrm{t}}(\mathrm{m} / \mathrm{s})$ & $P_{\mathrm{P}}(\mathrm{W})$ & $\Delta P_{\mathrm{P}}(\mathrm{W})$ \\
\hline $1.780 \mathrm{E}+04$ & $2.740 \mathrm{E}+00$ & $2.02 \mathrm{E}-02$ & $4.966 \mathrm{E}+02$ & $7.29 \mathrm{E}+00$ & $9.866 \mathrm{E}+01$ & $1.113 \mathrm{E}+02$ & $1.34 \mathrm{E}+00$ \\
\hline $1.764 \mathrm{E}+04$ & $5.354 \mathrm{E}+00$ & $2.20 \mathrm{E}-02$ & $4.847 \mathrm{E}+02$ & $7.23 \mathrm{E}+00$ & $9.790 \mathrm{E}+01$ & $1.095 \mathrm{E}+02$ & $1.36 \mathrm{E}+00$ \\
\hline $1.805 \mathrm{E}+04$ & $8.438 \mathrm{E}+00$ & $2.71 \mathrm{E}-02$ & $4.944 \mathrm{E}+02$ & $7.27 \mathrm{E}+00$ & $1.004 \mathrm{E}+02$ & $1.188 \mathrm{E}+02$ & $1.35 \mathrm{E}+00$ \\
\hline $1.798 \mathrm{E}+04$ & $1.158 \mathrm{E}+01$ & $3.26 \mathrm{E}-02$ & $4.678 \mathrm{E}+02$ & $7.25 \mathrm{E}+00$ & $1.003 \mathrm{E}+02$ & $1.190 \mathrm{E}+02$ & $1.34 \mathrm{E}+00$ \\
\hline $1.796 \mathrm{E}+04$ & $1.480 \mathrm{E}+01$ & $3.54 \mathrm{E}-02$ & $4.349 \mathrm{E}+02$ & $7.28 \mathrm{E}+00$ & $1.006 \mathrm{E}+02$ & $1.192 \mathrm{E}+02$ & $1.34 \mathrm{E}+00$ \\
\hline $1.802 \mathrm{E}+04$ & $1.806 \mathrm{E}+01$ & $3.78 \mathrm{E}-02$ & $3.952 \mathrm{E}+02$ & $7.32 \mathrm{E}+00$ & $1.015 \mathrm{E}+02$ & $1.197 \mathrm{E}+02$ & $1.34 \mathrm{E}+00$ \\
\hline $1.792 \mathrm{E}+04$ & $2.128 \mathrm{E}+01$ & $4.16 \mathrm{E}-02$ & $3.422 \mathrm{E}+02$ & $7.26 \mathrm{E}+00$ & $1.016 \mathrm{E}+02$ & $1.123 \mathrm{E}+02$ & $1.32 \mathrm{E}+00$ \\
\hline $1.809 \mathrm{E}+04$ & $2.452 \mathrm{E}+01$ & $4.63 \mathrm{E}-02$ & $3.028 \mathrm{E}+02$ & $7.33 \mathrm{E}+00$ & $1.032 \mathrm{E}+02$ & $1.090 \mathrm{E}+02$ & $1.42 \mathrm{E}+00$ \\
\hline $1.806 \mathrm{E}+04$ & $2.772 \mathrm{E}+01$ & $5.23 \mathrm{E}-02$ & $2.450 \mathrm{E}+02$ & $7.45 \mathrm{E}+00$ & $1.039 \mathrm{E}+02$ & $9.597 \mathrm{E}+01$ & $1.35 \mathrm{E}+00$ \\
\hline $1.802 \mathrm{E}+04$ & $3.092 \mathrm{E}+01$ & $5.72 \mathrm{E}-02$ & $1.803 \mathrm{E}+02$ & $7.34 \mathrm{E}+00$ & $1.045 \mathrm{E}+02$ & $7.895 \mathrm{E}+01$ & $1.37 \mathrm{E}+00$ \\
\hline$P_{\mathrm{e}}(\mathrm{W})$ & $\Delta P_{\mathrm{e}}(\mathrm{W})$ & $C_{\mathrm{T}}$ & $\Delta C_{\mathrm{T}}$ & $C_{\mathrm{P}}$ & $\Delta C_{\mathrm{P}}$ & $C_{\mathrm{Q}}$ & $\Delta C_{\mathrm{Q}}$ \\
\hline $1.708 \mathrm{E}+02$ & $1.86 \mathrm{E}+00$ & $1.207 \mathrm{E}-01$ & $1.77 \mathrm{E}-03$ & $6.592 \mathrm{E}-02$ & $7.98 \mathrm{E}-04$ & $1.049 \mathrm{E}-02$ & $1.27 \mathrm{E}-04$ \\
\hline $1.679 \mathrm{E}+02$ & $1.85 \mathrm{E}+00$ & 1.199E-01 & $1.79 \mathrm{E}-03$ & $6.661 \mathrm{E}-02$ & $8.31 \mathrm{E}-04$ & $1.060 \mathrm{E}-02$ & $1.32 \mathrm{E}-04$ \\
\hline $1.837 \mathrm{E}+02$ & $1.89 \mathrm{E}+00$ & $1.169 \mathrm{E}-01$ & $1.72 \mathrm{E}-03$ & $6.751 \mathrm{E}-02$ & 7.70E-04 & $1.074 \mathrm{E}-02$ & $1.22 \mathrm{E}-04$ \\
\hline $1.849 \mathrm{E}+02$ & $1.90 \mathrm{E}+00$ & $1.116 \mathrm{E}-01$ & $1.73 \mathrm{E}-03$ & $6.850 \mathrm{E}-02$ & $7.75 \mathrm{E}-04$ & $1.090 \mathrm{E}-02$ & $1.23 \mathrm{E}-04$ \\
\hline $1.850 \mathrm{E}+02$ & $1.91 \mathrm{E}+00$ & $1.039 \mathrm{E}-01$ & $1.74 \mathrm{E}-03$ & $6.879 \mathrm{E}-02$ & 7.73E-04 & $1.095 \mathrm{E}-02$ & $1.23 \mathrm{E}-04$ \\
\hline $1.841 \mathrm{E}+02$ & $1.90 \mathrm{E}+00$ & $9.386 \mathrm{E}-02$ & $1.74 \mathrm{E}-03$ & $6.841 \mathrm{E}-02$ & $7.68 \mathrm{E}-04$ & $1.089 \mathrm{E}-02$ & $1.22 \mathrm{E}-04$ \\
\hline $1.694 \mathrm{E}+02$ & $1.75 \mathrm{E}+00$ & $8.221 \mathrm{E}-02$ & $1.74 \mathrm{E}-03$ & $6.530 \mathrm{E}-02$ & $7.67 \mathrm{E}-04$ & $1.039 \mathrm{E}-02$ & $1.22 \mathrm{E}-04$ \\
\hline $1.595 \mathrm{E}+02$ & $1.76 \mathrm{E}+00$ & 7.139E-02 & $1.73 \mathrm{E}-03$ & $6.156 \mathrm{E}-02$ & 8.07E-04 & $9.798 \mathrm{E}-03$ & $1.28 \mathrm{E}-04$ \\
\hline $1.361 \mathrm{E}+02$ & $1.43 \mathrm{E}+00$ & $5.797 \mathrm{E}-02$ & $1.76 \mathrm{E}-03$ & $5.452 \mathrm{E}-02$ & $7.70 \mathrm{E}-04$ & $8.677 \mathrm{E}-03$ & $1.22 \mathrm{E}-04$ \\
\hline $1.082 \mathrm{E}+02$ & $1.16 \mathrm{E}+00$ & $4.287 \mathrm{E}-02$ & $1.75 \mathrm{E}-03$ & $4.518 \mathrm{E}-02$ & $7.88 \mathrm{E}-04$ & $7.190 \mathrm{E}-03$ & $1.25 \mathrm{E}-04$ \\
\hline$\rho\left(\mathrm{kg} / \mathrm{m}^{\wedge} 3\right)$ & $\eta_{\mathrm{T}}$ & $\Delta \eta_{\mathrm{T}}$ & $J$ & $\Delta J$ & $\eta_{\mathrm{P}}$ & $\Delta \eta_{\mathrm{P}}$ & $R e_{0.75}$ \\
\hline $1.156 \mathrm{E}+00$ & $6.516 \mathrm{E}-01$ & $1.06 \mathrm{E}-02$ & $6.547 \mathrm{E}-02$ & $4.82 \mathrm{E}-04$ & $1.199 \mathrm{E}-01$ & $2.45 \mathrm{E}-03$ & $6.588 \mathrm{E}+04$ \\
\hline $1.157 \mathrm{E}+00$ & $6.523 \mathrm{E}-01$ & $1.08 \mathrm{E}-02$ & $1.291 \mathrm{E}-01$ & $5.31 \mathrm{E}-04$ & $2.323 \mathrm{E}-01$ & $4.62 \mathrm{E}-03$ & $6.541 \mathrm{E}+04$ \\
\hline $1.156 \mathrm{E}+00$ & $6.468 \mathrm{E}-01$ & $9.92 \mathrm{E}-03$ & $1.988 \mathrm{E}-01$ & $6.42 \mathrm{E}-04$ & $3.442 \mathrm{E}-01$ & $6.51 \mathrm{E}-03$ & $6.698 \mathrm{E}+04$ \\
\hline $1.155 \mathrm{E}+00$ & $6.438 \mathrm{E}-01$ & $9.84 \mathrm{E}-03$ & $2.740 \mathrm{E}-01$ & $7.73 \mathrm{E}-04$ & 4.464E-01 & $8.67 \mathrm{E}-03$ & $6.685 \mathrm{E}+04$ \\
\hline $1.155 \mathrm{E}+00$ & $6.445 \mathrm{E}-01$ & $9.81 \mathrm{E}-03$ & $3.505 \mathrm{E}-01$ & $8.41 \mathrm{E}-04$ & $5.295 \mathrm{E}-01$ & $1.08 \mathrm{E}-02$ & $6.705 \mathrm{E}+04$ \\
\hline $1.155 \mathrm{E}+00$ & $6.504 \mathrm{E}-01$ & $9.88 \mathrm{E}-03$ & 4.261E-01 & $9.01 \mathrm{E}-04$ & $5.845 \mathrm{E}-01$ & $1.27 \mathrm{E}-02$ & $6.759 \mathrm{E}+04$ \\
\hline $1.154 \mathrm{E}+00$ & $6.631 \mathrm{E}-01$ & $1.04 \mathrm{E}-02$ & 5.049E-01 & $9.93 \mathrm{E}-04$ & $6.357 \mathrm{E}-01$ & $1.55 \mathrm{E}-02$ & $6.762 \mathrm{E}+04$ \\
\hline $1.154 \mathrm{E}+00$ & $6.830 \mathrm{E}-01$ & $1.17 \mathrm{E}-02$ & $5.762 \mathrm{E}-01$ & $1.11 \mathrm{E}-03$ & $6.681 \mathrm{E}-01$ & $1.84 \mathrm{E}-02$ & $6.869 \mathrm{E}+04$ \\
\hline $1.154 \mathrm{E}+00$ & $7.051 \mathrm{E}-01$ & $1.24 \mathrm{E}-02$ & $6.527 \mathrm{E}-01$ & $1.25 \mathrm{E}-03$ & $6.941 \mathrm{E}-01$ & $2.33 \mathrm{E}-02$ & $6.908 \mathrm{E}+04$ \\
\hline $1.153 \mathrm{E}+00$ & $7.296 \mathrm{E}-01$ & $1.49 \mathrm{E}-02$ & 7.297E-01 & $1.37 \mathrm{E}-03$ & $6.924 \mathrm{E}-01$ & 3.07E-02 & $6.949 \mathrm{E}+04$ \\
\hline
\end{tabular}




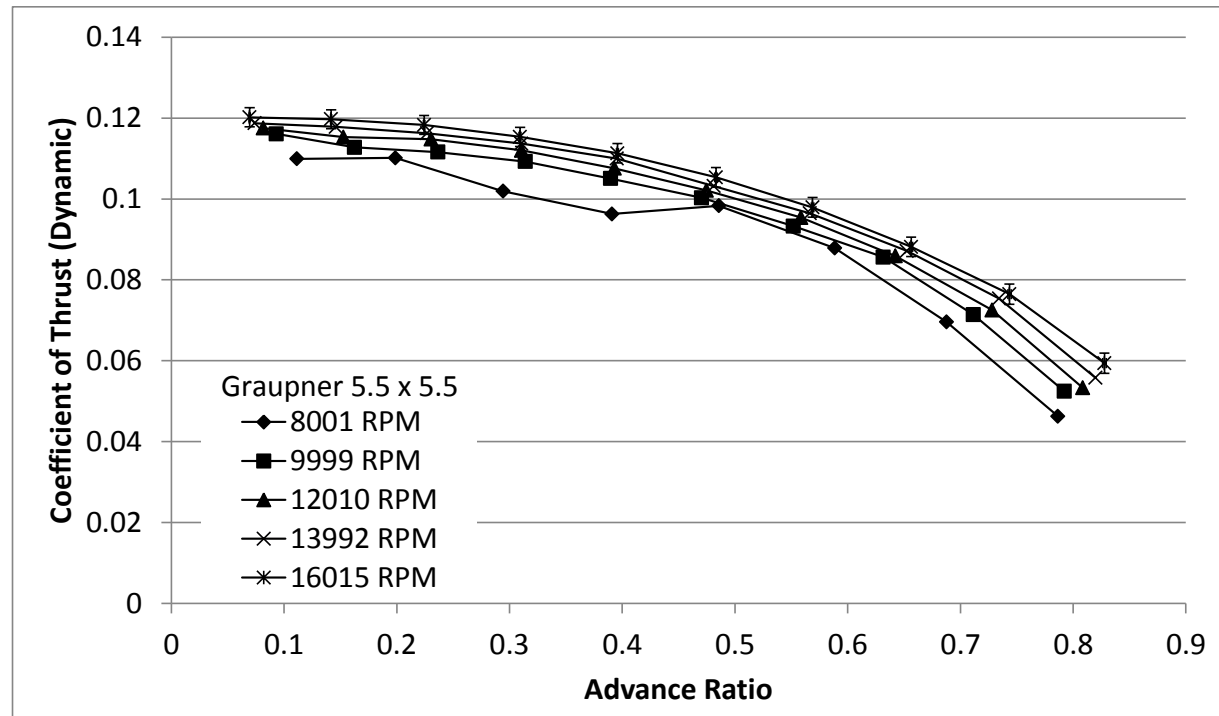

(a)

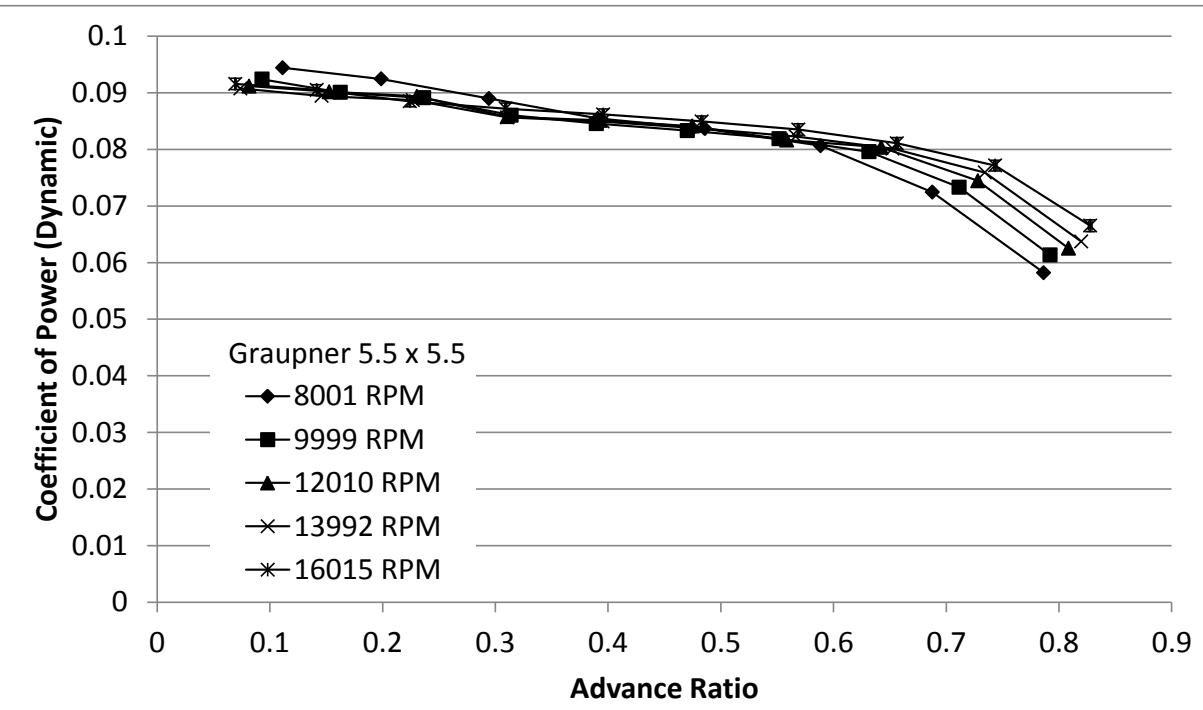

(b)

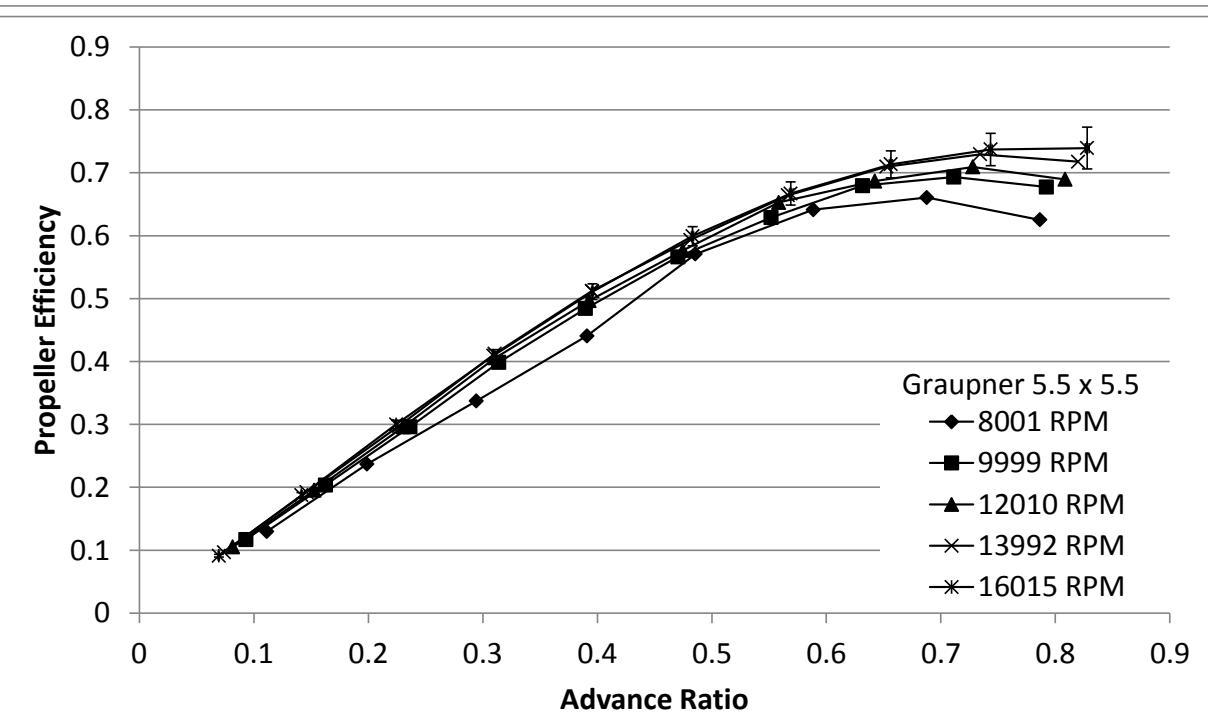

(c) 


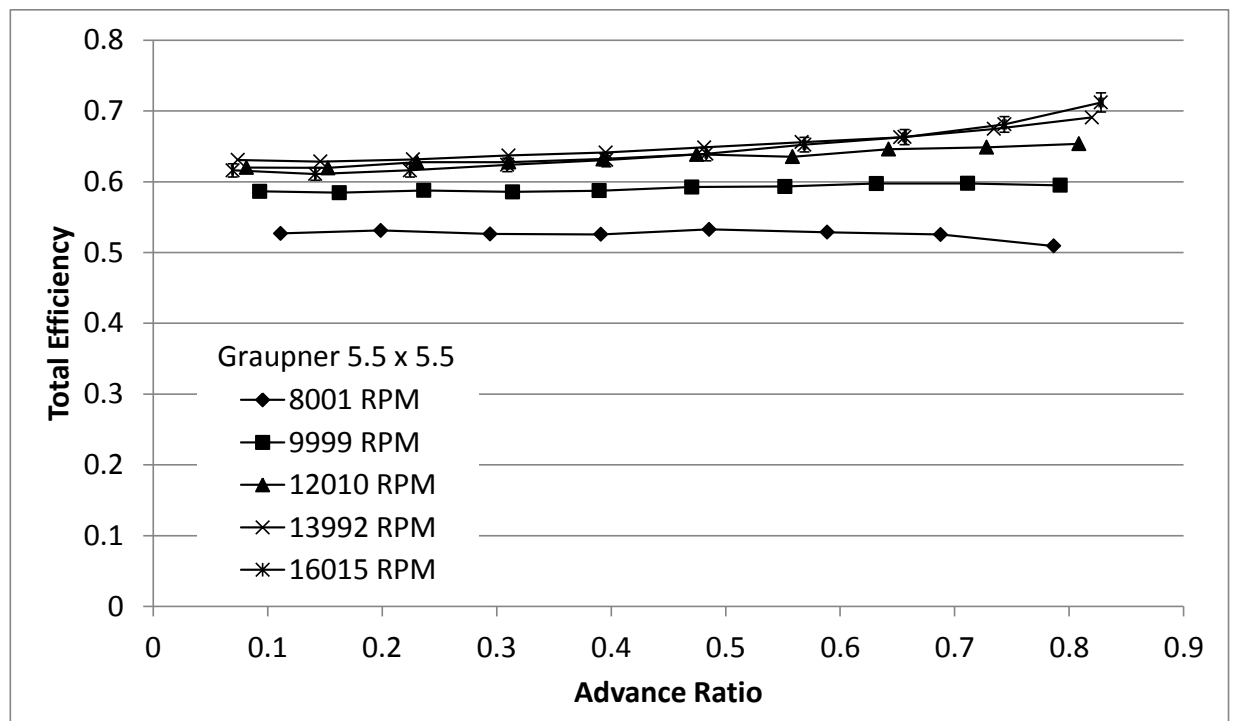

(d)

Figure 97: Graupner 5.5 x 5.5 Dynamic Test Results: (a) Coefficient of Thrust, (b) Coefficient of Power, (c) Propeller Efficiency, (d) Total Efficiency.

Table 226: Graupner 5.5 x 5.5 Dynamic Measured Values - 8001 RPM

\begin{tabular}{|c|c|c|c|c|c|c|c|}
\hline$n(\mathrm{RPM})$ & $Q(\mathrm{~g}-\mathrm{m})$ & $\Delta Q(\mathrm{~g}-\mathrm{m})$ & $V(\mathrm{~V})$ & $I(\mathrm{~A})$ & $T_{\mathrm{atm}}\left({ }^{\circ} \mathrm{C}\right)$ & $P_{\text {atm }}(\mathrm{Pa})$ & $P_{\text {diff }}(\mathrm{Pa})$ \\
\hline $7.965 \mathrm{E}+03$ & $1.697 \mathrm{E}+00$ & $6.26 \mathrm{E}-02$ & $1.105 \mathrm{E}+01$ & $2.384 \mathrm{E}+00$ & $2.245 \mathrm{E}+01$ & $9.895 \mathrm{E}+04$ & $2.746 \mathrm{E}+00$ \\
\hline $8.025 \mathrm{E}+03$ & $1.686 \mathrm{E}+00$ & $6.98 \mathrm{E}-02$ & $1.105 \mathrm{E}+01$ & $2.367 \mathrm{E}+00$ & $2.248 \mathrm{E}+01$ & $9.895 \mathrm{E}+04$ & $8.498 \mathrm{E}+00$ \\
\hline $7.975 \mathrm{E}+03$ & $1.602 \mathrm{E}+00$ & $6.39 \mathrm{E}-02$ & $1.105 \mathrm{E}+01$ & $2.256 \mathrm{E}+00$ & $2.256 \mathrm{E}+01$ & $9.894 \mathrm{E}+04$ & $1.803 \mathrm{E}+01$ \\
\hline $8.010 \mathrm{E}+03$ & $1.552 \mathrm{E}+00$ & $6.75 \mathrm{E}-02$ & $1.105 \mathrm{E}+01$ & $2.198 \mathrm{E}+00$ & $2.257 \mathrm{E}+01$ & $9.893 \mathrm{E}+04$ & $3.179 \mathrm{E}+01$ \\
\hline $8.035 \mathrm{E}+03$ & $1.528 \mathrm{E}+00$ & $6.58 \mathrm{E}-02$ & $1.105 \mathrm{E}+01$ & $2.141 \mathrm{E}+00$ & $2.268 \mathrm{E}+01$ & $9.893 \mathrm{E}+04$ & $4.906 \mathrm{E}+01$ \\
\hline $7.993 \mathrm{E}+03$ & $1.457 \mathrm{E}+00$ & $6.43 \mathrm{E}-02$ & $1.106 \mathrm{E}+01$ & $2.046 \mathrm{E}+00$ & $2.276 \mathrm{E}+01$ & $9.892 \mathrm{E}+04$ & $7.094 \mathrm{E}+01$ \\
\hline $7.999 \mathrm{E}+03$ & $1.311 \mathrm{E}+00$ & $6.31 \mathrm{E}-02$ & $1.106 \mathrm{E}+01$ & $1.852 \mathrm{E}+00$ & $2.279 \mathrm{E}+01$ & $9.893 \mathrm{E}+04$ & $9.665 \mathrm{E}+01$ \\
\hline $8.009 \mathrm{E}+03$ & $1.056 \mathrm{E}+00$ & $6.42 \mathrm{E}-02$ & $1.107 \mathrm{E}+01$ & $1.540 \mathrm{E}+00$ & $2.283 \mathrm{E}+01$ & $9.892 \mathrm{E}+04$ & $1.263 \mathrm{E}+02$ \\
\hline
\end{tabular}


Table 227: Graupner 5.5 x 5.5 Dynamic Calculated Values - 8001 RPM

\begin{tabular}{|c|c|c|c|c|c|c|c|}
\hline$n(\mathrm{RPM})$ & $V_{\infty}^{\prime}(\mathrm{m} / \mathrm{s})$ & $\Delta V_{\infty}^{\prime}(\mathrm{m} / \mathrm{s})$ & $T^{\prime}(\mathrm{g})$ & $\Delta T^{\prime}(\mathrm{g})$ & $V_{\mathrm{t}}(\mathrm{m} / \mathrm{s})$ & $P_{\mathrm{P}}(\mathrm{W})$ & $\Delta P_{\mathrm{P}}(\mathrm{W})$ \\
\hline $7.965 \mathrm{E}+03$ & $2.071 \mathrm{E}+00$ & $1.27 \mathrm{E}-02$ & $8.863 \mathrm{E}+01$ & $9.87 \mathrm{E}+00$ & $4.386 \mathrm{E}+01$ & $1.388 \mathrm{E}+01$ & $5.12 \mathrm{E}-01$ \\
\hline $8.025 \mathrm{E}+03$ & $3.722 \mathrm{E}+00$ & $1.35 \mathrm{E}-02$ & $9.011 \mathrm{E}+01$ & $9.96 \mathrm{E}+00$ & $4.429 \mathrm{E}+01$ & $1.389 \mathrm{E}+01$ & $5.75 \mathrm{E}-01$ \\
\hline $7.975 \mathrm{E}+03$ & $5.478 \mathrm{E}+00$ & $1.56 \mathrm{E}-02$ & $8.233 \mathrm{E}+01$ & $9.94 \mathrm{E}+00$ & $4.420 \mathrm{E}+01$ & $1.312 \mathrm{E}+01$ & $5.24 \mathrm{E}-01$ \\
\hline $8.010 \mathrm{E}+03$ & $7.311 \mathrm{E}+00$ & $1.83 \mathrm{E}-02$ & $7.848 \mathrm{E}+01$ & $9.89 \mathrm{E}+00$ & $4.466 \mathrm{E}+01$ & $1.277 \mathrm{E}+01$ & $5.55 \mathrm{E}-01$ \\
\hline $8.035 \mathrm{E}+03$ & $9.108 \mathrm{E}+00$ & $2.06 \mathrm{E}-02$ & $8.059 \mathrm{E}+01$ & $9.91 \mathrm{E}+00$ & $4.512 \mathrm{E}+01$ & $1.261 \mathrm{E}+01$ & $5.43 \mathrm{E}-01$ \\
\hline $7.993 \mathrm{E}+03$ & $1.098 \mathrm{E}+01$ & 2.35E-02 & $7.122 \mathrm{E}+01$ & $9.91 \mathrm{E}+00$ & $4.531 \mathrm{E}+01$ & $1.196 \mathrm{E}+01$ & $5.28 \mathrm{E}-01$ \\
\hline $7.999 \mathrm{E}+03$ & $1.284 \mathrm{E}+01$ & 2.63E-02 & $5.648 \mathrm{E}+01$ & $9.98 \mathrm{E}+00$ & $4.583 \mathrm{E}+01$ & $1.076 \mathrm{E}+01$ & 5.19E-01 \\
\hline $8.009 \mathrm{E}+03$ & $1.470 \mathrm{E}+01$ & $2.96 \mathrm{E}-02$ & $3.764 \mathrm{E}+01$ & $9.92 \mathrm{E}+00$ & $4.644 \mathrm{E}+01$ & $8.682 \mathrm{E}+00$ & $5.28 \mathrm{E}-01$ \\
\hline$P_{\mathrm{e}}(\mathrm{W})$ & $\Delta P_{\mathrm{e}}(\mathrm{W})$ & $C_{\mathrm{T}}$ & $\Delta C_{\mathrm{T}}$ & $C_{\mathrm{P}}$ & $\Delta C_{\mathrm{P}}$ & $C_{\mathrm{Q}}$ & $\Delta C_{\mathrm{Q}}$ \\
\hline $2.633 \mathrm{E}+01$ & $3.03 \mathrm{E}-01$ & $1.099 \mathrm{E}-01$ & $1.22 \mathrm{E}-02$ & $9.441 \mathrm{E}-02$ & $3.49 \mathrm{E}-03$ & $1.503 \mathrm{E}-02$ & $5.55 \mathrm{E}-04$ \\
\hline $2.615 \mathrm{E}+01$ & $3.01 \mathrm{E}-01$ & $1.101 \mathrm{E}-01$ & $1.22 \mathrm{E}-02$ & $9.243 \mathrm{E}-02$ & $3.83 \mathrm{E}-03$ & $1.471 \mathrm{E}-02$ & $6.09 \mathrm{E}-04$ \\
\hline $2.493 \mathrm{E}+01$ & $2.87 \mathrm{E}-01$ & $1.019 \mathrm{E}-01$ & $1.23 \mathrm{E}-02$ & 8.897E-02 & $3.55 \mathrm{E}-03$ & $1.416 \mathrm{E}-02$ & $5.65 \mathrm{E}-04$ \\
\hline $2.429 \mathrm{E}+01$ & $2.81 \mathrm{E}-01$ & $9.630 \mathrm{E}-02$ & $1.21 \mathrm{E}-02$ & $8.545 \mathrm{E}-02$ & $3.72 \mathrm{E}-03$ & $1.360 \mathrm{E}-02$ & $5.91 \mathrm{E}-04$ \\
\hline $2.367 \mathrm{E}+01$ & 2.73E-01 & $9.833 \mathrm{E}-02$ & $1.21 \mathrm{E}-02$ & $8.366 \mathrm{E}-02$ & $3.61 \mathrm{E}-03$ & $1.332 \mathrm{E}-02$ & 5.74E-04 \\
\hline $2.263 \mathrm{E}+01$ & $2.63 \mathrm{E}-01$ & $8.785 \mathrm{E}-02$ & $1.22 \mathrm{E}-02$ & $8.063 \mathrm{E}-02$ & $3.56 \mathrm{E}-03$ & $1.283 \mathrm{E}-02$ & $5.66 \mathrm{E}-04$ \\
\hline $2.049 \mathrm{E}+01$ & $2.40 \mathrm{E}-01$ & $6.956 \mathrm{E}-02$ & $1.23 \mathrm{E}-02$ & $7.242 \mathrm{E}-02$ & $3.49 \mathrm{E}-03$ & $1.153 \mathrm{E}-02$ & $5.55 \mathrm{E}-04$ \\
\hline $1.705 \mathrm{E}+01$ & $2.03 \mathrm{E}-01$ & $4.625 \mathrm{E}-02$ & $1.22 \mathrm{E}-02$ & 5.819E-02 & $3.54 \mathrm{E}-03$ & $9.261 \mathrm{E}-03$ & $5.63 \mathrm{E}-04$ \\
\hline$\rho\left(\mathrm{kg} / \mathrm{m}^{\wedge} 3\right)$ & $\eta_{\mathrm{T}}$ & $\Delta \eta_{\mathrm{T}}$ & $J$ & $\Delta J$ & $\eta_{\mathrm{P}}$ & $\Delta \eta_{\mathrm{P}}$ & $R e_{0.75}$ \\
\hline $1.166 \mathrm{E}+00$ & $5.271 \mathrm{E}-01$ & $2.04 \mathrm{E}-02$ & $1.114 \mathrm{E}-01$ & $6.88 \mathrm{E}-04$ & $1.297 \mathrm{E}-01$ & $1.52 \mathrm{E}-02$ & $2.780 \mathrm{E}+04$ \\
\hline $1.166 \mathrm{E}+00$ & $5.311 \mathrm{E}-01$ & $2.28 \mathrm{E}-02$ & $1.987 \mathrm{E}-01$ & $7.32 \mathrm{E}-04$ & $2.368 \mathrm{E}-01$ & $2.80 \mathrm{E}-02$ & $2.806 \mathrm{E}+04$ \\
\hline $1.166 \mathrm{E}+00$ & $5.262 \mathrm{E}-01$ & 2.19E-02 & $2.943 \mathrm{E}-01$ & $8.56 \mathrm{E}-04$ & $3.372 \mathrm{E}-01$ & $4.29 \mathrm{E}-02$ & $2.799 \mathrm{E}+04$ \\
\hline $1.165 \mathrm{E}+00$ & $5.257 \mathrm{E}-01$ & $2.36 \mathrm{E}-02$ & $3.910 \mathrm{E}-01$ & $1.00 \mathrm{E}-03$ & $4.407 \mathrm{E}-01$ & $5.88 \mathrm{E}-02$ & $2.828 \mathrm{E}+04$ \\
\hline $1.165 \mathrm{E}+00$ & $5.328 \mathrm{E}-01$ & $2.38 \mathrm{E}-02$ & $4.856 \mathrm{E}-01$ & $1.13 \mathrm{E}-03$ & $5.708 \mathrm{E}-01$ & $7.44 \mathrm{E}-02$ & $2.855 \mathrm{E}+04$ \\
\hline $1.165 \mathrm{E}+00$ & $5.286 \mathrm{E}-01$ & $2.41 \mathrm{E}-02$ & $5.886 \mathrm{E}-01$ & $1.30 \mathrm{E}-03$ & $6.412 \mathrm{E}-01$ & $9.36 \mathrm{E}-02$ & $2.866 \mathrm{E}+04$ \\
\hline $1.165 \mathrm{E}+00$ & $5.254 \mathrm{E}-01$ & $2.61 \mathrm{E}-02$ & $6.878 \mathrm{E}-01$ & $1.46 \mathrm{E}-03$ & $6.607 \mathrm{E}-01$ & $1.21 \mathrm{E}-01$ & $2.898 \mathrm{E}+04$ \\
\hline $1.164 \mathrm{E}+00$ & $5.093 \mathrm{E}-01$ & $3.16 \mathrm{E}-02$ & $7.865 \mathrm{E}-01$ & $1.64 \mathrm{E}-03$ & $6.251 \mathrm{E}-01$ & $1.69 \mathrm{E}-01$ & $2.936 \mathrm{E}+04$ \\
\hline
\end{tabular}


Table 228: Graupner 5.5 x 5.5 Dynamic Measured Values - 9999 RPM

\begin{tabular}{|c|c|c|c|c|c|c|c|}
\hline$n(\mathrm{RPM})$ & $Q(\mathrm{~g}-\mathrm{m})$ & $\Delta Q(\mathrm{~g}-\mathrm{m})$ & $V(\mathrm{~V})$ & $I(\mathrm{~A})$ & $T_{\text {atm }}\left({ }^{\circ} \mathrm{C}\right)$ & $P_{\text {atm }}(\mathrm{Pa})$ & $P_{\text {diff }}(\mathrm{Pa})$ \\
\hline $9.990 \mathrm{E}+03$ & $2.609 \mathrm{E}+00$ & $6.96 \mathrm{E}-02$ & $1.099 \mathrm{E}+01$ & $4.151 \mathrm{E}+00$ & $2.255 \mathrm{E}+01$ & $9.888 \mathrm{E}+04$ & $3.079 \mathrm{E}+00$ \\
\hline $9.981 \mathrm{E}+03$ & $2.537 \mathrm{E}+00$ & $7.00 \mathrm{E}-02$ & $1.100 \mathrm{E}+01$ & $4.046 \mathrm{E}+00$ & $2.262 \mathrm{E}+01$ & $9.887 \mathrm{E}+04$ & $8.908 \mathrm{E}+00$ \\
\hline $1.001 \mathrm{E}+04$ & $2.523 \mathrm{E}+00$ & $7.18 \mathrm{E}-02$ & $1.100 \mathrm{E}+01$ & $4.012 \mathrm{E}+00$ & $2.274 \mathrm{E}+01$ & $9.885 \mathrm{E}+04$ & $1.852 \mathrm{E}+01$ \\
\hline $9.998 \mathrm{E}+03$ & $2.431 \mathrm{E}+00$ & $6.88 \mathrm{E}-02$ & $1.100 \mathrm{E}+01$ & $3.873 \mathrm{E}+00$ & $2.282 \mathrm{E}+01$ & $9.885 \mathrm{E}+04$ & $3.217 \mathrm{E}+01$ \\
\hline $1.003 \mathrm{E}+04$ & $2.405 \mathrm{E}+00$ & $8.14 \mathrm{E}-02$ & $1.100 \mathrm{E}+01$ & $3.836 \mathrm{E}+00$ & $2.284 \mathrm{E}+01$ & $9.885 \mathrm{E}+04$ & $4.952 \mathrm{E}+01$ \\
\hline $1.001 \mathrm{E}+04$ & $2.359 \mathrm{E}+00$ & $7.11 \mathrm{E}-02$ & $1.101 \mathrm{E}+01$ & $3.717 \mathrm{E}+00$ & $2.291 \mathrm{E}+01$ & $9.885 \mathrm{E}+04$ & $7.141 \mathrm{E}+01$ \\
\hline $9.989 \mathrm{E}+03$ & $2.307 \mathrm{E}+00$ & $6.56 \mathrm{E}-02$ & $1.101 \mathrm{E}+01$ & $3.623 \mathrm{E}+00$ & $2.298 \mathrm{E}+01$ & $9.885 \mathrm{E}+04$ & $9.739 \mathrm{E}+01$ \\
\hline $9.995 \mathrm{E}+03$ & $2.244 \mathrm{E}+00$ & $6.47 \mathrm{E}-02$ & $1.101 \mathrm{E}+01$ & $3.500 \mathrm{E}+00$ & $2.306 \mathrm{E}+01$ & $9.884 \mathrm{E}+04$ & $1.273 \mathrm{E}+02$ \\
\hline $9.996 \mathrm{E}+03$ & $2.067 \mathrm{E}+00$ & $6.24 \mathrm{E}-02$ & $1.102 \mathrm{E}+01$ & $3.221 \mathrm{E}+00$ & $2.314 \mathrm{E}+01$ & $9.882 \mathrm{E}+04$ & $1.611 \mathrm{E}+02$ \\
\hline $9.983 \mathrm{E}+03$ & $1.725 \mathrm{E}+00$ & $5.99 \mathrm{E}-02$ & $1.104 \mathrm{E}+01$ & $2.693 \mathrm{E}+00$ & $2.318 \mathrm{E}+01$ & $9.881 \mathrm{E}+04$ & $1.987 \mathrm{E}+02$ \\
\hline
\end{tabular}


Table 229: Graupner 5.5 x 5.5 Dynamic Calculated Values - 9999 RPM

\begin{tabular}{|c|c|c|c|c|c|c|c|}
\hline$n(\mathrm{RPM})$ & $V_{\infty}^{\prime}(\mathrm{m} / \mathrm{s})$ & $\Delta V_{\infty}^{\prime}(\mathrm{m} / \mathrm{s})$ & $T^{\prime}(\mathrm{g})$ & $\Delta T^{\prime}(\mathrm{g})$ & $V_{\mathrm{t}}(\mathrm{m} / \mathrm{s})$ & $P_{\mathrm{P}}(\mathrm{W})$ & $\Delta P_{\mathrm{P}}(\mathrm{W})$ \\
\hline $9.990 \mathrm{E}+03$ & $2.170 \mathrm{E}+00$ & $1.37 \mathrm{E}-02$ & $1.471 \mathrm{E}+02$ & $8.95 \mathrm{E}+00$ & $5.499 \mathrm{E}+01$ & $2.676 \mathrm{E}+01$ & 7.14E-01 \\
\hline $9.981 \mathrm{E}+03$ & $3.788 \mathrm{E}+00$ & $1.46 \mathrm{E}-02$ & $1.425 \mathrm{E}+02$ & $8.93 \mathrm{E}+00$ & $5.502 \mathrm{E}+01$ & $2.601 \mathrm{E}+01$ & 7.17E-01 \\
\hline $1.001 \mathrm{E}+04$ & $5.524 \mathrm{E}+00$ & $1.63 \mathrm{E}-02$ & $1.418 \mathrm{E}+02$ & $8.92 \mathrm{E}+00$ & $5.533 \mathrm{E}+01$ & $2.594 \mathrm{E}+01$ & $7.38 \mathrm{E}-01$ \\
\hline $9.998 \mathrm{E}+03$ & $7.327 \mathrm{E}+00$ & $1.94 \mathrm{E}-02$ & $1.385 \mathrm{E}+02$ & $9.11 \mathrm{E}+00$ & $5.548 \mathrm{E}+01$ & $2.496 \mathrm{E}+01$ & $7.06 \mathrm{E}-01$ \\
\hline $1.003 \mathrm{E}+04$ & $9.127 \mathrm{E}+00$ & $2.14 \mathrm{E}-02$ & $1.341 \mathrm{E}+02$ & $8.99 \mathrm{E}+00$ & $5.593 \mathrm{E}+01$ & $2.479 \mathrm{E}+01$ & 8.39E-01 \\
\hline $1.001 \mathrm{E}+04$ & $1.099 \mathrm{E}+01$ & $2.44 \mathrm{E}-02$ & $1.274 \mathrm{E}+02$ & $8.98 \mathrm{E}+00$ & $5.615 \mathrm{E}+01$ & $2.425 \mathrm{E}+01$ & 7.31E-01 \\
\hline $9.989 \mathrm{E}+03$ & $1.286 \mathrm{E}+01$ & 2.72E-02 & $1.179 \mathrm{E}+02$ & $9.01 \mathrm{E}+00$ & $5.642 \mathrm{E}+01$ & $2.367 \mathrm{E}+01$ & $6.72 \mathrm{E}-01$ \\
\hline $9.995 \mathrm{E}+03$ & $1.473 \mathrm{E}+01$ & $2.98 \mathrm{E}-02$ & $1.083 \mathrm{E}+02$ & $8.95 \mathrm{E}+00$ & $5.691 \mathrm{E}+01$ & $2.304 \mathrm{E}+01$ & $6.65 \mathrm{E}-01$ \\
\hline $9.996 \mathrm{E}+03$ & $1.660 \mathrm{E}+01$ & $3.27 \mathrm{E}-02$ & $9.035 \mathrm{E}+01$ & $8.95 \mathrm{E}+00$ & $5.743 \mathrm{E}+01$ & $2.122 \mathrm{E}+01$ & $6.41 \mathrm{E}-01$ \\
\hline $9.983 \mathrm{E}+03$ & $1.846 \mathrm{E}+01$ & $3.55 \mathrm{E}-02$ & $6.615 \mathrm{E}+01$ & $8.93 \mathrm{E}+00$ & $5.793 \mathrm{E}+01$ & $1.768 \mathrm{E}+01$ & $6.14 \mathrm{E}-01$ \\
\hline$P_{\mathrm{e}}(\mathrm{W})$ & $\Delta P_{\mathrm{e}}(\mathrm{W})$ & $C_{\mathrm{T}}$ & $\Delta C_{\mathrm{T}}$ & $C_{\mathrm{P}}$ & $\Delta C_{\mathrm{P}}$ & $C_{\mathrm{Q}}$ & $\Delta C_{\mathrm{Q}}$ \\
\hline $4.564 \mathrm{E}+01$ & $5.41 \mathrm{E}-01$ & $1.161 \mathrm{E}-01$ & $7.07 \mathrm{E}-03$ & $9.238 \mathrm{E}-02$ & $2.47 \mathrm{E}-03$ & $1.470 \mathrm{E}-02$ & $3.92 \mathrm{E}-04$ \\
\hline $4.449 \mathrm{E}+01$ & $5.26 \mathrm{E}-01$ & $1.128 \mathrm{E}-01$ & $7.06 \mathrm{E}-03$ & $9.004 \mathrm{E}-02$ & $2.49 \mathrm{E}-03$ & $1.433 \mathrm{E}-02$ & $3.96 \mathrm{E}-04$ \\
\hline $4.412 \mathrm{E}+01$ & $5.30 \mathrm{E}-01$ & $1.116 \mathrm{E}-01$ & $7.02 \mathrm{E}-03$ & $8.910 \mathrm{E}-02$ & $2.54 \mathrm{E}-03$ & $1.418 \mathrm{E}-02$ & $4.04 \mathrm{E}-04$ \\
\hline $4.261 \mathrm{E}+01$ & $5.04 \mathrm{E}-01$ & $1.093 \mathrm{E}-01$ & $7.19 \mathrm{E}-03$ & $8.603 \mathrm{E}-02$ & $2.44 \mathrm{E}-03$ & $1.369 \mathrm{E}-02$ & $3.88 \mathrm{E}-04$ \\
\hline $4.220 \mathrm{E}+01$ & $5.03 \mathrm{E}-01$ & $1.050 \mathrm{E}-01$ & 7.04E-03 & $8.455 \mathrm{E}-02$ & $2.86 \mathrm{E}-03$ & $1.346 \mathrm{E}-02$ & $4.56 \mathrm{E}-04$ \\
\hline $4.092 \mathrm{E}+01$ & $4.86 \mathrm{E}-01$ & $1.003 \mathrm{E}-01$ & $7.07 \mathrm{E}-03$ & $8.330 \mathrm{E}-02$ & $2.51 \mathrm{E}-03$ & $1.326 \mathrm{E}-02$ & $4.00 \mathrm{E}-04$ \\
\hline $3.989 \mathrm{E}+01$ & $4.65 \mathrm{E}-01$ & $9.330 \mathrm{E}-02$ & 7.13E-03 & 8.187E-02 & $2.33 \mathrm{E}-03$ & $1.303 \mathrm{E}-02$ & $3.70 \mathrm{E}-04$ \\
\hline $3.855 \mathrm{E}+01$ & 4.48E-01 & $8.561 \mathrm{E}-02$ & $7.08 \mathrm{E}-03$ & 7.957E-02 & $2.30 \mathrm{E}-03$ & $1.266 \mathrm{E}-02$ & $3.66 \mathrm{E}-04$ \\
\hline $3.550 \mathrm{E}+01$ & $4.02 \mathrm{E}-01$ & $7.142 \mathrm{E}-02$ & $7.07 \mathrm{E}-03$ & $7.330 \mathrm{E}-02$ & $2.22 \mathrm{E}-03$ & $1.167 \mathrm{E}-02$ & $3.53 \mathrm{E}-04$ \\
\hline $2.972 \mathrm{E}+01$ & $3.37 \mathrm{E}-01$ & $5.243 \mathrm{E}-02$ & $7.08 \mathrm{E}-03$ & $6.133 \mathrm{E}-02$ & $2.13 \mathrm{E}-03$ & $9.762 \mathrm{E}-03$ & $3.39 \mathrm{E}-04$ \\
\hline$\rho\left(\mathrm{kg} / \mathrm{m}^{\wedge} 3\right)$ & $\eta_{\mathrm{T}}$ & $\Delta \eta_{\mathrm{T}}$ & $J$ & $\Delta J$ & $\eta_{\mathrm{P}}$ & $\Delta \eta_{\mathrm{P}}$ & $R e_{0.75}$ \\
\hline $1.165 \mathrm{E}+00$ & 5.864E-01 & $1.71 \mathrm{E}-02$ & $9.305 \mathrm{E}-02$ & $5.90 \mathrm{E}-04$ & $1.169 \mathrm{E}-01$ & $7.81 \mathrm{E}-03$ & $3.481 \mathrm{E}+04$ \\
\hline $1.164 \mathrm{E}+00$ & $5.845 \mathrm{E}-01$ & $1.75 \mathrm{E}-02$ & $1.626 \mathrm{E}-01$ & $6.28 \mathrm{E}-04$ & $2.036 \mathrm{E}-01$ & $1.40 \mathrm{E}-02$ & $3.481 \mathrm{E}+04$ \\
\hline $1.164 \mathrm{E}+00$ & $5.878 \mathrm{E}-01$ & $1.82 \mathrm{E}-02$ & $2.364 \mathrm{E}-01$ & 7.01E-04 & $2.961 \mathrm{E}-01$ & $2.05 \mathrm{E}-02$ & $3.497 \mathrm{E}+04$ \\
\hline $1.164 \mathrm{E}+00$ & $5.857 \mathrm{E}-01$ & $1.80 \mathrm{E}-02$ & $3.140 \mathrm{E}-01$ & $8.40 \mathrm{E}-04$ & $3.987 \mathrm{E}-01$ & $2.86 \mathrm{E}-02$ & $3.505 \mathrm{E}+04$ \\
\hline $1.163 \mathrm{E}+00$ & $5.873 \mathrm{E}-01$ & $2.11 \mathrm{E}-02$ & $3.897 \mathrm{E}-01$ & $9.24 \mathrm{E}-04$ & 4.842E-01 & $3.64 \mathrm{E}-02$ & $3.533 \mathrm{E}+04$ \\
\hline $1.163 \mathrm{E}+00$ & $5.926 \mathrm{E}-01$ & $1.92 \mathrm{E}-02$ & 4.704E-01 & $1.06 \mathrm{E}-03$ & 5.664E-01 & 4.34E-02 & $3.545 \mathrm{E}+04$ \\
\hline $1.163 \mathrm{E}+00$ & $5.933 \mathrm{E}-01$ & $1.82 \mathrm{E}-02$ & $5.518 \mathrm{E}-01$ & $1.18 \mathrm{E}-03$ & $6.288 \mathrm{E}-01$ & $5.13 \mathrm{E}-02$ & $3.561 \mathrm{E}+04$ \\
\hline $1.162 \mathrm{E}+00$ & $5.975 \mathrm{E}-01$ & $1.86 \mathrm{E}-02$ & $6.316 \mathrm{E}-01$ & $1.30 \mathrm{E}-03$ & $6.795 \mathrm{E}-01$ & $5.95 \mathrm{E}-02$ & $3.590 \mathrm{E}+04$ \\
\hline $1.162 \mathrm{E}+00$ & $5.977 \mathrm{E}-01$ & $1.93 \mathrm{E}-02$ & $7.115 \mathrm{E}-01$ & $1.43 \mathrm{E}-03$ & $6.932 \mathrm{E}-01$ & $7.18 \mathrm{E}-02$ & $3.620 \mathrm{E}+04$ \\
\hline $1.162 \mathrm{E}+00$ & $5.950 \mathrm{E}-01$ & 2.17E-02 & 7.922E-01 & $1.55 \mathrm{E}-03$ & $6.772 \mathrm{E}-01$ & $9.44 \mathrm{E}-02$ & $3.651 \mathrm{E}+04$ \\
\hline
\end{tabular}


Table 230: Graupner 5.5 x 5.5 Dynamic Measured Values - 12010 RPM

\begin{tabular}{|c|c|c|c|c|c|c|c|}
\hline$n(\mathrm{RPM})$ & $Q(\mathrm{~g}-\mathrm{m})$ & $\Delta Q(\mathrm{~g}-\mathrm{m})$ & $V(\mathrm{~V})$ & $I(\mathrm{~A})$ & $T_{\text {atm }}\left({ }^{\circ} \mathrm{C}\right)$ & $P_{\text {atm }}(\mathrm{Pa})$ & $P_{\text {diff }}(\mathrm{Pa})$ \\
\hline $1.197 \mathrm{E}+04$ & $3.688 \mathrm{E}+00$ & $7.38 \mathrm{E}-02$ & $1.092 \mathrm{E}+01$ & $6.696 \mathrm{E}+00$ & $2.300 \mathrm{E}+01$ & $9.879 \mathrm{E}+04$ & $3.449 \mathrm{E}+00$ \\
\hline $1.201 \mathrm{E}+04$ & $3.671 \mathrm{E}+00$ & $7.42 \mathrm{E}-02$ & $1.092 \mathrm{E}+01$ & $6.693 \mathrm{E}+00$ & $2.301 \mathrm{E}+01$ & $9.879 \mathrm{E}+04$ & $1.141 \mathrm{E}+01$ \\
\hline $1.201 \mathrm{E}+04$ & $3.640 \mathrm{E}+00$ & $7.47 \mathrm{E}-02$ & $1.092 \mathrm{E}+01$ & $6.549 \mathrm{E}+00$ & $2.306 \mathrm{E}+01$ & $9.879 \mathrm{E}+04$ & $2.535 \mathrm{E}+01$ \\
\hline $1.204 \mathrm{E}+04$ & $3.506 \mathrm{E}+00$ & $7.95 \mathrm{E}-02$ & $1.093 \mathrm{E}+01$ & $6.316 \mathrm{E}+00$ & $2.304 \mathrm{E}+01$ & $9.878 \mathrm{E}+04$ & $4.561 \mathrm{E}+01$ \\
\hline $1.200 \mathrm{E}+04$ & $3.456 \mathrm{E}+00$ & $7.16 \mathrm{E}-02$ & $1.093 \mathrm{E}+01$ & $6.159 \mathrm{E}+00$ & $2.310 \mathrm{E}+01$ & $9.876 \mathrm{E}+04$ & $7.188 \mathrm{E}+01$ \\
\hline $1.204 \mathrm{E}+04$ & $3.441 \mathrm{E}+00$ & $7.84 \mathrm{E}-02$ & $1.094 \mathrm{E}+01$ & $6.092 \mathrm{E}+00$ & $2.311 \mathrm{E}+01$ & $9.877 \mathrm{E}+04$ & $1.051 \mathrm{E}+02$ \\
\hline $1.203 \mathrm{E}+04$ & $3.334 \mathrm{E}+00$ & $7.66 \mathrm{E}-02$ & $1.094 \mathrm{E}+01$ & $5.927 \mathrm{E}+00$ & $2.312 \mathrm{E}+01$ & $9.876 \mathrm{E}+04$ & $1.446 \mathrm{E}+02$ \\
\hline $1.203 \mathrm{E}+04$ & $3.279 \mathrm{E}+00$ & $7.97 \mathrm{E}-02$ & $1.095 \mathrm{E}+01$ & $5.725 \mathrm{E}+00$ & $2.305 \mathrm{E}+01$ & $9.877 \mathrm{E}+04$ & $1.904 \mathrm{E}+02$ \\
\hline $1.198 \mathrm{E}+04$ & $3.013 \mathrm{E}+00$ & $7.08 \mathrm{E}-02$ & $1.096 \mathrm{E}+01$ & $5.212 \mathrm{E}+00$ & $2.303 \mathrm{E}+01$ & $9.877 \mathrm{E}+04$ & $2.421 \mathrm{E}+02$ \\
\hline $1.200 \mathrm{E}+04$ & $2.540 \mathrm{E}+00$ & $7.18 \mathrm{E}-02$ & $1.099 \mathrm{E}+01$ & $4.357 \mathrm{E}+00$ & $2.290 \mathrm{E}+01$ & $9.877 \mathrm{E}+04$ & $2.991 \mathrm{E}+02$ \\
\hline
\end{tabular}


Table 231: Graupner 5.5 x 5.5 Dynamic Calculated Values - 12010 RPM

\begin{tabular}{|c|c|c|c|c|c|c|c|}
\hline$n(\mathrm{RPM})$ & $V_{\infty}^{\prime}(\mathrm{m} / \mathrm{s})$ & $\Delta V_{\infty}^{\prime}(\mathrm{m} / \mathrm{s})$ & $T^{\prime}(\mathrm{g})$ & $\Delta T^{\prime}(\mathrm{g})$ & $V_{\mathrm{t}}(\mathrm{m} / \mathrm{s})$ & $P_{\mathrm{P}}(\mathrm{W})$ & $\Delta P_{\mathrm{P}}(\mathrm{W})$ \\
\hline $1.197 \mathrm{E}+04$ & $2.280 \mathrm{E}+00$ & $1.49 \mathrm{E}-02$ & $2.133 \mathrm{E}+02$ & $8.39 \mathrm{E}+00$ & $6.588 \mathrm{E}+01$ & $4.535 \mathrm{E}+01$ & 9.07E-01 \\
\hline $1.201 \mathrm{E}+04$ & $4.280 \mathrm{E}+00$ & $1.62 \mathrm{E}-02$ & $2.106 \mathrm{E}+02$ & $8.39 \mathrm{E}+00$ & $6.619 \mathrm{E}+01$ & $4.527 \mathrm{E}+01$ & $9.15 \mathrm{E}-01$ \\
\hline $1.201 \mathrm{E}+04$ & $6.462 \mathrm{E}+00$ & $1.88 \mathrm{E}-02$ & $2.097 \mathrm{E}+02$ & $8.35 \mathrm{E}+00$ & $6.639 \mathrm{E}+01$ & $4.491 \mathrm{E}+01$ & $9.22 \mathrm{E}-01$ \\
\hline $1.204 \mathrm{E}+04$ & $8.727 \mathrm{E}+00$ & $2.26 \mathrm{E}-02$ & $2.054 \mathrm{E}+02$ & $8.37 \mathrm{E}+00$ & $6.677 \mathrm{E}+01$ & $4.333 \mathrm{E}+01$ & $9.83 \mathrm{E}-01$ \\
\hline $1.200 \mathrm{E}+04$ & $1.100 \mathrm{E}+01$ & $2.61 \mathrm{E}-02$ & $1.960 \mathrm{E}+02$ & $8.33 \mathrm{E}+00$ & $6.689 \mathrm{E}+01$ & $4.257 \mathrm{E}+01$ & $8.83 \mathrm{E}-01$ \\
\hline $1.204 \mathrm{E}+04$ & $1.335 \mathrm{E}+01$ & $2.92 \mathrm{E}-02$ & $1.875 \mathrm{E}+02$ & $8.32 \mathrm{E}+00$ & $6.757 \mathrm{E}+01$ & $4.255 \mathrm{E}+01$ & $9.69 \mathrm{E}-01$ \\
\hline $1.203 \mathrm{E}+04$ & $1.568 \mathrm{E}+01$ & $3.20 \mathrm{E}-02$ & $1.748 \mathrm{E}+02$ & $8.26 \mathrm{E}+00$ & $6.801 \mathrm{E}+01$ & $4.120 \mathrm{E}+01$ & $9.46 \mathrm{E}-01$ \\
\hline $1.203 \mathrm{E}+04$ & $1.803 \mathrm{E}+01$ & $3.52 \mathrm{E}-02$ & $1.573 \mathrm{E}+02$ & $8.25 \mathrm{E}+00$ & $6.855 \mathrm{E}+01$ & $4.049 \mathrm{E}+01$ & $9.85 \mathrm{E}-01$ \\
\hline $1.198 \mathrm{E}+04$ & $2.035 \mathrm{E}+01$ & $3.89 \mathrm{E}-02$ & $1.317 \mathrm{E}+02$ & $8.33 \mathrm{E}+00$ & $6.894 \mathrm{E}+01$ & $3.706 \mathrm{E}+01$ & $8.71 \mathrm{E}-01$ \\
\hline $1.200 \mathrm{E}+04$ & $2.264 \mathrm{E}+01$ & $4.27 \mathrm{E}-02$ & $9.717 \mathrm{E}+01$ & $8.31 \mathrm{E}+00$ & $6.976 \mathrm{E}+01$ & $3.130 \mathrm{E}+01$ & $8.84 \mathrm{E}-01$ \\
\hline$P_{\mathrm{e}}(\mathrm{W})$ & $\Delta P_{\mathrm{e}}(\mathrm{W})$ & $C_{\mathrm{T}}$ & $\Delta C_{\mathrm{T}}$ & $C_{\mathrm{P}}$ & $\Delta C_{\mathrm{P}}$ & $C_{\mathrm{Q}}$ & $\Delta C_{\mathrm{Q}}$ \\
\hline $7.311 \mathrm{E}+01$ & $8.30 \mathrm{E}-01$ & $1.175 \mathrm{E}-01$ & $4.62 \mathrm{E}-03$ & $9.118 \mathrm{E}-02$ & $1.83 \mathrm{E}-03$ & $1.451 \mathrm{E}-02$ & $2.91 \mathrm{E}-04$ \\
\hline 7.307E+01 & $8.23 \mathrm{E}-01$ & $1.153 \mathrm{E}-01$ & $4.59 \mathrm{E}-03$ & $9.018 \mathrm{E}-02$ & $1.82 \mathrm{E}-03$ & $1.435 \mathrm{E}-02$ & $2.90 \mathrm{E}-04$ \\
\hline $7.154 \mathrm{E}+01$ & $7.92 \mathrm{E}-01$ & $1.148 \mathrm{E}-01$ & $4.57 \mathrm{E}-03$ & 8.937E-02 & $1.84 \mathrm{E}-03$ & $1.422 \mathrm{E}-02$ & $2.92 \mathrm{E}-04$ \\
\hline $6.902 \mathrm{E}+01$ & $7.70 \mathrm{E}-01$ & $1.120 \mathrm{E}-01$ & $4.57 \mathrm{E}-03$ & $8.577 \mathrm{E}-02$ & $1.95 \mathrm{E}-03$ & $1.365 \mathrm{E}-02$ & $3.10 \mathrm{E}-04$ \\
\hline $6.734 \mathrm{E}+01$ & $7.53 \mathrm{E}-01$ & $1.076 \mathrm{E}-01$ & $4.57 \mathrm{E}-03$ & $8.512 \mathrm{E}-02$ & $1.77 \mathrm{E}-03$ & $1.355 \mathrm{E}-02$ & $2.81 \mathrm{E}-04$ \\
\hline $6.662 \mathrm{E}+01$ & $7.45 \mathrm{E}-01$ & $1.021 \mathrm{E}-01$ & $4.53 \mathrm{E}-03$ & $8.410 \mathrm{E}-02$ & $1.92 \mathrm{E}-03$ & $1.338 \mathrm{E}-02$ & $3.05 \mathrm{E}-04$ \\
\hline $6.484 \mathrm{E}+01$ & 7.31E-01 & $9.543 \mathrm{E}-02$ & $4.51 \mathrm{E}-03$ & $8.164 \mathrm{E}-02$ & $1.88 \mathrm{E}-03$ & $1.299 \mathrm{E}-02$ & $2.99 \mathrm{E}-04$ \\
\hline $6.267 \mathrm{E}+01$ & $7.06 \mathrm{E}-01$ & $8.592 \mathrm{E}-02$ & $4.51 \mathrm{E}-03$ & $8.036 \mathrm{E}-02$ & $1.96 \mathrm{E}-03$ & $1.279 \mathrm{E}-02$ & $3.11 \mathrm{E}-04$ \\
\hline $5.713 \mathrm{E}+01$ & $6.49 \mathrm{E}-01$ & 7.254E-02 & $4.59 \mathrm{E}-03$ & $7.445 \mathrm{E}-02$ & $1.75 \mathrm{E}-03$ & $1.185 \mathrm{E}-02$ & $2.79 \mathrm{E}-04$ \\
\hline $4.787 \mathrm{E}+01$ & $5.50 \mathrm{E}-01$ & $5.331 \mathrm{E}-02$ & $4.56 \mathrm{E}-03$ & $6.252 \mathrm{E}-02$ & $1.77 \mathrm{E}-03$ & $9.951 \mathrm{E}-03$ & $2.81 \mathrm{E}-04$ \\
\hline$\rho\left(\mathrm{kg} / \mathrm{m}^{\wedge} 3\right)$ & $\eta_{\mathrm{T}}$ & $\Delta \eta_{\mathrm{T}}$ & $J$ & $\Delta J$ & $\eta_{\mathrm{P}}$ & $\Delta \eta_{\mathrm{P}}$ & $R e_{0.75}$ \\
\hline $1.162 \mathrm{E}+00$ & $6.202 \mathrm{E}-01$ & $1.43 \mathrm{E}-02$ & $8.159 \mathrm{E}-02$ & $5.33 \mathrm{E}-04$ & $1.052 \mathrm{E}-01$ & 4.69E-03 & $4.155 \mathrm{E}+04$ \\
\hline $1.162 \mathrm{E}+00$ & $6.195 \mathrm{E}-01$ & $1.43 \mathrm{E}-02$ & $1.527 \mathrm{E}-01$ & $5.81 \mathrm{E}-04$ & $1.952 \mathrm{E}-01$ & $8.75 \mathrm{E}-03$ & $4.174 \mathrm{E}+04$ \\
\hline $1.162 \mathrm{E}+00$ & $6.277 \mathrm{E}-01$ & $1.46 \mathrm{E}-02$ & 2.304E-01 & $6.76 \mathrm{E}-04$ & $2.960 \mathrm{E}-01$ & $1.33 \mathrm{E}-02$ & $4.185 \mathrm{E}+04$ \\
\hline $1.162 \mathrm{E}+00$ & $6.277 \mathrm{E}-01$ & $1.59 \mathrm{E}-02$ & 3.107E-01 & 8.11E-04 & 4.057E-01 & $1.90 \mathrm{E}-02$ & $4.209 \mathrm{E}+04$ \\
\hline $1.161 \mathrm{E}+00$ & $6.322 \mathrm{E}-01$ & $1.49 \mathrm{E}-02$ & $3.930 \mathrm{E}-01$ & $9.38 \mathrm{E}-04$ & $4.968 \mathrm{E}-01$ & $2.35 \mathrm{E}-02$ & $4.215 \mathrm{E}+04$ \\
\hline $1.161 \mathrm{E}+00$ & $6.387 \mathrm{E}-01$ & $1.62 \mathrm{E}-02$ & 4.747E-01 & $1.05 \mathrm{E}-03$ & $5.765 \mathrm{E}-01$ & $2.88 \mathrm{E}-02$ & $4.258 \mathrm{E}+04$ \\
\hline $1.161 \mathrm{E}+00$ & $6.353 \mathrm{E}-01$ & $1.63 \mathrm{E}-02$ & $5.585 \mathrm{E}-01$ & $1.16 \mathrm{E}-03$ & $6.528 \mathrm{E}-01$ & $3.43 \mathrm{E}-02$ & $4.285 \mathrm{E}+04$ \\
\hline $1.162 \mathrm{E}+00$ & $6.460 \mathrm{E}-01$ & $1.73 \mathrm{E}-02$ & $6.422 \mathrm{E}-01$ & $1.27 \mathrm{E}-03$ & $6.867 \mathrm{E}-01$ & $3.98 \mathrm{E}-02$ & $4.321 \mathrm{E}+04$ \\
\hline $1.162 \mathrm{E}+00$ & $6.487 \mathrm{E}-01$ & $1.69 \mathrm{E}-02$ & $7.280 \mathrm{E}-01$ & $1.41 \mathrm{E}-03$ & $7.093 \mathrm{E}-01$ & $4.79 \mathrm{E}-02$ & $4.347 \mathrm{E}+04$ \\
\hline $1.162 \mathrm{E}+00$ & $6.538 \mathrm{E}-01$ & $1.99 \mathrm{E}-02$ & $8.086 \mathrm{E}-01$ & $1.54 \mathrm{E}-03$ & $6.895 \mathrm{E}-01$ & $6.21 \mathrm{E}-02$ & $4.401 \mathrm{E}+04$ \\
\hline
\end{tabular}


Table 232: Graupner 5.5 x 5.5 Dynamic Measured Values - 13992 RPM

\begin{tabular}{|l|l|l|l|l|l|l|l|}
\hline$n(\mathrm{RPM})$ & $Q(\mathrm{~g}-\mathrm{m})$ & $\Delta Q(\mathrm{~g}-\mathrm{m})$ & $V(\mathrm{~V})$ & $I(\mathrm{~A})$ & $T_{\text {atm }}\left({ }^{\circ} \mathrm{C}\right)$ & $P_{\text {atm }}(\mathrm{Pa})$ & $P_{\text {diff }}(\mathrm{Pa})$ \\
\hline $1.162 \mathrm{E}+00$ & $6.202 \mathrm{E}-01$ & $1.43 \mathrm{E}-02$ & $8.160 \mathrm{E}-02$ & $3.72 \mathrm{E}-02$ & $1.047 \mathrm{E}-01$ & $4.80 \mathrm{E}-02$ & $4.155 \mathrm{E}+04$ \\
\hline $1.162 \mathrm{E}+00$ & $6.195 \mathrm{E}-01$ & $1.43 \mathrm{E}-02$ & $1.527 \mathrm{E}-01$ & $3.66 \mathrm{E}-02$ & $1.913 \mathrm{E}-01$ & $4.66 \mathrm{E}-02$ & $4.174 \mathrm{E}+04$ \\
\hline $1.162 \mathrm{E}+00$ & $6.277 \mathrm{E}-01$ & $1.46 \mathrm{E}-02$ & $2.306 \mathrm{E}-01$ & $3.64 \mathrm{E}-02$ & $2.808 \mathrm{E}-01$ & $4.62 \mathrm{E}-02$ & $4.186 \mathrm{E}+04$ \\
\hline $1.162 \mathrm{E}+00$ & $6.277 \mathrm{E}-01$ & $1.59 \mathrm{E}-02$ & $3.110 \mathrm{E}-01$ & $3.62 \mathrm{E}-02$ & $3.650 \mathrm{E}-01$ & $4.64 \mathrm{E}-02$ & $4.209 \mathrm{E}+04$ \\
\hline $1.161 \mathrm{E}+00$ & $6.322 \mathrm{E}-01$ & $1.49 \mathrm{E}-02$ & $3.935 \mathrm{E}-01$ & $3.62 \mathrm{E}-02$ & $4.111 \mathrm{E}-01$ & $4.42 \mathrm{E}-02$ & $4.216 \mathrm{E}+04$ \\
\hline $1.161 \mathrm{E}+00$ & $6.387 \mathrm{E}-01$ & $1.62 \mathrm{E}-02$ & $4.756 \mathrm{E}-01$ & $3.60 \mathrm{E}-02$ & $4.206 \mathrm{E}-01$ & $4.20 \mathrm{E}-02$ & $4.258 \mathrm{E}+04$ \\
\hline $1.161 \mathrm{E}+00$ & $6.353 \mathrm{E}-01$ & $1.63 \mathrm{E}-02$ & $5.596 \mathrm{E}-01$ & $3.59 \mathrm{E}-02$ & $3.877 \mathrm{E}-01$ & $4.06 \mathrm{E}-02$ & $4.286 \mathrm{E}+04$ \\
\hline $1.162 \mathrm{E}+00$ & $6.460 \mathrm{E}-01$ & $1.73 \mathrm{E}-02$ & $6.438 \mathrm{E}-01$ & $3.58 \mathrm{E}-02$ & $2.730 \mathrm{E}-01$ & $3.97 \mathrm{E}-02$ & $4.322 \mathrm{E}+04$ \\
\hline $1.162 \mathrm{E}+00$ & $6.487 \mathrm{E}-01$ & $1.69 \mathrm{E}-02$ & $7.300 \mathrm{E}-01$ & $3.58 \mathrm{E}-02$ & $5.387 \mathrm{E}-02$ & $4.51 \mathrm{E}-02$ & $4.348 \mathrm{E}+04$ \\
\hline $1.162 \mathrm{E}+00$ & $6.538 \mathrm{E}-01$ & $1.99 \mathrm{E}-02$ & $8.112 \mathrm{E}-01$ & $3.56 \mathrm{E}-02$ & $-3.865 \mathrm{E}-01$ & $6.25 \mathrm{E}-02$ & $4.403 \mathrm{E}+04$ \\
\hline
\end{tabular}


Table 233: Graupner 5.5 x 5.5 Dynamic Calculated Values - 13992 RPM

\begin{tabular}{|c|c|c|c|c|c|c|c|}
\hline$n(\mathrm{RPM})$ & $V_{\infty}^{\prime}(\mathrm{m} / \mathrm{s})$ & $\Delta V_{\infty}^{\prime}(\mathrm{m} / \mathrm{s})$ & $T^{\prime}(\mathrm{g})$ & $\Delta T^{\prime}(\mathrm{g})$ & $V_{\mathrm{t}}(\mathrm{m} / \mathrm{s})$ & $P_{\mathrm{P}}(\mathrm{W})$ & $\Delta P_{\mathrm{P}}(\mathrm{W})$ \\
\hline $1.392 \mathrm{E}+04$ & $2.404 \mathrm{E}+00$ & $1.64 \mathrm{E}-02$ & $2.919 \mathrm{E}+02$ & $8.73 \mathrm{E}+00$ & $7.658 \mathrm{E}+01$ & $7.107 \mathrm{E}+01$ & $9.56 \mathrm{E}-01$ \\
\hline $1.402 \mathrm{E}+04$ & $4.776 \mathrm{E}+00$ & $1.83 \mathrm{E}-02$ & $2.939 \mathrm{E}+02$ & $8.78 \mathrm{E}+00$ & $7.724 \mathrm{E}+01$ & $7.159 \mathrm{E}+01$ & $9.70 \mathrm{E}-01$ \\
\hline $1.400 \mathrm{E}+04$ & $7.411 \mathrm{E}+00$ & $2.12 \mathrm{E}-02$ & $2.891 \mathrm{E}+02$ & $8.79 \mathrm{E}+00$ & $7.733 \mathrm{E}+01$ & $7.062 \mathrm{E}+01$ & $9.76 \mathrm{E}-01$ \\
\hline $1.400 \mathrm{E}+04$ & $1.014 \mathrm{E}+01$ & $2.69 \mathrm{E}-02$ & $2.826 \mathrm{E}+02$ & $8.80 \mathrm{E}+00$ & $7.765 \mathrm{E}+01$ & $6.819 \mathrm{E}+01$ & $9.78 \mathrm{E}-01$ \\
\hline $1.398 \mathrm{E}+04$ & $1.290 \mathrm{E}+01$ & $3.06 \mathrm{E}-02$ & $2.724 \mathrm{E}+02$ & $8.82 \mathrm{E}+00$ & $7.794 \mathrm{E}+01$ & $6.725 \mathrm{E}+01$ & $9.87 \mathrm{E}-01$ \\
\hline $1.399 \mathrm{E}+04$ & $1.571 \mathrm{E}+01$ & 3.33E-02 & $2.557 \mathrm{E}+02$ & $8.83 \mathrm{E}+00$ & $7.851 \mathrm{E}+01$ & $6.640 \mathrm{E}+01$ & $9.94 \mathrm{E}-01$ \\
\hline $1.400 \mathrm{E}+04$ & $1.852 \mathrm{E}+01$ & $3.67 \mathrm{E}-02$ & $2.396 \mathrm{E}+02$ & $8.85 \mathrm{E}+00$ & $7.920 \mathrm{E}+01$ & $6.549 \mathrm{E}+01$ & $1.01 \mathrm{E}+00$ \\
\hline $1.398 \mathrm{E}+04$ & $2.128 \mathrm{E}+01$ & $4.09 \mathrm{E}-02$ & $2.155 \mathrm{E}+02$ & $8.85 \mathrm{E}+00$ & $7.976 \mathrm{E}+01$ & $6.339 \mathrm{E}+01$ & $1.00 \mathrm{E}+00$ \\
\hline $1.403 \mathrm{E}+04$ & $2.406 \mathrm{E}+01$ & $4.58 \mathrm{E}-02$ & $1.880 \mathrm{E}+02$ & $8.85 \mathrm{E}+00$ & $8.085 \mathrm{E}+01$ & $6.080 \mathrm{E}+01$ & $1.03 \mathrm{E}+00$ \\
\hline $1.402 \mathrm{E}+04$ & $2.683 \mathrm{E}+01$ & $5.02 \mathrm{E}-02$ & $1.388 \mathrm{E}+02$ & $8.74 \mathrm{E}+00$ & $8.165 \mathrm{E}+01$ & $5.089 \mathrm{E}+01$ & $1.06 \mathrm{E}+00$ \\
\hline$P_{\mathrm{e}}(\mathrm{W})$ & $\Delta P_{\mathrm{e}}(\mathrm{W})$ & $C_{\mathrm{T}}$ & $\Delta C_{\mathrm{T}}$ & $C_{\mathrm{P}}$ & $\Delta C_{\mathrm{P}}$ & $C_{\mathrm{Q}}$ & $\Delta C_{\mathrm{Q}}$ \\
\hline $1.126 \mathrm{E}+02$ & $1.22 \mathrm{E}+00$ & $1.187 \mathrm{E}-01$ & $3.55 \mathrm{E}-03$ & $9.074 \mathrm{E}-02$ & $1.22 \mathrm{E}-03$ & $1.444 \mathrm{E}-02$ & $1.95 \mathrm{E}-04$ \\
\hline $1.139 \mathrm{E}+02$ & $1.23 \mathrm{E}+00$ & $1.178 \mathrm{E}-01$ & $3.52 \mathrm{E}-03$ & $8.944 \mathrm{E}-02$ & $1.22 \mathrm{E}-03$ & $1.423 \mathrm{E}-02$ & $1.93 \mathrm{E}-04$ \\
\hline $1.118 \mathrm{E}+02$ & $1.20 \mathrm{E}+00$ & $1.163 \mathrm{E}-01$ & $3.54 \mathrm{E}-03$ & $8.868 \mathrm{E}-02$ & $1.23 \mathrm{E}-03$ & $1.411 \mathrm{E}-02$ & $1.95 \mathrm{E}-04$ \\
\hline $1.070 \mathrm{E}+02$ & $1.16 \mathrm{E}+00$ & $1.137 \mathrm{E}-01$ & $3.54 \mathrm{E}-03$ & $8.563 \mathrm{E}-02$ & $1.23 \mathrm{E}-03$ & $1.363 \mathrm{E}-02$ & $1.96 \mathrm{E}-04$ \\
\hline $1.048 \mathrm{E}+02$ & $1.14 \mathrm{E}+00$ & $1.100 \mathrm{E}-01$ & $3.56 \mathrm{E}-03$ & $8.491 \mathrm{E}-02$ & $1.25 \mathrm{E}-03$ & $1.351 \mathrm{E}-02$ & $1.99 \mathrm{E}-04$ \\
\hline $1.024 \mathrm{E}+02$ & $1.11 \mathrm{E}+00$ & $1.032 \mathrm{E}-01$ & $3.56 \mathrm{E}-03$ & $8.370 \mathrm{E}-02$ & $1.26 \mathrm{E}-03$ & $1.332 \mathrm{E}-02$ & $2.00 \mathrm{E}-04$ \\
\hline $9.983 \mathrm{E}+01$ & $1.08 \mathrm{E}+00$ & $9.647 \mathrm{E}-02$ & $3.57 \mathrm{E}-03$ & $8.228 \mathrm{E}-02$ & $1.27 \mathrm{E}-03$ & $1.310 \mathrm{E}-02$ & $2.02 \mathrm{E}-04$ \\
\hline $9.563 \mathrm{E}+01$ & $1.04 \mathrm{E}+00$ & $8.715 \mathrm{E}-02$ & $3.58 \mathrm{E}-03$ & $8.012 \mathrm{E}-02$ & $1.27 \mathrm{E}-03$ & $1.275 \mathrm{E}-02$ & $2.02 \mathrm{E}-04$ \\
\hline $9.013 \mathrm{E}+01$ & $9.80 \mathrm{E}-01$ & $7.539 \mathrm{E}-02$ & $3.55 \mathrm{E}-03$ & $7.590 \mathrm{E}-02$ & $1.29 \mathrm{E}-03$ & $1.208 \mathrm{E}-02$ & $2.06 \mathrm{E}-04$ \\
\hline $7.367 \mathrm{E}+01$ & $8.15 \mathrm{E}-01$ & $5.579 \mathrm{E}-02$ & $3.51 \mathrm{E}-03$ & $6.374 \mathrm{E}-02$ & $1.32 \mathrm{E}-03$ & $1.014 \mathrm{E}-02$ & $2.11 \mathrm{E}-04$ \\
\hline$\rho\left(\mathrm{kg} / \mathrm{m}^{\wedge} 3\right)$ & $\eta_{\mathrm{T}}$ & $\Delta \eta_{\mathrm{T}}$ & $J$ & $\Delta J$ & $\eta_{\mathrm{P}}$ & $\Delta \eta_{\mathrm{P}}$ & $R e_{0.75}$ \\
\hline $1.165 \mathrm{E}+00$ & $6.309 \mathrm{E}-01$ & $1.09 \mathrm{E}-02$ & 7.399E-02 & $5.07 \mathrm{E}-04$ & $9.681 \mathrm{E}-02$ & $3.25 \mathrm{E}-03$ & $4.851 \mathrm{E}+04$ \\
\hline $1.165 \mathrm{E}+00$ & $6.285 \mathrm{E}-01$ & $1.09 \mathrm{E}-02$ & $1.460 \mathrm{E}-01$ & $5.60 \mathrm{E}-04$ & $1.923 \mathrm{E}-01$ & $6.36 \mathrm{E}-03$ & $4.895 \mathrm{E}+04$ \\
\hline $1.165 \mathrm{E}+00$ & $6.316 \mathrm{E}-01$ & $1.11 \mathrm{E}-02$ & $2.269 \mathrm{E}-01$ & $6.53 \mathrm{E}-04$ & $2.974 \mathrm{E}-01$ & $9.98 \mathrm{E}-03$ & $4.898 \mathrm{E}+04$ \\
\hline $1.164 \mathrm{E}+00$ & $6.371 \mathrm{E}-01$ & $1.14 \mathrm{E}-02$ & $3.105 \mathrm{E}-01$ & $8.29 \mathrm{E}-04$ & $4.122 \mathrm{E}-01$ & $1.42 \mathrm{E}-02$ & $4.913 \mathrm{E}+04$ \\
\hline $1.163 \mathrm{E}+00$ & $6.414 \mathrm{E}-01$ & $1.17 \mathrm{E}-02$ & $3.953 \mathrm{E}-01$ & $9.45 \mathrm{E}-04$ & $5.122 \mathrm{E}-01$ & $1.83 \mathrm{E}-02$ & $4.925 \mathrm{E}+04$ \\
\hline $1.163 \mathrm{E}+00$ & $6.485 \mathrm{E}-01$ & $1.20 \mathrm{E}-02$ & 4.813E-01 & $1.03 \mathrm{E}-03$ & $5.932 \mathrm{E}-01$ & $2.24 \mathrm{E}-02$ & $4.958 \mathrm{E}+04$ \\
\hline $1.162 \mathrm{E}+00$ & $6.561 \mathrm{E}-01$ & $1.24 \mathrm{E}-02$ & $5.665 \mathrm{E}-01$ & $1.14 \mathrm{E}-03$ & $6.642 \mathrm{E}-01$ & $2.66 \mathrm{E}-02$ & $5.001 \mathrm{E}+04$ \\
\hline $1.162 \mathrm{E}+00$ & $6.629 \mathrm{E}-01$ & $1.27 \mathrm{E}-02$ & $6.524 \mathrm{E}-01$ & $1.27 \mathrm{E}-03$ & $7.096 \mathrm{E}-01$ & $3.13 \mathrm{E}-02$ & $5.033 \mathrm{E}+04$ \\
\hline $1.162 \mathrm{E}+00$ & $6.746 \mathrm{E}-01$ & $1.36 \mathrm{E}-02$ & $7.343 \mathrm{E}-01$ & $1.42 \mathrm{E}-03$ & 7.294E-01 & $3.66 \mathrm{E}-02$ & $5.100 \mathrm{E}+04$ \\
\hline $1.161 \mathrm{E}+00$ & $6.908 \mathrm{E}-01$ & $1.62 \mathrm{E}-02$ & 8.198E-01 & $1.56 \mathrm{E}-03$ & $7.176 \mathrm{E}-01$ & $4.76 \mathrm{E}-02$ & $5.148 \mathrm{E}+04$ \\
\hline
\end{tabular}


Table 234: Graupner 5.5 x 5.5 Dynamic Measured Values - 16015 RPM

\begin{tabular}{|c|c|c|c|c|c|c|c|}
\hline$n(\mathrm{RPM})$ & $Q(\mathrm{~g}-\mathrm{m})$ & $\Delta Q(\mathrm{~g}-\mathrm{m})$ & $V(\mathrm{~V})$ & $I(\mathrm{~A})$ & $T_{\text {atm }}\left({ }^{\circ} \mathrm{C}\right)$ & $P_{\text {atm }}(\mathrm{Pa})$ & $P_{\text {diff }}(\mathrm{Pa})$ \\
\hline $1.593 \mathrm{E}+04$ & $6.572 \mathrm{E}+00$ & $7.09 \mathrm{E}-02$ & $1.061 \mathrm{E}+01$ & $1.644 \mathrm{E}+01$ & $2.220 \mathrm{E}+01$ & $9.867 \mathrm{E}+04$ & $4.544 \mathrm{E}+00$ \\
\hline $1.600 \mathrm{E}+04$ & $6.552 \mathrm{E}+00$ & $6.79 \mathrm{E}-02$ & $1.061 \mathrm{E}+01$ & $1.662 \mathrm{E}+01$ & $2.213 \mathrm{E}+01$ & $9.865 \mathrm{E}+04$ & $1.761 \mathrm{E}+01$ \\
\hline $1.602 \mathrm{E}+04$ & $6.411 \mathrm{E}+00$ & $6.91 \mathrm{E}-02$ & $1.062 \mathrm{E}+01$ & $1.611 \mathrm{E}+01$ & $2.238 \mathrm{E}+01$ & $9.864 \mathrm{E}+04$ & $4.283 \mathrm{E}+01$ \\
\hline $1.601 \mathrm{E}+04$ & $6.306 \mathrm{E}+00$ & $6.94 \mathrm{E}-02$ & $1.064 \mathrm{E}+01$ & $1.562 \mathrm{E}+01$ & $2.261 \mathrm{E}+01$ & $9.864 \mathrm{E}+04$ & $8.007 \mathrm{E}+01$ \\
\hline $1.603 \mathrm{E}+04$ & $6.248 \mathrm{E}+00$ & $7.02 \mathrm{E}-02$ & $1.065 \mathrm{E}+01$ & $1.531 \mathrm{E}+01$ & $2.265 \mathrm{E}+01$ & $9.864 \mathrm{E}+04$ & $1.302 \mathrm{E}+02$ \\
\hline $1.603 \mathrm{E}+04$ & $6.155 \mathrm{E}+00$ & $6.99 \mathrm{E}-02$ & $1.066 \mathrm{E}+01$ & $1.485 \mathrm{E}+01$ & $2.268 \mathrm{E}+01$ & $9.863 \mathrm{E}+04$ & $1.928 \mathrm{E}+02$ \\
\hline $1.605 \mathrm{E}+04$ & $6.070 \mathrm{E}+00$ & $7.09 \mathrm{E}-02$ & $1.068 \mathrm{E}+01$ & $1.436 \mathrm{E}+01$ & $2.274 \mathrm{E}+01$ & $9.863 \mathrm{E}+04$ & $2.670 \mathrm{E}+02$ \\
\hline $1.603 \mathrm{E}+04$ & $5.878 \mathrm{E}+00$ & $7.12 \mathrm{E}-02$ & $1.070 \mathrm{E}+01$ & $1.363 \mathrm{E}+01$ & $2.273 \mathrm{E}+01$ & $9.862 \mathrm{E}+04$ & $3.533 \mathrm{E}+02$ \\
\hline $1.601 \mathrm{E}+04$ & $5.570 \mathrm{E}+00$ & $6.96 \mathrm{E}-02$ & $1.074 \mathrm{E}+01$ & $1.252 \mathrm{E}+01$ & $2.290 \mathrm{E}+01$ & $9.861 \mathrm{E}+04$ & $4.505 \mathrm{E}+02$ \\
\hline $1.604 \mathrm{E}+04$ & $4.820 \mathrm{E}+00$ & $7.58 \mathrm{E}-02$ & $1.081 \mathrm{E}+01$ & $1.031 \mathrm{E}+01$ & $2.300 \mathrm{E}+01$ & $9.861 \mathrm{E}+04$ & $5.591 \mathrm{E}+02$ \\
\hline
\end{tabular}


Table 235: Graupner 5.5 x 5.5 Dynamic Calculated Values - 16015 RPM

\begin{tabular}{|c|c|c|c|c|c|c|c|}
\hline$n(\mathrm{RPM})$ & $V_{\infty}^{\prime}(\mathrm{m} / \mathrm{s})$ & $\Delta V_{\infty}^{\prime}(\mathrm{m} / \mathrm{s})$ & $T^{\prime}(\mathrm{g})$ & $\Delta T^{\prime}(\mathrm{g})$ & $V_{\mathrm{t}}(\mathrm{m} / \mathrm{s})$ & $P_{\mathrm{P}}(\mathrm{W})$ & $\Delta P_{\mathrm{P}}(\mathrm{W})$ \\
\hline $1.593 \mathrm{E}+04$ & $2.583 \mathrm{E}+00$ & $1.81 \mathrm{E}-02$ & $3.870 \mathrm{E}+02$ & $7.61 \mathrm{E}+00$ & $8.767 \mathrm{E}+01$ & $1.075 \mathrm{E}+02$ & $1.16 \mathrm{E}+00$ \\
\hline $1.600 \mathrm{E}+04$ & $5.294 \mathrm{E}+00$ & $2.03 \mathrm{E}-02$ & $3.889 \mathrm{E}+02$ & $7.60 \mathrm{E}+00$ & $8.818 \mathrm{E}+01$ & $1.077 \mathrm{E}+02$ & $1.12 \mathrm{E}+00$ \\
\hline $1.602 \mathrm{E}+04$ & $8.386 \mathrm{E}+00$ & $2.45 \mathrm{E}-02$ & $3.846 \mathrm{E}+02$ & $7.56 \mathrm{E}+00$ & $8.849 \mathrm{E}+01$ & $1.055 \mathrm{E}+02$ & $1.14 \mathrm{E}+00$ \\
\hline $1.601 \mathrm{E}+04$ & $1.156 \mathrm{E}+01$ & $3.10 \mathrm{E}-02$ & $3.743 \mathrm{E}+02$ & $7.67 \mathrm{E}+00$ & $8.880 \mathrm{E}+01$ & $1.037 \mathrm{E}+02$ & $1.14 \mathrm{E}+00$ \\
\hline $1.603 \mathrm{E}+04$ & $1.481 \mathrm{E}+01$ & $3.40 \mathrm{E}-02$ & $3.621 \mathrm{E}+02$ & $7.84 \mathrm{E}+00$ & $8.940 \mathrm{E}+01$ & $1.029 \mathrm{E}+02$ & $1.16 \mathrm{E}+00$ \\
\hline $1.603 \mathrm{E}+04$ & $1.808 \mathrm{E}+01$ & $3.72 \mathrm{E}-02$ & $3.425 \mathrm{E}+02$ & $7.83 \mathrm{E}+00$ & $8.998 \mathrm{E}+01$ & $1.013 \mathrm{E}+02$ & $1.15 \mathrm{E}+00$ \\
\hline $1.605 \mathrm{E}+04$ & $2.132 \mathrm{E}+01$ & $4.16 \mathrm{E}-02$ & $3.194 \mathrm{E}+02$ & $7.87 \mathrm{E}+00$ & $9.083 \mathrm{E}+01$ & $1.001 \mathrm{E}+02$ & $1.17 \mathrm{E}+00$ \\
\hline $1.603 \mathrm{E}+04$ & $2.456 \mathrm{E}+01$ & $4.71 \mathrm{E}-02$ & $2.867 \mathrm{E}+02$ & $7.86 \mathrm{E}+00$ & $9.154 \mathrm{E}+01$ & $9.678 \mathrm{E}+01$ & $1.17 \mathrm{E}+00$ \\
\hline $1.601 \mathrm{E}+04$ & $2.778 \mathrm{E}+01$ & $5.26 \mathrm{E}-02$ & $2.477 \mathrm{E}+02$ & $8.02 \mathrm{E}+00$ & $9.231 \mathrm{E}+01$ & $9.155 \mathrm{E}+01$ & $1.14 \mathrm{E}+00$ \\
\hline $1.604 \mathrm{E}+04$ & $3.099 \mathrm{E}+01$ & $5.80 \mathrm{E}-02$ & $1.931 \mathrm{E}+02$ & $8.10 \mathrm{E}+00$ & $9.349 \mathrm{E}+01$ & $7.938 \mathrm{E}+01$ & $1.25 \mathrm{E}+00$ \\
\hline$P_{\mathrm{e}}(\mathrm{W})$ & $\Delta P_{\mathrm{e}}(\mathrm{W})$ & $C_{\mathrm{T}}$ & $\Delta C_{\mathrm{T}}$ & $C_{\mathrm{P}}$ & $\Delta C_{\mathrm{P}}$ & $C_{\mathrm{Q}}$ & $\Delta C_{\mathrm{Q}}$ \\
\hline $1.745 \mathrm{E}+02$ & $1.84 \mathrm{E}+00$ & $1.202 \mathrm{E}-01$ & $2.37 \mathrm{E}-03$ & $9.156 \mathrm{E}-02$ & $9.93 \mathrm{E}-04$ & $1.457 \mathrm{E}-02$ & $1.58 \mathrm{E}-04$ \\
\hline $1.762 \mathrm{E}+02$ & $1.82 \mathrm{E}+00$ & $1.197 \mathrm{E}-01$ & $2.34 \mathrm{E}-03$ & $9.050 \mathrm{E}-02$ & $9.41 \mathrm{E}-04$ & $1.440 \mathrm{E}-02$ & $1.50 \mathrm{E}-04$ \\
\hline $1.712 \mathrm{E}+02$ & $1.78 \mathrm{E}+00$ & $1.183 \mathrm{E}-01$ & $2.33 \mathrm{E}-03$ & $8.848 \mathrm{E}-02$ & $9.56 \mathrm{E}-04$ & $1.408 \mathrm{E}-02$ & $1.52 \mathrm{E}-04$ \\
\hline $1.662 \mathrm{E}+02$ & $1.73 \mathrm{E}+00$ & $1.154 \mathrm{E}-01$ & $2.37 \mathrm{E}-03$ & $8.720 \mathrm{E}-02$ & $9.63 \mathrm{E}-04$ & $1.388 \mathrm{E}-02$ & $1.53 \mathrm{E}-04$ \\
\hline $1.631 \mathrm{E}+02$ & $1.70 \mathrm{E}+00$ & $1.113 \mathrm{E}-01$ & $2.41 \mathrm{E}-03$ & $8.617 \mathrm{E}-02$ & $9.71 \mathrm{E}-04$ & $1.372 \mathrm{E}-02$ & $1.54 \mathrm{E}-04$ \\
\hline $1.584 \mathrm{E}+02$ & $1.66 \mathrm{E}+00$ & $1.053 \mathrm{E}-01$ & $2.41 \mathrm{E}-03$ & $8.494 \mathrm{E}-02$ & $9.67 \mathrm{E}-04$ & $1.352 \mathrm{E}-02$ & $1.54 \mathrm{E}-04$ \\
\hline $1.534 \mathrm{E}+02$ & $1.62 \mathrm{E}+00$ & $9.794 \mathrm{E}-02$ & $2.41 \mathrm{E}-03$ & $8.351 \mathrm{E}-02$ & $9.78 \mathrm{E}-04$ & $1.329 \mathrm{E}-02$ & $1.56 \mathrm{E}-04$ \\
\hline $1.459 \mathrm{E}+02$ & $1.54 \mathrm{E}+00$ & $8.816 \mathrm{E}-02$ & $2.42 \mathrm{E}-03$ & $8.108 \mathrm{E}-02$ & $9.84 \mathrm{E}-04$ & $1.290 \mathrm{E}-02$ & $1.57 \mathrm{E}-04$ \\
\hline $1.344 \mathrm{E}+02$ & $1.42 \mathrm{E}+00$ & $7.647 \mathrm{E}-02$ & $2.48 \mathrm{E}-03$ & $7.713 \mathrm{E}-02$ & $9.66 \mathrm{E}-04$ & $1.228 \mathrm{E}-02$ & $1.54 \mathrm{E}-04$ \\
\hline $1.115 \mathrm{E}+02$ & $1.20 \mathrm{E}+00$ & $5.941 \mathrm{E}-02$ & $2.49 \mathrm{E}-03$ & $6.652 \mathrm{E}-02$ & $1.05 \mathrm{E}-03$ & $1.059 \mathrm{E}-02$ & $1.67 \mathrm{E}-04$ \\
\hline$\rho\left(\mathrm{kg} / \mathrm{m}^{\wedge} 3\right)$ & $\eta_{\mathrm{T}}$ & $\Delta \eta_{\mathrm{T}}$ & $J$ & $\Delta J$ & $\eta_{\mathrm{P}}$ & $\Delta \eta_{\mathrm{P}}$ & $R e_{0.75}$ \\
\hline $1.164 \mathrm{E}+00$ & $6.161 \mathrm{E}-01$ & $9.30 \mathrm{E}-03$ & $6.945 \mathrm{E}-02$ & $4.88 \mathrm{E}-04$ & $9.116 \mathrm{E}-02$ & $2.15 \mathrm{E}-03$ & $5.550 \mathrm{E}+04$ \\
\hline $1.164 \mathrm{E}+00$ & $6.110 \mathrm{E}-01$ & $8.95 \mathrm{E}-03$ & $1.417 \mathrm{E}-01$ & $5.45 \mathrm{E}-04$ & $1.875 \mathrm{E}-01$ & $4.21 \mathrm{E}-03$ & $5.583 \mathrm{E}+04$ \\
\hline $1.163 \mathrm{E}+00$ & $6.161 \mathrm{E}-01$ & $9.22 \mathrm{E}-03$ & 2.243E-01 & $6.59 \mathrm{E}-04$ & $2.999 \mathrm{E}-01$ & $6.79 \mathrm{E}-03$ & $5.594 \mathrm{E}+04$ \\
\hline $1.162 \mathrm{E}+00$ & $6.239 \mathrm{E}-01$ & $9.46 \mathrm{E}-03$ & $3.093 \mathrm{E}-01$ & $8.33 \mathrm{E}-04$ & $4.093 \mathrm{E}-01$ & $9.60 \mathrm{E}-03$ & $5.605 \mathrm{E}+04$ \\
\hline $1.162 \mathrm{E}+00$ & $6.307 \mathrm{E}-01$ & $9.67 \mathrm{E}-03$ & $3.957 \mathrm{E}-01$ & $9.12 \mathrm{E}-04$ & 5.112E-01 & $1.25 \mathrm{E}-02$ & $5.642 \mathrm{E}+04$ \\
\hline $1.161 \mathrm{E}+00$ & $6.396 \mathrm{E}-01$ & $9.88 \mathrm{E}-03$ & 4.832E-01 & $9.99 \mathrm{E}-04$ & 5.992E-01 & $1.54 \mathrm{E}-02$ & $5.677 \mathrm{E}+04$ \\
\hline $1.161 \mathrm{E}+00$ & $6.524 \mathrm{E}-01$ & $1.03 \mathrm{E}-02$ & $5.688 \mathrm{E}-01$ & $1.12 \mathrm{E}-03$ & $6.671 \mathrm{E}-01$ & $1.83 \mathrm{E}-02$ & $5.728 \mathrm{E}+04$ \\
\hline $1.161 \mathrm{E}+00$ & $6.632 \mathrm{E}-01$ & $1.07 \mathrm{E}-02$ & $6.563 \mathrm{E}-01$ & $1.27 \mathrm{E}-03$ & $7.136 \mathrm{E}-01$ & $2.15 \mathrm{E}-02$ & $5.772 \mathrm{E}+04$ \\
\hline $1.160 \mathrm{E}+00$ & $6.811 \mathrm{E}-01$ & $1.11 \mathrm{E}-02$ & $7.435 \mathrm{E}-01$ & $1.42 \mathrm{E}-03$ & $7.370 \mathrm{E}-01$ & $2.56 \mathrm{E}-02$ & $5.815 \mathrm{E}+04$ \\
\hline $1.160 \mathrm{E}+00$ & $7.120 \mathrm{E}-01$ & $1.36 \mathrm{E}-02$ & $8.278 \mathrm{E}-01$ & $1.56 \mathrm{E}-03$ & 7.393E-01 & $3.32 \mathrm{E}-02$ & $5.886 \mathrm{E}+04$ \\
\hline
\end{tabular}




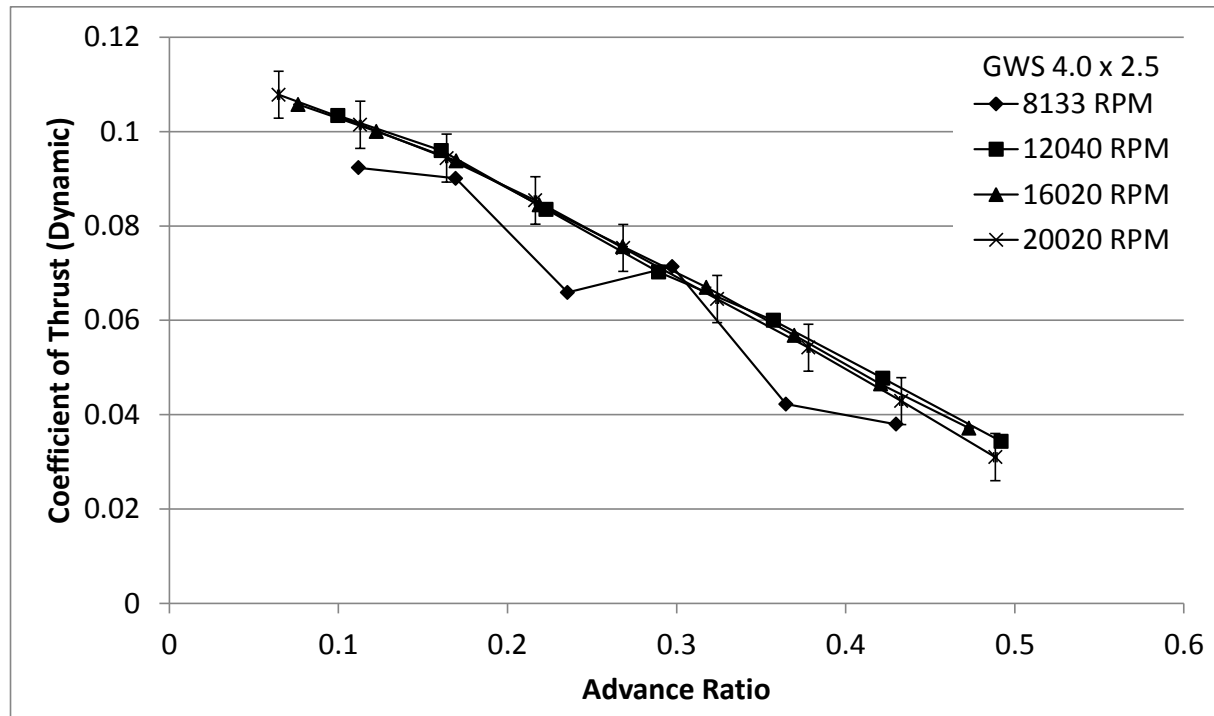

(a)

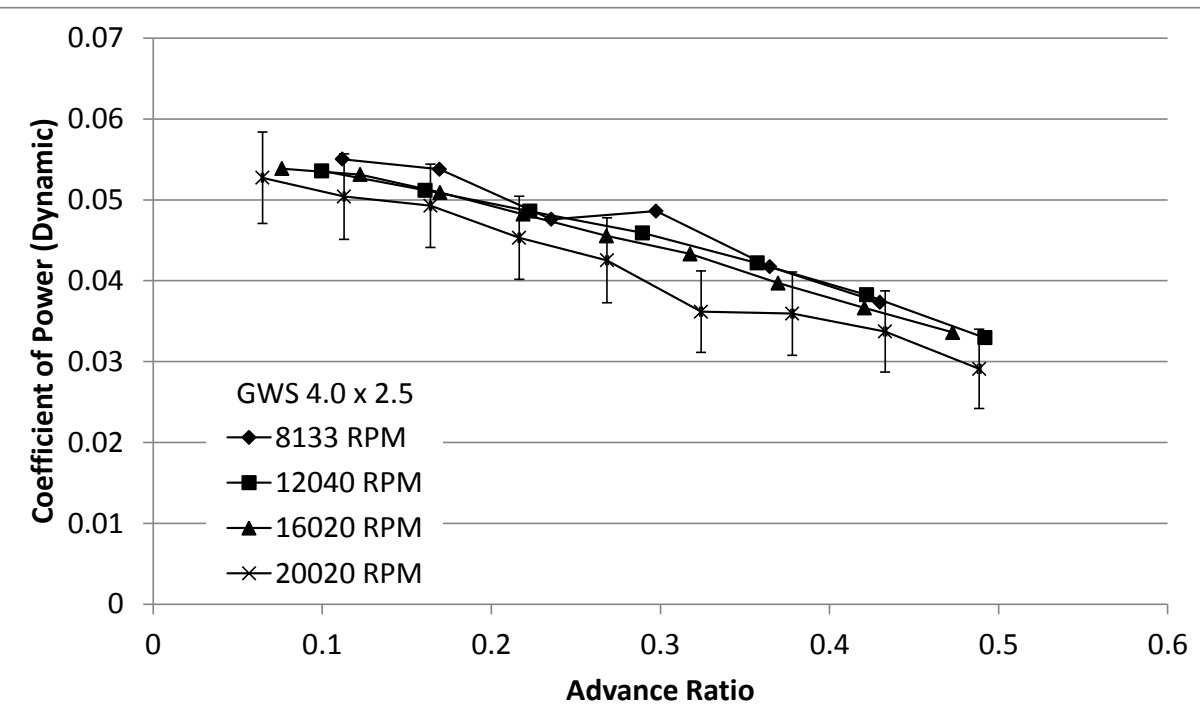

(b)

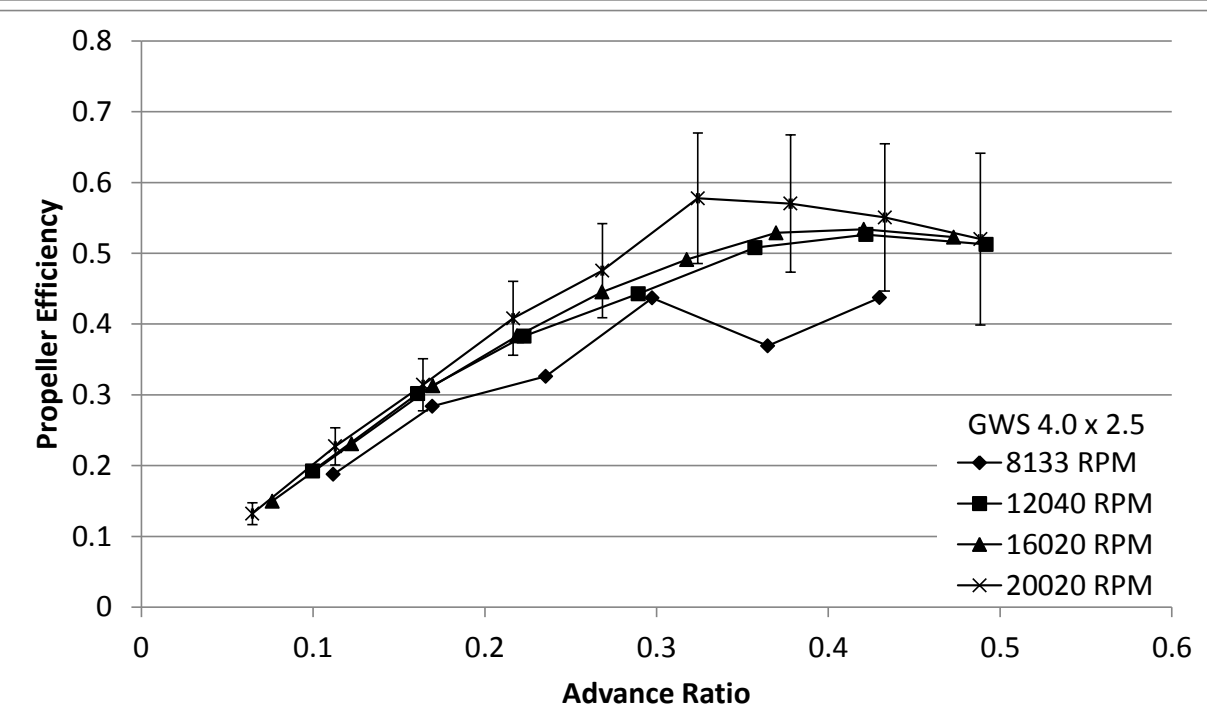

(c) 


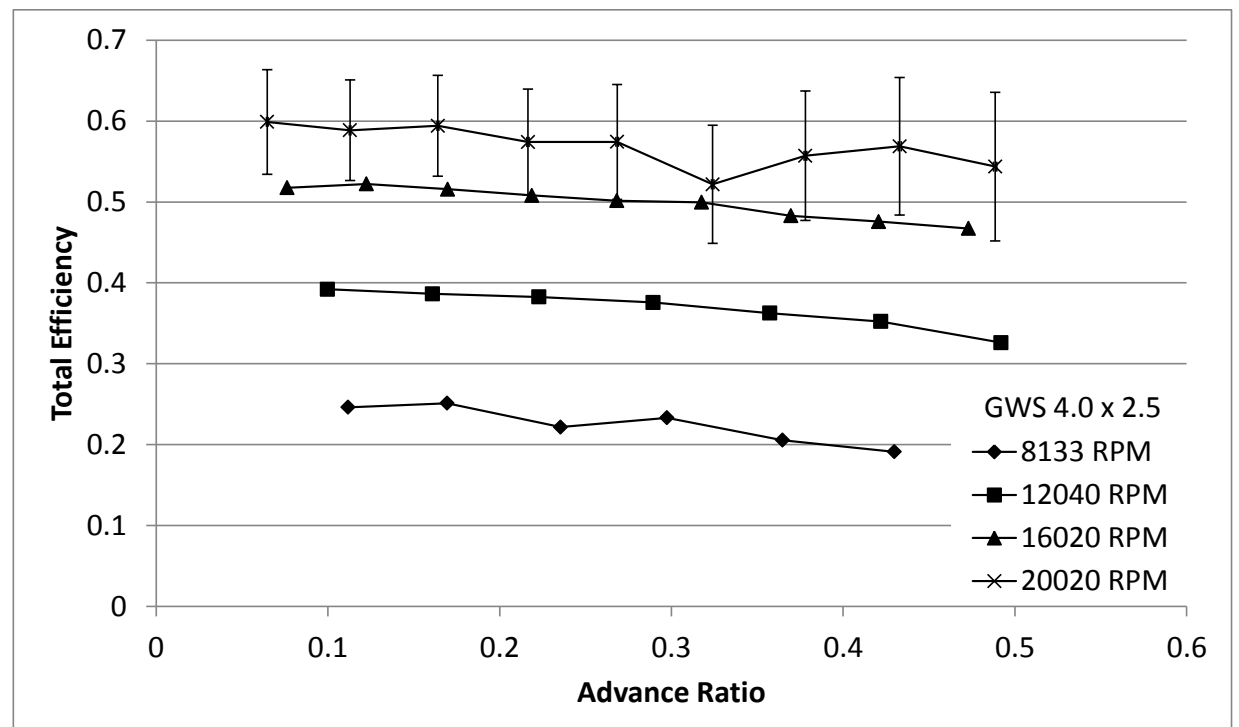

(d)

Figure 98: GWS 4.0 x 2.5 Dynamic Test Results: (a) Coefficient of Thrust, (b)

Coefficient of Power, (c) Propeller Efficiency, (d) Total Efficiency.

Table 236: GWS 4.0 x 2.5 Dynamic Measured Values - 8133 RPM

\begin{tabular}{|c|c|c|c|c|c|c|c|}
\hline$n(\mathrm{RPM})$ & $Q(\mathrm{~g}-\mathrm{m})$ & $\Delta Q(\mathrm{~g}-\mathrm{m})$ & $V(\mathrm{~V})$ & $I(\mathrm{~A})$ & $T_{\text {atm }}\left({ }^{\circ} \mathrm{C}\right)$ & $P_{\text {atm }}(\mathrm{Pa})$ & $P_{\text {diff }}(\mathrm{Pa})$ \\
\hline $8.146 \mathrm{E}+03$ & $2.072 \mathrm{E}-01$ & $6.15 \mathrm{E}-02$ & $1.110 \mathrm{E}+01$ & $6.346 \mathrm{E}-01$ & $2.183 \mathrm{E}+01$ & $9.864 \mathrm{E}+04$ & $1.449 \mathrm{E}+00$ \\
\hline $8.258 \mathrm{E}+03$ & $2.082 \mathrm{E}-01$ & $5.83 \mathrm{E}-02$ & $1.110 \mathrm{E}+01$ & $6.331 \mathrm{E}-01$ & $2.171 \mathrm{E}+01$ & $9.864 \mathrm{E}+04$ & $3.363 \mathrm{E}+00$ \\
\hline $8.085 \mathrm{E}+03$ & $1.766 \mathrm{E}-01$ & $6.36 \mathrm{E}-02$ & $1.110 \mathrm{E}+01$ & $5.959 \mathrm{E}-01$ & $2.170 \mathrm{E}+01$ & $9.864 \mathrm{E}+04$ & $6.151 \mathrm{E}+00$ \\
\hline $8.129 \mathrm{E}+03$ & $1.823 \mathrm{E}-01$ & $6.21 \mathrm{E}-02$ & $1.110 \mathrm{E}+01$ & $5.881 \mathrm{E}-01$ & $2.175 \mathrm{E}+01$ & $9.863 \mathrm{E}+04$ & $9.884 \mathrm{E}+00$ \\
\hline $8.087 \mathrm{E}+03$ & $1.549 \mathrm{E}-01$ & $6.54 \mathrm{E}-02$ & $1.110 \mathrm{E}+01$ & $5.640 \mathrm{E}-01$ & $2.176 \mathrm{E}+01$ & $9.863 \mathrm{E}+04$ & $1.462 \mathrm{E}+01$ \\
\hline $8.096 \mathrm{E}+03$ & $1.388 \mathrm{E}-01$ & $6.44 \mathrm{E}-02$ & $1.110 \mathrm{E}+01$ & $5.440 \mathrm{E}-01$ & $2.185 \mathrm{E}+01$ & $9.863 \mathrm{E}+04$ & $2.030 \mathrm{E}+01$ \\
\hline
\end{tabular}


Table 237: GWS 4.0 x 2.5 Dynamic Calculated Values - 8133 RPM

\begin{tabular}{|c|c|c|c|c|c|c|c|}
\hline$n(\mathrm{RPM})$ & $V_{\infty}^{\prime}(\mathrm{m} / \mathrm{s})$ & $\Delta V_{\infty}^{\prime}(\mathrm{m} / \mathrm{s})$ & $T^{\prime}(\mathrm{g})$ & $\Delta T^{\prime}(\mathrm{g})$ & $V_{\mathrm{t}}(\mathrm{m} / \mathrm{s})$ & $P_{\mathrm{P}}(\mathrm{W})$ & $\Delta P_{\mathrm{P}}(\mathrm{W})$ \\
\hline $8.146 \mathrm{E}+03$ & $1.542 \mathrm{E}+00$ & $1.34 \mathrm{E}-02$ & $2.151 \mathrm{E}+01$ & $9.99 \mathrm{E}+00$ & $3.252 \mathrm{E}+01$ & $1.733 \mathrm{E}+00$ & $5.14 \mathrm{E}-01$ \\
\hline $8.258 \mathrm{E}+03$ & $2.368 \mathrm{E}+00$ & $1.32 \mathrm{E}-02$ & $2.157 \mathrm{E}+01$ & $9.89 \mathrm{E}+00$ & $3.302 \mathrm{E}+01$ & $1.765 \mathrm{E}+00$ & 4.95E-01 \\
\hline $8.085 \mathrm{E}+03$ & $3.223 \mathrm{E}+00$ & $1.46 \mathrm{E}-02$ & $1.513 \mathrm{E}+01$ & $9.95 \mathrm{E}+00$ & $3.240 \mathrm{E}+01$ & $1.466 \mathrm{E}+00$ & $5.28 \mathrm{E}-01$ \\
\hline $8.129 \mathrm{E}+03$ & $4.093 \mathrm{E}+00$ & $1.51 \mathrm{E}-02$ & $1.656 \mathrm{E}+01$ & $1.01 \mathrm{E}+01$ & $3.267 \mathrm{E}+01$ & $1.522 \mathrm{E}+00$ & $5.18 \mathrm{E}-01$ \\
\hline $8.087 \mathrm{E}+03$ & $4.993 \mathrm{E}+00$ & $1.76 \mathrm{E}-02$ & $9.699 \mathrm{E}+00$ & $1.01 \mathrm{E}+01$ & $3.263 \mathrm{E}+01$ & $1.286 \mathrm{E}+00$ & $5.43 \mathrm{E}-01$ \\
\hline $8.096 \mathrm{E}+03$ & $5.890 \mathrm{E}+00$ & $1.81 \mathrm{E}-02$ & $8.734 \mathrm{E}+00$ & $1.01 \mathrm{E}+01$ & $3.282 \mathrm{E}+01$ & $1.154 \mathrm{E}+00$ & $5.35 \mathrm{E}-01$ \\
\hline$P_{\mathrm{e}}(\mathrm{W})$ & $\Delta P_{\mathrm{e}}(\mathrm{W})$ & $C_{\mathrm{T}}$ & $\Delta C_{\mathrm{T}}$ & $C_{\mathrm{P}}$ & $\Delta C_{\mathrm{P}}$ & $C_{\mathrm{Q}}$ & $\Delta C_{\mathrm{Q}}$ \\
\hline $7.043 \mathrm{E}+00$ & $9.41 \mathrm{E}-02$ & $9.234 \mathrm{E}-02$ & $4.29 \mathrm{E}-02$ & $5.503 \mathrm{E}-02$ & $1.63 \mathrm{E}-02$ & $8.758 \mathrm{E}-03$ & $2.60 \mathrm{E}-03$ \\
\hline $7.027 \mathrm{E}+00$ & $9.39 \mathrm{E}-02$ & $9.008 \mathrm{E}-02$ & $4.13 \mathrm{E}-02$ & $5.378 \mathrm{E}-02$ & $1.51 \mathrm{E}-02$ & $8.559 \mathrm{E}-03$ & $2.40 \mathrm{E}-03$ \\
\hline $6.615 \mathrm{E}+00$ & $9.11 \mathrm{E}-02$ & $6.590 \mathrm{E}-02$ & 4.33E-02 & 4.759E-02 & $1.71 \mathrm{E}-02$ & $7.575 \mathrm{E}-03$ & 2.73E-03 \\
\hline $6.528 \mathrm{E}+00$ & 8.94E-02 & 7.140E-02 & $4.34 \mathrm{E}-02$ & 4.862E-02 & $1.66 \mathrm{E}-02$ & $7.738 \mathrm{E}-03$ & $2.63 \mathrm{E}-03$ \\
\hline $6.261 \mathrm{E}+00$ & $8.56 \mathrm{E}-02$ & 4.224E-02 & $4.40 \mathrm{E}-02$ & 4.173E-02 & $1.76 \mathrm{E}-02$ & $6.641 \mathrm{E}-03$ & $2.81 \mathrm{E}-03$ \\
\hline $6.038 \mathrm{E}+00$ & 8.37E-02 & $3.796 \mathrm{E}-02$ & 4.37E-02 & $3.732 \mathrm{E}-02$ & $1.73 \mathrm{E}-02$ & $5.940 \mathrm{E}-03$ & $2.76 \mathrm{E}-03$ \\
\hline$\rho\left(\mathrm{kg} / \mathrm{m}^{\wedge} 3\right)$ & $\eta_{\mathrm{T}}$ & $\Delta \eta_{\mathrm{T}}$ & $J$ & $\Delta J$ & $\eta_{\mathrm{P}}$ & $\Delta \eta_{\mathrm{P}}$ & $R e_{0.75}$ \\
\hline $1.165 \mathrm{E}+00$ & $2.461 \mathrm{E}-01$ & 7.31E-02 & $1.118 \mathrm{E}-01$ & $9.79 \mathrm{E}-04$ & $1.876 \mathrm{E}-01$ & $1.03 \mathrm{E}-01$ & $2.579 \mathrm{E}+04$ \\
\hline $1.165 \mathrm{E}+00$ & $2.513 \mathrm{E}-01$ & 7.05E-02 & $1.694 \mathrm{E}-01$ & $9.56 \mathrm{E}-04$ & $2.838 \mathrm{E}-01$ & $1.52 \mathrm{E}-01$ & $2.620 \mathrm{E}+04$ \\
\hline $1.165 \mathrm{E}+00$ & $2.217 \mathrm{E}-01$ & 7.99E-02 & $2.355 \mathrm{E}-01$ & $1.08 \mathrm{E}-03$ & $3.261 \mathrm{E}-01$ & $2.44 \mathrm{E}-01$ & $2.571 \mathrm{E}+04$ \\
\hline $1.165 \mathrm{E}+00$ & $2.332 \mathrm{E}-01$ & 7.95E-02 & $2.975 \mathrm{E}-01$ & $1.12 \mathrm{E}-03$ & $4.368 \mathrm{E}-01$ & 3.04E-01 & $2.592 \mathrm{E}+04$ \\
\hline $1.165 \mathrm{E}+00$ & $2.054 \mathrm{E}-01$ & $8.68 \mathrm{E}-02$ & $3.647 \mathrm{E}-01$ & $1.31 \mathrm{E}-03$ & 3.693E-01 & $4.15 \mathrm{E}-01$ & $2.589 \mathrm{E}+04$ \\
\hline $1.165 \mathrm{E}+00$ & $1.911 \mathrm{E}-01$ & 8.87E-02 & 4.298E-01 & $1.36 \mathrm{E}-03$ & 4.372E-01 & $5.43 \mathrm{E}-01$ & $2.602 \mathrm{E}+04$ \\
\hline
\end{tabular}


Table 238: GWS 4.0 x 2.5 Dynamic Measured Values - 12040 RPM

\begin{tabular}{|c|c|c|c|c|c|c|c|}
\hline$n(\mathrm{RPM})$ & $Q(\mathrm{~g}-\mathrm{m})$ & $\Delta Q(\mathrm{~g}-\mathrm{m})$ & $V(\mathrm{~V})$ & $I(\mathrm{~A})$ & $T_{\text {atm }}\left({ }^{\circ} \mathrm{C}\right)$ & $P_{\text {atm }}(\mathrm{Pa})$ & $P_{\text {diff }}(\mathrm{Pa})$ \\
\hline $1.208 \mathrm{E}+04$ & $4.426 \mathrm{E}-01$ & $5.85 \mathrm{E}-02$ & $1.108 \mathrm{E}+01$ & $1.263 \mathrm{E}+00$ & $2.218 \mathrm{E}+01$ & $9.858 \mathrm{E}+04$ & $2.549 \mathrm{E}+00$ \\
\hline $1.201 \mathrm{E}+04$ & $4.181 \mathrm{E}-01$ & $5.76 \mathrm{E}-02$ & $1.108 \mathrm{E}+01$ & $1.204 \mathrm{E}+00$ & $2.215 \mathrm{E}+01$ & $9.858 \mathrm{E}+04$ & $6.422 \mathrm{E}+00$ \\
\hline $1.208 \mathrm{E}+04$ & $4.018 \mathrm{E}-01$ & $5.82 \mathrm{E}-02$ & $1.108 \mathrm{E}+01$ & $1.176 \mathrm{E}+00$ & $2.212 \mathrm{E}+01$ & $9.857 \mathrm{E}+04$ & $1.232 \mathrm{E}+01$ \\
\hline $1.203 \mathrm{E}+04$ & $3.764 \mathrm{E}-01$ & $5.86 \mathrm{E}-02$ & $1.109 \mathrm{E}+01$ & $1.116 \mathrm{E}+00$ & $2.215 \mathrm{E}+01$ & $9.858 \mathrm{E}+04$ & $2.047 \mathrm{E}+01$ \\
\hline $1.202 \mathrm{E}+04$ & $3.448 \mathrm{E}-01$ & $5.73 \mathrm{E}-02$ & $1.109 \mathrm{E}+01$ & $1.058 \mathrm{E}+00$ & $2.229 \mathrm{E}+01$ & $9.857 \mathrm{E}+04$ & $3.096 \mathrm{E}+01$ \\
\hline $1.205 \mathrm{E}+04$ & $3.144 \mathrm{E}-01$ & $5.75 \mathrm{E}-02$ & $1.109 \mathrm{E}+01$ & $9.961 \mathrm{E}-01$ & $2.236 \mathrm{E}+01$ & $9.857 \mathrm{E}+04$ & $4.327 \mathrm{E}+01$ \\
\hline $1.201 \mathrm{E}+04$ & $2.692 \mathrm{E}-01$ & $5.70 \mathrm{E}-02$ & $1.109 \mathrm{E}+01$ & $9.185 \mathrm{E}-01$ & $2.240 \mathrm{E}+01$ & $9.856 \mathrm{E}+04$ & $5.832 \mathrm{E}+01$ \\
\hline
\end{tabular}


Table 239: GWS 4.0 x 2.5 Dynamic Calculated Values - 12040 RPM

\begin{tabular}{|c|c|c|c|c|c|c|c|}
\hline$n(\mathrm{RPM})$ & $V_{\infty}^{\prime}(\mathrm{m} / \mathrm{s})$ & $\Delta V_{\infty}^{\prime}(\mathrm{m} / \mathrm{s})$ & $T^{\prime}(\mathrm{g})$ & $\Delta T^{\prime}(\mathrm{g})$ & $V_{\mathrm{t}}(\mathrm{m} / \mathrm{s})$ & $P_{\mathrm{P}}(\mathrm{W})$ & $\Delta P_{\mathrm{P}}(\mathrm{W})$ \\
\hline $1.208 \mathrm{E}+04$ & $2.038 \mathrm{E}+00$ & $1.26 \mathrm{E}-02$ & $5.285 \mathrm{E}+01$ & $8.15 \mathrm{E}+00$ & $4.820 \mathrm{E}+01$ & $5.489 \mathrm{E}+00$ & $7.25 \mathrm{E}-01$ \\
\hline $1.201 \mathrm{E}+04$ & $3.271 \mathrm{E}+00$ & $1.27 \mathrm{E}-02$ & $4.850 \mathrm{E}+01$ & $8.15 \mathrm{E}+00$ & $4.800 \mathrm{E}+01$ & $5.157 \mathrm{E}+00$ & $7.10 \mathrm{E}-01$ \\
\hline $1.208 \mathrm{E}+04$ & $4.558 \mathrm{E}+00$ & $1.38 \mathrm{E}-02$ & $4.272 \mathrm{E}+01$ & $8.13 \mathrm{E}+00$ & $4.840 \mathrm{E}+01$ & $4.985 \mathrm{E}+00$ & 7.22E-01 \\
\hline $1.203 \mathrm{E}+04$ & $5.894 \mathrm{E}+00$ & $1.55 \mathrm{E}-02$ & $3.562 \mathrm{E}+01$ & $8.16 \mathrm{E}+00$ & $4.834 \mathrm{E}+01$ & $4.651 \mathrm{E}+00$ & 7.24E-01 \\
\hline $1.202 \mathrm{E}+04$ & $7.267 \mathrm{E}+00$ & $1.74 \mathrm{E}-02$ & $3.033 \mathrm{E}+01$ & $8.14 \mathrm{E}+00$ & $4.846 \mathrm{E}+01$ & $4.255 \mathrm{E}+00$ & 7.07E-01 \\
\hline $1.205 \mathrm{E}+04$ & $8.606 \mathrm{E}+00$ & $1.93 \mathrm{E}-02$ & $2.427 \mathrm{E}+01$ & $8.16 \mathrm{E}+00$ & $4.881 \mathrm{E}+01$ & $3.891 \mathrm{E}+00$ & 7.12E-01 \\
\hline $1.201 \mathrm{E}+04$ & $1.000 \mathrm{E}+01$ & $2.11 \mathrm{E}-02$ & $1.735 \mathrm{E}+01$ & $8.19 \mathrm{E}+00$ & $4.894 \mathrm{E}+01$ & $3.322 \mathrm{E}+00$ & $7.03 \mathrm{E}-01$ \\
\hline$P_{\mathrm{e}}(\mathrm{W})$ & $\Delta P_{\mathrm{e}}(\mathrm{W})$ & $C_{\mathrm{T}}$ & $\Delta C_{\mathrm{T}}$ & $C_{\mathrm{P}}$ & $\Delta C_{\mathrm{P}}$ & $C_{\mathrm{Q}}$ & $\Delta C_{\mathrm{Q}}$ \\
\hline $1.400 \mathrm{E}+01$ & $1.67 \mathrm{E}-01$ & $1.034 \mathrm{E}-01$ & $1.60 \mathrm{E}-02$ & $5.358 \mathrm{E}-02$ & $7.08 \mathrm{E}-03$ & $8.527 \mathrm{E}-03$ & $1.13 \mathrm{E}-03$ \\
\hline $1.334 \mathrm{E}+01$ & $1.60 \mathrm{E}-01$ & $9.596 \mathrm{E}-02$ & $1.61 \mathrm{E}-02$ & $5.118 \mathrm{E}-02$ & $7.05 \mathrm{E}-03$ & $8.146 \mathrm{E}-03$ & $1.12 \mathrm{E}-03$ \\
\hline $1.303 \mathrm{E}+01$ & $1.56 \mathrm{E}-01$ & $8.350 \mathrm{E}-02$ & $1.59 \mathrm{E}-02$ & 4.859E-02 & 7.03E-03 & 7.733E-03 & $1.12 \mathrm{E}-03$ \\
\hline $1.238 \mathrm{E}+01$ & $1.49 \mathrm{E}-01$ & $7.021 \mathrm{E}-02$ & $1.61 \mathrm{E}-02$ & 4.591E-02 & $7.15 \mathrm{E}-03$ & 7.307E-03 & $1.14 \mathrm{E}-03$ \\
\hline $1.173 \mathrm{E}+01$ & $1.43 \mathrm{E}-01$ & $5.997 \mathrm{E}-02$ & $1.61 \mathrm{E}-02$ & $4.218 \mathrm{E}-02$ & 7.01E-03 & $6.714 \mathrm{E}-03$ & $1.12 \mathrm{E}-03$ \\
\hline $1.104 \mathrm{E}+01$ & $1.36 \mathrm{E}-01$ & $4.773 \mathrm{E}-02$ & $1.61 \mathrm{E}-02$ & $3.826 \mathrm{E}-02$ & $7.00 \mathrm{E}-03$ & $6.090 \mathrm{E}-03$ & $1.11 \mathrm{E}-03$ \\
\hline $1.019 \mathrm{E}+01$ & $1.26 \mathrm{E}-01$ & $3.434 \mathrm{E}-02$ & $1.62 \mathrm{E}-02$ & $3.296 \mathrm{E}-02$ & $6.97 \mathrm{E}-03$ & $5.246 \mathrm{E}-03$ & $1.11 \mathrm{E}-03$ \\
\hline$\rho\left(\mathrm{kg} / \mathrm{m}^{\wedge} 3\right)$ & $\eta_{\mathrm{T}}$ & $\Delta \eta_{\mathrm{T}}$ & $J$ & $\Delta J$ & $\eta_{\mathrm{P}}$ & $\Delta \eta_{\mathrm{P}}$ & $R e_{0.75}$ \\
\hline $1.163 \mathrm{E}+00$ & $3.922 \mathrm{E}-01$ & $5.20 \mathrm{E}-02$ & $9.969 \mathrm{E}-02$ & $6.18 \mathrm{E}-04$ & $1.924 \mathrm{E}-01$ & $3.91 \mathrm{E}-02$ & $3.812 \mathrm{E}+04$ \\
\hline $1.163 \mathrm{E}+00$ & $3.865 \mathrm{E}-01$ & $5.34 \mathrm{E}-02$ & $1.609 \mathrm{E}-01$ & $6.29 \mathrm{E}-04$ & $3.017 \mathrm{E}-01$ & $6.55 \mathrm{E}-02$ & $3.797 \mathrm{E}+04$ \\
\hline $1.163 \mathrm{E}+00$ & $3.826 \mathrm{E}-01$ & $5.56 \mathrm{E}-02$ & $2.229 \mathrm{E}-01$ & $6.84 \mathrm{E}-04$ & $3.830 \mathrm{E}-01$ & $9.16 \mathrm{E}-02$ & $3.829 \mathrm{E}+04$ \\
\hline $1.163 \mathrm{E}+00$ & $3.758 \mathrm{E}-01$ & $5.87 \mathrm{E}-02$ & $2.894 \mathrm{E}-01$ & 7.73E-04 & 4.427E-01 & $1.23 \mathrm{E}-01$ & $3.823 \mathrm{E}+04$ \\
\hline $1.162 \mathrm{E}+00$ & $3.626 \mathrm{E}-01$ & $6.04 \mathrm{E}-02$ & $3.573 \mathrm{E}-01$ & $8.70 \mathrm{E}-04$ & $5.080 \mathrm{E}-01$ & $1.60 \mathrm{E}-01$ & $3.830 \mathrm{E}+04$ \\
\hline $1.162 \mathrm{E}+00$ & $3.523 \mathrm{E}-01$ & $6.46 \mathrm{E}-02$ & 4.219E-01 & $9.66 \mathrm{E}-04$ & $5.263 \mathrm{E}-01$ & $2.02 \mathrm{E}-01$ & $3.856 \mathrm{E}+04$ \\
\hline $1.162 \mathrm{E}+00$ & $3.261 \mathrm{E}-01$ & $6.91 \mathrm{E}-02$ & 4.919E-01 & $1.06 \mathrm{E}-03$ & $5.125 \mathrm{E}-01$ & $2.65 \mathrm{E}-01$ & $3.865 \mathrm{E}+04$ \\
\hline
\end{tabular}


Table 240: GWS 4.0 x 2.5 Dynamic Measured Values - 16020 RPM

\begin{tabular}{|c|c|c|c|c|c|c|c|}
\hline$n(\mathrm{RPM})$ & $Q(\mathrm{~g}-\mathrm{m})$ & $\Delta Q(\mathrm{~g}-\mathrm{m})$ & $V(\mathrm{~V})$ & $I(\mathrm{~A})$ & $T_{\text {atm }}\left({ }^{\circ} \mathrm{C}\right)$ & $P_{\text {atm }}(\mathrm{Pa})$ & $P_{\text {diff }}(\mathrm{Pa})$ \\
\hline $1.608 \mathrm{E}+04$ & $7.886 \mathrm{E}-01$ & $6.37 \mathrm{E}-02$ & $1.105 \mathrm{E}+01$ & $2.276 \mathrm{E}+00$ & $2.211 \mathrm{E}+01$ & $9.854 \mathrm{E}+04$ & $2.689 \mathrm{E}+00$ \\
\hline $1.603 \mathrm{E}+04$ & $7.728 \mathrm{E}-01$ & $6.26 \mathrm{E}-02$ & $1.105 \mathrm{E}+01$ & $2.202 \mathrm{E}+00$ & $2.203 \mathrm{E}+01$ & $9.854 \mathrm{E}+04$ & $6.699 \mathrm{E}+00$ \\
\hline $1.602 \mathrm{E}+04$ & $7.400 \mathrm{E}-01$ & $6.24 \mathrm{E}-02$ & $1.106 \mathrm{E}+01$ & $2.135 \mathrm{E}+00$ & $2.208 \mathrm{E}+01$ & $9.853 \mathrm{E}+04$ & $1.268 \mathrm{E}+01$ \\
\hline $1.599 \mathrm{E}+04$ & $6.981 \mathrm{E}-01$ & $6.18 \mathrm{E}-02$ & $1.106 \mathrm{E}+01$ & $2.040 \mathrm{E}+00$ & $2.215 \mathrm{E}+01$ & $9.853 \mathrm{E}+04$ & $2.080 \mathrm{E}+01$ \\
\hline $1.603 \mathrm{E}+04$ & $6.622 \mathrm{E}-01$ & $6.24 \mathrm{E}-02$ & $1.106 \mathrm{E}+01$ & $1.964 \mathrm{E}+00$ & $2.222 \mathrm{E}+01$ & $9.853 \mathrm{E}+04$ & $3.122 \mathrm{E}+01$ \\
\hline $1.601 \mathrm{E}+04$ & $6.284 \mathrm{E}-01$ & $6.21 \mathrm{E}-02$ & $1.106 \mathrm{E}+01$ & $1.870 \mathrm{E}+00$ & $2.231 \mathrm{E}+01$ & $9.853 \mathrm{E}+04$ & $4.353 \mathrm{E}+01$ \\
\hline $1.601 \mathrm{E}+04$ & $5.760 \mathrm{E}-01$ & $6.19 \mathrm{E}-02$ & $1.107 \mathrm{E}+01$ & $1.772 \mathrm{E}+00$ & $2.234 \mathrm{E}+01$ & $9.852 \mathrm{E}+04$ & $5.875 \mathrm{E}+01$ \\
\hline $1.603 \mathrm{E}+04$ & $5.327 \mathrm{E}-01$ & $6.17 \mathrm{E}-02$ & $1.107 \mathrm{E}+01$ & $1.665 \mathrm{E}+00$ & $2.236 \mathrm{E}+01$ & $9.851 \mathrm{E}+04$ & $7.608 \mathrm{E}+01$ \\
\hline $1.599 \mathrm{E}+04$ & $4.861 \mathrm{E}-01$ & $6.18 \mathrm{E}-02$ & $1.107 \mathrm{E}+01$ & $1.543 \mathrm{E}+00$ & $2.240 \mathrm{E}+01$ & $9.851 \mathrm{E}+04$ & $9.553 \mathrm{E}+01$ \\
\hline
\end{tabular}


Table 241: GWS 4.0 x 2.5 Dynamic Calculated Values - 16020 RPM

\begin{tabular}{|c|c|c|c|c|c|c|c|}
\hline$n(\mathrm{RPM})$ & $V_{\infty}^{\prime}(\mathrm{m} / \mathrm{s})$ & $\Delta V_{\infty}^{\prime}(\mathrm{m} / \mathrm{s})$ & $T^{\prime}(\mathrm{g})$ & $\Delta T^{\prime}(\mathrm{g})$ & $V_{\mathrm{t}}(\mathrm{m} / \mathrm{s})$ & $P_{\mathrm{P}}(\mathrm{W})$ & $\Delta P_{\mathrm{P}}(\mathrm{W})$ \\
\hline $1.608 \mathrm{E}+04$ & $2.075 \mathrm{E}+00$ & $1.42 \mathrm{E}-02$ & $9.577 \mathrm{E}+01$ & $7.51 \mathrm{E}+00$ & $6.415 \mathrm{E}+01$ & $1.302 \mathrm{E}+01$ & $1.05 \mathrm{E}+00$ \\
\hline $1.603 \mathrm{E}+04$ & $3.322 \mathrm{E}+00$ & $1.45 \mathrm{E}-02$ & $9.002 \mathrm{E}+01$ & $7.57 \mathrm{E}+00$ & $6.399 \mathrm{E}+01$ & $1.272 \mathrm{E}+01$ & $1.03 \mathrm{E}+00$ \\
\hline $1.602 \mathrm{E}+04$ & $4.603 \mathrm{E}+00$ & $1.52 \mathrm{E}-02$ & $8.432 \mathrm{E}+01$ & $7.54 \mathrm{E}+00$ & $6.405 \mathrm{E}+01$ & $1.217 \mathrm{E}+01$ & $1.03 \mathrm{E}+00$ \\
\hline $1.599 \mathrm{E}+04$ & $5.921 \mathrm{E}+00$ & $1.67 \mathrm{E}-02$ & $7.562 \mathrm{E}+01$ & $7.56 \mathrm{E}+00$ & $6.403 \mathrm{E}+01$ & $1.146 \mathrm{E}+01$ & $1.01 \mathrm{E}+00$ \\
\hline $1.603 \mathrm{E}+04$ & $7.275 \mathrm{E}+00$ & $1.85 \mathrm{E}-02$ & $6.802 \mathrm{E}+01$ & $7.55 \mathrm{E}+00$ & $6.432 \mathrm{E}+01$ & $1.090 \mathrm{E}+01$ & $1.03 \mathrm{E}+00$ \\
\hline $1.601 \mathrm{E}+04$ & $8.609 \mathrm{E}+00$ & 2.02E-02 & $6.012 \mathrm{E}+01$ & $7.62 \mathrm{E}+00$ & $6.443 \mathrm{E}+01$ & $1.033 \mathrm{E}+01$ & $1.02 \mathrm{E}+00$ \\
\hline $1.601 \mathrm{E}+04$ & $1.002 \mathrm{E}+01$ & $2.13 \mathrm{E}-02$ & $5.098 \mathrm{E}+01$ & $7.58 \mathrm{E}+00$ & $6.463 \mathrm{E}+01$ & $9.470 \mathrm{E}+00$ & $1.02 \mathrm{E}+00$ \\
\hline $1.603 \mathrm{E}+04$ & $1.141 \mathrm{E}+01$ & $2.35 \mathrm{E}-02$ & $4.181 \mathrm{E}+01$ & $7.55 \mathrm{E}+00$ & $6.493 \mathrm{E}+01$ & $8.769 \mathrm{E}+00$ & $1.02 \mathrm{E}+00$ \\
\hline $1.599 \mathrm{E}+04$ & $1.280 \mathrm{E}+01$ & $2.62 \mathrm{E}-02$ & $3.324 \mathrm{E}+01$ & $7.63 \mathrm{E}+00$ & $6.504 \mathrm{E}+01$ & $7.983 \mathrm{E}+00$ & $1.02 \mathrm{E}+00$ \\
\hline$P_{\mathrm{e}}(\mathrm{W})$ & $\Delta P_{\mathrm{e}}(\mathrm{W})$ & $C_{\mathrm{T}}$ & $\Delta C_{\mathrm{T}}$ & $C_{\mathrm{P}}$ & $\Delta C_{\mathrm{P}}$ & $C_{\mathrm{Q}}$ & $\Delta C_{\mathrm{Q}}$ \\
\hline $2.515 \mathrm{E}+01$ & 2.91E-01 & $1.057 \mathrm{E}-01$ & $8.29 \mathrm{E}-03$ & 5.387E-02 & $4.35 \mathrm{E}-03$ & 8.574E-03 & $6.92 \mathrm{E}-04$ \\
\hline $2.435 \mathrm{E}+01$ & $2.78 \mathrm{E}-01$ & $1.000 \mathrm{E}-01$ & $8.41 \mathrm{E}-03$ & $5.312 \mathrm{E}-02$ & $4.30 \mathrm{E}-03$ & $8.455 \mathrm{E}-03$ & $6.85 \mathrm{E}-04$ \\
\hline $2.360 \mathrm{E}+01$ & $2.71 \mathrm{E}-01$ & $9.378 \mathrm{E}-02$ & $8.39 \mathrm{E}-03$ & $5.092 \mathrm{E}-02$ & $4.29 \mathrm{E}-03$ & $8.104 \mathrm{E}-03$ & $6.83 \mathrm{E}-04$ \\
\hline $2.256 \mathrm{E}+01$ & $2.59 \mathrm{E}-01$ & 8.444E-02 & 8.44E-03 & 4.823E-02 & 4.27E-03 & 7.677E-03 & $6.79 \mathrm{E}-04$ \\
\hline $2.173 \mathrm{E}+01$ & $2.52 \mathrm{E}-01$ & $7.563 \mathrm{E}-02$ & $8.40 \mathrm{E}-03$ & $4.555 \mathrm{E}-02$ & $4.29 \mathrm{E}-03$ & $7.250 \mathrm{E}-03$ & $6.83 \mathrm{E}-04$ \\
\hline $2.069 \mathrm{E}+01$ & $2.40 \mathrm{E}-01$ & $6.697 \mathrm{E}-02$ & $8.49 \mathrm{E}-03$ & $4.331 \mathrm{E}-02$ & $4.28 \mathrm{E}-03$ & $6.893 \mathrm{E}-03$ & $6.81 \mathrm{E}-04$ \\
\hline $1.961 \mathrm{E}+01$ & $2.28 \mathrm{E}-01$ & $5.682 \mathrm{E}-02$ & $8.45 \mathrm{E}-03$ & $3.971 \mathrm{E}-02$ & 4.27E-03 & $6.321 \mathrm{E}-03$ & $6.79 \mathrm{E}-04$ \\
\hline $1.843 \mathrm{E}+01$ & $2.15 \mathrm{E}-01$ & $4.650 \mathrm{E}-02$ & $8.40 \mathrm{E}-03$ & $3.665 \mathrm{E}-02$ & $4.25 \mathrm{E}-03$ & $5.833 \mathrm{E}-03$ & $6.76 \mathrm{E}-04$ \\
\hline $1.708 \mathrm{E}+01$ & $1.99 \mathrm{E}-01$ & $3.714 \mathrm{E}-02$ & $8.52 \mathrm{E}-03$ & $3.360 \mathrm{E}-02$ & $4.28 \mathrm{E}-03$ & $5.348 \mathrm{E}-03$ & $6.81 \mathrm{E}-04$ \\
\hline$\rho\left(\mathrm{kg} / \mathrm{m}^{\wedge} 3\right)$ & $\eta_{\mathrm{T}}$ & $\Delta \eta_{\mathrm{T}}$ & $J$ & $\Delta J$ & $\eta_{\mathrm{P}}$ & $\Delta \eta_{\mathrm{P}}$ & $R e_{0.75}$ \\
\hline $1.163 \mathrm{E}+00$ & $5.176 \mathrm{E}-01$ & $4.22 \mathrm{E}-02$ & $7.623 \mathrm{E}-02$ & $5.21 \mathrm{E}-04$ & $1.496 \mathrm{E}-01$ & $1.69 \mathrm{E}-02$ & $5.073 \mathrm{E}+04$ \\
\hline $1.163 \mathrm{E}+00$ & $5.223 \mathrm{E}-01$ & 4.27E-02 & $1.225 \mathrm{E}-01$ & 5.34E-04 & $2.306 \mathrm{E}-01$ & $2.69 \mathrm{E}-02$ & $5.063 \mathrm{E}+04$ \\
\hline $1.163 \mathrm{E}+00$ & $5.158 \mathrm{E}-01$ & 4.39E-02 & $1.698 \mathrm{E}-01$ & $5.64 \mathrm{E}-04$ & $3.127 \mathrm{E}-01$ & $3.85 \mathrm{E}-02$ & $5.066 \mathrm{E}+04$ \\
\hline $1.162 \mathrm{E}+00$ & $5.082 \mathrm{E}-01$ & $4.53 \mathrm{E}-02$ & $2.188 \mathrm{E}-01$ & $6.19 \mathrm{E}-04$ & $3.830 \mathrm{E}-01$ & $5.11 \mathrm{E}-02$ & $5.063 \mathrm{E}+04$ \\
\hline $1.162 \mathrm{E}+00$ & $5.016 \mathrm{E}-01$ & $4.76 \mathrm{E}-02$ & $2.682 \mathrm{E}-01$ & $6.84 \mathrm{E}-04$ & 4.453E-01 & $6.48 \mathrm{E}-02$ & $5.083 \mathrm{E}+04$ \\
\hline $1.162 \mathrm{E}+00$ & 4.996E-01 & 4.97E-02 & $3.176 \mathrm{E}-01$ & $7.50 \mathrm{E}-04$ & 4.911E-01 & 7.89E-02 & $5.089 \mathrm{E}+04$ \\
\hline $1.161 \mathrm{E}+00$ & $4.829 \mathrm{E}-01$ & $5.22 \mathrm{E}-02$ & $3.697 \mathrm{E}-01$ & 7.89E-04 & $5.288 \mathrm{E}-01$ & $9.70 \mathrm{E}-02$ & $5.103 \mathrm{E}+04$ \\
\hline $1.161 \mathrm{E}+00$ & $4.758 \mathrm{E}-01$ & $5.54 \mathrm{E}-02$ & 4.207E-01 & $8.71 \mathrm{E}-04$ & 5.337E-01 & $1.15 \mathrm{E}-01$ & $5.127 \mathrm{E}+04$ \\
\hline $1.161 \mathrm{E}+00$ & 4.673E-01 & 5.97E-02 & 4.730E-01 & $9.75 \mathrm{E}-04$ & $5.228 \mathrm{E}-01$ & $1.37 \mathrm{E}-01$ & $5.134 \mathrm{E}+04$ \\
\hline
\end{tabular}


Table 242: GWS 4.0 x 2.5 Dynamic Measured Values - 20020 RPM

\begin{tabular}{|c|c|c|c|c|c|c|c|}
\hline$n(\mathrm{RPM})$ & $Q(\mathrm{~g}-\mathrm{m})$ & $\Delta Q(\mathrm{~g}-\mathrm{m})$ & $V(\mathrm{~V})$ & $I(\mathrm{~A})$ & $T_{\text {atm }}\left({ }^{\circ} \mathrm{C}\right)$ & $P_{\text {atm }}(\mathrm{Pa})$ & $P_{\text {diff }}(\mathrm{Pa})$ \\
\hline $2.011 \mathrm{E}+04$ & $1.208 \mathrm{E}+00$ & $1.30 \mathrm{E}-01$ & $1.101 \mathrm{E}+01$ & $3.784 \mathrm{E}+00$ & $2.197 \mathrm{E}+01$ & $9.847 \mathrm{E}+04$ & $3.069 \mathrm{E}+00$ \\
\hline $2.001 \mathrm{E}+04$ & $1.143 \mathrm{E}+00$ & $1.20 \mathrm{E}-01$ & $1.101 \mathrm{E}+01$ & $3.623 \mathrm{E}+00$ & $2.200 \mathrm{E}+01$ & $9.847 \mathrm{E}+04$ & $8.909 \mathrm{E}+00$ \\
\hline $1.996 \mathrm{E}+04$ & $1.111 \mathrm{E}+00$ & $1.16 \mathrm{E}-01$ & $1.102 \mathrm{E}+01$ & $3.480 \mathrm{E}+00$ & $2.210 \mathrm{E}+01$ & $9.846 \mathrm{E}+04$ & $1.840 \mathrm{E}+01$ \\
\hline $1.998 \mathrm{E}+04$ & $1.023 \mathrm{E}+00$ & $1.16 \mathrm{E}-01$ & $1.102 \mathrm{E}+01$ & $3.315 \mathrm{E}+00$ & $2.218 \mathrm{E}+01$ & $9.846 \mathrm{E}+04$ & $3.178 \mathrm{E}+01$ \\
\hline $2.002 \mathrm{E}+04$ & $9.646 \mathrm{E}-01$ & $1.19 \mathrm{E}-01$ & $1.103 \mathrm{E}+01$ & $3.132 \mathrm{E}+00$ & $2.222 \mathrm{E}+01$ & $9.845 \mathrm{E}+04$ & $4.879 \mathrm{E}+01$ \\
\hline $1.999 \mathrm{E}+04$ & $8.177 \mathrm{E}-01$ & $1.14 \mathrm{E}-01$ & $1.103 \mathrm{E}+01$ & $2.915 \mathrm{E}+00$ & $2.225 \mathrm{E}+01$ & $9.846 \mathrm{E}+04$ & $7.053 \mathrm{E}+01$ \\
\hline $2.004 \mathrm{E}+04$ & $8.164 \mathrm{E}-01$ & $1.17 \mathrm{E}-01$ & $1.104 \mathrm{E}+01$ & $2.733 \mathrm{E}+00$ & $2.228 \mathrm{E}+01$ & $9.845 \mathrm{E}+04$ & $9.619 \mathrm{E}+01$ \\
\hline $2.004 \mathrm{E}+04$ & $7.659 \mathrm{E}-01$ & $1.14 \mathrm{E}-01$ & $1.105 \mathrm{E}+01$ & $2.509 \mathrm{E}+00$ & $2.230 \mathrm{E}+01$ & $9.845 \mathrm{E}+04$ & $1.258 \mathrm{E}+02$ \\
\hline $2.001 \mathrm{E}+04$ & $6.590 \mathrm{E}-01$ & $1.11 \mathrm{E}-01$ & $1.105 \mathrm{E}+01$ & $2.254 \mathrm{E}+00$ & $2.242 \mathrm{E}+01$ & $9.845 \mathrm{E}+04$ & $1.594 \mathrm{E}+02$ \\
\hline
\end{tabular}


Table 243: GWS 4.0 x 2.5 Dynamic Calculated Values - 20020 RPM

\begin{tabular}{|c|c|c|c|c|c|c|c|}
\hline$n(\mathrm{RPM})$ & $V_{\infty}^{\prime}(\mathrm{m} / \mathrm{s})$ & $\Delta V_{\infty}^{\prime}(\mathrm{m} / \mathrm{s})$ & $T^{\prime}(\mathrm{g})$ & $\Delta T^{\prime}(\mathrm{g})$ & $V_{\mathrm{t}}(\mathrm{m} / \mathrm{s})$ & $P_{\mathrm{P}}(\mathrm{W})$ & $\Delta P_{\mathrm{P}}(\mathrm{W})$ \\
\hline $2.011 \mathrm{E}+04$ & $2.201 \mathrm{E}+00$ & $1.66 \mathrm{E}-02$ & $1.528 \mathrm{E}+02$ & $7.02 \mathrm{E}+00$ & $8.024 \mathrm{E}+01$ & $2.496 \mathrm{E}+01$ & $2.68 \mathrm{E}+00$ \\
\hline $2.001 \mathrm{E}+04$ & $3.824 \mathrm{E}+00$ & $1.65 \mathrm{E}-02$ & $1.423 \mathrm{E}+02$ & $7.03 \mathrm{E}+00$ & $7.989 \mathrm{E}+01$ & $2.349 \mathrm{E}+01$ & $2.47 \mathrm{E}+00$ \\
\hline $1.996 \mathrm{E}+04$ & $5.542 \mathrm{E}+00$ & $1.81 \mathrm{E}-02$ & $1.317 \mathrm{E}+02$ & $7.08 \mathrm{E}+00$ & $7.980 \mathrm{E}+01$ & $2.278 \mathrm{E}+01$ & $2.38 \mathrm{E}+00$ \\
\hline $1.998 \mathrm{E}+04$ & $7.320 \mathrm{E}+00$ & $1.98 \mathrm{E}-02$ & $1.193 \mathrm{E}+02$ & $7.05 \mathrm{E}+00$ & $8.000 \mathrm{E}+01$ & $2.098 \mathrm{E}+01$ & $2.38 \mathrm{E}+00$ \\
\hline $2.002 \mathrm{E}+04$ & $9.099 \mathrm{E}+00$ & $2.25 \mathrm{E}-02$ & $1.057 \mathrm{E}+02$ & $7.01 \mathrm{E}+00$ & $8.037 \mathrm{E}+01$ & $1.984 \mathrm{E}+01$ & $2.44 \mathrm{E}+00$ \\
\hline $1.999 \mathrm{E}+04$ & $1.096 \mathrm{E}+01$ & $2.42 \mathrm{E}-02$ & $9.020 \mathrm{E}+01$ & $7.01 \mathrm{E}+00$ & $8.047 \mathrm{E}+01$ & $1.679 \mathrm{E}+01$ & $2.34 \mathrm{E}+00$ \\
\hline $2.004 \mathrm{E}+04$ & $1.283 \mathrm{E}+01$ & 2.69E-02 & $7.619 \mathrm{E}+01$ & $6.98 \mathrm{E}+00$ & $8.096 \mathrm{E}+01$ & $1.681 \mathrm{E}+01$ & $2.41 \mathrm{E}+00$ \\
\hline $2.004 \mathrm{E}+04$ & $1.468 \mathrm{E}+01$ & $2.95 \mathrm{E}-02$ & $6.026 \mathrm{E}+01$ & $6.99 \mathrm{E}+00$ & $8.125 \mathrm{E}+01$ & $1.576 \mathrm{E}+01$ & $2.35 \mathrm{E}+00$ \\
\hline $2.001 \mathrm{E}+04$ & $1.655 \mathrm{E}+01$ & $3.26 \mathrm{E}-02$ & $4.341 \mathrm{E}+01$ & $7.01 \mathrm{E}+00$ & $8.151 \mathrm{E}+01$ & $1.355 \mathrm{E}+01$ & $2.28 \mathrm{E}+00$ \\
\hline$P_{\mathrm{e}}(\mathrm{W})$ & $\Delta P_{\mathrm{e}}(\mathrm{W})$ & $C_{\mathrm{T}}$ & $\Delta C_{\mathrm{T}}$ & $C_{\mathrm{P}}$ & $\Delta C_{\mathrm{P}}$ & $C_{\mathrm{Q}}$ & $\Delta C_{\mathrm{Q}}$ \\
\hline $4.166 \mathrm{E}+01$ & 4.91E-01 & $1.078 \mathrm{E}-01$ & $4.96 \mathrm{E}-03$ & $5.274 \mathrm{E}-02$ & $5.66 \mathrm{E}-03$ & $8.393 \mathrm{E}-03$ & $9.01 \mathrm{E}-04$ \\
\hline $3.990 \mathrm{E}+01$ & $4.54 \mathrm{E}-01$ & $1.014 \mathrm{E}-01$ & $5.01 \mathrm{E}-03$ & $5.041 \mathrm{E}-02$ & $5.29 \mathrm{E}-03$ & $8.023 \mathrm{E}-03$ & $8.42 \mathrm{E}-04$ \\
\hline $3.834 \mathrm{E}+01$ & 4.42E-01 & $9.438 \mathrm{E}-02$ & $5.08 \mathrm{E}-03$ & 4.927E-02 & $5.14 \mathrm{E}-03$ & 7.842E-03 & $8.18 \mathrm{E}-04$ \\
\hline $3.654 \mathrm{E}+01$ & $4.19 \mathrm{E}-01$ & $8.541 \mathrm{E}-02$ & $5.05 \mathrm{E}-03$ & $4.531 \mathrm{E}-02$ & $5.14 \mathrm{E}-03$ & $7.211 \mathrm{E}-03$ & $8.17 \mathrm{E}-04$ \\
\hline $3.454 \mathrm{E}+01$ & $3.89 \mathrm{E}-01$ & $7.534 \mathrm{E}-02$ & 4.99E-03 & $4.253 \mathrm{E}-02$ & $5.24 \mathrm{E}-03$ & $6.769 \mathrm{E}-03$ & 8.34E-04 \\
\hline $3.217 \mathrm{E}+01$ & $3.61 \mathrm{E}-01$ & $6.451 \mathrm{E}-02$ & $5.01 \mathrm{E}-03$ & $3.618 \mathrm{E}-02$ & $5.04 \mathrm{E}-03$ & $5.758 \mathrm{E}-03$ & $8.02 \mathrm{E}-04$ \\
\hline $3.016 \mathrm{E}+01$ & $3.40 \mathrm{E}-01$ & $5.421 \mathrm{E}-02$ & $4.97 \mathrm{E}-03$ & $3.593 \mathrm{E}-02$ & $5.15 \mathrm{E}-03$ & $5.719 \mathrm{E}-03$ & $8.19 \mathrm{E}-04$ \\
\hline $2.771 \mathrm{E}+01$ & $3.14 \mathrm{E}-01$ & $4.289 \mathrm{E}-02$ & $4.98 \mathrm{E}-03$ & 3.373E-02 & $5.03 \mathrm{E}-03$ & $5.368 \mathrm{E}-03$ & $8.00 \mathrm{E}-04$ \\
\hline $2.491 \mathrm{E}+01$ & $2.85 \mathrm{E}-01$ & 3.099E-02 & $5.01 \mathrm{E}-03$ & $2.911 \mathrm{E}-02$ & $4.90 \mathrm{E}-03$ & $4.633 \mathrm{E}-03$ & $7.80 \mathrm{E}-04$ \\
\hline$\rho\left(\mathrm{kg} / \mathrm{m}^{\wedge} 3\right)$ & $\eta_{\mathrm{T}}$ & $\Delta \eta_{\mathrm{T}}$ & $J$ & $\Delta J$ & $\eta_{\mathrm{P}}$ & $\Delta \eta_{\mathrm{P}}$ & $R e_{0.75}$ \\
\hline $1.162 \mathrm{E}+00$ & $5.990 \mathrm{E}-01$ & $6.47 \mathrm{E}-02$ & $6.466 \mathrm{E}-02$ & 4.87E-04 & $1.322 \mathrm{E}-01$ & $1.55 \mathrm{E}-02$ & $6.347 \mathrm{E}+04$ \\
\hline $1.162 \mathrm{E}+00$ & $5.886 \mathrm{E}-01$ & $6.22 \mathrm{E}-02$ & $1.129 \mathrm{E}-01$ & 4.89E-04 & $2.272 \mathrm{E}-01$ & $2.64 \mathrm{E}-02$ & $6.318 \mathrm{E}+04$ \\
\hline $1.162 \mathrm{E}+00$ & $5.942 \mathrm{E}-01$ & $6.23 \mathrm{E}-02$ & $1.640 \mathrm{E}-01$ & $5.39 \mathrm{E}-04$ & $3.142 \mathrm{E}-01$ & $3.69 \mathrm{E}-02$ & $6.307 \mathrm{E}+04$ \\
\hline $1.161 \mathrm{E}+00$ & $5.742 \mathrm{E}-01$ & $6.54 \mathrm{E}-02$ & $2.165 \mathrm{E}-01$ & $5.89 \mathrm{E}-04$ & $4.081 \mathrm{E}-01$ & $5.22 \mathrm{E}-02$ & $6.319 \mathrm{E}+04$ \\
\hline $1.161 \mathrm{E}+00$ & 5.743E-01 & $7.11 \mathrm{E}-02$ & 2.684E-01 & $6.68 \mathrm{E}-04$ & $4.755 \mathrm{E}-01$ & $6.66 \mathrm{E}-02$ & $6.347 \mathrm{E}+04$ \\
\hline $1.161 \mathrm{E}+00$ & $5.219 \mathrm{E}-01$ & $7.29 \mathrm{E}-02$ & $3.240 \mathrm{E}-01$ & $7.23 \mathrm{E}-04$ & $5.777 \mathrm{E}-01$ & $9.22 \mathrm{E}-02$ & $6.353 \mathrm{E}+04$ \\
\hline $1.161 \mathrm{E}+00$ & $5.571 \mathrm{E}-01$ & $8.00 \mathrm{E}-02$ & $3.780 \mathrm{E}-01$ & $8.04 \mathrm{E}-04$ & 5.702E-01 & $9.70 \mathrm{E}-02$ & $6.391 \mathrm{E}+04$ \\
\hline $1.161 \mathrm{E}+00$ & $5.688 \mathrm{E}-01$ & $8.50 \mathrm{E}-02$ & 4.329E-01 & $8.83 \mathrm{E}-04$ & $5.506 \mathrm{E}-01$ & $1.04 \mathrm{E}-01$ & $6.413 \mathrm{E}+04$ \\
\hline $1.160 \mathrm{E}+00$ & $5.437 \mathrm{E}-01$ & $9.18 \mathrm{E}-02$ & $4.885 \mathrm{E}-01$ & $9.76 \mathrm{E}-04$ & $5.201 \mathrm{E}-01$ & $1.21 \mathrm{E}-01$ & $6.429 \mathrm{E}+04$ \\
\hline
\end{tabular}




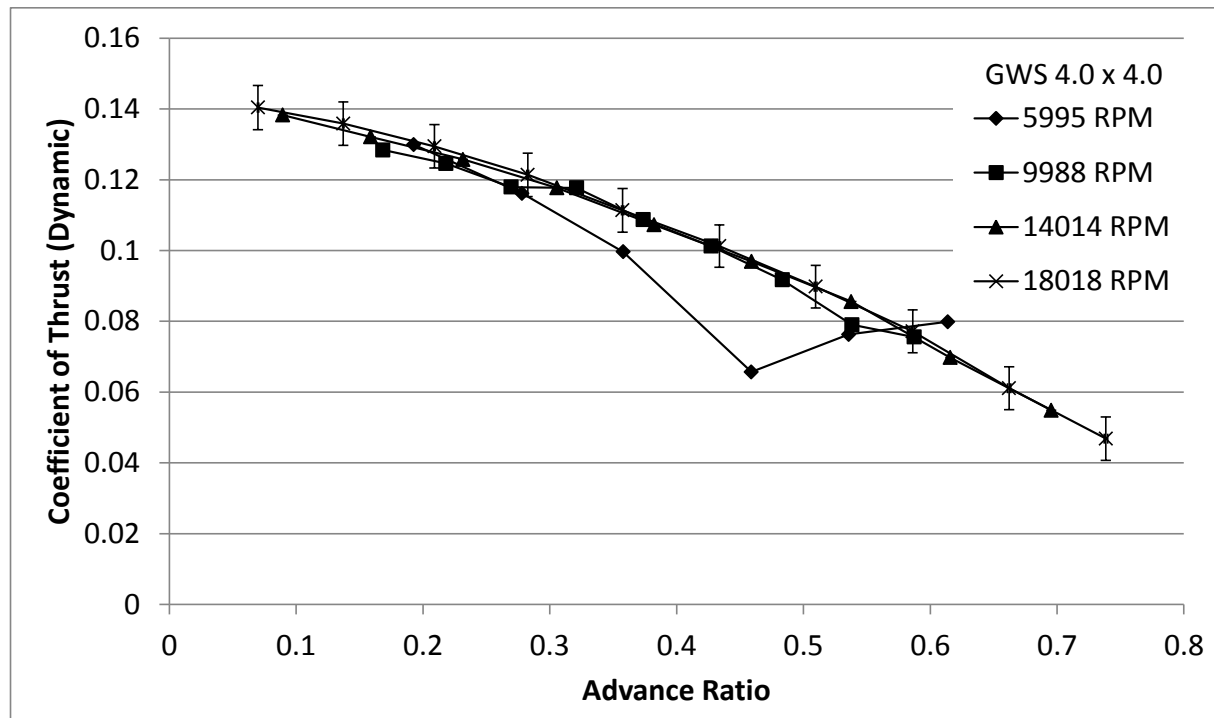

(a)

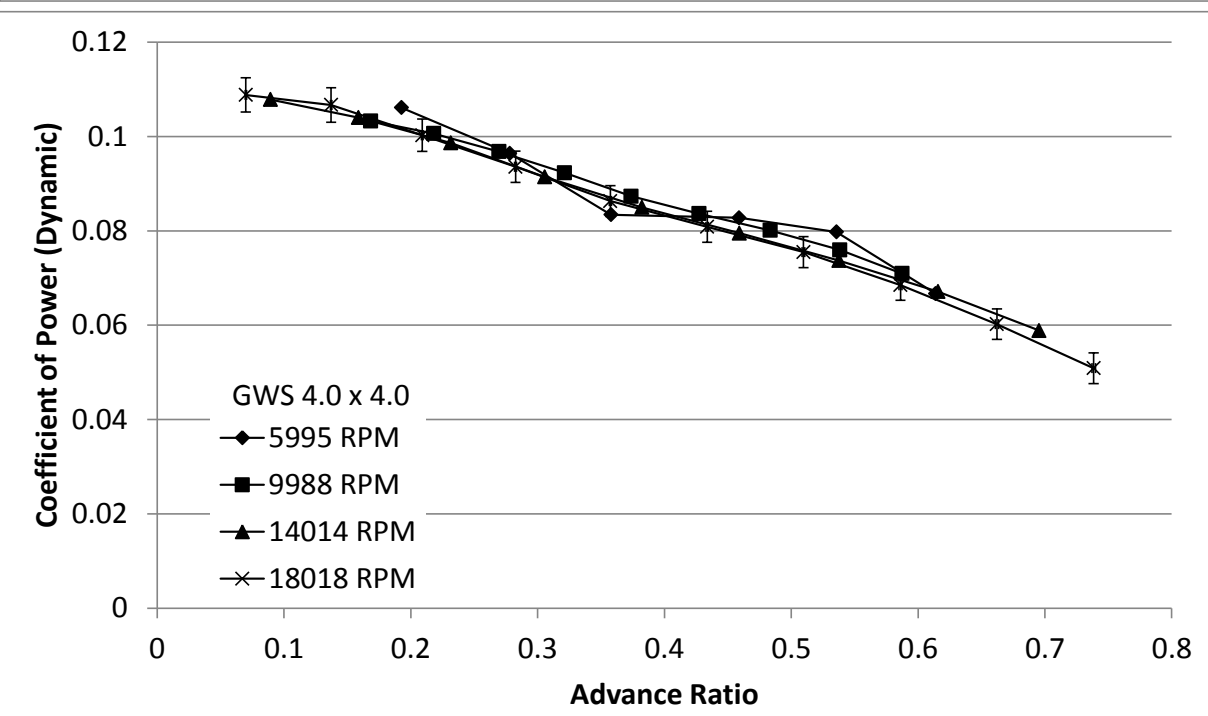

(b)

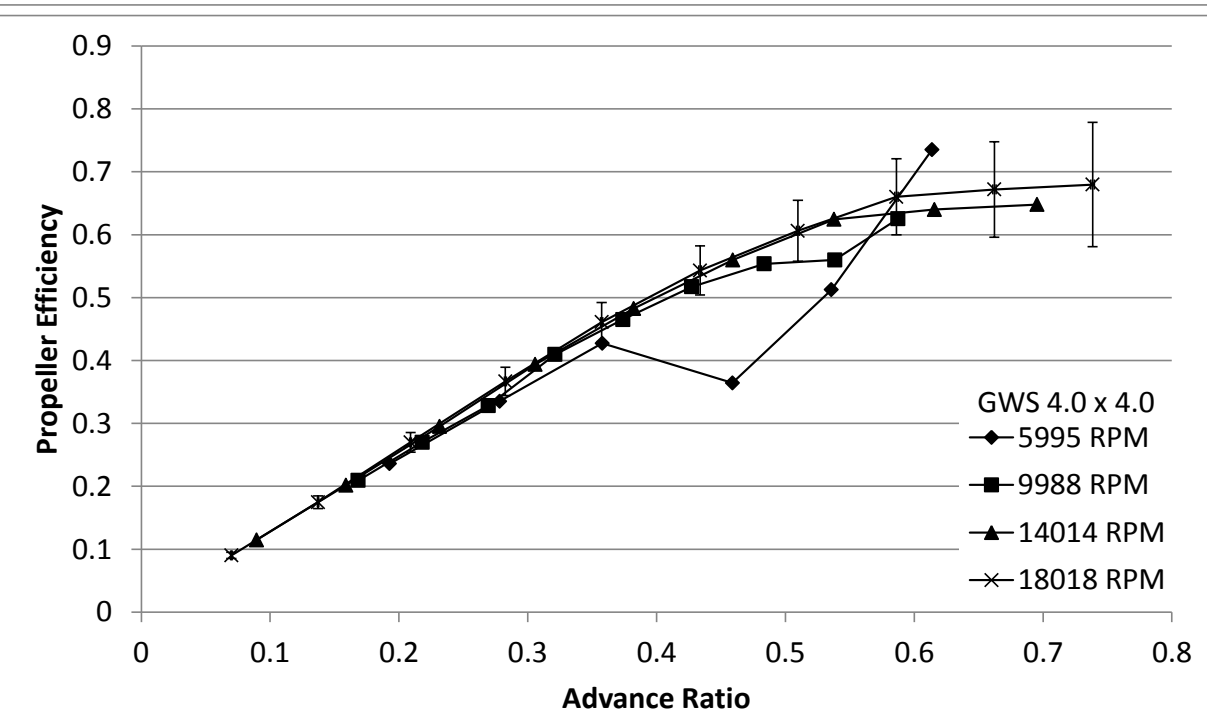

(c) 


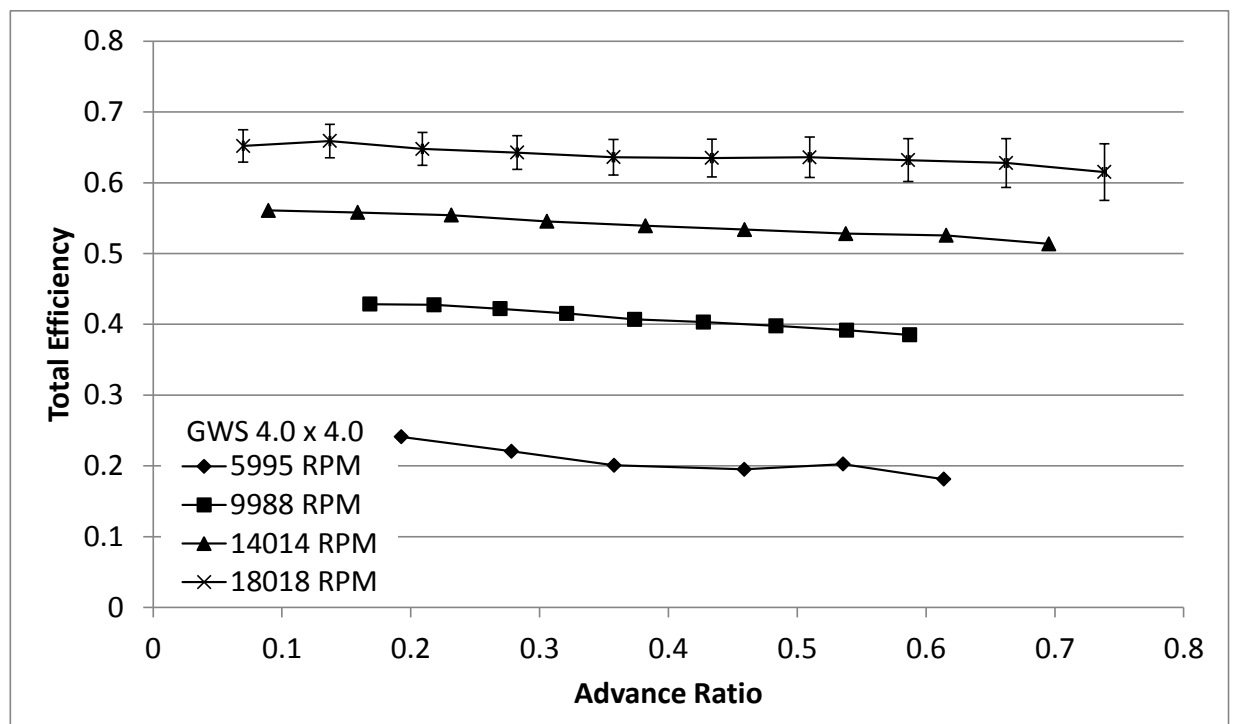

(d)

Figure 99: GWS 4.0 x 4.0 Dynamic Test Results: (a) Coefficient of Thrust, (b)

Coefficient of Power, (c) Propeller Efficiency, (d) Total Efficiency.

Table 244: GWS 4.0 x 4.0 Dynamic Measured Values - 5995 RPM

\begin{tabular}{|c|c|c|c|c|c|c|c|}
\hline$n(\mathrm{RPM})$ & $Q(\mathrm{~g}-\mathrm{m})$ & $\Delta Q(\mathrm{~g}-\mathrm{m})$ & $V(\mathrm{~V})$ & $I(\mathrm{~A})$ & $T_{\text {atm }}\left({ }^{\circ} \mathrm{C}\right)$ & $P_{\text {atm }}(\mathrm{Pa})$ & $P_{\text {diff }}(\mathrm{Pa})$ \\
\hline $6.028 \mathrm{E}+03$ & $2.228 \mathrm{E}-01$ & $7.56 \mathrm{E}-02$ & $1.110 \mathrm{E}+01$ & $5.155 \mathrm{E}-01$ & $2.211 \mathrm{E}+01$ & $9.844 \mathrm{E}+04$ & $2.335 \mathrm{E}+00$ \\
\hline $5.960 \mathrm{E}+03$ & $1.979 \mathrm{E}-01$ & $7.34 \mathrm{E}-02$ & $1.110 \mathrm{E}+01$ & $4.946 \mathrm{E}-01$ & $2.195 \mathrm{E}+01$ & $9.844 \mathrm{E}+04$ & $4.703 \mathrm{E}+00$ \\
\hline $6.054 \mathrm{E}+03$ & $1.767 \mathrm{E}-01$ & $7.42 \mathrm{E}-02$ & $1.110 \mathrm{E}+01$ & $4.927 \mathrm{E}-01$ & $2.202 \mathrm{E}+01$ & $9.843 \mathrm{E}+04$ & $7.975 \mathrm{E}+00$ \\
\hline $5.850 \mathrm{E}+03$ & $1.636 \mathrm{E}-01$ & $6.34 \mathrm{E}-02$ & $1.110 \mathrm{E}+01$ & $4.542 \mathrm{E}-01$ & $2.203 \mathrm{E}+01$ & $9.843 \mathrm{E}+04$ & $1.217 \mathrm{E}+01$ \\
\hline $5.987 \mathrm{E}+03$ & $1.651 \mathrm{E}-01$ & $7.58 \mathrm{E}-02$ & $1.110 \mathrm{E}+01$ & $4.516 \mathrm{E}-01$ & $2.216 \mathrm{E}+01$ & $9.842 \mathrm{E}+04$ & $1.736 \mathrm{E}+01$ \\
\hline $6.091 \mathrm{E}+03$ & $1.429 \mathrm{E}-01$ & $7.22 \mathrm{E}-02$ & $1.110 \mathrm{E}+01$ & $4.446 \mathrm{E}-01$ & $2.226 \mathrm{E}+01$ & $9.841 \mathrm{E}+04$ & $2.355 \mathrm{E}+01$ \\
\hline
\end{tabular}


Table 245: GWS 4.0 x 4.0 Dynamic Calculated Values - 5995 RPM

\begin{tabular}{|c|c|c|c|c|c|c|c|}
\hline$n(\mathrm{RPM})$ & $V_{\infty}^{\prime}(\mathrm{m} / \mathrm{s})$ & $\Delta V_{\infty}^{\prime}(\mathrm{m} / \mathrm{s})$ & $T^{\prime}(\mathrm{g})$ & $\Delta T^{\prime}(\mathrm{g})$ & $V_{\mathrm{t}}(\mathrm{m} / \mathrm{s})$ & $P_{\mathrm{P}}(\mathrm{W})$ & $\Delta P_{\mathrm{P}}(\mathrm{W})$ \\
\hline $6.028 \mathrm{E}+03$ & $1.975 \mathrm{E}+00$ & $1.56 \mathrm{E}-02$ & $1.681 \mathrm{E}+01$ & $1.22 \mathrm{E}+01$ & $2.423 \mathrm{E}+01$ & $1.379 \mathrm{E}+00$ & $4.68 \mathrm{E}-01$ \\
\hline $5.960 \mathrm{E}+03$ & $2.818 \mathrm{E}+00$ & $1.54 \mathrm{E}-02$ & $1.469 \mathrm{E}+01$ & $1.10 \mathrm{E}+01$ & $2.404 \mathrm{E}+01$ & $1.211 \mathrm{E}+00$ & 4.49E-01 \\
\hline $6.054 \mathrm{E}+03$ & $3.682 \mathrm{E}+00$ & $1.81 \mathrm{E}-02$ & $1.300 \mathrm{E}+01$ & $1.27 \mathrm{E}+01$ & $2.453 \mathrm{E}+01$ & $1.098 \mathrm{E}+00$ & 4.62E-01 \\
\hline $5.850 \mathrm{E}+03$ & $4.563 \mathrm{E}+00$ & $1.78 \mathrm{E}-02$ & $8.003 \mathrm{E}+00$ & $9.77 \mathrm{E}+00$ & $2.387 \mathrm{E}+01$ & $9.830 \mathrm{E}-01$ & $3.81 \mathrm{E}-01$ \\
\hline $5.987 \mathrm{E}+03$ & $5.452 \mathrm{E}+00$ & $1.90 \mathrm{E}-02$ & $9.730 \mathrm{E}+00$ & $1.14 \mathrm{E}+01$ & $2.459 \mathrm{E}+01$ & $1.015 \mathrm{E}+00$ & $4.66 \mathrm{E}-01$ \\
\hline $6.091 \mathrm{E}+03$ & $6.356 \mathrm{E}+00$ & $2.06 \mathrm{E}-02$ & $1.054 \mathrm{E}+01$ & $1.31 \mathrm{E}+01$ & $2.521 \mathrm{E}+01$ & 8.937E-01 & $4.51 \mathrm{E}-01$ \\
\hline$P_{\mathrm{e}}(\mathrm{W})$ & $\Delta P_{\mathrm{e}}(\mathrm{W})$ & $C_{\mathrm{T}}$ & $\Delta C_{\mathrm{T}}$ & $C_{\mathrm{P}}$ & $\Delta C_{\mathrm{P}}$ & $C_{\mathrm{Q}}$ & $\Delta C_{\mathrm{Q}}$ \\
\hline $5.723 \mathrm{E}+00$ & $8.14 \mathrm{E}-02$ & $1.299 \mathrm{E}-01$ & $9.46 \mathrm{E}-02$ & $1.061 \mathrm{E}-01$ & $3.60 \mathrm{E}-02$ & $1.689 \mathrm{E}-02$ & $5.73 \mathrm{E}-03$ \\
\hline $5.491 \mathrm{E}+00$ & $8.11 \mathrm{E}-02$ & $1.161 \mathrm{E}-01$ & $8.68 \mathrm{E}-02$ & $9.639 \mathrm{E}-02$ & $3.58 \mathrm{E}-02$ & $1.534 \mathrm{E}-02$ & $5.69 \mathrm{E}-03$ \\
\hline $5.470 \mathrm{E}+00$ & 7.76E-02 & $9.963 \mathrm{E}-02$ & $9.74 \mathrm{E}-02$ & $8.342 \mathrm{E}-02$ & $3.50 \mathrm{E}-02$ & $1.328 \mathrm{E}-02$ & $5.58 \mathrm{E}-03$ \\
\hline $5.043 \mathrm{E}+00$ & 7.56E-02 & $6.569 \mathrm{E}-02$ & $8.02 \mathrm{E}-02$ & $8.275 \mathrm{E}-02$ & $3.20 \mathrm{E}-02$ & $1.317 \mathrm{E}-02$ & $5.10 \mathrm{E}-03$ \\
\hline $5.014 \mathrm{E}+00$ & 7.44E-02 & $7.628 \mathrm{E}-02$ & $8.95 \mathrm{E}-02$ & 7.975E-02 & $3.66 \mathrm{E}-02$ & $1.269 \mathrm{E}-02$ & $5.83 \mathrm{E}-03$ \\
\hline $4.937 \mathrm{E}+00$ & 7.50E-02 & 7.987E-02 & $9.93 \mathrm{E}-02$ & $6.669 \mathrm{E}-02$ & 3.37E-02 & $1.061 \mathrm{E}-02$ & $5.36 \mathrm{E}-03$ \\
\hline$\rho\left(\mathrm{kg} / \mathrm{m}^{\wedge} 3\right)$ & $\eta_{\mathrm{T}}$ & $\Delta \eta_{\mathrm{T}}$ & $J$ & $\Delta J$ & $\eta_{\mathrm{P}}$ & $\Delta \eta_{\mathrm{P}}$ & $R e_{0.75}$ \\
\hline $1.161 \mathrm{E}+00$ & $2.410 \mathrm{E}-01$ & 8.19E-02 & $1.927 \mathrm{E}-01$ & $1.53 \mathrm{E}-03$ & $2.359 \mathrm{E}-01$ & $1.90 \mathrm{E}-01$ & $1.613 \mathrm{E}+04$ \\
\hline $1.162 \mathrm{E}+00$ & $2.206 \mathrm{E}-01$ & 8.19E-02 & $2.782 \mathrm{E}-01$ & $1.53 \mathrm{E}-03$ & $3.352 \mathrm{E}-01$ & $2.80 \mathrm{E}-01$ & $1.602 \mathrm{E}+04$ \\
\hline $1.162 \mathrm{E}+00$ & $2.008 \mathrm{E}-01$ & $8.44 \mathrm{E}-02$ & $3.578 \mathrm{E}-01$ & $1.78 \mathrm{E}-03$ & 4.274E-01 & $4.55 \mathrm{E}-01$ & $1.633 \mathrm{E}+04$ \\
\hline $1.162 \mathrm{E}+00$ & $1.949 \mathrm{E}-01$ & $7.55 \mathrm{E}-02$ & 4.589E-01 & $1.81 \mathrm{E}-03$ & $3.643 \mathrm{E}-01$ & 4.67E-01 & $1.589 \mathrm{E}+04$ \\
\hline $1.161 \mathrm{E}+00$ & $2.025 \mathrm{E}-01$ & $9.30 \mathrm{E}-02$ & $5.357 \mathrm{E}-01$ & $1.89 \mathrm{E}-03$ & $5.124 \mathrm{E}-01$ & $6.45 \mathrm{E}-01$ & $1.636 \mathrm{E}+04$ \\
\hline $1.161 \mathrm{E}+00$ & $1.810 \mathrm{E}-01$ & $9.15 \mathrm{E}-02$ & $6.138 \mathrm{E}-01$ & $2.13 \mathrm{E}-03$ & 7.352E-01 & $9.87 \mathrm{E}-01$ & $1.676 \mathrm{E}+04$ \\
\hline
\end{tabular}


Table 246: GWS 4.0 x 4.0 Dynamic Measured Values - 9988 RPM

\begin{tabular}{|c|c|c|c|c|c|c|c|}
\hline$n(\mathrm{RPM})$ & $Q(\mathrm{~g}-\mathrm{m})$ & $\Delta Q(\mathrm{~g}-\mathrm{m})$ & $V(\mathrm{~V})$ & $I(\mathrm{~A})$ & $T_{\text {atm }}\left({ }^{\circ} \mathrm{C}\right)$ & $P_{\text {atm }}(\mathrm{Pa})$ & $P_{\text {diff }}(\mathrm{Pa})$ \\
\hline $9.972 \mathrm{E}+03$ & $5.934 \mathrm{E}-01$ & $5.67 \mathrm{E}-02$ & $1.108 \mathrm{E}+01$ & $1.280 \mathrm{E}+00$ & $2.203 \mathrm{E}+01$ & $9.841 \mathrm{E}+04$ & $4.902 \mathrm{E}+00$ \\
\hline $9.988 \mathrm{E}+03$ & $5.803 \mathrm{E}-01$ & $5.57 \mathrm{E}-02$ & $1.108 \mathrm{E}+01$ & $1.257 \mathrm{E}+00$ & $2.187 \mathrm{E}+01$ & $9.840 \mathrm{E}+04$ & $8.173 \mathrm{E}+00$ \\
\hline $9.966 \mathrm{E}+03$ & $5.559 \mathrm{E}-01$ & $5.68 \mathrm{E}-02$ & $1.108 \mathrm{E}+01$ & $1.216 \mathrm{E}+00$ & $2.184 \mathrm{E}+01$ & $9.840 \mathrm{E}+04$ & $1.235 \mathrm{E}+01$ \\
\hline $9.984 \mathrm{E}+03$ & $5.318 \mathrm{E}-01$ & $5.56 \mathrm{E}-02$ & $1.108 \mathrm{E}+01$ & $1.184 \mathrm{E}+00$ & $2.189 \mathrm{E}+01$ & $9.839 \mathrm{E}+04$ & $1.753 \mathrm{E}+01$ \\
\hline $1.000 \mathrm{E}+04$ & $5.049 \mathrm{E}-01$ & $5.45 \mathrm{E}-02$ & $1.108 \mathrm{E}+01$ & $1.150 \mathrm{E}+00$ & $2.199 \mathrm{E}+01$ & $9.839 \mathrm{E}+04$ & $2.375 \mathrm{E}+01$ \\
\hline $1.002 \mathrm{E}+04$ & $4.846 \mathrm{E}-01$ & $5.48 \mathrm{E}-02$ & $1.109 \mathrm{E}+01$ & $1.115 \mathrm{E}+00$ & $2.215 \mathrm{E}+01$ & $9.839 \mathrm{E}+04$ & $3.101 \mathrm{E}+01$ \\
\hline $9.964 \mathrm{E}+03$ & $4.592 \mathrm{E}-01$ & $5.56 \mathrm{E}-02$ & $1.109 \mathrm{E}+01$ & $1.066 \mathrm{E}+00$ & $2.223 \mathrm{E}+01$ & $9.838 \mathrm{E}+04$ & $3.920 \mathrm{E}+01$ \\
\hline $9.970 \mathrm{E}+03$ & $4.358 \mathrm{E}-01$ & $5.57 \mathrm{E}-02$ & $1.109 \mathrm{E}+01$ & $1.027 \mathrm{E}+00$ & $2.231 \mathrm{E}+01$ & $9.839 \mathrm{E}+04$ & $4.855 \mathrm{E}+01$ \\
\hline $1.003 \mathrm{E}+04$ & $4.119 \mathrm{E}-01$ & $5.53 \mathrm{E}-02$ & $1.109 \mathrm{E}+01$ & $9.938 \mathrm{E}-01$ & $2.238 \mathrm{E}+01$ & $9.838 \mathrm{E}+04$ & $5.843 \mathrm{E}+01$ \\
\hline
\end{tabular}


Table 247: GWS 4.0 x 4.0 Dynamic Calculated Values - 9988 RPM

\begin{tabular}{|c|c|c|c|c|c|c|c|}
\hline$n(\mathrm{RPM})$ & $V_{\infty}^{\prime}(\mathrm{m} / \mathrm{s})$ & $\Delta V_{\infty}^{\prime}(\mathrm{m} / \mathrm{s})$ & $T^{\prime}(\mathrm{g})$ & $\Delta T^{\prime}(\mathrm{g})$ & $V_{\mathrm{t}}(\mathrm{m} / \mathrm{s})$ & $P_{\mathrm{P}}(\mathrm{W})$ & $\Delta P_{\mathrm{P}}(\mathrm{W})$ \\
\hline $9.972 \mathrm{E}+03$ & $2.855 \mathrm{E}+00$ & $1.25 \mathrm{E}-02$ & $4.544 \mathrm{E}+01$ & $7.71 \mathrm{E}+00$ & $4.004 \mathrm{E}+01$ & $6.077 \mathrm{E}+00$ & $5.81 \mathrm{E}-01$ \\
\hline $9.988 \mathrm{E}+03$ & $3.702 \mathrm{E}+00$ & $1.28 \mathrm{E}-02$ & $4.426 \mathrm{E}+01$ & $7.73 \mathrm{E}+00$ & $4.018 \mathrm{E}+01$ & $5.953 \mathrm{E}+00$ & $5.72 \mathrm{E}-01$ \\
\hline $9.966 \mathrm{E}+03$ & $4.565 \mathrm{E}+00$ & $1.37 \mathrm{E}-02$ & $4.172 \mathrm{E}+01$ & $7.71 \mathrm{E}+00$ & $4.018 \mathrm{E}+01$ & $5.689 \mathrm{E}+00$ & $5.82 \mathrm{E}-01$ \\
\hline $9.984 \mathrm{E}+03$ & $5.450 \mathrm{E}+00$ & $1.50 \mathrm{E}-02$ & $4.177 \mathrm{E}+01$ & $7.73 \mathrm{E}+00$ & $4.036 \mathrm{E}+01$ & $5.452 \mathrm{E}+00$ & $5.70 \mathrm{E}-01$ \\
\hline $1.000 \mathrm{E}+04$ & $6.355 \mathrm{E}+00$ & $1.59 \mathrm{E}-02$ & $3.871 \mathrm{E}+01$ & $7.72 \mathrm{E}+00$ & $4.057 \mathrm{E}+01$ & $5.186 \mathrm{E}+00$ & $5.60 \mathrm{E}-01$ \\
\hline $1.002 \mathrm{E}+04$ & $7.274 \mathrm{E}+00$ & $1.69 \mathrm{E}-02$ & $3.613 \mathrm{E}+01$ & $7.71 \mathrm{E}+00$ & $4.077 \mathrm{E}+01$ & $4.984 \mathrm{E}+00$ & $5.63 \mathrm{E}-01$ \\
\hline $9.964 \mathrm{E}+03$ & $8.188 \mathrm{E}+00$ & $1.81 \mathrm{E}-02$ & $3.239 \mathrm{E}+01$ & $7.72 \mathrm{E}+00$ & $4.074 \mathrm{E}+01$ & $4.699 \mathrm{E}+00$ & $5.69 \mathrm{E}-01$ \\
\hline $9.970 \mathrm{E}+03$ & $9.122 \mathrm{E}+00$ & $1.98 \mathrm{E}-02$ & $2.792 \mathrm{E}+01$ & $7.73 \mathrm{E}+00$ & $4.096 \mathrm{E}+01$ & $4.461 \mathrm{E}+00$ & $5.70 \mathrm{E}-01$ \\
\hline $1.003 \mathrm{E}+04$ & $1.001 \mathrm{E}+01$ & $2.17 \mathrm{E}-02$ & $2.701 \mathrm{E}+01$ & $7.72 \mathrm{E}+00$ & $4.141 \mathrm{E}+01$ & $4.243 \mathrm{E}+00$ & $5.69 \mathrm{E}-01$ \\
\hline$P_{\mathrm{e}}(\mathrm{W})$ & $\Delta P_{\mathrm{e}}(\mathrm{W})$ & $C_{\mathrm{T}}$ & $\Delta C_{\mathrm{T}}$ & $C_{\mathrm{P}}$ & $\Delta C_{\mathrm{P}}$ & $C_{\mathrm{Q}}$ & $\Delta C_{\mathrm{Q}}$ \\
\hline $1.418 \mathrm{E}+01$ & $1.71 \mathrm{E}-01$ & $1.284 \mathrm{E}-01$ & $2.18 \mathrm{E}-02$ & $1.033 \mathrm{E}-01$ & $9.87 \mathrm{E}-03$ & $1.644 \mathrm{E}-02$ & $1.57 \mathrm{E}-03$ \\
\hline $1.393 \mathrm{E}+01$ & $1.66 \mathrm{E}-01$ & $1.246 \mathrm{E}-01$ & $2.18 \mathrm{E}-02$ & $1.006 \mathrm{E}-01$ & $9.67 \mathrm{E}-03$ & $1.602 \mathrm{E}-02$ & $1.54 \mathrm{E}-03$ \\
\hline $1.348 \mathrm{E}+01$ & $1.64 \mathrm{E}-01$ & $1.179 \mathrm{E}-01$ & $2.18 \mathrm{E}-02$ & $9.681 \mathrm{E}-02$ & $9.90 \mathrm{E}-03$ & $1.541 \mathrm{E}-02$ & $1.58 \mathrm{E}-03$ \\
\hline $1.313 \mathrm{E}+01$ & $1.58 \mathrm{E}-01$ & $1.177 \mathrm{E}-01$ & $2.18 \mathrm{E}-02$ & $9.231 \mathrm{E}-02$ & $9.65 \mathrm{E}-03$ & $1.469 \mathrm{E}-02$ & $1.54 \mathrm{E}-03$ \\
\hline $1.274 \mathrm{E}+01$ & $1.54 \mathrm{E}-01$ & $1.087 \mathrm{E}-01$ & $2.17 \mathrm{E}-02$ & 8.734E-02 & $9.43 \mathrm{E}-03$ & $1.390 \mathrm{E}-02$ & $1.50 \mathrm{E}-03$ \\
\hline $1.236 \mathrm{E}+01$ & $1.52 \mathrm{E}-01$ & $1.013 \mathrm{E}-01$ & $2.16 \mathrm{E}-02$ & $8.366 \mathrm{E}-02$ & $9.46 \mathrm{E}-03$ & $1.331 \mathrm{E}-02$ & $1.51 \mathrm{E}-03$ \\
\hline $1.181 \mathrm{E}+01$ & $1.46 \mathrm{E}-01$ & $9.173 \mathrm{E}-02$ & $2.19 \mathrm{E}-02$ & $8.012 \mathrm{E}-02$ & $9.70 \mathrm{E}-03$ & $1.275 \mathrm{E}-02$ & $1.54 \mathrm{E}-03$ \\
\hline $1.139 \mathrm{E}+01$ & $1.41 \mathrm{E}-01$ & $7.900 \mathrm{E}-02$ & $2.19 \mathrm{E}-02$ & $7.596 \mathrm{E}-02$ & $9.71 \mathrm{E}-03$ & $1.209 \mathrm{E}-02$ & $1.54 \mathrm{E}-03$ \\
\hline $1.102 \mathrm{E}+01$ & $1.36 \mathrm{E}-01$ & 7.554E-02 & $2.16 \mathrm{E}-02$ & $7.096 \mathrm{E}-02$ & $9.52 \mathrm{E}-03$ & $1.129 \mathrm{E}-02$ & $1.52 \mathrm{E}-03$ \\
\hline$\rho\left(\mathrm{kg} / \mathrm{m}^{\wedge} 3\right)$ & $\eta_{\mathrm{T}}$ & $\Delta \eta_{\mathrm{T}}$ & $J$ & $\Delta J$ & $\eta_{\mathrm{P}}$ & $\Delta \eta_{\mathrm{P}}$ & $\operatorname{Re}_{0.75}$ \\
\hline $1.161 \mathrm{E}+00$ & $4.285 \mathrm{E}-01$ & 4.13E-02 & $1.684 \mathrm{E}-01$ & 7.39E-04 & $2.093 \mathrm{E}-01$ & $4.08 \mathrm{E}-02$ & $2.666 \mathrm{E}+04$ \\
\hline $1.162 \mathrm{E}+00$ & 4.274E-01 & 4.14E-02 & $2.180 \mathrm{E}-01$ & $7.56 \mathrm{E}-04$ & $2.699 \mathrm{E}-01$ & $5.38 \mathrm{E}-02$ & $2.677 \mathrm{E}+04$ \\
\hline $1.162 \mathrm{E}+00$ & $4.220 \mathrm{E}-01$ & $4.35 \mathrm{E}-02$ & $2.695 \mathrm{E}-01$ & $8.12 \mathrm{E}-04$ & $3.283 \mathrm{E}-01$ & $6.93 \mathrm{E}-02$ & $2.678 \mathrm{E}+04$ \\
\hline $1.162 \mathrm{E}+00$ & $4.154 \mathrm{E}-01$ & $4.37 \mathrm{E}-02$ & $3.211 \mathrm{E}-01$ & $8.91 \mathrm{E}-04$ & $4.095 \mathrm{E}-01$ & $8.71 \mathrm{E}-02$ & $2.689 \mathrm{E}+04$ \\
\hline $1.161 \mathrm{E}+00$ & $4.070 \mathrm{E}-01$ & 4.42E-02 & 3.737E-01 & $9.46 \mathrm{E}-04$ & $4.651 \mathrm{E}-01$ & $1.06 \mathrm{E}-01$ & $2.701 \mathrm{E}+04$ \\
\hline $1.161 \mathrm{E}+00$ & $4.031 \mathrm{E}-01$ & $4.58 \mathrm{E}-02$ & $4.273 \mathrm{E}-01$ & $1.00 \mathrm{E}-03$ & $5.171 \mathrm{E}-01$ & $1.25 \mathrm{E}-01$ & $2.712 \mathrm{E}+04$ \\
\hline $1.160 \mathrm{E}+00$ & 3.977E-01 & 4.84E-02 & 4.834E-01 & $1.08 \mathrm{E}-03$ & $5.535 \mathrm{E}-01$ & $1.48 \mathrm{E}-01$ & $2.709 \mathrm{E}+04$ \\
\hline $1.160 \mathrm{E}+00$ & $3.916 \mathrm{E}-01$ & $5.03 \mathrm{E}-02$ & 5.383E-01 & $1.18 \mathrm{E}-03$ & $5.598 \mathrm{E}-01$ & $1.71 \mathrm{E}-01$ & $2.722 \mathrm{E}+04$ \\
\hline $1.160 \mathrm{E}+00$ & $3.850 \mathrm{E}-01$ & $5.19 \mathrm{E}-02$ & $5.873 \mathrm{E}-01$ & $1.29 \mathrm{E}-03$ & $6.252 \mathrm{E}-01$ & $1.97 \mathrm{E}-01$ & $2.750 \mathrm{E}+04$ \\
\hline
\end{tabular}


Table 248: GWS 4.0 x 4.0 Dynamic Measured Values - 14014 RPM

\begin{tabular}{|c|c|c|c|c|c|c|c|}
\hline$n(\mathrm{RPM})$ & $Q(\mathrm{~g}-\mathrm{m})$ & $\Delta Q(\mathrm{~g}-\mathrm{m})$ & $V(\mathrm{~V})$ & $I(\mathrm{~A})$ & $T_{\text {atm }}\left({ }^{\circ} \mathrm{C}\right)$ & $P_{\text {atm }}(\mathrm{Pa})$ & $P_{\text {diff }}(\mathrm{Pa})$ \\
\hline $1.404 \mathrm{E}+04$ & $1.228 \mathrm{E}+00$ & $5.83 \mathrm{E}-02$ & $1.104 \mathrm{E}+01$ & $2.861 \mathrm{E}+00$ & $2.201 \mathrm{E}+01$ & $9.834 \mathrm{E}+04$ & $2.842 \mathrm{E}+00$ \\
\hline $1.398 \mathrm{E}+04$ & $1.174 \mathrm{E}+00$ & $5.81 \mathrm{E}-02$ & $1.104 \mathrm{E}+01$ & $2.736 \mathrm{E}+00$ & $2.189 \mathrm{E}+01$ & $9.833 \mathrm{E}+04$ & $8.597 \mathrm{E}+00$ \\
\hline $1.397 \mathrm{E}+04$ & $1.112 \mathrm{E}+00$ & $5.72 \mathrm{E}-02$ & $1.104 \mathrm{E}+01$ & $2.606 \mathrm{E}+00$ & $2.190 \mathrm{E}+01$ & $9.834 \mathrm{E}+04$ & $1.800 \mathrm{E}+01$ \\
\hline $1.402 \mathrm{E}+04$ & $1.038 \mathrm{E}+00$ & $5.71 \mathrm{E}-02$ & $1.105 \mathrm{E}+01$ & $2.481 \mathrm{E}+00$ & $2.206 \mathrm{E}+01$ & $9.834 \mathrm{E}+04$ & $3.135 \mathrm{E}+01$ \\
\hline $1.405 \mathrm{E}+04$ & $9.677 \mathrm{E}-01$ & $5.70 \mathrm{E}-02$ & $1.105 \mathrm{E}+01$ & $2.344 \mathrm{E}+00$ & $2.221 \mathrm{E}+01$ & $9.833 \mathrm{E}+04$ & $4.893 \mathrm{E}+01$ \\
\hline $1.403 \mathrm{E}+04$ & $9.026 \mathrm{E}-01$ & $5.68 \mathrm{E}-02$ & $1.105 \mathrm{E}+01$ & $2.204 \mathrm{E}+00$ & $2.231 \mathrm{E}+01$ & $9.834 \mathrm{E}+04$ & $7.013 \mathrm{E}+01$ \\
\hline $1.403 \mathrm{E}+04$ & $8.358 \mathrm{E}-01$ & $5.65 \mathrm{E}-02$ & $1.106 \mathrm{E}+01$ & $2.061 \mathrm{E}+00$ & $2.245 \mathrm{E}+01$ & $9.834 \mathrm{E}+04$ & $9.583 \mathrm{E}+01$ \\
\hline $1.402 \mathrm{E}+04$ & $7.593 \mathrm{E}-01$ & $5.66 \mathrm{E}-02$ & $1.106 \mathrm{E}+01$ & $1.880 \mathrm{E}+00$ & $2.293 \mathrm{E}+01$ & $9.834 \mathrm{E}+04$ & $1.251 \mathrm{E}+02$ \\
\hline $1.399 \mathrm{E}+04$ & $6.626 \mathrm{E}-01$ & $5.74 \mathrm{E}-02$ & $1.107 \mathrm{E}+01$ & $1.674 \mathrm{E}+00$ & $2.311 \mathrm{E}+01$ & $9.833 \mathrm{E}+04$ & $1.585 \mathrm{E}+02$ \\
\hline
\end{tabular}


Table 249: GWS 4.0 x 4.0 Dynamic Calculated Values - 14014 RPM

\begin{tabular}{|c|c|c|c|c|c|c|c|}
\hline$n(\mathrm{RPM})$ & $V_{\infty}^{\prime}(\mathrm{m} / \mathrm{s})$ & $\Delta V_{\infty}^{\prime}(\mathrm{m} / \mathrm{s})$ & $T^{\prime}(\mathrm{g})$ & $\Delta T^{\prime}(\mathrm{g})$ & $V_{\mathrm{t}}(\mathrm{m} / \mathrm{s})$ & $P_{\mathrm{P}}(\mathrm{W})$ & $\Delta P_{\mathrm{P}}(\mathrm{W})$ \\
\hline $1.404 \mathrm{E}+04$ & $2.136 \mathrm{E}+00$ & $1.42 \mathrm{E}-02$ & $9.700 \mathrm{E}+01$ & $7.49 \mathrm{E}+00$ & $5.629 \mathrm{E}+01$ & $1.771 \mathrm{E}+01$ & $8.40 \mathrm{E}-01$ \\
\hline $1.398 \mathrm{E}+04$ & $3.775 \mathrm{E}+00$ & $1.46 \mathrm{E}-02$ & $9.187 \mathrm{E}+01$ & $7.47 \mathrm{E}+00$ & $5.612 \mathrm{E}+01$ & $1.685 \mathrm{E}+01$ & 8.34E-01 \\
\hline $1.397 \mathrm{E}+04$ & $5.500 \mathrm{E}+00$ & $1.63 \mathrm{E}-02$ & $8.733 \mathrm{E}+01$ & $7.46 \mathrm{E}+00$ & $5.622 \mathrm{E}+01$ & $1.595 \mathrm{E}+01$ & $8.21 \mathrm{E}-01$ \\
\hline $1.402 \mathrm{E}+04$ & $7.288 \mathrm{E}+00$ & $1.82 \mathrm{E}-02$ & $8.233 \mathrm{E}+01$ & $7.47 \mathrm{E}+00$ & $5.665 \mathrm{E}+01$ & $1.495 \mathrm{E}+01$ & $8.23 \mathrm{E}-01$ \\
\hline $1.405 \mathrm{E}+04$ & $9.130 \mathrm{E}+00$ & $2.11 \mathrm{E}-02$ & $7.528 \mathrm{E}+01$ & $7.46 \mathrm{E}+00$ & $5.702 \mathrm{E}+01$ & $1.396 \mathrm{E}+01$ & $8.23 \mathrm{E}-01$ \\
\hline $1.403 \mathrm{E}+04$ & $1.095 \mathrm{E}+01$ & $2.36 \mathrm{E}-02$ & $6.779 \mathrm{E}+01$ & $7.46 \mathrm{E}+00$ & $5.725 \mathrm{E}+01$ & $1.301 \mathrm{E}+01$ & $8.19 \mathrm{E}-01$ \\
\hline $1.403 \mathrm{E}+04$ & $1.282 \mathrm{E}+01$ & $2.64 \mathrm{E}-02$ & $5.978 \mathrm{E}+01$ & $7.48 \mathrm{E}+00$ & $5.762 \mathrm{E}+01$ & $1.204 \mathrm{E}+01$ & $8.14 \mathrm{E}-01$ \\
\hline $1.402 \mathrm{E}+04$ & $1.467 \mathrm{E}+01$ & 2.97E-02 & $4.862 \mathrm{E}+01$ & $7.47 \mathrm{E}+00$ & $5.804 \mathrm{E}+01$ & $1.093 \mathrm{E}+01$ & $8.15 \mathrm{E}-01$ \\
\hline $1.399 \mathrm{E}+04$ & $1.654 \mathrm{E}+01$ & $3.26 \mathrm{E}-02$ & $3.804 \mathrm{E}+01$ & $7.46 \mathrm{E}+00$ & $5.842 \mathrm{E}+01$ & $9.519 \mathrm{E}+00$ & $8.25 \mathrm{E}-01$ \\
\hline$P_{\mathrm{e}}(\mathrm{W})$ & $\Delta P_{\mathrm{e}}(\mathrm{W})$ & $C_{\mathrm{T}}$ & $\Delta C_{\mathrm{T}}$ & $C_{\mathrm{P}}$ & $\Delta C_{\mathrm{P}}$ & $C_{\mathrm{Q}}$ & $\Delta C_{\mathrm{Q}}$ \\
\hline $3.157 \mathrm{E}+01$ & $3.51 \mathrm{E}-01$ & $1.383 \mathrm{E}-01$ & $1.07 \mathrm{E}-02$ & $1.078 \mathrm{E}-01$ & $5.12 \mathrm{E}-03$ & $1.716 \mathrm{E}-02$ & $8.14 \mathrm{E}-04$ \\
\hline $3.020 \mathrm{E}+01$ & $3.38 \mathrm{E}-01$ & $1.321 \mathrm{E}-01$ & $1.07 \mathrm{E}-02$ & $1.040 \mathrm{E}-01$ & $5.15 \mathrm{E}-03$ & $1.655 \mathrm{E}-02$ & 8.19E-04 \\
\hline $2.878 \mathrm{E}+01$ & $3.24 \mathrm{E}-01$ & $1.258 \mathrm{E}-01$ & $1.07 \mathrm{E}-02$ & $9.863 \mathrm{E}-02$ & $5.08 \mathrm{E}-03$ & $1.570 \mathrm{E}-02$ & $8.08 \mathrm{E}-04$ \\
\hline $2.741 \mathrm{E}+01$ & $3.11 \mathrm{E}-01$ & $1.177 \mathrm{E}-01$ & $1.07 \mathrm{E}-02$ & $9.141 \mathrm{E}-02$ & $5.03 \mathrm{E}-03$ & $1.455 \mathrm{E}-02$ & $8.01 \mathrm{E}-04$ \\
\hline $2.590 \mathrm{E}+01$ & $2.96 \mathrm{E}-01$ & $1.073 \mathrm{E}-01$ & $1.06 \mathrm{E}-02$ & $8.495 \mathrm{E}-02$ & $5.01 \mathrm{E}-03$ & $1.352 \mathrm{E}-02$ & 7.97E-04 \\
\hline $2.436 \mathrm{E}+01$ & $2.77 \mathrm{E}-01$ & $9.691 \mathrm{E}-02$ & $1.07 \mathrm{E}-02$ & $7.950 \mathrm{E}-02$ & $5.01 \mathrm{E}-03$ & $1.265 \mathrm{E}-02$ & $7.97 \mathrm{E}-04$ \\
\hline $2.279 \mathrm{E}+01$ & $2.60 \mathrm{E}-01$ & $8.556 \mathrm{E}-02$ & $1.07 \mathrm{E}-02$ & $7.369 \mathrm{E}-02$ & 4.99E-03 & $1.173 \mathrm{E}-02$ & 7.94E-04 \\
\hline $2.079 \mathrm{E}+01$ & 2.39E-01 & $6.978 \mathrm{E}-02$ & $1.07 \mathrm{E}-02$ & $6.713 \mathrm{E}-02$ & $5.01 \mathrm{E}-03$ & $1.068 \mathrm{E}-02$ & 7.97E-04 \\
\hline $1.853 \mathrm{E}+01$ & $2.14 \mathrm{E}-01$ & $5.486 \mathrm{E}-02$ & $1.08 \mathrm{E}-02$ & $5.887 \mathrm{E}-02$ & $5.10 \mathrm{E}-03$ & $9.369 \mathrm{E}-03$ & $8.12 \mathrm{E}-04$ \\
\hline$\rho\left(\mathrm{kg} / \mathrm{m}^{\wedge} 3\right)$ & $\eta_{\mathrm{T}}$ & $\Delta \eta_{\mathrm{T}}$ & $J$ & $\Delta J$ & $\eta_{\mathrm{P}}$ & $\Delta \eta_{\mathrm{P}}$ & $R e_{0.75}$ \\
\hline $1.161 \mathrm{E}+00$ & $5.610 \mathrm{E}-01$ & $2.73 \mathrm{E}-02$ & 8.949E-02 & $5.95 \mathrm{E}-04$ & $1.147 \mathrm{E}-01$ & $1.04 \mathrm{E}-02$ & $3.745 \mathrm{E}+04$ \\
\hline $1.161 \mathrm{E}+00$ & $5.580 \mathrm{E}-01$ & $2.83 \mathrm{E}-02$ & $1.589 \mathrm{E}-01$ & $6.16 \mathrm{E}-04$ & $2.018 \mathrm{E}-01$ & $1.92 \mathrm{E}-02$ & $3.737 \mathrm{E}+04$ \\
\hline $1.161 \mathrm{E}+00$ & $5.541 \mathrm{E}-01$ & $2.92 \mathrm{E}-02$ & $2.316 \mathrm{E}-01$ & $6.91 \mathrm{E}-04$ & $2.953 \mathrm{E}-01$ & $2.95 \mathrm{E}-02$ & $3.743 \mathrm{E}+04$ \\
\hline $1.160 \mathrm{E}+00$ & $5.454 \mathrm{E}-01$ & $3.07 \mathrm{E}-02$ & $3.057 \mathrm{E}-01$ & $7.70 \mathrm{E}-04$ & $3.936 \mathrm{E}-01$ & $4.18 \mathrm{E}-02$ & $3.768 \mathrm{E}+04$ \\
\hline $1.160 \mathrm{E}+00$ & 5.391E-01 & $3.24 \mathrm{E}-02$ & $3.823 \mathrm{E}-01$ & $8.92 \mathrm{E}-04$ & 4.827E-01 & $5.57 \mathrm{E}-02$ & $3.789 \mathrm{E}+04$ \\
\hline $1.159 \mathrm{E}+00$ & 5.338E-01 & $3.42 \mathrm{E}-02$ & 4.592E-01 & $9.98 \mathrm{E}-04$ & $5.598 \mathrm{E}-01$ & $7.10 \mathrm{E}-02$ & $3.803 \mathrm{E}+04$ \\
\hline $1.159 \mathrm{E}+00$ & $5.281 \mathrm{E}-01$ & $3.62 \mathrm{E}-02$ & 5.377E-01 & $1.12 \mathrm{E}-03$ & $6.243 \mathrm{E}-01$ & $8.88 \mathrm{E}-02$ & $3.824 \mathrm{E}+04$ \\
\hline $1.157 \mathrm{E}+00$ & $5.257 \mathrm{E}-01$ & $3.97 \mathrm{E}-02$ & $6.158 \mathrm{E}-01$ & $1.26 \mathrm{E}-03$ & $6.401 \mathrm{E}-01$ & $1.09 \mathrm{E}-01$ & $3.840 \mathrm{E}+04$ \\
\hline $1.156 \mathrm{E}+00$ & 5.137E-01 & 4.49E-02 & $6.954 \mathrm{E}-01$ & $1.38 \mathrm{E}-03$ & $6.480 \mathrm{E}-01$ & $1.39 \mathrm{E}-01$ & $3.861 \mathrm{E}+04$ \\
\hline
\end{tabular}


Table 250: GWS 4.0 x 4.0 Dynamic Measured Values - 18018 RPM

\begin{tabular}{|c|c|c|c|c|c|c|c|}
\hline$n(\mathrm{RPM})$ & $Q(\mathrm{~g}-\mathrm{m})$ & $\Delta Q(\mathrm{~g}-\mathrm{m})$ & $V(\mathrm{~V})$ & $I(\mathrm{~A})$ & $T_{\text {atm }}\left({ }^{\circ} \mathrm{C}\right)$ & $P_{\text {atm }}(\mathrm{Pa})$ & $P_{\text {diff }}(\mathrm{Pa})$ \\
\hline $1.801 \mathrm{E}+04$ & $2.032 \mathrm{E}+00$ & $6.80 \mathrm{E}-02$ & $1.097 \mathrm{E}+01$ & $5.256 \mathrm{E}+00$ & $2.280 \mathrm{E}+01$ & $9.834 \mathrm{E}+04$ & $2.904 \mathrm{E}+00$ \\
\hline $1.804 \mathrm{E}+04$ & $1.997 \mathrm{E}+00$ & $6.83 \mathrm{E}-02$ & $1.097 \mathrm{E}+01$ & $5.119 \mathrm{E}+00$ & $2.307 \mathrm{E}+01$ & $9.834 \mathrm{E}+04$ & $1.071 \mathrm{E}+01$ \\
\hline $1.800 \mathrm{E}+04$ & $1.868 \mathrm{E}+00$ & $6.39 \mathrm{E}-02$ & $1.098 \mathrm{E}+01$ & $4.856 \mathrm{E}+00$ & $2.332 \mathrm{E}+01$ & $9.834 \mathrm{E}+04$ & $2.432 \mathrm{E}+01$ \\
\hline $1.803 \mathrm{E}+04$ & $1.748 \mathrm{E}+00$ & $6.16 \mathrm{E}-02$ & $1.099 \mathrm{E}+01$ & $4.584 \mathrm{E}+00$ & $2.329 \mathrm{E}+01$ & $9.834 \mathrm{E}+04$ & $4.418 \mathrm{E}+01$ \\
\hline $1.802 \mathrm{E}+04$ & $1.612 \mathrm{E}+00$ & $6.11 \mathrm{E}-02$ & $1.100 \mathrm{E}+01$ & $4.265 \mathrm{E}+00$ & $2.325 \mathrm{E}+01$ & $9.834 \mathrm{E}+04$ & $7.020 \mathrm{E}+01$ \\
\hline $1.803 \mathrm{E}+04$ & $1.510 \mathrm{E}+00$ & $6.10 \mathrm{E}-02$ & $1.100 \mathrm{E}+01$ & $4.002 \mathrm{E}+00$ & $2.347 \mathrm{E}+01$ & $9.834 \mathrm{E}+04$ & $1.030 \mathrm{E}+02$ \\
\hline $1.803 \mathrm{E}+04$ & $1.410 \mathrm{E}+00$ & $6.13 \mathrm{E}-02$ & $1.101 \mathrm{E}+01$ & $3.726 \mathrm{E}+00$ & $2.350 \mathrm{E}+01$ & $9.834 \mathrm{E}+04$ & $1.418 \mathrm{E}+02$ \\
\hline $1.802 \mathrm{E}+04$ & $1.276 \mathrm{E}+00$ & $5.96 \mathrm{E}-02$ & $1.102 \mathrm{E}+01$ & $3.391 \mathrm{E}+00$ & $2.364 \mathrm{E}+01$ & $9.834 \mathrm{E}+04$ & $1.869 \mathrm{E}+02$ \\
\hline $1.801 \mathrm{E}+04$ & $1.121 \mathrm{E}+00$ & $6.00 \mathrm{E}-02$ & $1.103 \mathrm{E}+01$ & $2.995 \mathrm{E}+00$ & $2.382 \mathrm{E}+01$ & $9.834 \mathrm{E}+04$ & $2.379 \mathrm{E}+02$ \\
\hline $1.799 \mathrm{E}+04$ & $9.454 \mathrm{E}-01$ & $6.05 \mathrm{E}-02$ & $1.104 \mathrm{E}+01$ & $2.571 \mathrm{E}+00$ & $2.392 \mathrm{E}+01$ & $9.834 \mathrm{E}+04$ & $2.948 \mathrm{E}+02$ \\
\hline
\end{tabular}


Table 251: GWS 4.0 x 4.0 Dynamic Calculated Values - 18018 RPM

\begin{tabular}{|c|c|c|c|c|c|c|c|}
\hline$n(\mathrm{RPM})$ & $V_{\infty}^{\prime}(\mathrm{m} / \mathrm{s})$ & $\Delta V_{\infty}^{\prime}(\mathrm{m} / \mathrm{s})$ & $T^{\prime}(\mathrm{g})$ & $\Delta T^{\prime}(\mathrm{g})$ & $V_{\mathrm{t}}(\mathrm{m} / \mathrm{s})$ & $P_{\mathrm{P}}(\mathrm{W})$ & $\Delta P_{\mathrm{P}}(\mathrm{W})$ \\
\hline $1.801 \mathrm{E}+04$ & $2.140 \mathrm{E}+00$ & $1.65 \mathrm{E}-02$ & $1.616 \mathrm{E}+02$ & $7.16 \mathrm{E}+00$ & $7.217 \mathrm{E}+01$ & $3.759 \mathrm{E}+01$ & $1.26 \mathrm{E}+00$ \\
\hline $1.804 \mathrm{E}+04$ & $4.207 \mathrm{E}+00$ & $1.73 \mathrm{E}-02$ & $1.568 \mathrm{E}+02$ & $7.07 \mathrm{E}+00$ & $7.238 \mathrm{E}+01$ & $3.700 \mathrm{E}+01$ & $1.26 \mathrm{E}+00$ \\
\hline $1.800 \mathrm{E}+04$ & $6.398 \mathrm{E}+00$ & $1.95 \mathrm{E}-02$ & $1.486 \mathrm{E}+02$ & $7.05 \mathrm{E}+00$ & $7.240 \mathrm{E}+01$ & $3.454 \mathrm{E}+01$ & $1.18 \mathrm{E}+00$ \\
\hline $1.803 \mathrm{E}+04$ & $8.660 \mathrm{E}+00$ & $2.22 \mathrm{E}-02$ & $1.397 \mathrm{E}+02$ & $7.06 \mathrm{E}+00$ & $7.272 \mathrm{E}+01$ & $3.236 \mathrm{E}+01$ & $1.14 \mathrm{E}+00$ \\
\hline $1.802 \mathrm{E}+04$ & $1.095 \mathrm{E}+01$ & $2.57 \mathrm{E}-02$ & $1.281 \mathrm{E}+02$ & $7.07 \mathrm{E}+00$ & $7.301 \mathrm{E}+01$ & $2.983 \mathrm{E}+01$ & $1.13 \mathrm{E}+00$ \\
\hline $1.803 \mathrm{E}+04$ & $1.329 \mathrm{E}+01$ & $2.87 \mathrm{E}-02$ & $1.165 \mathrm{E}+02$ & $6.91 \mathrm{E}+00$ & $7.343 \mathrm{E}+01$ & $2.796 \mathrm{E}+01$ & $1.13 \mathrm{E}+00$ \\
\hline $1.803 \mathrm{E}+04$ & $1.562 \mathrm{E}+01$ & $3.17 \mathrm{E}-02$ & $1.033 \mathrm{E}+02$ & $6.93 \mathrm{E}+00$ & $7.388 \mathrm{E}+01$ & $2.610 \mathrm{E}+01$ & $1.14 \mathrm{E}+00$ \\
\hline $1.802 \mathrm{E}+04$ & $1.795 \mathrm{E}+01$ & $3.74 \mathrm{E}-02$ & $8.858 \mathrm{E}+01$ & $6.97 \mathrm{E}+00$ & $7.436 \mathrm{E}+01$ & $2.362 \mathrm{E}+01$ & $1.10 \mathrm{E}+00$ \\
\hline $1.801 \mathrm{E}+04$ & $2.028 \mathrm{E}+01$ & $3.87 \mathrm{E}-02$ & $7.011 \mathrm{E}+01$ & $6.97 \mathrm{E}+00$ & $7.495 \mathrm{E}+01$ & $2.074 \mathrm{E}+01$ & $1.11 \mathrm{E}+00$ \\
\hline $1.799 \mathrm{E}+04$ & $2.259 \mathrm{E}+01$ & $4.25 \mathrm{E}-02$ & $5.361 \mathrm{E}+01$ & $7.01 \mathrm{E}+00$ & $7.553 \mathrm{E}+01$ & $1.747 \mathrm{E}+01$ & $1.12 \mathrm{E}+00$ \\
\hline$P_{\mathrm{e}}(\mathrm{W})$ & $\Delta P_{\mathrm{e}}(\mathrm{W})$ & $C_{\mathrm{T}}$ & $\Delta C_{\mathrm{T}}$ & $C_{\mathrm{P}}$ & $\Delta C_{\mathrm{P}}$ & $C_{\mathrm{Q}}$ & $\Delta C_{\mathrm{Q}}$ \\
\hline $5.764 \mathrm{E}+01$ & $6.47 \mathrm{E}-01$ & $1.404 \mathrm{E}-01$ & $6.22 \mathrm{E}-03$ & $1.088 \mathrm{E}-01$ & $3.64 \mathrm{E}-03$ & $1.732 \mathrm{E}-02$ & $5.80 \mathrm{E}-04$ \\
\hline $5.616 \mathrm{E}+01$ & $6.28 \mathrm{E}-01$ & $1.359 \mathrm{E}-01$ & $6.13 \mathrm{E}-03$ & $1.067 \mathrm{E}-01$ & $3.65 \mathrm{E}-03$ & $1.698 \mathrm{E}-02$ & $5.81 \mathrm{E}-04$ \\
\hline $5.332 \mathrm{E}+01$ & 5.92E-01 & $1.295 \mathrm{E}-01$ & $6.14 \mathrm{E}-03$ & $1.003 \mathrm{E}-01$ & $3.43 \mathrm{E}-03$ & $1.596 \mathrm{E}-02$ & $5.46 \mathrm{E}-04$ \\
\hline $5.036 \mathrm{E}+01$ & $5.61 \mathrm{E}-01$ & $1.214 \mathrm{E}-01$ & $6.13 \mathrm{E}-03$ & $9.358 \mathrm{E}-02$ & $3.30 \mathrm{E}-03$ & $1.489 \mathrm{E}-02$ & $5.25 \mathrm{E}-04$ \\
\hline $4.689 \mathrm{E}+01$ & $5.22 \mathrm{E}-01$ & $1.114 \mathrm{E}-01$ & $6.14 \mathrm{E}-03$ & $8.632 \mathrm{E}-02$ & $3.27 \mathrm{E}-03$ & $1.374 \mathrm{E}-02$ & $5.21 \mathrm{E}-04$ \\
\hline $4.403 \mathrm{E}+01$ & 4.91E-01 & $1.013 \mathrm{E}-01$ & $6.01 \mathrm{E}-03$ & 8.086E-02 & $3.27 \mathrm{E}-03$ & $1.287 \mathrm{E}-02$ & $5.20 \mathrm{E}-04$ \\
\hline $4.103 \mathrm{E}+01$ & $4.55 \mathrm{E}-01$ & $8.980 \mathrm{E}-02$ & $6.02 \mathrm{E}-03$ & 7.549E-02 & $3.29 \mathrm{E}-03$ & $1.202 \mathrm{E}-02$ & $5.23 \mathrm{E}-04$ \\
\hline $3.736 \mathrm{E}+01$ & $4.15 \mathrm{E}-01$ & $7.715 \mathrm{E}-02$ & $6.07 \mathrm{E}-03$ & $6.849 \mathrm{E}-02$ & $3.20 \mathrm{E}-03$ & $1.090 \mathrm{E}-02$ & $5.09 \mathrm{E}-04$ \\
\hline $3.304 \mathrm{E}+01$ & $3.68 \mathrm{E}-01$ & $6.111 \mathrm{E}-02$ & $6.08 \mathrm{E}-03$ & $6.021 \mathrm{E}-02$ & $3.22 \mathrm{E}-03$ & $9.583 \mathrm{E}-03$ & $5.13 \mathrm{E}-04$ \\
\hline $2.839 \mathrm{E}+01$ & $3.20 \mathrm{E}-01$ & $4.685 \mathrm{E}-02$ & $6.13 \mathrm{E}-03$ & $5.090 \mathrm{E}-02$ & $3.26 \mathrm{E}-03$ & $8.101 \mathrm{E}-03$ & 5.19E-04 \\
\hline$\rho\left(\mathrm{kg} / \mathrm{m}^{\wedge} 3\right)$ & $\eta_{\mathrm{T}}$ & $\Delta \eta_{\mathrm{T}}$ & $J$ & $\Delta J$ & $\eta_{\mathrm{P}}$ & $\Delta \eta_{\mathrm{P}}$ & $R e_{0.75}$ \\
\hline $1.158 \mathrm{E}+00$ & $6.521 \mathrm{E}-01$ & $2.30 \mathrm{E}-02$ & $6.990 \mathrm{E}-02$ & $5.39 \mathrm{E}-04$ & $9.020 \mathrm{E}-02$ & $5.06 \mathrm{E}-03$ & $4.779 \mathrm{E}+04$ \\
\hline $1.157 \mathrm{E}+00$ & $6.590 \mathrm{E}-01$ & $2.37 \mathrm{E}-02$ & $1.372 \mathrm{E}-01$ & $5.66 \mathrm{E}-04$ & $1.748 \mathrm{E}-01$ & $9.92 \mathrm{E}-03$ & $4.786 \mathrm{E}+04$ \\
\hline $1.156 \mathrm{E}+00$ & $6.479 \mathrm{E}-01$ & $2.33 \mathrm{E}-02$ & $2.091 \mathrm{E}-01$ & $6.38 \mathrm{E}-04$ & $2.699 \mathrm{E}-01$ & $1.58 \mathrm{E}-02$ & $4.780 \mathrm{E}+04$ \\
\hline $1.156 \mathrm{E}+00$ & $6.427 \mathrm{E}-01$ & $2.38 \mathrm{E}-02$ & $2.826 \mathrm{E}-01$ & $7.28 \mathrm{E}-04$ & 3.667E-01 & $2.26 \mathrm{E}-02$ & $4.802 \mathrm{E}+04$ \\
\hline $1.156 \mathrm{E}+00$ & $6.361 \mathrm{E}-01$ & $2.51 \mathrm{E}-02$ & $3.574 \mathrm{E}-01$ & $8.43 \mathrm{E}-04$ & $4.611 \mathrm{E}-01$ & $3.09 \mathrm{E}-02$ & $4.822 \mathrm{E}+04$ \\
\hline $1.155 \mathrm{E}+00$ & $6.350 \mathrm{E}-01$ & $2.66 \mathrm{E}-02$ & 4.337E-01 & $9.41 \mathrm{E}-04$ & $5.431 \mathrm{E}-01$ & $3.90 \mathrm{E}-02$ & $4.843 \mathrm{E}+04$ \\
\hline $1.155 \mathrm{E}+00$ & $6.360 \mathrm{E}-01$ & $2.86 \mathrm{E}-02$ & $5.097 \mathrm{E}-01$ & $1.04 \mathrm{E}-03$ & $6.062 \mathrm{E}-01$ & 4.85E-02 & $4.872 \mathrm{E}+04$ \\
\hline $1.154 \mathrm{E}+00$ & $6.320 \mathrm{E}-01$ & $3.03 \mathrm{E}-02$ & $5.862 \mathrm{E}-01$ & $1.23 \mathrm{E}-03$ & $6.602 \mathrm{E}-01$ & $6.04 \mathrm{E}-02$ & $4.900 \mathrm{E}+04$ \\
\hline $1.154 \mathrm{E}+00$ & $6.279 \mathrm{E}-01$ & $3.43 \mathrm{E}-02$ & $6.622 \mathrm{E}-01$ & $1.27 \mathrm{E}-03$ & $6.720 \mathrm{E}-01$ & $7.59 \mathrm{E}-02$ & $4.933 \mathrm{E}+04$ \\
\hline $1.153 \mathrm{E}+00$ & $6.153 \mathrm{E}-01$ & $4.00 \mathrm{E}-02$ & 7.384E-01 & $1.40 \mathrm{E}-03$ & $6.798 \mathrm{E}-01$ & $9.90 \mathrm{E}-02$ & $4.969 \mathrm{E}+04$ \\
\hline
\end{tabular}




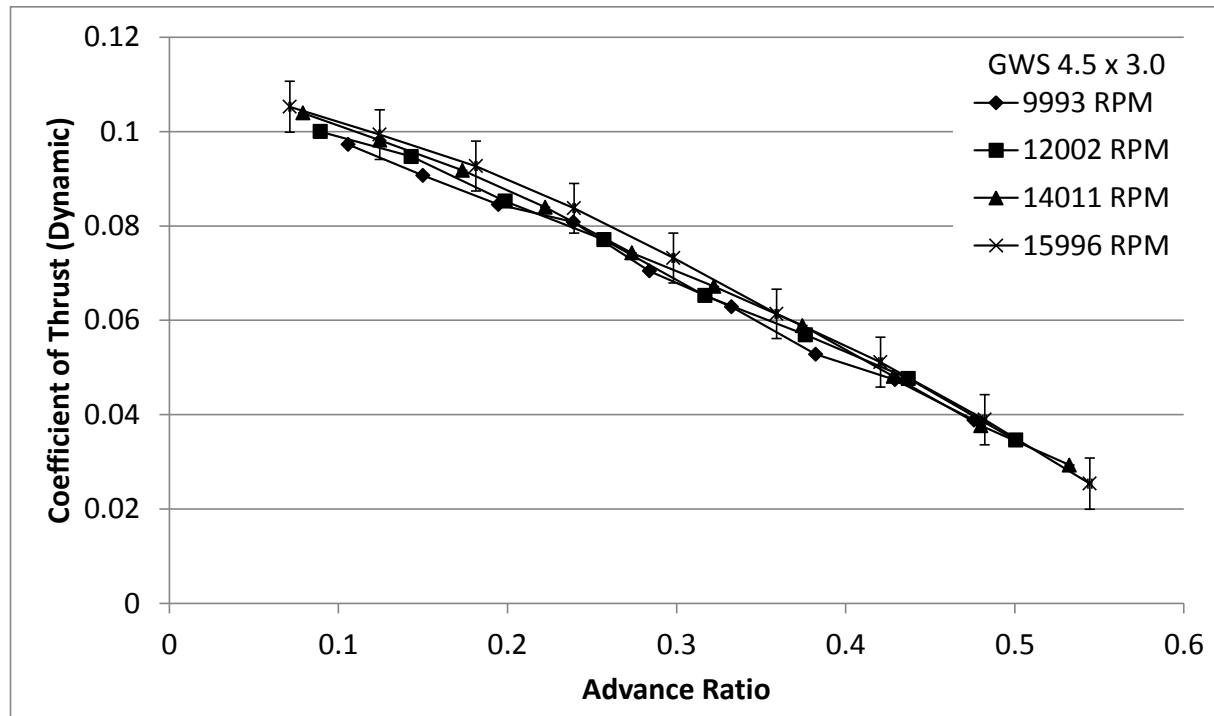

(a)

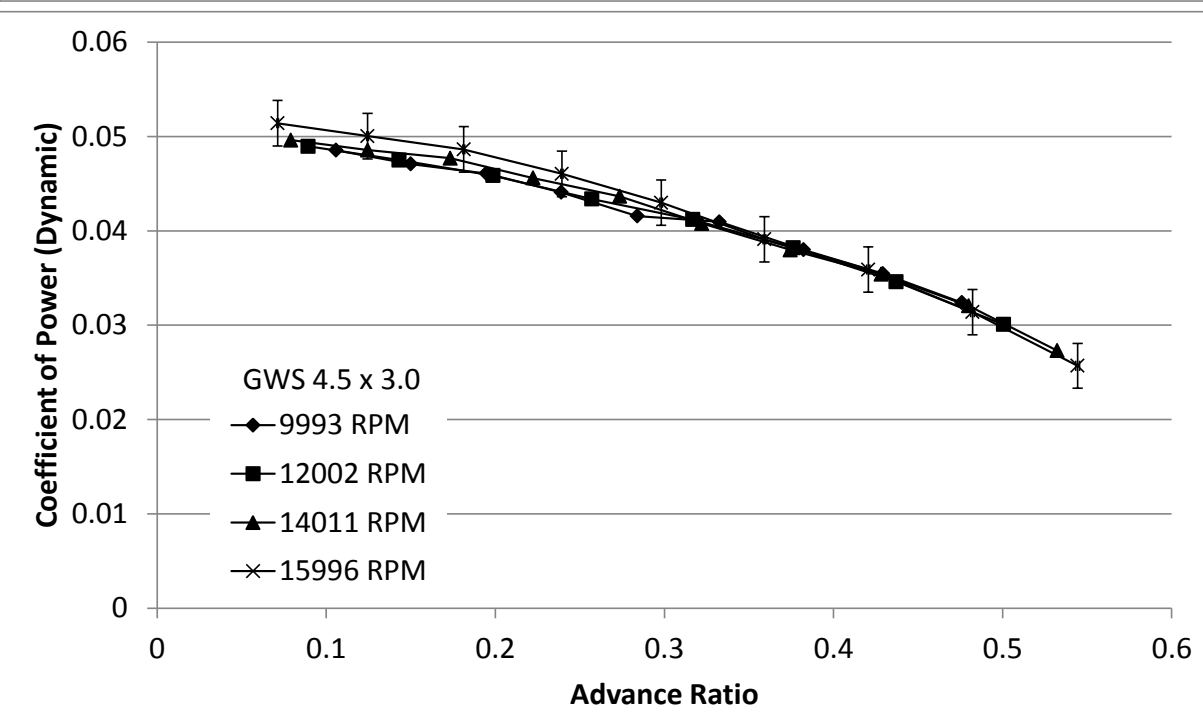

(b)

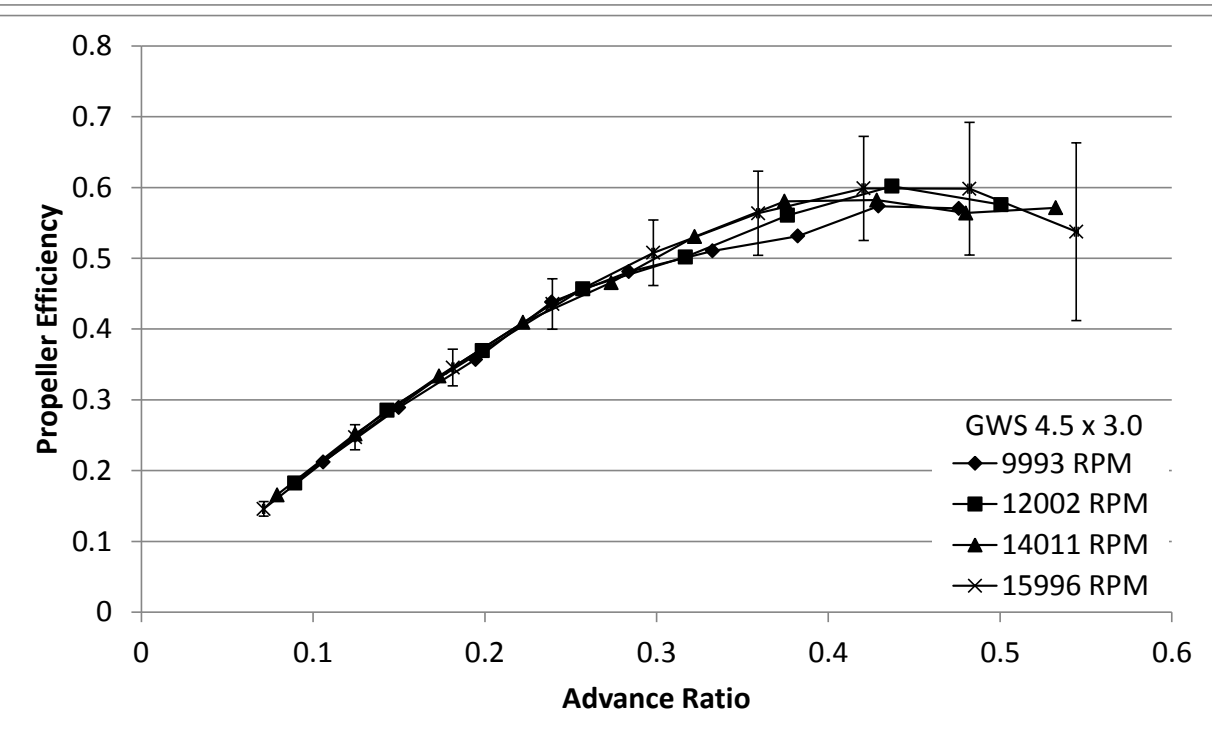

(c) 


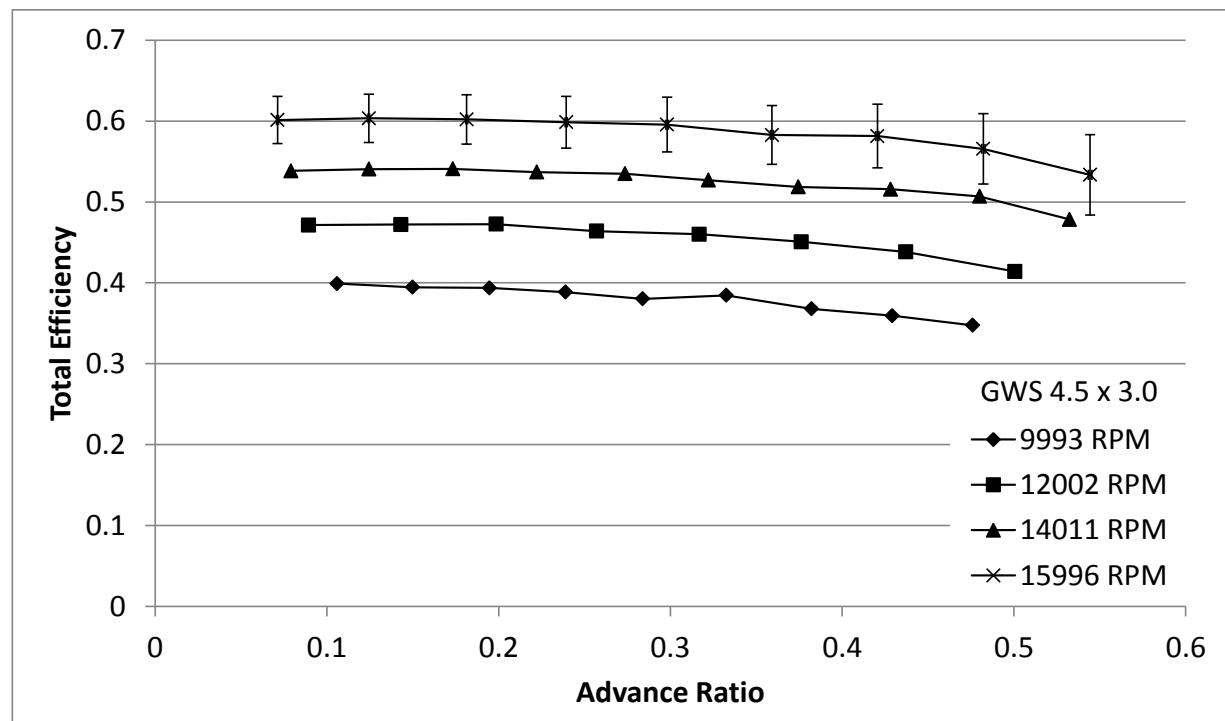

(d)

Figure 100: GWS 4.5 x 3.0 Dynamic Test Results: (a) Coefficient of Thrust, (b)

Coefficient of Power, (c) Propeller Efficiency, (d) Total Efficiency.

Table 252: GWS 4.5 x 3.0 Dynamic Measured Values - 9993 RPM

\begin{tabular}{|c|c|c|c|c|c|c|c|}
\hline$n(\mathrm{RPM})$ & $Q(\mathrm{~g}-\mathrm{m})$ & $\Delta Q(\mathrm{~g}-\mathrm{m})$ & $V(\mathrm{~V})$ & $I(\mathrm{~A})$ & $T_{\mathrm{atm}}\left({ }^{\circ} \mathrm{C}\right)$ & $P_{\text {atm }}(\mathrm{Pa})$ & $P_{\text {diff }}(\mathrm{Pa})$ \\
\hline $9.969 \mathrm{E}+03$ & $4.931 \mathrm{E}-01$ & $5.63 \mathrm{E}-02$ & $1.108 \mathrm{E}+01$ & $1.141 \mathrm{E}+00$ & $2.166 \mathrm{E}+01$ & $9.841 \mathrm{E}+04$ & $2.501 \mathrm{E}+00$ \\
\hline $9.963 \mathrm{E}+03$ & $4.780 \mathrm{E}-01$ & $5.66 \mathrm{E}-02$ & $1.108 \mathrm{E}+01$ & $1.118 \mathrm{E}+00$ & $2.149 \mathrm{E}+01$ & $9.841 \mathrm{E}+04$ & $4.912 \mathrm{E}+00$ \\
\hline $9.949 \mathrm{E}+03$ & $4.665 \mathrm{E}-01$ & $5.63 \mathrm{E}-02$ & $1.109 \mathrm{E}+01$ & $1.092 \mathrm{E}+00$ & $2.147 \mathrm{E}+01$ & $9.841 \mathrm{E}+04$ & $8.153 \mathrm{E}+00$ \\
\hline $1.001 \mathrm{E}+04$ & $4.515 \mathrm{E}-01$ & $5.53 \mathrm{E}-02$ & $1.109 \mathrm{E}+01$ & $1.077 \mathrm{E}+00$ & $2.142 \mathrm{E}+01$ & $9.840 \mathrm{E}+04$ & $1.235 \mathrm{E}+01$ \\
\hline $1.006 \mathrm{E}+04$ & $4.307 \mathrm{E}-01$ & $5.82 \mathrm{E}-02$ & $1.109 \mathrm{E}+01$ & $1.056 \mathrm{E}+00$ & $2.143 \mathrm{E}+01$ & $9.840 \mathrm{E}+04$ & $1.753 \mathrm{E}+01$ \\
\hline $1.004 \mathrm{E}+04$ & $4.224 \mathrm{E}-01$ & $5.63 \mathrm{E}-02$ & $1.109 \mathrm{E}+01$ & $1.022 \mathrm{E}+00$ & $2.153 \mathrm{E}+01$ & $9.839 \mathrm{E}+04$ & $2.384 \mathrm{E}+01$ \\
\hline $9.974 \mathrm{E}+03$ & $3.865 \mathrm{E}-01$ & $5.49 \mathrm{E}-02$ & $1.109 \mathrm{E}+01$ & $9.703 \mathrm{E}-01$ & $2.157 \mathrm{E}+01$ & $9.840 \mathrm{E}+04$ & $3.095 \mathrm{E}+01$ \\
\hline $9.957 \mathrm{E}+03$ & $3.593 \mathrm{E}-01$ & $5.52 \mathrm{E}-02$ & $1.109 \mathrm{E}+01$ & $9.218 \mathrm{E}-01$ & $2.166 \mathrm{E}+01$ & $9.840 \mathrm{E}+04$ & $3.878 \mathrm{E}+01$ \\
\hline $1.001 \mathrm{E}+04$ & $3.314 \mathrm{E}-01$ & $5.43 \mathrm{E}-02$ & $1.109 \mathrm{E}+01$ & $8.838 \mathrm{E}-01$ & $2.177 \mathrm{E}+01$ & $9.840 \mathrm{E}+04$ & $4.809 \mathrm{E}+01$ \\
\hline
\end{tabular}


Table 253: GWS 4.5 x 3.0 Dynamic Calculated Values - 9993 RPM

\begin{tabular}{|c|c|c|c|c|c|c|c|}
\hline$n(\mathrm{RPM})$ & $V_{\infty}^{\prime}(\mathrm{m} / \mathrm{s})$ & $\Delta V_{\infty}^{\prime}(\mathrm{m} / \mathrm{s})$ & $T^{\prime}(\mathrm{g})$ & $\Delta T^{\prime}(\mathrm{g})$ & $V_{\mathrm{t}}(\mathrm{m} / \mathrm{s})$ & $P_{\mathrm{P}}(\mathrm{W})$ & $\Delta P_{\mathrm{P}}(\mathrm{W})$ \\
\hline $9.969 \mathrm{E}+03$ & $2.010 \mathrm{E}+00$ & $1.26 \mathrm{E}-02$ & $5.431 \mathrm{E}+01$ & $8.99 \mathrm{E}+00$ & $4.479 \mathrm{E}+01$ & $5.048 \mathrm{E}+00$ & $5.77 \mathrm{E}-01$ \\
\hline $9.963 \mathrm{E}+03$ & $2.846 \mathrm{E}+00$ & $1.28 \mathrm{E}-02$ & $5.063 \mathrm{E}+01$ & $9.02 \mathrm{E}+00$ & $4.481 \mathrm{E}+01$ & $4.891 \mathrm{E}+00$ & $5.79 \mathrm{E}-01$ \\
\hline $9.949 \mathrm{E}+03$ & $3.688 \mathrm{E}+00$ & $1.30 \mathrm{E}-02$ & $4.706 \mathrm{E}+01$ & $8.99 \mathrm{E}+00$ & $4.481 \mathrm{E}+01$ & $4.767 E+00$ & $5.75 \mathrm{E}-01$ \\
\hline $1.001 \mathrm{E}+04$ & $4.555 \mathrm{E}+00$ & $1.41 \mathrm{E}-02$ & $4.551 \mathrm{E}+01$ & $9.02 \mathrm{E}+00$ & $4.514 \mathrm{E}+01$ & $4.640 \mathrm{E}+00$ & $5.68 \mathrm{E}-01$ \\
\hline $1.006 \mathrm{E}+04$ & $5.442 \mathrm{E}+00$ & $1.56 \mathrm{E}-02$ & $4.012 \mathrm{E}+01$ & $9.01 \mathrm{E}+00$ & $4.549 \mathrm{E}+01$ & $4.451 \mathrm{E}+00$ & $6.01 \mathrm{E}-01$ \\
\hline $1.004 \mathrm{E}+04$ & $6.363 \mathrm{E}+00$ & $1.70 \mathrm{E}-02$ & $3.564 \mathrm{E}+01$ & $9.00 \mathrm{E}+00$ & $4.553 \mathrm{E}+01$ & $4.357 \mathrm{E}+00$ & $5.80 \mathrm{E}-01$ \\
\hline $9.974 \mathrm{E}+03$ & $7.263 \mathrm{E}+00$ & $1.85 \mathrm{E}-02$ & $2.954 \mathrm{E}+01$ & $8.97 \mathrm{E}+00$ & $4.535 \mathrm{E}+01$ & $3.959 \mathrm{E}+00$ & $5.62 \mathrm{E}-01$ \\
\hline $9.957 \mathrm{E}+03$ & $8.139 \mathrm{E}+00$ & $1.85 \mathrm{E}-02$ & $2.640 \mathrm{E}+01$ & $9.00 \mathrm{E}+00$ & $4.542 \mathrm{E}+01$ & $3.674 \mathrm{E}+00$ & $5.64 \mathrm{E}-01$ \\
\hline $1.001 \mathrm{E}+04$ & $9.074 \mathrm{E}+00$ & $2.00 \mathrm{E}-02$ & $2.184 \mathrm{E}+01$ & $8.98 \mathrm{E}+00$ & $4.583 \mathrm{E}+01$ & $3.407 \mathrm{E}+00$ & $5.58 \mathrm{E}-01$ \\
\hline$P_{\mathrm{e}}(\mathrm{W})$ & $\Delta P_{\mathrm{e}}(\mathrm{W})$ & $C_{\mathrm{T}}$ & $\Delta C_{\mathrm{T}}$ & $C_{\mathrm{P}}$ & $\Delta C_{\mathrm{P}}$ & $C_{\mathrm{Q}}$ & $\Delta C_{\mathrm{Q}}$ \\
\hline $1.265 \mathrm{E}+01$ & $1.55 \mathrm{E}-01$ & $9.724 \mathrm{E}-02$ & $1.61 \mathrm{E}-02$ & $4.854 \mathrm{E}-02$ & $5.54 \mathrm{E}-03$ & $7.725 \mathrm{E}-03$ & $8.82 \mathrm{E}-04$ \\
\hline $1.239 \mathrm{E}+01$ & $1.51 \mathrm{E}-01$ & $9.070 \mathrm{E}-02$ & $1.62 \mathrm{E}-02$ & $4.708 \mathrm{E}-02$ & $5.58 \mathrm{E}-03$ & $7.493 \mathrm{E}-03$ & 8.87E-04 \\
\hline $1.211 \mathrm{E}+01$ & $1.48 \mathrm{E}-01$ & $8.454 \mathrm{E}-02$ & $1.62 \mathrm{E}-02$ & $4.608 \mathrm{E}-02$ & $5.56 \mathrm{E}-03$ & 7.333E-03 & $8.85 \mathrm{E}-04$ \\
\hline $1.194 \mathrm{E}+01$ & $1.48 \mathrm{E}-01$ & $8.081 \mathrm{E}-02$ & $1.60 \mathrm{E}-02$ & $4.408 \mathrm{E}-02$ & $5.39 \mathrm{E}-03$ & $7.015 \mathrm{E}-03$ & $8.59 \mathrm{E}-04$ \\
\hline $1.170 \mathrm{E}+01$ & $1.44 \mathrm{E}-01$ & $7.045 \mathrm{E}-02$ & $1.58 \mathrm{E}-02$ & $4.157 \mathrm{E}-02$ & $5.62 \mathrm{E}-03$ & $6.617 \mathrm{E}-03$ & 8.94E-04 \\
\hline $1.133 \mathrm{E}+01$ & $1.39 \mathrm{E}-01$ & $6.284 \mathrm{E}-02$ & $1.59 \mathrm{E}-02$ & $4.095 \mathrm{E}-02$ & $5.46 \mathrm{E}-03$ & $6.517 \mathrm{E}-03$ & $8.68 \mathrm{E}-04$ \\
\hline $1.076 \mathrm{E}+01$ & $1.34 \mathrm{E}-01$ & $5.282 \mathrm{E}-02$ & $1.60 \mathrm{E}-02$ & $3.799 \mathrm{E}-02$ & $5.40 \mathrm{E}-03$ & $6.047 \mathrm{E}-03$ & $8.59 \mathrm{E}-04$ \\
\hline $1.022 \mathrm{E}+01$ & $1.28 \mathrm{E}-01$ & 4.740E-02 & $1.62 \mathrm{E}-02$ & $3.546 \mathrm{E}-02$ & $5.45 \mathrm{E}-03$ & $5.643 \mathrm{E}-03$ & 8.67E-04 \\
\hline $9.802 \mathrm{E}+00$ & $1.25 \mathrm{E}-01$ & $3.882 \mathrm{E}-02$ & $1.60 \mathrm{E}-02$ & $3.239 \mathrm{E}-02$ & 5.31E-03 & $5.154 \mathrm{E}-03$ & 8.44E-04 \\
\hline$\rho\left(\mathrm{kg} / \mathrm{m}^{\wedge} 3\right)$ & $\eta_{\mathrm{T}}$ & $\Delta \eta_{\mathrm{T}}$ & $J$ & $\Delta J$ & $\eta_{\mathrm{P}}$ & $\Delta \eta_{\mathrm{P}}$ & $\operatorname{Re}_{0.75}$ \\
\hline $1.163 \mathrm{E}+00$ & 3.991E-01 & $4.58 \mathrm{E}-02$ & $1.059 \mathrm{E}-01$ & $6.63 \mathrm{E}-04$ & $2.121 \mathrm{E}-01$ & 4.27E-02 & $3.112 \mathrm{E}+04$ \\
\hline $1.164 \mathrm{E}+00$ & $3.946 \mathrm{E}-01$ & 4.70E-02 & 1.499E-01 & $6.74 \mathrm{E}-04$ & $2.889 \mathrm{E}-01$ & $6.18 \mathrm{E}-02$ & $3.116 \mathrm{E}+04$ \\
\hline $1.164 \mathrm{E}+00$ & $3.938 \mathrm{E}-01$ & $4.77 \mathrm{E}-02$ & $1.946 \mathrm{E}-01$ & $6.92 \mathrm{E}-04$ & $3.570 \mathrm{E}-01$ & $8.07 \mathrm{E}-02$ & $3.116 \mathrm{E}+04$ \\
\hline $1.164 \mathrm{E}+00$ & $3.885 \mathrm{E}-01$ & $4.78 \mathrm{E}-02$ & $2.390 \mathrm{E}-01$ & $7.43 \mathrm{E}-04$ & $4.381 \mathrm{E}-01$ & $1.02 \mathrm{E}-01$ & $3.141 \mathrm{E}+04$ \\
\hline $1.164 \mathrm{E}+00$ & 3.803E-01 & $5.16 \mathrm{E}-02$ & 2.839E-01 & 8.20E-04 & $4.811 \mathrm{E}-01$ & $1.26 \mathrm{E}-01$ & $3.165 \mathrm{E}+04$ \\
\hline $1.163 \mathrm{E}+00$ & $3.846 \mathrm{E}-01$ & $5.15 \mathrm{E}-02$ & $3.326 \mathrm{E}-01$ & 8.97E-04 & $5.104 \mathrm{E}-01$ & $1.46 \mathrm{E}-01$ & $3.165 E+04$ \\
\hline $1.163 \mathrm{E}+00$ & $3.680 \mathrm{E}-01$ & $5.25 \mathrm{E}-02$ & $3.823 \mathrm{E}-01$ & $9.83 \mathrm{E}-04$ & 5.314E-01 & $1.78 \mathrm{E}-01$ & $3.152 \mathrm{E}+04$ \\
\hline $1.163 \mathrm{E}+00$ & $3.594 \mathrm{E}-01$ & $5.54 \mathrm{E}-02$ & $4.291 \mathrm{E}-01$ & 9.89E-04 & $5.736 \mathrm{E}-01$ & $2.14 \mathrm{E}-01$ & $3.155 \mathrm{E}+04$ \\
\hline $1.162 \mathrm{E}+00$ & $3.475 \mathrm{E}-01$ & $5.71 \mathrm{E}-02$ & $4.760 \mathrm{E}-01$ & $1.06 \mathrm{E}-03$ & $5.705 \mathrm{E}-01$ & $2.52 \mathrm{E}-01$ & $3.181 \mathrm{E}+04$ \\
\hline
\end{tabular}


Table 254: GWS 4.5 x 3.0 Dynamic Measured Values - 12002 RPM

\begin{tabular}{|c|c|c|c|c|c|c|c|}
\hline$n(\mathrm{RPM})$ & $Q(\mathrm{~g}-\mathrm{m})$ & $\Delta Q(\mathrm{~g}-\mathrm{m})$ & $V(\mathrm{~V})$ & $I(\mathrm{~A})$ & $T_{\text {atm }}\left({ }^{\circ} \mathrm{C}\right)$ & $P_{\text {atm }}(\mathrm{Pa})$ & $P_{\text {diff }}(\mathrm{Pa})$ \\
\hline $1.192 \mathrm{E}+04$ & $7.110 \mathrm{E}-01$ & $5.75 \mathrm{E}-02$ & $1.107 \mathrm{E}+01$ & $1.668 \mathrm{E}+00$ & $2.162 \mathrm{E}+01$ & $9.838 \mathrm{E}+04$ & $2.577 \mathrm{E}+00$ \\
\hline $1.202 \mathrm{E}+04$ & $7.012 \mathrm{E}-01$ & $5.89 \mathrm{E}-02$ & $1.107 \mathrm{E}+01$ & $1.656 \mathrm{E}+00$ & $2.149 \mathrm{E}+01$ & $9.838 \mathrm{E}+04$ & $6.526 \mathrm{E}+00$ \\
\hline $1.208 \mathrm{E}+04$ & $6.838 \mathrm{E}-01$ & $5.88 \mathrm{E}-02$ & $1.107 \mathrm{E}+01$ & $1.621 \mathrm{E}+00$ & $2.148 \mathrm{E}+01$ & $9.839 \mathrm{E}+04$ & $1.250 \mathrm{E}+01$ \\
\hline $1.206 \mathrm{E}+04$ & $6.444 \mathrm{E}-01$ & $5.86 \mathrm{E}-02$ & $1.107 \mathrm{E}+01$ & $1.553 \mathrm{E}+00$ & $2.155 \mathrm{E}+01$ & $9.838 \mathrm{E}+04$ & $2.069 \mathrm{E}+01$ \\
\hline $1.198 \mathrm{E}+04$ & $6.038 \mathrm{E}-01$ & $5.79 \mathrm{E}-02$ & $1.107 \mathrm{E}+01$ & $1.458 \mathrm{E}+00$ & $2.169 \mathrm{E}+01$ & $9.838 \mathrm{E}+04$ & $3.078 \mathrm{E}+01$ \\
\hline $1.201 \mathrm{E}+04$ & $5.627 \mathrm{E}-01$ & $5.75 \mathrm{E}-02$ & $1.108 \mathrm{E}+01$ & $1.390 \mathrm{E}+00$ & $2.180 \mathrm{E}+01$ & $9.837 \mathrm{E}+04$ & $4.347 \mathrm{E}+01$ \\
\hline $1.201 \mathrm{E}+04$ & $5.097 \mathrm{E}-01$ & $5.73 \mathrm{E}-02$ & $1.108 \mathrm{E}+01$ & $1.295 \mathrm{E}+00$ & $2.190 \mathrm{E}+01$ & $9.838 \mathrm{E}+04$ & $5.846 \mathrm{E}+01$ \\
\hline $1.195 \mathrm{E}+04$ & $4.383 \mathrm{E}-01$ & $5.69 \mathrm{E}-02$ & $1.108 \mathrm{E}+01$ & $1.172 \mathrm{E}+00$ & $2.209 \mathrm{E}+01$ & $9.837 \mathrm{E}+04$ & $7.566 \mathrm{E}+01$ \\
\hline
\end{tabular}


Table 255: GWS 4.5 x 3.0 Dynamic Calculated Values - 12002 RPM

\begin{tabular}{|c|c|c|c|c|c|c|c|}
\hline$n(\mathrm{RPM})$ & $V_{\infty}^{\prime}(\mathrm{m} / \mathrm{s})$ & $\Delta V_{\infty}^{\prime}(\mathrm{m} / \mathrm{s})$ & $T^{\prime}(\mathrm{g})$ & $\Delta T^{\prime}(\mathrm{g})$ & $V_{\mathrm{t}}(\mathrm{m} / \mathrm{s})$ & $P_{\mathrm{P}}(\mathrm{W})$ & $\Delta P_{\mathrm{P}}(\mathrm{W})$ \\
\hline $1.192 \mathrm{E}+04$ & $2.028 \mathrm{E}+00$ & $1.32 \mathrm{E}-02$ & $7.982 \mathrm{E}+01$ & $8.17 \mathrm{E}+00$ & $5.353 \mathrm{E}+01$ & $8.702 \mathrm{E}+00$ & 7.04E-01 \\
\hline $1.202 \mathrm{E}+04$ & $3.276 \mathrm{E}+00$ & $1.31 \mathrm{E}-02$ & $7.688 \mathrm{E}+01$ & $8.16 \mathrm{E}+00$ & $5.403 \mathrm{E}+01$ & $8.653 \mathrm{E}+00$ & $7.26 \mathrm{E}-01$ \\
\hline $1.208 \mathrm{E}+04$ & $4.568 \mathrm{E}+00$ & $1.44 \mathrm{E}-02$ & $6.991 \mathrm{E}+01$ & $8.13 \mathrm{E}+00$ & $5.440 \mathrm{E}+01$ & $8.480 \mathrm{E}+00$ & $7.29 \mathrm{E}-01$ \\
\hline $1.206 \mathrm{E}+04$ & $5.904 \mathrm{E}+00$ & $1.62 \mathrm{E}-02$ & $6.294 \mathrm{E}+01$ & $8.15 \mathrm{E}+00$ & $5.443 \mathrm{E}+01$ & $7.978 \mathrm{E}+00$ & $7.26 \mathrm{E}-01$ \\
\hline $1.198 \mathrm{E}+04$ & $7.227 \mathrm{E}+00$ & $1.82 \mathrm{E}-02$ & $5.258 \mathrm{E}+01$ & $8.15 \mathrm{E}+00$ & $5.424 \mathrm{E}+01$ & $7.427 \mathrm{E}+00$ & $7.13 \mathrm{E}-01$ \\
\hline $1.201 \mathrm{E}+04$ & $8.607 \mathrm{E}+00$ & $1.91 \mathrm{E}-02$ & $4.609 \mathrm{E}+01$ & $8.15 \mathrm{E}+00$ & $5.458 \mathrm{E}+01$ & $6.939 \mathrm{E}+00$ & 7.09E-01 \\
\hline $1.201 \mathrm{E}+04$ & $9.999 \mathrm{E}+00$ & $2.11 \mathrm{E}-02$ & $3.859 \mathrm{E}+01$ & $8.16 \mathrm{E}+00$ & $5.483 \mathrm{E}+01$ & $6.287 \mathrm{E}+00$ & 7.07E-01 \\
\hline $1.195 \mathrm{E}+04$ & $1.139 \mathrm{E}+01$ & $2.35 \mathrm{E}-02$ & $2.771 \mathrm{E}+01$ & $8.18 \mathrm{E}+00$ & $5.484 \mathrm{E}+01$ & $5.379 \mathrm{E}+00$ & $6.98 \mathrm{E}-01$ \\
\hline$P_{\mathrm{e}}(\mathrm{W})$ & $\Delta P_{\mathrm{e}}(\mathrm{W})$ & $C_{\mathrm{T}}$ & $\Delta C_{\mathrm{T}}$ & $C_{\mathrm{P}}$ & $\Delta C_{\mathrm{P}}$ & $C_{\mathrm{Q}}$ & $\Delta C_{\mathrm{Q}}$ \\
\hline $1.846 \mathrm{E}+01$ & $2.14 \mathrm{E}-01$ & $9.999 \mathrm{E}-02$ & $1.02 \mathrm{E}-02$ & $4.896 \mathrm{E}-02$ & $3.96 \mathrm{E}-03$ & $7.792 \mathrm{E}-03$ & $6.31 \mathrm{E}-04$ \\
\hline $1.833 \mathrm{E}+01$ & $2.14 \mathrm{E}-01$ & $9.472 \mathrm{E}-02$ & $1.01 \mathrm{E}-02$ & $4.750 \mathrm{E}-02$ & $3.99 \mathrm{E}-03$ & $7.560 \mathrm{E}-03$ & $6.35 \mathrm{E}-04$ \\
\hline $1.794 \mathrm{E}+01$ & $2.10 \mathrm{E}-01$ & $8.526 \mathrm{E}-02$ & 9.91E-03 & $4.585 \mathrm{E}-02$ & $3.95 \mathrm{E}-03$ & 7.297E-03 & $6.28 \mathrm{E}-04$ \\
\hline $1.720 \mathrm{E}+01$ & $2.03 \mathrm{E}-01$ & $7.706 \mathrm{E}-02$ & $9.98 \mathrm{E}-03$ & 4.337E-02 & $3.95 \mathrm{E}-03$ & $6.903 \mathrm{E}-03$ & $6.28 \mathrm{E}-04$ \\
\hline $1.614 \mathrm{E}+01$ & $1.91 \mathrm{E}-01$ & $6.525 \mathrm{E}-02$ & $1.01 \mathrm{E}-02$ & $4.120 \mathrm{E}-02$ & $3.95 \mathrm{E}-03$ & $6.557 \mathrm{E}-03$ & $6.29 \mathrm{E}-04$ \\
\hline $1.539 \mathrm{E}+01$ & $1.82 \mathrm{E}-01$ & $5.691 \mathrm{E}-02$ & $1.01 \mathrm{E}-02$ & $3.819 \mathrm{E}-02$ & $3.91 \mathrm{E}-03$ & $6.079 \mathrm{E}-03$ & $6.22 \mathrm{E}-04$ \\
\hline $1.435 \mathrm{E}+01$ & $1.72 \mathrm{E}-01$ & $4.766 \mathrm{E}-02$ & $1.01 \mathrm{E}-02$ & $3.460 \mathrm{E}-02$ & $3.89 \mathrm{E}-03$ & $5.507 \mathrm{E}-03$ & $6.19 \mathrm{E}-04$ \\
\hline $1.299 \mathrm{E}+01$ & $1.58 \mathrm{E}-01$ & $3.459 \mathrm{E}-02$ & $1.02 \mathrm{E}-02$ & $3.007 \mathrm{E}-02$ & $3.90 \mathrm{E}-03$ & $4.786 \mathrm{E}-03$ & $6.21 \mathrm{E}-04$ \\
\hline$\rho\left(\mathrm{kg} / \mathrm{m}^{\wedge} 3\right)$ & $\eta_{\mathrm{T}}$ & $\Delta \eta_{\mathrm{T}}$ & $J$ & $\Delta J$ & $\eta_{\mathrm{P}}$ & $\Delta \eta_{\mathrm{P}}$ & $R e_{0.75}$ \\
\hline $1.163 \mathrm{E}+00$ & 4.714E-01 & $3.85 \mathrm{E}-02$ & $8.930 \mathrm{E}-02$ & $5.81 \mathrm{E}-04$ & $1.824 \mathrm{E}-01$ & $2.38 \mathrm{E}-02$ & $3.719 \mathrm{E}+04$ \\
\hline $1.163 \mathrm{E}+00$ & $4.721 \mathrm{E}-01$ & $4.00 \mathrm{E}-02$ & $1.431 \mathrm{E}-01$ & $5.89 \mathrm{E}-04$ & $2.854 \mathrm{E}-01$ & $3.86 \mathrm{E}-02$ & $3.756 \mathrm{E}+04$ \\
\hline $1.163 \mathrm{E}+00$ & $4.727 \mathrm{E}-01$ & $4.10 \mathrm{E}-02$ & $1.986 \mathrm{E}-01$ & $6.54 \mathrm{E}-04$ & $3.693 \mathrm{E}-01$ & $5.34 \mathrm{E}-02$ & $3.782 \mathrm{E}+04$ \\
\hline $1.163 \mathrm{E}+00$ & 4.639E-01 & $4.26 \mathrm{E}-02$ & $2.571 \mathrm{E}-01$ & 7.47E-04 & $4.568 \mathrm{E}-01$ & 7.23E-02 & $3.783 \mathrm{E}+04$ \\
\hline $1.162 \mathrm{E}+00$ & $4.601 \mathrm{E}-01$ & $4.45 \mathrm{E}-02$ & $3.168 \mathrm{E}-01$ & $8.03 \mathrm{E}-04$ & $5.018 \mathrm{E}-01$ & $9.14 \mathrm{E}-02$ & $3.766 \mathrm{E}+04$ \\
\hline $1.162 \mathrm{E}+00$ & $4.508 \mathrm{E}-01$ & $4.64 \mathrm{E}-02$ & $3.763 \mathrm{E}-01$ & $8.93 \mathrm{E}-04$ & $5.607 \mathrm{E}-01$ & $1.15 \mathrm{E}-01$ & $3.788 \mathrm{E}+04$ \\
\hline $1.162 \mathrm{E}+00$ & 4.382E-01 & $4.96 \mathrm{E}-02$ & $4.370 \mathrm{E}-01$ & $1.00 \mathrm{E}-03$ & 6.019E-01 & $1.44 \mathrm{E}-01$ & $3.802 \mathrm{E}+04$ \\
\hline $1.161 \mathrm{E}+00$ & $4.140 \mathrm{E}-01$ & $5.40 \mathrm{E}-02$ & $5.005 \mathrm{E}-01$ & $1.05 \mathrm{E}-03$ & $5.757 \mathrm{E}-01$ & $1.86 \mathrm{E}-01$ & $3.798 \mathrm{E}+04$ \\
\hline
\end{tabular}


Table 256: GWS 4.5 x 3.0 Dynamic Measured Values - 14011 RPM

\begin{tabular}{|c|c|c|c|c|c|c|c|}
\hline$n(\mathrm{RPM})$ & $Q(\mathrm{~g}-\mathrm{m})$ & $\Delta Q(\mathrm{~g}-\mathrm{m})$ & $V(\mathrm{~V})$ & $I(\mathrm{~A})$ & $T_{\text {atm }}\left({ }^{\circ} \mathrm{C}\right)$ & $P_{\text {atm }}(\mathrm{Pa})$ & $P_{\text {diff }}(\mathrm{Pa})$ \\
\hline $1.401 \mathrm{E}+04$ & $9.936 \mathrm{E}-01$ & $6.05 \mathrm{E}-02$ & $1.105 \mathrm{E}+01$ & $2.403 \mathrm{E}+00$ & $2.199 \mathrm{E}+01$ & $9.837 \mathrm{E}+04$ & $2.817 \mathrm{E}+00$ \\
\hline $1.405 \mathrm{E}+04$ & $9.783 \mathrm{E}-01$ & $6.06 \mathrm{E}-02$ & $1.105 \mathrm{E}+01$ & $2.362 \mathrm{E}+00$ & $2.201 \mathrm{E}+01$ & $9.838 \mathrm{E}+04$ & $6.801 \mathrm{E}+00$ \\
\hline $1.399 \mathrm{E}+04$ & $9.522 \mathrm{E}-01$ & $6.04 \mathrm{E}-02$ & $1.105 \mathrm{E}+01$ & $2.288 \mathrm{E}+00$ & $2.218 \mathrm{E}+01$ & $9.838 \mathrm{E}+04$ & $1.282 \mathrm{E}+01$ \\
\hline $1.401 \mathrm{E}+04$ & $9.125 \mathrm{E}-01$ & $6.03 \mathrm{E}-02$ & $1.105 \mathrm{E}+01$ & $2.212 \mathrm{E}+00$ & $2.230 \mathrm{E}+01$ & $9.838 \mathrm{E}+04$ & $2.094 \mathrm{E}+01$ \\
\hline $1.397 \mathrm{E}+04$ & $8.687 \mathrm{E}-01$ & $6.02 \mathrm{E}-02$ & $1.106 \mathrm{E}+01$ & $2.107 \mathrm{E}+00$ & $2.242 \mathrm{E}+01$ & $9.838 \mathrm{E}+04$ & $3.132 \mathrm{E}+01$ \\
\hline $1.403 \mathrm{E}+04$ & $8.186 \mathrm{E}-01$ & $6.03 \mathrm{E}-02$ & $1.106 \mathrm{E}+01$ & $2.024 \mathrm{E}+00$ & $2.249 \mathrm{E}+01$ & $9.837 \mathrm{E}+04$ & $4.359 \mathrm{E}+01$ \\
\hline $1.404 \mathrm{E}+04$ & $7.625 \mathrm{E}-01$ & $5.96 \mathrm{E}-02$ & $1.106 \mathrm{E}+01$ & $1.916 \mathrm{E}+00$ & $2.251 \mathrm{E}+01$ & $9.837 \mathrm{E}+04$ & $5.870 \mathrm{E}+01$ \\
\hline $1.399 \mathrm{E}+04$ & $7.059 \mathrm{E}-01$ & $6.03 \mathrm{E}-02$ & $1.107 \mathrm{E}+01$ & $1.777 \mathrm{E}+00$ & $2.254 \mathrm{E}+01$ & $9.838 \mathrm{E}+04$ & $7.602 \mathrm{E}+01$ \\
\hline $1.401 \mathrm{E}+04$ & $6.405 \mathrm{E}-01$ & $5.99 \mathrm{E}-02$ & $1.107 \mathrm{E}+01$ & $1.642 \mathrm{E}+00$ & $2.265 \mathrm{E}+01$ & $9.838 \mathrm{E}+04$ & $9.550 \mathrm{E}+01$ \\
\hline $1.401 \mathrm{E}+04$ & $5.458 \mathrm{E}-01$ & $5.97 \mathrm{E}-02$ & $1.107 \mathrm{E}+01$ & $1.482 \mathrm{E}+00$ & $2.269 \mathrm{E}+01$ & $9.838 \mathrm{E}+04$ & $1.173 \mathrm{E}+02$ \\
\hline
\end{tabular}


Table 257: GWS 4.5 x 3.0 Dynamic Calculated Values - 14011 RPM

\begin{tabular}{|c|c|c|c|c|c|c|c|}
\hline$n(\mathrm{RPM})$ & $V_{\infty}^{\prime}(\mathrm{m} / \mathrm{s})$ & $\Delta V_{\infty}^{\prime}(\mathrm{m} / \mathrm{s})$ & $T^{\prime}(\mathrm{g})$ & $\Delta T^{\prime}(\mathrm{g})$ & $V_{\mathrm{t}}(\mathrm{m} / \mathrm{s})$ & $P_{\mathrm{P}}(\mathrm{W})$ & $\Delta P_{\mathrm{P}}(\mathrm{W})$ \\
\hline $1.401 \mathrm{E}+04$ & $2.109 \mathrm{E}+00$ & $1.40 \mathrm{E}-02$ & $1.144 \mathrm{E}+02$ & $8.59 \mathrm{E}+00$ & $6.290 \mathrm{E}+01$ & $1.429 \mathrm{E}+01$ & $8.70 \mathrm{E}-01$ \\
\hline $1.405 \mathrm{E}+04$ & $3.333 \mathrm{E}+00$ & $1.42 \mathrm{E}-02$ & $1.087 \mathrm{E}+02$ & $8.53 \mathrm{E}+00$ & $6.314 \mathrm{E}+01$ & $1.411 \mathrm{E}+01$ & $8.75 \mathrm{E}-01$ \\
\hline $1.399 \mathrm{E}+04$ & $4.618 \mathrm{E}+00$ & $1.51 \mathrm{E}-02$ & $1.007 \mathrm{E}+02$ & $8.53 \mathrm{E}+00$ & $6.295 \mathrm{E}+01$ & $1.368 \mathrm{E}+01$ & $8.68 \mathrm{E}-01$ \\
\hline $1.401 \mathrm{E}+04$ & $5.931 \mathrm{E}+00$ & $1.68 \mathrm{E}-02$ & $9.237 \mathrm{E}+01$ & $8.59 \mathrm{E}+00$ & $6.315 \mathrm{E}+01$ & $1.313 \mathrm{E}+01$ & $8.68 \mathrm{E}-01$ \\
\hline $1.397 \mathrm{E}+04$ & $7.282 \mathrm{E}+00$ & $2.06 \mathrm{E}-02$ & $8.128 \mathrm{E}+01$ & $8.56 \mathrm{E}+00$ & $6.314 \mathrm{E}+01$ & $1.247 \mathrm{E}+01$ & 8.64E-01 \\
\hline $1.403 \mathrm{E}+04$ & $8.612 \mathrm{E}+00$ & $2.00 \mathrm{E}-02$ & $7.413 \mathrm{E}+01$ & $8.60 \mathrm{E}+00$ & $6.358 \mathrm{E}+01$ & $1.180 \mathrm{E}+01$ & $8.69 \mathrm{E}-01$ \\
\hline $1.404 \mathrm{E}+04$ & $1.001 \mathrm{E}+01$ & 2.18E-02 & $6.498 \mathrm{E}+01$ & $8.55 \mathrm{E}+00$ & $6.379 \mathrm{E}+01$ & $1.099 \mathrm{E}+01$ & 8.59E-01 \\
\hline $1.399 \mathrm{E}+04$ & $1.141 \mathrm{E}+01$ & 2.38E-02 & $5.278 \mathrm{E}+01$ & $8.56 \mathrm{E}+00$ & $6.383 \mathrm{E}+01$ & $1.014 \mathrm{E}+01$ & $8.66 \mathrm{E}-01$ \\
\hline $1.401 \mathrm{E}+04$ & $1.281 \mathrm{E}+01$ & $2.62 \mathrm{E}-02$ & $4.137 \mathrm{E}+01$ & $8.57 \mathrm{E}+00$ & $6.417 \mathrm{E}+01$ & $9.214 \mathrm{E}+00$ & $8.62 \mathrm{E}-01$ \\
\hline $1.401 \mathrm{E}+04$ & $1.421 \mathrm{E}+01$ & 2.87E-02 & $3.221 \mathrm{E}+01$ & $8.56 \mathrm{E}+00$ & $6.447 \mathrm{E}+01$ & $7.853 \mathrm{E}+00$ & 8.59E-01 \\
\hline$P_{\mathrm{e}}(\mathrm{W})$ & $\Delta P_{\mathrm{e}}(\mathrm{W})$ & $C_{\mathrm{T}}$ & $\Delta C_{\mathrm{T}}$ & $C_{\mathrm{P}}$ & $\Delta C_{\mathrm{P}}$ & $C_{\mathrm{Q}}$ & $\Delta C_{\mathrm{Q}}$ \\
\hline $2.654 \mathrm{E}+01$ & $2.98 \mathrm{E}-01$ & $1.039 \mathrm{E}-01$ & $7.81 \mathrm{E}-03$ & 4.962E-02 & $3.03 \mathrm{E}-03$ & 7.897E-03 & 4.81E-04 \\
\hline $2.610 \mathrm{E}+01$ & $2.95 \mathrm{E}-01$ & $9.816 \mathrm{E}-02$ & 7.70E-03 & 4.856E-02 & $3.01 \mathrm{E}-03$ & 7.729E-03 & 4.79E-04 \\
\hline $2.529 \mathrm{E}+01$ & $2.86 \mathrm{E}-01$ & $9.180 \mathrm{E}-02$ & 7.77E-03 & 4.770E-02 & $3.03 \mathrm{E}-03$ & $7.592 \mathrm{E}-03$ & 4.82E-04 \\
\hline $2.445 \mathrm{E}+01$ & $2.78 \mathrm{E}-01$ & 8.397E-02 & $7.81 \mathrm{E}-03$ & $4.560 \mathrm{E}-02$ & $3.02 \mathrm{E}-03$ & $7.257 \mathrm{E}-03$ & $4.80 \mathrm{E}-04$ \\
\hline $2.330 \mathrm{E}+01$ & 2.67E-01 & 7.429E-02 & 7.82E-03 & 4.365E-02 & $3.03 \mathrm{E}-03$ & $6.947 \mathrm{E}-03$ & 4.81E-04 \\
\hline $2.239 \mathrm{E}+01$ & $2.56 \mathrm{E}-01$ & $6.718 \mathrm{E}-02$ & $7.80 \mathrm{E}-03$ & 4.079E-02 & $3.01 \mathrm{E}-03$ & $6.491 \mathrm{E}-03$ & 4.78E-04 \\
\hline $2.119 \mathrm{E}+01$ & $2.44 \mathrm{E}-01$ & $5.887 \mathrm{E}-02$ & $7.75 \mathrm{E}-03$ & $3.798 \mathrm{E}-02$ & $2.97 \mathrm{E}-03$ & $6.045 \mathrm{E}-03$ & 4.73E-04 \\
\hline $1.966 \mathrm{E}+01$ & $2.28 \mathrm{E}-01$ & 4.812E-02 & 7.80E-03 & $3.539 \mathrm{E}-02$ & $3.02 \mathrm{E}-03$ & $5.632 \mathrm{E}-03$ & 4.81E-04 \\
\hline $1.818 \mathrm{E}+01$ & $2.13 \mathrm{E}-01$ & $3.765 \mathrm{E}-02$ & 7.80E-03 & $3.204 \mathrm{E}-02$ & $3.00 \mathrm{E}-03$ & $5.100 \mathrm{E}-03$ & 4.77E-04 \\
\hline $1.642 \mathrm{E}+01$ & $1.92 \mathrm{E}-01$ & $2.930 \mathrm{E}-02$ & 7.79E-03 & $2.730 \mathrm{E}-02$ & $2.99 \mathrm{E}-03$ & $4.345 \mathrm{E}-03$ & 4.75E-04 \\
\hline$\rho\left(\mathrm{kg} / \mathrm{m}^{\wedge} 3\right)$ & $\eta_{\mathrm{T}}$ & $\Delta \eta_{\mathrm{T}}$ & $J$ & $\Delta J$ & $\eta_{\mathrm{P}}$ & $\Delta \eta_{\mathrm{P}}$ & $R e_{0.75}$ \\
\hline $1.161 \mathrm{E}+00$ & $5.385 \mathrm{E}-01$ & 3.33E-02 & $7.906 \mathrm{E}-02$ & $5.32 \mathrm{E}-04$ & $1.656 \mathrm{E}-01$ & $1.61 \mathrm{E}-02$ & $4.360 \mathrm{E}+04$ \\
\hline $1.161 \mathrm{E}+00$ & $5.407 \mathrm{E}-01$ & $3.41 \mathrm{E}-02$ & $1.246 \mathrm{E}-01$ & $5.44 \mathrm{E}-04$ & $2.518 \mathrm{E}-01$ & $2.52 \mathrm{E}-02$ & $4.376 \mathrm{E}+04$ \\
\hline $1.160 \mathrm{E}+00$ & $5.409 \mathrm{E}-01$ & $3.49 \mathrm{E}-02$ & $1.733 \mathrm{E}-01$ & $5.91 \mathrm{E}-04$ & $3.335 \mathrm{E}-01$ & $3.53 \mathrm{E}-02$ & $4.359 \mathrm{E}+04$ \\
\hline $1.160 \mathrm{E}+00$ & $5.369 \mathrm{E}-01$ & $3.60 \mathrm{E}-02$ & $2.223 \mathrm{E}-01$ & $6.64 \mathrm{E}-04$ & 4.093E-01 & $4.68 \mathrm{E}-02$ & $4.369 \mathrm{E}+04$ \\
\hline $1.159 \mathrm{E}+00$ & 5.350E-01 & $3.76 \mathrm{E}-02$ & $2.736 \mathrm{E}-01$ & $8.19 \mathrm{E}-04$ & $4.656 \mathrm{E}-01$ & $5.87 \mathrm{E}-02$ & $4.365 \mathrm{E}+04$ \\
\hline $1.159 \mathrm{E}+00$ & $5.270 \mathrm{E}-01$ & 3.93E-02 & $3.221 \mathrm{E}-01$ & 8.08E-04 & $5.306 \mathrm{E}-01$ & 7.30E-02 & $4.393 E+04$ \\
\hline $1.159 \mathrm{E}+00$ & $5.186 \mathrm{E}-01$ & 4.10E-02 & $3.745 \mathrm{E}-01$ & 8.92E-04 & $5.805 \mathrm{E}-01$ & 8.89E-02 & $4.408 \mathrm{E}+04$ \\
\hline $1.159 \mathrm{E}+00$ & 5.159E-01 & $4.45 \mathrm{E}-02$ & 4.282E-01 & $9.81 \mathrm{E}-04$ & $5.823 \mathrm{E}-01$ & $1.07 \mathrm{E}-01$ & $4.410 \mathrm{E}+04$ \\
\hline $1.159 \mathrm{E}+00$ & $5.070 \mathrm{E}-01$ & $4.78 \mathrm{E}-02$ & 4.800E-01 & $1.09 \mathrm{E}-03$ & $5.640 \mathrm{E}-01$ & $1.28 \mathrm{E}-01$ & $4.430 \mathrm{E}+04$ \\
\hline $1.158 \mathrm{E}+00$ & 4.784E-01 & $5.26 \mathrm{E}-02$ & 5.324E-01 & $1.19 \mathrm{E}-03$ & 5.714E-01 & $1.64 \mathrm{E}-01$ & $4.450 \mathrm{E}+04$ \\
\hline
\end{tabular}


Table 258: GWS 4.5 x 3.0 Dynamic Measured Values - 15996 RPM

\begin{tabular}{|c|c|c|c|c|c|c|c|}
\hline$n(\mathrm{RPM})$ & $Q(\mathrm{~g}-\mathrm{m})$ & $\Delta Q(\mathrm{~g}-\mathrm{m})$ & $V(\mathrm{~V})$ & $I(\mathrm{~A})$ & $T_{\text {atm }}\left({ }^{\circ} \mathrm{C}\right)$ & $P_{\text {atm }}(\mathrm{Pa})$ & $P_{\text {diff }}(\mathrm{Pa})$ \\
\hline $1.594 \mathrm{E}+04$ & $1.332 \mathrm{E}+00$ & $6.29 \mathrm{E}-02$ & $1.102 \mathrm{E}+01$ & $3.290 \mathrm{E}+00$ & $2.219 \mathrm{E}+01$ & $9.838 \mathrm{E}+04$ & $2.992 \mathrm{E}+00$ \\
\hline $1.601 \mathrm{E}+04$ & $1.309 \mathrm{E}+00$ & $6.30 \mathrm{E}-02$ & $1.102 \mathrm{E}+01$ & $3.236 \mathrm{E}+00$ & $2.218 \mathrm{E}+01$ & $9.839 \mathrm{E}+04$ & $8.817 \mathrm{E}+00$ \\
\hline $1.601 \mathrm{E}+04$ & $1.270 \mathrm{E}+00$ & $6.30 \mathrm{E}-02$ & $1.103 \mathrm{E}+01$ & $3.146 \mathrm{E}+00$ & $2.234 \mathrm{E}+01$ & $9.838 \mathrm{E}+04$ & $1.835 \mathrm{E}+01$ \\
\hline $1.604 \mathrm{E}+04$ & $1.207 \mathrm{E}+00$ & $6.34 \mathrm{E}-02$ & $1.103 \mathrm{E}+01$ & $3.012 \mathrm{E}+00$ & $2.249 \mathrm{E}+01$ & $9.837 \mathrm{E}+04$ & $3.174 \mathrm{E}+01$ \\
\hline $1.602 \mathrm{E}+04$ & $1.123 \mathrm{E}+00$ & $6.26 \mathrm{E}-02$ & $1.104 \mathrm{E}+01$ & $2.810 \mathrm{E}+00$ & $2.260 \mathrm{E}+01$ & $9.837 \mathrm{E}+04$ & $4.869 \mathrm{E}+01$ \\
\hline $1.602 \mathrm{E}+04$ & $1.022 \mathrm{E}+00$ & $6.28 \mathrm{E}-02$ & $1.104 \mathrm{E}+01$ & $2.613 \mathrm{E}+00$ & $2.267 \mathrm{E}+01$ & $9.837 \mathrm{E}+04$ & $7.036 \mathrm{E}+01$ \\
\hline $1.600 \mathrm{E}+04$ & $9.356 \mathrm{E}-01$ & $6.22 \mathrm{E}-02$ & $1.105 \mathrm{E}+01$ & $2.392 \mathrm{E}+00$ & $2.271 \mathrm{E}+01$ & $9.838 \mathrm{E}+04$ & $9.585 \mathrm{E}+01$ \\
\hline $1.598 \mathrm{E}+04$ & $8.165 \mathrm{E}-01$ & $6.23 \mathrm{E}-02$ & $1.105 \mathrm{E}+01$ & $2.143 \mathrm{E}+00$ & $2.273 \mathrm{E}+01$ & $9.837 \mathrm{E}+04$ & $1.254 \mathrm{E}+02$ \\
\hline $1.595 \mathrm{E}+04$ & $6.666 \mathrm{E}-01$ & $6.17 \mathrm{E}-02$ & $1.106 \mathrm{E}+01$ & $1.850 \mathrm{E}+00$ & $2.241 \mathrm{E}+01$ & $9.838 \mathrm{E}+04$ & $1.590 \mathrm{E}+02$ \\
\hline
\end{tabular}


Table 259: GWS 4.5 x 3.0 Dynamic Calculated Values - 15996 RPM

\begin{tabular}{|c|c|c|c|c|c|c|c|}
\hline$n(\mathrm{RPM})$ & $V_{\infty}^{\prime}(\mathrm{m} / \mathrm{s})$ & $\Delta V_{\infty}^{\prime}(\mathrm{m} / \mathrm{s})$ & $T^{\prime}(\mathrm{g})$ & $\Delta T^{\prime}(\mathrm{g})$ & $V_{\mathrm{t}}(\mathrm{m} / \mathrm{s})$ & $P_{\mathrm{P}}(\mathrm{W})$ & $\Delta P_{\mathrm{P}}(\mathrm{W})$ \\
\hline $1.594 \mathrm{E}+04$ & $2.163 \mathrm{E}+00$ & $1.51 \mathrm{E}-02$ & $1.500 \mathrm{E}+02$ & $7.67 \mathrm{E}+00$ & $7.157 \mathrm{E}+01$ & $2.180 \mathrm{E}+01$ & $1.03 \mathrm{E}+00$ \\
\hline $1.601 \mathrm{E}+04$ & $3.795 \mathrm{E}+00$ & $1.57 \mathrm{E}-02$ & $1.429 \mathrm{E}+02$ & $7.57 \mathrm{E}+00$ & $7.197 \mathrm{E}+01$ & $2.152 \mathrm{E}+01$ & $1.04 \mathrm{E}+00$ \\
\hline $1.601 \mathrm{E}+04$ & $5.529 \mathrm{E}+00$ & $1.71 \mathrm{E}-02$ & $1.331 \mathrm{E}+02$ & $7.56 \mathrm{E}+00$ & $7.205 \mathrm{E}+01$ & $2.088 \mathrm{E}+01$ & $1.03 \mathrm{E}+00$ \\
\hline $1.604 \mathrm{E}+04$ & $7.314 \mathrm{E}+00$ & $1.91 \mathrm{E}-02$ & $1.208 \mathrm{E}+02$ & $7.58 \mathrm{E}+00$ & $7.237 \mathrm{E}+01$ & $1.989 \mathrm{E}+01$ & $1.04 \mathrm{E}+00$ \\
\hline $1.602 \mathrm{E}+04$ & $9.093 \mathrm{E}+00$ & $2.17 \mathrm{E}-02$ & $1.052 \mathrm{E}+02$ & $7.59 \mathrm{E}+00$ & $7.245 \mathrm{E}+01$ & $1.847 \mathrm{E}+01$ & $1.03 \mathrm{E}+00$ \\
\hline $1.602 \mathrm{E}+04$ & $1.096 \mathrm{E}+01$ & 2.37E-02 & $8.820 \mathrm{E}+01$ & $7.55 \mathrm{E}+00$ & $7.274 \mathrm{E}+01$ & $1.682 \mathrm{E}+01$ & $1.03 \mathrm{E}+00$ \\
\hline $1.600 \mathrm{E}+04$ & $1.281 \mathrm{E}+01$ & $2.65 \mathrm{E}-02$ & $7.323 \mathrm{E}+01$ & $7.56 \mathrm{E}+00$ & $7.294 \mathrm{E}+01$ & $1.537 \mathrm{E}+01$ & $1.02 \mathrm{E}+00$ \\
\hline $1.598 \mathrm{E}+04$ & $1.468 \mathrm{E}+01$ & $2.98 \mathrm{E}-02$ & $5.570 \mathrm{E}+01$ & $7.61 \mathrm{E}+00$ & $7.323 \mathrm{E}+01$ & $1.340 \mathrm{E}+01$ & $1.02 \mathrm{E}+00$ \\
\hline $1.595 \mathrm{E}+04$ & $1.654 \mathrm{E}+01$ & $3.23 \mathrm{E}-02$ & $3.619 \mathrm{E}+01$ & $7.77 \mathrm{E}+00$ & $7.348 \mathrm{E}+01$ & $1.092 \mathrm{E}+01$ & $1.01 \mathrm{E}+00$ \\
\hline$P_{\mathrm{e}}(\mathrm{W})$ & $\Delta P_{\mathrm{e}}(\mathrm{W})$ & $C_{\mathrm{T}}$ & $\Delta C_{\mathrm{T}}$ & $C_{\mathrm{P}}$ & $\Delta C_{\mathrm{P}}$ & $C_{\mathrm{Q}}$ & $\Delta C_{\mathrm{Q}}$ \\
\hline $3.626 \mathrm{E}+01$ & 4.06E-01 & $1.053 \mathrm{E}-01$ & $5.38 \mathrm{E}-03$ & $5.140 \mathrm{E}-02$ & $2.43 \mathrm{E}-03$ & 8.181E-03 & $3.86 \mathrm{E}-04$ \\
\hline $3.567 \mathrm{E}+01$ & $4.02 \mathrm{E}-01$ & $9.936 \mathrm{E}-02$ & $5.27 \mathrm{E}-03$ & $5.004 \mathrm{E}-02$ & $2.41 \mathrm{E}-03$ & 7.964E-03 & $3.84 \mathrm{E}-04$ \\
\hline $3.469 \mathrm{E}+01$ & $3.88 \mathrm{E}-01$ & $9.269 \mathrm{E}-02$ & $5.27 \mathrm{E}-03$ & $4.863 \mathrm{E}-02$ & $2.41 \mathrm{E}-03$ & $7.740 \mathrm{E}-03$ & $3.84 \mathrm{E}-04$ \\
\hline $3.323 \mathrm{E}+01$ & $3.74 \mathrm{E}-01$ & 8.377E-02 & $5.26 \mathrm{E}-03$ & 4.604E-02 & $2.42 \mathrm{E}-03$ & $7.328 \mathrm{E}-03$ & $3.85 \mathrm{E}-04$ \\
\hline $3.101 \mathrm{E}+01$ & $3.51 \mathrm{E}-01$ & $7.321 \mathrm{E}-02$ & $5.28 \mathrm{E}-03$ & 4.298E-02 & $2.40 \mathrm{E}-03$ & $6.841 \mathrm{E}-03$ & $3.81 \mathrm{E}-04$ \\
\hline $2.886 \mathrm{E}+01$ & $3.30 \mathrm{E}-01$ & $6.137 \mathrm{E}-02$ & $5.25 \mathrm{E}-03$ & $3.910 \mathrm{E}-02$ & $2.40 \mathrm{E}-03$ & $6.223 \mathrm{E}-03$ & $3.82 \mathrm{E}-04$ \\
\hline $2.643 \mathrm{E}+01$ & $3.01 \mathrm{E}-01$ & $5.111 \mathrm{E}-02$ & $5.28 \mathrm{E}-03$ & $3.590 \mathrm{E}-02$ & 2.39E-03 & $5.714 \mathrm{E}-03$ & $3.80 \mathrm{E}-04$ \\
\hline $2.369 \mathrm{E}+01$ & $2.71 \mathrm{E}-01$ & $3.895 \mathrm{E}-02$ & $5.32 \mathrm{E}-03$ & $3.139 \mathrm{E}-02$ & $2.39 \mathrm{E}-03$ & $4.995 \mathrm{E}-03$ & $3.81 \mathrm{E}-04$ \\
\hline $2.046 \mathrm{E}+01$ & $2.35 \mathrm{E}-01$ & $2.538 \mathrm{E}-02$ & $5.45 \mathrm{E}-03$ & $2.570 \mathrm{E}-02$ & $2.38 \mathrm{E}-03$ & $4.090 \mathrm{E}-03$ & $3.78 \mathrm{E}-04$ \\
\hline$\rho\left(\mathrm{kg} / \mathrm{m}^{\wedge} 3\right)$ & $\eta_{\mathrm{T}}$ & $\Delta \eta_{\mathrm{T}}$ & $J$ & $\Delta J$ & $\eta_{\mathrm{P}}$ & $\Delta \eta_{\mathrm{P}}$ & $R e_{0.75}$ \\
\hline $1.160 \mathrm{E}+00$ & $6.013 \mathrm{E}-01$ & $2.92 \mathrm{E}-02$ & $7.126 \mathrm{E}-02$ & 4.99E-04 & $1.459 \mathrm{E}-01$ & $1.02 \mathrm{E}-02$ & $4.955 \mathrm{E}+04$ \\
\hline $1.161 \mathrm{E}+00$ & $6.035 \mathrm{E}-01$ & $2.98 \mathrm{E}-02$ & $1.244 \mathrm{E}-01$ & $5.14 \mathrm{E}-04$ & $2.471 \mathrm{E}-01$ & $1.77 \mathrm{E}-02$ & $4.983 \mathrm{E}+04$ \\
\hline $1.160 \mathrm{E}+00$ & $6.021 \mathrm{E}-01$ & $3.06 \mathrm{E}-02$ & $1.814 \mathrm{E}-01$ & $5.64 \mathrm{E}-04$ & $3.456 \mathrm{E}-01$ & 2.61E-02 & 4.984E+04 \\
\hline $1.159 \mathrm{E}+00$ & $5.986 \mathrm{E}-01$ & $3.21 \mathrm{E}-02$ & $2.394 \mathrm{E}-01$ & $6.27 \mathrm{E}-04$ & $4.355 \mathrm{E}-01$ & $3.57 \mathrm{E}-02$ & $5.001 \mathrm{E}+04$ \\
\hline $1.159 \mathrm{E}+00$ & $5.956 \mathrm{E}-01$ & $3.39 \mathrm{E}-02$ & $2.981 \mathrm{E}-01$ & $7.14 \mathrm{E}-04$ & $5.077 \mathrm{E}-01$ & $4.63 \mathrm{E}-02$ & $5.004 \mathrm{E}+04$ \\
\hline $1.158 \mathrm{E}+00$ & 5.829E-01 & $3.64 \mathrm{E}-02$ & 3.591E-01 & $7.81 \mathrm{E}-04$ & $5.636 \mathrm{E}-01$ & $5.94 \mathrm{E}-02$ & $5.021 \mathrm{E}+04$ \\
\hline $1.158 \mathrm{E}+00$ & $5.815 \mathrm{E}-01$ & $3.93 \mathrm{E}-02$ & $4.205 \mathrm{E}-01$ & $8.74 \mathrm{E}-04$ & $5.988 \mathrm{E}-01$ & $7.36 \mathrm{E}-02$ & $5.034 \mathrm{E}+04$ \\
\hline $1.158 \mathrm{E}+00$ & $5.657 \mathrm{E}-01$ & $4.36 \mathrm{E}-02$ & $4.822 \mathrm{E}-01$ & $9.83 \mathrm{E}-04$ & $5.983 \mathrm{E}-01$ & $9.36 \mathrm{E}-02$ & $5.053 \mathrm{E}+04$ \\
\hline $1.160 \mathrm{E}+00$ & $5.336 \mathrm{E}-01$ & 4.97E-02 & 5.443E-01 & $1.07 \mathrm{E}-03$ & $5.376 \mathrm{E}-01$ & $1.26 \mathrm{E}-01$ & $5.080 \mathrm{E}+04$ \\
\hline
\end{tabular}




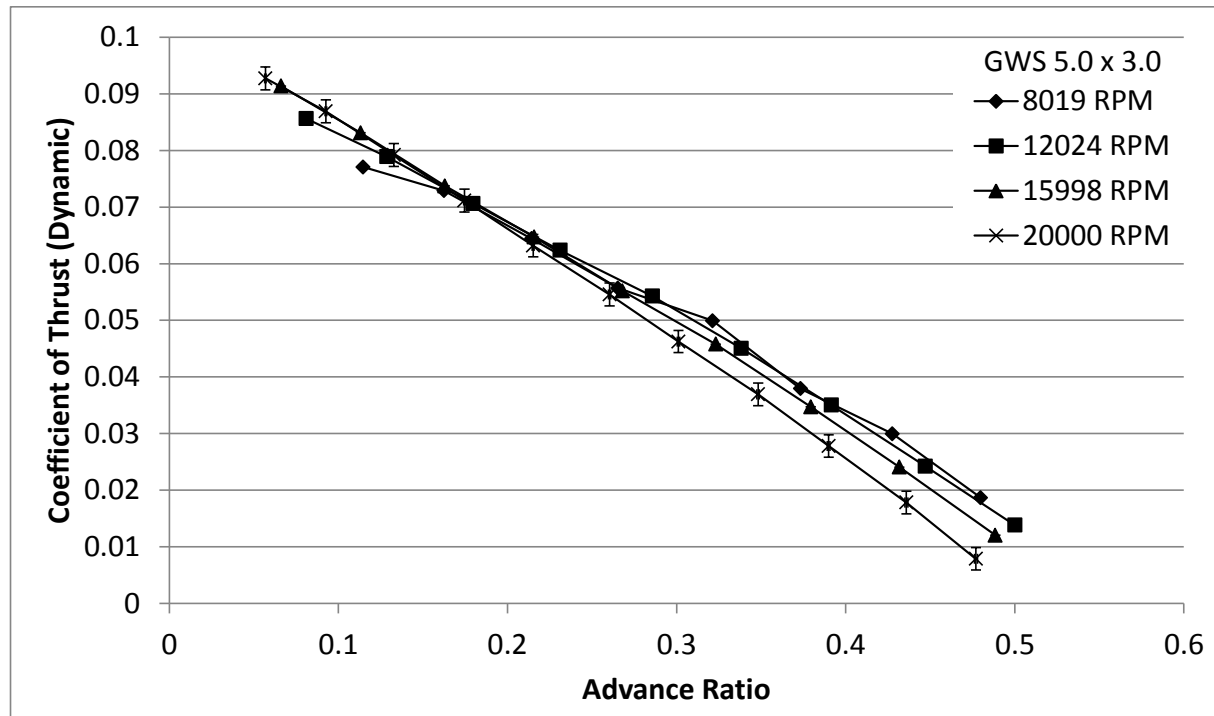

(a)

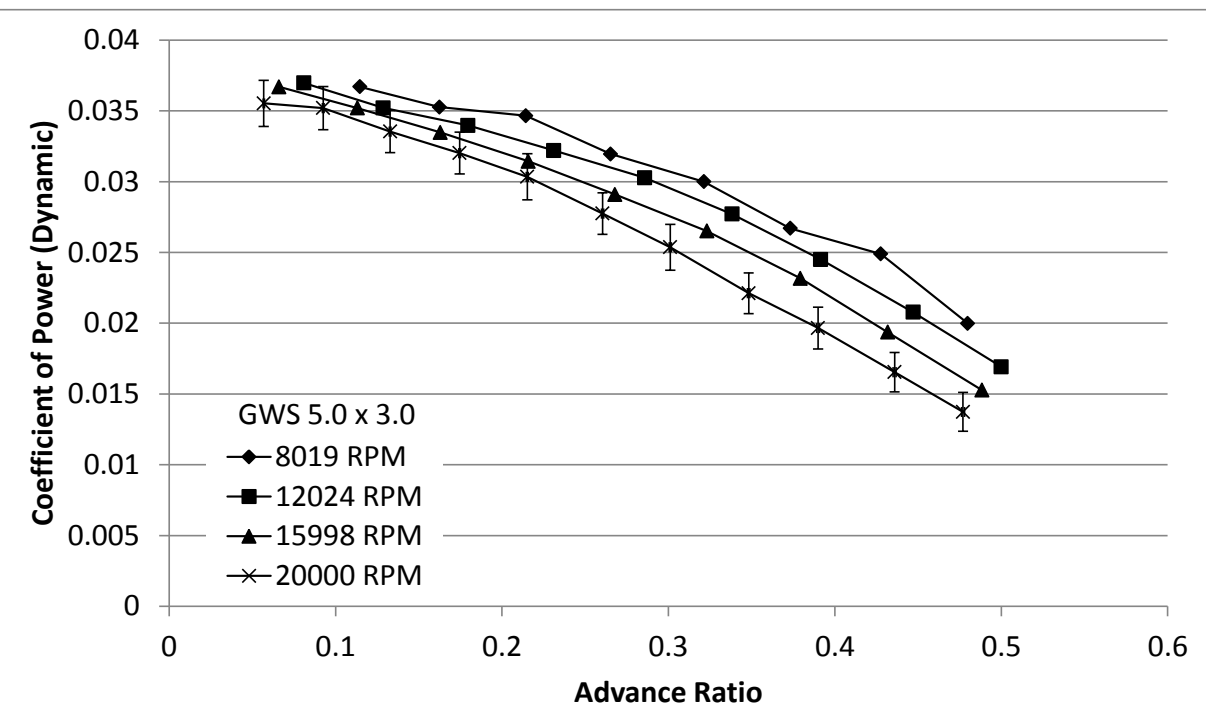

(b)

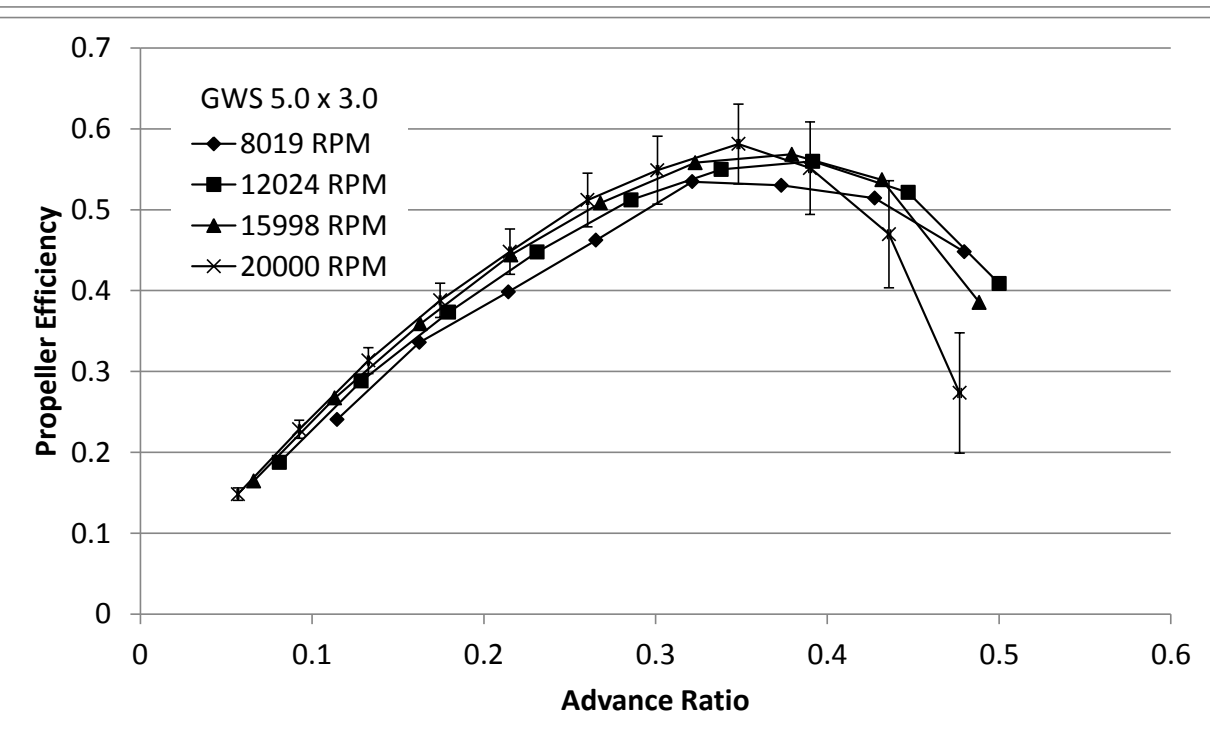

(c) 


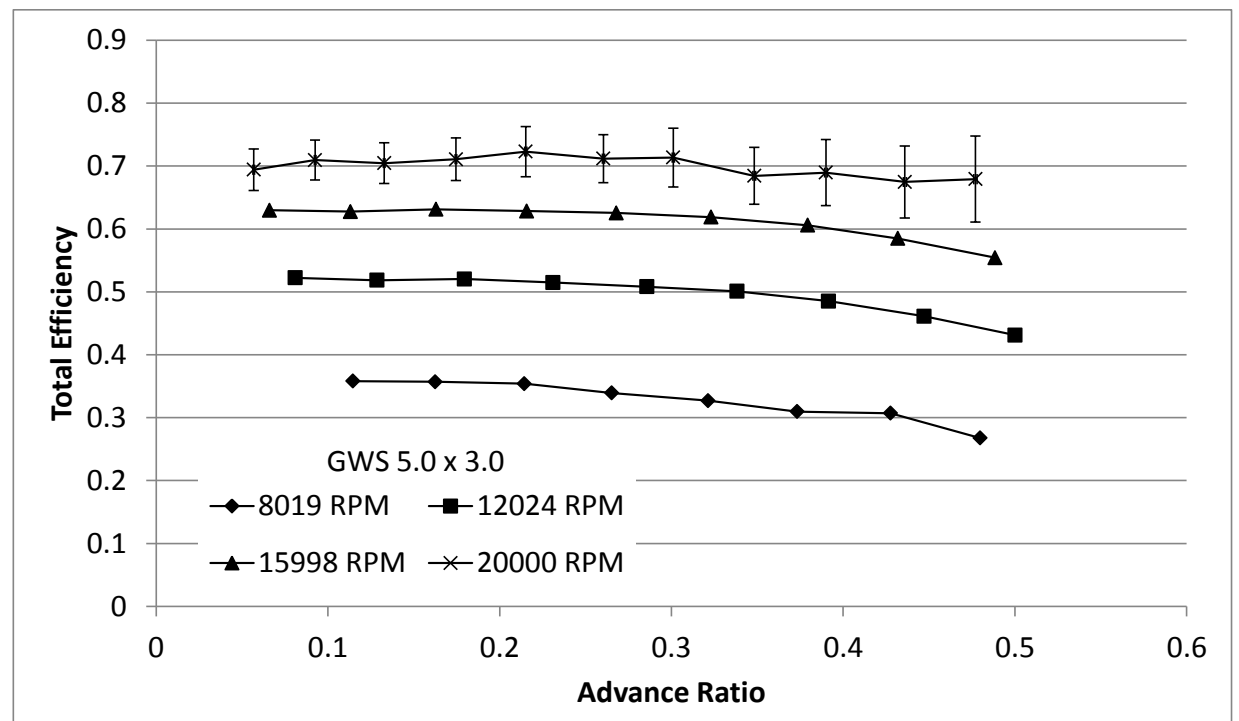

(d)

Figure 101: GWS 5.0 x 3.0 Dynamic Test Results: (a) Coefficient of Thrust, (b)

Coefficient of Power, (c) Propeller Efficiency, (d) Total Efficiency.

Table 260: GWS 5.0 x 3.0 Dynamic Measured Values - 8019 RPM

\begin{tabular}{|c|c|c|c|c|c|c|c|}
\hline$n(\mathrm{RPM})$ & $Q(\mathrm{~g}-\mathrm{m})$ & $\Delta Q(\mathrm{~g}-\mathrm{m})$ & $V(\mathrm{~V})$ & $I(\mathrm{~A})$ & $T_{\text {atm }}\left({ }^{\circ} \mathrm{C}\right)$ & $P_{\text {atm }}(\mathrm{Pa})$ & $P_{\text {diff }}(\mathrm{Pa})$ \\
\hline $8.005 \mathrm{E}+03$ & $4.099 \mathrm{E}-01$ & $6.54 \mathrm{E}-02$ & $1.109 \mathrm{E}+01$ & $8.483 \mathrm{E}-01$ & $2.188 \mathrm{E}+01$ & $9.840 \mathrm{E}+04$ & $2.340 \mathrm{E}+00$ \\
\hline $8.098 \mathrm{E}+03$ & $4.032 \mathrm{E}-01$ & $6.29 \mathrm{E}-02$ & $1.109 \mathrm{E}+01$ & $8.465 \mathrm{E}-01$ & $2.176 \mathrm{E}+01$ & $9.840 \mathrm{E}+04$ & $4.711 \mathrm{E}+00$ \\
\hline $8.032 \mathrm{E}+03$ & $3.896 \mathrm{E}-01$ & $6.53 \mathrm{E}-02$ & $1.109 \mathrm{E}+01$ & $8.182 \mathrm{E}-01$ & $2.188 \mathrm{E}+01$ & $9.839 \mathrm{E}+04$ & $7.956 \mathrm{E}+00$ \\
\hline $8.043 \mathrm{E}+03$ & $3.603 \mathrm{E}-01$ & $6.61 \mathrm{E}-02$ & $1.109 \mathrm{E}+01$ & $7.906 \mathrm{E}-01$ & $2.183 \mathrm{E}+01$ & $9.840 \mathrm{E}+04$ & $1.213 \mathrm{E}+01$ \\
\hline $7.961 \mathrm{E}+03$ & $3.313 \mathrm{E}-01$ & $6.84 \mathrm{E}-02$ & $1.109 \mathrm{E}+01$ & $7.465 \mathrm{E}-01$ & $2.183 \mathrm{E}+01$ & $9.840 \mathrm{E}+04$ & $1.732 \mathrm{E}+01$ \\
\hline $8.013 \mathrm{E}+03$ & $2.989 \mathrm{E}-01$ & $6.54 \mathrm{E}-02$ & $1.110 \mathrm{E}+01$ & $7.160 \mathrm{E}-01$ & $2.186 \mathrm{E}+01$ & $9.840 \mathrm{E}+04$ & $2.356 \mathrm{E}+01$ \\
\hline $7.988 \mathrm{E}+03$ & $2.767 \mathrm{E}-01$ & $6.74 \mathrm{E}-02$ & $1.110 \mathrm{E}+01$ & $6.662 \mathrm{E}-01$ & $2.203 \mathrm{E}+01$ & $9.841 \mathrm{E}+04$ & $3.062 \mathrm{E}+01$ \\
\hline $8.010 \mathrm{E}+03$ & $2.233 \mathrm{E}-01$ & $6.51 \mathrm{E}-02$ & $1.110 \mathrm{E}+01$ & $6.185 \mathrm{E}-01$ & $2.215 \mathrm{E}+01$ & $9.840 \mathrm{E}+04$ & $3.865 \mathrm{E}+01$ \\
\hline
\end{tabular}


Table 261: GWS 5.0 x 3.0 Dynamic Calculated Values - 8019 RPM

\begin{tabular}{|c|c|c|c|c|c|c|c|}
\hline$n(\mathrm{RPM})$ & $V_{\infty}^{\prime}(\mathrm{m} / \mathrm{s})$ & $\Delta V_{\infty}^{\prime}(\mathrm{m} / \mathrm{s})$ & $T^{\prime}(\mathrm{g})$ & $\Delta T^{\prime}(\mathrm{g})$ & $V_{\mathrm{t}}(\mathrm{m} / \mathrm{s})$ & $P_{\mathrm{P}}(\mathrm{W})$ & $\Delta P_{\mathrm{P}}(\mathrm{W})$ \\
\hline $8.005 \mathrm{E}+03$ & $1.945 \mathrm{E}+00$ & $1.27 \mathrm{E}-02$ & $4.251 \mathrm{E}+01$ & $8.51 \mathrm{E}+00$ & $4.003 \mathrm{E}+01$ & $3.369 \mathrm{E}+00$ & $5.37 \mathrm{E}-01$ \\
\hline $8.098 \mathrm{E}+03$ & $2.788 \mathrm{E}+00$ & $1.23 \mathrm{E}-02$ & $4.118 \mathrm{E}+01$ & $8.51 \mathrm{E}+00$ & $4.055 \mathrm{E}+01$ & $3.353 \mathrm{E}+00$ & $5.23 \mathrm{E}-01$ \\
\hline $8.032 \mathrm{E}+03$ & $3.649 \mathrm{E}+00$ & $1.32 \mathrm{E}-02$ & $3.578 \mathrm{E}+01$ & $8.52 \mathrm{E}+00$ & $4.029 \mathrm{E}+01$ & $3.214 \mathrm{E}+00$ & 5.39E-01 \\
\hline $8.043 \mathrm{E}+03$ & $4.524 \mathrm{E}+00$ & $1.43 \mathrm{E}-02$ & $3.101 \mathrm{E}+01$ & $8.50 \mathrm{E}+00$ & $4.043 \mathrm{E}+01$ & $2.976 \mathrm{E}+00$ & $5.46 \mathrm{E}-01$ \\
\hline $7.961 \mathrm{E}+03$ & $5.422 \mathrm{E}+00$ & $1.55 \mathrm{E}-02$ & $2.724 \mathrm{E}+01$ & $8.62 \mathrm{E}+00$ & $4.013 \mathrm{E}+01$ & $2.709 \mathrm{E}+00$ & $5.59 \mathrm{E}-01$ \\
\hline $8.013 E+03$ & $6.340 \mathrm{E}+00$ & $1.70 \mathrm{E}-02$ & $2.097 \mathrm{E}+01$ & $8.53 \mathrm{E}+00$ & $4.053 \mathrm{E}+01$ & $2.460 \mathrm{E}+00$ & $5.38 \mathrm{E}-01$ \\
\hline $7.988 \mathrm{E}+03$ & $7.240 \mathrm{E}+00$ & $1.85 \mathrm{E}-02$ & $1.644 \mathrm{E}+01$ & $8.56 \mathrm{E}+00$ & $4.055 \mathrm{E}+01$ & $2.270 \mathrm{E}+00$ & $5.53 \mathrm{E}-01$ \\
\hline $8.010 \mathrm{E}+03$ & $8.147 \mathrm{E}+00$ & $1.97 \mathrm{E}-02$ & $1.030 \mathrm{E}+01$ & $8.57 \mathrm{E}+00$ & $4.083 \mathrm{E}+01$ & $1.837 \mathrm{E}+00$ & $5.35 \mathrm{E}-01$ \\
\hline$P_{\mathrm{e}}(\mathrm{W})$ & $\Delta P_{\mathrm{e}}(\mathrm{W})$ & $C_{\mathrm{T}}$ & $\Delta C_{\mathrm{T}}$ & $C_{\mathrm{P}}$ & $\Delta C_{\mathrm{P}}$ & $C_{\mathrm{Q}}$ & $\Delta C_{\mathrm{Q}}$ \\
\hline $9.409 \mathrm{E}+00$ & $1.19 \mathrm{E}-01$ & 7.706E-02 & $1.54 \mathrm{E}-02$ & $3.671 \mathrm{E}-02$ & $5.86 \mathrm{E}-03$ & $5.842 \mathrm{E}-03$ & 9.32E-04 \\
\hline $9.389 \mathrm{E}+00$ & $1.18 \mathrm{E}-01$ & 7.291E-02 & $1.51 \mathrm{E}-02$ & $3.526 \mathrm{E}-02$ & $5.50 \mathrm{E}-03$ & $5.612 \mathrm{E}-03$ & 8.75E-04 \\
\hline $9.075 \mathrm{E}+00$ & $1.16 \mathrm{E}-01$ & $6.441 \mathrm{E}-02$ & $1.53 \mathrm{E}-02$ & $3.465 \mathrm{E}-02$ & 5.81E-03 & $5.515 \mathrm{E}-03$ & $9.24 \mathrm{E}-04$ \\
\hline $8.771 \mathrm{E}+00$ & $1.12 \mathrm{E}-01$ & $5.567 \mathrm{E}-02$ & $1.52 \mathrm{E}-02$ & $3.195 \mathrm{E}-02$ & 5.87E-03 & $5.085 \mathrm{E}-03$ & 9.33E-04 \\
\hline $8.283 \mathrm{E}+00$ & $1.08 \mathrm{E}-01$ & 4.991E-02 & $1.58 \mathrm{E}-02$ & $2.999 \mathrm{E}-02$ & $6.19 \mathrm{E}-03$ & $4.774 \mathrm{E}-03$ & $9.86 \mathrm{E}-04$ \\
\hline $7.945 \mathrm{E}+00$ & $1.04 \mathrm{E}-01$ & 3.793E-02 & $1.54 \mathrm{E}-02$ & $2.670 \mathrm{E}-02$ & $5.84 \mathrm{E}-03$ & $4.250 \mathrm{E}-03$ & $9.30 \mathrm{E}-04$ \\
\hline $7.392 \mathrm{E}+00$ & $9.96 \mathrm{E}-02$ & $2.994 \mathrm{E}-02$ & $1.56 \mathrm{E}-02$ & $2.489 \mathrm{E}-02$ & $6.06 \mathrm{E}-03$ & $3.962 \mathrm{E}-03$ & $9.65 \mathrm{E}-04$ \\
\hline $6.865 \mathrm{E}+00$ & $9.54 \mathrm{E}-02$ & $1.865 \mathrm{E}-02$ & $1.55 \mathrm{E}-02$ & $1.998 \mathrm{E}-02$ & $5.82 \mathrm{E}-03$ & $3.180 \mathrm{E}-03$ & $9.27 \mathrm{E}-04$ \\
\hline$\rho\left(\mathrm{kg} / \mathrm{m}^{\wedge} 3\right)$ & $\eta_{\mathrm{T}}$ & $\Delta \eta_{\mathrm{T}}$ & $J$ & $\Delta J$ & $\eta_{\mathrm{P}}$ & $\Delta \eta_{\mathrm{P}}$ & $\operatorname{Re}_{0.75}$ \\
\hline $1.162 \mathrm{E}+00$ & $3.581 \mathrm{E}-01$ & $5.73 \mathrm{E}-02$ & $1.146 \mathrm{E}-01$ & 7.57E-04 & $2.406 \mathrm{E}-01$ & $6.16 \mathrm{E}-02$ & $3.171 \mathrm{E}+04$ \\
\hline $1.162 \mathrm{E}+00$ & $3.571 \mathrm{E}-01$ & $5.59 \mathrm{E}-02$ & $1.624 \mathrm{E}-01$ & $7.28 \mathrm{E}-04$ & $3.358 \mathrm{E}-01$ & $8.70 \mathrm{E}-02$ & $3.214 \mathrm{E}+04$ \\
\hline $1.162 \mathrm{E}+00$ & $3.541 \mathrm{E}-01$ & $5.95 \mathrm{E}-02$ & $2.143 \mathrm{E}-01$ & 7.91E-04 & $3.984 \mathrm{E}-01$ & $1.16 \mathrm{E}-01$ & $3.191 \mathrm{E}+04$ \\
\hline $1.162 \mathrm{E}+00$ & 3.393E-01 & $6.24 \mathrm{E}-02$ & $2.653 \mathrm{E}-01$ & $8.60 \mathrm{E}-04$ & 4.623E-01 & $1.52 \mathrm{E}-01$ & $3.204 \mathrm{E}+04$ \\
\hline $1.162 \mathrm{E}+00$ & $3.271 \mathrm{E}-01$ & $6.77 \mathrm{E}-02$ & $3.213 \mathrm{E}-01$ & $9.47 \mathrm{E}-04$ & $5.347 \mathrm{E}-01$ & $2.02 \mathrm{E}-01$ & $3.180 \mathrm{E}+04$ \\
\hline $1.162 \mathrm{E}+00$ & $3.096 \mathrm{E}-01$ & 6.79E-02 & $3.732 \mathrm{E}-01$ & $1.04 \mathrm{E}-03$ & $5.301 \mathrm{E}-01$ & $2.45 \mathrm{E}-01$ & $3.211 \mathrm{E}+04$ \\
\hline $1.161 \mathrm{E}+00$ & $3.071 \mathrm{E}-01$ & 7.49E-02 & $4.276 \mathrm{E}-01$ & $1.14 \mathrm{E}-03$ & $5.143 \mathrm{E}-01$ & $2.96 \mathrm{E}-01$ & $3.210 \mathrm{E}+04$ \\
\hline $1.161 \mathrm{E}+00$ & $2.675 \mathrm{E}-01$ & $7.80 \mathrm{E}-02$ & 4.798E-01 & $1.21 \mathrm{E}-03$ & $4.479 \mathrm{E}-01$ & $3.95 \mathrm{E}-01$ & $3.229 \mathrm{E}+04$ \\
\hline
\end{tabular}


Table 262: GWS 5.0 x 3.0 Dynamic Measured Values - 12024 RPM

\begin{tabular}{|c|c|c|c|c|c|c|c|}
\hline$n(\mathrm{RPM})$ & $Q(\mathrm{~g}-\mathrm{m})$ & $\Delta Q(\mathrm{~g}-\mathrm{m})$ & $V(\mathrm{~V})$ & $I(\mathrm{~A})$ & $T_{\text {atm }}\left({ }^{\circ} \mathrm{C}\right)$ & $P_{\text {atm }}(\mathrm{Pa})$ & $P_{\text {diff }}(\mathrm{Pa})$ \\
\hline $1.196 \mathrm{E}+04$ & $9.218 \mathrm{E}-01$ & $5.67 \mathrm{E}-02$ & $1.106 \mathrm{E}+01$ & $1.960 \mathrm{E}+00$ & $2.183 \mathrm{E}+01$ & $9.840 \mathrm{E}+04$ & $2.693 \mathrm{E}+00$ \\
\hline $1.203 \mathrm{E}+04$ & $8.885 \mathrm{E}-01$ & $5.83 \mathrm{E}-02$ & $1.106 \mathrm{E}+01$ & $1.914 \mathrm{E}+00$ & $2.173 \mathrm{E}+01$ & $9.840 \mathrm{E}+04$ & $6.617 \mathrm{E}+00$ \\
\hline $1.203 \mathrm{E}+04$ & $8.567 \mathrm{E}-01$ & $5.78 \mathrm{E}-02$ & $1.106 \mathrm{E}+01$ & $1.838 \mathrm{E}+00$ & $2.173 \mathrm{E}+01$ & $9.839 \mathrm{E}+04$ & $1.263 \mathrm{E}+01$ \\
\hline $1.204 \mathrm{E}+04$ & $8.140 \mathrm{E}-01$ & $5.74 \mathrm{E}-02$ & $1.107 \mathrm{E}+01$ & $1.767 \mathrm{E}+00$ & $2.181 \mathrm{E}+01$ & $9.840 \mathrm{E}+04$ & $2.074 \mathrm{E}+01$ \\
\hline $1.199 \mathrm{E}+04$ & $7.585 \mathrm{E}-01$ & $5.59 \mathrm{E}-02$ & $1.107 \mathrm{E}+01$ & $1.661 \mathrm{E}+00$ & $2.194 \mathrm{E}+01$ & $9.840 \mathrm{E}+04$ & $3.118 \mathrm{E}+01$ \\
\hline $1.202 \mathrm{E}+04$ & $6.971 \mathrm{E}-01$ & $5.71 \mathrm{E}-02$ & $1.107 \mathrm{E}+01$ & $1.552 \mathrm{E}+00$ & $2.213 \mathrm{E}+01$ & $9.840 \mathrm{E}+04$ & $4.361 \mathrm{E}+01$ \\
\hline $1.204 \mathrm{E}+04$ & $6.187 \mathrm{E}-01$ & $5.80 \mathrm{E}-02$ & $1.108 \mathrm{E}+01$ & $1.423 \mathrm{E}+00$ & $2.216 \mathrm{E}+01$ & $9.840 \mathrm{E}+04$ & $5.843 \mathrm{E}+01$ \\
\hline $1.203 \mathrm{E}+04$ & $5.229 \mathrm{E}-01$ & $5.77 \mathrm{E}-02$ & $1.108 \mathrm{E}+01$ & $1.264 \mathrm{E}+00$ & $2.229 \mathrm{E}+01$ & $9.840 \mathrm{E}+04$ & $7.569 \mathrm{E}+01$ \\
\hline $1.207 \mathrm{E}+04$ & $4.292 \mathrm{E}-01$ & $5.58 \mathrm{E}-02$ & $1.108 \mathrm{E}+01$ & $1.113 \mathrm{E}+00$ & $2.230 \mathrm{E}+01$ & $9.841 \mathrm{E}+04$ & $9.522 \mathrm{E}+01$ \\
\hline
\end{tabular}


Table 263: GWS 5.0 x 3.0 Dynamic Calculated Values - 12024 RPM

\begin{tabular}{|c|c|c|c|c|c|c|c|}
\hline$n(\mathrm{RPM})$ & $V_{\infty}^{\prime}(\mathrm{m} / \mathrm{s})$ & $\Delta V_{\infty}^{\prime}(\mathrm{m} / \mathrm{s})$ & $T^{\prime}(\mathrm{g})$ & $\Delta T^{\prime}(\mathrm{g})$ & $V_{\mathrm{t}}(\mathrm{m} / \mathrm{s})$ & $P_{\mathrm{P}}(\mathrm{W})$ & $\Delta P_{\mathrm{P}}(\mathrm{W})$ \\
\hline $1.196 \mathrm{E}+04$ & $2.053 \mathrm{E}+00$ & $1.30 \mathrm{E}-02$ & $1.054 \mathrm{E}+02$ & $7.61 \mathrm{E}+00$ & $5.977 \mathrm{E}+01$ & $1.132 \mathrm{E}+01$ & $6.96 \mathrm{E}-01$ \\
\hline $1.203 \mathrm{E}+04$ & $3.280 \mathrm{E}+00$ & $1.33 \mathrm{E}-02$ & $9.837 \mathrm{E}+01$ & $7.57 \mathrm{E}+00$ & $6.018 \mathrm{E}+01$ & $1.098 \mathrm{E}+01$ & $7.21 \mathrm{E}-01$ \\
\hline $1.203 \mathrm{E}+04$ & $4.577 \mathrm{E}+00$ & $1.43 \mathrm{E}-02$ & $8.800 \mathrm{E}+01$ & $7.56 \mathrm{E}+00$ & $6.025 \mathrm{E}+01$ & $1.058 \mathrm{E}+01$ & 7.14E-01 \\
\hline $1.204 \mathrm{E}+04$ & $5.900 \mathrm{E}+00$ & $1.58 \mathrm{E}-02$ & $7.791 \mathrm{E}+01$ & $7.57 \mathrm{E}+00$ & $6.044 \mathrm{E}+01$ & $1.007 \mathrm{E}+01$ & 7.10E-01 \\
\hline $1.199 \mathrm{E}+04$ & $7.263 \mathrm{E}+00$ & $1.78 \mathrm{E}-02$ & $6.717 \mathrm{E}+01$ & $7.57 \mathrm{E}+00$ & $6.034 \mathrm{E}+01$ & $9.341 \mathrm{E}+00$ & $6.88 \mathrm{E}-01$ \\
\hline $1.202 \mathrm{E}+04$ & $8.616 \mathrm{E}+00$ & $1.94 \mathrm{E}-02$ & $5.599 \mathrm{E}+01$ & $7.55 \mathrm{E}+00$ & $6.065 \mathrm{E}+01$ & $8.604 \mathrm{E}+00$ & 7.05E-01 \\
\hline $1.204 \mathrm{E}+04$ & $9.994 \mathrm{E}+00$ & $2.10 \mathrm{E}-02$ & $4.370 \mathrm{E}+01$ & $7.57 \mathrm{E}+00$ & $6.098 \mathrm{E}+01$ & $7.652 \mathrm{E}+00$ & 7.17E-01 \\
\hline $1.203 \mathrm{E}+04$ & $1.140 \mathrm{E}+01$ & $2.33 \mathrm{E}-02$ & $3.012 \mathrm{E}+01$ & $7.55 \mathrm{E}+00$ & $6.114 \mathrm{E}+01$ & $6.457 \mathrm{E}+00$ & $7.13 \mathrm{E}-01$ \\
\hline $1.207 \mathrm{E}+04$ & $1.280 \mathrm{E}+01$ & $2.58 \mathrm{E}-02$ & $1.733 \mathrm{E}+01$ & $7.57 \mathrm{E}+00$ & $6.165 \mathrm{E}+01$ & $5.321 \mathrm{E}+00$ & $6.92 \mathrm{E}-01$ \\
\hline$P_{\mathrm{e}}(\mathrm{W})$ & $\Delta P_{\mathrm{e}}(\mathrm{W})$ & $C_{\mathrm{T}}$ & $\Delta C_{\mathrm{T}}$ & $C_{\mathrm{P}}$ & $\Delta C_{\mathrm{P}}$ & $C_{\mathrm{Q}}$ & $\Delta C_{\mathrm{Q}}$ \\
\hline $2.168 \mathrm{E}+01$ & $2.48 \mathrm{E}-01$ & 8.561E-02 & $6.18 \mathrm{E}-03$ & 3.697E-02 & $2.27 \mathrm{E}-03$ & 5.884E-03 & $3.62 \mathrm{E}-04$ \\
\hline $2.118 \mathrm{E}+01$ & $2.44 \mathrm{E}-01$ & $7.889 \mathrm{E}-02$ & $6.07 \mathrm{E}-03$ & $3.521 \mathrm{E}-02$ & $2.31 \mathrm{E}-03$ & $5.603 \mathrm{E}-03$ & $3.68 \mathrm{E}-04$ \\
\hline $2.033 \mathrm{E}+01$ & $2.34 \mathrm{E}-01$ & $7.062 \mathrm{E}-02$ & $6.07 \mathrm{E}-03$ & $3.397 \mathrm{E}-02$ & $2.29 \mathrm{E}-03$ & $5.406 \mathrm{E}-03$ & $3.65 \mathrm{E}-04$ \\
\hline $1.956 \mathrm{E}+01$ & $2.29 \mathrm{E}-01$ & $6.237 \mathrm{E}-02$ & $6.06 \mathrm{E}-03$ & $3.220 \mathrm{E}-02$ & $2.27 \mathrm{E}-03$ & $5.124 \mathrm{E}-03$ & $3.62 \mathrm{E}-04$ \\
\hline $1.838 \mathrm{E}+01$ & $2.13 \mathrm{E}-01$ & $5.426 \mathrm{E}-02$ & $6.11 \mathrm{E}-03$ & $3.027 \mathrm{E}-02$ & $2.23 \mathrm{E}-03$ & $4.818 \mathrm{E}-03$ & $3.55 \mathrm{E}-04$ \\
\hline $1.718 \mathrm{E}+01$ & $2.00 \mathrm{E}-01$ & $4.506 \mathrm{E}-02$ & $6.07 \mathrm{E}-03$ & $2.772 \mathrm{E}-02$ & $2.27 \mathrm{E}-03$ & $4.411 \mathrm{E}-03$ & $3.61 \mathrm{E}-04$ \\
\hline $1.577 \mathrm{E}+01$ & $1.88 \mathrm{E}-01$ & $3.503 \mathrm{E}-02$ & $6.07 \mathrm{E}-03$ & $2.450 \mathrm{E}-02$ & $2.30 \mathrm{E}-03$ & $3.900 \mathrm{E}-03$ & $3.66 \mathrm{E}-04$ \\
\hline $1.400 \mathrm{E}+01$ & $1.66 \mathrm{E}-01$ & $2.422 \mathrm{E}-02$ & $6.07 \mathrm{E}-03$ & $2.077 \mathrm{E}-02$ & $2.29 \mathrm{E}-03$ & $3.306 \mathrm{E}-03$ & $3.65 \mathrm{E}-04$ \\
\hline $1.234 \mathrm{E}+01$ & $1.50 \mathrm{E}-01$ & $1.383 \mathrm{E}-02$ & $6.04 \mathrm{E}-03$ & $1.692 \mathrm{E}-02$ & $2.20 \mathrm{E}-03$ & $2.693 \mathrm{E}-03$ & $3.50 \mathrm{E}-04$ \\
\hline$\rho\left(\mathrm{kg} / \mathrm{m}^{\wedge} 3\right)$ & $\eta_{\mathrm{T}}$ & $\Delta \eta_{\mathrm{T}}$ & $J$ & $\Delta J$ & $\eta_{\mathrm{P}}$ & $\Delta \eta_{\mathrm{P}}$ & $R e_{0.75}$ \\
\hline $1.162 \mathrm{E}+00$ & $5.222 \mathrm{E}-01$ & $3.27 \mathrm{E}-02$ & $8.101 \mathrm{E}-02$ & $5.14 \mathrm{E}-04$ & $1.876 \mathrm{E}-01$ & $1.78 \mathrm{E}-02$ & $4.736 \mathrm{E}+04$ \\
\hline $1.162 \mathrm{E}+00$ & 5.184E-01 & $3.46 \mathrm{E}-02$ & $1.286 \mathrm{E}-01$ & $5.25 \mathrm{E}-04$ & $2.883 \mathrm{E}-01$ & $2.92 \mathrm{E}-02$ & $4.772 \mathrm{E}+04$ \\
\hline $1.162 \mathrm{E}+00$ & 5.204E-01 & $3.56 \mathrm{E}-02$ & $1.795 \mathrm{E}-01$ & $5.67 \mathrm{E}-04$ & 3.733E-01 & $4.08 \mathrm{E}-02$ & 4.777E+04 \\
\hline $1.162 \mathrm{E}+00$ & $5.147 \mathrm{E}-01$ & $3.68 \mathrm{E}-02$ & $2.311 \mathrm{E}-01$ & $6.30 \mathrm{E}-04$ & $4.478 \mathrm{E}-01$ & $5.38 \mathrm{E}-02$ & $4.790 \mathrm{E}+04$ \\
\hline $1.162 \mathrm{E}+00$ & $5.081 \mathrm{E}-01$ & $3.79 \mathrm{E}-02$ & 2.857E-01 & $7.04 \mathrm{E}-04$ & $5.122 \mathrm{E}-01$ & $6.90 \mathrm{E}-02$ & $4.778 \mathrm{E}+04$ \\
\hline $1.161 \mathrm{E}+00$ & $5.008 \mathrm{E}-01$ & 4.14E-02 & 3.382E-01 & $7.78 \mathrm{E}-04$ & 5.499E-01 & $8.68 \mathrm{E}-02$ & 4.797E+04 \\
\hline $1.161 \mathrm{E}+00$ & $4.853 \mathrm{E}-01$ & $4.59 \mathrm{E}-02$ & $3.915 \mathrm{E}-01$ & $8.44 \mathrm{E}-04$ & $5.598 \mathrm{E}-01$ & $1.10 \mathrm{E}-01$ & $4.822 \mathrm{E}+04$ \\
\hline $1.160 \mathrm{E}+00$ & $4.611 \mathrm{E}-01$ & $5.12 \mathrm{E}-02$ & 4.471E-01 & $9.39 \mathrm{E}-04$ & $5.213 \mathrm{E}-01$ & $1.43 \mathrm{E}-01$ & $4.831 \mathrm{E}+04$ \\
\hline $1.160 \mathrm{E}+00$ & 4.312E-01 & $5.64 \mathrm{E}-02$ & $5.000 \mathrm{E}-01$ & $1.04 \mathrm{E}-03$ & $4.087 \mathrm{E}-01$ & $1.86 \mathrm{E}-01$ & $4.872 \mathrm{E}+04$ \\
\hline
\end{tabular}


Table 264: GWS 5.0 x 3.0 Dynamic Measured Values - 15998 RPM

\begin{tabular}{|c|c|c|c|c|c|c|c|}
\hline$n(\mathrm{RPM})$ & $Q(\mathrm{~g}-\mathrm{m})$ & $\Delta Q(\mathrm{~g}-\mathrm{m})$ & $V(\mathrm{~V})$ & $I(\mathrm{~A})$ & $T_{\text {atm }}\left({ }^{\circ} \mathrm{C}\right)$ & $P_{\text {atm }}(\mathrm{Pa})$ & $P_{\text {diff }}(\mathrm{Pa})$ \\
\hline $1.600 \mathrm{E}+04$ & $1.637 \mathrm{E}+00$ & $6.15 \mathrm{E}-02$ & $1.100 \mathrm{E}+01$ & $3.881 \mathrm{E}+00$ & $2.181 \mathrm{E}+01$ & $9.841 \mathrm{E}+04$ & $3.285 \mathrm{E}+00$ \\
\hline $1.599 \mathrm{E}+04$ & $1.569 \mathrm{E}+00$ & $6.09 \mathrm{E}-02$ & $1.101 \mathrm{E}+01$ & $3.727 \mathrm{E}+00$ & $2.170 \mathrm{E}+01$ & $9.840 \mathrm{E}+04$ & $9.131 \mathrm{E}+00$ \\
\hline $1.602 \mathrm{E}+04$ & $1.499 \mathrm{E}+00$ & $6.05 \mathrm{E}-02$ & $1.101 \mathrm{E}+01$ & $3.547 \mathrm{E}+00$ & $2.174 \mathrm{E}+01$ & $9.840 \mathrm{E}+04$ & $1.857 \mathrm{E}+01$ \\
\hline $1.600 \mathrm{E}+04$ & $1.402 \mathrm{E}+00$ & $6.04 \mathrm{E}-02$ & $1.102 \mathrm{E}+01$ & $3.326 \mathrm{E}+00$ & $2.185 \mathrm{E}+01$ & $9.841 \mathrm{E}+04$ & $3.200 \mathrm{E}+01$ \\
\hline $1.601 \mathrm{E}+04$ & $1.299 \mathrm{E}+00$ & $6.05 \mathrm{E}-02$ & $1.103 \mathrm{E}+01$ & $3.096 \mathrm{E}+00$ & $2.208 \mathrm{E}+01$ & $9.841 \mathrm{E}+04$ & $4.892 \mathrm{E}+01$ \\
\hline $1.601 \mathrm{E}+04$ & $1.183 \mathrm{E}+00$ & $5.97 \mathrm{E}-02$ & $1.103 \mathrm{E}+01$ & $2.849 \mathrm{E}+00$ & $2.214 \mathrm{E}+01$ & $9.842 \mathrm{E}+04$ & $7.073 \mathrm{E}+01$ \\
\hline $1.595 \mathrm{E}+04$ & $1.026 \mathrm{E}+00$ & $5.96 \mathrm{E}-02$ & $1.104 \mathrm{E}+01$ & $2.510 \mathrm{E}+00$ & $2.224 \mathrm{E}+01$ & $9.841 \mathrm{E}+04$ & $9.620 \mathrm{E}+01$ \\
\hline $1.603 \mathrm{E}+04$ & $8.669 \mathrm{E}-01$ & $5.95 \mathrm{E}-02$ & $1.105 \mathrm{E}+01$ & $2.208 \mathrm{E}+00$ & $2.220 \mathrm{E}+01$ & $9.841 \mathrm{E}+04$ & $1.256 \mathrm{E}+02$ \\
\hline $1.598 \mathrm{E}+04$ & $6.782 \mathrm{E}-01$ & $5.90 \mathrm{E}-02$ & $1.106 \mathrm{E}+01$ & $1.814 \mathrm{E}+00$ & $2.227 \mathrm{E}+01$ & $9.842 \mathrm{E}+04$ & $1.591 \mathrm{E}+02$ \\
\hline
\end{tabular}


Table 265: GWS 5.0 x 3.0 Dynamic Calculated Values - 15998 RPM

\begin{tabular}{|c|c|c|c|c|c|c|c|}
\hline$n(\mathrm{RPM})$ & $V_{\infty}^{\prime}(\mathrm{m} / \mathrm{s})$ & $\Delta V_{\infty}^{\prime}(\mathrm{m} / \mathrm{s})$ & $T^{\prime}(\mathrm{g})$ & $\Delta T^{\prime}(\mathrm{g})$ & $V_{\mathrm{t}}(\mathrm{m} / \mathrm{s})$ & $P_{\mathrm{P}}(\mathrm{W})$ & $\Delta P_{\mathrm{P}}(\mathrm{W})$ \\
\hline $1.600 \mathrm{E}+04$ & $2.239 \mathrm{E}+00$ & $1.58 \mathrm{E}-02$ & $2.014 \mathrm{E}+02$ & $7.32 \mathrm{E}+00$ & $7.994 \mathrm{E}+01$ & $2.690 \mathrm{E}+01$ & $1.01 \mathrm{E}+00$ \\
\hline $1.599 \mathrm{E}+04$ & $3.834 \mathrm{E}+00$ & $1.60 \mathrm{E}-02$ & $1.830 \mathrm{E}+02$ & $7.30 \mathrm{E}+00$ & $7.994 \mathrm{E}+01$ & $2.575 \mathrm{E}+01$ & $9.99 \mathrm{E}-01$ \\
\hline $1.602 \mathrm{E}+04$ & $5.535 \mathrm{E}+00$ & $1.72 \mathrm{E}-02$ & $1.631 \mathrm{E}+02$ & $7.65 \mathrm{E}+00$ & $8.023 \mathrm{E}+01$ & $2.466 \mathrm{E}+01$ & $9.96 \mathrm{E}-01$ \\
\hline $1.600 \mathrm{E}+04$ & $7.318 \mathrm{E}+00$ & $1.91 \mathrm{E}-02$ & $1.426 \mathrm{E}+02$ & $7.45 \mathrm{E}+00$ & $8.024 \mathrm{E}+01$ & $2.304 \mathrm{E}+01$ & $9.93 \mathrm{E}-01$ \\
\hline $1.601 \mathrm{E}+04$ & $9.091 \mathrm{E}+00$ & 2.19E-02 & $1.217 \mathrm{E}+02$ & $7.39 \mathrm{E}+00$ & $8.048 \mathrm{E}+01$ & $2.135 \mathrm{E}+01$ & $9.94 \mathrm{E}-01$ \\
\hline $1.601 \mathrm{E}+04$ & $1.097 \mathrm{E}+01$ & 2.39E-02 & $1.010 \mathrm{E}+02$ & $7.39 \mathrm{E}+00$ & $8.072 \mathrm{E}+01$ & $1.946 \mathrm{E}+01$ & $9.81 \mathrm{E}-01$ \\
\hline $1.595 \mathrm{E}+04$ & $1.282 \mathrm{E}+01$ & $2.63 \mathrm{E}-02$ & $7.593 \mathrm{E}+01$ & $7.47 \mathrm{E}+00$ & $8.069 \mathrm{E}+01$ & $1.679 \mathrm{E}+01$ & $9.77 \mathrm{E}-01$ \\
\hline $1.603 \mathrm{E}+04$ & $1.468 \mathrm{E}+01$ & 2.94E-02 & $5.326 \mathrm{E}+01$ & $7.38 \mathrm{E}+00$ & $8.143 \mathrm{E}+01$ & $1.427 \mathrm{E}+01$ & $9.80 \mathrm{E}-01$ \\
\hline $1.598 \mathrm{E}+04$ & $1.654 \mathrm{E}+01$ & $3.21 \mathrm{E}-02$ & $2.644 \mathrm{E}+01$ & $7.49 \mathrm{E}+00$ & $8.150 \mathrm{E}+01$ & $1.113 \mathrm{E}+01$ & $9.67 \mathrm{E}-01$ \\
\hline$P_{\mathrm{e}}(\mathrm{W})$ & $\Delta P_{\mathrm{e}}(\mathrm{W})$ & $C_{\mathrm{T}}$ & $\Delta C_{\mathrm{T}}$ & $C_{\mathrm{P}}$ & $\Delta C_{\mathrm{P}}$ & $C_{\mathrm{Q}}$ & $\Delta C_{\mathrm{Q}}$ \\
\hline $4.271 \mathrm{E}+01$ & $4.76 \mathrm{E}-01$ & $9.138 \mathrm{E}-02$ & $3.32 \mathrm{E}-03$ & $3.669 \mathrm{E}-02$ & $1.38 \mathrm{E}-03$ & $5.840 \mathrm{E}-03$ & $2.20 \mathrm{E}-04$ \\
\hline $4.103 \mathrm{E}+01$ & $4.55 \mathrm{E}-01$ & $8.312 \mathrm{E}-02$ & $3.32 \mathrm{E}-03$ & $3.520 \mathrm{E}-02$ & $1.37 \mathrm{E}-03$ & $5.602 \mathrm{E}-03$ & $2.17 \mathrm{E}-04$ \\
\hline $3.907 \mathrm{E}+01$ & 4.32E-01 & $7.372 \mathrm{E}-02$ & $3.46 \mathrm{E}-03$ & $3.347 \mathrm{E}-02$ & $1.35 \mathrm{E}-03$ & $5.328 \mathrm{E}-03$ & $2.15 \mathrm{E}-04$ \\
\hline $3.665 \mathrm{E}+01$ & $4.10 \mathrm{E}-01$ & $6.470 \mathrm{E}-02$ & $3.38 \mathrm{E}-03$ & $3.144 \mathrm{E}-02$ & $1.35 \mathrm{E}-03$ & $5.004 \mathrm{E}-03$ & $2.16 \mathrm{E}-04$ \\
\hline $3.414 \mathrm{E}+01$ & $3.82 \mathrm{E}-01$ & $5.519 \mathrm{E}-02$ & $3.35 \mathrm{E}-03$ & 2.909E-02 & $1.36 \mathrm{E}-03$ & 4.631E-03 & $2.16 \mathrm{E}-04$ \\
\hline $3.144 \mathrm{E}+01$ & $3.51 \mathrm{E}-01$ & $4.579 \mathrm{E}-02$ & $3.35 \mathrm{E}-03$ & $2.651 \mathrm{E}-02$ & $1.34 \mathrm{E}-03$ & 4.219E-03 & $2.13 \mathrm{E}-04$ \\
\hline $2.772 \mathrm{E}+01$ & $3.14 \mathrm{E}-01$ & $3.471 \mathrm{E}-02$ & $3.41 \mathrm{E}-03$ & $2.316 \mathrm{E}-02$ & $1.35 \mathrm{E}-03$ & $3.686 \mathrm{E}-03$ & $2.14 \mathrm{E}-04$ \\
\hline $2.440 \mathrm{E}+01$ & $2.79 \mathrm{E}-01$ & $2.408 \mathrm{E}-02$ & 3.34E-03 & $1.936 \mathrm{E}-02$ & $1.33 \mathrm{E}-03$ & $3.082 \mathrm{E}-03$ & 2.12E-04 \\
\hline $2.007 \mathrm{E}+01$ & 2.32E-01 & $1.204 \mathrm{E}-02$ & $3.41 \mathrm{E}-03$ & $1.527 \mathrm{E}-02$ & $1.33 \mathrm{E}-03$ & $2.430 \mathrm{E}-03$ & $2.11 \mathrm{E}-04$ \\
\hline$\rho\left(\mathrm{kg} / \mathrm{m}^{\wedge} 3\right)$ & $\eta_{\mathrm{T}}$ & $\Delta \eta_{\mathrm{T}}$ & $J$ & $\Delta J$ & $\eta_{\mathrm{P}}$ & $\Delta \eta_{\mathrm{P}}$ & $R e_{0.75}$ \\
\hline $1.162 \mathrm{E}+00$ & $6.298 \mathrm{E}-01$ & $2.47 \mathrm{E}-02$ & $6.603 \mathrm{E}-02$ & $4.65 \mathrm{E}-04$ & $1.644 \mathrm{E}-01$ & $8.68 \mathrm{E}-03$ & $6.336 \mathrm{E}+04$ \\
\hline $1.163 \mathrm{E}+00$ & $6.276 \mathrm{E}-01$ & $2.53 \mathrm{E}-02$ & $1.132 \mathrm{E}-01$ & 4.72E-04 & 2.672E-01 & $1.49 \mathrm{E}-02$ & $6.339 \mathrm{E}+04$ \\
\hline $1.163 \mathrm{E}+00$ & $6.313 \mathrm{E}-01$ & $2.64 \mathrm{E}-02$ & $1.630 \mathrm{E}-01$ & $5.07 \mathrm{E}-04$ & $3.589 \mathrm{E}-01$ & $2.22 \mathrm{E}-02$ & $6.361 \mathrm{E}+04$ \\
\hline $1.162 \mathrm{E}+00$ & $6.285 \mathrm{E}-01$ & $2.80 \mathrm{E}-02$ & $2.158 \mathrm{E}-01$ & $5.66 \mathrm{E}-04$ & $4.441 \mathrm{E}-01$ & $3.01 \mathrm{E}-02$ & $6.358 \mathrm{E}+04$ \\
\hline $1.161 \mathrm{E}+00$ & $6.254 \mathrm{E}-01$ & $3.00 \mathrm{E}-02$ & $2.679 \mathrm{E}-01$ & $6.49 \mathrm{E}-04$ & $5.083 \mathrm{E}-01$ & 3.89E-02 & $6.368 \mathrm{E}+04$ \\
\hline $1.161 \mathrm{E}+00$ & $6.188 \mathrm{E}-01$ & $3.20 \mathrm{E}-02$ & $3.231 \mathrm{E}-01$ & $7.08 \mathrm{E}-04$ & $5.581 \mathrm{E}-01$ & $4.96 \mathrm{E}-02$ & $6.385 \mathrm{E}+04$ \\
\hline $1.161 \mathrm{E}+00$ & $6.059 \mathrm{E}-01$ & $3.59 \mathrm{E}-02$ & $3.793 \mathrm{E}-01$ & $7.83 \mathrm{E}-04$ & $5.685 \mathrm{E}-01$ & $6.50 \mathrm{E}-02$ & $6.378 \mathrm{E}+04$ \\
\hline $1.161 \mathrm{E}+00$ & 5.850E-01 & 4.07E-02 & $4.318 \mathrm{E}-01$ & $8.69 \mathrm{E}-04$ & 5.369E-01 & $8.30 \mathrm{E}-02$ & $6.439 \mathrm{E}+04$ \\
\hline $1.161 \mathrm{E}+00$ & $5.544 \mathrm{E}-01$ & $4.86 \mathrm{E}-02$ & $4.885 \mathrm{E}-01$ & $9.54 \mathrm{E}-04$ & $3.854 \mathrm{E}-01$ & $1.14 \mathrm{E}-01$ & $6.442 \mathrm{E}+04$ \\
\hline
\end{tabular}


Table 266: GWS 5.0 x 3.0 Dynamic Measured Values - 20000 RPM

\begin{tabular}{|c|c|c|c|c|c|c|c|}
\hline$n(\mathrm{RPM})$ & $Q(\mathrm{~g}-\mathrm{m})$ & $\Delta Q(\mathrm{~g}-\mathrm{m})$ & $V(\mathrm{~V})$ & $I(\mathrm{~A})$ & $T_{\text {atm }}\left({ }^{\circ} \mathrm{C}\right)$ & $P_{\text {atm }}(\mathrm{Pa})$ & $P_{\text {diff }}(\mathrm{Pa})$ \\
\hline $2.005 \mathrm{E}+04$ & $2.497 \mathrm{E}+00$ & $1.14 \mathrm{E}-01$ & $1.092 \mathrm{E}+01$ & $6.782 \mathrm{E}+00$ & $2.210 \mathrm{E}+01$ & $9.883 \mathrm{E}+04$ & $3.911 \mathrm{E}+00$ \\
\hline $1.998 \mathrm{E}+04$ & $2.456 \mathrm{E}+00$ & $1.06 \mathrm{E}-01$ & $1.093 \mathrm{E}+01$ & $6.498 \mathrm{E}+00$ & $2.218 \mathrm{E}+01$ & $9.884 \mathrm{E}+04$ & $9.729 \mathrm{E}+00$ \\
\hline $1.994 \mathrm{E}+04$ & $2.330 \mathrm{E}+00$ & $1.03 \mathrm{E}-01$ & $1.094 \mathrm{E}+01$ & $6.189 \mathrm{E}+00$ & $2.230 \mathrm{E}+01$ & $9.883 \mathrm{E}+04$ & $1.937 \mathrm{E}+01$ \\
\hline $1.991 \mathrm{E}+04$ & $2.219 \mathrm{E}+00$ & $1.02 \mathrm{E}-01$ & $1.095 \mathrm{E}+01$ & $5.830 \mathrm{E}+00$ & $2.225 \mathrm{E}+01$ & $9.882 \mathrm{E}+04$ & $3.285 \mathrm{E}+01$ \\
\hline $2.010 \mathrm{E}+04$ & $2.143 \mathrm{E}+00$ & $1.15 \mathrm{E}-01$ & $1.095 \mathrm{E}+01$ & $5.584 \mathrm{E}+00$ & $2.222 \mathrm{E}+01$ & $9.882 \mathrm{E}+04$ & $5.036 \mathrm{E}+01$ \\
\hline $1.992 \mathrm{E}+04$ & $1.925 \mathrm{E}+00$ & $1.01 \mathrm{E}-01$ & $1.097 \mathrm{E}+01$ & $5.043 \mathrm{E}+00$ & $2.228 \mathrm{E}+01$ & $9.882 \mathrm{E}+04$ & $7.185 \mathrm{E}+01$ \\
\hline $2.013 \mathrm{E}+04$ & $1.797 \mathrm{E}+00$ & $1.15 \mathrm{E}-01$ & $1.098 \mathrm{E}+01$ & $4.742 \mathrm{E}+00$ & $2.227 \mathrm{E}+01$ & $9.882 \mathrm{E}+04$ & $9.757 \mathrm{E}+01$ \\
\hline $1.994 \mathrm{E}+04$ & $1.537 \mathrm{E}+00$ & $1.00 \mathrm{E}-01$ & $1.100 \mathrm{E}+01$ & $4.180 \mathrm{E}+00$ & $2.229 \mathrm{E}+01$ & $9.882 \mathrm{E}+04$ & $1.274 \mathrm{E}+02$ \\
\hline $2.005 \mathrm{E}+04$ & $1.381 \mathrm{E}+00$ & $1.04 \mathrm{E}-01$ & $1.101 \mathrm{E}+01$ & $3.745 \mathrm{E}+00$ & $2.235 \mathrm{E}+01$ & $9.882 \mathrm{E}+04$ & $1.610 \mathrm{E}+02$ \\
\hline $1.995 \mathrm{E}+04$ & $1.150 \mathrm{E}+00$ & $9.67 \mathrm{E}-02$ & $1.102 \mathrm{E}+01$ & $3.169 \mathrm{E}+00$ & $2.239 \mathrm{E}+01$ & $9.882 \mathrm{E}+04$ & $1.986 \mathrm{E}+02$ \\
\hline $2.005 \mathrm{E}+04$ & $9.638 \mathrm{E}-01$ & $9.65 \mathrm{E}-02$ & $1.104 \mathrm{E}+01$ & $2.646 \mathrm{E}+00$ & $2.255 \mathrm{E}+01$ & $9.882 \mathrm{E}+04$ & $2.395 \mathrm{E}+02$ \\
\hline
\end{tabular}


Table 267: GWS 5.0 x 3.0 Dynamic Calculated Values - 20000 RPM

\begin{tabular}{|c|c|c|c|c|c|c|c|}
\hline$n(\mathrm{RPM})$ & $V_{\infty}^{\prime}(\mathrm{m} / \mathrm{s})$ & $\Delta V_{\infty}^{\prime}(\mathrm{m} / \mathrm{s})$ & $T^{\prime}(\mathrm{g})$ & $\Delta T^{\prime}(\mathrm{g})$ & $V_{\mathrm{t}}(\mathrm{m} / \mathrm{s})$ & $P_{\mathrm{P}}(\mathrm{W})$ & $\Delta P_{\mathrm{P}}(\mathrm{W})$ \\
\hline $2.005 \mathrm{E}+04$ & $2.415 \mathrm{E}+00$ & $1.84 \mathrm{E}-02$ & $3.220 \mathrm{E}+02$ & $6.98 \mathrm{E}+00$ & $1.002 \mathrm{E}+02$ & $5.141 \mathrm{E}+01$ & $2.36 \mathrm{E}+00$ \\
\hline $1.998 \mathrm{E}+04$ & $3.919 \mathrm{E}+00$ & $1.89 \mathrm{E}-02$ & $2.996 \mathrm{E}+02$ & $6.93 \mathrm{E}+00$ & $9.985 \mathrm{E}+01$ & $5.038 \mathrm{E}+01$ & $2.18 \mathrm{E}+00$ \\
\hline $1.994 \mathrm{E}+04$ & $5.611 \mathrm{E}+00$ & $1.96 \mathrm{E}-02$ & $2.718 \mathrm{E}+02$ & $6.95 \mathrm{E}+00$ & $9.974 \mathrm{E}+01$ & $4.770 \mathrm{E}+01$ & $2.12 \mathrm{E}+00$ \\
\hline $1.991 \mathrm{E}+04$ & 7.367E+00 & $2.12 \mathrm{E}-02$ & $2.436 \mathrm{E}+02$ & $6.95 \mathrm{E}+00$ & $9.973 \mathrm{E}+01$ & $4.536 \mathrm{E}+01$ & $2.09 \mathrm{E}+00$ \\
\hline $2.010 \mathrm{E}+04$ & $9.168 \mathrm{E}+00$ & $2.40 \mathrm{E}-02$ & $2.204 \mathrm{E}+02$ & $6.92 \mathrm{E}+00$ & $1.008 \mathrm{E}+02$ & $4.422 \mathrm{E}+01$ & $2.38 \mathrm{E}+00$ \\
\hline $1.992 \mathrm{E}+04$ & $1.100 \mathrm{E}+01$ & 2.67E-02 & $1.870 \mathrm{E}+02$ & $6.96 \mathrm{E}+00$ & $1.001 \mathrm{E}+02$ & $3.937 \mathrm{E}+01$ & $2.07 \mathrm{E}+00$ \\
\hline $2.013 \mathrm{E}+04$ & $1.285 \mathrm{E}+01$ & 2.84E-02 & $1.618 \mathrm{E}+02$ & $6.86 \mathrm{E}+00$ & $1.014 \mathrm{E}+02$ & $3.714 \mathrm{E}+01$ & $2.38 \mathrm{E}+00$ \\
\hline $1.994 \mathrm{E}+04$ & $1.472 \mathrm{E}+01$ & $3.02 \mathrm{E}-02$ & $1.267 \mathrm{E}+02$ & $6.91 \mathrm{E}+00$ & $1.007 \mathrm{E}+02$ & $3.146 \mathrm{E}+01$ & $2.05 \mathrm{E}+00$ \\
\hline $2.005 \mathrm{E}+04$ & $1.657 \mathrm{E}+01$ & $3.26 \mathrm{E}-02$ & $9.646 \mathrm{E}+01$ & $6.90 \mathrm{E}+00$ & $1.015 \mathrm{E}+02$ & $2.843 \mathrm{E}+01$ & $2.14 \mathrm{E}+00$ \\
\hline $1.995 \mathrm{E}+04$ & $1.843 \mathrm{E}+01$ & $3.55 \mathrm{E}-02$ & $6.124 \mathrm{E}+01$ & $6.93 \mathrm{E}+00$ & $1.014 \mathrm{E}+02$ & $2.357 \mathrm{E}+01$ & $1.98 \mathrm{E}+00$ \\
\hline $2.005 \mathrm{E}+04$ & $2.027 \mathrm{E}+01$ & $3.81 \mathrm{E}-02$ & $2.730 \mathrm{E}+01$ & $6.90 \mathrm{E}+00$ & $1.022 \mathrm{E}+02$ & $1.984 \mathrm{E}+01$ & $1.99 \mathrm{E}+00$ \\
\hline$P_{\mathrm{e}}(\mathrm{W})$ & $\Delta P_{\mathrm{e}}(\mathrm{W})$ & $C_{\mathrm{T}}$ & $\Delta C_{\mathrm{T}}$ & $C_{\mathrm{P}}$ & $\Delta C_{\mathrm{P}}$ & $C_{\mathrm{Q}}$ & $\Delta C_{\mathrm{Q}}$ \\
\hline $7.406 \mathrm{E}+01$ & 8.83E-01 & $9.273 \mathrm{E}-02$ & $2.01 \mathrm{E}-03$ & $3.553 \mathrm{E}-02$ & $1.63 \mathrm{E}-03$ & $5.655 \mathrm{E}-03$ & $2.59 \mathrm{E}-04$ \\
\hline $7.101 \mathrm{E}+01$ & $7.72 \mathrm{E}-01$ & $8.691 \mathrm{E}-02$ & $2.01 \mathrm{E}-03$ & $3.520 \mathrm{E}-02$ & $1.52 \mathrm{E}-03$ & $5.602 \mathrm{E}-03$ & $2.42 \mathrm{E}-04$ \\
\hline $6.769 \mathrm{E}+01$ & 7.41E-01 & 7.919E-02 & $2.03 \mathrm{E}-03$ & $3.354 \mathrm{E}-02$ & $1.49 \mathrm{E}-03$ & $5.338 \mathrm{E}-03$ & $2.37 \mathrm{E}-04$ \\
\hline $6.382 \mathrm{E}+01$ & 7.02E-01 & 7.115E-02 & 2.03E-03 & $3.202 \mathrm{E}-02$ & $1.48 \mathrm{E}-03$ & $5.095 \mathrm{E}-03$ & $2.35 \mathrm{E}-04$ \\
\hline $6.117 \mathrm{E}+01$ & 7.60E-01 & $6.318 \mathrm{E}-02$ & $1.99 \mathrm{E}-03$ & $3.034 \mathrm{E}-02$ & $1.63 \mathrm{E}-03$ & $4.829 \mathrm{E}-03$ & $2.59 \mathrm{E}-04$ \\
\hline $5.532 \mathrm{E}+01$ & $6.27 \mathrm{E}-01$ & $5.456 \mathrm{E}-02$ & 2.03E-03 & $2.775 \mathrm{E}-02$ & $1.46 \mathrm{E}-03$ & $4.416 \mathrm{E}-03$ & $2.32 \mathrm{E}-04$ \\
\hline $5.206 \mathrm{E}+01$ & $6.72 \mathrm{E}-01$ & 4.624E-02 & $1.96 \mathrm{E}-03$ & $2.537 \mathrm{E}-02$ & $1.62 \mathrm{E}-03$ & 4.037E-03 & $2.58 \mathrm{E}-04$ \\
\hline $4.596 \mathrm{E}+01$ & $5.29 \mathrm{E}-01$ & $3.692 \mathrm{E}-02$ & 2.01E-03 & $2.212 \mathrm{E}-02$ & $1.44 \mathrm{E}-03$ & $3.521 \mathrm{E}-03$ & $2.29 \mathrm{E}-04$ \\
\hline $4.123 \mathrm{E}+01$ & 4.73E-01 & $2.780 \mathrm{E}-02$ & $1.99 \mathrm{E}-03$ & $1.966 \mathrm{E}-02$ & $1.48 \mathrm{E}-03$ & $3.129 \mathrm{E}-03$ & $2.36 \mathrm{E}-04$ \\
\hline $3.493 \mathrm{E}+01$ & $3.95 \mathrm{E}-01$ & $1.782 \mathrm{E}-02$ & $2.02 \mathrm{E}-03$ & $1.654 \mathrm{E}-02$ & $1.39 \mathrm{E}-03$ & $2.632 \mathrm{E}-03$ & $2.21 \mathrm{E}-04$ \\
\hline $2.921 \mathrm{E}+01$ & $3.31 \mathrm{E}-01$ & 7.874E-03 & $1.99 \mathrm{E}-03$ & $1.373 \mathrm{E}-02$ & $1.38 \mathrm{E}-03$ & $2.186 \mathrm{E}-03$ & $2.19 \mathrm{E}-04$ \\
\hline$\rho\left(\mathrm{kg} / \mathrm{m}^{\wedge} 3\right)$ & $\eta_{\mathrm{T}}$ & $\Delta \eta_{\mathrm{T}}$ & $J$ & $\Delta J$ & $\eta_{\mathrm{P}}$ & $\Delta \eta_{\mathrm{P}}$ & $R e_{0.75}$ \\
\hline $1.166 \mathrm{E}+00$ & $6.942 \mathrm{E}-01$ & $3.29 \mathrm{E}-02$ & $5.684 \mathrm{E}-02$ & 4.35E-04 & $1.483 \mathrm{E}-01$ & 7.61E-03 & $7.958 \mathrm{E}+04$ \\
\hline $1.166 \mathrm{E}+00$ & $7.095 \mathrm{E}-01$ & $3.16 \mathrm{E}-02$ & $9.255 \mathrm{E}-02$ & 4.47E-04 & $2.285 \mathrm{E}-01$ & $1.13 \mathrm{E}-02$ & $7.931 \mathrm{E}+04$ \\
\hline $1.165 \mathrm{E}+00$ & $7.046 \mathrm{E}-01$ & 3.22E-02 & $1.328 \mathrm{E}-01$ & $4.67 \mathrm{E}-04$ & $3.135 \mathrm{E}-01$ & $1.61 \mathrm{E}-02$ & $7.915 \mathrm{E}+04$ \\
\hline $1.165 \mathrm{E}+00$ & 7.108E-01 & $3.37 \mathrm{E}-02$ & $1.746 \mathrm{E}-01$ & $5.06 \mathrm{E}-04$ & 3.879E-01 & $2.11 \mathrm{E}-02$ & $7.916 \mathrm{E}+04$ \\
\hline $1.166 \mathrm{E}+00$ & $7.230 \mathrm{E}-01$ & 3.99E-02 & $2.152 \mathrm{E}-01$ & $5.70 \mathrm{E}-04$ & $4.481 \mathrm{E}-01$ & 2.79E-02 & $8.004 \mathrm{E}+04$ \\
\hline $1.165 \mathrm{E}+00$ & 7.117E-01 & $3.83 \mathrm{E}-02$ & $2.604 \mathrm{E}-01$ & $6.39 \mathrm{E}-04$ & $5.120 \mathrm{E}-01$ & $3.31 \mathrm{E}-02$ & $7.945 \mathrm{E}+04$ \\
\hline $1.165 \mathrm{E}+00$ & 7.135E-01 & 4.65E-02 & $3.011 \mathrm{E}-01$ & $6.75 \mathrm{E}-04$ & $5.489 \mathrm{E}-01$ & $4.21 \mathrm{E}-02$ & $8.045 \mathrm{E}+04$ \\
\hline $1.165 \mathrm{E}+00$ & $6.844 \mathrm{E}-01$ & $4.52 \mathrm{E}-02$ & $3.482 \mathrm{E}-01$ & $7.26 \mathrm{E}-04$ & $5.812 \mathrm{E}-01$ & 4.94E-02 & $7.988 \mathrm{E}+04$ \\
\hline $1.165 \mathrm{E}+00$ & $6.896 \mathrm{E}-01$ & $5.25 \mathrm{E}-02$ & 3.899E-01 & 7.79E-04 & $5.514 \mathrm{E}-01$ & $5.73 \mathrm{E}-02$ & $8.053 \mathrm{E}+04$ \\
\hline $1.165 \mathrm{E}+00$ & $6.747 \mathrm{E}-01$ & $5.72 \mathrm{E}-02$ & $4.359 \mathrm{E}-01$ & $8.54 \mathrm{E}-04$ & 4.697E-01 & $6.62 \mathrm{E}-02$ & $8.038 \mathrm{E}+04$ \\
\hline $1.164 \mathrm{E}+00$ & $6.792 \mathrm{E}-01$ & $6.84 \mathrm{E}-02$ & $4.770 \mathrm{E}-01$ & $9.13 \mathrm{E}-04$ & $2.735 \mathrm{E}-01$ & 7.44E-02 & $8.095 \mathrm{E}+04$ \\
\hline
\end{tabular}




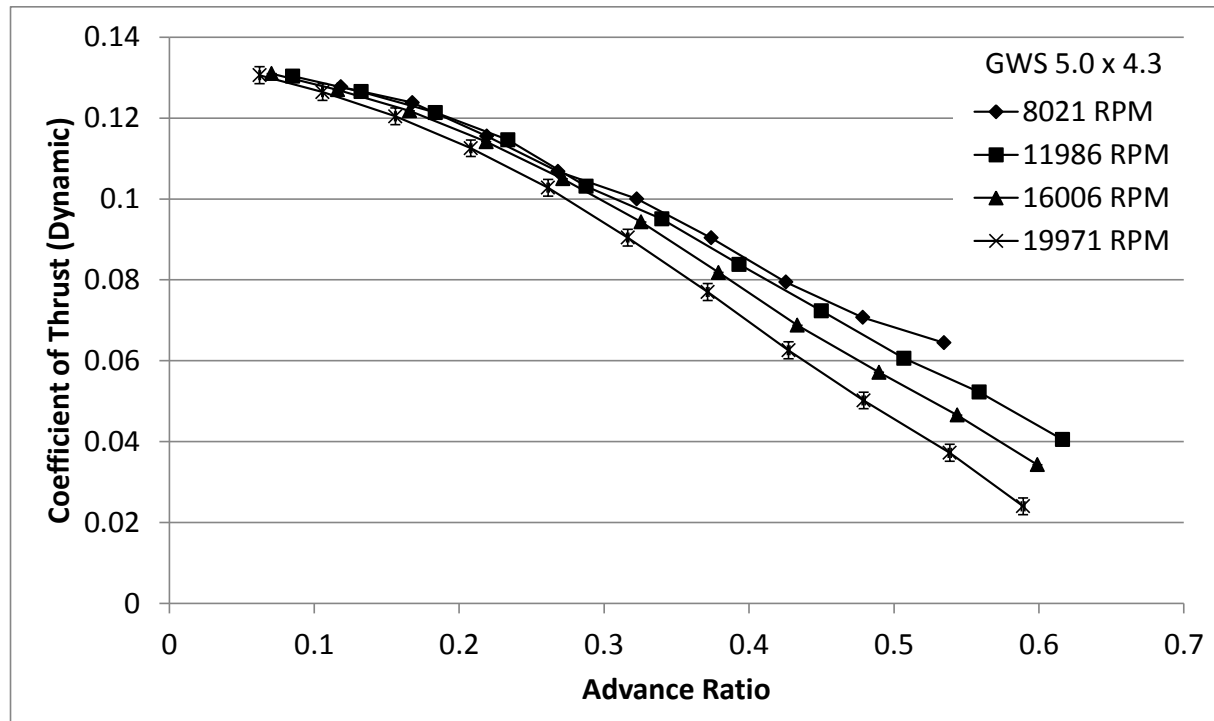

(a)

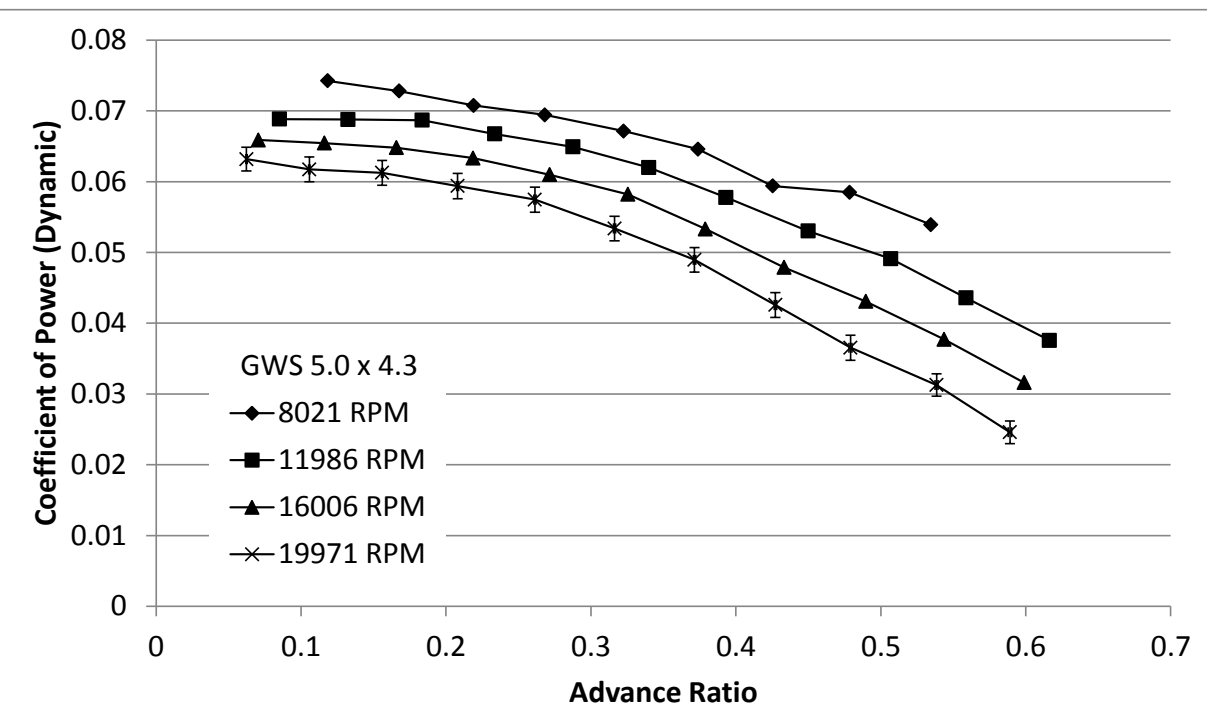

(b)

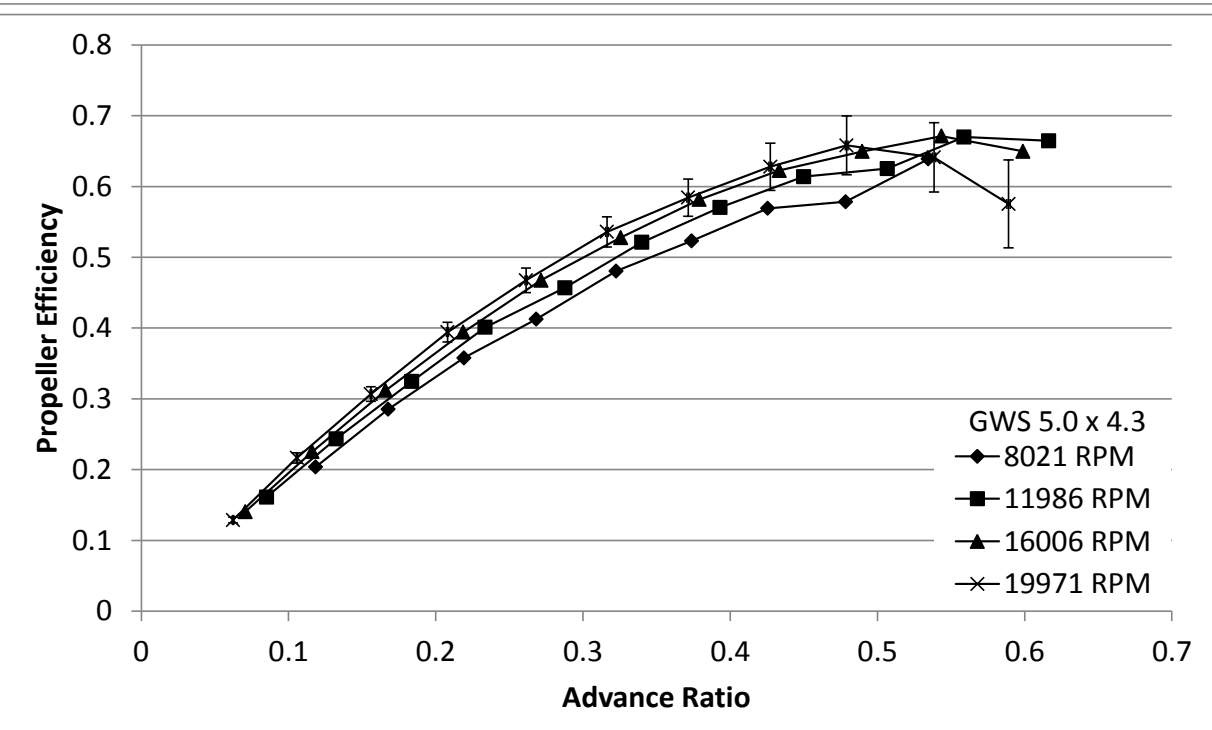

(c) 


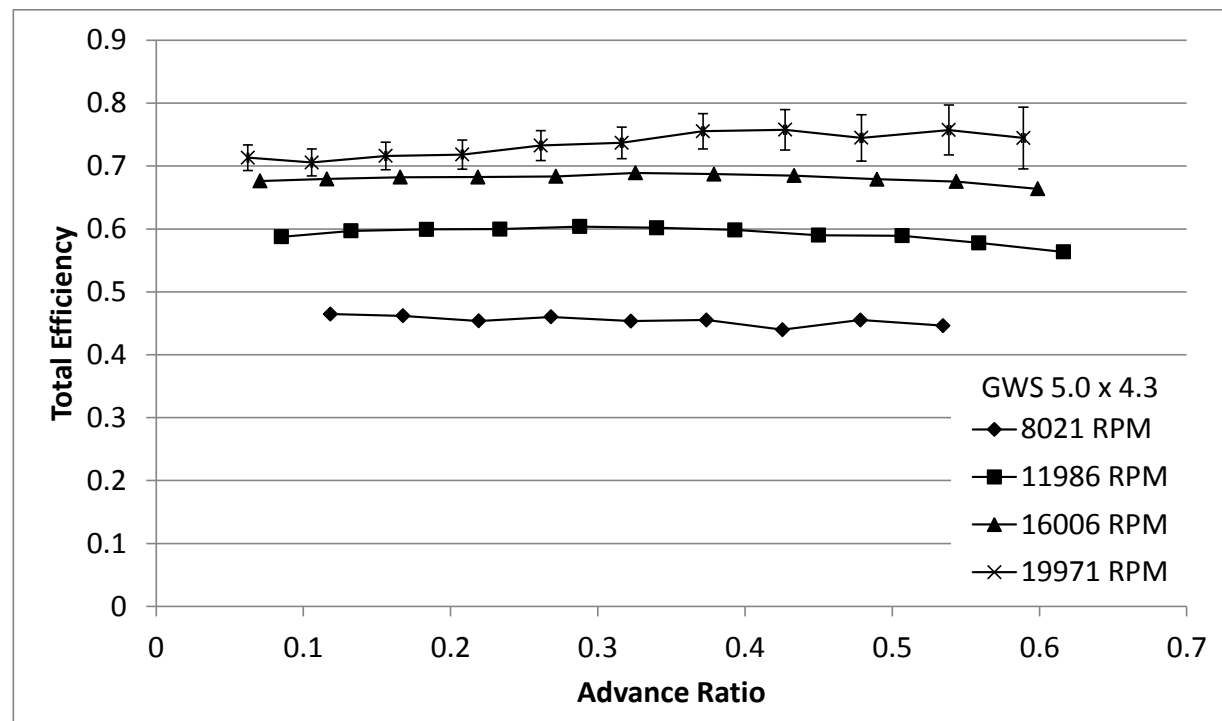

(d)

Figure 102: GWS 5.0 x 4.3 Dynamic Test Results: (a) Coefficient of Thrust, (b) Coefficient of Power, (c) Propeller Efficiency, (d) Total Efficiency.

Table 268: GWS 5.0 x 4.3 Dynamic Measured Values - 8021 RPM

\begin{tabular}{|c|c|c|c|c|c|c|c|}
\hline$n(\mathrm{RPM})$ & $Q(\mathrm{~g}-\mathrm{m})$ & $\Delta Q(\mathrm{~g}-\mathrm{m})$ & $V(\mathrm{~V})$ & $I(\mathrm{~A})$ & $T_{\text {atm }}\left({ }^{\circ} \mathrm{C}\right)$ & $P_{\text {atm }}(\mathrm{Pa})$ & $P_{\text {diff }}(\mathrm{Pa})$ \\
\hline $8.014 \mathrm{E}+03$ & $8.244 \mathrm{E}-01$ & $7.05 \mathrm{E}-02$ & $1.108 \mathrm{E}+01$ & $1.318 \mathrm{E}+00$ & $2.225 \mathrm{E}+01$ & $9.873 \mathrm{E}+04$ & $2.535 \mathrm{E}+00$ \\
\hline $8.039 \mathrm{E}+03$ & $8.127 \mathrm{E}-01$ & $7.17 \mathrm{E}-02$ & $1.108 \mathrm{E}+01$ & $1.311 \mathrm{E}+00$ & $2.247 \mathrm{E}+01$ & $9.873 \mathrm{E}+04$ & $4.989 \mathrm{E}+00$ \\
\hline $7.956 \mathrm{E}+03$ & $7.732 \mathrm{E}-01$ & $7.25 \mathrm{E}-02$ & $1.108 \mathrm{E}+01$ & $1.257 \mathrm{E}+00$ & $2.263 \mathrm{E}+01$ & $9.872 \mathrm{E}+04$ & $8.218 \mathrm{E}+00$ \\
\hline $8.025 \mathrm{E}+03$ & $7.716 \mathrm{E}-01$ & $7.07 \mathrm{E}-02$ & $1.108 \mathrm{E}+01$ & $1.248 \mathrm{E}+00$ & $2.277 \mathrm{E}+01$ & $9.872 \mathrm{E}+04$ & $1.241 \mathrm{E}+01$ \\
\hline $7.952 \mathrm{E}+03$ & $7.328 \mathrm{E}-01$ & $7.24 \mathrm{E}-02$ & $1.108 \mathrm{E}+01$ & $1.191 \mathrm{E}+00$ & $2.273 \mathrm{E}+01$ & $9.872 \mathrm{E}+04$ & $1.750 \mathrm{E}+01$ \\
\hline $8.006 \mathrm{E}+03$ & $7.142 \mathrm{E}-01$ & $7.06 \mathrm{E}-02$ & $1.108 \mathrm{E}+01$ & $1.164 \mathrm{E}+00$ & $2.281 \mathrm{E}+01$ & $9.871 \mathrm{E}+04$ & $2.369 \mathrm{E}+01$ \\
\hline $8.054 \mathrm{E}+03$ & $6.644 \mathrm{E}-01$ & $6.97 \mathrm{E}-02$ & $1.108 \mathrm{E}+01$ & $1.127 \mathrm{E}+00$ & $2.283 \mathrm{E}+01$ & $9.871 \mathrm{E}+04$ & $3.091 \mathrm{E}+01$ \\
\hline $8.079 \mathrm{E}+03$ & $6.585 \mathrm{E}-01$ & $6.79 \mathrm{E}-02$ & $1.108 \mathrm{E}+01$ & $1.083 \mathrm{E}+00$ & $2.285 \mathrm{E}+01$ & $9.871 \mathrm{E}+04$ & $3.921 \mathrm{E}+01$ \\
\hline $8.061 \mathrm{E}+03$ & $6.043 \mathrm{E}-01$ & $6.93 \mathrm{E}-02$ & $1.109 \mathrm{E}+01$ & $1.011 \mathrm{E}+00$ & $2.283 \mathrm{E}+01$ & $9.870 \mathrm{E}+04$ & $4.863 \mathrm{E}+01$ \\
\hline
\end{tabular}


Table 269: GWS 5.0 x 4.3 Dynamic Calculated Values - 8021 RPM

\begin{tabular}{|c|c|c|c|c|c|c|c|}
\hline$n(\mathrm{RPM})$ & $V_{\infty}^{\prime}(\mathrm{m} / \mathrm{s})$ & $\Delta V_{\infty}^{\prime}(\mathrm{m} / \mathrm{s})$ & $T^{\prime}(\mathrm{g})$ & $\Delta T^{\prime}(\mathrm{g})$ & $V_{\mathrm{t}}(\mathrm{m} / \mathrm{s})$ & $P_{\mathrm{P}}(\mathrm{W})$ & $\Delta P_{\mathrm{P}}(\mathrm{W})$ \\
\hline $8.014 \mathrm{E}+03$ & $2.006 \mathrm{E}+00$ & $1.26 \mathrm{E}-02$ & $7.019 \mathrm{E}+01$ & $9.15 \mathrm{E}+00$ & $3.999 \mathrm{E}+01$ & $6.784 \mathrm{E}+00$ & $5.80 \mathrm{E}-01$ \\
\hline $8.039 E+03$ & $2.851 \mathrm{E}+00$ & $1.27 \mathrm{E}-02$ & $6.843 \mathrm{E}+01$ & $9.07 \mathrm{E}+00$ & $4.017 \mathrm{E}+01$ & $6.709 \mathrm{E}+00$ & $5.92 \mathrm{E}-01$ \\
\hline $7.956 \mathrm{E}+03$ & $3.688 \mathrm{E}+00$ & $1.31 \mathrm{E}-02$ & $6.248 \mathrm{E}+01$ & $9.28 \mathrm{E}+00$ & $3.982 \mathrm{E}+01$ & $6.317 \mathrm{E}+00$ & $5.92 \mathrm{E}-01$ \\
\hline $8.025 \mathrm{E}+03$ & $4.554 \mathrm{E}+00$ & $1.41 \mathrm{E}-02$ & $5.875 \mathrm{E}+01$ & $9.09 \mathrm{E}+00$ & $4.025 \mathrm{E}+01$ & $6.360 \mathrm{E}+00$ & $5.83 \mathrm{E}-01$ \\
\hline $7.952 \mathrm{E}+03$ & $5.427 \mathrm{E}+00$ & $1.54 \mathrm{E}-02$ & $5.404 \mathrm{E}+01$ & $9.34 \mathrm{E}+00$ & $4.000 \mathrm{E}+01$ & $5.985 \mathrm{E}+00$ & $5.91 \mathrm{E}-01$ \\
\hline $8.006 \mathrm{E}+03$ & $6.332 \mathrm{E}+00$ & $1.64 \mathrm{E}-02$ & $4.948 \mathrm{E}+01$ & $9.23 \mathrm{E}+00$ & $4.040 \mathrm{E}+01$ & $5.872 \mathrm{E}+00$ & $5.80 \mathrm{E}-01$ \\
\hline $8.054 \mathrm{E}+03$ & $7.248 \mathrm{E}+00$ & $1.73 \mathrm{E}-02$ & $4.400 \mathrm{E}+01$ & $9.19 \mathrm{E}+00$ & $4.079 \mathrm{E}+01$ & $5.495 \mathrm{E}+00$ & $5.77 \mathrm{E}-01$ \\
\hline $8.079 \mathrm{E}+03$ & $8.176 \mathrm{E}+00$ & $1.87 \mathrm{E}-02$ & $3.941 \mathrm{E}+01$ & $9.25 \mathrm{E}+00$ & $4.108 \mathrm{E}+01$ & $5.464 \mathrm{E}+00$ & $5.64 \mathrm{E}-01$ \\
\hline $8.061 \mathrm{E}+03$ & $9.115 \mathrm{E}+00$ & $2.02 \mathrm{E}-02$ & $3.575 \mathrm{E}+01$ & $9.17 \mathrm{E}+00$ & $4.119 \mathrm{E}+01$ & $5.002 \mathrm{E}+00$ & $5.74 \mathrm{E}-01$ \\
\hline$P_{\mathrm{e}}(\mathrm{W})$ & $\Delta P_{\mathrm{e}}(\mathrm{W})$ & $C_{\mathrm{T}}$ & $\Delta C_{\mathrm{T}}$ & $C_{\mathrm{P}}$ & $\Delta C_{\mathrm{P}}$ & $C_{\mathrm{Q}}$ & $\Delta C_{\mathrm{Q}}$ \\
\hline $1.460 \mathrm{E}+01$ & $1.74 \mathrm{E}-01$ & $1.277 \mathrm{E}-01$ & $1.66 \mathrm{E}-02$ & $7.426 \mathrm{E}-02$ & $6.35 \mathrm{E}-03$ & $1.182 \mathrm{E}-02$ & $1.01 \mathrm{E}-03$ \\
\hline $1.453 \mathrm{E}+01$ & $1.75 \mathrm{E}-01$ & $1.238 \mathrm{E}-01$ & $1.64 \mathrm{E}-02$ & $7.280 \mathrm{E}-02$ & $6.43 \mathrm{E}-03$ & $1.159 \mathrm{E}-02$ & $1.02 \mathrm{E}-03$ \\
\hline $1.393 \mathrm{E}+01$ & $1.69 \mathrm{E}-01$ & $1.155 \mathrm{E}-01$ & $1.72 \mathrm{E}-02$ & 7.077E-02 & $6.64 \mathrm{E}-03$ & $1.126 \mathrm{E}-02$ & $1.06 \mathrm{E}-03$ \\
\hline $1.382 \mathrm{E}+01$ & $1.68 \mathrm{E}-01$ & $1.068 \mathrm{E}-01$ & $1.65 \mathrm{E}-02$ & $6.943 \mathrm{E}-02$ & $6.36 \mathrm{E}-03$ & $1.105 \mathrm{E}-02$ & $1.01 \mathrm{E}-03$ \\
\hline $1.320 \mathrm{E}+01$ & $1.61 \mathrm{E}-01$ & $1.000 \mathrm{E}-01$ & $1.73 \mathrm{E}-02$ & $6.715 \mathrm{E}-02$ & $6.64 \mathrm{E}-03$ & $1.069 \mathrm{E}-02$ & $1.06 \mathrm{E}-03$ \\
\hline $1.290 \mathrm{E}+01$ & $1.62 \mathrm{E}-01$ & $9.039 \mathrm{E}-02$ & $1.69 \mathrm{E}-02$ & $6.459 \mathrm{E}-02$ & $6.38 \mathrm{E}-03$ & $1.028 \mathrm{E}-02$ & $1.02 \mathrm{E}-03$ \\
\hline $1.249 \mathrm{E}+01$ & $1.54 \mathrm{E}-01$ & 7.944E-02 & $1.66 \mathrm{E}-02$ & $5.938 \mathrm{E}-02$ & $6.23 \mathrm{E}-03$ & $9.451 \mathrm{E}-03$ & $9.92 \mathrm{E}-04$ \\
\hline $1.200 \mathrm{E}+01$ & $1.52 \mathrm{E}-01$ & 7.072E-02 & $1.66 \mathrm{E}-02$ & $5.850 \mathrm{E}-02$ & $6.04 \mathrm{E}-03$ & $9.310 \mathrm{E}-03$ & $9.61 \mathrm{E}-04$ \\
\hline $1.121 \mathrm{E}+01$ & $1.39 \mathrm{E}-01$ & $6.444 \mathrm{E}-02$ & $1.65 \mathrm{E}-02$ & $5.392 \mathrm{E}-02$ & 6.19E-03 & $8.582 \mathrm{E}-03$ & $9.85 \mathrm{E}-04$ \\
\hline$\rho\left(\mathrm{kg} / \mathrm{m}^{\wedge} 3\right)$ & $\eta_{\mathrm{T}}$ & $\Delta \eta_{\mathrm{T}}$ & $J$ & $\Delta J$ & $\eta_{\mathrm{P}}$ & $\Delta \eta_{\mathrm{P}}$ & $\operatorname{Re}_{0.75}$ \\
\hline $1.164 \mathrm{E}+00$ & 4.647E-01 & 4.01E-02 & $1.184 \mathrm{E}-01$ & $7.50 \mathrm{E}-04$ & $2.036 \mathrm{E}-01$ & $3.18 \mathrm{E}-02$ & $3.217 \mathrm{E}+04$ \\
\hline $1.163 \mathrm{E}+00$ & 4.619E-01 & 4.12E-02 & $1.676 \mathrm{E}-01$ & 7.62E-04 & $2.851 \mathrm{E}-01$ & 4.54E-02 & $3.227 \mathrm{E}+04$ \\
\hline $1.163 \mathrm{E}+00$ & $4.536 \mathrm{E}-01$ & $4.29 \mathrm{E}-02$ & $2.191 \mathrm{E}-01$ & $7.97 \mathrm{E}-04$ & $3.577 \mathrm{E}-01$ & $6.28 \mathrm{E}-02$ & $3.196 \mathrm{E}+04$ \\
\hline $1.162 \mathrm{E}+00$ & $4.601 \mathrm{E}-01$ & $4.25 \mathrm{E}-02$ & $2.682 \mathrm{E}-01$ & $8.57 \mathrm{E}-04$ & $4.125 \mathrm{E}-01$ & $7.42 \mathrm{E}-02$ & $3.228 \mathrm{E}+04$ \\
\hline $1.162 \mathrm{E}+00$ & 4.534E-01 & $4.51 \mathrm{E}-02$ & $3.226 \mathrm{E}-01$ & $9.55 \mathrm{E}-04$ & $4.805 \mathrm{E}-01$ & $9.57 \mathrm{E}-02$ & $3.209 \mathrm{E}+04$ \\
\hline $1.162 \mathrm{E}+00$ & $4.552 \mathrm{E}-01$ & $4.53 \mathrm{E}-02$ & $3.739 \mathrm{E}-01$ & $1.02 \mathrm{E}-03$ & $5.232 \mathrm{E}-01$ & $1.10 \mathrm{E}-01$ & $3.239 E+04$ \\
\hline $1.162 \mathrm{E}+00$ & $4.398 \mathrm{E}-01$ & $4.65 \mathrm{E}-02$ & 4.254E-01 & $1.07 \mathrm{E}-03$ & $5.691 \mathrm{E}-01$ & $1.33 \mathrm{E}-01$ & $3.269 \mathrm{E}+04$ \\
\hline $1.162 \mathrm{E}+00$ & $4.552 \mathrm{E}-01$ & $4.73 \mathrm{E}-02$ & 4.784E-01 & $1.16 \mathrm{E}-03$ & $5.784 \mathrm{E}-01$ & $1.48 \mathrm{E}-01$ & $3.293 \mathrm{E}+04$ \\
\hline $1.162 \mathrm{E}+00$ & $4.461 \mathrm{E}-01$ & $5.15 \mathrm{E}-02$ & $5.345 \mathrm{E}-01$ & $1.26 \mathrm{E}-03$ & $6.389 \mathrm{E}-01$ & $1.80 \mathrm{E}-01$ & $3.302 \mathrm{E}+04$ \\
\hline
\end{tabular}


Table 270: GWS 5.0 x 4.3 Dynamic Measured Values - 11986 RPM

\begin{tabular}{|c|c|c|c|c|c|c|c|}
\hline$n(\mathrm{RPM})$ & $Q(\mathrm{~g}-\mathrm{m})$ & $\Delta Q(\mathrm{~g}-\mathrm{m})$ & $V(\mathrm{~V})$ & $I(\mathrm{~A})$ & $T_{\text {atm }}\left({ }^{\circ} \mathrm{C}\right)$ & $P_{\text {atm }}(\mathrm{Pa})$ & $P_{\text {diff }}(\mathrm{Pa})$ \\
\hline $1.188 \mathrm{E}+04$ & $1.680 \mathrm{E}+00$ & $5.76 \mathrm{E}-02$ & $1.103 \mathrm{E}+01$ & $3.166 \mathrm{E}+00$ & $2.172 \mathrm{E}+01$ & $9.853 \mathrm{E}+04$ & $2.976 \mathrm{E}+00$ \\
\hline $1.202 \mathrm{E}+04$ & $1.720 \mathrm{E}+00$ & $6.33 \mathrm{E}-02$ & $1.102 \mathrm{E}+01$ & $3.227 \mathrm{E}+00$ & $2.143 \mathrm{E}+01$ & $9.852 \mathrm{E}+04$ & $7.077 \mathrm{E}+00$ \\
\hline $1.195 \mathrm{E}+04$ & $1.695 \mathrm{E}+00$ & $5.84 \mathrm{E}-02$ & $1.103 \mathrm{E}+01$ & $3.147 \mathrm{E}+00$ & $2.169 \mathrm{E}+01$ & $9.851 \mathrm{E}+04$ & $1.316 \mathrm{E}+01$ \\
\hline $1.206 \mathrm{E}+04$ & $1.668 \mathrm{E}+00$ & $6.05 \mathrm{E}-02$ & $1.103 \mathrm{E}+01$ & $3.123 \mathrm{E}+00$ & $2.355 \mathrm{E}+01$ & $9.851 \mathrm{E}+04$ & $2.129 \mathrm{E}+01$ \\
\hline $1.197 \mathrm{E}+04$ & $1.604 \mathrm{E}+00$ & $5.93 \mathrm{E}-02$ & $1.103 \mathrm{E}+01$ & $2.959 \mathrm{E}+00$ & $2.230 \mathrm{E}+01$ & $9.851 \mathrm{E}+04$ & $3.162 \mathrm{E}+01$ \\
\hline $1.201 \mathrm{E}+04$ & $1.542 \mathrm{E}+00$ & $6.08 \mathrm{E}-02$ & $1.103 \mathrm{E}+01$ & $2.864 \mathrm{E}+00$ & $2.254 \mathrm{E}+01$ & $9.850 \mathrm{E}+04$ & $4.417 \mathrm{E}+01$ \\
\hline $1.203 \mathrm{E}+04$ & $1.437 \mathrm{E}+00$ & $6.14 \mathrm{E}-02$ & $1.104 \mathrm{E}+01$ & $2.687 \mathrm{E}+00$ & $2.309 \mathrm{E}+01$ & $9.850 \mathrm{E}+04$ & $5.882 \mathrm{E}+01$ \\
\hline $1.199 \mathrm{E}+04$ & $1.311 \mathrm{E}+00$ & $5.95 \mathrm{E}-02$ & $1.105 \mathrm{E}+01$ & $2.477 \mathrm{E}+00$ & $2.318 \mathrm{E}+01$ & $9.849 \mathrm{E}+04$ & $7.625 \mathrm{E}+01$ \\
\hline $1.195 \mathrm{E}+04$ & $1.205 \mathrm{E}+00$ & $5.83 \mathrm{E}-02$ & $1.105 \mathrm{E}+01$ & $2.271 \mathrm{E}+00$ & $2.328 \mathrm{E}+01$ & $9.849 \mathrm{E}+04$ & $9.575 \mathrm{E}+01$ \\
\hline $1.201 \mathrm{E}+04$ & $1.082 \mathrm{E}+00$ & $5.84 \mathrm{E}-02$ & $1.106 \mathrm{E}+01$ & $2.089 \mathrm{E}+00$ & $2.293 \mathrm{E}+01$ & $9.849 \mathrm{E}+04$ & $1.176 \mathrm{E}+02$ \\
\hline $1.197 \mathrm{E}+04$ & $9.255 \mathrm{E}-01$ & $5.77 \mathrm{E}-02$ & $1.106 \mathrm{E}+01$ & $1.824 \mathrm{E}+00$ & $2.289 \mathrm{E}+01$ & $9.849 \mathrm{E}+04$ & $1.416 \mathrm{E}+02$ \\
\hline
\end{tabular}


Table 271: GWS 5.0 x 4.3 Dynamic Calculated Values - 11986 RPM

\begin{tabular}{|c|c|c|c|c|c|c|c|}
\hline$n(\mathrm{RPM})$ & $V_{\infty}^{\prime}(\mathrm{m} / \mathrm{s})$ & $\Delta V_{\infty}^{\prime}(\mathrm{m} / \mathrm{s})$ & $T^{\prime}(\mathrm{g})$ & $\Delta T^{\prime}(\mathrm{g})$ & $V_{\mathrm{t}}(\mathrm{m} / \mathrm{s})$ & $P_{\mathrm{P}}(\mathrm{W})$ & $\Delta P_{\mathrm{P}}(\mathrm{W})$ \\
\hline $1.188 \mathrm{E}+04$ & $2.140 \mathrm{E}+00$ & $1.45 \mathrm{E}-02$ & $1.575 \mathrm{E}+02$ & $7.88 \mathrm{E}+00$ & $5.927 \mathrm{E}+01$ & $2.051 \mathrm{E}+01$ & 7.03E-01 \\
\hline $1.202 \mathrm{E}+04$ & $3.366 \mathrm{E}+00$ & $1.55 \mathrm{E}-02$ & $1.566 \mathrm{E}+02$ & $7.91 \mathrm{E}+00$ & $6.002 \mathrm{E}+01$ & $2.124 \mathrm{E}+01$ & $7.82 \mathrm{E}-01$ \\
\hline $1.195 \mathrm{E}+04$ & $4.642 \mathrm{E}+00$ & $1.59 \mathrm{E}-02$ & $1.482 \mathrm{E}+02$ & $7.95 \mathrm{E}+00$ & $5.973 \mathrm{E}+01$ & $2.079 \mathrm{E}+01$ & 7.17E-01 \\
\hline $1.206 \mathrm{E}+04$ & $5.959 \mathrm{E}+00$ & $1.73 \mathrm{E}-02$ & $1.418 \mathrm{E}+02$ & $7.86 \mathrm{E}+00$ & $6.041 \mathrm{E}+01$ & $2.066 \mathrm{E}+01$ & $7.50 \mathrm{E}-01$ \\
\hline $1.197 \mathrm{E}+04$ & $7.283 \mathrm{E}+00$ & $1.87 \mathrm{E}-02$ & $1.261 \mathrm{E}+02$ & $7.86 \mathrm{E}+00$ & $6.009 \mathrm{E}+01$ & $1.971 \mathrm{E}+01$ & 7.29E-01 \\
\hline $1.201 \mathrm{E}+04$ & $8.637 \mathrm{E}+00$ & $2.12 \mathrm{E}-02$ & $1.170 \mathrm{E}+02$ & $7.84 \mathrm{E}+00$ & $6.049 \mathrm{E}+01$ & $1.902 \mathrm{E}+01$ & $7.50 \mathrm{E}-01$ \\
\hline $1.203 \mathrm{E}+04$ & $1.000 \mathrm{E}+01$ & $2.28 \mathrm{E}-02$ & $1.032 \mathrm{E}+02$ & $7.84 \mathrm{E}+00$ & $6.077 \mathrm{E}+01$ & $1.775 \mathrm{E}+01$ & $7.59 \mathrm{E}-01$ \\
\hline $1.199 \mathrm{E}+04$ & $1.141 \mathrm{E}+01$ & $2.44 \mathrm{E}-02$ & $8.855 \mathrm{E}+01$ & $7.85 \mathrm{E}+00$ & $6.084 \mathrm{E}+01$ & $1.614 \mathrm{E}+01$ & 7.33E-01 \\
\hline $1.195 \mathrm{E}+04$ & $1.281 \mathrm{E}+01$ & $2.63 \mathrm{E}-02$ & $7.362 \mathrm{E}+01$ & $7.84 \mathrm{E}+00$ & $6.092 \mathrm{E}+01$ & $1.479 \mathrm{E}+01$ & $7.16 \mathrm{E}-01$ \\
\hline $1.201 \mathrm{E}+04$ & $1.420 \mathrm{E}+01$ & $2.87 \mathrm{E}-02$ & $6.420 \mathrm{E}+01$ & $7.85 \mathrm{E}+00$ & $6.153 \mathrm{E}+01$ & $1.335 \mathrm{E}+01$ & $7.20 \mathrm{E}-01$ \\
\hline $1.197 \mathrm{E}+04$ & $1.560 \mathrm{E}+01$ & $3.12 \mathrm{E}-02$ & $4.940 \mathrm{E}+01$ & $7.84 \mathrm{E}+00$ & $6.164 \mathrm{E}+01$ & $1.137 \mathrm{E}+01$ & 7.09E-01 \\
\hline$P_{\mathrm{e}}(\mathrm{W})$ & $\Delta P_{\mathrm{e}}(\mathrm{W})$ & $C_{\mathrm{T}}$ & $\Delta C_{\mathrm{T}}$ & $C_{\mathrm{P}}$ & $\Delta C_{\mathrm{P}}$ & $C_{\mathrm{Q}}$ & $\Delta C_{\mathrm{Q}}$ \\
\hline $3.491 \mathrm{E}+01$ & $3.88 \mathrm{E}-01$ & $1.304 \mathrm{E}-01$ & $6.52 \mathrm{E}-03$ & $6.883 \mathrm{E}-02$ & $2.36 \mathrm{E}-03$ & $1.095 \mathrm{E}-02$ & $3.75 \mathrm{E}-04$ \\
\hline $3.558 \mathrm{E}+01$ & $3.98 \mathrm{E}-01$ & $1.265 \mathrm{E}-01$ & $6.39 \mathrm{E}-03$ & $6.878 \mathrm{E}-02$ & $2.53 \mathrm{E}-03$ & $1.095 \mathrm{E}-02$ & $4.03 \mathrm{E}-04$ \\
\hline $3.470 \mathrm{E}+01$ & $3.86 \mathrm{E}-01$ & $1.214 \mathrm{E}-01$ & $6.51 \mathrm{E}-03$ & $6.868 \mathrm{E}-02$ & $2.37 \mathrm{E}-03$ & $1.093 \mathrm{E}-02$ & 3.77E-04 \\
\hline $3.444 \mathrm{E}+01$ & $3.86 \mathrm{E}-01$ & $1.146 \mathrm{E}-01$ & $6.36 \mathrm{E}-03$ & $6.675 \mathrm{E}-02$ & $2.42 \mathrm{E}-03$ & $1.062 \mathrm{E}-02$ & $3.86 \mathrm{E}-04$ \\
\hline $3.264 \mathrm{E}+01$ & $3.63 \mathrm{E}-01$ & $1.031 \mathrm{E}-01$ & $6.43 \mathrm{E}-03$ & $6.492 \mathrm{E}-02$ & $2.40 \mathrm{E}-03$ & $1.033 \mathrm{E}-02$ & $3.82 \mathrm{E}-04$ \\
\hline $3.161 \mathrm{E}+01$ & $3.52 \mathrm{E}-01$ & $9.507 \mathrm{E}-02$ & $6.37 \mathrm{E}-03$ & $6.201 \mathrm{E}-02$ & $2.45 \mathrm{E}-03$ & $9.868 \mathrm{E}-03$ & $3.89 \mathrm{E}-04$ \\
\hline $2.967 \mathrm{E}+01$ & 3.34E-01 & $8.383 \mathrm{E}-02$ & $6.36 \mathrm{E}-03$ & $5.777 \mathrm{E}-02$ & $2.47 \mathrm{E}-03$ & $9.195 \mathrm{E}-03$ & 3.93E-04 \\
\hline $2.736 \mathrm{E}+01$ & $3.10 \mathrm{E}-01$ & $7.235 \mathrm{E}-02$ & $6.42 \mathrm{E}-03$ & $5.302 \mathrm{E}-02$ & $2.41 \mathrm{E}-03$ & $8.438 \mathrm{E}-03$ & $3.83 \mathrm{E}-04$ \\
\hline $2.510 \mathrm{E}+01$ & $2.88 \mathrm{E}-01$ & $6.060 \mathrm{E}-02$ & $6.46 \mathrm{E}-03$ & 4.911E-02 & $2.38 \mathrm{E}-03$ & $7.816 \mathrm{E}-03$ & $3.78 \mathrm{E}-04$ \\
\hline $2.309 \mathrm{E}+01$ & $2.62 \mathrm{E}-01$ & $5.223 \mathrm{E}-02$ & 6.39E-03 & 4.357E-02 & $2.35 \mathrm{E}-03$ & $6.934 \mathrm{E}-03$ & $3.74 \mathrm{E}-04$ \\
\hline $2.018 \mathrm{E}+01$ & $2.33 \mathrm{E}-01$ & $4.050 \mathrm{E}-02$ & $6.43 \mathrm{E}-03$ & $3.756 \mathrm{E}-02$ & $2.34 \mathrm{E}-03$ & 5.979E-03 & $3.73 \mathrm{E}-04$ \\
\hline$\rho\left(\mathrm{kg} / \mathrm{m}^{\wedge} 3\right)$ & $\eta_{\mathrm{T}}$ & $\Delta \eta_{\mathrm{T}}$ & $J$ & $\Delta J$ & $\eta_{\mathrm{P}}$ & $\Delta \eta_{\mathrm{P}}$ & $R e_{0.75}$ \\
\hline $1.164 \mathrm{E}+00$ & $5.875 \mathrm{E}-01$ & $2.12 \mathrm{E}-02$ & 8.510E-02 & $5.76 \mathrm{E}-04$ & $1.612 \mathrm{E}-01$ & $9.83 \mathrm{E}-03$ & $4.774 \mathrm{E}+04$ \\
\hline $1.165 \mathrm{E}+00$ & $5.969 \mathrm{E}-01$ & $2.30 \mathrm{E}-02$ & $1.323 \mathrm{E}-01$ & $6.12 \mathrm{E}-04$ & $2.435 \mathrm{E}-01$ & $1.53 \mathrm{E}-02$ & $4.842 \mathrm{E}+04$ \\
\hline $1.164 \mathrm{E}+00$ & 5.992E-01 & $2.17 \mathrm{E}-02$ & $1.836 \mathrm{E}-01$ & $6.30 \mathrm{E}-04$ & $3.245 \mathrm{E}-01$ & $2.07 \mathrm{E}-02$ & $4.811 \mathrm{E}+04$ \\
\hline $1.157 \mathrm{E}+00$ & $5.998 \mathrm{E}-01$ & $2.28 \mathrm{E}-02$ & $2.336 \mathrm{E}-01$ & $6.91 \mathrm{E}-04$ & 4.011E-01 & $2.66 \mathrm{E}-02$ & $4.812 \mathrm{E}+04$ \\
\hline $1.162 \mathrm{E}+00$ & $6.039 \mathrm{E}-01$ & $2.33 \mathrm{E}-02$ & $2.877 \mathrm{E}-01$ & 7.44E-04 & $4.569 \mathrm{E}-01$ & $3.31 \mathrm{E}-02$ & $4.822 \mathrm{E}+04$ \\
\hline $1.161 \mathrm{E}+00$ & $6.018 \mathrm{E}-01$ & $2.47 \mathrm{E}-02$ & $3.399 \mathrm{E}-01$ & $8.55 \mathrm{E}-04$ & $5.212 \mathrm{E}-01$ & $4.05 \mathrm{E}-02$ & $4.847 \mathrm{E}+04$ \\
\hline $1.158 \mathrm{E}+00$ & 5.984E-01 & $2.64 \mathrm{E}-02$ & $3.931 \mathrm{E}-01$ & $9.19 \mathrm{E}-04$ & $5.705 \mathrm{E}-01$ & $4.97 \mathrm{E}-02$ & $4.853 \mathrm{E}+04$ \\
\hline $1.158 \mathrm{E}+00$ & $5.900 \mathrm{E}-01$ & $2.76 \mathrm{E}-02$ & 4.499E-01 & $9.77 \mathrm{E}-04$ & $6.139 \mathrm{E}-01$ & $6.12 \mathrm{E}-02$ & $4.856 \mathrm{E}+04$ \\
\hline $1.157 \mathrm{E}+00$ & 5.893E-01 & $2.93 \mathrm{E}-02$ & $5.068 \mathrm{E}-01$ & $1.05 \mathrm{E}-03$ & $6.254 \mathrm{E}-01$ & 7.32E-02 & $4.859 \mathrm{E}+04$ \\
\hline $1.159 \mathrm{E}+00$ & 5.779E-01 & $3.19 \mathrm{E}-02$ & $5.588 \mathrm{E}-01$ & $1.17 \mathrm{E}-03$ & $6.699 \mathrm{E}-01$ & $8.95 \mathrm{E}-02$ & $4.919 \mathrm{E}+04$ \\
\hline $1.159 \mathrm{E}+00$ & $5.636 \mathrm{E}-01$ & $3.57 \mathrm{E}-02$ & $6.164 \mathrm{E}-01$ & $1.24 \mathrm{E}-03$ & $6.646 \mathrm{E}-01$ & $1.13 \mathrm{E}-01$ & $4.928 \mathrm{E}+04$ \\
\hline
\end{tabular}


Table 272: GWS 5.0 x 4.3 Dynamic Measured Values - 16006 RPM

\begin{tabular}{|c|c|c|c|c|c|c|c|}
\hline$n(\mathrm{RPM})$ & $Q(\mathrm{~g}-\mathrm{m})$ & $\Delta Q(\mathrm{~g}-\mathrm{m})$ & $V(\mathrm{~V})$ & $I(\mathrm{~A})$ & $T_{\text {atm }}\left({ }^{\circ} \mathrm{C}\right)$ & $P_{\text {atm }}(\mathrm{Pa})$ & $P_{\text {diff }}(\mathrm{Pa})$ \\
\hline $1.599 \mathrm{E}+04$ & $2.900 \mathrm{E}+00$ & $6.75 \mathrm{E}-02$ & $1.093 \mathrm{E}+01$ & $6.441 \mathrm{E}+00$ & $2.265 \mathrm{E}+01$ & $9.850 \mathrm{E}+04$ & $3.783 \mathrm{E}+00$ \\
\hline $1.603 \mathrm{E}+04$ & $2.898 \mathrm{E}+00$ & $6.84 \mathrm{E}-02$ & $1.093 \mathrm{E}+01$ & $6.425 \mathrm{E}+00$ & $2.259 \mathrm{E}+01$ & $9.849 \mathrm{E}+04$ & $9.738 \mathrm{E}+00$ \\
\hline $1.606 \mathrm{E}+04$ & $2.880 \mathrm{E}+00$ & $7.07 \mathrm{E}-02$ & $1.093 \mathrm{E}+01$ & $6.371 \mathrm{E}+00$ & $2.263 \mathrm{E}+01$ & $9.850 \mathrm{E}+04$ & $1.945 \mathrm{E}+01$ \\
\hline $1.599 \mathrm{E}+04$ & $2.792 \mathrm{E}+00$ & $6.81 \mathrm{E}-02$ & $1.094 \mathrm{E}+01$ & $6.142 \mathrm{E}+00$ & $2.213 \mathrm{E}+01$ & $9.849 \mathrm{E}+04$ & $3.301 \mathrm{E}+01$ \\
\hline $1.599 \mathrm{E}+04$ & $2.683 \mathrm{E}+00$ & $7.20 \mathrm{E}-02$ & $1.095 \mathrm{E}+01$ & $5.887 \mathrm{E}+00$ & $2.289 \mathrm{E}+01$ & $9.849 \mathrm{E}+04$ & $5.028 \mathrm{E}+01$ \\
\hline $1.595 \mathrm{E}+04$ & $2.545 \mathrm{E}+00$ & $6.91 \mathrm{E}-02$ & $1.096 \mathrm{E}+01$ & $5.522 \mathrm{E}+00$ & $2.310 \mathrm{E}+01$ & $9.849 \mathrm{E}+04$ & $7.135 \mathrm{E}+01$ \\
\hline $1.602 \mathrm{E}+04$ & $2.351 \mathrm{E}+00$ & $6.86 \mathrm{E}-02$ & $1.097 \mathrm{E}+01$ & $5.132 \mathrm{E}+00$ & $2.321 \mathrm{E}+01$ & $9.849 \mathrm{E}+04$ & $9.698 \mathrm{E}+01$ \\
\hline $1.605 \mathrm{E}+04$ & $2.118 \mathrm{E}+00$ & $6.91 \mathrm{E}-02$ & $1.098 \mathrm{E}+01$ & $4.640 \mathrm{E}+00$ & $2.329 \mathrm{E}+01$ & $9.848 \mathrm{E}+04$ & $1.266 \mathrm{E}+02$ \\
\hline $1.599 \mathrm{E}+04$ & $1.894 \mathrm{E}+00$ & $6.35 \mathrm{E}-02$ & $1.100 \mathrm{E}+01$ & $4.165 \mathrm{E}+00$ & $2.304 \mathrm{E}+01$ & $9.847 \mathrm{E}+04$ & $1.601 \mathrm{E}+02$ \\
\hline $1.602 \mathrm{E}+04$ & $1.664 \mathrm{E}+00$ & $6.37 \mathrm{E}-02$ & $1.101 \mathrm{E}+01$ & $3.682 \mathrm{E}+00$ & $2.324 \mathrm{E}+01$ & $9.847 \mathrm{E}+04$ & $1.976 \mathrm{E}+02$ \\
\hline $1.599 \mathrm{E}+04$ & $1.387 \mathrm{E}+00$ & $6.43 \mathrm{E}-02$ & $1.103 \mathrm{E}+01$ & $3.111 \mathrm{E}+00$ & $2.332 \mathrm{E}+01$ & $9.846 \mathrm{E}+04$ & $2.382 \mathrm{E}+02$ \\
\hline
\end{tabular}


Table 273: GWS 5.0 x 4.3 Dynamic Calculated Values - 16006 RPM

\begin{tabular}{|c|c|c|c|c|c|c|c|}
\hline$n(\mathrm{RPM})$ & $V_{\infty}^{\prime}(\mathrm{m} / \mathrm{s})$ & $\Delta V_{\infty}^{\prime}(\mathrm{m} / \mathrm{s})$ & $T^{\prime}(\mathrm{g})$ & $\Delta T^{\prime}(\mathrm{g})$ & $V_{\mathrm{t}}(\mathrm{m} / \mathrm{s})$ & $P_{\mathrm{P}}(\mathrm{W})$ & $\Delta P_{\mathrm{P}}(\mathrm{W})$ \\
\hline $1.599 \mathrm{E}+04$ & $2.389 \mathrm{E}+00$ & $1.76 \mathrm{E}-02$ & $2.855 \mathrm{E}+02$ & $7.40 \mathrm{E}+00$ & $7.971 \mathrm{E}+01$ & $4.761 \mathrm{E}+01$ & $1.11 \mathrm{E}+00$ \\
\hline $1.603 \mathrm{E}+04$ & $3.937 \mathrm{E}+00$ & $1.84 \mathrm{E}-02$ & $2.784 \mathrm{E}+02$ & $7.21 \mathrm{E}+00$ & $8.000 \mathrm{E}+01$ & $4.772 \mathrm{E}+01$ & $1.13 \mathrm{E}+00$ \\
\hline $1.606 \mathrm{E}+04$ & $5.637 \mathrm{E}+00$ & $1.96 \mathrm{E}-02$ & $2.680 \mathrm{E}+02$ & $7.21 \mathrm{E}+00$ & $8.025 \mathrm{E}+01$ & $4.751 \mathrm{E}+01$ & $1.17 \mathrm{E}+00$ \\
\hline $1.599 \mathrm{E}+04$ & $7.394 \mathrm{E}+00$ & $2.15 \mathrm{E}-02$ & $2.492 \mathrm{E}+02$ & $7.32 \mathrm{E}+00$ & $8.002 \mathrm{E}+01$ & $4.585 \mathrm{E}+01$ & $1.12 \mathrm{E}+00$ \\
\hline $1.599 \mathrm{E}+04$ & $9.184 \mathrm{E}+00$ & $2.45 \mathrm{E}-02$ & $2.287 \mathrm{E}+02$ & $7.37 \mathrm{E}+00$ & $8.021 \mathrm{E}+01$ & $4.405 \mathrm{E}+01$ & $1.18 \mathrm{E}+00$ \\
\hline $1.595 \mathrm{E}+04$ & $1.098 \mathrm{E}+01$ & $2.69 \mathrm{E}-02$ & $2.042 \mathrm{E}+02$ & $7.41 \mathrm{E}+00$ & $8.023 \mathrm{E}+01$ & $4.169 \mathrm{E}+01$ & $1.13 \mathrm{E}+00$ \\
\hline $1.602 \mathrm{E}+04$ & $1.284 \mathrm{E}+01$ & $2.88 \mathrm{E}-02$ & $1.787 \mathrm{E}+02$ & 7.37E+00 & $8.086 \mathrm{E}+01$ & $3.869 \mathrm{E}+01$ & $1.13 \mathrm{E}+00$ \\
\hline $1.605 \mathrm{E}+04$ & $1.471 \mathrm{E}+01$ & $3.04 \mathrm{E}-02$ & $1.506 \mathrm{E}+02$ & $7.32 \mathrm{E}+00$ & $8.131 \mathrm{E}+01$ & $3.490 \mathrm{E}+01$ & $1.14 \mathrm{E}+00$ \\
\hline $1.599 \mathrm{E}+04$ & $1.656 \mathrm{E}+01$ & $3.30 \mathrm{E}-02$ & $1.244 \mathrm{E}+02$ & $7.42 \mathrm{E}+00$ & $8.139 \mathrm{E}+01$ & $3.110 \mathrm{E}+01$ & $1.04 \mathrm{E}+00$ \\
\hline $1.602 \mathrm{E}+04$ & $1.842 \mathrm{E}+01$ & $3.61 \mathrm{E}-02$ & $1.017 \mathrm{E}+02$ & $7.42 \mathrm{E}+00$ & $8.195 \mathrm{E}+01$ & $2.738 \mathrm{E}+01$ & $1.05 \mathrm{E}+00$ \\
\hline $1.599 \mathrm{E}+04$ & $2.025 \mathrm{E}+01$ & 3.91E-02 & $7.451 \mathrm{E}+01$ & $7.51 \mathrm{E}+00$ & $8.221 \mathrm{E}+01$ & $2.278 \mathrm{E}+01$ & $1.06 \mathrm{E}+00$ \\
\hline$P_{\mathrm{e}}(\mathrm{W})$ & $\Delta P_{\mathrm{e}}(\mathrm{W})$ & $C_{\mathrm{T}}$ & $\Delta C_{\mathrm{T}}$ & $C_{\mathrm{P}}$ & $\Delta C_{\mathrm{P}}$ & $C_{\mathrm{Q}}$ & $\Delta C_{\mathrm{Q}}$ \\
\hline $7.040 \mathrm{E}+01$ & 7.87E-01 & $1.310 \mathrm{E}-01$ & $3.40 \mathrm{E}-03$ & $6.588 \mathrm{E}-02$ & $1.53 \mathrm{E}-03$ & 1.049E-02 & $2.44 \mathrm{E}-04$ \\
\hline $7.023 \mathrm{E}+01$ & $7.86 \mathrm{E}-01$ & $1.270 \mathrm{E}-01$ & $3.29 \mathrm{E}-03$ & $6.544 \mathrm{E}-02$ & $1.55 \mathrm{E}-03$ & $1.042 \mathrm{E}-02$ & $2.46 \mathrm{E}-04$ \\
\hline $6.964 \mathrm{E}+01$ & $7.81 \mathrm{E}-01$ & $1.218 \mathrm{E}-01$ & $3.28 \mathrm{E}-03$ & $6.479 \mathrm{E}-02$ & $1.59 \mathrm{E}-03$ & $1.031 \mathrm{E}-02$ & $2.53 \mathrm{E}-04$ \\
\hline $6.719 \mathrm{E}+01$ & 7.53E-01 & $1.142 \mathrm{E}-01$ & $3.36 \mathrm{E}-03$ & $6.333 \mathrm{E}-02$ & $1.55 \mathrm{E}-03$ & $1.008 \mathrm{E}-02$ & $2.46 \mathrm{E}-04$ \\
\hline $6.444 \mathrm{E}+01$ & $7.50 \mathrm{E}-01$ & $1.050 \mathrm{E}-01$ & $3.38 \mathrm{E}-03$ & 6.099E-02 & $1.64 \mathrm{E}-03$ & $9.707 \mathrm{E}-03$ & $2.61 \mathrm{E}-04$ \\
\hline $6.050 \mathrm{E}+01$ & $6.79 \mathrm{E}-01$ & $9.434 \mathrm{E}-02$ & $3.42 \mathrm{E}-03$ & $5.821 \mathrm{E}-02$ & $1.58 \mathrm{E}-03$ & $9.265 \mathrm{E}-03$ & $2.52 \mathrm{E}-04$ \\
\hline $5.629 \mathrm{E}+01$ & $6.37 \mathrm{E}-01$ & $8.181 \mathrm{E}-02$ & $3.38 \mathrm{E}-03$ & $5.331 \mathrm{E}-02$ & $1.56 \mathrm{E}-03$ & 8.484E-03 & $2.48 \mathrm{E}-04$ \\
\hline $5.096 \mathrm{E}+01$ & $5.78 \mathrm{E}-01$ & $6.878 \mathrm{E}-02$ & $3.34 \mathrm{E}-03$ & $4.788 \mathrm{E}-02$ & $1.56 \mathrm{E}-03$ & $7.620 \mathrm{E}-03$ & 2.49E-04 \\
\hline $4.580 \mathrm{E}+01$ & $5.10 \mathrm{E}-01$ & $5.714 \mathrm{E}-02$ & $3.41 \mathrm{E}-03$ & 4.307E-02 & $1.44 \mathrm{E}-03$ & $6.854 \mathrm{E}-03$ & $2.30 \mathrm{E}-04$ \\
\hline $4.054 \mathrm{E}+01$ & $4.48 \mathrm{E}-01$ & $4.658 \mathrm{E}-02$ & $3.40 \mathrm{E}-03$ & $3.772 \mathrm{E}-02$ & $1.44 \mathrm{E}-03$ & $6.003 \mathrm{E}-03$ & $2.30 \mathrm{E}-04$ \\
\hline $3.431 \mathrm{E}+01$ & $3.86 \mathrm{E}-01$ & $3.428 \mathrm{E}-02$ & $3.46 \mathrm{E}-03$ & $3.160 \mathrm{E}-02$ & $1.46 \mathrm{E}-03$ & $5.029 \mathrm{E}-03$ & 2.33E-04 \\
\hline$\rho\left(\mathrm{kg} / \mathrm{m}^{\wedge} 3\right)$ & $\eta_{\mathrm{T}}$ & $\Delta \eta_{\mathrm{T}}$ & $J$ & $\Delta J$ & $\eta_{\mathrm{P}}$ & $\Delta \eta_{\mathrm{P}}$ & $R e_{0.75}$ \\
\hline $1.160 \mathrm{E}+00$ & $6.762 \mathrm{E}-01$ & $1.75 \mathrm{E}-02$ & $7.065 \mathrm{E}-02$ & $5.22 \mathrm{E}-04$ & $1.405 \mathrm{E}-01$ & $5.01 \mathrm{E}-03$ & $6.382 \mathrm{E}+04$ \\
\hline $1.160 \mathrm{E}+00$ & $6.795 \mathrm{E}-01$ & $1.78 \mathrm{E}-02$ & $1.161 \mathrm{E}-01$ & $5.44 \mathrm{E}-04$ & $2.252 \mathrm{E}-01$ & 7.97E-03 & $6.408 \mathrm{E}+04$ \\
\hline $1.160 \mathrm{E}+00$ & $6.821 \mathrm{E}-01$ & $1.84 \mathrm{E}-02$ & $1.659 \mathrm{E}-01$ & $5.79 \mathrm{E}-04$ & $3.118 \mathrm{E}-01$ & $1.14 \mathrm{E}-02$ & $6.427 \mathrm{E}+04$ \\
\hline $1.162 \mathrm{E}+00$ & $6.824 \mathrm{E}-01$ & $1.83 \mathrm{E}-02$ & $2.186 \mathrm{E}-01$ & $6.37 \mathrm{E}-04$ & $3.941 \mathrm{E}-01$ & $1.51 \mathrm{E}-02$ & $6.426 \mathrm{E}+04$ \\
\hline $1.159 \mathrm{E}+00$ & $6.835 \mathrm{E}-01$ & $2.00 \mathrm{E}-02$ & $2.715 \mathrm{E}-01$ & $7.29 \mathrm{E}-04$ & 4.675E-01 & $1.97 \mathrm{E}-02$ & $6.413 \mathrm{E}+04$ \\
\hline $1.158 \mathrm{E}+00$ & $6.890 \mathrm{E}-01$ & $2.02 \mathrm{E}-02$ & $3.256 \mathrm{E}-01$ & $8.02 \mathrm{E}-04$ & $5.277 \mathrm{E}-01$ & $2.40 \mathrm{E}-02$ & $6.406 \mathrm{E}+04$ \\
\hline $1.158 \mathrm{E}+00$ & $6.873 \mathrm{E}-01$ & $2.15 \mathrm{E}-02$ & $3.790 \mathrm{E}-01$ & $8.56 \mathrm{E}-04$ & $5.816 \mathrm{E}-01$ & $2.94 \mathrm{E}-02$ & $6.453 \mathrm{E}+04$ \\
\hline $1.157 \mathrm{E}+00$ & $6.849 \mathrm{E}-01$ & 2.37E-02 & 4.334E-01 & $9.02 \mathrm{E}-04$ & $6.226 \mathrm{E}-01$ & $3.65 \mathrm{E}-02$ & $6.485 \mathrm{E}+04$ \\
\hline $1.158 \mathrm{E}+00$ & 6.790E-01 & $2.40 \mathrm{E}-02$ & 4.896E-01 & $9.82 \mathrm{E}-04$ & $6.496 \mathrm{E}-01$ & $4.45 \mathrm{E}-02$ & $6.501 \mathrm{E}+04$ \\
\hline $1.157 \mathrm{E}+00$ & $6.753 \mathrm{E}-01$ & $2.69 \mathrm{E}-02$ & $5.436 \mathrm{E}-01$ & $1.07 \mathrm{E}-03$ & $6.712 \mathrm{E}-01$ & $5.53 \mathrm{E}-02$ & $6.537 \mathrm{E}+04$ \\
\hline $1.157 \mathrm{E}+00$ & $6.639 \mathrm{E}-01$ & $3.17 \mathrm{E}-02$ & 5.989E-01 & $1.17 \mathrm{E}-03$ & $6.498 \mathrm{E}-01$ & 7.21E-02 & $6.554 \mathrm{E}+04$ \\
\hline
\end{tabular}


Table 274: GWS 5.0 x 4.3 Dynamic Measured Values - 19971 RPM

\begin{tabular}{|c|c|c|c|c|c|c|c|}
\hline$n(\mathrm{RPM})$ & $Q(\mathrm{~g}-\mathrm{m})$ & $\Delta Q(\mathrm{~g}-\mathrm{m})$ & $V(\mathrm{~V})$ & $I(\mathrm{~A})$ & $T_{\text {atm }}\left({ }^{\circ} \mathrm{C}\right)$ & $P_{\text {atm }}(\mathrm{Pa})$ & $P_{\text {diff }}(\mathrm{Pa})$ \\
\hline $1.990 \mathrm{E}+04$ & $4.300 \mathrm{E}+00$ & $1.14 \mathrm{E}-01$ & $1.078 \mathrm{E}+01$ & $1.143 \mathrm{E}+01$ & $2.317 \mathrm{E}+01$ & $9.847 \mathrm{E}+04$ & $4.628 \mathrm{E}+00$ \\
\hline $1.998 \mathrm{E}+04$ & $4.232 \mathrm{E}+00$ & $1.20 \mathrm{E}-01$ & $1.078 \mathrm{E}+01$ & $1.141 \mathrm{E}+01$ & $2.346 \mathrm{E}+01$ & $9.847 \mathrm{E}+04$ & $1.260 \mathrm{E}+01$ \\
\hline $1.999 \mathrm{E}+04$ & $4.198 \mathrm{E}+00$ & $1.20 \mathrm{E}-01$ & $1.079 \mathrm{E}+01$ & $1.115 \mathrm{E}+01$ & $2.372 \mathrm{E}+01$ & $9.847 \mathrm{E}+04$ & $2.660 \mathrm{E}+01$ \\
\hline $2.004 \mathrm{E}+04$ & $4.088 \mathrm{E}+00$ & $1.24 \mathrm{E}-01$ & $1.080 \mathrm{E}+01$ & $1.084 \mathrm{E}+01$ & $2.385 \mathrm{E}+01$ & $9.847 \mathrm{E}+04$ & $4.673 \mathrm{E}+01$ \\
\hline $2.000 \mathrm{E}+04$ & $3.941 \mathrm{E}+00$ & $1.21 \mathrm{E}-01$ & $1.082 \mathrm{E}+01$ & $1.021 \mathrm{E}+01$ & $2.391 \mathrm{E}+01$ & $9.848 \mathrm{E}+04$ & $7.267 \mathrm{E}+01$ \\
\hline $1.997 \mathrm{E}+04$ & $3.650 \mathrm{E}+00$ & $1.18 \mathrm{E}-01$ & $1.084 \mathrm{E}+01$ & $9.365 \mathrm{E}+00$ & $2.382 \mathrm{E}+01$ & $9.848 \mathrm{E}+04$ & $1.053 \mathrm{E}+02$ \\
\hline $1.997 \mathrm{E}+04$ & $3.346 \mathrm{E}+00$ & $1.19 \mathrm{E}-01$ & $1.087 \mathrm{E}+01$ & $8.356 \mathrm{E}+00$ & $2.398 \mathrm{E}+01$ & $9.848 \mathrm{E}+04$ & $1.443 \mathrm{E}+02$ \\
\hline $1.994 \mathrm{E}+04$ & $2.900 \mathrm{E}+00$ & $1.19 \mathrm{E}-01$ & $1.091 \mathrm{E}+01$ & $7.185 \mathrm{E}+00$ & $2.416 \mathrm{E}+01$ & $9.848 \mathrm{E}+04$ & $1.894 \mathrm{E}+02$ \\
\hline $2.004 \mathrm{E}+04$ & $2.517 \mathrm{E}+00$ & $1.21 \mathrm{E}-01$ & $1.093 \mathrm{E}+01$ & $6.362 \mathrm{E}+00$ & $2.376 \mathrm{E}+01$ & $9.848 \mathrm{E}+04$ & $2.401 \mathrm{E}+02$ \\
\hline $1.986 \mathrm{E}+04$ & $2.116 \mathrm{E}+00$ & $1.08 \mathrm{E}-01$ & $1.097 \mathrm{E}+01$ & $5.198 \mathrm{E}+00$ & $2.367 \mathrm{E}+01$ & $9.848 \mathrm{E}+04$ & $2.972 \mathrm{E}+02$ \\
\hline $2.001 \mathrm{E}+04$ & $1.689 \mathrm{E}+00$ & $1.09 \mathrm{E}-01$ & $1.099 \mathrm{E}+01$ & $4.240 \mathrm{E}+00$ & $2.363 \mathrm{E}+01$ & $9.848 \mathrm{E}+04$ & $3.602 \mathrm{E}+02$ \\
\hline
\end{tabular}


Table 275: GWS 5.0 x 4.3 Dynamic Calculated Values - 19971 RPM

\begin{tabular}{|c|c|c|c|c|c|c|c|}
\hline$n(\mathrm{RPM})$ & $V_{\infty}^{\prime}(\mathrm{m} / \mathrm{s})$ & $\Delta V_{\infty}^{\prime}(\mathrm{m} / \mathrm{s})$ & $T^{\prime}(\mathrm{g})$ & $\Delta T^{\prime}(\mathrm{g})$ & $V_{\mathrm{t}}(\mathrm{m} / \mathrm{s})$ & $P_{\mathrm{P}}(\mathrm{W})$ & $\Delta P_{\mathrm{P}}(\mathrm{W})$ \\
\hline $1.990 \mathrm{E}+04$ & $2.622 \mathrm{E}+00$ & $2.08 \mathrm{E}-02$ & $4.401 \mathrm{E}+02$ & $7.07 \mathrm{E}+00$ & $9.921 \mathrm{E}+01$ & $8.788 \mathrm{E}+01$ & $2.33 \mathrm{E}+00$ \\
\hline $1.998 \mathrm{E}+04$ & $4.468 \mathrm{E}+00$ & $2.22 \mathrm{E}-02$ & $4.291 \mathrm{E}+02$ & $7.05 \mathrm{E}+00$ & $9.968 \mathrm{E}+01$ & $8.684 \mathrm{E}+01$ & $2.46 \mathrm{E}+00$ \\
\hline $1.999 \mathrm{E}+04$ & $6.593 \mathrm{E}+00$ & $2.35 \mathrm{E}-02$ & $4.086 \mathrm{E}+02$ & $7.01 \mathrm{E}+00$ & $9.983 \mathrm{E}+01$ & $8.617 \mathrm{E}+01$ & $2.47 \mathrm{E}+00$ \\
\hline $2.004 \mathrm{E}+04$ & $8.816 \mathrm{E}+00$ & $2.68 \mathrm{E}-02$ & $3.836 \mathrm{E}+02$ & $7.02 \mathrm{E}+00$ & $1.002 \mathrm{E}+02$ & $8.412 \mathrm{E}+01$ & $2.55 \mathrm{E}+00$ \\
\hline $2.000 \mathrm{E}+04$ & $1.105 \mathrm{E}+01$ & $3.03 \mathrm{E}-02$ & $3.490 \mathrm{E}+02$ & $7.01 \mathrm{E}+00$ & $1.003 \mathrm{E}+02$ & $8.093 \mathrm{E}+01$ & $2.49 \mathrm{E}+00$ \\
\hline $1.997 \mathrm{E}+04$ & $1.336 \mathrm{E}+01$ & $3.25 \mathrm{E}-02$ & $3.061 \mathrm{E}+02$ & $7.02 \mathrm{E}+00$ & $1.004 \mathrm{E}+02$ & $7.484 \mathrm{E}+01$ & $2.42 \mathrm{E}+00$ \\
\hline $1.997 \mathrm{E}+04$ & $1.569 \mathrm{E}+01$ & $3.40 \mathrm{E}-02$ & $2.606 \mathrm{E}+02$ & $7.04 \mathrm{E}+00$ & $1.007 \mathrm{E}+02$ & $6.862 \mathrm{E}+01$ & $2.45 \mathrm{E}+00$ \\
\hline $1.994 \mathrm{E}+04$ & $1.802 \mathrm{E}+01$ & $3.62 \mathrm{E}-02$ & $2.110 \mathrm{E}+02$ & $7.09 \mathrm{E}+00$ & $1.010 \mathrm{E}+02$ & $5.938 \mathrm{E}+01$ & $2.44 \mathrm{E}+00$ \\
\hline $2.004 \mathrm{E}+04$ & $2.031 \mathrm{E}+01$ & $3.96 \mathrm{E}-02$ & $1.711 \mathrm{E}+02$ & $6.98 \mathrm{E}+00$ & $1.019 \mathrm{E}+02$ & $5.180 \mathrm{E}+01$ & $2.49 \mathrm{E}+00$ \\
\hline $1.986 \mathrm{E}+04$ & $2.262 \mathrm{E}+01$ & $4.34 \mathrm{E}-02$ & $1.248 \mathrm{E}+02$ & $7.09 \mathrm{E}+00$ & $1.015 \mathrm{E}+02$ & $4.317 \mathrm{E}+01$ & $2.20 \mathrm{E}+00$ \\
\hline $2.001 \mathrm{E}+04$ & $2.493 \mathrm{E}+01$ & 4.72E-02 & $8.168 \mathrm{E}+01$ & $7.06 \mathrm{E}+00$ & $1.028 \mathrm{E}+02$ & $3.471 \mathrm{E}+01$ & $2.25 \mathrm{E}+00$ \\
\hline$P_{\mathrm{e}}(\mathrm{W})$ & $\Delta P_{\mathrm{e}}(\mathrm{W})$ & $C_{\mathrm{T}}$ & $\Delta C_{\mathrm{T}}$ & $C_{\mathrm{P}}$ & $\Delta C_{\mathrm{P}}$ & $C_{\mathrm{Q}}$ & $\Delta C_{\mathrm{Q}}$ \\
\hline $1.232 \mathrm{E}+02$ & $1.31 \mathrm{E}+00$ & $1.306 \mathrm{E}-01$ & $2.10 \mathrm{E}-03$ & $6.318 \mathrm{E}-02$ & $1.67 \mathrm{E}-03$ & $1.006 \mathrm{E}-02$ & $2.66 \mathrm{E}-04$ \\
\hline $1.231 \mathrm{E}+02$ & $1.28 \mathrm{E}+00$ & $1.264 \mathrm{E}-01$ & $2.08 \mathrm{E}-03$ & $6.173 \mathrm{E}-02$ & $1.75 \mathrm{E}-03$ & $9.825 \mathrm{E}-03$ & $2.79 \mathrm{E}-04$ \\
\hline $1.203 \mathrm{E}+02$ & $1.26 \mathrm{E}+00$ & $1.204 \mathrm{E}-01$ & 2.07E-03 & $6.125 \mathrm{E}-02$ & $1.76 \mathrm{E}-03$ & $9.748 \mathrm{E}-03$ & $2.80 \mathrm{E}-04$ \\
\hline $1.171 \mathrm{E}+02$ & $1.28 \mathrm{E}+00$ & $1.125 \mathrm{E}-01$ & $2.06 \mathrm{E}-03$ & $5.938 \mathrm{E}-02$ & $1.80 \mathrm{E}-03$ & $9.451 \mathrm{E}-03$ & $2.86 \mathrm{E}-04$ \\
\hline $1.105 \mathrm{E}+02$ & $1.16 \mathrm{E}+00$ & $1.028 \mathrm{E}-01$ & $2.07 \mathrm{E}-03$ & $5.747 \mathrm{E}-02$ & $1.77 \mathrm{E}-03$ & $9.146 \mathrm{E}-03$ & $2.82 \mathrm{E}-04$ \\
\hline $1.016 \mathrm{E}+02$ & $1.07 \mathrm{E}+00$ & $9.045 \mathrm{E}-02$ & $2.07 \mathrm{E}-03$ & 5.339E-02 & $1.73 \mathrm{E}-03$ & 8.497E-03 & $2.75 \mathrm{E}-04$ \\
\hline $9.086 \mathrm{E}+01$ & 9.94E-01 & $7.700 \mathrm{E}-02$ & $2.08 \mathrm{E}-03$ & 4.895E-02 & $1.75 \mathrm{E}-03$ & 7.791E-03 & $2.78 \mathrm{E}-04$ \\
\hline $7.837 \mathrm{E}+01$ & $8.44 \mathrm{E}-01$ & $6.258 \mathrm{E}-02$ & $2.10 \mathrm{E}-03$ & $4.258 \mathrm{E}-02$ & $1.75 \mathrm{E}-03$ & $6.777 \mathrm{E}-03$ & $2.78 \mathrm{E}-04$ \\
\hline $6.955 \mathrm{E}+01$ & $7.58 \mathrm{E}-01$ & $5.018 \mathrm{E}-02$ & $2.05 \mathrm{E}-03$ & $3.653 \mathrm{E}-02$ & $1.76 \mathrm{E}-03$ & 5.814E-03 & $2.80 \mathrm{E}-04$ \\
\hline $5.700 \mathrm{E}+01$ & $6.65 \mathrm{E}-01$ & $3.723 \mathrm{E}-02$ & 2.12E-03 & $3.126 \mathrm{E}-02$ & $1.60 \mathrm{E}-03$ & $4.976 \mathrm{E}-03$ & $2.54 \mathrm{E}-04$ \\
\hline $4.662 \mathrm{E}+01$ & $5.32 \mathrm{E}-01$ & $2.402 \mathrm{E}-02$ & $2.08 \mathrm{E}-03$ & $2.459 \mathrm{E}-02$ & $1.59 \mathrm{E}-03$ & $3.914 \mathrm{E}-03$ & $2.53 \mathrm{E}-04$ \\
\hline$\rho\left(\mathrm{kg} / \mathrm{m}^{\wedge} 3\right)$ & $\eta_{\mathrm{T}}$ & $\Delta \eta_{\mathrm{T}}$ & $J$ & $\Delta J$ & $\eta_{\mathrm{P}}$ & $\Delta \eta_{\mathrm{P}}$ & $R e_{0.75}$ \\
\hline $1.158 \mathrm{E}+00$ & $7.133 \mathrm{E}-01$ & $2.03 \mathrm{E}-02$ & $6.230 \mathrm{E}-02$ & $4.96 \mathrm{E}-04$ & $1.288 \mathrm{E}-01$ & $4.12 \mathrm{E}-03$ & $7.917 \mathrm{E}+04$ \\
\hline $1.157 \mathrm{E}+00$ & 7.057E-01 & $2.13 \mathrm{E}-02$ & $1.057 \mathrm{E}-01$ & $5.28 \mathrm{E}-04$ & $2.165 \mathrm{E}-01$ & 7.19E-03 & $7.941 \mathrm{E}+04$ \\
\hline $1.155 \mathrm{E}+00$ & $7.160 \mathrm{E}-01$ & $2.19 \mathrm{E}-02$ & $1.559 \mathrm{E}-01$ & $5.59 \mathrm{E}-04$ & $3.066 \mathrm{E}-01$ & $1.03 \mathrm{E}-02$ & $7.941 \mathrm{E}+04$ \\
\hline $1.155 \mathrm{E}+00$ & 7.183E-01 & $2.31 \mathrm{E}-02$ & $2.080 \mathrm{E}-01$ & $6.39 \mathrm{E}-04$ & 3.942E-01 & $1.40 \mathrm{E}-02$ & $7.968 \mathrm{E}+04$ \\
\hline $1.155 \mathrm{E}+00$ & 7.325E-01 & $2.38 \mathrm{E}-02$ & 2.613E-01 & $7.25 \mathrm{E}-04$ & 4.674E-01 & $1.72 \mathrm{E}-02$ & $7.968 \mathrm{E}+04$ \\
\hline $1.155 \mathrm{E}+00$ & 7.369E-01 & $2.51 \mathrm{E}-02$ & 3.163E-01 & $7.82 \mathrm{E}-04$ & 5.359E-01 & $2.13 \mathrm{E}-02$ & $7.982 \mathrm{E}+04$ \\
\hline $1.155 \mathrm{E}+00$ & $7.552 \mathrm{E}-01$ & $2.82 \mathrm{E}-02$ & $3.715 \mathrm{E}-01$ & $8.22 \mathrm{E}-04$ & $5.843 \mathrm{E}-01$ & $2.62 \mathrm{E}-02$ & $8.002 \mathrm{E}+04$ \\
\hline $1.154 \mathrm{E}+00$ & $7.577 \mathrm{E}-01$ & $3.21 \mathrm{E}-02$ & 4.273E-01 & $8.77 \mathrm{E}-04$ & $6.280 \mathrm{E}-01$ & $3.34 \mathrm{E}-02$ & $8.013 \mathrm{E}+04$ \\
\hline $1.155 \mathrm{E}+00$ & $7.448 \mathrm{E}-01$ & 3.67E-02 & 4.791E-01 & $9.56 \mathrm{E}-04$ & $6.580 \mathrm{E}-01$ & 4.15E-02 & $8.106 \mathrm{E}+04$ \\
\hline $1.156 \mathrm{E}+00$ & $7.572 \mathrm{E}-01$ & $3.96 \mathrm{E}-02$ & $5.385 \mathrm{E}-01$ & $1.07 \mathrm{E}-03$ & $6.412 \mathrm{E}-01$ & $4.90 \mathrm{E}-02$ & $8.080 \mathrm{E}+04$ \\
\hline $1.156 \mathrm{E}+00$ & $7.445 \mathrm{E}-01$ & $4.89 \mathrm{E}-02$ & $5.891 \mathrm{E}-01$ & $1.14 \mathrm{E}-03$ & 5.753E-01 & $6.22 \mathrm{E}-02$ & $8.181 \mathrm{E}+04$ \\
\hline
\end{tabular}




\section{REFERENCES}

Brandt, J.B. and Selig, M.S. "Propeller Performance Data at Low Reynolds Number." 49th AIAA Aerospace Sciences Meeting. 2011. AIAA 2011-1255.

Corrigan, E.K. and Altman, A. "Survey of Small Unmanned Aerial Vehicle Electric Propulsion Systems." 46th AIAA Aerospace Sciences Meeting. 2008. AIAA 2008179.

Deters, R.W. and Selig, M.S. "Static Testing of Micro Propellers." 26th AIAA Applied Aerodynamics Conference. 2008. AIAA 2008-6246.

Gamble, D.E. "Automated Dynamic Propeller Testing at Low Reynolds Numbers." M.S. Thesis. Stillwater, OK: Oklahoma State University, 2009.

Glauert, H.. The Elements of Aerofoil and Airscrew Theory. Cambridge: Cambridge University Press, 1926.

Hackett, J.E., Lilley, D.E., and Wilsden, D.J. Estimation of Tunnel Blockage from Wall Pressure Signatures: A Review and Data Correlation . NASA CR15-2241, 1979.

Hepperle, M. PropellerScanner Software. 2003. http://www.mh-aerotools.de/.

Kline, S. J., and F. A. McClintock. "Describing Uncertainties in Single-Sample Experiments." Mech. Eng., 1953: 3.

Merchant, M.P. and Miller, L.S. "Propeller Performance Measurement for Low Reynolds Number UAV Applications." 44th AIAA Aerospace Sciences Meeting. 2006. AIAA 2006-1127.

NACA. Equations, Tables, and Charts for Compressible Flow, NACA Report 1135. Washington, D.C.: U.S. Government Printing Office, 1953. 
Ol, M., Zeune, C., and Logan, M. "Analytical - Experimental Comparison for Small Electric Unmanned Air Vehicle Propellers." 26th AIAA Applied Aerodynamics Conference. 2008. AIAA 2008-7345.

Selig, M. UIUC Propeller Database. 2012. http://www.ae.illinois.edu/mselig/props/propDB.html.

Selig, M., and Ananda, G. Low Reynolds Number Propeller Performance Data: Wind Tunnel Corrections for Motor Fixture Drag. 2011. http://www.ae.illinois.edu/mselig/props/uiuc-props-wind-tunnel-correction.pdf. 